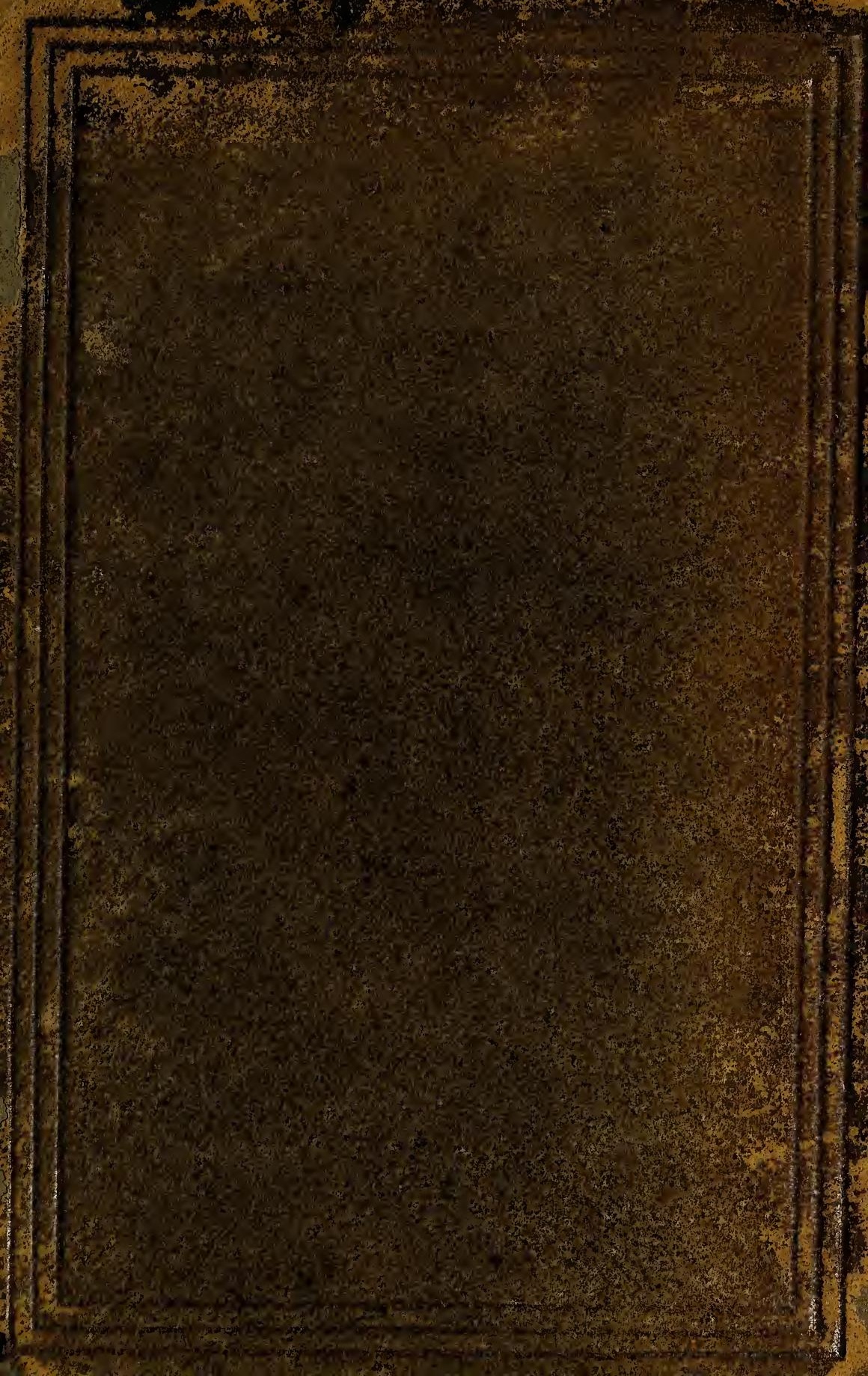



Lang. 



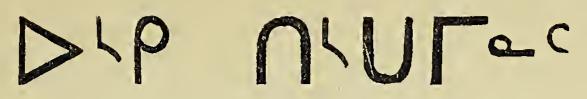

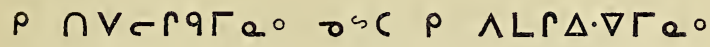

$$
\text { rhe b乌̧ } 4 c_{x}
$$

\section{THE NEW/TESTAMENT, TRANSLATED/INTO THE CREE/LANGUAGE}

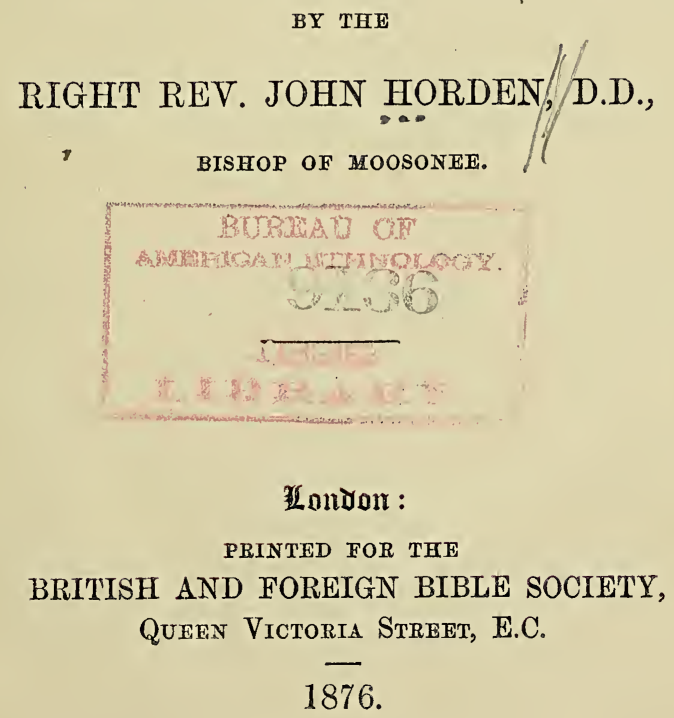


pM989 . 348 1826

LONDON
GILBERT AND RITINGTON, PRINTERS,
ST. JOHN'S SRUARE, AND 28, WHTTEPRIARS STREET. 


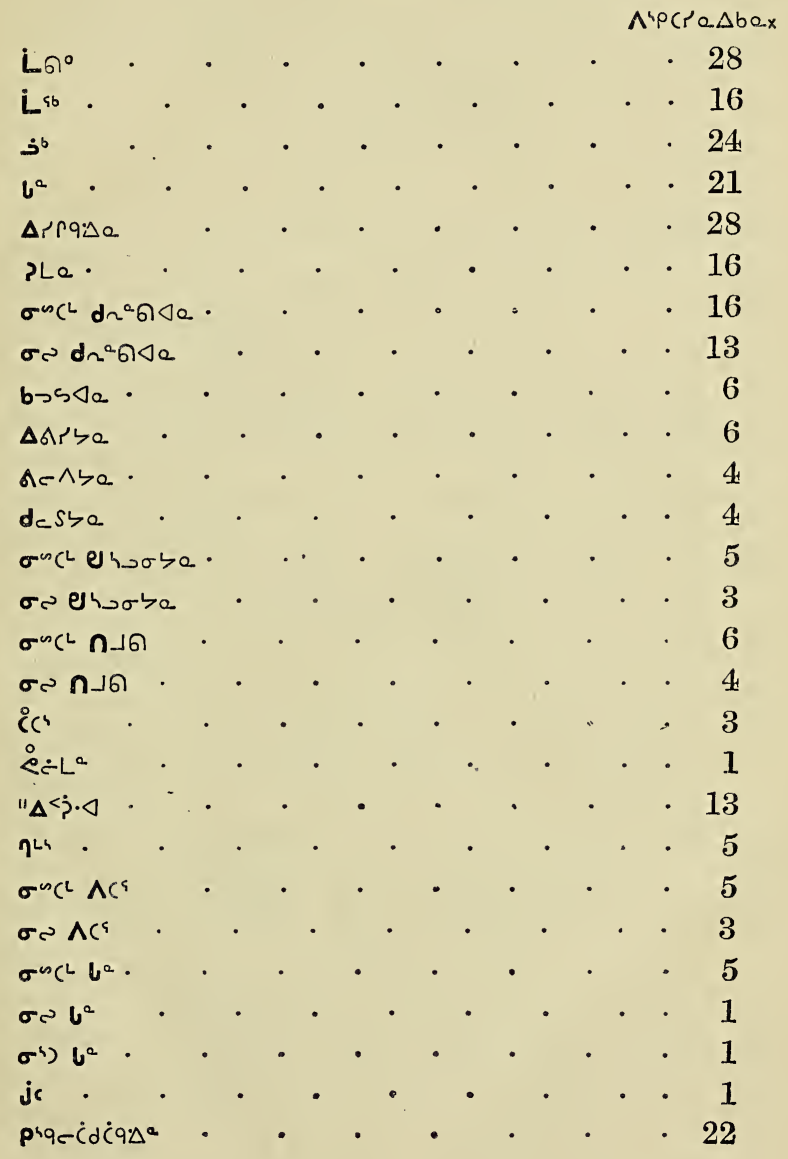





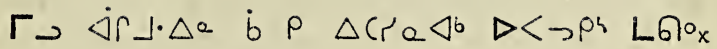

\section{A'pcre $\triangle b^{2} 1$.}

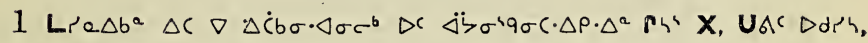
$\nabla<v<i \quad d$ drhx

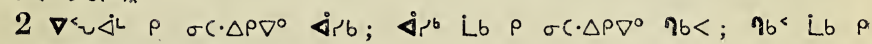
$\sigma C \cdot \Delta P \nabla^{\circ} j(h$ o"c $\Delta r \dot{s} \sigma c \cdot \Delta$;

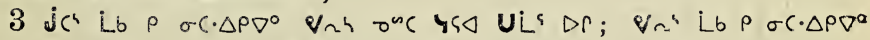
$\nabla \backslash \zeta L ; \nabla \backslash \zeta L$ Lb $P \sigma C \cdot \triangle P \nabla^{\circ} \nabla \zeta L$;

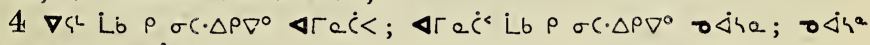
Lb $\rho$ oC. $\triangle P \nabla^{\circ}$ hं L.Le;

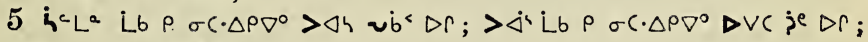

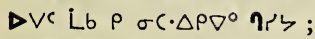

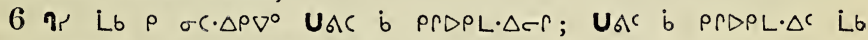
$\rho \sigma c \cdot \Delta P \nabla^{\circ}$ he Le $\Delta \sigma \Delta D \Gamma \dot{b} \rho \Delta \Delta C r$ jish;

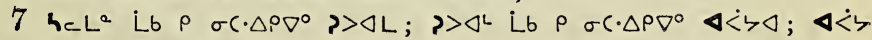
L.b $\rho$ oc. $\Delta P \nabla^{\circ} \nabla h \triangleleft$;

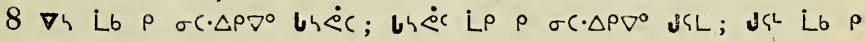
$\sigma C \cdot \Delta P \nabla^{\circ} \Delta i \zeta h$;

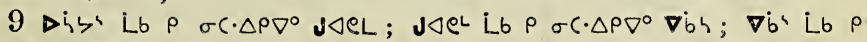

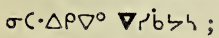

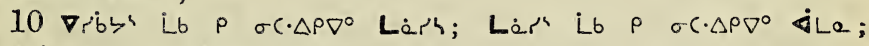
SLa Lb $P$ oC. $\triangle P \nabla^{0}$ Jibh;

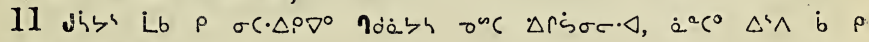
$\Delta x<\Delta b \sigma^{\circ} \Delta r^{b}<\wedge c \sigma^{b}$;

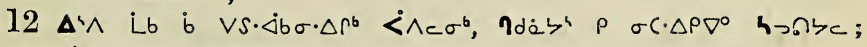

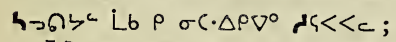

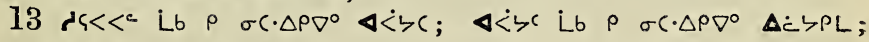
$\Delta \dot{C} \zeta P L$ L L $P$ OC. $\Delta P \nabla^{\circ} \nabla h 5$;

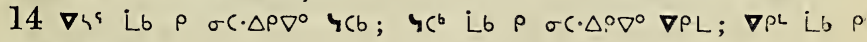
$\sigma C \cdot \Delta P \nabla^{\circ} \Delta \dot{C} \backslash C ;$

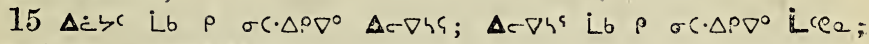
LCea Lb $\rho$ oc. $\triangle P \nabla^{c} \eta p<$; 


\section{$D<\neg P^{4}$ L̊० $1,2$.}

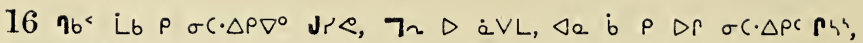

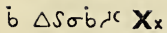

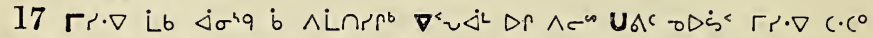

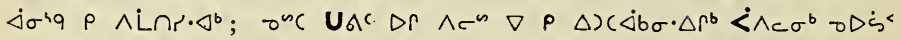

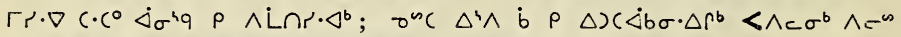

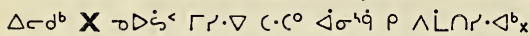

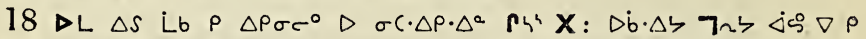

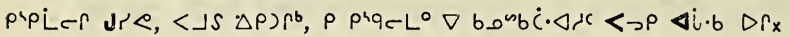

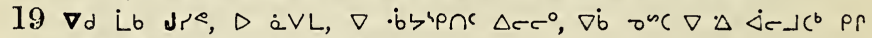

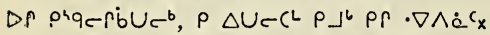

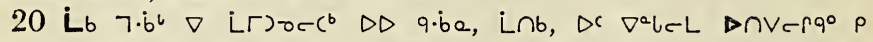

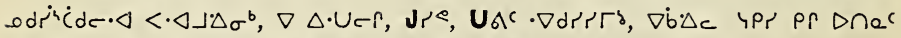

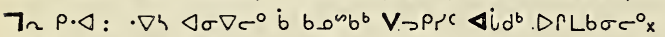

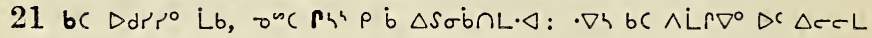
$\Delta L \Gamma \cdot C \cdot \Delta \sigma c^{b} D R_{x}$

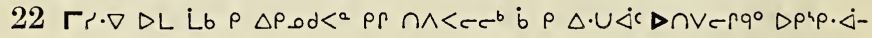
$\triangle q \cdot \triangleleft, \nabla \Delta \cdot U \hookrightarrow$,

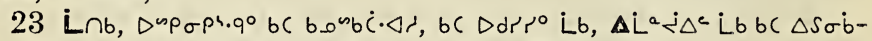
$\left.\cap 7 \cdot \triangleleft^{b}, \dot{b} \Delta \cdot U L b^{b}, \nabla \Delta \cdot U^{\prime} r \dot{b} U^{b}, p r L \sigma\right) \rho \Delta r\left(S 9 \Gamma d a^{\circ} \times\right.$

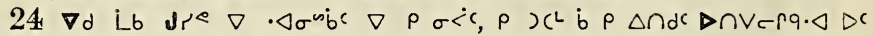
$\nabla \cdot L e \Gamma e \cdot \triangleleft, \rho \triangleright \cap 0^{\circ}$ Lb $\Delta \cdot \triangleleft$ :

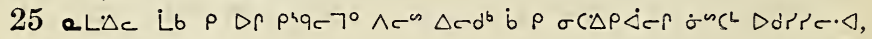
P'L L $P \triangle S \sigma \dot{Q} \cap \cap 7^{\circ} x$

\section{$\Lambda^{\prime} p\left(r e \triangle b^{2} 2\right.$.}

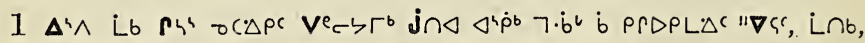

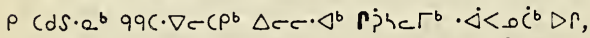

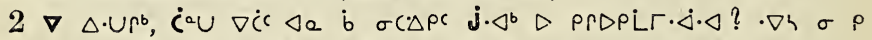

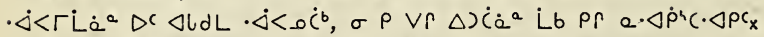

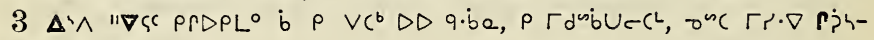

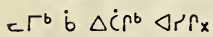

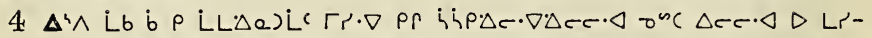

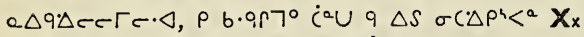

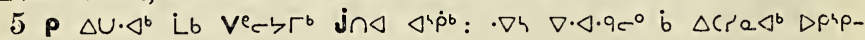
$\cdot \dot{\Delta} \Delta q^{\circ}$,

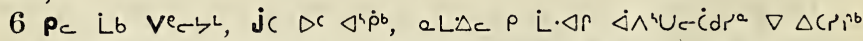

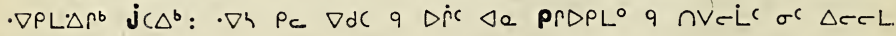
$\Delta^{4} \sim \Delta c x$

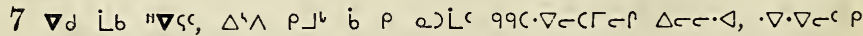

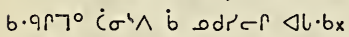

8 p $\Delta S \cap s \cdot \nabla^{0}$ Lb $V^{e} c \zeta \Gamma^{b}, p \Delta U^{0}$ Lb, $\left.\left.\rho\right) U^{b}, \cdot \nabla \cdot \nabla c^{c} L_{b} b \dot{a}_{a}\right) a^{b} \Delta a$

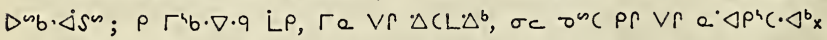

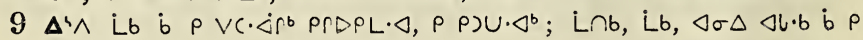
2 


\section{$B<\rho^{h} \mathrm{R}^{\circ} 2,3$.}

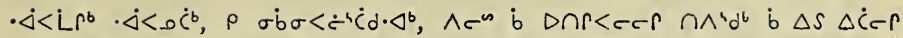
$\varangle \sigma \Delta \nabla^{\circ b} \cdot \dot{s s s}$

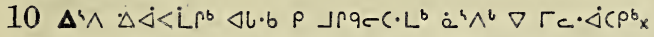

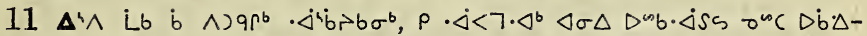

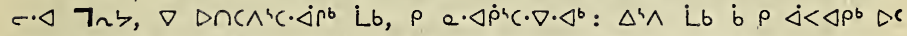

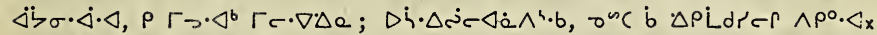

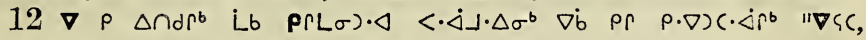
$\wedge$ J $\Delta S \rho \rho \cdot \nabla \cdot \Delta^{b} \cap \wedge c \cdot \nabla \Delta c \Delta^{b} \rho \cdot \dot{j}^{b} x$

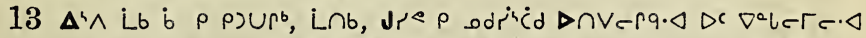

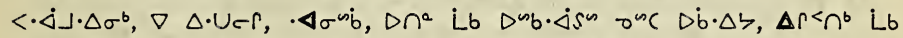

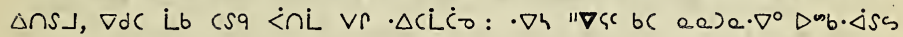
Pr $\sigma S \cdot \Delta \dot{\alpha} r \dot{\Delta} c_{x}$

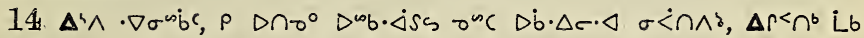
$\rho \Delta U^{\circ}$ :

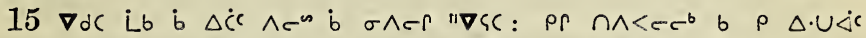

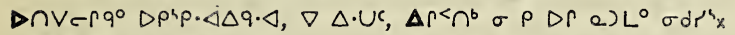

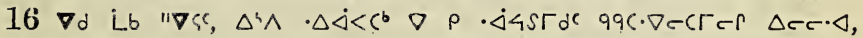

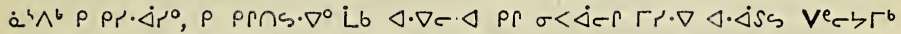

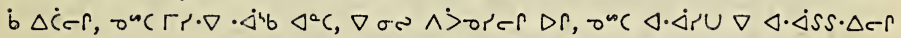

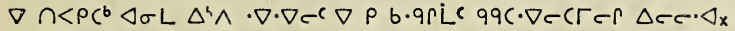

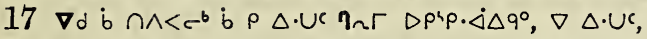

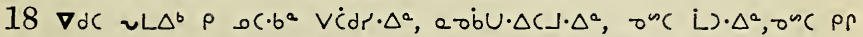
$\dot{L} \cdot \Delta \dot{b} s \cdot \nabla \cdot \Delta a, u r^{a} \nabla L \cdot \Delta b \dot{c} c \Delta c \Delta \cdot \Delta s r s, a L \cdot \Delta c$ Lb $\left.\rho \cdot \Delta \Gamma \cdot s c\right\lrcorner \Delta d r^{\circ}, \nabla \dot{b}$ $\nabla \Delta \dot{C} c \Gamma_{x}$

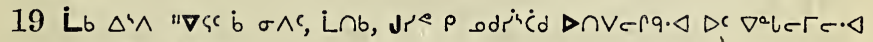
$<\cdot \dot{\lrcorner}\lrcorner \Delta \sigma^{b} \Delta^{a} C \Delta r^{<} n^{b}$,

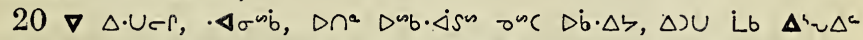

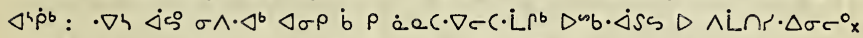

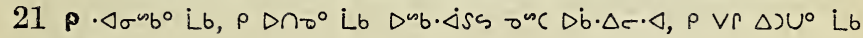
$\Delta^{4} u \Delta^{c} \Delta^{s} \dot{p}^{b} x$

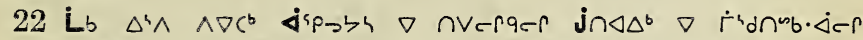

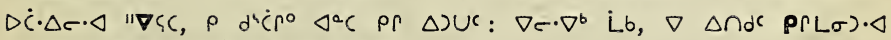
$<\cdot \dot{\Delta}\lrcorner \cdot \Delta \sigma^{b} \rho<<^{4} q^{\circ} b-c^{b}$ pr $\Delta u^{c}$ :

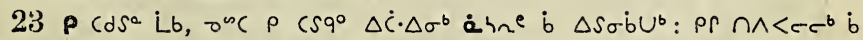
$p \Delta \cdot U p^{b} \Delta p^{4} p \cdot \dot{j} \Delta q \cdot \Delta^{b}, \dot{a}^{b} \dot{n}^{a} b C \Delta S \sigma \dot{b} \dot{c} b \sigma \cdot \Delta^{\circ} x$

\section{$\Lambda^{4} p C r e \triangle b^{2} 3$.}

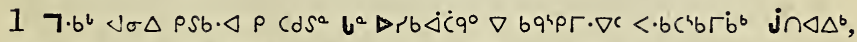

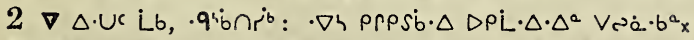

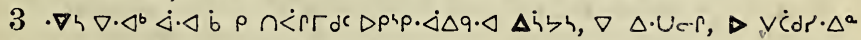

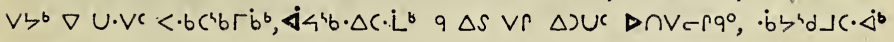
$\nabla 7^{2} b e \cdot \nabla_{x}$ 


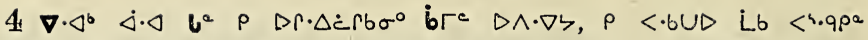

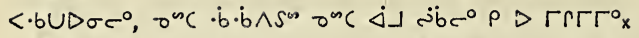

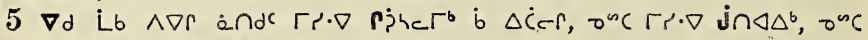

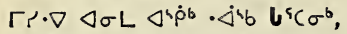

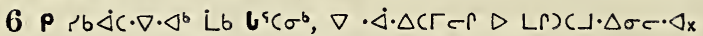

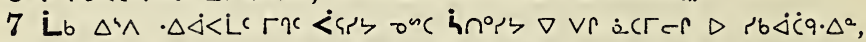

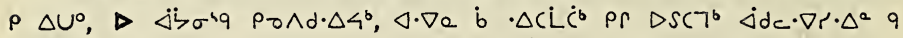
$\Delta \cap r<c^{b}$ ?

8 คd $\dot{C}^{b} \Delta h \nabla \Delta S \Gamma \sigma s \cdot \Delta^{b} \cdot q^{b} b \cap \Omega \cdot \Delta^{a}:$

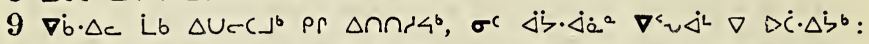

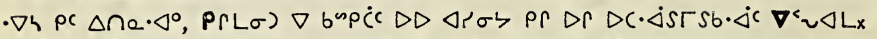

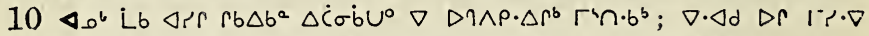

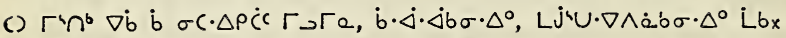

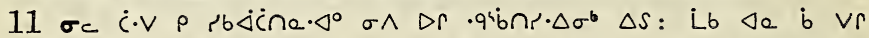

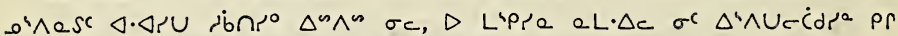

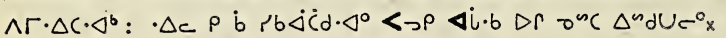

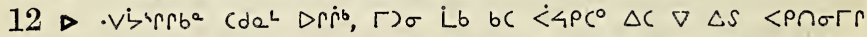

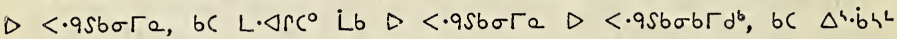
Lb $\mathrm{Lb} \cdot \nabla<c r b \sigma c^{\circ} \Delta^{a} d U^{b} \nabla \dot{b} q \dot{\Delta}^{c} c \cdot \nabla c^{b} x$

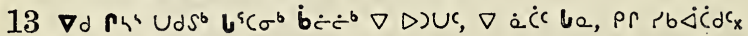

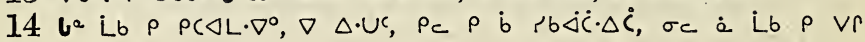
isa?

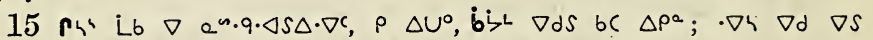

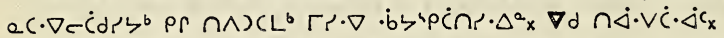

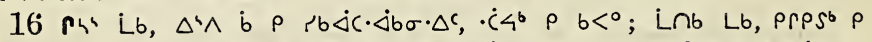

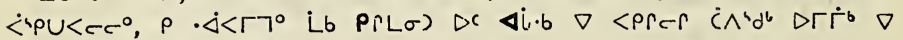
$\triangle S \dot{a} d e r$, on $^{n} \nabla \cdot U \Delta \dot{C} d^{c}:$

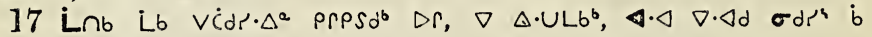
i $\rho \Delta^{b}, \dot{a}^{\prime} \wedge^{b}$ b $a \Delta c \cdot \nabla \Delta c x$

\section{APcre $\triangle b^{a} 4$}

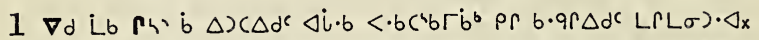

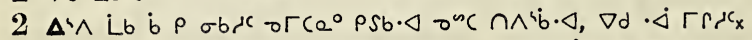

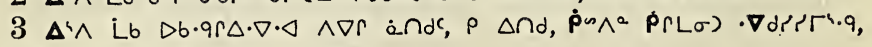
$\Delta c \omega \cdot \nabla \nabla d \Delta r \sigma^{b} b r \Delta c d e \cdot \Delta r^{b} x$

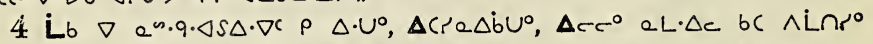

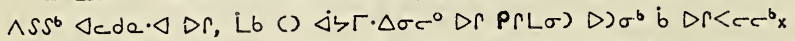

$5 \nabla d$ Lb LrL $\sigma) \nabla)\left(\dot{<} c<s \rho \Delta \dot{C} \Delta \sigma^{b}, \rho \cup()^{0}\right.$ Lb $\left(d^{b} \Delta c \nabla \Delta \Delta^{a}<c^{b}\right.$ $\rho \rho \dot{\rho} \zeta \Gamma \nabla \cdot \Delta b \Gamma d^{b}$,

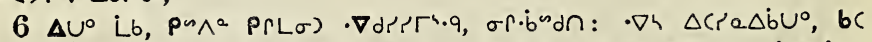

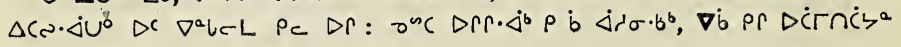
$\operatorname{Prc} \varangle r \sigma^{b} x$ 


\section{$\Delta<\rightarrow$ P L $\Omega^{\circ} 4,5$.}

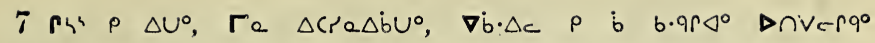
P PRLOTLx

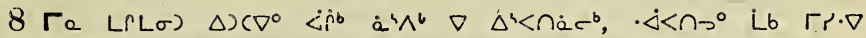

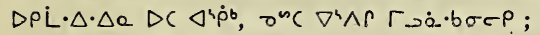

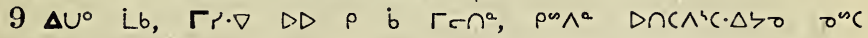

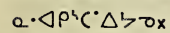

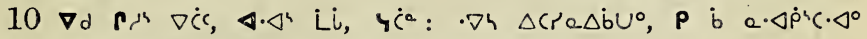

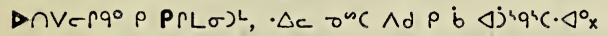

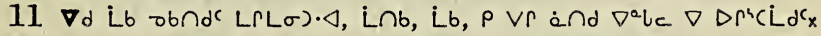

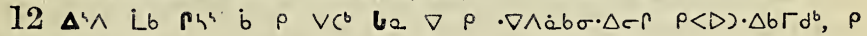
$\Delta u^{\circ} \dot{b} r \dot{c}^{\circ}$;

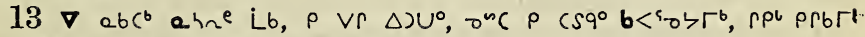

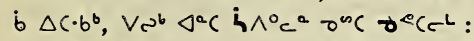

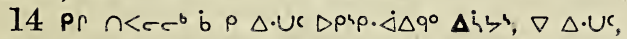

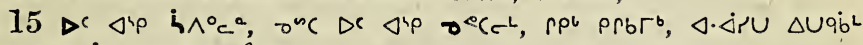

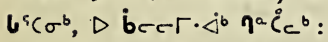

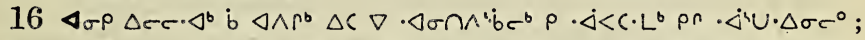

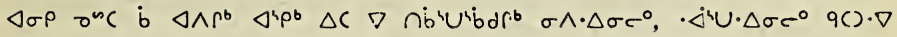
Dnnd. $\triangleleft b_{x}$

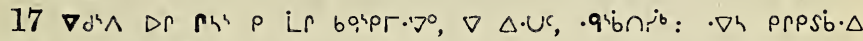
$D P L \cdot \Delta \cdot \Delta a$ VNáb $b^{a} x$

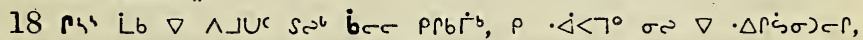

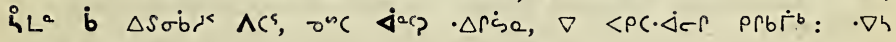
$\triangle 0) 74 \cdot \Delta \cdot \Delta^{b} x$

$19 P \Delta U^{0} L b, 0^{4} \wedge a j^{b} \nabla d q \Delta S \Delta C \cdot b^{b}$ pr $\wedge C D_{>} \cdot b^{b} \Delta r r \cdot \Delta^{b} x$

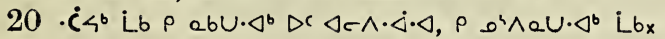

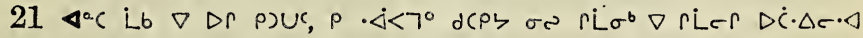

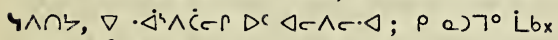

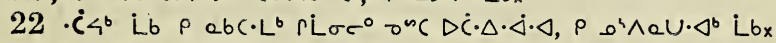

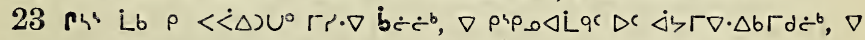

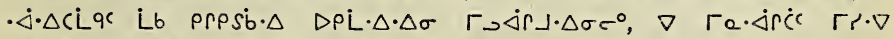

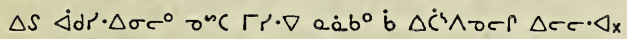

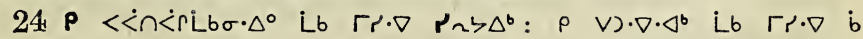

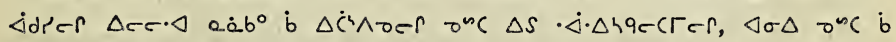

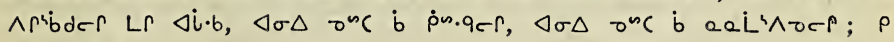
$\Gamma \mathrm{e} \cdot \Delta r \nabla^{\circ} \mathrm{L} b_{\mathrm{x}}$

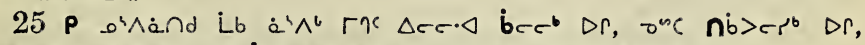

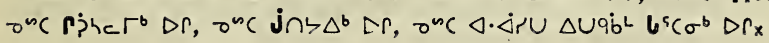

\section{$\Lambda^{4} p c r a \triangle b^{a} 5$.}

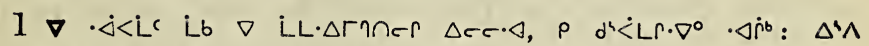
Lb $b$ b $a \triangleleft \wedge c, p \vee r$ and $D$ php. $\Delta \triangleleft L \cdot \Delta b a x$ 


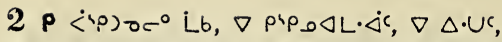

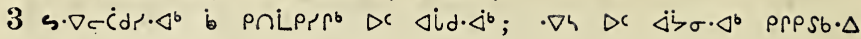
$D P L \cdot \Delta \cdot \Delta \Delta^{a} x$

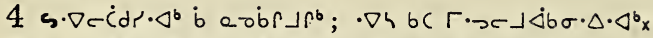

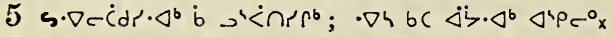

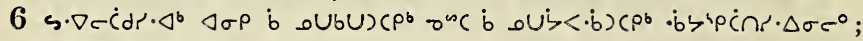
$\cdot \nabla h b c \dot{\rho} n>\Delta \cdot \Delta^{b} x$

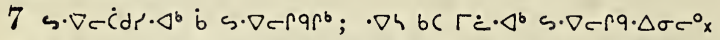

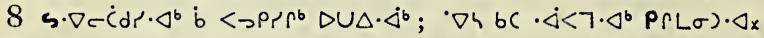

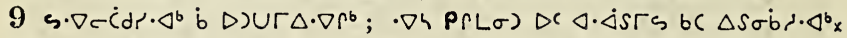

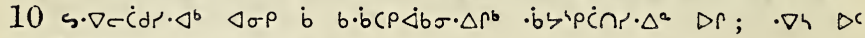

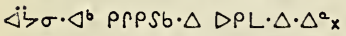

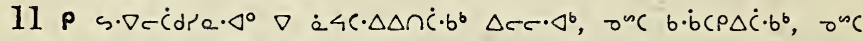

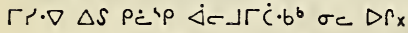

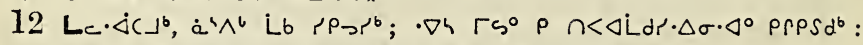

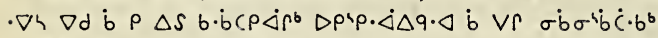

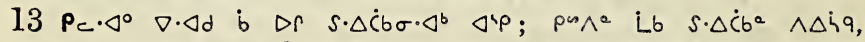

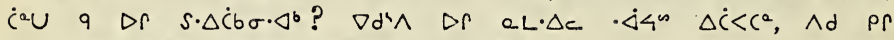

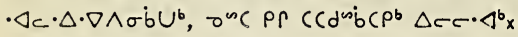

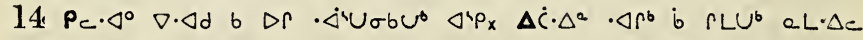
bc $\rho \dot{b} \dot{C} \sigma \cdot \triangleleft^{a} x$

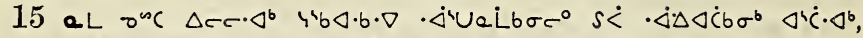

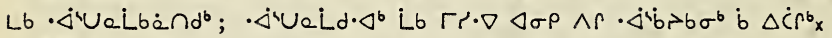

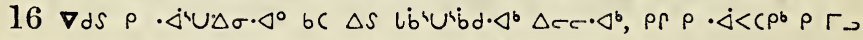

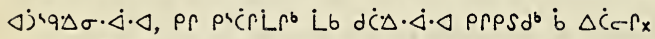

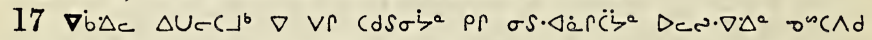

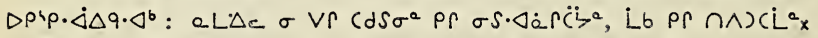

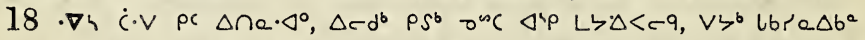

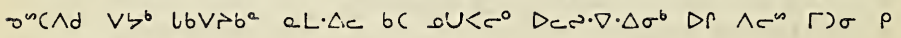
nAsiubx

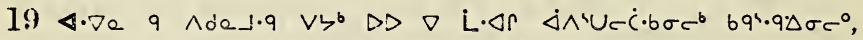

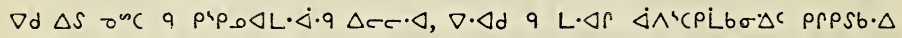

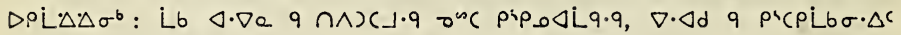
PrPSib $\triangle P$ L $\triangle \Delta \sigma^{b} x$

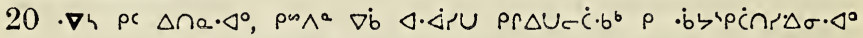

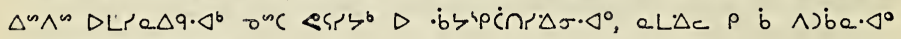

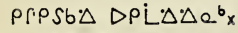

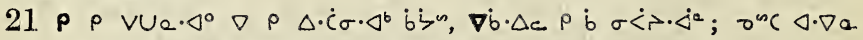

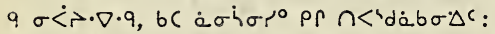

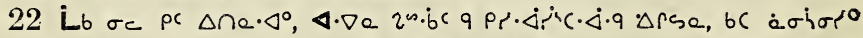

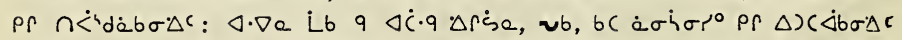

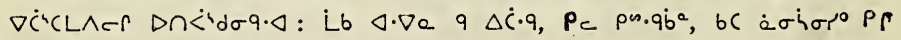
$\cdot \nabla \wedge \dot{a} b \sigma \cdot \Delta c L r \Delta \Delta^{\infty} d U^{b} x$ 


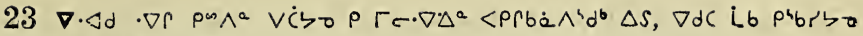

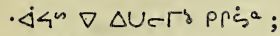

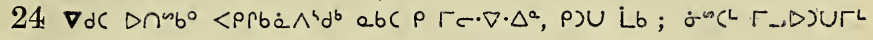
Prsंa, $\nabla d$ Lb $\vee r<P \cap \sigma q \rho \Gamma c \cdot \nabla \cdot \Delta^{a} x$

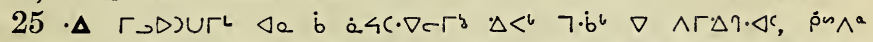

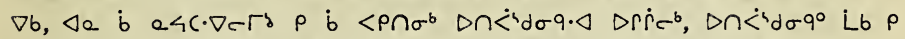

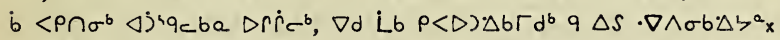

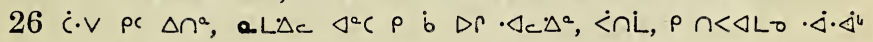

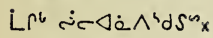

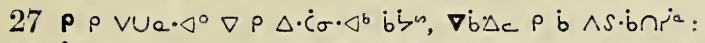

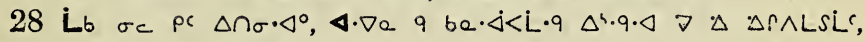
¿द $\rho \Delta r \wedge s \cdot b \cap r^{\circ} 7^{\circ} \Delta U \Delta^{b} x$

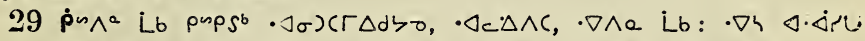

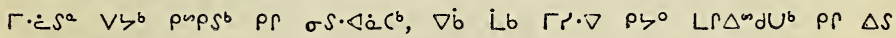
$\cdot \nabla \wedge \sigma \dot{b} U^{b} x$

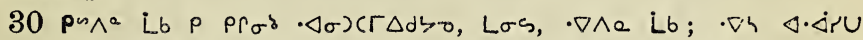

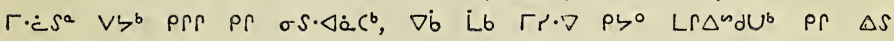
$-\nabla \wedge \sigma \dot{b} U^{b} x$

31 p $\Delta \cdot \dot{C} \sigma \cdot \nabla^{a}, \varangle \cdot \nabla e q \cdot \nabla \wedge \dot{a} \cdot q \Delta \cdot \Delta, \nabla d S$ bc $\Gamma$ >0 Lre $\Delta q \cdot \Delta \sigma \sigma^{\circ} \nabla$ $\Delta c^{b} \nabla \cdot \nabla \wedge \dot{a}^{c}:$

32 Lb $\sigma_{c} \rho c \Delta \cap a \cdot \triangleleft^{\circ}, \triangleleft \cdot \nabla e q \cdot \nabla \wedge \dot{a} \cdot q \cdot \Delta \cdot \triangleleft \wedge d \wedge s \cdot \dot{b} \cap \cdot \Delta \sigma c^{\circ} \nabla r$,

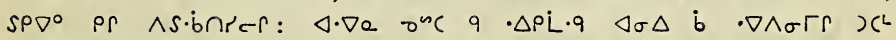
$\wedge S \cdot \dot{b} \cap r \cdot \Delta \sigma r^{\circ} x$

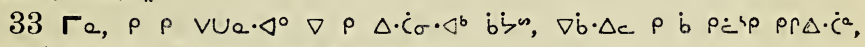
Lb $P$ b $\cap A)\left(L \cdot \Delta^{\circ} \Delta \cap V-r q^{\circ} p\right.$ pr $\Delta \cdot U \cdot \Delta e$;

34 Lb oc pe $\left.\Delta \cap a \cdot \Delta^{\circ}, \nabla \dot{b} \cdot \Delta_{c} \cdot \Delta^{4} b^{c} \rho \Gamma \Delta \cdot U^{b} ; \nabla \dot{b} \rho \Gamma P S^{b} \nabla \nabla^{b} \wedge\right\lrcorner_{4^{b}}$, $\cdot \nabla 4 \nabla \cdot \nabla \cdot 9 c^{\circ} P(L \sigma) \Delta\left(\Delta P L \cdot \Delta \wedge \cdot \Delta^{a}\right.$;

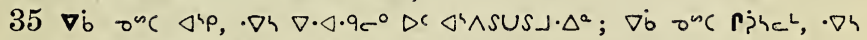
$\nabla \cdot \Delta \cdot 9 c^{\circ} q^{4} \cap \nabla^{\circ}$ PRDPLO $D C \Delta \dot{C} \cdot \Delta^{a}:$

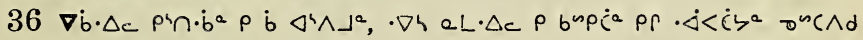

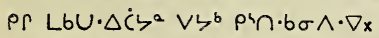

37 íb $\Delta L \Delta s \rho \dot{b} \Delta \cdot \dot{C} a \cdot \Delta^{\circ} \nabla$ jhr $\left.\Delta\right) 4^{b}, \nabla \nabla, \nabla \nabla ; a L \cdot \Delta c, a L \cdot \Delta c$ :

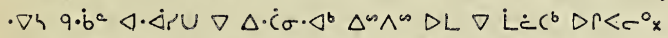

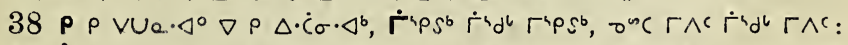

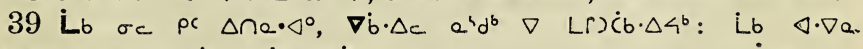

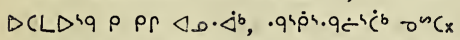

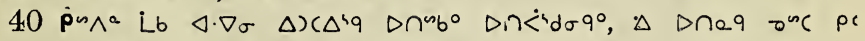

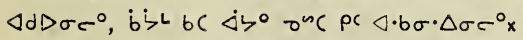

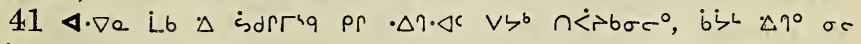
กंiेbex

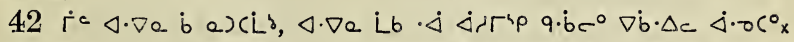

$43 \rho \dot{\rho} \vee U_{a} \cdot \Delta^{\circ} \nabla \rho \Delta \cdot \dot{C} \sigma \cdot \triangleleft^{b}, \rho$ b $i \rho \Delta^{\circ} \dot{\rho} r \Delta c^{\circ}, \rho \dot{b}<\cdot \dot{b} c^{\circ} \quad L_{k}$

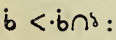




$$
D<\rightarrow \text { pᄂ L० } 5,6 \text {. }
$$

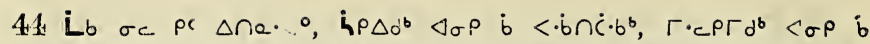

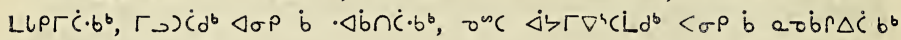
onc b. bC $\bar{C} \Delta \dot{C} \cdot b^{b}$;

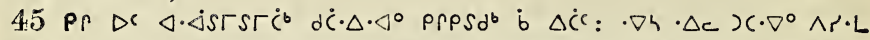

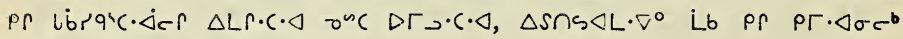

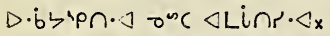

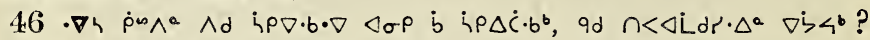
CL a $D L \Delta S) C \cdot L^{b}<<<e^{\circ} a^{b}$ ?

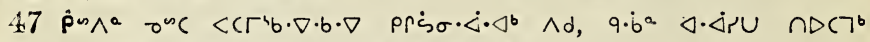

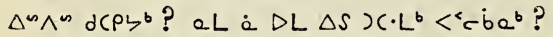

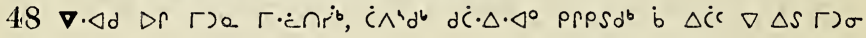
$r \cdot \dot{c} \cap \dot{c}_{x}$

\section{A $p c r a \triangle b^{a} 6$.}

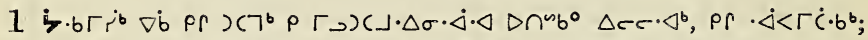

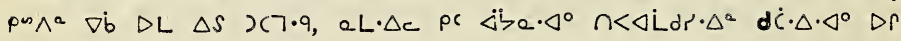
PCPSd b $\triangle C^{\circ} c_{x}$

$\left.2 \nabla \cdot \Delta d \quad D r \Delta^{\prime} \wedge \cap D C L \sigma \rho \Gamma \rightarrow\right)(\lrcorner \cdot \Delta a, \nabla \dot{b} \cdot \Delta c \wedge C$. $\dot{C} d \dot{C}>\dot{C} r b^{a} ; \dot{C} \wedge \cdot d^{b}$

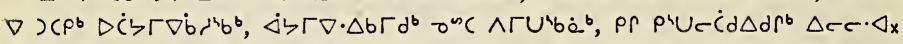

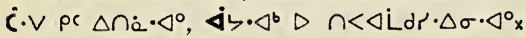

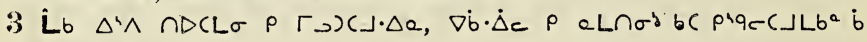

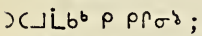

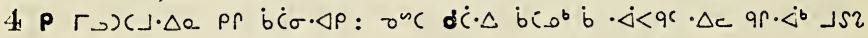
$\rho \dot{b} \cap<\Delta \dot{L}^{b} x$

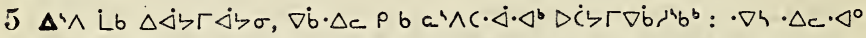

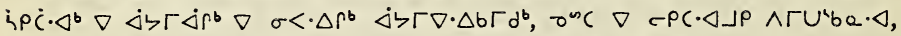

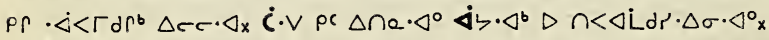

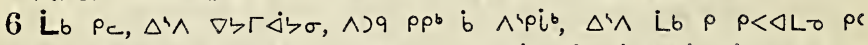

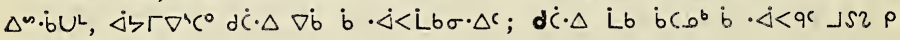
b $n<\Delta L^{b} x$

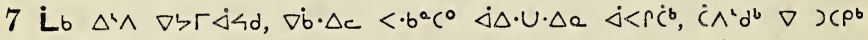

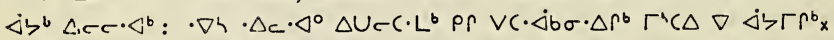

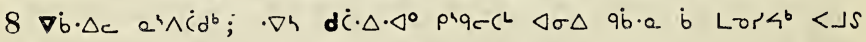
a) $\left(L \cdot \nabla^{b} \times\right.$ 。

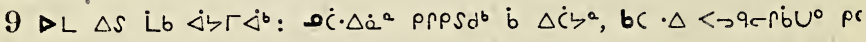
$\triangle S \sigma \dot{b} r \cdot \Delta \Delta^{a} \times$

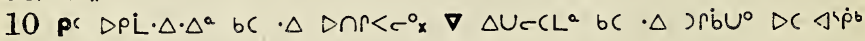

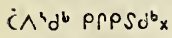

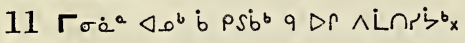

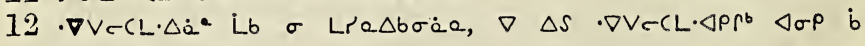
Lra $\triangle q)\left(\cdot \Delta \zeta \Gamma r^{b}\right.$ x

$13 \nabla \dot{b} \cdot \Delta c$ Lb $\Delta)\left(\Delta \dot{a}^{a} q \quad \Delta S \quad b \cdot 9 r \Delta b \cdot \Delta \dot{b} b ; \dot{L} b \Gamma \dot{C} \cdot q a \cdot L \cdot \Delta \dot{a}^{a}\right.$ Lr $q \cdot \dot{b} a:$ 8 


\section{D< $<>\rho^{\prime}$ Lด० 6 .}

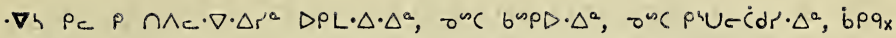
$\nabla 7^{2} x$

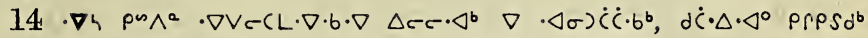
o"C $\rho$ bे $\cdot \nabla V C C$ L $d \cdot \triangleleft^{\circ}:$

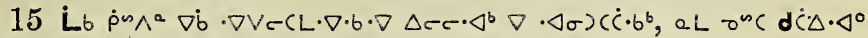
$\left.\rho \dot{b} \cdot \nabla \vee-C L \dot{d} \cdot \Delta^{0} \nabla \cdot \triangleleft \sigma\right)\left(\cdot \nabla^{b} x\right.$

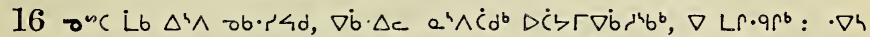

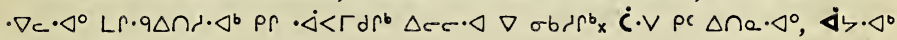
$\nabla n<\Delta L d r \cdot \Delta \sigma \cdot \Delta^{\circ} x$

17 Lb $\rho_{c}, \Delta^{4} \wedge$-b.rbo, J⿸厂

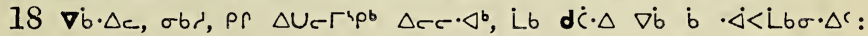
$d \dot{C} \cdot \Delta \dot{L} b \dot{b} C .^{b} \dot{b} \cdot\langle<q c,-\lrcorner S 2 \rho \dot{b} \cap<\Delta L^{b} x$

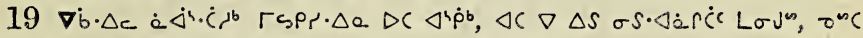

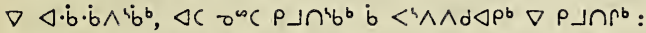

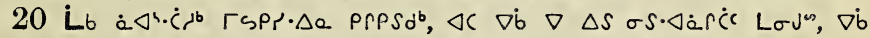

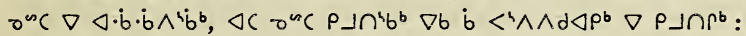

$21 \cdot \nabla h \Delta C \nabla \Delta S C \cdot b^{b} P \Gamma S P^{b} \cdot \Delta^{a}, \nabla d C \sigma^{n C} q \Delta C \cdot b^{b} p U \Delta x$

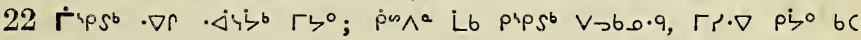

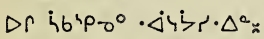

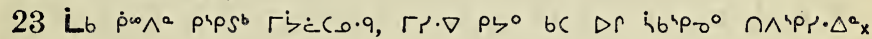

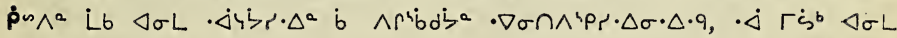

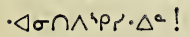

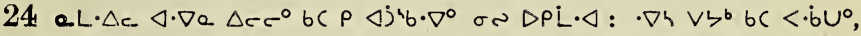

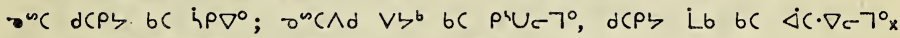

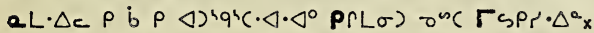

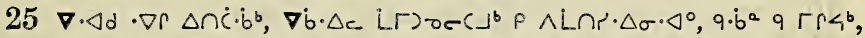

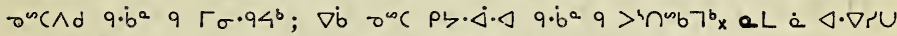

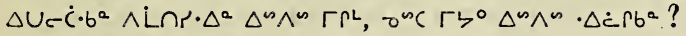

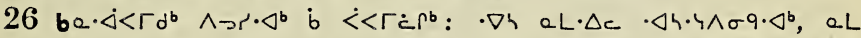

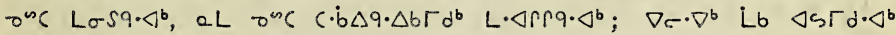

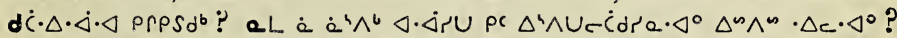

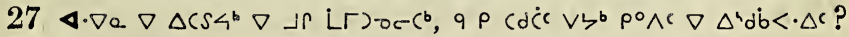

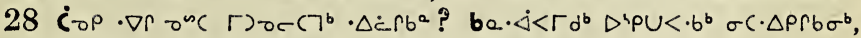

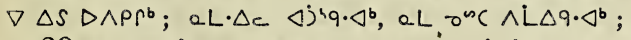

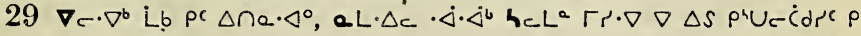
$\triangle S D \dot{C} \wedge^{4} d^{b} \vee L^{b} D d x$

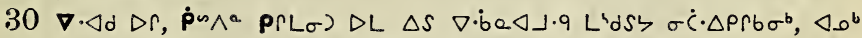

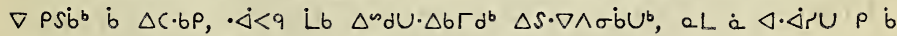

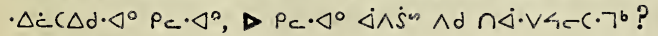

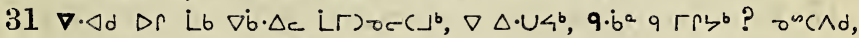

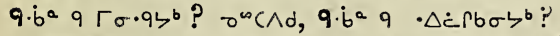

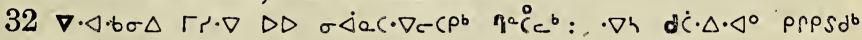

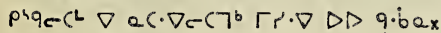




\section{D<>PS Lด० 6,7 .}

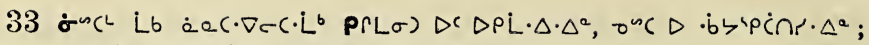

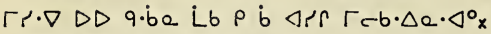

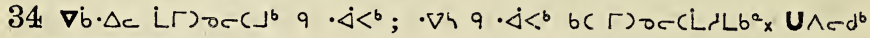
bc $\dot{\Delta C L^{a}} \nabla$ PSib $x$

\section{A'pc?a $\triangle b^{2} 7$.}

$1 \nabla \dot{\nabla} \cdot \Delta c \cap \dot{<} d \sigma^{b}, \nabla \dot{b} p_{c} \cdot \triangleleft^{\circ} p \rho \cap \dot{<}^{\prime} d \sigma b \cdot \Delta \zeta^{b} x$

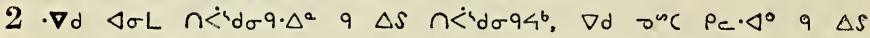

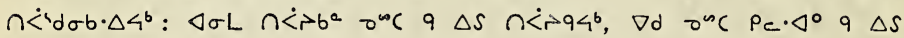
$n<\Delta L b \cdot \Delta \zeta^{b} x$

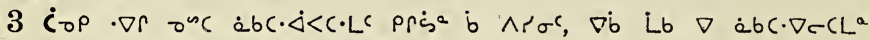
$\wedge \Gamma c \cdot \Delta<a \cap \wedge \Lambda_{c} \cdot \nabla$ piss $d^{b} \dot{b} \Delta c \cdot b^{b}$ ?

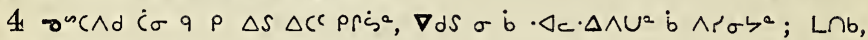

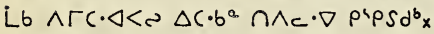

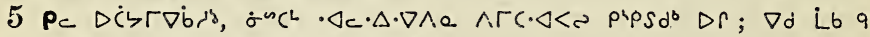

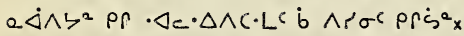

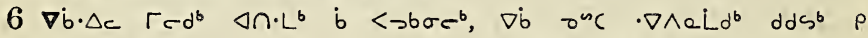
$\cdot \dot{\Delta} \cdot \nabla S D \sigma \cdot \dot{\Delta} \cdot \Delta, L^{\prime} d^{b} \cdot \Delta c b C c C d^{b} b C \cdot L^{b}, \sigma^{n} C \nabla \Delta<\dot{\Gamma}^{\circ} C \dot{C} \cdot b^{b} \operatorname{pr} D S d \Delta \dot{C} \cdot b^{b} x$

7 a) $\left(L q^{b}, \rho\right.$ b $\Gamma c b \cdot \Delta \dot{a} \cdot \Delta^{\circ} L b ; \dot{L} a(\cdot \nabla c-\lrcorner^{b}, \rho$ b $\Gamma^{\natural} q a \cdot \triangleleft^{\circ} \quad L b$

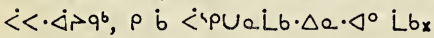

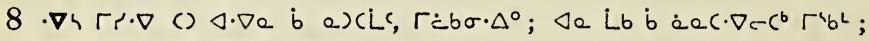

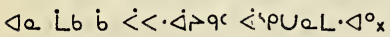

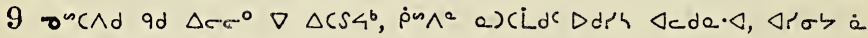
bC $\Gamma>^{\circ}$ ?

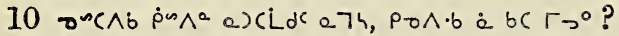

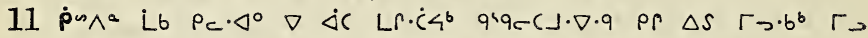

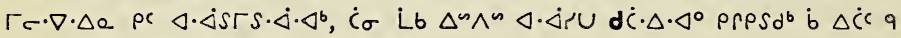

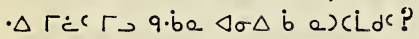

$12 \nabla \cdot \triangleleft d \quad$ or Lb $r s \cdot \nabla$ a.be $\nabla \Delta S$ ec $\nabla c 7 \cdot b^{b} \Delta r c \cdot \Delta^{b}$ pr $x \dot{C} \cdot b^{b}, \nabla d$

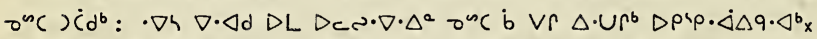

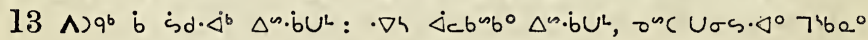
$\sigma s \cdot \Delta \dot{a} \cap r \cdot \Delta \sigma^{b}$ bे $\left.\Delta c\right\lrcorner^{b}, \Gamma \eta \cap \cdot \Delta^{b}$ Lb $\varangle \sigma \nabla \sigma^{\circ}$ b $\Gamma \cap 7 \Gamma^{b} x$

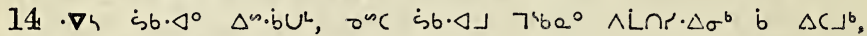

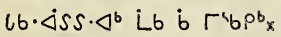

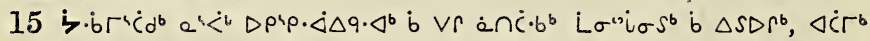

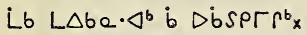

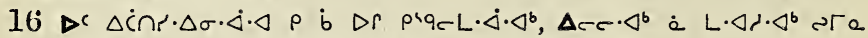

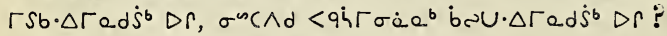

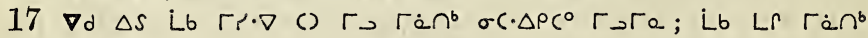
$\sigma C \cdot \triangle P C^{\circ}$ LRT $\mathrm{CX}$

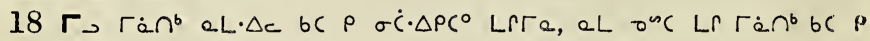
$\sigma C^{\circ} \triangle P C^{\circ} \Gamma \Delta \Gamma Q_{x}$ 


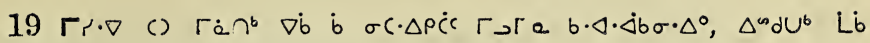
$\cdot \nabla \wedge \dot{a} b \sigma \cdot \Delta^{\circ} \mathrm{x}$

$20 \triangleleft \sigma \Delta \Delta S \cdot \dot{C} \cdot \Delta a \dot{b} \sigma c \cdot \Delta p \dot{C} r^{b} p \dot{b} \Delta r p^{r} q c L \cdot \dot{\Delta} \cdot \Delta^{b} x$

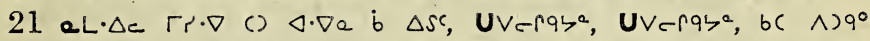

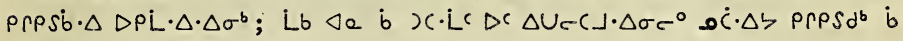
$\triangle \dot{C}-\Gamma_{x}$

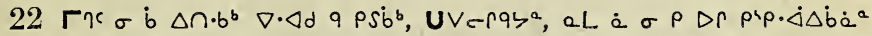

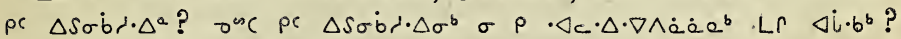

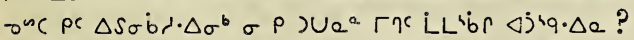

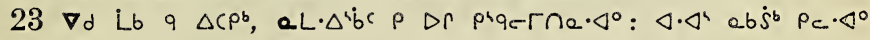
b $L \Gamma)\left(7^{b x}\right.$

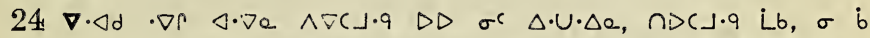

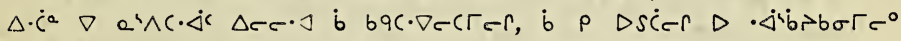
Uं $\wedge^{4} b^{b} \mathrm{x}$

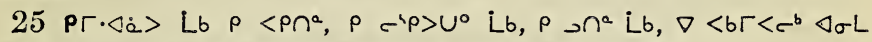

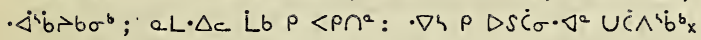

$26 \Gamma_{r} \cdot \nabla(c) \triangleleft \cdot \nabla e$ Lb b $\vee c^{b} \sigma^{c} \Delta \cdot U \cdot \Delta a, \nabla \dot{b}$ Lb b $)^{b}, a^{\prime} \wedge\left(\cdot \nabla^{\circ}\right.$

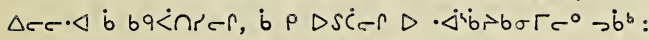

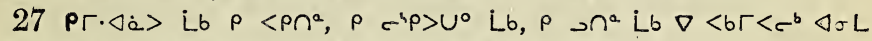

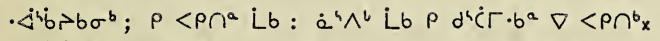

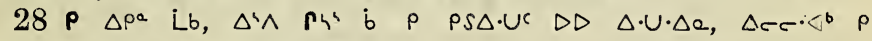

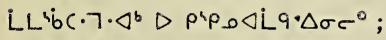

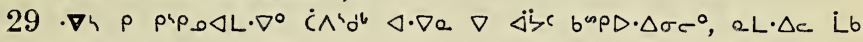
$\dot{C} \wedge^{4} d^{6} D L P Q \triangle q \cdot \Delta^{b} x$

\section{Arpcra $\Delta b^{a} 8$.}

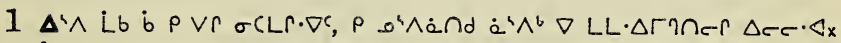

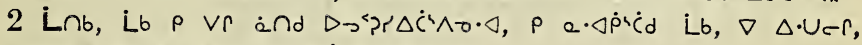

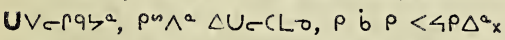

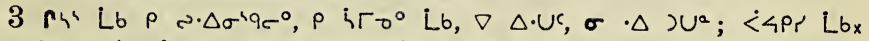

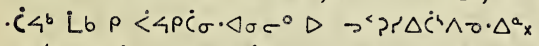

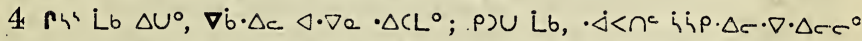

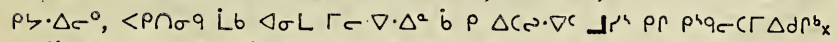

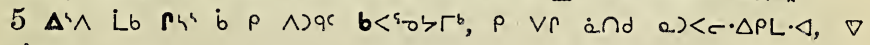
e) $C L^{d} d$,

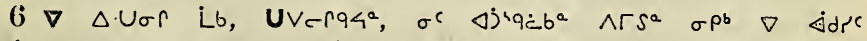

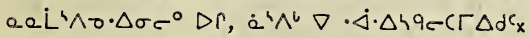

$7 r^{\prime} L b \Delta U^{\circ}, \sigma \dot{b} \vee r \Gamma \mathrm{a} \cdot \Delta r \Delta 0^{\circ}$

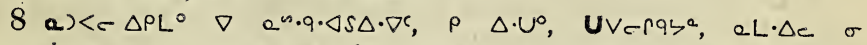

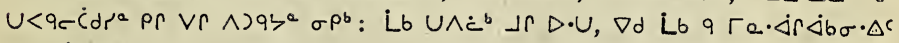
$\sigma^{c} \triangleleft j a \dot{c} b^{4} x$

$9 \cdot \nabla b \quad \sigma_{c}$ o $\left.\cap V e r^{b}, \nabla \cap V-L \rho^{b} a\right)<c \cdot \Delta c c \cdot \triangleleft^{b}: \Delta \cdot \triangleleft \Delta c c^{0} L b$ 11 


\section{$\triangleright<\rightarrow \rho^{4}$ Lด० 8.}

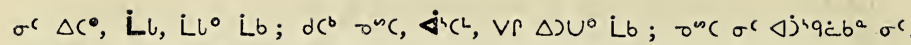
$\left.\Delta c^{\circ}, \Delta L\right)(,)^{L} L b_{x}$

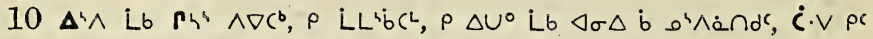

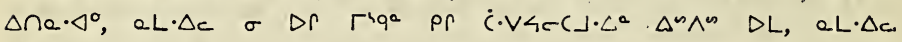
$\Delta \cdot v \Delta r^{b} x$

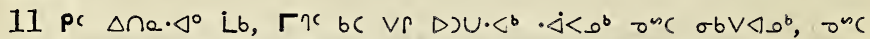

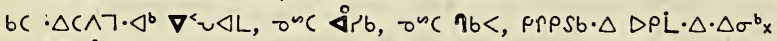

12 Lb $\left\langle\sigma \rho\right.$ b $\Delta c \triangleleft \cdot\left\langle S \Gamma S \Gamma d \rho^{b} \triangleleft \sigma \nabla c^{\circ} \Delta \rho L \cdot \Delta \cdot \Delta \sigma c^{\circ}, b c \cdot \varangle c \cdot \Delta \cdot \nabla \wedge a \cdot \triangleleft^{b}\right.$

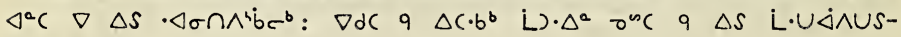
$\sigma \dot{\alpha} \sigma \cdot<b x$

$13 \nabla d \quad h^{4} \nabla \dot{C} c a j<r \cdot \Delta P L \cdot \triangleleft, P \cdot \nabla \Delta h ; \cdot 7 r$ b $\Delta S \dot{C} \cdot V C L^{a}, \nabla d a$

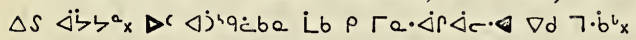

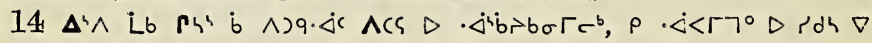

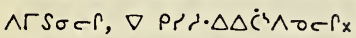

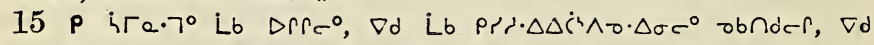

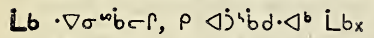

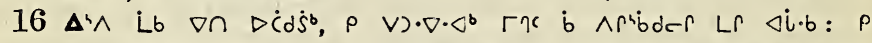

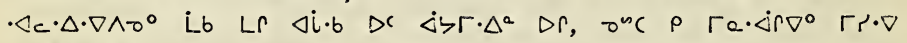
i $\dot{j}$ drer:

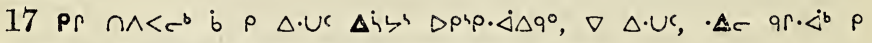

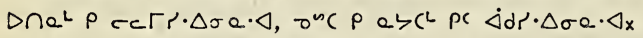

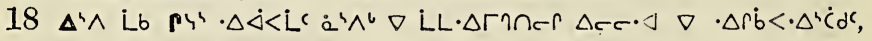

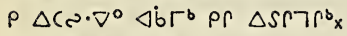

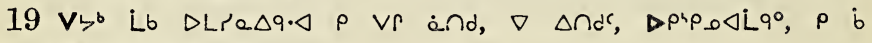

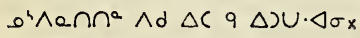

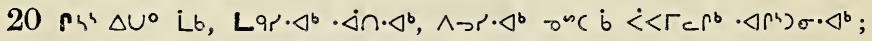

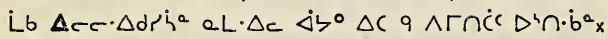

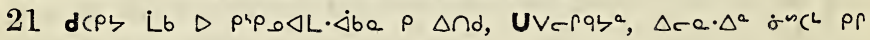
ec $\cdot \Delta a \Delta b \cdot \Delta b$. $\dot{C} \cdot \Delta x$

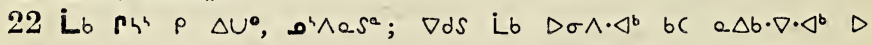
$\sigma \wedge \Gamma \cdot \dot{\Delta} \cdot \triangleleft x$

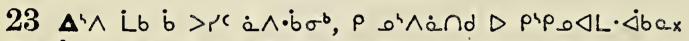

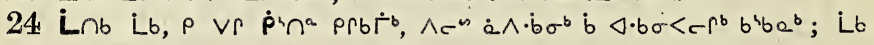
- $\triangle c \rho \sigma<<a x$

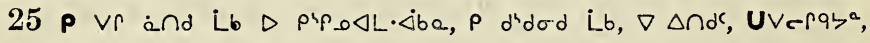
$\wedge \dot{L} \wedge \Delta \dot{a}^{a} ; \sigma \sigma S \cdot \Delta \dot{Q} \cap \pi \dot{a}^{a} x$

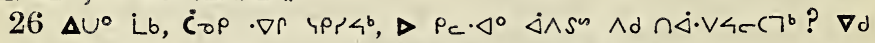

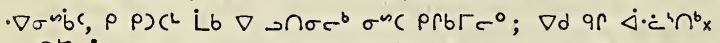

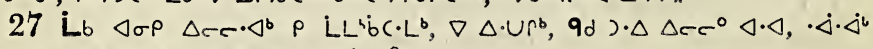

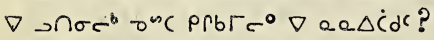

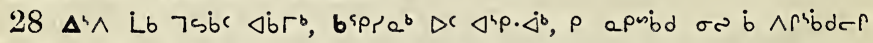

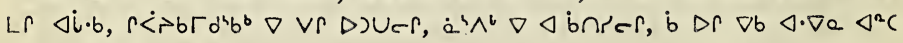
$\nabla P \wedge\lrcorner \cup C_{x}$ 


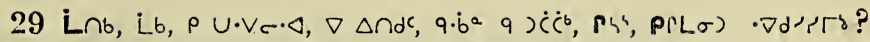

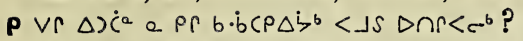

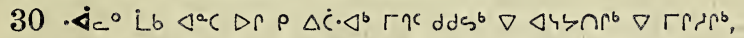

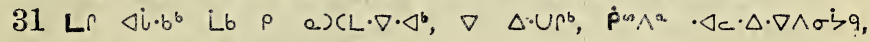
$\triangle c a \cdot \Delta a^{a} p p \wedge>q^{a} b \cdot \Delta p p^{b} d d s^{b} x$

$32 \rho \Delta U^{0}$ Lb, $\Delta U^{b}{ }^{b} \Delta^{\prime} \wedge$ Lb b $\rho \cdot \Delta c \cdot \Delta r^{b}, \rho \wedge q^{4} b \cdot \nabla \cdot \triangleleft^{b} d d s:$ Lnb,

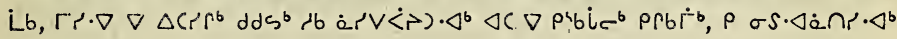
Lb $\sigma \wedge^{6} x$

$33 \varangle \sigma^{\rho} \rho$ L.b $\left.\quad b \quad b e \cdot \nabla c L \rho^{b} \rho \Delta S\right\lrcorner \cdot \Delta^{b}, \Delta c \Delta \sigma^{b} \Delta S$ Lb $\rho \quad \Delta U \cdot \Delta^{b}, \rho$

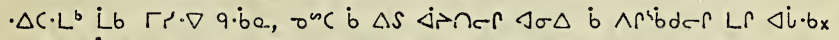

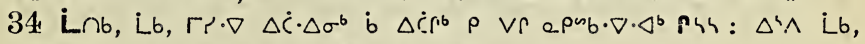

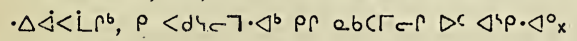

\section{Ápcra $\triangle b^{2} 9$.}

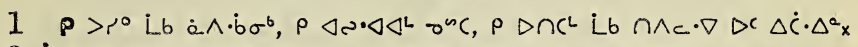

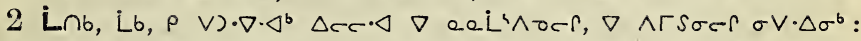

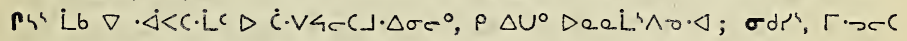
$\Delta \zeta ; p$ LP) $(J \cdot \Delta e, p \cdot \nabla V \in C L b \cdot \Delta a x$

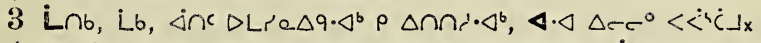

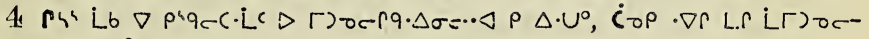
$\left(7^{\circ} P \cup \triangle \cdot \Delta^{6}\right.$ ?

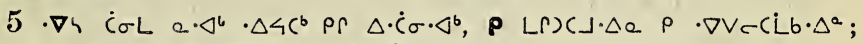

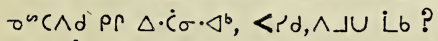

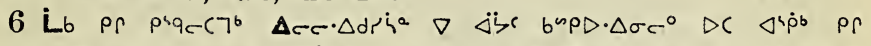

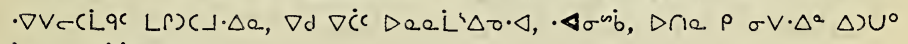
Li $\rho \cdot \Delta \dot{s} b \vec{b} b \sigma \Gamma^{b} x$

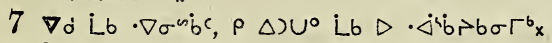

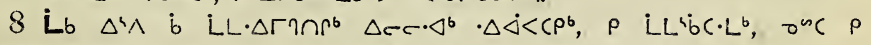

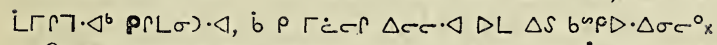

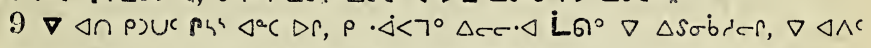

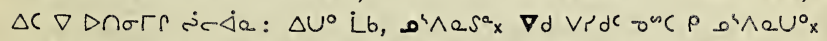

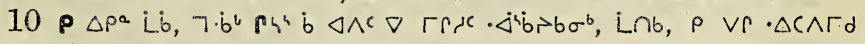

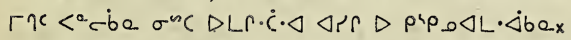

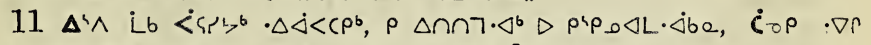

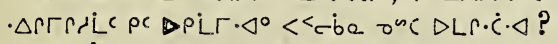

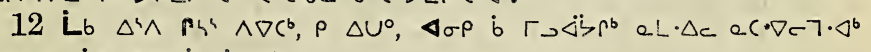
ecdce, Lb $\triangleleft \sigma \rho$ b $\dot{j d r} \rho^{b} x$

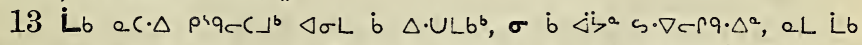

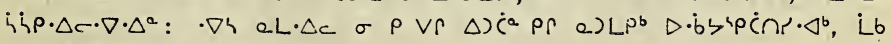

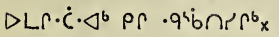

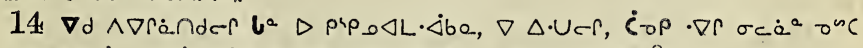

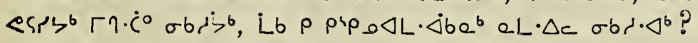

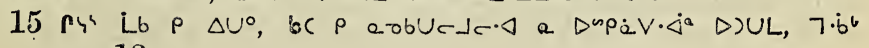
13 


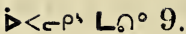

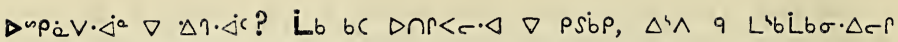

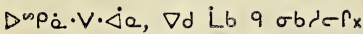

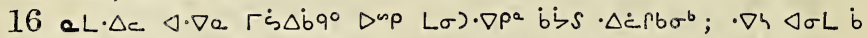
$\left.\left.\Gamma \dot{s} \Delta \dot{b} \dot{b} \sigma \cdot \triangleleft^{b} \dot{C}\right) \wedge(\lrcorner L b^{a}, \nabla d \triangleleft(\nabla \dot{C})<\sigma^{b} \triangleleft \cdot \Delta \beta U\right\urcorner \dot{s} b^{b} x$

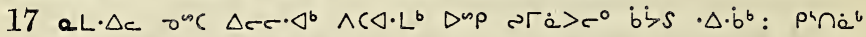

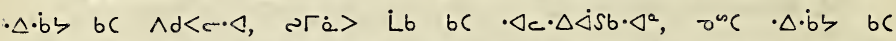

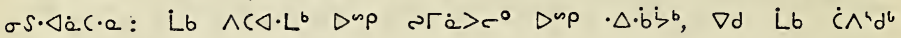
qa. $\nabla r$ ribuc $p_{x}$

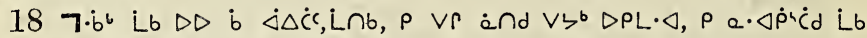

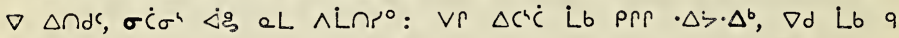
$\wedge \operatorname{Ln} \cap c_{x}$

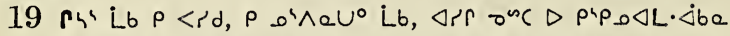

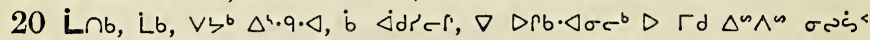
$\Lambda>\cdot a, \Delta^{4} \Lambda^{4} \cdot b \sigma^{b} \rho \vee r$ a $n d, \nabla i \Gamma a \Gamma c r \Delta \cdot \Delta \dot{\varepsilon} r b^{a} \cap n \Lambda^{4} c \Delta b \sigma^{b}$ :

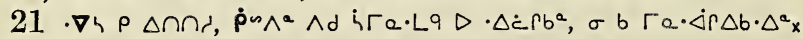

22 Lb $p^{4} \rho \cdot q^{4} p b<\cdot \Delta^{\circ}, \Delta^{4} \wedge$ Lb $\Delta \Delta^{\prime}<L<, \rho \Delta \cdot U^{\circ}, \sigma i \sigma^{4}, \Gamma \cdot \sec \Delta 4$;

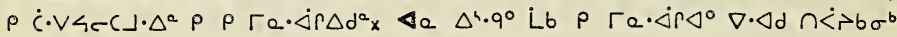
$\wedge r\lrcorner b \sigma^{b} x$

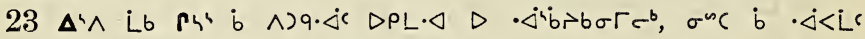

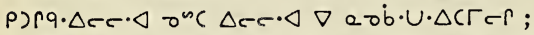

24. $\rho \Delta U^{0}, \dot{C} \cdot \Delta^{b} ; \cdot \nabla h$ aL. $\Delta c \quad \sigma \wedge^{0} \dot{\Delta} \cdot \Delta \Delta^{4} \cdot 9 S^{\mu n}$, Lb $\sigma<^{\circ} x \quad \nabla d$ Lb

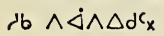

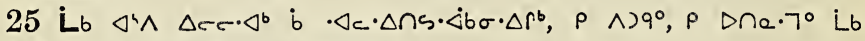
$\Delta r r c^{\circ}, \nabla d$ Lb $\cdot \nabla \sigma^{\infty} b^{c}<\Delta e \Delta^{\prime} \cdot 9 S^{\infty} x$

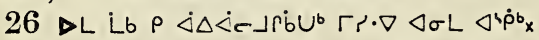

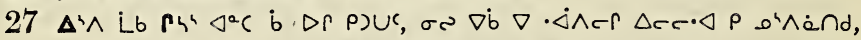

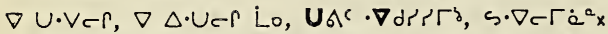

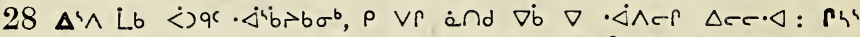

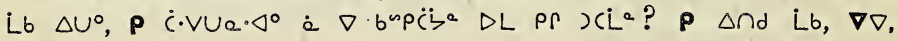
UV $\sim r q L^{\circ} x$

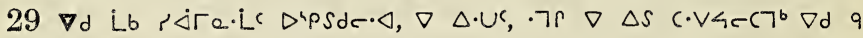
$\Delta S \triangleleft \ddot{b} \dot{\zeta}^{b} \mathrm{x}$

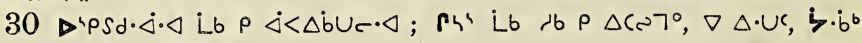
$\nabla \dot{b} \cdot \Delta c . \Delta \cdot \nabla a$ er prqec bx

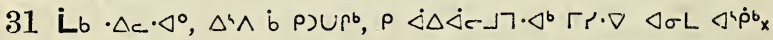

$\left.32 \nabla \triangleleft \cap \cdot \Delta c \cdot \Delta \rho^{b} L b, L \cap \cap b, \rho \vee v\right) \cdot \nabla \cdot \triangleleft^{b} \Delta c c \cdot \triangleleft \nabla b \quad \nabla \sigma c \cdot \nabla c \beta \nabla$ $\wedge$ sibóar Lr $\Delta i \cdot b x$

$33 \Delta^{4} \wedge$ Lb $\cdot \nabla c \cdot J \cdot \nabla \wedge \dot{a} b \sigma \cdot \Delta c$ Lr $\varangle \dot{b} b, \nabla \dot{b} \dot{b} \sigma \dot{c} \cdot \nabla c \rho \dot{\Delta b} \Gamma^{\circ}: \Gamma \dot{b} L \cdot \Delta-$

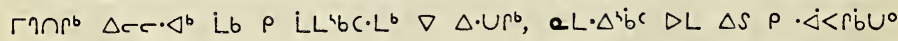
$\Delta \cdot u \Delta r^{b} x$

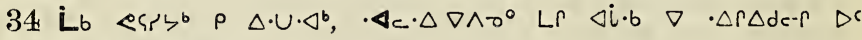
$D P L \Gamma \cdot \dot{\Delta} \cdot \triangleleft \operatorname{Lr} \triangleleft i \cdot b^{b} x$

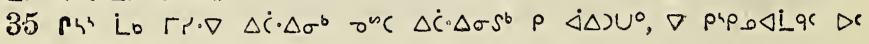
14 


\section{¿<cค' LQ $9,10$.}

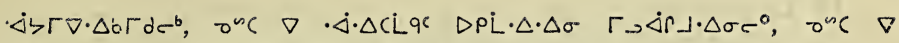

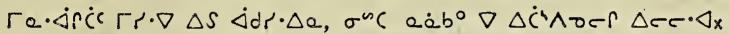

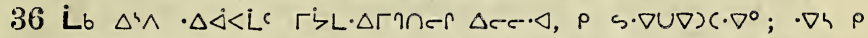

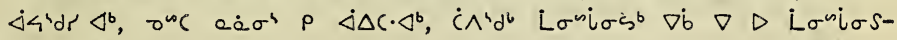
$\cdot \Delta c c r b^{x} x$

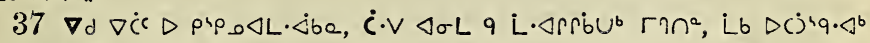
เ $6 \cdot \Delta s S \cdot \triangleleft^{\mathrm{b}}:$

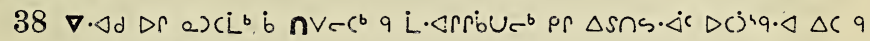
$\triangle S L \cdot \Delta r \dot{b} b \sigma \cdot \triangleleft \sigma c^{b} x$

\section{N'pcra $\triangle b^{\circ} 10$.}

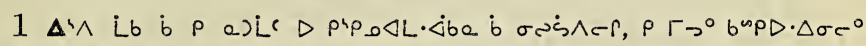

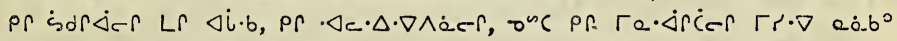

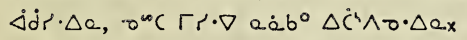

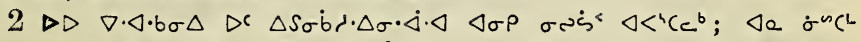

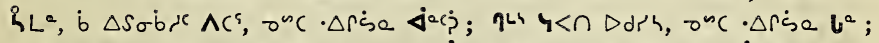

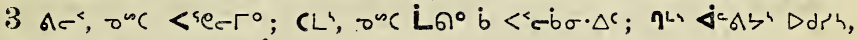

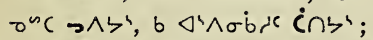

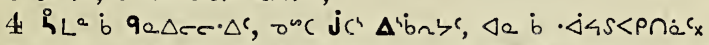

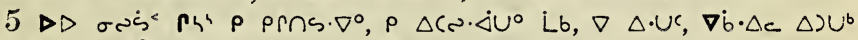

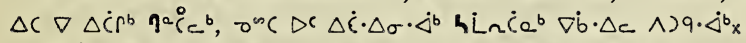

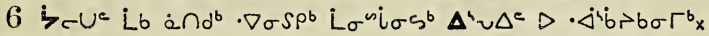

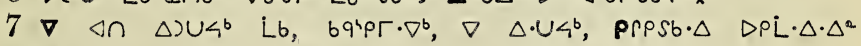
VNa. $\cdot b^{2} x$

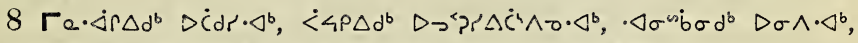
$\cdot \Delta c \cdot \Delta \cdot \nabla \wedge \sigma d^{b}$ Lr $\left.\left.\Delta \dot{l} \cdot b^{b}:\right\lrcorner 2 \rho \rho \Gamma-\dot{b} \cdot \Delta a \cdot \Delta^{0},\right\lrcorner 2 \Gamma \tau \cdot \nabla^{b} x$

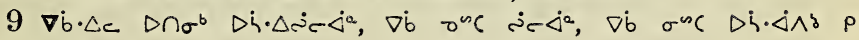
$\wedge \dot{C} \cdot \cdot \Delta \sigma \cdot \dot{\Delta}^{b}$,

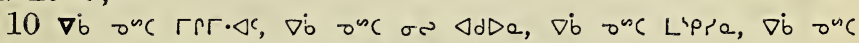

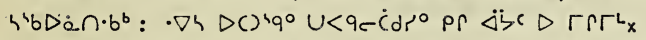

$11 \varangle C \wedge d$ pr $\Delta \dot{C} \cdot \Delta^{a}$ osc $\left.\left(\wedge d \Delta \dot{C} \cdot \Delta^{a} q \Delta S \wedge\right) q \cdot \nabla \cdot q, b \cdot 9 r q\right\lrcorner^{b} \triangleleft \cdot \nabla a . \nabla d C$

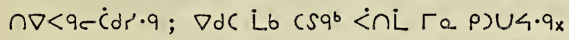

$12 \Delta^{4} \wedge$ L.b $\wedge>94 \cdot q \cdot \Delta^{j} b r b \sigma^{b}, \Delta C \Gamma^{4} b J^{b} x$

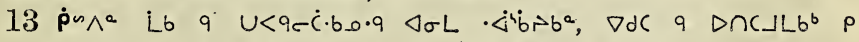

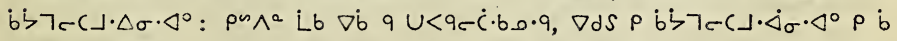
p. $\nabla \dot{x} \dot{(d e} \cdot \triangleleft{ }^{\circ} x$

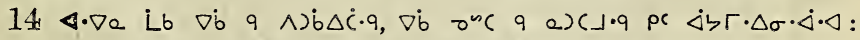

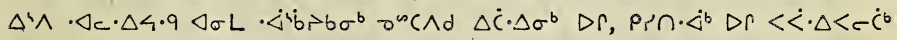
LP.bं Sx

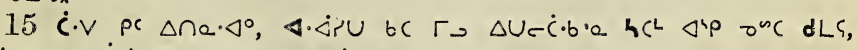

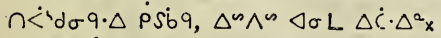

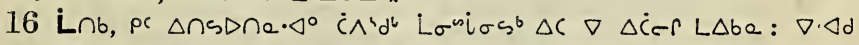

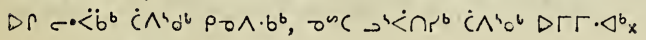




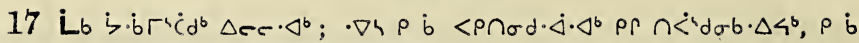
$<<4 \cup \Delta d \cdot \dot{\Delta} \cdot \Delta^{b} L b \quad \Delta C \Delta b \Gamma \nabla \cdot \Delta b \Gamma d \cdot \dot{\Delta}^{b}$;

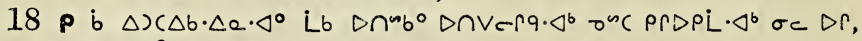

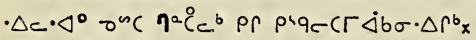

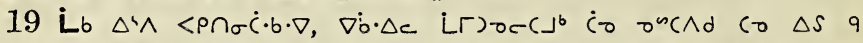

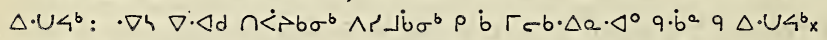

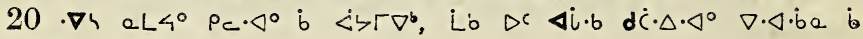
$\dot{\Delta b} \Gamma^{i} \dot{b} \dot{c}^{b} x$

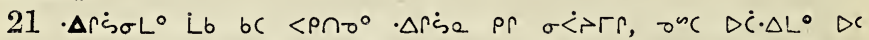

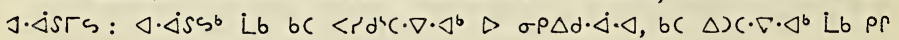
$\sigma \dot{<}>\Gamma r_{x}$

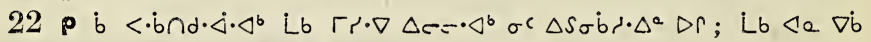
q $\dot{s} d r \dot{\Delta} b \sigma \cdot \Delta c \nabla \cdot \Delta \cdot \dot{b} e$ q $\wedge \dot{L} r \dot{\Delta} b \sigma \cdot \Delta c_{x}$

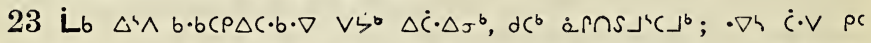

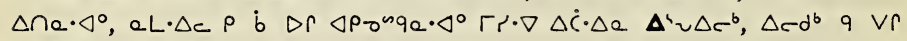

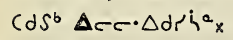

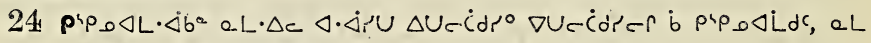

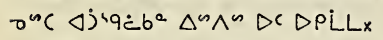

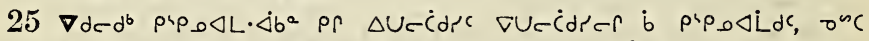

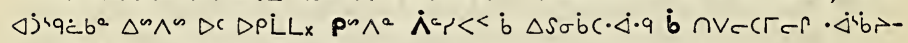

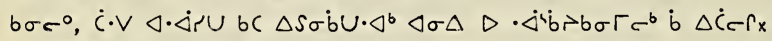

$26 \nabla \cdot \Delta d \quad \nabla r \quad \nabla \dot{b} \cdot \Delta c \quad d^{\prime} \cap d^{b}: \cdot \nabla h, a L \cdot \Delta c \quad q \cdot b^{a} \quad \forall \cdot b c \Delta b U^{\circ}, \quad \nabla b \quad q$

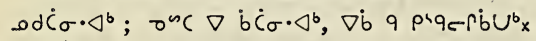

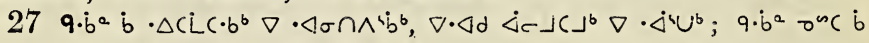

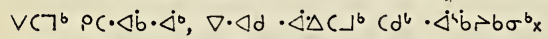

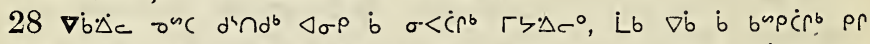

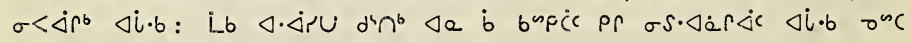
$\Gamma \sqcup \Delta c^{\circ}$ Lr $\Delta \backsim d U^{b} x$

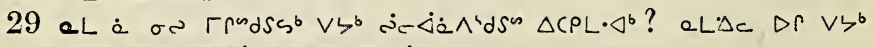

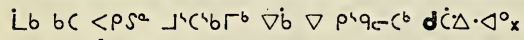

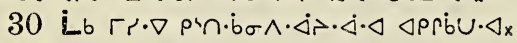

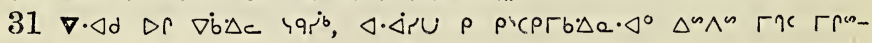
dss ${ }^{\mathrm{x}} \mathrm{x}$

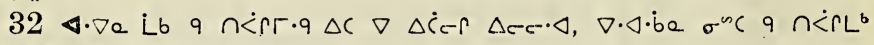

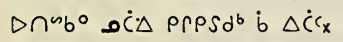

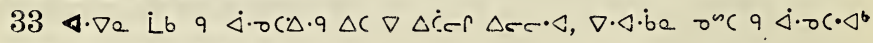
$\Delta \cap^{\circ} b^{\circ} \circ \dot{C} \triangle$ PRPSO $^{b} \dot{b} \Delta \dot{C}_{x}$

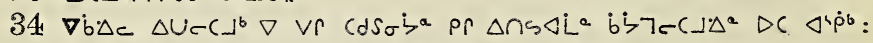

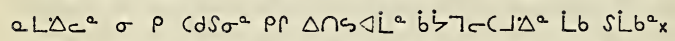

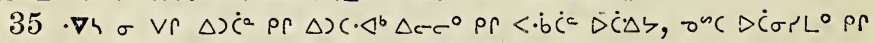

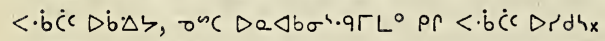

$36 \Delta c^{\circ} c^{\circ}$ Lb bC $<\cdot \dot{b} \cap d \Delta \sigma \Delta \dot{b} \Delta \dot{C} e r \cap \Lambda c \cdot \nabla \cdot \Delta \rho^{b} x$

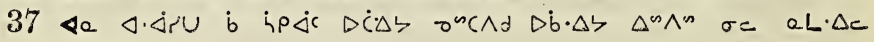
16 


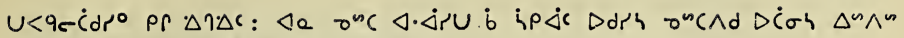
$\sigma c$, eL $\Delta c \quad U<q-\dot{C} d p^{\circ}$ pr $\Delta 1 \Delta c_{x}$

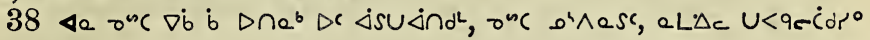
pr $\Delta \eta \Delta c_{x}$

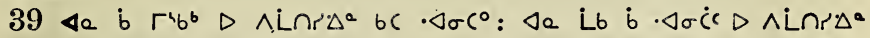
$\sigma c \Delta r b C r^{4} b L_{x}$

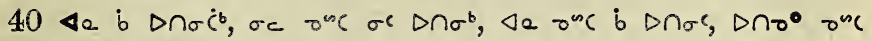
$\Delta \sigma \Delta$ b VRnSDCrx

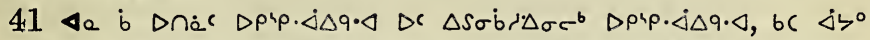

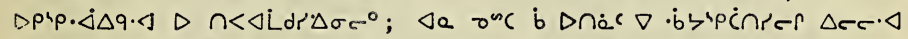

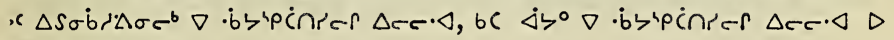
$n<\Delta L d d^{\circ} \Delta \sigma \sigma^{\circ} x$

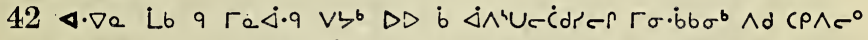

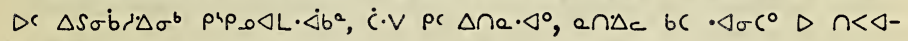
Ldr. $\Delta a^{a}$

\section{niscra $\triangle b_{0} 11$.}

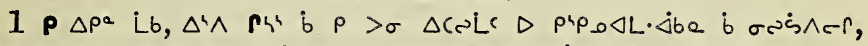

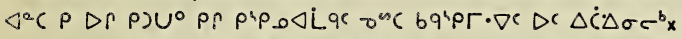

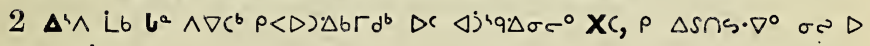
PיP_o $\Delta L \cdot \Delta b a$,

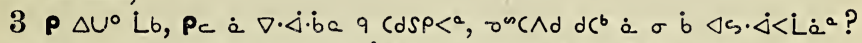

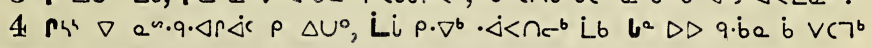
onc $\dot{b} \cdot \dot{<}<C 7^{\circ}$ :

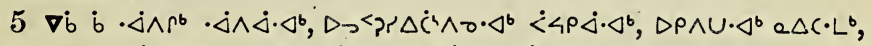

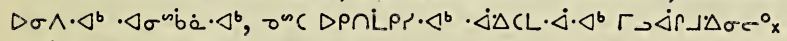

6 s. $\nabla c \dot{C} d p^{\circ} \mathrm{Lb}, \varangle \cdot \nabla c \nabla \dot{b} 9 \dot{a} 4 c \cdot \nabla c r \cdot q_{x}$

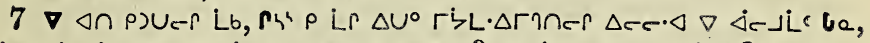
$\left.9 \cdot b^{a} \cdot \dot{\Delta} \cdot \dot{\Delta}<c 7^{b} \Delta^{4} \wedge \dot{b} \Delta\right) \cup<^{b}<\cdot b c^{b} b r b^{b} ? \cdot \Delta \dot{<} b \dot{a} \nabla \cdot \nabla \cdot \nabla<<^{b}$ ?

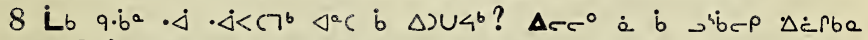

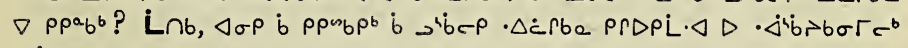
$\triangle \dot{C} \cdot \Delta^{b} \mathrm{x}$

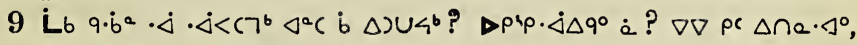

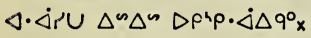

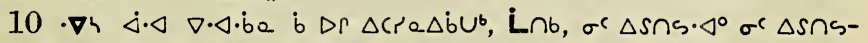

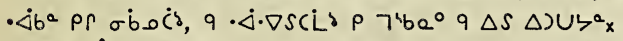

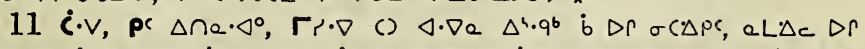

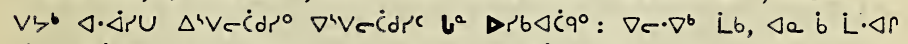

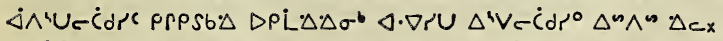

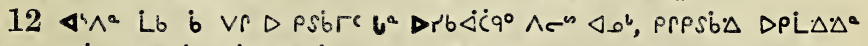

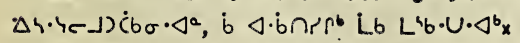

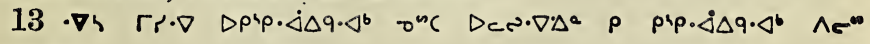
$U d S^{b} l^{2} x$

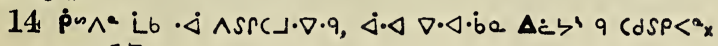
17 
$15 \varangle a \dot{b} \nabla \dot{C} \cdot \Delta \dot{b} c \operatorname{pr} V C^{b}, \nabla d S b C \vee C^{L} \times$

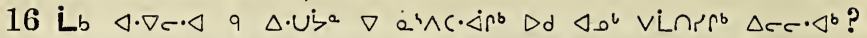

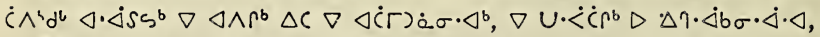

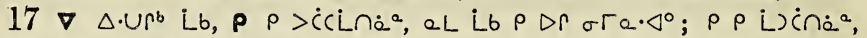

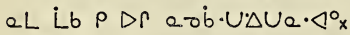

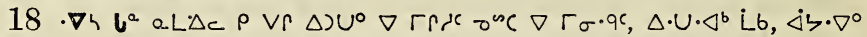
Lr $\triangleleft i \cdot b_{x}$

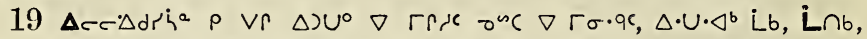

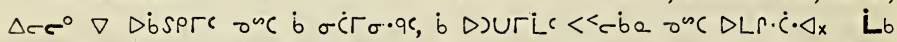

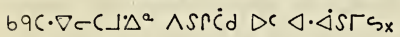

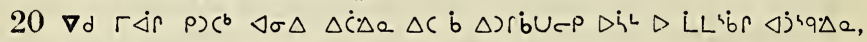

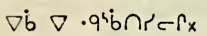

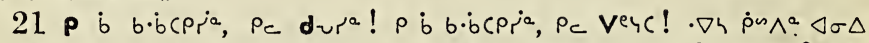

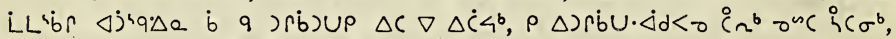

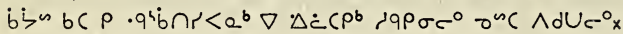

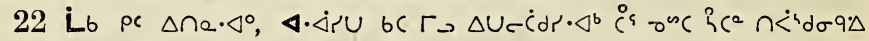
psise $\Delta s \wedge ⿻ \rho^{\infty} \rho_{c} \cdot \triangleleft^{\circ} \times$

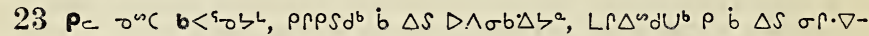

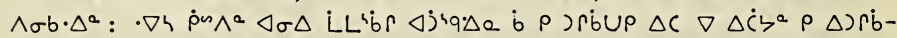

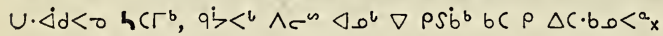

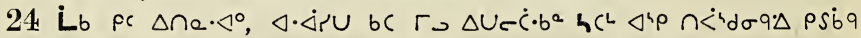
$\Delta m \wedge ⿻ p_{c x}$

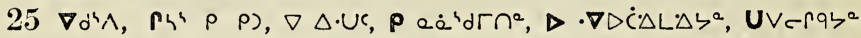

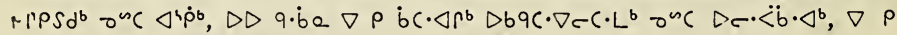

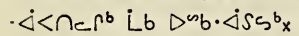

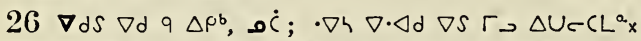

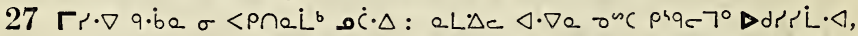

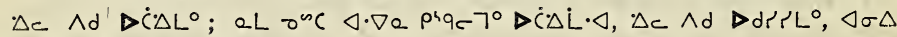

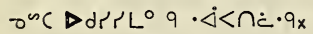

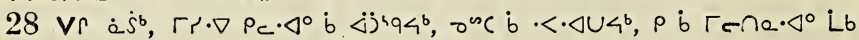
pr $\dot{\Delta} \cdot \supset \wedge \Delta^{b} \mathrm{x}$

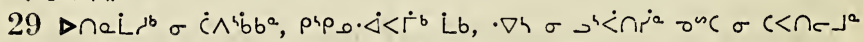

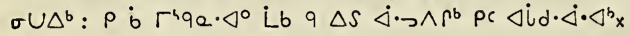

$30 \cdot \nabla h \sigma \dot{C} \wedge^{4} b b^{a} \cdot \nabla C^{a}, \nabla^{n c} \sigma \Delta \cdot \Delta S \Delta^{a} \dot{c} b S^{a} x$

\section{Ápcra $\triangle b^{a} 12$.}

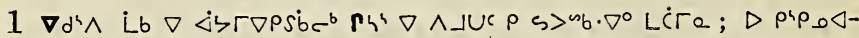

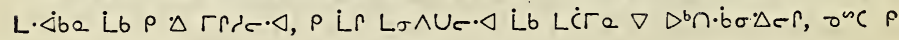
\lrcorner$\cdot \nabla c \cdot \triangleleft x$.

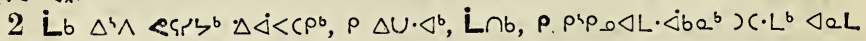

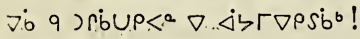

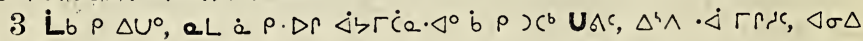
osc b $\triangle 12 \Delta d c ;$ 


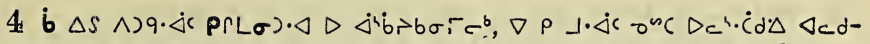

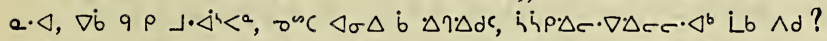

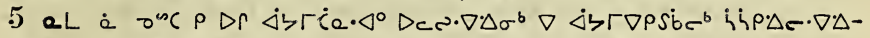

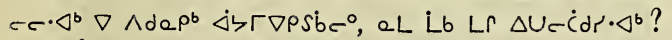

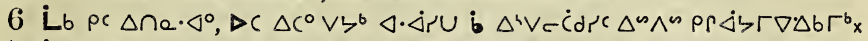

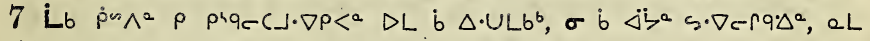

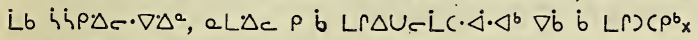

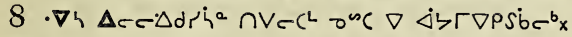

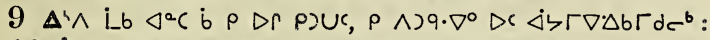

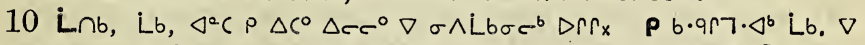

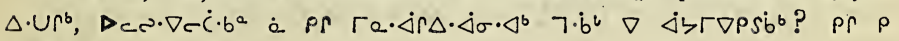
LTSLR'x

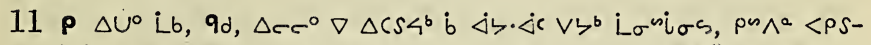

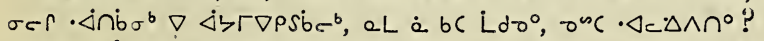

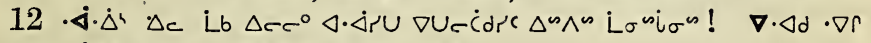

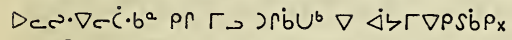

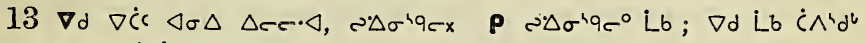

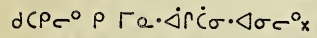

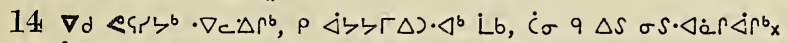

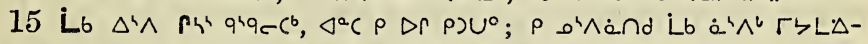

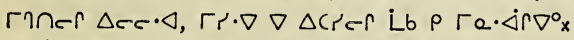

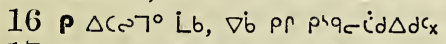

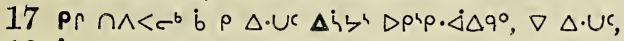

18 Lnb $\triangleleft \cdot \triangleleft$ oc $\varangle j 4 q \dot{c} b^{a}, \dot{b} \rho \cdot \nabla \cdot \nabla \dot{c}<L^{b} ; \sigma i \rho \dot{\Delta} b^{a}, b \quad b r \dot{a}^{4} \wedge^{6}$

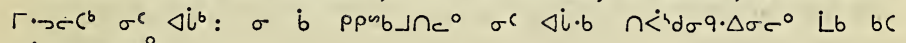
$\cdot \dot{<<n \rightarrow 0} \eta^{\circ}{ }^{\circ} \mathrm{C} x$

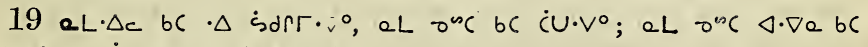
$V C \cdot 7^{\circ} D V \dot{C} d r \cdot \Delta \sigma c^{\circ} \wedge J U^{\prime} b a^{b} x$

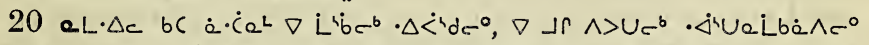

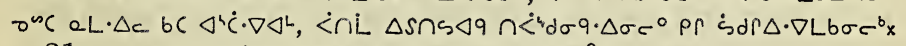

$21 \cdot \Delta c \Delta c \Delta S \sigma b r \cdot \Delta \sigma^{b}\left[b b c \Delta^{b} V c\right\lrcorner c \cdot \Delta \eta^{\circ}{ }^{\circ} c x$

$22 \nabla d \wedge \nabla\rangle \cdot\left\langle b c \cdot \Delta c \triangleleft \cdot \nabla c \cdot \triangleleft b \quad \wedge r^{4} b d e r\right.$ Lr $\Delta i \cdot b, \nabla b \nabla \cdot \Delta \wedge c r, \nabla b$

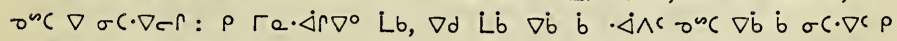

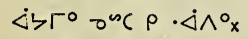

$23 \Gamma \cdot \cdot \nabla \Delta c c \cdot \Delta^{b}$ Lb. $P$ LLbbC.Lb, $\rho \quad \Delta \cdot U \cdot \Delta^{b}$ Lb, aL a $\triangleleft \cdot \triangleleft$ UAC Dor's?

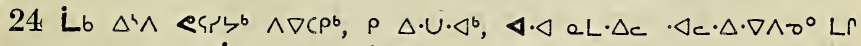
$\Delta i \cdot b, \nabla \cdot \Delta r \Delta d c \wedge d \dot{\Lambda} c r<<L r \quad \Delta i \cdot b \Delta c \Delta p L i r c \cdot \Delta x$

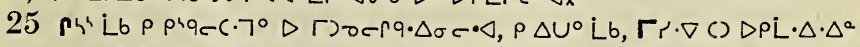

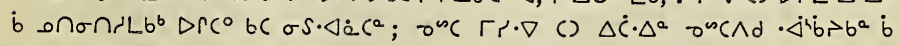

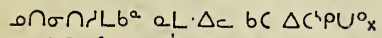

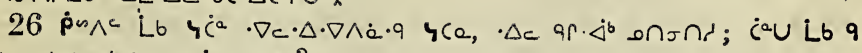
Dr $\triangle C^{i} P U C^{b} D C D F L \cdot \Delta \cdot \Delta^{a}$ ? 


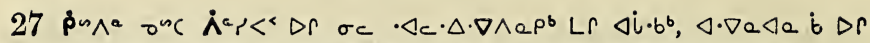

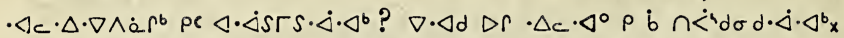

28 Lb pas $\left.\wedge^{a} \rho \Gamma L \sigma\right) \Delta c \Delta i \cdot b \Delta r \cdot \Delta c \cdot \Delta \cdot \nabla \wedge a \rho^{b}$ Lr $\Delta i \cdot b^{b}, \nabla d \cdot \nabla \cap n d-$ - $\left.\nabla^{b} P C L \sigma\right) D\left(D P L \cdot \Delta \cdot \Delta^{a} x\right.$

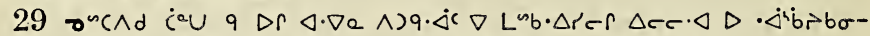

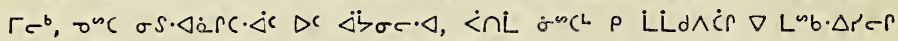

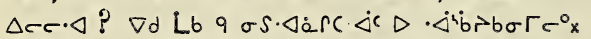

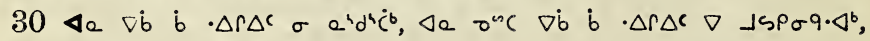
$\cdot \triangleleft 4 \cdot 4 \wedge \sigma 9^{\circ} x$

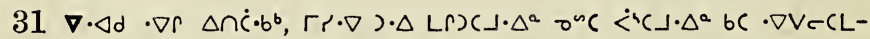
$\cdot \dot{\Delta} \cdot \Delta^{b} \Delta c c \cdot \Delta^{b}:$ Lb $\nabla \dot{<} \dot{<} C L b \sigma \cdot \Delta c$ Vspre $\Delta i b$, aL. $\Delta c$ bC $\cdot \nabla V c C L-$

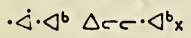

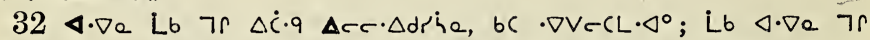

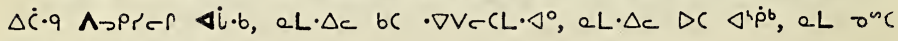
$\triangle$ यें $q \Delta \cap r<c^{b} x$

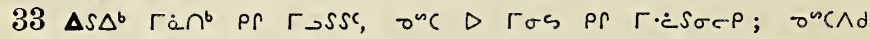

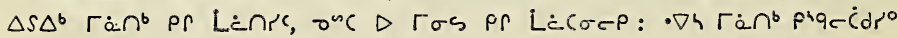
$\nabla \Delta S \Gamma \sigma s \cdot \Delta c_{x}$

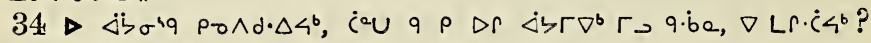

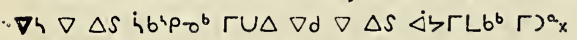

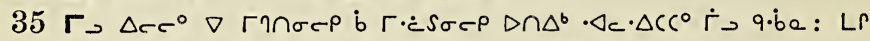

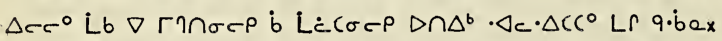

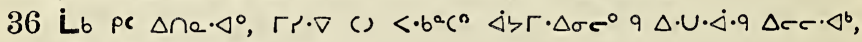

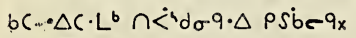

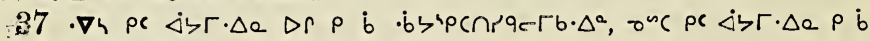

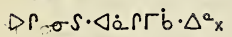

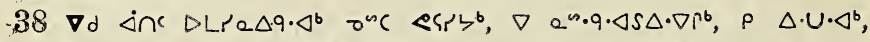

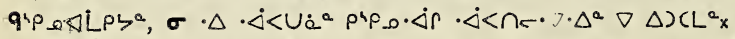

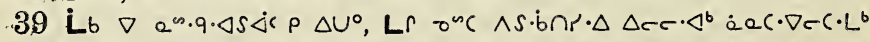

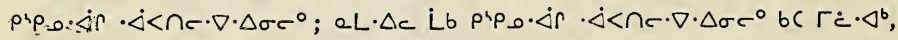

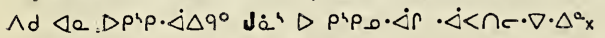

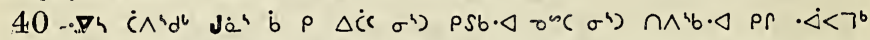

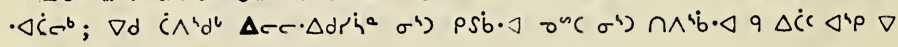
$\Delta \cup \triangle L_{b} \operatorname{coc}^{b} \mathrm{x}$

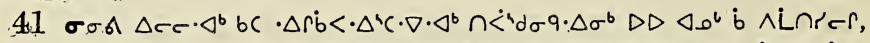

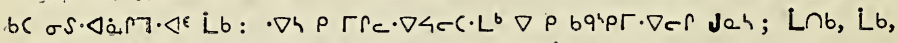

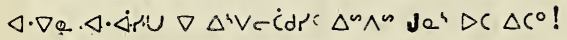

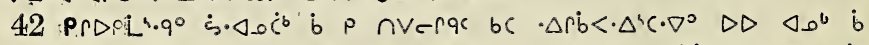

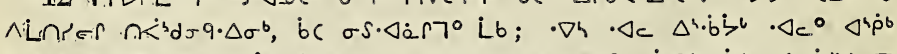

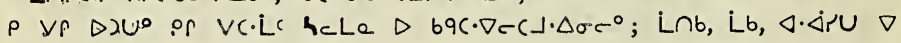

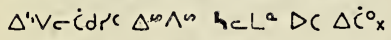

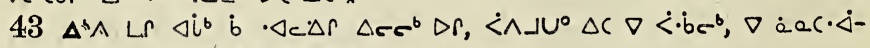

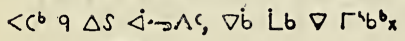




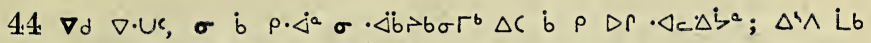
"UdSb, $\Gamma^{b} b^{L} \nabla \wedge S S \cdot b c^{b}, \nabla \cdot \nabla<\Delta \dot{b} U c^{b}, \sigma^{n c} \nabla \cdot \dot{\Delta} \cdot \nabla S \rho \dot{b} U c^{b} x$

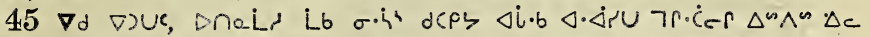

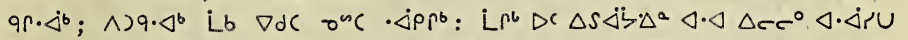

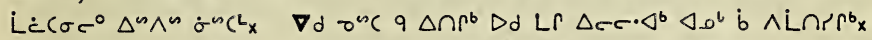

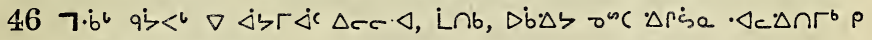
$\sigma<\Delta r \cdot \triangleleft \nabla \Delta \dot{\langle}>r \Delta d c^{\prime} x$

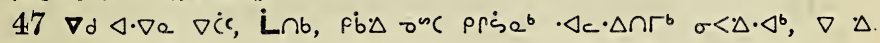

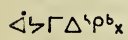

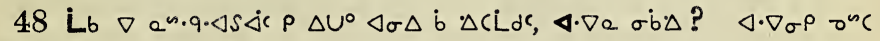
orseb?

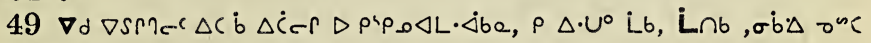
orseb!

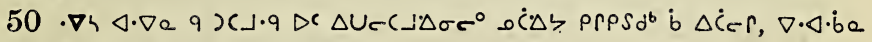
oría, onc orisos.ql onc $\sigma \dot{b} \Delta x$

\section{Aspera $\Delta b^{a} 13 x$}

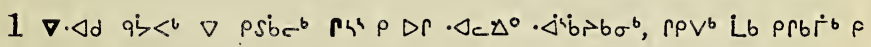
$\triangleleft \wedge{ }^{\circ} \mathrm{x}$

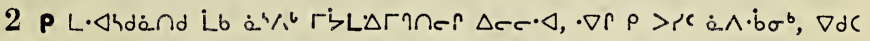

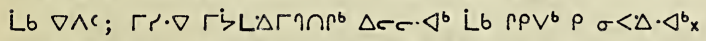

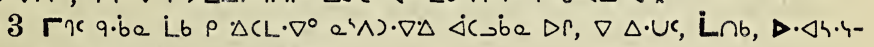
$\left.\wedge \sigma q^{\circ} \rho \rho\right) U^{\circ} \rho \varphi \cdot \Delta 4 \cdot \zeta \wedge \sigma q c$ :

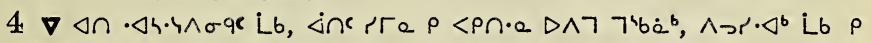
$\operatorname{vr} \dot{\rho} \dot{c} \cdot \triangleleft^{b}:$

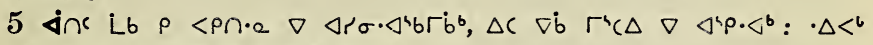

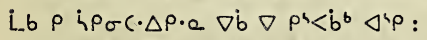

$6 \Delta^{\wedge} \wedge$ Lb $\wedge^{\prime L}$ b idUc, $\rho \Delta^{4} \cdot \dot{b} U \cdot \triangleleft ; \nabla b$ Lb $\nabla \cdot \Delta C \wedge \cdot \Delta p, \rho \& n$ $\sigma \wedge L b \cdot a x$

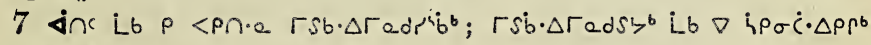
$\rho \rho \wedge \mathrm{s} b \cdot L^{b} \mathrm{P} x$

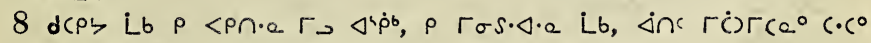

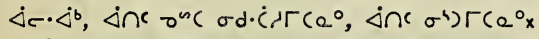

$9 \varangle \cdot \nabla e^{2} \cdot \nabla \dot{C} \cdot \Delta b \cdot q \rho f \vee C^{b}, \nabla d S$ bc $V C^{L} x$

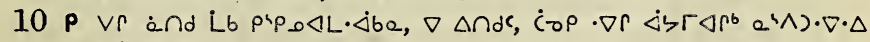
¿cube Dr?

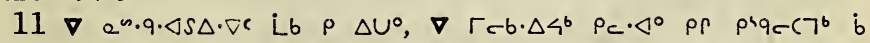

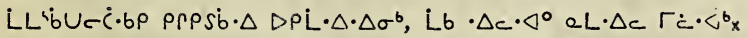

$12 \cdot \nabla h \quad \triangleleft \cdot \nabla e \quad \nabla \dot{\zeta} \cdot q, \nabla \cdot \triangleleft \cdot b e q \Gamma \dot{c} b \sigma \cdot \Delta c, \varangle \cdot \Delta p U$ Lb bc $\cdot \nabla \rightarrow n r^{\circ}:$ Lb $\triangleleft \cdot \nabla e \nabla b \quad \nabla \dot{b} \cdot q, \nabla \cdot \Delta \cdot b e q$ L'bLbo $\Delta c q \cdot b \sigma^{\circ} b \dot{b}\left\langle\dot{b} c_{x}\right.$

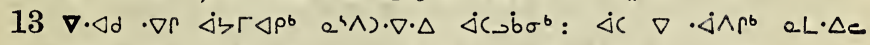

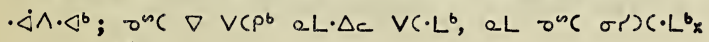




\section{D<P Lด 13.}

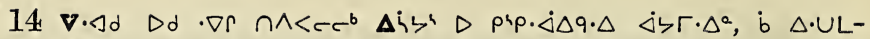

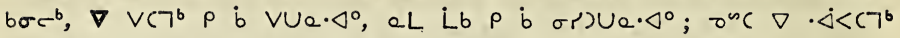

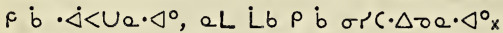

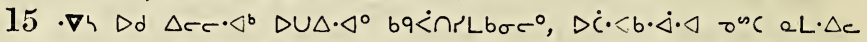

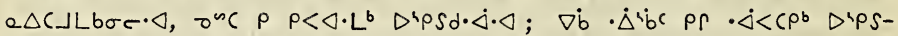

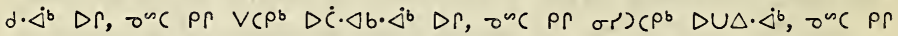

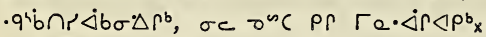

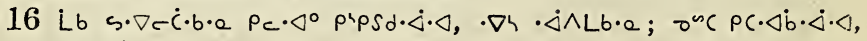
$\cdot \nabla 4$ VCJLb.ax

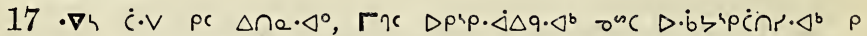

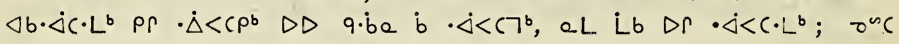
pr $V C \rho^{b} \triangle D$ a.be b $V C 7^{b}$, eL Lb $F \vee C \cdot L^{b} x$

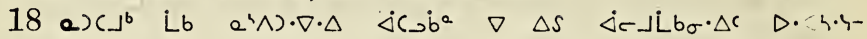
$\wedge \sigma^{\circ} \mathrm{x}$

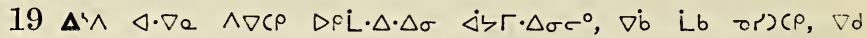

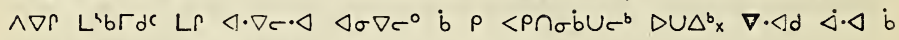

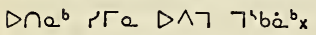

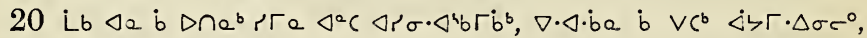

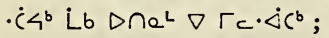

21 aL. $\Delta c$ Lb $D C \wedge \triangle^{\circ} \wedge \Gamma^{b}$, Lb $\Delta \Gamma_{c}^{\circ} \wedge d \quad \sigma<\cdot \Delta^{0}:$. $\nabla 4 \quad \Delta^{4} \wedge$

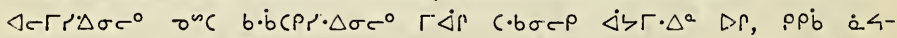
$C \cdot \nabla \leftarrow C^{2} x$

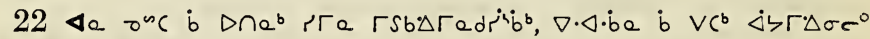

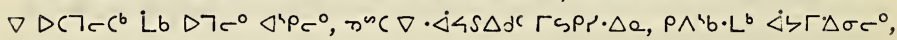
e L $\Delta c$ L L.b $\Gamma \sigma s \Delta^{\circ} x$

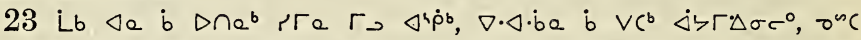

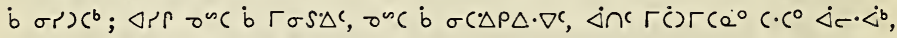
inc o.d. $\dot{\lambda} \Gamma\left(e^{\circ}, \dot{\Delta i n} \sigma^{4}\right) \Gamma\left(e^{\circ} x\right.$

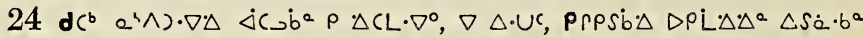

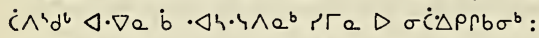

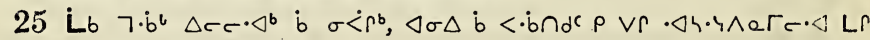
L'dSA rre, $\nabla d$ Lb $\Gamma \Delta i_{c} r_{x}$

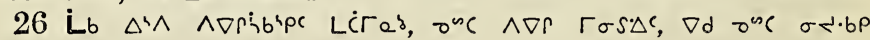
Lr L'dSSX

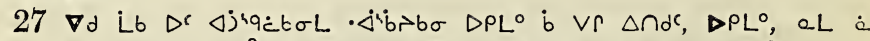

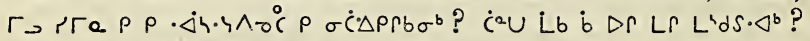

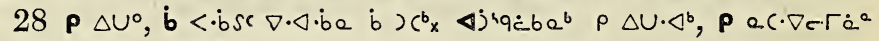
a $\operatorname{pr}$ aC $\triangle \mathrm{L} \sigma \wedge C \mathrm{~L}^{b}$ ?

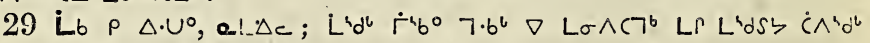

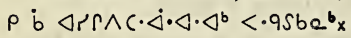

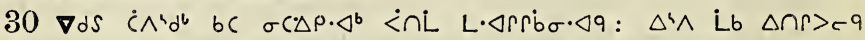

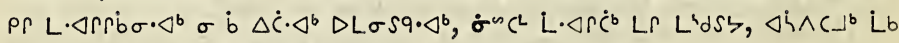
pr $\Delta^{b} \cdot b_{b} 7^{b} ; \quad L b \quad L \cdot \Delta r \Delta d^{b}<\cdot 95 b a^{b} \sigma c \cdot b \Delta 9 \Delta b \Gamma d^{b} x$ 


$$
\triangleright<>\text { P Lด } 13 .
$$

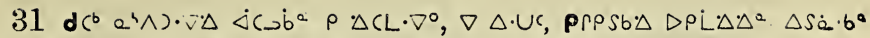

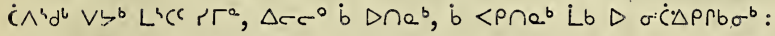

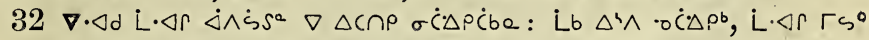

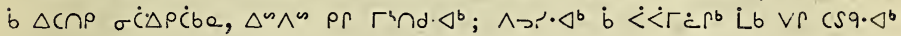
$\nabla \cdot \Delta \cap \cdot b \sigma^{b} x$

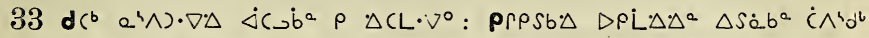

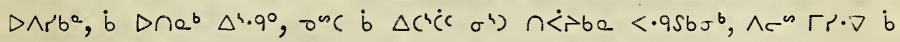
$D \wedge<<c_{e} x$

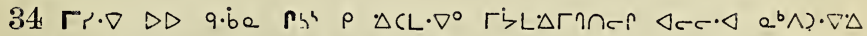

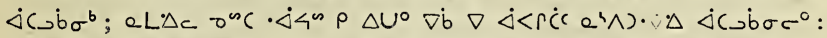

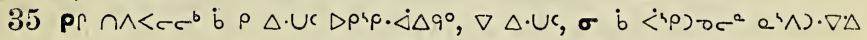
$\dot{\Delta c} \vec{b} b \sigma^{b} ; \sigma \dot{b} \Delta U^{a} q \cdot \dot{b} a \dot{b} \rho \dot{b} r \dot{b} \cup \rho \Delta^{4} \wedge^{a} \dot{b} \vee r \quad \Delta^{4} \rho \cdot \Delta^{b} x$

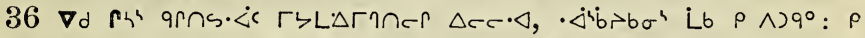

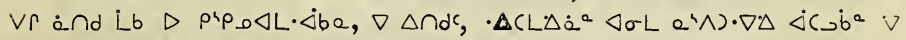

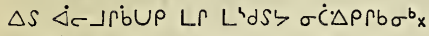

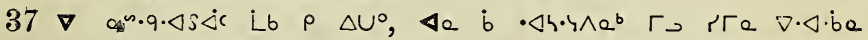
$\Delta r-\Delta d r^{2} b^{2}$;

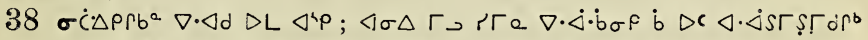

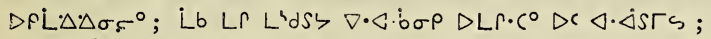

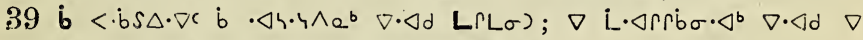

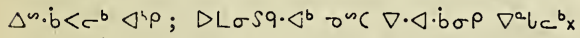

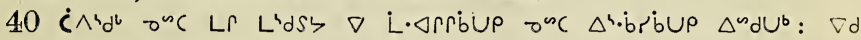

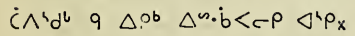

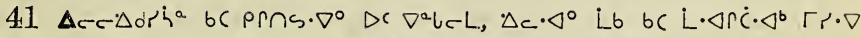
$q \cdot \dot{b} c^{\circ} \dot{b} \dot{a} 4 c \cdot \nabla c \dot{c} \cdot b \tau c^{b}, \Delta \sigma \Delta \sigma^{\infty} c \dot{b}$ Lr) $(\Gamma e r$;

42 bc $\Delta S \cdot \nabla \wedge_{0} \cdot \Delta^{b}$ Lb $\Delta^{a} d U \Delta b \Gamma d^{b}: \nabla d c$ q $\Delta\left(\cdot b^{b}\right.$ L $) \Delta^{a}$ occ $9 \Delta S$ L.U $\cup \dot{\Delta} \wedge U S \sigma \dot{\alpha} \sigma \cdot \triangleleft b x$

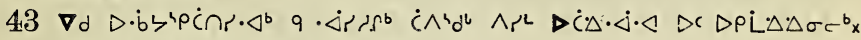
$\varangle \cdot \nabla e \cdot \nabla \dot{C} \cdot \Delta \dot{b} \cdot q$ Pr $V C^{b}, \nabla d S$ bC $V C C^{L} x$

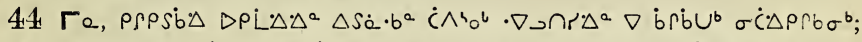

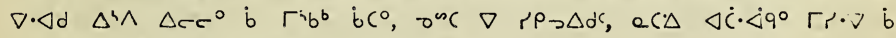

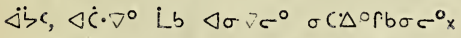

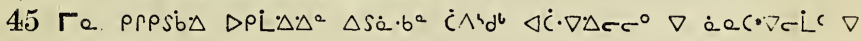
rosser $\nabla u$;

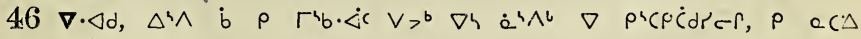

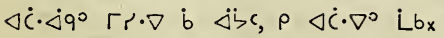

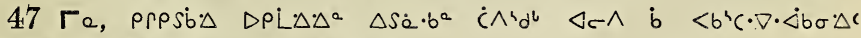

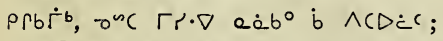

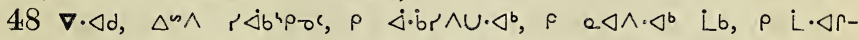

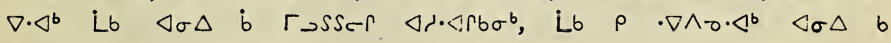
Lintertx

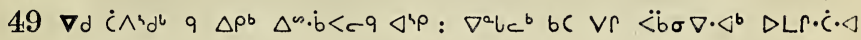
$D \cdot \dot{b}$ ipn. $\triangleleft$ Dr; 
$50 \Delta \Delta^{\omega} d U \Delta b r d^{b}$ L.b bC $\Delta S \cdot \nabla \Lambda_{0} \cdot \Delta^{b}: \nabla d C \quad 9 \quad \Delta\left(\cdot b^{b} L\right) \Delta^{a}$ owe 9

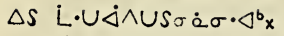

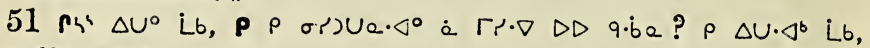

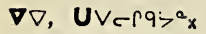

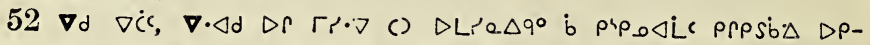

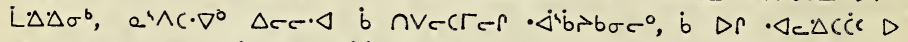
- $\nabla$ anis $\Delta \sigma^{b}$ dup qobe onc bisn $q . \dot{b}$ ex

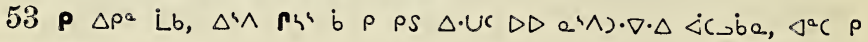
Dr $p) \cup^{\circ} \times$

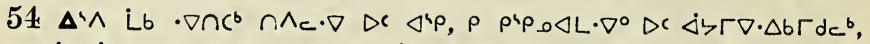

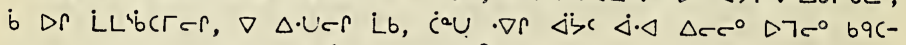
- $\nabla c c\lrcorner \Delta \sigma c^{\circ}, \sigma^{s c} \Delta D$ LL'br $<j i q \Delta a$ ?

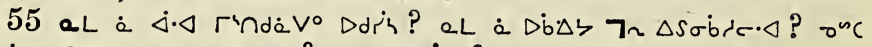

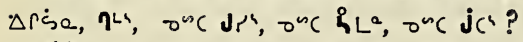

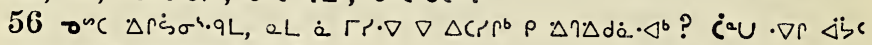

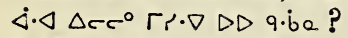

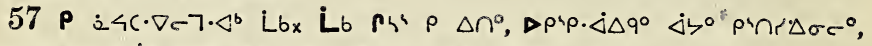

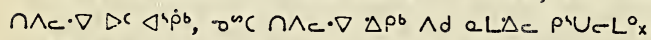

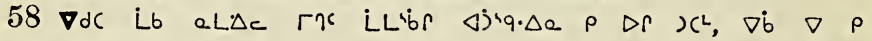
$\dot{c} \cdot \mathrm{V} \in T r r_{x}$

Aיpcre $\Delta b^{\circ} 14$.

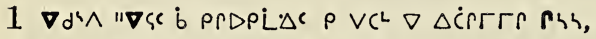

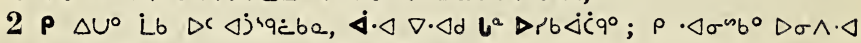

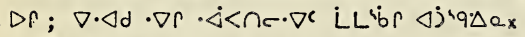

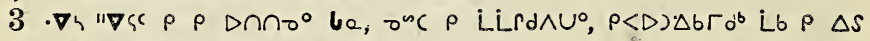
$<P \cap 0^{\circ} " \Delta ? \cap S^{\prime} \Delta r, \Delta r \dot{s}$ a $\Delta_{c}<\Delta \Delta r \cdot \Delta x$

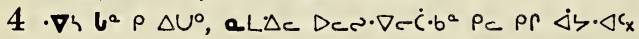

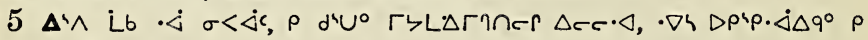
$\Delta U \leftarrow \cdot c \cdot \Delta x$

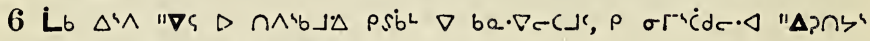
$\Delta \dot{C} \sigma \zeta, \rho a \Delta c \cdot \nabla \Delta d c \cdot \triangleleft \dot{L} b " \nabla \varsigma_{x}$

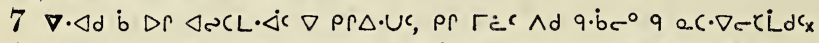

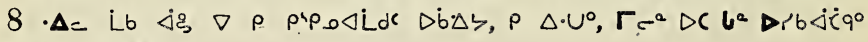
$D^{\prime} n \cdot b^{a} D \dot{c} b \sigma^{b} x$

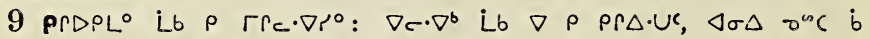

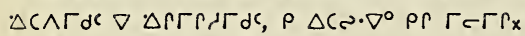

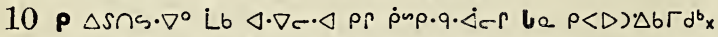

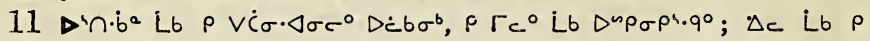
v) $\cdot \nabla^{\circ} \Delta \dot{b} \Delta b x$

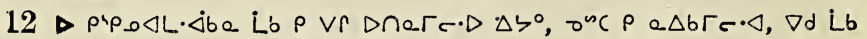
$O C \Delta \triangle C L \cdot \dot{C} C \Gamma M B X$ 
$13 \Delta^{4} \wedge$ Lb $\left.m^{4} \wedge \nabla C^{b}, \Delta^{a} c \rho D r \rho\right) U^{0} a \wedge \cdot b \sigma^{b}<\cdot b\left(b \Gamma b^{b} \nabla \Delta\right) U<<b^{a}$ :

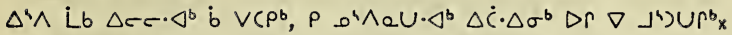

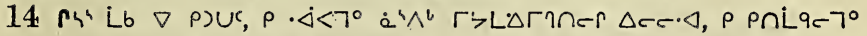
Lb, $\rho \Gamma a \cdot \Delta r \Delta 7^{\circ}$ Lb $\Delta<\dot{\Delta d r} \Gamma \cdot \Delta \cdot \Delta x$

$15 \Delta^{i} \wedge L b \cdot \nabla \dot{C} d S^{b}, \rho \vee r$ and $D$ pip.o $\Delta L \cdot \dot{b} b e, \rho \Delta n d \quad L b,<\cdot b c^{4} b \Gamma b^{\circ}$

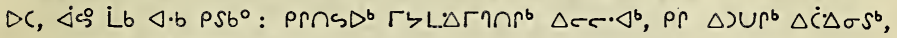

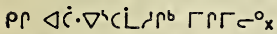

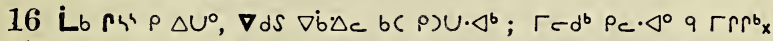

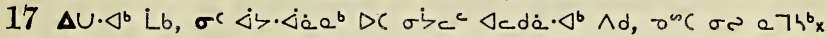

$18 p \Delta \cdot v^{\circ}, \Delta(V) \Delta^{b} x$

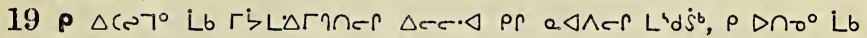

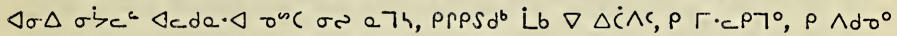

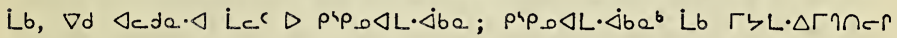
$\Delta r c \cdot \Delta x$

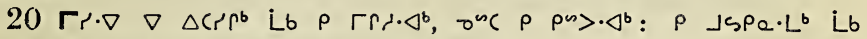

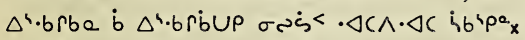

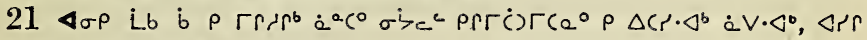

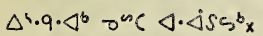

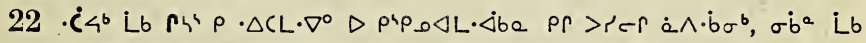

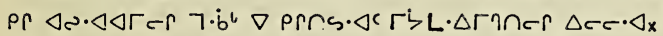

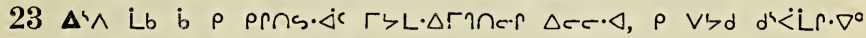
- $\Delta \dot{r}^{b}: \Delta 4 \wedge$ Lb $\nabla \cap \Delta \dot{C} d S^{b}, \Delta d C \nabla \dot{C} C \nabla \vee b d^{d} x_{x}$

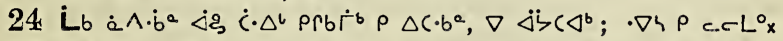

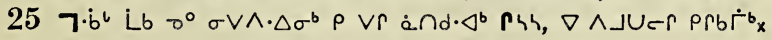

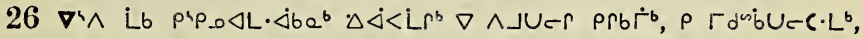

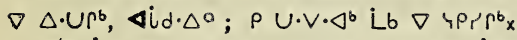

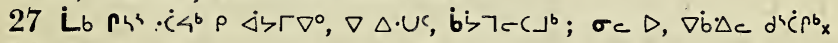

$28 \wedge c^{s}$ Lb $\nabla$ a $a^{n . q .} \Delta S \Delta c \rho \Delta U^{0}, U \vee c r q b^{a}, \dot{\rho}^{n} \wedge^{a} \rho_{c}, \Delta C L \Delta^{a} \rho r$ $\dot{\mathrm{e}} \dot{\mathrm{C}}^{\mathrm{a}} \sigma \dot{\lambda}^{\mathrm{b}} \mathrm{x}$

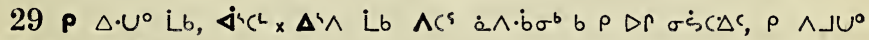
$\sigma \dot{\lambda}^{b}$, pr aंc $\operatorname{mihx}$

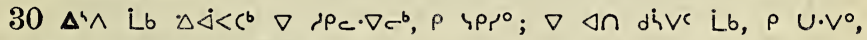

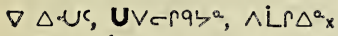

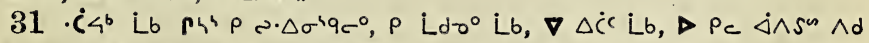

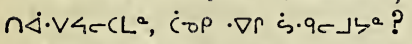

$32 \Delta^{i} \wedge$ L $b \quad b>r r^{b} a \wedge \cdot \dot{a} \sigma_{b}, \rho>\sigma c \cdot \nabla^{\circ} x$

$33 \nabla d \dot{L} b \wedge \nabla r$ and $\triangleleft \sigma \Delta \dot{a} \wedge \cdot b \sigma^{b}$ b $\Delta \dot{C} e r, \rho a \cdot \Delta \dot{\rho} r \dot{C} d \dot{L} b, \nabla \Delta \cdot U=r$, C.V $\left.\rho_{C} P \rho L \sigma\right) D d P h x$

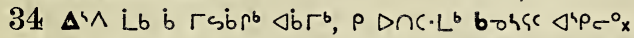

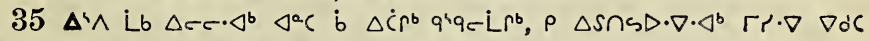

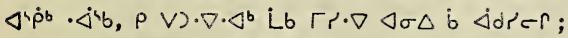

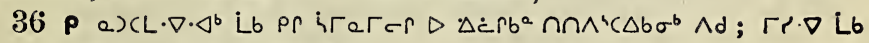

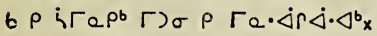


Ápcra $\triangle b^{a} 15$.

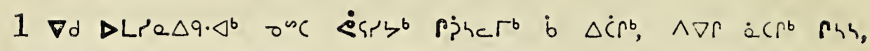
$\nabla \Delta \cdot u^{b}$,

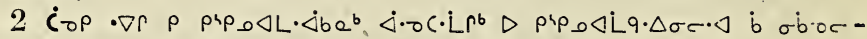

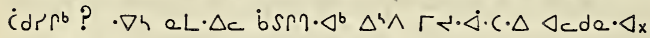

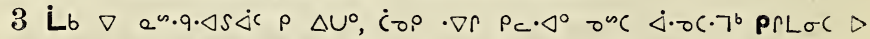

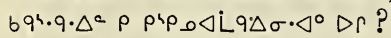

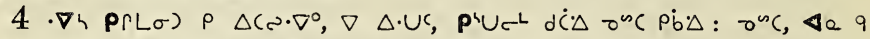
LLPLC $D \dot{C} \Delta \zeta \sigma^{\omega} C \wedge d \Delta \dot{b} \Delta \zeta, \nabla d S$ bC $\sigma<<^{\circ}$ x

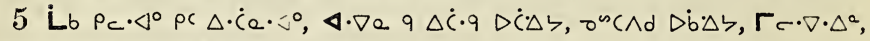
J L $q \Delta S p^{r} \dot{C}<r \Delta \dot{b} \cdot \Delta \zeta^{a} \sigma c \Delta r$;

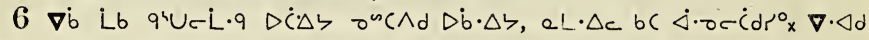

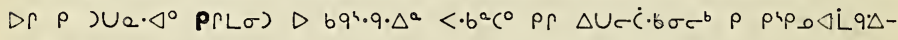
$\sigma \cdot \triangleleft^{\circ} \Delta \Gamma_{x}$

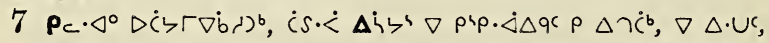

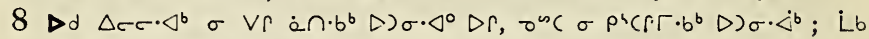
$\Delta \cup \Delta \cdot \dot{\Delta} \cdot \triangleleft$ a $c \cdot \dot{\Delta<}<r d \cdot \dot{\Delta}^{a} x$

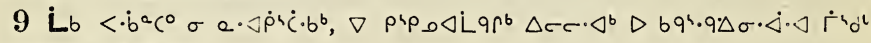

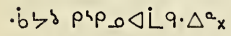

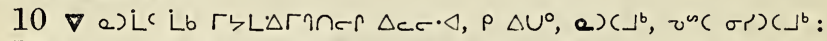

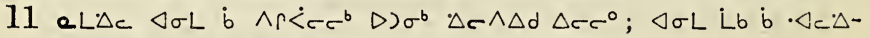

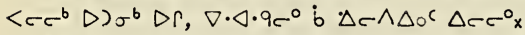

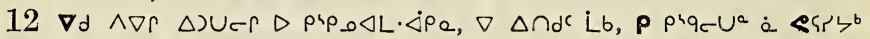
$\nabla \rho \dot{a}<c \cdot \nabla e c \rho b, \dot{b} \vee\left(\rho b \triangleleft \sigma \nabla c^{\circ} \Delta \cdot U \Delta \sigma c^{\circ}\right.$ ?

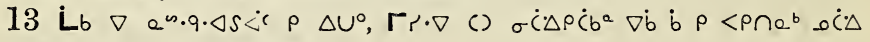

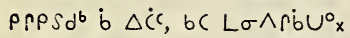

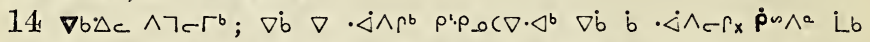

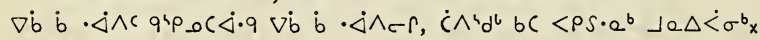

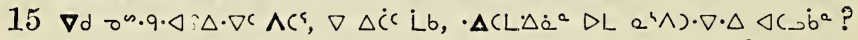

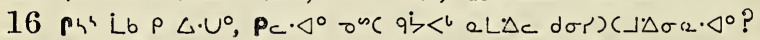

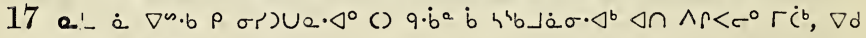
$\nabla \triangleleft n \cdot \Delta c \Delta<c^{b}$ ?

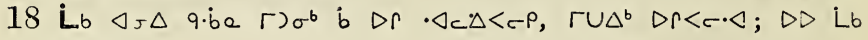
$\Delta c \wedge \Delta d \Delta c c^{\circ} x$

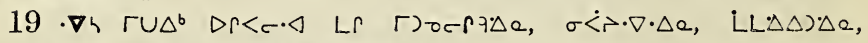

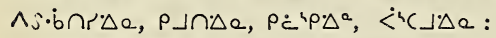

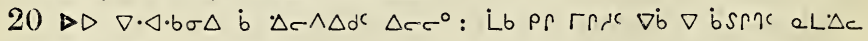
$\Delta \sim \wedge \Delta d \Delta c c^{\circ} x$

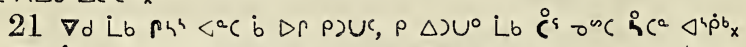

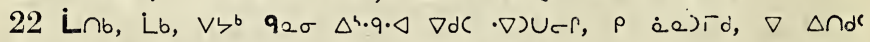

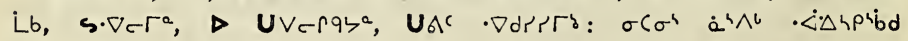
LS $\Delta i \cdot b_{x}$ 


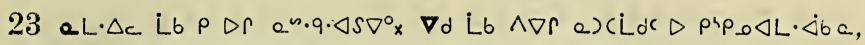

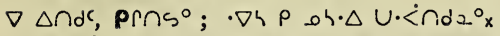

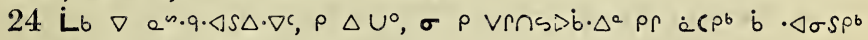

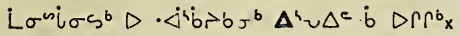

$25 \nabla d \wedge \nabla r a \cdot \Delta \rho^{r} c \cdot \dot{<c}, \nabla \Delta \cdot U c, U \vee r e q b^{a} \cdot \Delta r \Delta^{a} x$

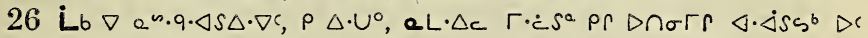

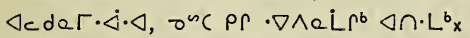

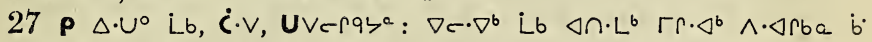

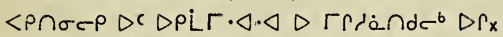

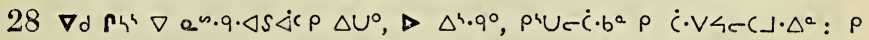

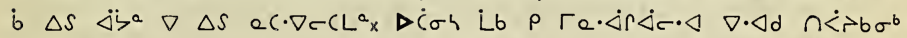
$\wedge r\lrcorner b \sigma^{b} x$

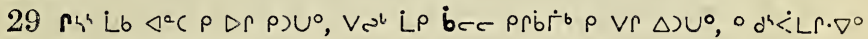
Lb $\cdot \Delta \dot{r} b, \nabla d C$ L $b \quad \nabla \Delta \wedge c_{x}$

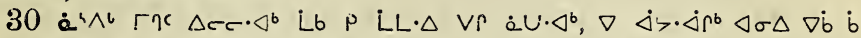

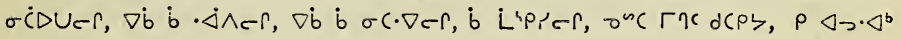

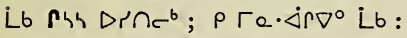

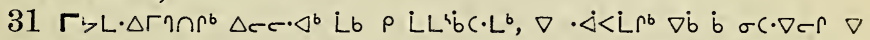

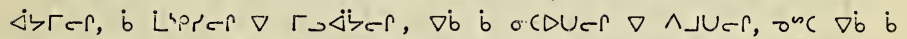

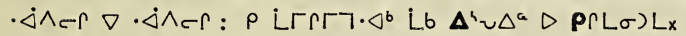

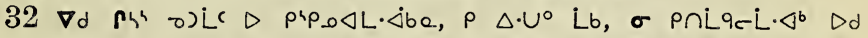

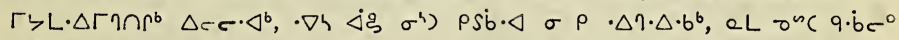

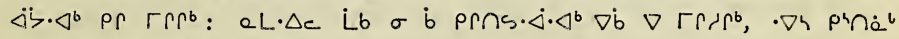
bC..$\cup S \cdot 0^{b} 7 \cdot b^{6} \nabla \wedge J U r^{b} x$

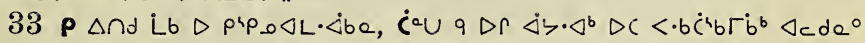

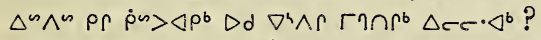

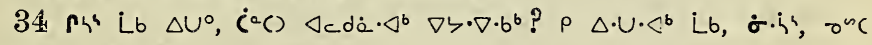
ince a $7 c^{b} x$

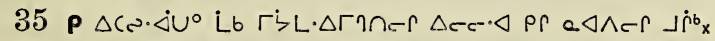

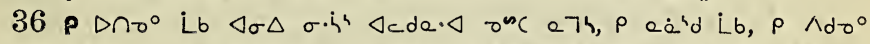

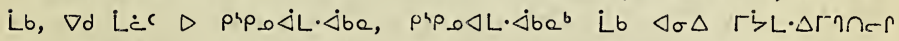
$\Delta c r \cdot \Delta x$

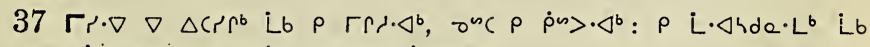

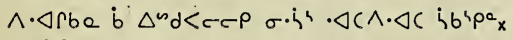

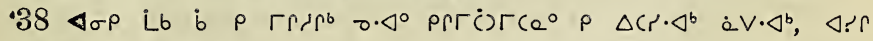
$\triangle 4 \cdot q \cdot \Delta^{b} \nabla^{n C} \mathrm{C} \Delta \cdot \Delta s s^{b} \mathrm{x}$

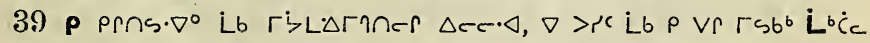
$\checkmark \dot{p} b_{x}$

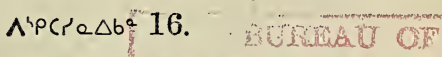

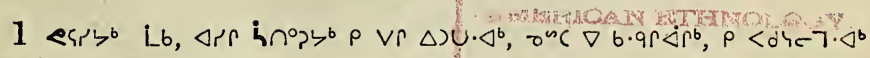

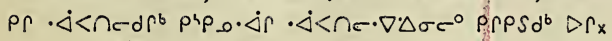

27 


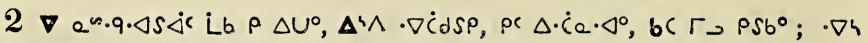
$\Gamma \cdot b \cdot \Delta^{4} \cdot 6^{2} x$

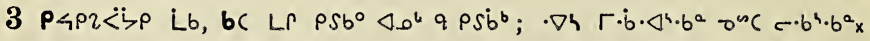

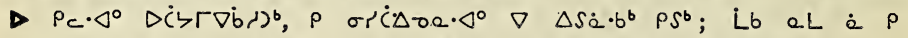

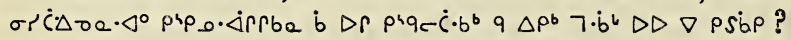

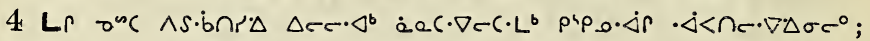

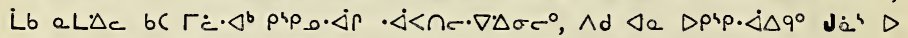

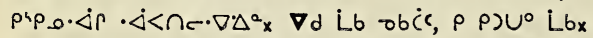

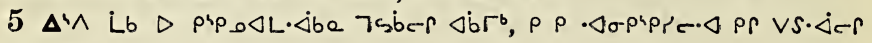
$\triangle c d a \cdot \Delta x$

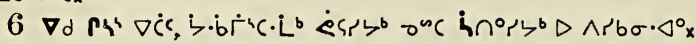

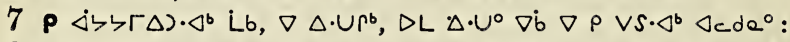

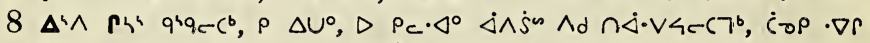

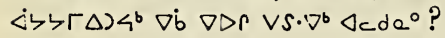

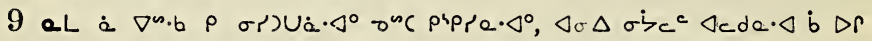

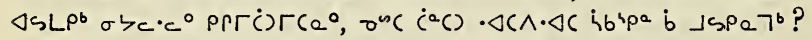

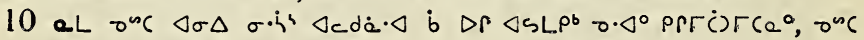
$\dot{c}^{a}() \cdot \Delta C \wedge \cdot \Delta C$ ibspa b jspa $]^{b}$ ?

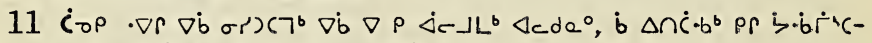

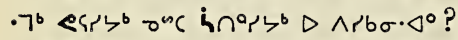

$12 \nabla d$ or)

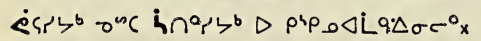

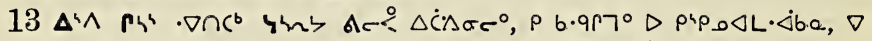

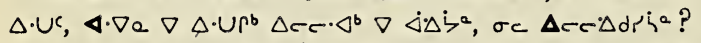

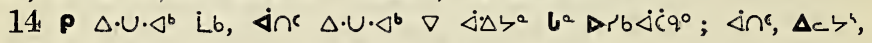

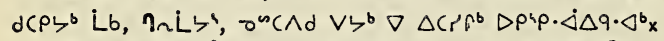

$15 \Delta U^{\circ} L_{b}, L_{b} \triangleleft \cdot \nabla a P_{c} \cdot \triangleleft^{\circ} \nabla \cdot \cup \cup^{b} \nabla \Delta \Delta \Delta^{\circ}$ ? ?

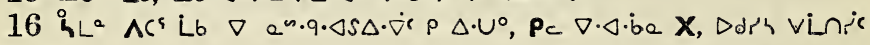
PSLO $)_{x}$

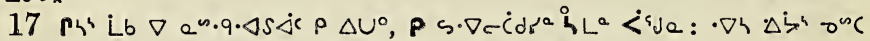

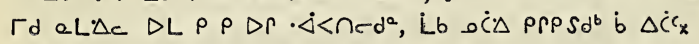

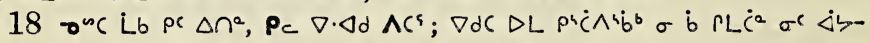
$\Gamma \nabla \Delta b \Gamma^{b} ; a L \Delta c$ Lb bC $\dot{c} d r \Delta d L b^{\circ} L \Gamma \Delta^{n} d U^{\circ} \Delta^{n}$.bULx

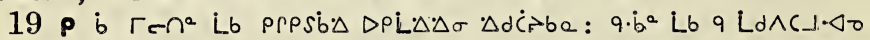

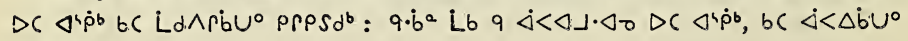
PRPS dbx

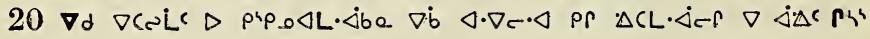
⿶e $\boldsymbol{X} \mathbf{x}$

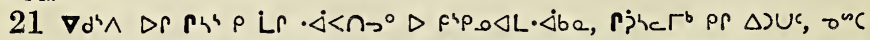

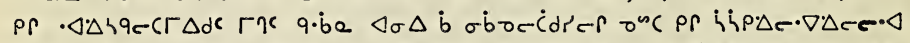

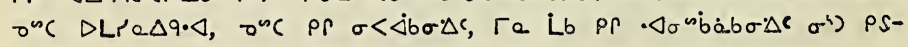
be q $^{x}$ 
$22 \nabla d \wedge c^{s} \cdot \nabla \cap \dot{a} c, \nabla \Delta P C \Delta L \cdot \dot{c}, \nabla \Delta \cdot U c, \Delta L \cdot \dot{<} c^{0} b c \Delta \Delta c \cdot b^{a}, U V-$ -rqל०: $D L Q L \Delta c \rho$ b $D \cap \cap d^{2} x$

$23 L_{b} \nabla \cdot q^{4} p b<\Delta c, \rho \Delta U^{0} \wedge\left(c, \sigma^{4} \wedge^{4} \cdot b \sigma^{b} \Delta S \Delta\right) U, 4 c^{a}: \rho: 4 c \cdot \nabla c c-$

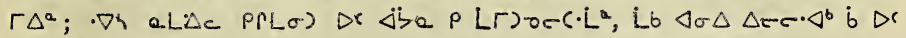
$\Delta \dot{\zeta} \sigma \rho^{b} b_{x}$

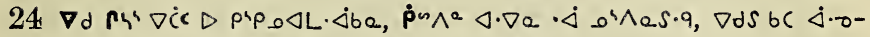

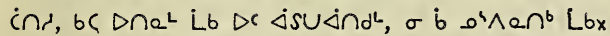

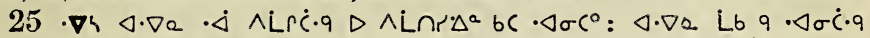
$\Delta \wedge L \cap \Delta^{2} \sigma c \Delta r, b c r^{4} b L^{2}$

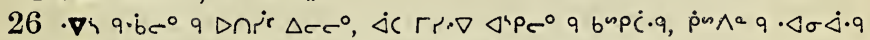

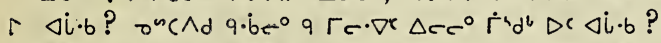

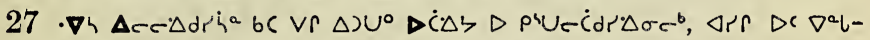

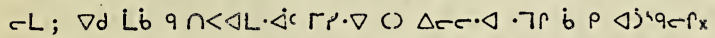

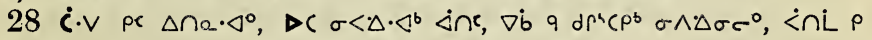

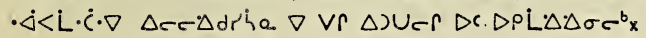

\section{A'pcre $\triangle b^{\circ}$ 17.}

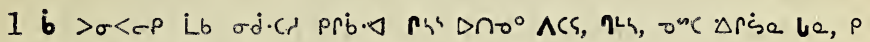

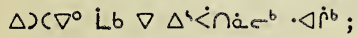

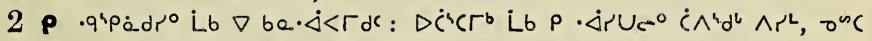

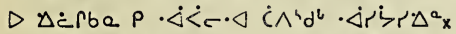

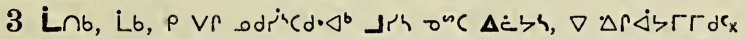

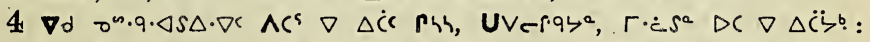

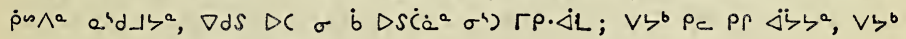
o"C $\perp$ "

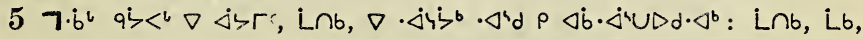

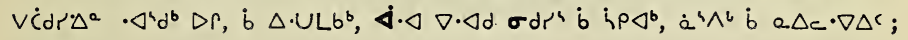
a) $\dot{c} \Delta^{b} \mathrm{x}$

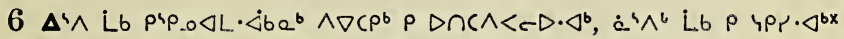

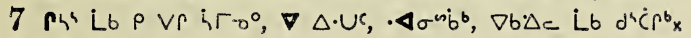

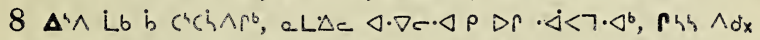

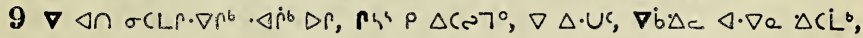

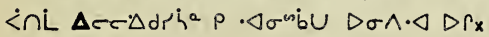

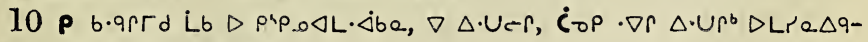

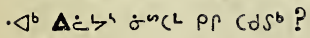

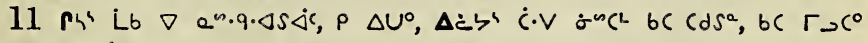
Le $T$ r. $\nabla 9 \cdot \dot{0}$ a:

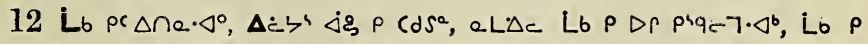

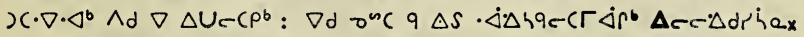

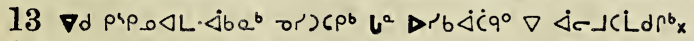

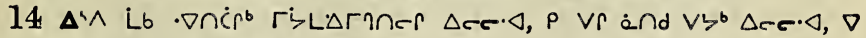
Drr.be $\dot{\wedge} \dot{C} d c, \nabla \cdot \Delta$ nde Lb, 


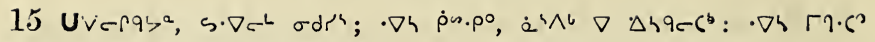

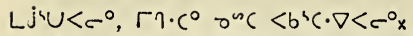

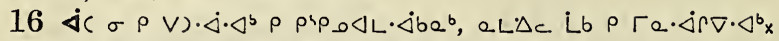

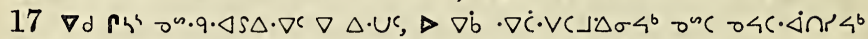

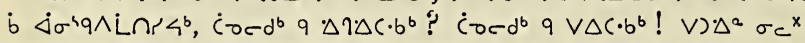

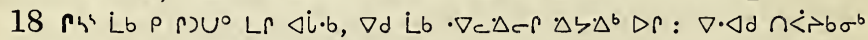

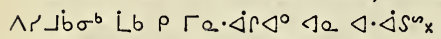

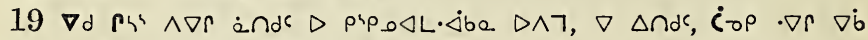

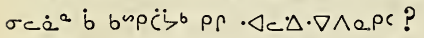

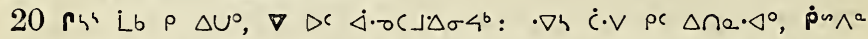

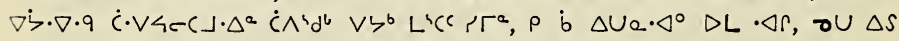

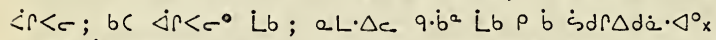

21 Lb $\Delta L) \cdot \Delta$ aL $\Delta c \cdot \Delta c \cdot \Delta<c^{\circ} \Delta b \Gamma \Delta \cdot \Delta^{a}$ onc $\sigma b r \cdot \Delta^{a} \Delta r \wedge d x$

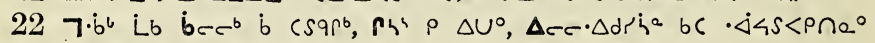
$\Delta c c \cdot \triangleleft \Delta R r^{\circ} c^{b} ;$

23 bc $\sigma<\nabla \cdot \triangleleft^{b}$ Lb, $\left.\sigma^{s}\right)$ psibeq Lb $\Gamma a$ bc $\triangleleft \sigma^{s} b a^{\circ} x$ a $\wedge^{\wedge} b$ Lb $\rho$ $\Gamma r_{c} \cdot \nabla r \cdot \Delta^{b} x$

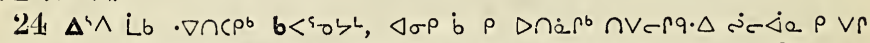

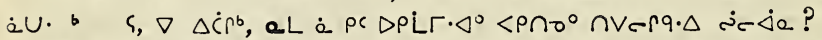

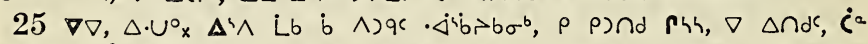

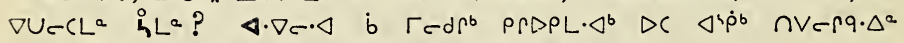

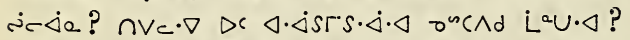

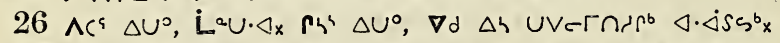

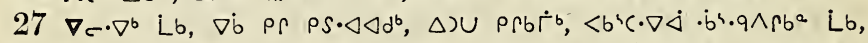

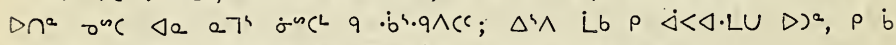

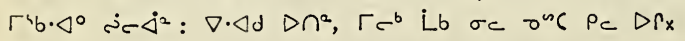

\section{A'pcra $\triangle b^{\circ} 18$.}

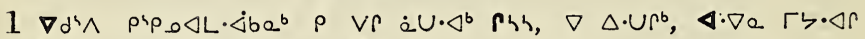

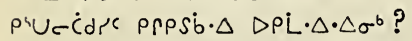

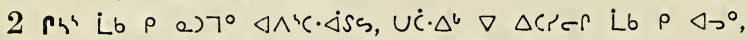

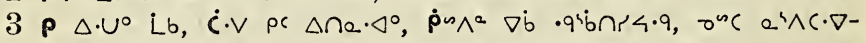
$\left.\cdot \dot{b} \cdot \nabla \Delta \Lambda^{4} c \cdot \Delta S S^{b}, a L \cdot \Delta c \rho \dot{b} \wedge\right) \dot{b} e \cdot \Delta^{\circ} \rho S \rho S b \cdot \Delta \Delta \rho L \cdot \Delta \cdot \Delta \sigma^{b} x$

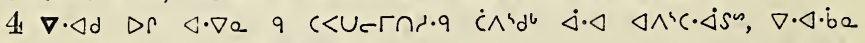

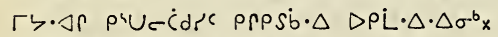

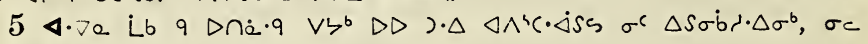
$\sigma^{c} \Delta \cap \sigma^{b} x$

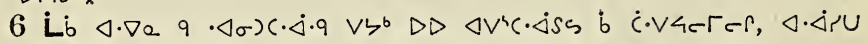

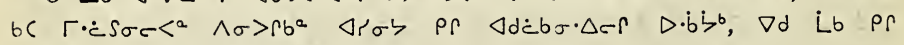

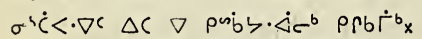

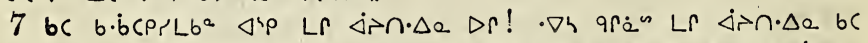

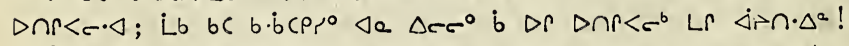

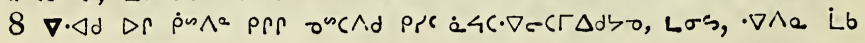
30 


\section{$\triangleright<\rightarrow$ p' L̊० 18.}

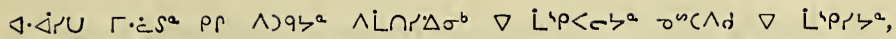

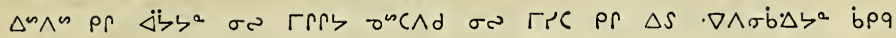
$\Delta n d U^{b x}$

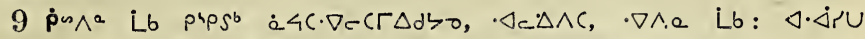

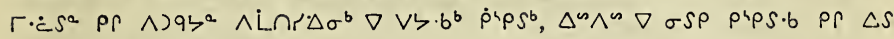
$\cdot \nabla \wedge \sigma \dot{b} \Delta \zeta^{a}$ Lr $\Delta u d U^{b} x$

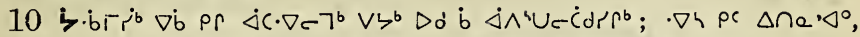

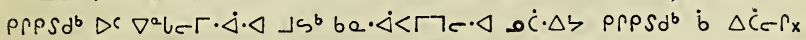

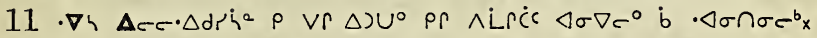

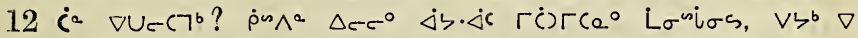

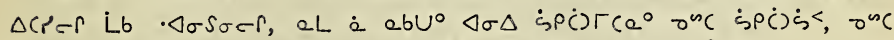

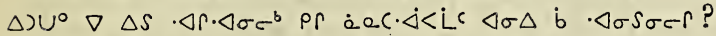

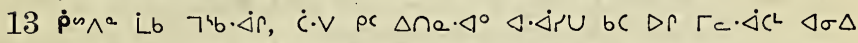

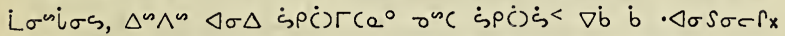

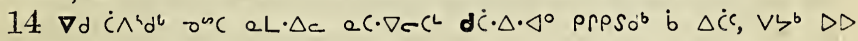

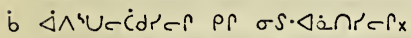

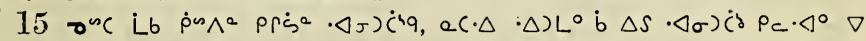

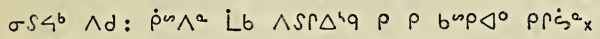

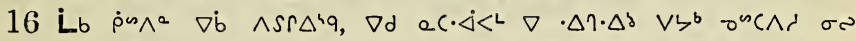

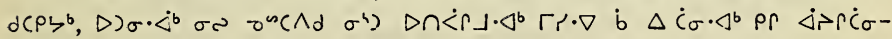
$\triangleleft \sigma c^{b} x$

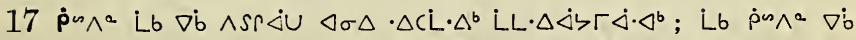

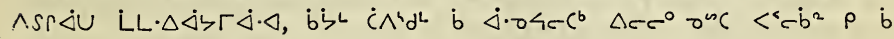
$\triangle U C L^{\circ} \times$

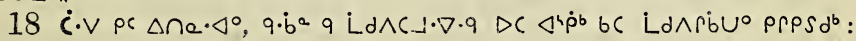

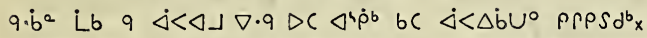

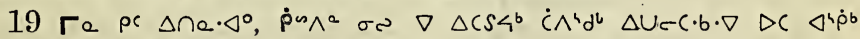

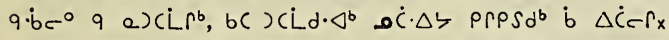

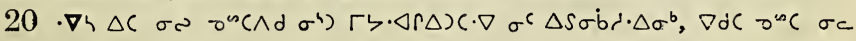
$9 \Delta \ddot{C}^{0} \times$

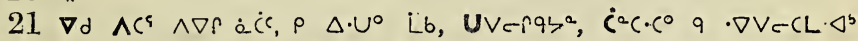

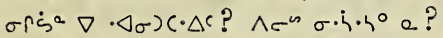

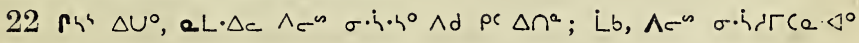
$\sigma \cdot i^{4} x$

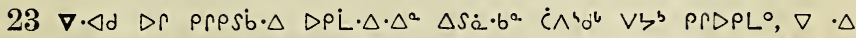

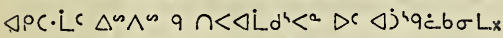

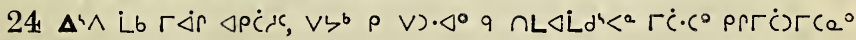
$\dot{\mathrm{C}} \mathrm{C}^{\mathrm{a}} \mathrm{C}$ :

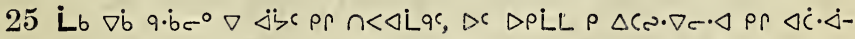

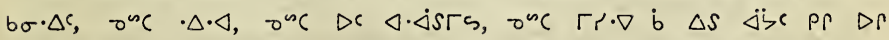
$n<\Delta L \operatorname{Lod}^{\prime} \mathrm{P}_{\mathrm{x}}$

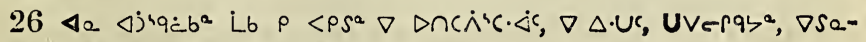

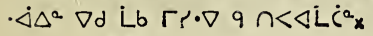




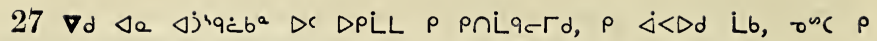
$\cdot \nabla V c C$ Ld $\Gamma r \cdot \nabla D$ Lre $\Delta b^{a} x$

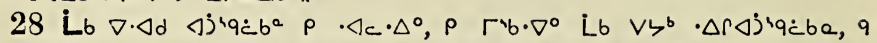

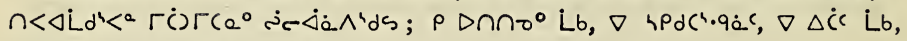
$n<\Delta L \cdot \Delta^{a} q \cap<\angle L \cdot \Delta b<^{a} x$

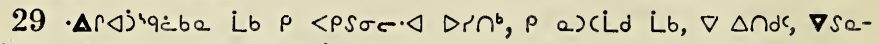

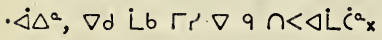

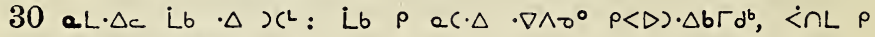
$n<\Delta r e r \Delta L R Q \Delta b^{2} x$

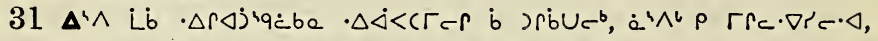

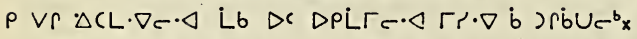

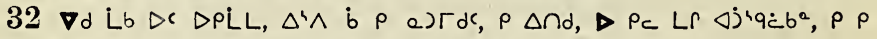

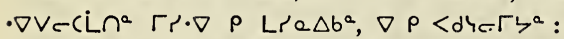

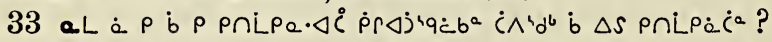

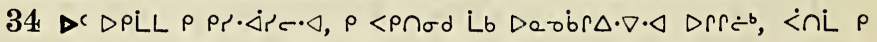
$n<\Delta q \Gamma r \cdot \nabla \dot{b} \Delta S$ Lre $\triangle q c_{x}$

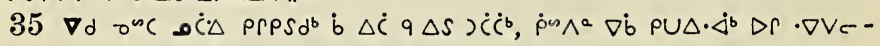
$\left.\mathrm{CL} \cdot \nabla \cdot b \cdot \nabla \rho P \operatorname{sis} \sigma \cdot \dot{\Delta} \cdot \Delta^{b} \nabla \cdot \Delta \sigma\right) \dot{C} \dot{C} \cdot b^{b} x$

\section{$\Lambda^{\prime} p\left(r \circ \triangle b^{\circ} 19\right.$.}

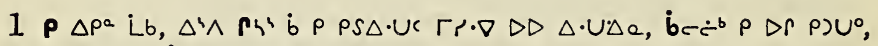
$\rho$ VR $\triangle U^{\circ}$ Lb ins $<^{4} \dot{b} b \Delta \dot{b} \Gamma^{b} l^{c} C \sigma^{b}$ :

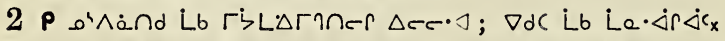

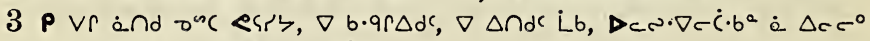
br $\cdot \nabla \wedge \dot{a}<\Delta \cdot \Delta T r \cdot \nabla q \cdot \dot{b} c_{0} \nabla r$ ?

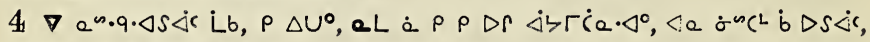
$\rho \triangle S \nabla^{\circ} \mathrm{i} V \cdot \triangleleft \nabla^{\infty} \mathrm{C} \Delta^{4} \cdot q \cdot \triangleleft$,

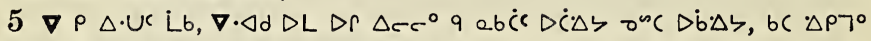
Lb $\Delta \cdot \triangleleft ; \Delta d$ Lb $\nabla \sigma S \rho^{b} q$ Vלd $\Delta \dot{\zeta} r r^{b} \times$

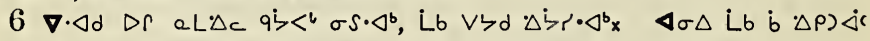
$\rho \rho L \sigma), \nabla \dot{b} \Delta c \Delta a c^{\circ} b\left(<\ddot{b} \sigma \nabla^{\circ} x\right.$

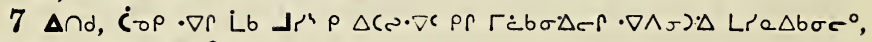
$\nabla d$ Lb $\operatorname{~pr} \cdot \nabla \wedge \sigma \Gamma r$ ?

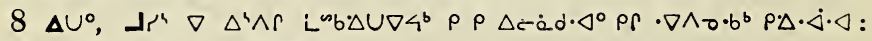
$\sigma^{n C L} \Delta r$ Ĺb eL $\Delta c$ Dr $\triangle p^{a} x$

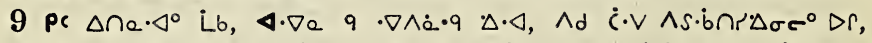

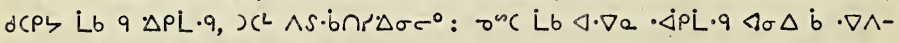

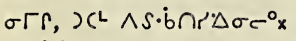

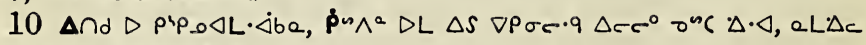
$r \cdot \dot{c} S^{a}$ pr $\left.\Delta \rho\right) \dot{\alpha} \sigma \cdot \Delta^{b} \mathbf{x}$

11 Lb $\rho \Delta U^{\circ}, a L \Delta c \Gamma r \cdot \nabla \Delta c c \cdot \Delta^{b} \rho \Delta \Omega_{a} \cdot L^{b} \nabla 7 \sigma^{\circ} \Delta \cdot U \Delta \sigma \sigma^{\circ}, \Delta \sigma \rho$ $\Lambda_{d} \dot{b} \Delta S \Gamma \dot{c} b \sigma \Delta r^{b} x$ 


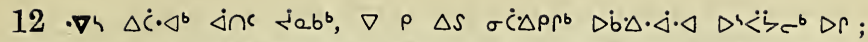

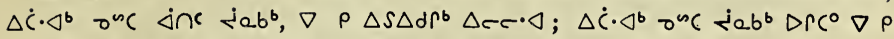

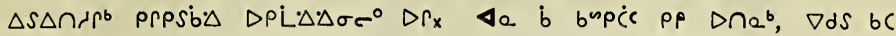
$D \cap e^{L x}$

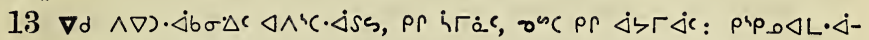
ba ${ }^{b}$ Lb $\rho$ PC $\Delta L \cdot \nabla \cdot \Delta b x$

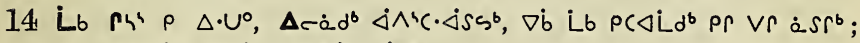
. $\nabla 4 \nabla d) \Delta \rho \rho \rho S i b \Delta D \rho \dot{L} \Delta \Delta \sigma^{b} \dot{b} \Delta \dot{C}^{b} \mathrm{x}$

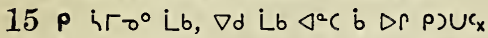

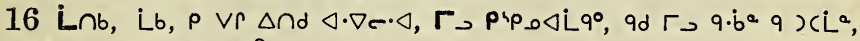

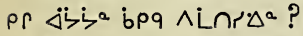

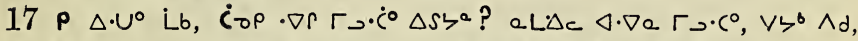

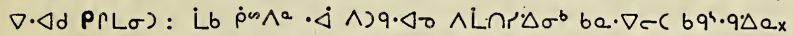

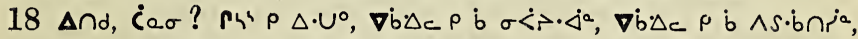

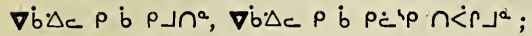

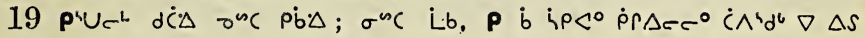
ip $\Delta \cap$ rbax $^{x}$

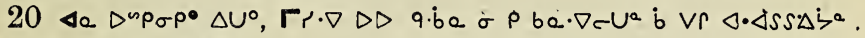
$\therefore \cdot b^{a} q \dot{b}<b \quad 0 U<-\dot{i} a$ ?

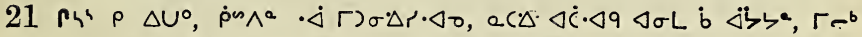

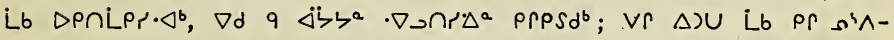
essax

22 Lb $\Delta^{b} \wedge \Delta \backsim \rho_{\sigma} \rho^{\circ} \wedge \nabla c^{b} \triangleleft \sigma \nabla \sigma^{\circ} \Delta \cdot U \cdot \Delta \sigma \sigma^{\circ}, \rho \rho j U^{\circ} \nabla \Gamma \Gamma_{c} \cdot \nabla r^{c} ; \cdot \nabla h$ pr $\nabla \rightarrow \cap n \cdot \Delta a . \rho<\zeta^{\circ} x$

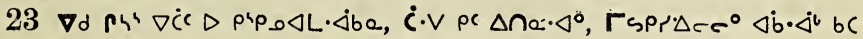
$\wedge 9^{\circ}$ PrPSib $\triangle$ DPL $\Delta \Delta \sigma^{b} x$

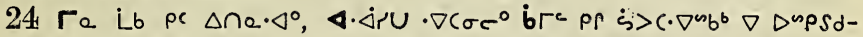

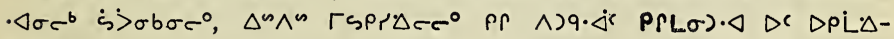
$\Delta \sigma c^{b} x$

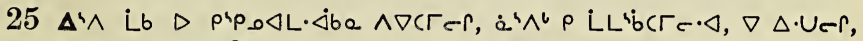
$4 \cdot \nabla a$ Lb $9 \rho \wedge$ L $P D C ?$

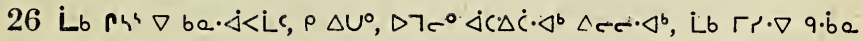
$\cdot \nabla\left(\sigma \sigma^{\circ} P \Gamma L \sigma\right)_{x}$

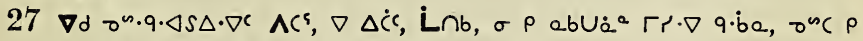

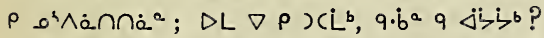

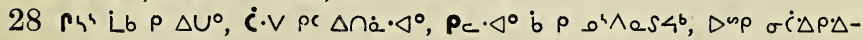

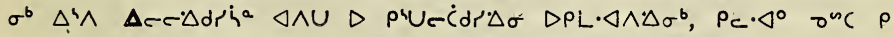

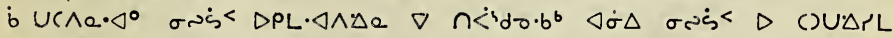
$\Delta \cdot v \Delta^{a x}$

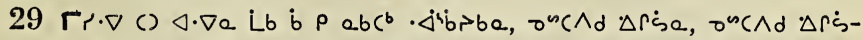

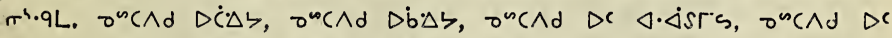

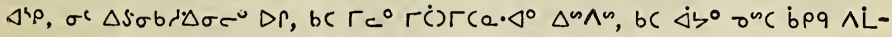
ni. $\Delta \sigma \sigma^{\top} x$ 


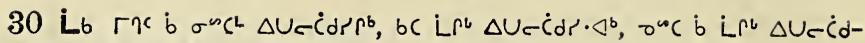

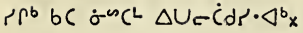

\section{A'pCra $\triangle b^{\circ} 20$.}

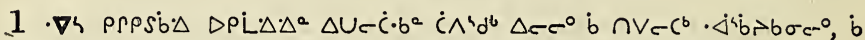

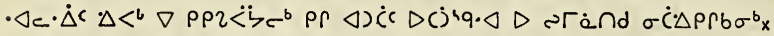

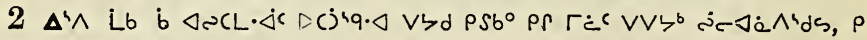

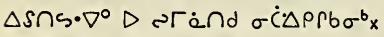

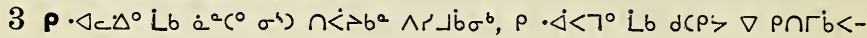
$\Delta r \Delta \Delta(\nabla \triangleleft \dot{C} r) \dot{\mathrm{a}} \sigma^{\circ} \cdot \Delta^{b}$,

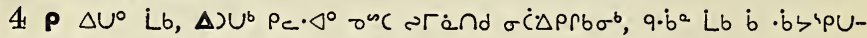

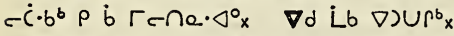

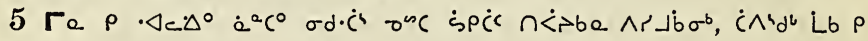
$x^{\mathrm{L}} \mathrm{x}$

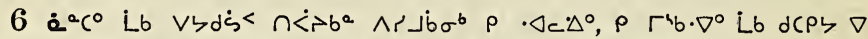

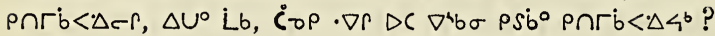

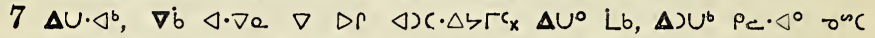

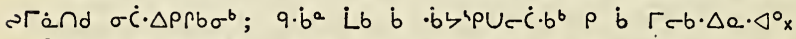

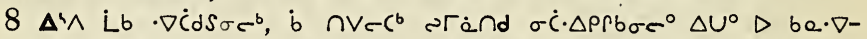

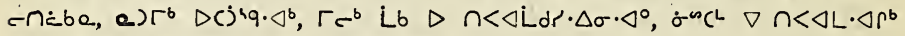

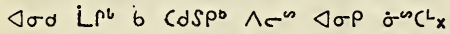

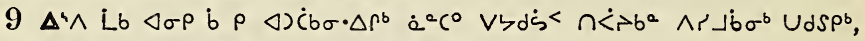

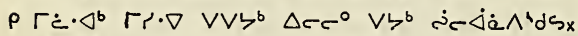

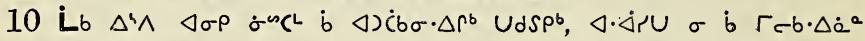

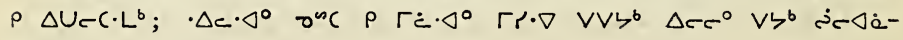
$\Lambda^{\prime} d \mathrm{~d} x$

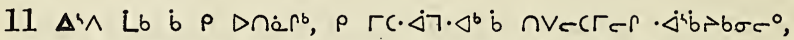

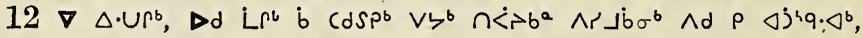

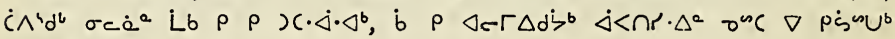
$\nabla \mathrm{V} b \sigma \rho \mathrm{C}^{\circ} \mathrm{x}$

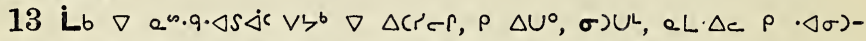

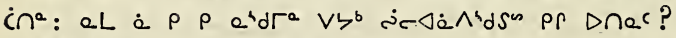

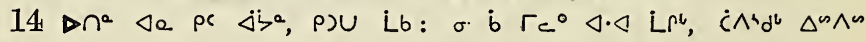
Pсx

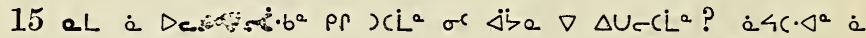

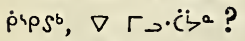

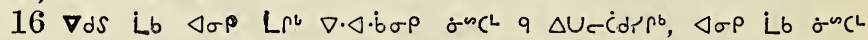

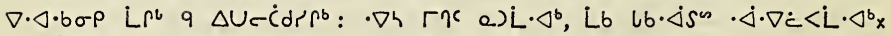

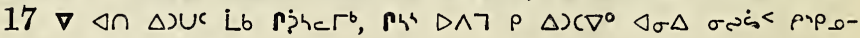

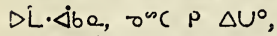

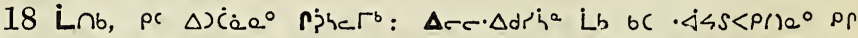

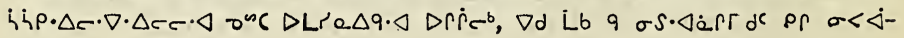
$b \sigma \cdot \Delta c$ 


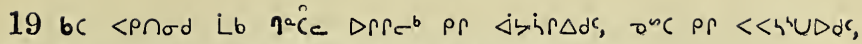

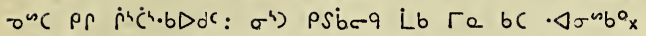

$20 \nabla d \wedge \nabla r$ ande $\Delta \dot{b} \cdot \Delta c \cdot \triangleleft 4 \wedge \cap \Delta c \triangleleft \cdot \Delta s \Gamma s \quad \Delta d r h \quad \varangle r f, \nabla a \cdot \triangleleft-$ picide, $\sigma^{\infty} \mathrm{C} \nabla$ ec $\nabla e c L d c q \cdot \dot{b} c^{\circ} x$

$21 P \Delta U^{0} L b, 9 \cdot b^{a} o C \cdot \nabla c C L^{a} ? \Delta \cap d, \Gamma c^{b} \Delta d \sigma d r r^{b} b$ os $\rho^{b} \rho f$

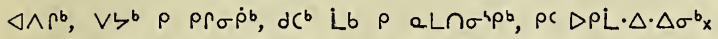

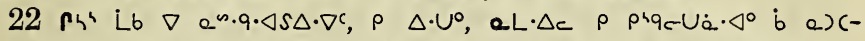

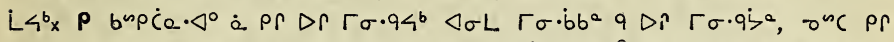

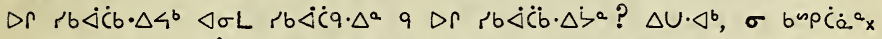

$23 \Delta U^{\circ} \dot{L} b, \dot{C} \cdot V \rho \dot{b} \Delta r \Gamma \sigma \cdot \dot{b} a \cdot \triangleleft^{\circ} \sigma \Gamma \sigma \cdot \dot{b} b \sigma^{b}$, onc $\rho \dot{b}$ Dr rb $\dot{\Delta} \dot{C} b-$

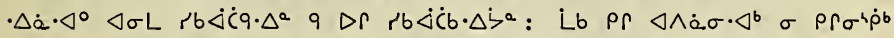

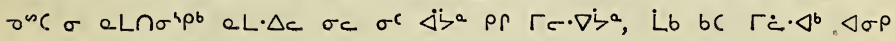
b. $\dot{\Delta}\left\langle^{4} b \cdot \Delta C L d \rho^{b}\right.$ o $\dot{C} \cdot \Delta b x$

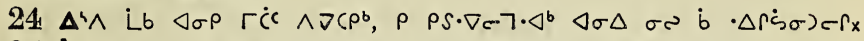

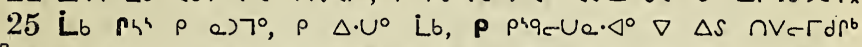

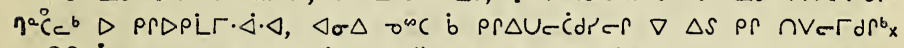

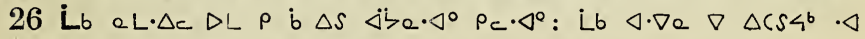

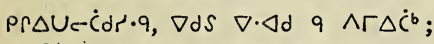

$27 \triangleleft \cdot \nabla e \nabla \Delta C S<^{b}$ Lb $\cdot \dot{\triangleleft} \Delta P L \cdot \Delta \cdot q, \nabla \cdot \Delta d q \Delta C \Delta j 4 q c b \sigma \cdot \Gamma<b$ :

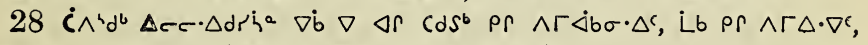

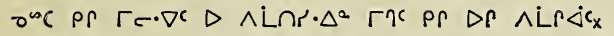

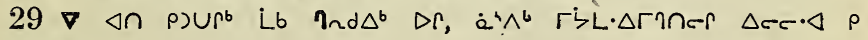

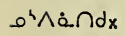

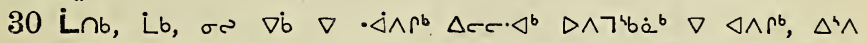

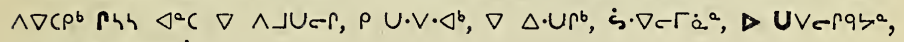

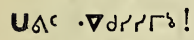

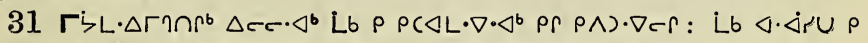

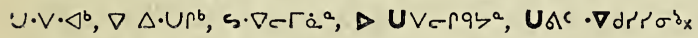

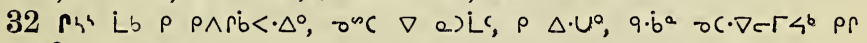
$x \dot{c} \cdot b^{b}$ ?

$33 \Delta U \cdot \triangleleft^{b}, U V e r q b^{a}$, ospsdie $p r<4 p U e L^{a} x$

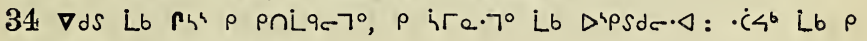
$\cdot \dot{\Delta \wedge \dot{\Delta} \cdot \triangleleft^{b}, \rho} \rho^{4} \wedge a U \cdot \triangleleft^{b} L b x$

\section{$A^{s} p c r e \triangle b^{a} 21$.}

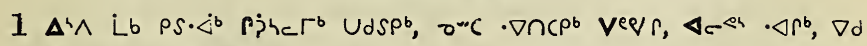

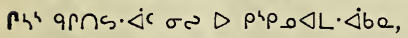

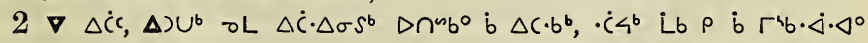

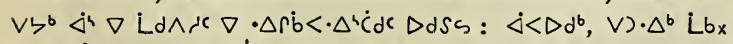

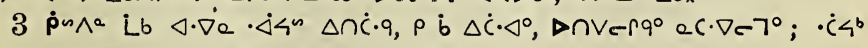
Lb oc Vrns. $\nabla^{\circ} x$

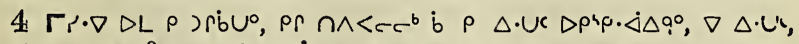

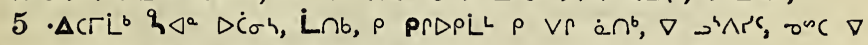

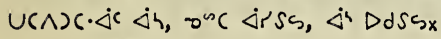




\section{$\triangleright<>\rho^{4}$ Lดค 21.}

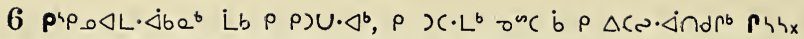

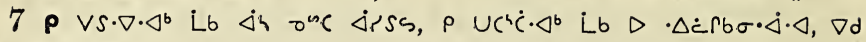
Lb $\cap \nabla C \dot{C} \Gamma^{b} \mathrm{x}$

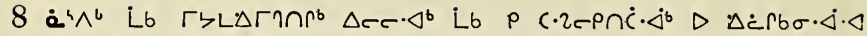

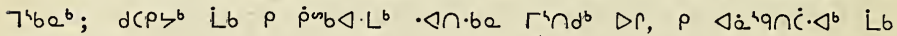
$7^{4} b a^{b} x$

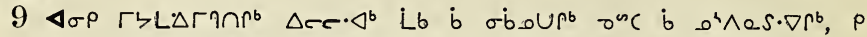

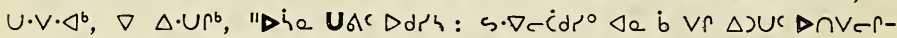

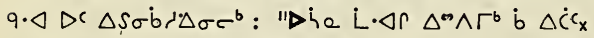

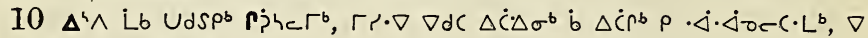
$\Delta \cdot U r^{b}, \triangleleft \cdot \nabla e \triangleleft \cdot \triangleleft ?$

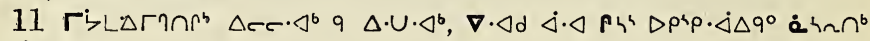
$\operatorname{Dr} \dot{b}_{e} c^{b} x$

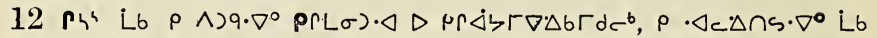

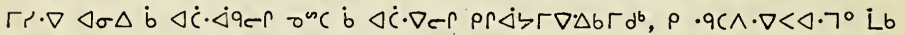

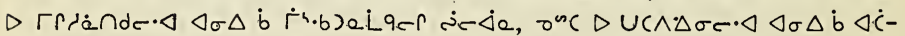
- jiqer $\operatorname{dr} \cdot \triangleleft$,

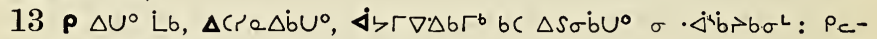
$\left.\cdot \triangleleft^{\circ} \mathrm{L} b \rho\right\lrcorner \cap^{6} b^{b} \nabla \cdot \Delta \dot{\Delta} \cap \cdot \nabla^{\circ} \rho \rho \Delta S \dot{C} a \cdot \triangleleft^{\circ} x$

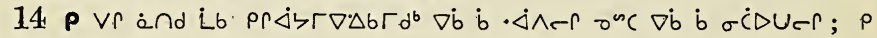
$\Gamma e \cdot \Delta r \nabla^{\circ} \mathrm{L} b x$

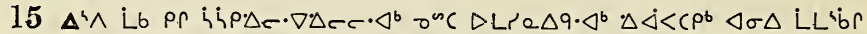

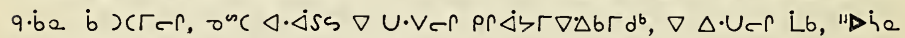
UAC $\triangle$ drh; $\dot{a}^{4} \wedge^{6}$ QL $P$ Q $Q \nabla C C \cdot L^{b}$,

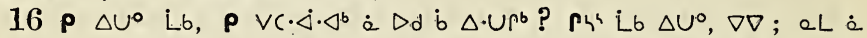

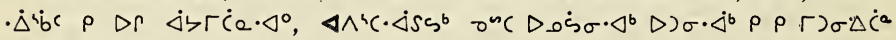
LLRT. $\nabla \cdot \Delta^{\circ}$ ?

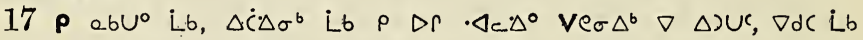
Usq.

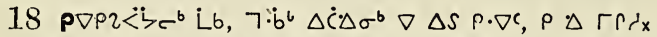

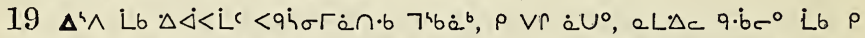

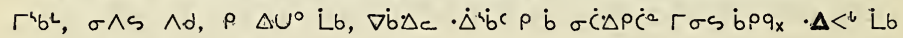
$<q h \sigma \Gamma \dot{a} \cap^{b} \rho \triangleleft \cap \dot{<} \dot{x}$

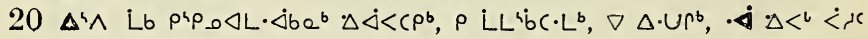
$\dot{\triangleleft} \triangleleft<q \dot{i} \sigma \Gamma \dot{a} \cap^{b}$ !

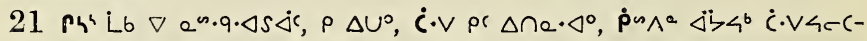

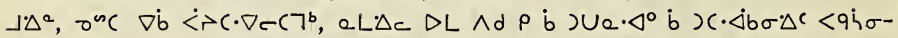

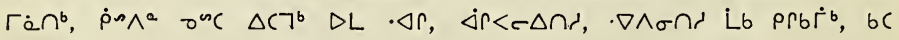
sigivex

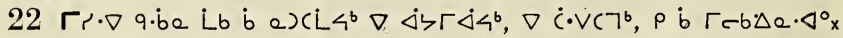

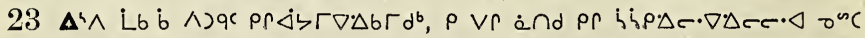

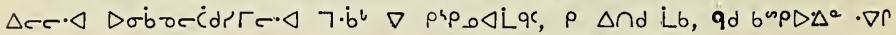

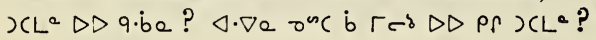




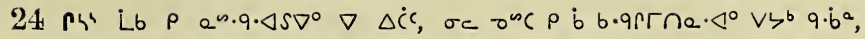

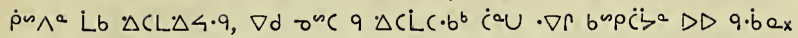

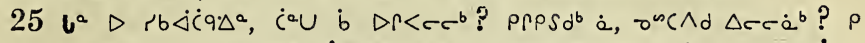

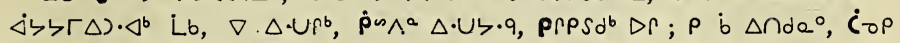
Lb $\cdot \nabla r \nabla \dot{b} \rho \dot{C} \cdot V C \cdot \nabla^{b}$ ?

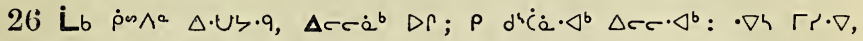

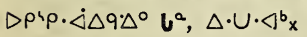

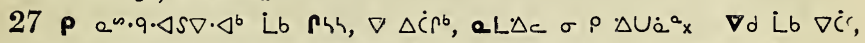

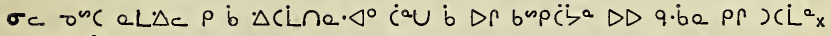

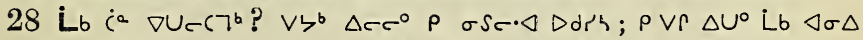

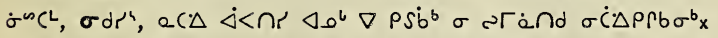

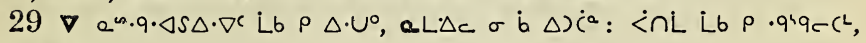
$\rho \Delta U^{0} L b_{x}$

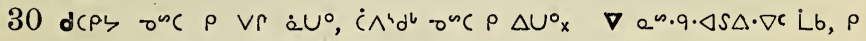
$\Delta \cdot \cup^{\circ}, \sigma b \Delta x \dot{C}^{a}: a L \cdot \Delta c$ Lb $D \Gamma \Delta U^{\circ} x_{x}$

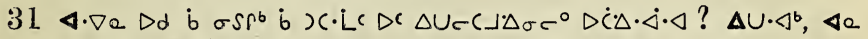

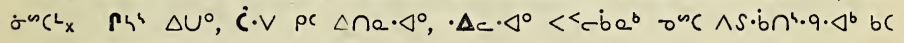
$\left.\wedge) 9 \cdot \nabla \cdot \Delta^{b} \rho P L \sigma\right) \cdot \Delta D C D \rho L \Delta \Delta \sigma \sigma^{b}<J S P_{C} \cdot \Delta^{\circ} x$

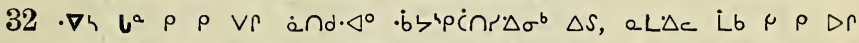

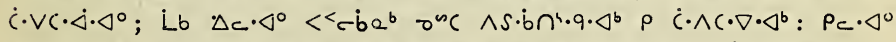

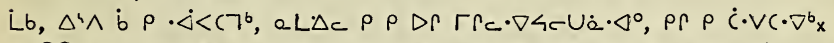

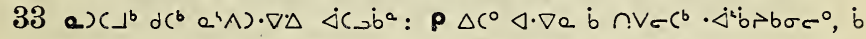

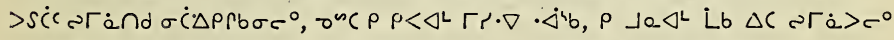

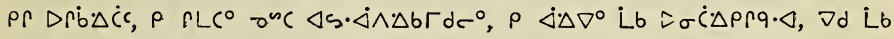
$\Delta \dot{\rho} b \cdot \dot{\Delta} c^{\circ} \nabla x u^{c} x$

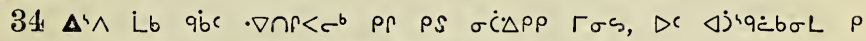

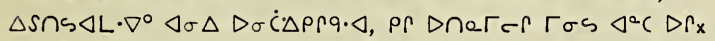

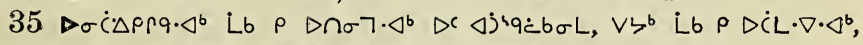

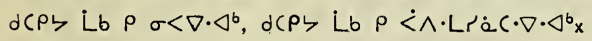

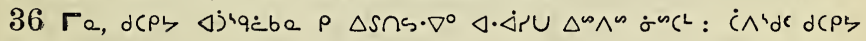
L.b $\rho)\left(\cdot \nabla \cdot \triangleleft^{b} x\right.$

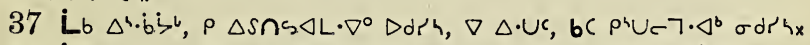

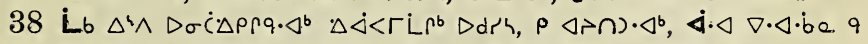

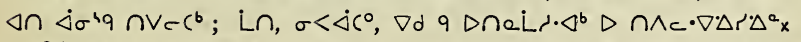

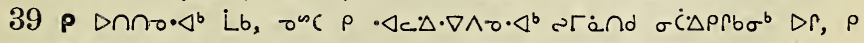
$\sigma<\nabla \cdot \triangleleft^{b} \mathrm{~L}_{b x}$

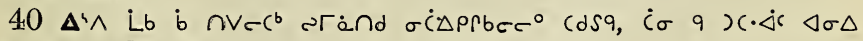
$\triangle \sigma i \Delta \rho \rho q \cdot \triangleleft$ ?

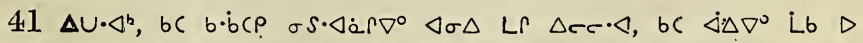

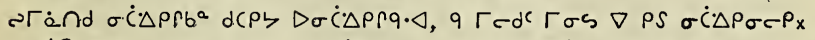

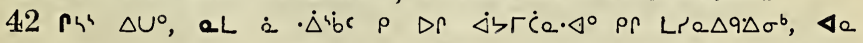

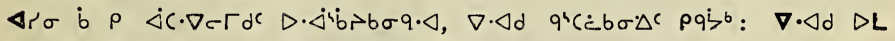
$\left.\Delta \cap V e r q^{\circ} \Delta c \Delta x\right\lrcorner \Delta^{a}, \rho$ LL Libroa $a^{\circ}$ Lbx 


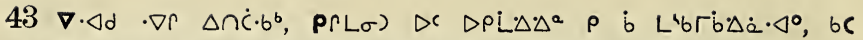

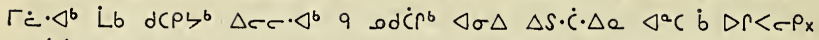

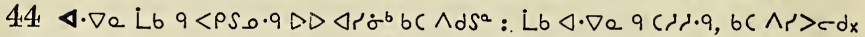

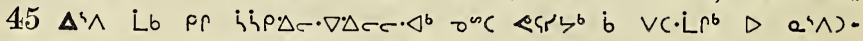

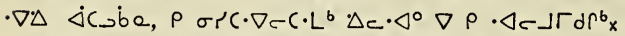

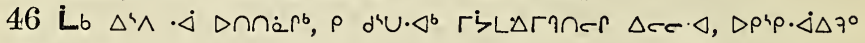
$\nabla \Delta U \sim L_{C} \Gamma_{x}$

\section{$\Lambda_{i} p\left(r a \Delta b^{\circ} 22\right.$.}

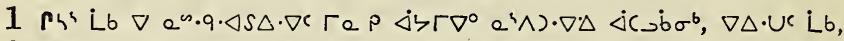

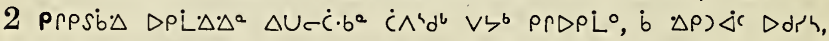

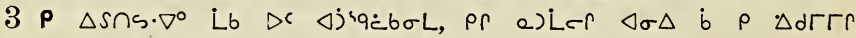
$\Delta \rho) \Delta \sigma^{b}:$ aL: $\Delta c$ Lb $\left.\Delta V P \quad \Delta\right) U c \cdot \Delta x$

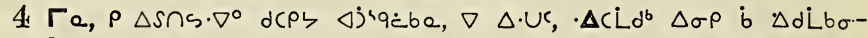

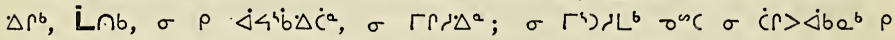

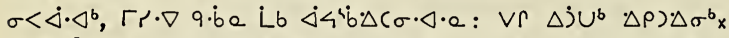

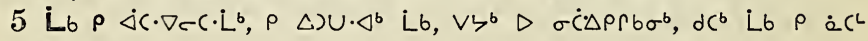
$\Delta C \triangleleft \dot{C} \cdot \dot{<b a x}$

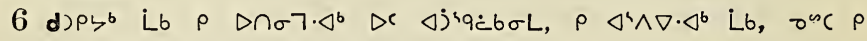
$\sigma<\nabla \cdot \Delta^{b} x$

7 Ĺb $\triangle^{3} \wedge \rho P D P L^{\circ} \wedge \nabla C^{b}, \rho \Delta d c \cdot \nabla r^{\circ}: \rho \triangle S \cap S \cdot \nabla^{\circ}$ Lb $D$ on $q \mathrm{qL}$,

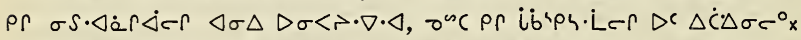

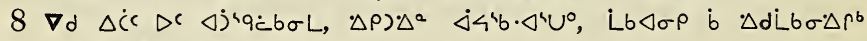

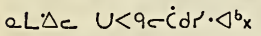

$9 \Delta) U^{b}$ Lb $\left.\rho f 7^{b} b a^{b}, \sigma^{a c} \Gamma r \cdot \nabla \quad q \Gamma_{b}^{b} \cdot \nabla \cdot b^{b}, \Delta d \Gamma d^{b} \Delta \rho\right) \Delta \sigma^{b} x$

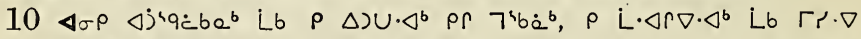

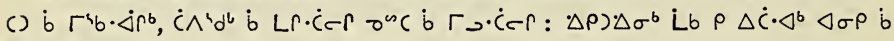
$\Delta d L b \sigma \cdot \Delta r^{b} x$

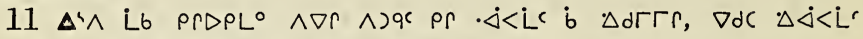

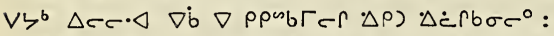

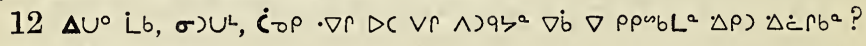
eL. $\Delta c$ L $b \Gamma \Gamma)_{x}$

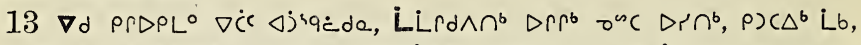
$\cdot \nabla \wedge \sigma^{b} L b \cdot \Delta c \Delta \cap \Gamma^{b} \nabla \Delta S \cdot \Delta \sigma \cap \Lambda^{b} b c^{b}: \nabla d c \quad q \Delta\left(\cdot b^{b} L\right) \Delta^{a} \sigma^{s c}$ q $\Delta S$ L.U $\dot{\Delta} \wedge U S \sigma \dot{\alpha} \sigma \cdot \triangleleft^{b} x$

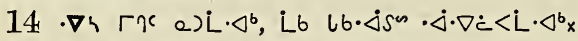

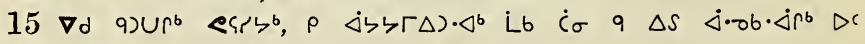
$\dot{\Delta \zeta} \Gamma \Delta \sigma c^{b} \mathrm{x}$

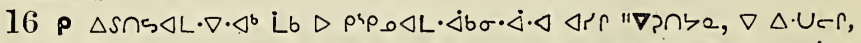

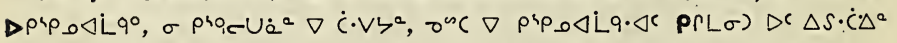

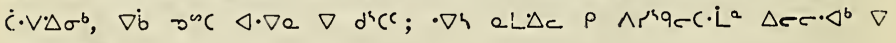
$\Delta s \dot{a} \operatorname{dr}^{b} \mathrm{p}_{\mathrm{x}}$

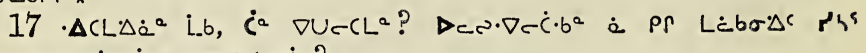

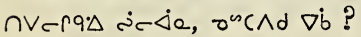




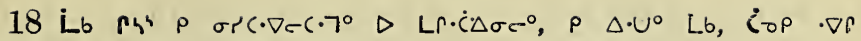

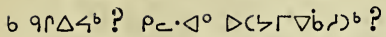

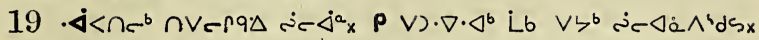

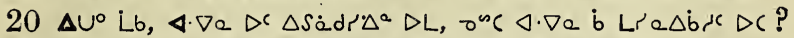

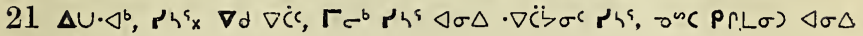
- $\left.\nabla \ddot{C} \dot{\zeta} \sigma^{(} P \rho L \sigma\right)_{x}$

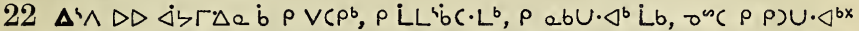

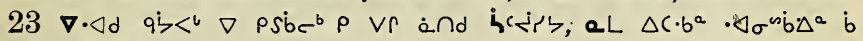
$\triangle \cdot U r^{b}, \rho$ b. $9 \Gamma \Gamma \mathrm{d}$ Lb.

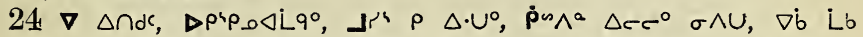

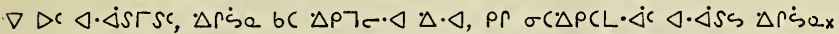

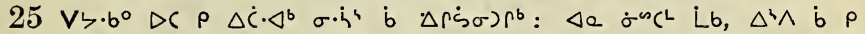

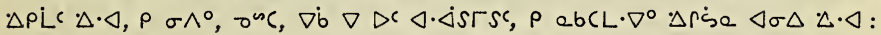

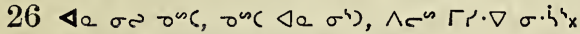

27 L $r^{n}$ L $b \triangleleft a \Delta^{n} \cdot q^{\circ}$ onc $^{n} \sigma \wedge^{\circ} \mathrm{x}$

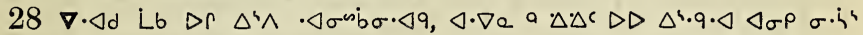

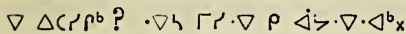

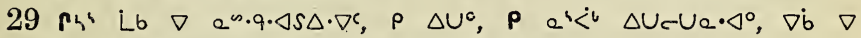
prqe $\left(7^{b} \text { pr Lre } \Delta q \Delta a, \nabla b \text { occ } D \text { bupD } \Delta^{a} p \rho L \sigma\right)_{x}$

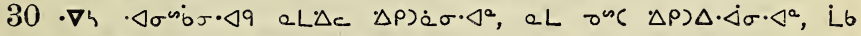

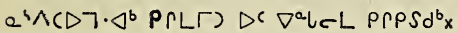

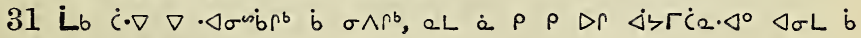
$\Delta \cap \dot{C}^{b} P(L \sigma), \nabla \Delta \cdot U$,

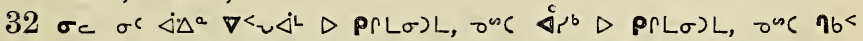

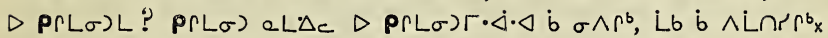

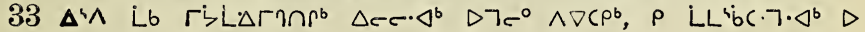
pro.o $\Delta L \mathrm{~L} q \cdot \Delta \sigma r^{\circ} x$

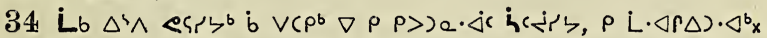

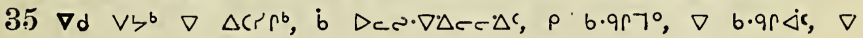
$\triangle \cdot U \times L b$,

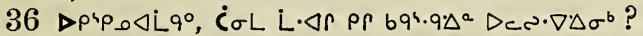

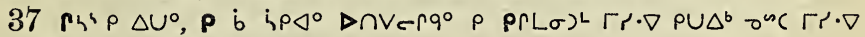

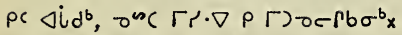

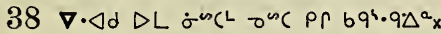

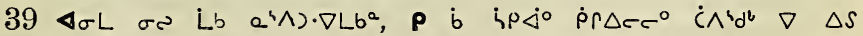
i $P \triangle \cap \dot{r}^{\circ} \mathrm{x}$

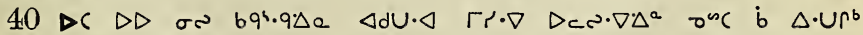
$D p^{\prime} p \cdot \Delta \Delta q \cdot \Delta b \mathrm{~b}$

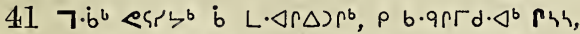

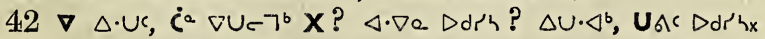

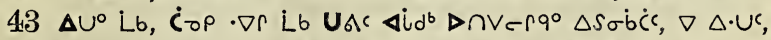

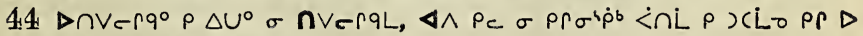

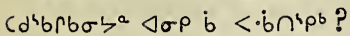




\section{$D<$ ค' Lด $22,23$.}

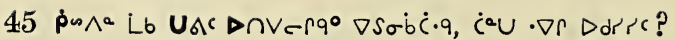

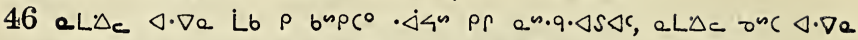

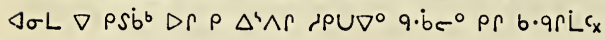

\section{Ápcra $\triangle b^{a} 23$.}

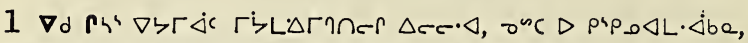

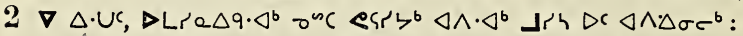

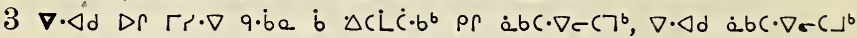

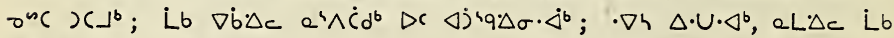
$x \cdot L^{b} x$

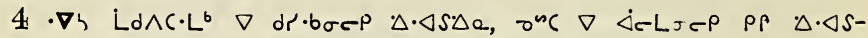

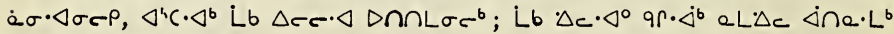

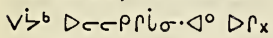

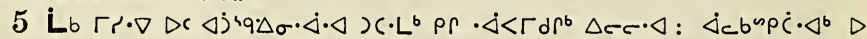

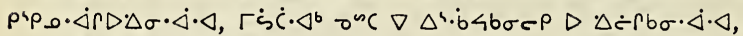

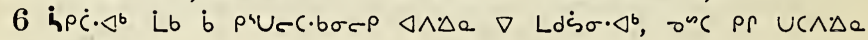
$\dot{\Delta}>\Gamma \nabla \Delta \Gamma^{\circ} \mathrm{d}^{b}$,

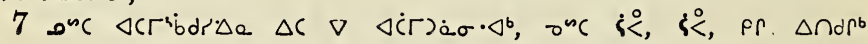
$\Delta r c \cdot \Delta x$

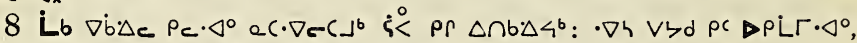

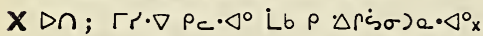

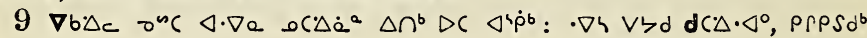
b) $\Delta \dot{c} c_{x}$

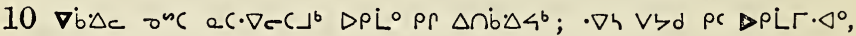
$\mathrm{X} \triangleright \cap_{\mathrm{x}}$

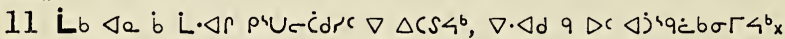

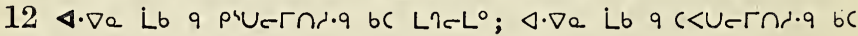
PiUcL ${ }^{\circ}$

13 Ĺb $\rho$ b b. b(

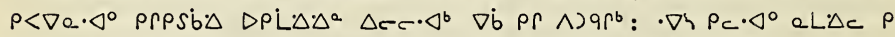

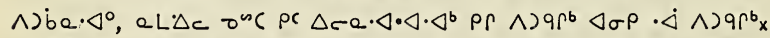

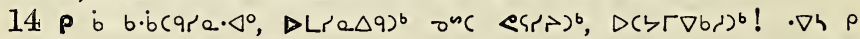

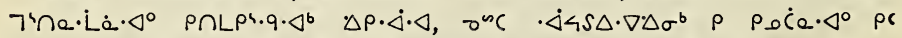

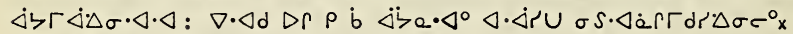

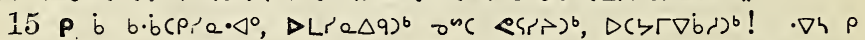

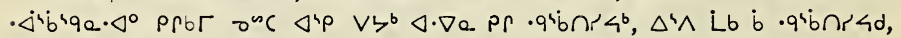

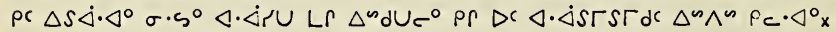

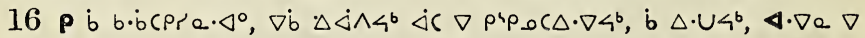

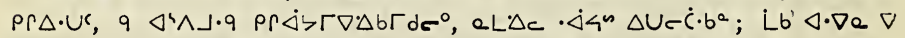

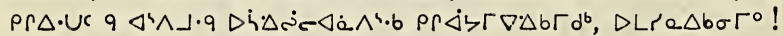

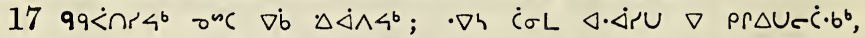

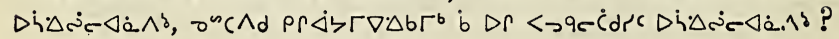




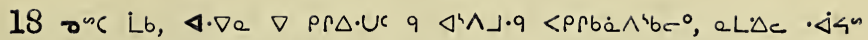

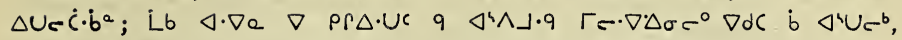
DLra $\Delta b \sigma \Gamma^{\circ} x$

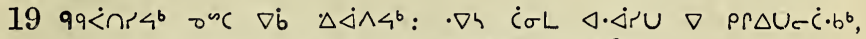

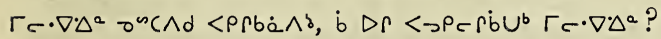

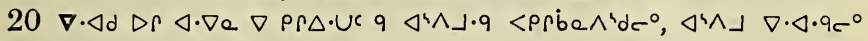

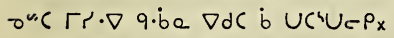

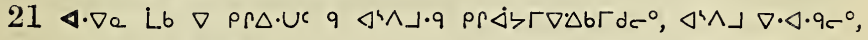
onc $\triangle \sigma \Delta \nabla d c \dot{b} \triangle P C \rho_{x}$

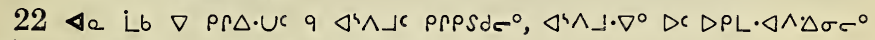
PrL $\sigma) \cdot \triangleleft$, o"c $\varangle \sigma \Delta \nabla d C \dot{b} \cup\left(\wedge c r_{x}\right.$

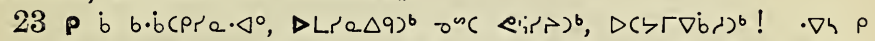

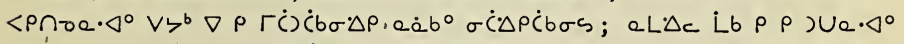

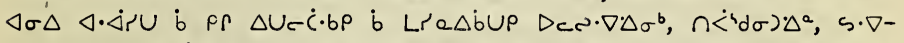

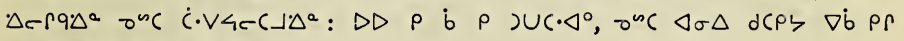
$\mathrm{ab}\left(7^{b} \nabla \dot{b} \nabla D r\right)\left(7^{b} x\right.$

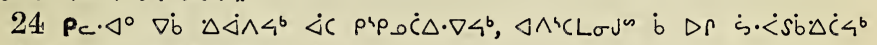
$q \cdot b^{a}, \dot{b}$ dr<e $<<^{b}$ Lb $\dot{b} \Gamma^{c} c_{x}$

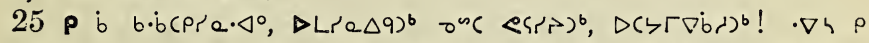

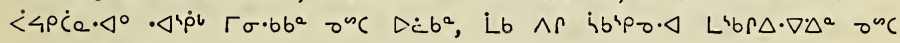
$\Delta i r \Delta r^{2} \Delta^{a} x$

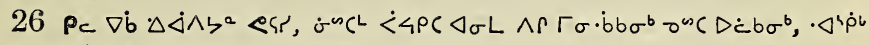
onc $\operatorname{Pr}<<<b^{b} x$

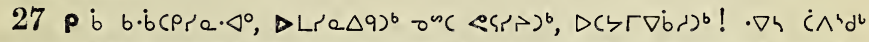

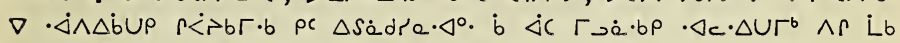

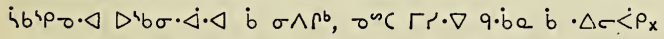

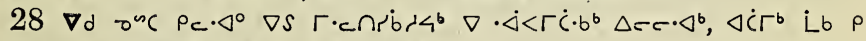

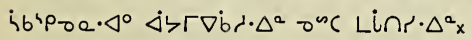

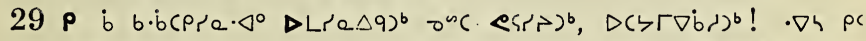

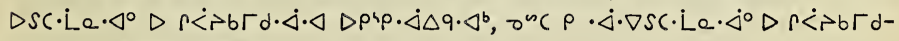

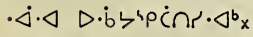

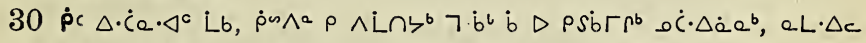
$\sigma \dot{b} \rho \cdot \Delta r \Delta \dot{C} \dot{a} a^{b} \nabla r b \triangleleft \cdot \dot{L} \rho^{b} \Delta \rho^{a} \rho \cdot \dot{\Delta} \Delta q \cdot \Delta^{b} D \Gamma d \sigma^{\circ} x$

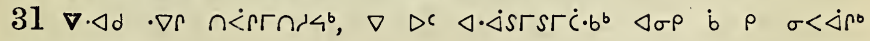
DPsp. $\Delta \triangle q \cdot \triangleleft x$

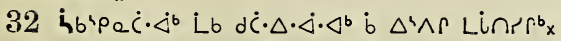

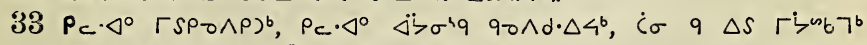

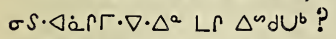

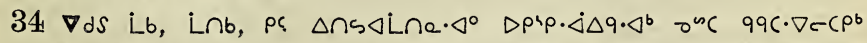

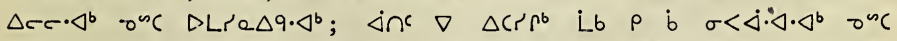

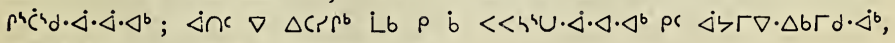

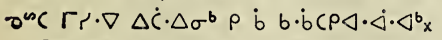

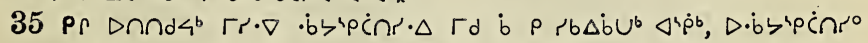
41 


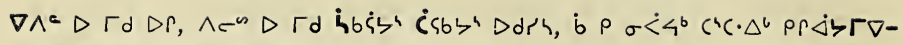

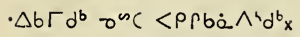

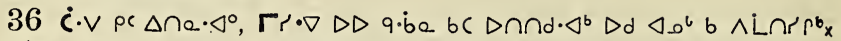

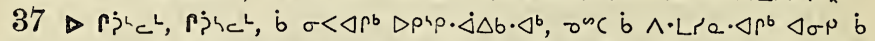

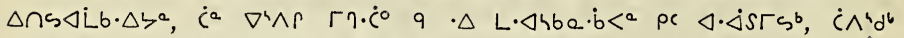

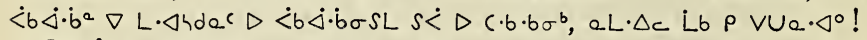

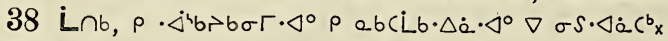

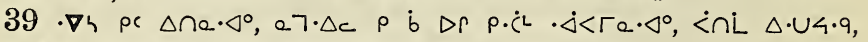

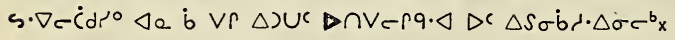

\section{Nיpcro $\triangle b^{\circ} 21$.}

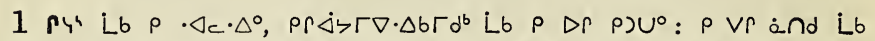

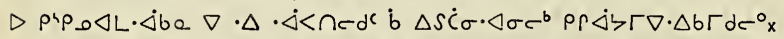

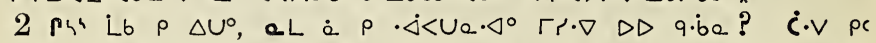

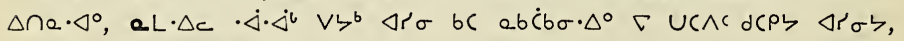
$\nabla \dot{b}$ व $\sigma \rho \cdot \nabla \wedge \dot{a} b \sigma \cdot \Delta c_{x}$

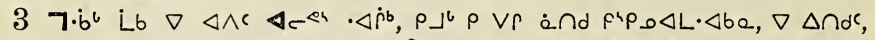

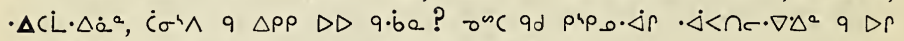

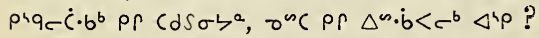

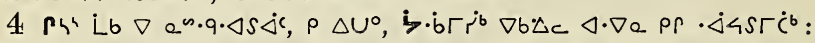

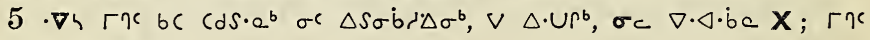
Lb $b C \cdot \ddot{\Delta} 4 S 7 \cdot \triangleleft^{b} x$

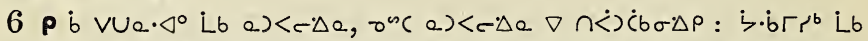

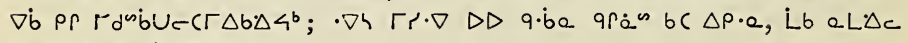
$\nabla n \cdot b$ bc $\Delta m \cdot b<c^{\circ} x$

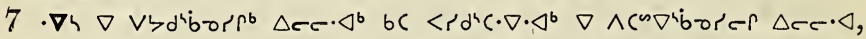

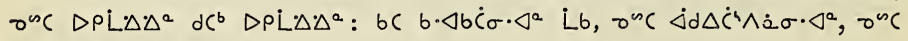
$b\left(\cdot \rho \cdot b^{a} a \dot{a}\right)^{b} \Delta S_{x}$

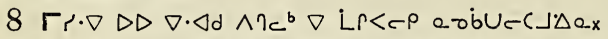

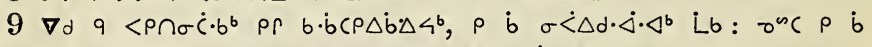

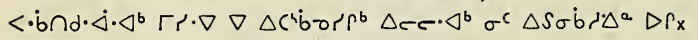

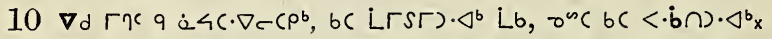

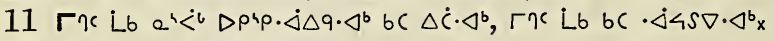

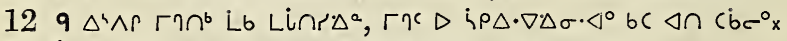

$13 \dot{L} b \Delta e \nabla \dot{b} q \dot{s} d r \dot{b b \sigma} \cdot \Delta c, \nabla \cdot \triangleleft \cdot \dot{b} a q \wedge \dot{L} r \Delta b \sigma \cdot \Delta c_{x}$

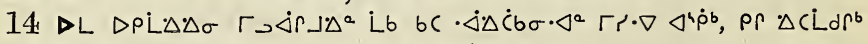

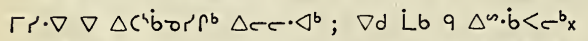

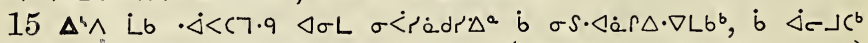

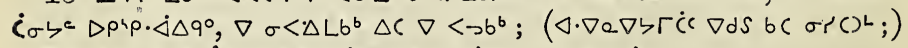

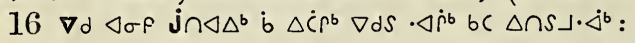

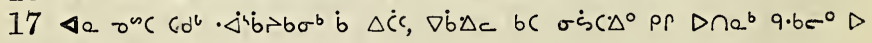
$\dot{\Delta} \dot{b} r b \sigma \Gamma^{b} \triangleright r:$ 


\section{$D<\rightarrow$ PS LQO 24.}

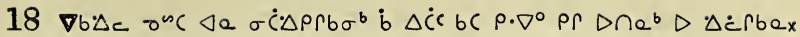

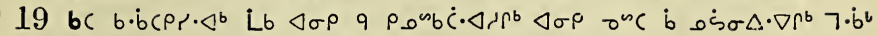
$\triangle \triangleright q$ psícp!

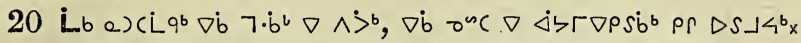

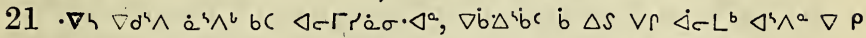

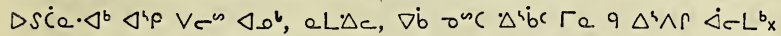

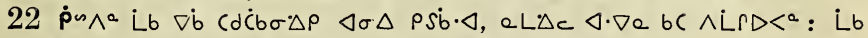

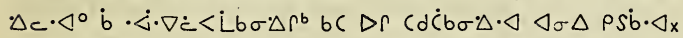

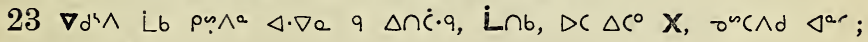
$\nabla \dot{b} \cdot \Delta c \dot{c} \cdot V C \cdot J^{b} x$

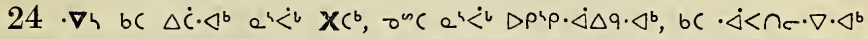

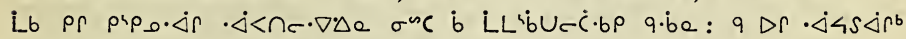

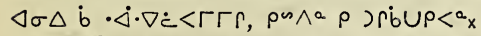

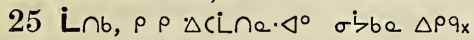

$\left.26 \nabla \cdot \Delta d \Delta \Gamma \rho^{a} \wedge^{a} \Delta \Gamma \dot{C} \cdot b \cdot \nabla, \dot{L} \cap b,<\cdot b c^{b} b \Gamma^{b} b^{b} \Delta c^{\circ} ; \nabla \dot{b} \Delta c \nabla^{a} c \Delta\right) U^{b}$ : Lnb, bc $\left.\left.c^{b} \wedge\right) b \Gamma^{b} \Delta c^{0} ; \nabla b \Delta c \dot{c} \cdot V c \cdot\right\lrcorner^{b} x$

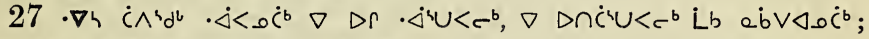

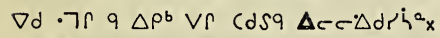

$\left.28 \cdot \nabla h \Delta C \nabla \Delta C \cdot b^{b} \Gamma \zeta^{\circ} b \quad \sigma \wedge L b^{b}, \nabla d C a L \cdot \Delta r \Delta\right)^{b} \Gamma \rho^{b} \cdot\left\langle b^{b}\right.$

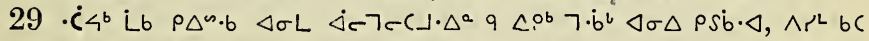

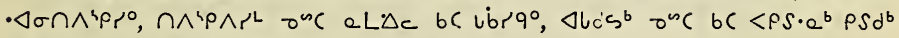

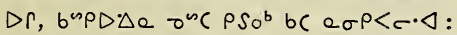

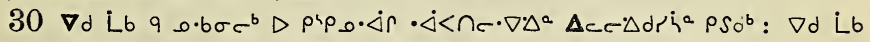

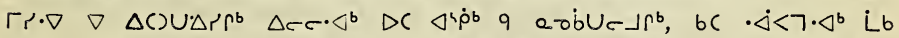

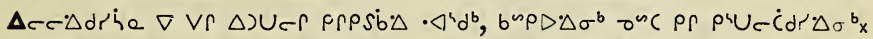

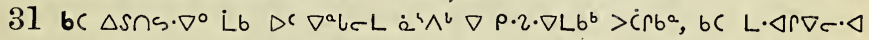

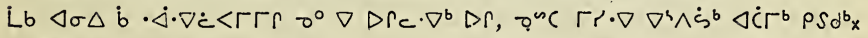

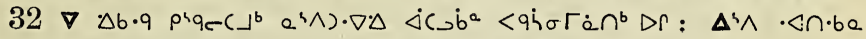

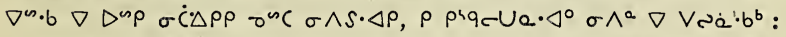

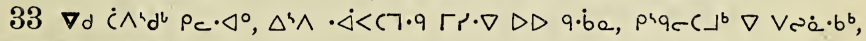
$\Delta u \cdot \dot{b} U \Gamma^{-b} \Delta u \wedge{ }^{n} x$

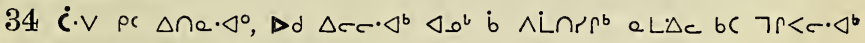
<ni $\Gamma r \cdot \nabla \Delta D q \cdot b_{e} \rho \cap \wedge<c \cdot b \cdot \nabla x$

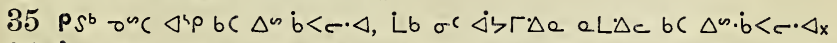

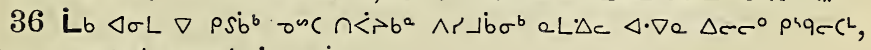

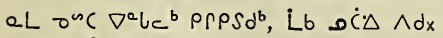

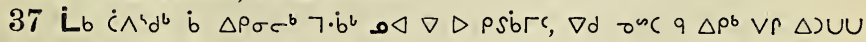
$\Delta-r \Delta d r i a_{x}$

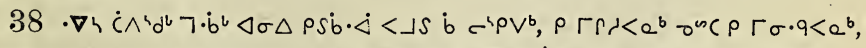

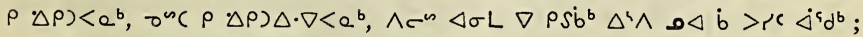

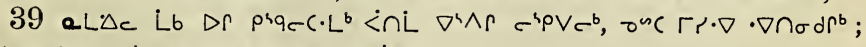

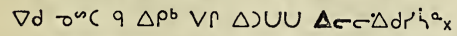

$40 \nabla d^{4} \wedge \sigma a b C \Delta \dot{C} \cdot \Delta^{b} \sigma \dot{C} \Delta \rho \rho b \sigma^{b} ; V^{b} b^{b} b C D \cap a^{\circ}, d C^{b}$ Lb bC abc ${ }^{\circ} x$ 43 


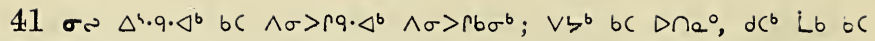
$a b c^{\circ} x$

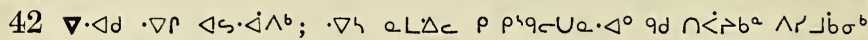

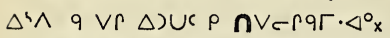

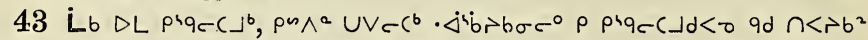

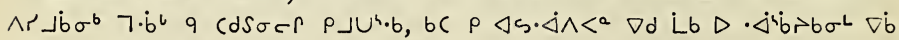
.q $p \wedge d \Delta \dot{b} \cup c^{4}<a$

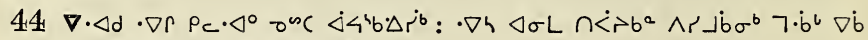
$\nabla \Delta U c C 7^{b} \Delta c r \Delta d r^{2} i^{a} b c\left(d S^{a} x\right.$

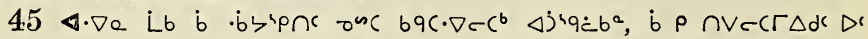

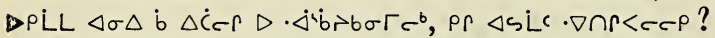

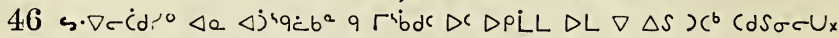

$47 \dot{c} \cdot \vee$ pc $\Delta \cap a \cdot \triangleleft^{\circ}, b c \cap \vee c c r \Delta d \Gamma r \cdot \nabla \Delta c \Delta \dot{\zeta} \sigma c \cdot \Delta x$

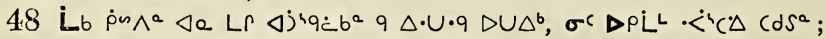

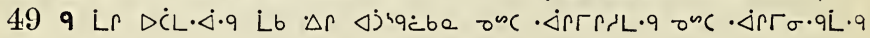

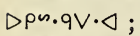

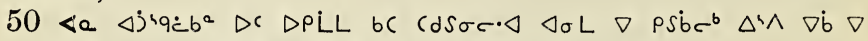

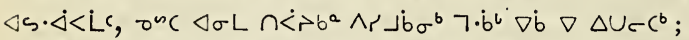

51 bc jupad Lb, onc bc $\triangle \cap S \Delta d$ Lb pp $\Delta 1 \cdot \dot{<c} \nabla C b \Gamma \nabla b d \cdot \triangleleft ; \nabla d C q$ $\Delta\left(\cdot b^{b} L\right) \Delta^{a} \sigma^{n c}$ a $\Delta S L \cdot \cup \dot{i} \wedge U S \sigma \dot{\alpha} \sigma \cdot \Delta^{b} x$

\section{A'pcra $\triangle b^{a} 25$.}

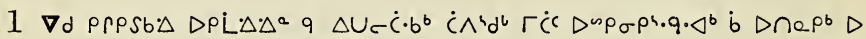

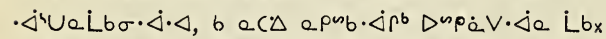

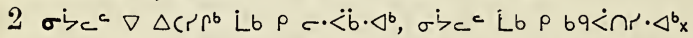

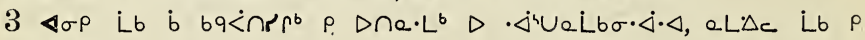
$D \cap e \cdot L^{b} \wedge \Gamma^{\circ}{ }^{\circ}$

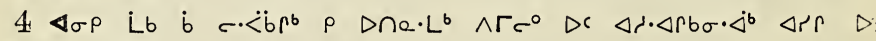

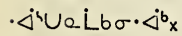

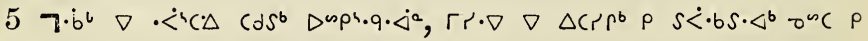
$\sigma \dot{<} \cdot \Delta^{\circ} x$

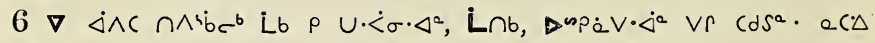
epribx

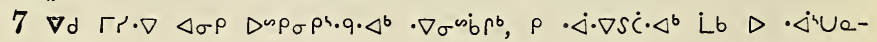
Ló $\sigma \cdot \dot{\Delta} \cdot \triangleleft x$

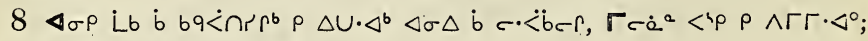

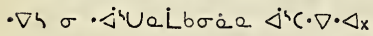

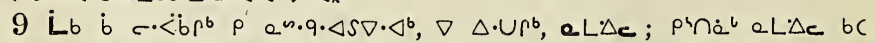

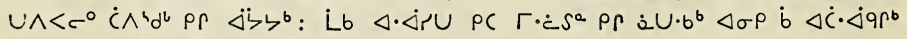

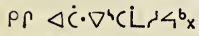

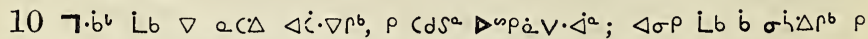
$\left.\wedge) 9 \Delta \eta \cdot \nabla \cdot \Delta^{b} \Delta \rho\right) \Delta \sigma^{b}: \nabla d$ Lb $\Delta$ c. bUL $q<\Delta \dot{b} L^{b} b_{x}$ 


\section{$\triangleright<\rightarrow$ Ṕ Lด 25.}

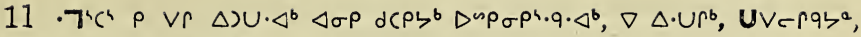

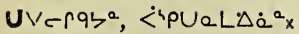

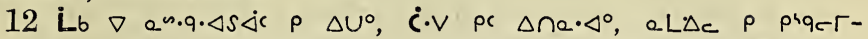
กอ. $\triangleleft{ }^{\circ} x$

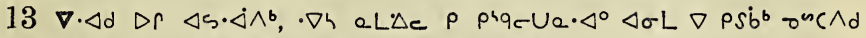

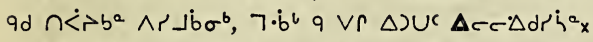

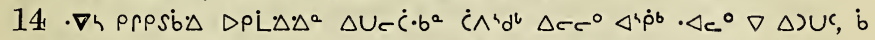

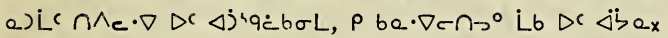

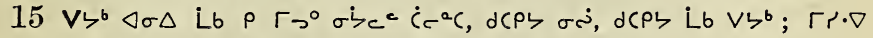

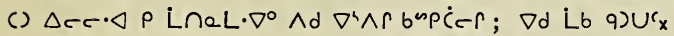

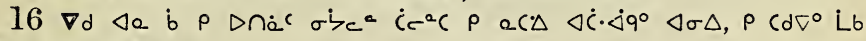
$d C P \zeta \sigma i c c^{c} \dot{C} c^{a} C_{x}$

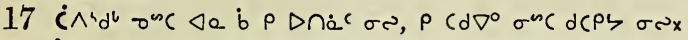

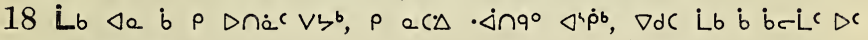
$\triangle P L L D \dot{S}_{c} \triangleleft \dot{j} \sigma L_{x}$

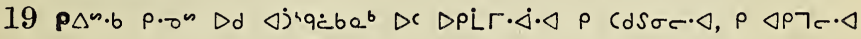
Lbx

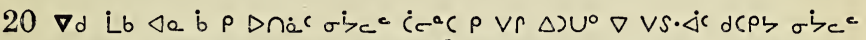

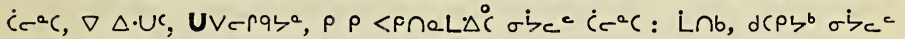

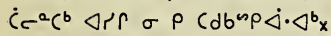

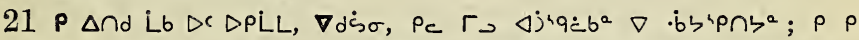

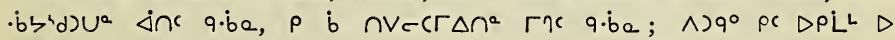
נrac $C \cdot \Delta \sigma^{b} x$

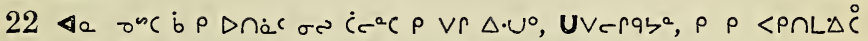

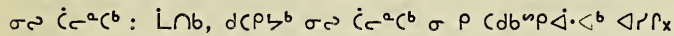

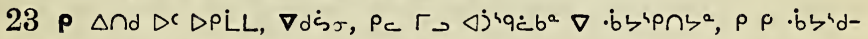

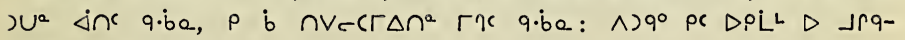
$c\lrcorner \Delta \sigma^{b} x$

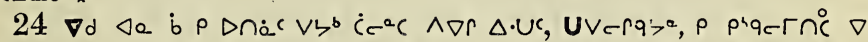

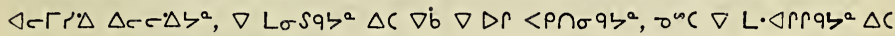
$\nabla \dot{b} \nabla \Delta r \cdot \Delta h \cdot 4 \wedge \sigma a \zeta^{a} ;$

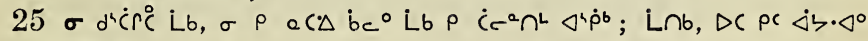

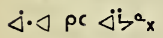

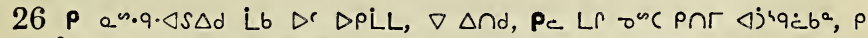

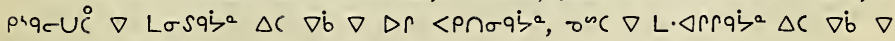
$\left.\Delta r \cdot \Delta 4 \cdot 4 \wedge_{\sigma} 9\right\rangle^{\circ}$;

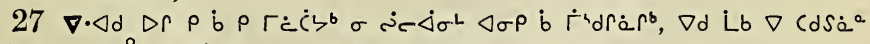

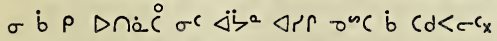

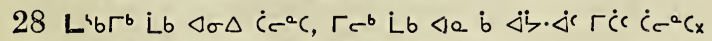

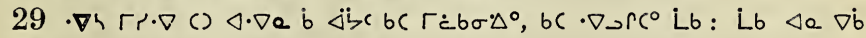

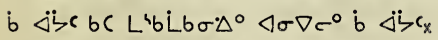

$30 \Delta S \cdot \nabla \wedge \sigma^{b}$ Ĺb $\nabla \dot{b} \dot{a}^{a} c^{\circ} \dot{b} \Delta \dot{c}<n r^{c} \Delta j 4 q \dot{c} b^{a} \cdot \Delta c \Delta \cap \Gamma^{b} \nabla \Delta S \cdot \Delta \sigma \cap-$

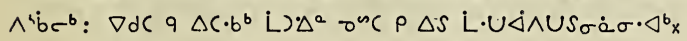




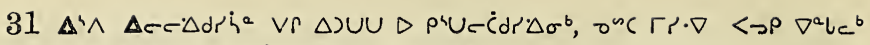

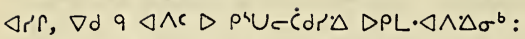

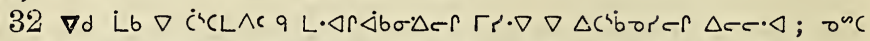

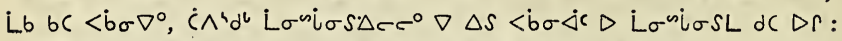

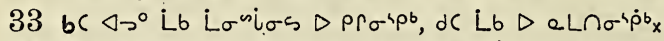

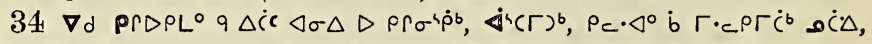

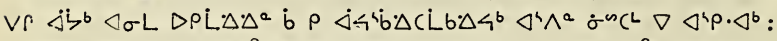

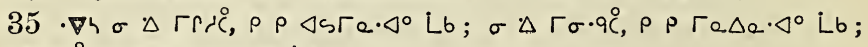
$\sigma$ L $a \Delta \dot{C}, \rho \rho \wedge) b \Delta e \cdot \Delta^{\circ} \dot{L} b$;

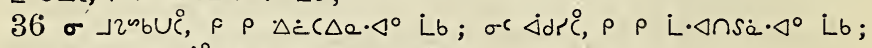
$\mu<\Delta) \Delta b \Gamma d^{b} \sigma^{c} \Delta \dot{C} \dot{C}, \rho \rho \vee r$ ese. $\Delta^{\circ} \dot{L} b_{x}$

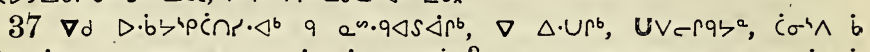

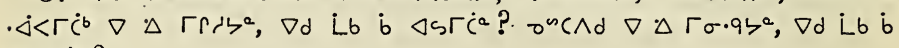
$\Gamma \circ \Delta \dot{C}^{b}$ ?

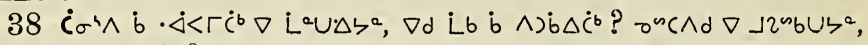
$\nabla d$ Lb b $\Delta \dot{c} c \Delta \dot{c}^{b}$ ?

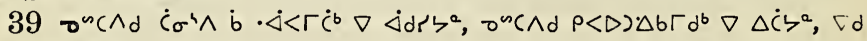
Lb 6 ancं ?

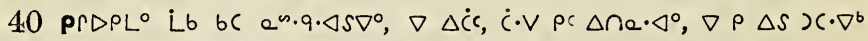

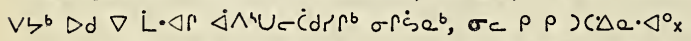

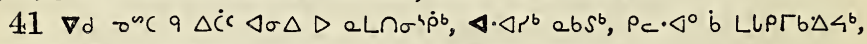

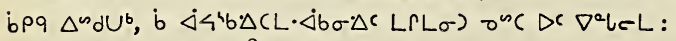

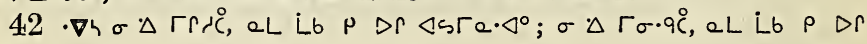
$\Gamma \circ \Delta e \cdot \Delta^{\circ}$;

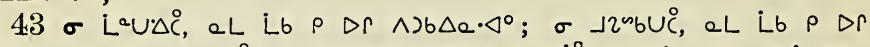

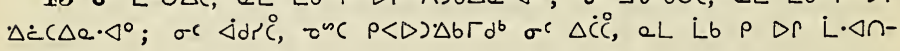
$S e \cdot \Delta \circ x$

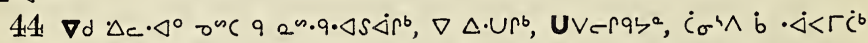

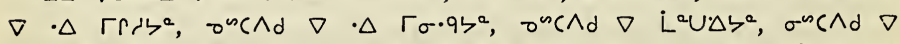

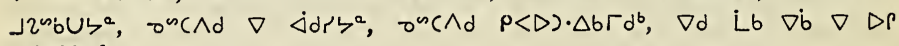
$<\dot{\varphi} \dot{C} \dot{C}^{\circ}$ ?

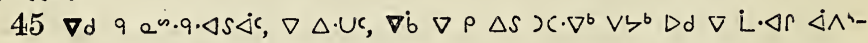

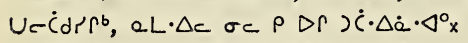

46 Dd ld bpq b.bcpr $\Delta \sigma^{b}$ bc $\Delta x \cdot \Delta^{b}$, Lb $D \cdot b b^{4} p i n a \cdot \Delta^{b}$ bpq $\wedge \operatorname{Ln} \Delta \sigma^{b} x$

\section{$A^{4} p c$ c $\triangle b^{\circ} 26$.}

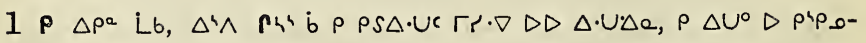
QL. ¿̇be,

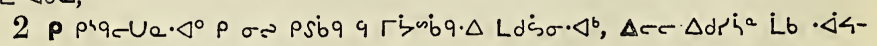

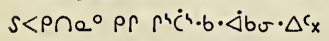

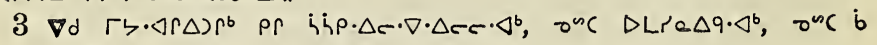
46 


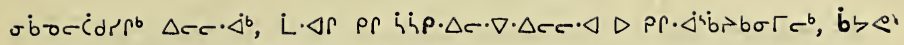
b $\Delta S \sigma \dot{b} d e r$,

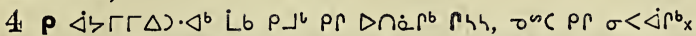

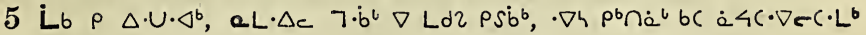
$\triangle c c \cdot \Delta^{b} \mathrm{x}$

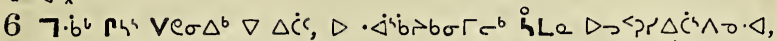

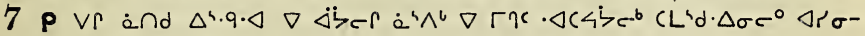

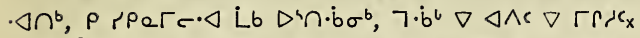

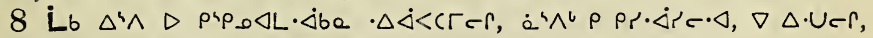

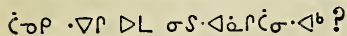

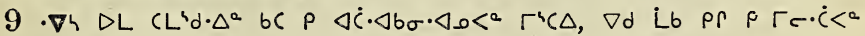

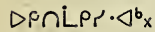

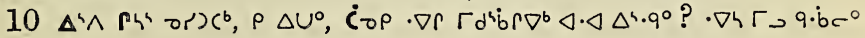
$\sigma \rho) \dot{x}^{b x}$

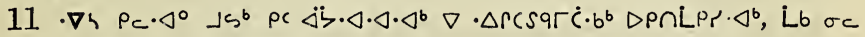

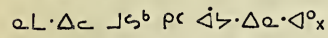

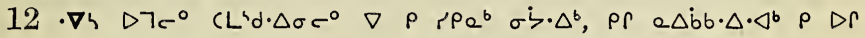
$\mathrm{CL}^{\mathrm{L}} \mathrm{x}$

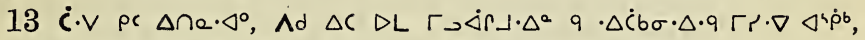

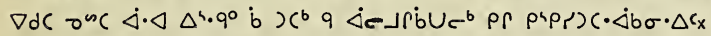

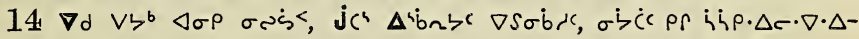
$\lessdot \odot \cdot \triangleleft$,

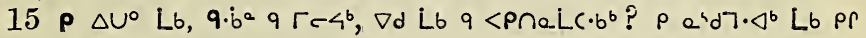

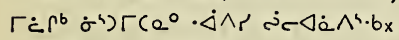

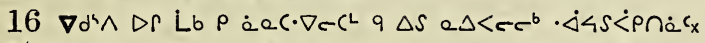

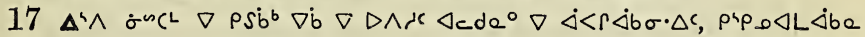

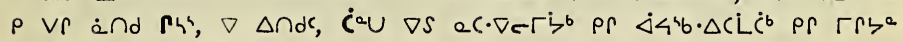

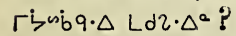

18 P $\Delta \cdot U^{0}$ Lb, $\left.\Delta\right) U^{b} \Delta \dot{C} \cdot \Delta \sigma^{b}, \dot{C}^{b}$ L.b Vלb $\Delta c c^{\circ}, \Delta L$ Lb $\rho$ b $\Delta \dot{C} \cdot \triangleleft^{\circ}$,

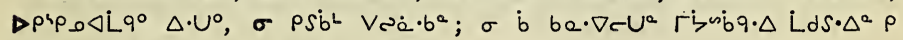

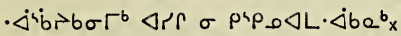

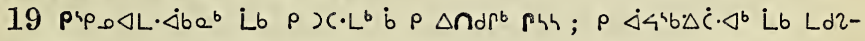
$\Delta \sigma c^{\circ} \mathrm{x}$

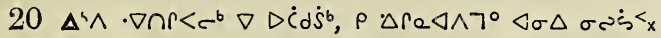

$217 \cdot \dot{b}^{b}$ Lb $\nabla \Gamma R \rho^{b}, \rho \Delta \cdot \cup^{0} i \cdot V$ pc $\Delta \cap a \cdot \Delta^{\circ}, V \zeta^{b} \nabla \Delta C S 4^{b} \sigma$ b $\cdot \dot{\Delta}\left\langle s<p \cap \sigma^{b} x\right.$

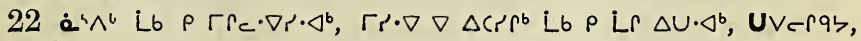
$\sigma c \dot{a}$ ?

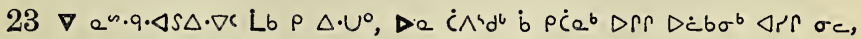
$\nabla \cdot \triangleleft \cdot$ be $q \cdot \triangleleft 4 S<p \cap \sigma c_{x}$

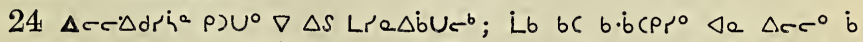

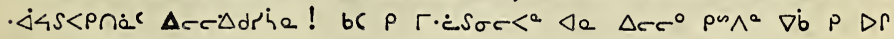
$\sigma \dot{C} \Delta P c_{x}$ 


\section{$\triangleright<\rightarrow \rho^{4}$ Lด 26.}

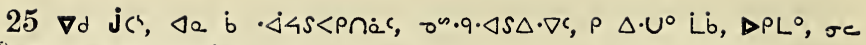
i. p $\Delta U^{0}, p \rho \Delta \cdot \dot{c}^{a} x$

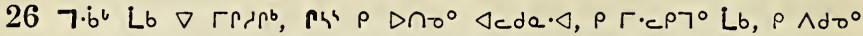

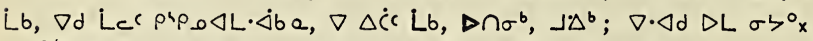

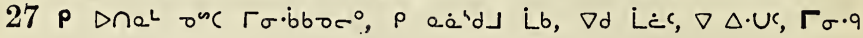
$\Gamma t \cdot \nabla \nabla \Delta C S 4^{b} x$

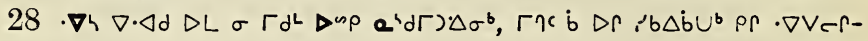
bUP LP.C.Aax

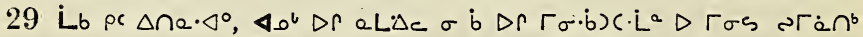

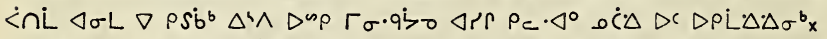

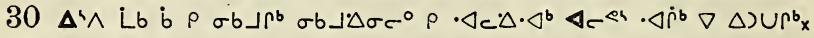

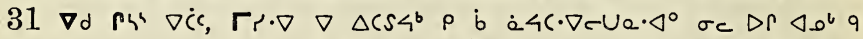

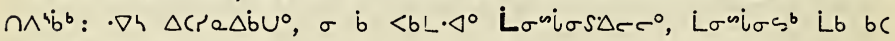
$\cdot \Delta 4 \cdot 4 \cap 5 \cdot \dot{\Delta} \cdot \Delta b x$

$32 \Delta^{\prime} \wedge$ Lb $\left.\rho \cdot \triangleleft \sigma^{n} b^{b}\right\rangle \sigma, \rho \dot{b} a c \Delta \vee \Delta \cap a \cdot \triangleleft^{\circ} \dot{b}_{c} c^{b} x$

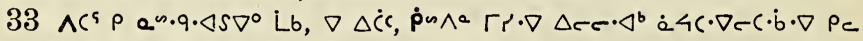

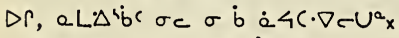

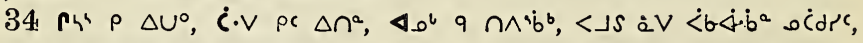

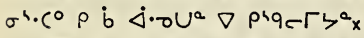

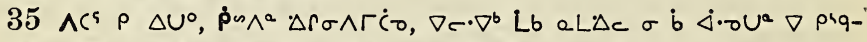

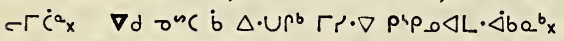

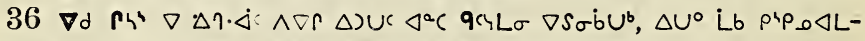

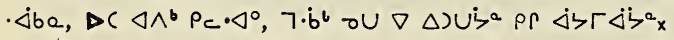

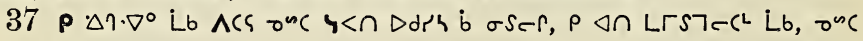
Q. $\backslash \wedge \varphi p$ drdLrDx

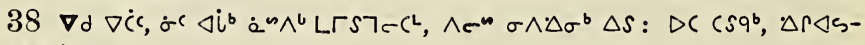
$\triangleleft \wedge \Gamma^{b}$ L bx

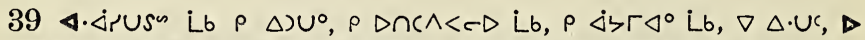

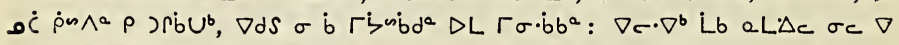
$\triangle U \leftarrow C L^{a}, L_{b} \nabla \Delta U \leftarrow L^{a} P_{C x}$

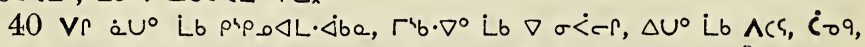

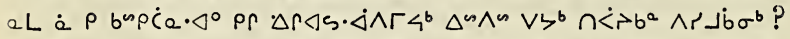

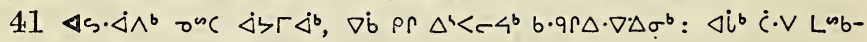
- $\nabla \subset C^{L}$, Lb $\Delta \dot{S}^{4}-C \Gamma P L b^{a} x$

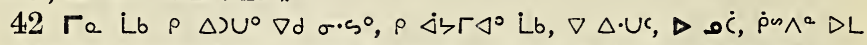

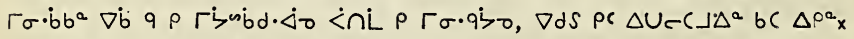

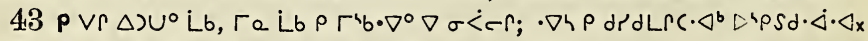

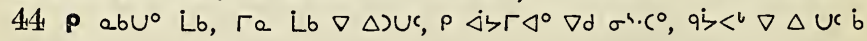
$\triangle \cdot U c_{x}$

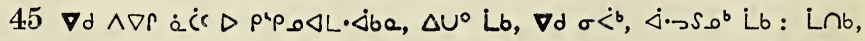

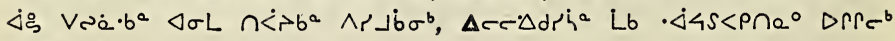
$\Delta L \Gamma \cdot \dot{C} \cdot \Delta_{x}$

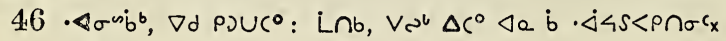
48 


\section{$D<\neg$ p' Lด๐ 26.}

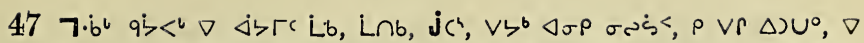

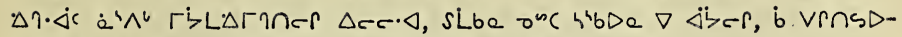

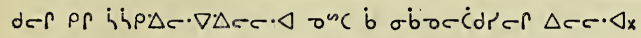

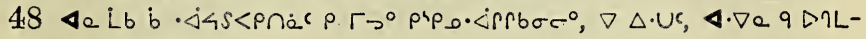
$\cdot \triangleleft 9, \nabla \cdot \triangleleft \cdot b e ; \Gamma r \sigma^{\cdot b x}$

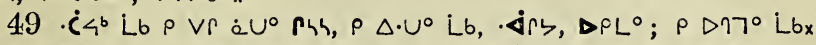

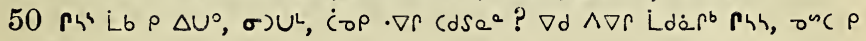
Dno. $\Delta^{b} x$

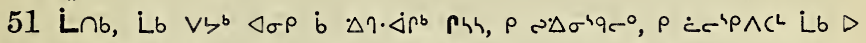

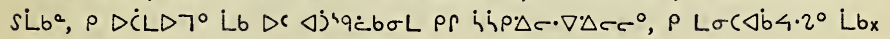

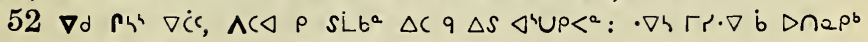

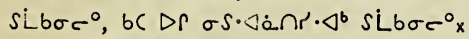

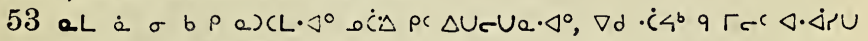
$\Delta \omega \wedge n \sigma s \dot{s}<$ cle $\nabla^{a} k$ ?

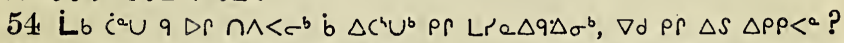

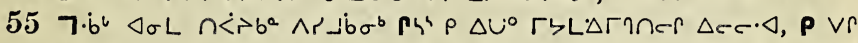

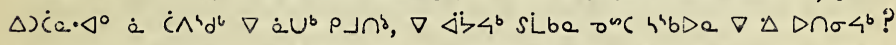

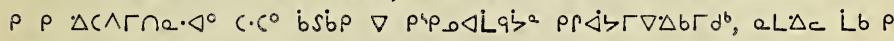
Dr $D \cap \sigma e \cdot \Delta^{\circ} x$

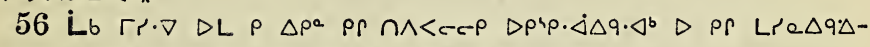

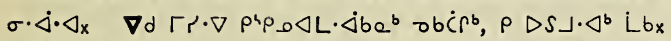

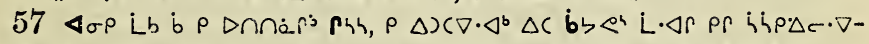

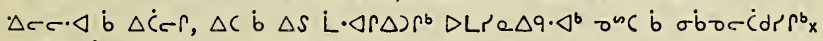

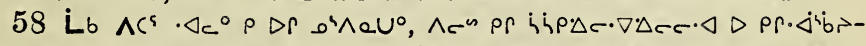

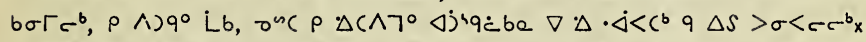

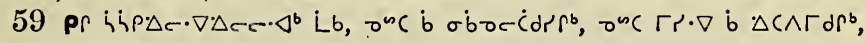

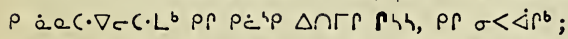

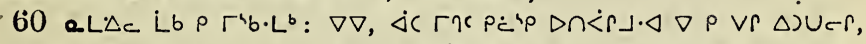

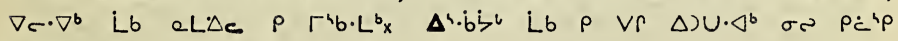
$\nabla \cap \dot{<}\lrcorner \cdot \triangleleft^{b}$,

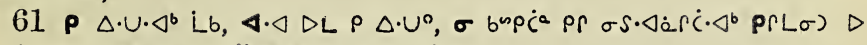

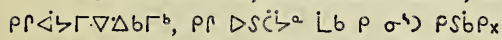

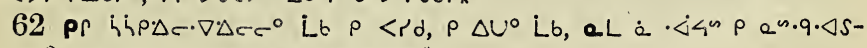

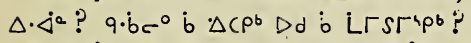

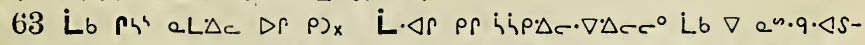

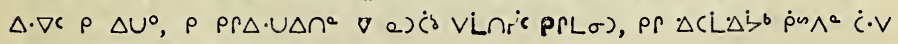
$\triangle \backslash \cdot \Delta \cdot \Delta 0 X, P r L \sigma) D d r h x$

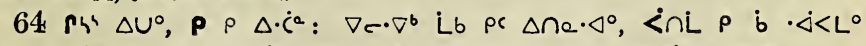

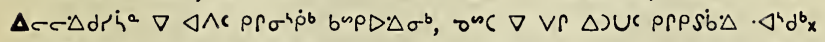

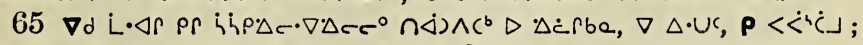

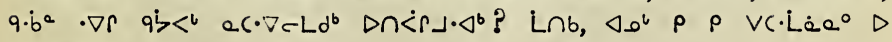
$\dot{<}(\lrcorner \cdot \Delta a x$

$66 c^{a} \nabla U e C 7^{b}$ ? $\nabla$ a $a \cdot q \cdot \Delta r \Delta \cdot \nabla r^{b}$ Lb, $\rho \Delta \cdot U \cdot \Delta^{b}, \Delta U=\dot{C} d r^{\circ}$ or $\sigma \wedge c_{x}$ 49 


$$
\triangleright<\rightarrow \text { ps Lด } 26,27 .
$$

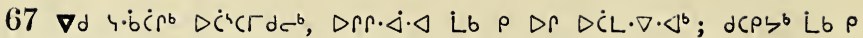
$<<\cdot \cdot q \cdot \nabla \cdot \Delta^{b}$,

$68 \nabla \Delta \cdot U r^{b},<\cdot b e^{\circ} \Delta C L \Delta a^{a}, p_{c} X, \Delta \cdot \nabla e$ b <p.qDb?

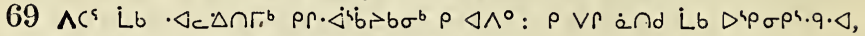

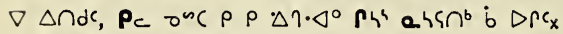

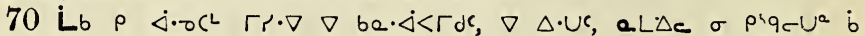
$\Delta \cdot U^{2} \mathrm{x}$

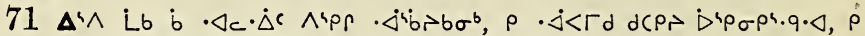

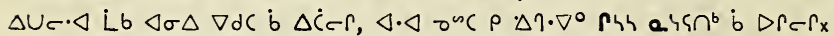

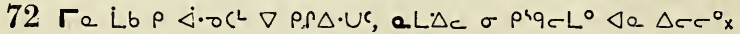

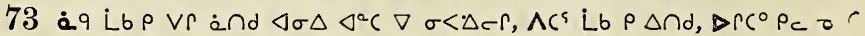

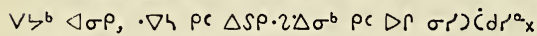

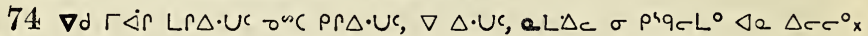

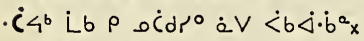

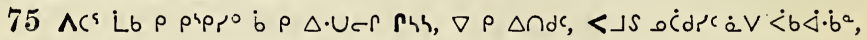
$\sigma^{4} \cdot c^{\circ} \rho \dot{b}<\cdot \sigma^{a} \nabla \rho^{4} q_{c} \Gamma \zeta^{a} x \quad \rho \cdot \Delta_{c} \Delta^{\circ}$ L.b, db Lb $\rho$ L. $)_{x}$

\section{$\Lambda$ A}

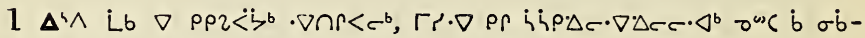

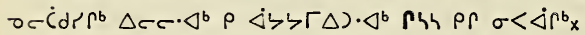

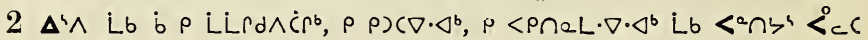
$\Delta \cap \vee\ulcorner q \cdot \triangleleft x$

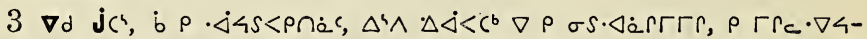

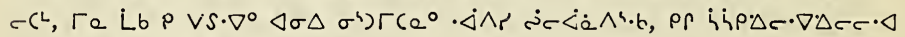
onc b obrocidrer $\nabla \wedge \Gamma \dot{c} c$,

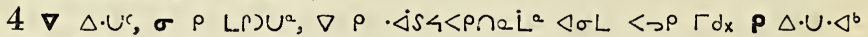
Lb, $\dot{C}_{\sigma}$ Lb $\left.q\right)\left(\dot{L}^{b} \sigma c \dot{a}^{a}\right.$ ? $\rho_{c} \nabla \cdot \triangleleft d$ be $\cdot \dot{<}<C_{x}$

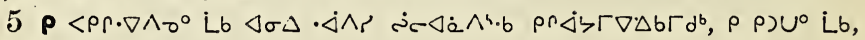
onc $\rho$ ec $\triangle \Delta d c \cap d x$

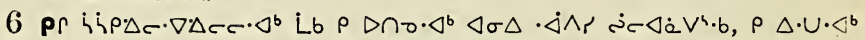

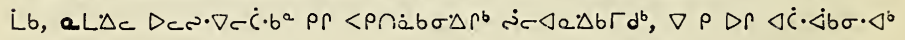
$\Gamma \mathrm{dx}$

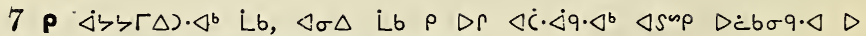

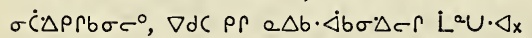

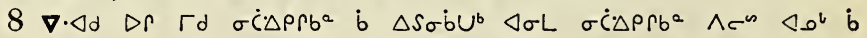
$\rho j \dot{b}^{b} \times$

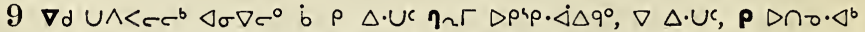

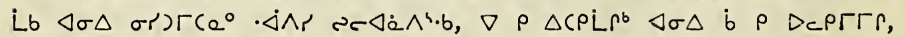

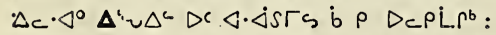

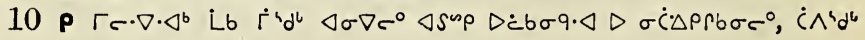
$\nabla \rho \Delta C \omega \cdot \Delta S^{c} \Delta \cap V \in \Gamma \rho^{\circ} x$ 


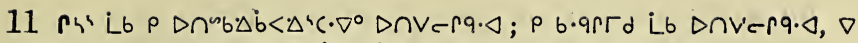

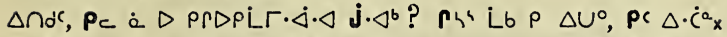

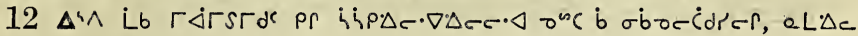

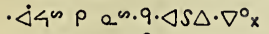

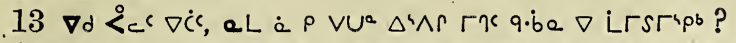

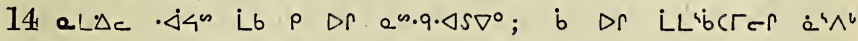
$\triangle \cap V e \uparrow q \cdot \triangleleft_{x}$

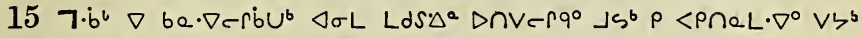
$\rho<\cdot<b e, \wedge d \triangleleft \sigma \Delta q a c \cdot \nabla r L-\Gamma_{x}$

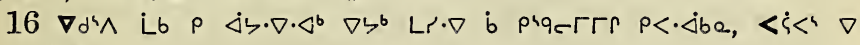

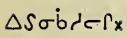

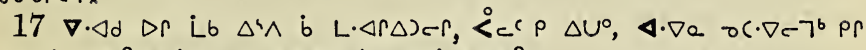

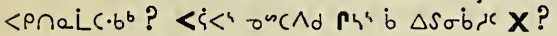

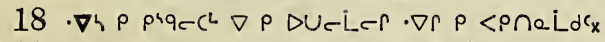

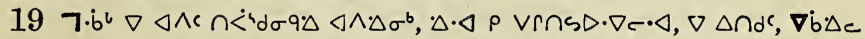

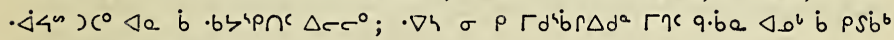
$<\cdot \triangleleft\lrcorner \Delta \sigma^{b} \Delta c \nabla \Gamma_{x}$

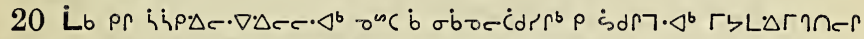

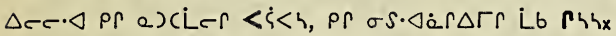

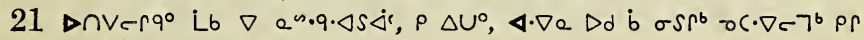
$<$ PกQL L $\cdot b^{b}$ ? $P \Delta \cdot U \cdot \Delta^{b},<\dot{\zeta}<{ }^{4} x$

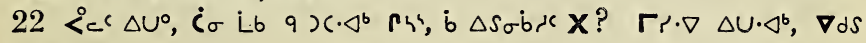
de ricis.b. $4{ }^{\circ} x$

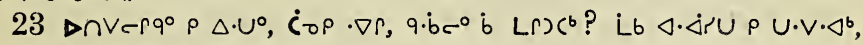
$\nabla \triangle \cdot U \mu^{b}, \nabla d S b C \dot{r}^{\prime} \dot{c}^{i} \cdot b \cdot \Delta^{\circ} x$

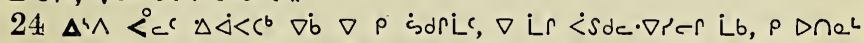

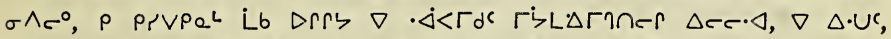

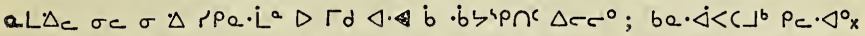

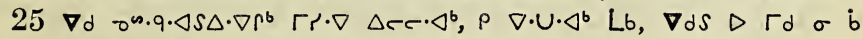

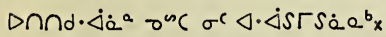

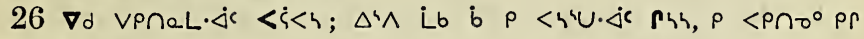
$\Gamma^{4} c^{4} \cdot b \cdot \Delta b \sigma \cdot \Delta r r_{x}$

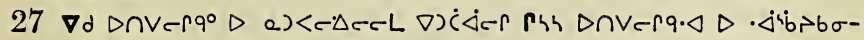

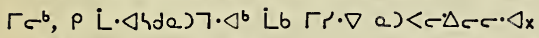

$28 \rho 9 C d V_{0} \cdot \triangleleft^{b}$ L.b, $\rho>c\left(d<\nabla \cdot \triangleleft^{b}\right.$ L $b \Gamma \cdot q \rho^{a} \triangleleft d D \sigma \sigma^{\circ} x$

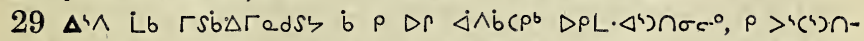

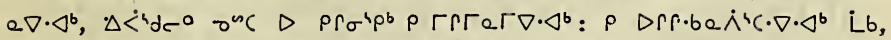

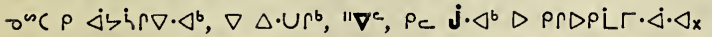

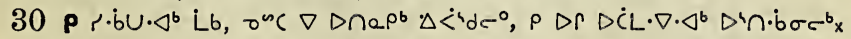

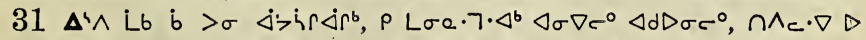
$\Delta c r b a$ Lb $\left.\left.\rho>>^{4} b\right\lrcorner 0 \cdot \Delta^{b}, \rho \rho\right)\left(\nabla \cdot \Delta^{b}\right.$ Lb $\rho r r^{4} \dot{c}^{4} \cdot b \cdot \Delta \rho^{b} x$

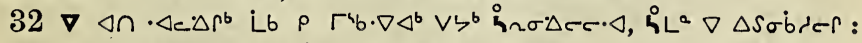

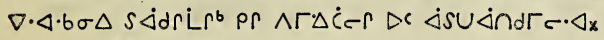




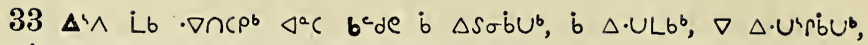
sin.boba,

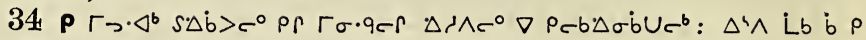
d $P^{4} C^{b}, Q L \Delta \Delta_{c} \rho \Delta \Gamma \sigma \cdot 9^{\circ} x$

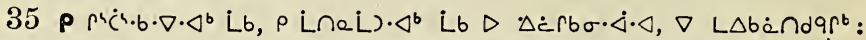

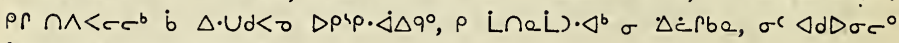
Lb $\rho$ $D \Gamma$ L $\Delta b \dot{a} \cap d q \cdot \Delta^{b} x$

$36 \nabla d C L b \nabla a \triangleleft \wedge r^{b}, \rho$ édbC. $\dot{\Delta}<7 \cdot \triangleleft^{b}$ :

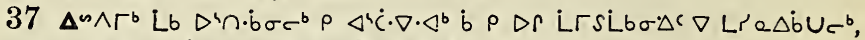

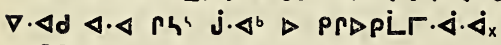

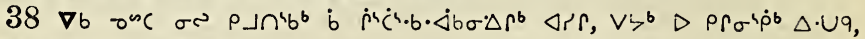
$d c^{b}$ Lb $D$ QLก $\sigma^{4} \dot{p}^{b} \Delta \cup q_{x}$

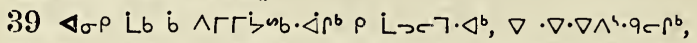

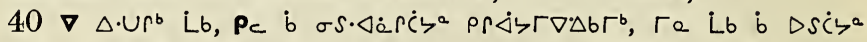

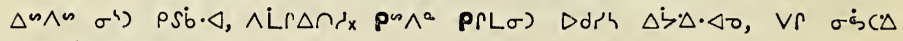
$\dot{\Delta S U}\left\langle\sin ^{b} \Delta r_{x}\right.$

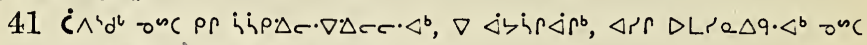

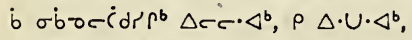

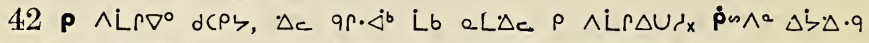

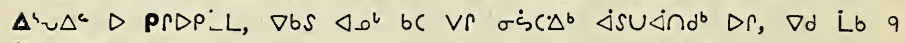
$\dot{C} \cdot V C \cdot \Delta^{b} x$

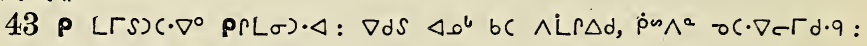
- $\nabla h \rho \Delta \cdot U^{\circ}, \sigma_{c} P(L \sigma) D d \rho h x$

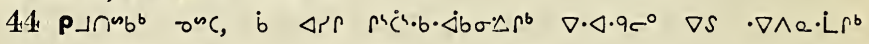
$\Delta \wedge \cap^{b} x$

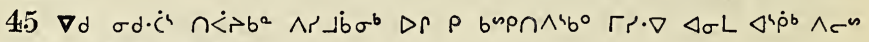

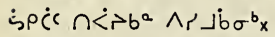

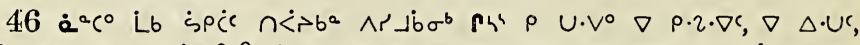

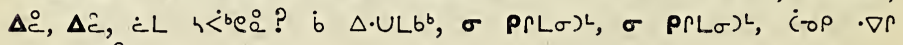
$\cdot \nabla \wedge \sigma \zeta a ?$

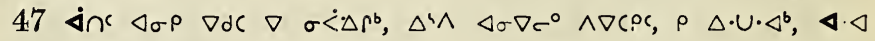
$\Delta c c^{\circ}$ a) $]^{\circ} \Delta \dot{c} \zeta h_{x}$

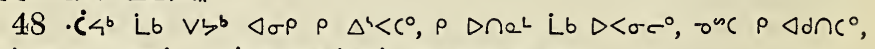

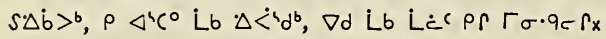

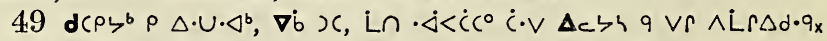

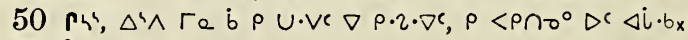

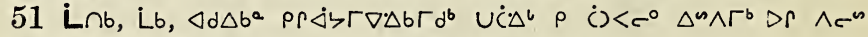

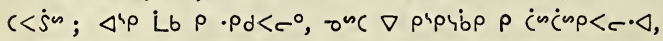

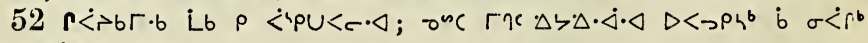
$\rho \cdot \Delta \sigma \omega \dot{b} L b \sigma r \cdot \triangleleft$,

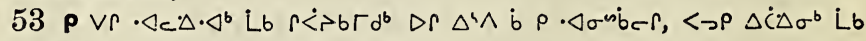

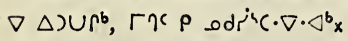

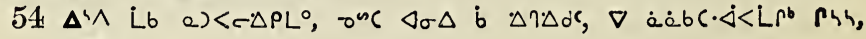




\section{\<>PS Lด゚ 27, 28.}

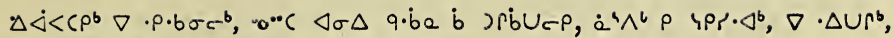

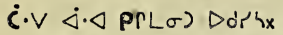

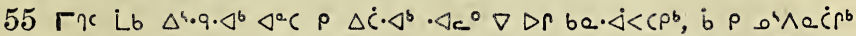
Phs ber ${ }^{b} D R, \nabla D \Gamma^{4} C L \cdot \Delta r^{b}$;

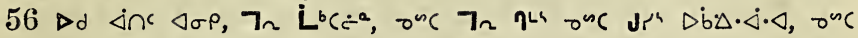
$4<n \Delta C \triangleleft \cdot \dot{S} S \Gamma S \Delta \dot{b} \Delta r \cdot \Delta x$

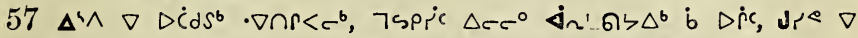

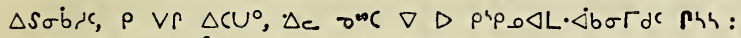

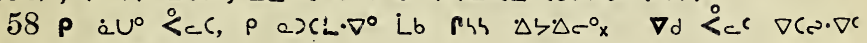

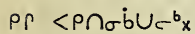

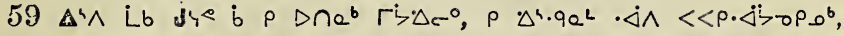

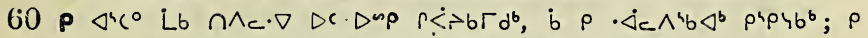

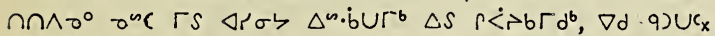

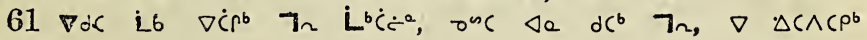
$r \dot{<} \vec{r} b \Gamma d \sigma^{\circ} x$

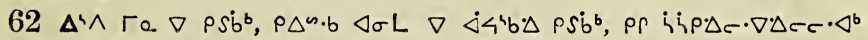

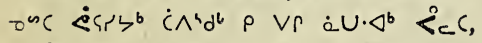

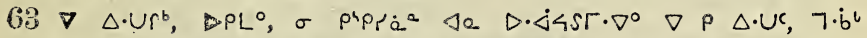

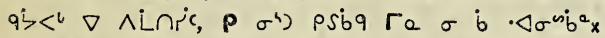

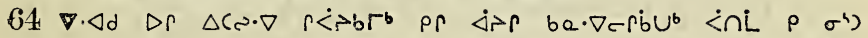

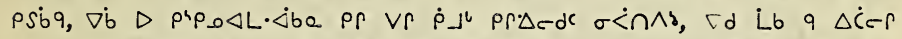

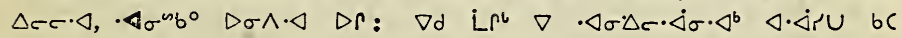
$\dot{\alpha}\left\langle\dot{C} \cdot \triangleleft^{a} \Delta \omega \wedge \omega \dot{\sigma}^{\infty} c^{2} x\right.$

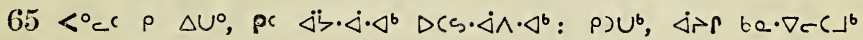
$\Delta u \wedge a$ bupicib

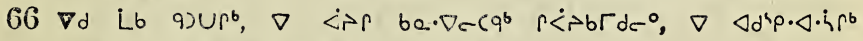

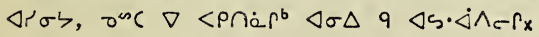

\section{$\Lambda^{4} p c r a \Delta b^{a} 28$.}

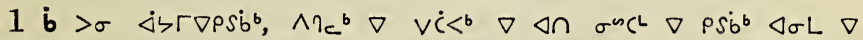

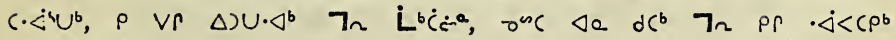
$r \dot{<}>b r \Gamma d c^{\circ} x$

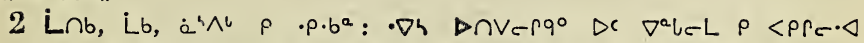

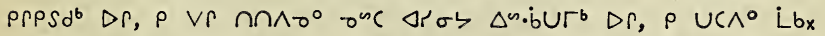

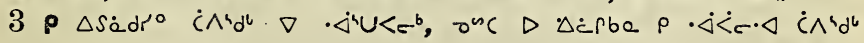
dex

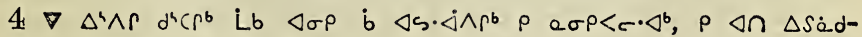

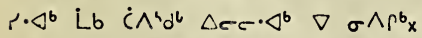

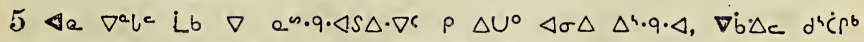

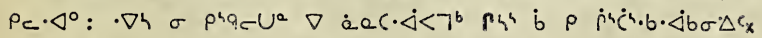

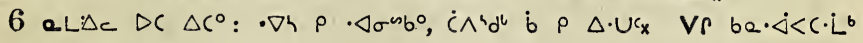
$\triangle c$ b $p \wedge r S^{b} \Delta \cap V r r q^{\circ}:$ 


\section{$\Delta<\neg$ p' Lsb 1 .}

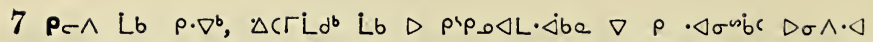

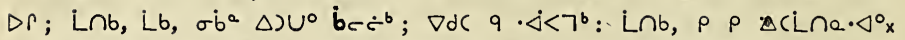

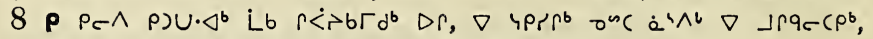

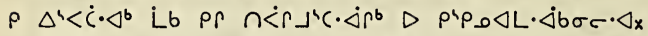

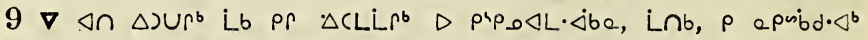

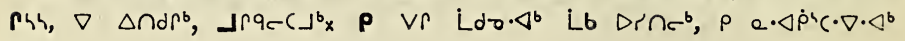
Lbx

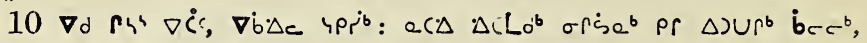
$\nabla d c$ Lb $9 \cdot j<\Gamma r^{b} x$

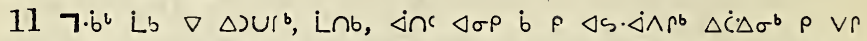

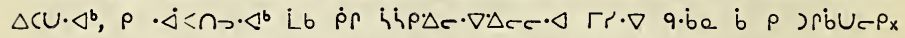

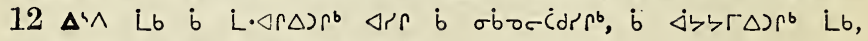

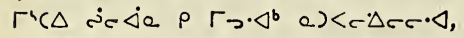

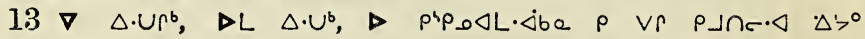
$\sigma \dot{<n \wedge d} 7 \cdot \dot{b} 6 \nabla \quad \sigma \dot{<} \dot{b} b_{x}$

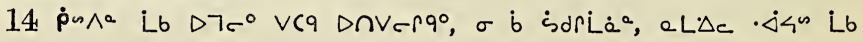
$\rho \dot{b}) \dot{c} b \Delta a \cdot \triangleleft^{\circ} x$

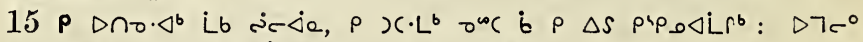

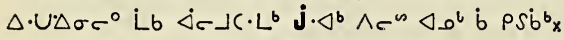

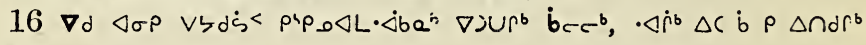
Pihx

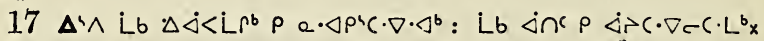

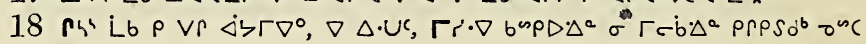
DC $\triangleleft \dot{\rho} \dot{b}_{x}$

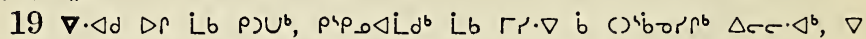

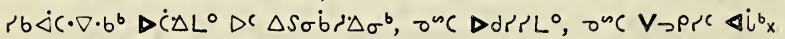

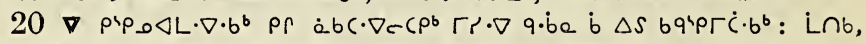
Lb, $\left.\rho \dot{b} \Delta \eta \Delta \cap \dot{a} \cdot \triangleleft^{0}\right\lrcorner \varsigma^{b} \wedge c^{n} q \Delta u \cdot \dot{b}<\sigma^{b} \triangleleft^{a} p_{x} \quad \nabla 7^{a} x$

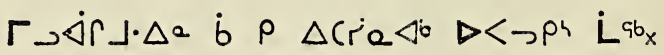

\section{Arpcea $\Delta b^{\circ} 1$.}

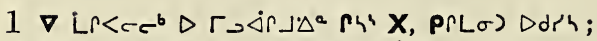

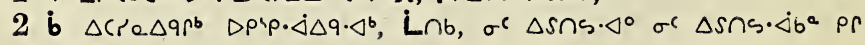
$\sigma \dot{b} \circ \dot{c}$ s, $q \sigma \dot{b} \sigma \cdot \dot{\Delta} \cdot \nabla S C L^{8} \rho 7^{\prime} b \dot{\alpha} c^{\circ} x$

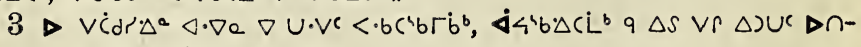

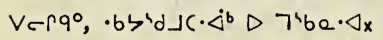

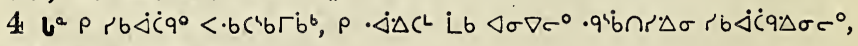

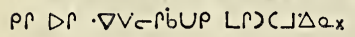

54 


\section{$\triangleright<>\rho^{4}$ L'sb 1.}

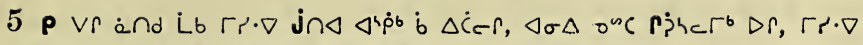

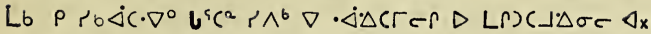

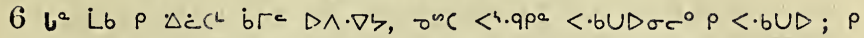

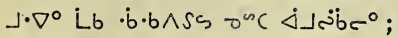

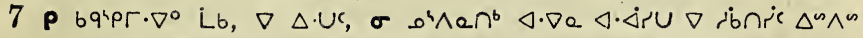

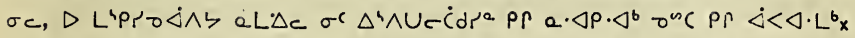

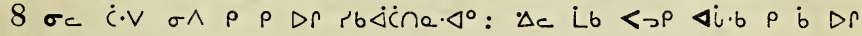
rb $\dot{\Delta} \dot{C} \mathrm{~d} \cdot \Delta^{\circ} \mathrm{x}$

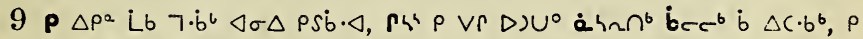
rb $\Delta \dot{C} d$ Lb le $l^{4} C \sigma^{b} x$

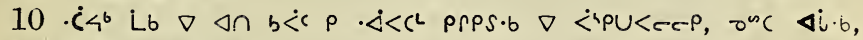

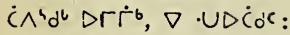

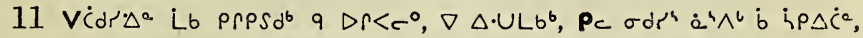
$a^{4} \wedge{ }^{\natural} b a \Delta c \cdot \nabla \Delta b^{a} x$.

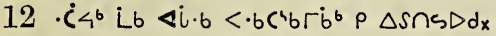

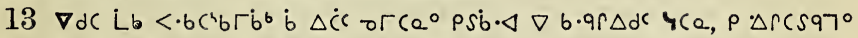

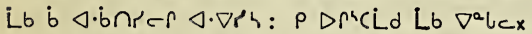

14. $\Delta^{4} \wedge$ Lb $b^{a} \dot{b} \Delta S \cdot \nabla \wedge \dot{a} b \sigma \Delta c p(\Delta) \Delta b \Gamma d^{b}, \mu^{4} \rho \vee r \Delta U^{0} b c c^{b}, \nabla$

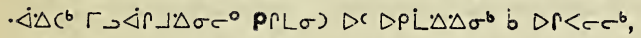

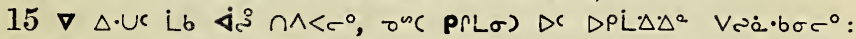

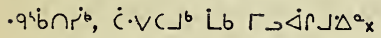

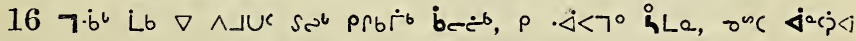

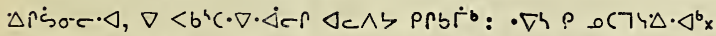

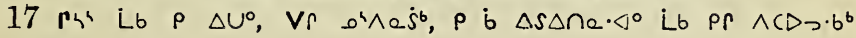
$\Delta c c \cdot \Delta \Delta^{b} \mathrm{x}$

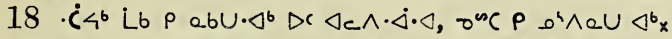

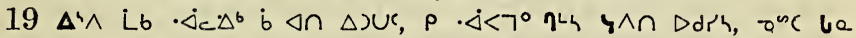
$\Delta r \dot{s} \sigma c \cdot \triangleleft, \dot{a} \wedge \cdot \dot{b} \sigma^{b} \dot{b} \Delta \dot{c} e r \nabla \cdot \dot{d} \wedge C e V c r_{x}$

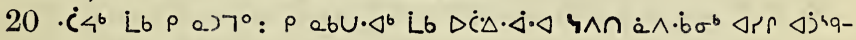
cbe, $P$. ${ }^{4} \wedge e U \cdot \Delta^{b} \mathrm{~L} b \mathrm{bx}$

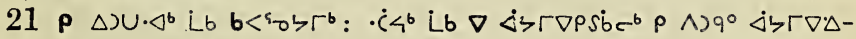
$b \Gamma d^{b}, p$ paposL $L^{\circ} L b_{x}$

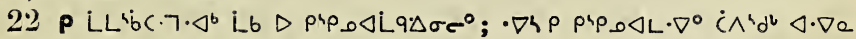

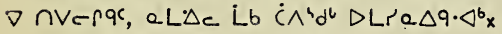

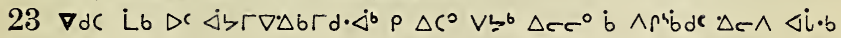
$\rho \cup \cdot V^{\circ}$ Lb,

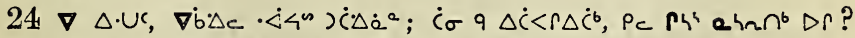

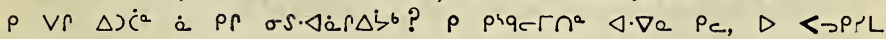
P $(L \sigma)_{x}$

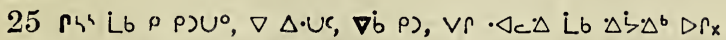

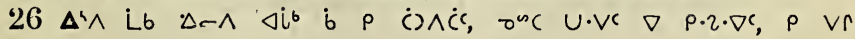
$-\Delta c \Delta^{\circ} x$

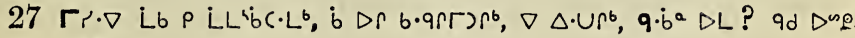
55 


\section{$\Delta<\neg \rho$ iss $1,2$.}

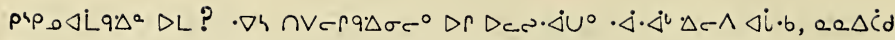
Lbx

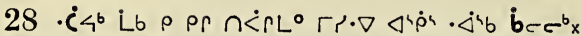

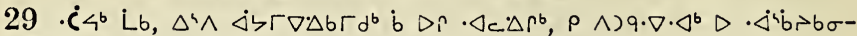

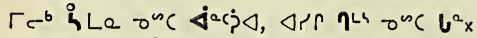

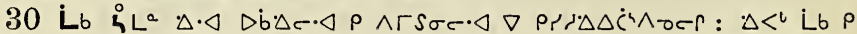
$\triangle C L \cdot \nabla \cdot \Delta^{b} x$

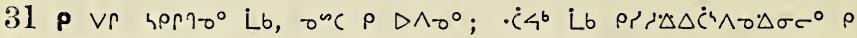
a.bnde. $\triangleleft, \rho \wedge \Gamma \nabla^{\circ}$ Lox

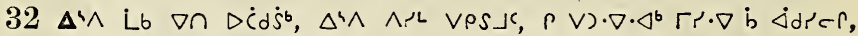
onc $\Delta \sigma \Delta \dot{b} \wedge \mu^{4} \dot{b} d e r$ Lr $\Delta i \cdot b x$

$33 \Gamma r \cdot \nabla \dot{L} \cdot b \quad \Delta \sigma L \Delta \dot{C} \Delta \sigma^{b} \dot{b} \Delta \dot{C}^{b} \rho$ LL $\left.\Delta L \cdot \Delta r \Delta\right) \cdot \Delta^{b} \Delta^{w} \cdot \dot{b} U \Gamma^{b} x$

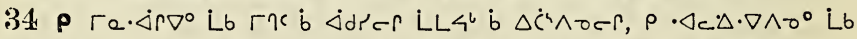

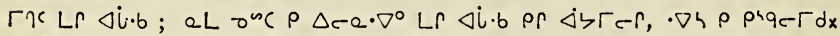

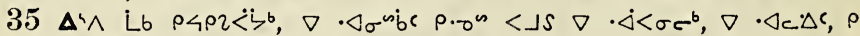
$\Delta) U^{\circ} \Delta(\nabla \sigma S b) \cdot \nabla c \dot{c} \cdot b \sigma c^{b}, \nabla d c$ Lb $\nabla \zeta \Gamma \dot{<} c_{x}$

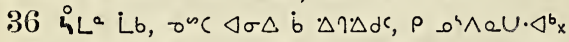

$37 \Delta^{4 \wedge}$ Lb b $\Gamma^{4 b} \cdot \Delta r^{b}, \rho \Delta U \cdot \Delta^{b}, \Gamma r \cdot \nabla \Delta c e^{\cdot} \cdot \Delta^{b} \rho \dot{a} a c \cdot \nabla c \Gamma \cdot b^{b} x$

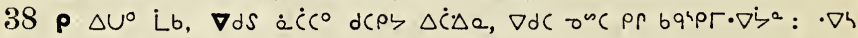
$\nabla \cdot \Delta d \cdot \nabla r \quad v r \Delta x U^{2} \zeta^{2} x$

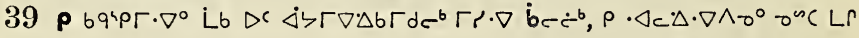
$\Delta i \cdot b x$

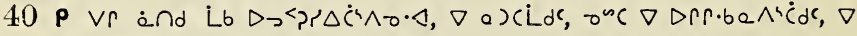
$\Delta$ nde Lb, posa $\Delta)\left(L-0, \rho\right.$ b $\rho<<<\Delta^{a} x$

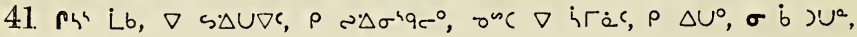
$\dot{<\langle\rho P x}$

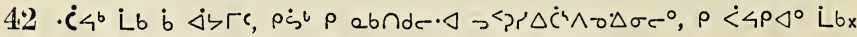

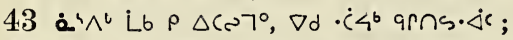

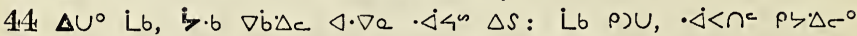

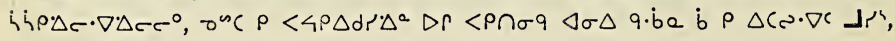

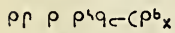

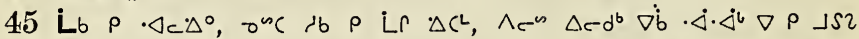

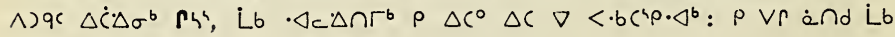
$r \cdot \nabla a \dot{Q})^{b} \nabla r_{x}$

\section{Aspcrasia 2}

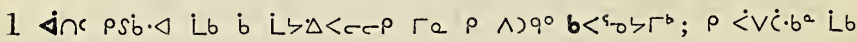
$\Lambda r \cdot \Delta \dot{u} b \vec{b} b \sigma^{b} \nabla \Delta \dot{c}_{x}$

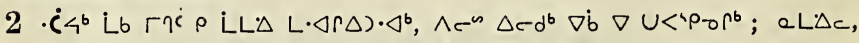

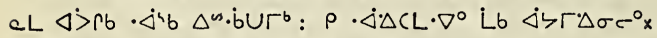

$3 \rho \vee r \dot{a} U \cdot \Delta^{b} L b, \nabla \vee s \cdot \Delta r^{b} \triangleleft \cdot \nabla \sigma \cdot \triangleleft \nabla a Q L^{4} \wedge \sigma r r, \nabla^{\circ} \nabla \wedge \Gamma \Delta \dot{\varepsilon} r^{b} x$

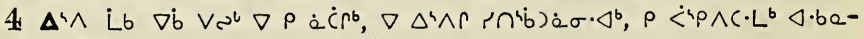
56 


\section{$\Delta<>$ ps Lis 2.}

$\Delta b \sigma c^{\circ} \Delta C \nabla \Delta \dot{C} c_{r}: \Delta^{4} \wedge$ Lb b $\rho C \Delta e p^{b}, \rho \dot{C} S e \cdot L^{b} \sigma V \Delta \sigma c^{\circ} \Delta C D Q Q L^{4}-$ $\wedge_{0} \cdot \Delta \nabla \wedge \Gamma S \sigma e r_{x}$

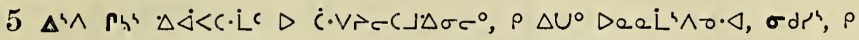
Lr) (\lrcorner$\Delta a \rho \cdot \nabla \vee-\left(L b \Delta^{a} x\right.$

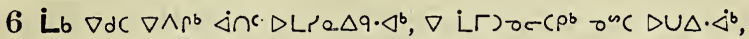

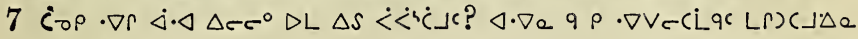
P(L $\sigma) \wedge d$ ?

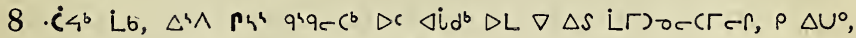

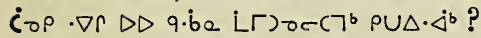

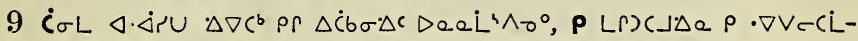

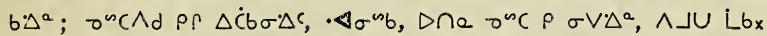

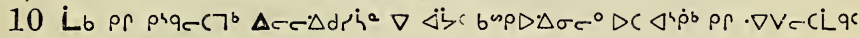

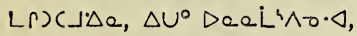

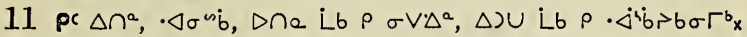

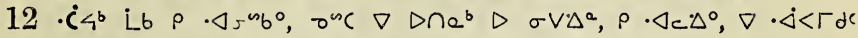

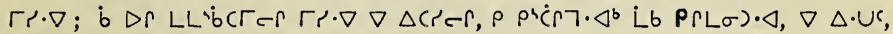

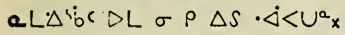

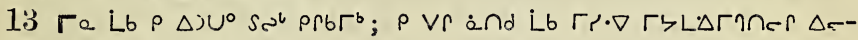
$\tau \cdot \triangleleft, \rho$ pip. $\rho_{0} \Delta L \cdot \nabla^{\circ} \mathrm{L} b_{x}$

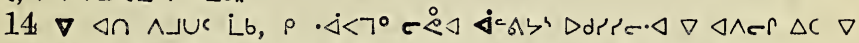

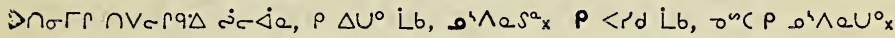

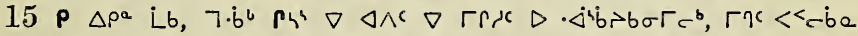

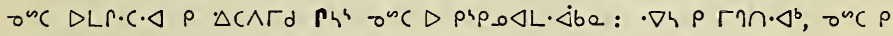
o $\wedge$ QU. $\Delta^{b} x$

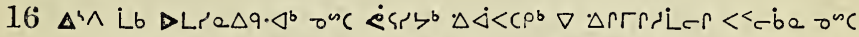

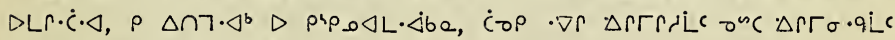
i< be onC $\triangle L C \cdot C \cdot \triangleleft$ ?

$17 \Delta^{\prime} \wedge \rho^{\prime} \wedge \nabla C^{b}, \Delta U^{0}, \Delta \sigma \rho$ b $\Gamma \rightarrow \Delta b^{b} \rho^{b}$ a $L \Delta c$ ec $\cdot \nabla c 7 \cdot \Delta^{b}$ eCdea, Lb

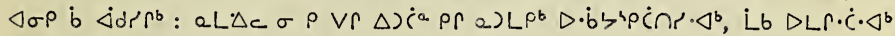
br apionspbx

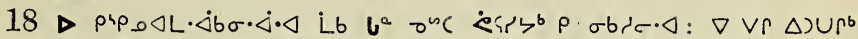

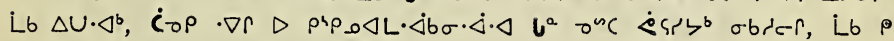

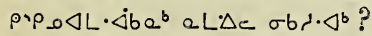

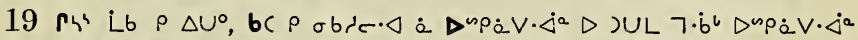

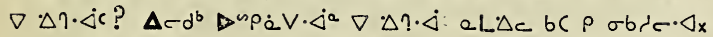

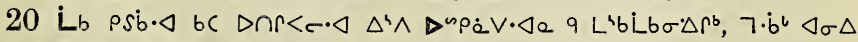
$\rho s \dot{s} \cdot \triangleleft$ Lb bC $\sigma b d \cdot \triangleleft^{b} x$

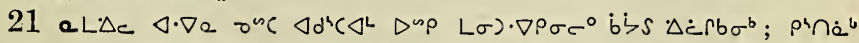

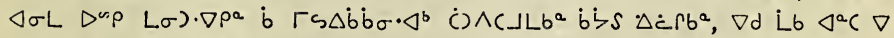
() $<\tau^{b} \triangleleft \cdot \dot{\Delta r} \cup \neg \dot{S} b{ }^{b}$

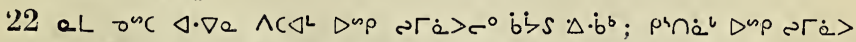

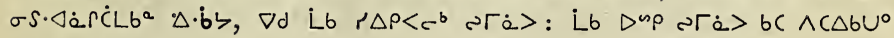
Dup $\triangle \cdot b^{b} \times$ 


$$
\Delta<>\text { pr Lsb } 2,3 .
$$

$23 \rho \triangle p^{a}$ Lb $\nabla \rho$ s>mb LCi

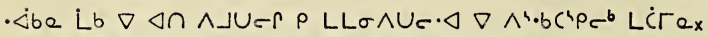

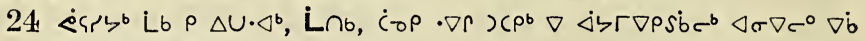
bे $\operatorname{le}_{c} \cdot \nabla c \dot{C} \cdot b \sigma c^{b}$ ?

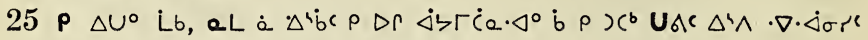

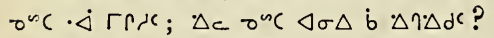

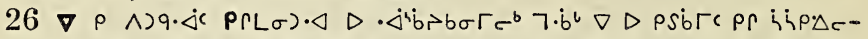

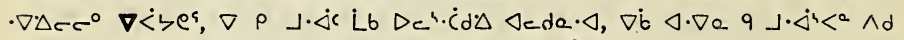

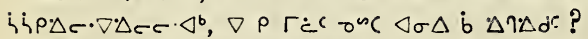

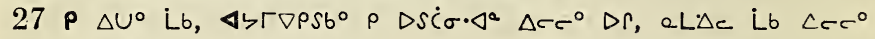
$\dot{\angle} \backslash \Gamma P S 6^{\circ} D \Gamma:$

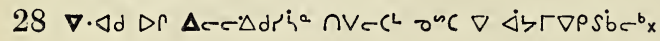

\section{A p p r $\triangle b^{\circ} 3$.}

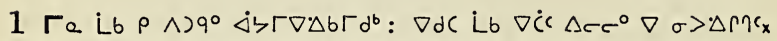

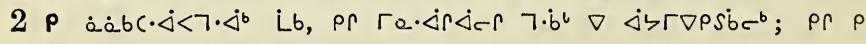
LITSinco ${ }^{b}$

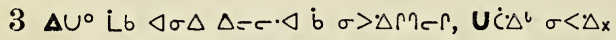

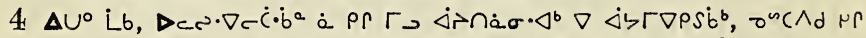

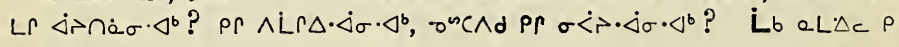
Dr $\rho) \cdot \Delta^{b} x$

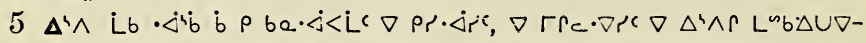

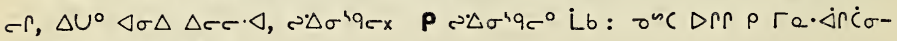
$-\Delta \sigma \sigma^{\circ} \dot{C} \wedge^{4} d_{l} \mathrm{~d}\left(P c^{\circ} x\right.$

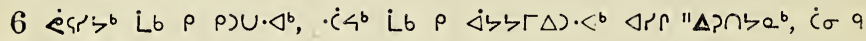
$\triangle S \sigma S \cdot \Delta \dot{\alpha} r \dot{\Delta} r^{b} x$

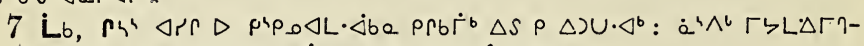

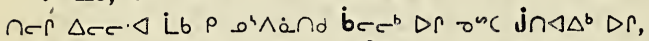

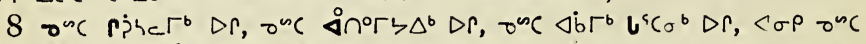

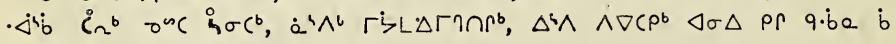

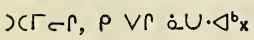

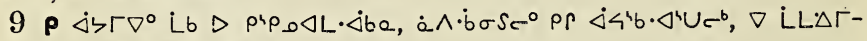
ince $D r, \nabla \dot{b}$ or rnsibdcx

$10 \cdot \nabla h \Gamma x \rho \Gamma a \cdot \Delta r \dot{j} \cdot \Delta^{b}, \dot{b}$ or rnubde $\nabla \Delta i \Gamma \sigma d c, \Gamma r \cdot \nabla \Delta \sigma \Delta b$ $\Delta r C^{\prime} \wedge$ or $r x$

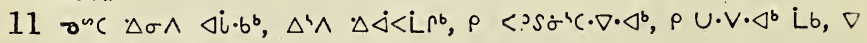
$\triangle \cdot U \Gamma^{b}, P_{C} P(L \sigma) D d r_{x}$

$12 \mathrm{~d}$ b Lb $\rho \Delta C \sim 7^{\circ} \nabla \dot{b}$ pp piqcid $\Delta d c_{x}$

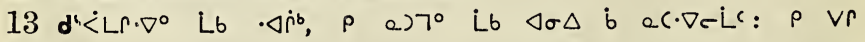
ind L bx

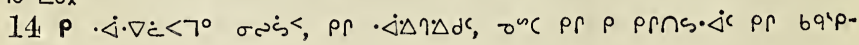
$\Gamma \cdot \nabla \subset \Gamma$, 


\section{$D<>$ ps L ${ }^{\text {sb }} 3,4$.}

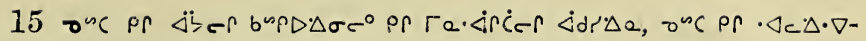
$\wedge \dot{a}-r$ Lr $\Delta i \cdot b x$

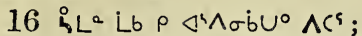

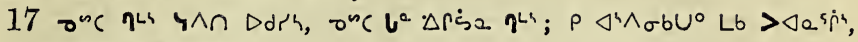

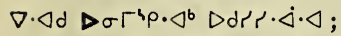

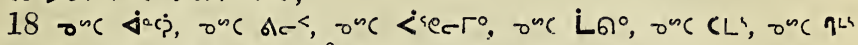

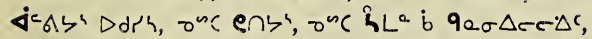

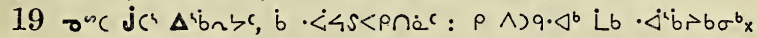

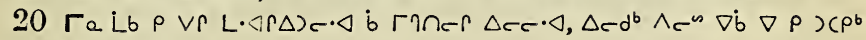
pr $\lrcorner \cdot \Delta r^{b} \varangle c d a \cdot \Delta x$

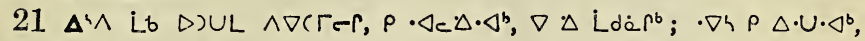

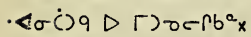

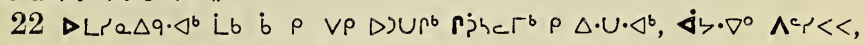

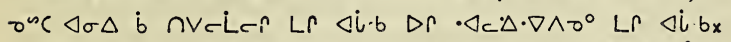

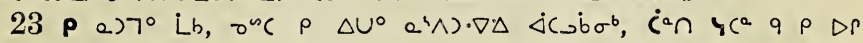

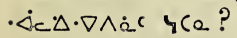

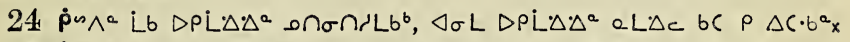

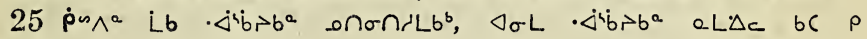
$\triangle C^{4}, \cup^{\circ}{ }^{\circ}$

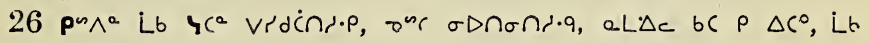
bc $>\sigma \Delta c^{\circ} x$

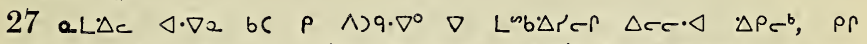

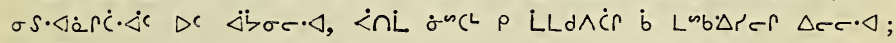

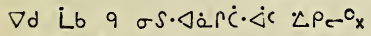

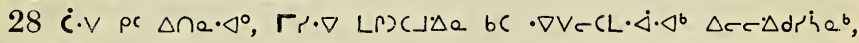

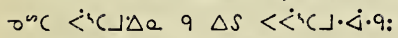

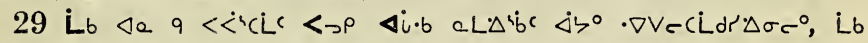

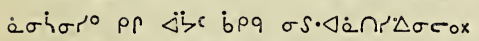

$30 \nabla \rho \Delta \cdot u r^{b}, \dot{\Delta} \zeta \cdot \nabla^{\circ} \Delta r \wedge \Delta i \cdot b x$

$31 \nabla d \wedge \nabla r \Delta x \cup r \quad \Delta r \dot{s} a$ occ $\Delta \dot{b} \Delta b, \Delta c \Delta \cap r^{b}$ Lb $\nabla \sigma \dot{<} \Delta c r, \rho$ $\triangle S \cap S D \cdot \nabla c \cdot \triangleleft, \nabla$ a) $\Gamma d^{b} \mathbf{x}$

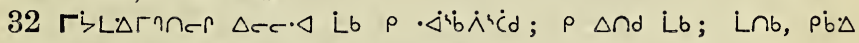

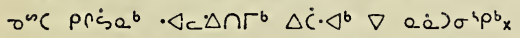

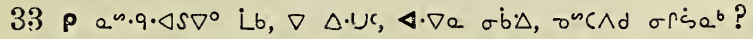

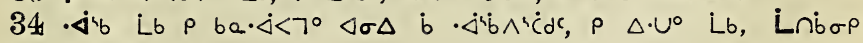
$\sigma \dot{b} \Delta \sigma^{\prime \prime C}$ orśab!

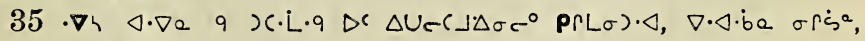
(⿻) $\sigma r \dot{s} \sigma^{4} \cdot q 4,0^{n c} \sigma \dot{b} \Delta x$

\section{Aspcra $\triangle b^{2} 4$.}

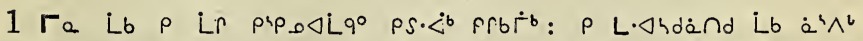

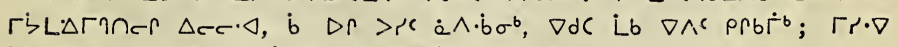

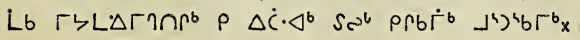




\section{$\Delta<>$ ps Lss 4.}

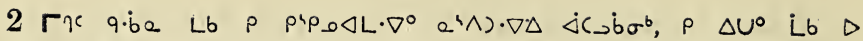

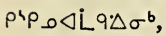

3 a) (\lrcorner$^{b} ; \operatorname{L} \cap b, \nabla \cdot \Delta h \cdot \zeta \wedge_{\sigma} q^{\circ} \rho \cdot \Delta c \Delta^{\circ} p r \cdot \triangleleft h \cdot \zeta \wedge \sigma q c$ :

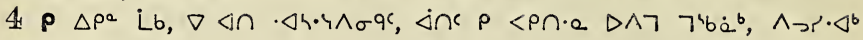
Lb $\rho \vee P r \rho \dot{C} \cdot \Delta b x$

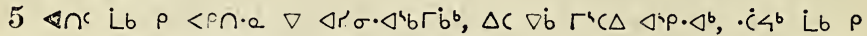
iं $\sigma \dot{c} \dot{C} \Delta \cdot a, \nabla \dot{b} \nabla \rho^{i}<\dot{b}^{b} \Delta^{i} p:$

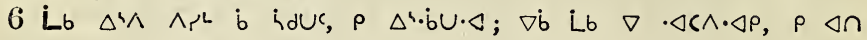
$\sigma \wedge L b \cdot a_{x}$

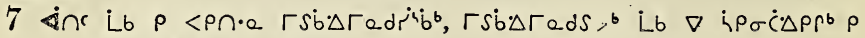
$r \Lambda^{4} b \cdot L^{b}, a L \Delta c$ Lb $\rho \Gamma \sigma s \cdot \Delta^{2} x$

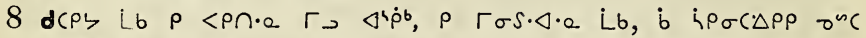

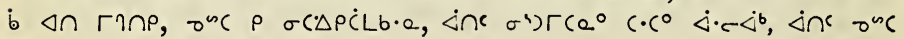

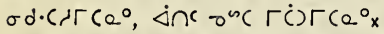

9 p $\triangle U^{\circ}$ Lb $\varangle a \dot{b} \Delta \dot{C} \cdot \Delta b^{c}$ of $V C^{b}, \nabla d S$ bc $V C C_{x}$

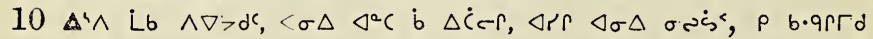

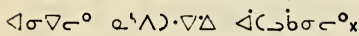

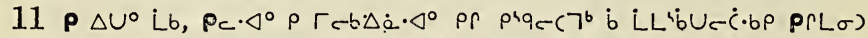

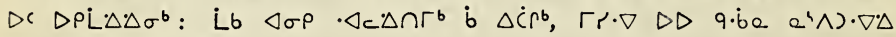
$<\left(\Delta \dot{b} \sigma^{b}\right)\left(L \cdot \dot{\Delta} \cdot \Delta^{b}\right.$ :

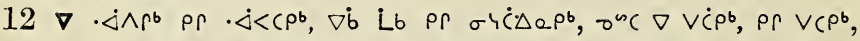

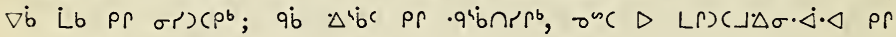
$\cdot \nabla V C C L \cdot<b \sigma \Delta \Delta r^{b} x_{x}$

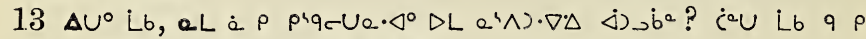

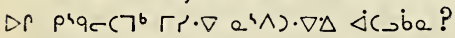

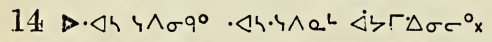

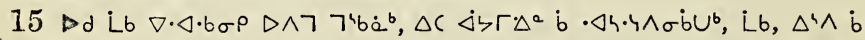

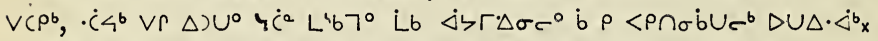

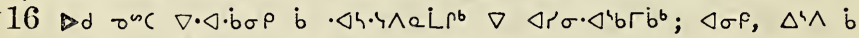

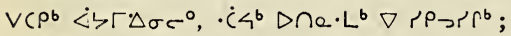

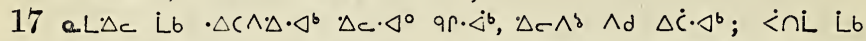

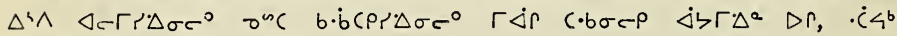
$\dot{\alpha} 4 C \cdot \nabla \Gamma C \cdot L^{b} x$

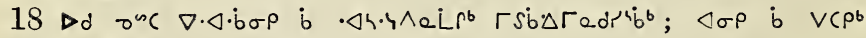

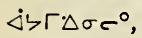

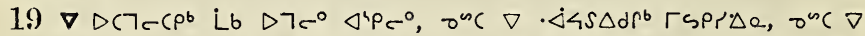

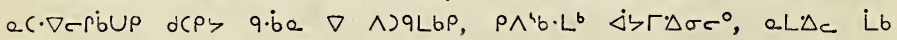
$\Gamma \sigma s \cdot<^{2} x$

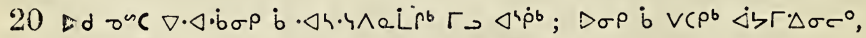

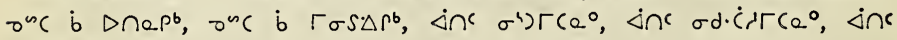
$\Gamma \dot{C}) \Gamma\left(0^{\circ} x\right.$

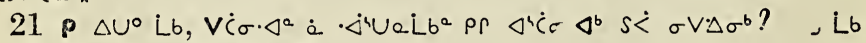

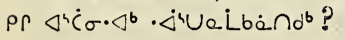




\section{$D<>$ p' L 4 \& 4,5 .}

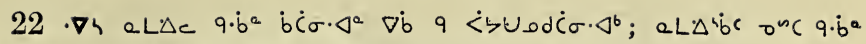
$\dot{\rho}\lrcorner^{l} \rho$ be $\cdot \nabla c r \dot{b} U^{\circ}, \nabla b$ q prqc $\dot{c} \cdot b^{b} x$

$23 \dot{\rho} \backsim \wedge^{a} \triangleleft \cdot \nabla a \cdot \nabla C \cdot \Delta b \cdot q$ pr $\vee C^{b}, \nabla d S b C \vee C C^{L} x$

$24 \Delta U^{\circ} L b, \dot{i} \cdot \dot{b} \Gamma r^{b} q \cdot b^{a} \dot{b} \vee C 7^{b}: \Delta \sigma L \cap \dot{<}>b^{a} q \Delta S \cap \dot{<}>q \zeta^{b}, \nabla d q$

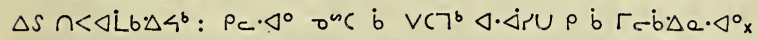

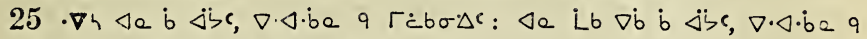
$L ' b L b \sigma \cdot \Delta c \cdot \dot{\Delta} \cdot \dot{b l} \Delta \sigma \nabla c^{\circ} \dot{b} \Delta \dot{b} c_{x}$

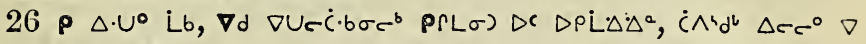
- $\nabla \wedge a^{b}$ r

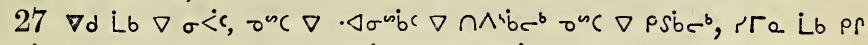

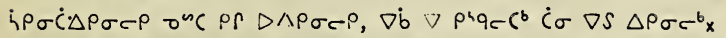

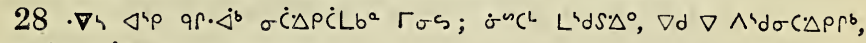
$\dot{<} \mathrm{L} L$ Lb LCं ${ }^{a} \nabla$ PS $\sigma C \triangle P C_{x}$

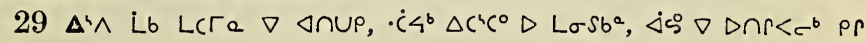
$L \cdot \Delta r r b \sigma \cdot \Delta b^{b} x$

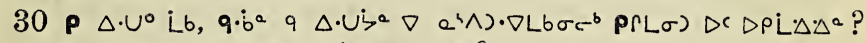

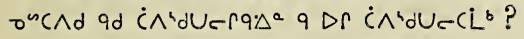

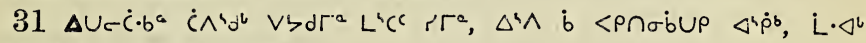

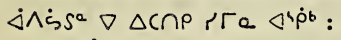

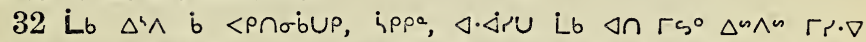

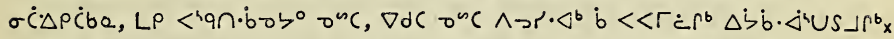

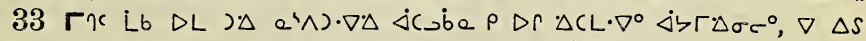

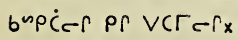

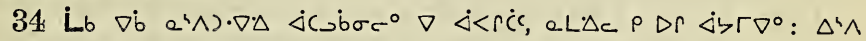

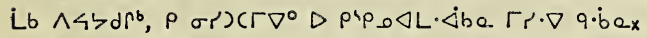

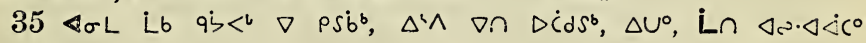
.q6ibl $\Delta s_{x}$

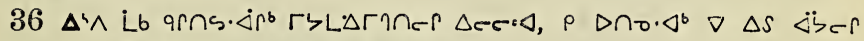
$\dot{a} \wedge \cdot \dot{b} \sigma^{b}: \rho \Delta C D \Gamma d$ onc dCPs $\dot{a} \wedge \cdot \dot{b} \sigma s_{x}$

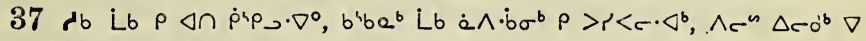
íb? 0 ob $x$

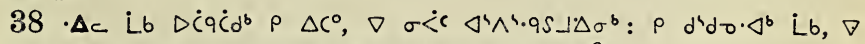

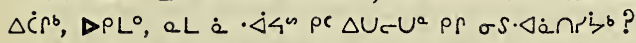

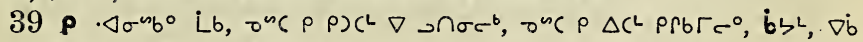

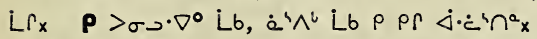

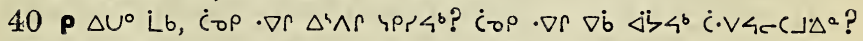

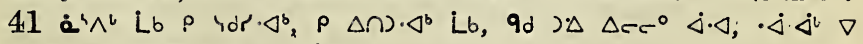

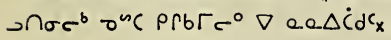

\section{A $P C r e \triangle b^{2} 5$.}

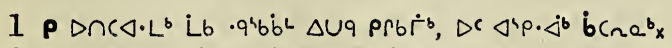

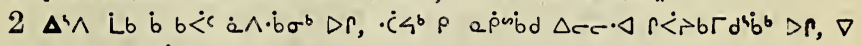

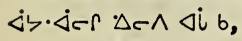




\section{$\Delta<\rightarrow$ ps Ls 5.}

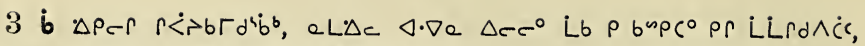

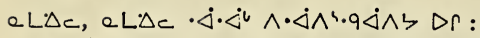

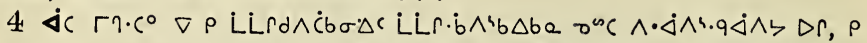

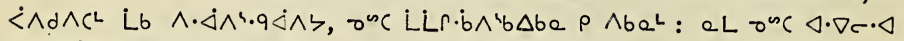

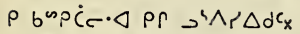

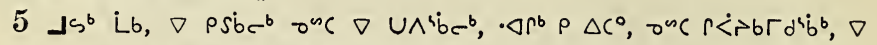

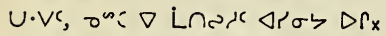

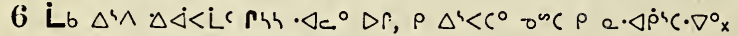

7 p U.Vo Lb $\nabla$ p.2. $\nabla c$, onc $\rho \Delta \cdot U^{\circ}, \dot{C}_{\sigma} \rho_{c} q \Delta \dot{C}<r \Delta C^{a}, \rho_{4} \rho_{c}$

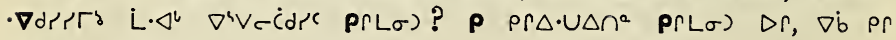
b $b C p \triangle \zeta^{2} x$

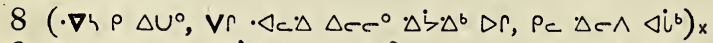

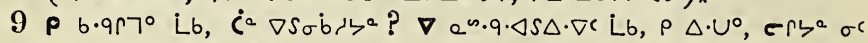
$\triangle S \sigma \dot{b} \gamma^{a} ; \cdot \nabla h \sigma \Gamma \eta \cap a^{a} x$

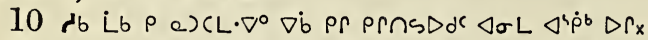

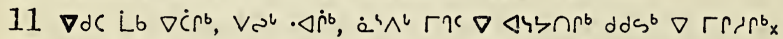

$12 \Gamma r \cdot \nabla$ Lb Lr $\Delta i \cdot b^{b} \rho$ a $)\left(L \cdot \nabla \cdot \Delta^{b}, \nabla \Delta \cdot U^{b}, \Delta S \cap S D \dot{a}^{a} \Delta C \nabla \Delta C^{b}\right.$ ddd $b$, $\rho \Gamma \wedge \supset q^{b} b \cdot \Delta \rho \rho^{b} x_{x}$

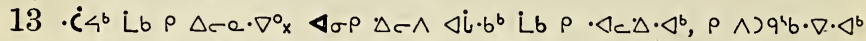

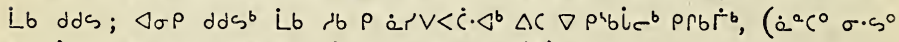

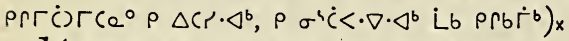

$14 \triangleleft \sigma^{\circ}$ Lb b $\Delta s \dot{L} \Gamma^{b}$ dds $\left.\rho \Delta S\right\lrcorner \cdot \Delta^{b}, \rho \Delta C \cdot L^{b}$ Lb $\Delta \dot{C} \Delta \sigma^{b}$, osc $<^{a} C$

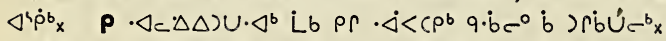

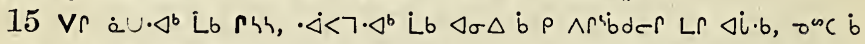

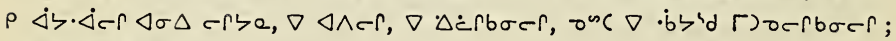

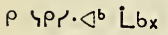

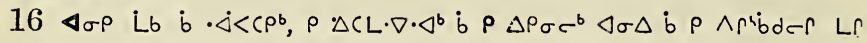
$\triangle i \cdot b, \Delta t r$ ons dds $\nabla r_{x}$

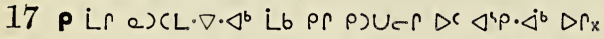

$18 \Delta^{4} \wedge$ Lb $\dot{b}>r^{c} \dot{a} \wedge \cdot \dot{b} \sigma^{b}$, $\Delta a b \quad \dot{b} \wedge r^{4} b d^{c}$ Lr $\left.\Delta i \cdot b \rho a\right)\left(L \cdot \nabla^{\circ} \rho \Gamma\right.$ $\Delta \eta \cdot \dot{\triangleleft} \dot{c}_{\mathrm{x}}$

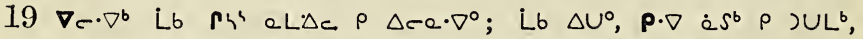

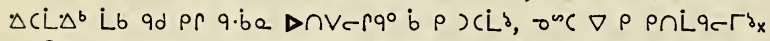

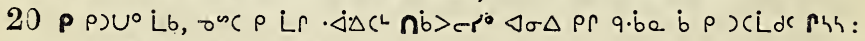

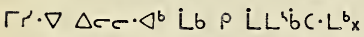

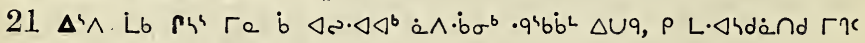

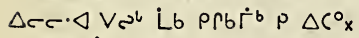

22 Lnb, Lb, $\rho \vee r$ and $\triangleleft \cdot \nabla c \cdot \triangleleft b$ bVecrer $\triangleleft b \Gamma \nabla \Delta b \Gamma d c^{\circ}, \eta \zeta^{4} \nabla$

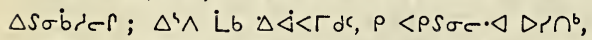

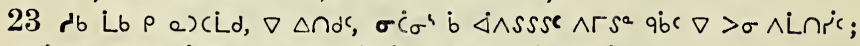

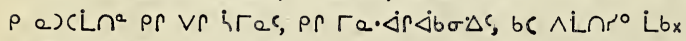

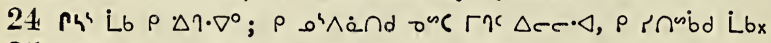

$25 V^{\prime b}$ Lb $\Delta^{4} \cdot 9^{\circ}, \nabla \Delta r b \cdot \triangleleft \sigma c^{b} \triangleright \Gamma d \sigma \sigma^{\circ}<\wedge>\cdot a$, 


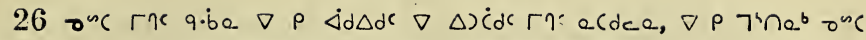

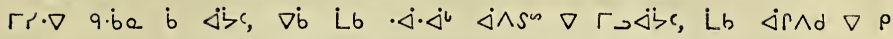
४doc,

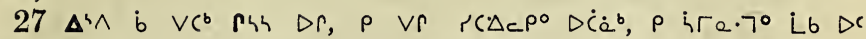
$\measuredangle d D \sigma \sigma^{\circ}$ :

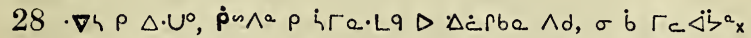

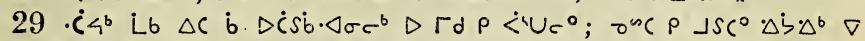

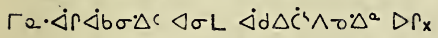

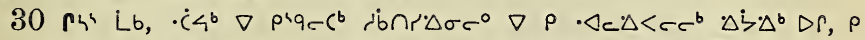

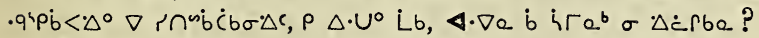

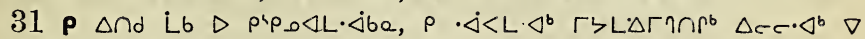
rnubipb, $\rho c \Delta \cdot \dot{c}^{a} \dot{a} L b, \varangle \cdot \nabla e \dot{b}$ i $\zeta \sigma c$ ?

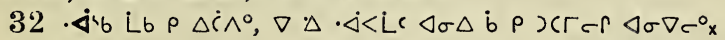

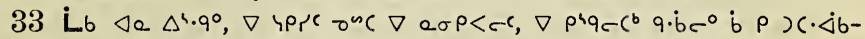

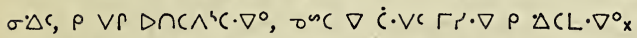

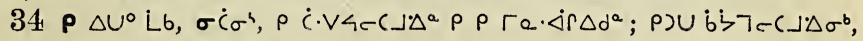

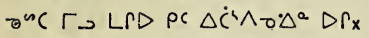

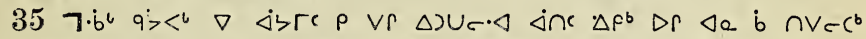

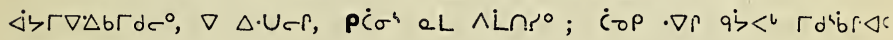
DPLO?

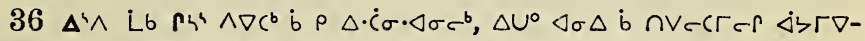

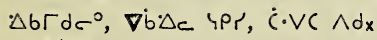

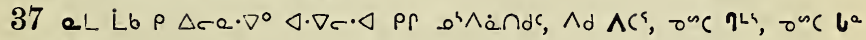
$\Delta$ risa $\eta^{L 4} \mathrm{x}$

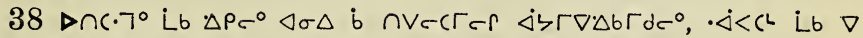

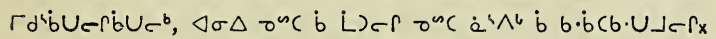

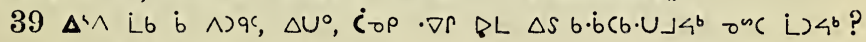
Dupops.qS" aLA $\Delta_{c} \sigma \wedge^{\circ}$, Lb $\sigma<^{c} x$

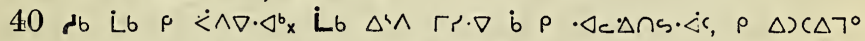

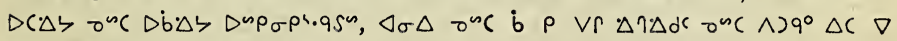
$\triangle S \wedge \Gamma S \sigma e r$ Dupoph.qSSx

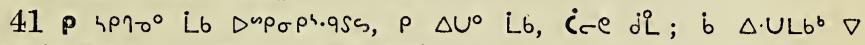

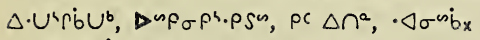

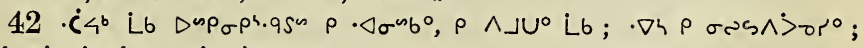

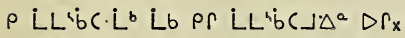

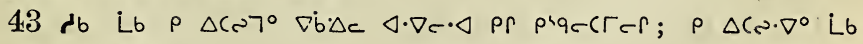
$q . e_{0} \operatorname{pr}<s t r r_{x}$

\section{A $P C$ P $\triangle b^{a} 6$.}

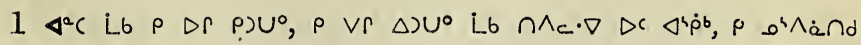
Lb $D$ p'p. $\Delta L \cdot \dot{\Delta b a x}$

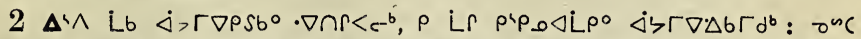

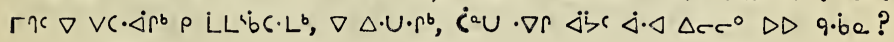
63 


\section{$\Delta<>$ ps Lis 6 .}

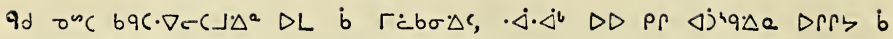
D.P $x^{\circ}$ ?

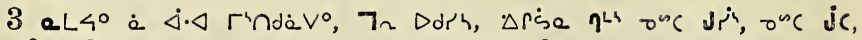

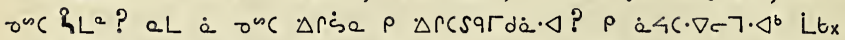

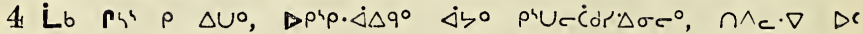

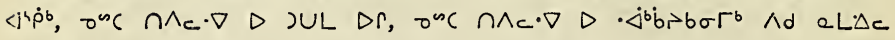
prUcLb $\sigma^{\circ} \Delta^{0} \mathrm{x}$

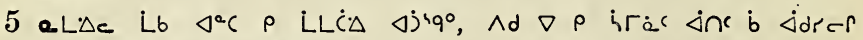
$\Delta r c \cdot \triangleleft$ rec $\nabla \rho \Gamma_{Q} \cdot \dot{\Delta r} \dot{\Delta c_{x}}$

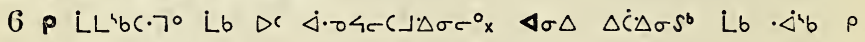

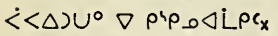

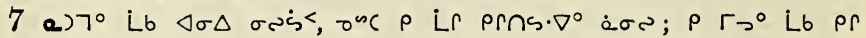
sंdricr $\Delta r \wedge \Delta i \cdot b$;

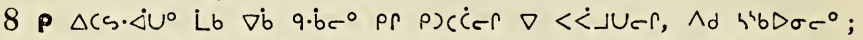

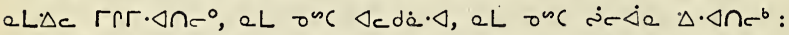

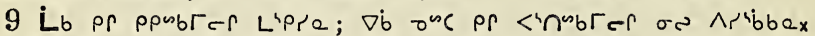

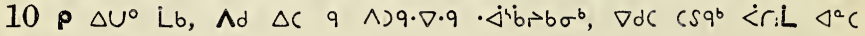
Ar p)U $4 \cdot q_{x}$

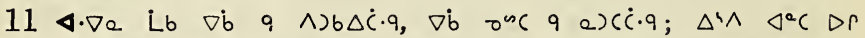

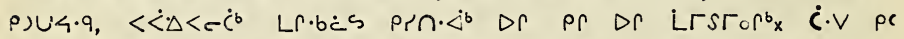

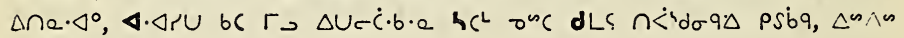
$\Delta \sigma L \Delta \dot{C} \Delta^{a} x$

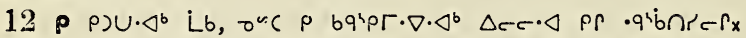

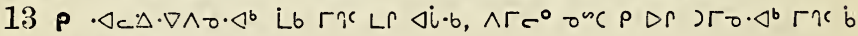

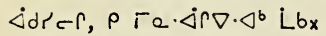

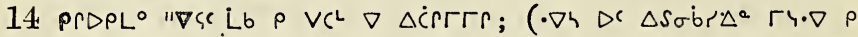

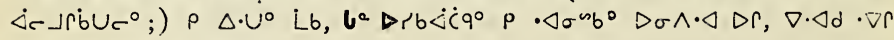

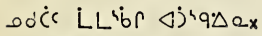

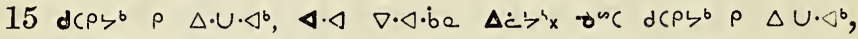

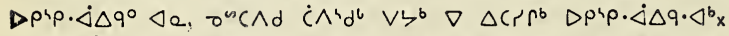

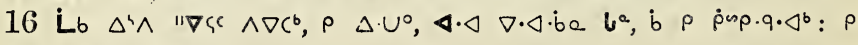
$\cdot \triangleleft \sigma^{m b}{ }^{\circ} \nabla \sigma \wedge \cdot \triangleleft D r_{x}$

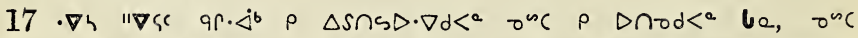

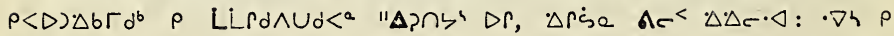
$\triangle P\urcorner d<0^{\circ} x$

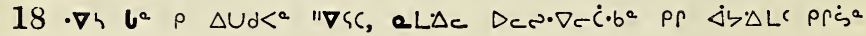
$\Delta \cdot \triangleleft_{x}$

$19 \nabla \cdot \Delta d \cdot \nabla f \quad \Delta ; \cap\rangle^{\prime} \rho<\cdot b \dot{c} c, b c \quad \rho \quad \sigma<\nabla<a \quad L b ; a L \Delta c$ Lb $\rho$ mpc ${ }^{\circ} x$

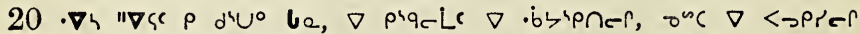

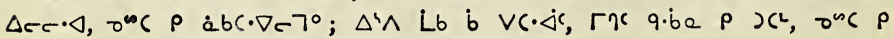
$s b \rightarrow r_{0} \nabla V C \cdot \Delta c^{x}$

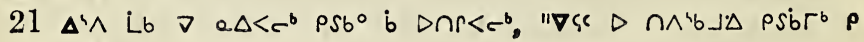
64t 


\section{$D<\rightarrow$ piss 6 .}

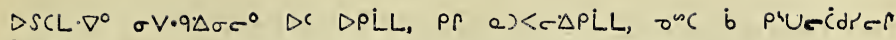
bcc $c^{b}$ :

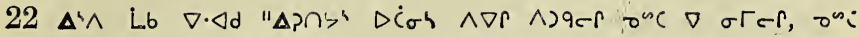

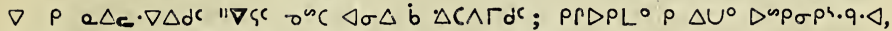
a) $C \dot{L} \Delta^{a} \wedge d \quad q \cdot b^{a} \dot{b}$ ac $\nabla c C L^{a}, \nabla d$ Lb $q \Gamma-c^{a} x$

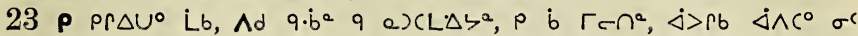
$\triangle P L \Delta \triangle \Delta^{a} x$

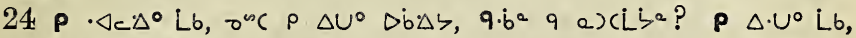

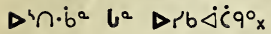

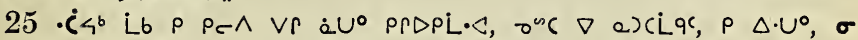

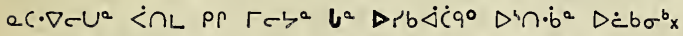

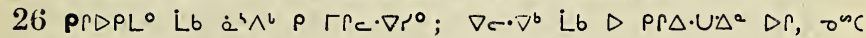
$\triangle \sigma \Delta D r \dot{b} \Delta C \wedge r d c$, eLAc $\Delta<\cdot \sigma c \cdot \nabla^{\circ} x$

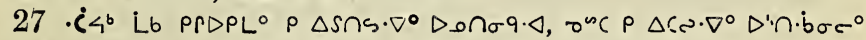

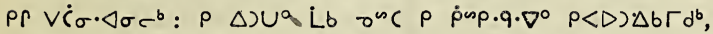

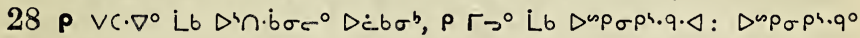
Lb $\rho \Gamma \supset^{\circ} \Delta \dot{b} \Delta \zeta^{x}$

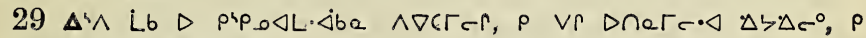
$\Delta^{4} \dot{C} \cdot \nabla \cdot \Delta^{b}$ Ĺb $r \dot{<}>b \Gamma d^{b} x$

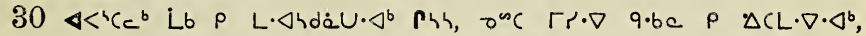

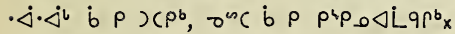

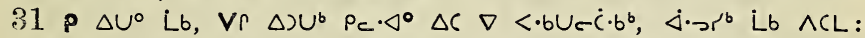

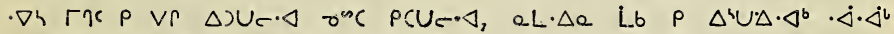

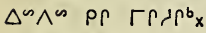

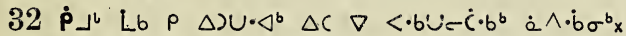

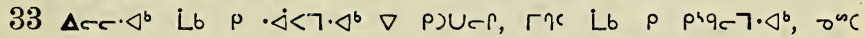

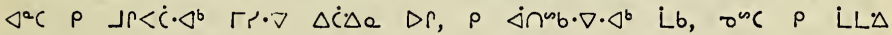
¿े $\cap \cdot \triangleleft^{b} \mathrm{x}$

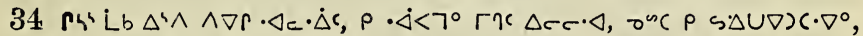

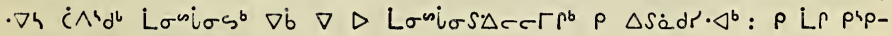

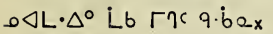

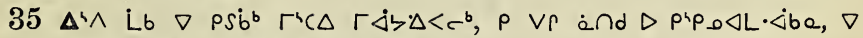

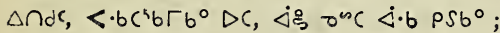

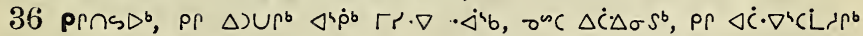

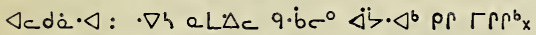

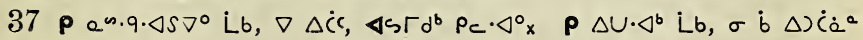

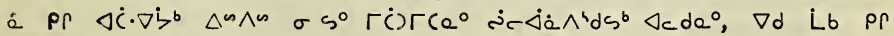
$\checkmark s L d^{b} !$

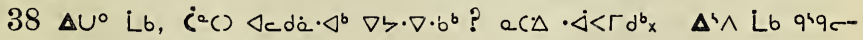
( $p^{b}, \Delta \cdot U \cdot \Delta^{b}, \sigma>c^{c}, \nabla^{\mu c}\left(\sigma a\right.$ a $7 b^{b} x$

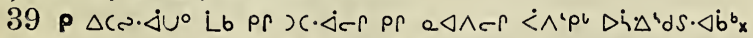

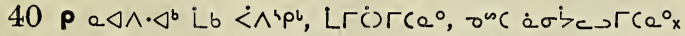

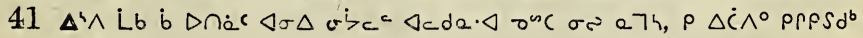
65 


$$
\Delta<-s \rho \text { ' Lsb } 6,7 .
$$

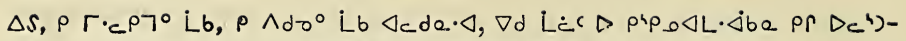
- $\Delta C r ;$;

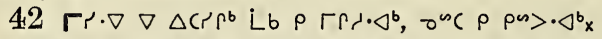

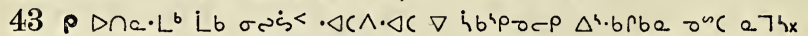

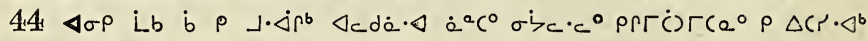
$\Delta r c \cdot \triangleleft^{b} \mathrm{x}$

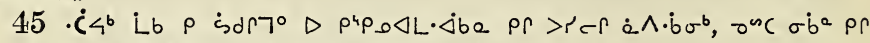

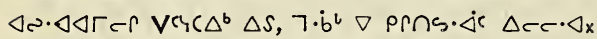

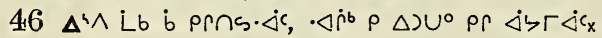

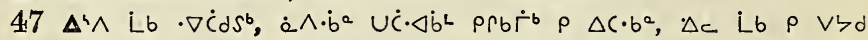
$J^{2} c^{4} b \Gamma^{b} x$

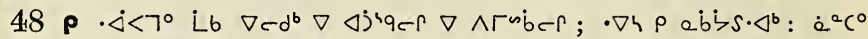

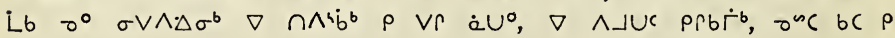

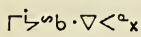

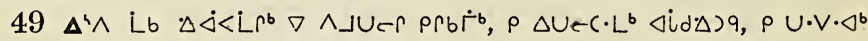
Lb ;

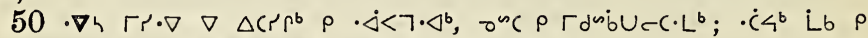

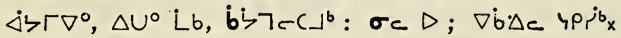

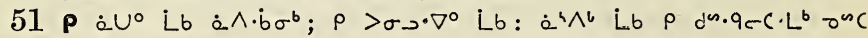
$\dot{\Delta} \cdot \cdot \dot{\Delta}^{b} \rho \dot{L} L^{b} b C \cdot L^{b} x$

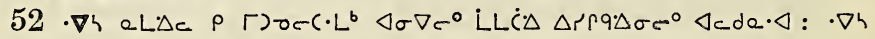

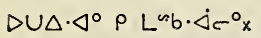

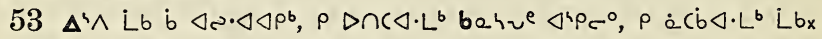

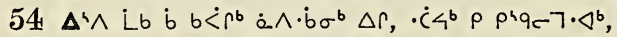

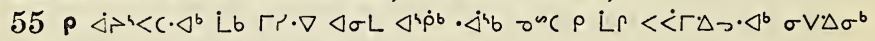
$\triangleleft \sigma \Delta \dot{b}<\operatorname{dicer} \Delta C \nabla \vee C \rho^{b} \nabla \Delta \dot{C}-r x$

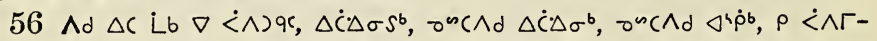

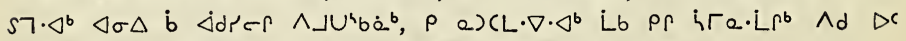
$\Delta d D \sigma \sigma^{\circ} \cap \cap \wedge^{4} c \Delta b \sigma^{b} ; \Gamma s \cdot \nabla$ Lb b $i \Gamma a \Gamma^{b} \rho \Gamma a \cdot \Delta r \dot{\Delta} \cdot \Delta^{b} x$

\section{A}

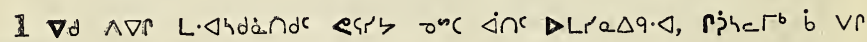
D) UES

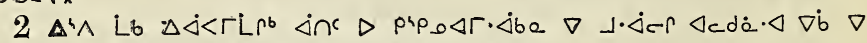

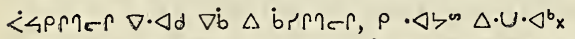

$3 \cdot \nabla h$ एद⿱

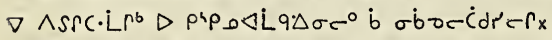

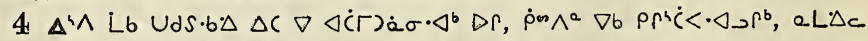

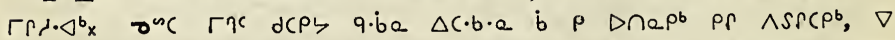

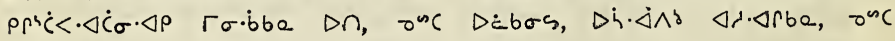
rrjén.px

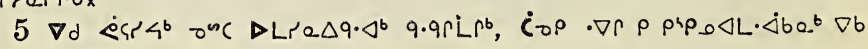
66 


\section{$\Delta<\rightarrow \rho^{4} \mathrm{~L}^{46} 7$.}

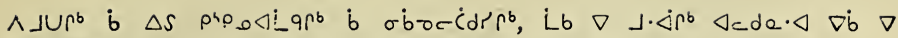
inrmps?

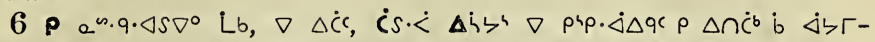

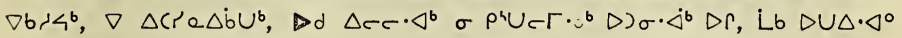
$\sigma c \cdot \dot{\Delta}<\Gamma d \cdot \dot{\Delta}^{a} x$

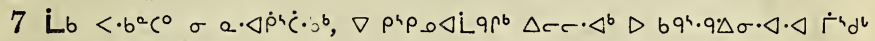
.bלs pip.o $\Delta$ Liq $\Delta^{a} x$

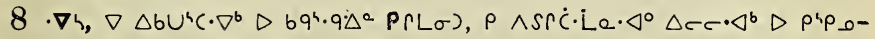

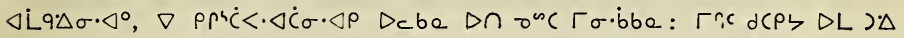

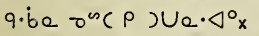

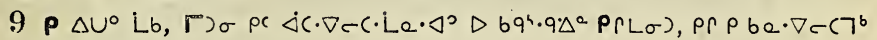

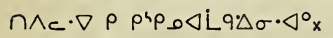

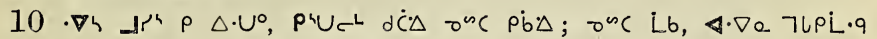

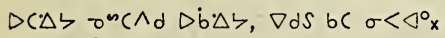

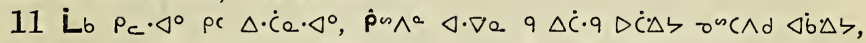
$d^{a} \dot{<}^{a}, \nabla \cdot \Delta \cdot b \sigma L \nabla \Delta \cdot U L b^{b}, \Gamma c \cdot \nabla \cdot \Delta^{a}, \Delta \sigma L a \Delta S$ ph $\dot{C}<r \Delta \dot{b} \Delta b^{a} \sigma c \Delta r$;

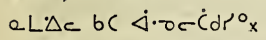

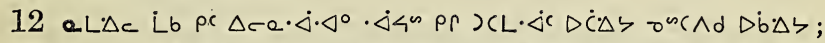

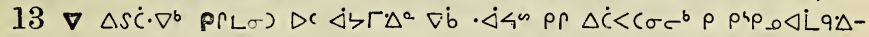

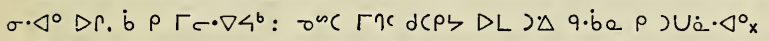

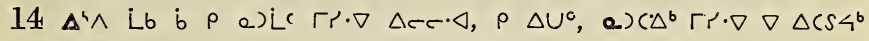
$\sigma r)(\lrcorner^{b} \mathrm{~L} b$ :

15 a $\left.\left.L \Delta c \Delta c \cdot b^{a} q \cdot b^{a} \cdot \triangleleft^{4} \dot{p}^{\natural} \Delta c c^{0}, \nabla \wedge\right) q L b \sigma c^{b} \Delta\right\rangle^{b} \Delta^{b} q \rho \Delta c \wedge \Delta d^{c}$ :

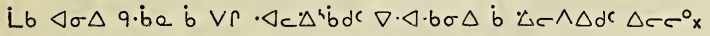

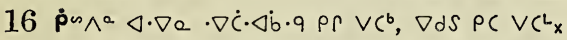

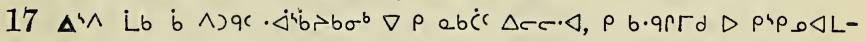

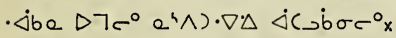

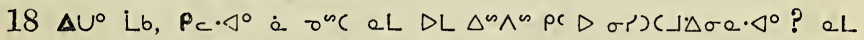

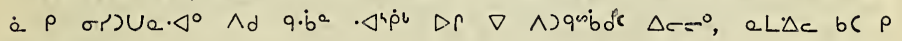
$\Delta r \wedge \Delta d$ :

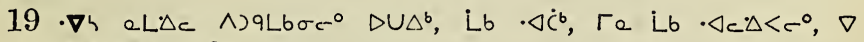

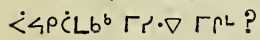

20 p $\Delta \cdot U^{\circ} L b, \varangle \sigma L b \cdot \Delta c \Delta^{4} b d c \Delta c c^{\circ} \nabla \cdot \Delta \cdot 9 c^{\circ} b \cdot \Delta r \wedge \Delta d^{c} \Delta c c^{\circ} x$

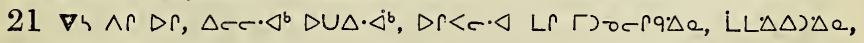
$V S \cdot b \cap r \cdot \Delta e, \quad \sigma \dot{<} \zeta \cdot \nabla \cdot \Delta e$,

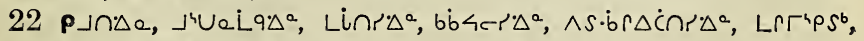

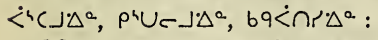

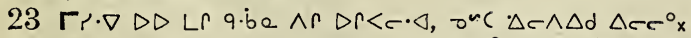

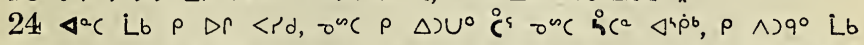

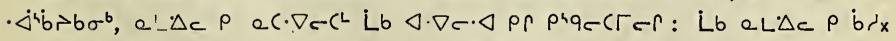

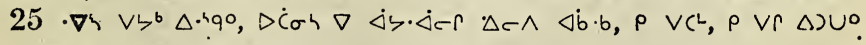
Lb, onc $\rho<\rho S^{a} D^{\text {sin }} \sigma^{b}$ :

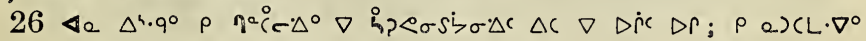
Lb pr $\nabla c \Delta \Delta \cdot \nabla \wedge \dot{a} r r$ Lr $\Delta \dot{i} \cdot b \quad \Delta \dot{C} a b \quad \Delta \dot{\zeta} \Delta c^{b} \Delta r_{x}$ 


$$
\text { D<sps L is } 7,8 \text {. }
$$

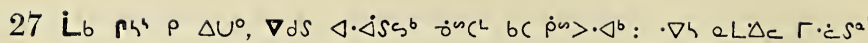

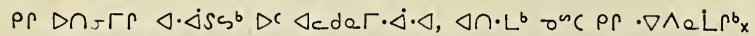

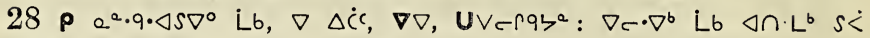

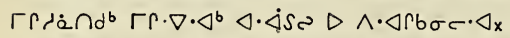

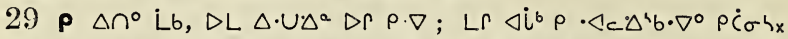

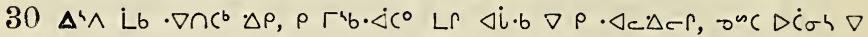
$\wedge \Gamma S \sigma c r \quad \sigma V \Delta \sigma^{b} x$

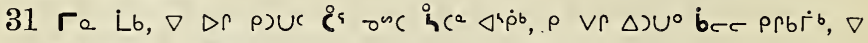
$\dot{s}>\left(\cdot \nabla^{m} b^{b} n \dot{b}\right) c^{4} \triangleleft b c^{\circ} c^{\circ} x$

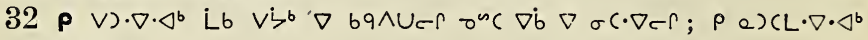
Ĺb $\operatorname{se}$ i

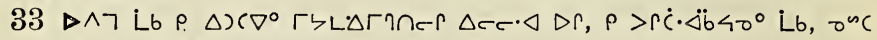

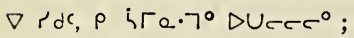

34 onc PrPSd $\nabla \Delta \dot{C} \wedge c, \quad \rho$ a $\triangleleft \dot{C}\lrcorner, \Delta U^{\circ}$ Lb, $\nabla<e, \quad \nabla \cdot \triangleleft \cdot b \sigma L$, $<\operatorname{PQU}<\mathrm{C}$

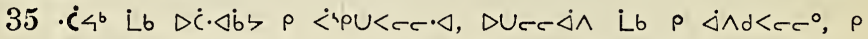

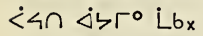

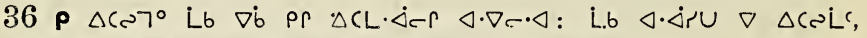
$\triangle \cdot \dot{\Delta} U \dot{a}^{4} \wedge^{6} \Gamma^{\prime} C \Delta \rho \cdot \dot{\Delta} \Delta C \cdot L^{b}$,

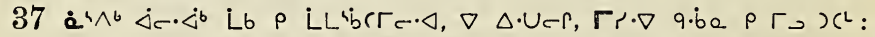

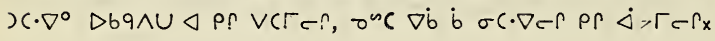

\section{Aיpcre $\triangle b^{a} 8$.}

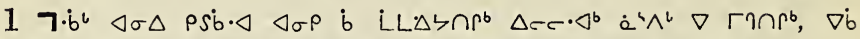

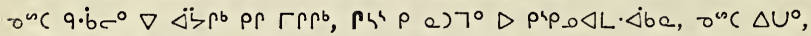

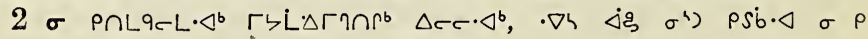

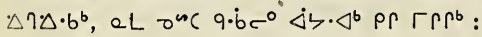

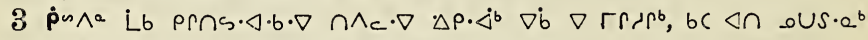
$\nabla \cdot \Delta) \cup p^{b} ; \cdot \nabla h \Gamma \eta c \nabla \Delta\left(r^{b} \cdot \dot{\Delta} c^{0} \rho \vee r \Delta\right) U \cdot \Delta^{b} x$

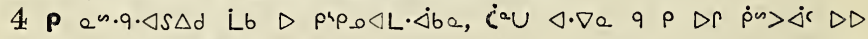
$\triangle c c \cdot \triangleleft D C<\cdot b c^{4} b \Gamma^{b} b^{b}$ ?

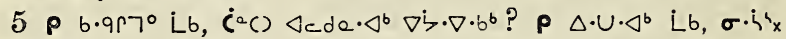

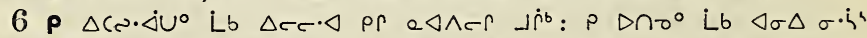

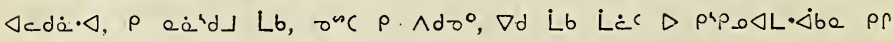

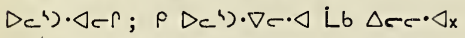

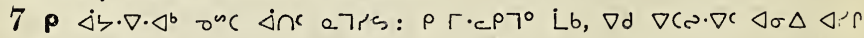

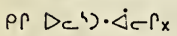

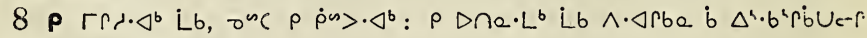

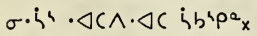

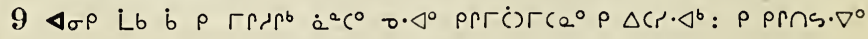
Lbx 


\section{$D<\rightarrow$ fí ${ }^{\text {sb }} 8$}

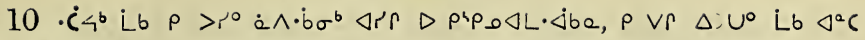
CiLoe $\triangle U q^{x}$

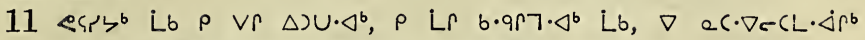

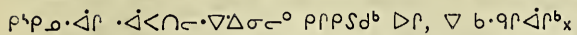

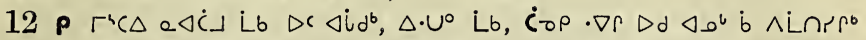

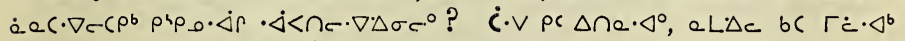

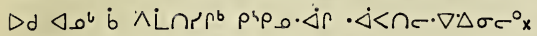

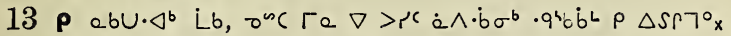

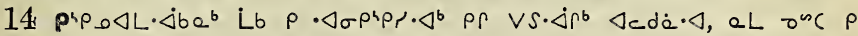

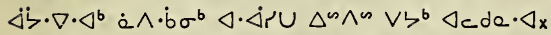

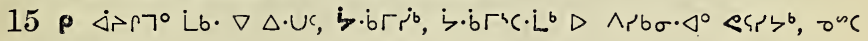
D $\wedge b_{0} " \nabla \varsigma_{x}$

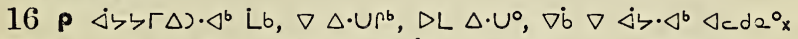

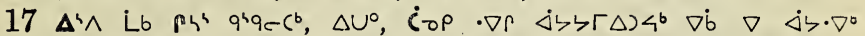

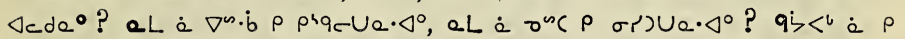
Lœb $\triangle U \nabla a \cdot \triangleleft^{\circ}$ ?

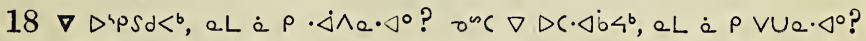
CL a onc $p$ pipre. $\nabla^{\circ}$ ?

$19 \Delta^{4} \wedge \dot{b} \wedge d a L \cdot \Delta \rho^{b} \sigma \zeta c^{c} \triangleleft c d a \cdot \triangleleft \triangleleft \sigma \rho \sigma b c \cdot c^{0} \operatorname{p\rho r}(\dot{C}) \Gamma\left(a^{\circ}, \dot{C}^{\circ}()\right.$

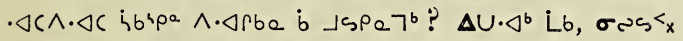

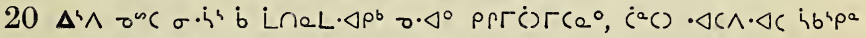
$\wedge \cdot \Delta r b e$ b Jspe $]^{b}$ ? $p \Delta \cdot U \cdot \Delta^{b} L b, \sigma \cdot h^{4} x$

$\left.21 \rho \Delta U^{\circ} L b, C_{o p} \cdot \nabla \rho \nabla \dot{b} \sigma r\right)\left(7^{\circ}\right.$ ?

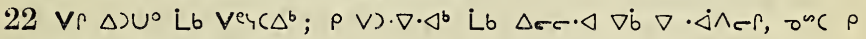

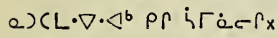

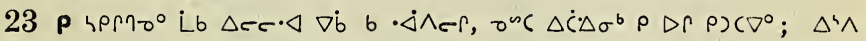

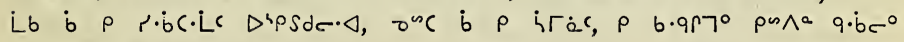

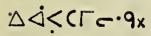

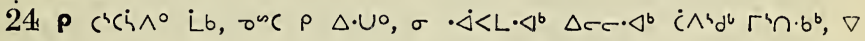
$\wedge\lrcorner \cup P^{b} \mathrm{x}$

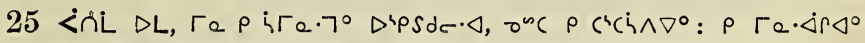
Lb,

$26 \rho \Delta S \cap s \cdot \nabla^{\circ}$ Lb $\Delta \rho_{c}^{b b}, \nabla \Delta \cdot U c, \nabla b \Delta c \Delta U \Delta \dot{C} \Delta \sigma^{b}, \nabla \cdot b \Delta c \sigma^{\infty c C}$ $\checkmark \cdot \supset$ Ja $\triangle C L^{\circ} \Delta C \Delta \sigma^{b} x$

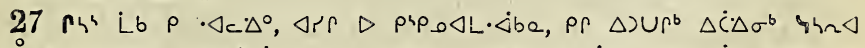

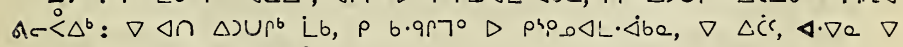
$\triangle \cdot \cup r^{b} \Delta r c \cdot \triangleleft^{b} \nabla \dot{\Delta} \Delta \dot{\Delta} \dot{c} ?$

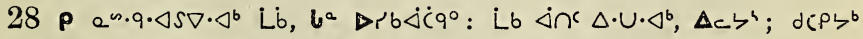
Lb, V $\zeta^{b} \nabla \Delta C p^{b} \Delta \rho^{b} \rho \cdot \dot{\Delta} \Delta q \cdot \triangleleft^{b} x$

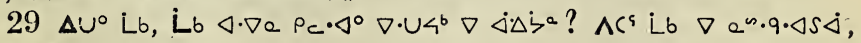
$\Delta U^{\circ}, P_{c} \nabla \cdot \triangleleft \cdot b_{e} X_{x}$

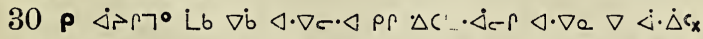

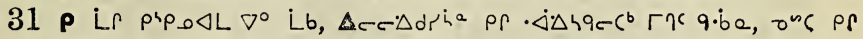
69 


\section{D< $>>\rho^{4} \dot{L}_{5 b} 8,9$.}

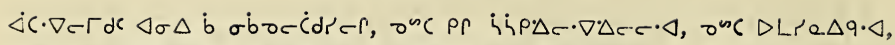

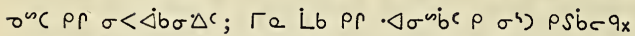

$32 \varangle \sigma \nabla \sigma^{\circ} \Delta \cdot U \Delta \sigma c^{\circ}$ Lb $\lrcorner S 2 \rho \Delta \cdot U^{\circ} x \quad \Lambda c^{s}$ Lb $\rho \Delta \cap \sigma^{\circ}, \rho$ L $\rho$ $P C \triangleleft L \cdot \nabla^{\circ} \mathrm{L} b_{x}$

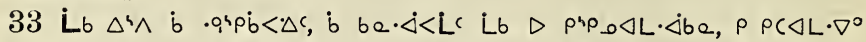

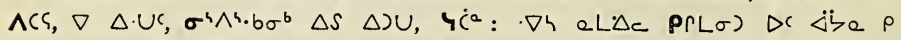

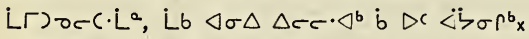

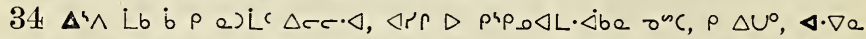

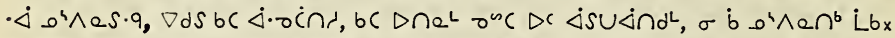

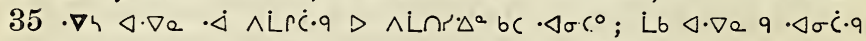

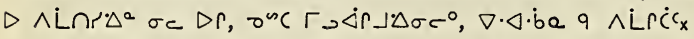

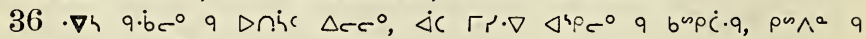
$\triangleleft \sigma \dot{\Delta} \cdot q \Delta<\Delta i \cdot b$ ?

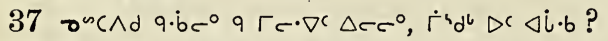

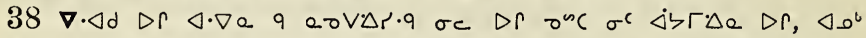

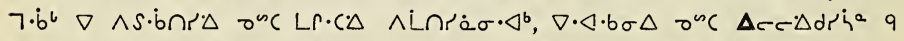

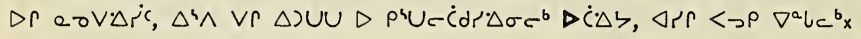

\section{N'pcre $\triangle b^{\circ} 9$.}

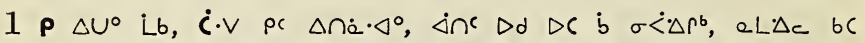
$\left.d \Gamma^{4} C \cdot L^{b} \quad \sigma \wedge \Delta \sigma c^{\circ}, \quad \dot{<} \cap \dot{L} \quad \rho \cdot \dot{\Delta}<C \cdot \dot{L} \cdot \dot{C} \cdot \nabla \quad P R L \sigma\right) \cdot \triangleleft \quad D C \quad D P \dot{L} \Delta \Delta \cdot \Delta \sigma c^{\circ}$ Pr $\nabla \cap<<c c^{b}$ bn $P D \Delta \sigma^{b} x$

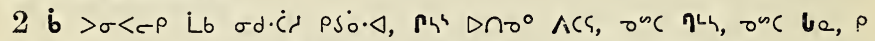

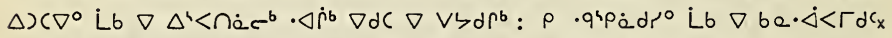

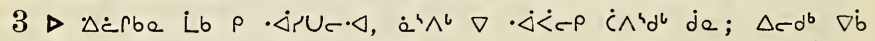

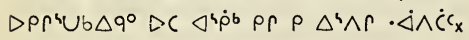

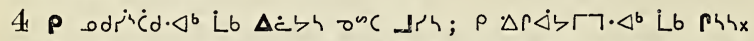

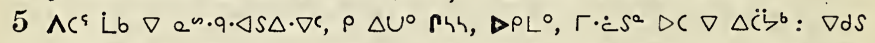

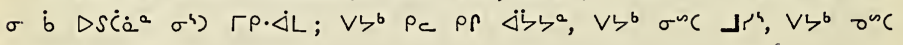
$\Delta \dot{c} \zeta^{4} \times$

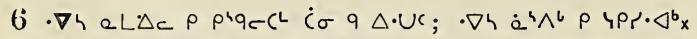

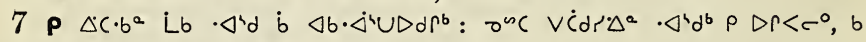
$\left.\triangle \cdot U L b^{b}, \varangle \cdot \triangleleft \nabla \cdot \triangleleft d \sigma d \rho^{b} b i \rho \varangle^{b} ; a\right) \dot{c} \Delta \Delta^{b} x$

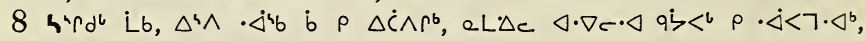

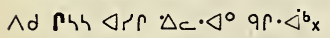

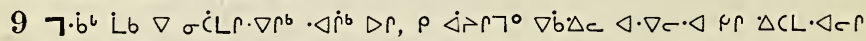

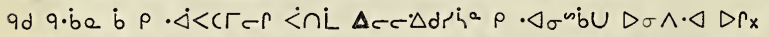

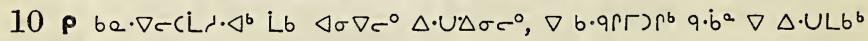
$\cdot \Delta \sigma^{n} \dot{b} \Delta^{a} \Delta \sigma \wedge \cdot \triangleleft^{b} \Delta \Gamma_{x}$

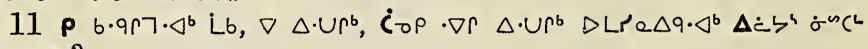
PP $C d S^{b}$ ?

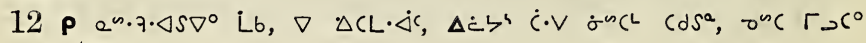
70 


\section{$\Delta<\rightarrow \rho^{4}$ Lst 9 .}

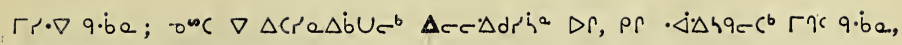

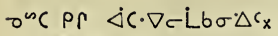

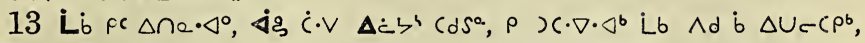
is $\Delta C$ Ca $\triangle \dot{b} U c^{b} \Delta c \Delta r_{x}$

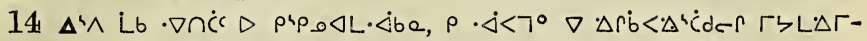
nner $\triangle e c \cdot \triangleleft$, onc $\nabla$ b.

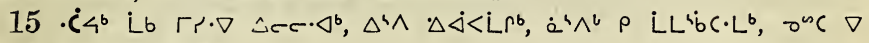

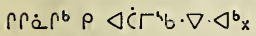

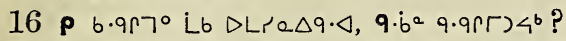

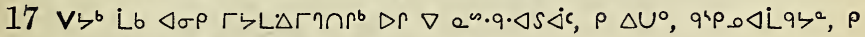

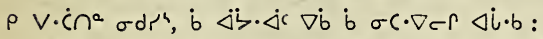

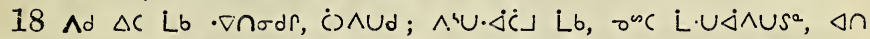

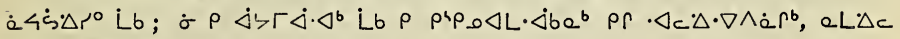
Lb $\rho$ bup $\cdot \cdot \int^{b} \mathrm{~b}$

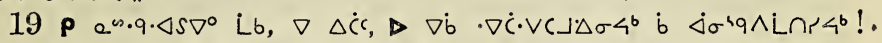

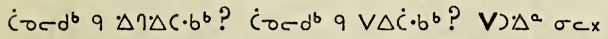

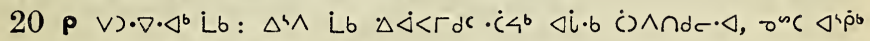

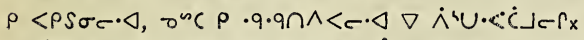

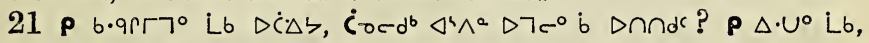
$\nabla \triangleleft \cdot \Delta s S \Delta r v r_{x}$

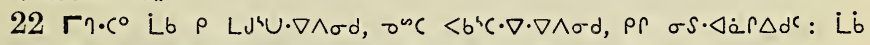

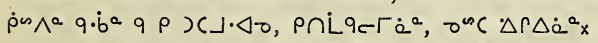

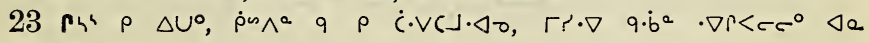
b $\dot{c} \cdot V c^{b} x$

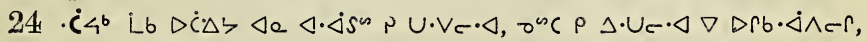

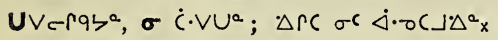

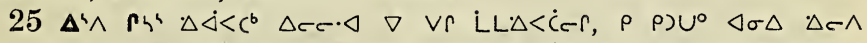

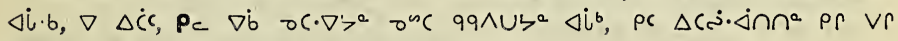

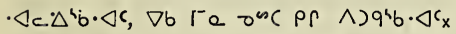

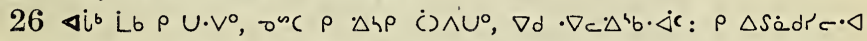
Lb $\dot{c} \wedge^{4} d^{l} \triangleleft \cdot \nabla e \nabla \sigma \wedge c ;$ b $D r \Delta \cdot U r^{b} \Gamma \eta c, \sigma \wedge^{\circ}{ }_{x}$

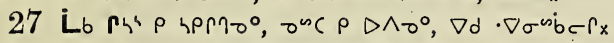

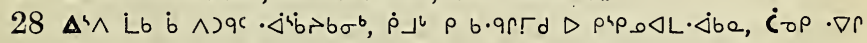

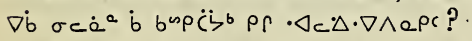

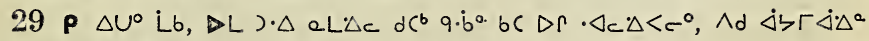

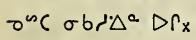

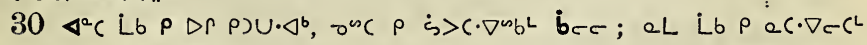

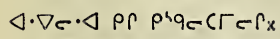

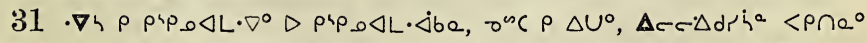
$\Delta r f c^{b} \Delta c c \cdot \triangleleft, b c \quad \sigma<r d$ Lb, $\Delta^{4} \wedge$ Lb $\left.\rho \quad \sigma<\Delta b \sigma \cdot \Delta U, b c \cdot \Delta \sigma^{\infty} b b^{\circ} \sigma^{4}\right)$ psibeqx

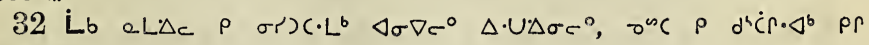
$6 \cdot 9 \operatorname{LL}^{6} \mathrm{x}$ 


\section{$\triangleright<>\rho^{4}$ Lsb 9, 10.}

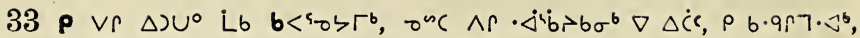

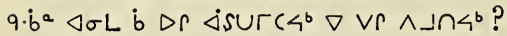

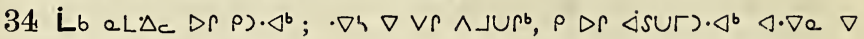

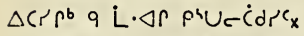

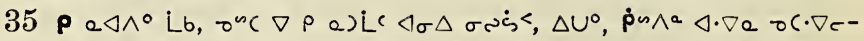

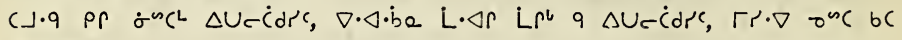
$\Delta)^{\mathrm{s}} \mathrm{b} \cdot \nabla^{\circ} \mathrm{x}$

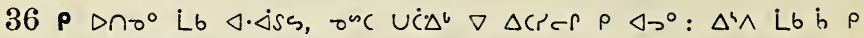
$\Delta \cap \dot{Q}\left(\Delta \wedge \wedge \sigma^{b}, \rho \Delta U^{\circ}\right.$,

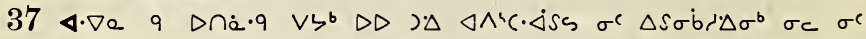

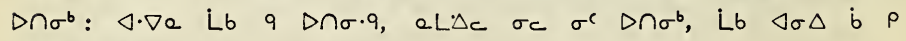
$\operatorname{Vrns} \operatorname{cr}_{\mathrm{x}}$

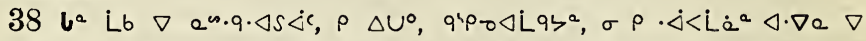

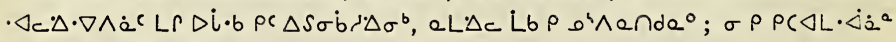

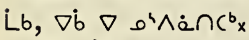

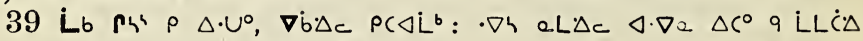

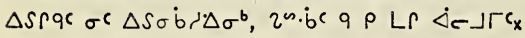

$40 \cdot \nabla h \triangleleft a \nabla b \dot{b} a^{4} d^{4} \dot{c} c^{b}, \rho \Delta r \Delta d a^{\circ} x$

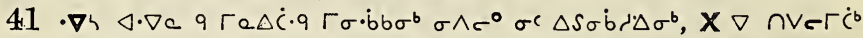
$\Delta r, \dot{C} \cdot \vee \rho c \Delta \cap a \cdot \triangleleft^{\circ}, a L \Delta c b c \cdot \Delta \sigma c^{\circ} \triangleright \cap<\Delta L d r \cdot \Delta^{a} x$

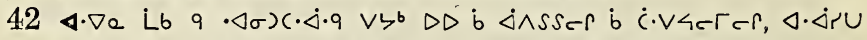

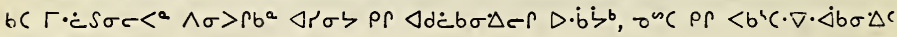

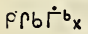

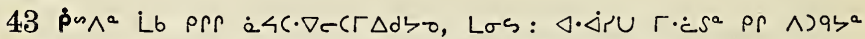

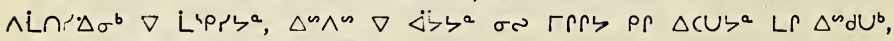
$\Delta \sigma \Gamma \Delta a d U^{b} \nabla \dot{b} \Delta^{b} b c a \Delta^{i} c \cdot \nabla^{b}$;

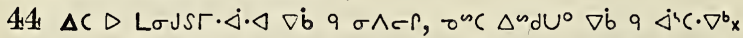

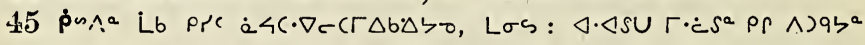

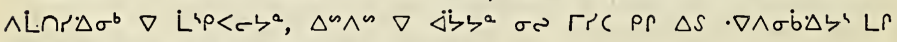

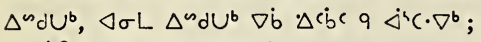

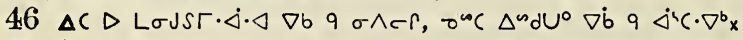

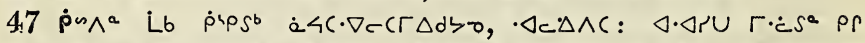

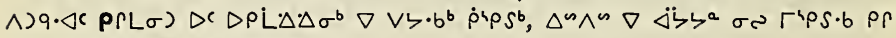
$\Delta S \cdot \nabla \wedge \sigma \dot{b} \Delta \zeta^{a} L \Gamma \Delta \Delta^{\omega} d U^{b}$;

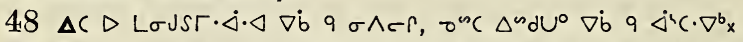

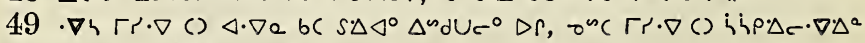
bC $s \Delta \dot{c} b \sigma \cdot \Delta^{a} s \Delta \dot{c}^{a} \Delta r_{x}$

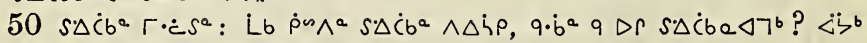

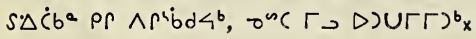

\section{Ápcre $\triangle b^{\circ} 10$.}

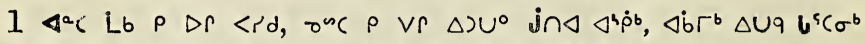

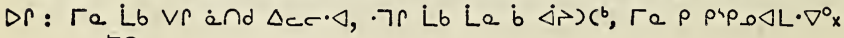




\section{$D<$ Ps Lsb 10.}

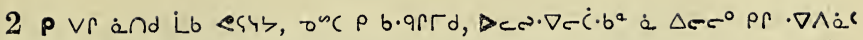
$\Delta \cdot \triangleleft ? \nabla$ ? $\nabla \cdot q r \Delta r^{b} x$

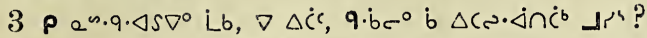

4 p $\Delta \cdot U \cdot \Delta^{b} L b, d r^{4} p \Delta r \dot{\alpha} q^{\circ}$ pr Lro $\left.\Delta b U c^{b} \cdot \nabla \wedge \sigma\right) \Delta L r a b \sigma \sigma c^{\circ}, p r$ $\cdot \nabla \wedge \sigma \Gamma r$ L.bx

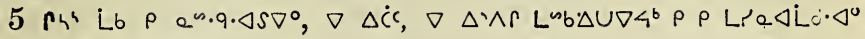
$\nabla 7 c^{\circ} \Delta C \cdot \nabla \Delta \sigma c^{\circ}:$

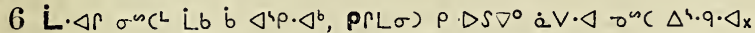

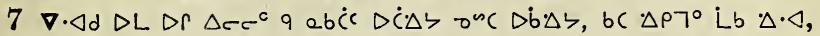

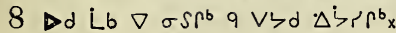

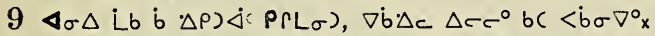

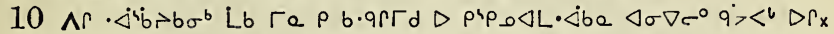

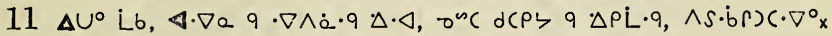

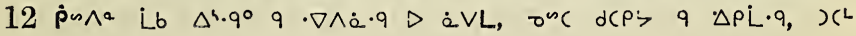
$\wedge s \cdot \dot{b} \cap r^{\prime} \Delta \sigma c^{\circ} x$

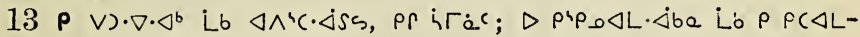
$\cdot \nabla c \cdot \triangleleft \triangleleft \sigma \Delta \dot{b} \vee s \cdot \dot{j} \Gamma x$

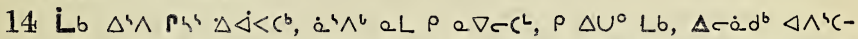

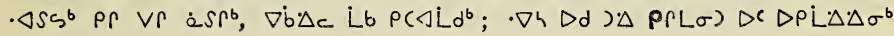
b) $\Delta \dot{C} r^{b} x$

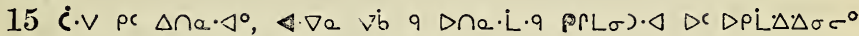

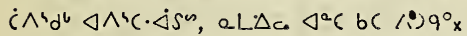

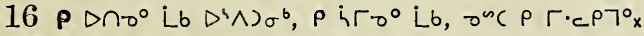

$17 \Delta^{4} \wedge$ Lb b $\Delta x \cup 7^{4} b \dot{a}^{b}, \rho \dot{<}<\dot{C} c \cdot \triangleleft \vee \zeta^{b} \triangleleft \cdot \nabla c \cdot \triangleleft \nabla \nabla r r \cdot b e \wedge^{4} \dot{C} d c$,

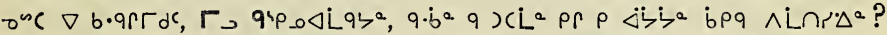

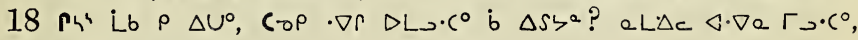
$\checkmark \zeta b \wedge d, \nabla \cdot \Delta d \rho(L \sigma) x$

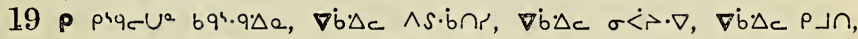

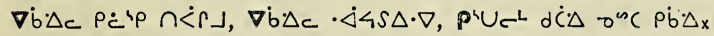

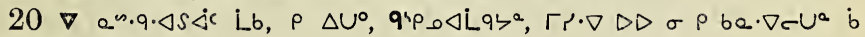
$\vee r \triangleleft \cdot \dot{\Delta s} s \Delta \dot{b} a^{a} x$

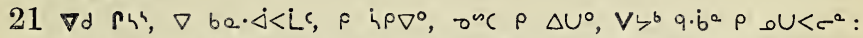

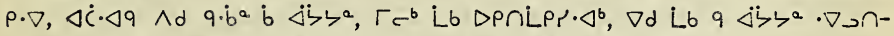

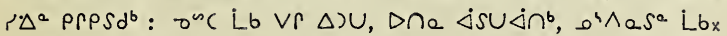

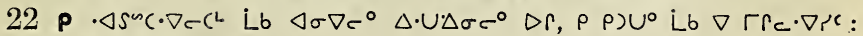
$\cdot \nabla h \dot{a}^{4} \wedge^{6} \rho \cdot \nabla \rightarrow \cap r^{\circ}{ }^{\circ}$

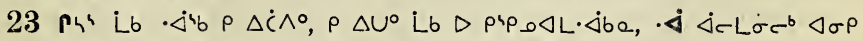

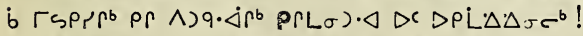

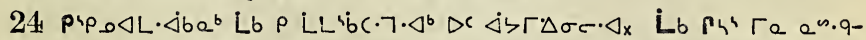

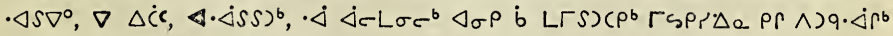
PRLO) $D C D P L \Delta \triangle \sigma \sigma^{b}$ !

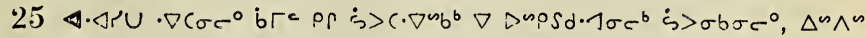

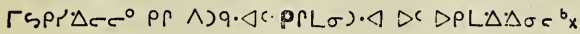




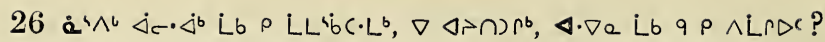

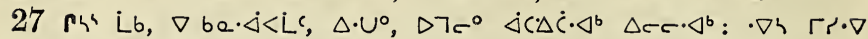
q.be $\cdot \nabla\left(\sigma c^{\circ} \text { PrL } \sigma\right)_{x}$

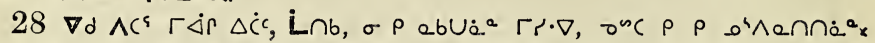

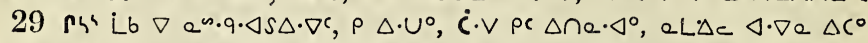

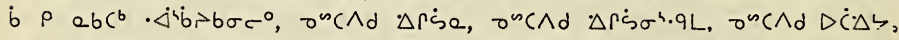

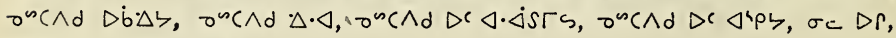
$\sigma^{\circ C} \Gamma \rightarrow \Delta \Gamma L \Delta \sigma \sigma^{\circ} \Delta \Gamma$,

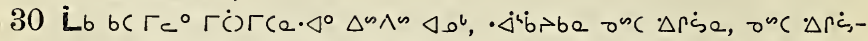

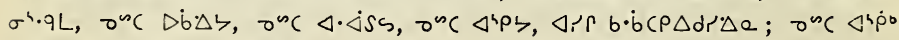

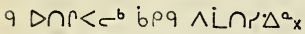

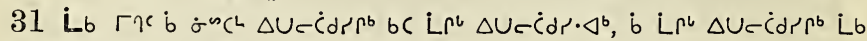

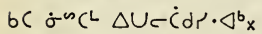

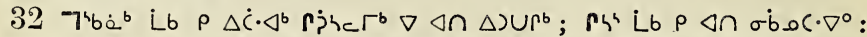

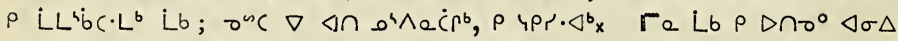

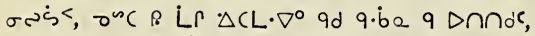

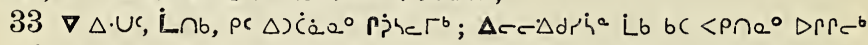

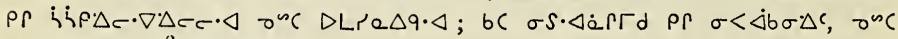

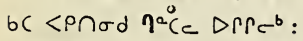

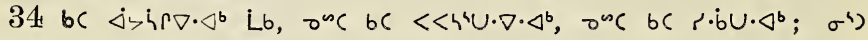
psibeq Lb $\Gamma \sigma b c \cdot \triangleleft \sigma^{m} b^{\circ} x$

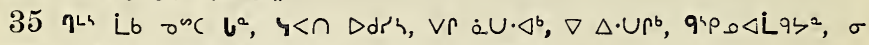

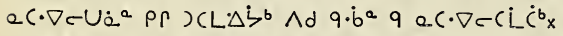

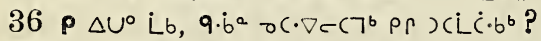

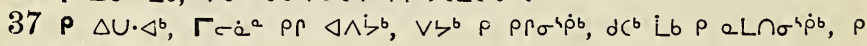

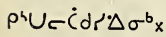

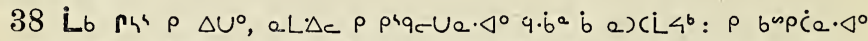

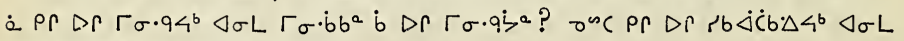

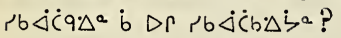

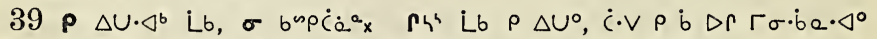

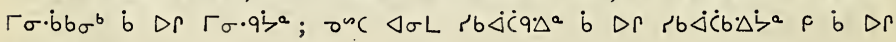
$r b \Delta \dot{C} b \cdot \Delta a \cdot \Delta^{\circ}:$

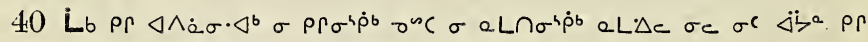

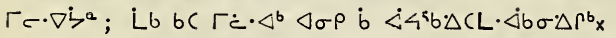

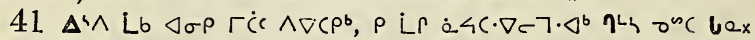

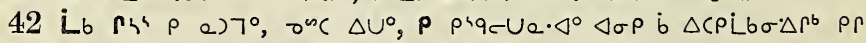

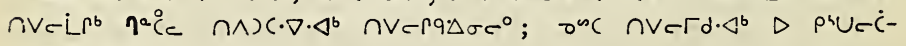
$\operatorname{dr} \Gamma \cdot \dot{\Delta} \cdot \Delta x$

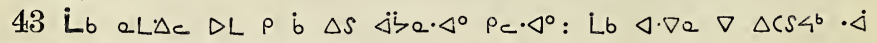

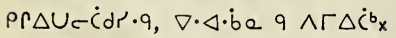

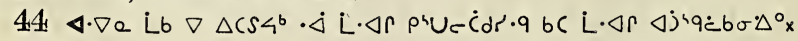

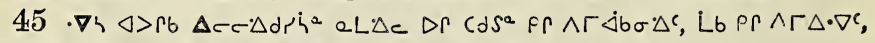

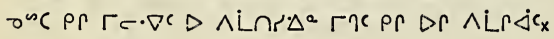




\section{$D<>$ pᄂ L's $10,11$.}

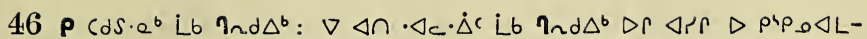

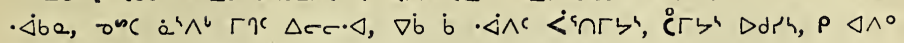
$\nabla \wedge 7^{4} b a^{b} \nabla<d s \cdot \dot{c}_{x}$

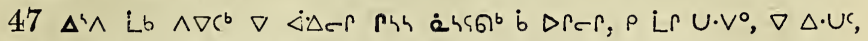

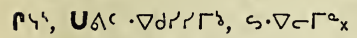

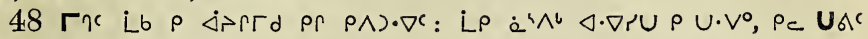

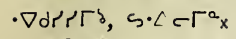

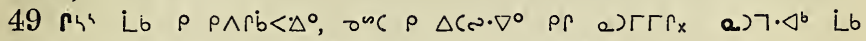

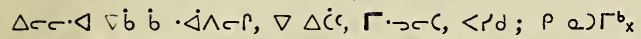

$50 \cdot \Delta_{c} L b, \nabla \cdot \nabla \wedge a^{b} \Delta C \triangleleft d D^{a}, \rho \vee r \dot{a} U^{0} p h b_{x}$

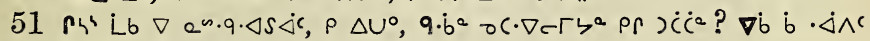

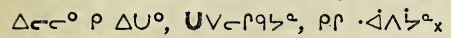

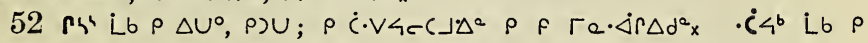

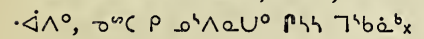

\section{Ápcre $\triangle b^{a} 11$}

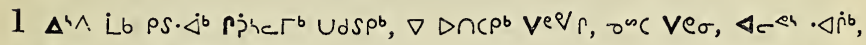

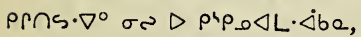

$2 \Delta U^{\circ}$ Lb, $\Delta U^{b}$ oL $\Delta \dot{C} \Delta \sigma S^{b} \nabla n^{a b} b^{\circ}$ b $\Delta C \cdot b^{b} ; \Delta<b$ L.b $\left.\rho \wedge\right) q 4 \cdot 9$,

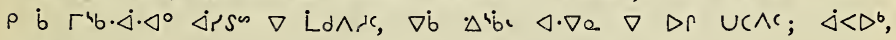
$V s^{b} L_{b x}$

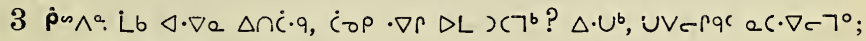

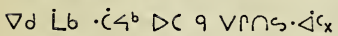

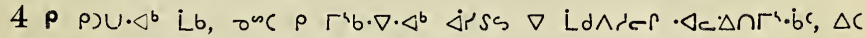

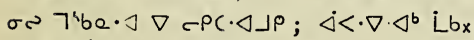

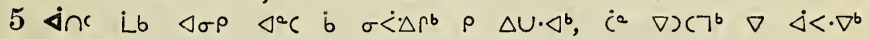
¿riss?

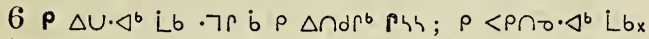

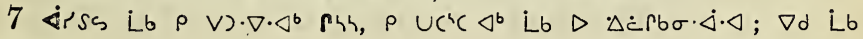
$\cap \nabla C \wedge C_{x}$

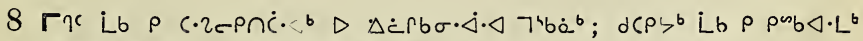

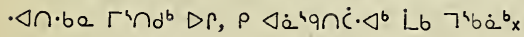

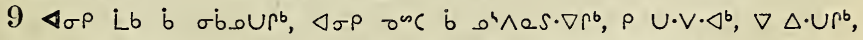

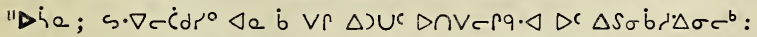

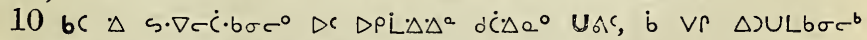

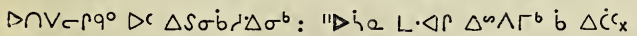

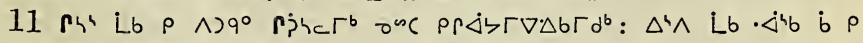

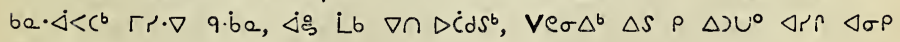
$\sigma_{e} \dot{c}^{2}<x$

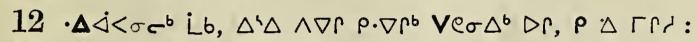

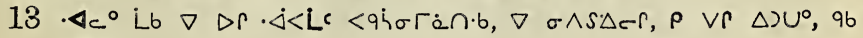
75 


\section{$D<s$ p' Lsb 11.}

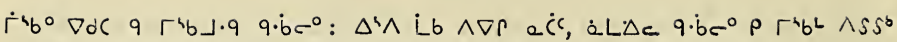

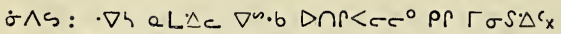

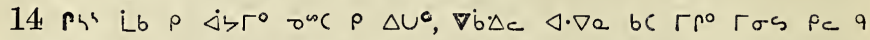

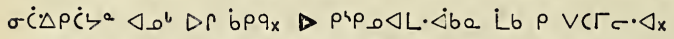

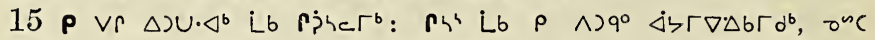

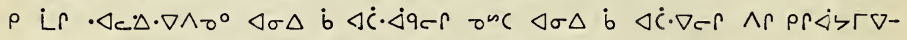

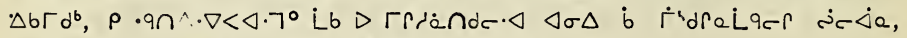
$\tau^{\circ} C D U C \wedge \Delta \sigma c \cdot \triangleleft \triangleleft \sigma \Delta \dot{b} \triangleleft \dot{C} \cdot \Delta q c r \Delta \Gamma \Gamma \cdot \triangleleft$;

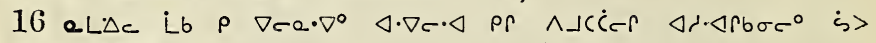
$\rho r \Delta \dot{b} \Gamma \nabla \Delta b \Gamma d^{b} \times$

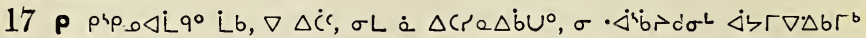

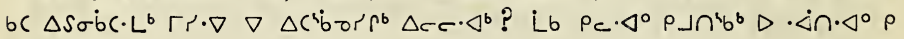
$\rho \triangle S \dot{C} a \cdot \Delta^{\circ} x$

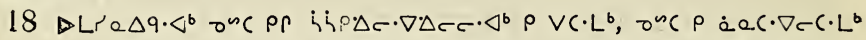

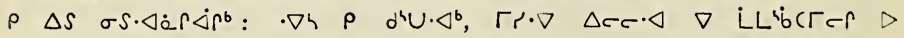

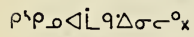

$19 \Delta^{\wedge} \wedge$ Lb $\nabla \cap \Delta \dot{C} d S^{b}, \Delta \dot{C} \Delta \sigma^{b} \rho \Delta \rho \cdot \Delta c \Delta^{\circ} x$

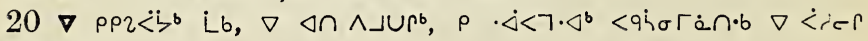
$\Delta\urcorner \wedge d^{b} \Delta r_{x}$

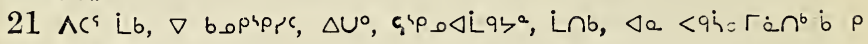
LLPLC $\dot{<} \dot{\text { <े }}$ !

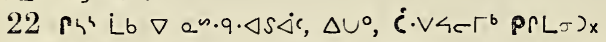

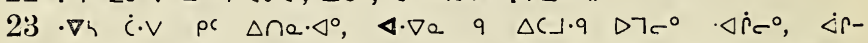

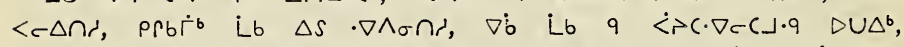

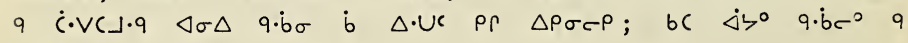
$\Delta \cdot \cup \cdot q_{x}$

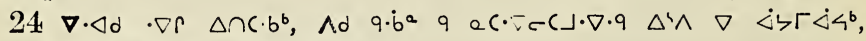

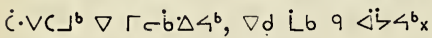

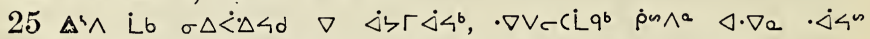

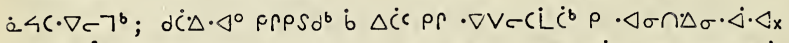

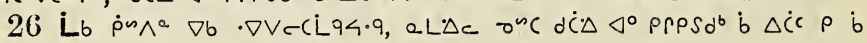
$\cdot \nabla \vee c C L d \cdot \triangleleft^{\circ} \rho \cdot\langle\sigma n \Delta \sigma \cdot \dot{\Delta} \cdot\langle x$

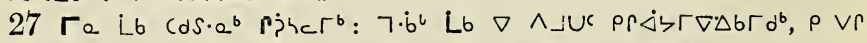

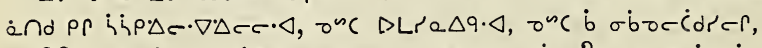

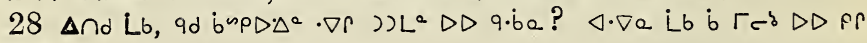
$\mathrm{CL}^{\circ}$ ?

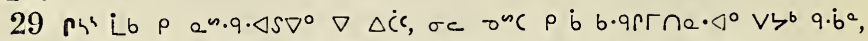

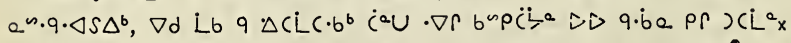

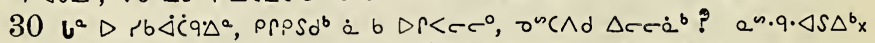

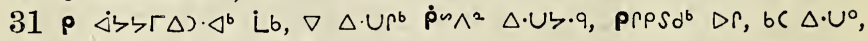
$\dot{C}^{\circ} \rho \cdot \nabla r$ L L $\nabla \dot{b} \rho \dot{C} \cdot V C \cdot \nabla^{b}$ ?

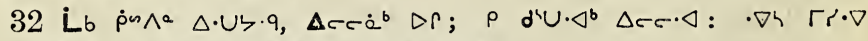

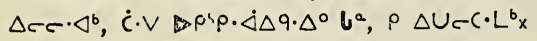




$$
\Delta<\rightarrow \text { ps Lst } 11,12 \text {. }
$$

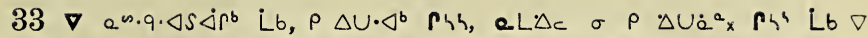

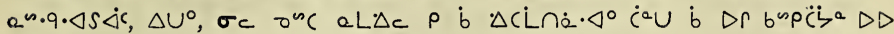
q.be $\operatorname{Pr} x \mathrm{CL}^{2} \mathrm{x}$

$$
\text { Aיpre } \triangle b^{2} 12 .
$$

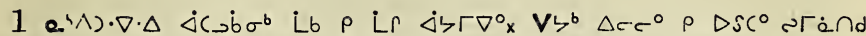

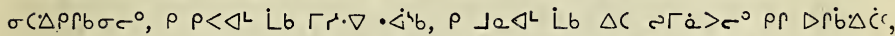

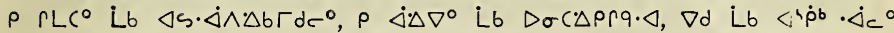
$\nabla) \cup x_{x}$

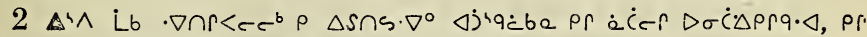

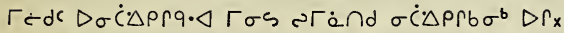

$3 \rho \Delta \cap \cap_{0} \cdot \Delta^{b} L b, \tau^{c} C \nabla \rho D C L \cdot\left\langle\rho^{b}, \wedge S S^{b} \rho \rho R \cap s \cdot \nabla \cdot \Delta^{b} x\right.$

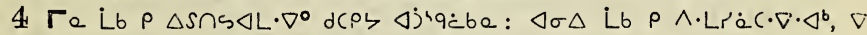

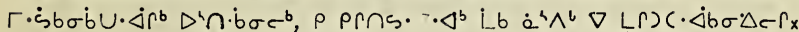

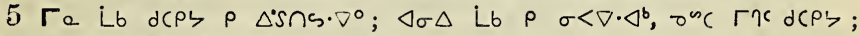

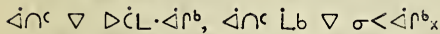

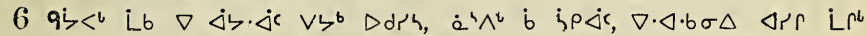
$\nabla \cap S<L \cdot \Delta c, \nabla \Delta \cdot U$, bC p'U $-7 \cdot \Delta^{b} \sigma d r^{\prime h x}$

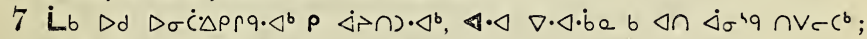

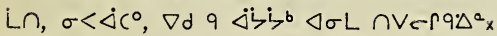

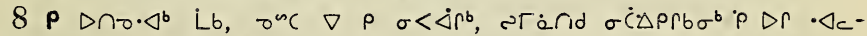
$\Delta \cdot \nabla \wedge^{0} \cdot \Delta^{b x}$

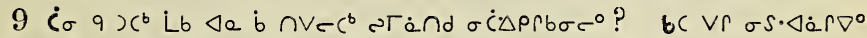

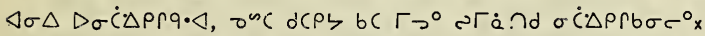

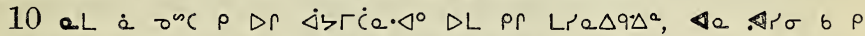

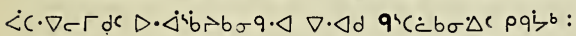

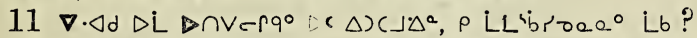

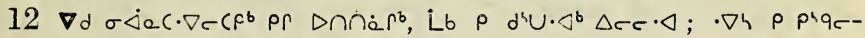

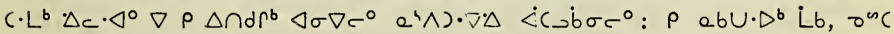
$\rho$ p) $U \cdot \Delta b x$

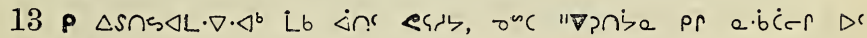
$j \zeta \Gamma \cdot \Delta \sigma c^{b} x$

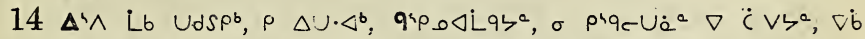

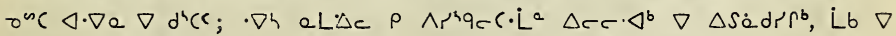

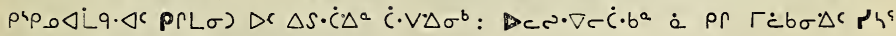

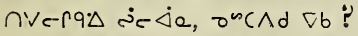

$15 \sigma$ b $\Gamma c \cdot \dot{\Delta a^{a}} \dot{a}, \sigma^{\infty} C V d \nabla d S$ a $e L_{\Delta c} \sigma$ b $\Gamma c \cdot \dot{\Delta a^{a}}$ ? Lb $\Delta_{c}, \nabla$

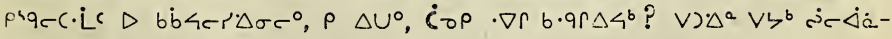

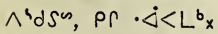

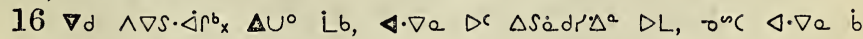
Lre $\Delta b$ r: $D C$ ? P $\Delta U \cdot \Delta^{b} L b^{x} h_{h^{c} x}$

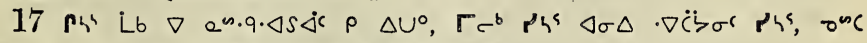

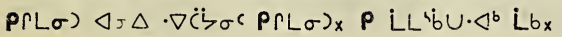




$$
\Delta<>\rho^{4} \text { L. is } 12 .
$$

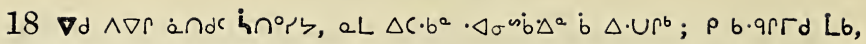
$\nabla \Delta \cap d^{c}$,

19 q

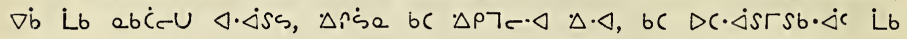
$\triangle r \dot{s} a x$

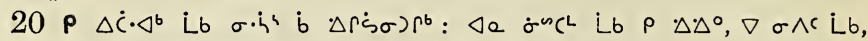
eLAC Dr ebU० $\triangleleft \cdot \dot{\Delta s} 5 x$

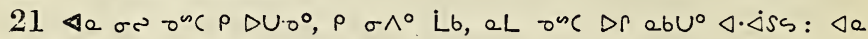
$\sigma^{3)} \nabla^{n}(x$

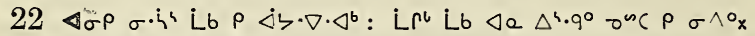

$23 \nabla \cdot \Delta d$ Lb $\Delta r \Delta^{4} \wedge \cdot \Delta \sigma^{\omega} \dot{b} \sigma \cdot \Delta q, \Delta^{4} \wedge \cdot \Delta \sigma^{n} \dot{b} \cdot c \cdot \nabla, \Delta \cdot \nabla e \nabla \cdot \Delta c r p^{b} \quad q$ $\triangle \Delta C D D \Delta^{4} \cdot q \cdot \Delta ? \cdot \nabla h \Delta \sigma \rho \sigma \cdot h^{4} \rho \Delta \Delta \cdot \Delta^{b} x$

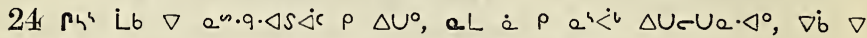

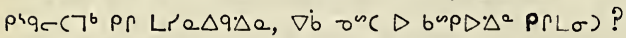

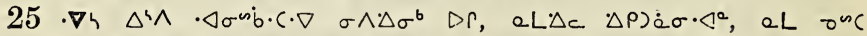
$\triangle \rho) \Delta \cdot \dot{\nabla} \sigma \cdot \nabla^{a}:$ Lb $a^{4} \wedge\left(\cdot \nabla \cdot \Delta^{b} \nabla^{a} l_{c} \rho \rho P S d^{b} x\right.$

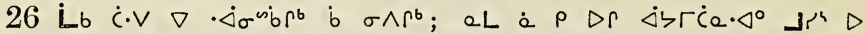

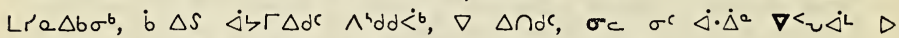

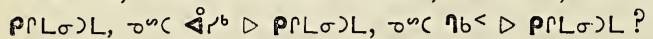

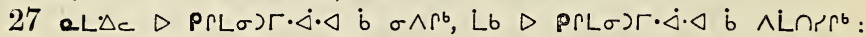

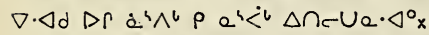

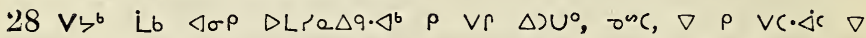

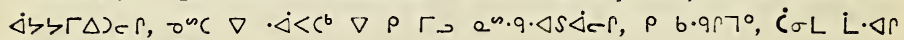

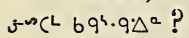

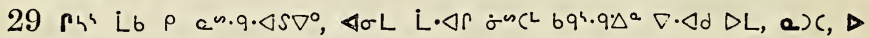

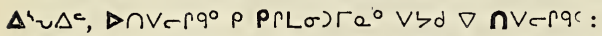

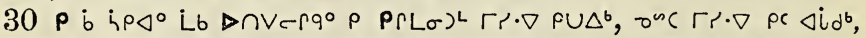

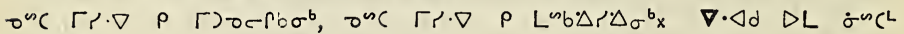
bqh. $9 . \Delta^{\circ} x$

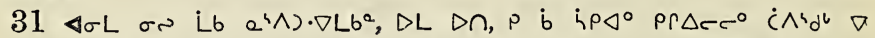

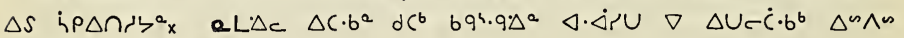
$D D_{x}$

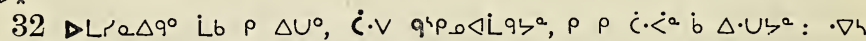

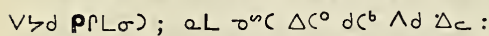

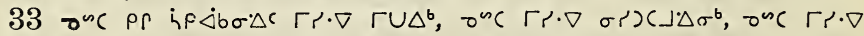

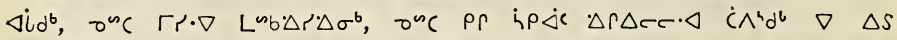

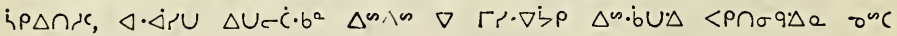

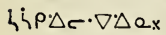

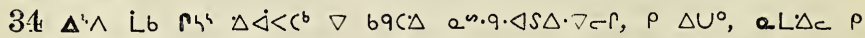

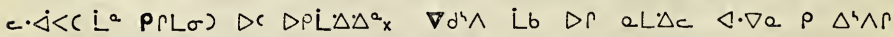
spu $\nabla_{0} q \cdot \dot{b}^{\circ} c^{\circ} \operatorname{pr} b \cdot q r \dot{L} c_{x}$

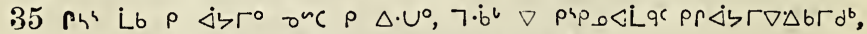

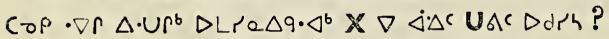




\section{$\Delta<>$ ps Ĺsb $12,13$.}

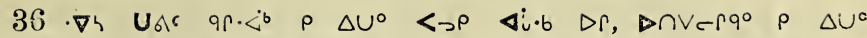

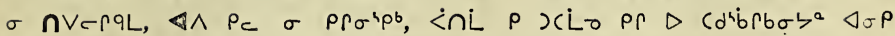
b $<\cdot \dot{b} \cap \cap^{b} b_{x}$

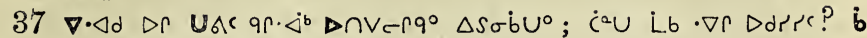

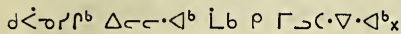

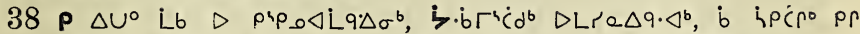

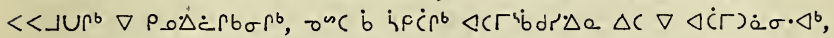

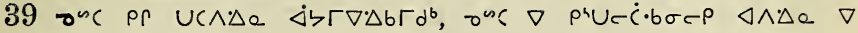
Ld $\dot{s} \sigma \cdot \triangleleft^{b}$;

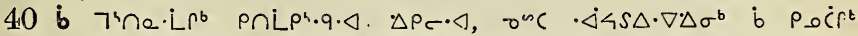

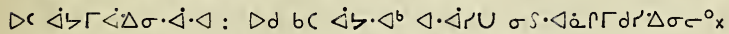

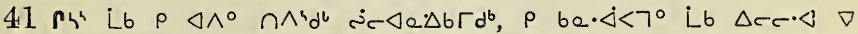

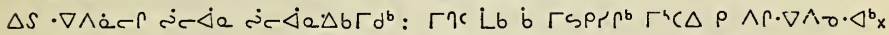

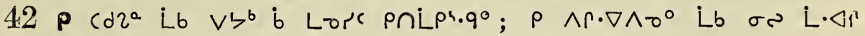

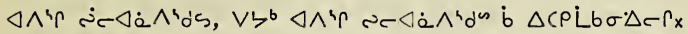

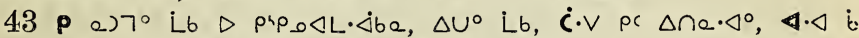

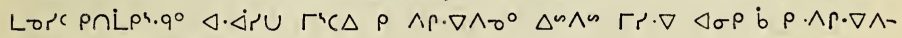

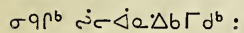

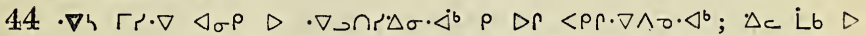

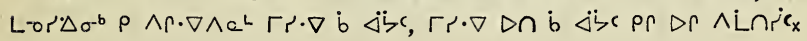

\section{A $p c \subset a \triangle b^{a} 13$.}

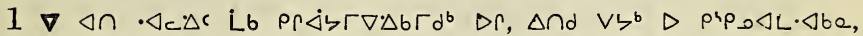

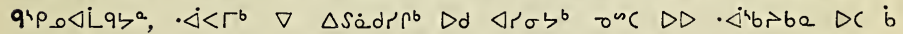
$\triangle C \cdot b P$ !

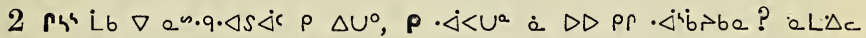

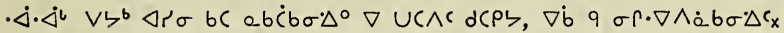

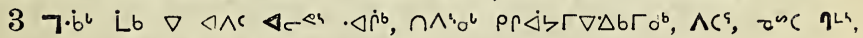

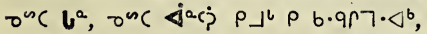

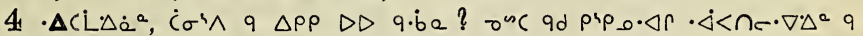

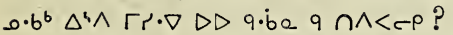

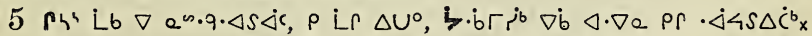

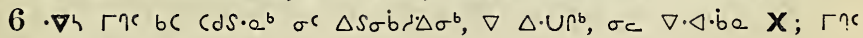
Lb $b c \cdot<<27 \cdot \triangleleft^{b} x$

$7 \Delta^{4} \wedge$ Lb $\vee\left(7.9\right.$ e) $\left.<c^{\Delta} \Delta e, \sigma^{n}(e)<c \Delta e \quad \nabla \cap \dot{<}\right) \dot{(b \sigma} \Delta \rho, \nabla \dot{b} \Delta c$

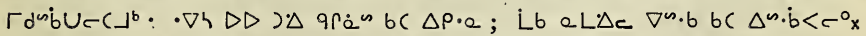

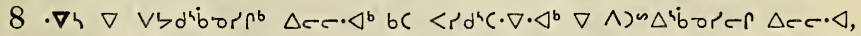

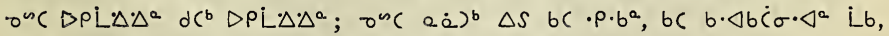

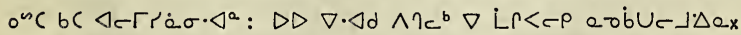

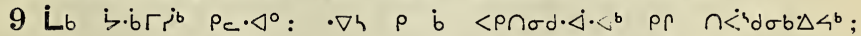

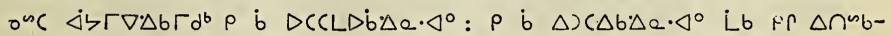

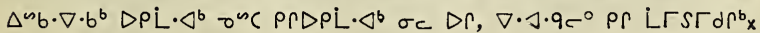




\section{$\triangleright<>$ p4 Lst 13.}

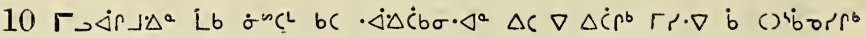
$\Delta-c^{-c} \cdot \Delta^{b} \mathrm{x}$

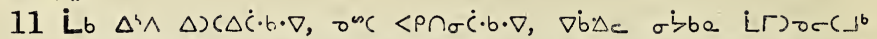

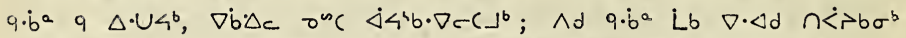

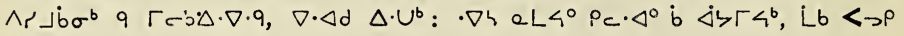
$\Delta i b_{x}$

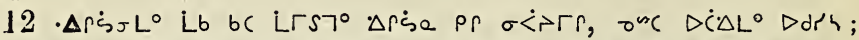

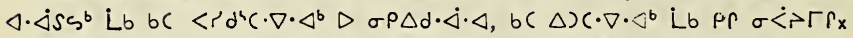

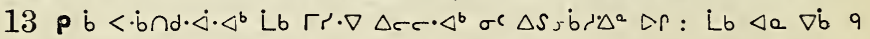

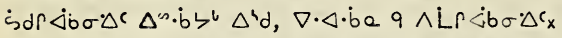

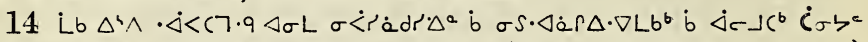

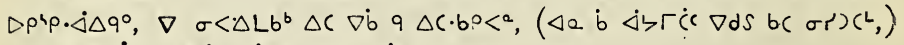
$\left.\nabla d \quad \varangle \sigma^{\rho} \dot{j} \cap \Delta \Delta^{b} \dot{b} \Delta \dot{C} \beta^{b} \nabla d S \cdot \Delta \dot{r}^{b} b c \Delta \cap S\right\lrcorner \cdot<^{b}$ :

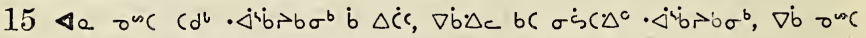
$\nabla d c b c \wedge) q^{\circ}$, pr $\Delta \cap a^{b} q \cdot b c^{\circ} D \cdot \Delta 4 b r b \sigma \Gamma^{b} D r:$

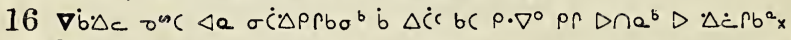

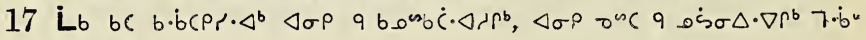
$\triangle \triangleright$ q PSice !

18 a) $\left\langle\dot{L} q^{b}\right.$ Lb $\nabla \dot{b} 7 \cdot b^{b} \nabla \wedge>b$ pr $\left.\Delta s\right\lrcorner 4^{b} x$

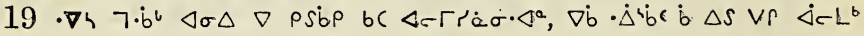

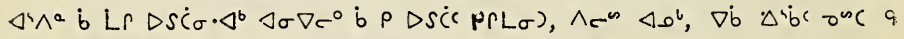
$\triangle \wedge r \dot{\Delta} \mathrm{CL}^{b} \mathrm{x}$

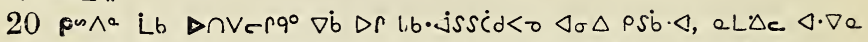

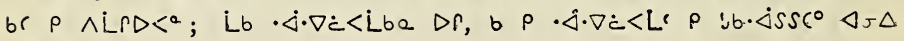
i $s \dot{b} \cdot \Delta x$

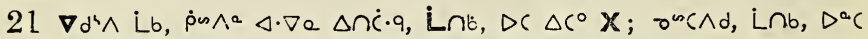
$\Delta c^{\circ} ; \nabla \dot{b} \Delta c \dot{c} \cdot v \dot{C}^{b} x$

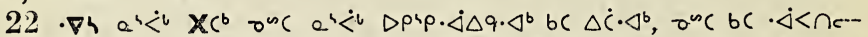

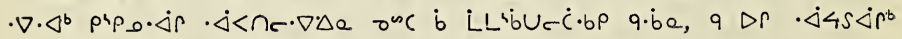

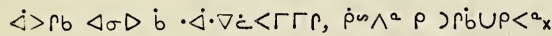

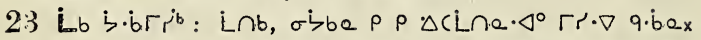

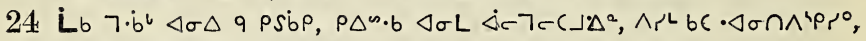

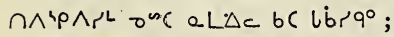

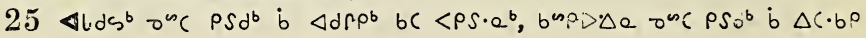
$b C$ e $\sigma p<c \cdot \Delta x$

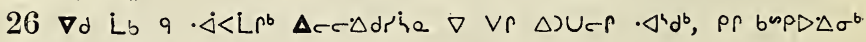
onc prucidd $\Delta \sigma^{b} x$

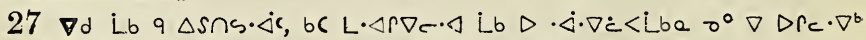

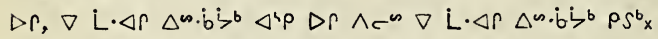

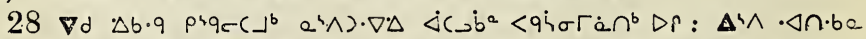

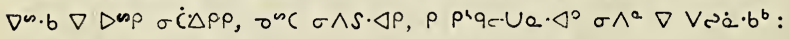

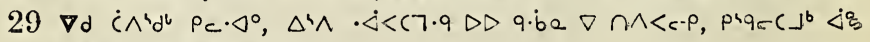
$\nabla \vee \omega_{\dot{a}} \cdot b^{b}, \Delta \omega \cdot \dot{b} U \Gamma^{b} \Delta \omega \wedge \omega_{x}$ 


\section{$\triangleright<\neg \rho^{4}$ L's $13,14$.}

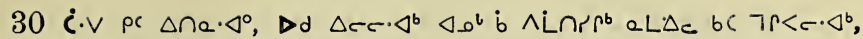

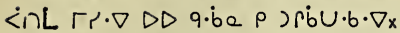

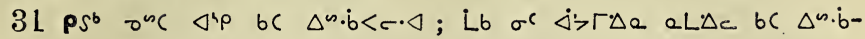
$<r \cdot \Delta:$

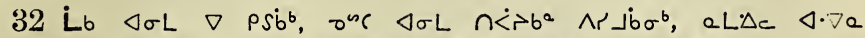

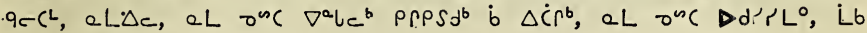
$>C \Delta L^{\circ} \wedge d x$

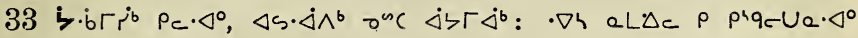
$\triangle n \wedge a q D \cap<<c^{b x}$

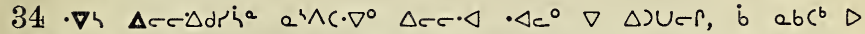

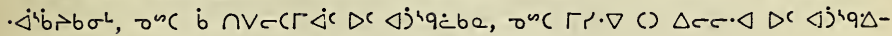

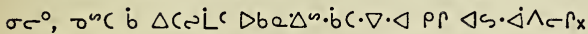

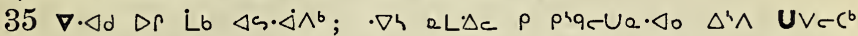

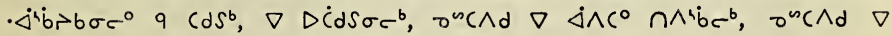

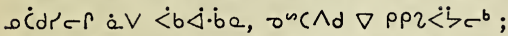

36 qb, qcc $\nabla \cos q, \rho$ b $\Gamma^{4} \dot{b} d \cdot \triangleleft^{\circ} \nabla \sigma<<<^{b} x$

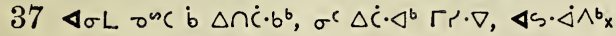

\section{Ápcre $\triangle b^{a} 14$.}

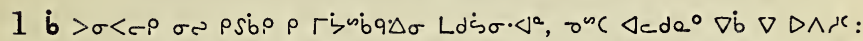

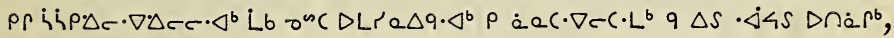
osc $\operatorname{pr} \sigma<\Delta \dot{\beta}^{b} \mathrm{x}$

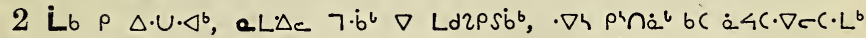
$\Delta r c \cdot \Delta^{b} x$

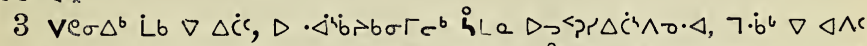

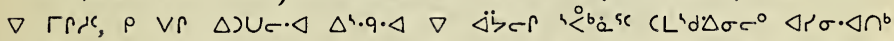

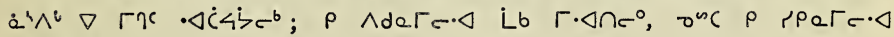
$D^{n} n \cdot b \sigma^{b} x$

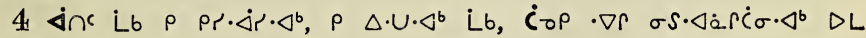
CL'd $d \Delta^{a}$ ?

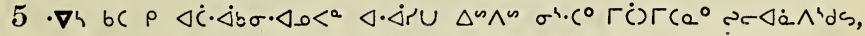

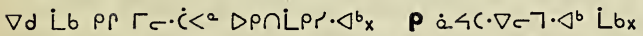

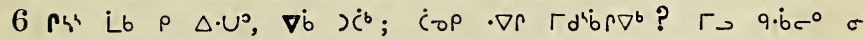
$p \dot{c}^{b x}$

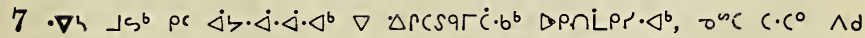

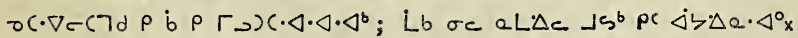

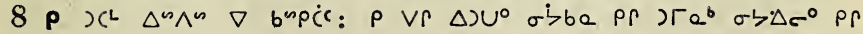
$e \Delta \dot{b} b \cdot \Delta \cdot \Delta^{b} x$

$9 \dot{C} \cdot \vee$ pc $\left.\Delta \cap a \cdot \triangleleft^{\circ}, \Lambda d \Delta C \Delta L \Gamma \Delta \dot{\Delta r}\right\lrcorner \Delta^{a} q \Delta \dot{C} b \sigma \Delta \cdot q \Gamma \cdot \cdot \nabla \triangleleft^{4} \dot{\rho} b, \nabla d C$

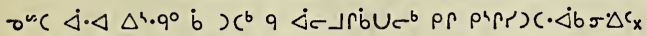

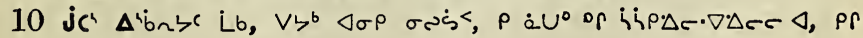

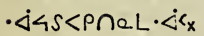




\section{$\Delta<>$ pr Lis 14 .}

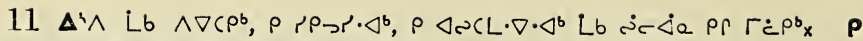
Q $2 C \cdot \nabla e C^{L}$ Lb $q \Delta S$ a $\Delta \cdot \dot{j} \angle S<P \cap \dot{C} C_{x}$

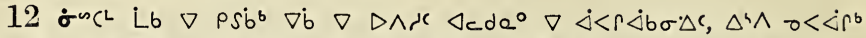

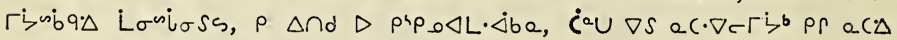

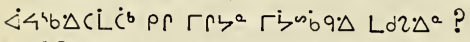

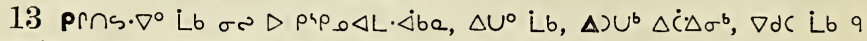

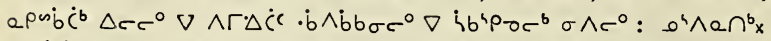

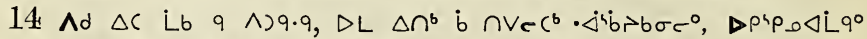

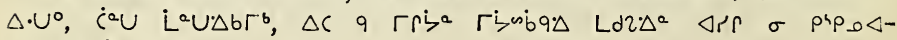

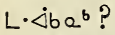

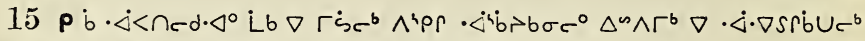

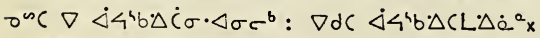

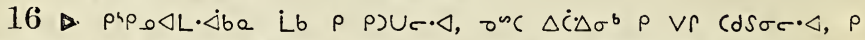

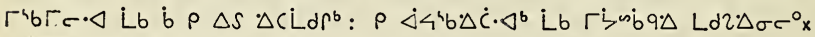

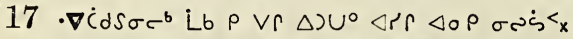

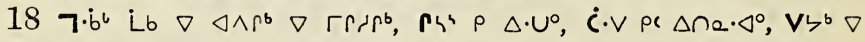

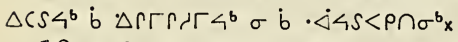

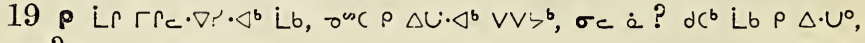
$\sigma<\dot{\alpha}$ ?

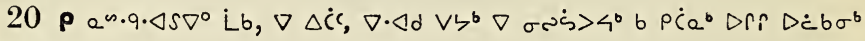
$\triangle \uparrow \rho \sigma_{c x}$

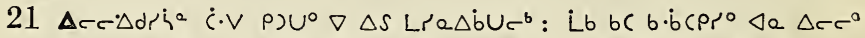

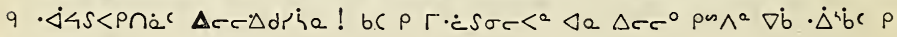
Dr $\sigma \dot{C} \Delta P c_{x}$

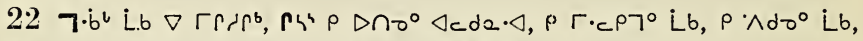

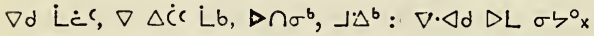

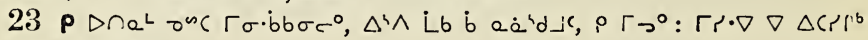
Lb $P \Gamma \sigma \cdot q \cdot \Delta^{b} x$

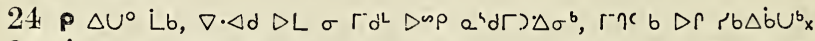

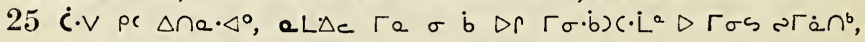

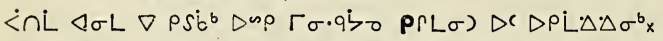

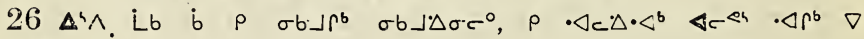
$\triangle u^{b} r^{b}$

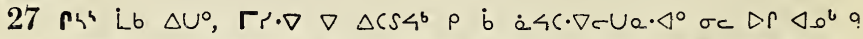

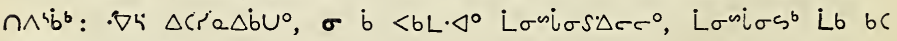
$\cdot \triangleleft 4 \cdot 4 \cap 5 \cdot \dot{\Delta} \cdot<^{b} x$

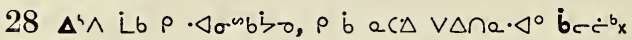

29 Lb $\wedge c^{s} \rho \Delta U^{0}, \dot{\rho} \omega \wedge^{a} \Gamma r \cdot \nabla \dot{c}\left\langle c \cdot \nabla c c \cdot b \cdot \nabla, \nabla c \cdot \nabla^{b}\right.$ Lb a L $\Delta c \sigma_{c} \sigma$ b $\dot{a} 4 c \cdot \nabla c U^{2} x$

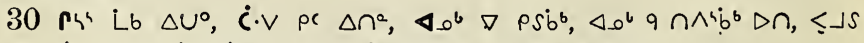

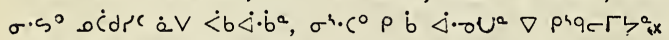

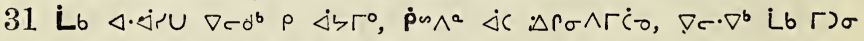

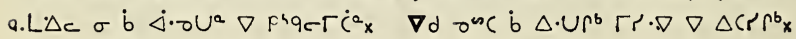




\section{$\Delta<>$ ps isb 14 .}

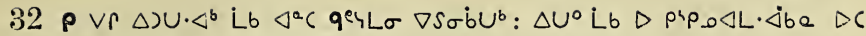

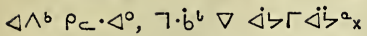

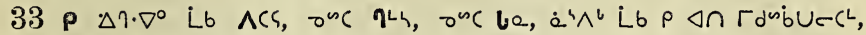

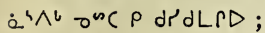

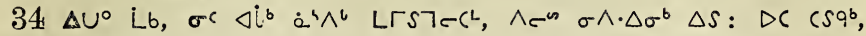
$\Delta s \cdot \Delta \wedge^{b}$ Lbx

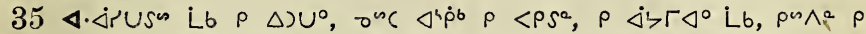

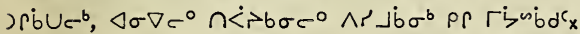

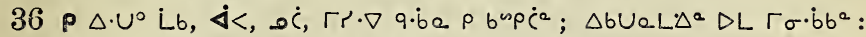
$\nabla c \cdot \nabla^{b}$ Lb, aL $\Delta c \sigma_{c} \nabla \Delta U_{c} C^{\circ} L^{a}$, Lb $\nabla \Delta U_{c} C L^{a} P_{c x}$

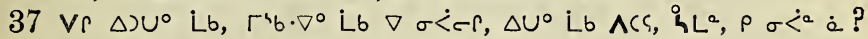

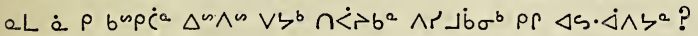

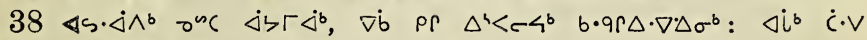
L"b. $\nabla c C L$, Lb $\triangle \zeta^{4} c c \Gamma r L b{ }^{a} x$

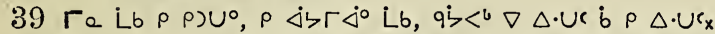

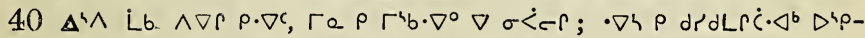

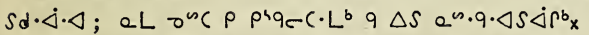

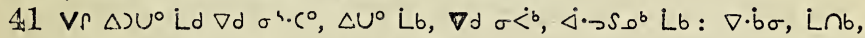

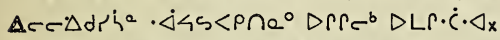

$\left.42 \cdot \Delta \sigma^{a b} b^{b}, \rho\right) \cup c^{\circ}:$ Lnb, $\Delta e \dot{b} \cdot\left\langle 4 S<p \cap \sigma^{c} \vee \omega^{b} \Delta c^{\circ}\right.$

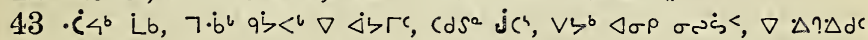

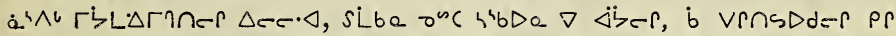

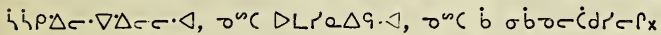

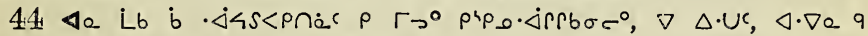
$\nabla \cap L \cdot \triangleleft \cdot 9, \nabla \cdot \triangleleft \cdot b e ; \nabla \cap \sigma^{b}, \tau^{n C} \rho \rho \Delta c^{b} x$

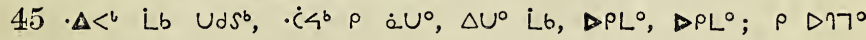
Lbx

$46 P \operatorname{L} d_{0} \cdot \nabla^{b} L b, \tau^{n C} \rho \triangleright \cap 0 \cdot \Delta^{b} x$

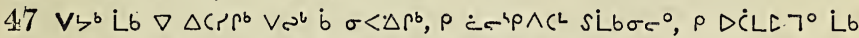

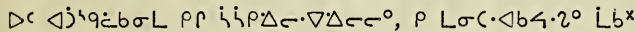

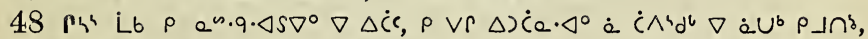

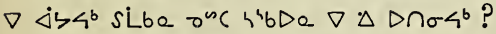

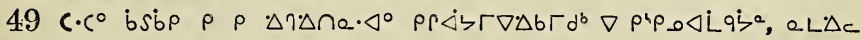

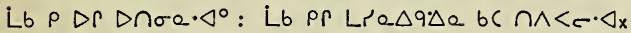

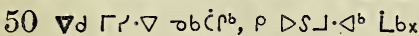

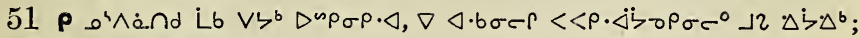
$\rho$ Ĺdod Ĺb $\triangleright$ is $\rho_{\sigma} \rho \cdot \triangleleft x$

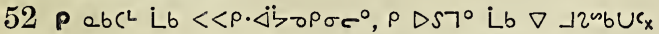

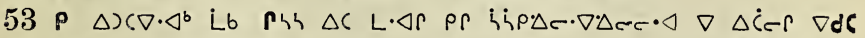

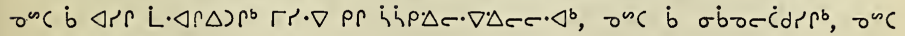
$\triangle L$ Re $\triangle q \cdot \triangleleft^{b} x$

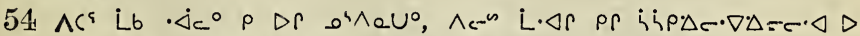

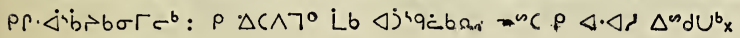




\section{$\triangle<>\rho^{2} \mathrm{~L}^{c b} 14,15$.}

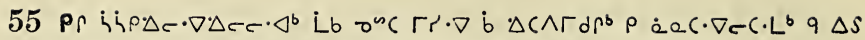

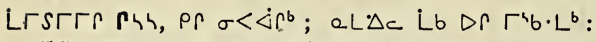

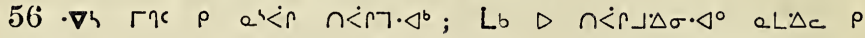
$a \Delta<c^{\circ} x$

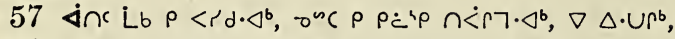

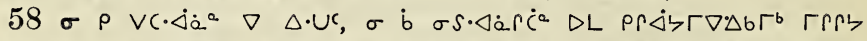

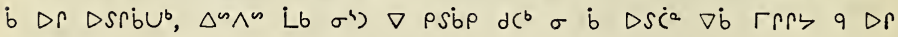
$D S r i b{ }^{2} x$

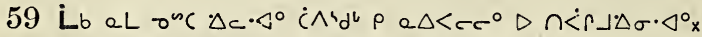

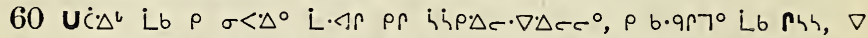

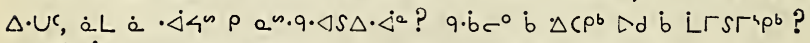

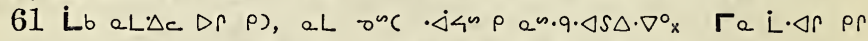

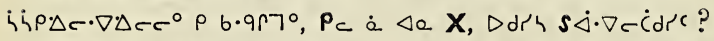

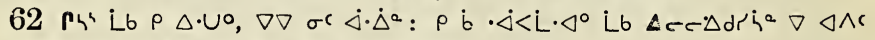

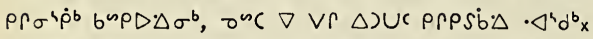

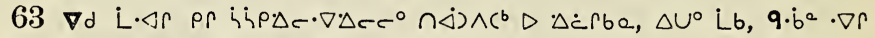

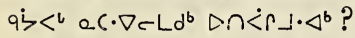

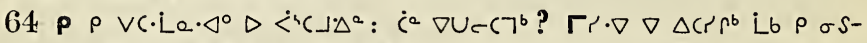

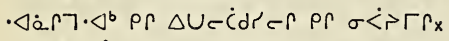

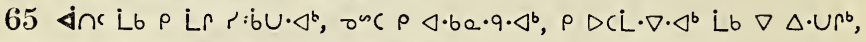
p'p. $\Delta \Delta q: \Delta j^{4} q \dot{\varepsilon} b a^{b} \dot{L} b \rho<<r \cdot q \cdot \nabla \cdot \Delta^{b} x$

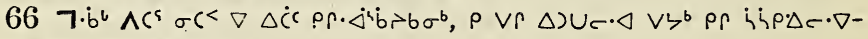
$\Delta C c^{\circ} \Delta C \Delta \dot{j}^{4} q \dot{q} b \sigma^{4} . q L$ :

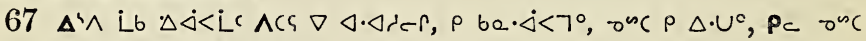

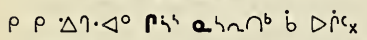

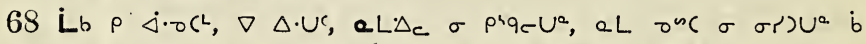

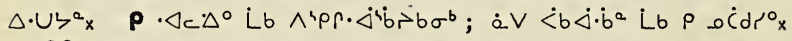

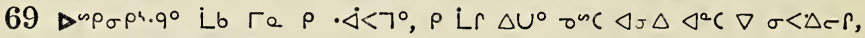
$\triangleleft \cdot \triangleleft \nabla \cdot \triangleleft d \vee \zeta^{b} \nabla \Delta C c^{b} b_{x}$

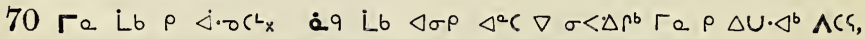

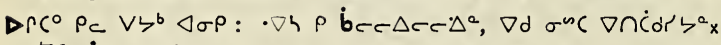

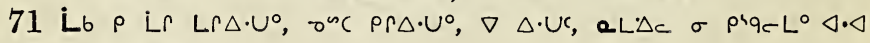
$\Delta r c^{\circ}$ b $\left.\left.\dot{\Delta}-\right\lrcorner\right]^{b} x$

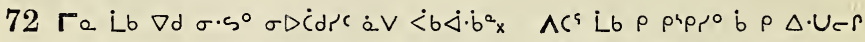

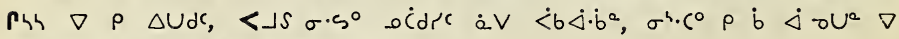

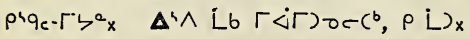

\section{A'p $\subset \propto \triangle \triangle b^{\circ} 15$.}

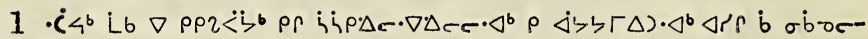

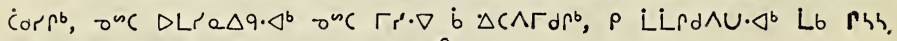

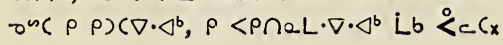




\section{$D<>$ p is 15 .}

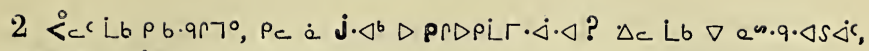
$\rho \angle U^{\circ}, p c \Delta \cdot \dot{c}^{\circ} x$

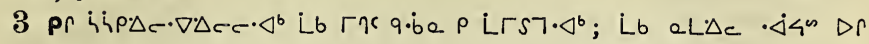
Q $\bar{c} \cdot 9 \cdot \Delta S \Delta \cdot \nabla^{\circ} \mathrm{x}$

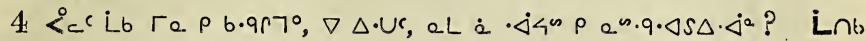

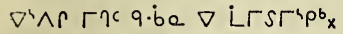

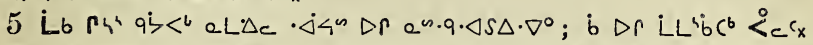

$6 \neg \cdot b^{b} \nabla$ be. $\nabla e r b U^{b} \triangleleft \sigma L L d \tau \Delta^{a} \rho<\rho \cap a L \cdot \nabla^{\circ} V b^{b} \rho<\cdot \Delta b e, \wedge d \triangleleft \sigma \Delta$ oc. $\nabla$ - L Le $\Gamma_{x}$

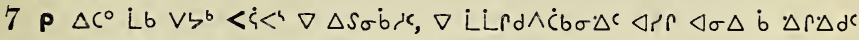

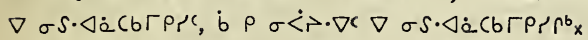

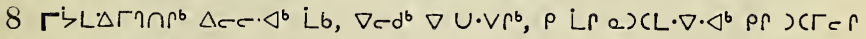

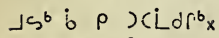

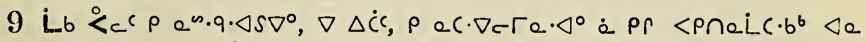
j. $\triangleleft^{b} \triangleright \operatorname{srDCLL} T \cdot \triangleleft \cdot \triangleleft$ ?

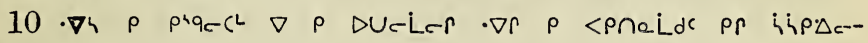
$\cdot \nabla \cdot \Delta r \cdot \Delta x$

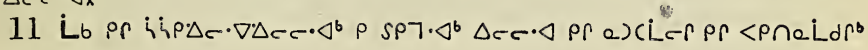
$<4<4 x$

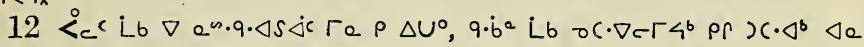
j. $\triangleleft^{b} \triangleright \rho P D \rho L T \cdot \dot{j} \cdot \triangleleft \dot{b} \Delta S \sigma \dot{b} U^{b}$ ?

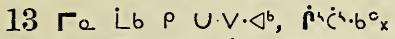

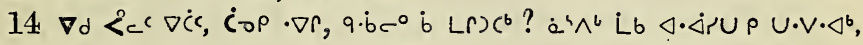

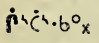

$15 \nabla d$ Lb $\mathcal{K}_{c} c, \nabla \Delta a \Delta c \cdot \nabla \dot{<}, \Delta c c \cdot \triangleleft, \rho<P \cap a L \cdot \nabla^{\circ}<\dot{\zeta}<h, \Delta^{4} \wedge$ Lb b

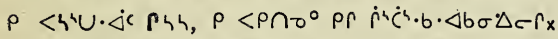

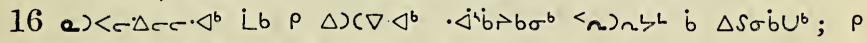

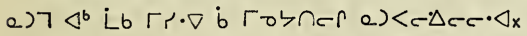

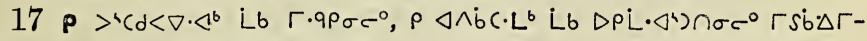

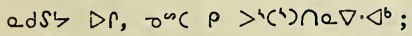

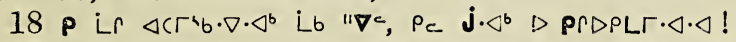

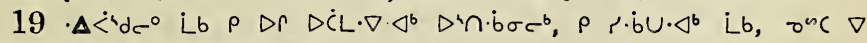

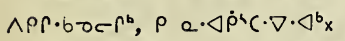

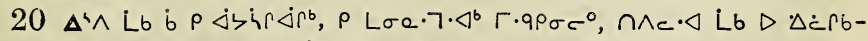

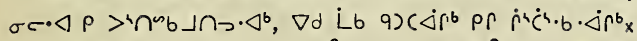

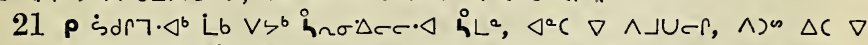

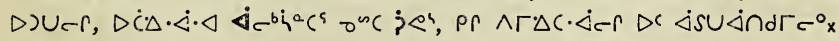

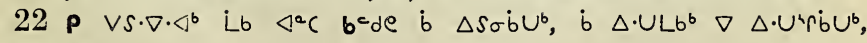
$\Delta \ln \cdot \dot{b} \sigma b^{2} x$

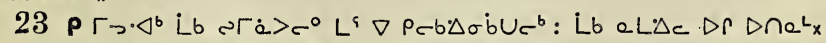

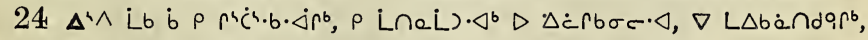

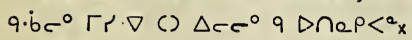

$\left.\left.25 \sigma^{4}\right) \cap \dot{<}>b^{a} \wedge r\right\lrcorner \dot{b} \sigma^{b} \rho \Delta^{4}<\tau^{0} ; \rho \dot{\rho} \dot{c}^{4} \cdot b \cdot \nabla \cdot \Delta^{b} \mathrm{~L} b x$ 


\section{$\triangleright<>p^{4}$ Lst 15.}

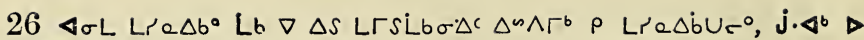
PrDPLL. $\cdot \varangle \cdot \varangle x$

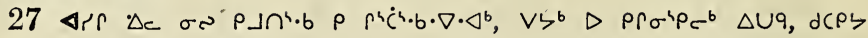

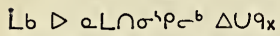

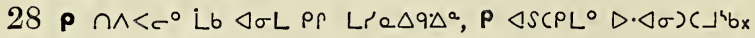

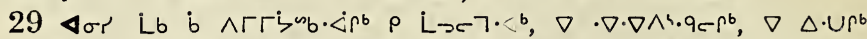

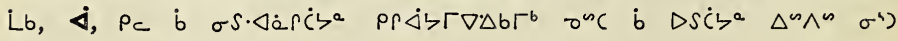
$\rho S \dot{b} \cdot \triangleleft$,

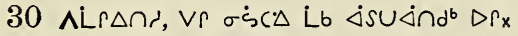

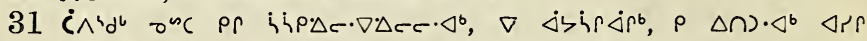

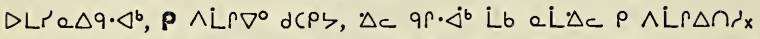

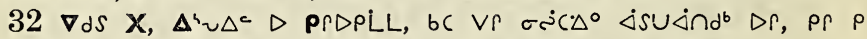

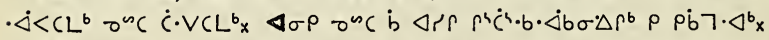

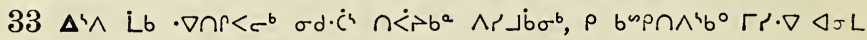

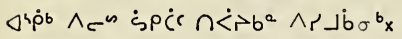

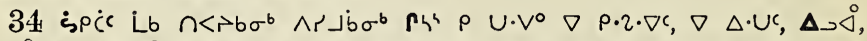

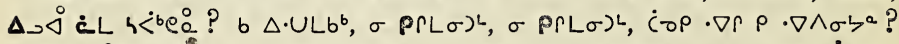

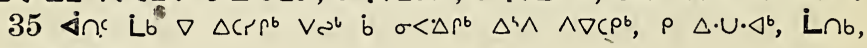
a) $7^{\circ} \Delta \dot{\dot{C}} \zeta$ hx

36 V $\zeta^{b}$ Lb $\rho \Delta^{4}<c^{\circ}, \rho \Delta d n c^{\circ}$ Lb $D<\sigma c^{\circ} s \Delta \dot{b}>^{b}, \rho \triangleleft^{4} c^{\circ}$ Lb $\Delta \dot{<}^{4} d^{b}$,

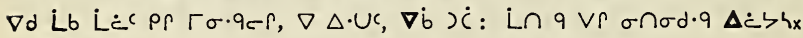

$37 r^{4}$ Lb $\rho U \cdot V^{\circ} \nabla \rho \cdot 2 \cdot \nabla c, \nabla d$ Lb $V P \cap \dot{c} c \Delta c \Delta i \cdot b x$

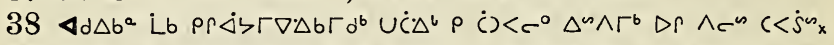

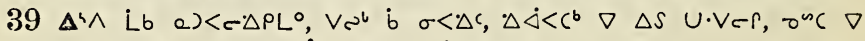

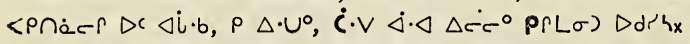

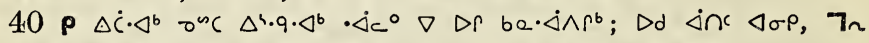

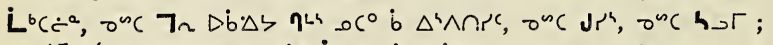

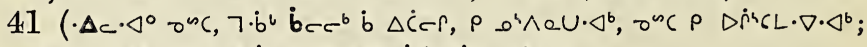

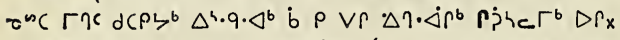

$42 \nabla d$ Lb $\Delta^{4} \wedge \cdot \nabla \dot{C} d \dot{S}^{b},\left(\nabla<\dot{s}^{b} b \Delta \rho S \dot{b}^{b}, \nabla \cdot \triangleleft d \quad \varangle \sigma L \nabla \rho S b^{b}<\right\lrcorner S$ ¿לr $\nabla$ Psib, )

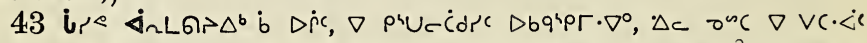

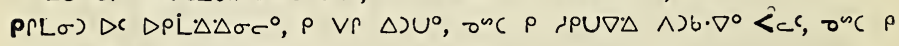
e.) $C L \cdot \nabla^{\circ}$ PhS $\triangle \dot{S} \Delta \boldsymbol{c}^{\circ} \mathrm{x}$

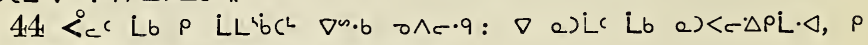

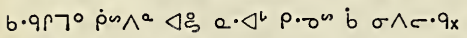

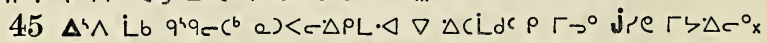

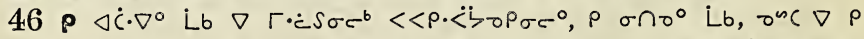

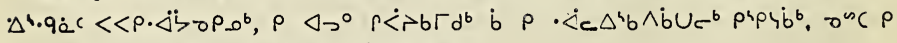

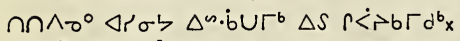

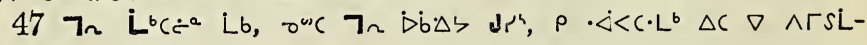
bo $\Delta r r_{x}$ 


\section{$\triangleright<\neg$ P' LsB 16.}

\section{A`pCra $\triangle b^{\circ} 16$.}

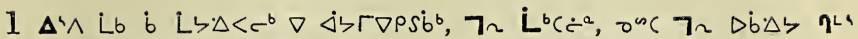

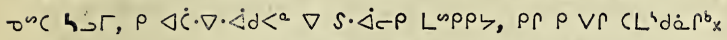

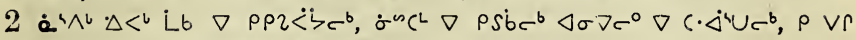

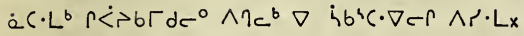

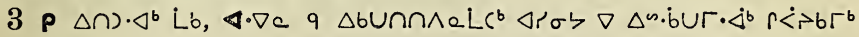
$\Delta \Gamma$ ?

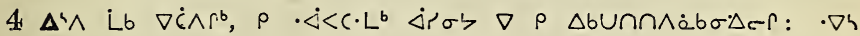
àn

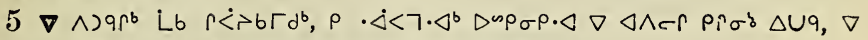

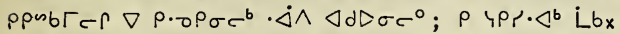

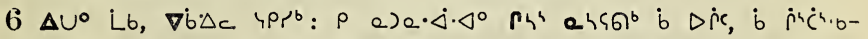
- $\Delta b \sigma^{\circ} \Delta c: \rho \cdot\left\langle\sigma^{\infty} d^{\circ}\right.$, aL $L c \Delta c \Delta \dot{C}^{\circ}: \operatorname{Lnb} \Delta c \dot{b} \Delta S \wedge \Gamma S L \Gamma^{b}{ }^{b}$

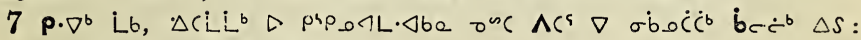
$\nabla d C q \cdot \dot{\Delta}<7^{b}, \dot{c} \wedge^{4} d^{b}$ b $\Delta \cap \dot{C}^{b} x$

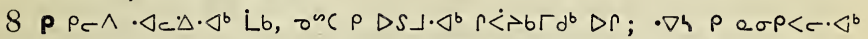

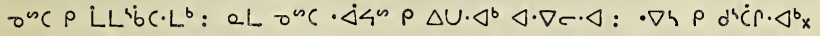

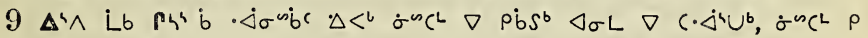

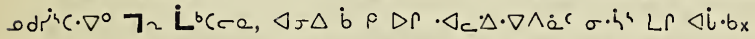

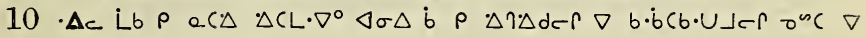
L) $\subset \Gamma x$

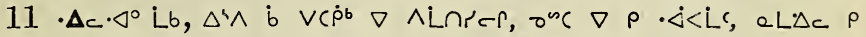
Dr $\dot{C} \cdot V C \cdot L^{b} x$

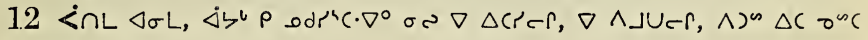
$\nabla \Delta U \Gamma_{x}$

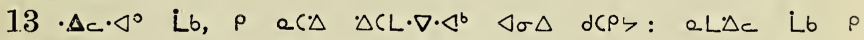
$\dot{c} \cdot v \dot{C} d \cdot \triangleleft^{b} x$

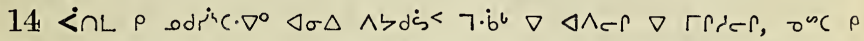

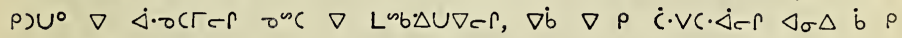
$\cdot \dot{\Delta}<\Gamma d d \dot{b} \cdot \Delta \sigma \dot{b} \dot{b} c_{x}$

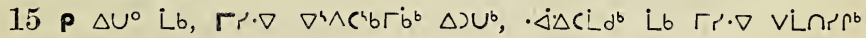
$r \Delta \Delta \dot{\Delta}\lrcorner \Delta \sigma c^{\circ} \mathrm{x}$

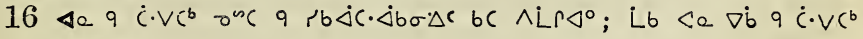
bC $\sigma S \cdot \triangleleft \dot{Q} \Gamma L^{\circ} \mathrm{X}$

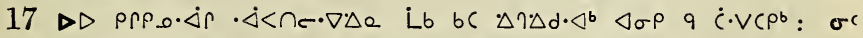

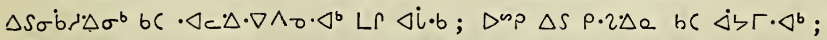

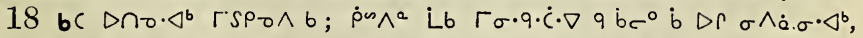

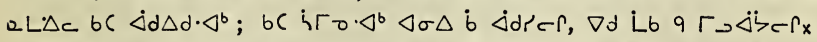

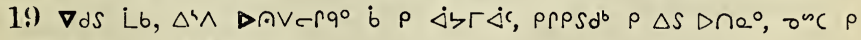
$\Delta \wedge \cdot \nabla^{\circ} D \rho\left(\sigma^{4} \rho \sigma^{b} P \Gamma L \sigma\right) \cdot \Delta x$

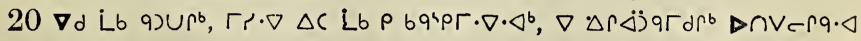

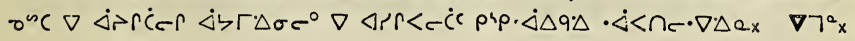




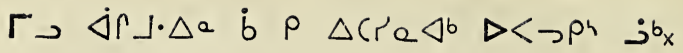

\section{Ápcre $\triangle b^{a} 1$.}

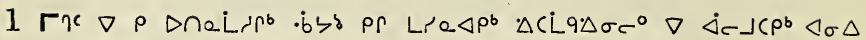
$9 \cdot 6$ e $9 \dot{\alpha}^{u} \cdot \dot{b} \dot{C} V^{\prime} C L^{b}$,

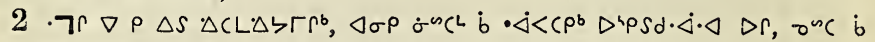
nरे) $\rho^{b}<\dot{\langle\zeta} \Delta \sigma \sigma^{\circ}$;

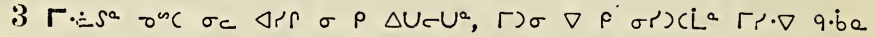

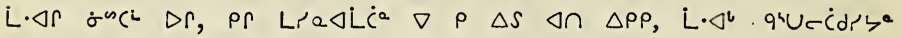
ถD $\Delta c^{4}$,

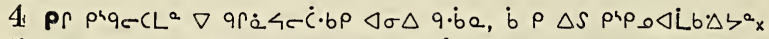

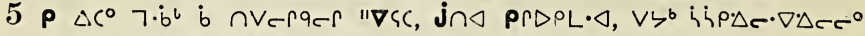

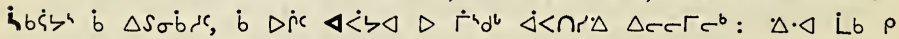
$\nabla \dot{C} \sigma r \Gamma d \nabla<a, \Delta c h \wedge e$ Lb $\rho \Delta S \sigma \dot{b} r c \cdot \Delta x$

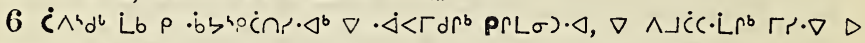

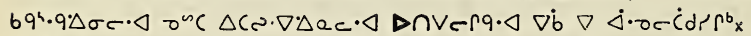

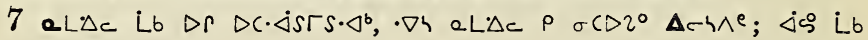

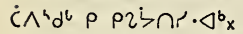

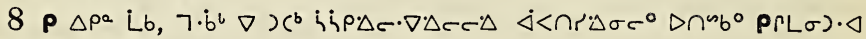

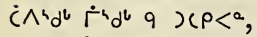

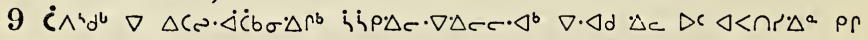

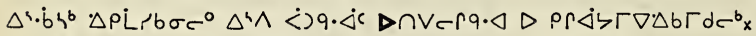

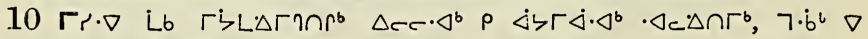

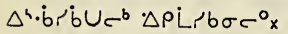

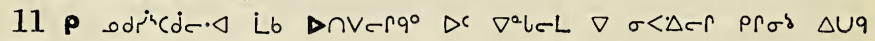
$\Delta d L_{1} d \sigma \cdot \Delta<P \rho b \dot{a} \wedge^{4} d^{b} x$

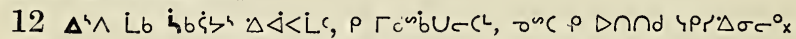

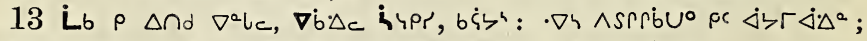
$\rho . \triangleleft \Delta c h \wedge e$ Lb $\rho \dot{b} \sigma \dot{C} \Delta P C L^{b} q \Delta d r P b^{a}$, la Lb $\rho$ b $\Delta S \sigma \dot{b} C^{\circ} x$

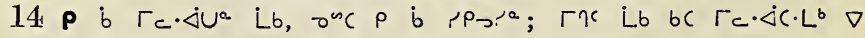
$\sigma C \Delta P_{C} \Gamma_{x}$

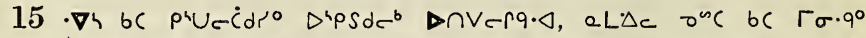

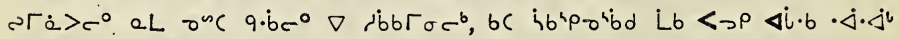
$\Delta \dot{b} \Delta \Delta^{4}<\dot{C} c^{b} \Delta \Gamma_{x}$

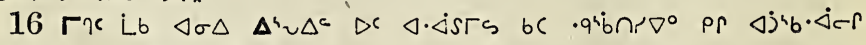
$\Delta \cap V-\Gamma q \cdot \triangleleft \nabla \operatorname{P}(\dot{L}) \Gamma c \cdot \Delta x$

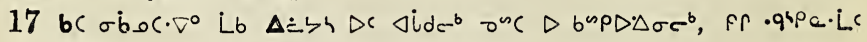
88 


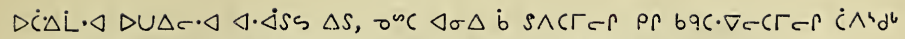

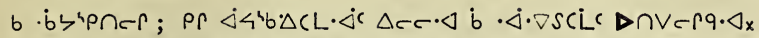

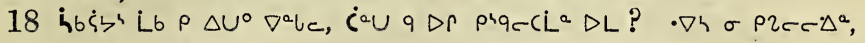
$\sigma \cdot \triangleleft$ onc $\Delta \dot{\Delta} \rho \zeta \zeta \cap r^{\circ} x$

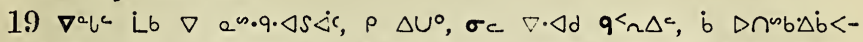

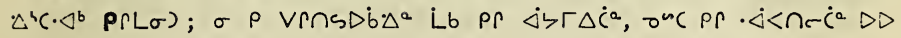
$\Gamma \rightarrow \Delta r\lrcorner \Delta e_{x}$

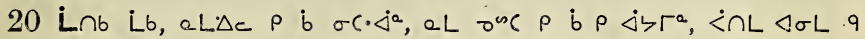

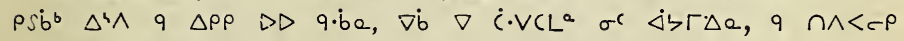
$a \Delta<c \cdot b \cdot \nabla x$

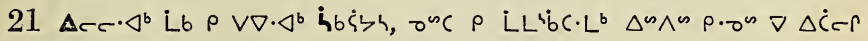
$\rho \Gamma \Delta \zeta \Gamma \nabla \Delta b \Gamma d^{b} x$

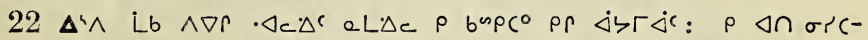

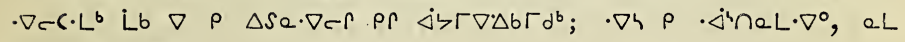
"uC $D r \Delta b \Gamma^{\circ} \times$

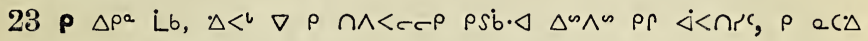
$\Delta U^{\circ} \cap \wedge_{c} \cdot \nabla \Delta \rho^{\circ} b_{x}$

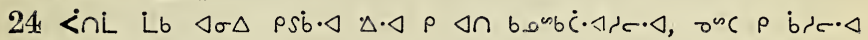

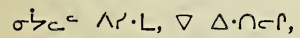

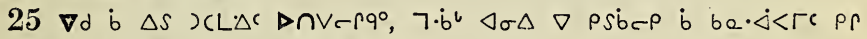

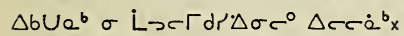

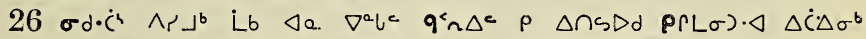
beceb, b $\Delta c \cdot b^{b}$, asce $\nabla \Delta S \sigma \dot{b} U^{b}$,

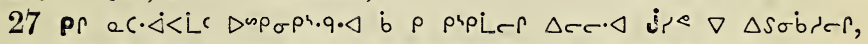

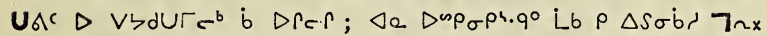

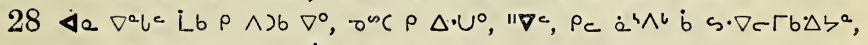

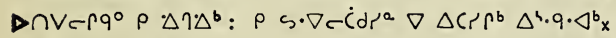

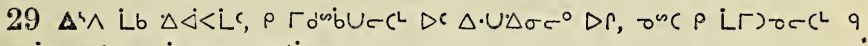

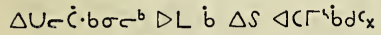

30 p $\Delta$ nd Lb $\nabla^{a} k_{c}, \nabla b \Delta c$ 4pr, ᄀn: $\nabla h \rho \rho s \cdot \nabla c \Gamma^{b} p(L \sigma \sigma) x$

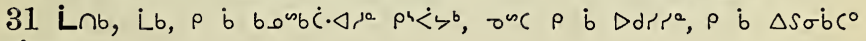
Lb $\dot{\Gamma}_{4}{ }^{4} x$

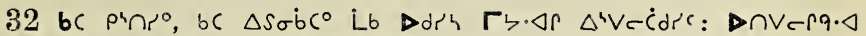

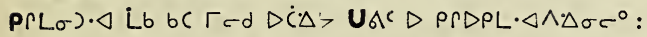

33 bc $\cap V c C \cdot 7^{\circ}$ Lb $\eta b<\Delta \rho c^{\circ}$ bPq: $D C D P L \Delta \Delta \Delta^{a}$ Lb eL. $\dot{\Delta}^{\circ} \cdot b c$ bc $\Delta n \cdot \dot{b}<c^{\circ} x$

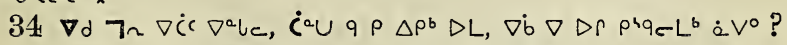

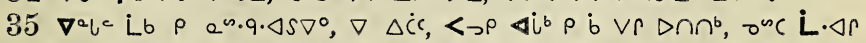

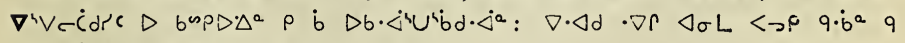
$\left.\sigma \dot{C} \Delta \rho \dot{C} \zeta^{a} P \Gamma L \sigma\right) D d r h b C \Delta S \sigma \dot{b} U^{\circ} x$

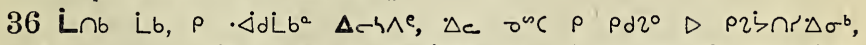

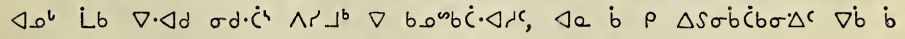
$\sigma C D 2 c_{x}$ 


$$
\Delta<>\rho^{4} \dot{j}^{b} 1 \text {. }
$$

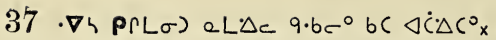

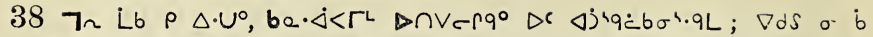

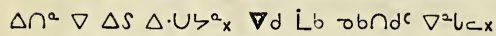

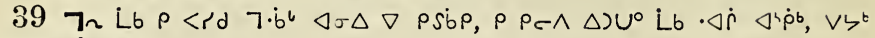
$\Delta \dot{C} \Delta \sigma^{b} \hat{j}\left(\Delta^{b}\right.$;

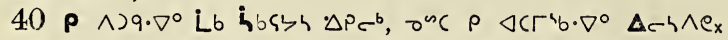

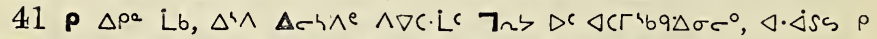

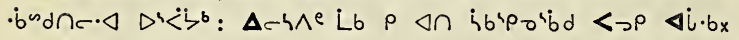

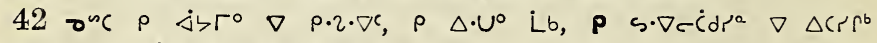

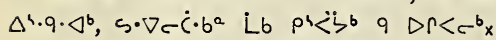

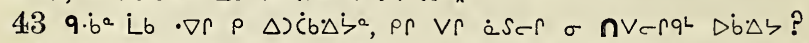

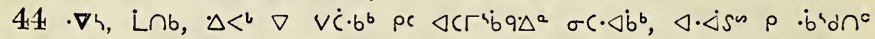
$\sigma^{2}<\ddot{b} b \quad \nabla i \wedge r \Gamma_{c} \cdot \dot{\Delta} c^{b} x$

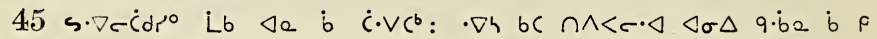
$\triangle C$ L de $\Delta \cap V \subset r q \cdot \Delta x$

46 ᄀน Lb $\rho \Delta \cdot U^{\circ}, \sigma \Gamma$ )

47 oc $\Delta i^{b}$ onc $\rho \Gamma c \cdot \Delta U^{\circ} P(L \sigma) \cdot \triangleleft \sigma \wedge L \Gamma \Delta \cdot \nabla L^{2} x$

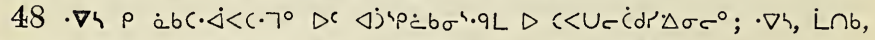

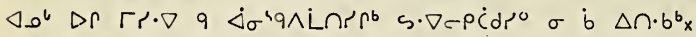

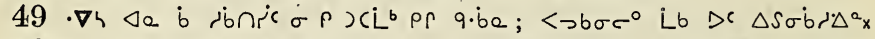

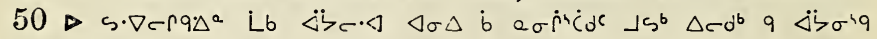
$\wedge \dot{L} \cap r \dot{Q} \sigma \cdot \nabla^{b} x$

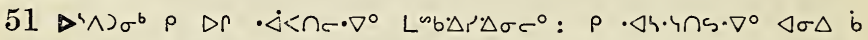

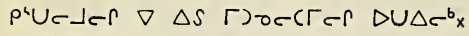

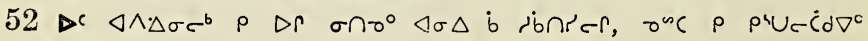

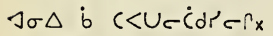

$53 \rho \dot{\rho} n>\nabla^{\circ} \varangle \sigma \Delta \dot{b}$ oUbUer $\Gamma_{2}$ q.be $D r$; Lb $\varangle \sigma \Delta$ b rsprer $\Lambda S S^{b} \rho \operatorname{PrnS} \cdot \nabla^{\circ} x$

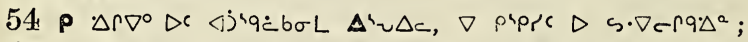

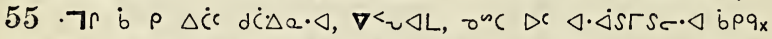

56 ᄀ几 Lb $\rho \cdot \Delta) 97^{\circ} \dot{a}^{a}\left({ }^{\circ} \sigma^{4}\right) \wedge r \cdot L, \nabla d \dot{b} \cdot \nabla c \cap \wedge c \cdot \nabla \Delta \nabla^{c} b_{x}$

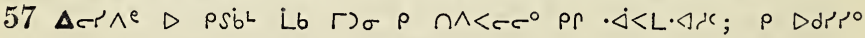
Lbx

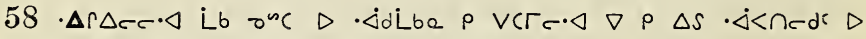
$\left.s \cdot \nabla c r q \Delta^{a} \Delta \cap V c r q \cdot \triangleleft, \rho \quad \Delta r \Gamma_{c} \cdot \Delta c\right] \Gamma_{d}$ L Lbx

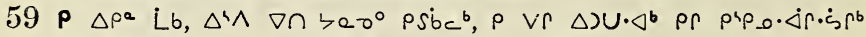

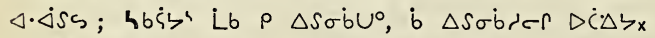

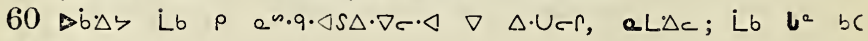
$\triangle S \sigma b^{\circ} c^{\circ} x$

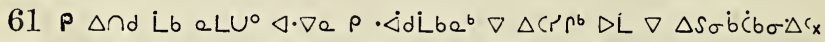

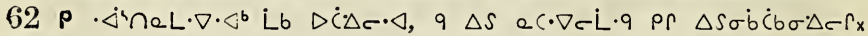

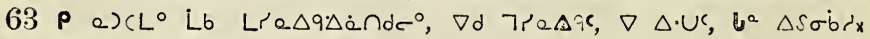

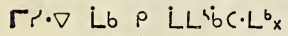




\section{$\Delta<\neg \rho^{4} \dot{j}^{6} 1,2$.}

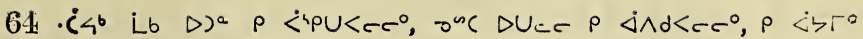

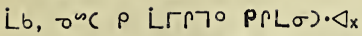

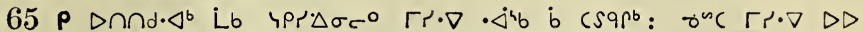

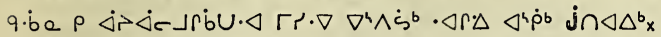

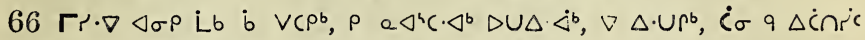

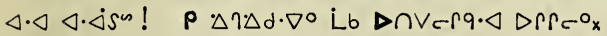

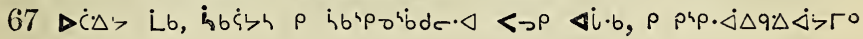
Lb, $\nabla \Delta \cdot u c$,

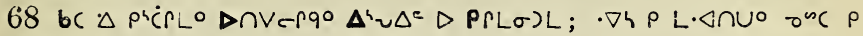

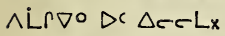

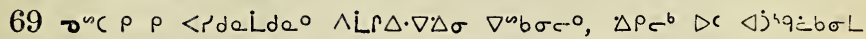
UAC;

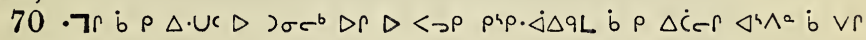

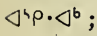

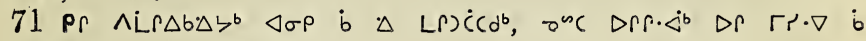
$<\cdot \dot{b} \cap \mathrm{o}^{\mathrm{b}}$;

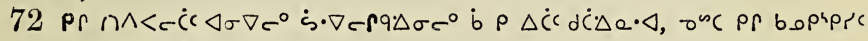
$D<\rightarrow Q^{i} \mathrm{~d} \Gamma \cdot \nabla \cdot \Delta^{a}$;

$73 \varangle \tau \nabla c^{\circ} \rho r \Delta \cdot U \Delta \sigma \sigma^{\circ}$ b $\rho$ pr $\Delta \dot{C} c$ d $\dot{C} \Delta e \cdot \triangleleft \nabla<\tau \Delta L$,

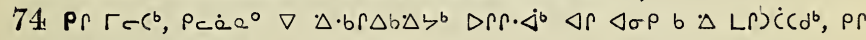

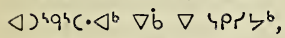

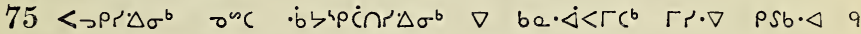
$\wedge \mathrm{L} \cap \cdot \triangleleft \cdot q^{x}$

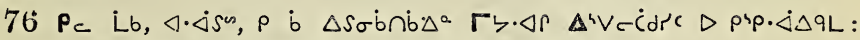

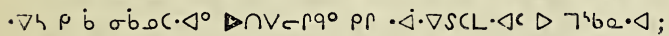

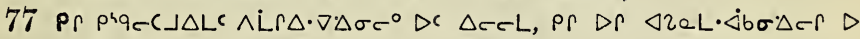
LRX $\lrcorner \Delta \sigma r \cdot \dot{\Delta}$,

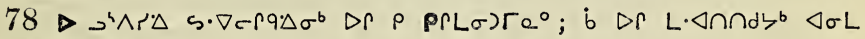
$.8<a$ erpsd $D r$,

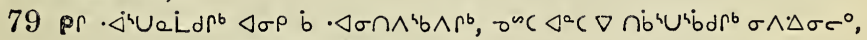

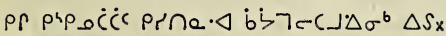

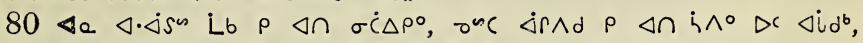

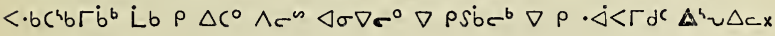

\section{N'pcra $\triangle b^{a} 2$.}

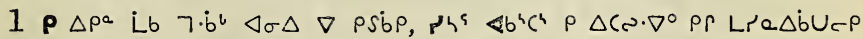

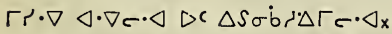

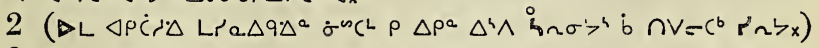

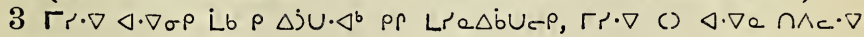
$\Delta C \Delta \dot{C} \Delta \sigma^{b} x$

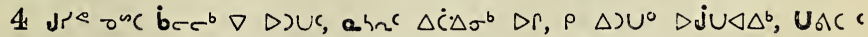

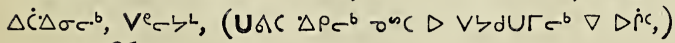

91 


\section{$D<\rightarrow \rho^{4} j^{b} 2$}

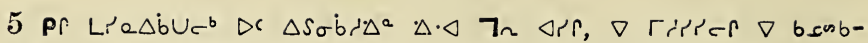
$\dot{c} \cdot \Delta 4-6 x$

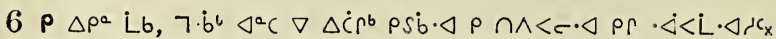

$7 P \sigma \dot{C} \Delta \rho \nabla^{\circ}$ Lb $\left.\nabla \dot{\sigma}^{n C}\right\lrcorner \dot{\zeta} a, \rho \cdot \nabla \cdot \nabla \rho_{0}^{\circ}$ Ĺb $\cdot \nabla \cdot \nabla \rho \sigma \Delta \Delta \dot{c} \rho b \sigma^{b}, \rho$ $\wedge \Gamma S 7^{\circ} \dot{L} b \Delta \cdot \Delta \dot{b} \sigma b \Gamma d^{b} ; \nabla \dot{b} \nabla \rho C \cdot<\sigma^{b} \rho \Gamma \Delta \dot{C} \Gamma^{b} \Delta \dot{C} \Delta b \Gamma d^{b x}$

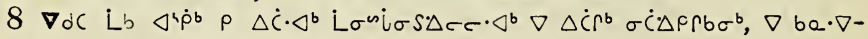
$\sim \operatorname{Lr}^{b} \triangleright \mathrm{L}^{u} \mathrm{i}_{\sigma} S \Gamma \cdot \dot{\Delta} \cdot \triangleleft x$

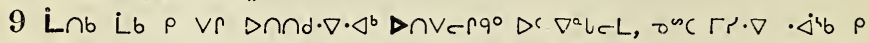

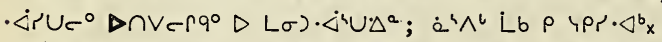

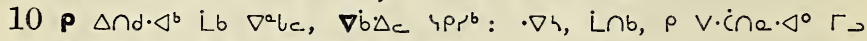
$\cap<r\lrcorner \Delta^{a} q \Delta r \dot{a}^{4} \wedge c \Gamma_{c} \cdot \dot{r} \dot{b} u^{b}, q \Delta \dot{\zeta} r^{b} \Gamma r \cdot \nabla \Delta c c^{\cdot} \cdot \Delta^{b} x$

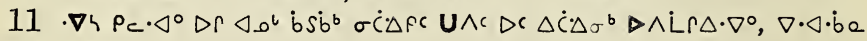
$x \rightarrow \cap V \in r^{\circ} x$

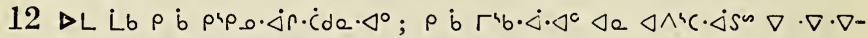
$\rho \dot{a} b \sigma \Delta c \cdot \nabla \cdot \nabla \rho \sigma^{*} \Delta \Delta \dot{c} \Gamma b \sigma^{b}, \nabla \wedge \Gamma S^{b} \triangleleft \cdot \Delta \dot{b} \sigma b \Gamma d^{b} x$

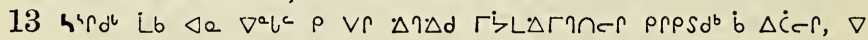

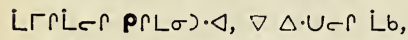

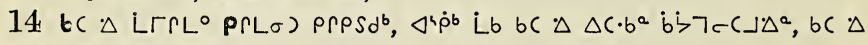
$\mathrm{s} \cdot \nabla c \mathrm{~L} \cdot \Delta^{b} \Delta r c \cdot \Delta^{b} \mathrm{x}$

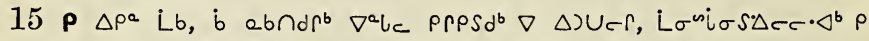

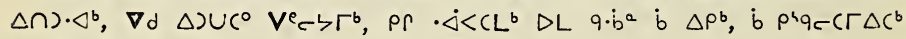
$\Delta \cap V-r^{\circ} \mathrm{x}$

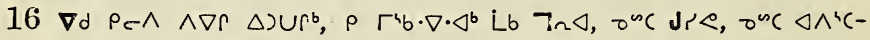

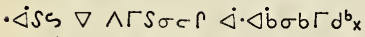

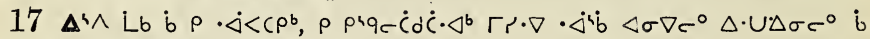
$\rho \Delta C L \cdot \Delta b \sigma \cdot \Delta r^{b} \Delta D \triangleleft \cdot \dot{\Delta} S \omega \Delta r_{x}$

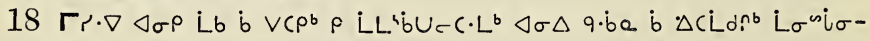
$s \cdot \Delta c c \cdot \Delta x$

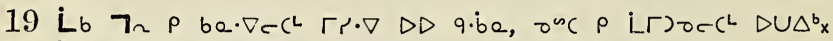

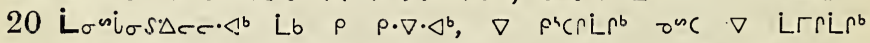

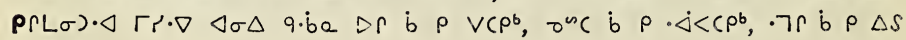
$\triangle C \dot{L} \Gamma^{b} x$

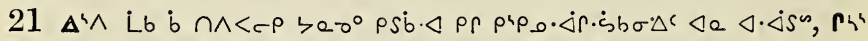

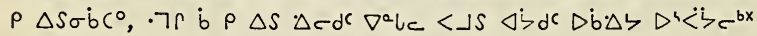

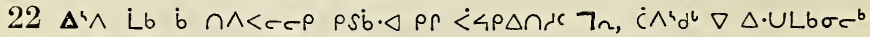

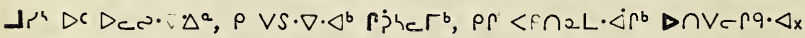

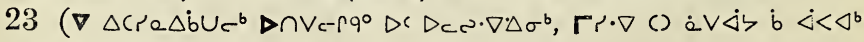

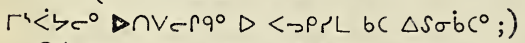

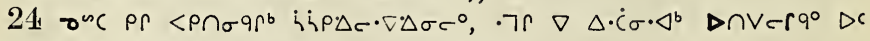

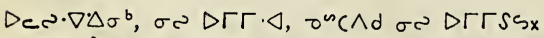

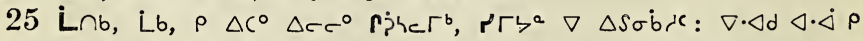

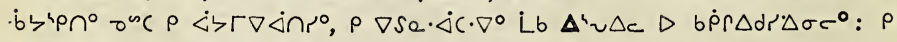
opuibd Lb <>p \&i.bx 


\section{$D<>\rho^{4}$ s. 2.}

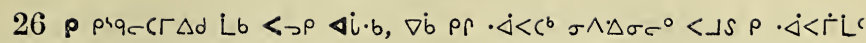
$\Delta \cap V e q^{\circ} D X \cap L x$

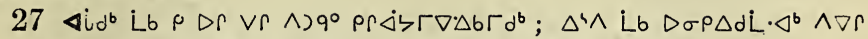

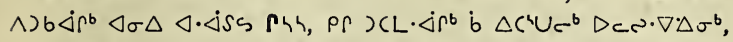

$28 \nabla d \cdot \nabla \cap \dot{a}\left(\Delta^{4} \wedge\right) \sigma^{b}, \sigma^{n}\left(\rho \operatorname{Lr} \rho 7^{\circ} P \Gamma L \sigma\right) \cdot \Delta, \nabla \Delta \cdot U c$,

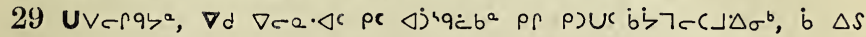
$\triangle \cdot \cup$ ப⿻;

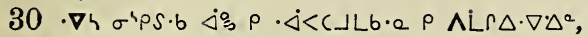

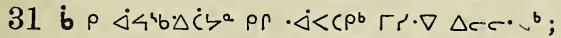

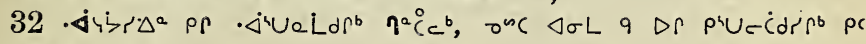
$\Delta c c^{\prime} \Delta^{4} v \Delta c x$

33 J $\sigma \cdot \Delta r_{x}$

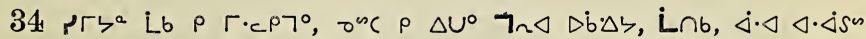

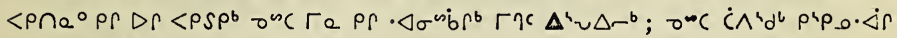

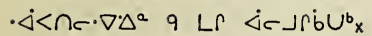

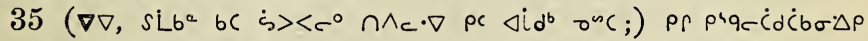
$\Delta U \leftarrow C\lrcorner \Delta e^{\circ}$ b $\Delta C \cdot b p \Gamma \eta c \Gamma U \Delta^{b} x$

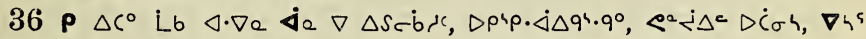

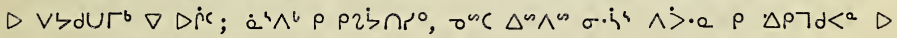
¿.VL $\nabla$ Dupops $q \triangle C>\Gamma$ :

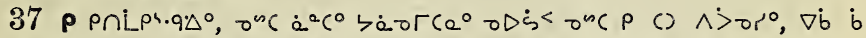

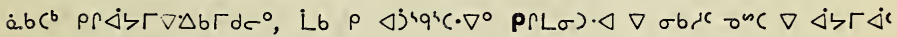

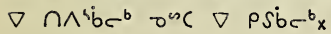

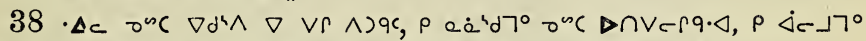

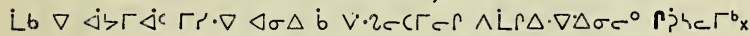

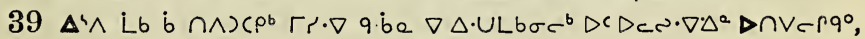
$\dot{b}_{c} \dot{c}^{b} \rho \Delta S \rho \cdot \nabla \cdot \Delta^{b}, \cap \Lambda_{c} \cdot \nabla \Delta c \Delta \dot{C} \Delta \sigma \cdot \dot{j}^{b} \dot{a}^{b} h_{n} e_{x}$

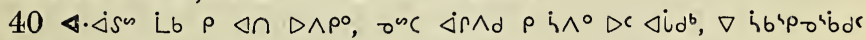

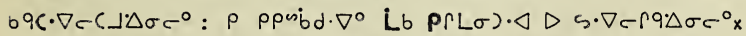

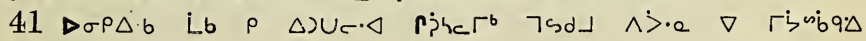
$L d \dot{s} \sigma \cdot \triangleleft \dot{\sigma} c^{b} x$

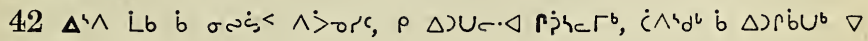
Ld $\dot{S} \sigma \cdot \Delta^{b} x$

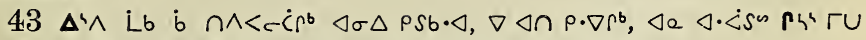

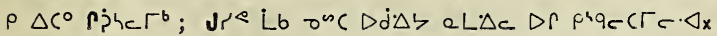

44 Lb $\left.\Delta_{c} \cdot \triangleleft^{\circ}, \Delta \uparrow \cdot \nabla\right) q \nabla$ LL $\Delta \wedge U_{c} \uparrow \Delta c c \cdot \triangleleft, \nabla \Delta U_{c}\left(\rho^{b}, \rho \wedge\right\lrcorner U \cdot \Delta^{b}$

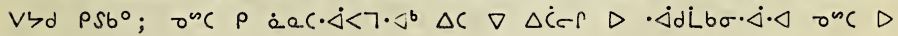
UU $\cdot \dot{\triangleleft} \cdot \Delta x$

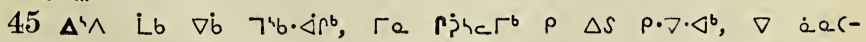
$\cdot \dot{i}<L^{b} r^{b} x$

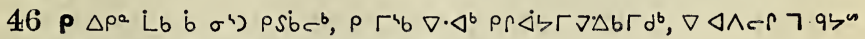

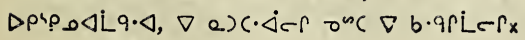




\section{$\Delta<$ or jo $2,3$.}

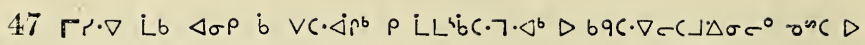

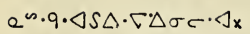

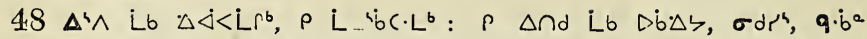

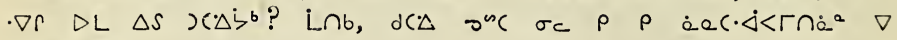
$\Gamma c, 7 \subset-L^{b}{ }^{b}$

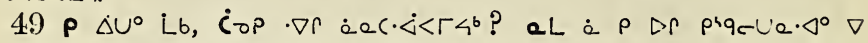

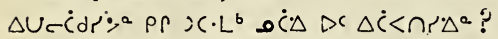

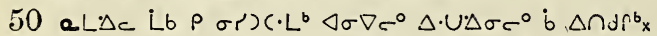

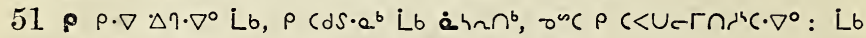

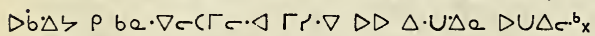

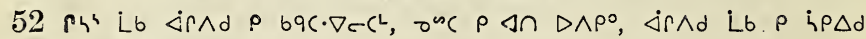
$P \Gamma L \sigma) \cdot \triangleleft$ on $\Delta r c \cdot \nabla x$

\section{Aspcre $\triangle b^{a} 3$.}

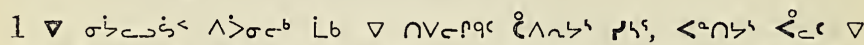

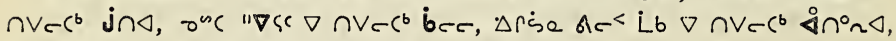

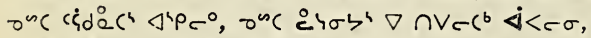

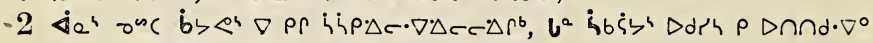
PrL $\sigma) \cdot \Delta$ Dr $\dot{\Delta L} \Gamma \Delta \sigma \sigma^{\circ}<\cdot b c^{4} b \Gamma \dot{b}^{b} x$

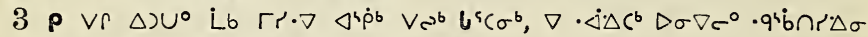

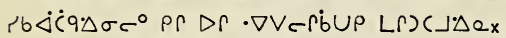

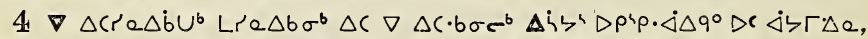

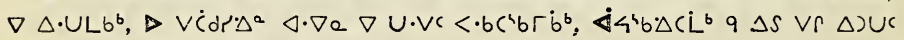

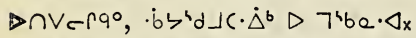

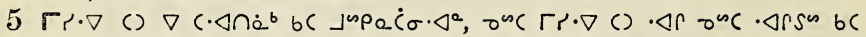

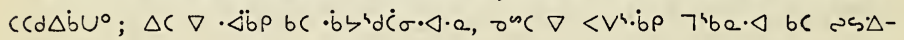
$\dot{c} \sigma \cdot \triangleleft \cdot a$;

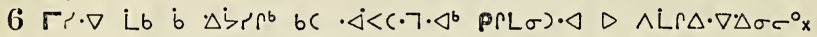

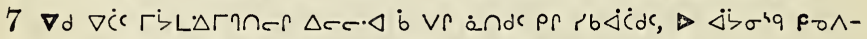

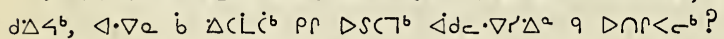

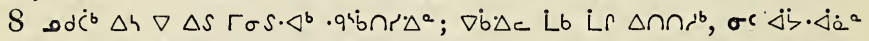

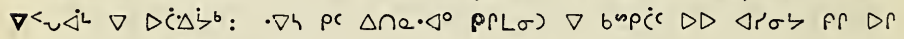
$D C \cdot \Delta S T S b \cdot \dot{i} \boldsymbol{\nabla}<\tau \triangleleft L_{x}$

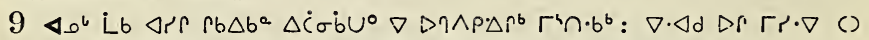

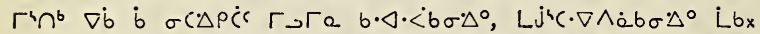

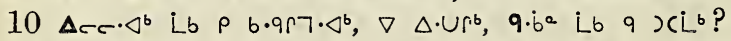

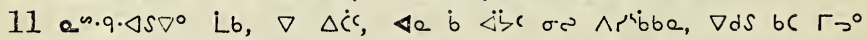

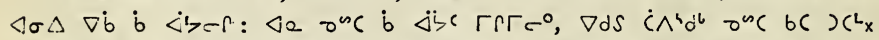

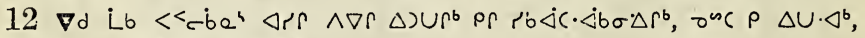

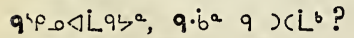

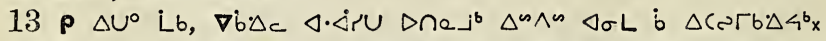

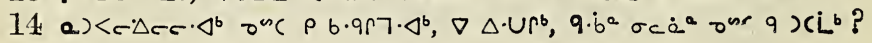
94 


\section{$D<p$ pr $\dot{j}^{6} 3$}

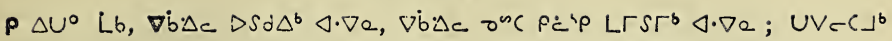
Lb $\rho \cap<\Delta L d r \Delta \sigma \cdot \Delta^{\circ} x$

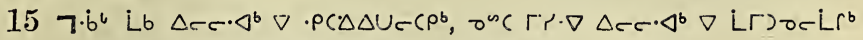

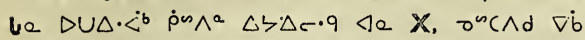

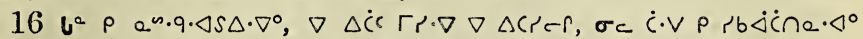

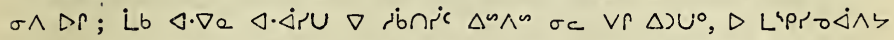

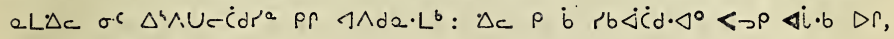
onc $\Delta$ u dUco;

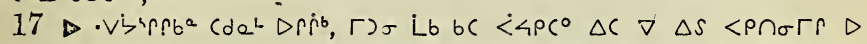

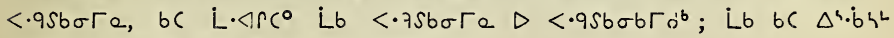

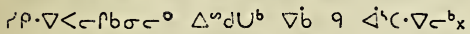

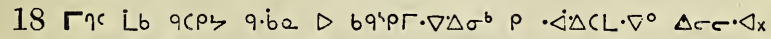

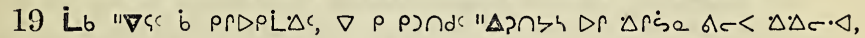
$\nabla^{n}(\Gamma \rho \cdot \nabla \triangleleft \sigma \Delta \dot{b}$ Lंc( $\sigma c \rho \dot{b} \rho)(b \| \nabla \varsigma$,

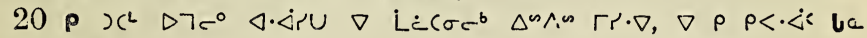
$\rho<\Delta) \Delta b \Gamma d^{b} x$

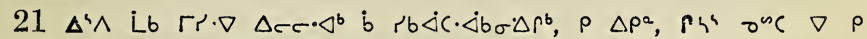

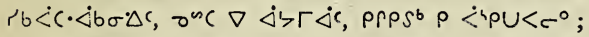

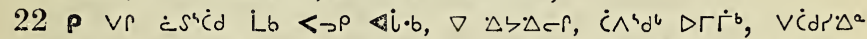

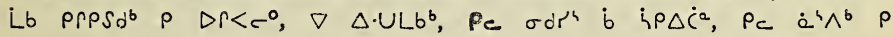
$c \Delta c \cdot \nabla \Delta^{a} x$

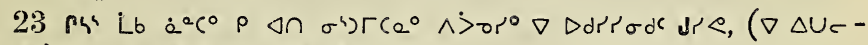

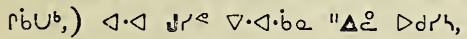

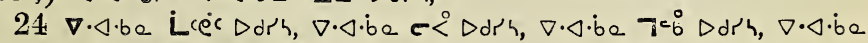
Le $\Delta d r^{\prime} h, \nabla \cdot \triangleleft \cdot b e$ Je $\nabla d r h$,

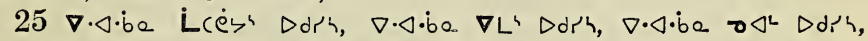

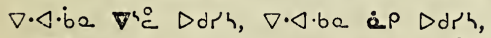

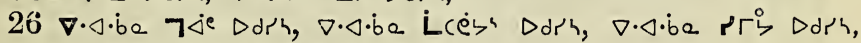
$\nabla \cdot \triangleleft \cdot b a$ J

$27 \nabla \cdot \Delta \cdot b_{e} j \triangleleft e$ $\nabla d r h, \nabla \cdot \Delta \cdot b e$ a $\nabla d r h, \nabla \cdot \nabla \cdot b e$ d $5<\wedge<\nabla d$ ih,

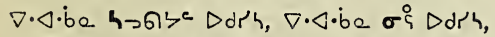

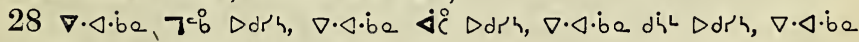

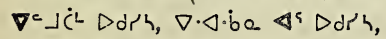

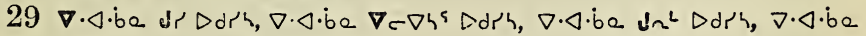

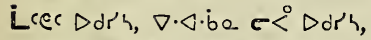

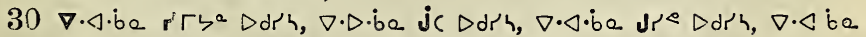

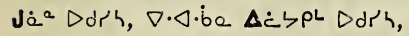

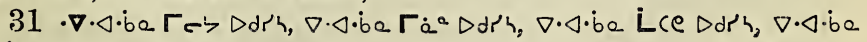

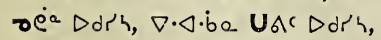

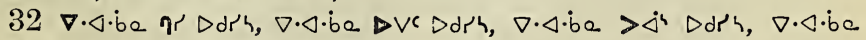

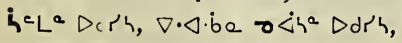

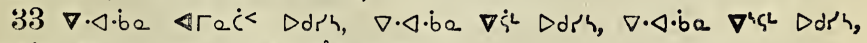
$\nabla \cdot \triangleleft b e v \dot{i}^{4} \nabla b r h, \nabla \cdot \triangleleft \cdot \dot{b} a$ jc $\nabla d r h$, 


\section{$\Delta<\neg$ p' jb 3,4 .}

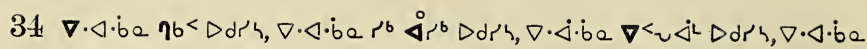
es $D$ dr',$\nabla \triangleleft \cdot b e$ dos $D$ br'

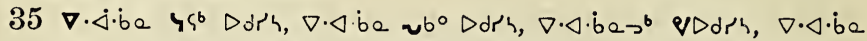
" $\Delta<s \nabla d r h, \nabla \cdot \triangleleft \cdot b e$ Yc $D d r h$,

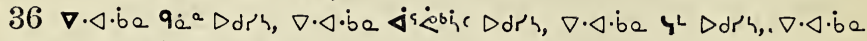
Q $\triangle \Delta d r h, \nabla \cdot \triangleleft \cdot \dot{b} a \supset 7^{b} \nabla d r h$,

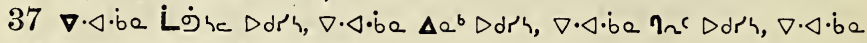

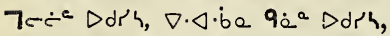

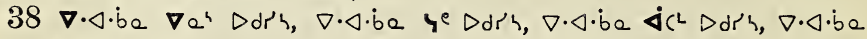
PrL $\sigma) \triangleright P h x$

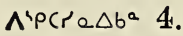

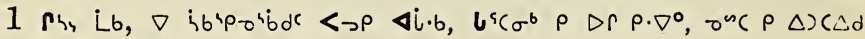
$\varangle i \cdot b<\cdot b c^{4} b \Gamma^{b} b$,

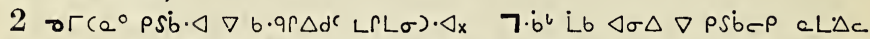

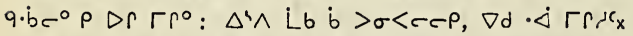

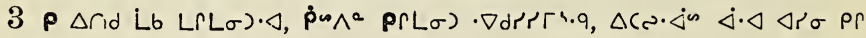
$\triangle c d i \Delta c_{x}$

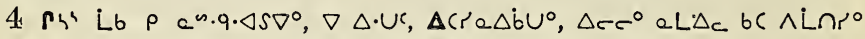

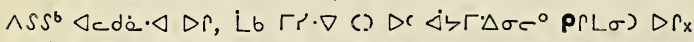

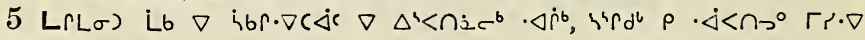
$\triangle P L \Delta \Delta Q \Delta \Delta^{4} \dot{p}^{b} x$

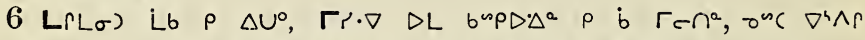

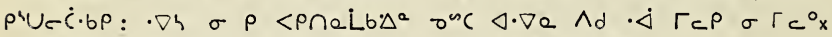

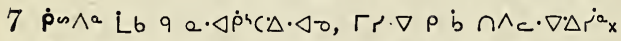

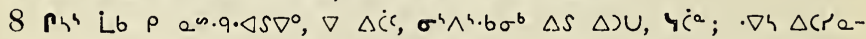

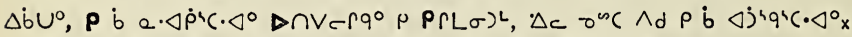

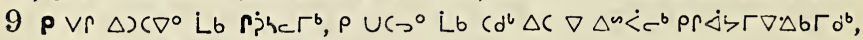

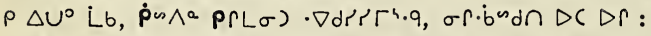

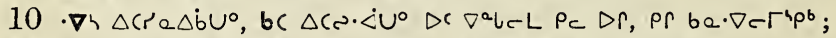

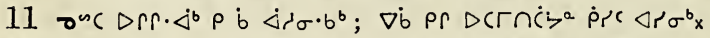

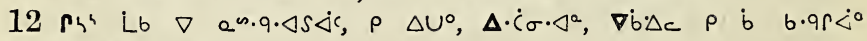
$\triangle \cap V C R Q^{\circ} P$ P $\left.R L \sigma\right)^{L} x$

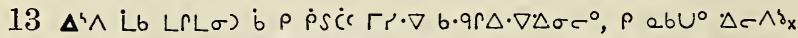

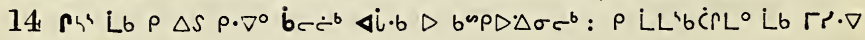

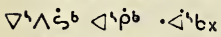

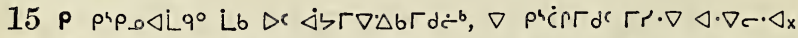

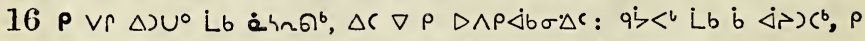

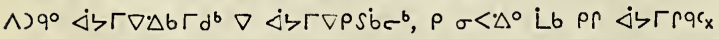

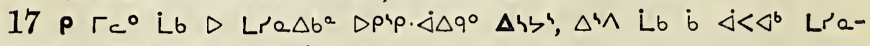
$\Delta b \sigma c^{\circ}, P \Gamma^{\mathrm{h}} \mathrm{b}^{\mathrm{L}} \Delta C \nabla$ Lre $\Delta \dot{b} U \mathrm{c}^{\mathrm{b}}$,

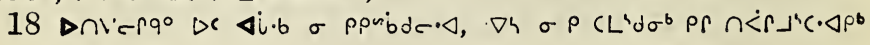
96 


\section{$D<>$ pi jo 4 .}

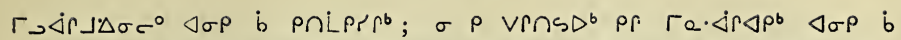

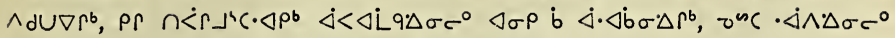

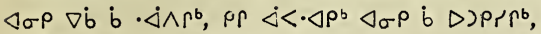

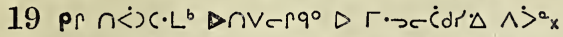

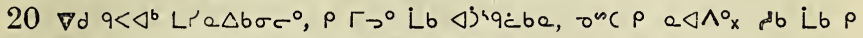
be. $\dot{\Delta}<\Gamma d \Gamma r \cdot \nabla \Delta \sigma \Delta \dot{b} \Delta \dot{C}-r \Delta \zeta \Gamma \nabla \Delta b \Gamma d^{b} x$

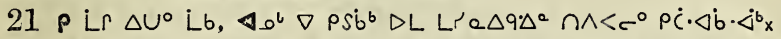

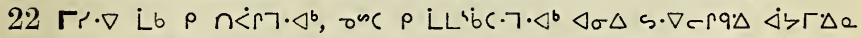

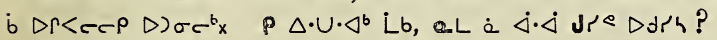

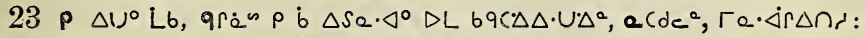

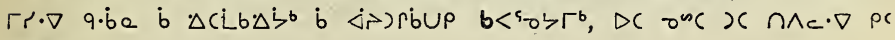
बipे $b_{x}$

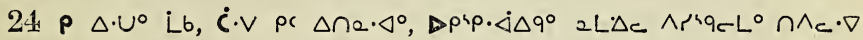
$\Delta r \triangleleft \dot{\rho} b_{x}$

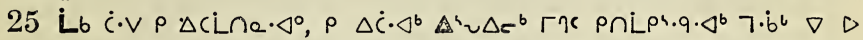

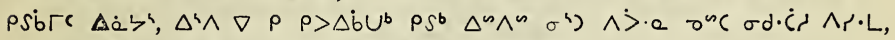

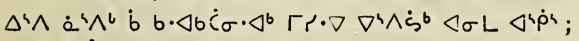

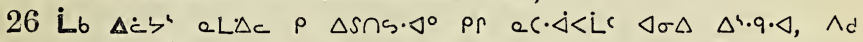

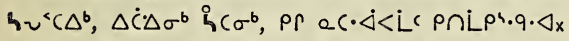

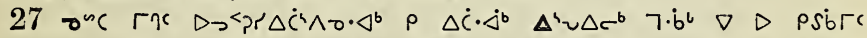

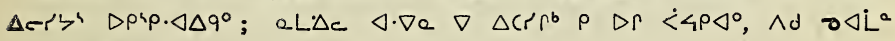
P $\triangle \Delta \triangle c^{\circ} x$

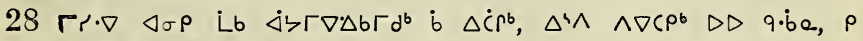

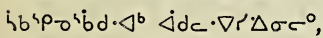

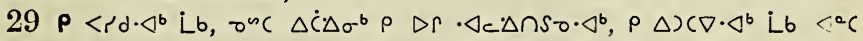

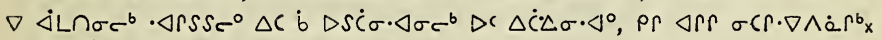

30 Lb $\left.\Delta c, \nabla \dot{s}>{ }_{b} \cdot<c \rho p\right)$,

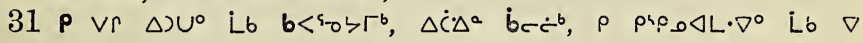

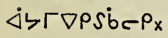

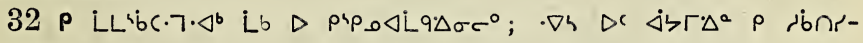
Lb $\sigma \sigma^{\circ} \mathrm{x}$

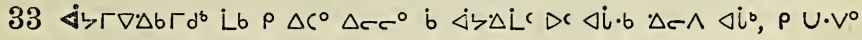
Lb, $\nabla \rho \cdot 2 \cdot \nabla^{c}$,

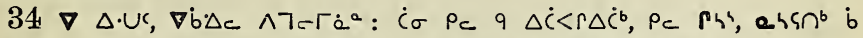

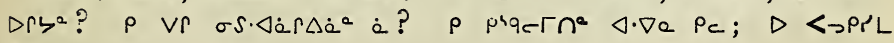
$P \Gamma L \sigma)_{x}$

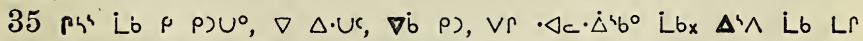

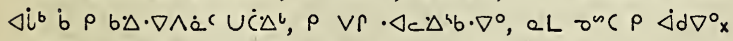

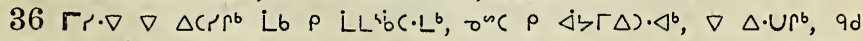

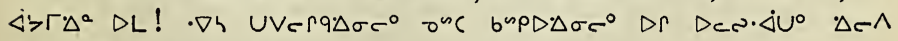
$\Delta i \cdot b, v r \cdot \Delta c \cdot \Delta<r \cdot \triangleleft i b x$

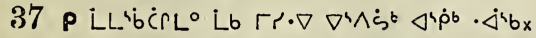

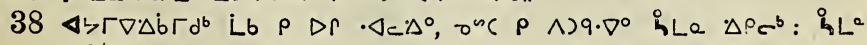




$$
D<>p^{4} \text {;b } 4,5 .
$$

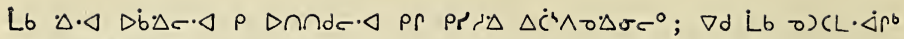
er $r e \cdot \Delta r \dot{\Delta} c_{x}$

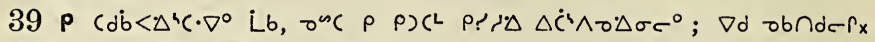
$\cdot C 4^{b}$ Lb $\rho \cdot \Delta \sigma^{\infty} b^{\circ}, \sigma^{\infty} C \rho<\Gamma \nabla^{\circ} x$

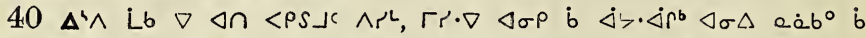

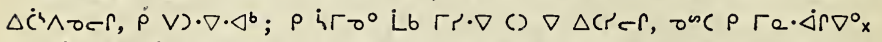

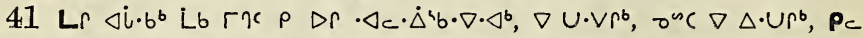

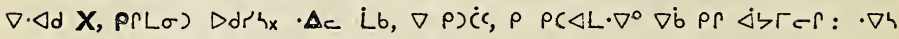

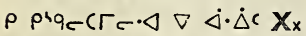

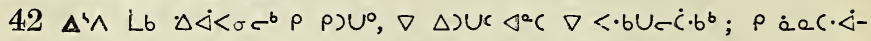

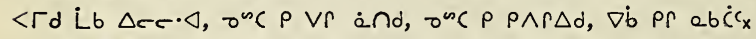

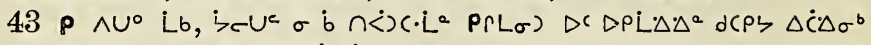

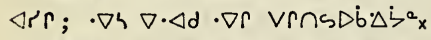

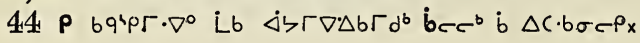

\section{A $P$ sp a $\triangle b^{a} 5$.}

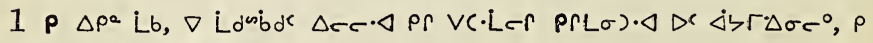

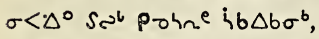

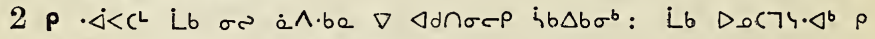

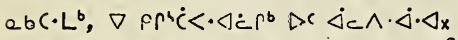

$3 \rho>r^{\circ}$ Lb $\vee b^{b} \triangleleft \sigma \Delta \dot{a} \wedge \cdot b \sigma^{b}$, hLe $\nabla \cap \wedge c \cdot \nabla \Delta r e r, \rho$ e) $\left(L \cdot \nabla^{\circ}\right.$ Lb

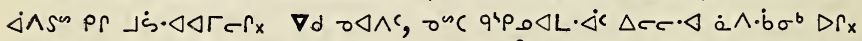

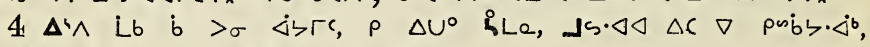

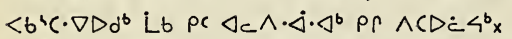

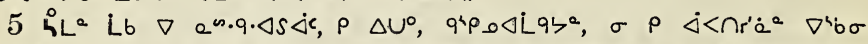
$\cap \wedge^{4} b^{b}$, aL Lb $\sigma P D \Gamma \wedge C D \dot{C} \dot{a}^{a} ; \nabla c \cdot \nabla^{b} L b \nabla \Delta \cdot U b^{a} P_{c}, \sigma \dot{b}<b^{4} C \cdot \nabla \cdot \triangleleft^{\circ}$ $D_{C} \wedge_{x}$

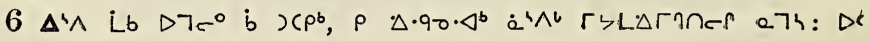

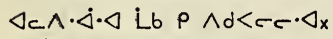

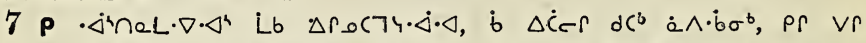

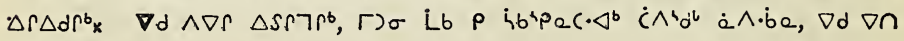
divepx

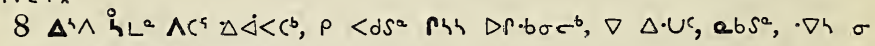

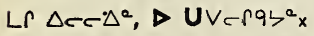

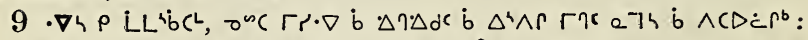

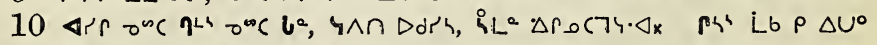

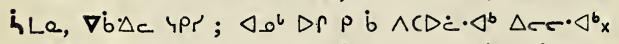

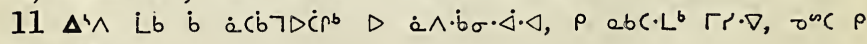
o $\wedge \circ U \cdot \Delta^{b} x$

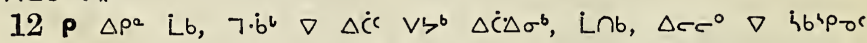

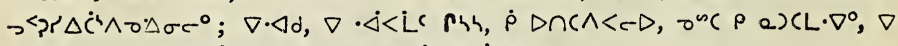

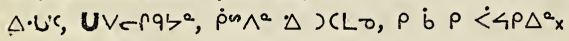




$$
\Delta<\neg \rho^{4} \dot{\Xi}^{6} 5 \text {. }
$$

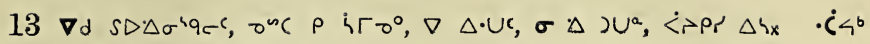

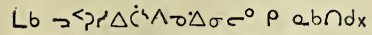

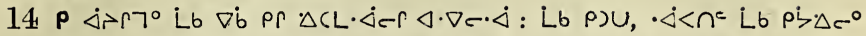

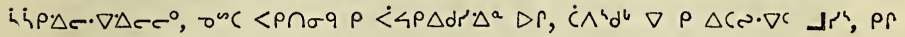
prqe $C \Gamma \Delta d \rho^{b} x$

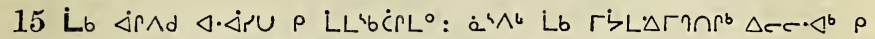

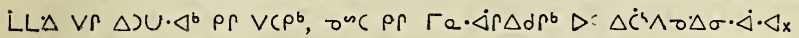

$16 \rho \Delta U^{\circ} L b<\cdot b c^{4} b \Gamma b^{b}, \nabla d \Delta \Delta b \Gamma \Delta c_{x}$

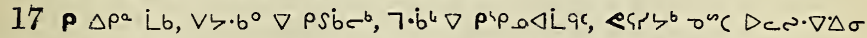

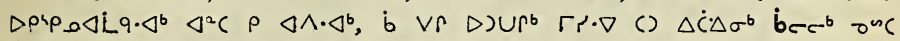

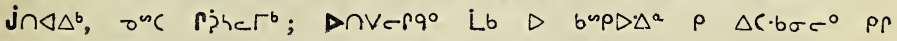
$r e \cdot \dot{s} \Delta d \rho^{b} x$

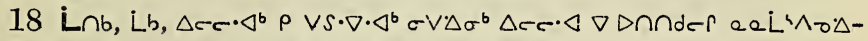

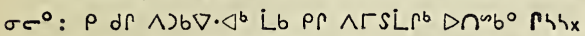

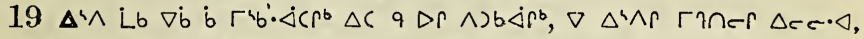

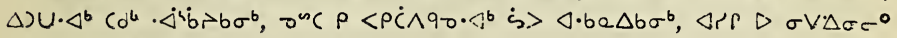

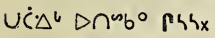

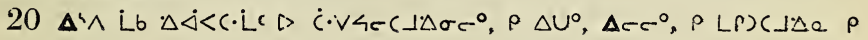
- $\nabla V e C L b \Delta^{a} x$

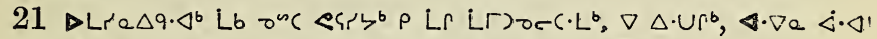

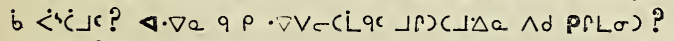

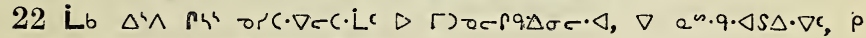

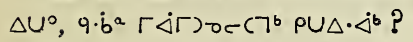

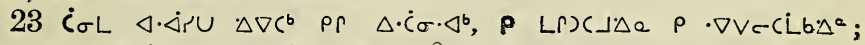

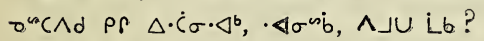

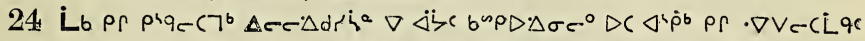

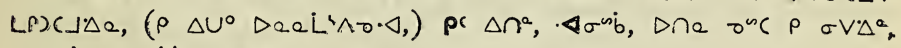

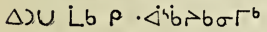

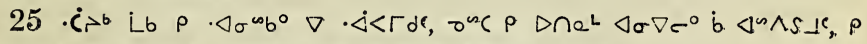

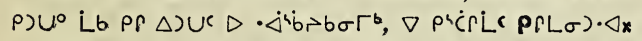

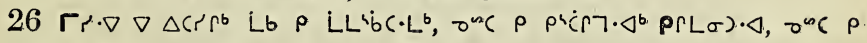

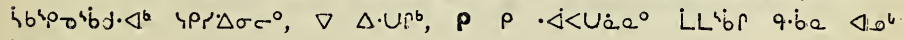
bs $b^{b} \times$

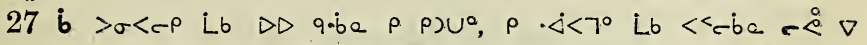

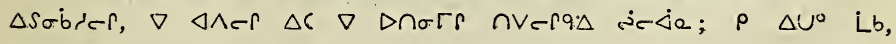
$a^{4} \wedge s^{a} x$

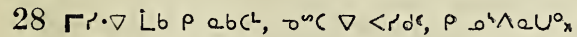

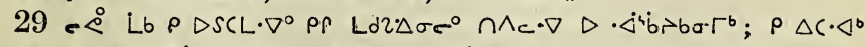

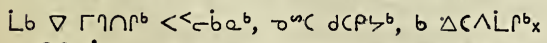

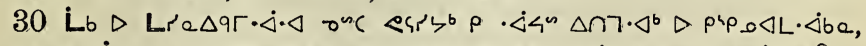

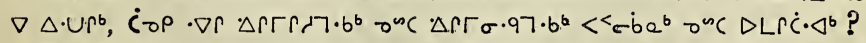

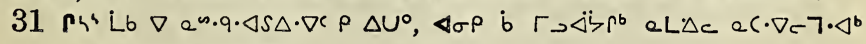
e(dce, Lb $\triangleleft \sigma \rho$ b $\left\langle\dot{d} \rho \rho^{b}{ }^{b}\right.$ 


\section{$\triangleright<\rightarrow$ pi jo 5,6 .}

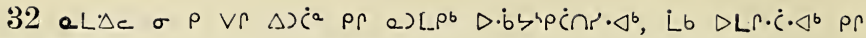
- gibneprox

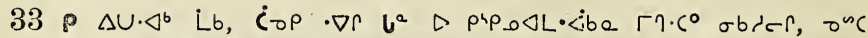

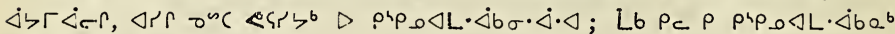

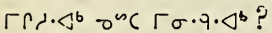

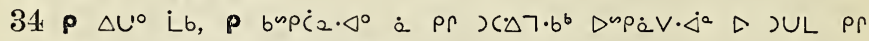

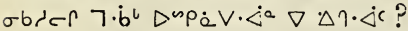

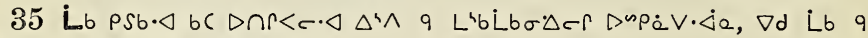
$\sigma b$ ic $\left.7 \cdot b^{6}\right\lrcorner \sigma \Delta \nabla \rho \operatorname{sib} P_{x}$

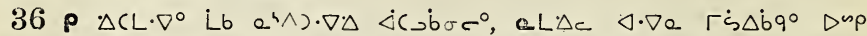

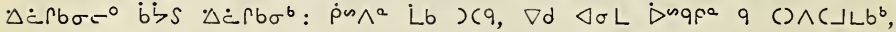

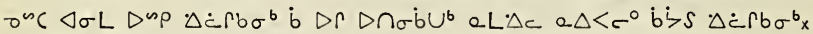

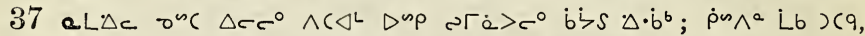

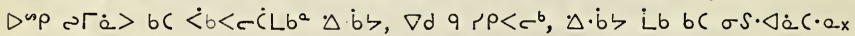

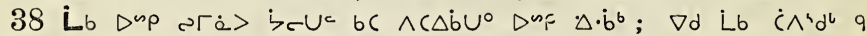
be. $\nabla e r \dot{r} \cup p_{x}$

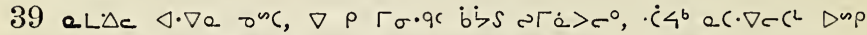

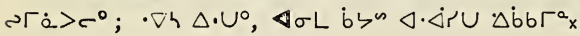

\section{Asprre $\triangle b^{\circ} \cdot 6$.}

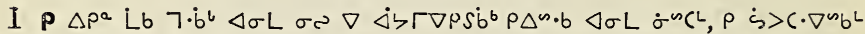

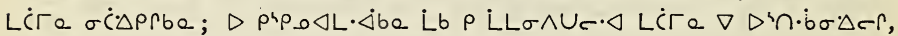

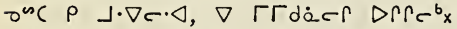

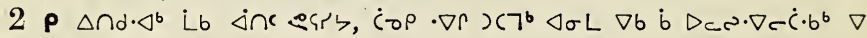
¿५r

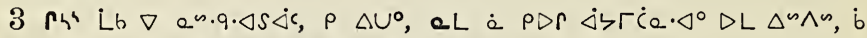

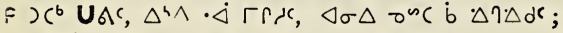

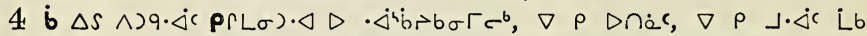

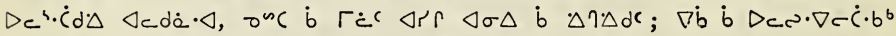

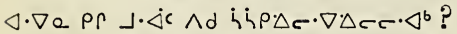

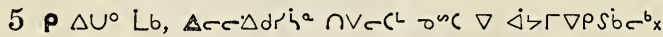

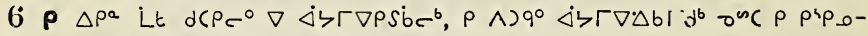
$\triangle \mathrm{L}^{\circ} ; \nabla d C$ Lb $\nabla \dot{C} C \Delta c c^{0} \nabla \sigma \wedge L b \sigma c^{b} \nabla \rho \rho \sigma^{b x}$

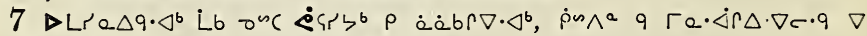

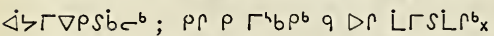

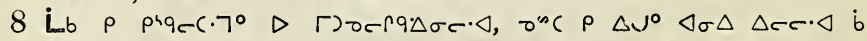

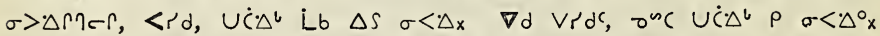

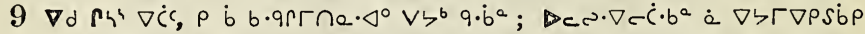

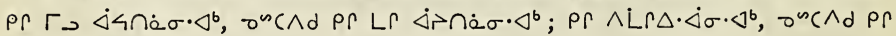
$\sigma \dot{<} \vec{r} \cdot \dot{\Delta} \sigma \cdot \Delta^{b}$ ?

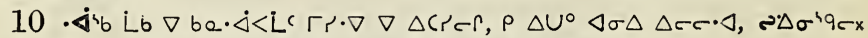
$\nabla d \nabla) c^{b}: \Delta \rho r$ Lb $\rho \Gamma e \cdot \Delta r \dot{C} \sigma \cdot \Delta \sigma \sigma^{\circ} \dot{C} \wedge^{h} d^{b} d\left(\rho c^{\circ} x\right.$ 


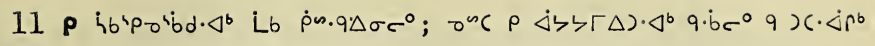
Phis

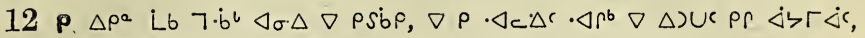
$\nabla 4 b \sigma \cap \wedge^{4} b c^{b} L b \rho \Delta \zeta \Gamma \nabla^{4}\left(\cdot \nabla^{\circ} \rho \Gamma L \sigma\right) \cdot \triangleleft x$

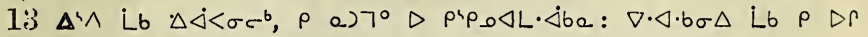

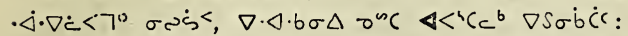

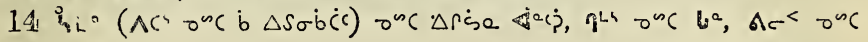
<'sect".

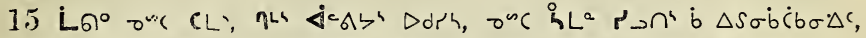

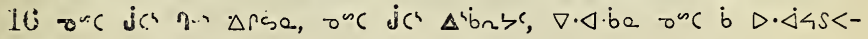
$p \cap \sigma \cdot \nabla C^{x}$

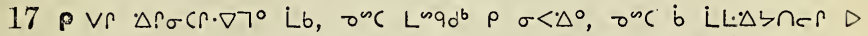

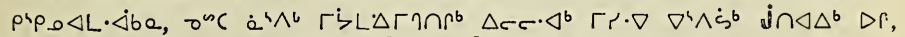

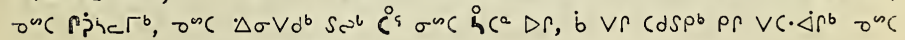

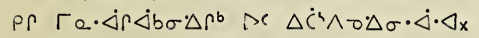

$18 \varangle \sigma \rho$ onc b $\triangleleft c \Gamma \Delta d^{b} \Delta-\wedge \Delta i \cdot b: \rho \Gamma a \cdot \Delta r \Delta \cdot \Delta^{b} L b x$

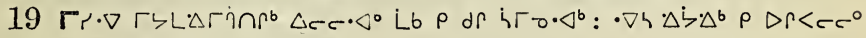

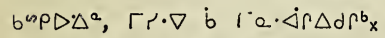

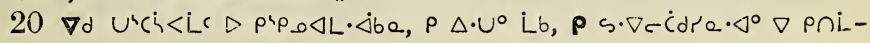

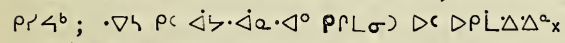

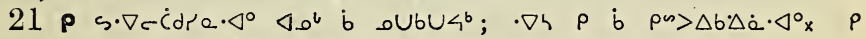

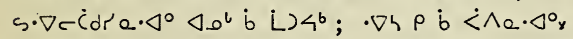

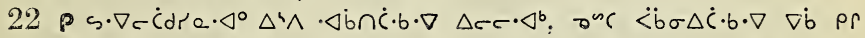

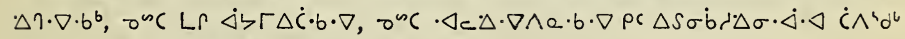
$\nabla \operatorname{Lic}\left(b, \Delta r c \Delta d r^{2}{ }^{\circ} \Delta r_{x}\right.$

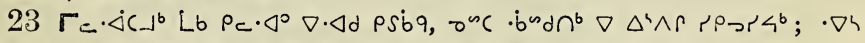

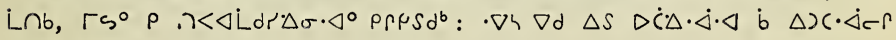
$\nabla \rho \uparrow \rho \cdot \Delta \Delta q \cdot \Delta x$

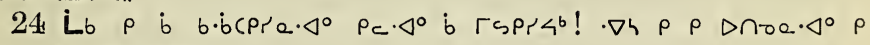
$\Gamma \cdot>c \cdot \Delta d^{\prime} \cdot \Delta \sigma \cdot \triangleleft 0^{\circ}$

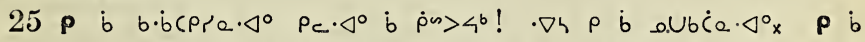

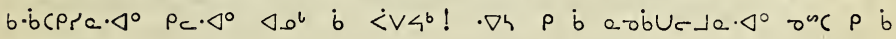
L) $a \cdot \Delta^{\circ} x$

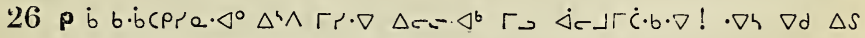

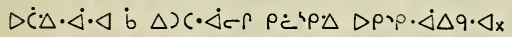

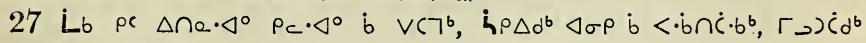
$\Delta \sigma \rho \dot{b} \cdot \Delta \dot{b} \cap \dot{C} b^{b}$,

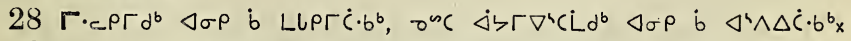

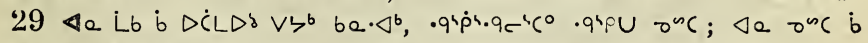

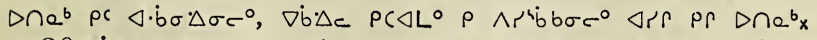

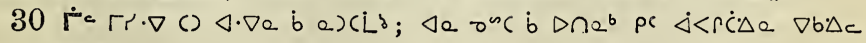
re e.SCLO ${ }^{\circ}$

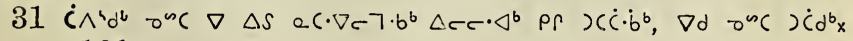
101 


\section{$\Delta<>$ ps jo 6.}

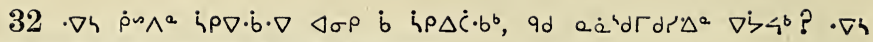

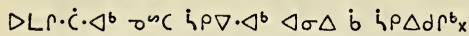

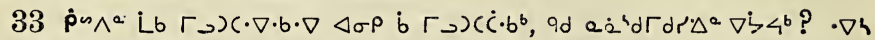

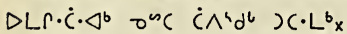

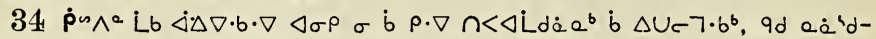

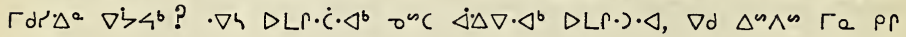
$r e d b^{b} x$

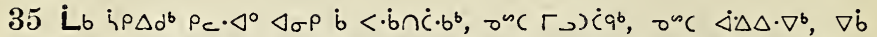

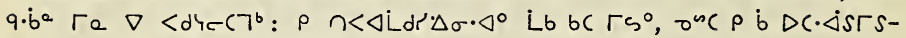

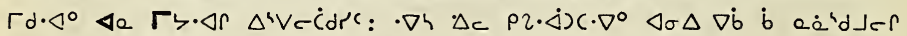

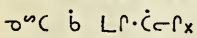

$36 \nabla \cdot \Delta d \Delta r s \cdot \nabla c r q^{b}, \dot{c} \wedge^{4} d^{l} d \dot{d} \dot{c} \Delta \cdot \Delta^{\circ} \nabla \Delta S s \cdot \nabla e r q c_{x}$

$37 \nabla \dot{b} \Delta c \cap \dot{<}^{4} d \sigma q^{b}, \nabla d \nabla \dot{b} q \cap \dot{<}^{b} d \sigma b \Delta 4^{b}: \nabla \dot{b} \Delta c \sigma s \cdot \Delta \dot{c} \mu r \cdot \nabla^{b}, \nabla d$ $\nabla \dot{b} q \sigma s \cdot \Delta \dot{a} \Gamma \Gamma b \Delta 4^{b}: \cdot \nabla V \in C L q^{b}, \nabla d \dot{L} b 9 \cdot \nabla V c C L b \Delta 4^{b} x$

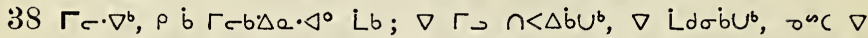

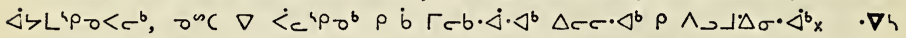

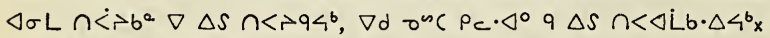

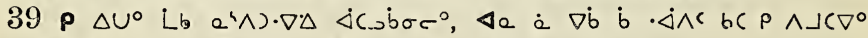
$\triangleleft \sigma \Delta \nabla \dot{b} \dot{b} \cdot \dot{\Delta \wedge} \wedge r$ ? $\left.a L \dot{a} \dot{C} \wedge^{4} d^{b} b C<\rho S \cdot a^{b}\right\rfloor \dot{a} \Delta \dot{<} \sigma^{b} x$

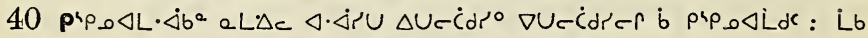

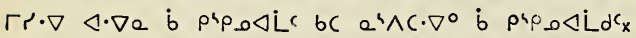

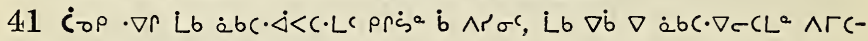

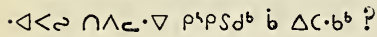

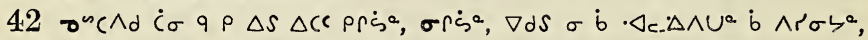

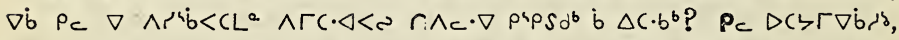

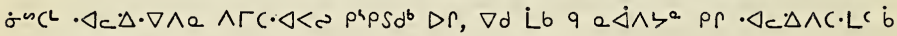
$\Lambda \sigma^{c} \operatorname{ses} e^{a} x$

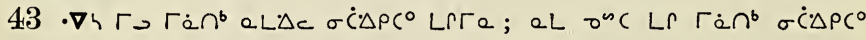
$\Gamma\lrcorner \Gamma \mathrm{ax}$

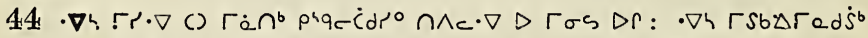

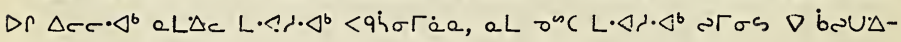

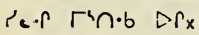

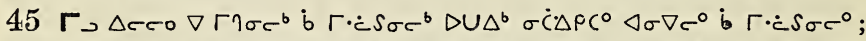

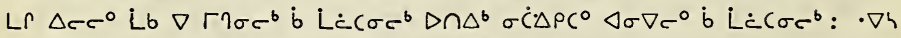

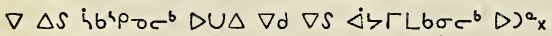

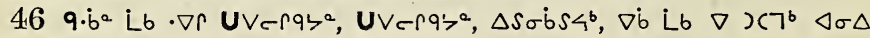

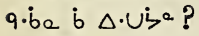

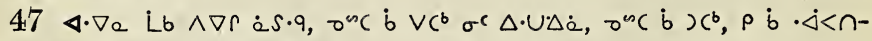

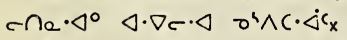

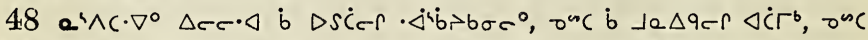

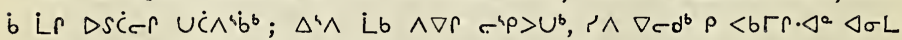

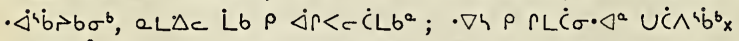

49 Ĺb $\triangleleft a b \dot{b} \vee c^{b}, \nabla \dot{b}$ Lb b $x^{b}, a^{4} \wedge c \cdot \nabla=\Delta c c \cdot \triangleleft \nabla \dot{b} \nabla \Delta c^{b} \dot{C}=r$ 102 


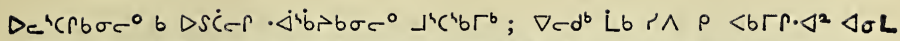

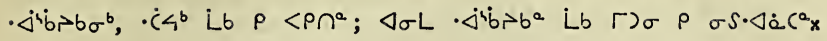

\section{A.pcre $\triangle b^{\circ} 7$.}

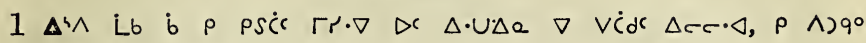
$b<50 b \Gamma^{b} x$

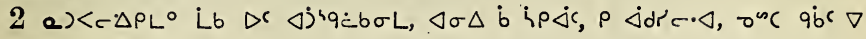
$\sigma \wedge-r_{x}$

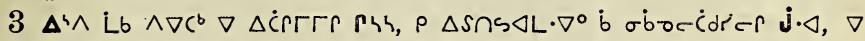

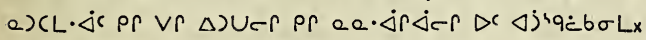

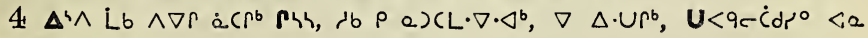
$\nabla 7 \odot^{\circ}$ q $\mathrm{XL} \cdot \triangleleft c$ :

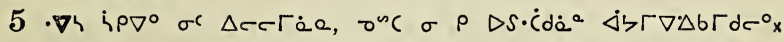

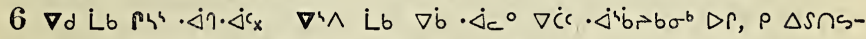

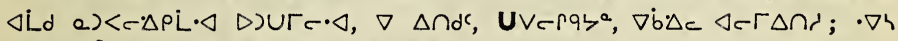

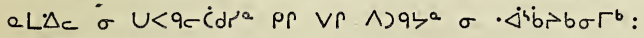

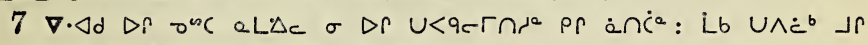
$\Delta \cdot U, \nabla d \dot{L} b$ oc $\varangle \dot{j} q^{\prime} \dot{c} b^{a} q \Gamma a \cdot \Delta r \Delta b \sigma \cdot \Delta c_{x}$

$8 \cdot \nabla h \sigma c \sigma^{n c} \sigma \cap V \in C\left(r \Delta b \Delta^{a}, \nabla \cap V-L \rho^{b} a\right)<c \Delta=c \cdot \Delta^{b} ; V \zeta^{b} L b \sigma^{c}$

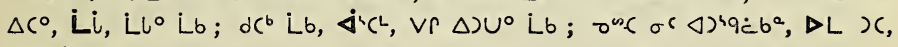
)L Lbx

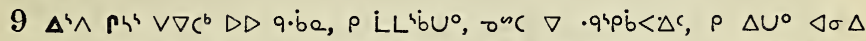

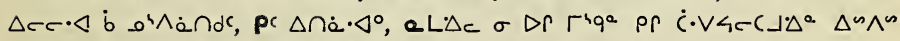
$\Delta L, \quad e L \Delta c, e L \Delta_{c} \Delta^{4} v \Delta c^{b} x$

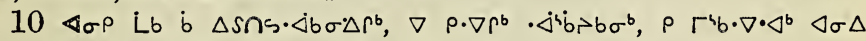

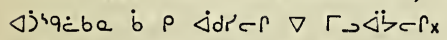

$11 \rho \Delta \rho^{a}$ Lb $\Delta \dot{i}<\sigma c^{b}, \rho \Delta U^{0} \Delta \dot{C} \cdot \Delta \sigma^{b} \partial \Delta^{a} \nabla \Delta S \sigma \dot{b} U^{b}: \rho \Delta 1 \Delta d \dot{L} b$

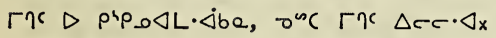

$12 \Delta^{b} \wedge$ Lb $\wedge \nabla \cdot s<c^{b} \Delta \dot{C} \Delta \sigma c^{\circ} \nabla \Delta s . \dot{b} U \Gamma \cdot \Delta \sigma c^{b}, L \cap b, \nabla \sigma \wedge c \Delta c c^{\circ} \rho$

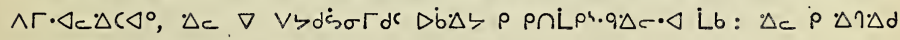
Lb $\Gamma \eta \Delta r c \cdot \Delta \Delta C \Delta \sigma^{b} D r_{x}$

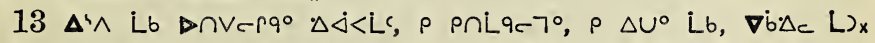

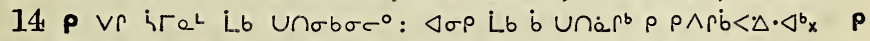

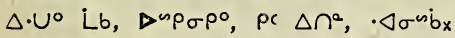

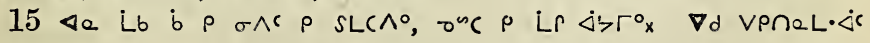
$\triangle \dot{b} \Delta r \cdot \Delta x$

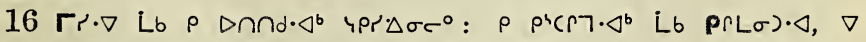

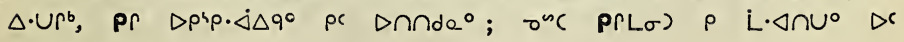
$\triangle r C L_{x}$

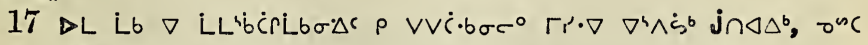

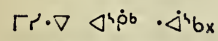

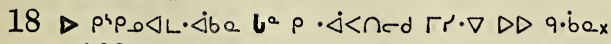
103 


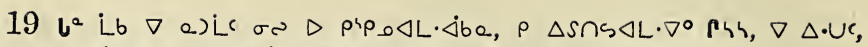

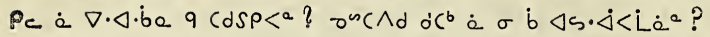

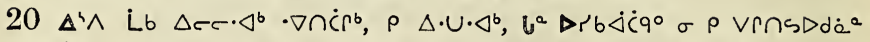

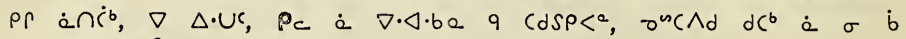
$\triangleleft s \cdot \Delta<\dot{L} \dot{a}{ }^{\circ}$ ?

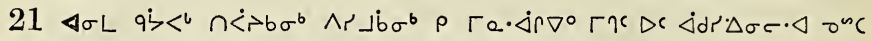

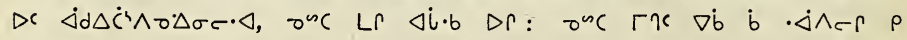
$\cdot \triangleleft \wedge \nabla^{\circ} x$

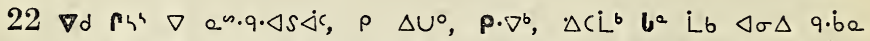

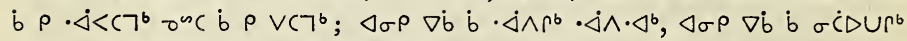

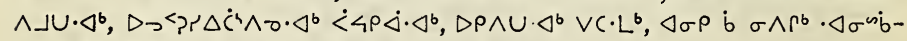

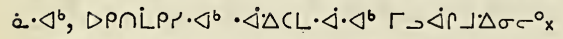

23 s. $\nabla c \dot{C} d d^{\circ}$ Lb, $\triangleleft \cdot \nabla e \nabla \dot{b} q$ a $4 C \cdot \nabla e \Gamma \cdot q_{x}$

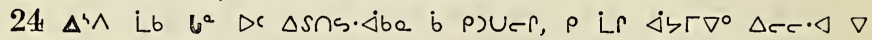

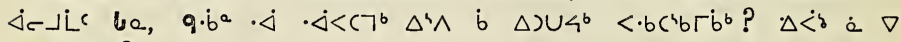
$\cdot \nabla \cdot \nabla \dot{<} \mathbf{C}^{b}$ ?

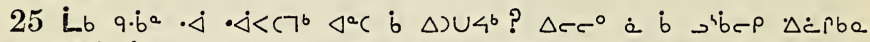

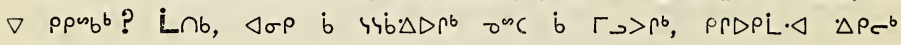
$\Delta \dot{C} \cdot \Delta^{b} \mathrm{x}$

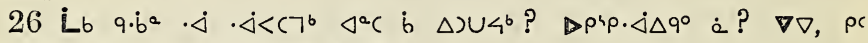

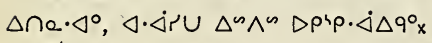

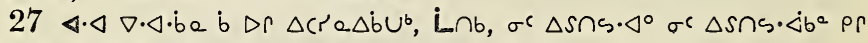

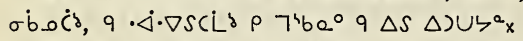

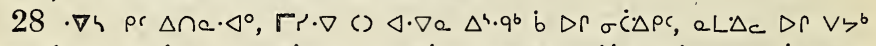

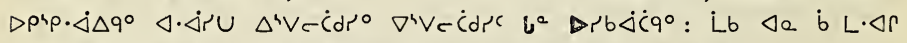

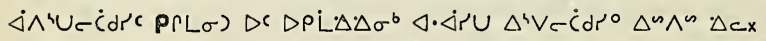

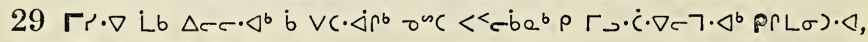

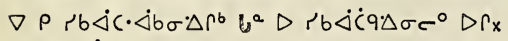

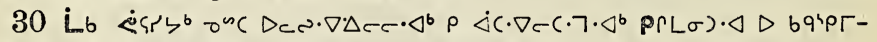
$\cdot \nabla \Delta \sigma \sigma^{\circ} \dot{b} \Delta s$ bq $p \Gamma d c r, \nabla \dot{b} \nabla \Delta r<b \Delta \dot{C} d r^{b} x$

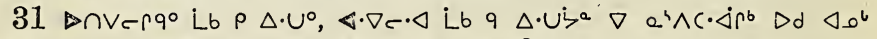

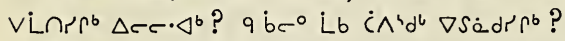

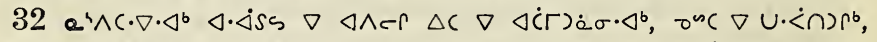

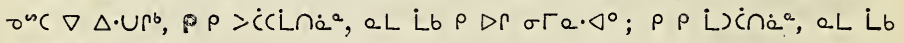
$b \quad P C L)_{0} \cdot \Delta^{\circ} x$

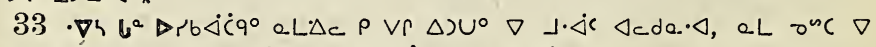

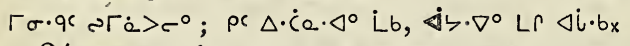

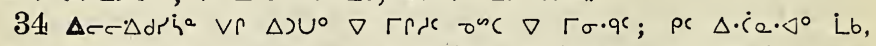

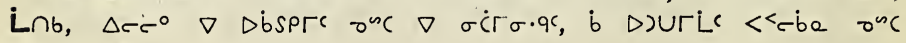
$\triangle L \Gamma \cdot C \cdot \triangleleft !$

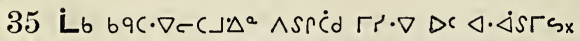

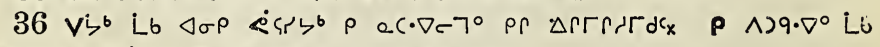

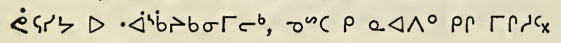

104 
37 Lnb, Lb, $\Delta^{4} \cdot q^{\circ} \Delta \dot{c} \Delta \sigma^{b} \Delta \Gamma, \dot{b} \quad L \Gamma \cdot \dot{c}^{c}, \Delta^{4} \wedge q^{4} q=c^{b} \nabla \Delta \wedge c \Gamma$ p

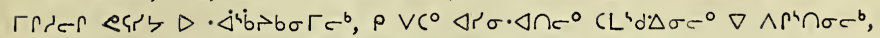

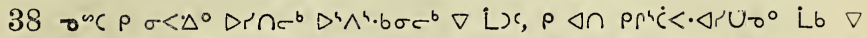

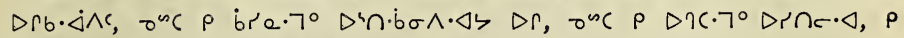
$C^{4} d a \cdot 7^{\circ}$ Lb CL'd $\Delta \sigma c^{\circ} D \Gamma_{x}$

$39 \Delta^{4} \wedge$ Lb $\triangleleft e$ es b

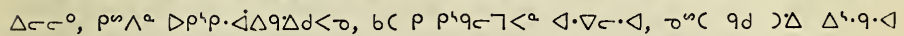
$\triangle D \dot{b}$ i $\Gamma \sigma \sigma^{\circ} ; \cdot \nabla h L \Gamma \cdot C^{\circ} x$

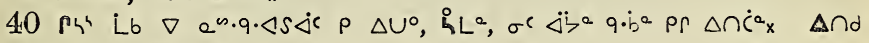
Lb, q $p_{0} \Delta \operatorname{Liq}^{\circ} \zeta^{a}, \Delta \cdot U_{x}$

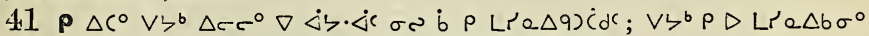

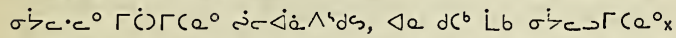

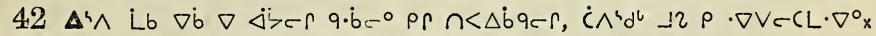
$\cdot \Delta C$ L $\Delta^{a}$ Lb,$\Delta \cdot \nabla c \cdot \triangleleft \nabla \sigma S e r \triangleleft \cdot \Delta i r U q$ h $\rho \Delta d^{d c}$ ?

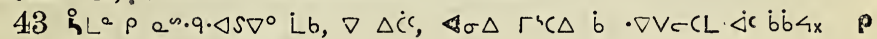

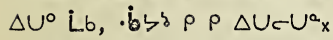

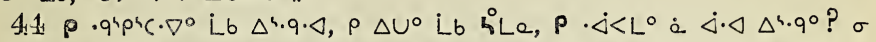

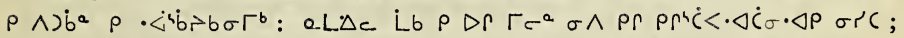

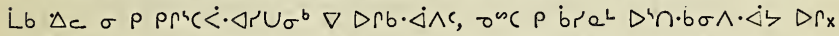

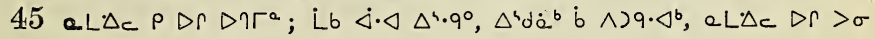
$D \cap C L$ or $C x$

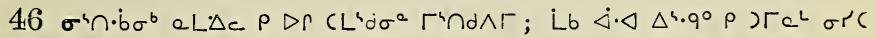
CL'd $\Delta \sigma \sigma^{\circ} x$

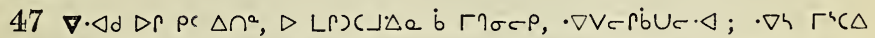

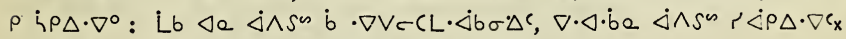

$48 \rho \Delta U^{\circ}$ Lb, $P \cdot \nabla V-\left(L b \Delta^{a} \rho\right.$ Lr) (\lrcorner$\Delta e_{x}$

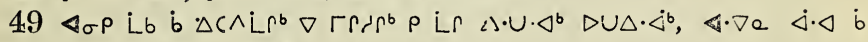
- $\nabla$ VeCLac LS)

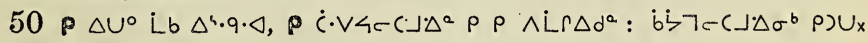

\section{Ápcre $\triangle b^{a} 8$.}

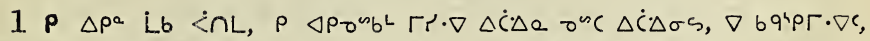

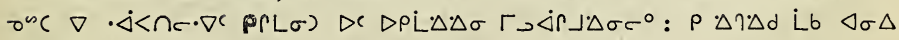
arsis $<_{x}$

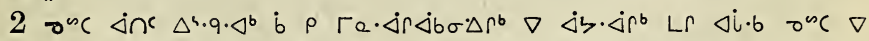

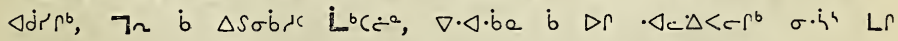
$\Delta i \cdot b^{b}$,

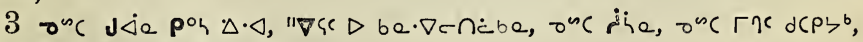

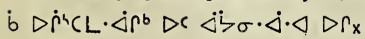

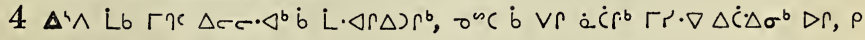

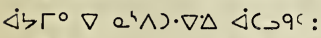

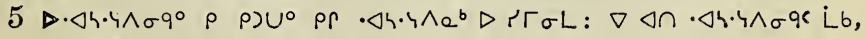
105 


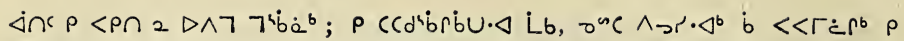
$\rho \dot{C} \cdot \triangleleft^{b} x$

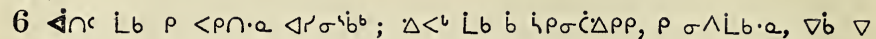
$\Gamma-L \cdot \Delta \rho_{x}$

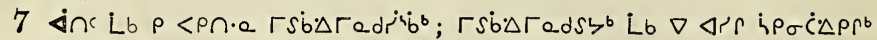
$\rho P \wedge^{\mathrm{C}} \mathrm{b} \cdot L^{\mathrm{b}} \mathrm{x}$

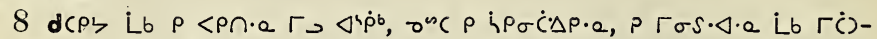

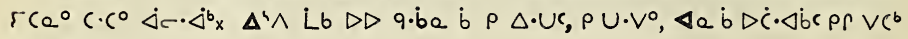
$\nabla d S$ bC $V C L^{L}$

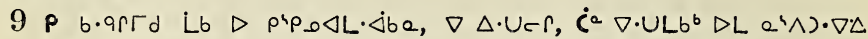
$\Delta c \rightarrow b^{\circ} ?$

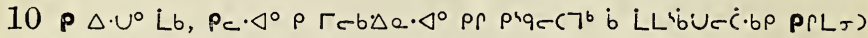

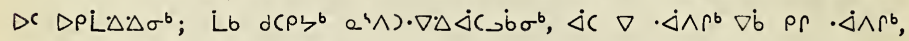
$\sigma^{n c} \nabla V\left(\rho^{b} \nabla b \operatorname{se} \sigma r\right)\left(\rho^{b} x_{x}\right.$

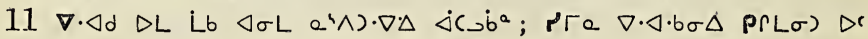
$\dot{\Delta b} \Gamma^{a} \mathrm{x}$

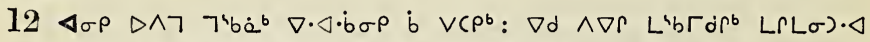

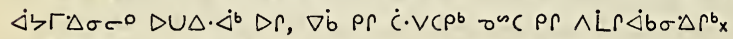

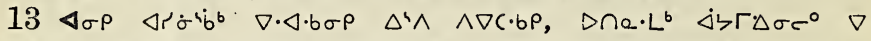

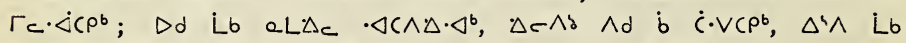

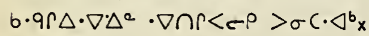

$14 \varangle \sigma L$ Lb $\Gamma S \dot{b} \Delta \Gamma$ adribb b $<\rho \cap b, \nabla \cdot \Delta \cdot b \sigma \rho, \Delta^{4} \wedge \wedge \nabla\left(\rho^{b}, \rho\right) \cup \cdot \triangleleft^{b}$,

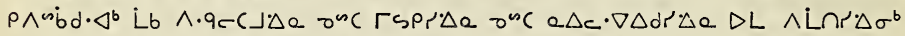
b $\Delta\left(\cdot b P, \text { QL. } \Delta_{c} \text { Lb } \Gamma\right)_{\sigma}$ PST $\sigma S \Delta \cdot \triangleleft^{b} \mathrm{x}$

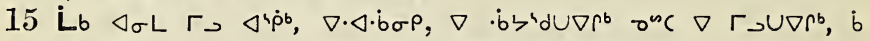
$V C \cdot b \Delta \Delta \zeta \Gamma \cdot \Delta \sigma \sigma^{0}, b e \cdot \nabla c c \cdot L^{b}, \sigma^{n c} C S \Lambda c \cdot \nabla r \cdot \Delta \sigma^{b} \Gamma \sigma S \Delta \cdot \Delta^{b} x$

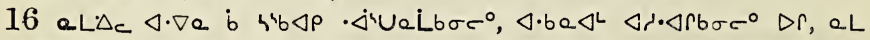

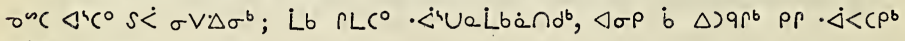
$\cdot \dot{\triangleleft} \cup \Delta \sigma \sigma^{\circ} \mathrm{x}$

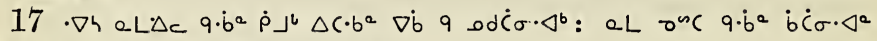

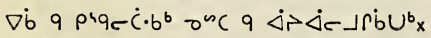

$18 \nabla \cdot \varangle d \Delta r \dot{b} \cdot b \Gamma r^{b} \nabla \Delta S \vee\left(7^{b}: \cdot \nabla h() \triangleleft \cdot \nabla a b \Delta \ddot{b c}, b c \Gamma_{c o} ;()\right.$

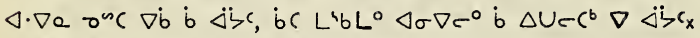

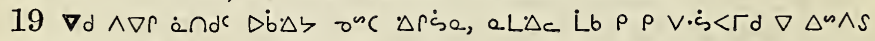
$\left\langle\cap \dot{a}_{b}\right) \dot{\alpha} \sigma \cdot \triangleleft \sigma c^{b} x$

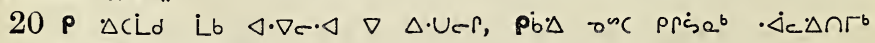

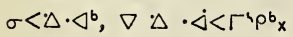

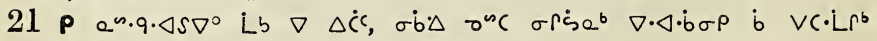

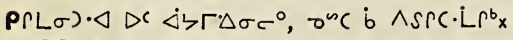

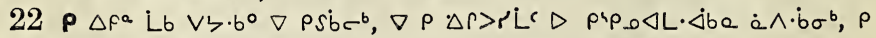
$\Delta U^{\circ} L b, \nabla d S \Delta c^{3} \cdot \Delta \Delta c^{\circ} . q^{4} b b^{b} i b \Delta b \sigma^{b x} \quad \nabla d \nabla \cap \Delta S r 7^{b x}$

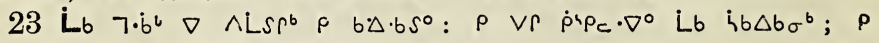

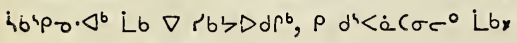




\section{$D<>\rho^{4}$; 8.}

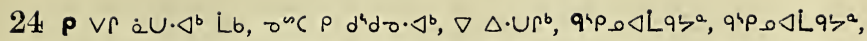

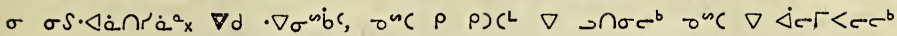

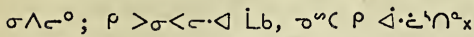

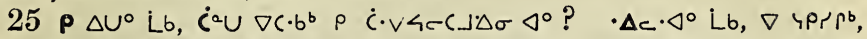

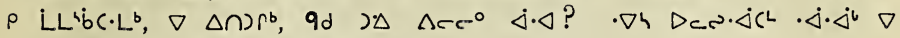
$\rightarrow \cap \sigma^{b} \sigma^{n C} \sigma \wedge c^{\circ}$, e. $\Delta \dot{C} d$ L Lbx

$26 \rho \Gamma s \dot{b} \cdot \Delta^{b} \dot{L} b \dot{b} c \sim e \Delta c \Delta^{4} \rho c^{b}, n \Lambda^{4} d^{b} b_{c}-c^{b} b \Delta c \cdot b \sigma c^{b} x$

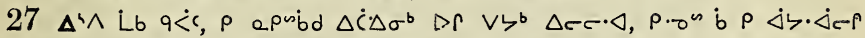

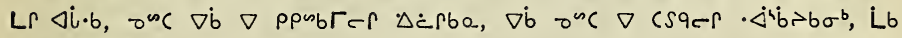
$r \dot{<r} \vec{b} r d^{i} b^{b} x$

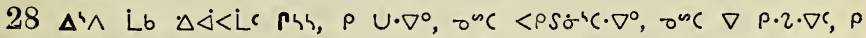

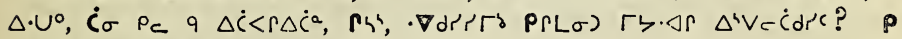

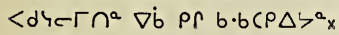

$29\left(\cdot \nabla \zeta \rho \quad \Delta C \cdot \Delta U^{\circ} \Delta r \wedge \Delta i \cdot b \quad \Delta \sigma \Delta \Delta=c \cdot \Delta \rho \rho \Delta r \cdot \Delta c \Delta r r_{x} \quad \cdot \nabla h\right.$

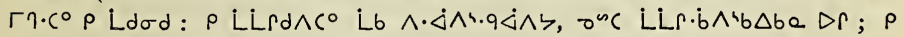

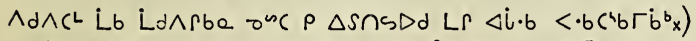

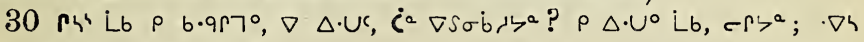

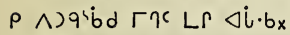

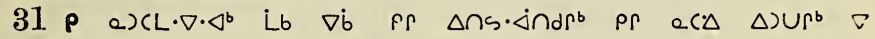
$n \Gamma c^{b} x$

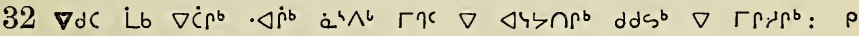
a) $C L \cdot \nabla \cdot \Delta^{b} L b$ pr $\left.\Delta-\dot{a} d p^{b} \Delta \sigma \Delta \rho \rho \wedge\right) q^{a} b \cdot \Delta \rho^{b} x_{x} p \Delta r a \cdot \nabla^{0} L b_{x}$

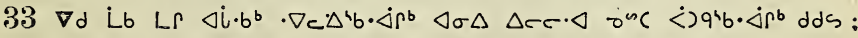

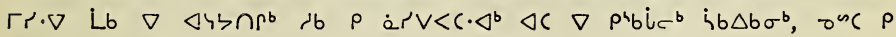
$\rho>C \dot{L} L<\cdot \nabla \cdot \triangleleft b x$

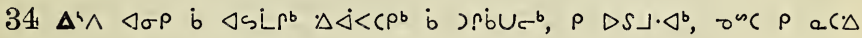
$\Delta C \cdot L^{b} \Delta \dot{C} \Delta \sigma^{b}, \sigma^{n C} \Delta^{4} \dot{p} b \cdot \Delta 4 b x$

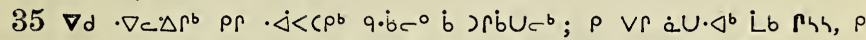

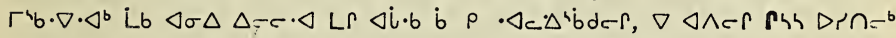

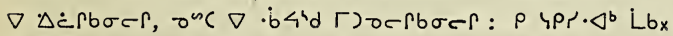

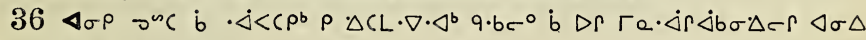
b $\wedge \Gamma^{4} \dot{b} d e r$ LS $\Delta i \cdot b x$

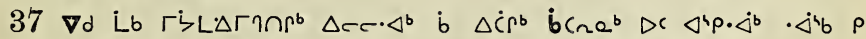

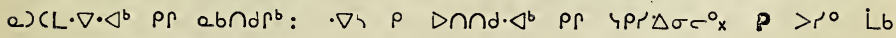
$\dot{a} \wedge \cdot \dot{b} \sigma^{b}, \sigma^{n C} C$ Г⿸ $P$ P. $\nabla D_{x}$

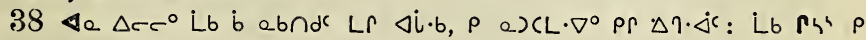
prns. $\nabla^{\circ}, \nabla \Delta \cdot u_{r_{x}}$

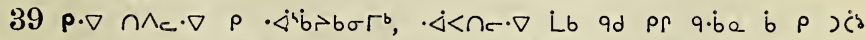

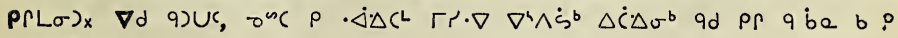

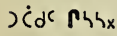

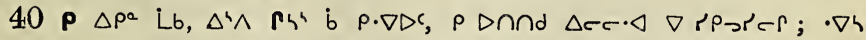
$\rho \vee \Delta d \Gamma r \cdot \nabla \nabla \Delta C r-r_{x}$

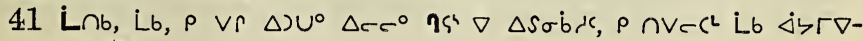
107 


$$
D<\rightarrow \rho^{4} \dot{j}^{b} 8,9 \text {. }
$$

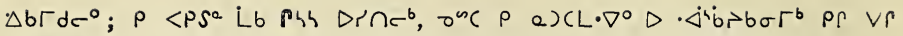
A) $q \subset r:$

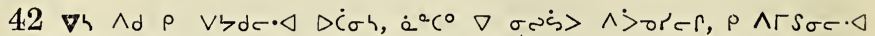

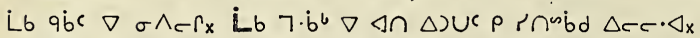

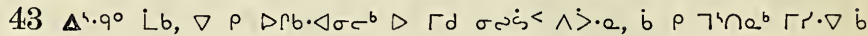

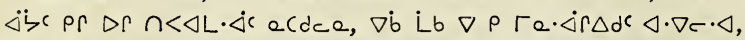

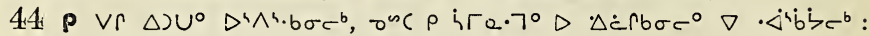
$\cdot \dot{C}^{b}{ }^{b} L b \nabla \nabla \Gamma b \cdot \triangleleft \sigma c^{b} \nabla \Gamma \dot{d} \rho \rho \wedge \Gamma b \cdot \triangleleft \sigma c^{\circ}{ }^{\circ}$

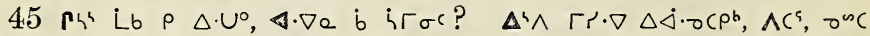

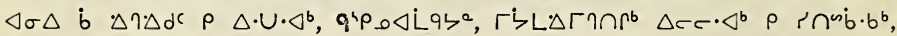

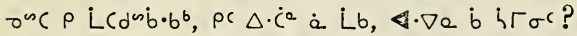

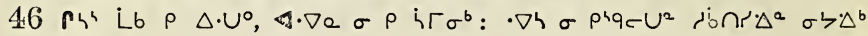
$\nabla \rho D r<c^{b} x$

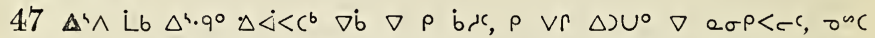

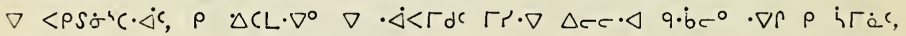

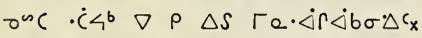

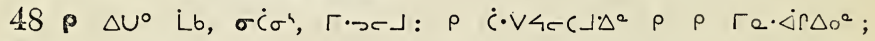

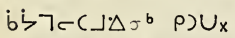

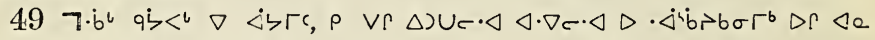

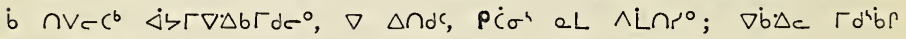
$\triangle$ PIP_O $\triangle L^{\circ} q^{\circ}$

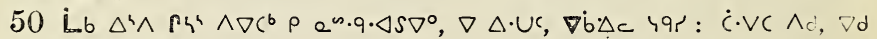
9. $\Gamma \mathrm{e} \cdot \dot{<r<b \sigma \sigma \Delta x}$

$\left.\left.51 \Delta^{4} \wedge L_{b} \dot{<}\right) q c \cdot \dot{\Delta}^{4} b>b \sigma^{b}, a L \Delta c \rho \Delta r a \cdot \nabla^{\circ} \triangleleft \cdot \nabla c \cdot \triangleleft \rho p \wedge\right) q c \uparrow \wedge d$

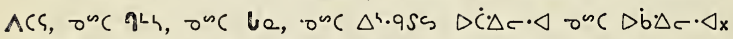

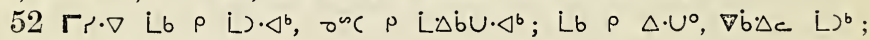
$Q L \Delta c \sigma \wedge^{\circ}, L_{b} \sigma<^{\circ} x$

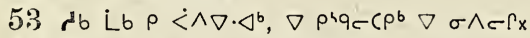

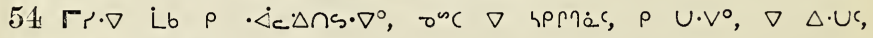
Dspops.qsu, $\triangleleft \sigma^{n i b x}$

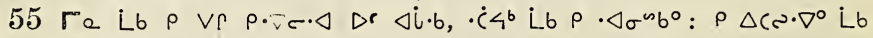
pr $\angle S T \Gamma \Gamma_{x}$

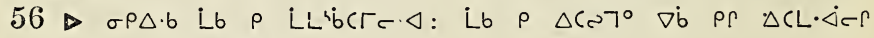
\lrcorner$\left.\nabla c \cdot \triangleleft q \dot{b} c^{\circ} \dot{b}\right) r \dot{b} \cup c^{-b} x$

Ápcra $\triangle b^{a} 9$.

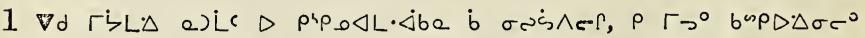

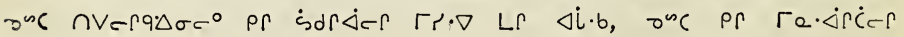
jdr. $\Delta e_{x}$

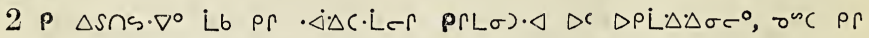

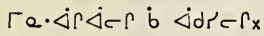

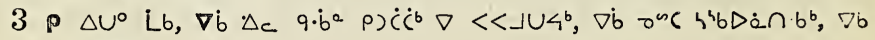
108 


\section{$\Delta<p^{4}$; 9 .}

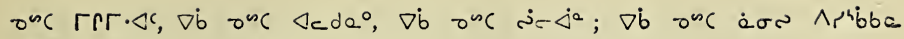
$\ddot{\Delta i b x}$

$4 \wedge d \Delta \sigma L \cdot \Delta 4 b>b^{a}$ q $\triangle S \wedge C q \cdot \nabla \cdot q, \quad \nabla d C \quad C S q^{b}$, onc $\nabla d C \quad D r$ p) $U^{b} x$

$5 \varangle \cdot \nabla a$ Lb $\nabla \dot{b} q \wedge) \dot{b} \Delta \dot{C} \cdot q, \Delta^{4} \wedge \cdot \Delta c \Delta 4 \cdot q \quad \Delta \sigma L \Delta \dot{C} \Delta \sigma^{b} \quad \nabla r, \Delta>r b$

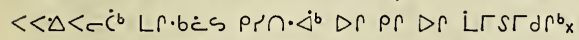

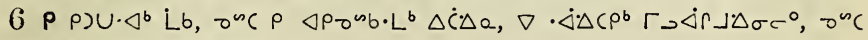
$\Gamma \dot{r} \cdot \nabla \Delta s \nabla \Gamma a \cdot \dot{\Delta} \Delta \cdot \nabla r^{b} x$

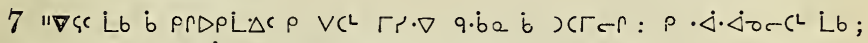

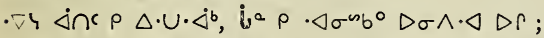

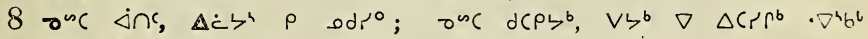

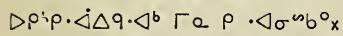

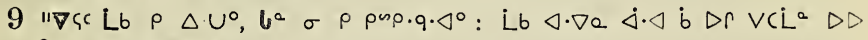
q.be? $\rho \Delta \cdot \dot{i}<7^{\circ}$ Lbx

$10 \Delta^{h} C^{b} c^{b} L b, \Delta^{h} \wedge$ b $\rho \cdot \nabla \rho^{b}, \rho \Delta C L \cdot \nabla \cdot \Delta^{b} \Gamma r \cdot \nabla q \cdot b a$ b $\left.\rho\right)\left(\rho^{b} x \quad \rho\right.$

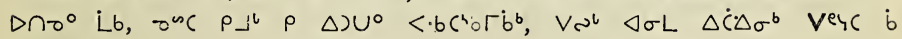
$\triangle S \sigma \dot{b} U^{b} x$

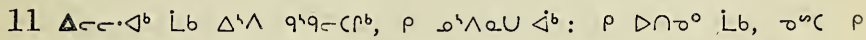

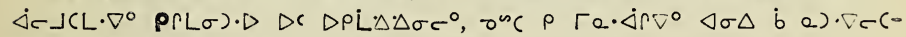
$\operatorname{rer} \operatorname{pr} \Gamma_{a} \cdot \dot{\Delta r} \dot{\Delta b \sigma} \Delta r r_{x}$

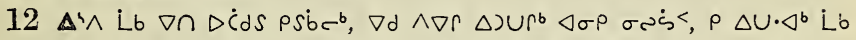

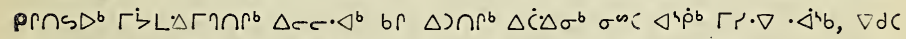

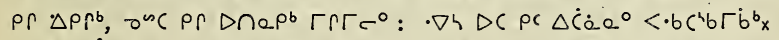

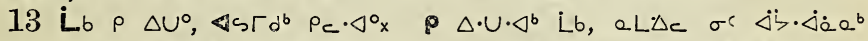

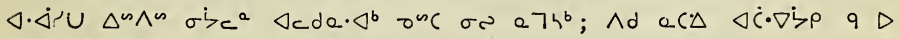

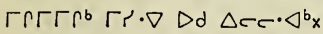

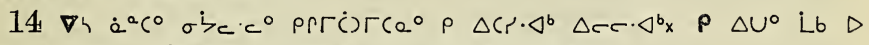

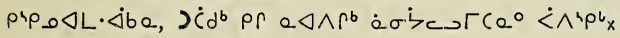

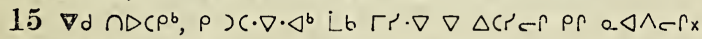

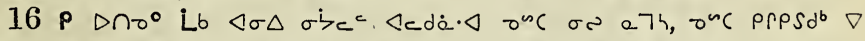

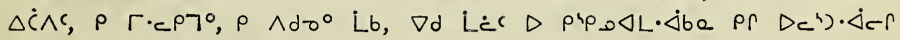
riL $\Delta \Gamma \cap \cap-r \Delta c c \cdot \Delta x$

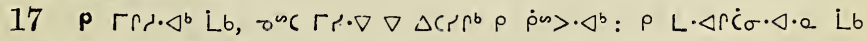
$\Delta^{4} \cdot b r b e$ b $\Delta^{4} \cdot b^{4} c \rho b, \sigma{ }^{2} \dot{s}<\cdot \Delta C \wedge \cdot \Delta C x$

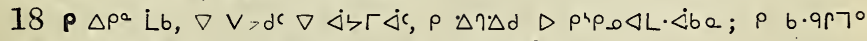
Lb, $\nabla \Delta \cdot U<, \varangle \cdot \nabla e \nabla \Delta \cdot U r^{b} \Delta c c \cdot \Delta^{b} \nabla \Delta \cdot \Delta \dot{\Delta} \cdot ?$

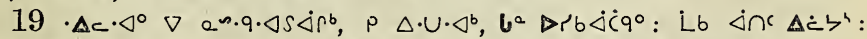

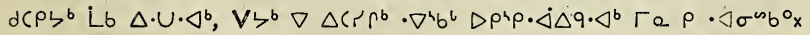

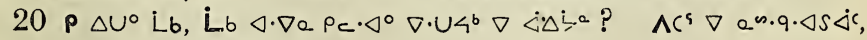
$P \triangle \cdot U^{\circ}, P(L \sigma) D X \cap L x$

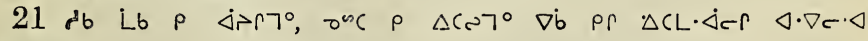
$\triangleleft \sigma \nabla \sigma^{\circ} x$

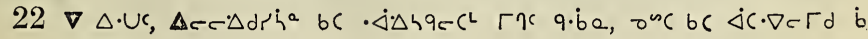
109 


\section{$D<\rightarrow$ ps jo 9 .}

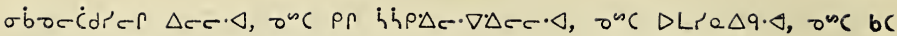
$\sigma<\dot{\Delta}^{\circ}, \sigma^{n}\left(b c \cdot \triangleleft \sigma^{n} \dot{b} a^{\circ} \sigma^{n}\right) \rho s \dot{b}-9 x$

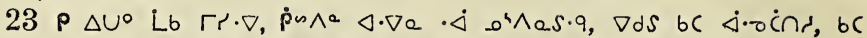

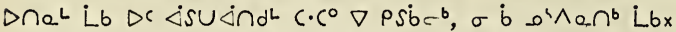

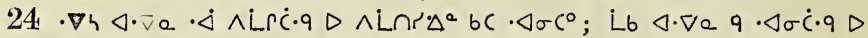
$\wedge i \cap r \cdot \Delta^{a} \sigma c \Delta r, \nabla \cdot \triangleleft \cdot b e q \wedge i r c c_{x}$

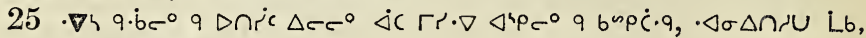
$\sigma^{n} \mathrm{C} \wedge d \cdot \nabla \wedge \dot{Q} b \sigma \cdot \Delta U_{x}$

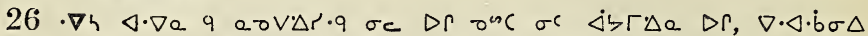

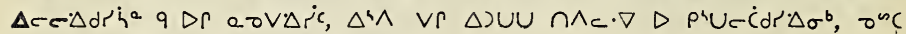

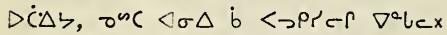

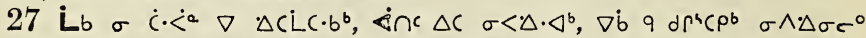

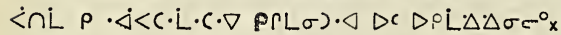

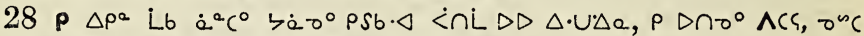

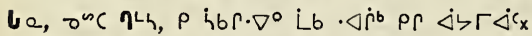

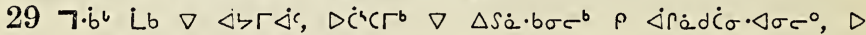

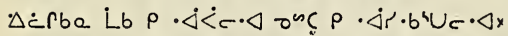

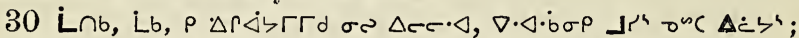

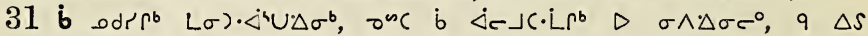
$\sigma \wedge e r$ pjhe $\Gamma^{b} x$

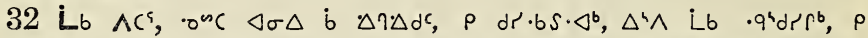

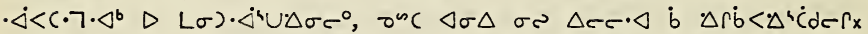

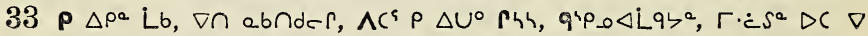

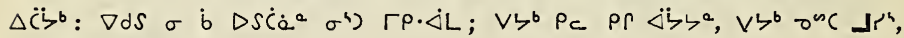

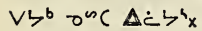

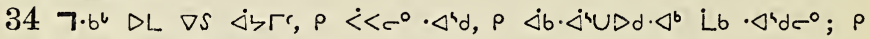
$4 \varphi^{\prime} \cdot \Delta^{b}$ Lb $\nabla \triangleleft \cap \triangle \dot{C} \Gamma^{b} \cdot \Delta^{b} d^{b} x$

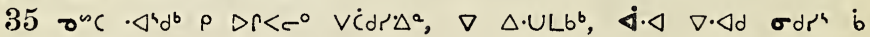
ip $\left.p \Delta^{b} ; a\right) \dot{C} \Delta^{b} x$

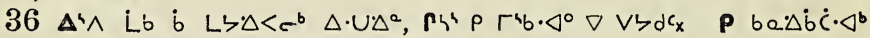

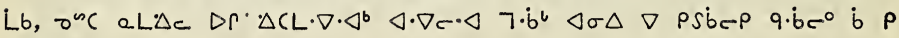
$\cdot \dot{j}<c \rho^{b} \mathrm{x}$

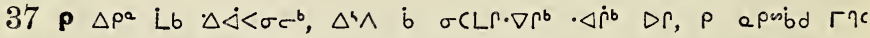
$\triangle c c \cdot \triangleleft x$

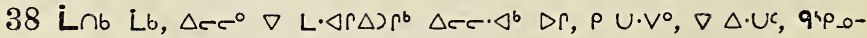

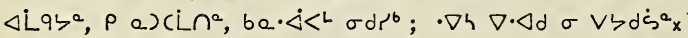

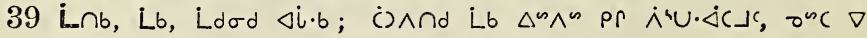
$\triangle \wedge C D d^{c} \Delta \dot{b} \cdot \dot{j}^{i} a b \cap d x$

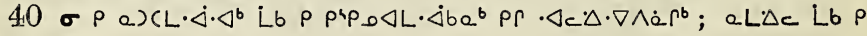
b) $p \dot{C} \cdot \triangleleft^{b} x$

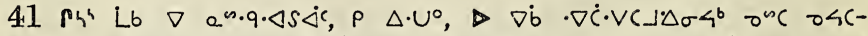

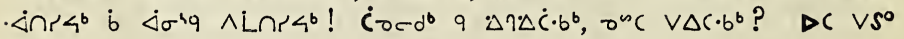
Pdres $x$ 


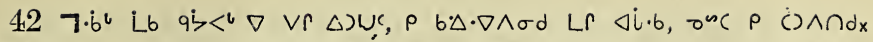

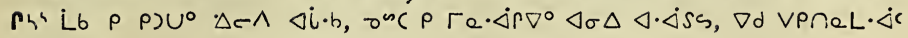
$\triangle \dot{C} \Delta r \cdot \Delta x$

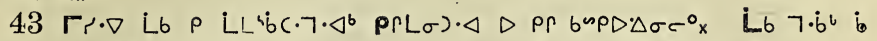

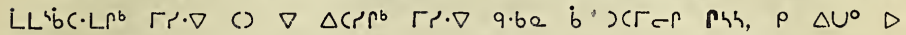
pip.o $\triangle L \cdot \Delta$. $\Delta b e^{x}$

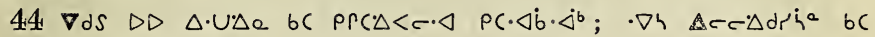
$<P \cap e^{\circ} \triangle r c \cdot \triangleleft \Delta R r c^{b x}$

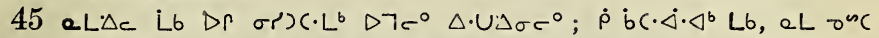

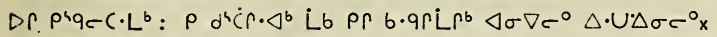

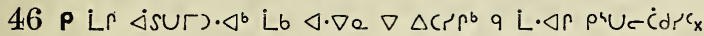

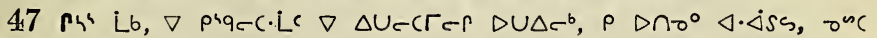
$\triangle \wedge \neg P \triangleleft \supset^{\circ}$,

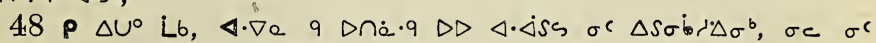

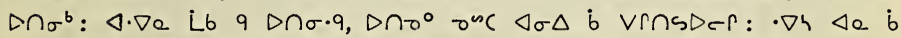

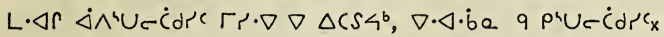

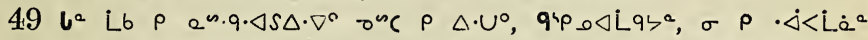

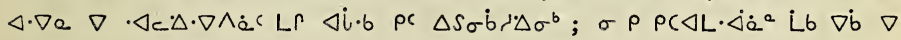

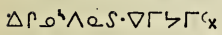

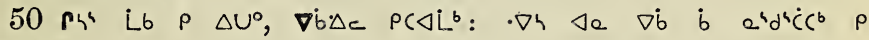
$\Delta r \Delta d e^{\circ} \mathrm{x}$

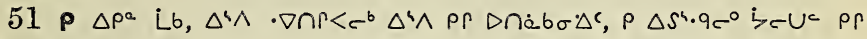
$\triangle \mathcal{A}$ rihac $\Gamma^{\mathrm{b} x}$

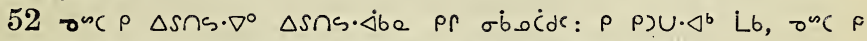

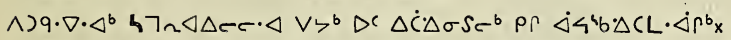

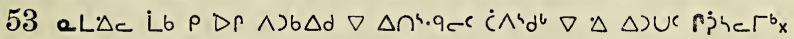

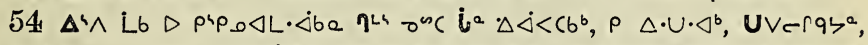

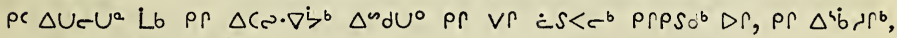

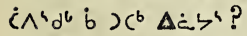

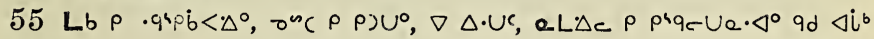
$\cdot \nabla\left(i, d \zeta^{b} x\right.$

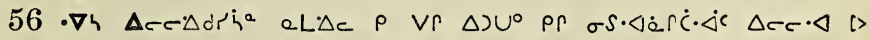

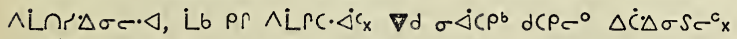

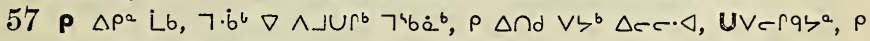

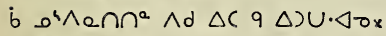

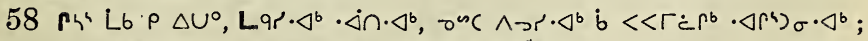

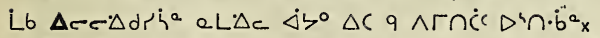

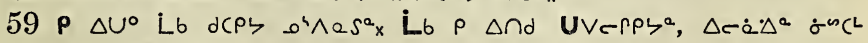

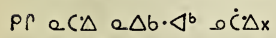

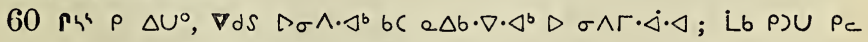

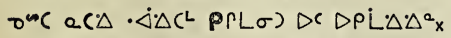

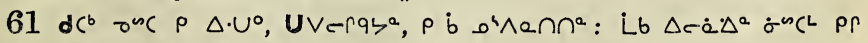

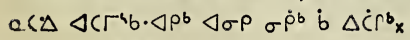

111 


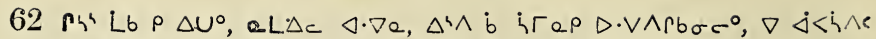

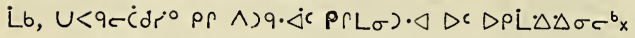

\section{Arpcra $\Delta b^{\circ} 10$.}

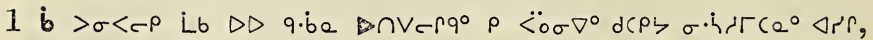

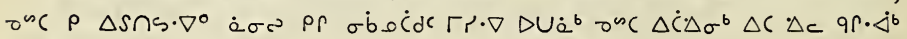
$\cdot \dot{\Delta} \Delta U \mathrm{Cx}_{\mathrm{x}}$

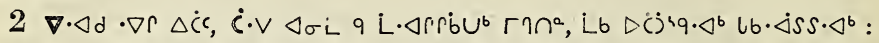

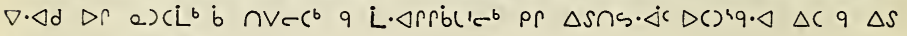
$L \cdot \Delta r i b \sigma \cdot \Delta \sigma c^{0} x$

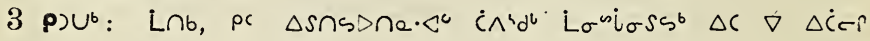
L $\triangle b e_{x}$

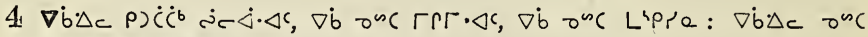
$\triangleleft \cdot \nabla e \triangleleft \dot{C} \Gamma^{i} b^{b} e^{b} b \cdot \nabla x$

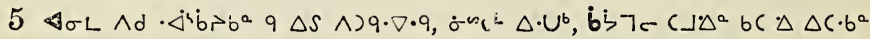
$D C \cdot \dot{\Delta l} b \dot{b} b \sigma^{b} x$

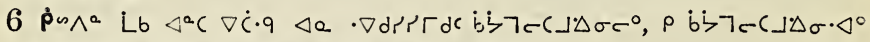
$\left.\nabla d c q \Delta c \cdot b^{b}: \dot{p} u \wedge^{a} \nabla \dot{b}, \Gamma a \rho b \dot{b} \rho \cdot \nabla\right) \dot{c} d a \cdot \Delta^{\circ} x$

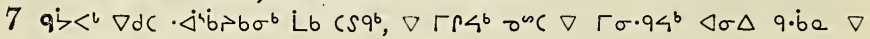

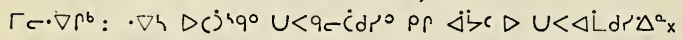

8 Ad $\left.\left.\Delta \sigma L \Delta \dot{C} \cdot \Delta^{a} q \Delta S \wedge\right) q \cdot \nabla \cdot q, \nu^{\omega c} \wedge\right) \dot{b} \Delta \dot{c} \cdot b \cdot \nabla, \Gamma r^{b} \Delta \sigma \Delta q \cdot \dot{b} a q$ $D_{c}^{4} \cdot \dot{C} \dot{C} \cdot b^{6} ;$

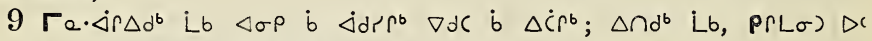

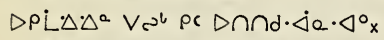

$10 \wedge d \Delta \sigma L \Delta \dot{C} \Delta^{a}$ Lb $\left.q \Delta S \wedge\right) q \cdot \nabla \cdot q, \nabla \dot{b}$ Lb $\left.\wedge\right) \dot{b} \Delta \dot{C} \cdot b \cdot \nabla, \cdot \Delta c \cdot \dot{\Delta}^{b} \nabla$ $\left.\Delta U<^{b} \wedge\right\lrcorner U^{b} b a^{b} \Delta^{a} C$ b $\Delta C \cdot b P, \Delta \cdot U^{b} L b$,

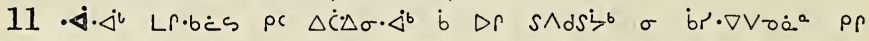

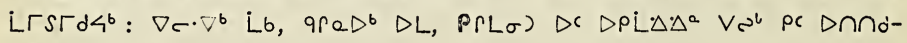
$\cdot \dot{\triangleleft} e \cdot \Delta^{\circ} x$

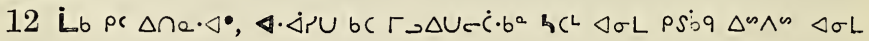
$\Delta \dot{c} \cdot \Delta^{a} x$

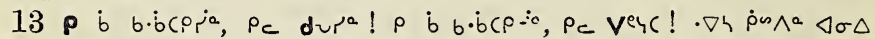

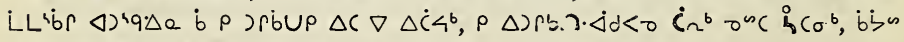

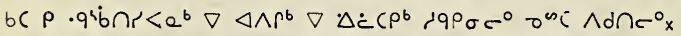

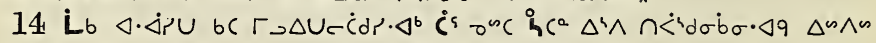
$\rho_{c} \cdot \triangleleft^{\circ} x$

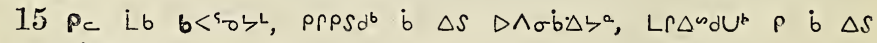
$\sigma \rho \cdot \nabla \wedge \sigma \dot{b} \cdot \Delta a x$

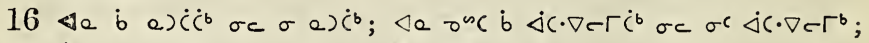

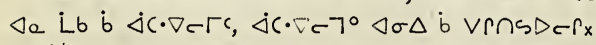

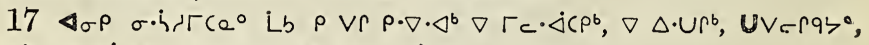

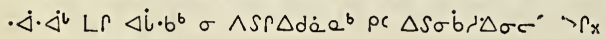




\section{$\triangleright<>\rho^{4}$ ib 10 .}

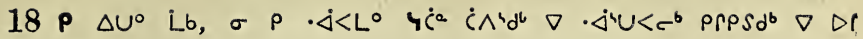
$<P S^{b} \times$

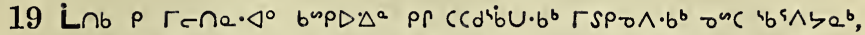

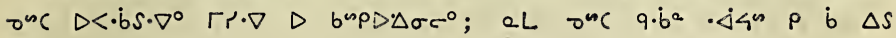
$\dot{\Delta d} \Delta d a \cdot \triangleleft^{\circ} x$

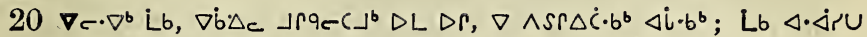

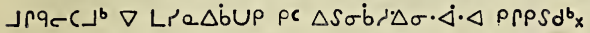

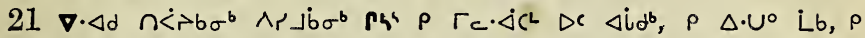

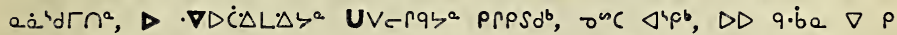

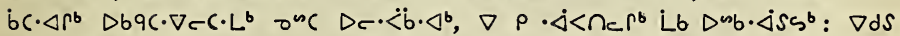
$\nabla d q \Delta \rho b, \rho \dot{C} ; \cdot \nabla h \nabla \cdot \Delta d \nabla S \Gamma, \Delta U-C L^{a} x$

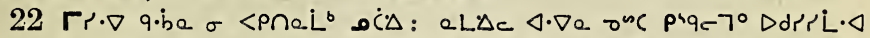

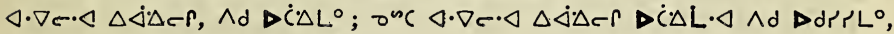

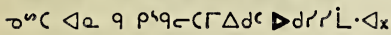

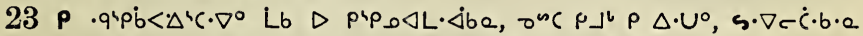

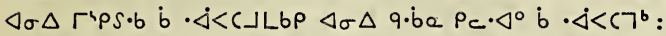

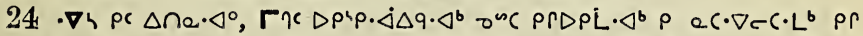

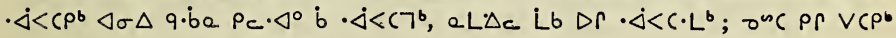
$\triangle \sigma \Delta q \cdot b_{e} P_{c} \cdot \triangleleft^{\circ} b \mathrm{~b} V C 7^{b}$, aLA $\Delta=$ Lb $D r V C \cdot L^{b} x$

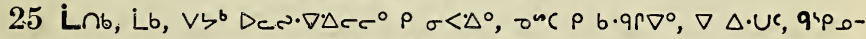

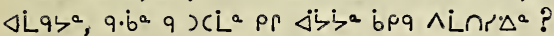

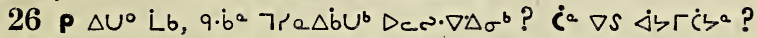

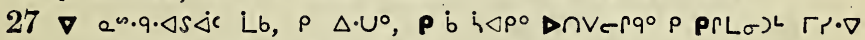

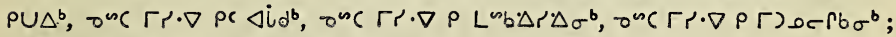

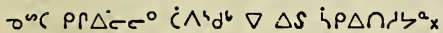

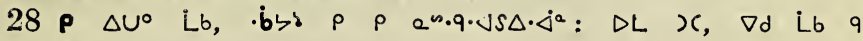
$\wedge \operatorname{Ln} \operatorname{Li}^{\circ}{ }^{\circ} x$

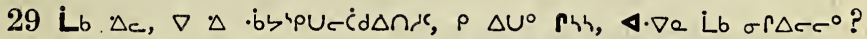

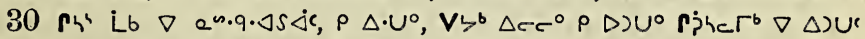

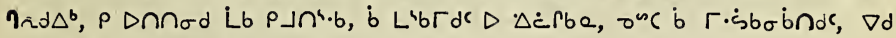
q) Ucr, $\nabla$ abnde $\dot{\Delta \wedge c^{\circ} \nabla} \sigma \wedge c_{x}$

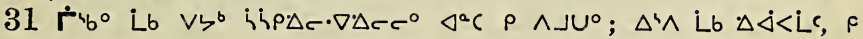

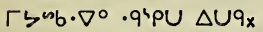

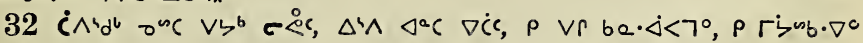
Lb $\cdot q^{\circ} P U \Delta \cup q_{x}$

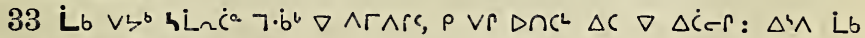

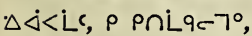

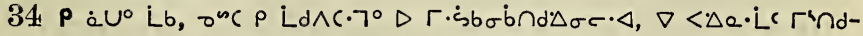

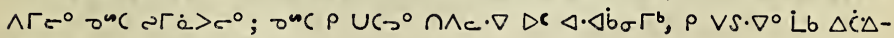
bГd $d^{b}, \tau^{n C} \rho$ ba. $\left.\nabla c\right]^{\circ} x$

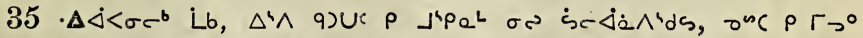

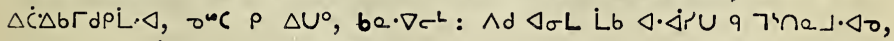

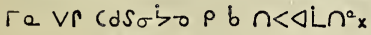




\section{$\Delta<$ P is 10, 11.}

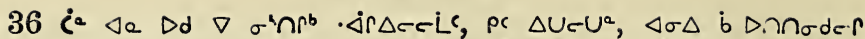
ค. กิ.6?

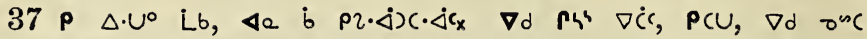
Pc $x_{x}$

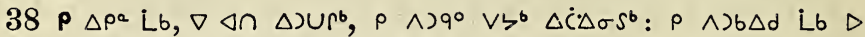

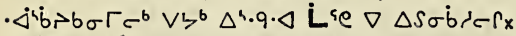

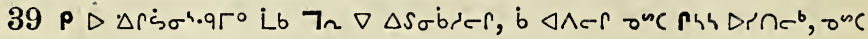

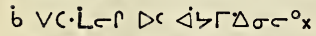

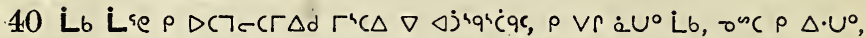

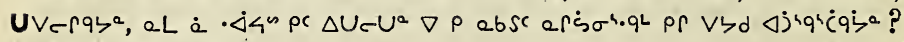
$\Delta C L^{\circ}$ Lb PS $\Delta r \Delta C_{x}$

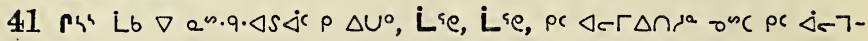
- $c r \Delta d^{a} \Gamma \eta q \cdot b a$;

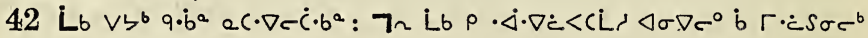
$\nabla$ b 9 L'b $L b \sigma \Delta c_{x}$

\section{Ápcre $\triangle b^{a} 11$.}

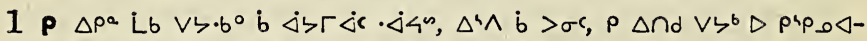

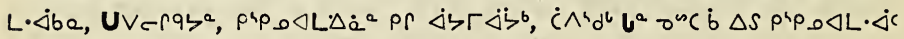
$\triangle$ pip. $\Delta L \cdot \Delta b e x$

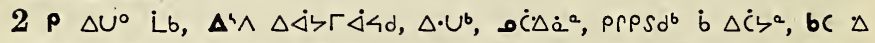

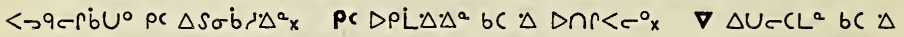

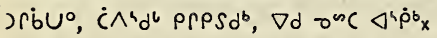

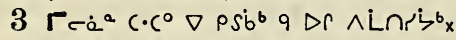

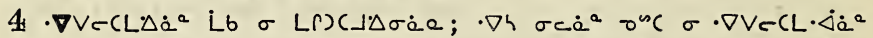

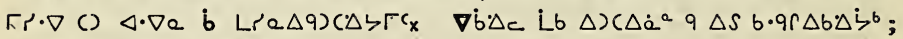
Lb $\Gamma \dot{C} \cdot q_{a} L \Delta \dot{a}^{a}$ Lr $q \cdot \dot{b} a x$

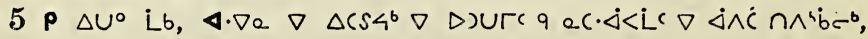

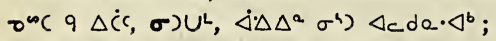

$6 \cdot \nabla h \sigma \rho \vee r \Delta \cap \cap^{b} \vee \zeta^{b} \sigma U^{L} \nabla \wedge \Gamma \wedge r c, a L \Delta c q \cdot b^{a}$ Lb $\sigma^{c} \Delta \ddot{b a} p \rho$ $\left.\Delta c^{i}\right) \cdot \triangleleft^{6}$ ?

$\left.7 \cdot \Delta c L_{b} \wedge\right) b \Gamma^{b} b C D r$ as.q. $\Delta S \Delta \cdot \nabla^{\circ}, \nabla \Delta \cdot U c, \nabla \dot{b} \Delta c r d^{4} b r \Delta^{a} ; \Delta^{\infty} \cdot \dot{b} U^{L}$

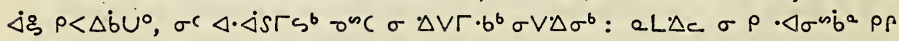
$\Gamma-c^{a} x$

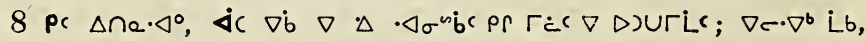
$\left.\Gamma \eta \cdot c^{\circ} \nabla a\right)\left(L d d, b c \cdot \Delta \sigma^{\infty} b^{\circ}\right.$, onc bc $\left.\Gamma>^{\circ} C\right)$ b a $a\left(\cdot \nabla c L-\Gamma_{x}\right.$

9 pc $\left.\Delta \cap a \cdot \triangleleft^{\circ} L b, a\right)\left(\dot{L} q^{b}, \rho b \Gamma-b \Delta a \cdot \triangleleft^{\circ} ; \dot{a} a c \cdot \nabla c c\right\lrcorner^{b}, \rho b \Gamma^{4} q a \cdot \triangleleft^{\circ}$

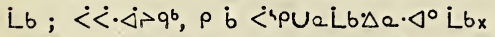

$10 \cdot \nabla 4 \Gamma r \cdot \nabla(c) \triangleleft \cdot \nabla a b$ b a $)\left(L c, \Gamma \dot{c} b \sigma \Delta^{\circ} ; \triangleleft a\right.$ Lb b $\dot{a} a c \cdot \nabla c c^{b}, \Gamma^{4} b^{2}$;

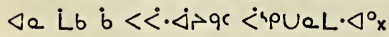

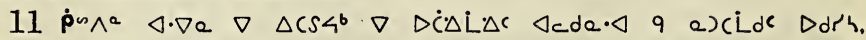

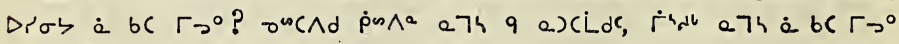
P०^•b?

\section{4}




\section{$\triangleright<-s \rho^{4} j^{6} 11$.}

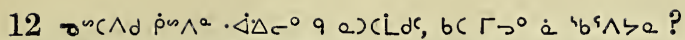

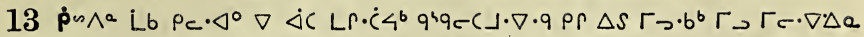

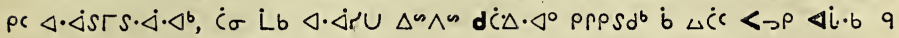
$\Gamma \dot{c}(\Delta \sigma \Delta \dot{b}$ a) $(\dot{L} d \boldsymbol{d}$ ?

14 $\rho \cdot \triangleleft c \Delta \cdot \nabla \Lambda_{0}<a$ Lb $\Gamma r \Delta i \cdot b$, aL Lb $\rho \sigma c \cdot \nabla c \cdot \Delta x p \Delta \rho^{a} L b$,

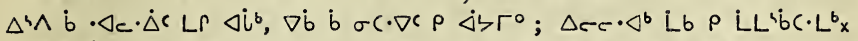

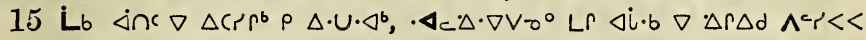
LR $\triangle i \cdot b^{b} D C D P L T \cdot \Delta \cdot \Delta x$

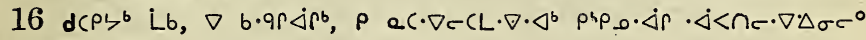
PPPSdb $D r_{x}$

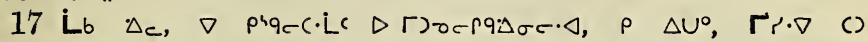

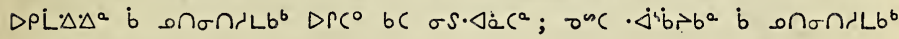
$\left\langle P \cap^{2} x\right.$

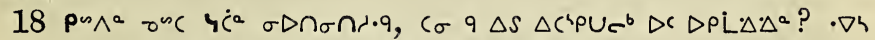
pc $\Delta \cdot \dot{C}_{0} \cdot \Delta^{\circ} \nabla \cdot \Delta c \Delta \cdot \nabla \Lambda e \rho^{b}$ Lr. $\Delta i \cdot b^{b} \nabla \Delta r \Delta c \dot{\Lambda} a r<<_{x}$

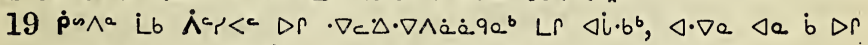

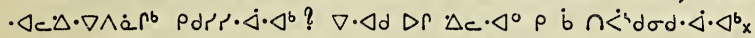

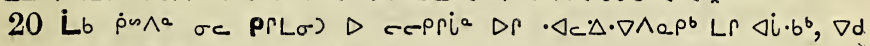

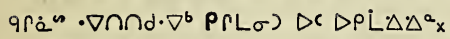

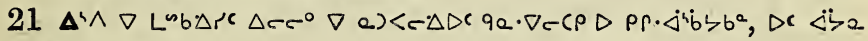
bं $\Delta C \cdot b \sigma c \cdot \triangleleft:$

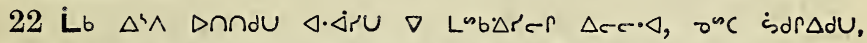

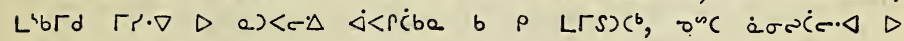
$L^{\prime} b \cdot U \Delta \sigma c \cdot \Delta^{x}$

23 ४e $\nabla \dot{b}$ b $\Delta r \Delta c \sigma a^{4} d^{4} c^{b}: \Delta e \sigma^{s c} \nabla b$ b $\Delta r \Delta c \nabla \mu s p \sigma q: \Delta^{b}$, $\cdot \Delta 4 \cdot \zeta \wedge \sigma 9^{\circ} \mathrm{x}$

24. $\Delta^{4} \wedge \Delta r \wedge \Delta i^{b} b \cdot \Delta c \Delta r \Delta r c^{b} \Delta r, \dot{<} \wedge J^{\circ} \Delta c \nabla \dot{<} \cdot b c^{b}, \nabla \dot{a} a c-$

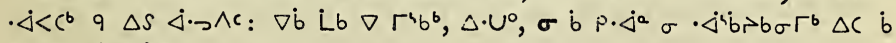
$\rho \Delta r \cdot \dot{\Delta} \dot{c} \Delta \dot{\zeta}^{2} x$

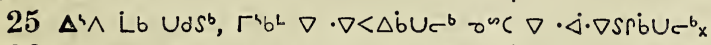

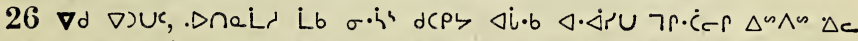

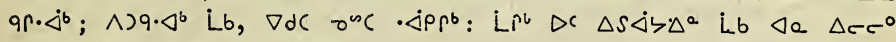

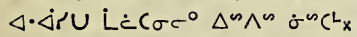

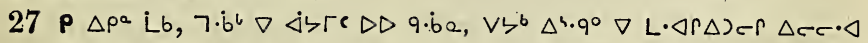

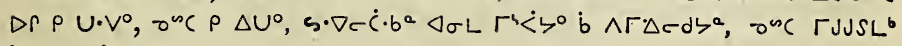

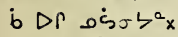

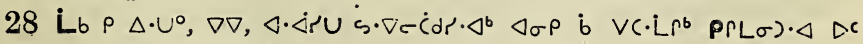

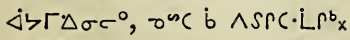

$29 \Delta^{4} \wedge$ Lb $\Delta r a \cdot \Delta^{b} \nabla$ rqu

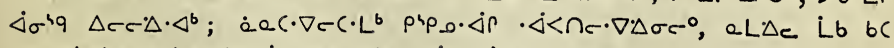

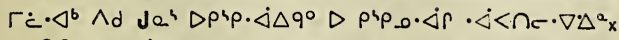

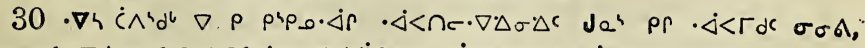

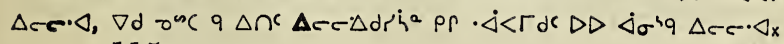
115 


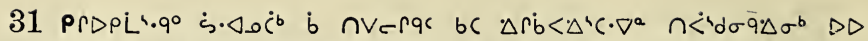

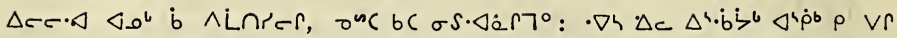

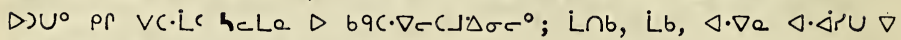

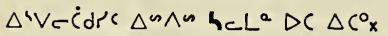

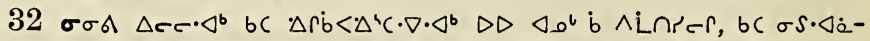

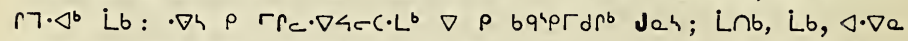

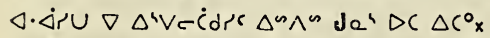

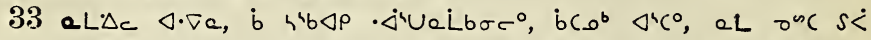

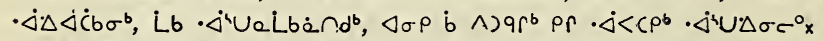

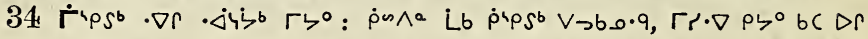

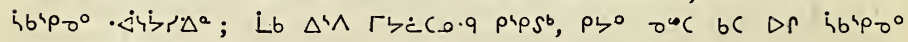
- $\Delta \sigma \cap \wedge i p r \cdot \Delta a x$

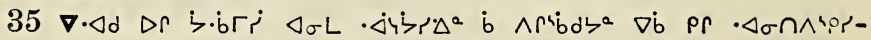
$\Delta \sigma \cdot \triangleleft^{b} \mathrm{x}$

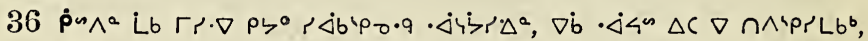

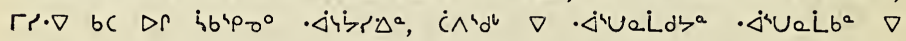
$\cdot \Delta 4 v^{b} x$

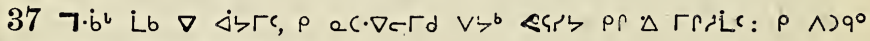

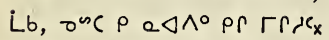

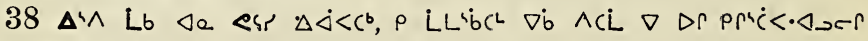

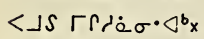

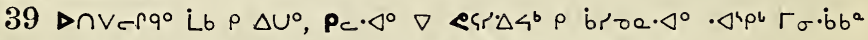

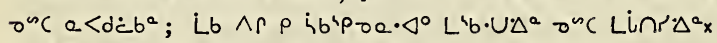

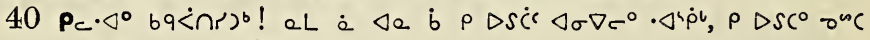
$\Delta \sigma \nabla c^{\circ} \wedge \Gamma$ b $\Delta c \cdot b \sigma c^{b}$ ?

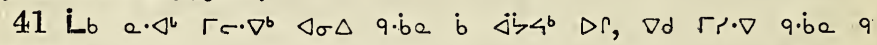
$\dot{<} \rho \cdot \dot{C} d \zeta^{b} x$

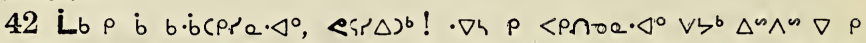

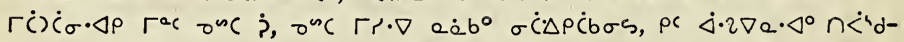

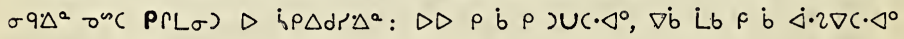
$\triangleleft \sigma L d C^{b} x$

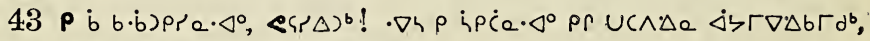

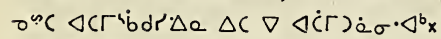

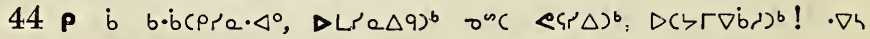

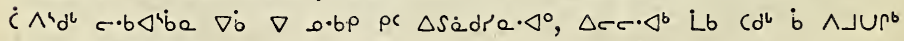
aLAC PlqCC.Lbx

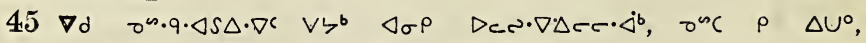

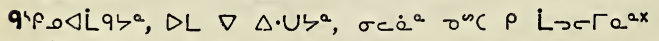

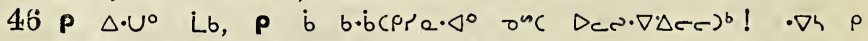

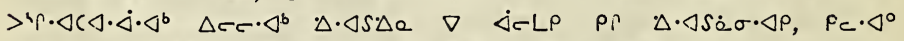

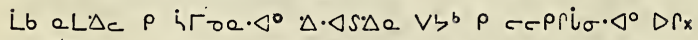

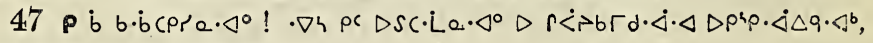
d $\dot{C} \Delta \cdot \triangleleft \cdot \triangleleft^{b}$ L L $\quad \rho \quad \sigma<\nabla \cdot \Delta^{b} x$ 


\section{$\triangleright<>\rho^{4}$ jb 11, 12.}

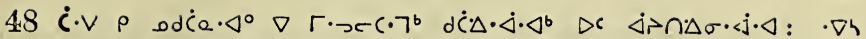

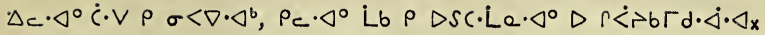

$\left.49 \nabla \cdot \Delta d \cdot \nabla r \quad \triangle \cdot U L b u \sigma^{b} P R L \sigma\right) \quad b \quad b(\cdot \nabla c c\lrcorner \Delta^{a}, \sigma \quad b \quad \Delta S \cap S \Delta L-$

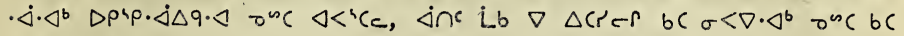
$b \cdot \dot{b}\left(\rho \nabla \cdot \triangleleft^{b} ;\right.$

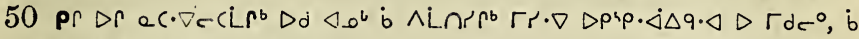

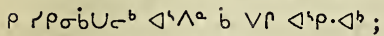

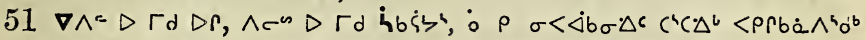

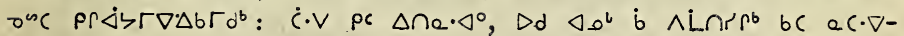
$-\mathrm{CL} \cdot \dot{\Delta} \cdot \triangleleft^{\mathrm{b}} \mathrm{x}$

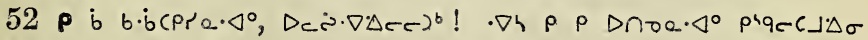

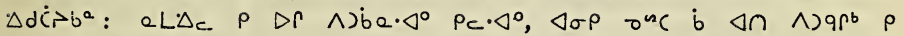

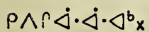

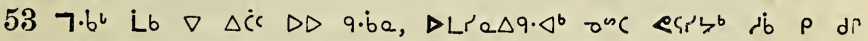

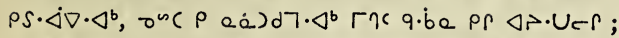

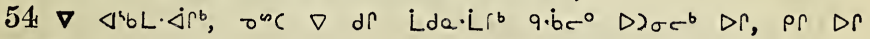
ĹSSL $\Gamma^{b}{ }_{x}$

\section{$\Lambda^{\prime} p\left(P^{2} \propto \Delta b^{2} 12\right.$.}

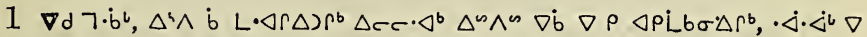

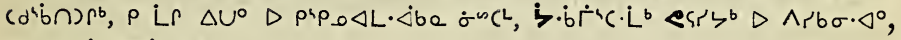
$\nabla \cdot \triangleleft d \dot{d}\left\langle\nabla \nabla \dot{b} \cdot \Delta^{a} x\right.$

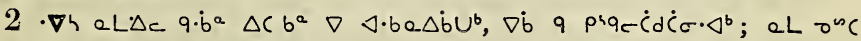
$q \cdot \dot{b}^{a} \dot{b} \dot{c} \sigma \cdot \nabla^{a} \cdot \nabla \dot{b} q$ prac $\dot{c} \cdot b^{b} x$

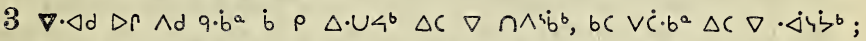

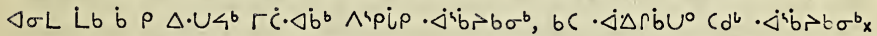

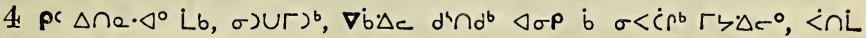
$\left.\Delta \sigma \nabla c^{\circ} \dot{L} b \nabla \dot{b} \dot{b} \quad \dot{b} \rho^{b} q \cdot \dot{b} c^{\circ} q \rho\right)\left(p^{b} x\right.$

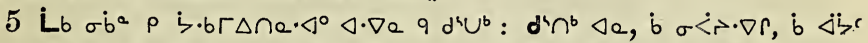

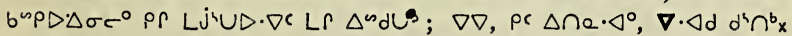

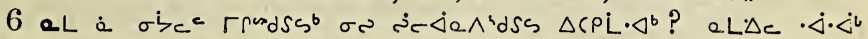

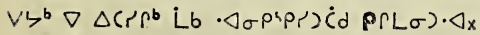

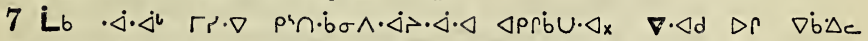

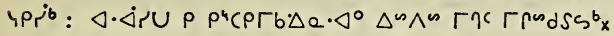

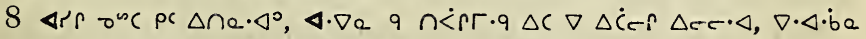

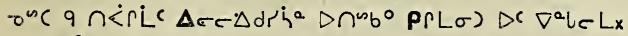

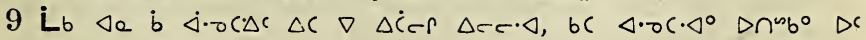
$\nabla$ Q $L C L P P L \sigma)_{x}$

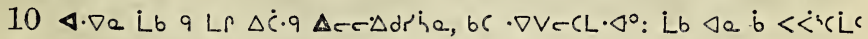
$<>P \varangle i \cdot b, a L \Delta c \quad b C \cdot \nabla V-C L \cdot \Delta 0_{x}$

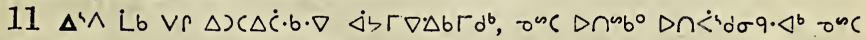

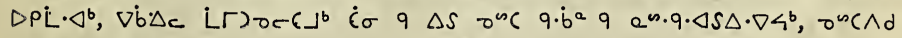
$q \cdot b^{a} q \Delta \cdot \cup<b^{b} x$ 


\section{$\Delta<>\rho^{4}$;b 12 .}

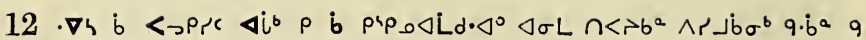
$\Delta \cdot U<d<<^{a} x$

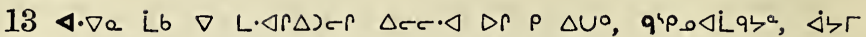

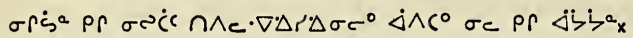

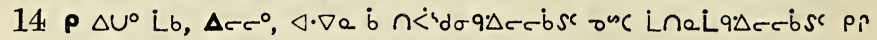
nV $-\Gamma \dot{C} \cdot b^{6}$ ?

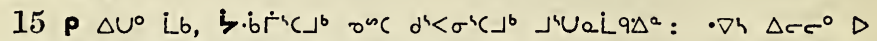

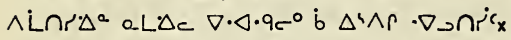

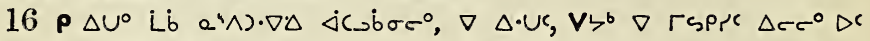
$\triangleleft^{\prime} \rho \Gamma^{\prime}\left(\Delta \rho \sigma \dot{C} \Delta \rho \dot{C} L b \sigma \sigma^{\circ} \mathrm{x}\right.$

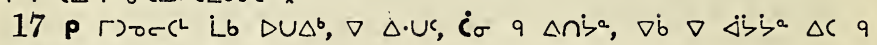
$\triangle \ddot{C} \dot{\zeta} \sigma$ o $\sigma \dot{C} \Delta \rho \dot{c} b a$ ?

$18 P \Delta \cdot U^{\circ}$ Lb, $\Delta L \quad \sigma$ b $) U^{a}: \sigma$ b $\sigma r \wedge U^{a} \sigma c \cdot b \Delta q \Delta b \Gamma \cdot b, \sigma^{a c} \sigma b$

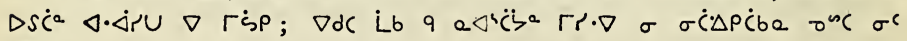
¿לंex

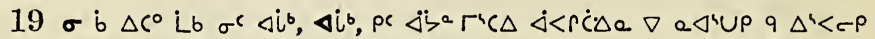

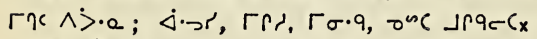

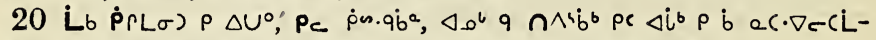

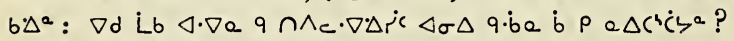

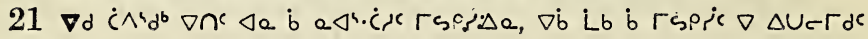
$P \Gamma(\sigma) \cdot \triangleleft x$

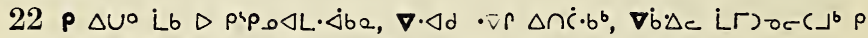

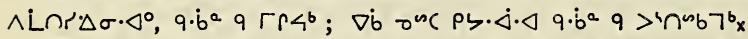

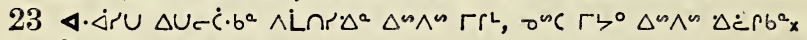

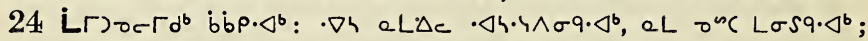

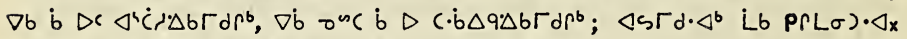

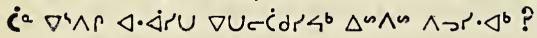

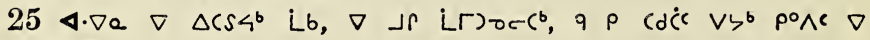
$\Delta$ 'd $b<\Delta<?$

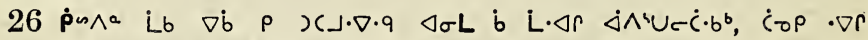
Lr)dec $]^{b} \triangleleft \sigma \Delta d(P)$ ?

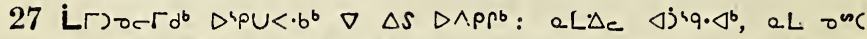

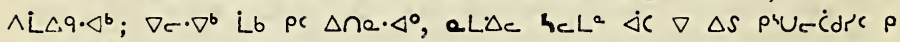
$\triangle S D \dot{C} \wedge$ ‘ $d^{l} \vee V \zeta^{b} D d x$

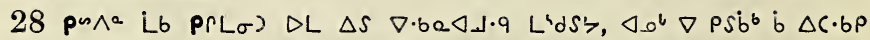

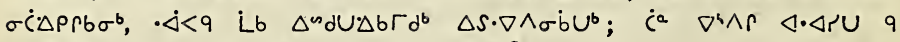

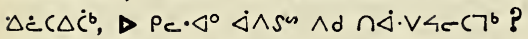

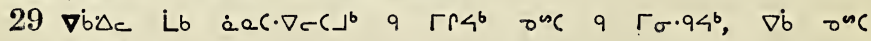
$\cdot p C \Delta \Delta U \subset-C \cdot J^{b} x$

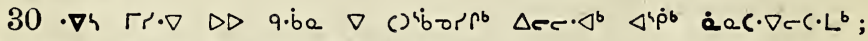

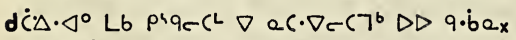

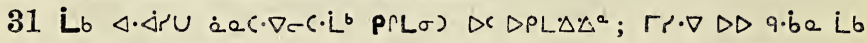
$\rho \dot{b}$ बrr $r-b \Delta z \cdot \Delta^{\circ} x$

118 


\section{$b<>p^{4}$ ib $^{\circ} 12$.}

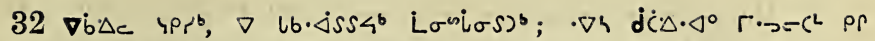
$\Gamma-\dot{C}^{b} \triangleleft \sigma \nabla \sigma^{\circ} \triangle \rho L \Delta \Delta \sigma \sigma^{\circ} x$

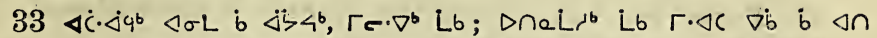

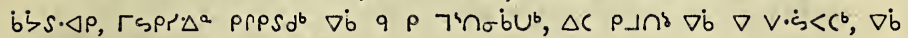
onC LOJus $\nabla \sigma s \cdot \Delta \dot{\alpha} r C_{x}$

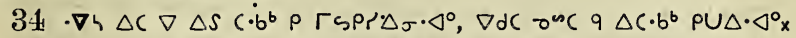

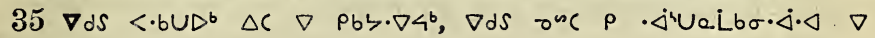
4 'b $\triangle \mathrm{b} \cup \mathrm{P}_{\mathrm{x}}$

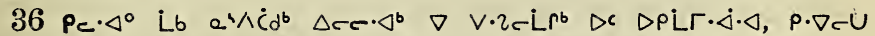

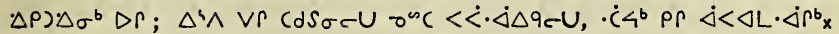

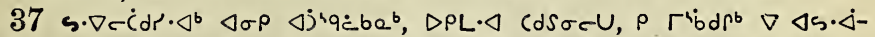

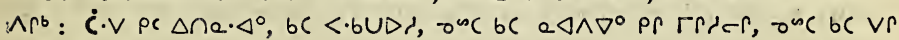
$\wedge \Gamma \nabla^{\circ} x$

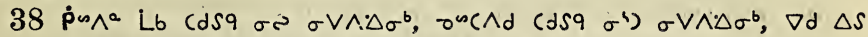

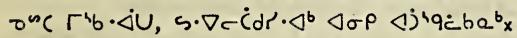

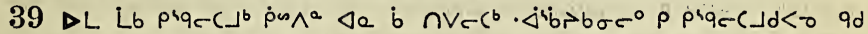

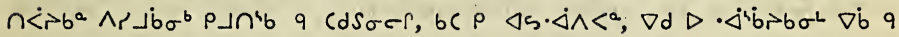
$b<i \wedge \wedge d \sigma \cdot \dot{b} \cup \sigma^{i}<{ }^{a} x$

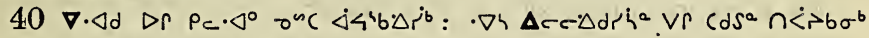
$\wedge r\lrcorner \dot{b} \sigma^{b} 7 \cdot b^{b} \nabla \dot{b} \nabla \Delta U \in C 7^{b} x$

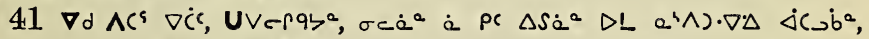
oncAd $L s \cdot \nabla$ a $b c \Delta \dot{C} \cdot \Delta^{b}$ ?

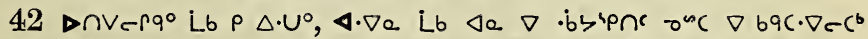

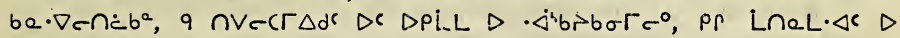
Lrtr $c_{0}, \cdot \nabla n k<\epsilon c b$ ?

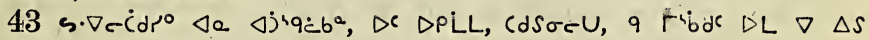
$x^{b} \times$

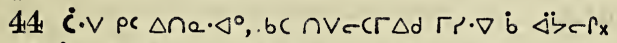

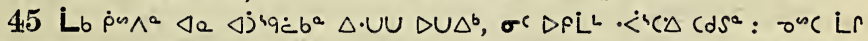

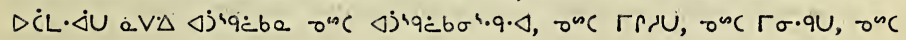
pंn.qvU;

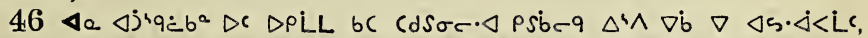

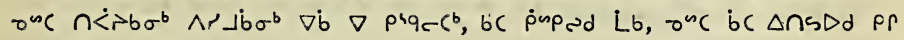
$\Delta \eta \cdot \dot{\Delta} \overrightarrow{\nabla b} \dot{b} \dot{b} \cdot V C \Gamma r r_{x}$

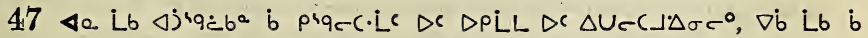

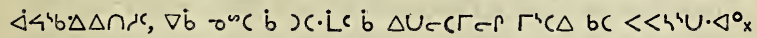

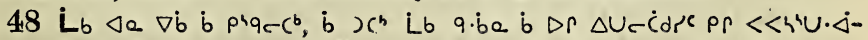

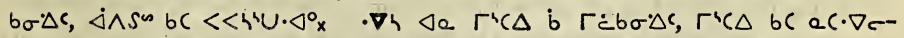

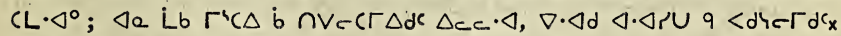

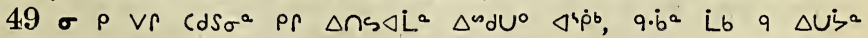

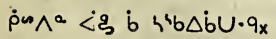

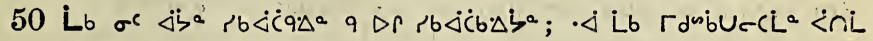
P $\cap \wedge<\uparrow$ ! 


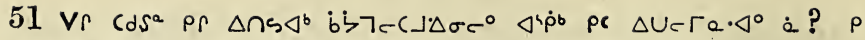

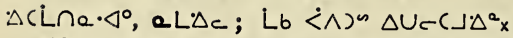

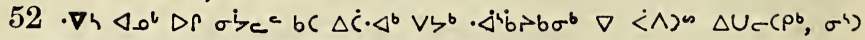
$\nabla \cdot \Delta \dot{b} \dot{C}^{b} \sigma a, \sigma^{n}\left(\sigma \omega \nabla \cdot \Delta \dot{b} \dot{C}^{b} \sigma^{-4}\right)^{x}$

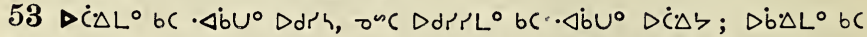

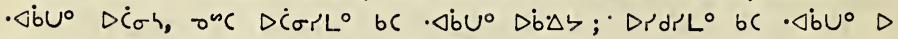
e $\Delta b \sigma^{4} \cdot q L, D_{e} \Delta b \sigma^{4} \cdot 9 \Gamma L^{\circ} L b b C \cdot \Delta b U^{0} D_{r d h x}$

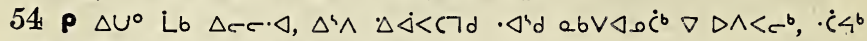
pc $\Delta \cdot \dot{C}_{a} \cdot \Delta^{\circ}, V r \rho \Gamma \cdot \nabla^{a} ; \nabla d$ onc $\nabla \rho b^{x}$

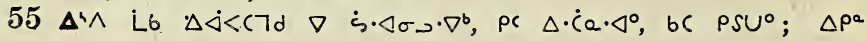
Lbx

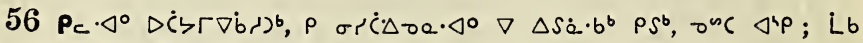

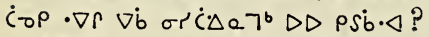

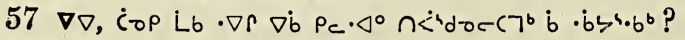

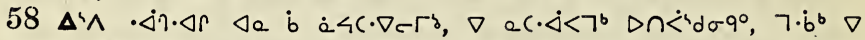

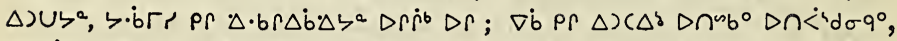

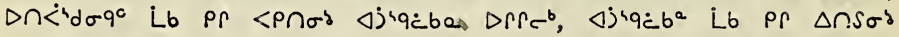
$P(D) \Delta b \Gamma d^{b} x$

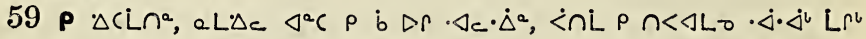
तेc $\triangleleft \dot{a} \wedge^{n} d S^{n} x$

\section{ÁpCra $\triangle b^{\circ} 13$.}

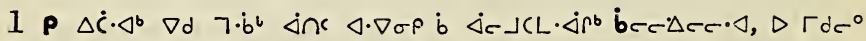

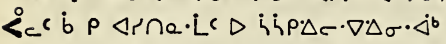

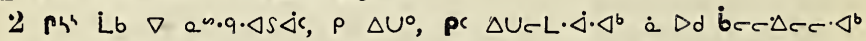

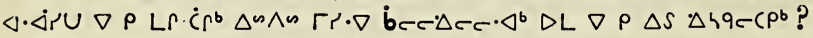

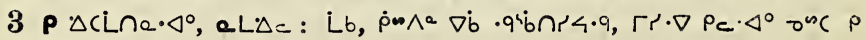
bे $\sigma s \cdot \Delta \dot{a} \cap r a \cdot \Delta^{\circ} x$

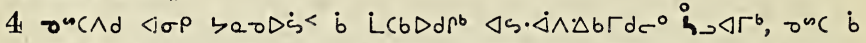

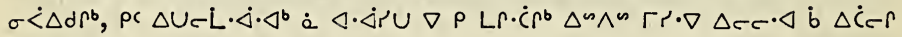
Pinct ${ }^{b}$ ?

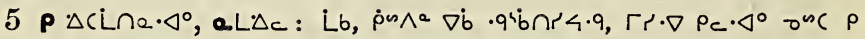
b $\quad \sigma s \cdot \Delta \dot{a} \cap r a \cdot \Delta^{\circ} x$

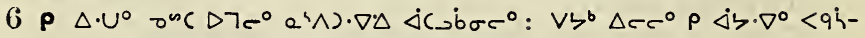

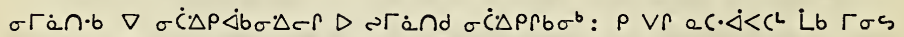
$\nabla^{a} C$, $Q L$ Lb $D r \Gamma^{\prime} b b^{2} x$

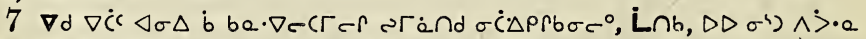

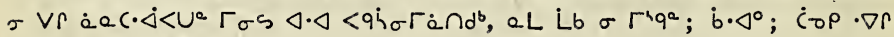

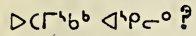

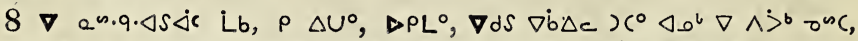

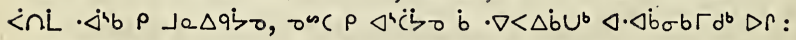

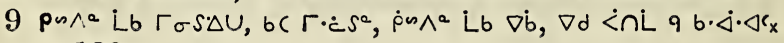




\section{$\triangleright<>$ p' jb 13 .}

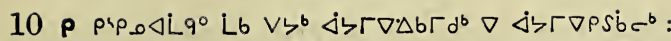

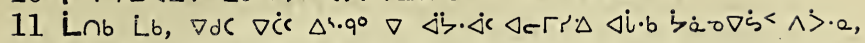

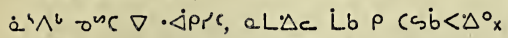

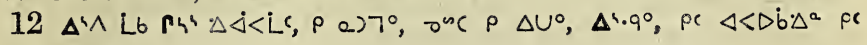
$\triangle C \Gamma P \cdot \Delta \sigma^{b} D r_{x}$

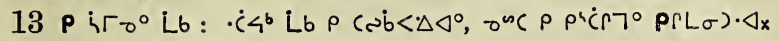

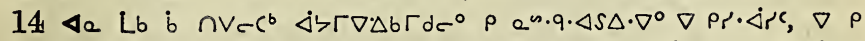

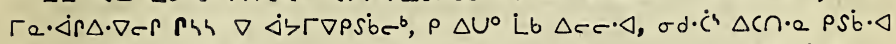

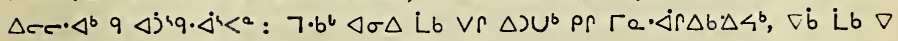
$\Delta \zeta \Gamma \nabla \rho S b^{b} x$

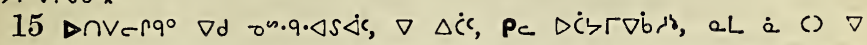

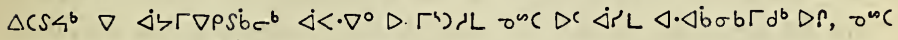
$\triangle)\left(\nabla^{\circ}\right.$ pr $\Gamma \sigma . q_{c} r$ ?

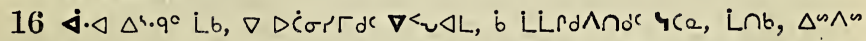

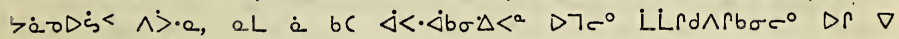
यंट $\nabla \rho S \dot{b} e^{\circ}$ ?

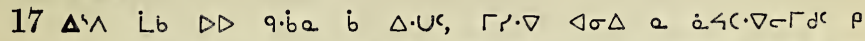

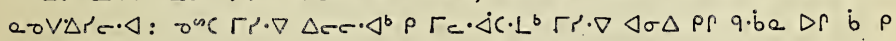
$x \Gamma-\Gamma_{x}$

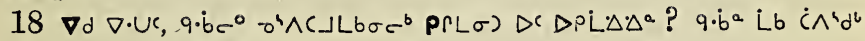
$\Delta S \dot{\alpha} \cdot b \sigma c^{\circ} q \Delta \cdot \cup \dot{\zeta} a$ ?

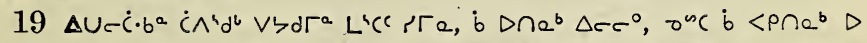

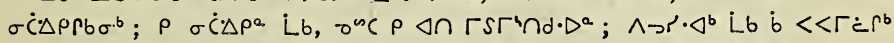
$\rho c s q \cdot \triangleleft^{b} \cdot \triangleleft \Omega \cdot b \sigma^{b} x$

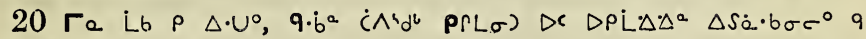
$\triangle \cdot \cup \zeta^{2}$ ?

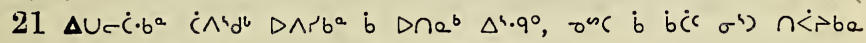

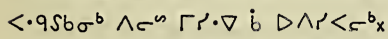

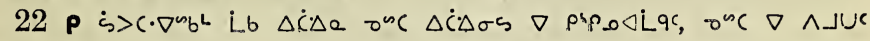
Pihe $\Gamma^{b} \nabla \Delta u^{\circ} e_{x}$

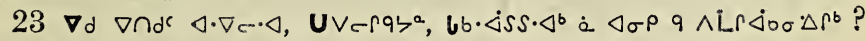
$p \Delta U^{\circ} \mathrm{Lb}$,

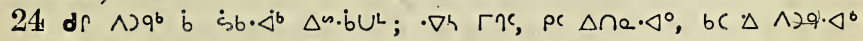
e L $\Delta c$ Lb b $\rho)\left(\cdot L^{b} x\right.$

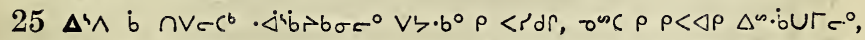

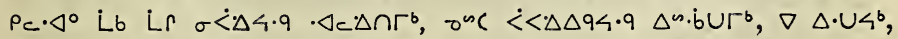

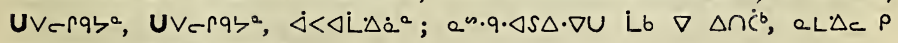
prqa $r \cap a \cdot \triangleleft^{\circ} \dot{c} \cup \cdot \nabla r<^{b}$;

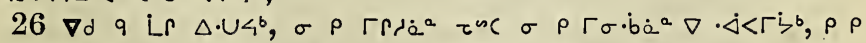

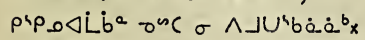

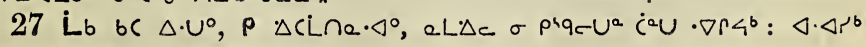

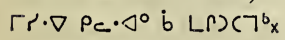

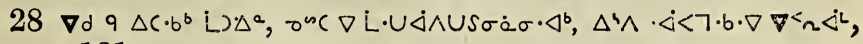
121 


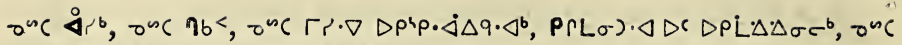
$\rho_{c} \cdot J^{\circ} q r \cdot \dot{\Delta}^{b} \nabla \cdot \Delta c \Delta \cap S \sigma b \cdot \Delta^{b} x$

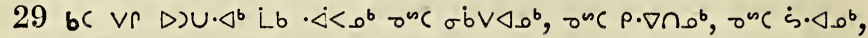
$b\left(a \triangleleft \wedge \cdot \Delta^{b} L b P \Gamma L \sigma\right) \cdot \triangleleft D C D P L \cdot \Delta \Delta \sigma c^{b} x$

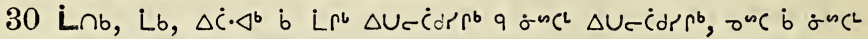

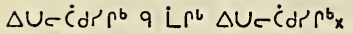

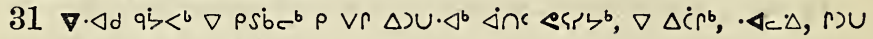
Lb : $\nabla 4$ " $\nabla \varsigma s \rho \dot{b} \sigma<\Delta^{b} x$

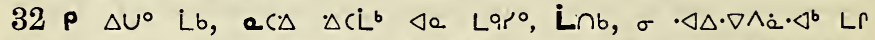

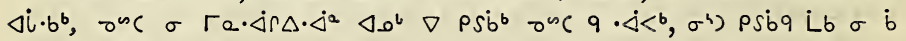
Г) $\sigma \Delta \Delta \Delta \dot{b}^{a} x$

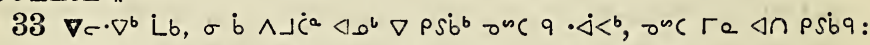

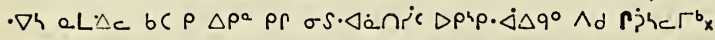

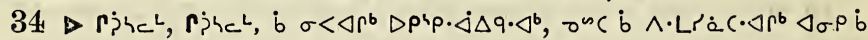

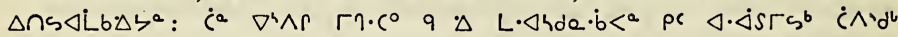

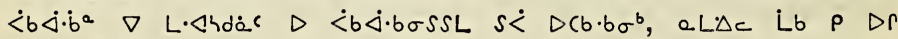
$\vee \cup \circ \cdot \triangleleft^{\circ}$ !

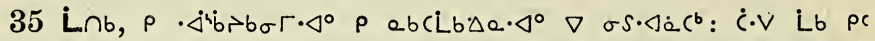

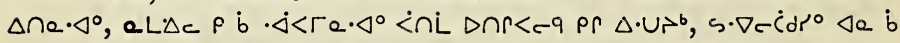

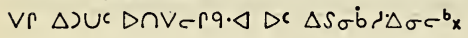

\section{$\Lambda^{4} p\left(r a \Delta b^{\circ} 14\right.$.}

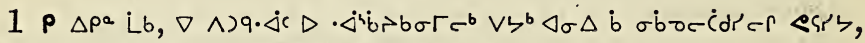
pr $\lrcorner \cdot\left\langle i \triangleleft c d a \cdot \Delta \nabla \Delta \zeta \Gamma \nabla \rho s \dot{b} c^{b} \rho\right.$ a.br $\Delta d x$

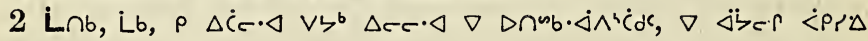
$\triangle \dot{c}^{\wedge} \wedge_{\sigma} \Delta \sigma \sigma^{\circ} \mathrm{x}$

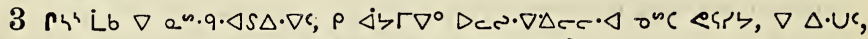

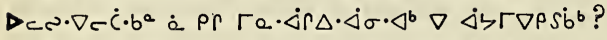

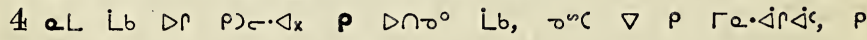
$<P \cap 0^{\circ}$;

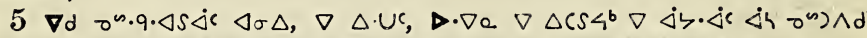

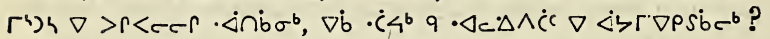

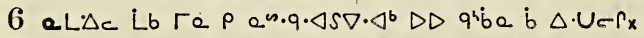

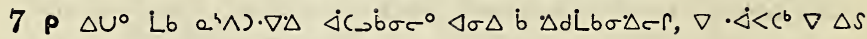
e. $\Delta r a r e r \operatorname{sr} \Delta \wedge \Delta a ; \nabla \Delta \dot{c}$, ,

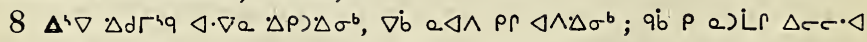

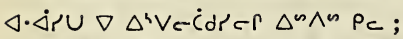

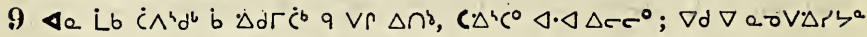

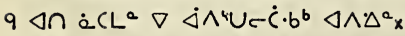

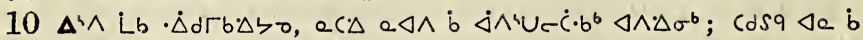

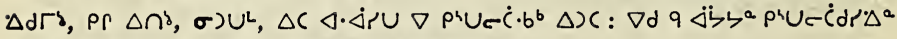

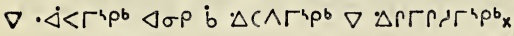




$$
\Delta<\rho^{4} \text {; } 14 .
$$

11 . PiUCLํ.

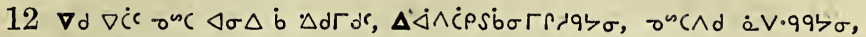

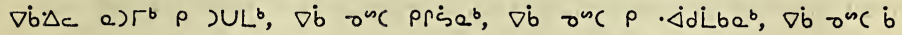

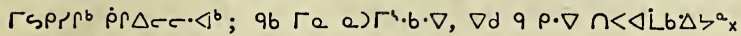

13 Lb $7 d 2 \zeta \sigma, a) \Gamma^{b}$ b PnLPppb. b L'pppb, $\nabla \dot{b}$ b $\sigma C D U p^{b}, \nabla \dot{b} b$ $\cdot \triangleleft \wedge \wedge^{b}$ :

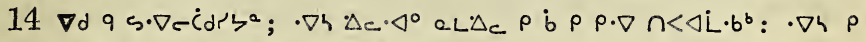

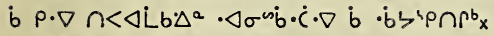

$15 \Delta^{4} \wedge$ Lb Vלb $\Delta \sigma \Delta$ b $\Delta C \wedge \Gamma d c \nabla \Gamma \Gamma d e \wedge \nabla c^{b} \Delta \sigma q \cdot \dot{d} a, \rho \Delta U^{\circ}$,

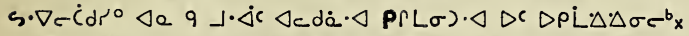

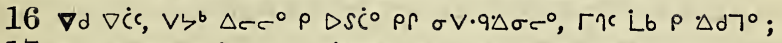

$17 \rho \Delta S \cap s \cdot \nabla^{\circ}$ Lb $\Delta C \quad \Delta j 4 \dot{4} b \sigma L \quad \Delta^{4} \wedge \cdot \nabla \cap r<c^{b}$ pr $\sigma V \cdot b_{\sigma} \cdot \Delta^{b}$ pr

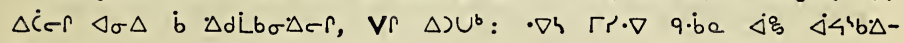
$\dot{\mathrm{C}} \sigma \cdot \triangleleft \cdot \mathrm{o}_{\mathrm{x}}$

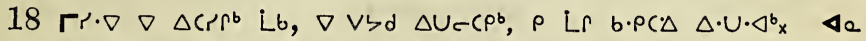

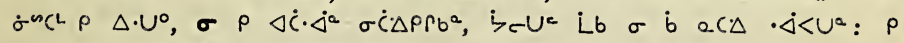
a) $C L n^{2}, L r_{0} \circ r^{a} x$

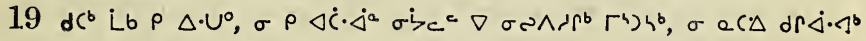
$\mathrm{Lb}: \rho$ a) $\left(\mathrm{L} \cap^{a}, \mathrm{~L} r r_{0} \Gamma^{a x}\right.$

$20 d c^{b}$ Lb $\rho \Delta \cdot U^{\circ}, \sigma \rho \Delta \Delta^{a} ; \nabla \cdot \Delta d \cdot \nabla r \nabla b \rho \vee r \Delta u U^{a}{ }^{a} x$

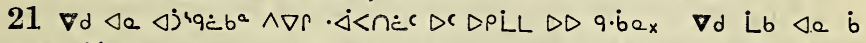

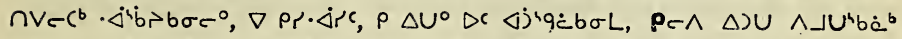

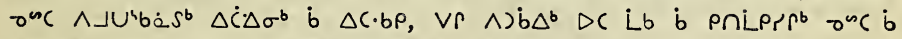

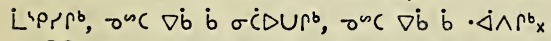

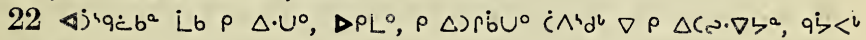
Lb $c \cdot \Delta^{\circ} x$

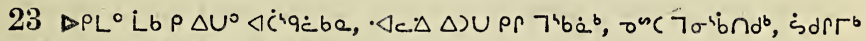

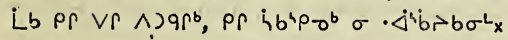

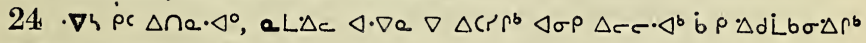
bC dri $^{\mathrm{C} C L} \sigma \sigma \cdot \mathrm{V} \cdot 9 \cdot \Delta \sigma c^{\circ} \mathrm{x}$

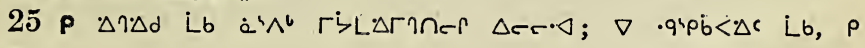
$\triangle u^{\circ}$,

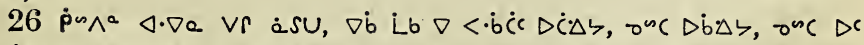

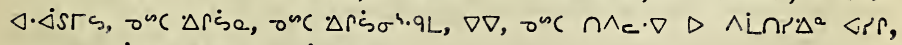
QL $\Delta=\sigma$ b $P$ P $\triangle P^{4} P . \Delta L \cdot \Delta b \sigma L^{\circ} \times$

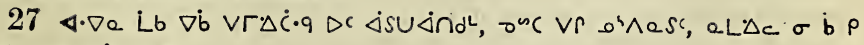

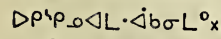

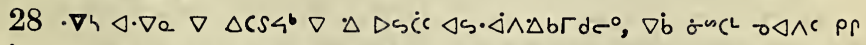

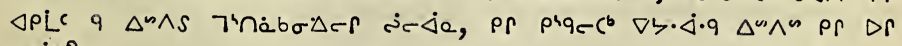
PSĊ?

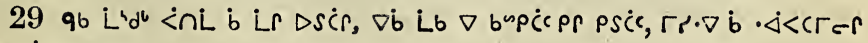
Lr <u $\Delta d c$, 


\section{$\triangleright<>b^{4} ; 14,15$.}

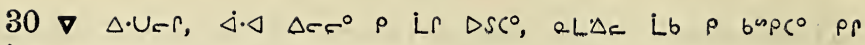
. $p$ Sic $x$

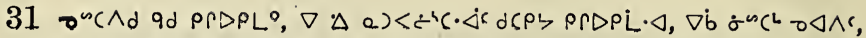

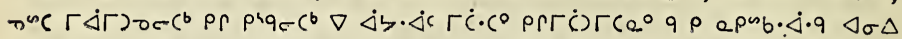

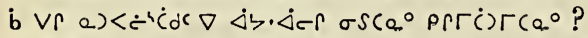

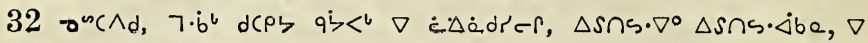
ec $\left(\cdot \nabla c c^{b} a \nabla c\right) \Delta \sigma \kappa^{\circ} x$

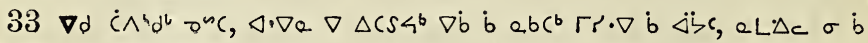
$P D P^{\prime} P \_\Delta L \cdot \Delta b \sigma L^{\circ} x$

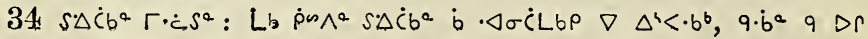
$s \Delta \dot{c} b \sigma \cdot \triangleleft^{b}$ ?

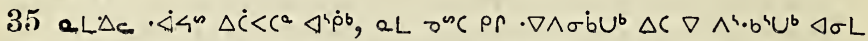

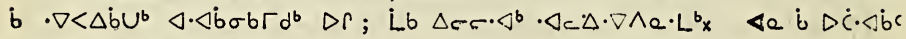
PS $V S^{b}, \nabla d S$ b $C \vee C^{b} x$

\section{Nipcra $\triangle b^{\circ} 15$.}

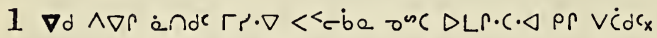

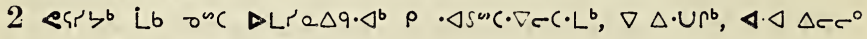

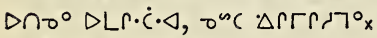

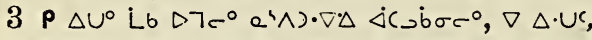

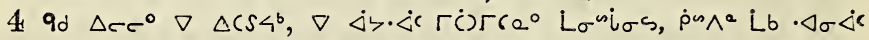

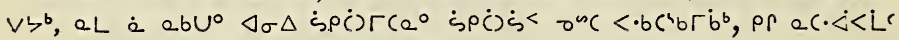
$\Delta \sigma \Delta \dot{b} \cdot \Delta \sigma s \sigma c r<i n L \Gamma^{\natural} b \cdot \Delta U$ ?

$5 \Delta^{4} \wedge$ Lb b $\Gamma^{b} b \cdot \dot{\Delta c}, \triangleleft \rho^{\circ} \Delta \cap \cap L \sigma^{b} \nabla \Gamma_{c} \cdot \dot{<} c^{b} x$

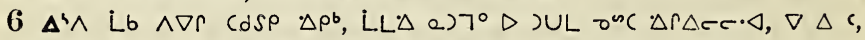

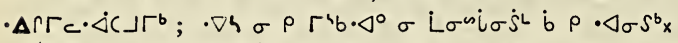

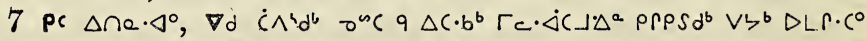

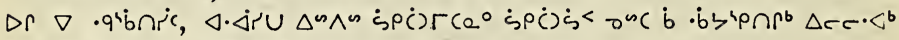

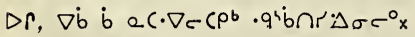

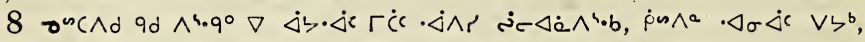

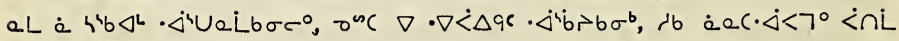
$\Gamma^{2} b \cdot \Delta U$ ?

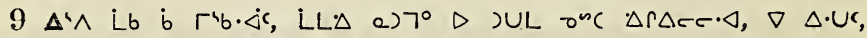
$\left.\Delta r \Gamma_{c} \cdot \Delta c\right\lrcorner \Gamma^{b} ; \cdot \nabla h \sigma \rho \Gamma^{\mathrm{b} b} \cdot \Delta^{\circ} \triangleleft a \dot{b} \rho \cdot \Delta \sigma \Delta^{b} \mathrm{x}$

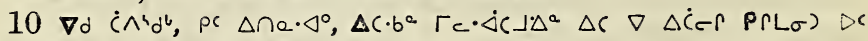

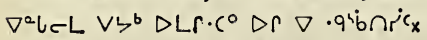

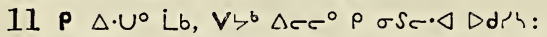

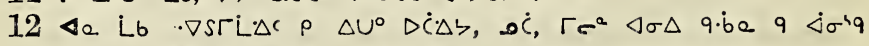

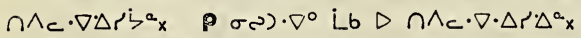

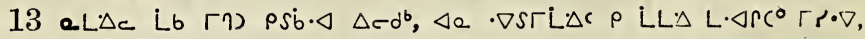

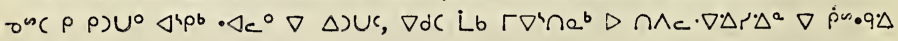
$\wedge L \cap i c_{x}$ 


\section{$\triangleright<>\rho^{4}$ jb 15.}

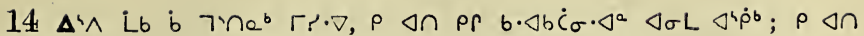
$\cdot \dot{\Delta} \cdot \dot{\Delta} \sigma p^{\circ} L b_{x}$

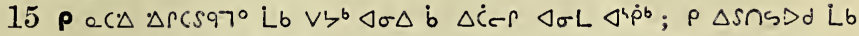

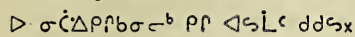

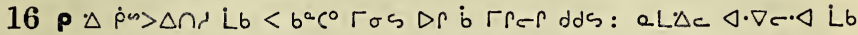
Dr $\Gamma e d q \cdot b c^{\circ} x$

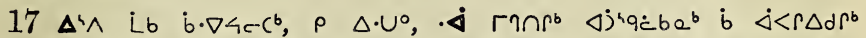

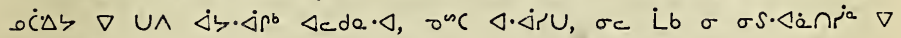
b. $\triangle b \cup i\rangle^{\circ}$ !

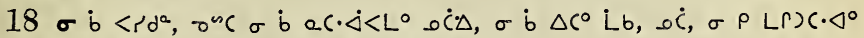

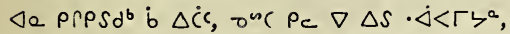

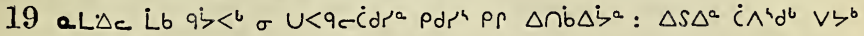

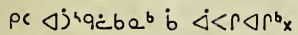

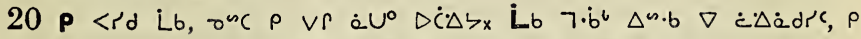

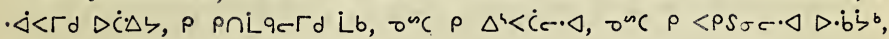
onc $P D T \Gamma d x$

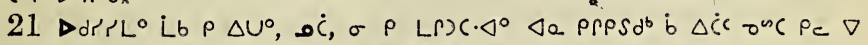

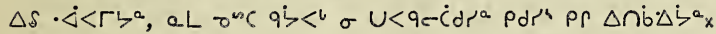

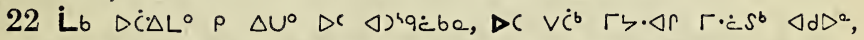

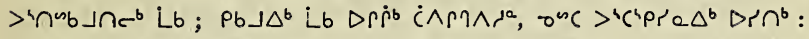

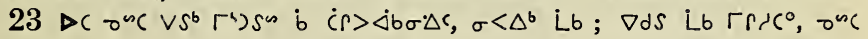
$\mathrm{JP} \mathrm{CC}^{\mathrm{C} C \mathrm{C}^{\circ} \mathrm{x}}$

$24 \cdot \nabla h \dot{\Delta} \triangleleft \sigma b r^{\prime \prime} \rho \sigma \wedge^{\circ}, \Gamma e$ Lb $\wedge i \cap r^{\circ} ; \rho \cdot \triangleleft_{\sigma} \triangleleft^{\circ}, \Gamma^{2} b \cdot \triangleleft^{\circ} L b_{x} \quad \nabla d$ $\Gamma<r \quad \mu r q C<\rho^{b} x$

$25 \nabla d r h$ Lb b DUULLCr $\rho \Delta \dot{C} \sigma \cdot \triangleleft \sigma \dot{C} \Delta \rho \rho b \sigma^{b}: \nabla V R \quad V \cdot \dot{s}<c^{b}$ Lb

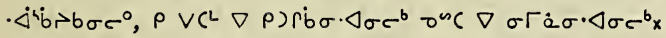

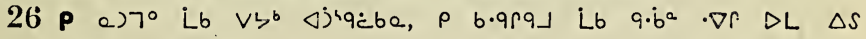
$\dot{\Delta} \vec{\cap} \cap \dot{a} \sigma \cdot \Delta^{b} \mathrm{x}$

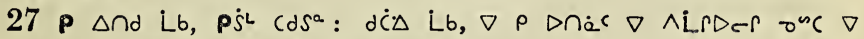

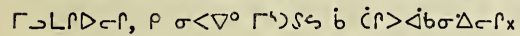

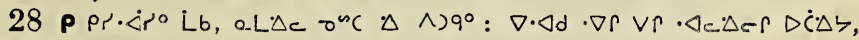

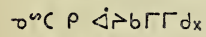

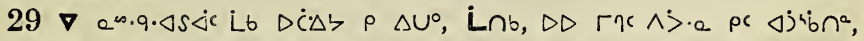

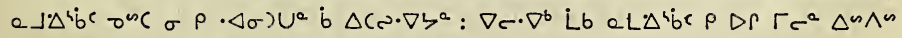

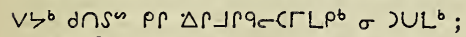

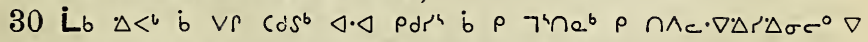

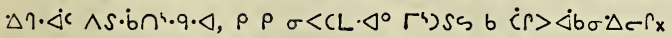

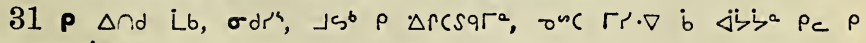
$\cap \wedge_{c} \cdot \nabla \cdot \Delta r^{a_{x}}$

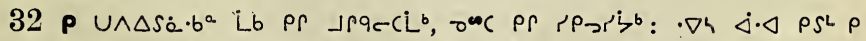

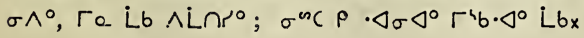




\section{$\triangleright<\rightarrow P^{4}$ jb $^{6} 16$.}

\section{A $P C$ $\triangle \triangle b^{2} 16$.}

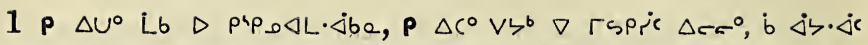

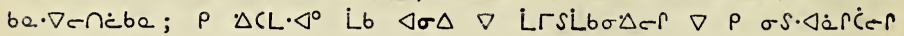
$\Delta C \Delta \dot{b a x}$

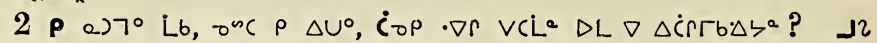

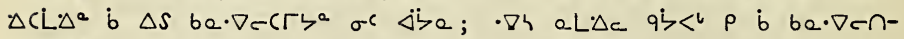
$\dot{c} b \cdot \sigma^{2} x$

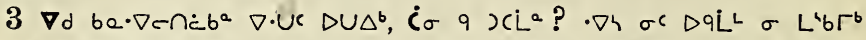

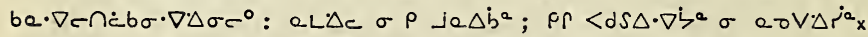

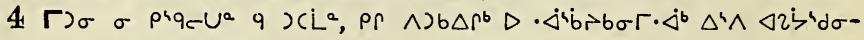
b. $\Delta$ לo be $\cdot \nabla e \cap \dot{c} b \sigma \cdot \nabla \Delta \sigma^{b} D \Gamma_{x}$

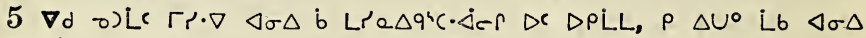

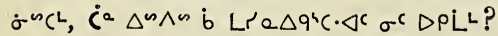

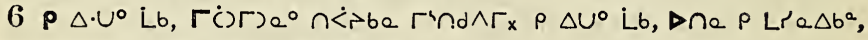

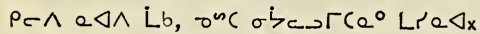

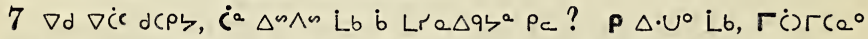

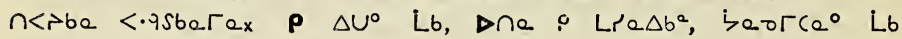
Lre $\triangleleft x$

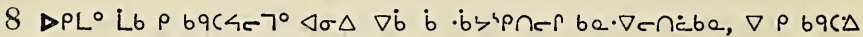

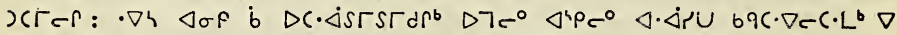

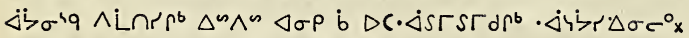

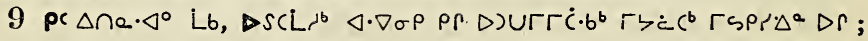
. $U<C 4 . q$ pr $\wedge>b \Delta \dot{C} \cdot b^{b}$ b $p q$ q $\Delta S \Delta r 4^{b}$ x

$10 \varangle a b$ b bל

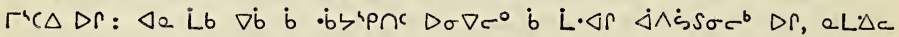
bל

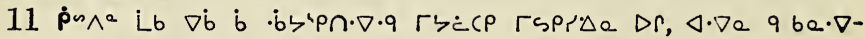

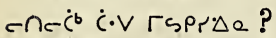

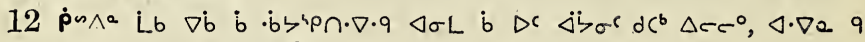
$\Gamma-\dot{C}^{b} \triangleleft \sigma L b$ b $\cap \Lambda_{c} \cdot \nabla \Delta r^{b} \dot{L}^{b}$ ?

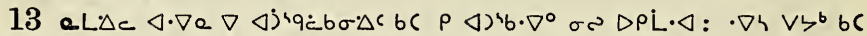

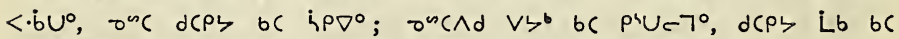

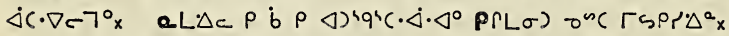

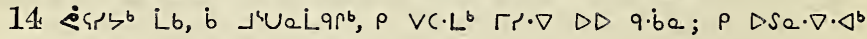
L.bx

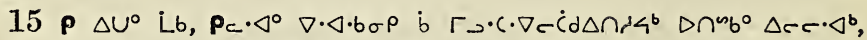

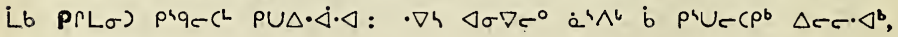
$L i<(L / P R L \sigma)_{x}$

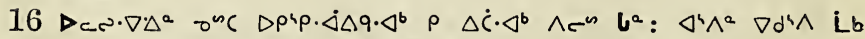

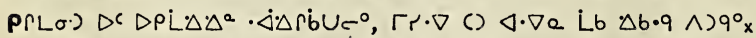

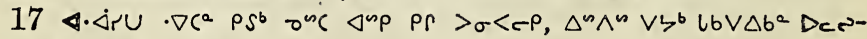
$\cdot \nabla \Delta \sigma^{b} \nabla \dot{b}$ pr $\cap \wedge<\sigma^{b} x$ 


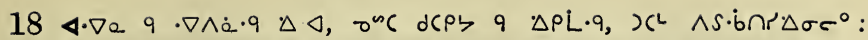

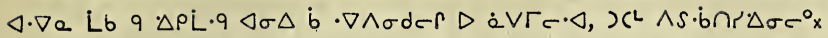

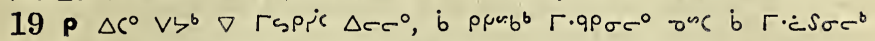

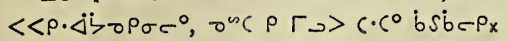

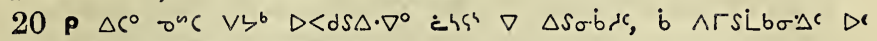

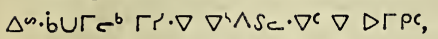

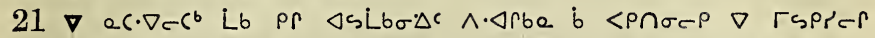

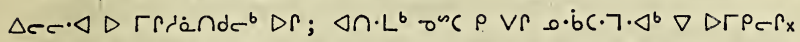

$22 \rho \Delta \rho^{a}$ Lb, $\nabla \rho \sigma \wedge c \Delta<d S \Delta \cdot \nabla^{\circ}, \nabla^{a}$ le Lb $\rho \Delta S \Delta c d \nabla^{<-J} \Delta L \quad D$

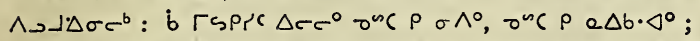

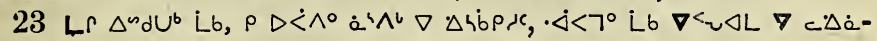
drer, onc $\approx 4 \& 4 \nabla \Lambda\lrcorner\lrcorner \Delta \sigma c^{b} x$

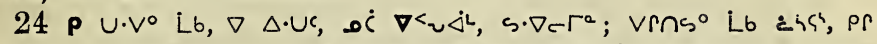

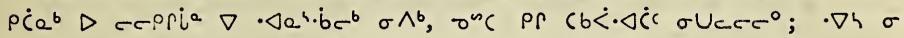
$\triangle 49-C r \Delta \dot{b} \Delta^{a} D C \nabla \cdot \Delta c U^{b} x$

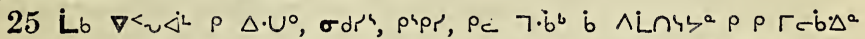

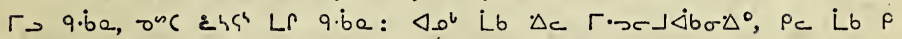
$\triangle 49-C r \Delta b \Delta^{a} x$

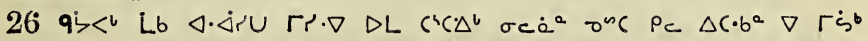

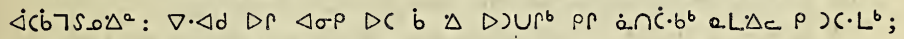

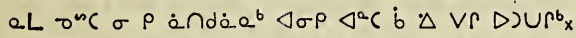

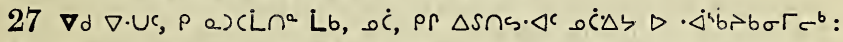

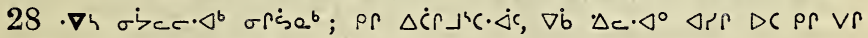
$\Delta u r^{s} \Delta C \nabla \cdot \dot{\Delta} \Delta 4 q c r b r^{b} \times$

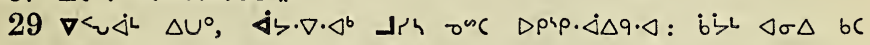
a) $C \cdot \nabla \cdot \Delta b x$

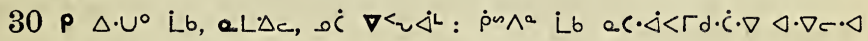
b $\sigma \wedge c r \Delta r, \quad b c \cdot q^{i} \dot{b} \cap r \cdot \Delta b^{b}$

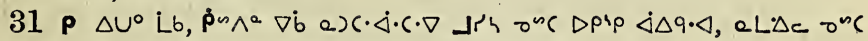

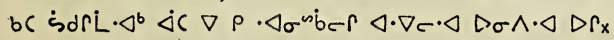

\section{A'pçе $\triangle b^{\circ} 17$.}

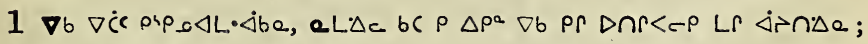

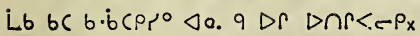

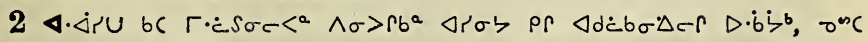

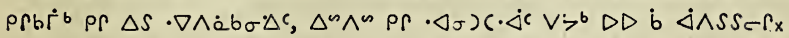

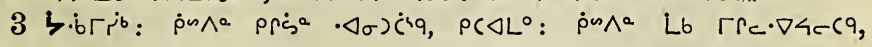
- $\nabla V \in C L^{\circ} x$

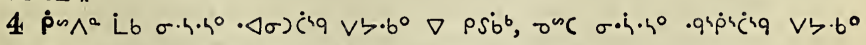

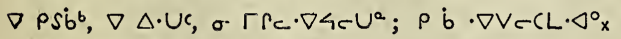

$5 \triangleleft<<^{4} C_{c^{b}} L_{b} \rho \Delta U \cdot \triangleleft^{b} \Delta \cap V e r q \cdot \triangleleft$, cPC $\left.\sigma \dot{C} \cdot V A C C\right\lrcorner \Delta \sigma \dot{a}^{a} x$ 127 


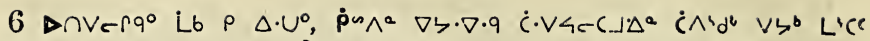

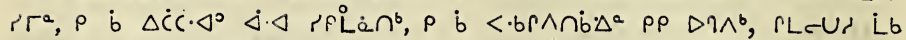

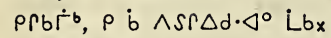

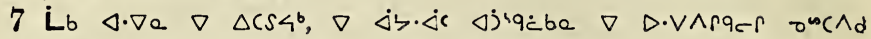

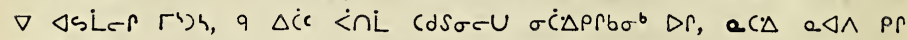
rrisa?

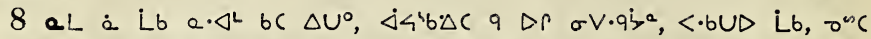

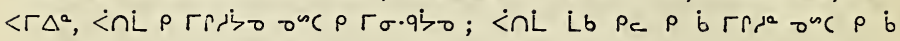
$\Gamma \sigma \cdot b^{\circ}$ ?

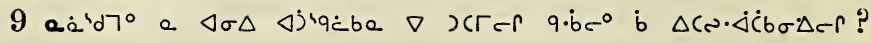
$\Delta \sim 9 b_{x}$

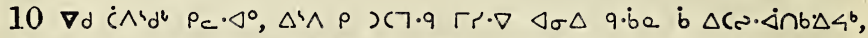

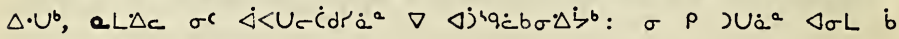
$\Delta U-\dot{C} d r^{\prime} \zeta$ b $\left.p r\right)\left(L^{b} x\right.$ becx

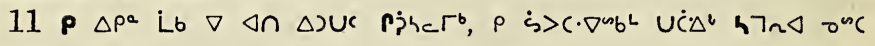

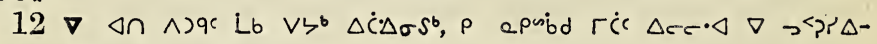

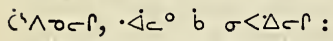

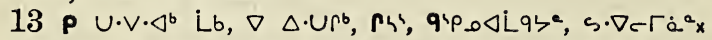

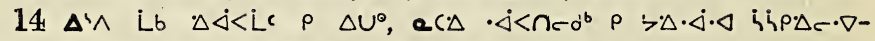

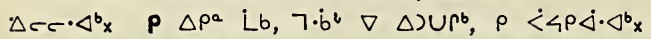

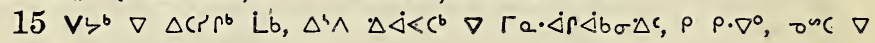

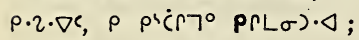

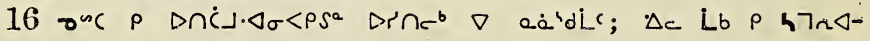
$\Delta r \Delta^{\circ} x$

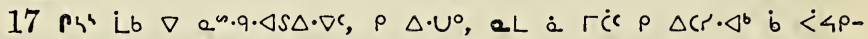

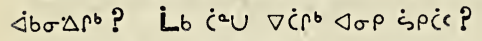

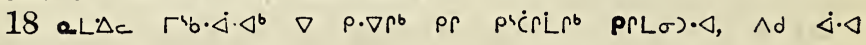
โ ${ }^{\circ}{ }^{\circ} x$

$\left.19 p \Delta U^{0} L b, \cdot \Delta \sigma^{n j} \dot{b}, \rho\right) U: \rho \dot{c} \cdot V 4 c\left(-j \Delta^{a} p \rho \Gamma a \cdot \Delta r \Delta d^{2} x\right.$

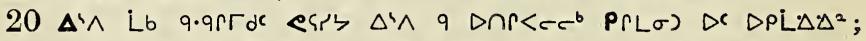

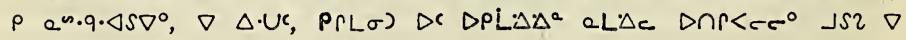
$\operatorname{\sigma r} C \cdot \Delta \dot{\alpha} \cdot b \sigma \sigma^{b} x$

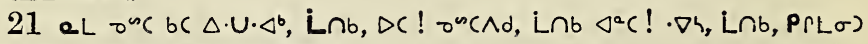
Dr $D P L \Delta \cdot \Delta^{a} P \wedge r^{b} b d \cdot \Delta a \cdot \Delta^{\circ}{ }_{x}$

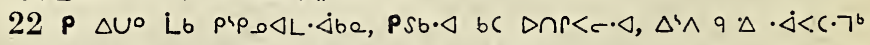

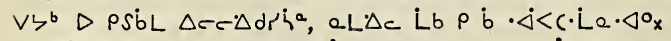

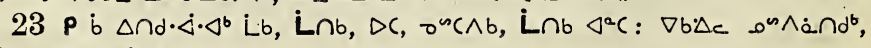
$\nabla \dot{b}$ onc 0 asibobx

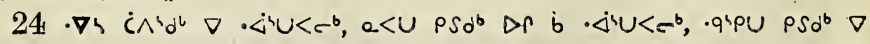

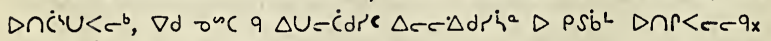

25 Lb $\wedge C L$ bc $\triangle 4 q c C L$ rqc $q \cdot \dot{b} a$, onc bc $\Delta c \cdot \nabla c \Gamma d$ d $D \dot{b}<\dot{j} \sigma^{4} q$ $\wedge$ Line $r_{x}$ 


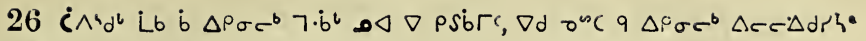
$\triangle$ PSíl $D \cap R<C C q_{x}$

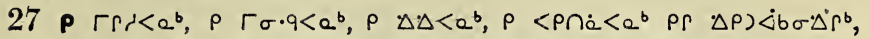

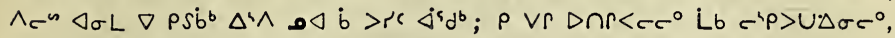

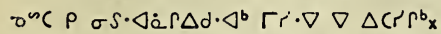

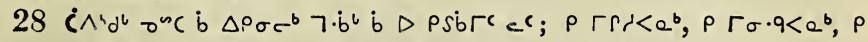

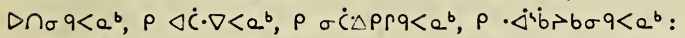

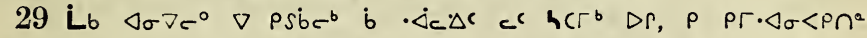

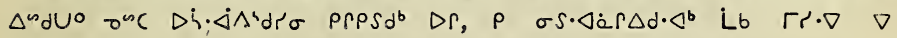
$\Delta C c_{s} p^{x}$

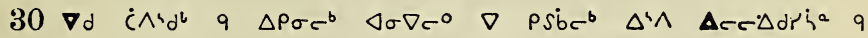
odr'c $x^{2}$

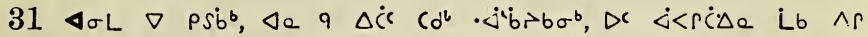

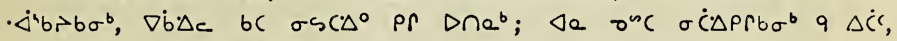
$\nabla \dot{b} \Delta c$ onc bc $V r \rho \cdot \nabla 0^{x}$

32 p'ps) $\left(L^{b}\right.$ es $\triangle . \Delta x$

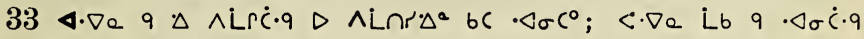
$D \wedge \operatorname{LnR} \triangle^{a}$, bc $\wedge . \operatorname{LrC}^{\circ} x$

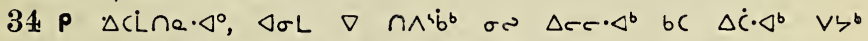
$\sigma V \Delta \sigma^{b} ; V r^{b} b C \Delta \cap e^{\circ}, d c^{b} L b$ bC $\Delta^{a} d a^{\circ} x$

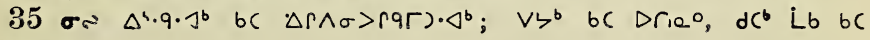
$\Delta n \operatorname{se}_{0} x$

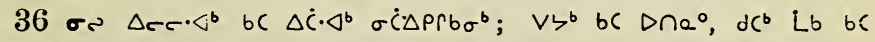
$\triangle n$ de ${ }^{x}$

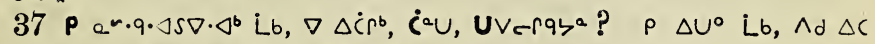

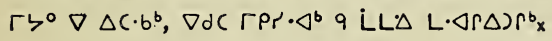

\section{$\Lambda^{\prime} p \in C_{Q} \triangle b^{\circ} 18$.}

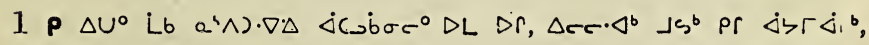
$\nabla \dot{L}$ Lb $\operatorname{Pr}$ L'brrb;

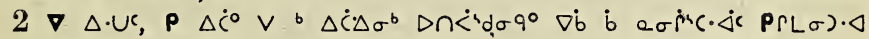
$\nabla \dot{b}$ onc b $\wedge^{n}$ iqcic $\Delta r c \cdot \Delta$ :

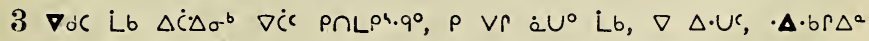
Se $b$ LRTCAC $D \Gamma_{x}$

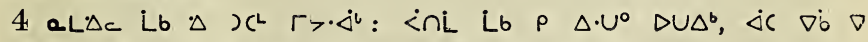

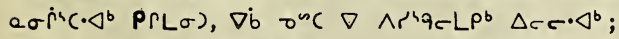

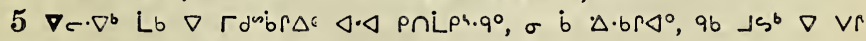
$\Delta u<\sigma$ b $\varangle 4^{i} d \Delta^{b} x$

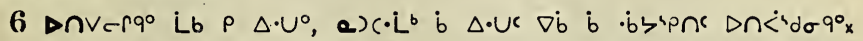

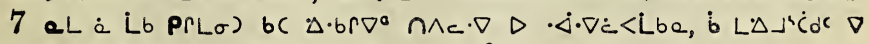

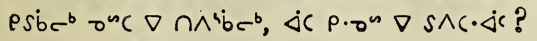




\section{$\Delta<>\rho^{4} j^{b} 18$.}

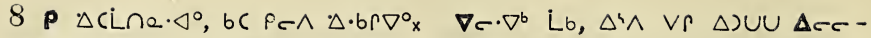

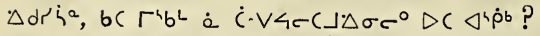

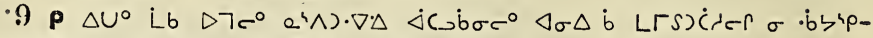

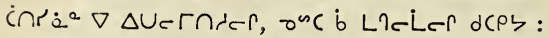

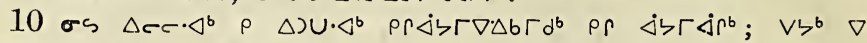
$<c \cdot \Delta c, d c^{b}$ Lb $\nabla<<<b \sigma^{*} \Delta c^{x}$

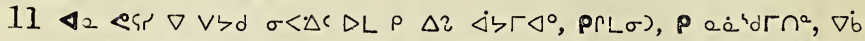

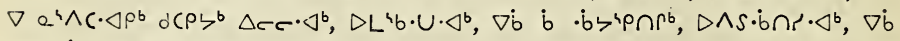

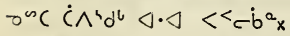

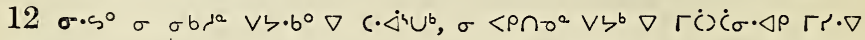

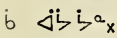

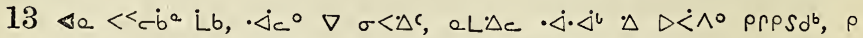

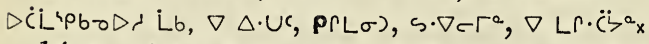

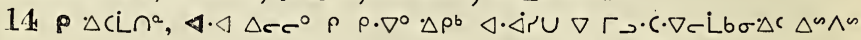

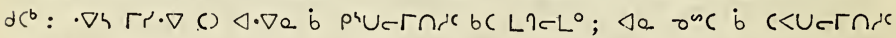
bC PrUCL ${ }^{\circ} x$

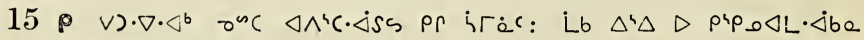
$\triangle \Delta<C \Gamma \tau r, \rho \rho C \Delta L \cdot \nabla c \cdot \Delta x$

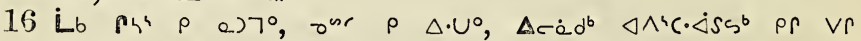

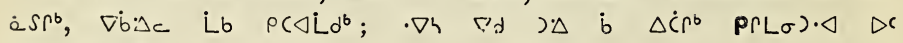
$\triangle P L \cdot \Delta \cdot \Delta \sigma \sigma^{b} x$

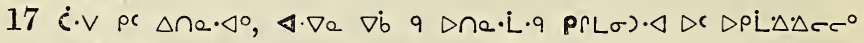

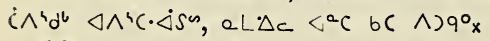

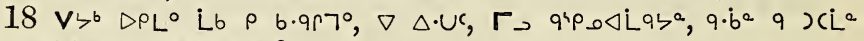

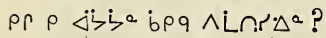

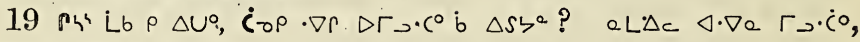
$\vee \zeta^{b} \wedge d, \nabla \cdot \Delta d P(L \sigma)_{x}$

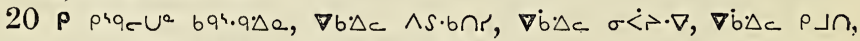

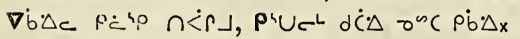

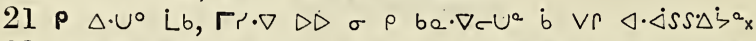

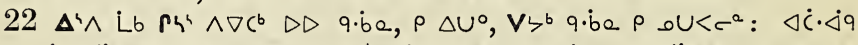

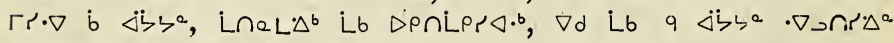

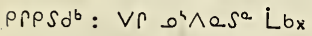

$23 \Delta^{4} \wedge$ Lb $\Delta 7 c^{\circ} \wedge \nabla C^{b}, \quad a^{4} \wedge^{6} \quad \rho \quad \Gamma \Gamma_{c} \cdot \nabla r^{\circ} ; \quad \nabla b \quad \dot{a}^{4} \wedge^{6} \quad \rho$ Lserox

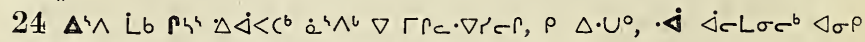

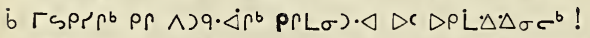

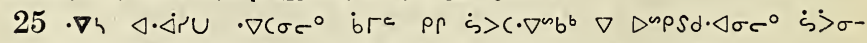

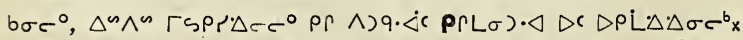

$26 \varangle \sigma \rho$ Lb b $\vee\left(\rho b \rho \Delta \cdot U \cdot \triangleleft^{b}, \varangle \cdot \nabla e\right.$ Lb $9 \rho \wedge L \Gamma \triangleright C$ ?

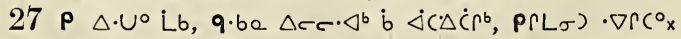

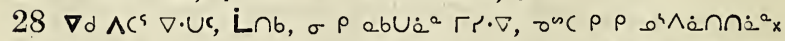

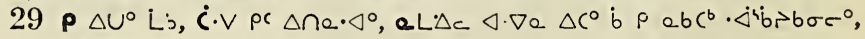
130 


\section{$D<\neg \rho^{4}$ j $18,19$.}

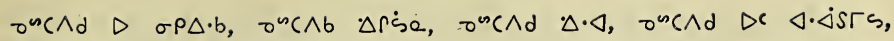
PrL $\sigma) \cdot \triangleleft D C D P L \Delta \Delta \sigma c^{\circ} \Delta R$,

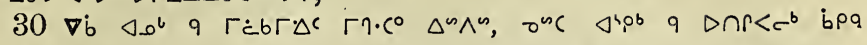
$\Lambda L \cap r \Delta \sigma \sigma^{\circ} x$

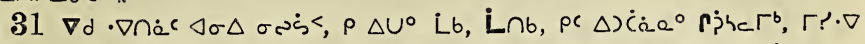

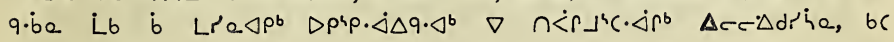
$\cap \wedge<c c \cdot \triangleleft x$

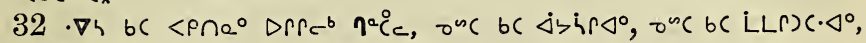
onc $b c r \cdot b c^{\circ}:$

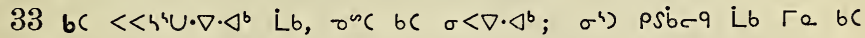
$\cdot \triangleleft \sigma^{\infty} b^{\circ} \mathrm{x}$

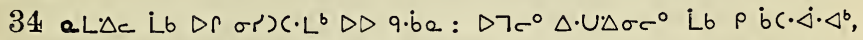

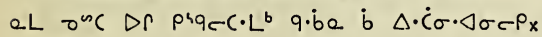

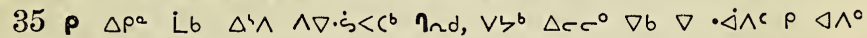

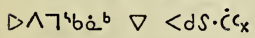

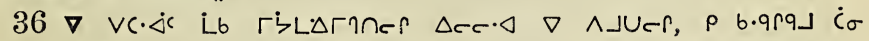

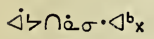

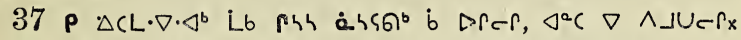

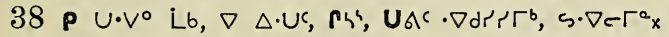

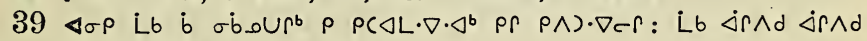

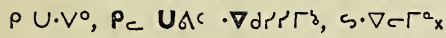

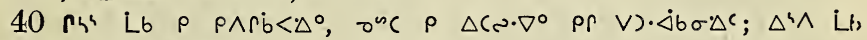
$\Lambda \nabla$ तèdtch, $b$ b. $967^{\circ}$,

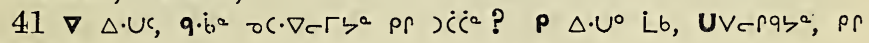
$\dot{\triangleleft} \wedge \zeta^{a} \mathrm{x}$

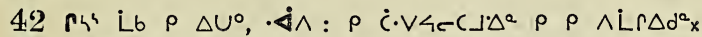

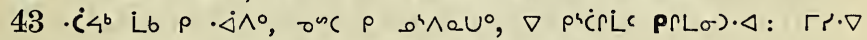

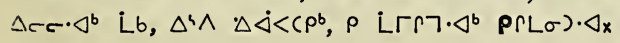

\section{Ápcre $\Delta b^{\circ} 19$.}

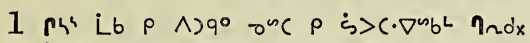

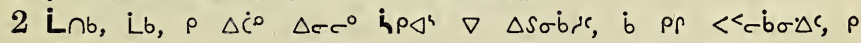
rspe Lbx

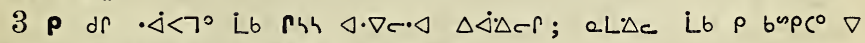
$\Delta \sim \wedge S \quad r n \omega b) \dot{a} \sigma \cdot \Delta \sigma c^{b}, \nabla h \rho\left(\Delta \dot{b}<\Delta \Delta^{\circ} x\right.$

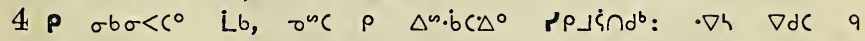
$\wedge \mathcal{J} U e \Gamma_{x}$

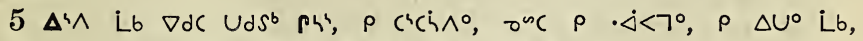

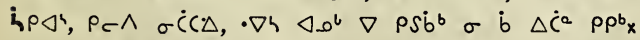

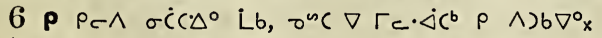

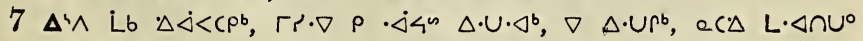
$\Delta r c \cdot \triangleleft \dot{b} L \Gamma \cdot \dot{C} c r_{x}$ 


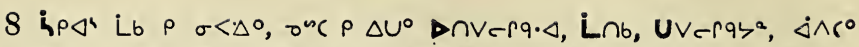

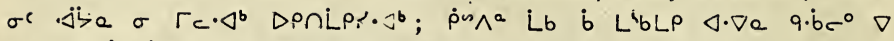

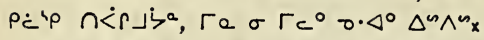

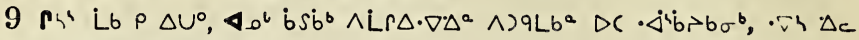
onc Dodtrid $\nabla^{\circ}<\Delta L x$

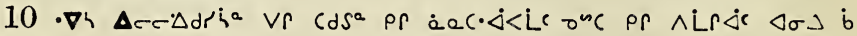
$\cdot \triangleleft \sigma S \sigma c r_{x}$

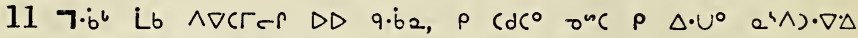

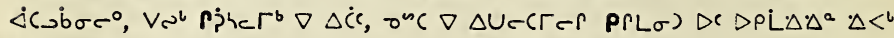
or $0 . b_{0} c^{b} x$

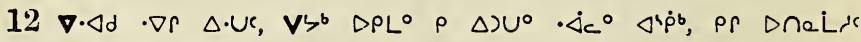
$D P L \Delta \Delta \sigma \sigma^{\circ}, \nabla d P R P \cdot \nabla c_{x}$

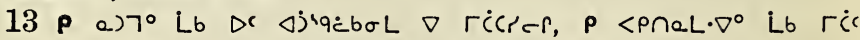

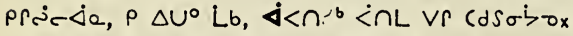

$14 \Delta \operatorname{ci} \Delta \sigma c c L$ Lb $\rho<\dot{b} \cap d$, onc $\rho<$ da $\Delta \Delta n d, \nabla \Delta \cdot U c r$, a $L \Delta c \sigma$

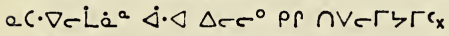

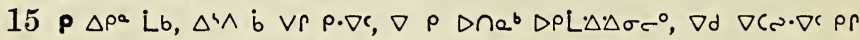

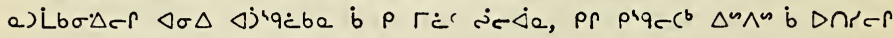
$r s \cdot \nabla() \triangle c c \cdot \Delta \nabla \Delta \dot{C} r \cdot \nabla c r$,

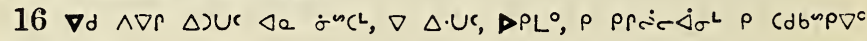
Tंc pricis $\dot{\Delta a x}$

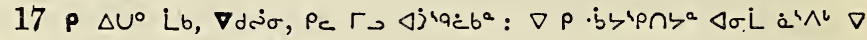
$\dot{\Delta} \wedge \dot{s} s^{b} \Delta r, \cap V \in C r \dot{C} \Delta \dot{C} \Delta e_{x}$

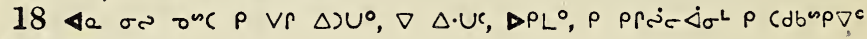
cice prise $\dot{x} e_{x}$

$19 P \Delta U^{\circ} \triangleleft \sigma \Delta$ onc, $n V \bar{c} P_{c}$ onc $\sigma^{i} c_{c}^{2} \Delta \dot{C} \Delta e_{x}$

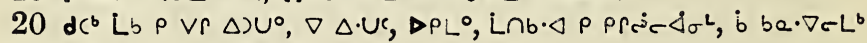

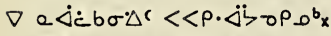

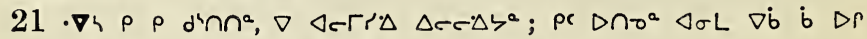

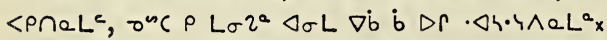

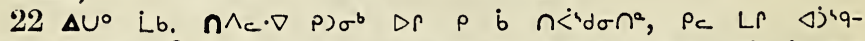
$\dot{\varepsilon} b^{a} \times \quad p$ p'q-Ui $\nabla$ बr

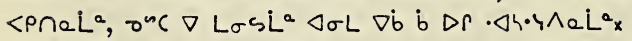

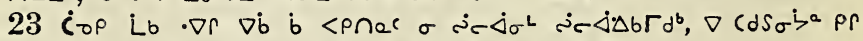

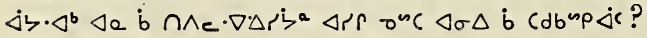

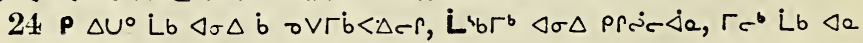

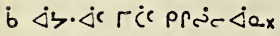

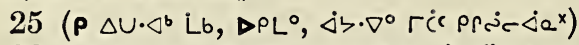

$26 \cdot \nabla h \rho c \Delta n_{a} \cdot \Delta^{\circ}, \Gamma \cdot \cdot \nabla() \triangleleft \cdot \nabla a b$ b $\Delta c b c \Gamma \dot{c} b \sigma \Delta^{\circ}: \Delta a$ Lh $\nabla \dot{b} b$

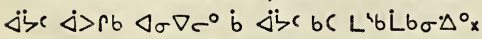

27 Lb $\varangle \sigma \rho b<\cdot b S r^{b}, \nabla \dot{b} b$ br $a C \cdot \nabla e r r^{b}$ pr $\cap V C L P b, D C V S 0^{b}$, $\sigma \dot{<} \Delta d^{b} \dot{L} b \nabla \cdot \dot{i}<L \rho^{b} x_{x}$

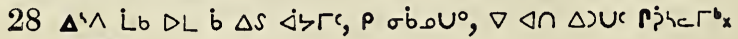
132 


\section{$\Delta<>$ Ps jb 19.}

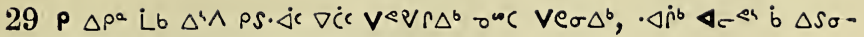

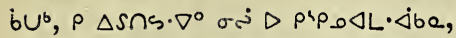

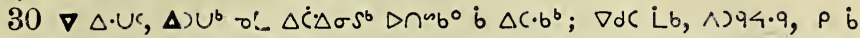

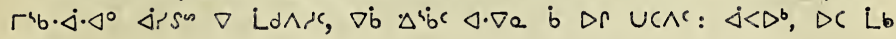
$\checkmark s^{b} x$

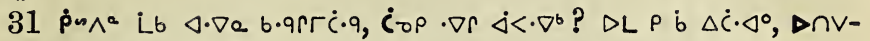
$-r q^{\circ}$ aC $\left.C \cdot \nabla C\right]^{\circ} x$

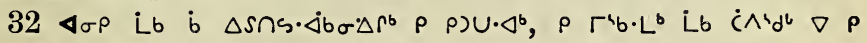
$\Delta \cap d r^{b} x$

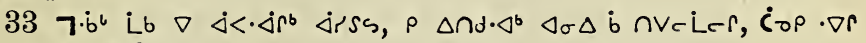

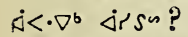

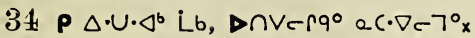

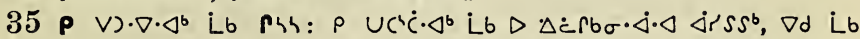

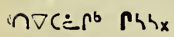

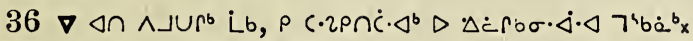

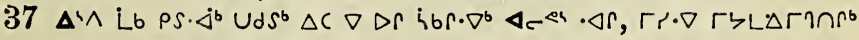

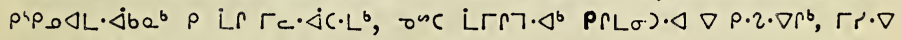

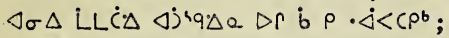

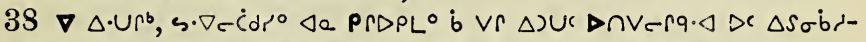

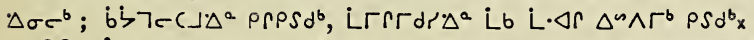

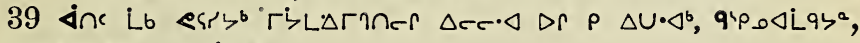
$P C \triangle L \triangle^{b} P P^{4} P \Delta \Delta L \cdot \Delta b Q^{b} x$

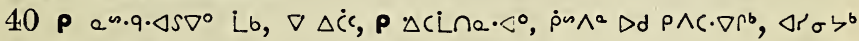
$\cdot \dot{i}<^{b} b C U \cdot v<a^{b} x$

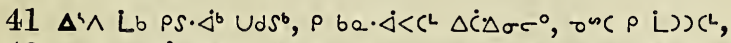

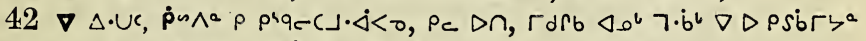

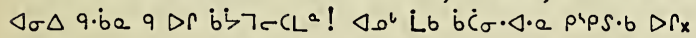

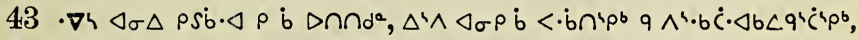

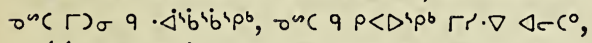

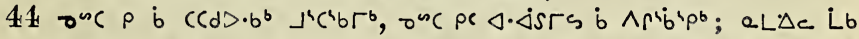

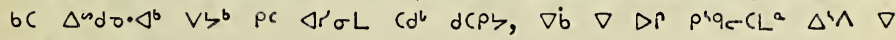
$L \cdot \triangleleft \cap \cap)^{\prime} \Delta \succ^{a} x$

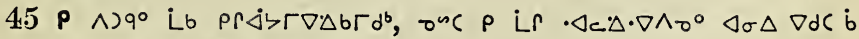
$\Delta \dot{C} \cdot \Delta \dot{q} r r, \sigma n c \Delta \sigma \Delta \dot{b} \Delta \dot{C} \cdot \nabla r r$;

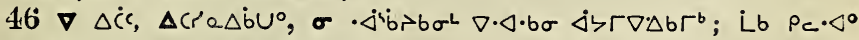

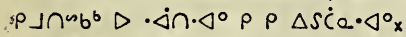

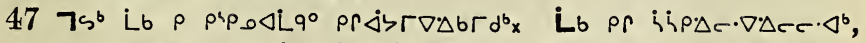

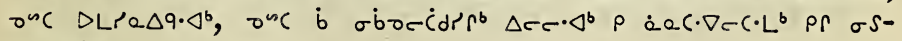
- $\Delta \dot{a} r \Delta r^{b}$;

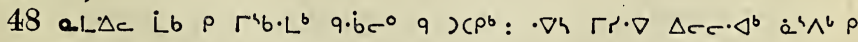
$d u \cdot b \cdot \Delta r a)\left(\cdot \nabla \cdot \Delta^{b} x\right.$ 


\section{$D<\neg$ Pb jb 20.}

\section{$\Lambda^{4} p c r e \Delta b^{a} 20$.}

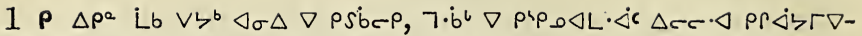

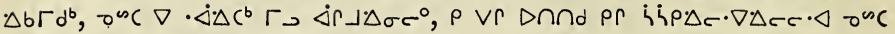

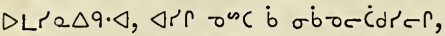

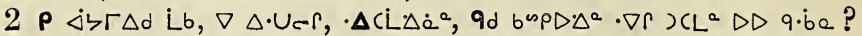

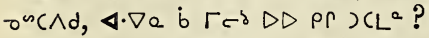

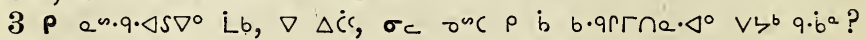

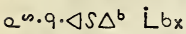

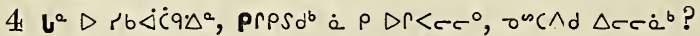

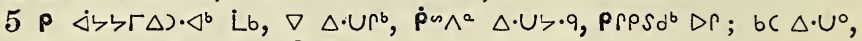
CंOP L L $\cdot \nabla C \nabla \dot{G} \rho \dot{C} \cdot V C \cdot \nabla b$ ?

6 Ĺb $\dot{\rho} \backsim \wedge^{a} \Delta \cdot U \zeta \cdot q, \Delta c c \dot{a}^{b} D \Gamma ; \Gamma r \cdot \nabla \Delta c c \cdot \Delta^{b} \rho \dot{b} \wedge \cdot L r \dot{a} C>d a \cdot \triangleleft^{b}:$ $\cdot \nabla h \dot{C} \cdot V \cdot \Delta q C C \cdot L^{b} \nabla$ D plp. $\Delta \Delta q \Delta r r$ lex

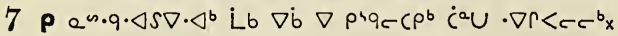

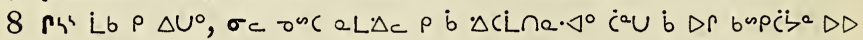
q.ba $P r) C L^{a} x$

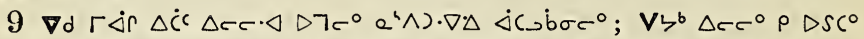

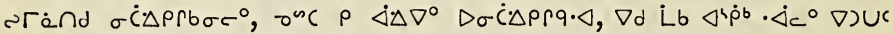
b.os pr $\Delta \dot{C} \wedge \dot{r} \dot{c}_{x}$

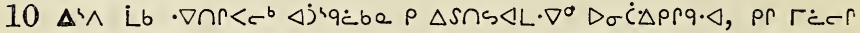

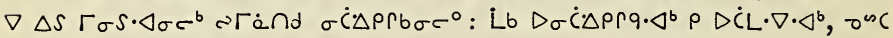
$\wedge s s^{b} \rho \operatorname{sins} \cdot \nabla \cdot \triangleleft^{b} x$

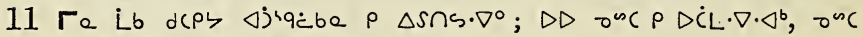

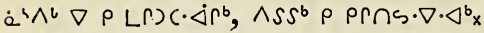

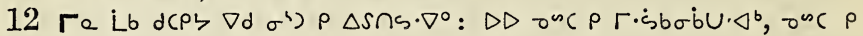
$\cdot \triangleleft c \Delta \cdot \nabla \wedge \tau \cdot \triangleleft^{b} \mathrm{x}$

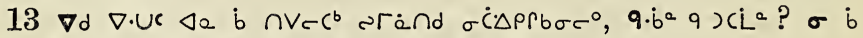

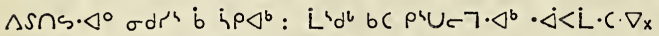

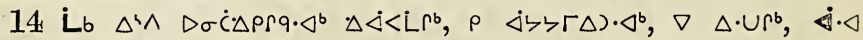

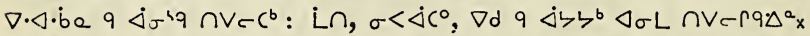

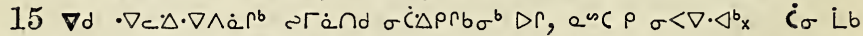

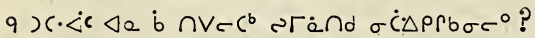

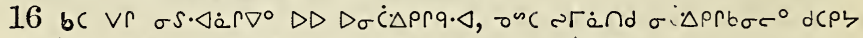
$b c \Gamma>^{\circ}{ }_{x} \quad \Delta^{\prime} \wedge L b \wedge \nabla c \rho b, \rho \Delta \cdot U \cdot \Delta^{b}, a L \Delta c b c \Delta \Delta p^{a} a_{x}$

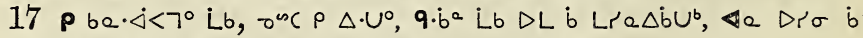

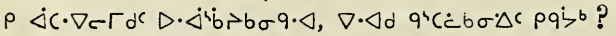

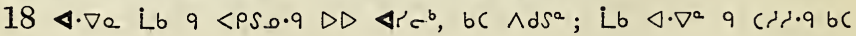
$\lambda r>c d x$

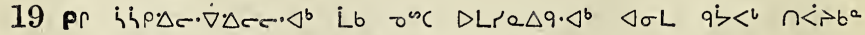

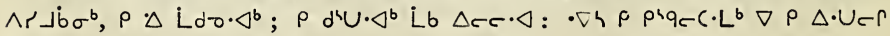

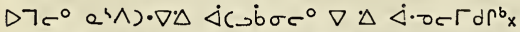

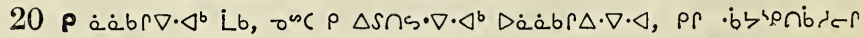
134 


\section{$\Delta<>\rho^{4} \neq 620$.}

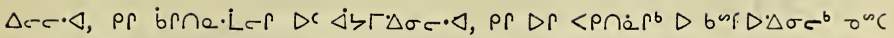
$\triangle \cap V \in \Gamma Q \Delta \sigma c^{b} D P L \cdot \triangleleft x$

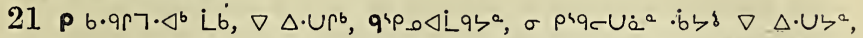

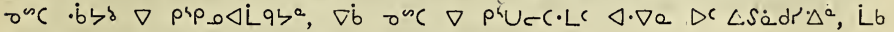

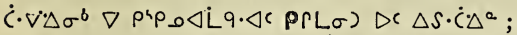

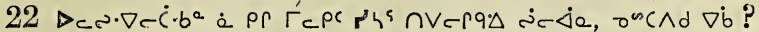

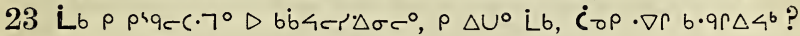

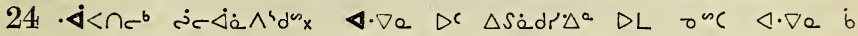

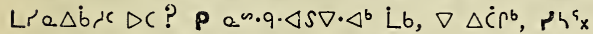

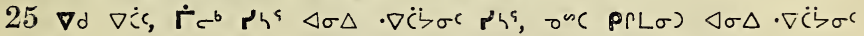
PrLo) $x$

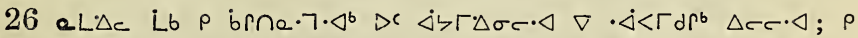

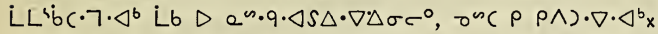

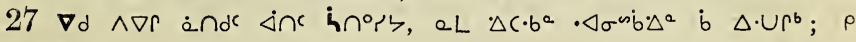
b.9r厂d Lb,

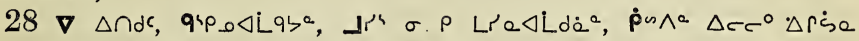

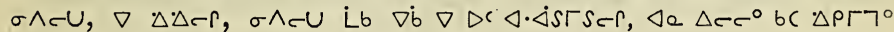

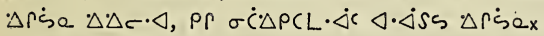

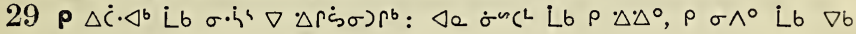
$\nabla$ D $\triangle \cdot \Delta S T S C^{x}$

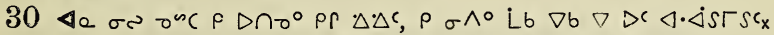

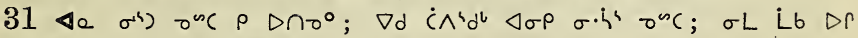
abU. $\triangleleft^{b} \triangleleft \cdot \triangleleft s s, \rho \sigma \wedge \cdot \triangleleft^{b}$ Lbx

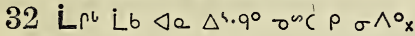

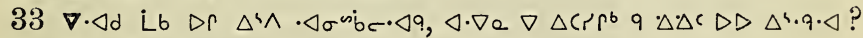

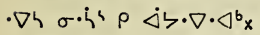

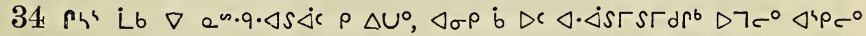
$\triangle P) \cdot \Delta^{b} \tau^{\infty}(\Delta P) \Delta b \sigma \Delta \cdot \nabla^{b}:$

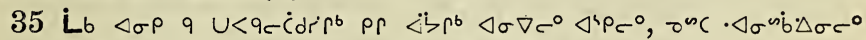

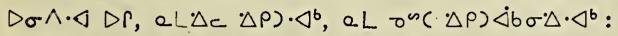

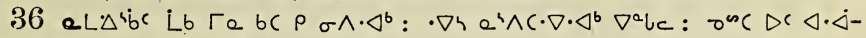

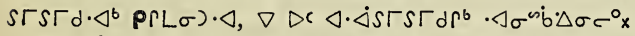

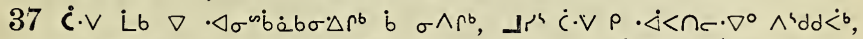

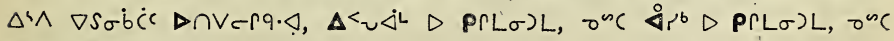
$\left.\eta_{6}<\Delta P \Gamma L\right) L x$

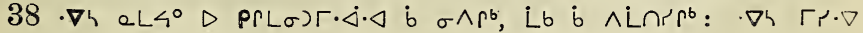
$\wedge i \cap r^{\circ} \dot{C} d x$

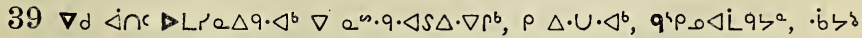
p $\rho \Delta \cdot \dot{c}^{a} x$

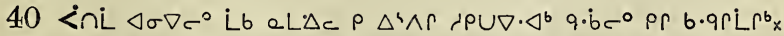

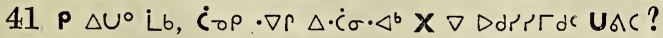

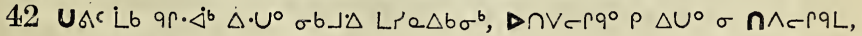
$\varangle \wedge \rho_{c} \sigma \rho \rho^{\prime} \dot{\rho} b$, 


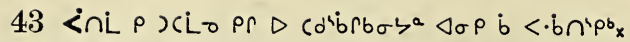

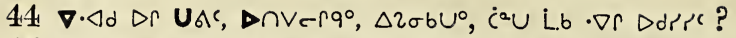

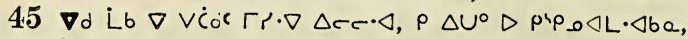

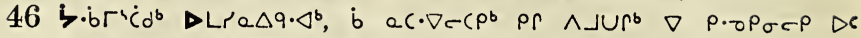

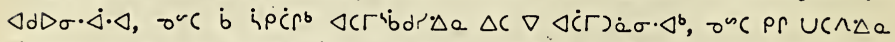

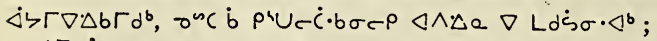

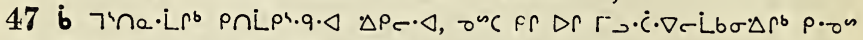

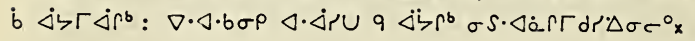

\section{$\Lambda^{\prime} p C r a \triangle b^{2} 21$.}

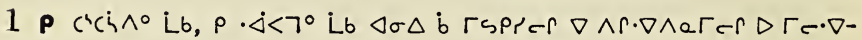
$\Delta \sigma c \triangleleft \dot{A}^{\circ} \Delta a \Delta b \Gamma d^{b} x$

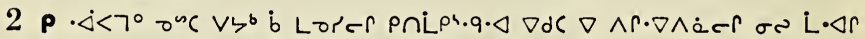

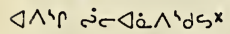

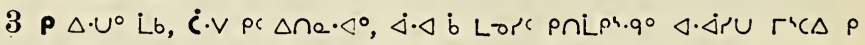

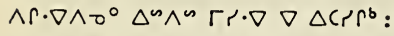

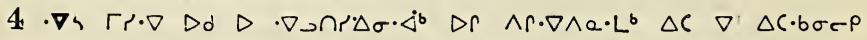

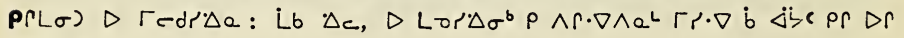
$\wedge \operatorname{Ln} r^{x}$

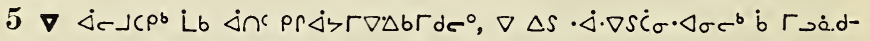

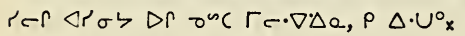

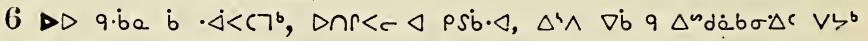
$\Delta r \sigma \nabla U C \wedge c^{\prime} d C P>\nabla \dot{b} q \sigma r \cdot \nabla \wedge \dot{a} b \sigma \cdot \Delta c_{x}$

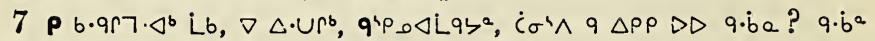

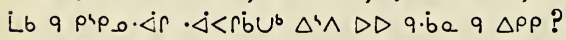

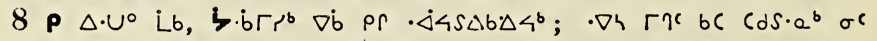

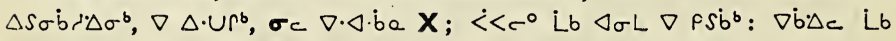
$0^{4} \wedge \dot{a} \cap d^{b} x$

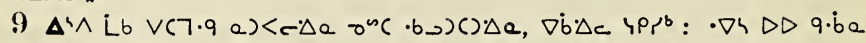

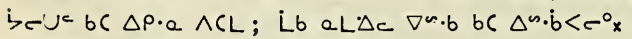

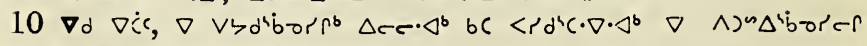

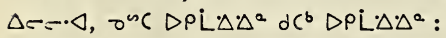

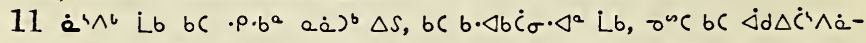

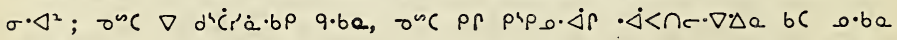
$b S d^{b} D r_{x}$

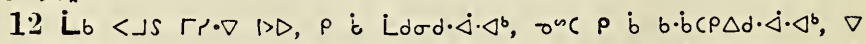

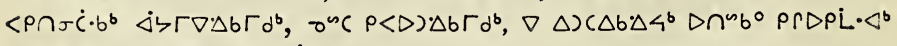
onc $\Delta \cap V e r q \cdot \Delta^{b} \sigma^{c} \Delta S_{\sigma} b_{i} \lambda^{2} \Delta r_{x}$

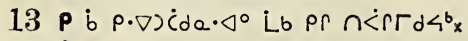

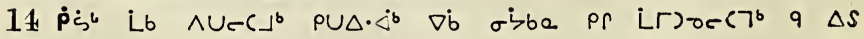
c $\backsim . q \cdot \triangleleft S \triangle \cdot \nabla 4^{b} \mathrm{x}$

\section{6}




$$
D<=\text { ps jb } 21 .
$$

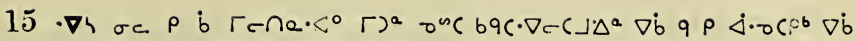

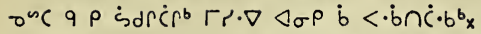

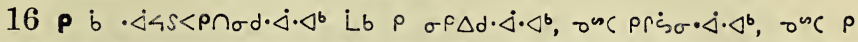

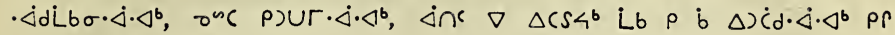
$\sigma \dot{<} \Delta b \Delta \zeta^{b} \mathrm{x}$

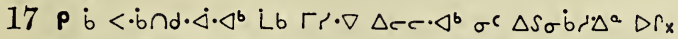

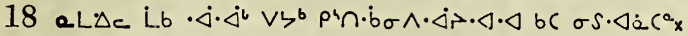

$19 \nabla s \wedge c \cdot \nabla r<^{b} b e \cdot \nabla c \Gamma d^{b} \rho c \Delta i d \cdot \Delta \cdot \Delta^{b} x$

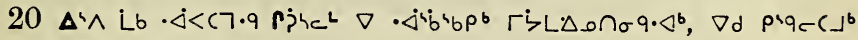
$\nabla \vee v \dot{a} \cdot b_{b}$ or $\sigma s \cdot \Delta \dot{s} C^{b} x$

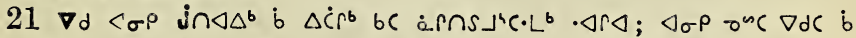

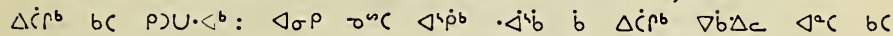
$\wedge \supset q \cdot \Delta^{b} x$

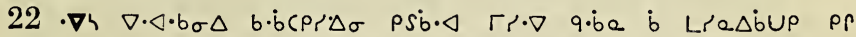
$\cap \wedge<-P_{x}$

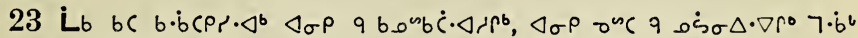
$\Delta>9$ psibe $\dot{\Delta d e} \cdot \nabla r \cdot \Delta \sigma \sigma^{\circ} \mathrm{x}$

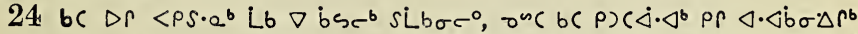

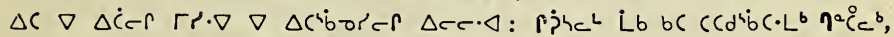
$\dot{<} \cap \mathrm{L} \cap \Lambda<c c \cdot b \cdot \nabla \eta^{\circ}{ }^{\circ} c_{c}^{b} \nabla \rho \operatorname{sib} \Gamma \cdot \dot{\Delta} \cdot \Delta_{x}$

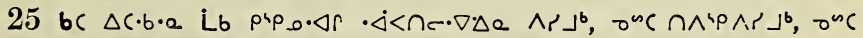

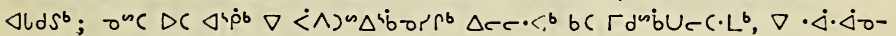
- $C^{b b} ; \operatorname{\rho rb\Gamma } \nabla \rho \cdot 2 \cdot \nabla L b^{b}, \tau^{n} C b^{b} b a^{b} \nabla \rho \cdot 2 \cdot \nabla \zeta b L \varangle \rho^{b}$;

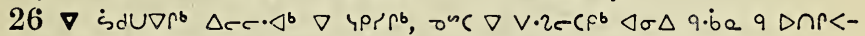

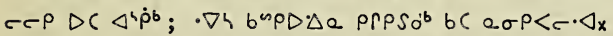

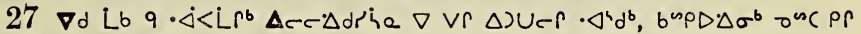
$\rho^{c} U \subset \dot{C} d r \Delta \sigma^{b} x$

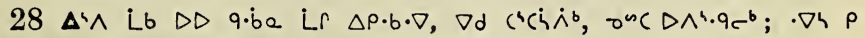
$\Lambda L r \Delta d r^{\prime} \Delta \sigma^{\cdot} \cdot \Delta^{\circ} \rho S \cdot \triangleleft^{b} \vee r \Delta\left(\cdot b^{a} \times\right.$

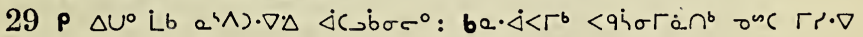
$r^{4} \cap \cdot 6^{6}$;

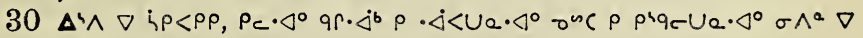

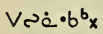

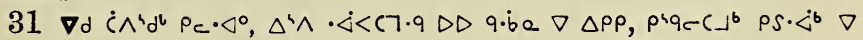
$\Delta\left(\cdot b \sigma \sigma^{b} p(L \sigma) D C D P L \Delta \Delta^{a} x\right.$

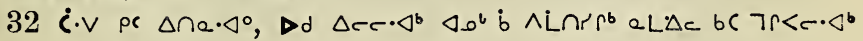
$\dot{<}$ L $\Gamma^{\prime} \cdot \nabla \rho \cap \wedge<-q_{x}$

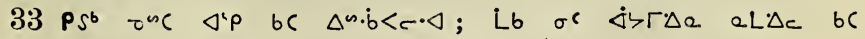
$\Delta u \cdot \dot{b}<c \cdot \Delta_{x}$

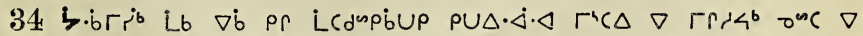

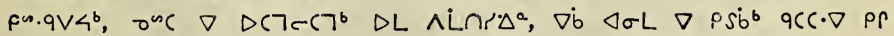
D) $\cap d^{b} \times$ 


$$
\Delta<\rightarrow \rho^{4} \text {; } 21,22 .
$$

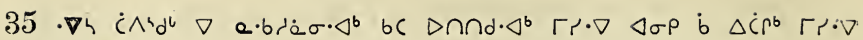
$\nabla 4 \wedge \dot{s}^{6} \triangleleft^{4} \dot{\rho}^{6}{ }^{6}$

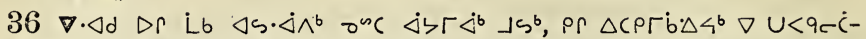

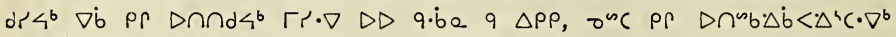
$\Delta r-\Delta d r i i^{2} x$

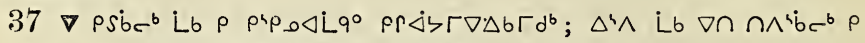

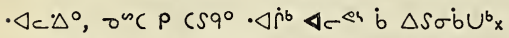

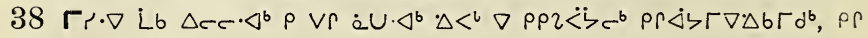
$v \cdot c \cdot \Delta r^{b} x$

\section{A'pcre $\triangle b^{\circ} 22$.}

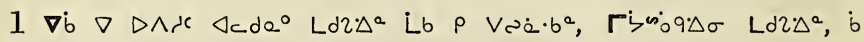
$\triangle S \sigma \dot{b} U^{b} \times$

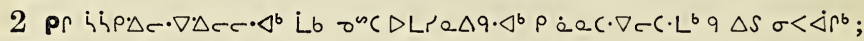
- $\nabla h \rho$ d $P \cdot \Delta^{b} \Delta r c \cdot \Delta x$

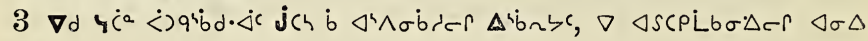
aतsं<x

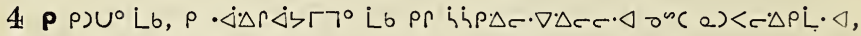
q $\triangle S \cdot \dot{\Delta} \angle S<P \cap Q L \cdot \dot{\Delta c_{x}}$

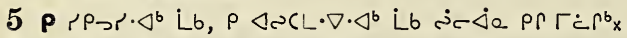

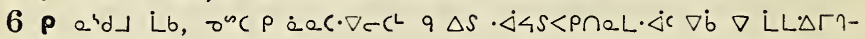
$\operatorname{ner} \wedge c c \cdot \Delta x$

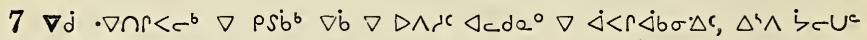

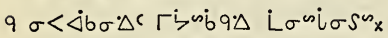

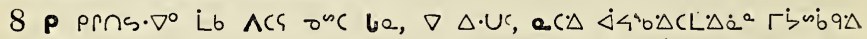

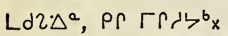

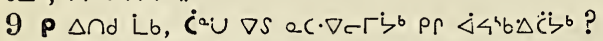

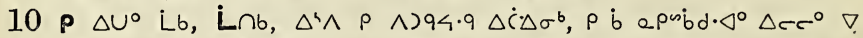

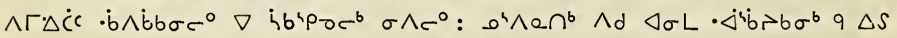
$\wedge) q_{x}$

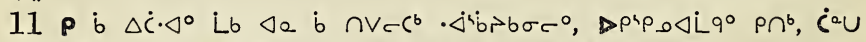

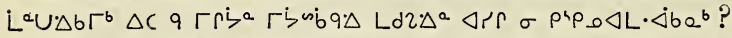

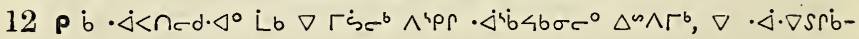
$U c^{b}: \nabla d C<s^{b} b \Delta \dot{c}^{b} x$

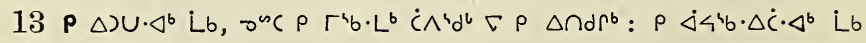

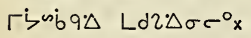

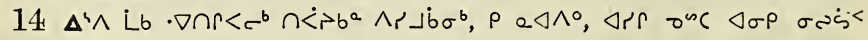
$\Delta<^{4} c c^{b} x$

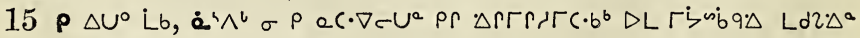
$<\lrcorner S \quad \triangle 49 C C^{\circ}{ }^{\circ}:$

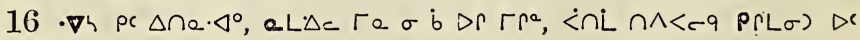
$\triangle P L \Delta \Delta \sigma^{b} x$ 


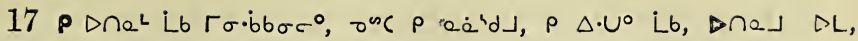
LneĹ') Lb :

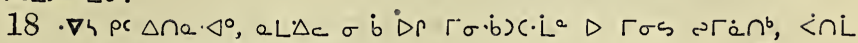
$D \cap R<C C$ P $P(\sigma)$ DC DPL $\Delta \Delta^{a} \times$

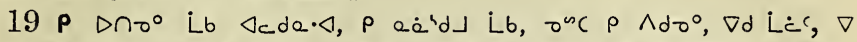

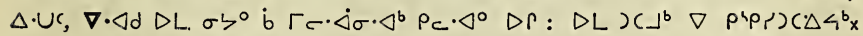

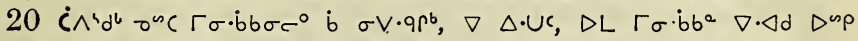
$\left.a^{4} d \Gamma\right) \Delta^{a} \sigma \Gamma d^{b}, b c \cdot \Delta^{\circ} b \quad b r<\rho \sigma b U^{b} x$

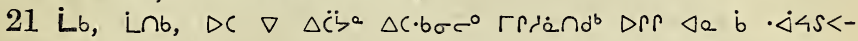
$P \cap \sigma^{C} x$

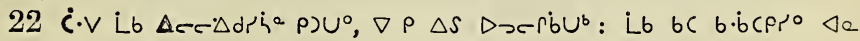
$\Delta r c^{\circ} q \cdot \dot{\Delta}\langle s<p \cap \dot{a} c$ !

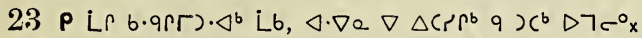

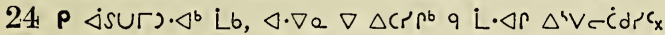

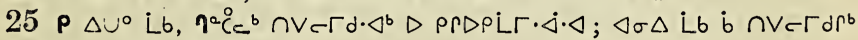
$\Delta S \sigma \dot{b} \dot{c} b \sigma \cdot \Delta c \cdot \triangleleft D \Gamma د) \dot{c} q \cdot \Delta b x$

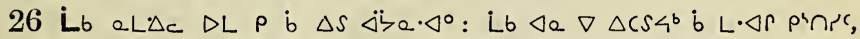

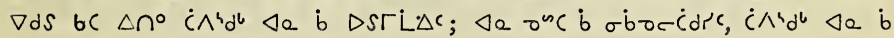
$\wedge \Gamma \triangle \cdot \nabla c_{x}$

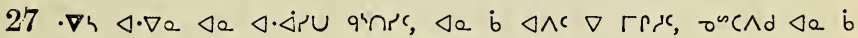

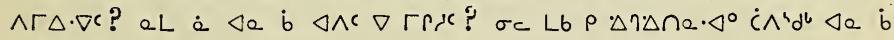
$\wedge \Gamma \triangle \cdot \nabla c_{x}$

$28 P_{c} \cdot \triangleleft^{0} \nabla \cdot \triangleleft \cdot b \sigma \rho$ b $\rho \Delta \eta \Delta\left\langle^{b} \sigma\right.$ b. $9 r \Delta d d^{\cdot} \Delta \sigma^{b}:$

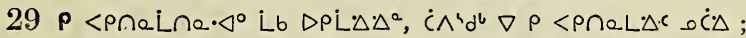

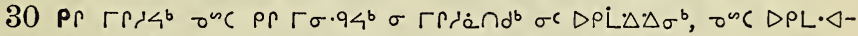

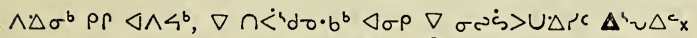

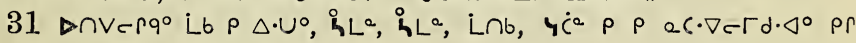

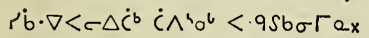

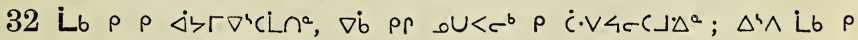

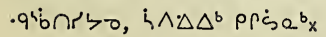

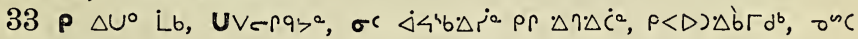
$\sigma \wedge \Delta \sigma^{b} \Delta S_{x}$

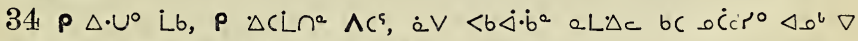

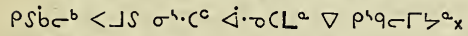

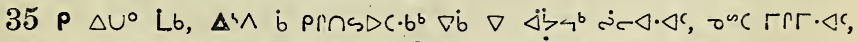

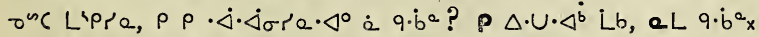

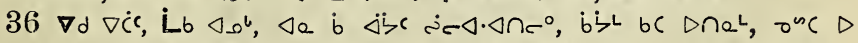

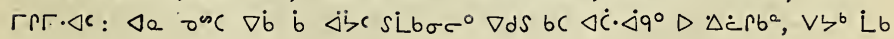
bc $\triangleleft \dot{C} \cdot \nabla^{\circ} x$

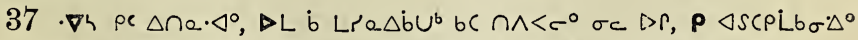
Lb $\triangleleft \sigma \Delta \dot{b} \cdot \triangleleft \sigma)(\Gamma c \rho: \cdot \nabla h \triangleleft \sigma \Delta q \cdot \dot{b} a \dot{b} \Delta \dot{C} \Gamma \Gamma b \cdot \dot{b} a b c \Delta u \cdot \dot{b}<\sigma \cdot \Delta x$

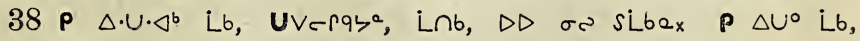
$\nabla \cdot \dot{b} \sigma x$ 


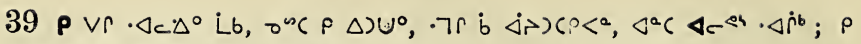

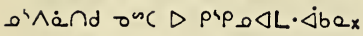

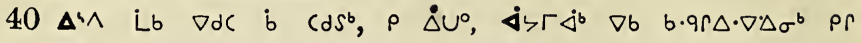
$\Delta x<c^{b} x$

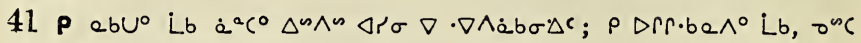
$\rho \dot{\triangleleft} \zeta \Gamma \dot{\triangleleft}^{\circ} x$

$\left.42 \nabla \Delta \cdot \cup c, 0 \dot{c}, \dot{\beta}^{\sim} \wedge^{a} \cdot \dot{\triangleleft} x\right\lrcorner \cdot \Delta_{\sigma}, \Delta b \cup a L \Delta^{a} \Delta L \Gamma_{\sigma} \cdot b b^{a}: \nabla c \cdot \nabla^{b} L b$, e L $\Delta c \sigma_{c} \nabla \Delta U-C L^{a}, L_{b} \nabla \Delta U c C L^{a} P_{c}, b c \Delta p^{a} x$

$43 P$ odricd Lb $\nabla^{a}$ le pripsdb $D \Gamma \nabla h \wedge \Delta \Delta d^{2} x$

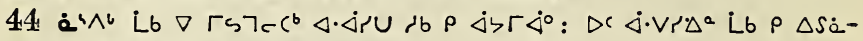

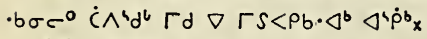

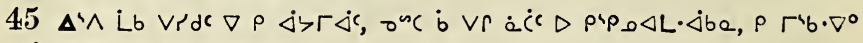

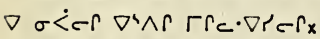

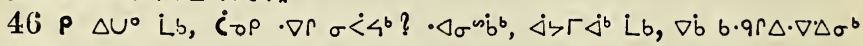
pr $\Delta$ is $<<^{b} \mathrm{x}$

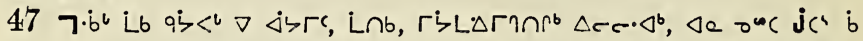

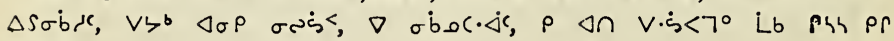
$\Delta>\mathrm{L} c_{x}$

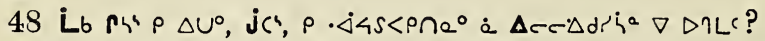

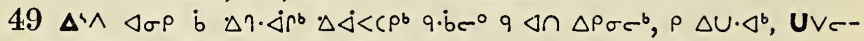

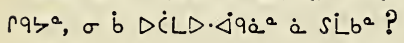

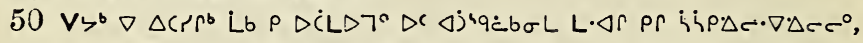
$\rho$ L $\sigma S \cdot 7^{\circ}$ Lb $D C \cdot \Delta b \sigma^{\circ} D$ Próp $\sigma^{b} \Delta U q_{x}$

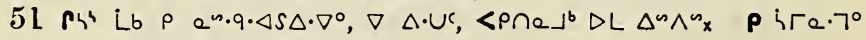
Lb $\triangle C \cdot \Delta b^{\circ} e^{\circ}$, onc $\rho \Gamma \mathrm{e} \cdot \Delta \mu C \cdot \nabla^{\circ} x$

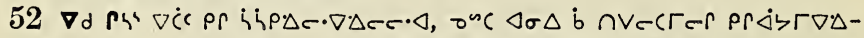

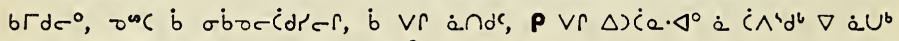

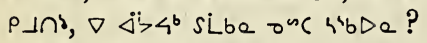

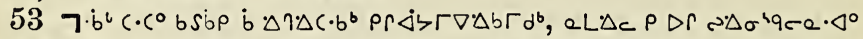

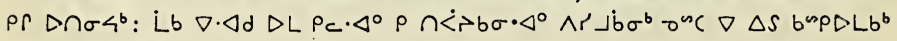

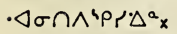

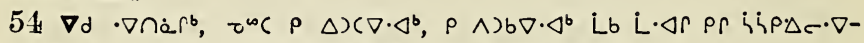

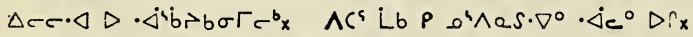

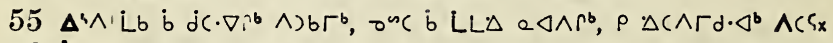

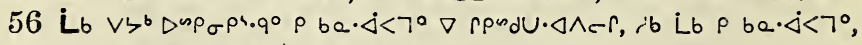

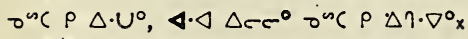

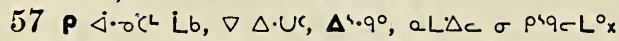

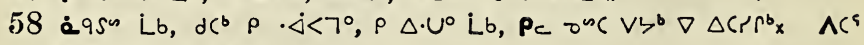
Lb $\rho \Delta \cdot \cup^{\circ}, \Delta c c^{\circ}, a L \Delta c \sigma c^{x}$

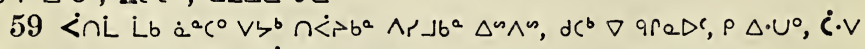

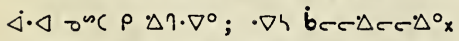

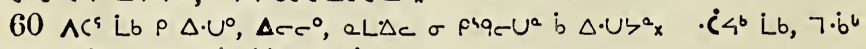

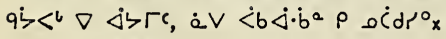




\section{$\Delta<\neg$ p' jb 22, 23.}

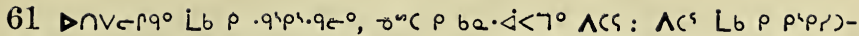

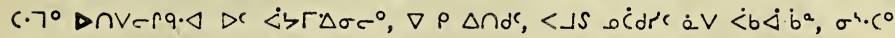
$\rho \dot{b} \dot{4} \cdot 0 V^{a} \nabla$ plqc $\Gamma b^{2} x$

$62 \wedge c^{s} L L_{b} \rho \cdot \Delta c \Delta^{\circ}, s^{\circ} L_{b} \rho L J_{x}$

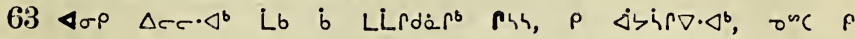
$D C L \cdot \nabla \cdot \triangleleft^{b} \mathrm{x}$

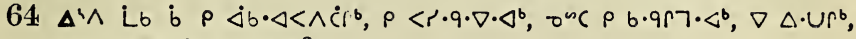
$<\cdot b a^{\circ} \Delta C, \triangle \cdot \nabla a$ b $<$ <. $9 D$ b?

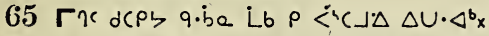

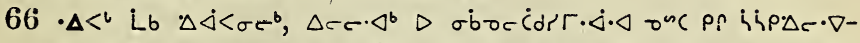

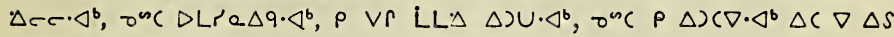

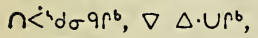

$67 p_{c} \dot{a} \Delta a X$ ? $\Delta C L \cdot \dot{a}^{a} x$ p $\Delta U^{\circ} L_{b}, \dot{p} \wedge \wedge^{a} \Delta C L C \cdot b q, a L_{c} \rho \dot{b}$

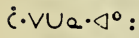

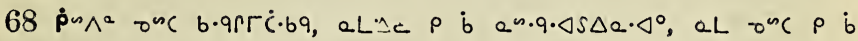
$<P \cap \sigma e \cdot \Delta^{\circ} x$

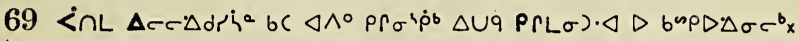

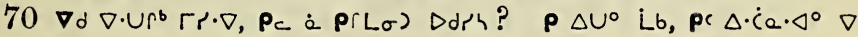
$\Delta \Delta \dot{\Delta} \mathrm{i} x$

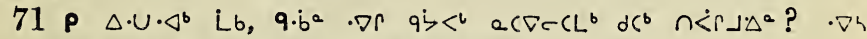

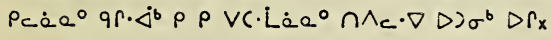

\section{A $P C$ PQ $\triangle b^{2} 23$.}

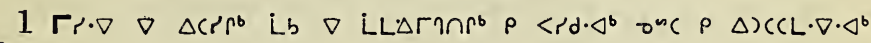
$<c<x$

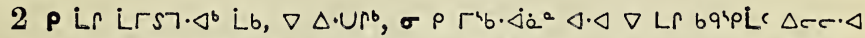

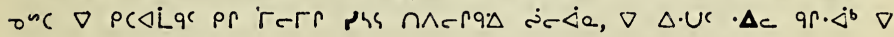
$X \cap \Delta C \nabla$ FrDPL $\triangle C_{X}$

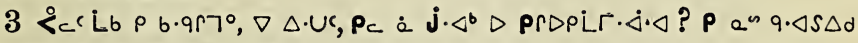
Lb, $\nabla \Delta \cdot U_{c} r, p_{c}$ pe $\Delta \cdot \dot{c}^{a} x$

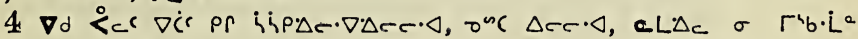
$L r \cdot c \Delta \sigma c^{\circ} \nabla \Delta \dot{\Delta C} \dot{\Delta} \cdot \Delta \Delta r c^{\circ} x$

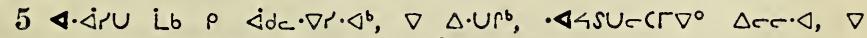

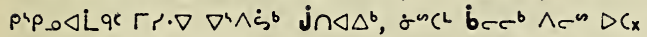

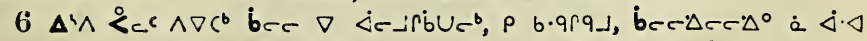
$\Delta r^{\circ}$ ?

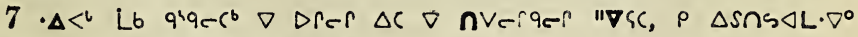

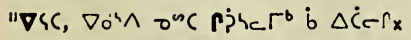

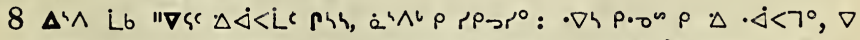

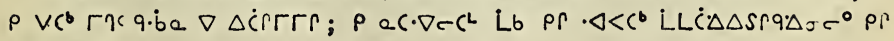
$x<r<x$

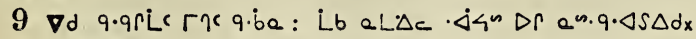
141 


\section{$\Delta<\rho^{4} j^{b} 23$.}

10 er hib. $\operatorname{LrS} 7 \cdot \triangleleft^{b} \mathrm{x}$

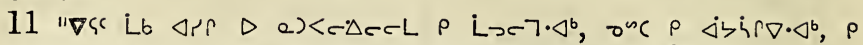

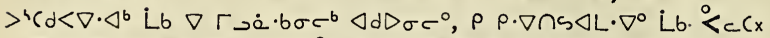

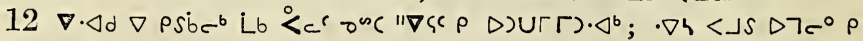
$<\cdot b \cap) \cdot \triangleleft b x$

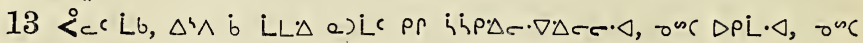
$\Delta r \cdot \cdot \triangleleft$,

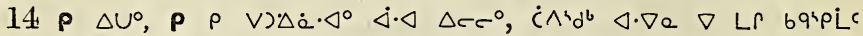
$\Delta c c \cdot \triangleleft:$ Lnb, Lb, $\sigma c, \nabla \rho \cap \dot{<}^{\prime} d a^{b} \nabla \cdot \dot{\Delta}<\Gamma \zeta^{b}$, a L $\Delta c \sigma \Gamma^{b} \cdot b \cdot L^{a}$

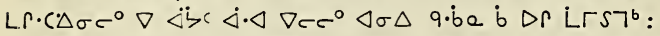

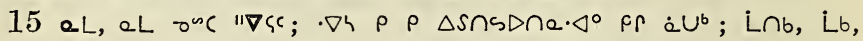

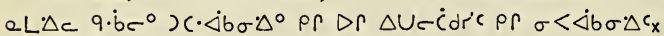

$16 \nabla \cdot \Delta d \Delta r_{G} \dot{b}<b^{4} U \cdot \Delta^{\circ}, \nabla d$ Lb $q<P \cap a^{b} x$

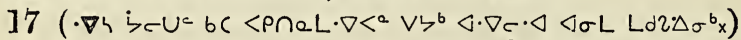

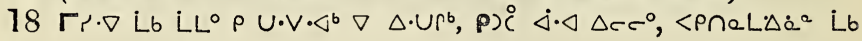
$<\dot{<}<$ :

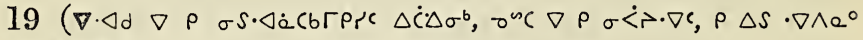
$\left.p<D>\Delta b \Gamma d^{b} x\right)$

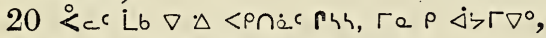

21 L $b \rho U \cdot V \cdot \Delta^{b}, \dot{r^{2}} \dot{c}^{c} \cdot b^{\circ}, r^{4} \dot{c}^{4} \cdot b^{\circ} x$

$22 \rho \Delta U^{0}$ Lb, $\nabla d \sigma^{4} \cdot c^{\circ}, \dot{c}_{-} \rho \cdot \nabla r, q \cdot b \sigma^{\circ} b$ Lr) $c^{b}$ ? a $L \Delta c \sigma \Delta r$

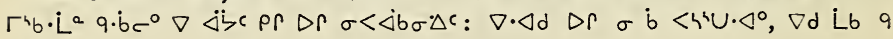
$<p\rangle_{a} b_{x}$

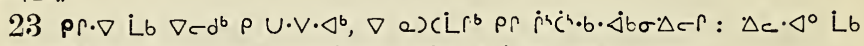

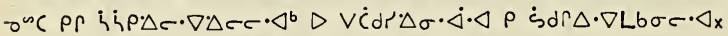

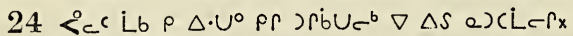

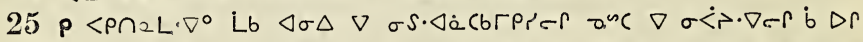

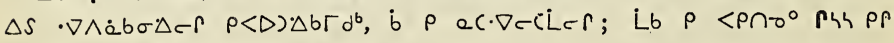
$x \cdot \Delta b \sigma \Delta c r \nabla \Delta S$ ec $\cdot \nabla-c r c r x$

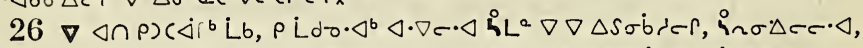

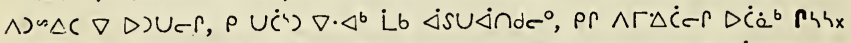

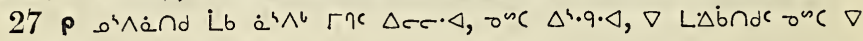

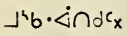

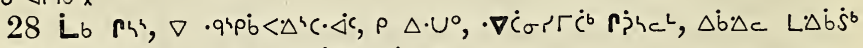

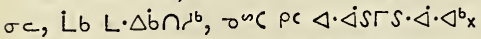

$29 \cdot \nabla h, \dot{L} \cap b, \dot{<}<c \cdot \triangleleft \rho S \dot{b} \cdot \triangleleft, \Delta^{4} \wedge q \Delta \cdot \dot{C} \sigma^{\cdot} \cdot \triangleleft^{b}, s \cdot \nabla-\dot{C} d r \cdot \triangleleft^{b} \triangleleft_{\sigma} P \nabla \dot{b} \dot{b}$

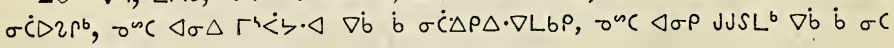
गंड $\sigma \Delta \cdot \nabla r^{b} \mathrm{x}$

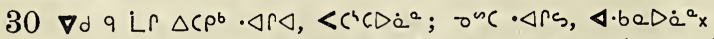

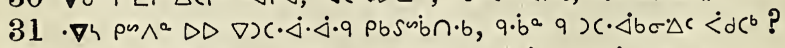

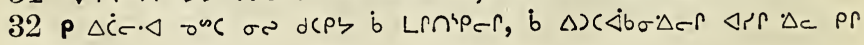

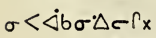




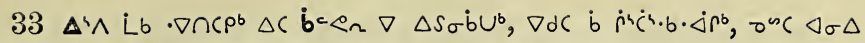

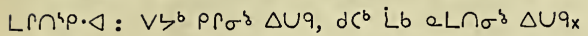

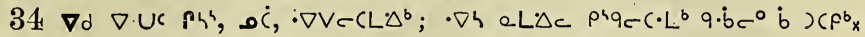

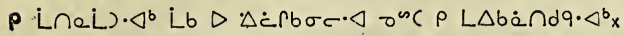

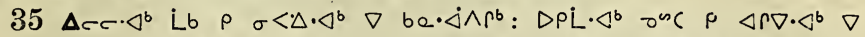

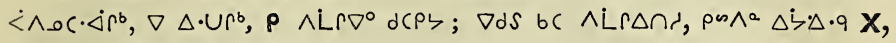
$\dot{b} \cdot \dot{\Delta} \cdot \nabla \dot{c}<\Gamma d^{\prime}(P r L \sigma) \cdot \Delta x$

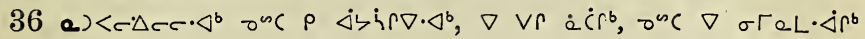
$s \Delta \dot{b}>c^{\circ} \times$

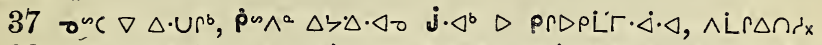

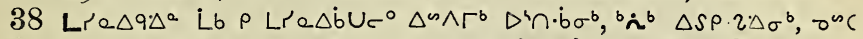

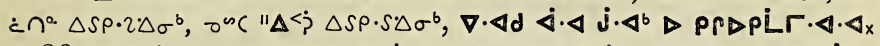

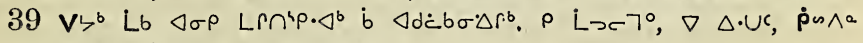

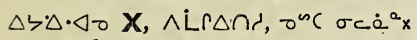

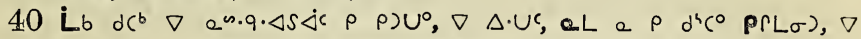

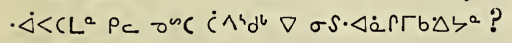

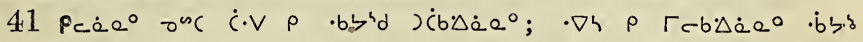

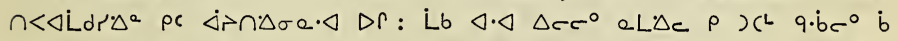
Lं $c\left(\sigma c^{b} x\right.$

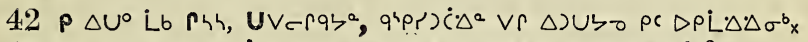

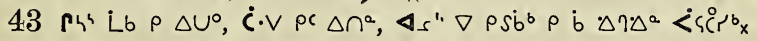

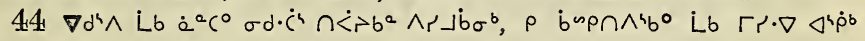
$\left.\wedge c^{c} \dot{s} \rho \dot{c} c \cap<\dot{<} b^{a} \wedge r\right\lrcorner \dot{b} b \sigma^{b} x$

$45 \wedge r^{L}$ Lb $\rho<\Delta \Delta b^{4} U r^{\circ}, \triangleleft d \Delta b^{a}$ Lb $\left.\rho \Gamma \dot{\Delta}>\Gamma \nabla \Delta b \Gamma d^{b} \cup \dot{C} \cdot \Delta^{b} \rho \dot{C}\right)<c^{\circ} x$

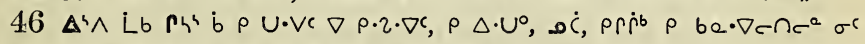
$\left.\Delta L^{b}: \Delta\right\urcorner r^{\circ} L b$ b $\rho \Delta \cdot \cap c, \rho<\rho \cap \sigma^{\circ} \Delta C \Delta i \cdot b_{x}$

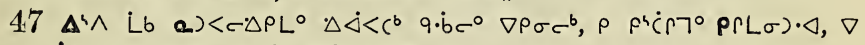

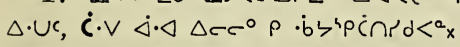

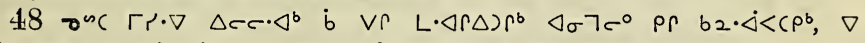

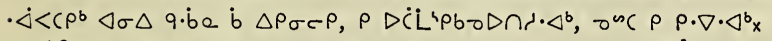

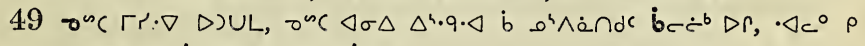
$\sigma<\Delta c \cdot \triangleleft, \nabla$ be $\cdot \dot{i}<c \Gamma e r \Delta D q \cdot \dot{b} e_{x}$

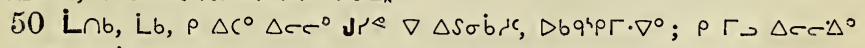
Lb, onc $\rho \cdot b^{2}$ is $p \cap^{\circ}$ :

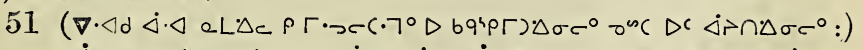

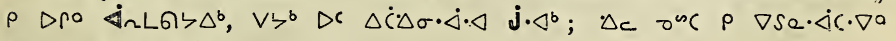
$P(L \sigma) \cdot \triangleleft \Delta C D P L \Delta \Delta \sigma \sigma^{\circ} x$

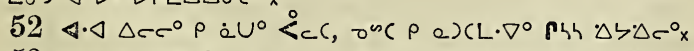

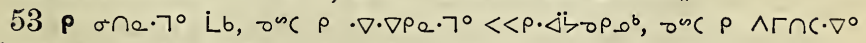
$\left.r \dot{<}>b \Gamma d^{b} \dot{b}\right\lrcorner a \wedge$ b $b \Delta \dot{b} U^{b} \triangleleft r \sigma^{b}, \Delta C \nabla \dot{b} \triangleleft \cdot \nabla a \nabla \sigma c \wedge \Gamma S \dot{L} b \sigma \Delta c_{x}$

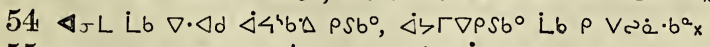

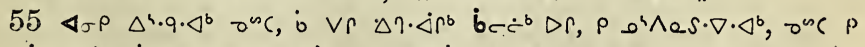

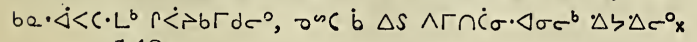




\section{$D<\rightarrow \rho^{4}$; $23,24$.}

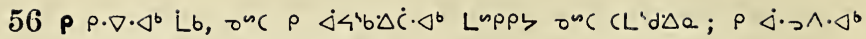
Lb $\nabla \dot{\Delta b} \nabla \nabla P S \dot{b} \sigma^{b}, \nabla \Delta C\left(\cdot \cdot \dot{<} \sigma \cdot \Delta^{b} x\right.$

\section{A p cra $\triangle b^{2} 24$}

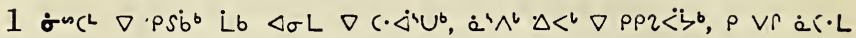

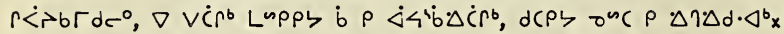

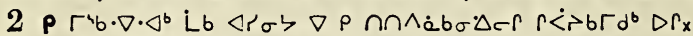

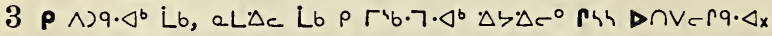

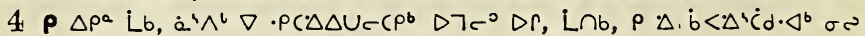

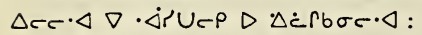

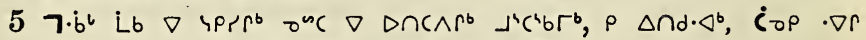
a a $c \cdot \dot{<}<7^{b} \triangleleft a \nabla \wedge$ Lnic $\Delta C \nabla \Delta \dot{C} e r \dot{b} \sigma \wedge c r$ ?

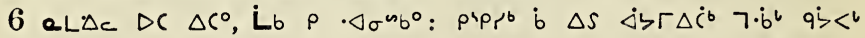
bec $c^{b} \nabla \Delta \dot{c} c$,

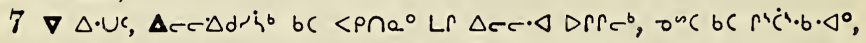
$\sigma^{4)}$ PSibcq $\mathrm{Lb} \Gamma \mathrm{a} b \mathrm{~b} \cdot \Delta \sigma^{n_{0}}{ }^{\circ} \mathrm{x}$

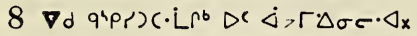

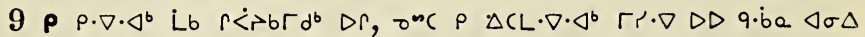

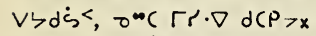

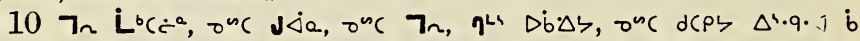

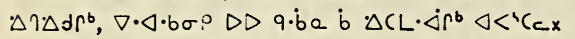

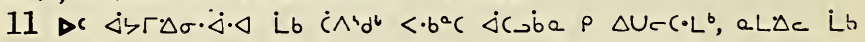
p $\dot{C} \cdot V C \cdot L^{b} x$

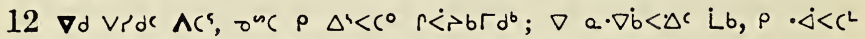

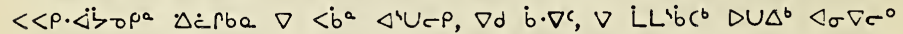
b) $\Delta \rho \sigma \sigma^{b} x$

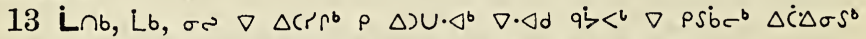

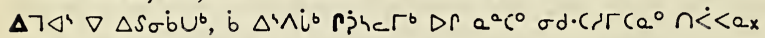

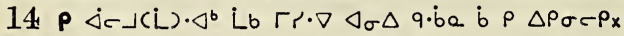

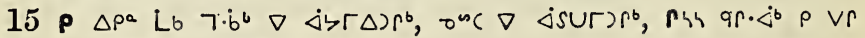
and. $\Delta^{b}, 0^{n c} \rho \Delta \rho^{\circ} \Delta d \cdot \Delta^{b}$;

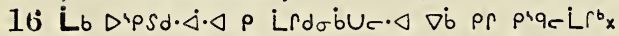

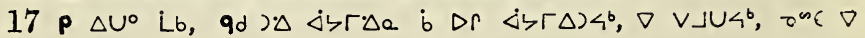
$\wedge$ Pribuec $77^{\circ}$ ?

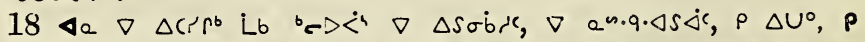

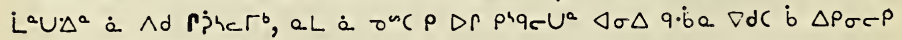
$7.6^{\circ} D D \nabla$ PSíp?

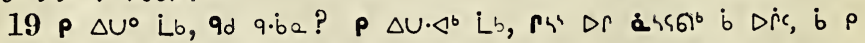

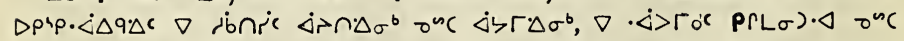
$r s \cdot \nabla \Delta c c \cdot \Delta:$

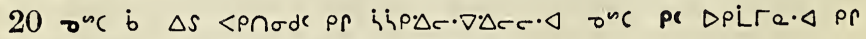

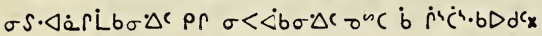
$14 \pm$ 


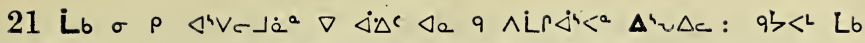

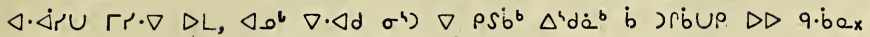

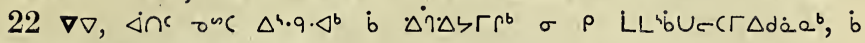
$\dot{\alpha}<\rho b r \dot{<}>b \Gamma d \sigma^{\circ} \Delta<\varphi \quad q \rho \tau^{<} x$

$23 \Delta^{4} \wedge$ Lb $\nabla b \quad 7^{b} b \cdot L r^{b} \Delta b^{b} \Delta c^{0}, \rho \quad \vee r \quad \Delta u \cdot \Delta^{b}, \nabla \Delta \cdot U r^{b}, \sigma \rho$ $\cdot \Delta<\dot{L} \dot{a} a^{b} \nabla^{a} b c^{b} \nabla$ odrac $\Delta b \Gamma r^{b}, \dot{b} \Delta \cdot U r^{b}, \lambda L n r^{\circ} x$

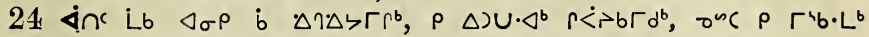

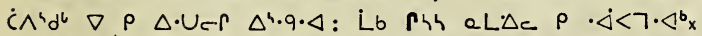

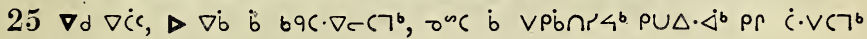

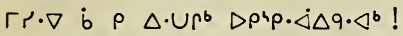

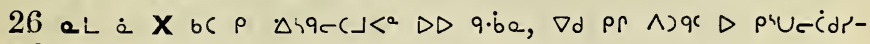
$\Delta \sigma^{b}$ ?

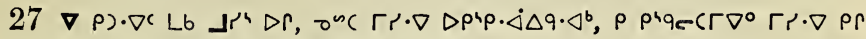
Lre $\Delta q \Delta \sigma^{b} \varangle \sigma \Delta q \cdot \dot{b} a$ b $\Delta S \Delta \dot{C} r$ L b $\sigma \Delta c_{x}$

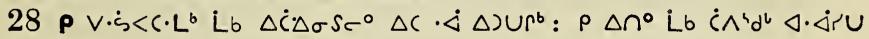
$\nabla \Delta \Delta u r_{x}$

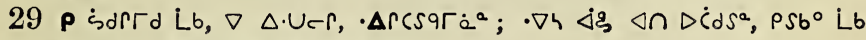
$\left.q \dot{b c} \Delta \Delta^{n} \cdot b_{b}<e^{\circ} x \quad \nabla d \dot{<}\right) q c$ pr $\Delta r c s q L c_{x}$

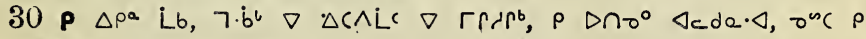
$\Gamma \cdot c \cdot \rho]^{\circ}, \rho \wedge d 0^{\circ} L b, \sigma^{n C} \rho \Gamma>^{\circ} x$

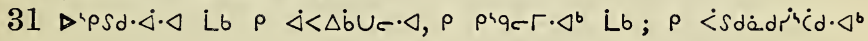
Lbx

$32 p \Delta \cap) \cdot \Delta^{b} L b, a L$ a $p U \Delta a^{\circ} p<r U^{\circ} \wedge r \rho b \Delta \dot{a}^{b}, 7 \cdot b^{b} \dot{b} \Delta b \Gamma \Delta c^{b}$ $\nabla \wedge\lrcorner U^{b}$, occ $7 \cdot b^{b} b \dot{b}<<\Delta L c^{b}$ pr Lre $\Delta 9 \Delta Q_{x}$

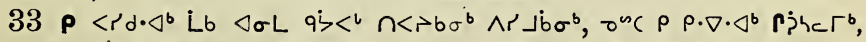

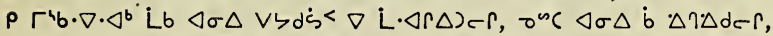

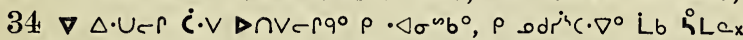

$35 \rho \Delta C \cdot L^{b}$ Lb $\Delta \sigma \Delta q \cdot \dot{b} a b \Delta \rho \sigma c \rho 7^{4} b a^{b}, \sigma^{a C} \nabla \rho \Delta S \rho^{4} q c L L^{b} \nabla$ $\wedge$ deer $\Delta c$ do. $\Delta x$

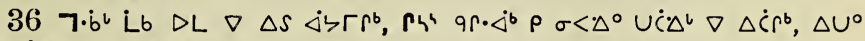

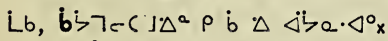

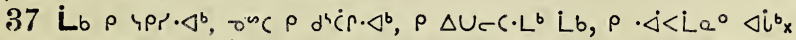

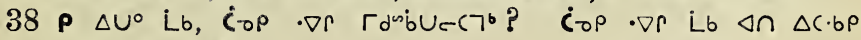

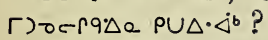

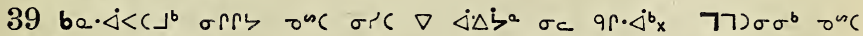

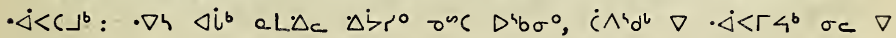
$\Delta ל \dot{\zeta} a^{x} x$

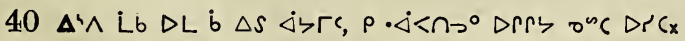

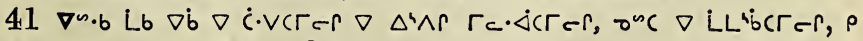
$\Delta U^{\circ}, \Delta C$ a $\Gamma \Gamma^{L} \rho C\left\langle i b a \cdot \Delta^{\circ}\right.$ ?

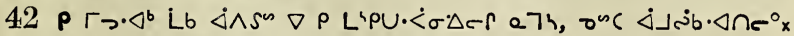

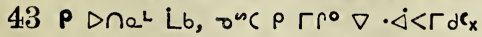

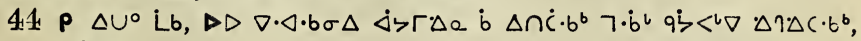
145 


$$
\Delta<>\rho^{4} \text { धa } 1 .
$$

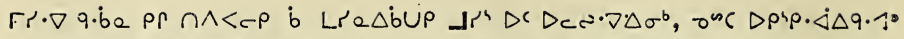

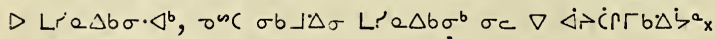

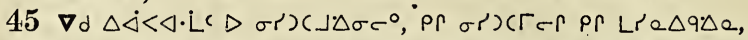

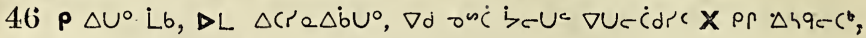

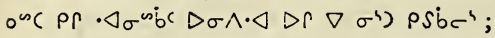

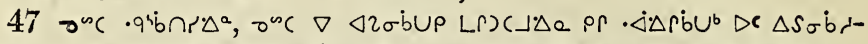

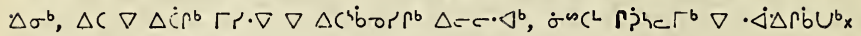

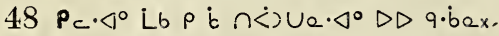

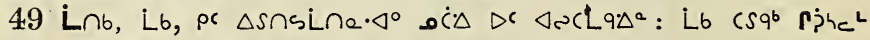

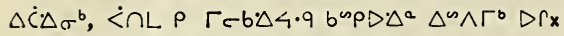

$50 \rho: \Delta c \Delta C \nabla^{\circ}$ Lb Ve $\sigma \Delta^{b} \Delta^{4} d: \rho \triangleright \wedge \sigma^{4} \cdot q^{\circ}\left[b\right.$, onc $\rho \Gamma \cdot c P 7^{\circ} x$

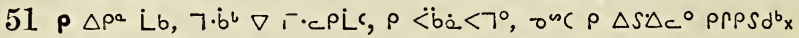

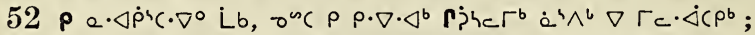

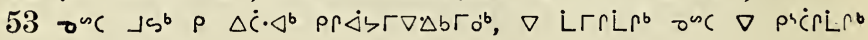
$\rho(L \sigma) \cdot \triangleleft x \nabla\urcorner^{a} x$

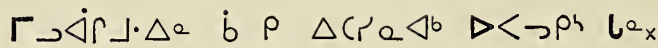

\section{A'pcre $\triangle b^{a} 1$.}

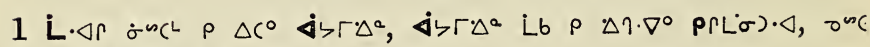
$\dot{\Delta} \succ \Gamma \Delta^{2} \rho \rho P(L \sigma) \Delta^{\circ} x$

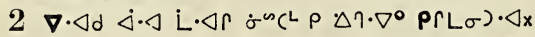

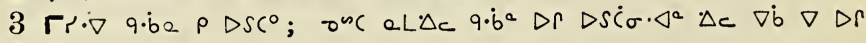
$D S \dot{c} x_{x}$

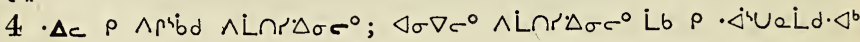
$\Delta c c^{\cdot} \cdot \Delta^{b} \mathrm{x}$

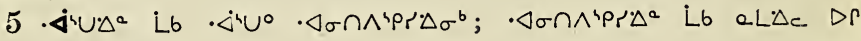
or $C \cdot \nabla \subset C \perp L b^{a} x$

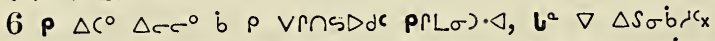

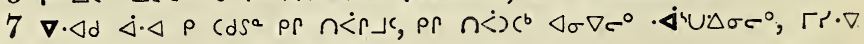

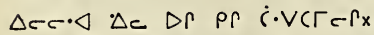

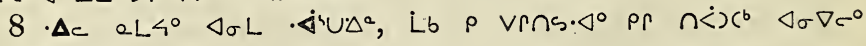
$\cdot \Delta \backsim \Delta \cdot \Delta \sigma c^{\circ} x$

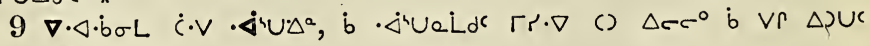
$D C \varangle \dot{s} b_{x}$

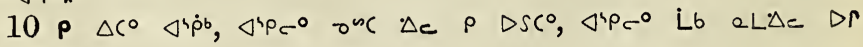
p'qc $\Gamma \mathrm{dx}$ 


\section{$\Delta<\neg \rho^{s}$ ba 1 .}

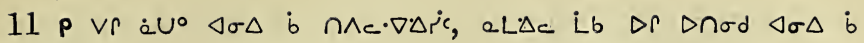
$n \wedge c \cdot \nabla \Delta r c^{\prime} c_{x}$

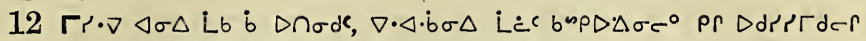

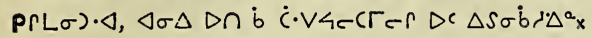

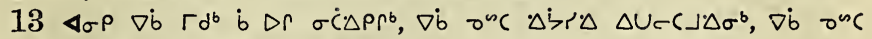

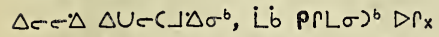

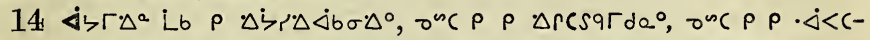

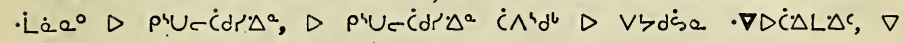

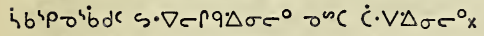

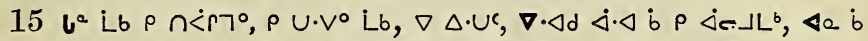

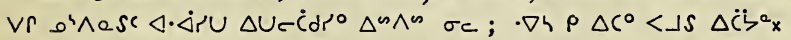

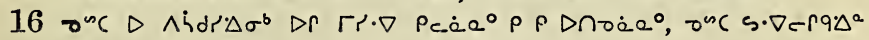
s. $\nabla e r q \Delta^{\circ} \Delta r^{x}$

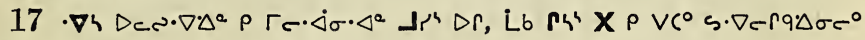
$\operatorname{sic} \dot{C} \cdot V \cdot \Delta \sigma \sigma^{\circ} \mathrm{x}$

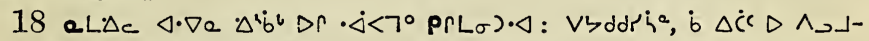

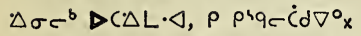

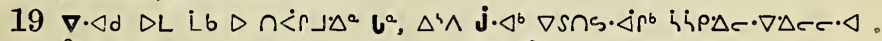

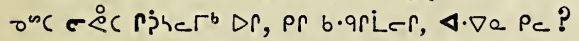

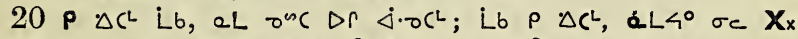

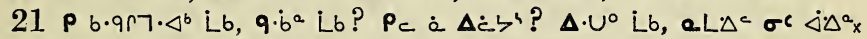

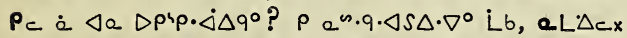

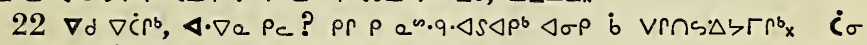

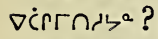

$\left.23 \rho \Delta \cdot U^{0}, \sigma c \nabla \cdot \triangleleft d \nabla V \dot{C} d r \cdot \Delta^{a} \triangleleft \cdot \nabla a \nabla U \cdot v c<\cdot b c^{4} b r^{b} b^{b}, \cdot b b^{4} d\right\lrcorner c \cdot \Delta^{b}$

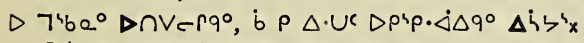

$24 \varangle \sigma \rho$ Lb b $\Delta S \cap S \cdot\left\langle b \sigma \cdot \Delta r^{b} \rho<S r^{\cdot} \Delta \cdot \Delta^{b} x\right.$

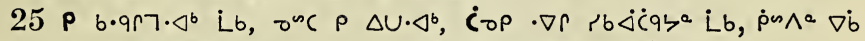

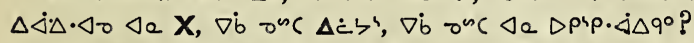

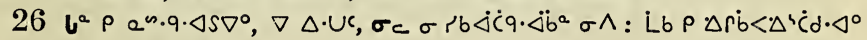
$\triangleleft \cdot \nabla e, \nabla b$ b $\rho^{r} q \sigma 7^{b} x$

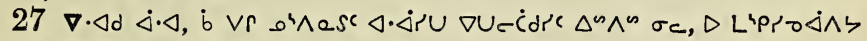
$\nabla b \cdot \dot{\Delta} \cdot \Delta^{i} \nabla \Delta U-\dot{C} d r \cdot \triangleleft^{b}$ br $\Delta \wedge$ da $\cdot L^{b} x$

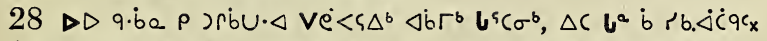

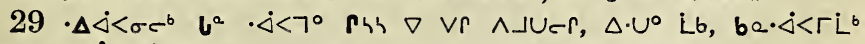

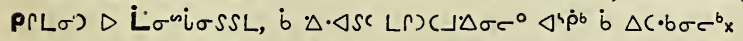

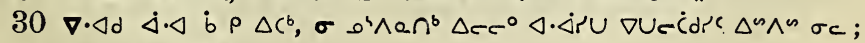
- $\left.\nabla 4 \rho \Delta C^{\circ}<\right\lrcorner S \Delta \ddot{C}^{\circ} \times$

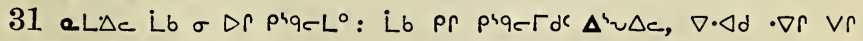
$r b \triangleleft \dot{c} q \cdot \triangleleft \dot{i} \dot{\zeta}^{a} \sigma \wedge x$

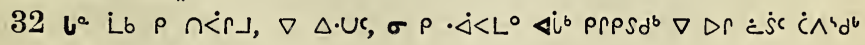
$\Delta \Gamma \Gamma^{b}, \rho \cdot U D C \cdot \nabla^{\circ} L b_{x}$

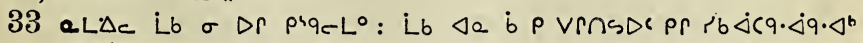
147 


\section{$\Delta<\rightarrow$ os $b^{2} 1$.}

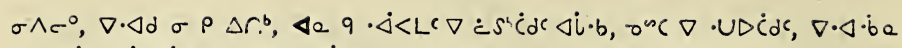
$q$ rb $\Delta c q \cdot \Delta q c \dot{b}<s p r e r \Delta i \cdot b x$

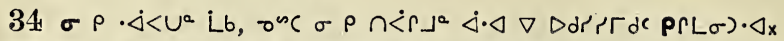

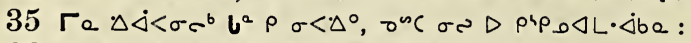

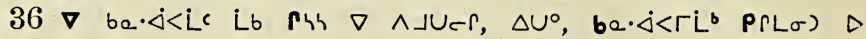
i $\sigma^{\sim i} i_{\sigma S S L}$ !

$37 \varangle \sigma \rho$ б Lb $\boldsymbol{P h i x}$

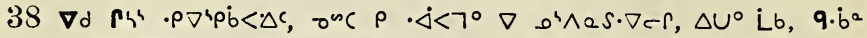
$\sigma<a c \cdot \nabla \subset c 7^{b}$ ? $p \Delta U \cdot \Delta^{b} L b, \dot{i}<,\left(b \Delta \cdot U L b^{b} \nabla \Delta \cdot U^{b} p b U^{b}, q^{b} p \circ \Delta L a b^{a},\right)$

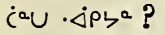

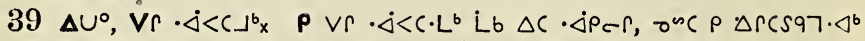

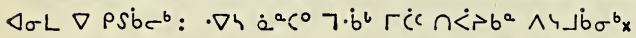

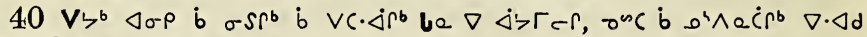
jarj, $L^{\circ} \Lambda C^{s} \Delta r \dot{s} a_{x}$

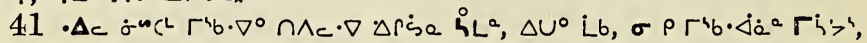
b $\triangle \cdot U L b^{b} \nabla \Delta \cdot U^{r} r \dot{b} U^{b}, X_{x}$

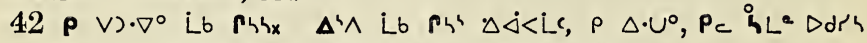

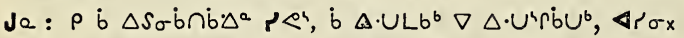

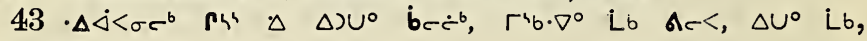
onesax

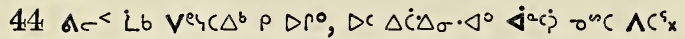

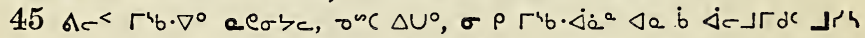

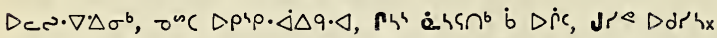

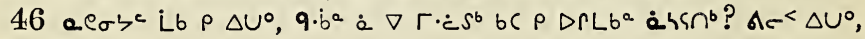
V $\Gamma \cdot \dot{j}<C_{x}$

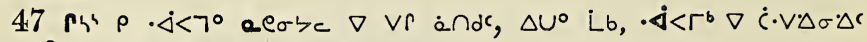

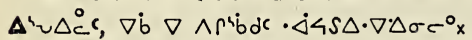

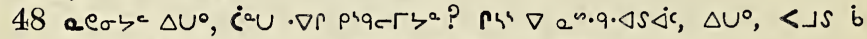
a) $\Gamma^{b} \Delta c^{<}, \Delta^{4} \wedge s \dot{<}<q i \sigma \Gamma^{\circ} \dot{a} \cap d^{b} \nabla \dot{C} \zeta^{a}, p \rho \cdot \dot{j}<\Gamma \cap^{a} x$

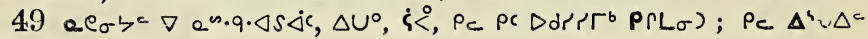
$D$ PRDPLLX

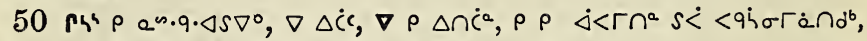

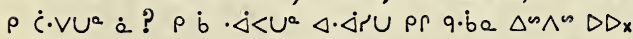

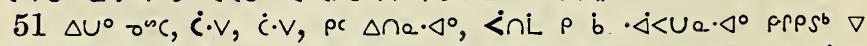

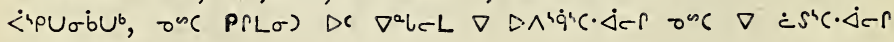
$\Delta c-\Delta d$ ílex

\section{Aspcre $\triangle b^{\circ} 2$.}

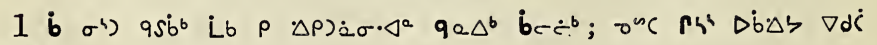
$\nabla \dot{C} \kappa r:$

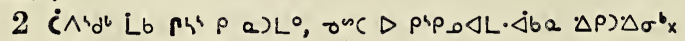
148 


$$
\Delta<>\text { pi ua } 2 .
$$

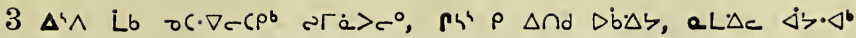
Nए $\dot{a}^{\prime}>c^{\circ} x$

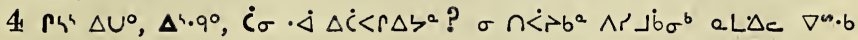
$D \cap R<e^{\circ} x$

$\left.5 \Delta \dot{b} \Delta \zeta \Delta U c \cdot \triangleleft \triangleleft \dot{j} q \dot{c} b e, \wedge d q \cdot \dot{b} c^{\circ} q \Delta \cap \dot{c} \cdot q, \nabla \cdot \Delta d \Delta x \cdot\right\lrcorner^{b} x$

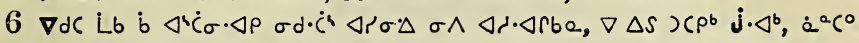

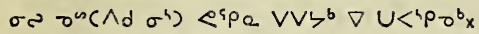

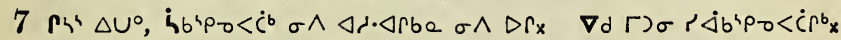

$\left.\left.8 \Delta \cup^{\circ} \mathrm{Lb}, \nabla \cap a\right\lrcorner^{b} \nabla d, \Delta\right)\left(\cdot \dot{C}^{b}\right.$ Lb LdZ $\left.\Delta P L^{\circ} \times \quad \rho \Delta\right)() \cdot \nabla \cdot \Delta^{b}$ Lbx

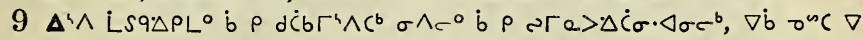

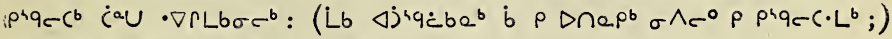
$L S 9 \Delta P L^{\circ} \rho$ Q $) 7^{\circ}$ Dup4.9. $\nabla \cdot \triangleleft$,

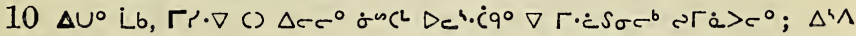
Lb $\Delta c c \cdot \triangleleft \Gamma^{h} c \Delta \dot{b} \Gamma \sigma \cdot q c \cdot c \cdot \nabla, \nabla d \Delta \sigma \nabla c^{\circ} \nabla \dot{b} b \Delta^{n} \Delta^{s} \Gamma \cdot \dot{c} S \sigma c^{b}:$ Lb $P_{c}$

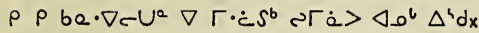

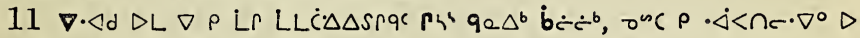

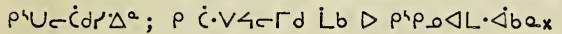

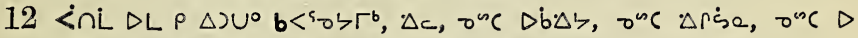

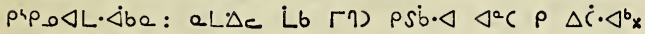

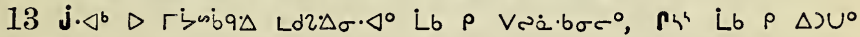
riscer $\Gamma^{b} x$

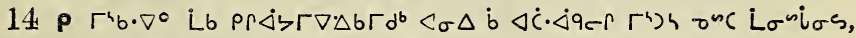

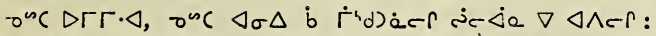

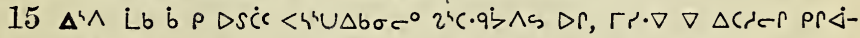

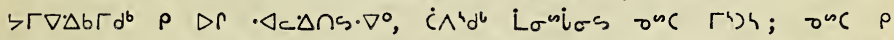

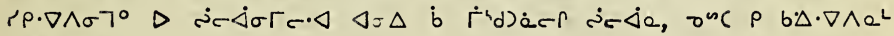
tryon.e;

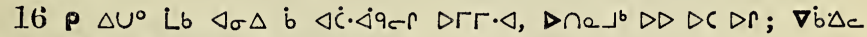

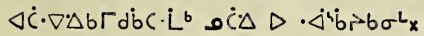

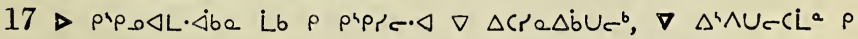
$\left.\cdot<4 b r b \sigma \sigma^{2} \sigma \quad \rho \quad \rho C\right\lrcorner d^{2} x$

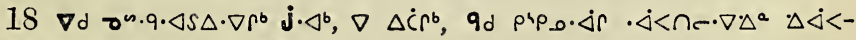
$\cap-\dot{b} b \triangleright$ q.be $\nabla \mathrm{CL}^{2}$ ?

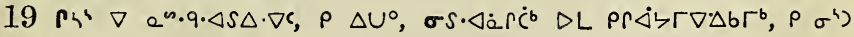
PSibq $L b \quad \sigma \quad b \cdot \Delta \sigma^{n} b \sigma^{a} x$

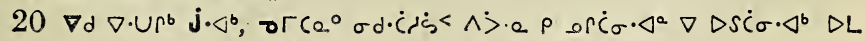

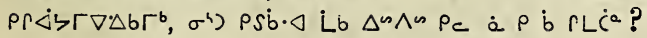

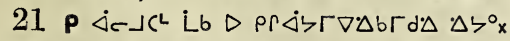

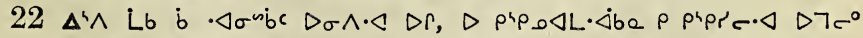

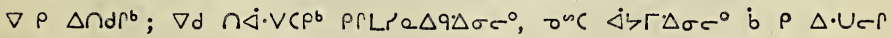
Phix

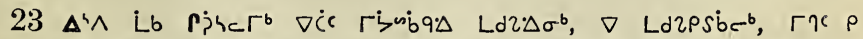

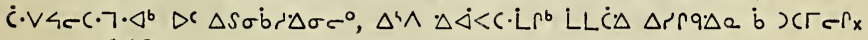
149 


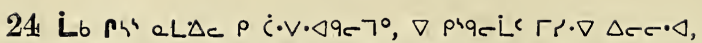

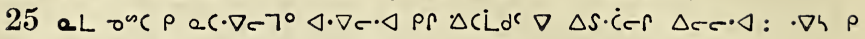
$\operatorname{prqc} c \cdot 7_{0}^{\circ} q \cdot \dot{b} c^{\circ} \dot{b} \wedge r i b d e r \Delta c c \cdot \Delta x$

\section{N}

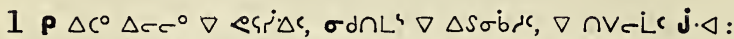

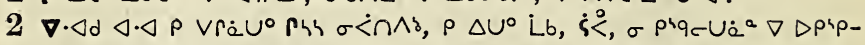

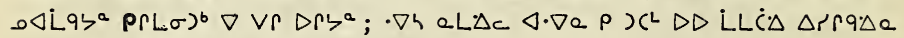

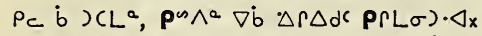

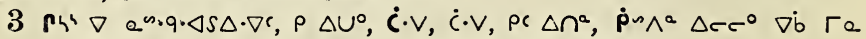
$\left.\sigma \dot{C} \Delta P U, Q L \Delta C b C \rho \cdot \dot{i}<C \cdot 7^{\circ} P \Gamma L \sigma\right) \cdot \triangleleft D C D P L \Delta \Delta \Delta \sigma \sigma^{\circ} x$

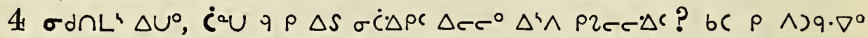

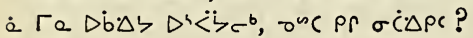

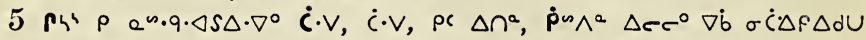
$\left.\left.\sigma \wedge \sigma^{\circ} \sigma^{n c} \varangle i \cdot b, a L \Delta c b c \rho \wedge\right) q \cdot \nabla^{\circ} p R L \sigma\right) \cdot \Delta D\left(D P L \Delta \Delta \sigma \sigma^{b} x\right.$

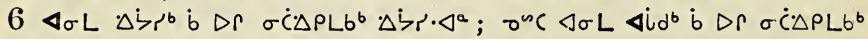
$\Delta i d \cdot \Delta^{a} x$

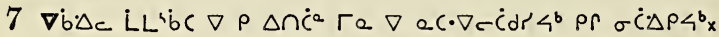

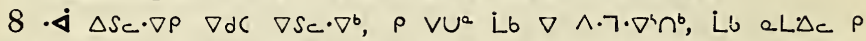

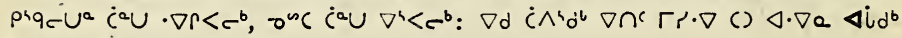
b $\operatorname{Dr} \sigma \dot{c} \Delta p c_{x}$

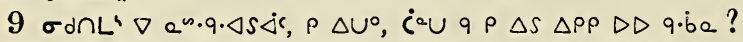

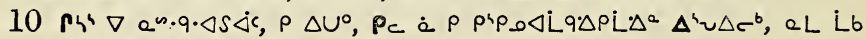

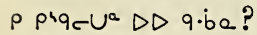

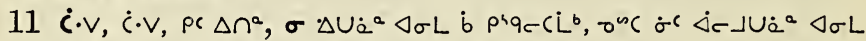

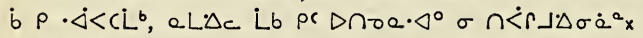

$12 \dot{p} \sim \wedge^{a} \nabla \Delta C L C \cdot b^{b} \triangleleft^{4} p \cdot \Delta q \cdot \dot{b} a, \nabla \dot{b} L b \nabla \dot{c} \cdot V C 7^{b}, \dot{c}^{a} \cup$ q $p \Delta S \dot{c} \cdot V C 7^{b}$ pus $\wedge^{a} \Delta C$ LC. 69 prpsib $\Delta q \cdot b a$ ?

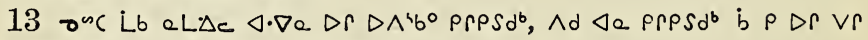

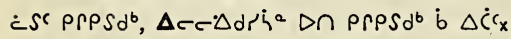

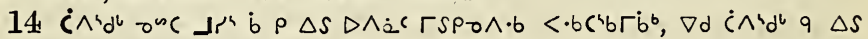
$\nabla \wedge \dot{b} b \sigma \Delta r \Delta c-\Delta d r i i^{2}:$

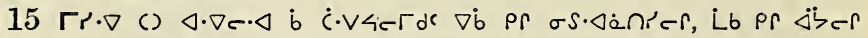
bpq $\lambda L \cap \Delta \cdot \Delta \sigma \sigma^{\circ} x$

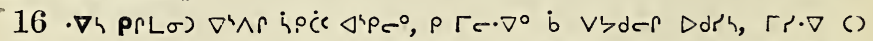

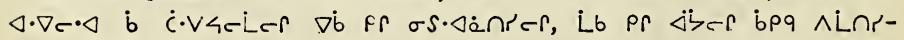
$\Delta \sigma \sigma^{\circ} \mathrm{x}$

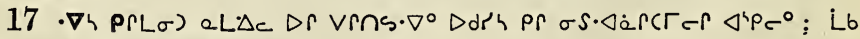
$\Delta c \operatorname{Pr} \Delta r \wedge \dot{L} \dot{C} \sigma \cdot \triangleleft^{b} \Delta^{4} p_{x}$

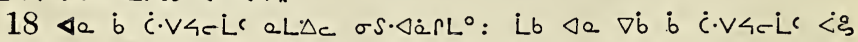

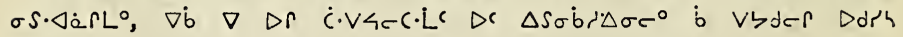
PrL $L$ ) $x$ 


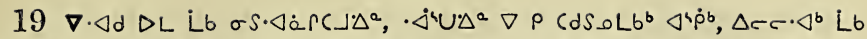

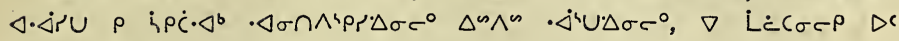
$\dot{\Delta} \sim \cap \cdot \Delta \sigma \cdot \dot{\Delta} \cdot \Delta x$

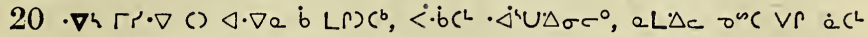

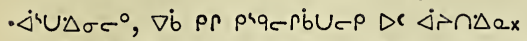

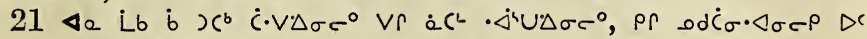

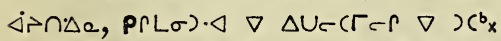

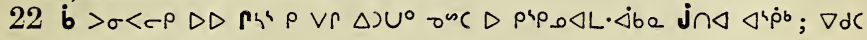

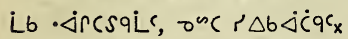

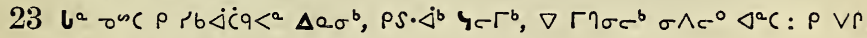

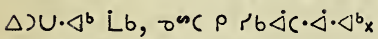

$\left.24 \cdot \nabla h l^{a} a L \Delta c \nabla^{a} \cdot b \rho \Delta S \cdot \nabla \wedge a^{\circ} p<D\right) \Delta b \Gamma d^{b} x$

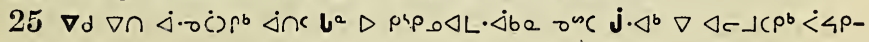
$\Delta \cdot \nabla \Delta \sigma c^{\circ} x$

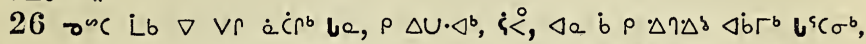

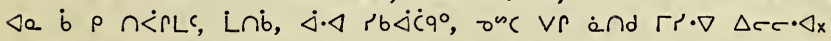

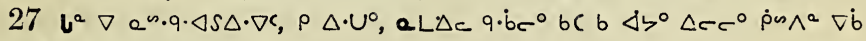
brPSd $D C r \dot{c} b \sigma . \triangle U^{x}$

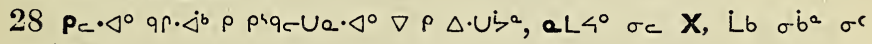
$\triangle \cap S \triangle i \Delta^{a} \mathrm{x}$

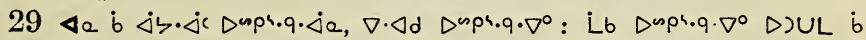

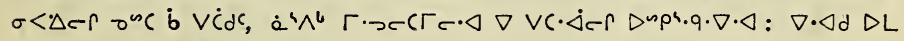
L $b \sigma \Gamma c \cdot \Delta c\lrcorner \Delta^{2} n \wedge<c^{\circ} x$

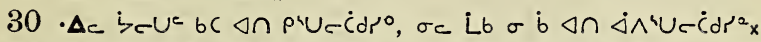

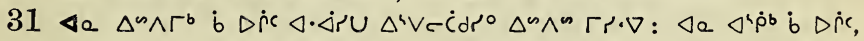

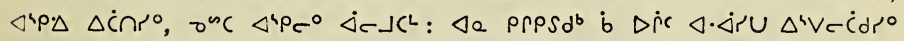
$\Delta \mu \wedge * i r \cdot \nabla x$

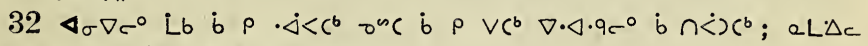
$\triangleleft \cdot \nabla$ e Lb $\left.D \cap a \cdot 7^{\circ} D \cap \dot{R}\right\lrcorner \Delta \sigma c^{\circ} x$

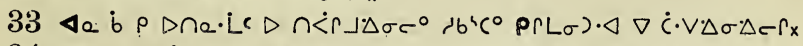

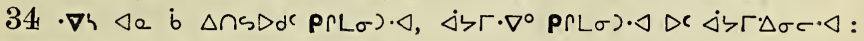

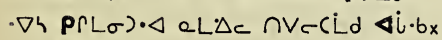

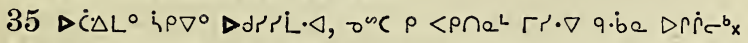

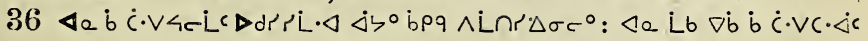

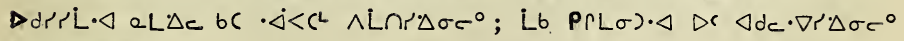
$L C d^{4} b d \cdot \nabla^{\circ} x$

\section{Nיpcra $\triangle b^{2} 4$.}

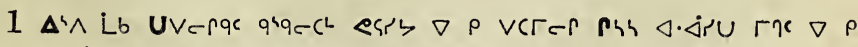

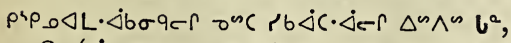

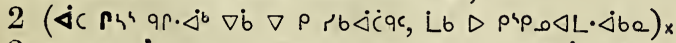

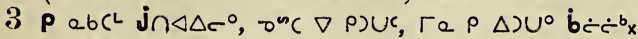

151 


\section{$D<>$ pi la $^{2} 4$.}

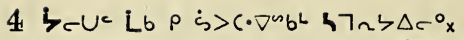

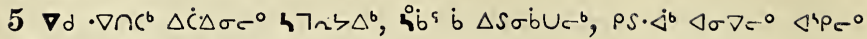

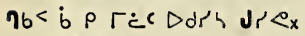

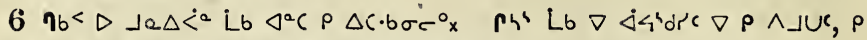

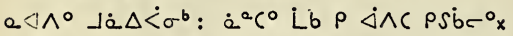

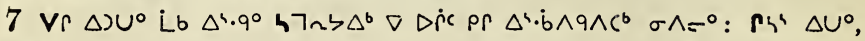
$\Gamma \circ \Delta^{a} x$

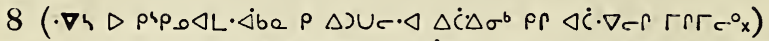

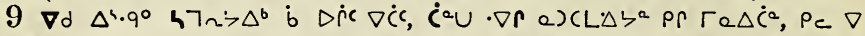

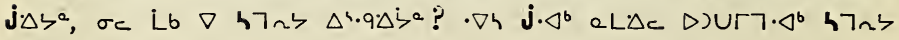
$\Delta r c \cdot \Delta x$

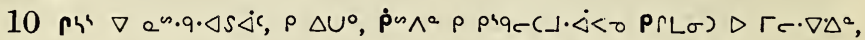

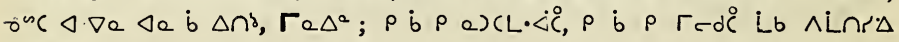
$\sigma \wedge c^{\circ} x$

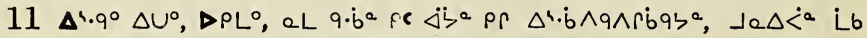

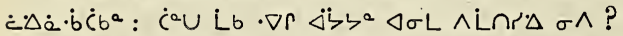

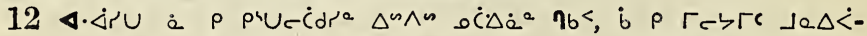

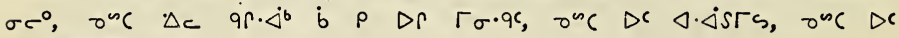
$\triangleleft \cdot \triangleleft b \sigma L$ ?

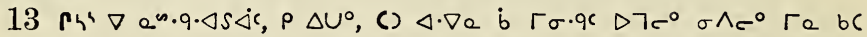
.Uं<<.$^{\circ}$ :

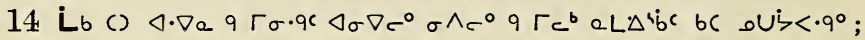

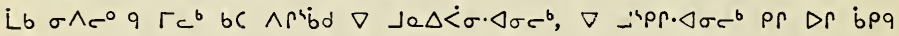
$\wedge \mathrm{L} \cap \mathrm{c}_{\mathrm{c}}$

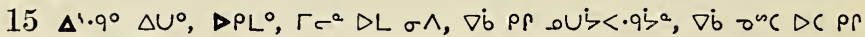

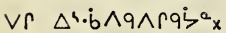

$\left.16 p^{4} \Delta U^{\circ}, \dot{L} i, a\right) L \rho \dot{a} V^{L}, V r \Delta J U$ Lbx

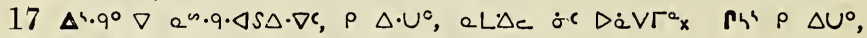

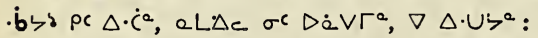

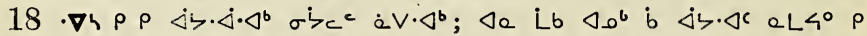
$\dot{a} V^{2}: \nabla d C \cap \Delta \cdot V \zeta^{\circ} \dot{b} \Delta \cdot U^{\circ} \times$

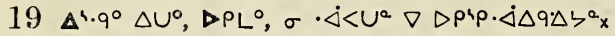

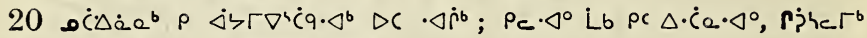

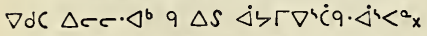

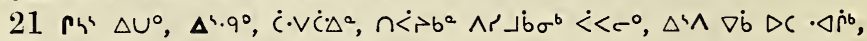

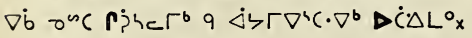

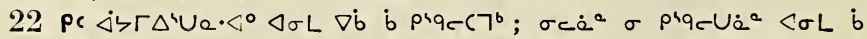
$\dot{<} \zeta \Gamma \nabla^{\top} C L^{b}: \cdot \nabla 4 \wedge \mathrm{L} \Gamma \Delta \cdot \nabla \cdot \Delta^{a} \cdot \mathbf{j} \cdot \Delta^{b} \nabla d C \cdot \nabla \Gamma L b^{b} \mathrm{x}$

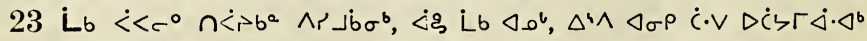

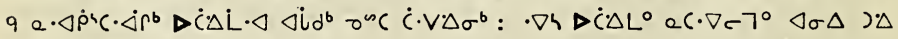

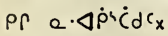

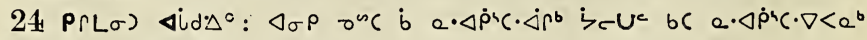
$\Delta i d^{b} \sigma^{n C} \dot{C} \cdot v \Delta \Delta \sigma^{b} x$ 


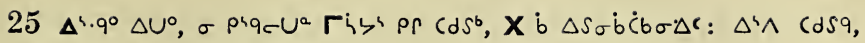
$\Gamma r \cdot \nabla$ q.be $\sigma$ b $\Delta C$ Ldd $^{a} x$

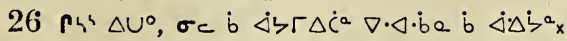

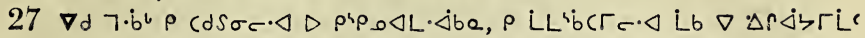

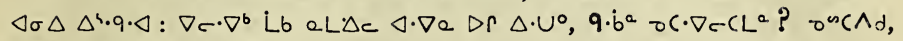
$9 \cdot \dot{b}^{a} \cdot \nabla r \Delta r \Delta \zeta r L C ?$

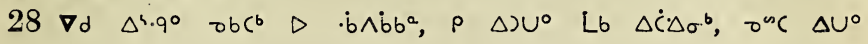
$\triangle c r \cdot \Delta$,

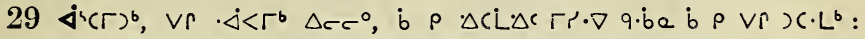
eL a $\nabla \cdot \triangleleft d \dot{d} \dot{j} \triangleleft \mathbf{X}$ ?

$30 \nabla d \cdot \nabla c \Delta r^{b} \Delta \dot{C} \Delta \sigma^{b} \Delta r$, onc $\rho \vee r \quad \Delta \cap U \cdot \triangleleft^{b} x$

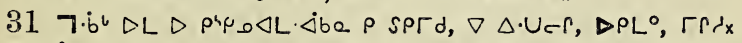

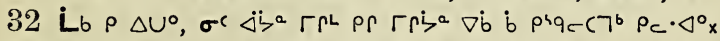

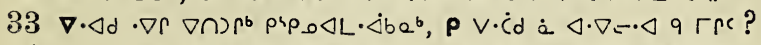

$34 r^{3} \Delta U^{\circ}, \nabla \cdot \Delta d$ o $r r r^{L}$ pr $x \cdot L^{b} D C \cdot \Delta U C C \cdot \Delta^{a}<l e$ b $p$ vrnsde,

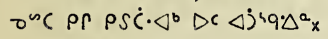

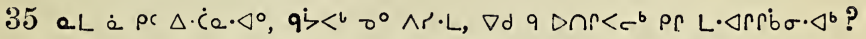

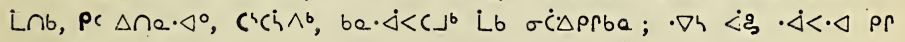
$L \cdot \Delta r r \dot{b} \sigma \cdot \Delta b_{x}$

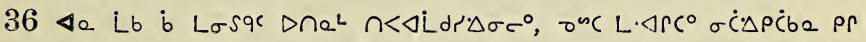

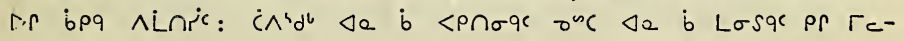
- $\Delta r . p b_{x}$

$37 \nabla d C D C U \wedge<C^{b} \varangle \sigma L \Delta \cdot U \Delta^{a}, V \succ^{b}<\rho \cap \sigma^{\circ}, d C^{b}$ Lb $L \sigma S 9^{\circ} x$

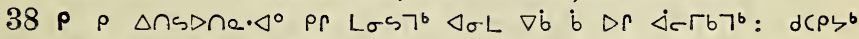

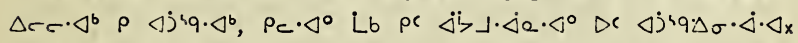

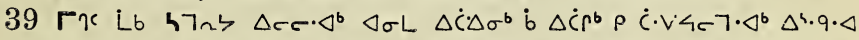

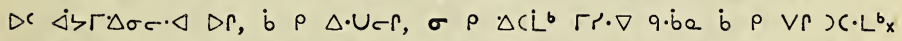

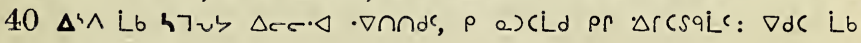

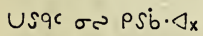

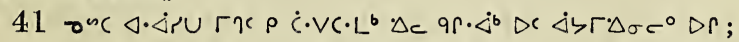

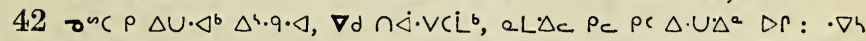

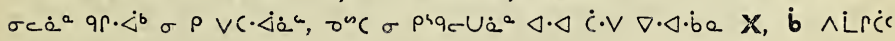
$\triangle A c^{\circ} x$

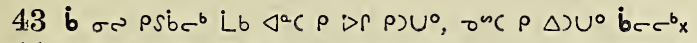

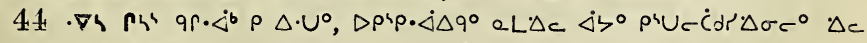
$\cap \wedge c \cdot \nabla$ De $\Delta^{4} \dot{p}^{b} x$

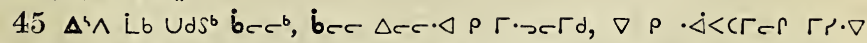

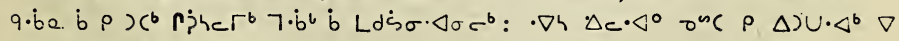

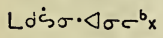

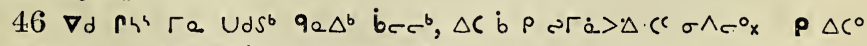

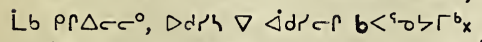

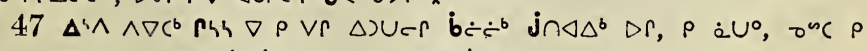

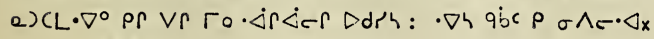




$$
\triangleright<\text { or に2 } 4,5 \text {. }
$$

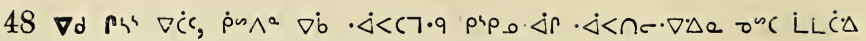
q.be, $a L \cdot \Delta c \rho \dot{b} \dot{c} \cdot V \cup e \cdot \Delta^{\circ} x$

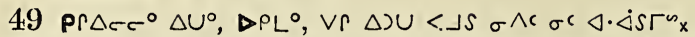

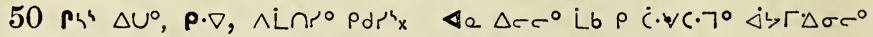

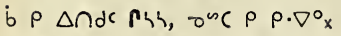

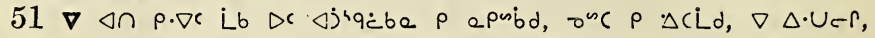
$\Lambda$ Linto $b$ dris $x$

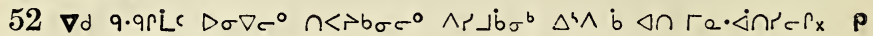

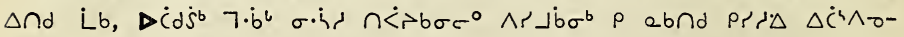
$\Delta \sigma c^{\circ} \mathrm{x}$

$23 \nabla d \quad \Delta \dot{C} \Delta L^{\circ} \quad q^{4} q_{c} c^{b} \quad \nabla \quad \rho \quad \Delta \rho_{\sigma} \sigma^{b} \quad 7 \cdot b^{b} \quad \Delta \sigma \nabla \sigma^{\circ} \cap>>b \sigma c^{\circ} \wedge r-$

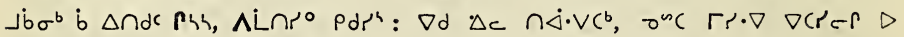
$\cdot \Delta b \dot{b} b \sigma \sigma \Gamma^{b} x$

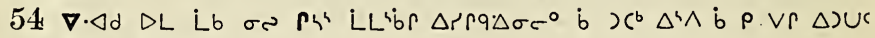
$\dot{b} c c^{b}, \dot{j} \cap \Delta \Delta^{b} \Delta r_{x}$

\section{$\Lambda^{4} p C r \propto \triangle b^{2} 5$.}

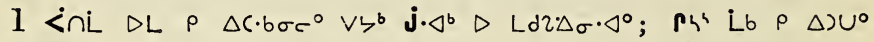
píte $\Gamma^{b} x$

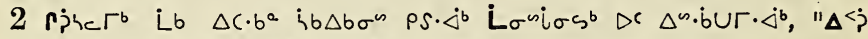
$\Delta S \rho \cdot 2 \Delta \sigma^{b} V e^{c} c \dot{b} \Delta S \sigma \dot{b} U^{b}, \sigma \dot{b} c^{c} \Lambda^{4} \rho \cdot \dot{c}^{4} b \Delta b \sigma s \nabla d c \nabla \Delta c \cdot b P_{x}$

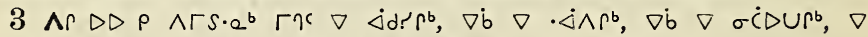
$\sigma>\Delta \dot{a} d r \rho^{b}, \nabla V \dot{C} \rho^{b} \rho \rho<\dot{\Delta}\left(b L \Delta b U c^{b} \sigma \wedge c^{\circ} x\right.$

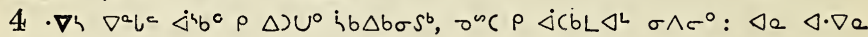

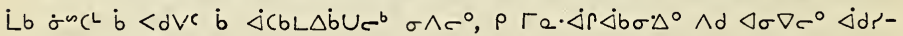

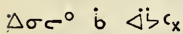

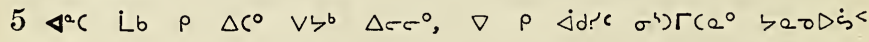
$\wedge>\cdot a_{x}$

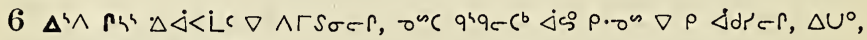
$p \Delta \Gamma e \cdot \Delta r \Delta d r^{a} \dot{a} ?$

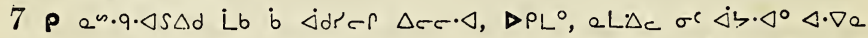

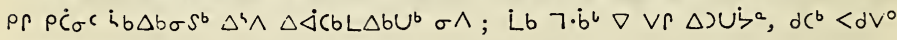
$<\lrcorner S \sigma c x$

8 ph $\Delta U^{\circ}, \cdot \triangleleft \sigma^{i b}$, Dne $\left.\rho \sigma V \Delta^{a}, \wedge\right\lrcorner U$ Lbx

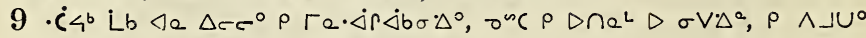
Lb: $\nabla \cdot \triangleleft \cdot b \sigma \nabla \rho S \dot{b} b$ Lb $\rho \dot{\Delta}>\Gamma \nabla P S \dot{b} \mathrm{C}_{x}$

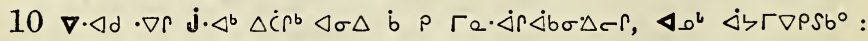
eL $\Delta_{c} \nabla_{c} \cdot \nabla \cdot \nabla c \dot{C} \cdot b^{a}$ or $\wedge \Gamma \cdot \dot{C} \zeta^{a} \rho \sigma V \cdot \Delta^{a} x$

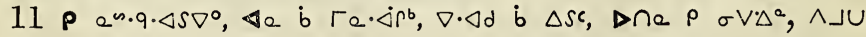
Lbx

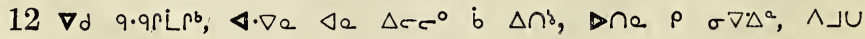
Lb? 


\section{$\triangleright<>$ p la 5.}

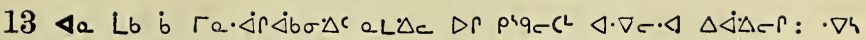

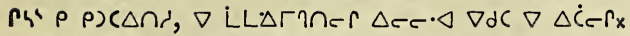

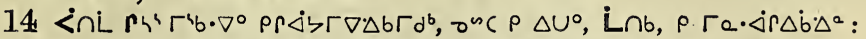

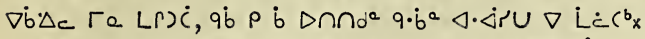

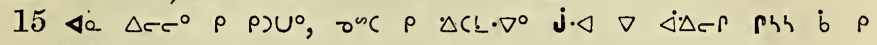
$\Gamma a \cdot \dot{<r} \Delta d d_{x}$

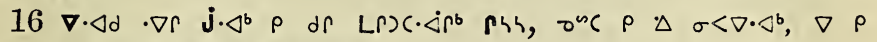

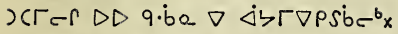

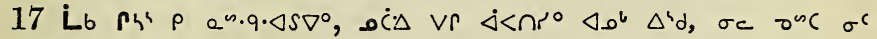
$\dot{\Delta}<n^{\circ} \times x$

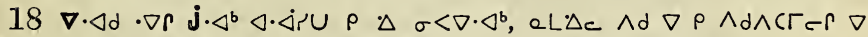

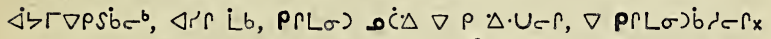

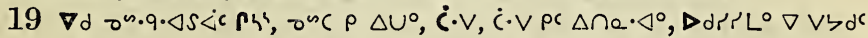

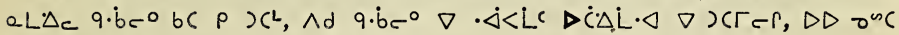
$x^{2} D$ dPSL ${ }^{\circ} x$

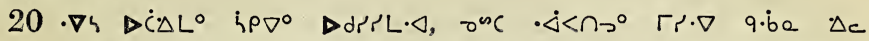

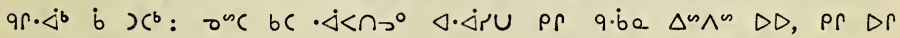
LL'bC $b 7^{b} x$

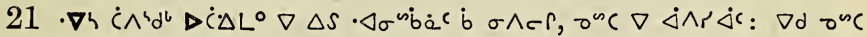

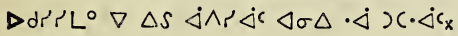

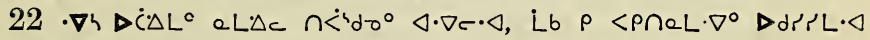

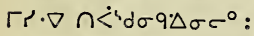

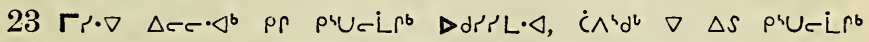

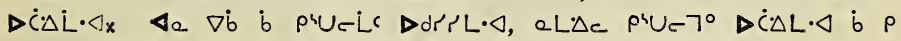
vrns. $\dot{\Delta}-r_{x}$

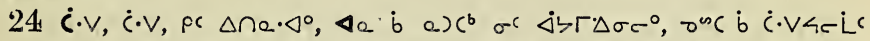

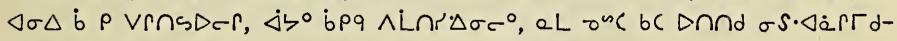

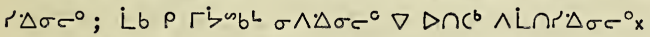

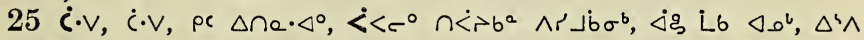

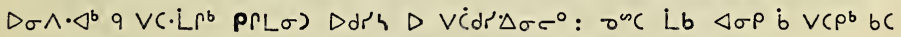
$\wedge \mathrm{L} \cap \mathrm{r}^{\circ} \cdot \Delta^{\mathrm{b} x}$

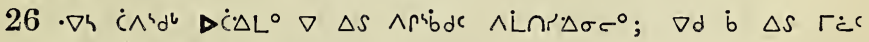

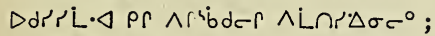

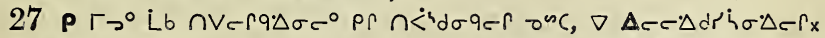

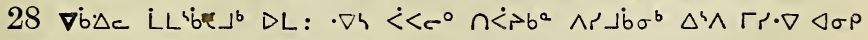

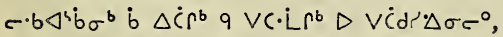

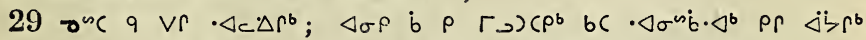

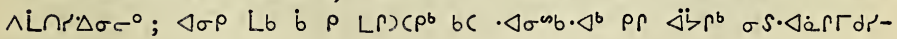
$\Delta \sigma c^{\circ} x$

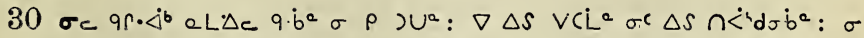

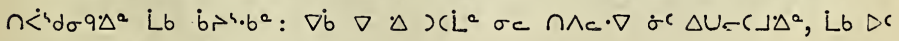
$\triangle U-C\lrcorner \cdot \Delta^{a} \Delta \dot{C} \Delta L^{\circ}$ b $\rho$ V $R \cap S D^{\prime} x$

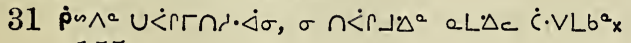
155 


$$
\triangleright<>\text { คr ๒ } 5,6 \text {. }
$$

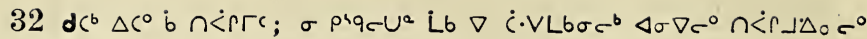
b) $\Delta$ crr $r c_{x}$

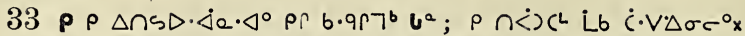

34 a L. $\Delta c$ Lb or $\left.\Delta \cap \sigma^{a} \cap \dot{<r}\right\lrcorner \Delta^{a} \Delta c^{b} \Delta r$ : Lb $\Delta D$ q.be oc $\Delta \cdot \dot{c}^{a}$ pr $\wedge \dot{L} \wedge b \Delta \Delta^{b} \mathrm{x}$

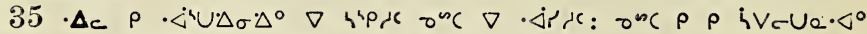

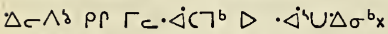

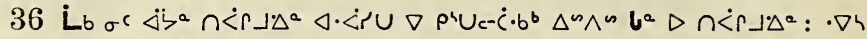

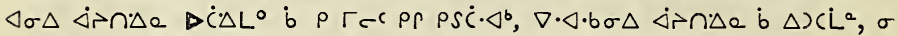
$n<i r d^{a}, \nabla \rho \vee r n a \Delta C \Delta C^{\circ} \Delta L^{\circ} x$

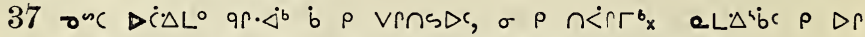

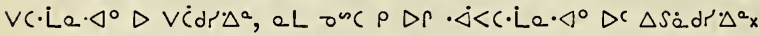

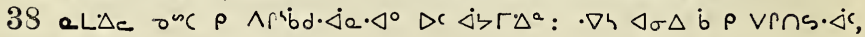
$\nabla \cdot \Delta d$ el: $\Delta c \rho \dot{c} \cdot V C \cdot \dot{\Delta} \cdot \triangleleft^{\circ} x$

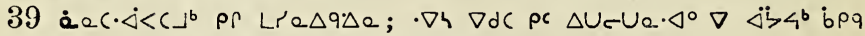

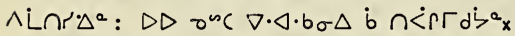

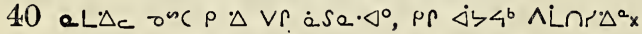

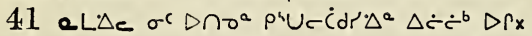

42 Lb $\rho$ prqc $\left.\Gamma \cap a \cdot \Omega_{0} \cdot \nabla \dot{b} \nabla \wedge \mu^{4} \dot{b} d \cdot \nabla^{b} p \rho L \sigma\right) \Delta i \rho \Delta \cdot \nabla \cdot \Delta^{a} x$

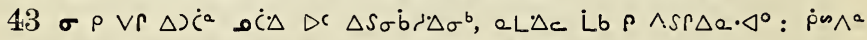

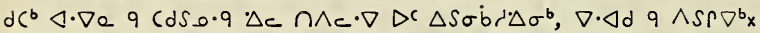

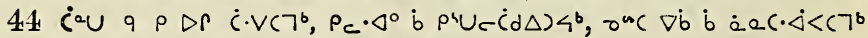

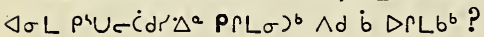

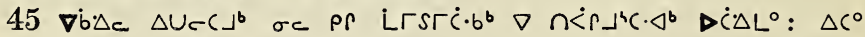
$\checkmark \cdot \nabla e$ b $\left.L \Gamma S \Gamma \dot{C}^{b}, J \mu^{\prime} D \cap \dot{b} L \Gamma S\right)\left(\cdot \nabla^{b} x\right.$

$46 \cdot \nabla h \dot{\rho} \omega \wedge^{a} \rho \dot{c} \cdot V C \cdot \nabla d<0 \perp \mu^{\prime}, \sigma_{c} \sigma^{n c} \rho$ b $\rho \dot{c} \cdot V C \Delta C \cdot \Delta^{\circ}: \cdot \nabla h \sigma \rho$ $\dot{\Delta}-\lrcorner \Gamma^{b} \nabla$ Lre $\Delta \mathrm{q}_{\mathrm{x}}$

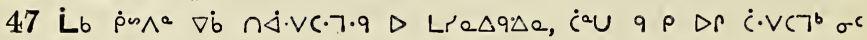

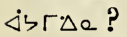

\section{Arpcre $\triangle b^{a} 6$.}

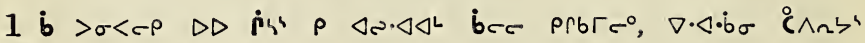
pib $\Gamma x$

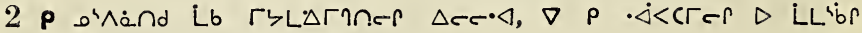

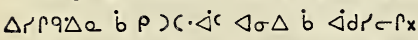

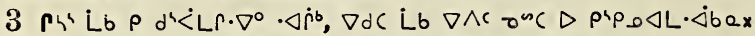

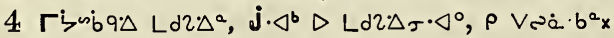

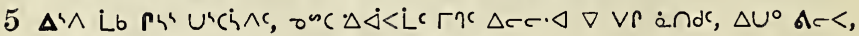

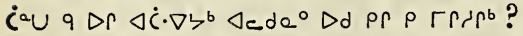

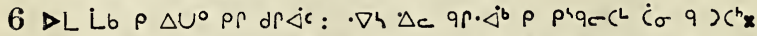

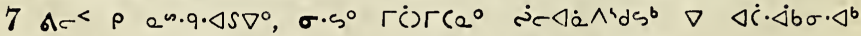

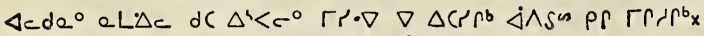

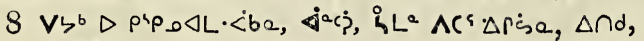

\section{6}




$$
><>\text { p ia } 6 \text {. }
$$

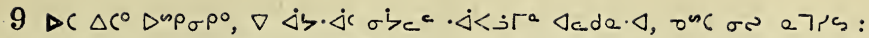
$9 \cdot \dot{b}^{a}$ L.b $D_{d} \nabla \Delta \backsim \wedge S \Gamma \eta \cap \dot{\alpha} \sigma \cdot \Delta^{b}$ ?

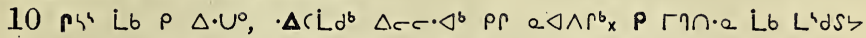

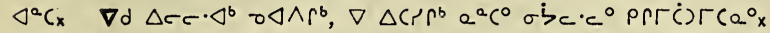

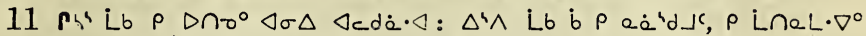

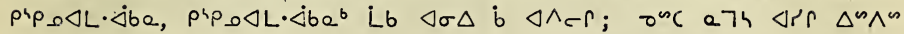
$\tau C \cdot \nabla-L_{-} \Gamma_{x}$

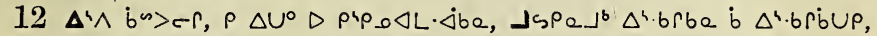
$\nabla \dot{b} q \cdot \dot{b}^{a} \rho \varphi \cdot \Delta \sigma \dot{C}_{\sigma} \cdot \Delta b x$

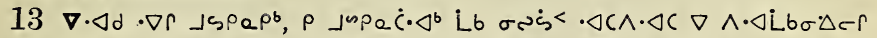

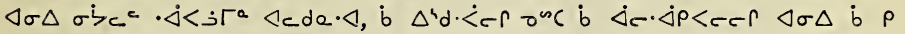
reserx

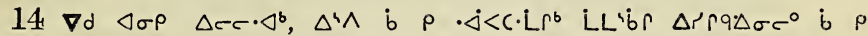

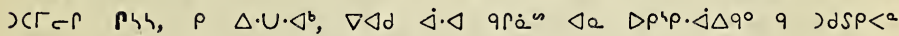
बip $\dot{\rho}^{6} \mathrm{x}$

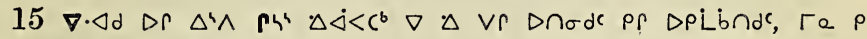
$\Delta U^{0} \nabla V{ }^{L}>d e \cdot \Delta \dot{r}^{b} x$

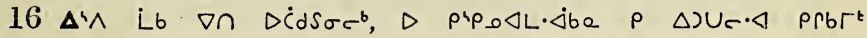
$\triangle S_{x}$

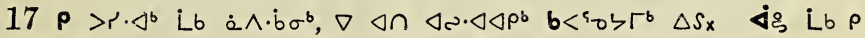

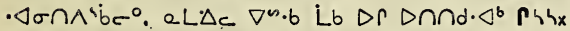

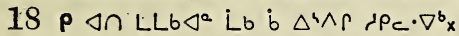

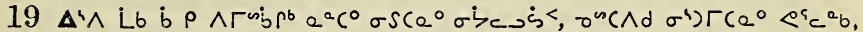

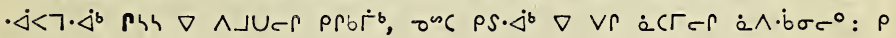
$d^{4} \dot{C} \varphi \cdot \triangleleft^{b} \dot{L} b x$

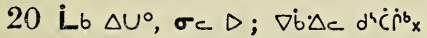

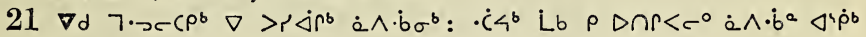
$\Delta C \cdot \dot{\Delta} \triangle S \sin ^{\circ} \mathrm{x}$

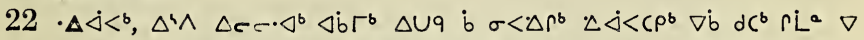

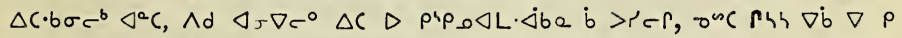

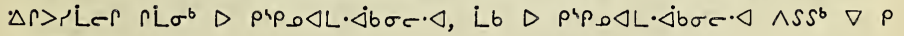

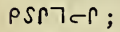

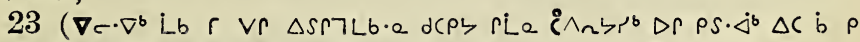

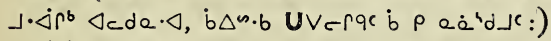

24. $\Delta^{4} \wedge$ Lb $\Delta c c \cdot \Delta^{b} \Delta \dot{j}<C \rho^{b}$ bib $\nabla \dot{b} \nabla^{a} C \nabla \Delta \dot{C}-r, \nabla \dot{b}$ onc $\nabla$

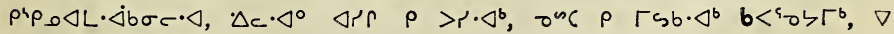
cec $c \cdot \dot{j}<\dot{L} \rho^{b} \boldsymbol{p}\left\langle b_{x}\right.$

$25 \Delta^{4} \wedge$ Lb b $\Gamma^{4} b \cdot\left\langle\rho^{b} . q^{4} b \dot{b} b \quad \Delta \sigma V d^{b}, \rho \Delta U \cdot \Delta^{b}, \dot{<}<, \dot{C} \sigma^{4} \wedge \Delta C\right.$ b $\vee r$ $\triangle U^{2}$ ?

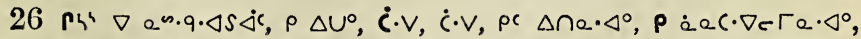

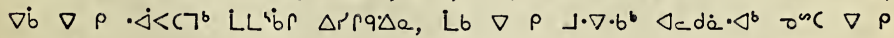
prs $>\zeta^{b} x$

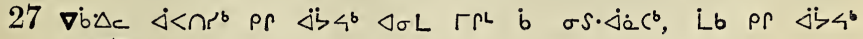
157 


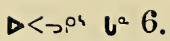

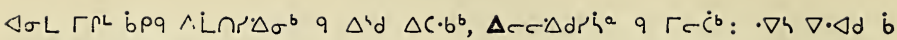
JbPrdC $\triangle \dot{C} \triangle L \cdot \Delta \cdot \Delta x$

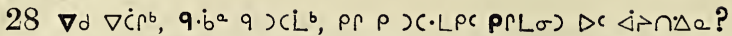

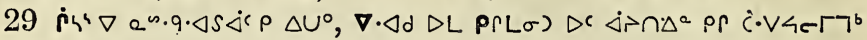
$\triangleleft \sigma \Delta \dot{b} \rho \vee v r \cap s \cdot \dot{i} c_{x}$

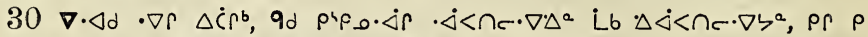

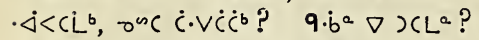

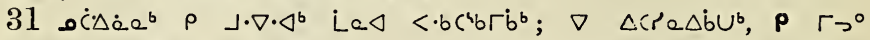

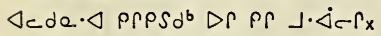

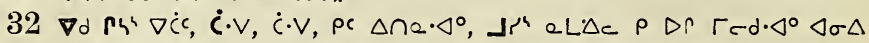

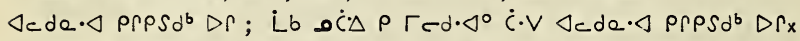

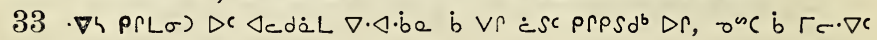

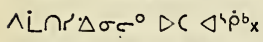

$34 \nabla \dot{0} \nabla \dot{C} \rho^{b} U \vee c i q \zeta^{a}$, b $\rho q \Gamma c \dot{a}^{a} \triangleleft \cdot \triangleleft \triangleleft c d e{ }^{\circ} x$

35 p' Lb $\rho \Delta U^{\circ}, \sigma c \nabla \cdot \Delta d \wedge i n \pi \Delta \sigma \quad \Delta c d a^{\circ}: \Delta e$ b $V r$ asc

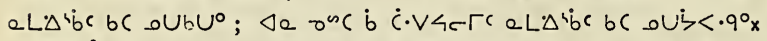

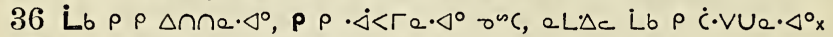

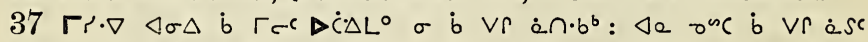
¿CUe el. $\Delta$ e $\sigma \dot{b} \cdot \Delta c \Delta \cdot \nabla \wedge e^{\circ} x$

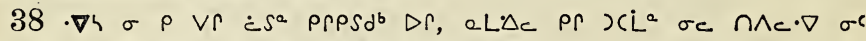

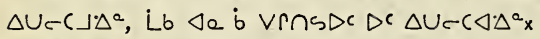

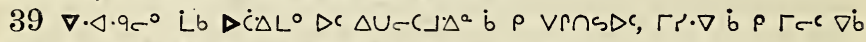

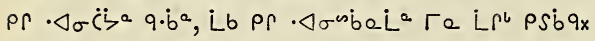

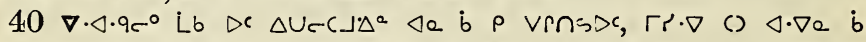

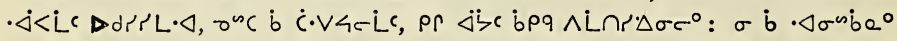
Lb L L

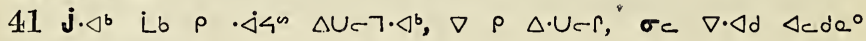
presdb $\dot{b} \Delta r<C^{-c_{x}}$

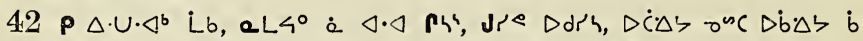

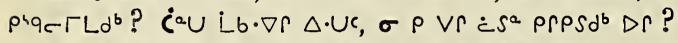

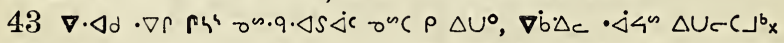

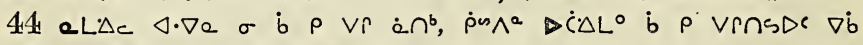

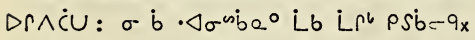

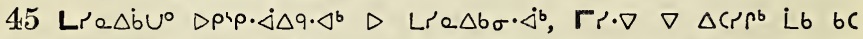

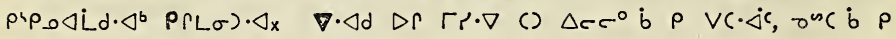
pip.o $\Delta \dot{L} d e \Delta \dot{C} \cdot \Delta L \cdot \triangleleft, \sigma \vee r$ a $\cap^{b} x$

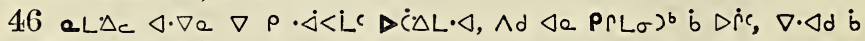
$\rho \cdot \dot{\Delta}<L C \Delta \dot{L} \Delta L \cdot \triangleleft x$

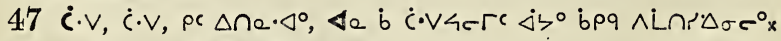

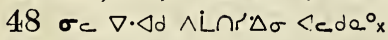

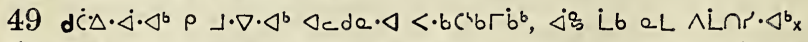

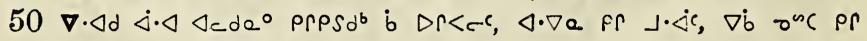
$\sigma \wedge c_{x}$ 


\section{$\triangleright<\rightarrow p^{4}$ しa 6.}

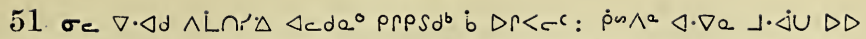

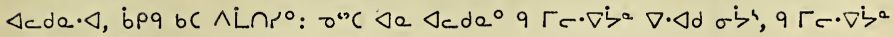

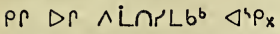

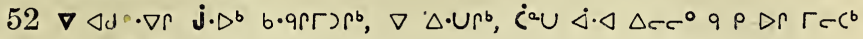

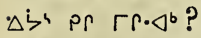

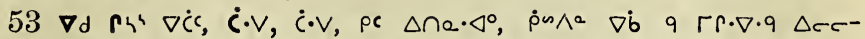

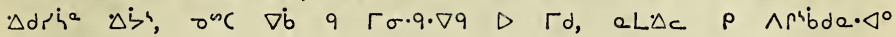
$\wedge \operatorname{Ln} \operatorname{La}^{a}{ }^{a} x$

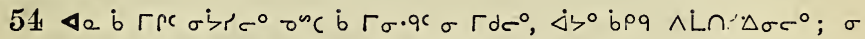

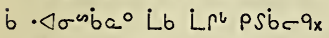

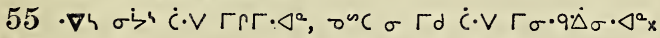

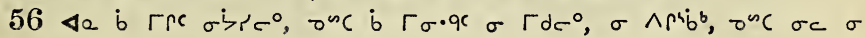
$\wedge \Gamma^{4} b \cdot \triangleleft{ }^{\circ} x$

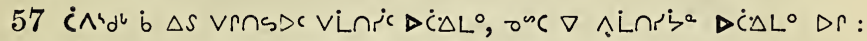

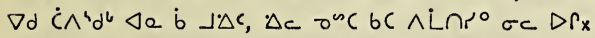

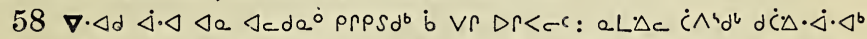

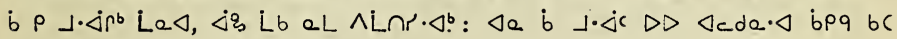
$\wedge \operatorname{Ln} 0^{\circ} x$

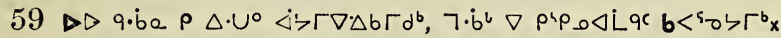

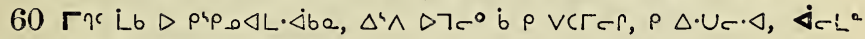
$D L \Delta \cdot U \cdot \Delta^{a} ; \triangleleft \cdot \nabla 0 q P V C^{b}$ ?

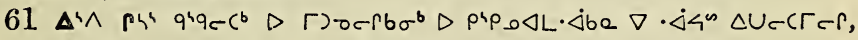

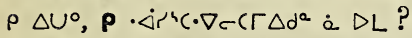

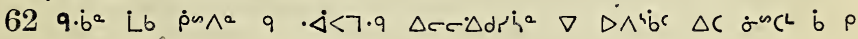
$\Delta \dot{c} c_{x}$

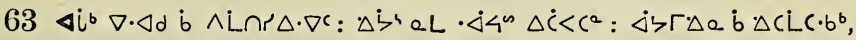

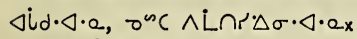

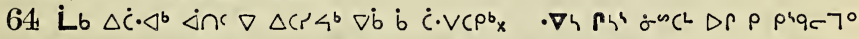

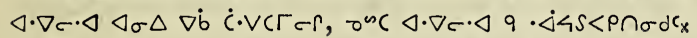

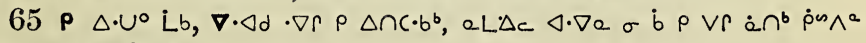
$\nabla \dot{b} \Gamma c d u$ ocं $\Delta x$

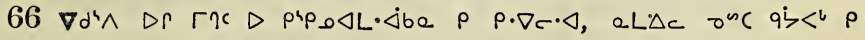
$\Delta\urcorner \Delta d x$

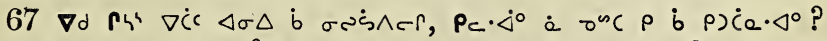

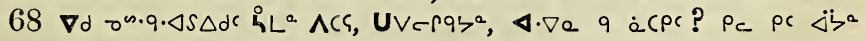

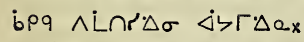

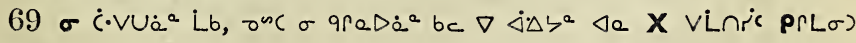
Ddrhx

70 p' $\rho$ Q Q $V \zeta^{b} \nabla \Delta\left(S \zeta^{b}\right.$ Lb $\nabla$ LrL $\left.\sigma\right) \Delta C$ ?

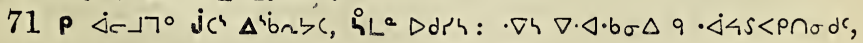
$\nabla \triangleleft S C P L b \sigma \Delta-r \Delta \sigma \Delta \sigma \sigma^{\circ} \dot{s}<_{x}$ 


\section{$D<>$ ps Ga 7.}

\section{A'pcra $\triangle b^{a} 7$}

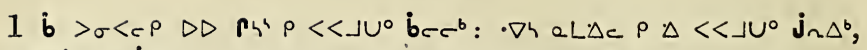
$\nabla \Delta \sigma \dot{r}>d c \dot{j} \cdot \Delta x$

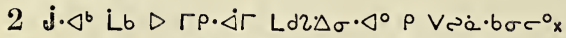

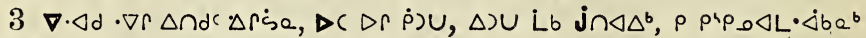
onc $p r \cdot \dot{\Delta<}<(\rho b \triangleleft \sigma \Delta \Delta \vec{i} \cap \Delta e \dot{b})\left(L^{a} \times\right.$

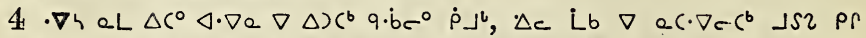

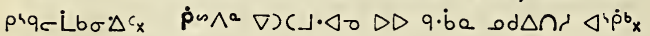

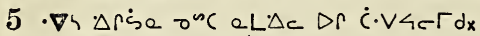

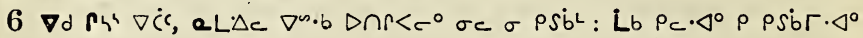
$J s^{x} \dot{\Delta} \Delta \Delta^{4} b \cdot \Delta^{a} x$

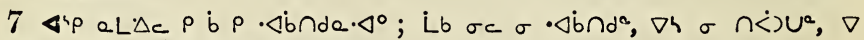

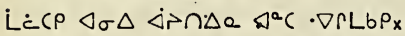

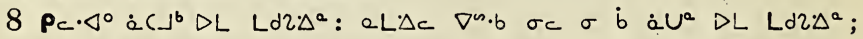
- $\nabla 4 \sigma$ PSibl aL $\left.\Delta c \nabla^{n} \cdot b \Gamma\right) \sigma D \cap K<\tau^{\circ} x$

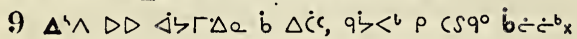

$10 \Delta^{\prime} \wedge$ Lb $\Delta r \dot{s} a$ b $\Delta U U c r, \nabla d$ onc $\Delta c$ oc $\Delta$ a $c^{b}$ Ld2 $\Delta \sigma c^{\circ}$, aL $\Delta c$ \lrcorner $S 2$, Lb $P J^{\prime \prime} \Delta S_{x}$

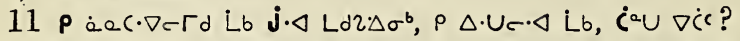

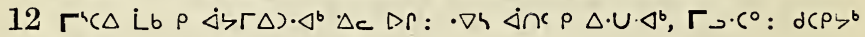
$P \Delta \cdot U \cdot \triangleleft^{b}, a L \Delta c ; L b \cdot \Delta 4 S \nabla^{\circ} \Delta r c \cdot \Delta x$

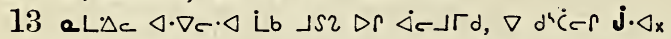

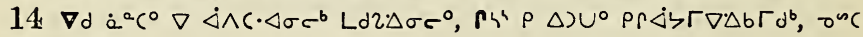
p pros $\Delta$ Li० ${ }^{\circ} x$

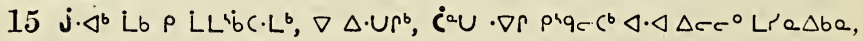
$\nabla \dot{b} \cdot \dot{\Delta} \dot{b} \vec{b} \dot{b} \nabla r$ prp_osic?

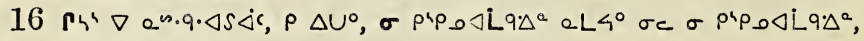

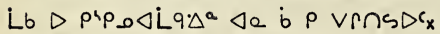

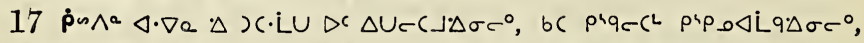

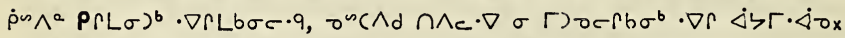

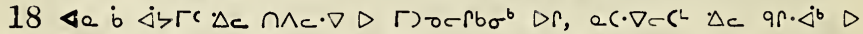

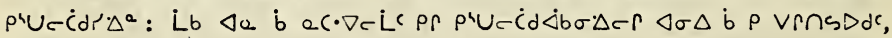

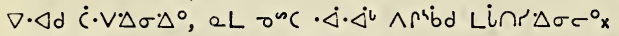

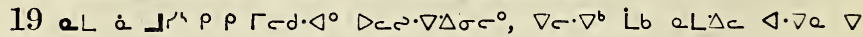

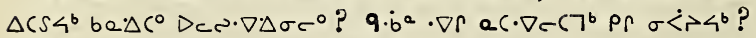

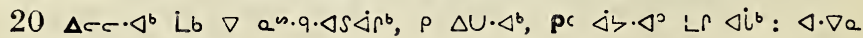

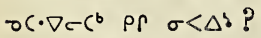

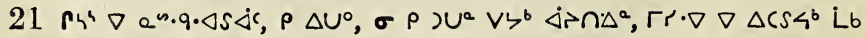
$\rho \quad L L$ 'bUa. $\triangleleft^{\circ} x$

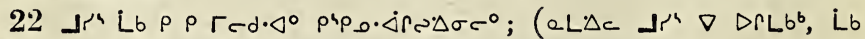

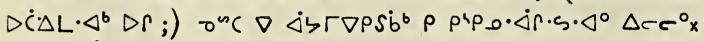

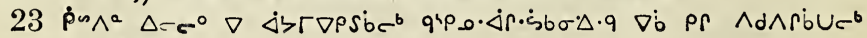
160 


\section{D<spa ve 7.}

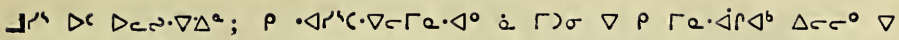

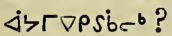

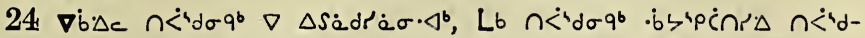
$\sigma 9 \Delta \Delta^{a} x$

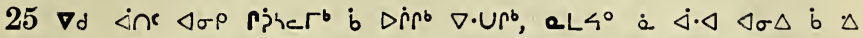
$\sigma<<\rho^{b}$ ?

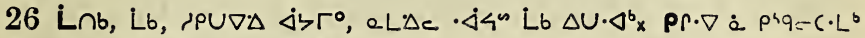
$\triangle P L \cdot \triangleleft^{b} D \supset \dot{C} \cdot V \nabla \triangleleft \Delta-r X$ ?

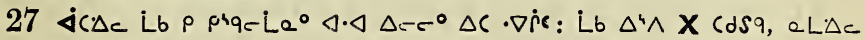
$\Delta \cdot \nabla$ e prqc-CL $\Delta C \cdot \nabla r c r x$

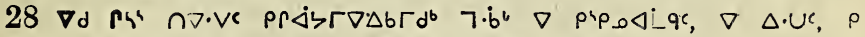

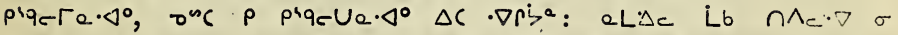

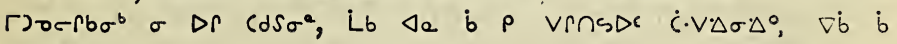
prq- $77^{6} x$

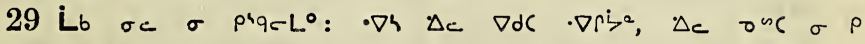
$\operatorname{Vrns} D^{b} x$

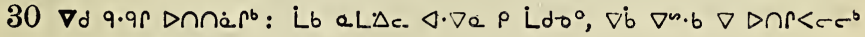
$\nabla \cap \dot{r} \vec{b}^{a} \wedge r j \dot{b} \sigma^{b} x$

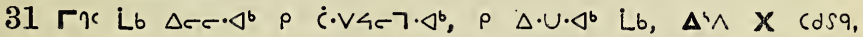

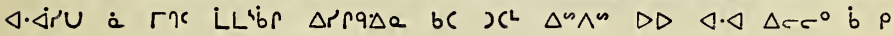
$x^{b}$ ?

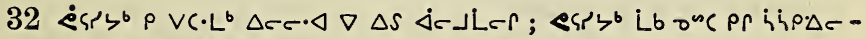

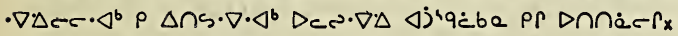

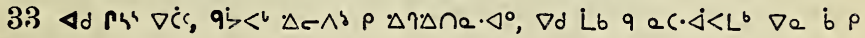
VRnSDCx

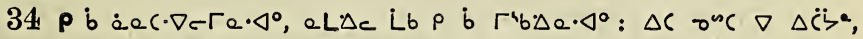
$\triangleleft^{2}(a L \Delta c \rho$ b $\vee r \Delta) \dot{c} a \cdot \Delta^{\circ} x$

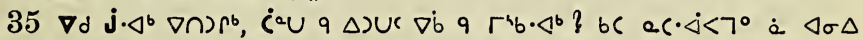

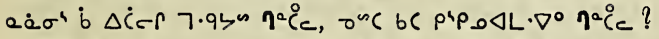

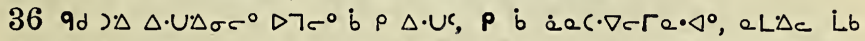

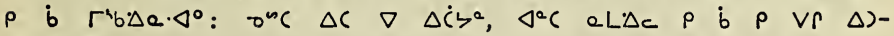
ce. $\triangleleft 0$ ?

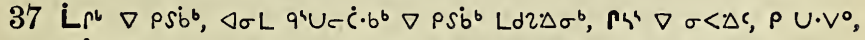

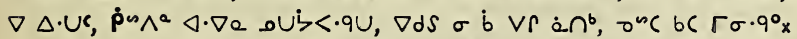

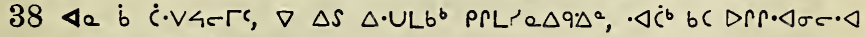
r^ל $\Delta C \nabla \Delta C \cdot b^{b} \wedge i \cap r \Delta \sigma \wedge x$

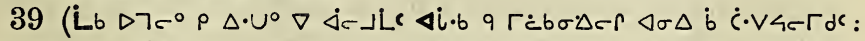

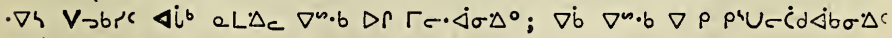
$\left(s^{4}\right) \times$

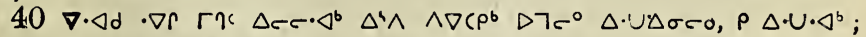
$\dot{C} \cdot \vee \triangleleft \cdot \triangleleft \triangleleft a \triangleright p^{\circ} \cdot \dot{\Delta} \Delta 9^{\circ} \times$

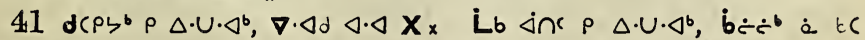
D po $\mathbf{X}$ ? 


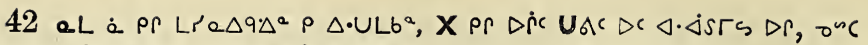
Vecbl $\Delta \dot{C} \Delta \sigma^{b} D r, \Delta C$ UAC b $\rho \Delta \dot{C}^{\prime} x$

$43 \nabla d \dot{<}<b^{a} \nabla \cdot U r^{b} \Delta c c \cdot \triangleleft^{b} \Delta c \Delta r_{x}$

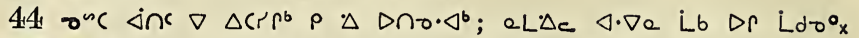

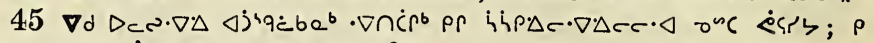
$\Delta \cap d \cdot \Delta^{b}$ Lb, $\dot{C}_{o p} \cdot \nabla r \nabla \dot{b} \rho \vee S \cdot \nabla^{b}$ ?

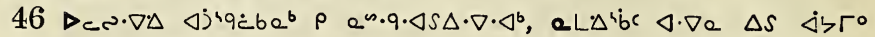

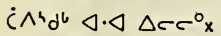

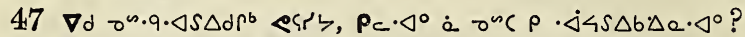

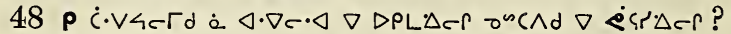

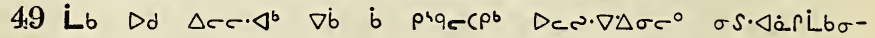
$\triangle \cdot \Delta b_{x}$

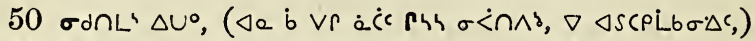

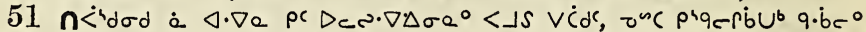
b) $x^{b}$ ?

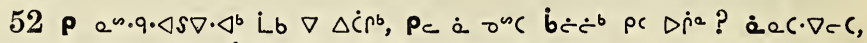

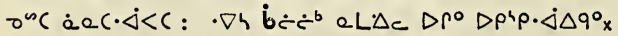

$53 \nabla d \nabla) \cup(r \cdot \nabla c) \triangleleft \cdot \nabla e \cap \wedge c \cdot \nabla \nabla \cdot\left\langle\dot{b} b r_{b} b \Gamma^{b} x\right.$

\section{nipcre $\triangle b^{a} 8$.}

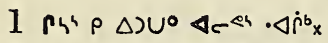

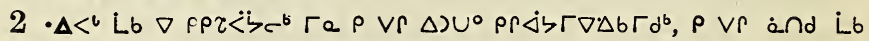

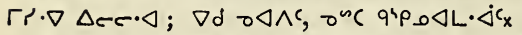

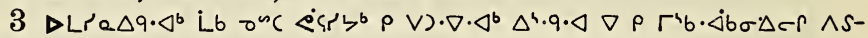

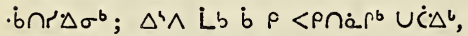

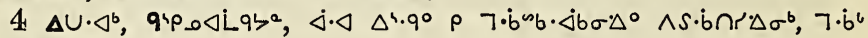
$\nabla \Delta)^{b} \times$

5 لـ L

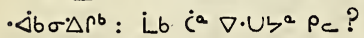

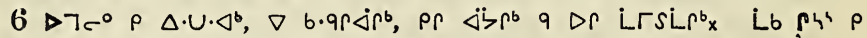

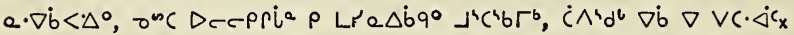

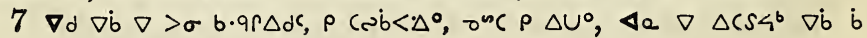
Linec, $\nabla \cdot \Delta d \dot{\sigma}^{n C L} q \wedge \cdot L_{c} \triangleleft r \sigma c^{\circ} x$

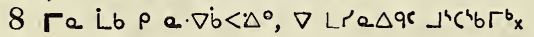

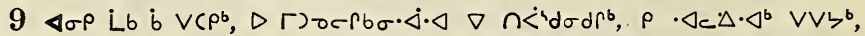

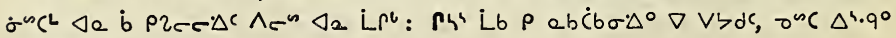
Uं் $\Delta \sigma \quad \sigma<\Delta c_{x}$

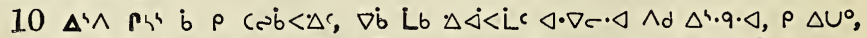

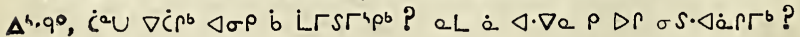

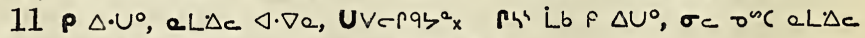

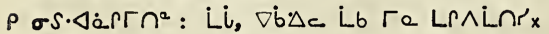

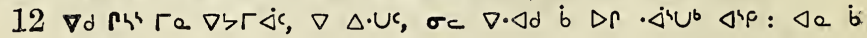
162 


\section{$\triangleright<\neg$ ๒゚ 8 .}

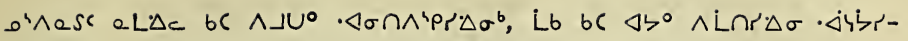
$\Delta \sigma c^{\circ} x$

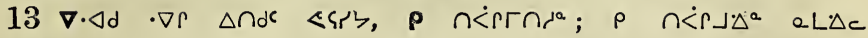
$\dot{C} \cdot V L b^{\circ} x$

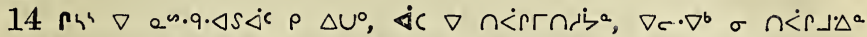

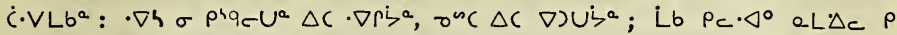

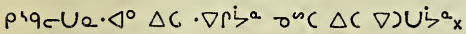

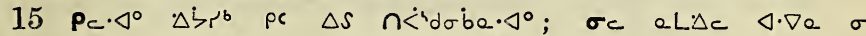

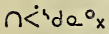

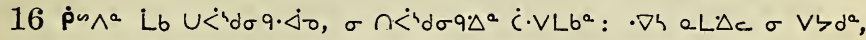
Lb $\sigma c$ o"c $D C \triangle L^{\circ}$ b $P V R \cap S D c_{x}$

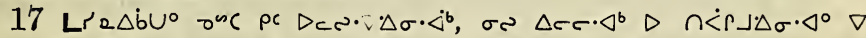
$\dot{C} \cdot V L b \sigma c^{b} \mathrm{x}$

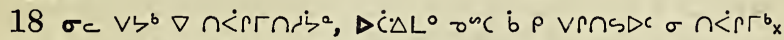

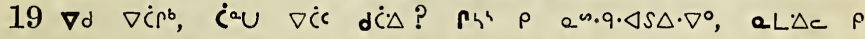

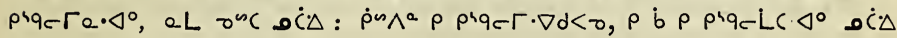
$\cos ^{n} \mathrm{x}$

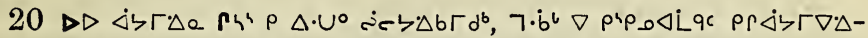

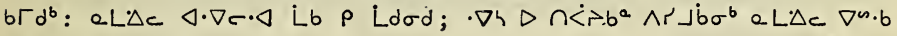
$b$ DOR<C- ${ }^{\circ} x$

$\left.21 \nabla d r^{4} \Gamma a \nabla \dot{c} c, \sigma \rho\right) \dot{c} a, \rho \dot{b}$ àec $\nabla c \Gamma a \cdot \triangleleft^{\circ} L b, \tau^{n c} \rho \dot{b} \sigma \wedge a \cdot \triangleleft^{\circ}$

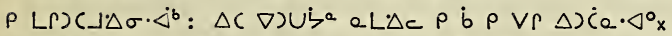

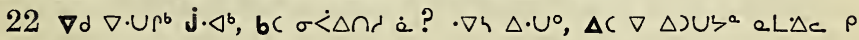
b $\rho \vee r \Delta \dot{x} a \cdot \Delta^{\circ} x$

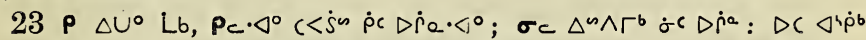

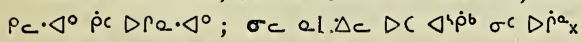

$24 \nabla \cdot \Delta d \cdot \nabla \rho \Delta \cap\left(\cdot b^{b}, \rho\right.$ b $\left.\sigma \wedge a \cdot \triangleleft^{0} \rho L \rho\right)(\lrcorner \Delta \sigma \cdot \dot{\Delta}^{b}: \cdot \nabla h \dot{\rho} \sim \wedge^{a} \nabla b$ $\dot{c} \cdot \vee\left(7 \cdot q \sigma c \nabla \cdot \triangleleft d \nabla \Delta \cdot \Delta \dot{b} a, \rho \dot{b} \sigma \wedge a \cdot \triangleleft^{0} \rho L \rho\right)(\lrcorner \Delta \sigma \cdot \sigma^{b} x$

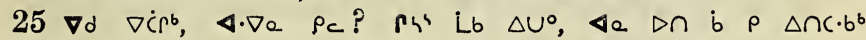
$\dot{\sigma}^{n} C L D r_{x}$

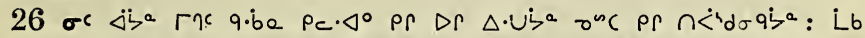

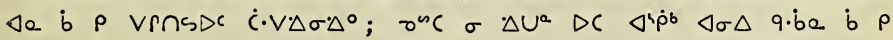
$\Delta C \dot{L} \cdot \Delta c_{x}$

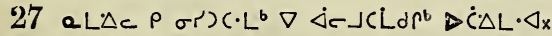

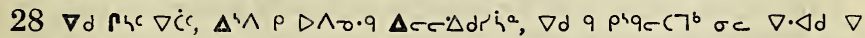

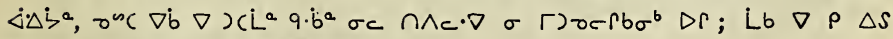

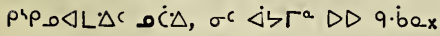

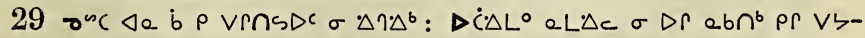
$\left.d \cdot \triangleleft^{b} ; \cdot \nabla h\right\lrcorner c^{b} \sigma^{c} \Delta x \cdot L^{a} \varangle \sigma \Delta q \cdot b e$ b $e \Delta c \nabla \Delta d d_{x}$

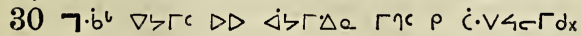

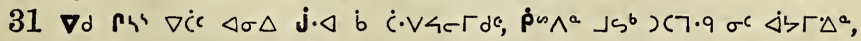
$\nabla d \dot{C} \cdot V \cdot \nabla \rho^{\prime} \rho_{0} \circ \Delta L \cdot \Delta \dot{b} b \sigma \Gamma C \cdot b^{b} ;$

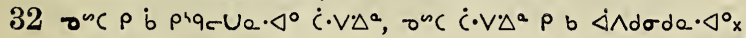
163 


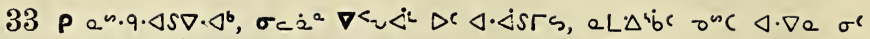

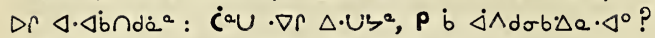

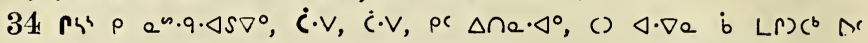

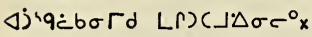

$35 \varangle j^{4} q \dot{c} b^{a}$ Lb aLAc bpq $\wedge r . \varangle b b p \rho \sigma^{b} \Delta c^{\circ}:$ Lb DdrRLf bคq $\Delta c^{\circ} x$

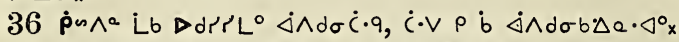

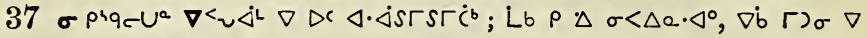
$\wedge r^{i b} \dot{b}<b$ oc $\dot{\forall}>\Gamma \cdot \Delta^{a} x$

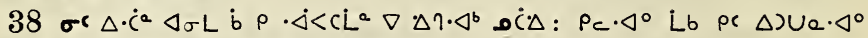
$\triangleleft \sigma L \dot{b} \rho \cdot \dot{j}<C 7^{b} \nabla \Delta \eta \cdot \nabla^{b} d \dot{C} \Delta \cdot \triangleleft^{\circ} x$

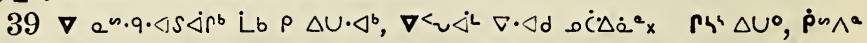

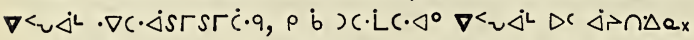

$40 \varangle 0^{\prime \prime} L_{b} \rho \Delta \sigma<\Delta a \cdot \triangleleft^{\circ}, \nabla \Delta r c \Delta^{\circ} b^{a} \nabla \Delta C L C \cdot b^{b} \dot{C} \cdot V \cdot \Delta^{a}$, b $\rho \Delta C L \cdot \Delta^{c}$

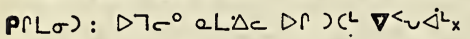

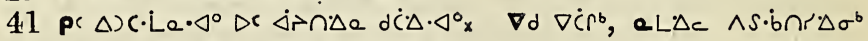

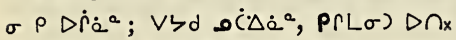

$\left.42 p^{3} \rho \Delta U^{0}, p^{n} \Lambda^{a} P R L \sigma\right) \cdot \nabla \dot{C} \Delta 4 d, \rho$ b $h \rho \Delta\left(\cdot \triangleleft^{\circ}: \cdot \nabla h P R L \sigma\right)^{b} \sigma^{\circ}$

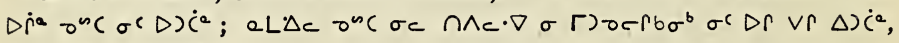
Lb $\sigma \rho V I \cap S D^{b} x$

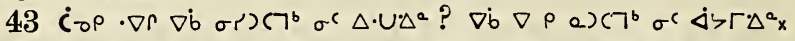

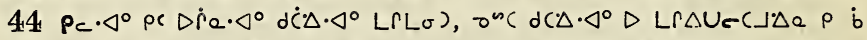

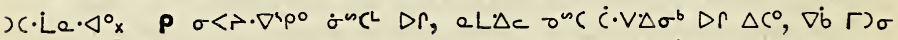

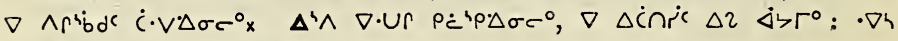

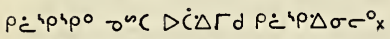

$45 \nabla \Delta C \dot{L} C \cdot b^{b} L b \dot{C} \cdot V \cdot \Delta^{a}, a L \Delta c \rho \dot{C} \cdot V C \cdot \Delta a \cdot \triangleleft^{\circ} x$

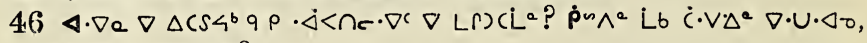
$\dot{C} \sigma \rho \cdot \nabla r \nabla \dot{C} \dot{C} \cdot V C \cdot \Delta \zeta^{b}$ ?

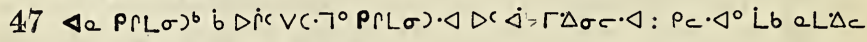
$\left.\rho \vee C \cdot L a \cdot \nabla^{\circ} \nabla \dot{b} P \Gamma L \sigma\right)^{b} \nabla \nabla \Gamma \zeta^{b} x$

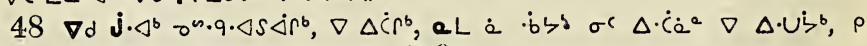

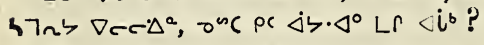

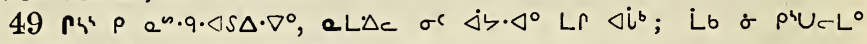

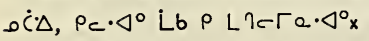

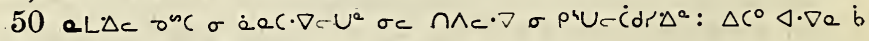

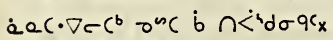

$51 \dot{c} \cdot \vee \dot{c} \cdot \vee, \rho c \Delta n_{a} \cdot \triangleleft^{\circ}, \dot{p} \backsim \wedge^{a} \triangleleft \cdot \nabla a b 2 \Delta \dot{C} U \sigma^{c} \Delta \cdot U \Delta \sigma c^{\circ}, a L \cdot \Delta b c b c$ $\cdot \dot{j<c} \sigma \wedge \Delta \sigma c^{\circ} \mathrm{x}$

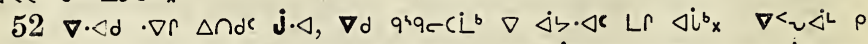

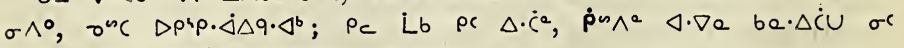
$\triangle \cdot \cup \cdot \Delta \sigma \sigma^{\circ}, a L \Delta \Delta^{4} b c b C d r^{3} C^{L} \sigma \wedge \Delta \sigma \sigma^{\circ} x$

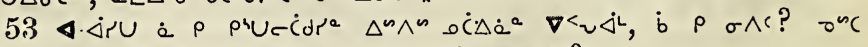

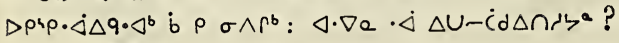




\section{$D<\rightarrow$ pr be 8,9}

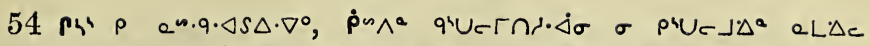

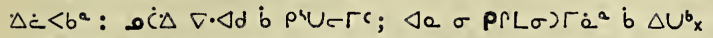

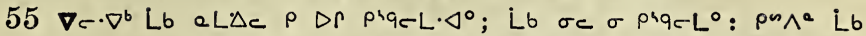

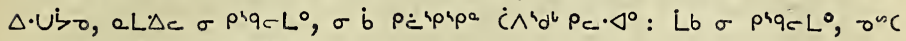
$\sigma$ be $\Delta C \cdot \dot{u}^{a} \Delta c \Delta \cdot U \cdot \Delta^{a} \mathrm{x}$

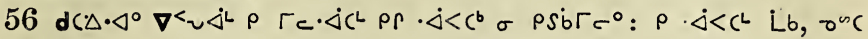
$\rho<p>\rho^{\circ} x$

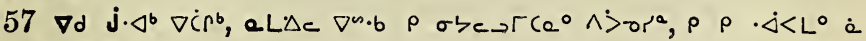
Lb $\nabla<u<i$ ?

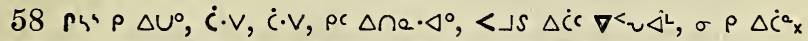

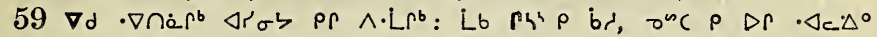

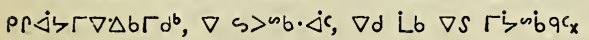

\section{nipcra $\triangle b^{a} 9$.}

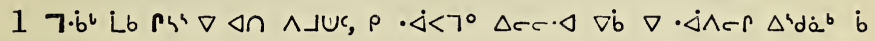
$\sigma \dot{C} \triangle P_{C}-r_{x}$

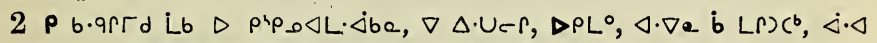
$\triangle c c^{\circ}, \sigma^{n} C \wedge d \Delta \sigma \rho \Delta \cdot b, \cdot \nabla r \nabla b \cdot j \wedge c$ oc $\Delta \rho c$ ?

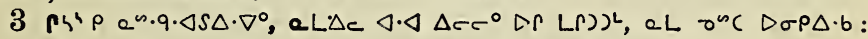

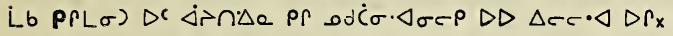

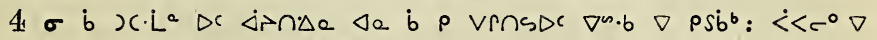
$\cap \wedge^{b} b^{b}, \Delta 4 \wedge \nabla \dot{b} \Delta \cdot \nabla a q \rho \quad \dot{\Delta}<\cap r c_{x}$

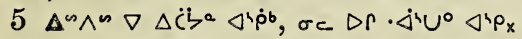

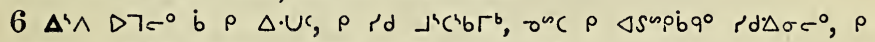

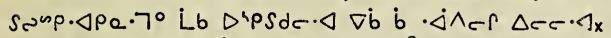

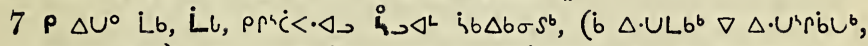

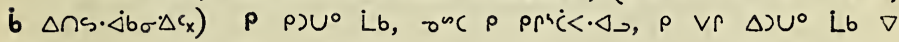
$\cdot \dot{\Delta} \wedge c_{x}$

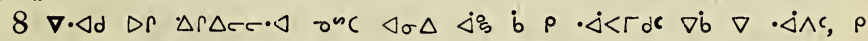
$\triangle \cdot U c \cdot \triangleleft, a L \zeta^{\circ} \dot{a} \triangleleft \cdot \triangleleft \triangleleft a$ b $\rho \triangleleft \wedge c \nabla<d S \cdot \dot{C} c$ ?

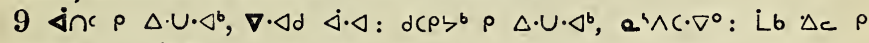
$\Delta \cdot U^{0}, \sigma c \sigma^{c} \Delta \Delta^{a} x$

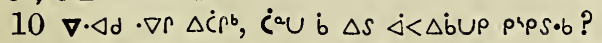

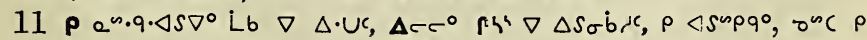

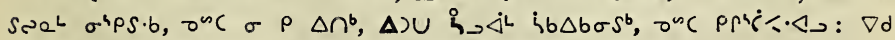

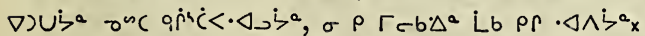

$12 \nabla d \nabla \dot{C} \kappa^{b}, \dot{c} u \nabla \dot{C}$ ? $\rho \Delta \cdot U^{\circ}$, el $\Delta c \sigma \rho^{4} q_{c} U^{a} x$

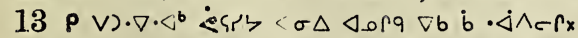

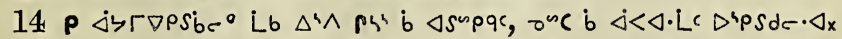

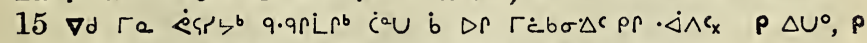

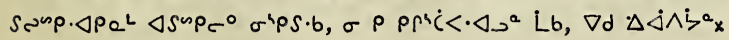




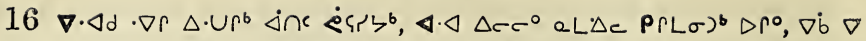

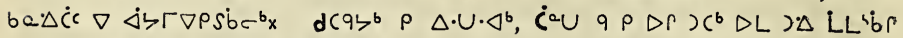

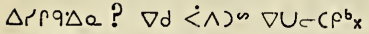

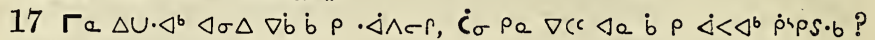
p $\Delta \cdot U^{\circ}, \Delta \rho \varphi \rho \cdot \dot{\Delta} \Delta q^{\circ} \Delta^{\circ} \mathrm{x}$

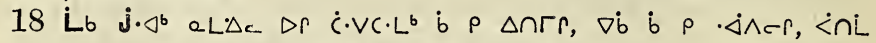

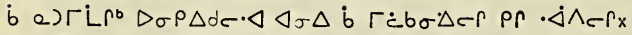

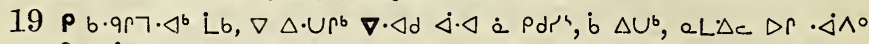

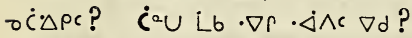

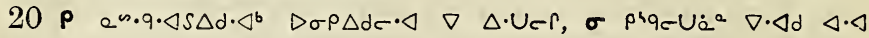

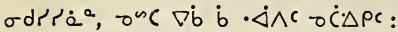

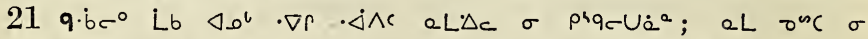

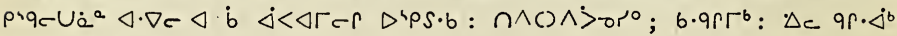
bC $\Delta \rightarrow \Gamma^{\prime} C L i d x$

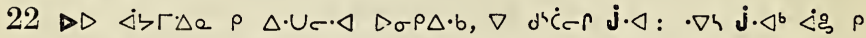

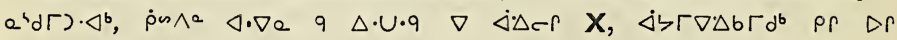
$\cdot \triangleleft c \cdot \Delta \cap S \dot{b} b \sigma \cdot \Delta c_{x}$

$23 \nabla \cdot \triangleleft d \cdot \nabla r \Delta \cdot U c r \Delta \sigma P \Delta \cdot b, n \wedge C \wedge>0 r^{\circ} ; b \cdot q r r^{b} x$

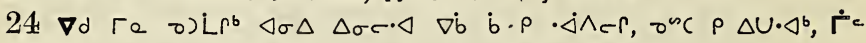

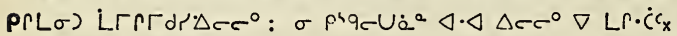

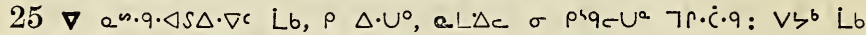
$q \cdot b^{a} \sigma$ prqc ${ }^{a}, \nabla \cdot \Delta d, \nabla b \nabla \Delta r \cdot \dot{\Delta} \wedge \dot{b} a, \Delta 0^{b} \sigma \cdot \dot{\Delta \Lambda^{a} x}$

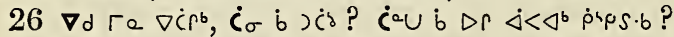

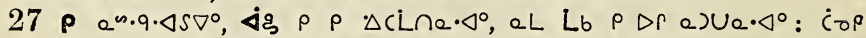

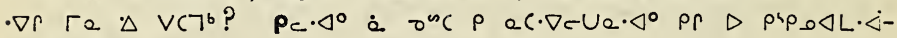
b $\sigma \Gamma^{\circ} \mathrm{c}$ ?

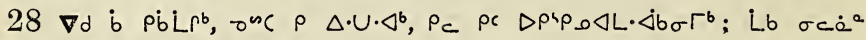

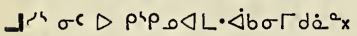

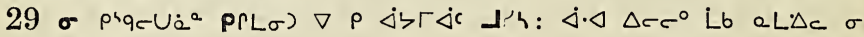
prac U $\dot{a}^{2} \Delta C \cdot \nabla \dot{r} c_{x}$

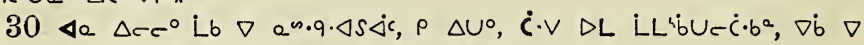
$\rho^{s} q_{c} c 7^{b} \Delta c \cdot \nabla \dot{r} c, \nabla c \cdot \nabla^{b} L b \nabla \rho<<<\Delta^{b} \sigma^{4} \rho s \cdot b x$

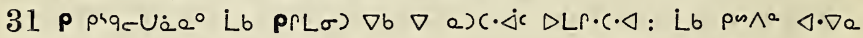

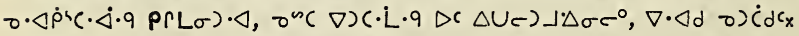

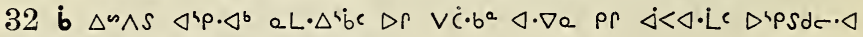
$\triangleleft \cdot \nabla c \cdot \triangleleft \nabla \dot{b} \nabla \Delta r \cdot \Delta \wedge c r \quad 0 C \Delta \rho_{c} r_{x}$

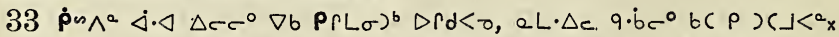

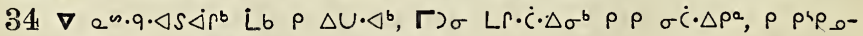
$\Delta L \cdot \Delta \dot{a}{ }^{a} \dot{a} L b \quad \sigma c \dot{a}^{a}$ ? $\nabla d \cdot \nabla c \Delta \cap S \dot{a} r^{b} x^{2}$

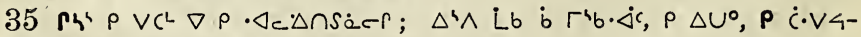
$c \Gamma L \cdot \triangleleft$ a $P(L / \sigma)$ Dorh?

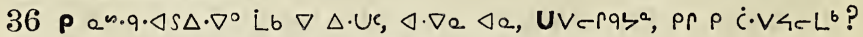

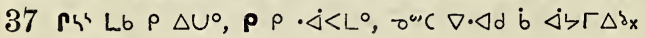




\section{$\Delta<>p^{4}$ ua 9, 10.}

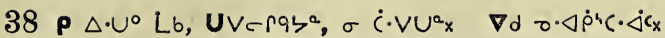

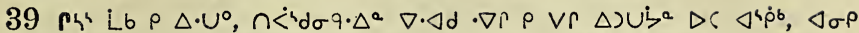

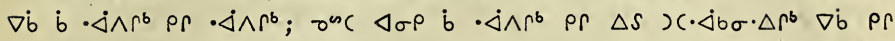

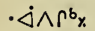

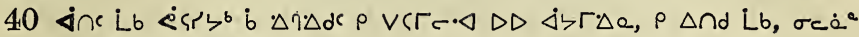

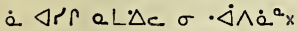

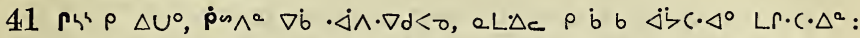

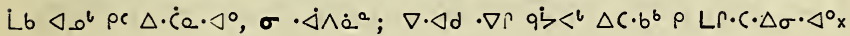

\section{Ápcre $\triangle b^{a} 10 x$}

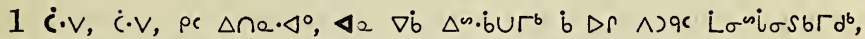

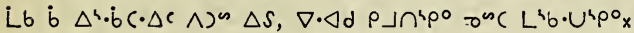

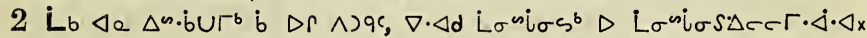

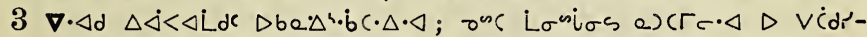

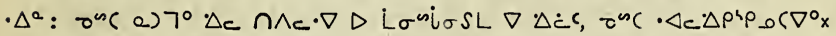

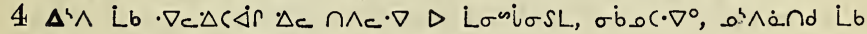
L $\left.\sigma^{u i} i_{\sigma} s: \cdot \nabla h \sigma r\right)\left(\Gamma c \cdot \triangleleft \Delta V \dot{C} d r \cdot \Delta^{a} x\right.$

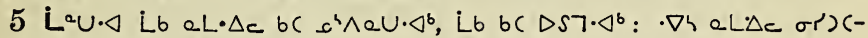
$\cdot 7 \cdot \Delta^{b}\left[{ }^{a} U \cdot \triangleleft \triangleright V \dot{C} d r \cdot \Delta \sigma c^{\circ} x\right.$

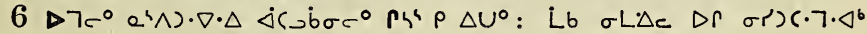

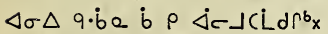

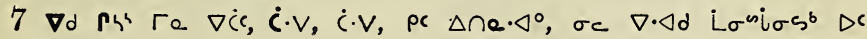

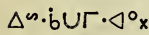

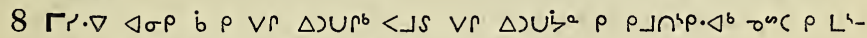

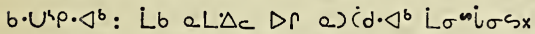

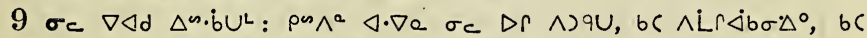
$\wedge) q^{\circ}$ Lb $\sigma^{\infty} C b c \cdot \Delta c \Delta^{\circ}, b c \Gamma^{\circ} b^{L}$ Lb $\Gamma \Gamma d \Delta \sigma c^{\circ} x$

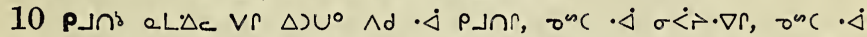

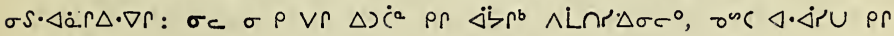
$\cdot \nabla \rightarrow r \dot{c} r^{6} x$

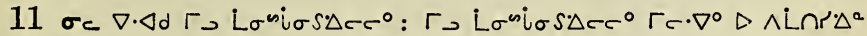
L $\sigma^{\operatorname{si} \sigma s} \nabla r_{x}$

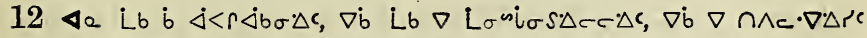

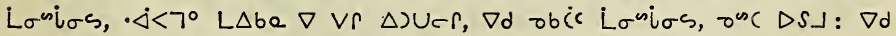

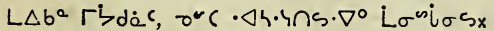

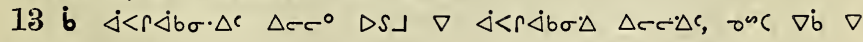
c.9cLe Losifosx

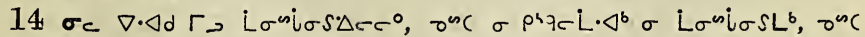
$\left.\sigma p^{p} q_{c} \Gamma \cdot b^{b} \Delta_{\sigma} p \dot{b} \cap \wedge c \cdot \nabla \cdot \Delta r^{i}\right\rangle^{2} x$

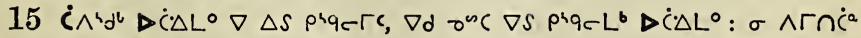

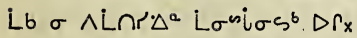

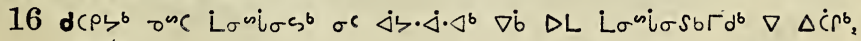
167 


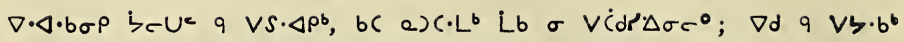

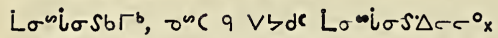

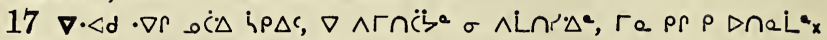

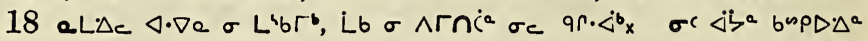

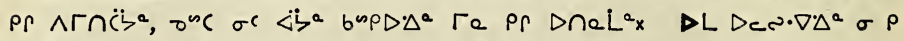
$\Gamma c^{b} \mathrm{C} \dot{\mathrm{C}} \Delta \mathrm{x}$

$19 \nabla \cdot \Delta d \cdot \nabla r \Gamma e<<b^{a} \Delta \cdot u r^{b} j \cdot \triangleleft^{b} . \Delta \triangleright\left\langle j b \Gamma \Delta e \Delta r_{x}\right.$

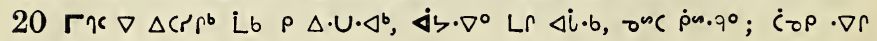
a) $C \cdot \nabla^{b}$ ?

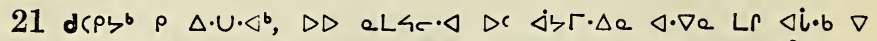

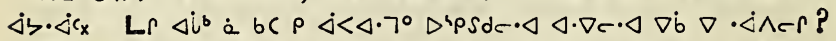

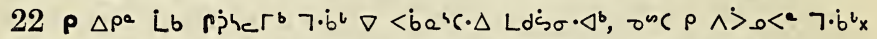

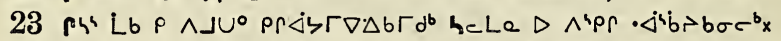

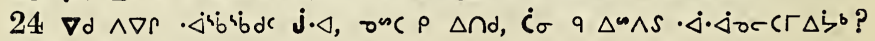
$\dot{\rho} \sim \wedge \wedge^{a} \Delta \dot{\Delta} \Delta \cdot \Delta 0 X, \mu S 2 \Delta C L \Delta \dot{Q}^{a} \times$

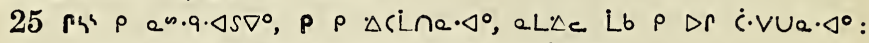

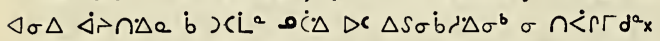

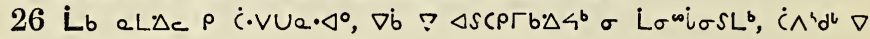
P $\triangle \cap C \cdot b^{b} x$

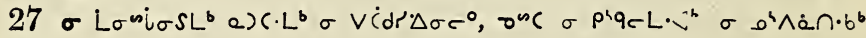
$\mathrm{J}^{\infty} \mathrm{c} x$

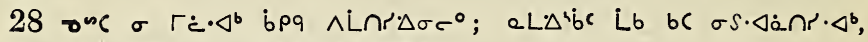

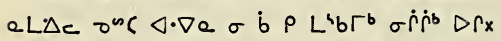

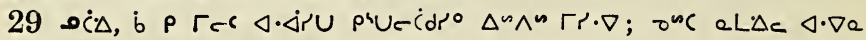

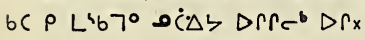

30 oc onc $0 \dot{c} \Delta \sigma$ Vלda ${ }^{a} x$

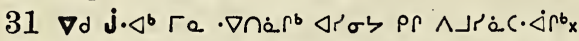

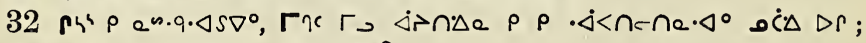

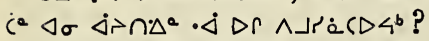

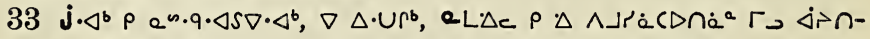

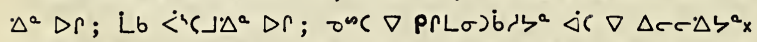

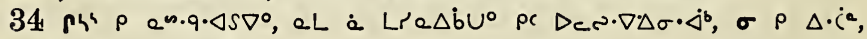
$\left.P_{c} \cdot \triangleleft^{\circ} p L \sigma\right\rangle \cdot Q \cdot \triangleleft^{\circ}$ ?

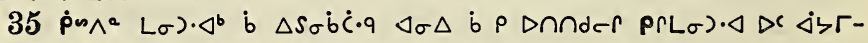
$\Delta \sigma c^{\circ}$, pr Lre $\Delta q^{\circ} \Delta^{\circ}$ Lb a L $\Delta_{c}$ bC $\rho \wedge d \wedge r b U^{\circ}$;

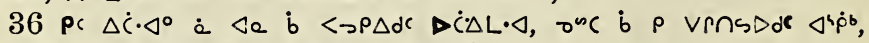

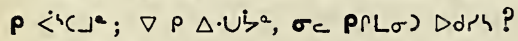

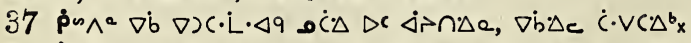

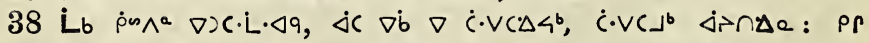

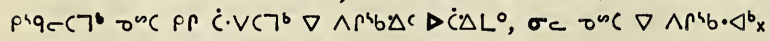

$39 \nabla \cdot \Delta d \cdot \nabla r \Gamma e \Delta \Delta \cap \cap \dot{Q} r^{b}:$ Lb $\rho \rho \Delta d \cdot<^{b}$

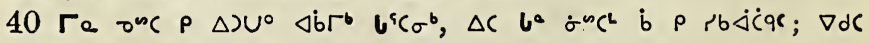
Lb Usq. 


$$
D<\rightarrow \text { P fe } 10,11 .
$$

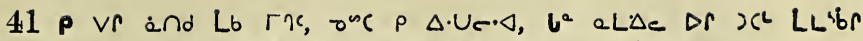

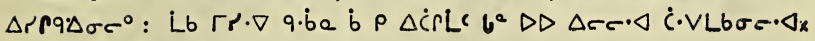
$42 \Gamma \eta \operatorname{Lb} \nabla d c \cap \Delta \cdot V 4 C \Gamma d x_{x}$

A pera $\Delta b^{\circ} 11$.

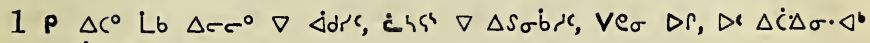
ᄀน o"c Lise $\Delta r \dot{s} \sigma^{4} \cdot 9 L x$

$2 \nabla \cdot \triangleleft d \triangleleft a$ ᄀn b $\rho$ CL'dác UVerqer CL'd $\Delta \sigma c^{\circ}$, omc b p brerUác

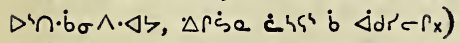

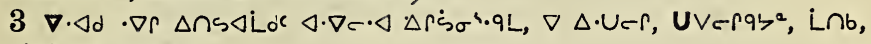

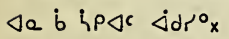

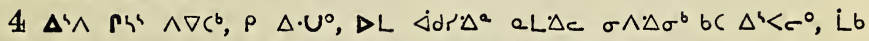

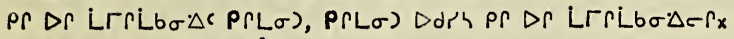

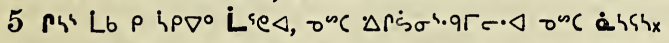

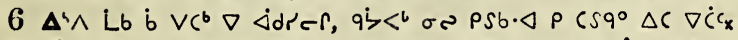

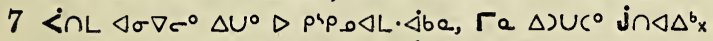

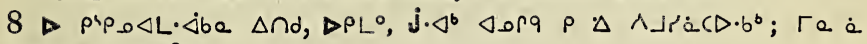
Lb $\triangleleft^{a} \mathrm{C}$ pe $\left.\Delta\right) \dot{C}^{a}$ ?

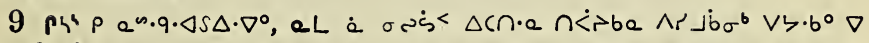

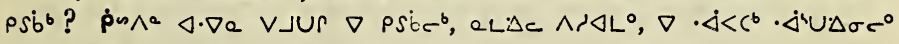
$\triangle C \Delta^{4} \dot{p} b$ b $\Delta C \cdot b \sigma \sigma^{b} x$

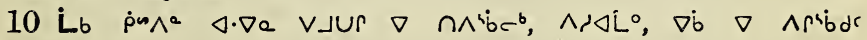
$\cdot \Delta 4 U \Delta \sigma \sigma^{\circ} x$

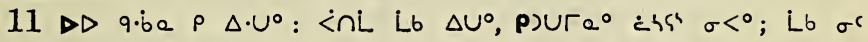

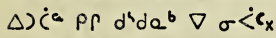

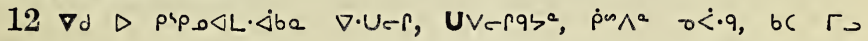
$\dot{4} \succ^{\circ} \mathrm{x}$

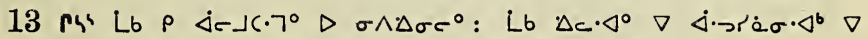
$\left.\sigma \dot{<} \sigma \cdot \Delta^{b} \dot{\Delta} \sigma\right\lrcorner C^{L}, P \Delta U \tau^{\prime} \cdot \cdot<^{b} x$

$\left.14 \nabla \partial \rho^{4}\right\lrcorner S 2 \nabla \dot{C}, \dot{C}, S^{4} \rho \sigma \Lambda^{\circ} \mathrm{x}$

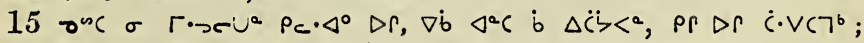
$\nabla c \cdot \nabla^{b} L b a c \cdot \dot{\Delta}<L C^{\circ}{ }^{\circ}$

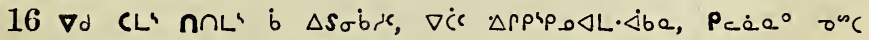
$\Delta U c^{\circ}$ or $\Delta r \sigma \wedge L^{b} x$

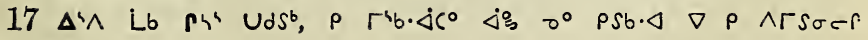
$\mathrm{i} \dot{<} \rightarrow b r d^{b} x$

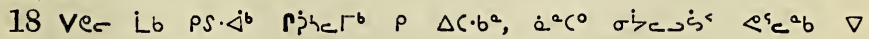
$\Delta x \wedge i b^{b}$

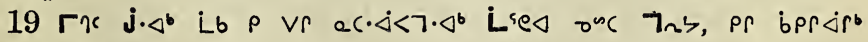
$\Delta r \dot{s}_{\alpha \sigma} \cdot \triangleleft \Delta r_{x}$

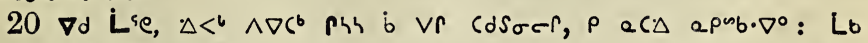
ᄀน $\rho \triangleleft \wedge^{0} \cdot \Delta^{j} b \vec{b} b \sigma^{b} x$

169 


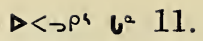

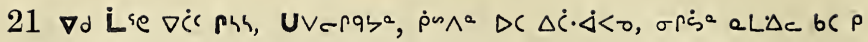
$\sigma \wedge<<^{a} x$

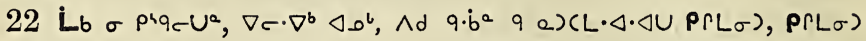
$\rho$ is $\Gamma \sigma^{b} x$

23 rs $\Delta U^{\circ}, p \Gamma \dot{s} a \Gamma a b C \cdot \Delta \sigma^{\infty} b^{\circ} x$

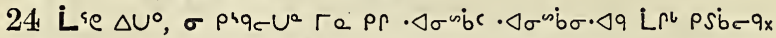

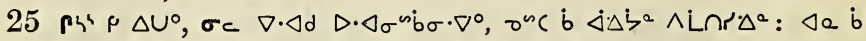

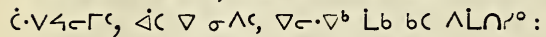

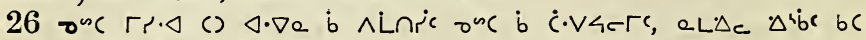
$\sigma \wedge^{\circ} \mathrm{P} \dot{C} \cdot V U^{a} \dot{a} D L$ ?

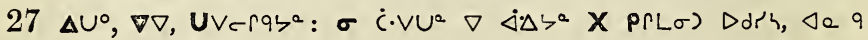
Vr $\Delta u^{h}<<^{a} \Delta C \Delta^{h} \dot{\rho}^{b} x$

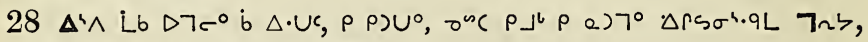
$\checkmark \triangle \cdot U c, D P L^{\circ}\left(d S^{a}, \tau^{n}\left(P\right.\right.$ a) $\Gamma^{b} x$

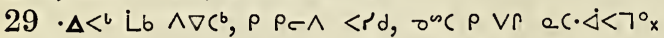

30 eL $\Delta c \nabla^{n \cdot b}$ Lb $\left.\rho^{4} \Delta r \wedge\right) q^{\circ} \Delta \dot{C} \Delta \sigma^{b}$, Ĺb $\rho \Delta c^{\circ} \Delta c \dot{b} \rho$ epubde isesx

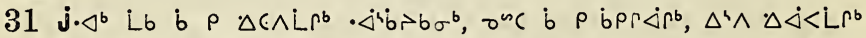

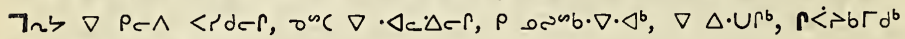
$\triangle U^{\circ} \nabla d C$ PS L $x_{x}$

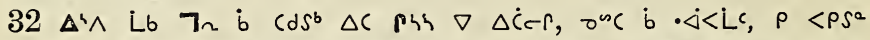

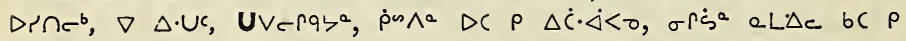
$\sigma \wedge<{ }^{a} x$

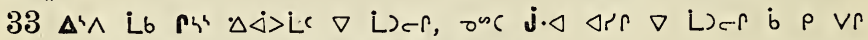

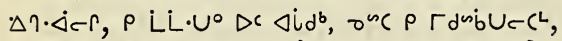

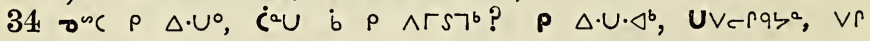
$\cdot \dot{j}<c x$

35 ps $p$ Ĺx

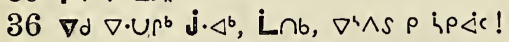

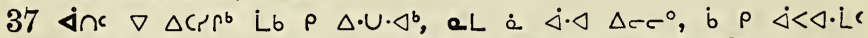

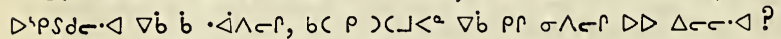

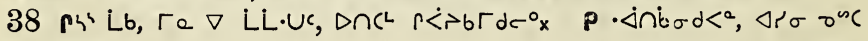
C $d^{l} b \triangleleft \wedge \times x$

39 pr $\rho \Delta \cdot U^{\circ}, \Delta b \cup \sigma^{b} \Delta r \sigma x$ Lse, $\Delta r \dot{s} \sigma^{4} .9 L \Delta e$ b $\sigma \wedge c, \Delta U^{\circ}$,

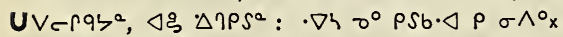

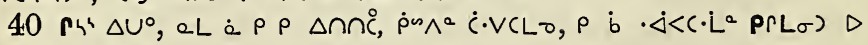
pivéंdr. $\triangle a ?$

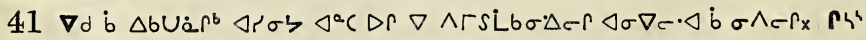

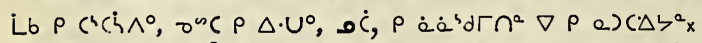

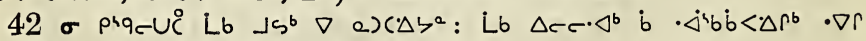

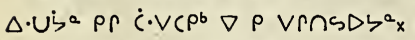

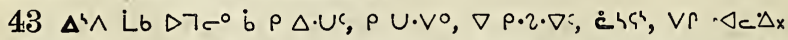

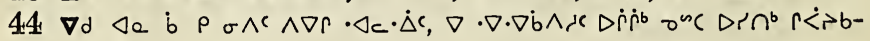
170 


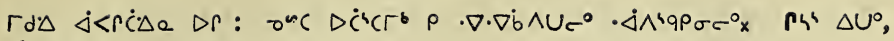

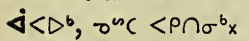

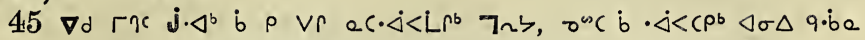
bus b $b$ )

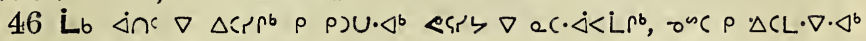
$\Delta \sigma \Delta q \cdot \dot{b}$ e $\rho_{4 h}$ b $\left.\rho\right)\left(\Gamma-r_{x}\right.$

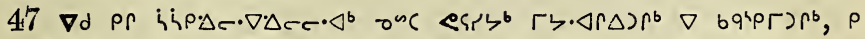

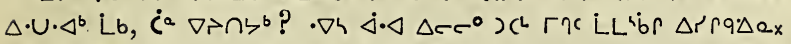

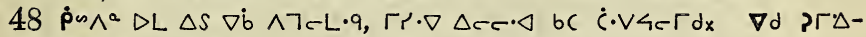

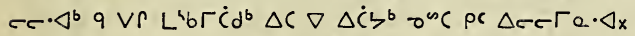

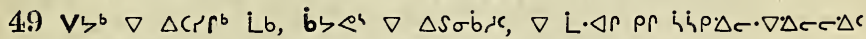

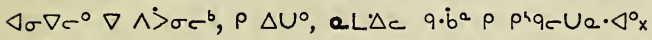

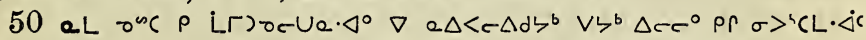

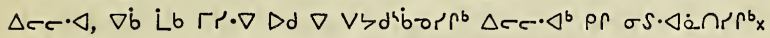

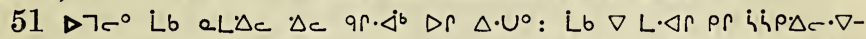

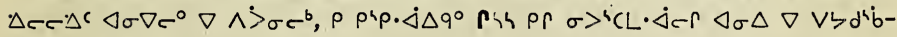
orer $\triangle r c \cdot \triangleleft$;

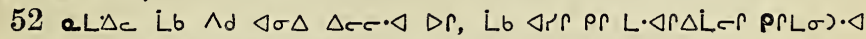

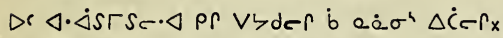

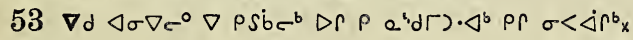

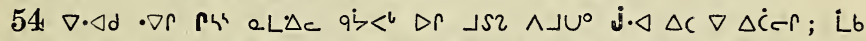

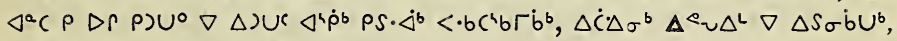

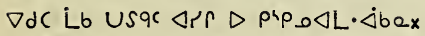

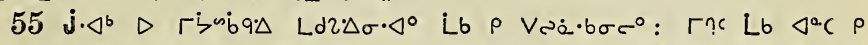

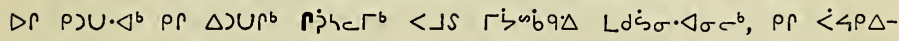
nrpbx

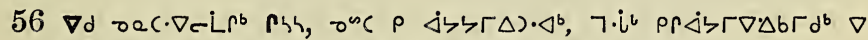
$\sigma<\Delta r^{b}, \dot{c}^{a} \nabla U c c 7^{b} \rho_{c} \cdot \triangleleft^{\circ}$, aL a bc Vr $\Delta U^{\circ} L d 2 \Delta \sigma^{b}$ ?

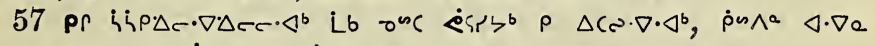

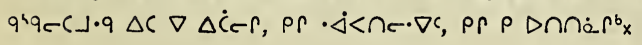

\section{A p cre $\triangle b^{\circ} 12$.}

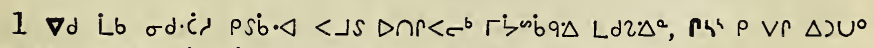

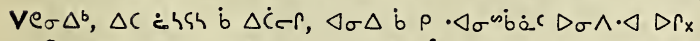

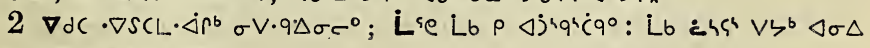
b. $\Delta C \wedge r d c r t r i a n d b x$

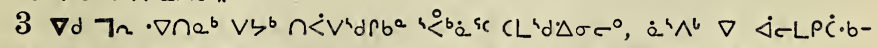

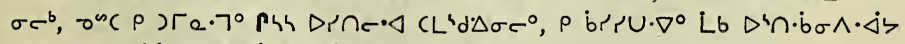

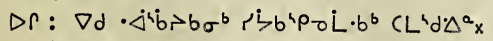

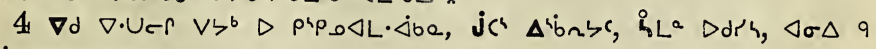
$\cdot \triangleleft 4 S<p \cap \sigma d c$, 


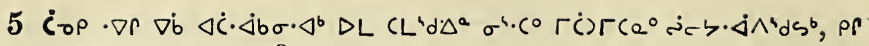
Lं்

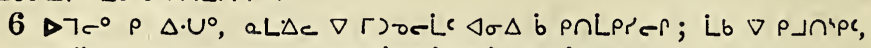

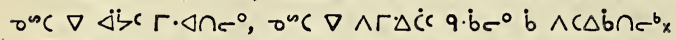

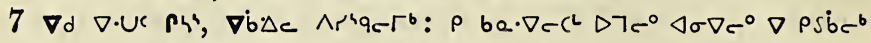
Dr $q a \Delta b b \cdot \Delta \cdot \Delta^{b} x$

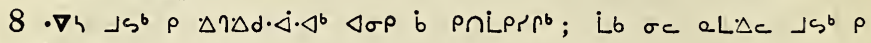
$\Delta\urcorner \Delta \cap a \cdot \triangleleft^{\circ} x$

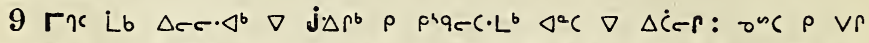

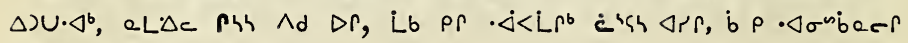
$\nabla \sigma \wedge \cdot \triangleleft \Delta r_{x}$

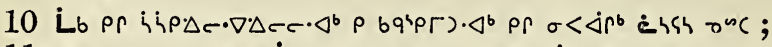

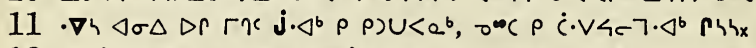

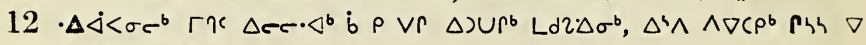
vr $\Delta$ Uuer ríke $\Gamma^{b}$,

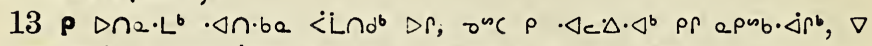

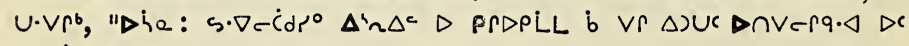
$\Delta S \sigma b \cdot d \sigma c^{b} x$

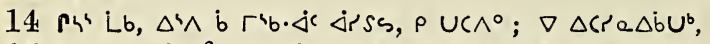

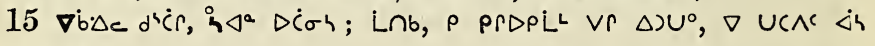
$D d S S c \cdot \triangleleft x$

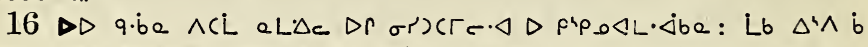

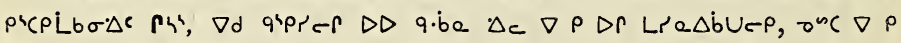
$x \cdot \dot{\Delta r^{6}} \Delta D q \cdot \dot{b} e_{x}$

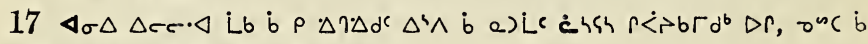

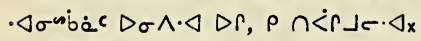

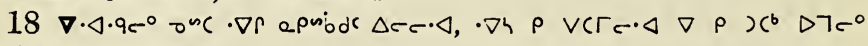
LL'br $\triangle r r q \Delta \sigma \sigma^{\circ} x$

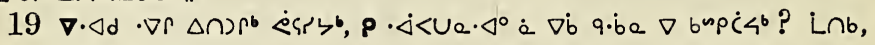

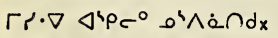

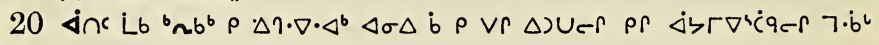
b) $L d \dot{S} \sigma \cdot \triangleleft \sigma \sigma^{b}$ :

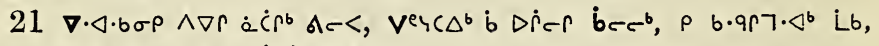
$\nabla \Delta \cdot U \mu^{b}, \Delta P L^{\circ}, \sigma \Delta \cdot \dot{j}<L \dot{Q}^{a} \Gamma^{4} \zeta^{b} x$

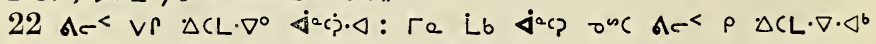
Pith

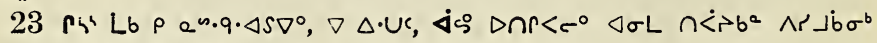

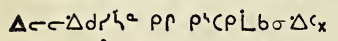

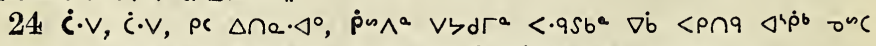

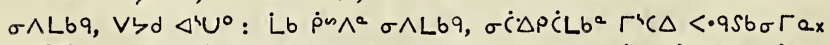

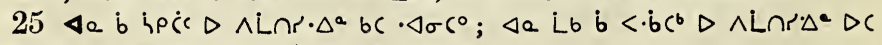

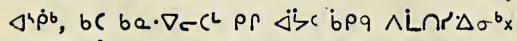

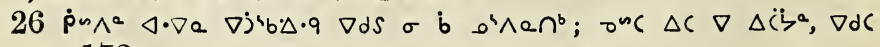
172 


\section{$\Delta<\supset \rho^{\natural}$ し́ 12 .}

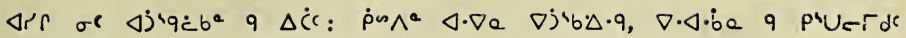
oi $\Delta b x$

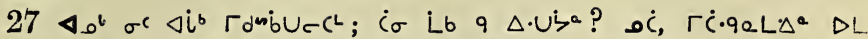

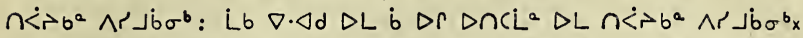

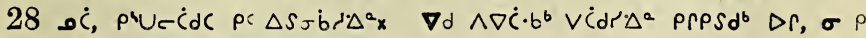

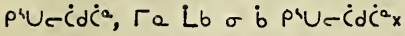

$29 \Delta-c \cdot \triangleleft^{b} L b \triangleleft^{a} c b \sigma<\Delta r^{b}$, rac b $\vee\left(\rho^{b}, \rho \Delta \cdot U \cdot \triangleleft^{b}, D \sigma \Gamma^{a} \rho \cdot \triangleleft^{b} \rho\right)-$

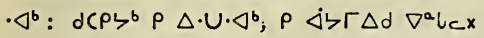

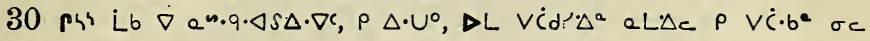
$\nabla r, L_{b} P_{c} \cdot \triangleleft^{\circ} \nabla R x$

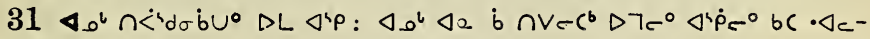
$\Delta \cdot \nabla \wedge \dot{a} b \sigma \cdot \Delta^{\circ} x$

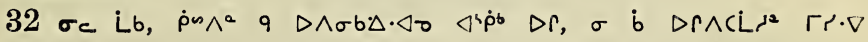
$\Delta r c \cdot \triangleleft^{b} \mathrm{x}$

$33 \triangleright 7 c^{\circ} \rho \Delta \cdot \cup^{0}, \nabla$ prqcidic $q d \sigma \wedge \Delta \sigma c^{\circ} q \Delta r \sigma \wedge c_{x}$

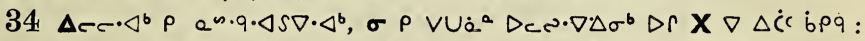

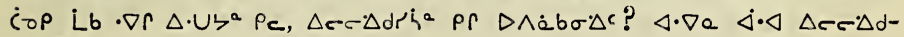
ía?

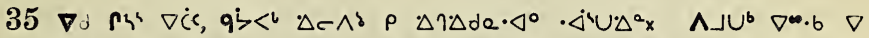

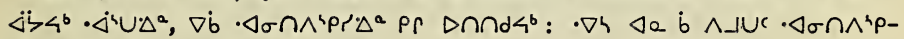

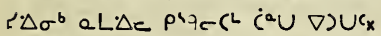

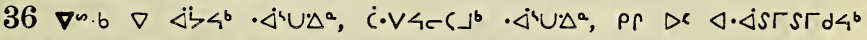

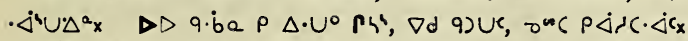

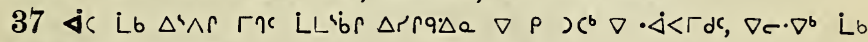
QLAC $\Delta r \dot{C} \cdot V A_{c} C \Gamma d x$

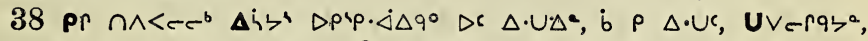

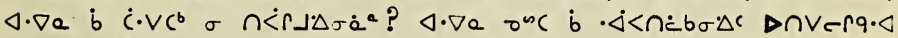
$\triangle \wedge \nu \sigma \Gamma^{\circ}$ ?

$39 \nabla \cdot \triangleleft d \cdot \nabla r \nabla \dot{b} \rho \dot{c} \cdot v C \rho^{b}, \nabla \rho \Delta \cdot U c \Delta i \zeta b \Gamma a$,

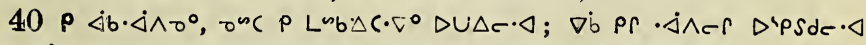

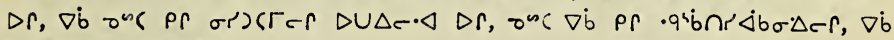
Lb $\operatorname{pr} \Gamma \mathrm{a} \cdot \Delta r \Delta \rho b_{x}$

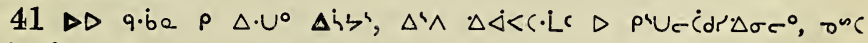
$\Delta \dot{\Delta}-J L C_{x}$

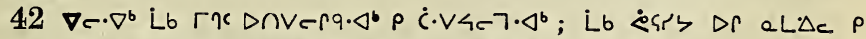

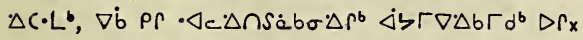

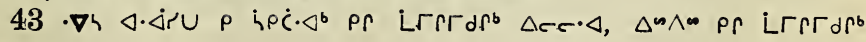
$\operatorname{Pr}(\sigma) \cdot \Delta x$

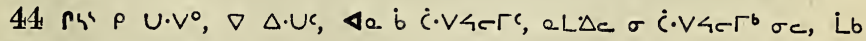
$\varangle \sigma \Delta \dot{b} \rho \operatorname{vinsDCr_{x}}$

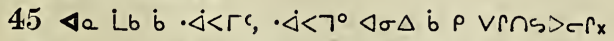

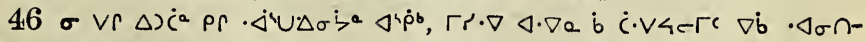
Nipp $\Delta \sigma^{6}$ pr $\Delta \dot{C} c_{x}$

173 


\section{$\triangleright<\supset$ ṕ ๒゙ 12, 13.}

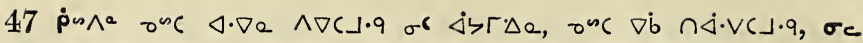

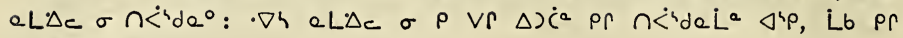

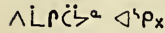

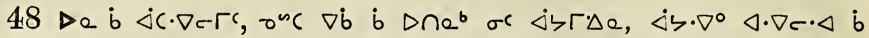

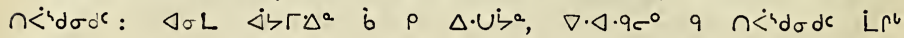
psibeqx

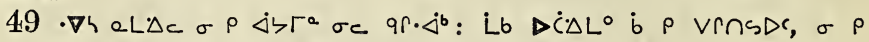

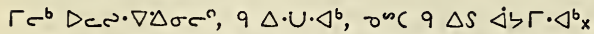

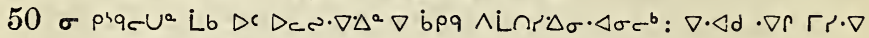

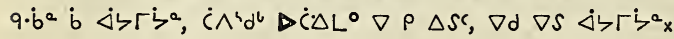

\section{$\Lambda$ pcra $\triangle b^{a} 13$.}

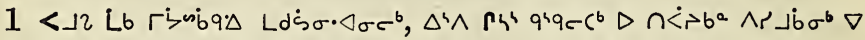

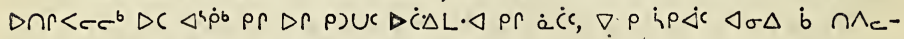

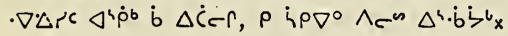

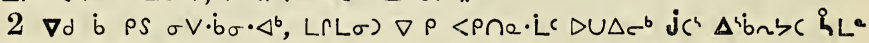

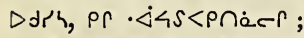

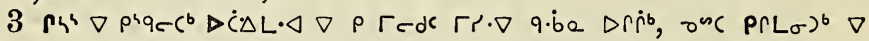
$\nabla \dot{C}$, os $\rho \Gamma L \sigma) \cdot \triangleleft \nabla \dot{a} \dot{C}$;

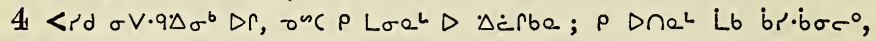
Dec $p<\cdot b U D_{x}$

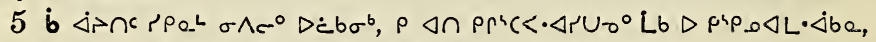

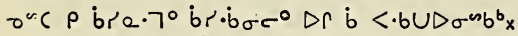

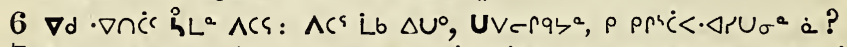

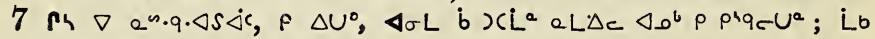
<nL $\rho$ b prqcuex

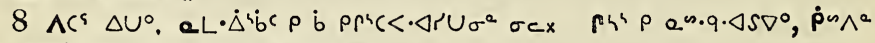
$\nabla \dot{b} \rho \rho^{i} \dot{C}<\cdot \Delta c \dot{c}-$, a $L \Delta c \rho \Delta \eta \Delta^{a} x$

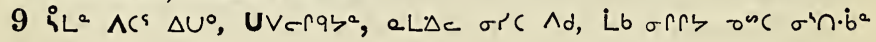
$\Delta r i x$

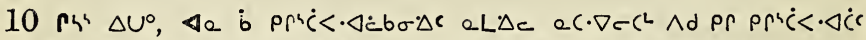

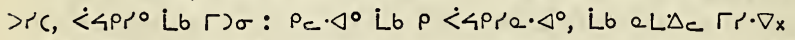

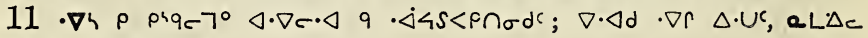

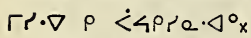

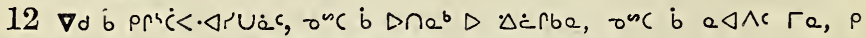

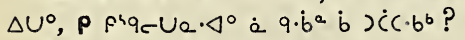

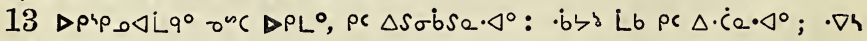
$\dot{\sigma} c \dot{\Delta} \cdot \dot{\Delta}^{a} x$

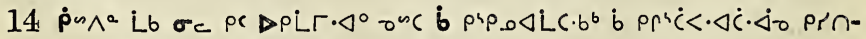

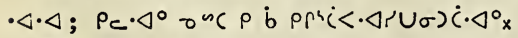

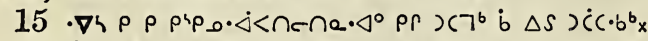

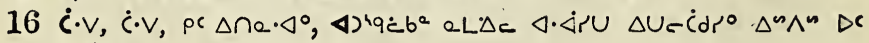
174 


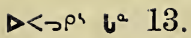

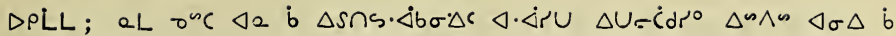
$\triangle \cap S D d^{C} x$

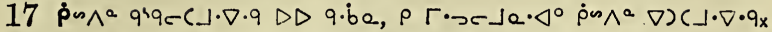

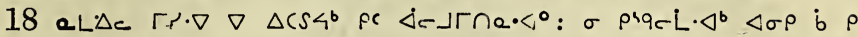

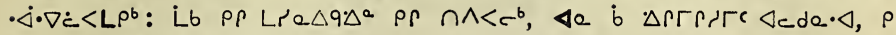

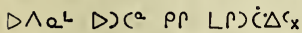

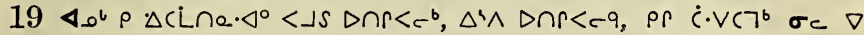
$\triangleleft \Delta \dot{i}^{a} \mathrm{x}$

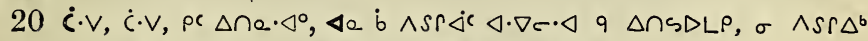
$\sigma c ; \Delta a \tau^{n c} \dot{b} \wedge S r \Delta c, \wedge S R \nabla^{\circ} \Delta \sigma \Delta \dot{b} \rho \operatorname{VrnSDCrx}$

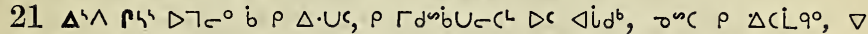

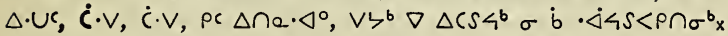

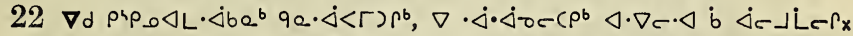

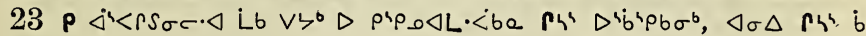
ip jcx

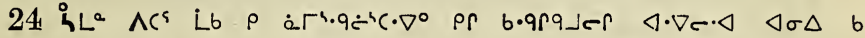

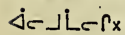

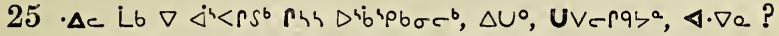

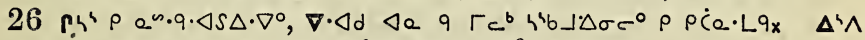

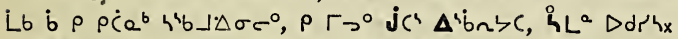

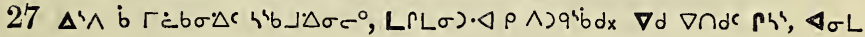
$9)\left(L_{a}^{a}, P \subset \wedge\right) C_{x}$

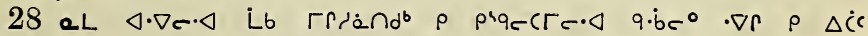
$\triangle 7 c^{\circ} x$

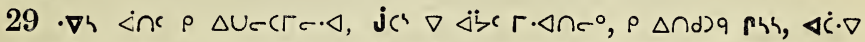

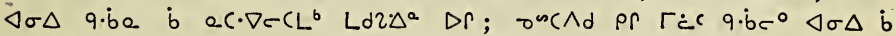
pกĹLrerx

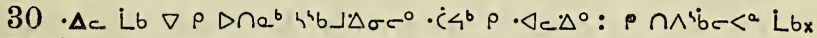

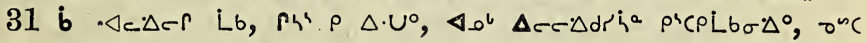

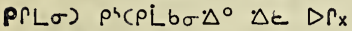

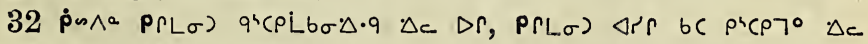

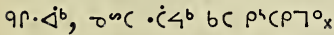

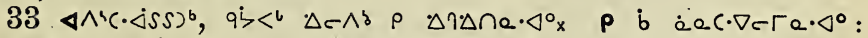

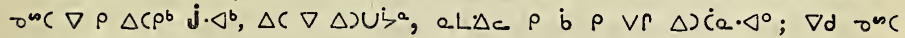
$\ln \left(\cdot b^{b} x\right.$

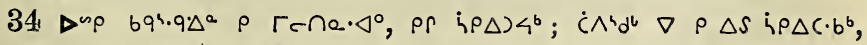

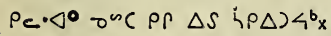

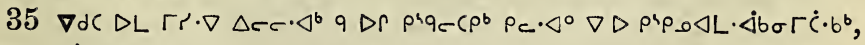
puna $r<p \Delta>4 \cdot q_{x}$

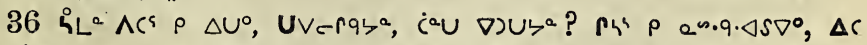

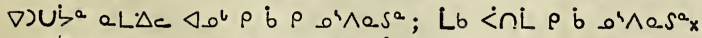

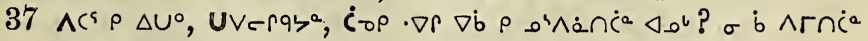
$\sigma \wedge L \cap \Delta^{a} \rho_{c} \Delta r_{x}$

\section{5}




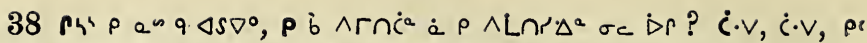

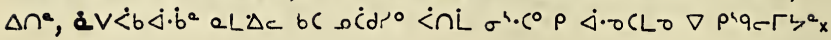

\section{A'pCra $\triangle b^{a} 14$.}

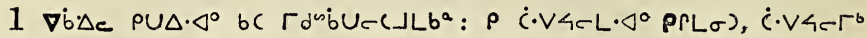
$\sigma c \gamma^{\omega} c_{x}$

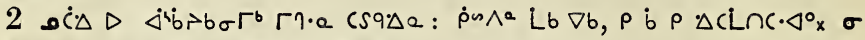
p) $\dot{C}^{a}$ pr $\dot{\Delta} \dot{4}^{\mathrm{b} b} \Delta \mathrm{CLC} \cdot \mathrm{b}^{b} \Delta C$ q $\Delta \dot{C} \dot{C}^{b} \mathrm{x}$

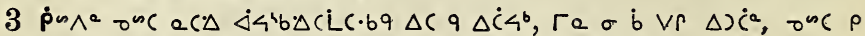

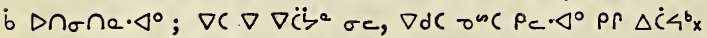

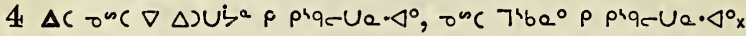

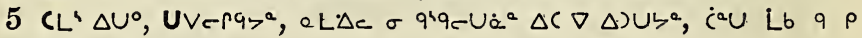
Dr prac CL' T'beo?

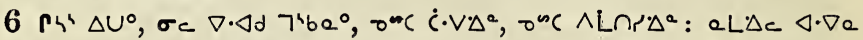
$\varepsilon U^{\circ} \Delta \dot{C} \Delta L \cdot \triangleleft, \wedge d \sigma_{c} \Delta \Gamma_{x}$

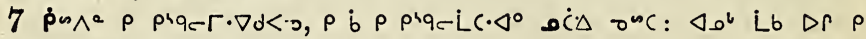

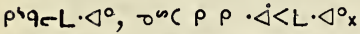

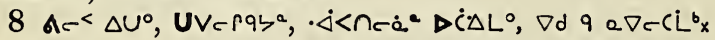

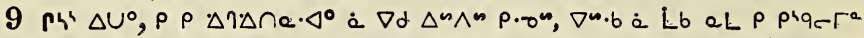

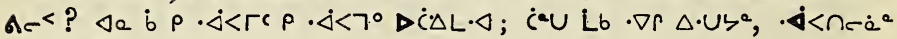
$\triangle C \triangle \Delta \circ$ ?

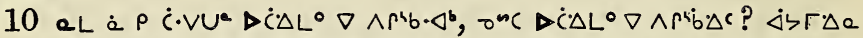

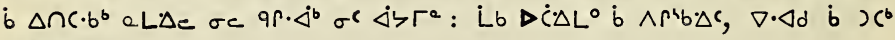

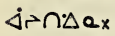

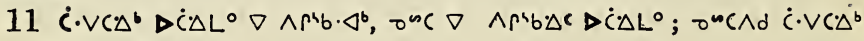
$\triangleleft \sigma \Delta \dot{\Delta r} \cap \Delta e \quad \nabla r_{x}$

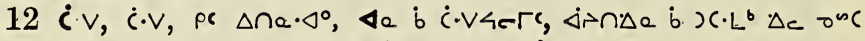

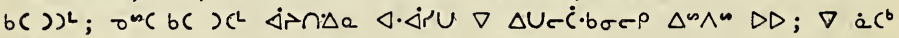
คं $\Delta x$

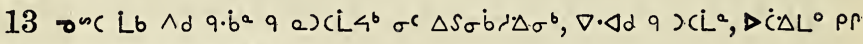

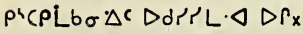

$\left.14 \dot{p} \sim \wedge^{a} a\right)\left(\dot{L} 4 \cdot 9 q \cdot b^{a} \sigma^{c} \Delta S \sigma \dot{b} \lambda^{4} \Delta \sigma^{b}, \sigma\right.$ b $u^{a} \times$

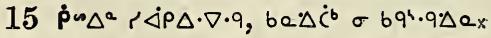

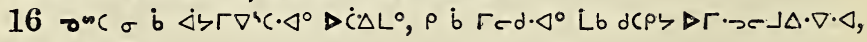
bคq $p \rho \Delta \eta \Delta \dot{C}^{b} x$

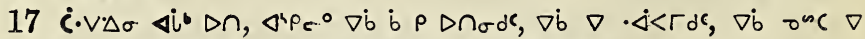

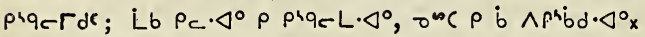

18 a L $\Delta c \rho$ b abnna. $\Delta^{\circ} \nabla \rho \cdot \Delta S \dot{s} \sigma \Delta 4^{\circ}: \rho$ b Vr anna $\cdot \Delta^{\circ} x$

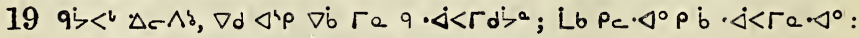

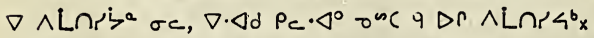

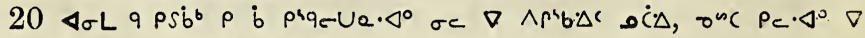

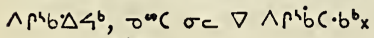




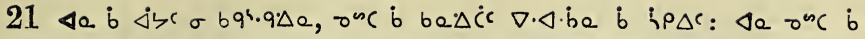

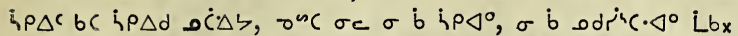

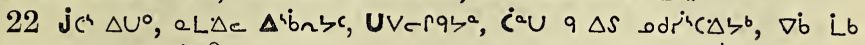
$p r \cdot \dot{i}<\Gamma b \Delta \zeta^{a} \triangleleft^{4} \dot{\rho} b$ ?

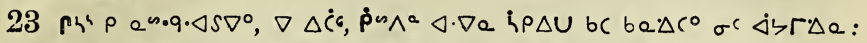

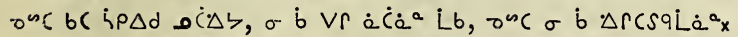

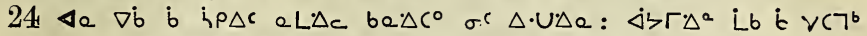

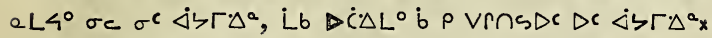

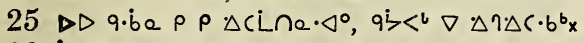

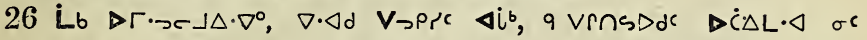

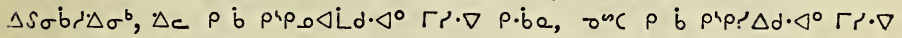
$9 \cdot \dot{b}$. $b$ b $\triangle \cap C \cdot b^{b} \mathrm{x}$

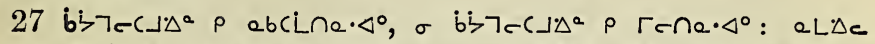

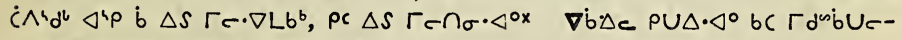
CJLba, $\nabla \dot{b} \Delta c$ onc bC dicrLbax

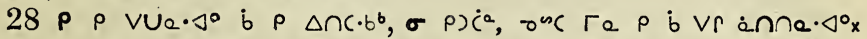

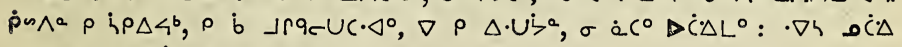

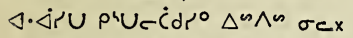

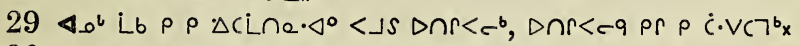

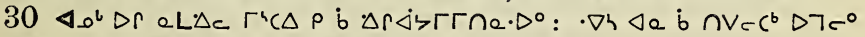

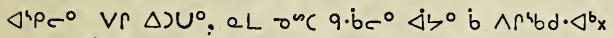

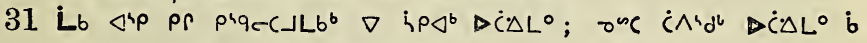
$\Delta\left(\omega \cdot\langle S c, \nabla d \nabla S) C^{a}{ }^{a} \quad<d^{b}, \rho\right) \cup C^{\circ} \times$

\section{A $p c r a-\triangle b^{a} 15$.}

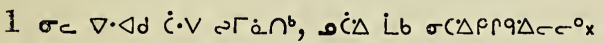

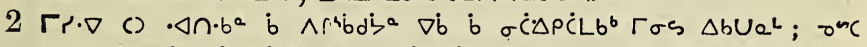

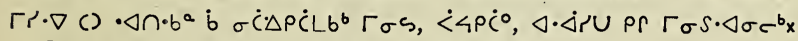

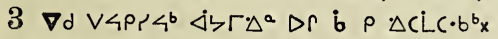

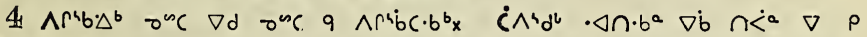

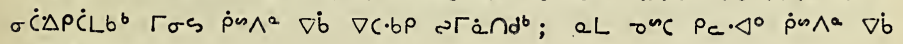
$\wedge \Gamma^{2} b \Delta 4 \cdot 9 x$

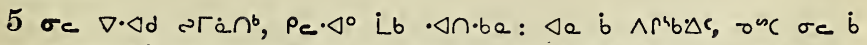

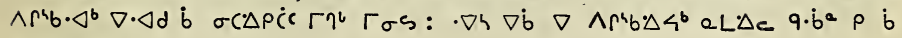
$\rho U_{0} \cdot \Delta^{\circ} x$

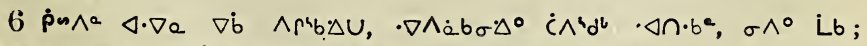

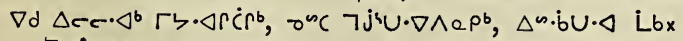

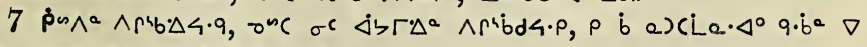
$\Delta S$ a $\left.c \cdot \nabla e c 7^{b}, \nabla d q\right) C L b \Delta<\zeta^{b} x$

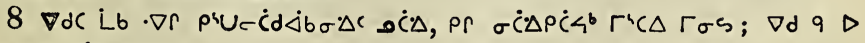
pip.o $\triangleleft L \cdot \Delta b \sigma \Gamma C \cdot b^{b} x$

$9 \Delta \dot{C} \Delta L^{\circ} \dot{b} \Delta s i p \Delta c, \nabla d \dot{c} \wedge^{4} d^{b}$ b $\Delta s i p \Delta c \cdot b^{b}=c s q^{b} \sigma i p \Delta \cdot \nabla \Delta \sigma^{b} x$ 177 


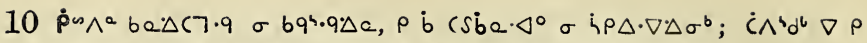

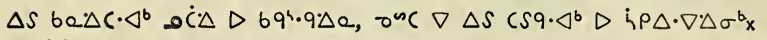

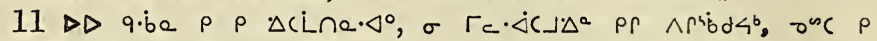

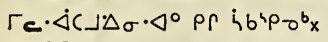

$\left.12 \nabla \cdot \Delta d \Delta L \sigma b q^{b} \cdot q \cdot \Delta^{a}, p \rho i p \Delta\right) \zeta^{b}, c^{2} \wedge^{b} d^{b} \nabla \rho \Delta S i p \Delta C \cdot b^{b} x$

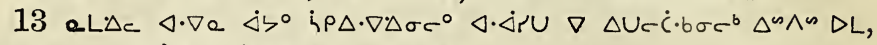

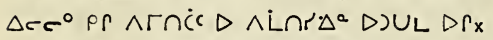

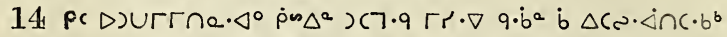

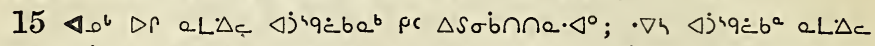
PrqeCL $\left.q \cdot b \sigma^{\circ} D C D P L L \quad \nabla\right)(\Gamma c r:$ Lb $\sigma) \cup L^{b} \rho \rho \Delta S \sigma b \cap \cap a \cdot \Delta^{\circ}, \cdot \nabla h \Gamma r \cdot \nabla$

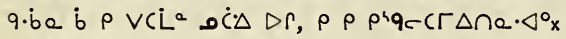

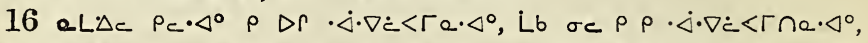

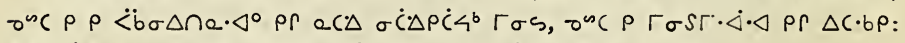

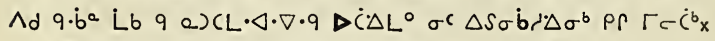

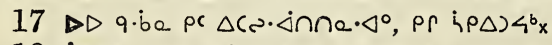

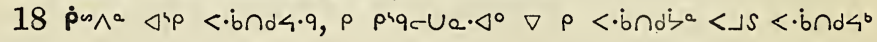
$\rho_{c} \cdot \Delta_{0} x$

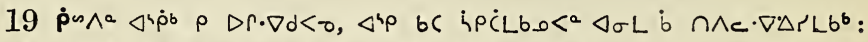

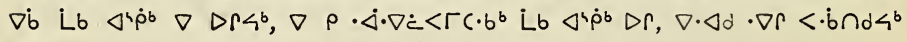
$\Delta$ spx

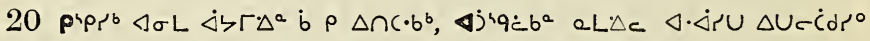

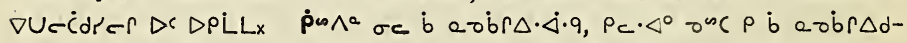

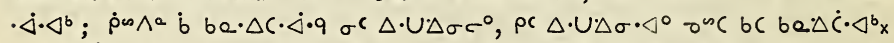

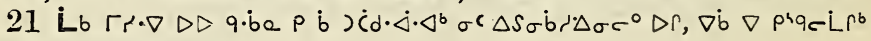

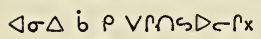

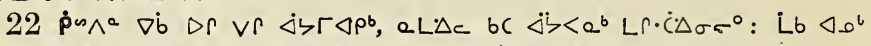

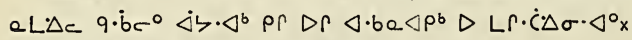

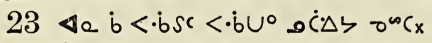

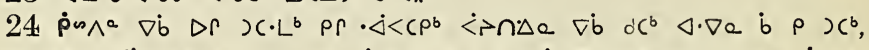

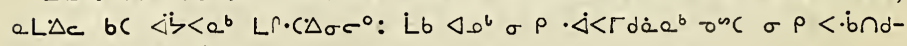

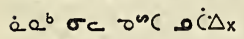

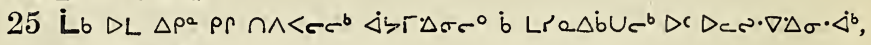
2c. bc $\sigma \rho<\cdot \dot{b} \cap \cdot b^{b} x$

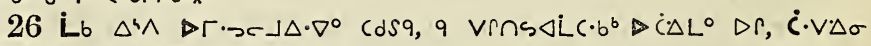

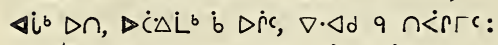

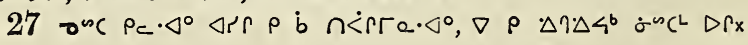

\section{$\Lambda^{4} p \subset\left\ulcorner\circ \wedge b^{2} 16\right.$.}

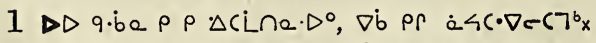

$2 \rho \dot{b} \cdot \Delta c \Delta \cdot \nabla \wedge \sigma d \cdot \dot{\Delta} \cdot \Delta^{b} \quad \dot{\Delta}\left\langle\Gamma \nabla \Delta b \Gamma d^{b} \quad \Delta R: \nabla \nabla, \cap \dot{<}>b^{a} \wedge r\right\lrcorner \dot{b} \sigma^{b}$

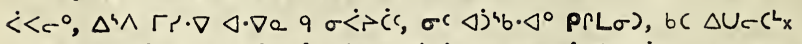

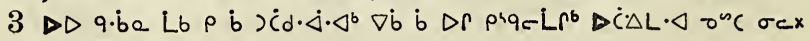
178 


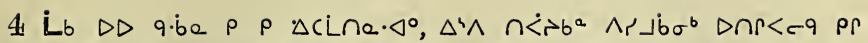

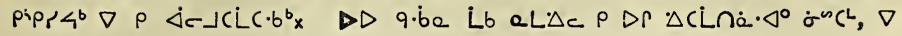
$\triangle \eta \triangle C \cdot b P<{ }^{a} \times$

5 Lb $\triangleleft 0^{b} \sigma$ a $c^{\circ} \triangleleft a$ b $P$ VRnSDC: eL Lb $\triangleleft \cdot \nabla e \nabla \Delta C s 4^{b} \sigma b \cdot 9 r r^{b}$, ¿थ $\nabla) \cup \zeta$ ?

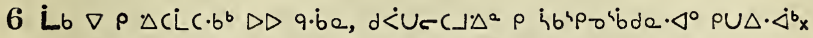

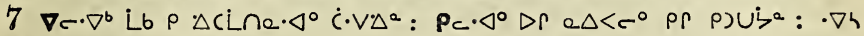

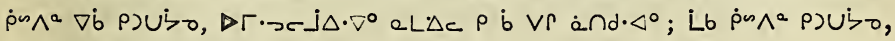
$\rho$ b VRns $\Delta$ Lina. $\triangleleft^{\circ} x$

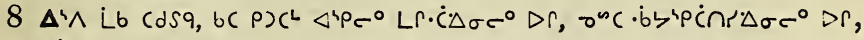

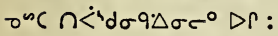

9 Lr.C. $\Delta \sigma c^{\circ} D r, \nabla \dot{b} \nabla \dot{C} \cdot V 4 c r r^{b}:$

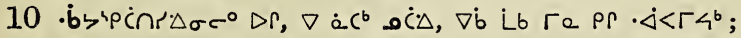

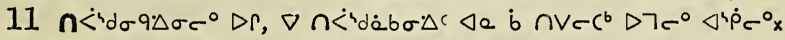

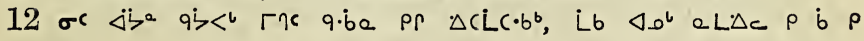
$\mathrm{VUe} \cdot \Delta^{\circ} \mathrm{x}$

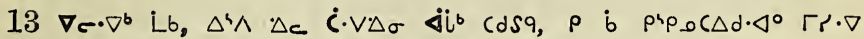

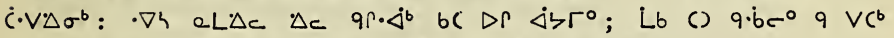

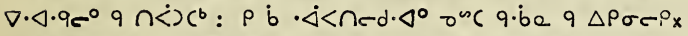

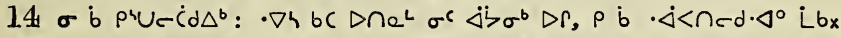

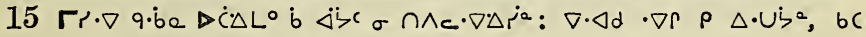

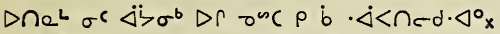

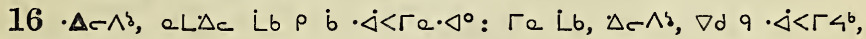
$\nabla \dot{a} C^{b} \Delta \dot{C} \Delta L^{\circ} \mathrm{x}$

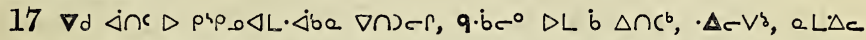

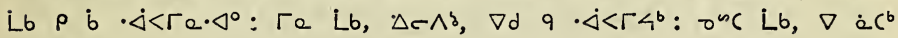
$\triangle \dot{C} \triangle L^{\circ}$ ?

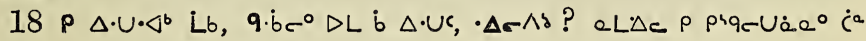
$\nabla \cdot \mathrm{U}_{\mathrm{x}}$

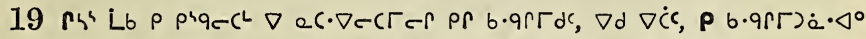

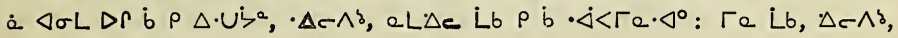
$\nabla d q \cdot \dot{<}<\Gamma<^{b}$ ?

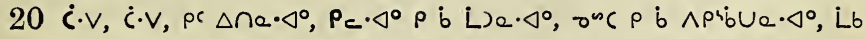

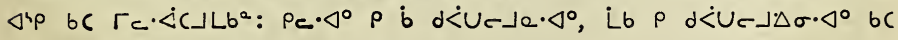

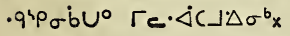

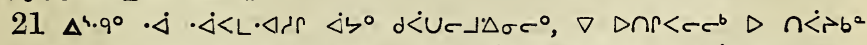

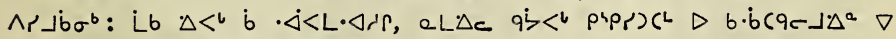

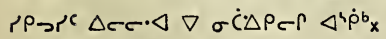

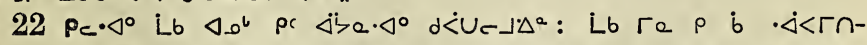

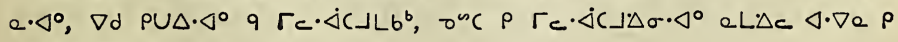
L'b $\Gamma d \cdot d^{\circ} x$

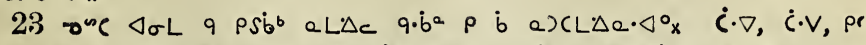

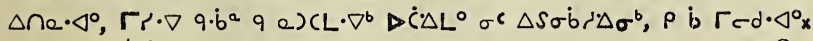




$$
\Delta<>\text { ps be } 16,17 .
$$

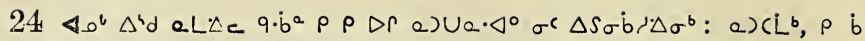

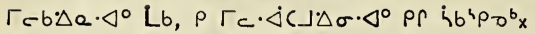

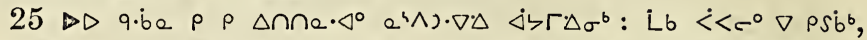

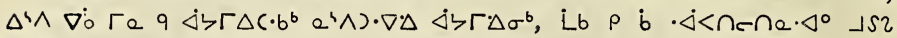
$\Delta \dot{C} \Delta L^{\circ} \Delta r_{x}$

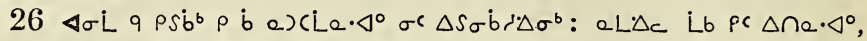
o b $\dot{b}\left\langle\Gamma \nabla^{\top} C \cdot \Delta^{\circ} \Delta \dot{C} \Delta L^{\circ} \rho_{C} \cdot \Delta^{\circ} \Delta r:\right.$

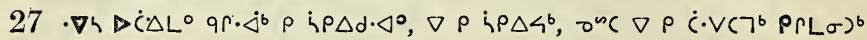
$\nabla \Delta \Gamma i{ }^{\circ} \times$

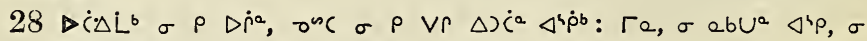
a. $C^{\circ} \mathrm{Lb} \Delta \mathrm{C}^{\circ} \Delta \mathrm{L}^{\circ} \mathrm{x}$

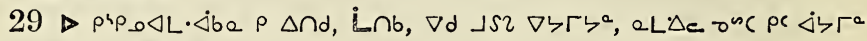

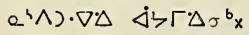

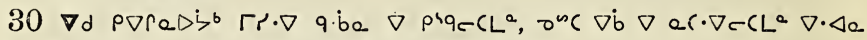

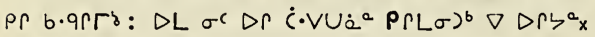

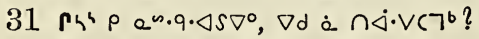

$\left.32 \dot{L} \cap b, \cap \dot{<}>b^{a} \wedge r\right\lrcorner \dot{b} \sigma^{b} \dot{<}<\sigma^{\circ}, \nabla \nabla,\left\langle\dot{z} \Delta \cap r<\sigma^{\circ}, \Delta^{4} \wedge q\right.$ a. $\sigma s \cap s-$

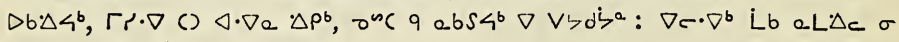

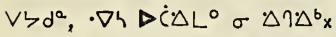

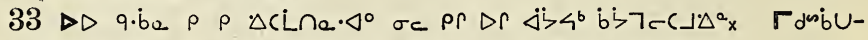

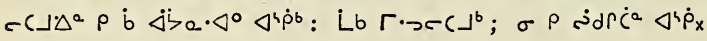

\section{Ápcre $\triangle b^{a} 17$.}

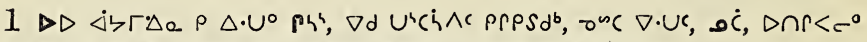

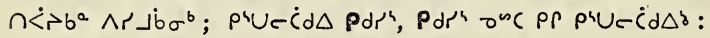

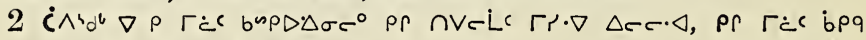
$\wedge L \cap \Delta \sigma c^{\circ}() \triangleleft \sigma \Delta b \rho \Gamma c^{C} x$

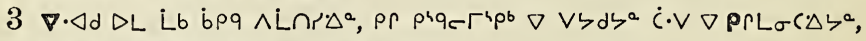

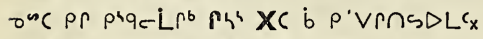

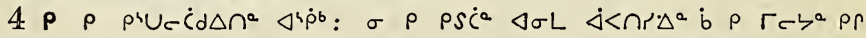
)$\left(L^{a} \times\right.$

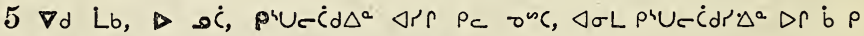

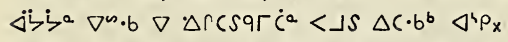

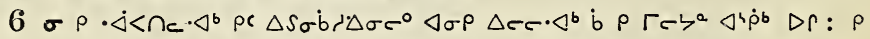

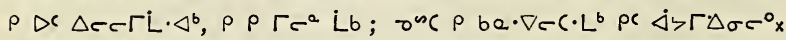

$7 \nabla d$ b prqc $\rho^{\circ b} \Gamma r \cdot \nabla q \cdot b e$ b $\rho \Gamma c \zeta^{a} \rho_{c} \nabla$. $\Delta r \Delta C \cdot b \rho_{x}$

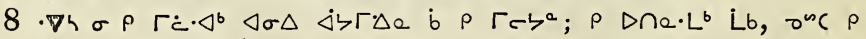
$\dot{C} \cdot V C \cdot L^{b} \nabla \rho V R \cap S \triangle \zeta^{\circ} x$

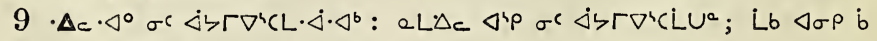
bГrל

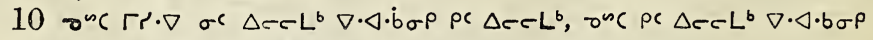
oc $\Delta C L^{b} ;$ onc o phUCंd $\Delta b^{b x}$

180 


\section{$\triangleright<>$ ps ia 17, 18.}

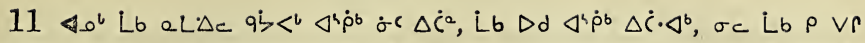

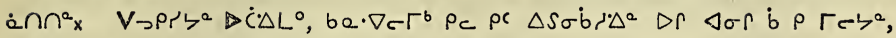

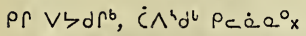

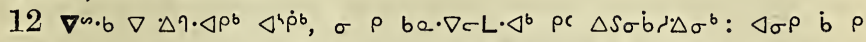

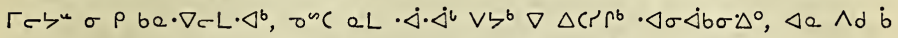

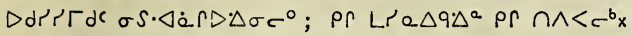

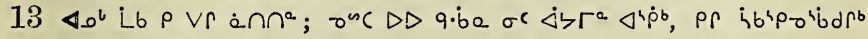
$\left.\sigma \Gamma_{c} \cdot \dot{\Delta C}\right\lrcorner \Delta \sigma \sigma^{\circ} \mathrm{x}$

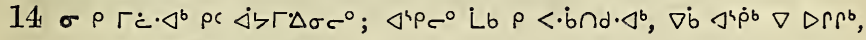

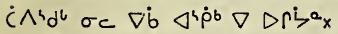

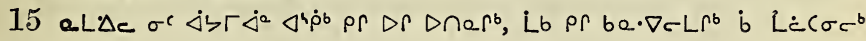
$D \Gamma x$

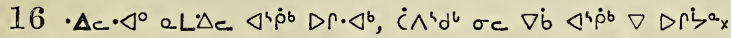

$17 \rho \dot{c} \cdot V \cdot \Delta \sigma c^{\circ} \Delta r<s \rho \Delta^{b}: \rho c \dot{\Delta b} \Gamma \cdot \Delta^{a} \dot{C} \cdot V \cdot \Delta \sigma \cdot \Delta^{a} x$

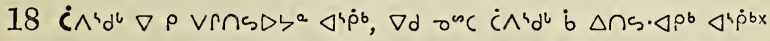

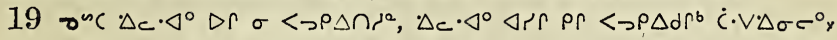

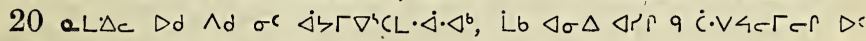

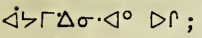

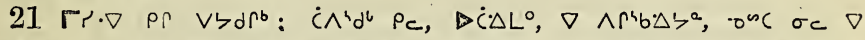

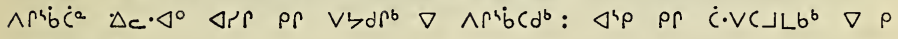
$\operatorname{VrnSD} b^{\circ} x$

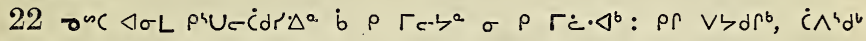
$\nabla \Delta S$ Vלdלb:

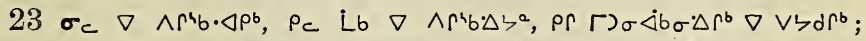

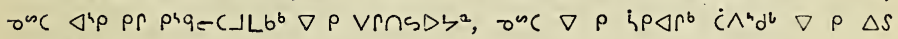

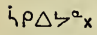

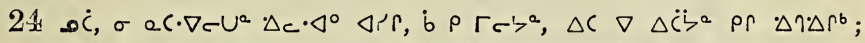

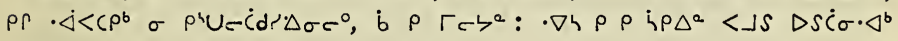
$\checkmark \mathrm{s} p_{x}$

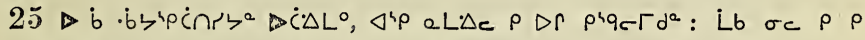
prqe $\Gamma \cap^{a}$, ouc $D d$ prqeC. $L^{b} \nabla \rho \wedge r \cap S D b^{\circ} x$

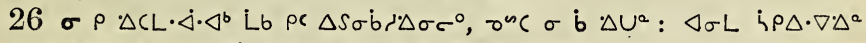

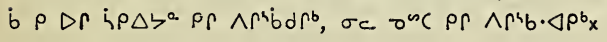

\section{N'pcra. $\Delta b^{a} 18$.}

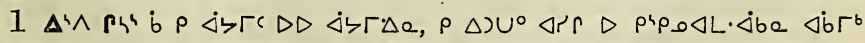

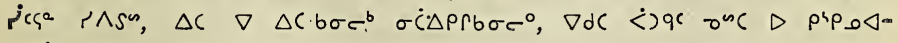
$L \cdot \Delta b e x$

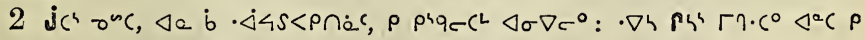

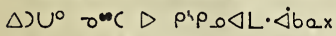

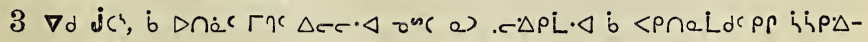
181 


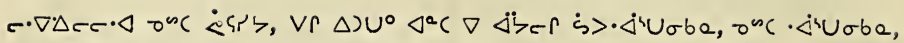
onc <bLbax

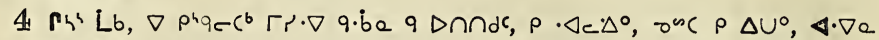
тec $(\cdot \nabla c]^{6}$ ?

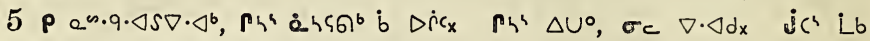

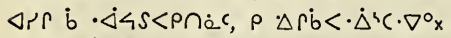

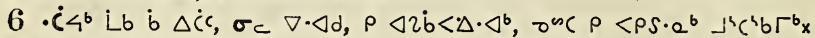

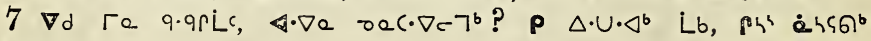
b $\Delta \dot{r} \dot{r}_{x}$

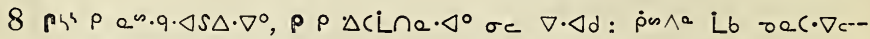
Г४.

9 p $\cap \wedge\left\langle\tau c^{b}\left\langle\sigma \nabla c^{\circ} \Delta \cdot U \Delta \sigma c^{\circ}\right.\right.$ b $\rho \quad \Delta \cdot U c, \Delta \sigma \rho b$ b $\rho \Gamma c \zeta^{a}$ a $L \Delta c$

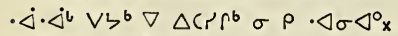

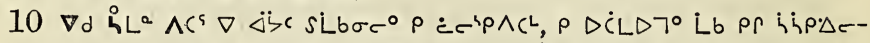

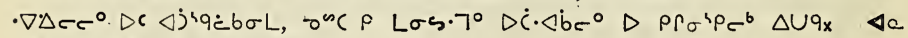
$\Delta \dot{j} q \dot{c} b^{a}$ Lebs $\rho \Delta \hat{j} \sigma \dot{b} d x$

$11 \nabla d \rho^{4} \nabla \dot{C} C \wedge C \zeta, \Lambda C \triangleleft \rho S \dot{L} b^{a} \wedge \Gamma d \dot{L} \dot{\alpha} \sigma^{b}: \Delta \sigma \nabla c^{\circ} \Gamma \sigma \cdot \dot{b} b \sigma \sigma^{\circ}{ }^{\circ} \dot{C} \Delta$ b $\rho \Gamma c c, a L d a \dot{b} \Gamma \sigma \cdot q \cdot \dot{\Delta}^{a}$ ?

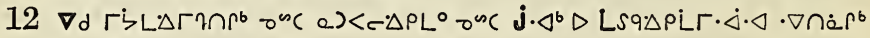
phi, ges $\rho$ LLSdAU $\triangleleft^{b}$,

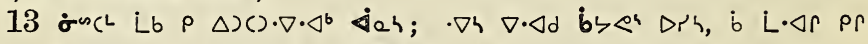

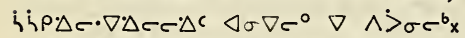

14 bל $e^{4}$ Lb $\nabla \cdot \triangleleft d$ b $\rho$ bq $\sigma>^{\top} C L \cdot \dot{d} \Delta r \cdot \nabla \cdot \Delta x$

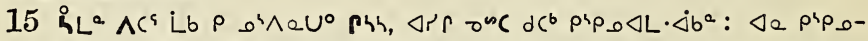

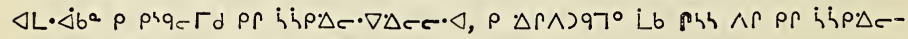

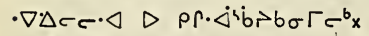

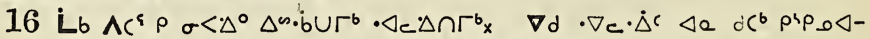

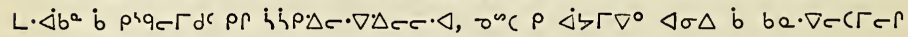
$\triangle \sim$ s.bU厂 $\left.\sigma^{\circ}, \rho \wedge\right) b \nabla^{\circ}$ Lb $\wedge\left(c_{x}\right.$

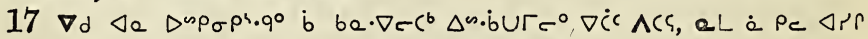

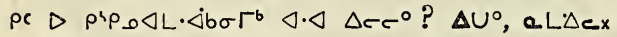

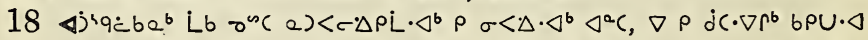

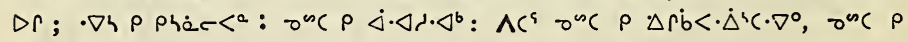
$\dot{\Delta \cdot \triangleleft \lambda x}$

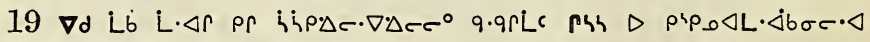

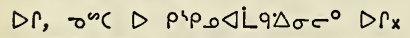

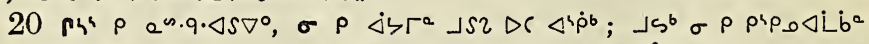

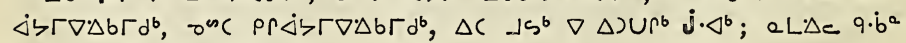

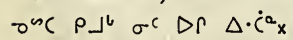

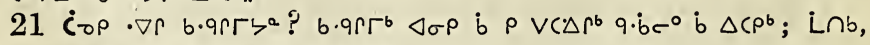
$\Delta c \cdot \Delta^{\circ} p^{2} q-C \cdot L^{b} q \cdot \dot{b} e^{\circ} \dot{b} \quad \Delta \cdot U \cdot \Delta^{b} x$

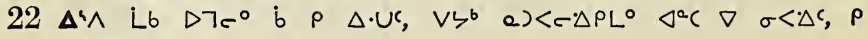
182 


\section{$\triangleright<\neg \rho^{2}$ ua 18.}

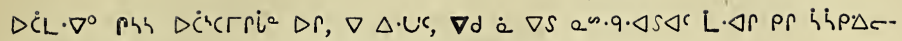
$\cdot \nabla \Delta c^{\circ}$ ?

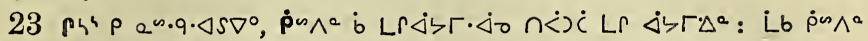

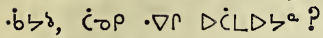

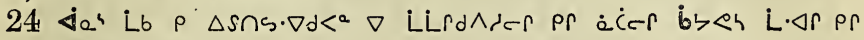
i i $\rho \cdot \Delta r \cdot \nabla \cdot \Delta r r \cdot \Delta x$

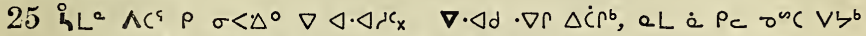

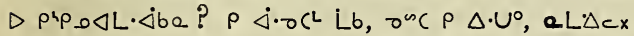

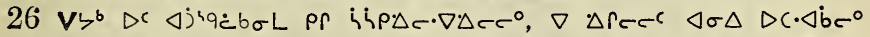

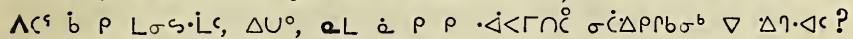

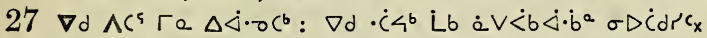

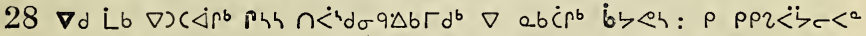

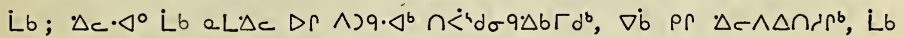

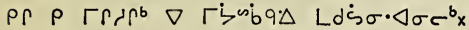

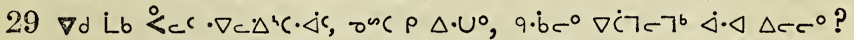

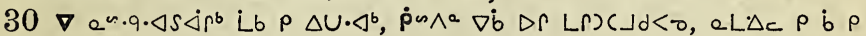
$v \cdot \dot{i n c} \dot{c}^{a} x$

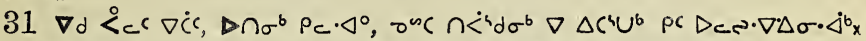

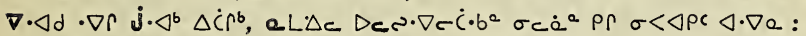

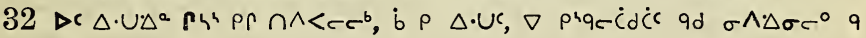
$D \Gamma \sigma \wedge c_{x}$

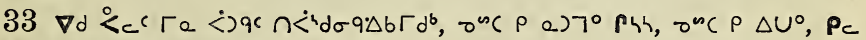
a $\mathbf{j} \cdot \triangleleft^{b} \triangleright \operatorname{PrDPL} \Gamma \cdot \dot{\Delta} \cdot \triangleleft$ ?

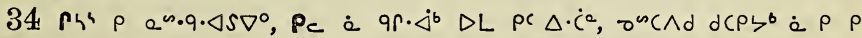
$\triangle C L \cdot b^{b} \nabla \Delta \dot{C} \Gamma\left\ulcorner b \Delta \zeta^{\circ}\right.$ ?

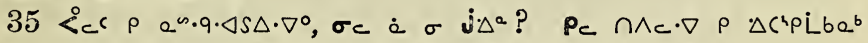

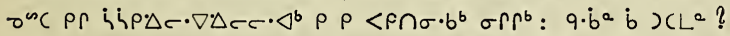

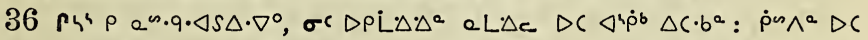

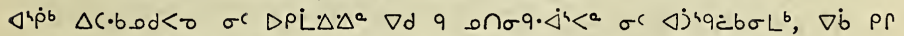

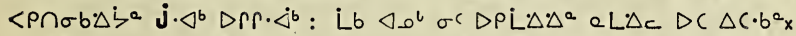

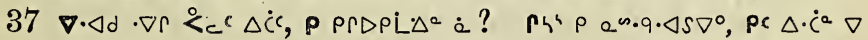

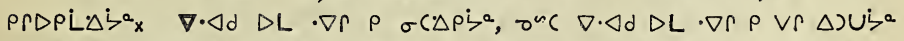

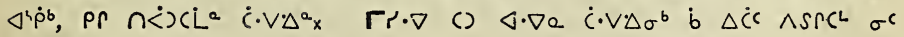

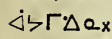

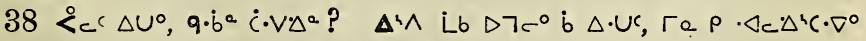
$j \cdot \triangleleft, \sigma^{n}\left(\Delta U^{0}\right.$, aL $\left.\left.\Gamma\right) \sigma \sigma \Gamma^{4} b \cdot L^{a} b \dot{b} L\right)()^{b} x$

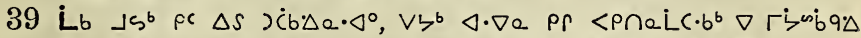

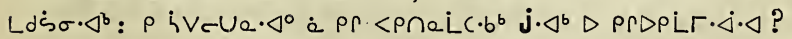

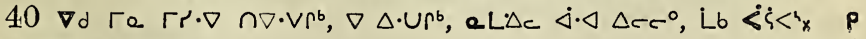
$L^{4} b \cdot$ U'po $L b<\dot{\zeta}<4 x$ 


\section{$\triangleright<>\rho^{4}$ ua 19.}

\section{A'pcre $\triangle b^{a} 19$.}

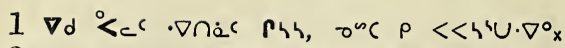

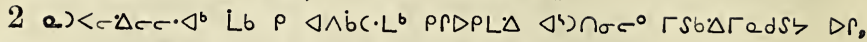

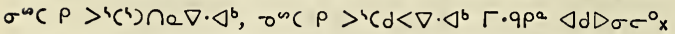

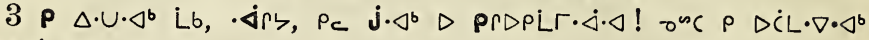
$\nabla r r \cdot \dot{\Delta} \cdot \Delta \nabla r^{x}$

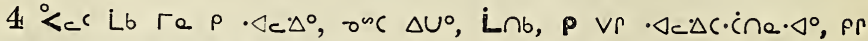

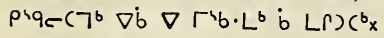

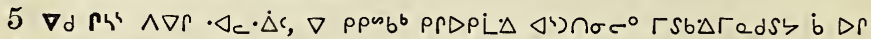

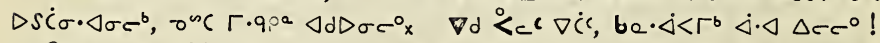

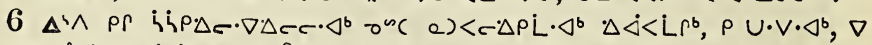

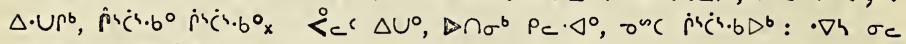

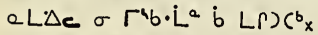

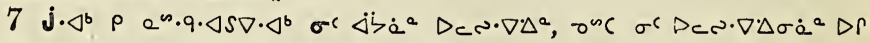
bC $\sigma \wedge<<^{a}, \cdot \nabla h P(L \sigma) D d d_{h} \rho \Delta U-\dot{C} d \Delta \cap R x$

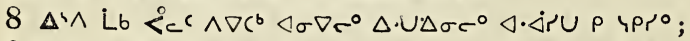

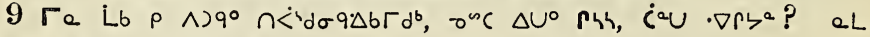

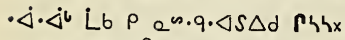

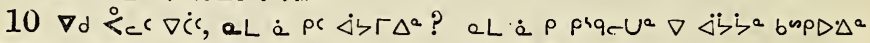

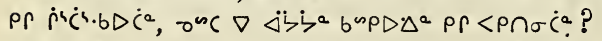

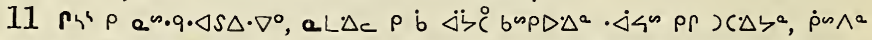

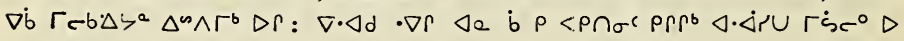
LR) (\lrcorner$\cdot \Delta^{a} x$

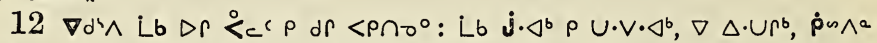

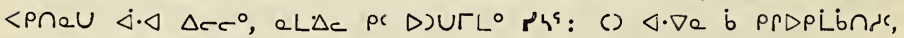

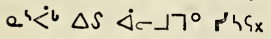

$13 \Delta^{\prime} \wedge$ Lb ${ }^{\circ}<c^{c} \wedge \nabla c^{b} \triangleleft \sigma \nabla c^{\circ} \Delta \cdot U \Delta \sigma c^{\circ}, \rho \vee V r \cdot \Delta c \Delta C \nabla^{\circ}$ phh, $\sigma^{\infty} C \rho$

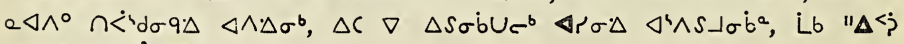
$\Delta S \rho \cdot 2 \Delta \sigma^{b}, \dot{b}<e_{x}$

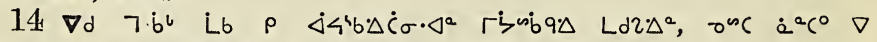

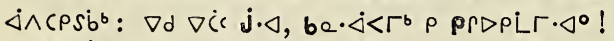

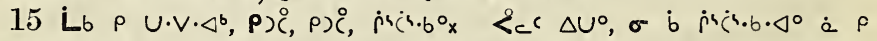

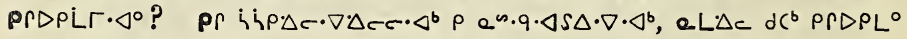

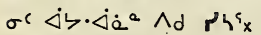

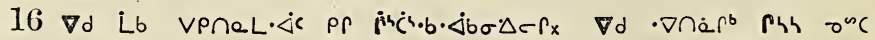
q) $\left(\Delta r^{b} \times\right.$

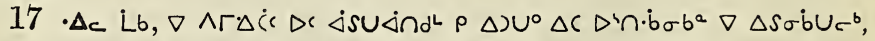
" $\Delta<j \Delta S \rho \cdot 2 \Delta \sigma^{b} d^{c}$ de b $\Delta S \sigma \cdot \dot{b} U^{b}$ :

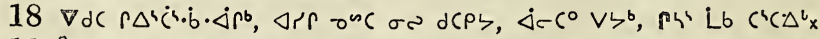

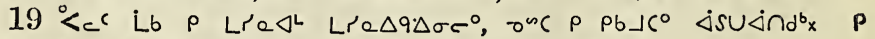

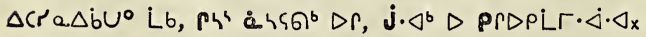

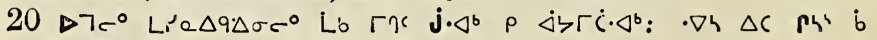
184 


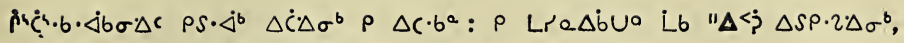
$\sigma^{a c}{ }^{b} \sim^{b} \Delta S \rho \cdot 2 \Delta \sigma^{b}, \sigma^{a c}$ ह $n^{a} \Delta 2 \rho \cdot S \Delta \sigma^{b} x$

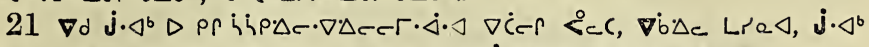

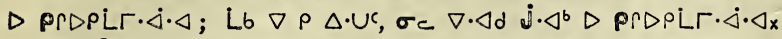

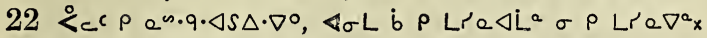

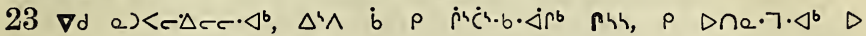

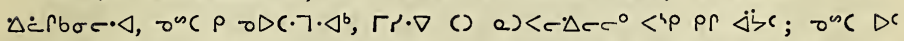

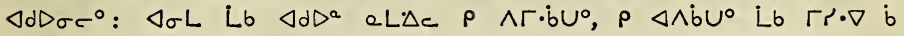
$\Delta \wedge \dot{S}^{b} \mathrm{x}$

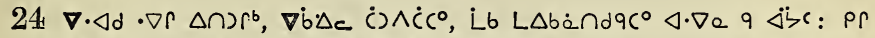

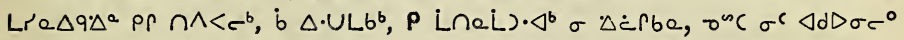

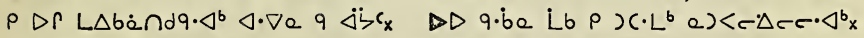

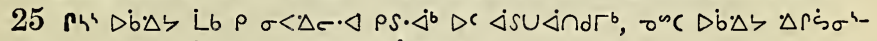
.

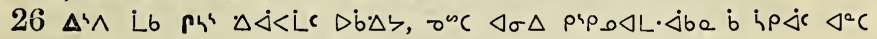

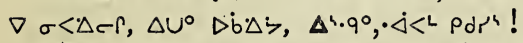

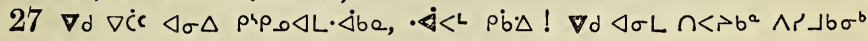

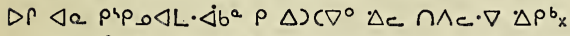

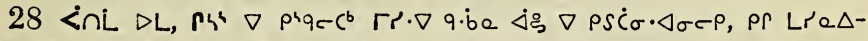
$q \Delta^{a}$ p $\rho \wedge \wedge<\tau^{b}, \Delta \cdot U^{\circ}, \sigma \Delta \Gamma \sigma \cdot b^{c} x$

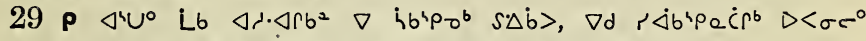

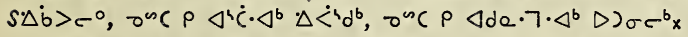

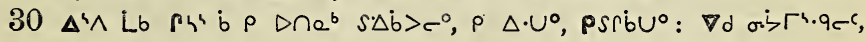
onC VPnác $D C \triangle i \cdot b x$

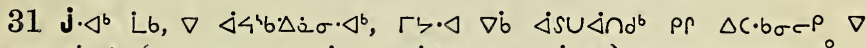

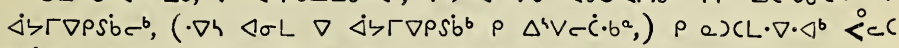

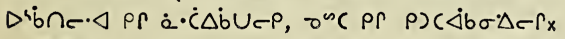

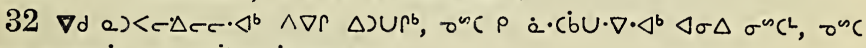

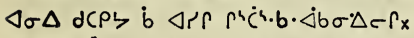

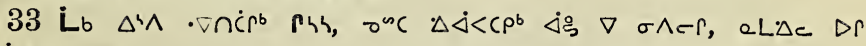
$\dot{\mathrm{i}} \cdot \dot{\mathrm{C}} \mathrm{QU} \cdot \nabla \cdot \Delta^{\mathrm{b}}$ :

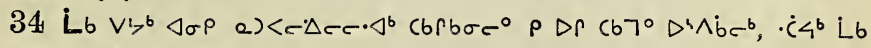
$\rho \supset r r \cdot \Delta \sigma \sigma^{\circ} \Gamma \mathrm{d} \sigma^{\mathrm{N} C} \sigma \Lambda_{x}$

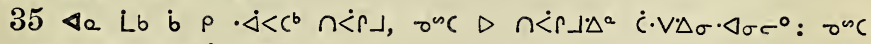
prqeCL $\nabla C \cdot V c$, pr $\dot{C} \cdot V C 7^{b} x$

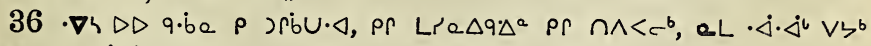
$\triangle b^{\circ} b C \dot{a} \cdot \dot{C} \Delta \dot{b} U c^{\circ} x$

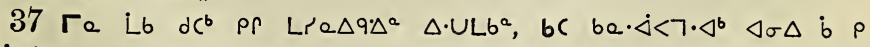
(biL $r^{b} x$

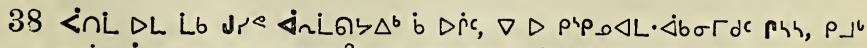

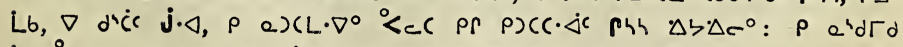

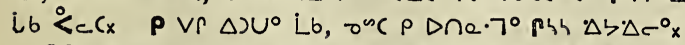

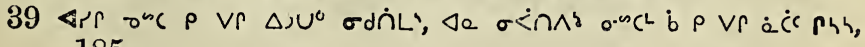
185 


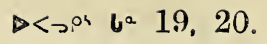

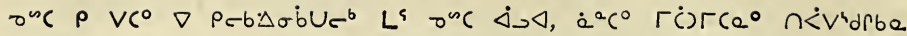
$\Delta \sim \wedge \omega_{x}$

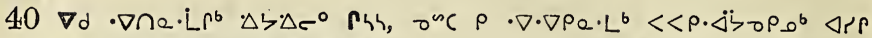
$\left\langle\sigma \Delta L\right.$ Lppל, $\nabla \Delta S$ a $\triangle \dot{b} q \rho^{b} \mathbf{j} \cdot \triangleleft^{b} \times$

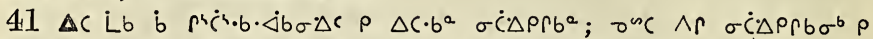
$\Delta c \cdot b^{a} \Delta m \rho<<r b \Gamma^{b}, \Delta c \nabla b \quad \nabla n \cdot b \triangleleft \cdot \nabla a b$ b $\Delta r \wedge \Gamma S L b \sigma \cdot \Delta c_{x}$

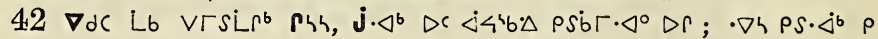
$\Delta C \cdot b^{a} r \dot{<} \vec{r} b \Gamma^{b} x_{x}$

\section{Ápcre $\Delta b^{2} 20$.}

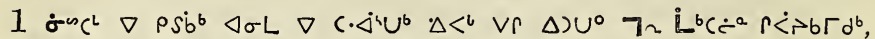

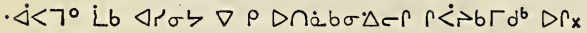

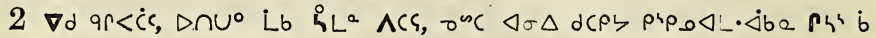

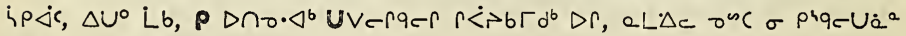
iaU b $A T S L r^{b} x$

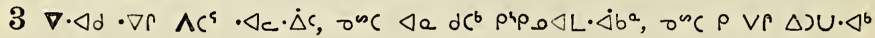
$r \dot{<}>b r^{b} x$

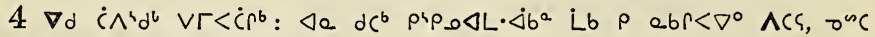
$\dot{\sigma} s C L P D C L \dot{K} P b \Gamma d \sigma^{\circ} x$

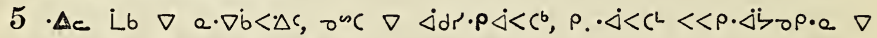

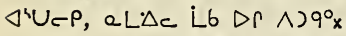

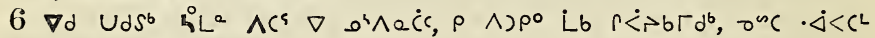

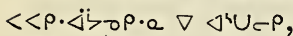

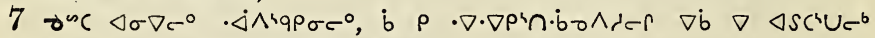
$<<\rho \cdot \Delta \dot{b}\rangle \rho \cdot a$, Lb $<\ddot{b}^{a} \nabla \cdot \nabla \cdot \nabla \rho_{\sigma} \dot{b} U \sigma^{b} x$

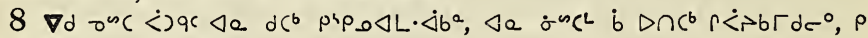
$\cdot \dot{\Delta<C L} \dot{L} b$, onc $\rho \dot{C} \cdot V C^{L} x$

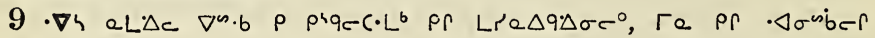
$\nabla \sigma \wedge \cdot \triangleleft \Delta r_{x}$

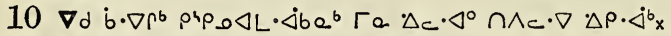

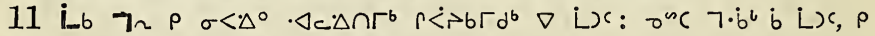

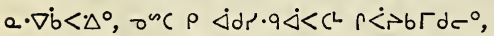

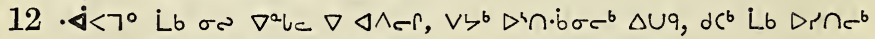
$\Delta \cup q, \Delta C P h^{4} \Delta \zeta^{\circ}$ b $\rho \wedge \Gamma \cap \sigma^{b} x$

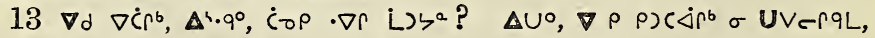

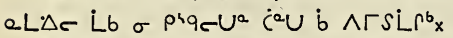

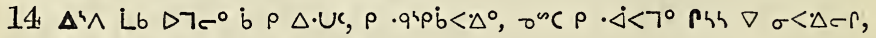

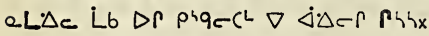

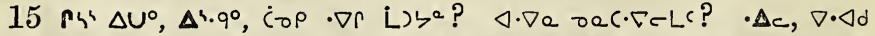

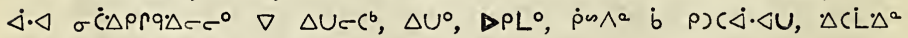
cंu b $\triangle \Gamma S L C, \nabla d$ q $D \cap a^{b} x$

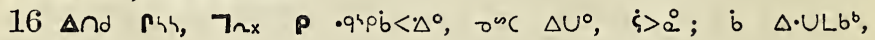

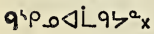




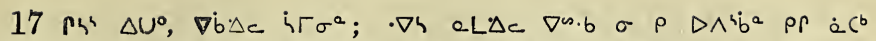

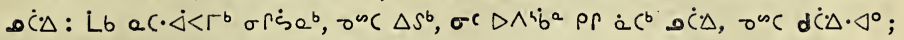
o" $\left.(\sigma P P L \sigma)^{L}, \rho P P L \sigma\right) \Gamma \cdot \triangleleft^{\circ} L b x$

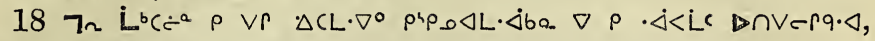
oesc $\nabla \rho \Delta C$ Lde $\triangle D$ q.bex

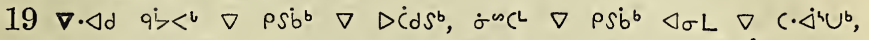

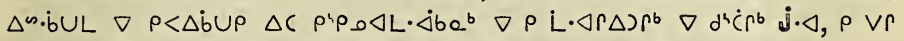

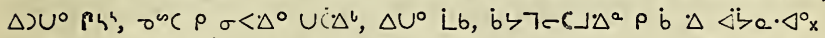

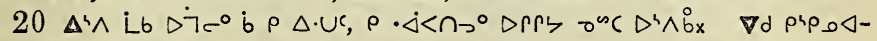

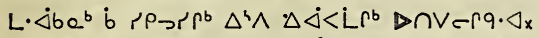

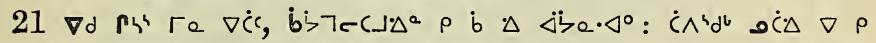
Vrns $D C, \nabla d$ onc $\nabla \triangle S \cap S D C \cdot b^{b} x$

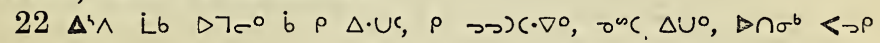
$\triangleleft i b$ :

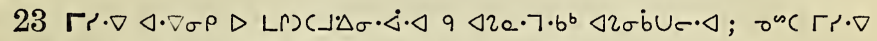

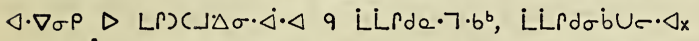

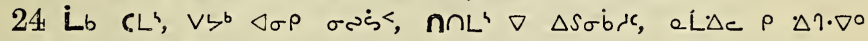
$\triangle \wedge$ b cosoer mibx

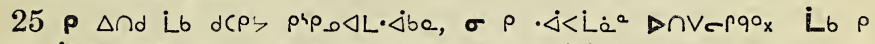

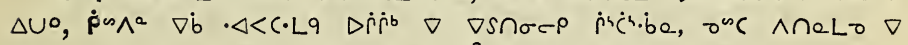

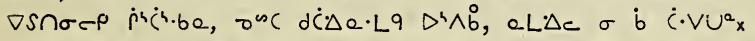

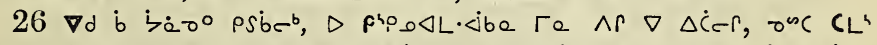

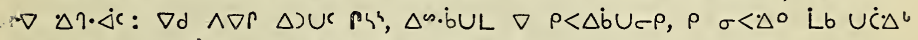

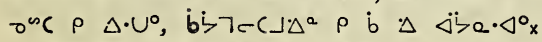

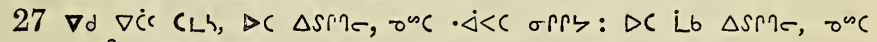

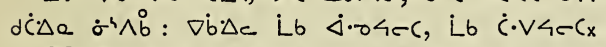

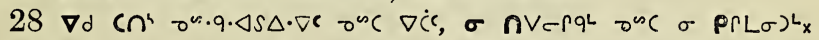

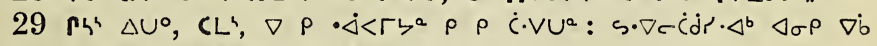
b $\Delta r \cdot \dot{\Delta}<\Gamma r^{b} ; \nabla r \cdot \nabla^{b}$ Lb b $\dot{c} \cdot V C \rho^{b} b_{x}$

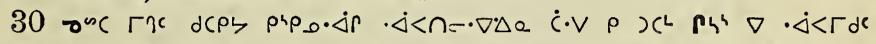

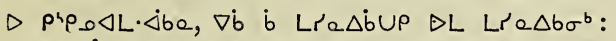

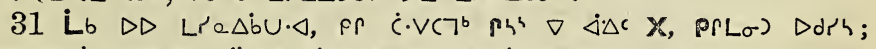

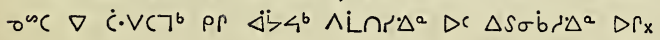

\section{Ápcra $\triangle b^{a-2} 21$.}

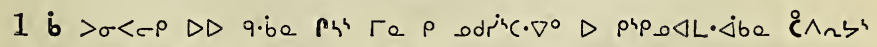
Prbib ; $D L$ Lb $\rho \Delta S$ od $\Delta \cap A x$

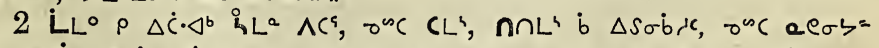

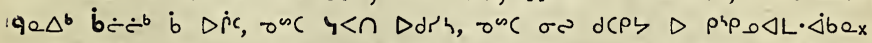

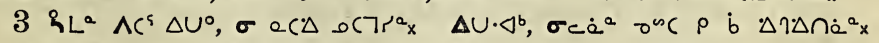

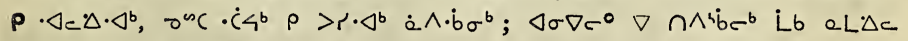
$q \cdot b r^{\circ}$ Dr $\wedge C D \dot{c} \cdot \triangleleft^{b} x$ 


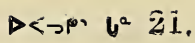

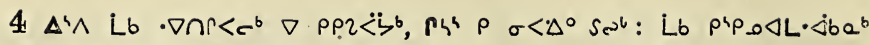

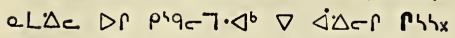

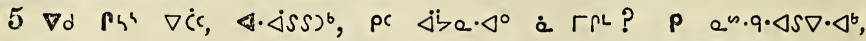
a.L $\Delta \Delta_{c x}$

$6 \nabla d \nabla \dot{c} c,\left\langle b^{4} c \cdot \nabla \nabla^{b} \triangleleft c \wedge \rho \rho \sigma^{4} \dot{\rho} b \quad \Delta U q \dot{a} \wedge \cdot \dot{b} \sigma^{b}, \nabla d \quad q \Gamma^{b} b 7^{b} x \quad \nabla \cdot \triangleleft d\right.$

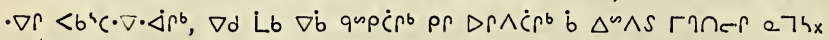

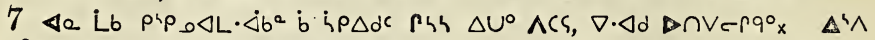
Lb $\left.h^{\circ} L^{a} \wedge C^{5} \wedge \nabla C^{b} \nabla \Delta \Delta-r \Delta \cap V e r q \cdot \triangleleft, \rho<\cdot b U D\right) C^{L} \triangleright .0\left(74 \cdot \Delta d^{<},(\cdot \nabla h \rho\right.$

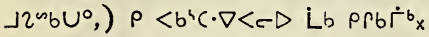

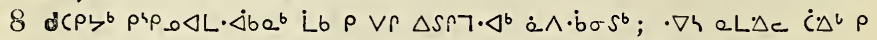

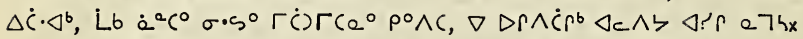

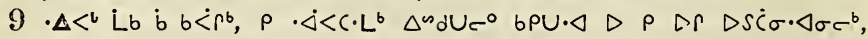
onc a $74 \nabla U C \dot{c} b \sigma \Delta c r$, onc $\Delta c d^{\circ} \cdot \Delta x$

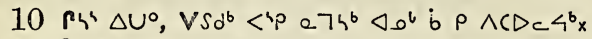

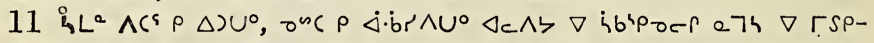

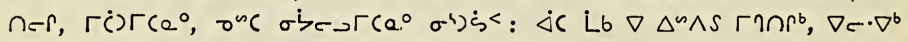
eL. $\Delta_{c}$ Dr $\wedge d<\sigma^{\circ} \triangleleft_{c} \wedge_{x}$

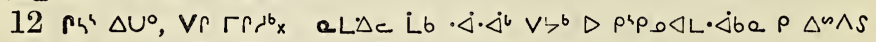

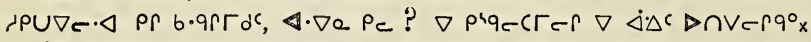

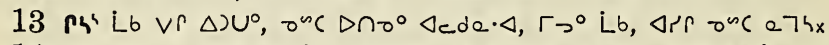

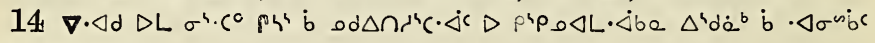
$\nabla \sigma \wedge \cdot \triangleleft \Delta r_{x}$

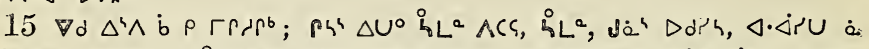

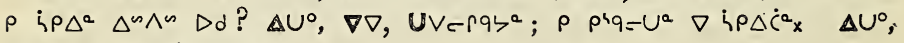
$\Delta S \Gamma^{b} \sigma$ L $\sigma^{n i} \operatorname{si} S S L^{b} \mathrm{x}$ 。

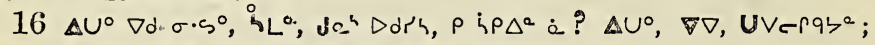
p prqe $U^{a} \nabla i \rho \Delta \dot{C}^{a} x \quad \Delta U^{\circ}, \varangle S \Gamma^{b} \sigma L^{n i} \sigma_{\sigma} S L^{b} x$

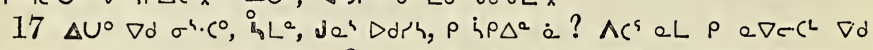

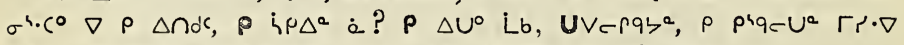

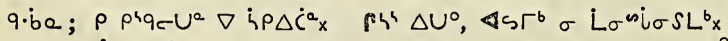

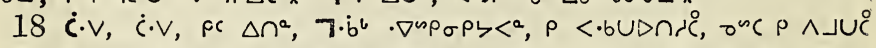

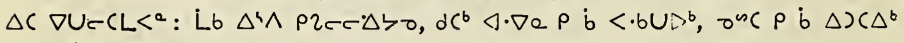
$\triangle C \nabla \dot{b} \nabla$ e $\nabla C C L^{2} x$

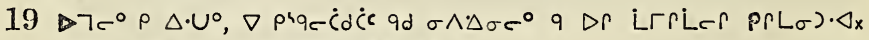

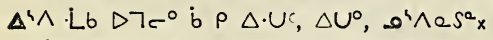

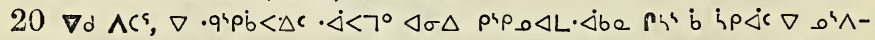

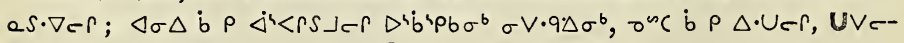

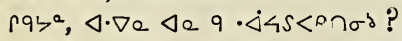

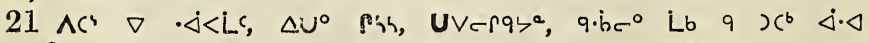
$\Delta c^{\circ}$ ?

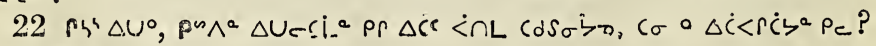
o'sesa pex

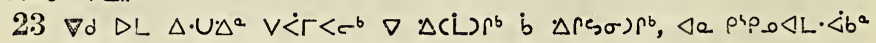
188 


\section{$\triangleleft<^{b} C c^{b} 1$.}

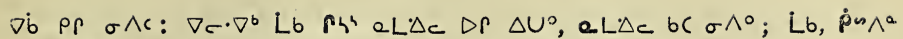
$\triangle U=C L^{a}$ pr $\Delta \dot{C}<\dot{<} \cap \dot{L}\left(d S \sigma \dot{\zeta} \sigma, \dot{C}_{\sigma} q \Delta \dot{C}<r \dot{C}\right\rangle^{a} \rho_{c}$ ?

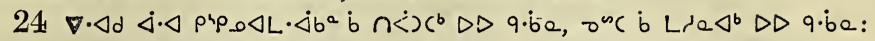

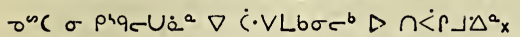

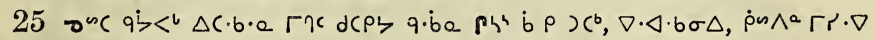

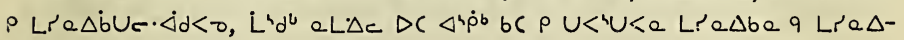
bu. $\left.\dot{d} d<\sigma_{x} \quad \nabla\right\urcorner^{\circ} x$

\section{$\triangleleft<^{4} c c^{b} \nabla^{c} \Delta r r q \cdot \Delta \sigma \cdot \triangleleft \cdot \triangleleft x$}

\section{A'pcre $\triangle b^{\circ} 1$.}

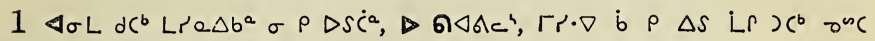
php.oषLqC $m$ h',

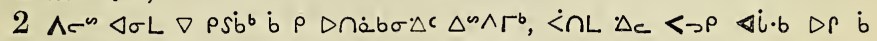

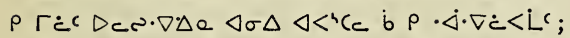

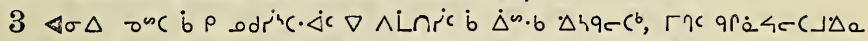

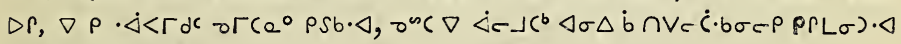
$\triangle C D P L \Delta \Delta \sigma \sigma^{b} \mathrm{x}$

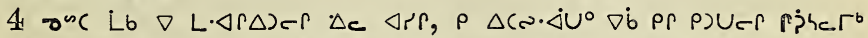

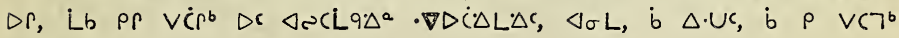
$\sigma c D p_{x}$

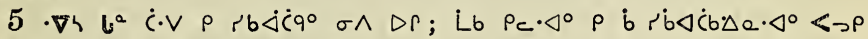

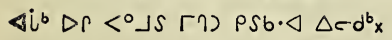

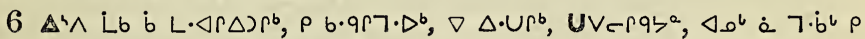
b $\rho \cdot \nabla \Gamma c^{\circ} \Delta^{4} \backsim \Delta^{c} \Delta P L \Delta \Delta \sigma \sigma^{\circ}$ ?

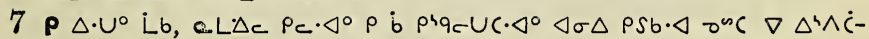
$\zeta \cdot \dot{j}^{b} \Delta \dot{C} \Delta L^{\circ}$ b $\rho$ बic $\cap \Lambda_{C} \cdot \nabla D$ b" $P D \Delta \sigma^{b}{ }_{x}$

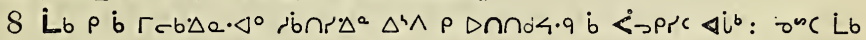

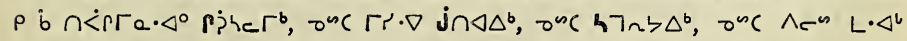
$\nabla \Delta u \cdot \dot{b} \zeta^{b} \triangleleft^{4} p b_{x}$

$9 \Delta^{4} \wedge$ Lb $\triangle D$ q.be b $\rho \Delta \cdot U c, 7 \cdot b^{6} \nabla$ be. $\Delta \wedge e r, \rho \Delta \wedge \dot{a} b \sigma \Delta^{\circ}, \sigma^{n c}$

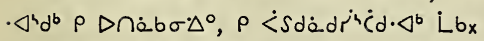

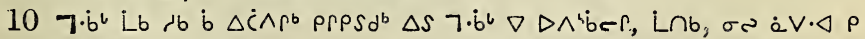

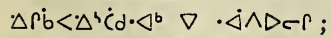

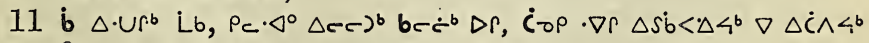

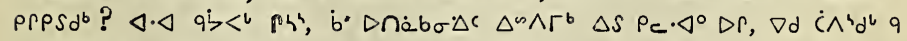
$\triangle S C d^{b}, \dot{b} \Delta S \cdot \dot{j}<7^{b} \nabla \Delta J U C P P P S d^{b} x$

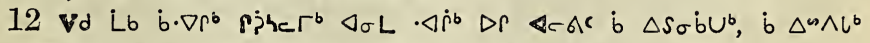

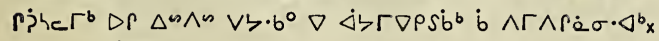




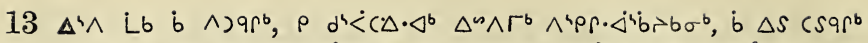

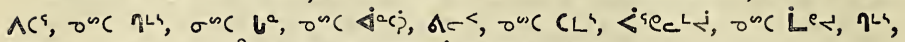

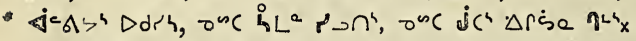

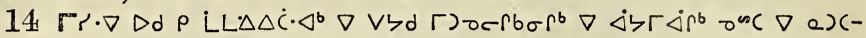

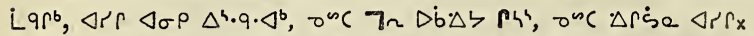

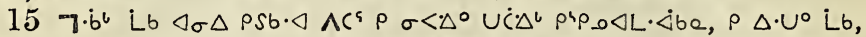

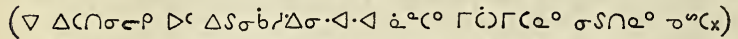

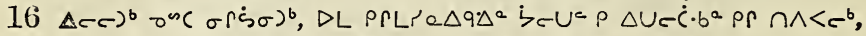

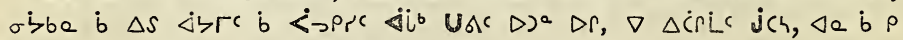

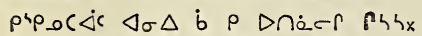

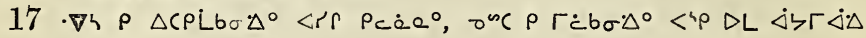
$\triangleleft j 49 \Delta^{\circ} \times$

$18 \varangle \cdot \triangleleft \Delta c c^{\circ}$ Lb $\rho \triangleleft \dot{C} \cdot \nabla^{\circ} \sigma c \Delta \rho \rho b \sigma c^{\circ} \triangleleft \sigma L \dot{b} \rho \cap\langle\Delta L \cdot \dot{\Delta} b \sigma \cdot \Delta c \Delta r \quad \nabla$

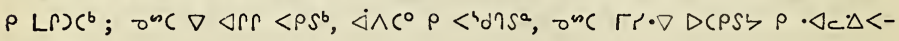
$\widetilde{c \cdot \triangleleft x}$

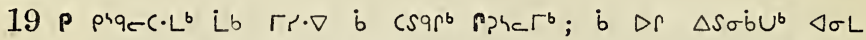

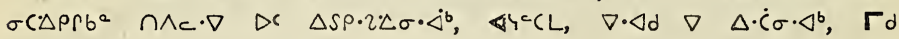
$\sigma\left(\Delta P \rho b^{2} x\right.$

$\left.20 \cdot \nabla h \Delta C\} a \Delta b U^{\circ} \sigma b\right\lrcorner \Delta$ Lre $\Delta b \sigma^{b}, \nabla d S \Delta C S 9 \Delta^{a}$ bC $\wedge S s \cdot b r^{\circ}$, aL

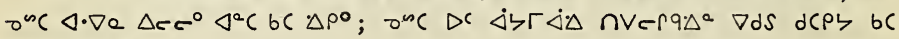
$\nabla \cap e r c \cdot \Delta x$

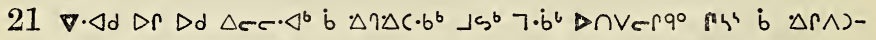
arco onc b $^{\circ} \triangle r \cdot \triangle c \Delta r c^{b}$,

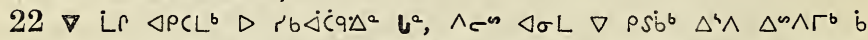

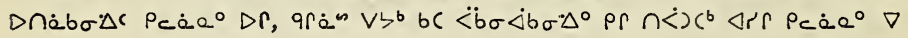
$\rho \cdot \Delta \sigma^{\omega b} c^{-r x}$

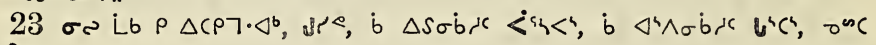
Lięs $x$

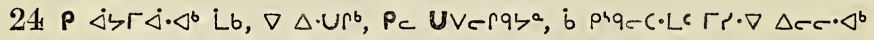

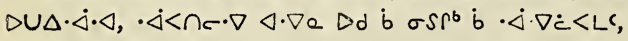

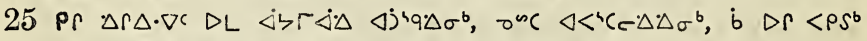

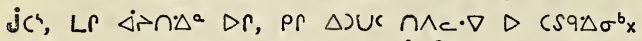

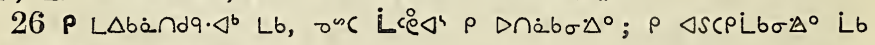
$\triangleleft \sigma \Delta \vee \zeta d \dot{s}<\triangleleft<h C \times x$

\section{${\text { AspCre } \triangle b^{2} 2 .}^{2}$.}

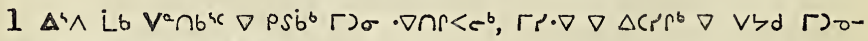

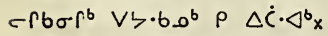

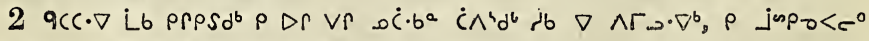

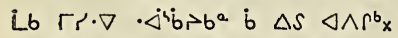

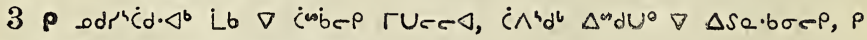

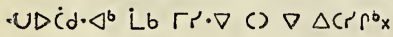




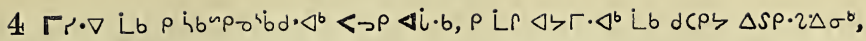
b $\Delta S \Gamma c d \rho^{b} \varangle i \cdot b x$

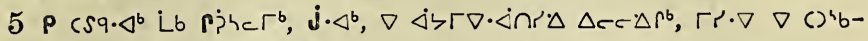

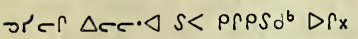

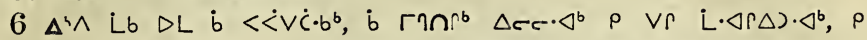

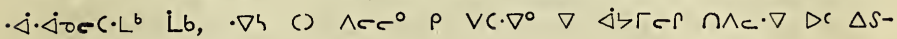
$\rho \cdot 2 \cdot \Delta \sigma^{b} x$

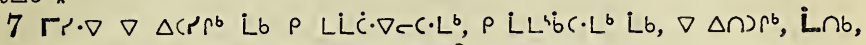

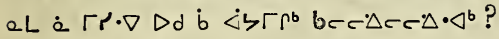

$8 \dot{c^{a} U}$ Lb $\cdot \nabla r \quad V\left(\cdot \Delta d^{b}\right.$ () $\Delta r c^{\circ} \cap \wedge_{c} \cdot \nabla$ pe $\Delta S \rho \cdot 2 \Delta \sigma \dot{\alpha}^{b}$, b $\Delta S$ $\sigma \dot{C} \triangle P \zeta^{\circ}$ ?

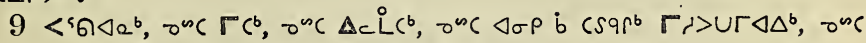

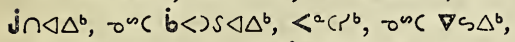

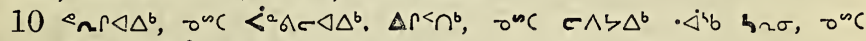

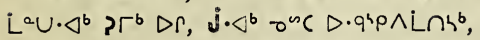

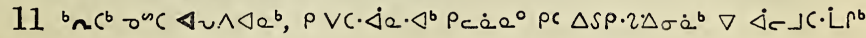

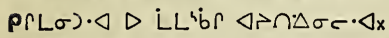

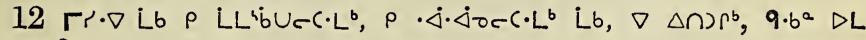
b $\triangle \rho^{b}$ ?

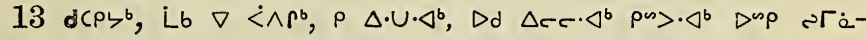
$>c^{\circ} \Delta \Gamma_{x}$

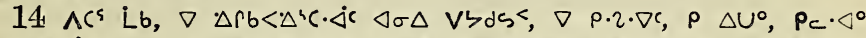

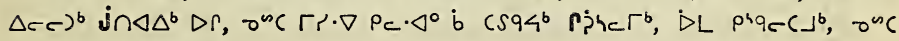
$V(\lrcorner^{b} \sigma^{6} \triangleleft \zeta \Gamma \Delta Q:$

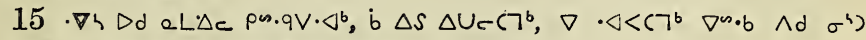
$\left.n<\Delta b^{a} \wedge r\right\lrcorner b \sigma^{b} \nabla \Delta^{b}<\sigma^{b} \nabla \rho s^{b} b^{b} x$

$16 \nabla \cdot \Delta d \dot{D}$ L Lb b $\Delta \cdot U d<-0 \rho^{4} \rho \cdot\left\langle\Delta q^{\circ} j \Delta^{c}\right.$ :

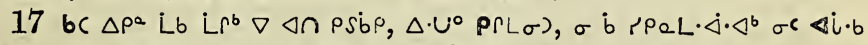

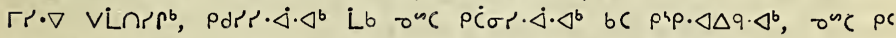

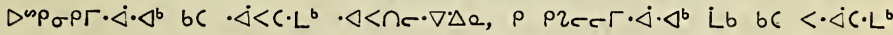
$<\cdot \triangleleft \cdot \Delta \mathrm{Q} Q$ :

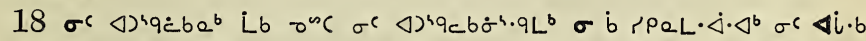
$7 \cdot b^{b} \triangleleft \sigma \Delta \rho S \dot{b} \cdot \triangleleft ; b c$ pip. $\triangleleft \Delta 9 \cdot \Delta^{b} \mathrm{~L} b$ :

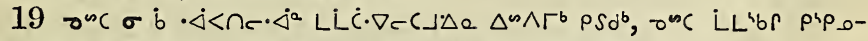

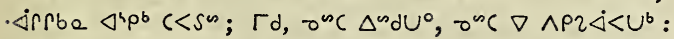

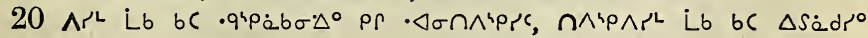

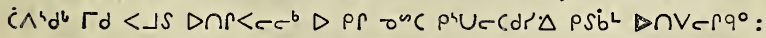

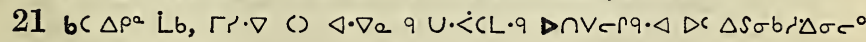
bc $\wedge L r b \sigma \Delta^{\circ} \times$

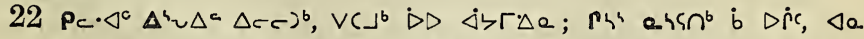

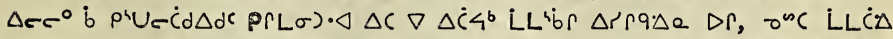

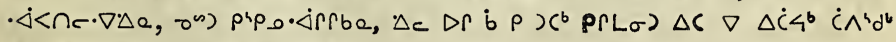
$\rho_{c} \cdot \Delta^{\circ} \sigma^{w C} \nabla \Delta S$ prqe $C 7^{b}$ :

191 


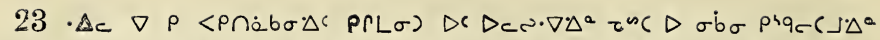

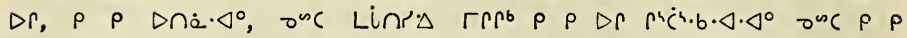
$\sigma<\dot{\Delta} \cdot \triangleleft^{\circ}:$

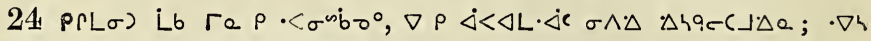
$c c \Delta c$ bc $\rho \Delta \rho^{a} \rho \Gamma \rho \Gamma r \Gamma \sigma d^{d} x_{x}$

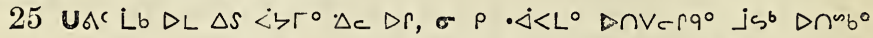

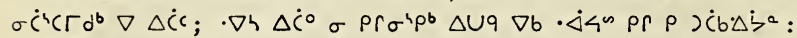

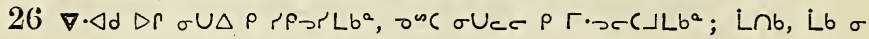
$\left.\triangle \zeta^{4} \sigma^{n c} b C \Delta \cdot \Omega \wedge L b^{a} \triangleleft^{4} \vee c\right\lrcorner \Delta \sigma^{b}$ :

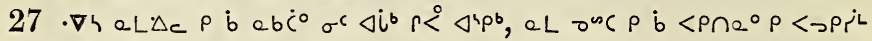
br $\cdot \dot{\Delta<}<c^{b} \sigma s \cdot \Delta a n \cdot \cdot \Delta \sigma c^{\circ} x$

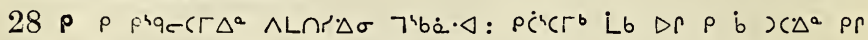

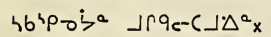

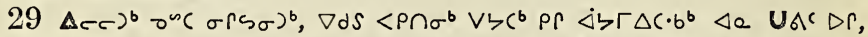

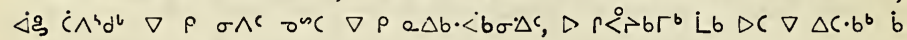

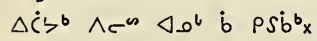

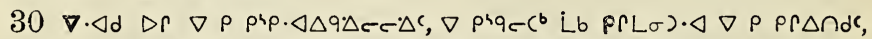

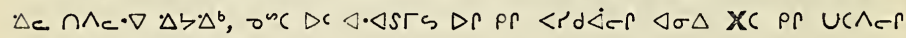
$\Delta C D P L \cdot \triangleleft \wedge \Delta \sigma c^{\circ}$;

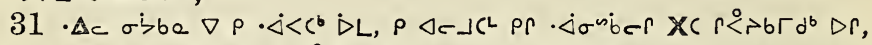

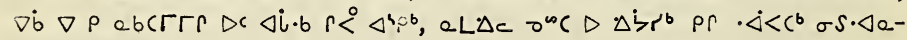
$n \pi \Delta \sigma \tau^{\circ} x$

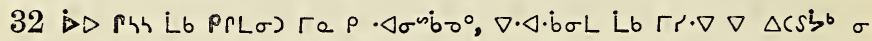
$n<\mathcal{U Q}{ }^{a} \times$

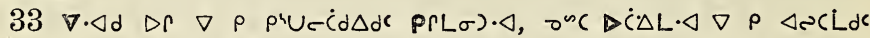

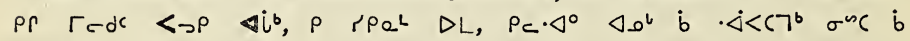
$v C 7^{b} x$

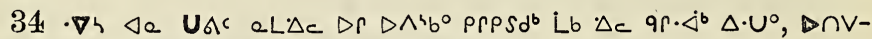

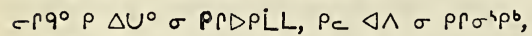

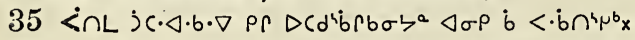

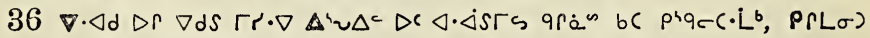

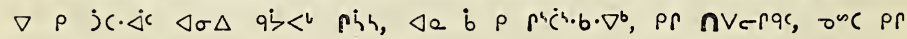
$X \cap \Delta c_{x}$

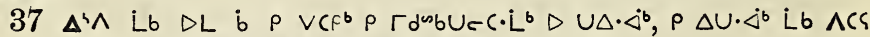

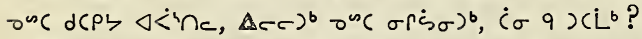

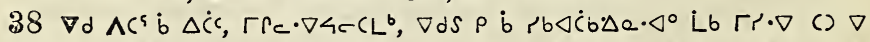

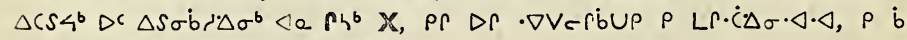

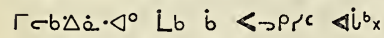

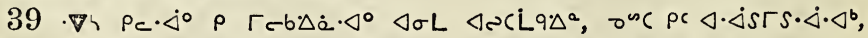

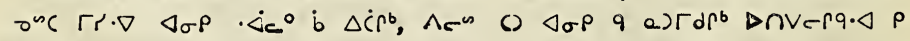
$P \Gamma L \sigma) \Gamma \mathrm{Q} \cdot \triangleleft_{\mathrm{x}}$

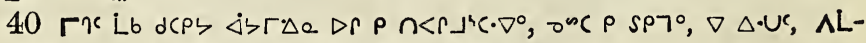

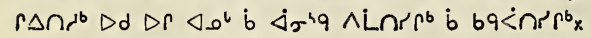




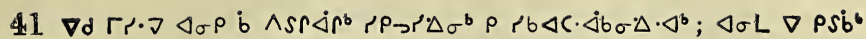

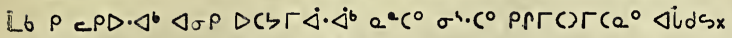

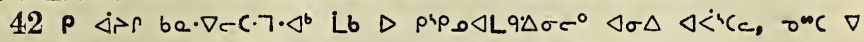

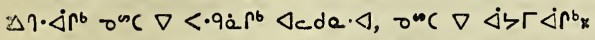

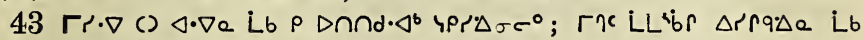

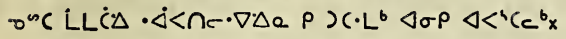

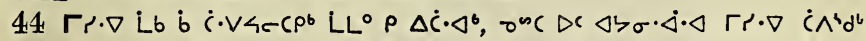

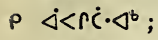

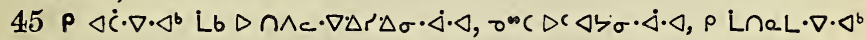

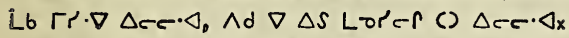

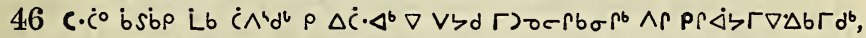

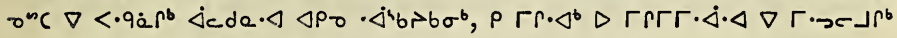

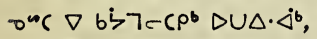

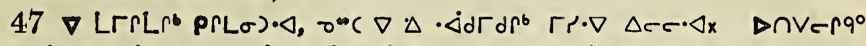
Lb $C \cdot \dot{C}^{\circ} \nabla \rho S \dot{b}^{6} \rho C d \nabla^{\circ} L L \Delta \Delta \dot{\Delta} \zeta \dot{\Delta} \cdot \triangleleft \triangleleft \sigma \Delta q \wedge L \Gamma \Delta b \sigma \Delta r r_{x}$

\section{$A^{3} p C r a \triangle b^{2} 3$.}

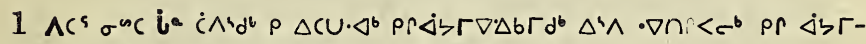

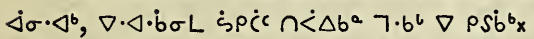

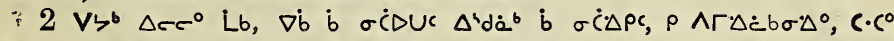

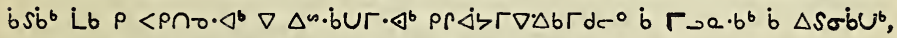

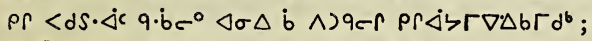

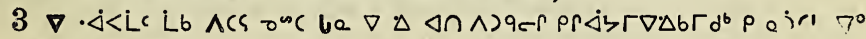
$q \cdot b r^{\circ}$ or $\Gamma e d c_{x}$

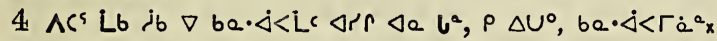

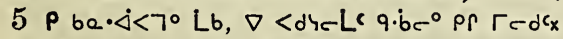

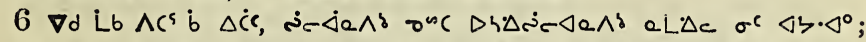

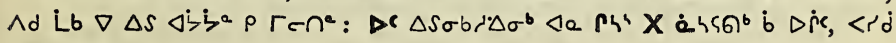
onc. $\wedge\lrcorner U_{\mathrm{x}}$

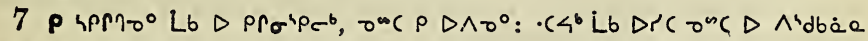
$\rho$ i $\wedge L b \sigma e \cdot \Delta x$

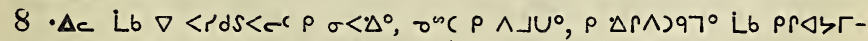
$\nabla \bullet \Delta b \Gamma d^{b}, \nabla \wedge J U c, \nabla \cdot b^{4} d \cap c, \sigma^{\text {sc }} \nabla$ LISLL $\left.P R L \sigma\right) \cdot \Delta x$

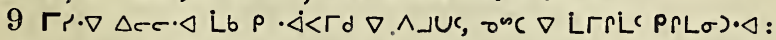

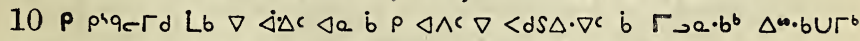
$\rho \Gamma \dot{b} \zeta \nabla \nabla \cdot \Delta b \Gamma d^{b}, a^{4} \wedge^{c}$ L Lb $\rho$ LL'bUec $\cdot L^{b} \triangleleft \sigma \nabla c^{0} b \Delta \rho \sigma c^{b x}$

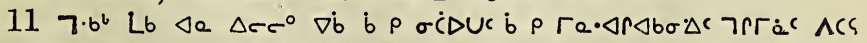

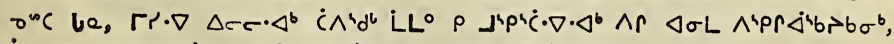

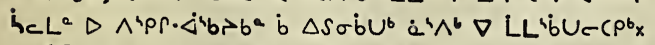

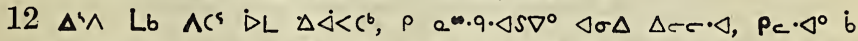

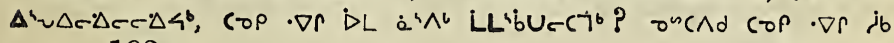
193 


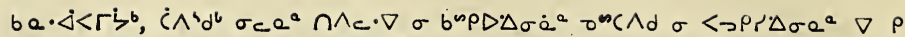

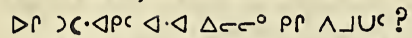

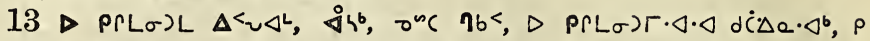

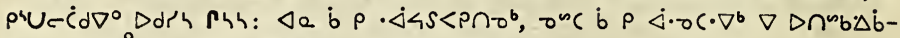

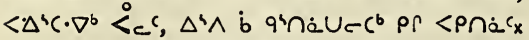

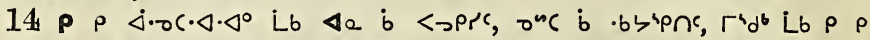
ec $\cdot \nabla-L \cdot \Delta^{\circ} \Delta \sigma<r \cdot \nabla^{\circ}$ pr $\Gamma-b \Delta<^{b}$;

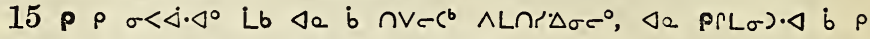

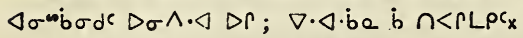

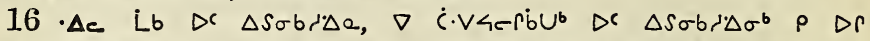

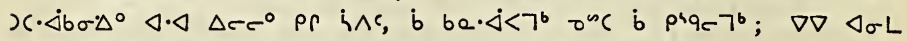

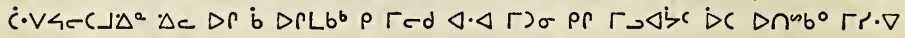
$\nabla \Delta C S 4^{b} x_{x}$

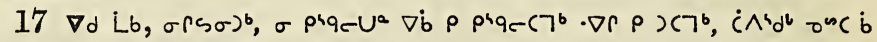
P $)$ <

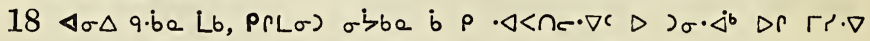

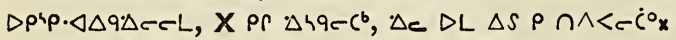

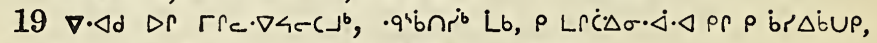

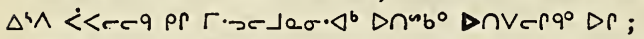

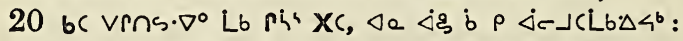

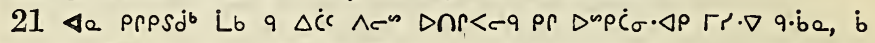

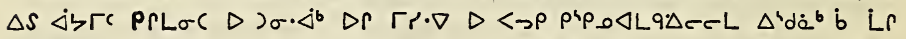
$\triangle 4 p \cdot \Delta^{b} x$

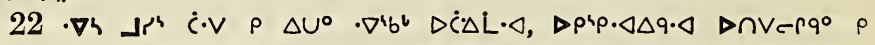

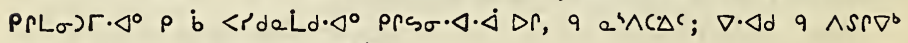
$\Gamma r \cdot \nabla$ q.be $\Delta r, \wedge d q \Delta S \triangle C L \dot{C}^{b} x$

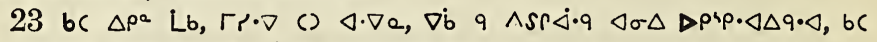
$\sigma s \cdot \Delta a r \dot{b} b \sigma \cdot \Delta^{\circ} \dot{\Delta}^{a} c \nabla \Delta \dot{C}-r \Delta c r \cdot \Delta \Delta r_{x}$

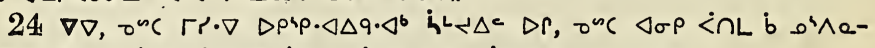

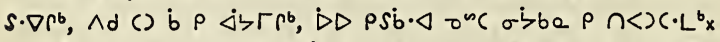

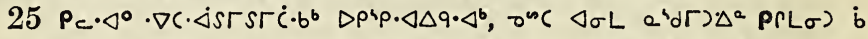

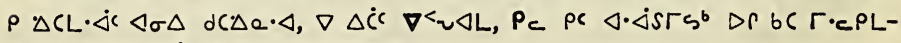

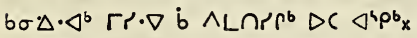

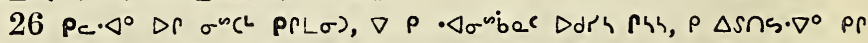

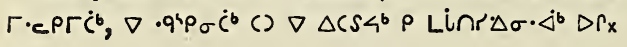

\section{$A^{s} p c r a \Delta b^{a} 4$.}

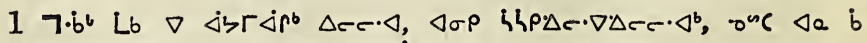

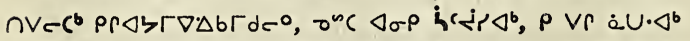

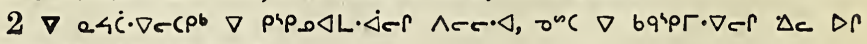
$M 4 \cdot \triangleleft \sigma^{\omega} b \Delta^{a} \nabla \sigma \wedge \cdot \Delta D r_{x}$

194 


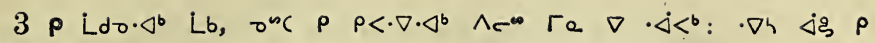
$D \dot{C} d S^{a} x$

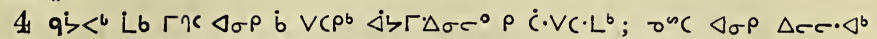
$\left.\dot{a}^{a} C^{\circ} \sigma b c \cdot \Delta^{\circ} \operatorname{prr} \dot{C}\right) \Gamma\left(a^{\circ} p \Delta\left(S \cdot \Delta^{b} x\right.\right.$

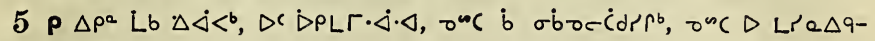
$\Delta \leftarrow r \cdot \dot{\Delta} \cdot \triangleleft$,

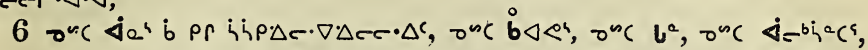

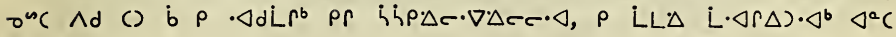
Prhe $\Gamma^{b} x$

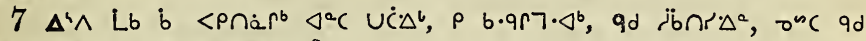
$\Delta S \sigma^{\circ} \dot{b} r^{\circ} \Delta a, b$ b $\left.P R\right)\left(7^{b} D L\right.$ ?

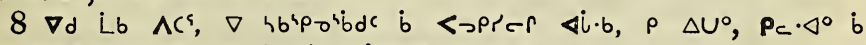

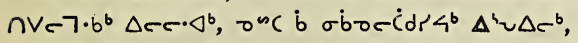

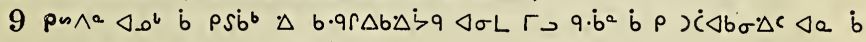

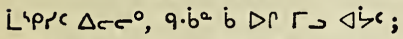

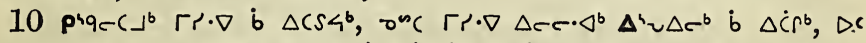

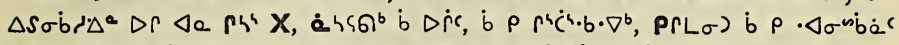

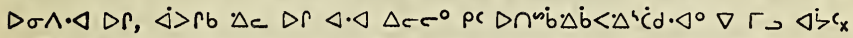

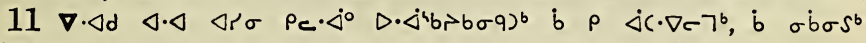
pqibे

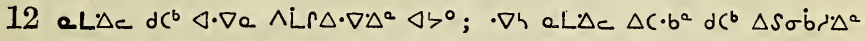
$s<\rho S d^{b} b \Gamma^{b} c^{b} \Delta c c \cdot \Delta^{b}, q \rho \quad \Delta r \wedge L r \Delta b \Delta b^{b} x$

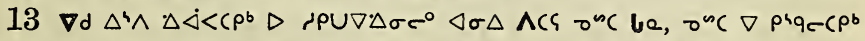

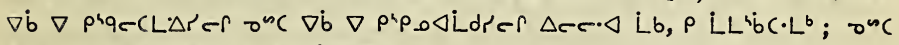
$\rho$ a $\dot{a} b c \cdot \nabla r-7 \cdot \Delta^{b} \nabla \rho \Delta \eta \cdot \Delta \beta^{b} p h h x$

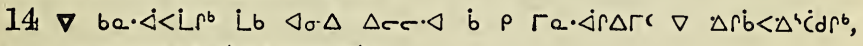

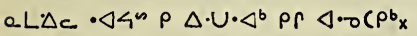

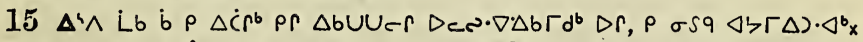

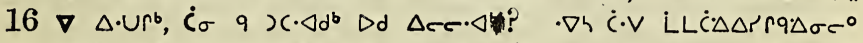

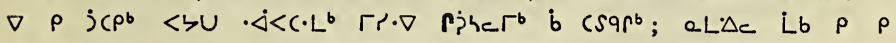
$\dot{\Delta} \cdot$ ovea $^{\circ} x$

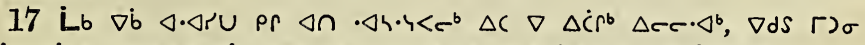

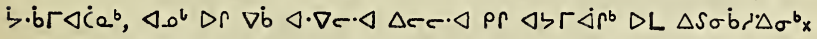

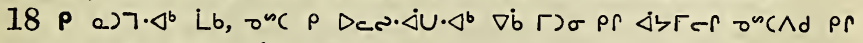

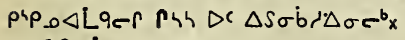

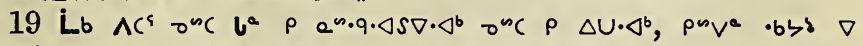

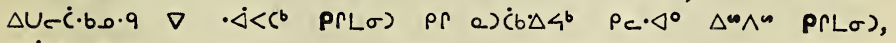
กरंde lb $D L x$

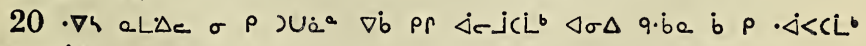
onc $V C L^{b} x$

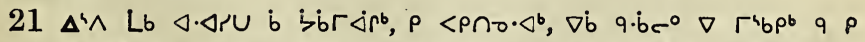

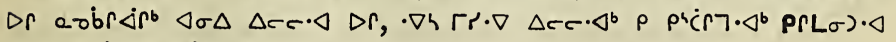
$\Delta \sigma \nabla \sigma^{\circ}$ b $\rho$ sibuc $\Delta r_{x}$ 


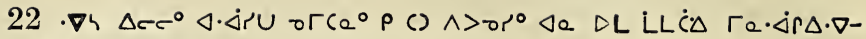
$\Delta \sigma c^{\circ}$ b $) C L \cdot \Delta b \sigma \Delta c_{x}$.

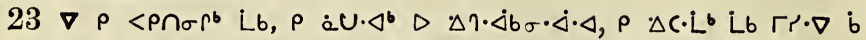

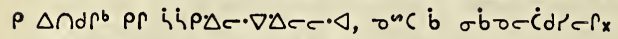

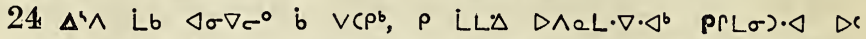

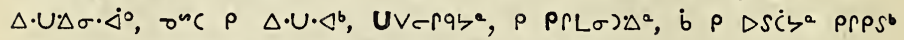

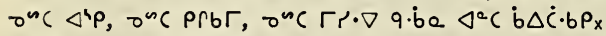

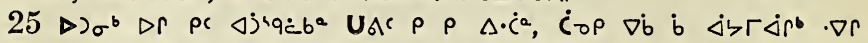

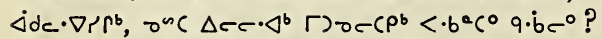

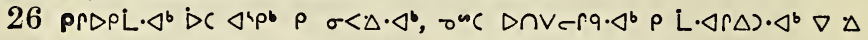

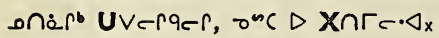

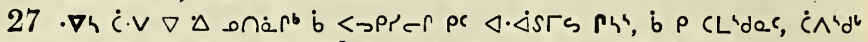

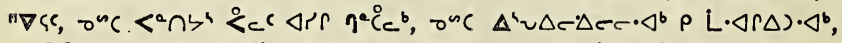

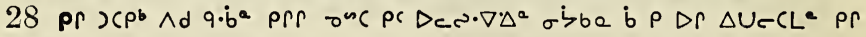
sibus

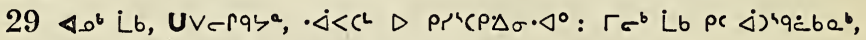

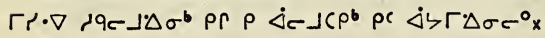

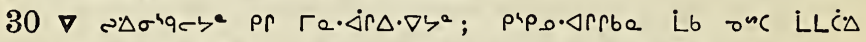

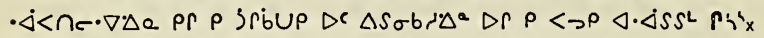

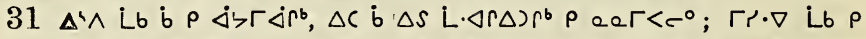

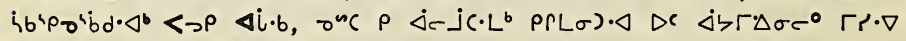
sac $\lrcorner \Delta \sigma^{b} x$

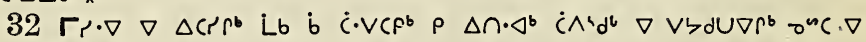

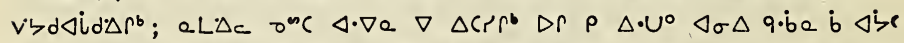

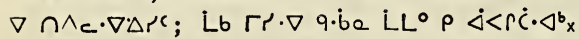

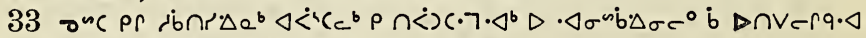

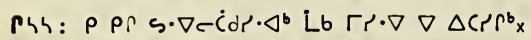

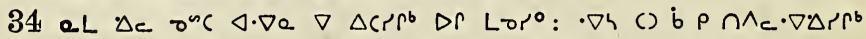

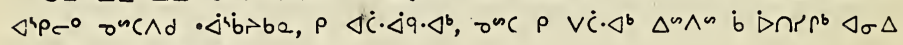

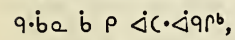

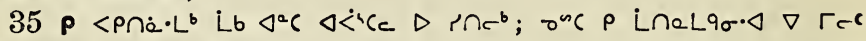
() $\triangle e c^{\circ} \wedge d \nabla \triangle S L_{d o r} C_{x}$

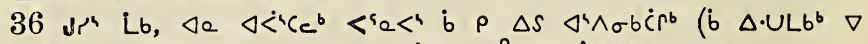

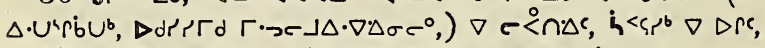

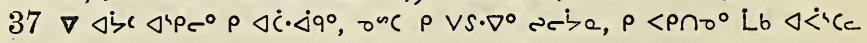
$\operatorname{Din} e^{b} x$

\section{$\Lambda^{4} p c r a \Delta b^{a} 5$.}

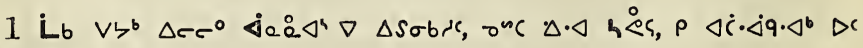
$\triangleleft \dot{\zeta} \sigma \cdot \dot{\Delta} \triangleleft$,

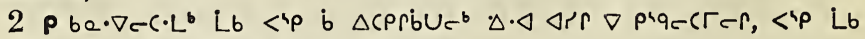

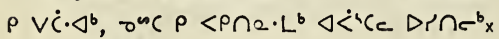




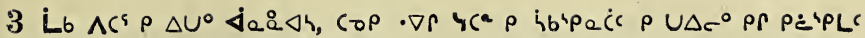

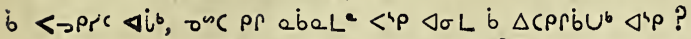

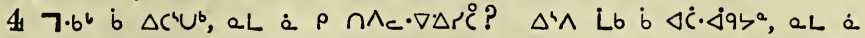

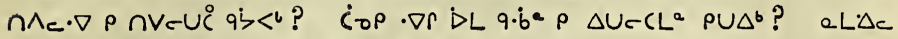

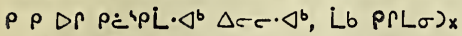

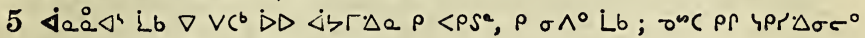

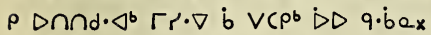

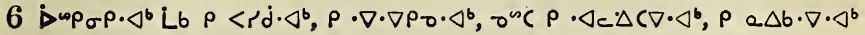
Lbx

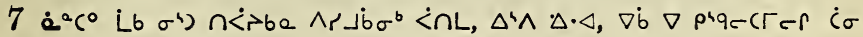

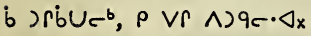

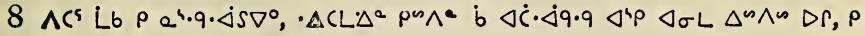
$\Delta \cdot U^{0} L b, \nabla \nabla, \nabla \cdot b \sigma \Delta r d^{b} x$

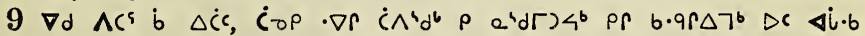

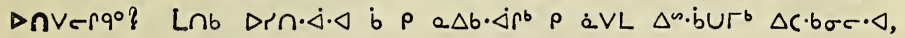
onc $\rho_{c} \rho$ b $\cdot \Delta_{c} \Delta C \Delta \cdot b^{b} x$

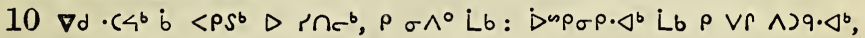

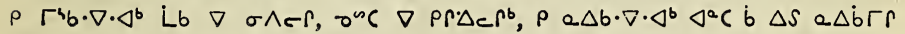
$D \dot{a} V L x$

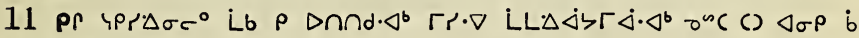
$V C \rho b$ D $\mathrm{q} \cdot \dot{b} \cdot \mathrm{x}$

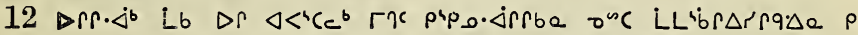

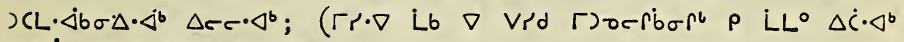

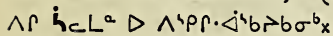

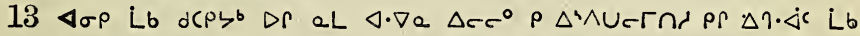
$\triangle r c \cdot \triangleleft p$ presr ${ }^{\prime} \cdot \Delta b^{b}$

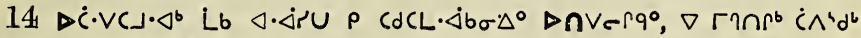

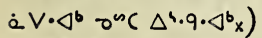

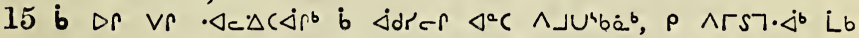

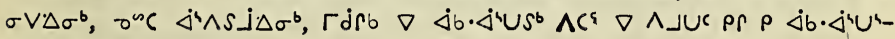

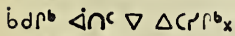

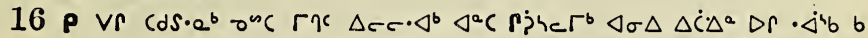

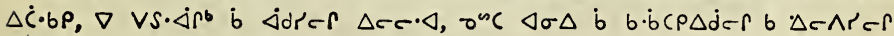

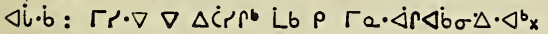

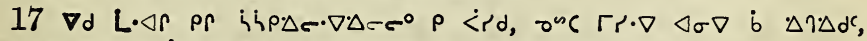

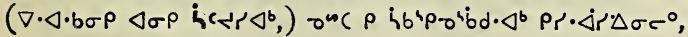

$18 \rho$ Ldo. $\left.\triangleleft^{b} L b<\sigma \Delta \triangleleft<^{4} C c, \sigma^{n} c \rho<\rho \cap 0 \cdot<^{b} \rho<D\right) \Delta b \Gamma d^{b} x$

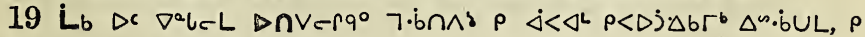
$V r \cdot \Delta c \Delta C \nabla^{\circ} L b, \sigma^{\omega C} \rho \Delta U^{\circ}$,

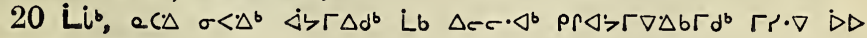

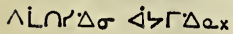

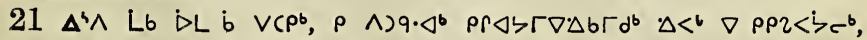

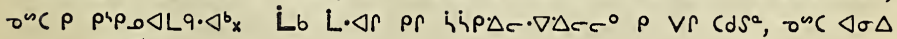
197 


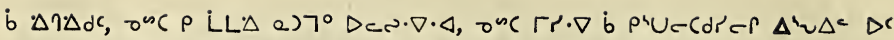

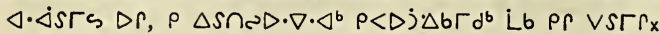

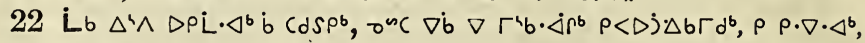
osC $P \Delta C \cdot L^{b}$,

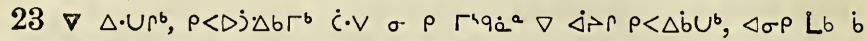

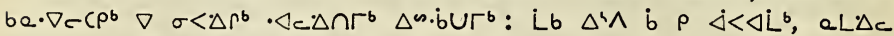
$\triangleleft \cdot \nabla e \Delta r c^{0} \wedge r \sigma \rho \Gamma^{\mathrm{u} b} b \cdot \triangleleft \dot{Q}^{a} \mathrm{x}$

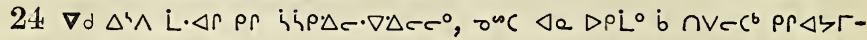

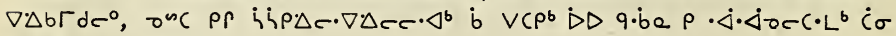
$q \triangleleft \cap \triangle P c c^{b} D L x$

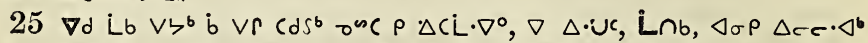

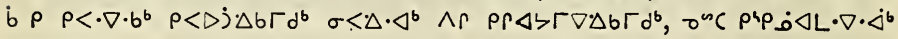
$\Delta r c \cdot \Delta x$

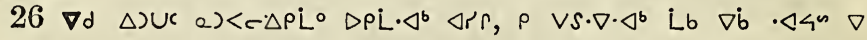

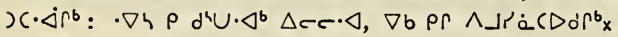

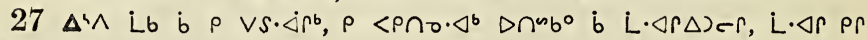
hi $\rho \cdot \Delta c \cdot \nabla \Delta c c^{\circ} \rho$ b.qr $7^{\circ}$ Lb,

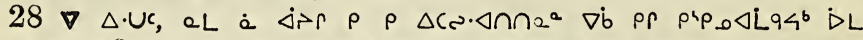

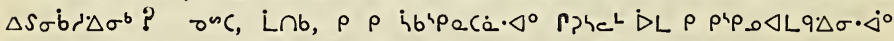

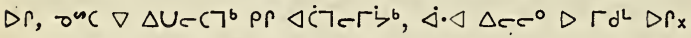

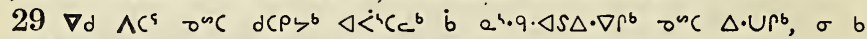

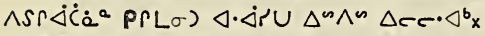

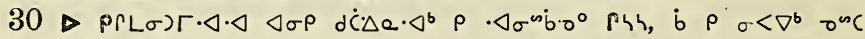
$\Gamma^{4} \cap d^{b} \dot{b} \rho \triangleleft \dot{d} \partial^{b} x$

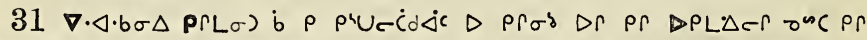

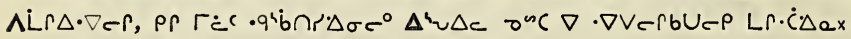

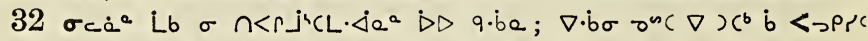
$\varangle i b, \Delta \sigma \Delta \rho \rho L \sigma)$ b $\rho \Gamma \dot{c}\left(\Gamma r \cdot \nabla()\right.$ b ea $\Delta \dot{C} d d_{x}$

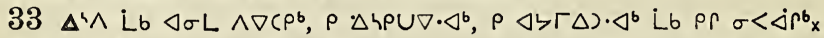

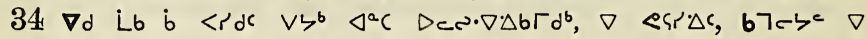

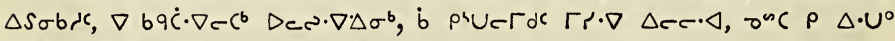
$\triangle \wedge \neg$ ses $p \rho \Delta)(\Delta \Gamma r \triangleleft \sigma \Delta \triangleleft \dot{<}(c ;$

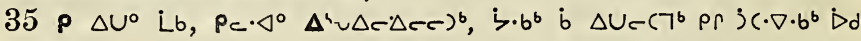
$\Delta c r \cdot \Delta^{b} \mathrm{x}$

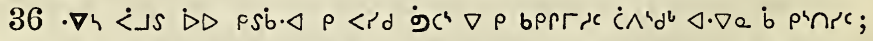

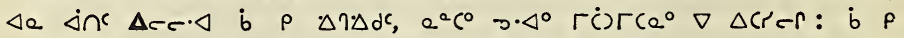

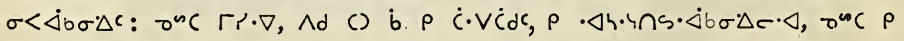
$<\cap 7 r<c \cdot \Delta x$

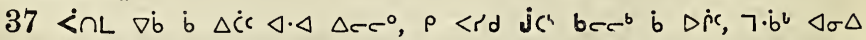

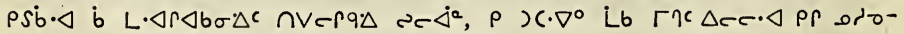

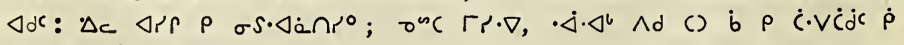
$\triangleleft 4 \cdot 4 \cap s \cdot \Delta b \sigma \cdot \Delta c \cdot \Delta_{x}$

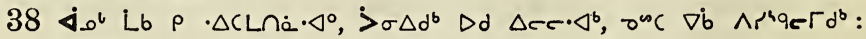
198 


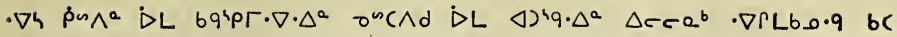
$\Delta \omega . \dot{b}<c^{0}:$

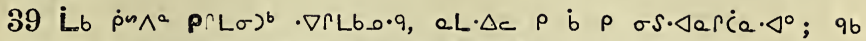

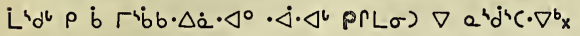

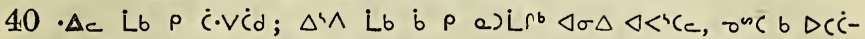

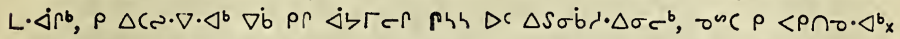

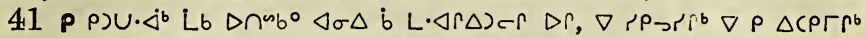

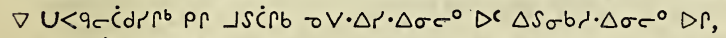

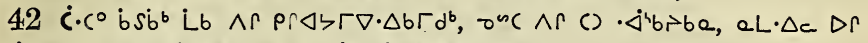

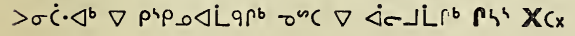

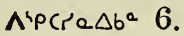

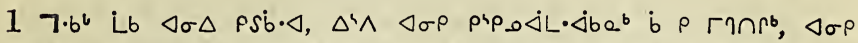

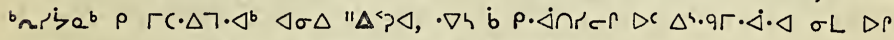

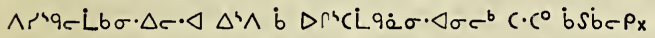

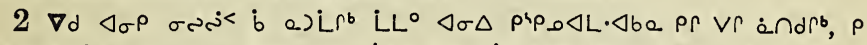

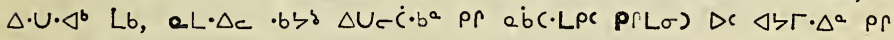

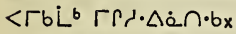

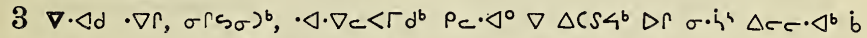

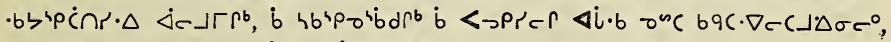
$\triangleleft \sigma \rho$ q $\rho \cap \operatorname{VV} \in C r \Delta \rho \rho^{b} \dot{D} L \Delta \dot{C}<\cap r \Delta^{a} x$

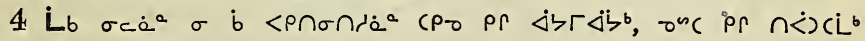
$\dot{\Delta} \zeta \Gamma^{a} \mathrm{x}$

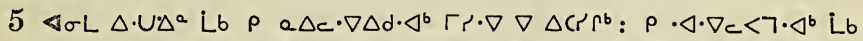

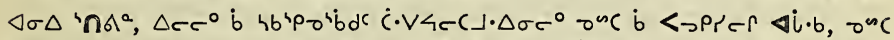

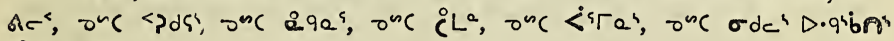
$\dot{\alpha} \cap \Delta \rho b \Delta r$;

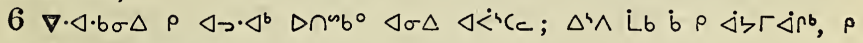

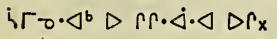

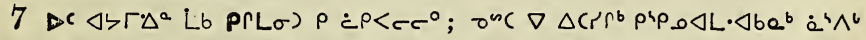

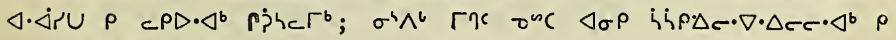
Q.ं $\left.\Delta C \cdot L^{b} \dot{C} \cdot V \angle C C\right\lrcorner \Delta \sigma c^{\circ} x$

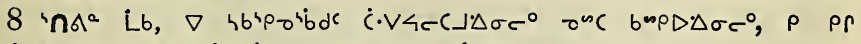
pr LL'br) CL owe $\rho$ LL'br$\Delta r r g^{\circ} \Delta C \nabla \Delta \dot{C}-r \Delta c c \cdot \Delta x$

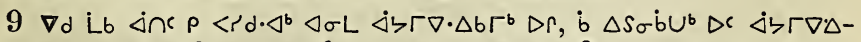

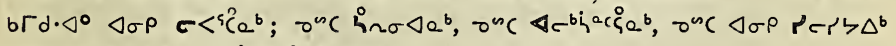
$\nabla C$ Juc $\nabla s \Delta^{b}, \nabla \Delta i s U L r^{b}$ index

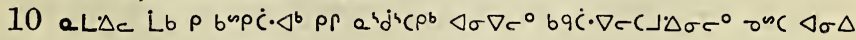

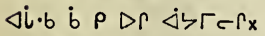

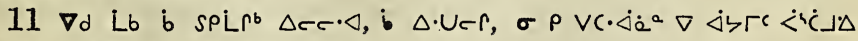

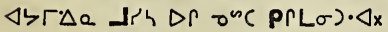




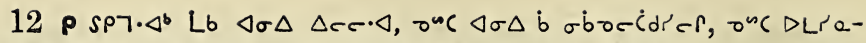

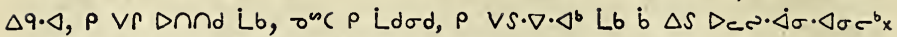

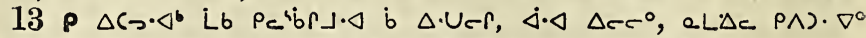

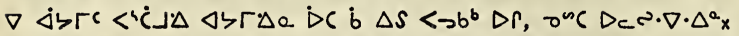

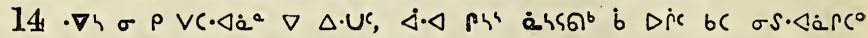

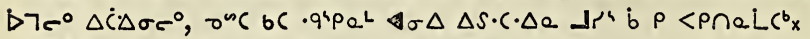

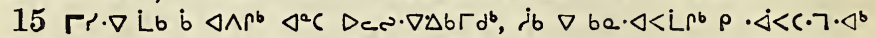
$\Delta \dot{C}^{4} C \Gamma d c^{0} \nabla \Delta S a \cdot b \sigma c^{b} \dot{C} \Lambda^{\prime} d^{b} \nabla^{a} l^{a} \Delta \dot{C}^{4} C \Gamma^{b} x$

\section{Nipcre $\triangle b^{*} 7$.}

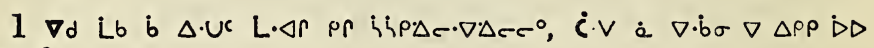
$q \cdot b a ?$

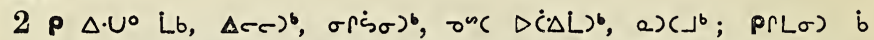

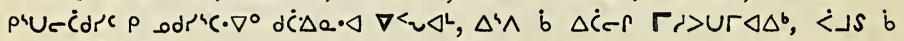
csacr bs $\sigma^{b}$,

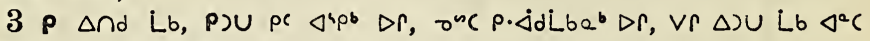

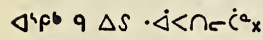

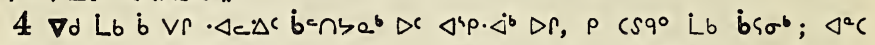

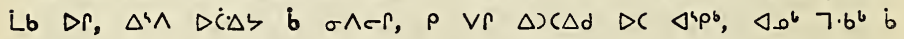

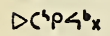

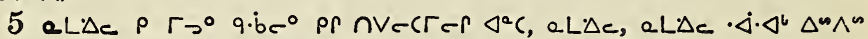

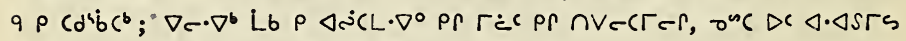
<ंกL, $\Delta$ in $\nabla \dot{b} \nabla^{n} \cdot b \triangleleft \cdot \Delta S S \nabla \triangleleft \dot{b} \cdot \triangleleft c \Gamma_{x}$

6 PrL $\sigma)$ Lb $D L \rho \Delta S \triangleleft b \Gamma^{\circ}, \triangleleft \sigma \Delta \Delta C \triangleleft \cdot \triangleleft s \Gamma S$ pr $\left(s q C r\right.$ LaU $\Delta \triangleleft^{4} p b$;

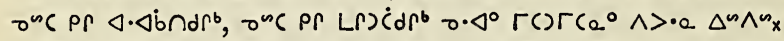

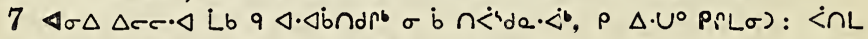

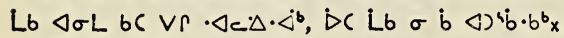

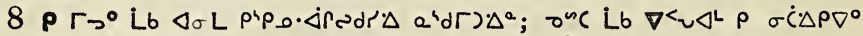

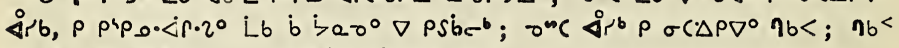
Lb $\rho \sigma \dot{C} \Delta P \nabla^{\circ} \triangleleft \sigma \Delta \sigma \sigma^{\dot{S}}<\dot{b} \Delta \dot{C} \Delta L \Delta r \Gamma_{x}$

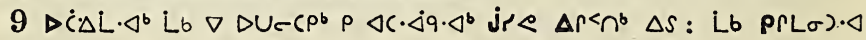
$\rho \Delta \eta \Delta d$,

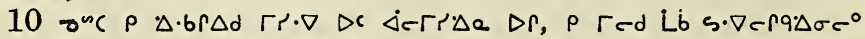

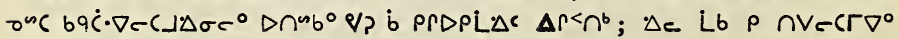

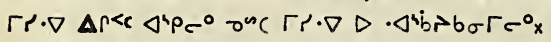

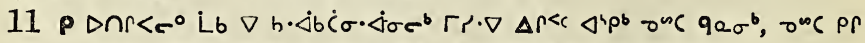

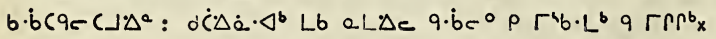

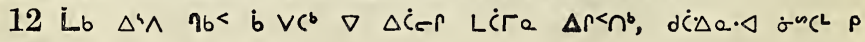
$\Delta s \cap s_{5} \cdot \nabla^{\circ} \mathrm{x}$

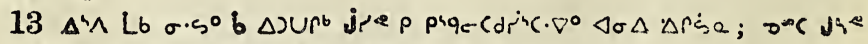

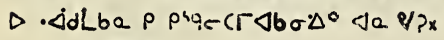




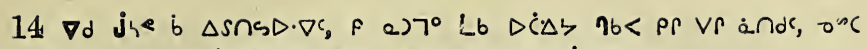

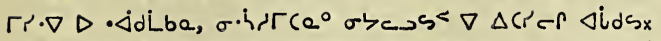

$15 \nabla d \eta_{b}<\dot{b} \Delta x \cup \Delta r^{<}<n^{b}, \rho \sigma \wedge^{0} L b, \Delta c$, onc $d \dot{C} \Delta \dot{a} \cdot \Delta^{b}$,

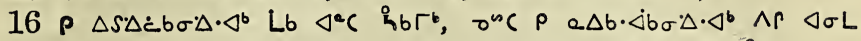

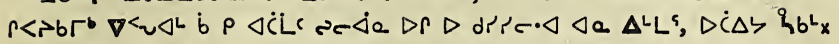

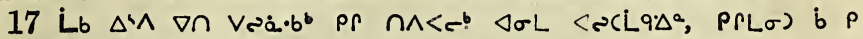

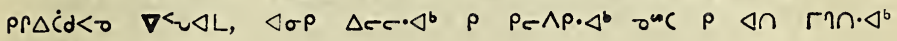
$\Delta r<n$,

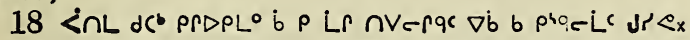

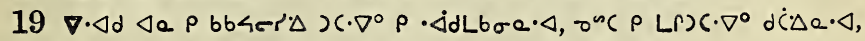

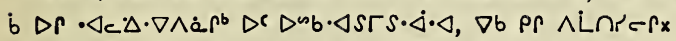

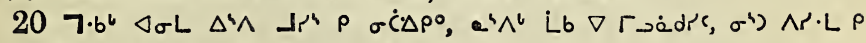
be. $\left.\nabla c L b \sigma \cdot \Delta^{\circ} D C \Delta\right\rangle D \cdot \dot{B} b+b \sigma \Gamma \sigma^{b}$ :

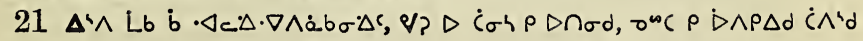
$\cap \wedge c \cdot \nabla D d r h x$

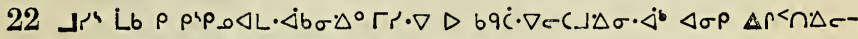

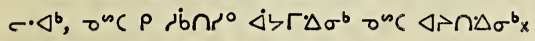

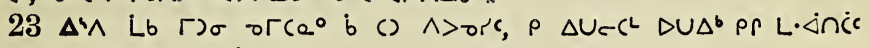
$\Delta r s e \Delta^{\prime} v \Delta^{c} D^{C} \Delta \cdot \Delta s r s_{x}$

$24 \nabla \cdot \Delta<L C$ Lb $\left.V \zeta^{b} \triangleleft \sigma \Delta \nabla \cdot \Delta \sigma\right)\left(\cdot \Delta b \sigma \Delta r r, \rho\right.$ a $C L \cdot \nabla^{0}, \rho \Delta \rho \nabla^{\circ}$ Lb b $\rho$ e obir $\Delta r r, \sigma^{n} C \rho \sigma<\nabla^{\circ} \triangleleft \sigma \Delta \Delta \beta<n \Delta c r \cdot \triangleleft$ :

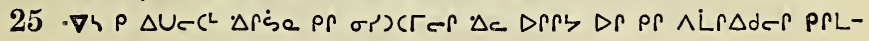
$\sigma) \cdot \triangleleft:$ Lb eLAc $D r$ or) $(T c \cdot \Delta x$

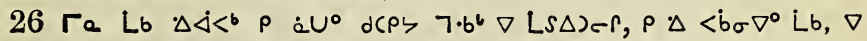

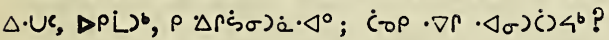

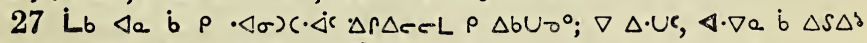

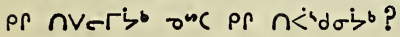

28 b b $\sigma<\Delta^{a}$ a b $\left.\Delta s\right)\left(\cdot \Delta c \Delta a \Delta r<n \Delta c c^{\circ} \Delta \dot{c} d s^{b}\right.$ ?

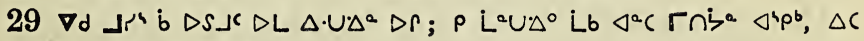
b $p$ ocisper or $D$ drhx

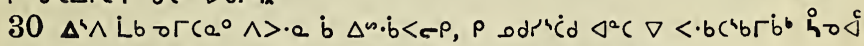

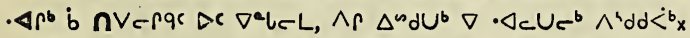

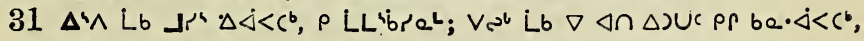

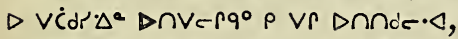

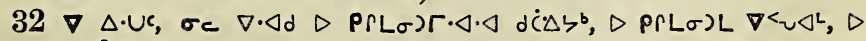

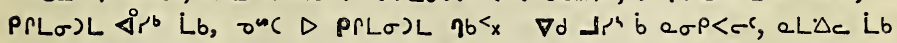
$\rho$ be. $\dot{\langle} \wedge{ }^{\circ} x$

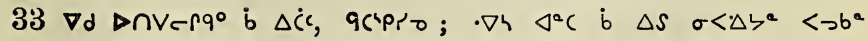
$\triangleleft 4 p_{x}$

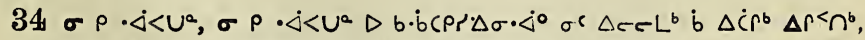

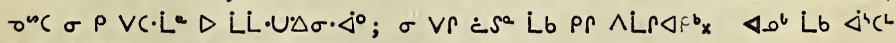
$\rho$ bे $\Delta S \cap \dot{s} \Delta \cap^{2} \Delta p^{<} \Omega^{b} x$

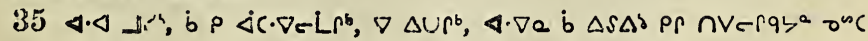
201 


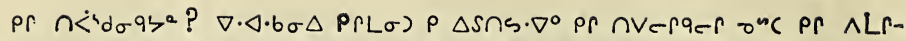

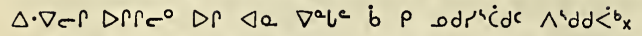

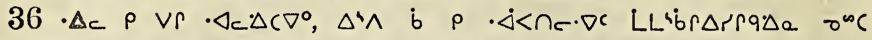

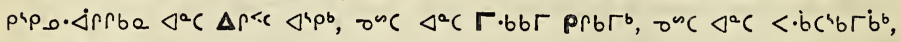
or $\mathrm{CQ}^{\circ} \wedge>\cdot \mathrm{ex}^{-x}$

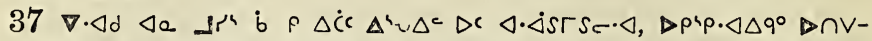

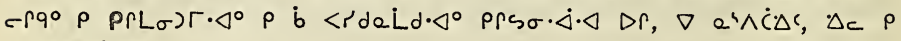
b $\wedge \operatorname{si\Delta } \Delta \cdot \triangleleft^{\circ} x$

$38 \nabla \cdot \triangleleft d \triangleleft \cdot \triangleleft \dot{b} \rho \Delta \dot{c} c \triangleleft b \Gamma \nabla \Delta b \Gamma d^{b} \nabla^{a} c<\cdot b c h b \Gamma b^{b}, \Delta r p \triangleleft \sigma \Delta \nabla^{a} l_{c} b$

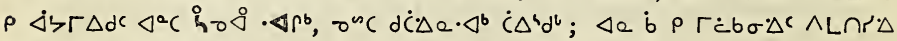
$\nabla c r \cdot \nabla \Delta e$ or $\Gamma_{C-C^{b}:}$

$\left.39 \varangle \sigma \Delta d \dot{C} \Delta a \cdot \Delta^{b} \nabla \dot{b} \dot{b} \Delta a\right)\left(\cdot \Delta r^{b}\right.$, Lb $\nabla \rho \Delta>b U \dot{a} r^{b}, \tau^{a c} \Delta U \Delta \cdot<^{b}$ $\Gamma e \rho \cdot \Delta \rho \cdot \nabla \cdot \dot{j}^{b} \Delta r^{<} \Omega^{b}$

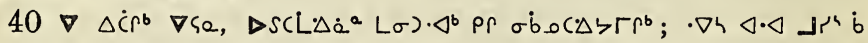

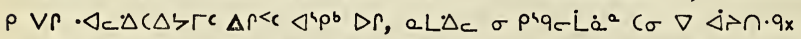

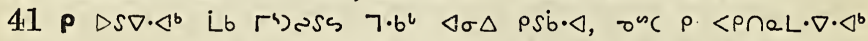

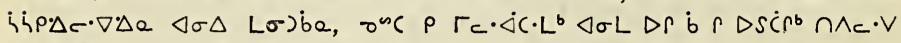
$\Delta r r \cdot \dot{b}^{b} \mathrm{x}$

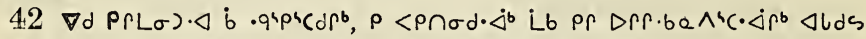

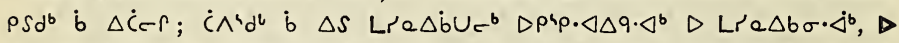

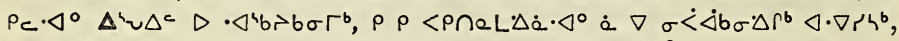

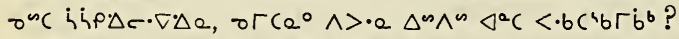

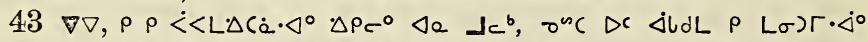

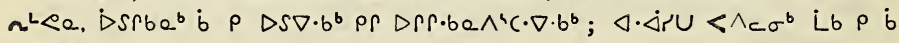
$\Delta x \Delta \cap \dot{0} \cdot \Delta^{\circ} x$

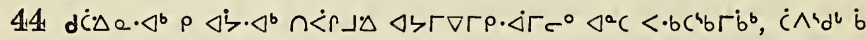

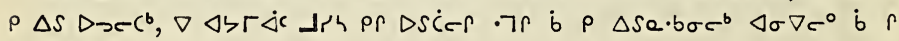
$\cdot \Delta<c^{b} x$

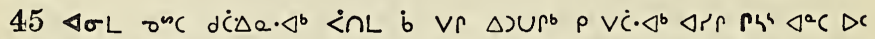

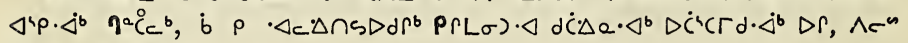
$U A C D$ PSBLX

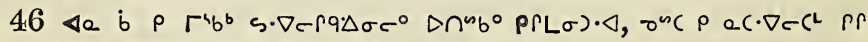

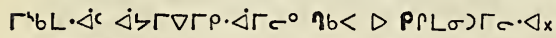

47 Lb he Le $P$ DSCL. $\nabla^{\circ} \cdot \Delta \triangleleft b r b \sigma c^{\circ} x$

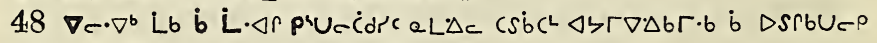

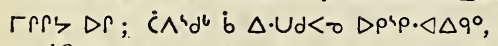

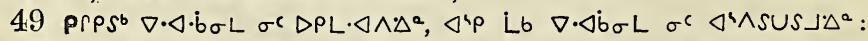

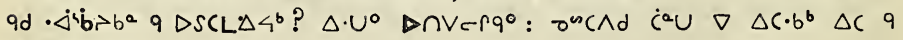

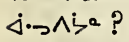

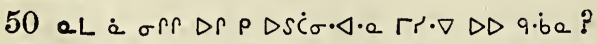

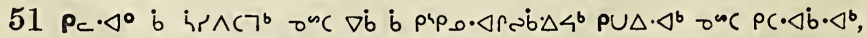

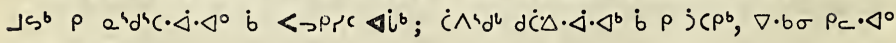

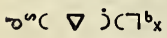




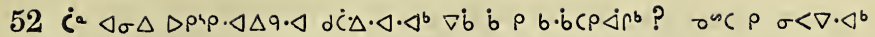

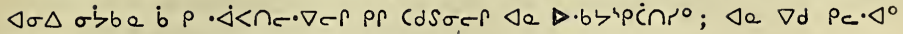
b) $\rho$ LTS T $]^{b} \sigma^{\text {cs } C} \sigma<\nabla^{b}$ :

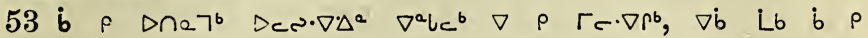
$b a \cdot \nabla c c 7^{b} x$

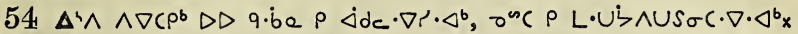

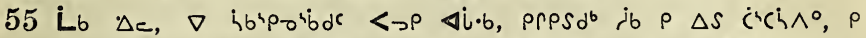

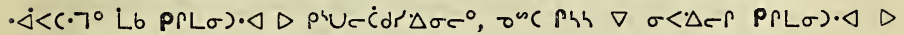
próp $\rho^{b}$,

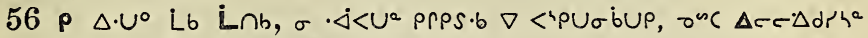
$\nabla \sigma<\Delta(P \rho L \sigma) \cdot \triangleleft D \rho P \sigma^{4} \rho_{C} \sigma^{b x}$

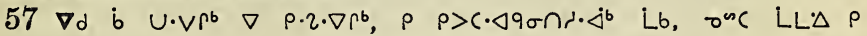
J'pr $C \cdot \nabla \cdot \Delta^{b}$,

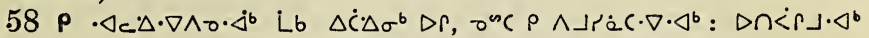

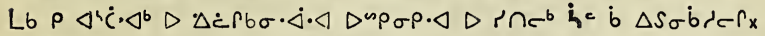

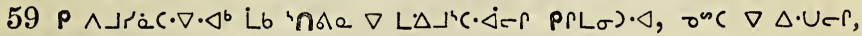
UVerqia $r^{4}, \Delta \cap^{a} \sigma^{c} \Delta i b_{x}$

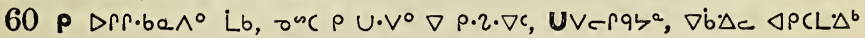
$D L L P)(\lrcorner \Delta \sigma \sigma^{\circ} x \quad \Delta^{\wedge} \wedge$ Lb $D L$ b $\rho \Delta \cdot U c, \rho b \Delta \cdot b S^{\circ} x$

\section{Aipcra $\triangle b^{a} 8$.}

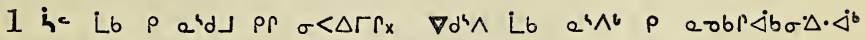

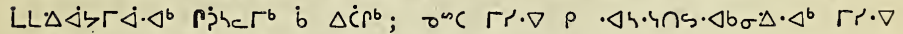

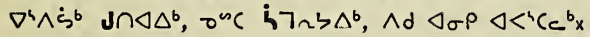

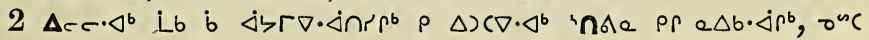
$a^{4} \wedge^{b} \rho L \Delta \Delta \dot{b} U \cdot \Delta^{b} x$

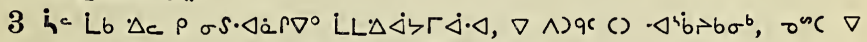

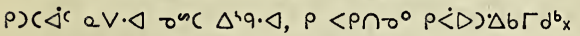

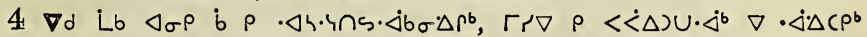
$\left\langle i\left\ulcorner\Delta \sigma \sigma^{\circ} x\right.\right.$

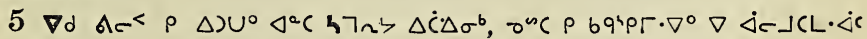
$\triangleleft \sigma \Delta \mathbf{X} C_{\mathrm{X}}$

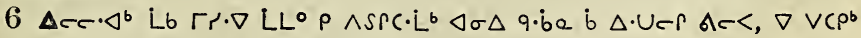
osc $\nabla \cdot \dot{\Delta}<C \rho^{b} \triangleleft \sigma \Delta$ LLCं $\Delta \Delta r \rho q \Delta e$ b ) $C \Gamma c r_{x}$

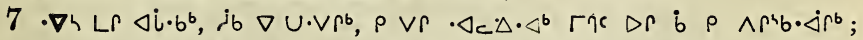

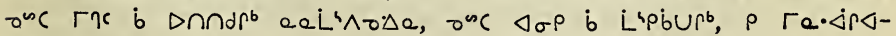
$b \sigma \Delta \cdot \Delta b^{b}$

$8 \dot{a}^{4} \wedge^{6} L b \rho \Gamma c \cdot \Delta \dot{\beta} b u^{\circ} \triangleleft \sigma L \Delta \dot{C} \Delta \sigma^{b} x$

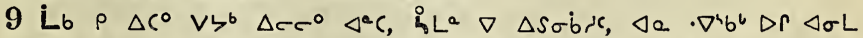

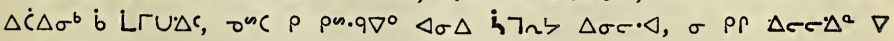
$\Delta \cdot \dot{c} \dot{r}^{\mathrm{c}}$ : 


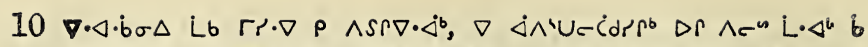

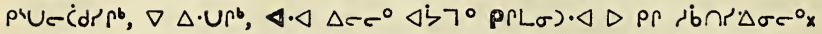

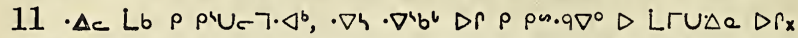

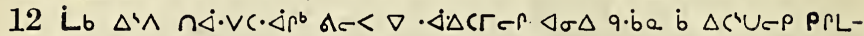

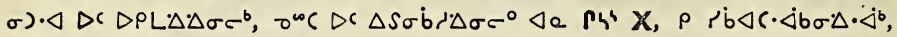

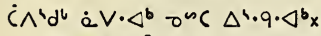

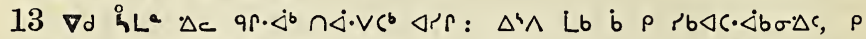

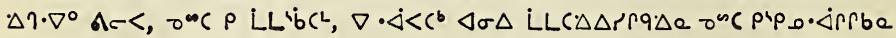
b) siburep

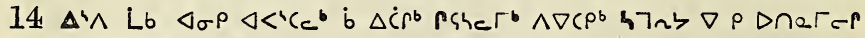

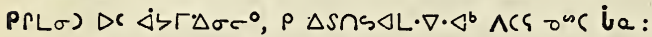

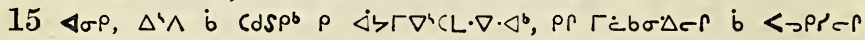
$\triangleleft i \cdot b$ :

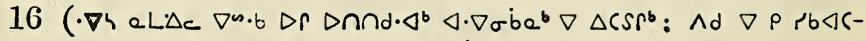

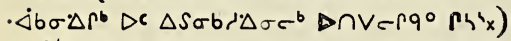

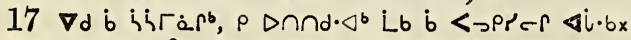

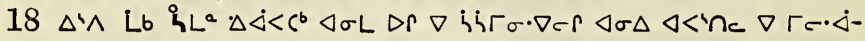

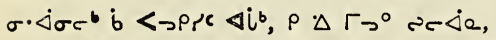

$19 \nabla \Delta \cdot U c, \Gamma \sigma^{b} \sigma c$ osc $D L$ bup $\nabla \Delta^{a}, \wedge d \triangleleft \cdot \nabla e q i \Gamma e \cdot \Delta q$ pr $p \Delta \cap \dot{c} c$ i <spter $\Delta i \cdot b x$

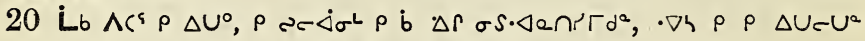

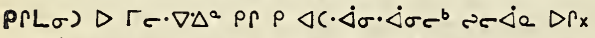

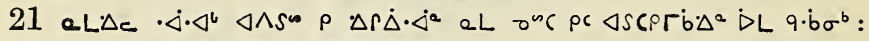

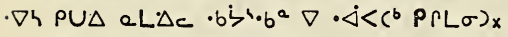

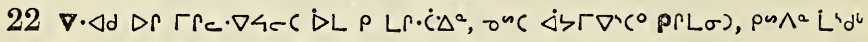
b $\Delta S$ T)

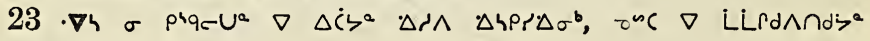
LinR $\Delta^{a} x$

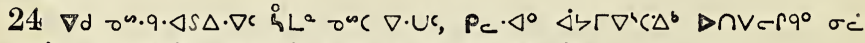

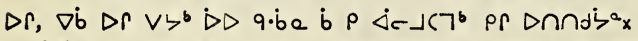

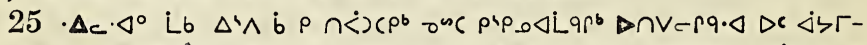

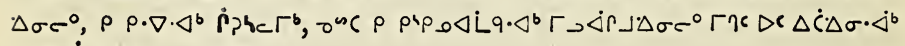

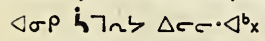

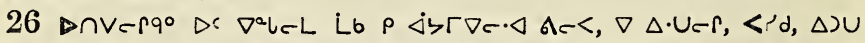

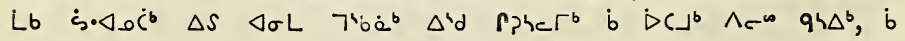
$<\cdot b\left(r p \cdot \triangleleft^{b} x\right.$

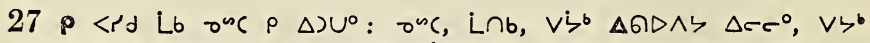

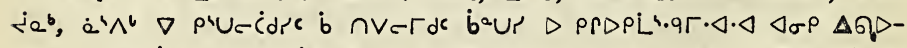

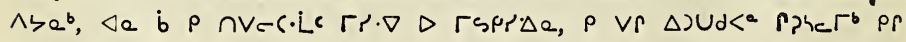
¿לr

$28 \nabla \triangleleft n \rho \cdot \nabla c$, J $\Delta 9 \cdot \Delta x$

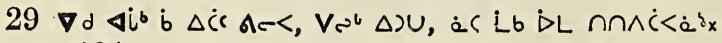
204 


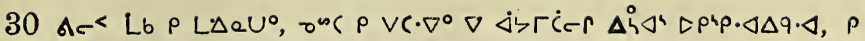

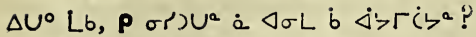

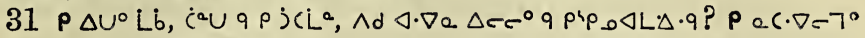
Lb Ac $<$ Pr $\vee r \Delta C A \Gamma d d_{x}$

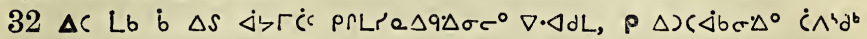

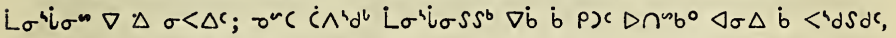

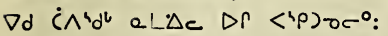

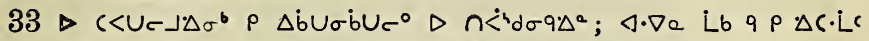

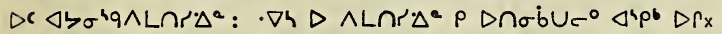

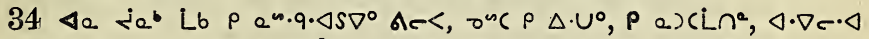

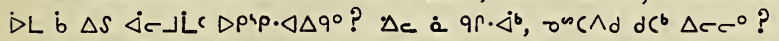

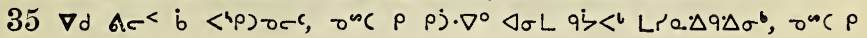

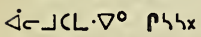

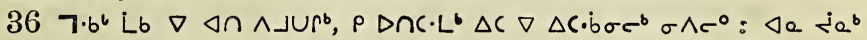

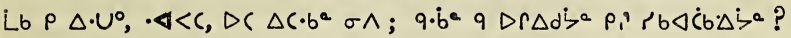

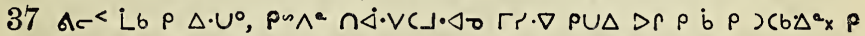

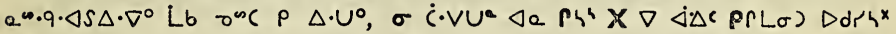

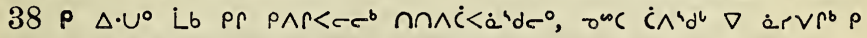

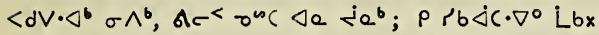

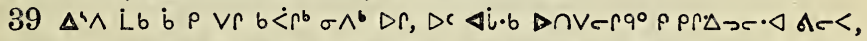

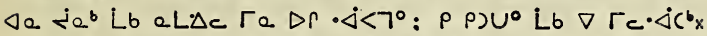

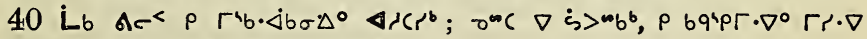
$\Delta \dot{C} \Delta a, \Lambda_{-\infty}^{-\infty} \dot{c} C S^{b} \Delta^{a} c$ phn $\Delta \Delta^{b} x$

\section{$\Lambda^{4} p c r a \Delta b^{a} 9$.}

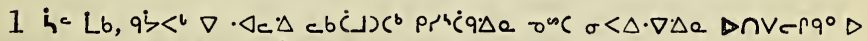

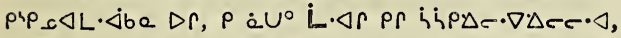

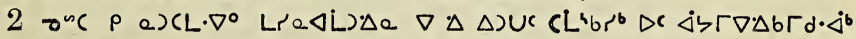

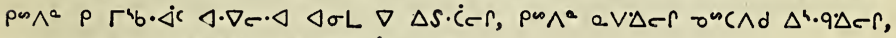

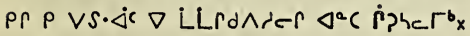

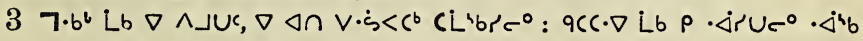

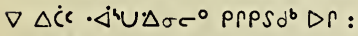

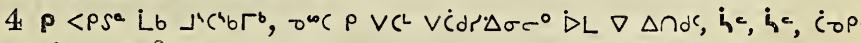
- $\nabla r \quad b \cdot \dot{b} C P \Delta \zeta^{2}$ ?

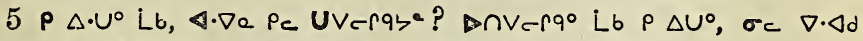

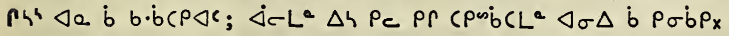

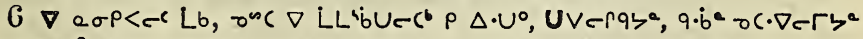

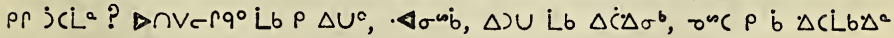
$9 \cdot b^{a} q \dot{ }{ }^{2} L^{2} x$

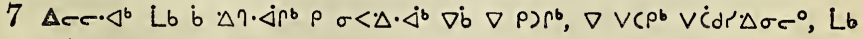
$\nabla \dot{b} \triangleleft \cdot \nabla c \cdot \triangleleft \nabla \cdot \triangleleft<L r^{b} x$

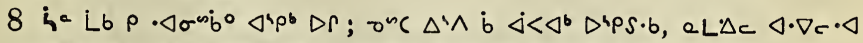

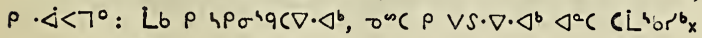




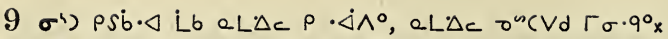

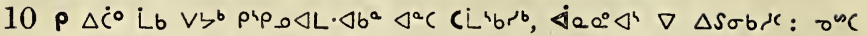

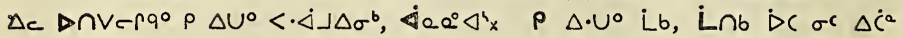
UVerab $x$

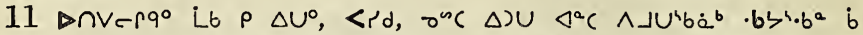

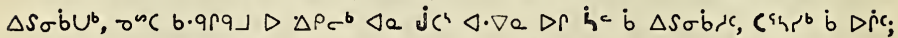

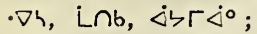

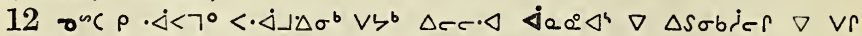

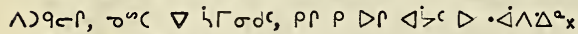

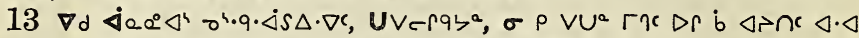

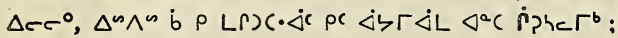

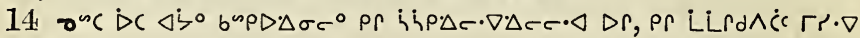
i $U \cdot<C r e r$ pe $\Delta s \sigma \dot{b} i \Delta \sigma c^{\circ} x$

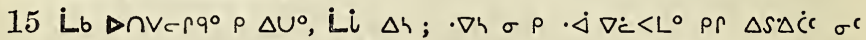

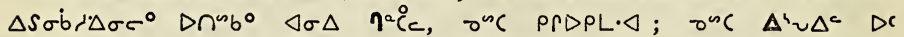
$\triangleleft \cdot \triangleleft s \Gamma s$ :

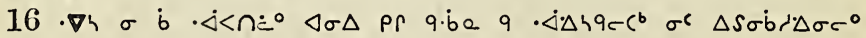
$\Delta r_{x}$

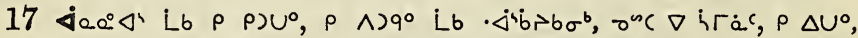

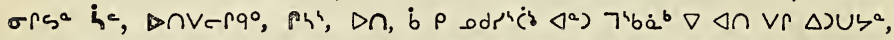

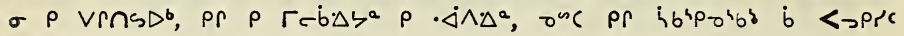
$\Delta i b_{x}$

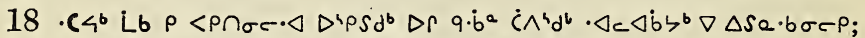

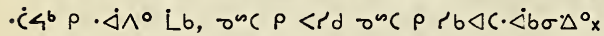

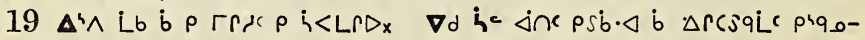
$\triangleleft L \cdot \Delta b e \triangleleft^{a} C C L^{\prime} b p^{b}$ b $\Delta \dot{C}-r_{x}$

$20 \cdot\left(4^{b}\right.$ Lb $\rho$ bq Ddrhx

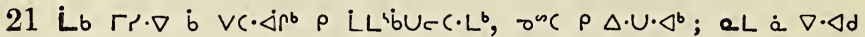

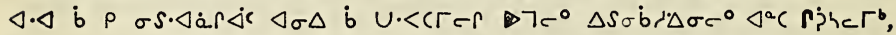

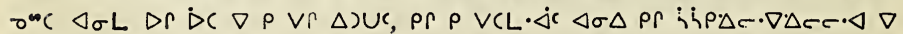
Lirdajer?

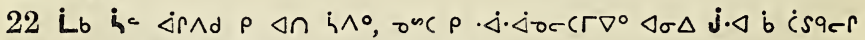

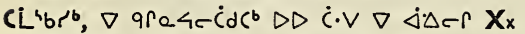

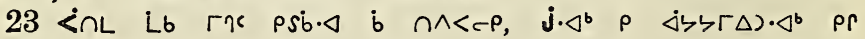

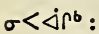

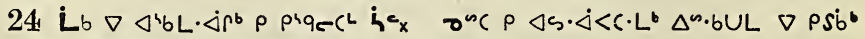

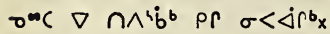

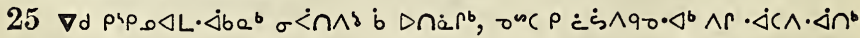
<s $\nabla$ ceclbc ${ }^{b} x$

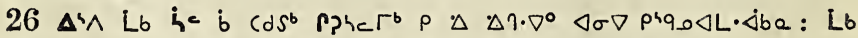

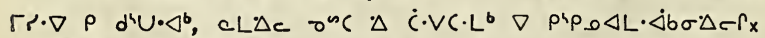

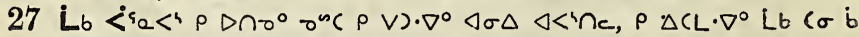
206 


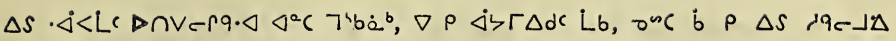

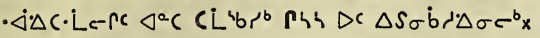

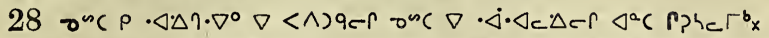

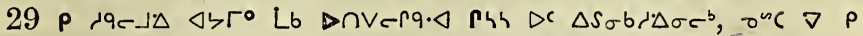

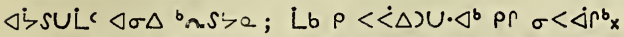

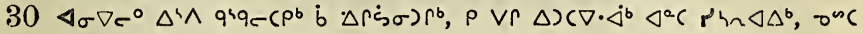
P $\triangle S \cap S \cdot \nabla \cdot \triangleleft^{b} \dot{C}^{s} h r^{b} \mathrm{x}$

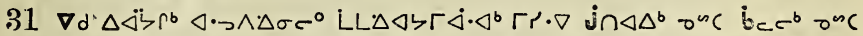

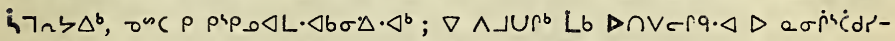

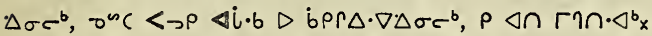

$32 \rho \Delta \rho^{a}$ Lb, $\left.7 \cdot b^{6} \wedge c^{s} \nabla<\dot{<} \Delta\right) \cup c \Gamma^{\circ} \nabla \Delta s, \rho \vee r \dot{\alpha} U^{\circ} \sigma^{n} \mathrm{C} \Delta \sigma \Delta$

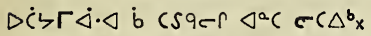

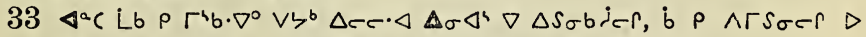

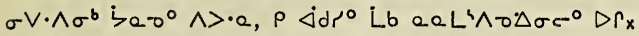

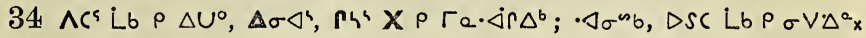
-c cb Ĺb $\rho \cdot \Delta \sigma^{-4 b}{ }^{\circ} x$

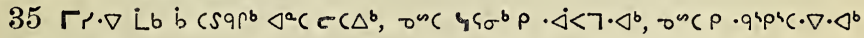
$\Delta \cap V e r q \cdot \triangleleft x$

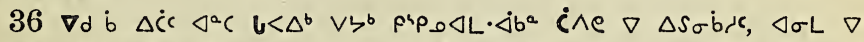

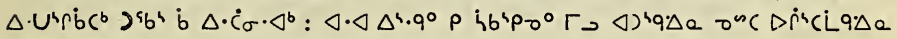
b $\rho) x^{b} x$

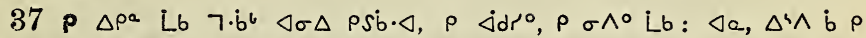

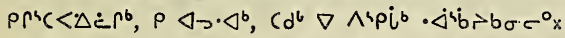

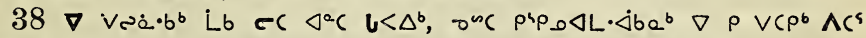

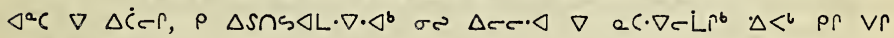
ec $c \cdot \dot{\Delta}<r d \rho^{b} x$

$39 \nabla d \wedge c^{s} \dot{b}<r d c, \rho \Delta \eta \cdot \nabla^{0} \dot{L} b x \quad \Delta^{s} \wedge \dot{b}\left(d S^{b}, \rho \vee r \wedge\right) b \nabla \cdot \Delta^{b} \nabla^{a} c$

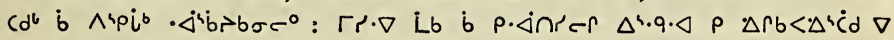

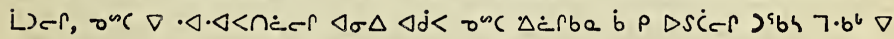
$\triangleleft \triangle 1 \Delta d r^{b} \times$

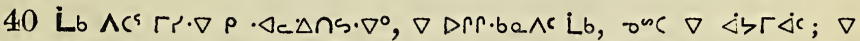

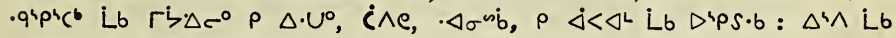
$\triangle \triangleleft<L C \wedge C \varsigma, P S L C \wedge^{\circ} x$

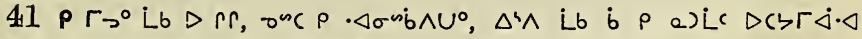

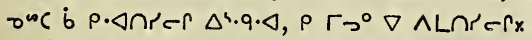

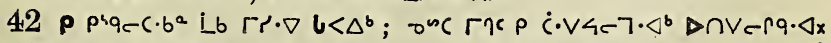

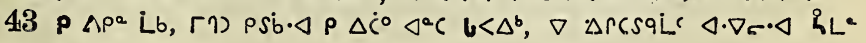
$D<4.9 p\lrcorner 97 x$

\section{Arpcraatoa 10.}

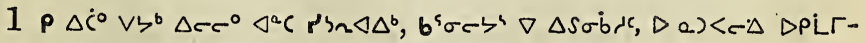

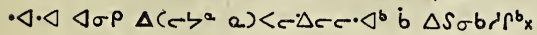

207 


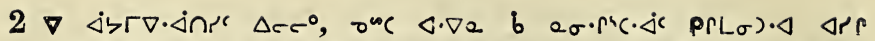

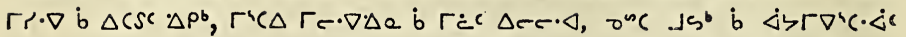
$P \Gamma(\sigma) \cdot \Delta x$

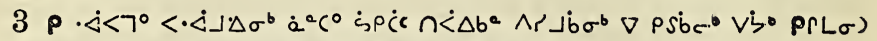

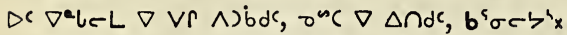

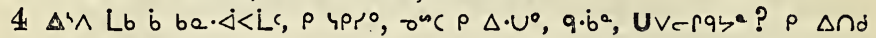

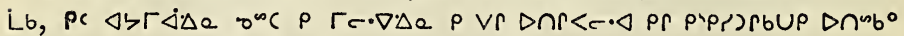
PrL $)_{x}$

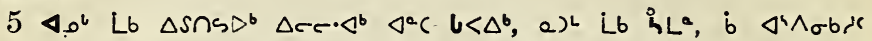
$\wedge C^{5}:$

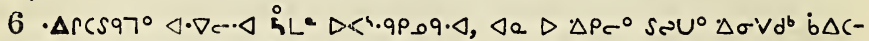
$\cdot b \sigma c^{b}: \Delta c \rho$ b $\left.\Delta C L^{b} q \cdot b^{a} q\right) C L<^{a} x$

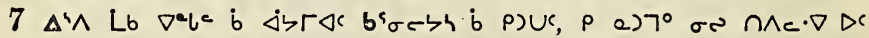

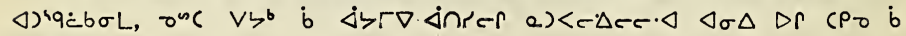
$\varangle$ ४ibdc;

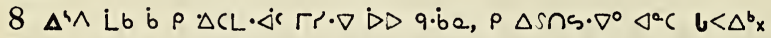

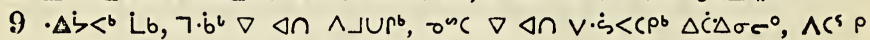

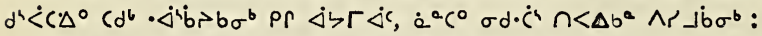

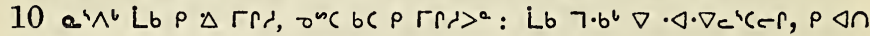
$\Delta S e \cdot \nabla^{\circ} x$

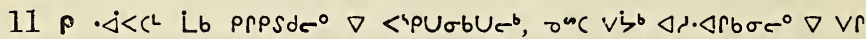

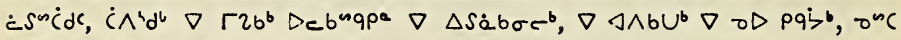
$\rho \dot{c} S \sigma b U \sigma^{\circ} \triangleleft^{4} \rho b$;

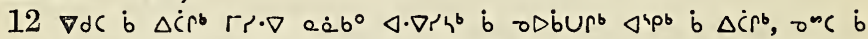

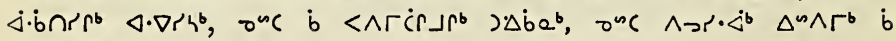
$<<\Gamma_{c} \Gamma^{b} x$

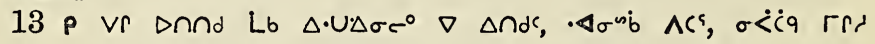
Lbx

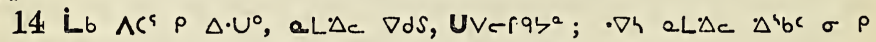
$r r^{a} a \cdot b^{a}<\cdot b^{a} c^{\circ}$ b $\Delta U-\dot{c} \cdot b^{b}, \tau^{\omega} c \wedge d \nabla b$ b $<\rightarrow b^{b} x$

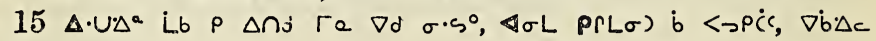
Lec $C^{a} \Delta S \sigma \dot{b} C_{x}$

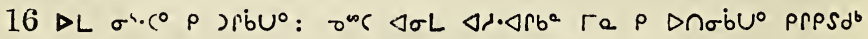
$\Delta S_{x}$

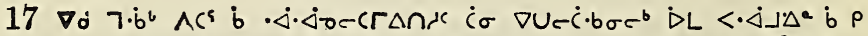

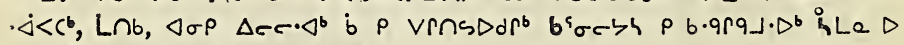
$\Delta P c^{b}, \rho \sigma<\Delta \cdot \Delta^{b}$ Lb $\Delta^{a} \cdot b U \Gamma^{b}$,

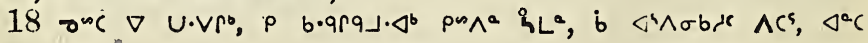
US $9 c \cdot 9 x$

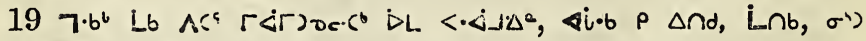
$\triangle c c \cdot \Delta^{b} p a \dot{a}^{2} \cdot b^{b} x$

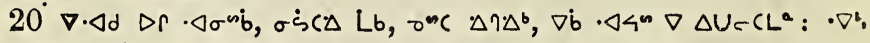
- $p \vee r \cap s \cdot \dot{4} \cdot \Delta^{b} x$ 


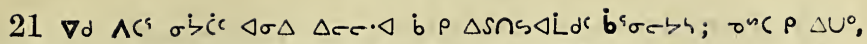
L $\cap b, \sigma c \nabla \cdot \triangleleft d \dot{b} a j a \cdot \nabla^{b}: 9 \cdot b^{a}$ Lb $\cdot \nabla r$ Vr $\Delta x U \zeta^{b} x$

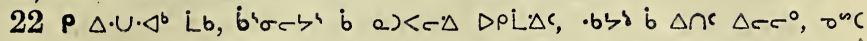

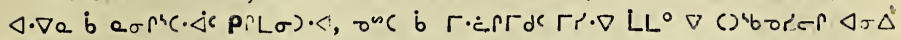

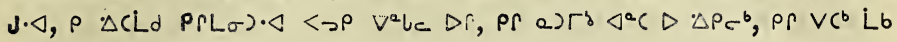
$\triangleleft \zeta \nabla_{e} P_{c} D r_{x}$

$23 \nabla d$ b $\wedge)$ a a) Lc, onc $\rho \Delta r\left(s q \Gamma d x \quad \cdot \Delta \dot{\Delta<b}\right.$ Lb $\wedge c^{s} \rho \Delta \eta \cdot \nabla^{\circ}$, onc

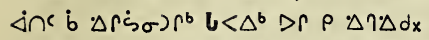

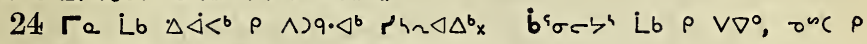

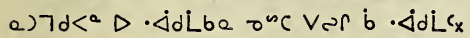

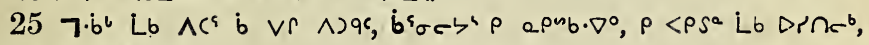

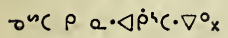

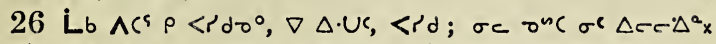

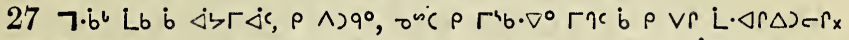

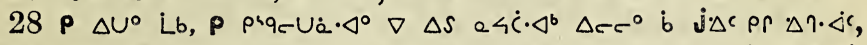

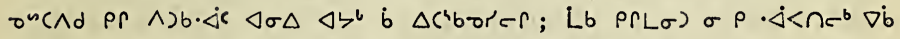

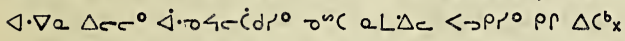

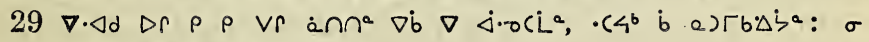

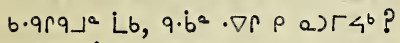

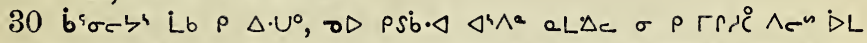

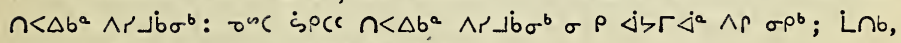
Lb $\Delta c c^{\circ} \sigma \rho \Delta \cap^{n} b \Delta \dot{b}<\Delta^{4} c^{b} \nabla \cdot \dot{\Delta} \wedge D c$,

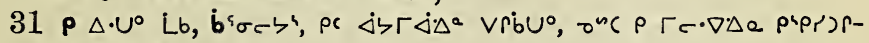
bU. $\triangleleft \nabla \cdot \Delta<\left\langle C^{b} \text { PrL } \sigma\right)_{x}$

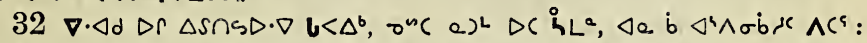

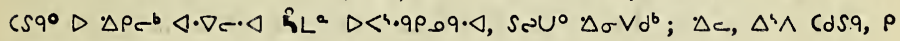
$\dot{b} \dot{i} \zeta \Gamma^{b} \mathrm{x}$

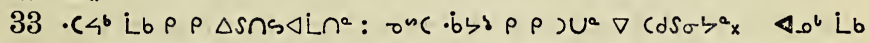

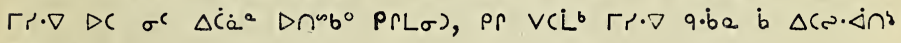
PrLG)x

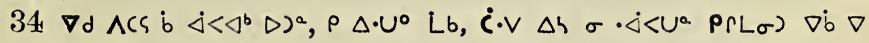

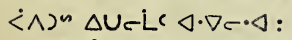

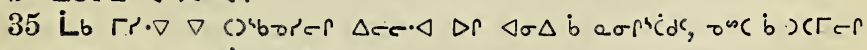
. bליc

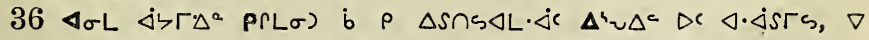

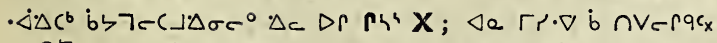

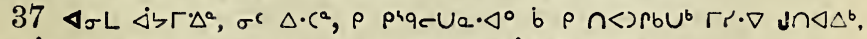

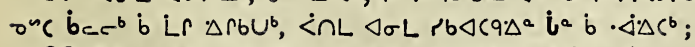

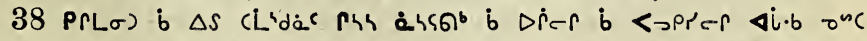

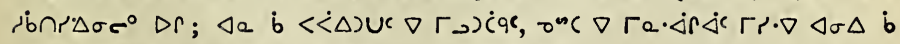
eobr $\Delta d e r L(L \sigma) \cdot \Delta: \cdot \nabla h \rho \Gamma L \sigma) \cdot \triangleleft \rho \Delta \eta \Delta d x$

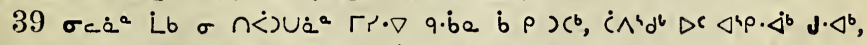

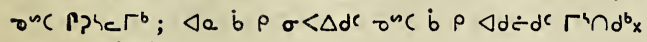




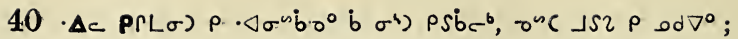

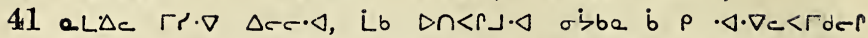

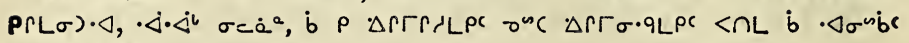
$D \sigma \wedge \cdot \Delta D r_{X}$

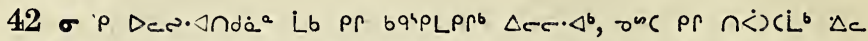

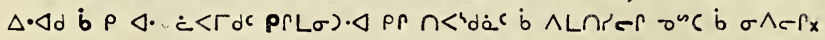

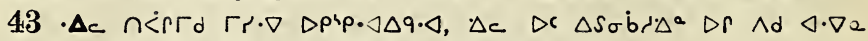

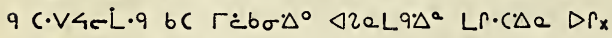

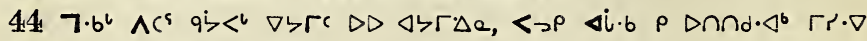
$\triangle \sigma \rho$ bे $V C \rho^{\circ} \Delta \backslash \Gamma \Delta \sigma c^{\circ} \mathrm{x}$

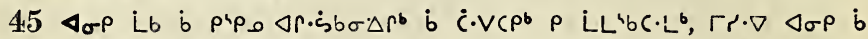

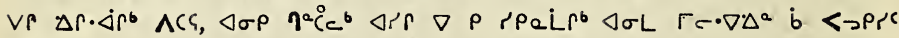
$\Delta i b_{x}$

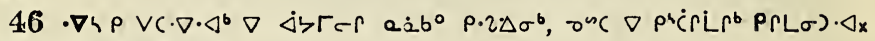

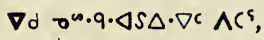

$47 \varangle \cdot \nabla a \Delta r c^{\circ}$ a bc $\rho \Delta r \Delta \cdot \nabla^{0} \sigma \wedge c^{\circ}, D_{d} \nabla \dot{b}$ pr $\rho$ rb $\Delta c \cdot \Delta b \sigma \angle r^{b}$, b

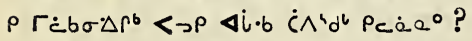

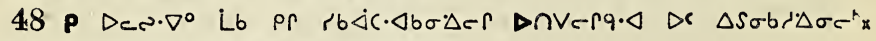

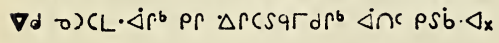

\section{Nipcre $\triangle b^{a} 11$.}

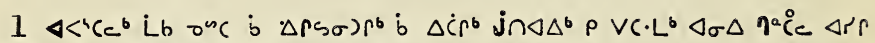
$\nabla$ P. Dne.Ler PRL $\sigma) \cdot \Delta D\left(\Delta \zeta \Gamma \Delta \sigma e^{\circ} x\right.$

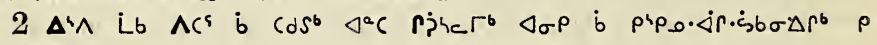
¿งบา. $\triangleleft^{6}$,

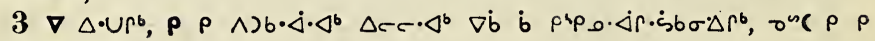

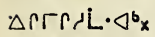

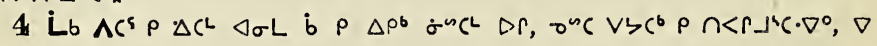
$\Delta \cdot U e$,

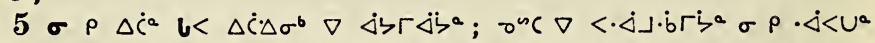

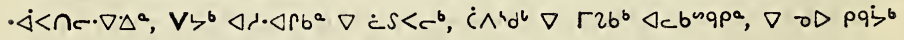

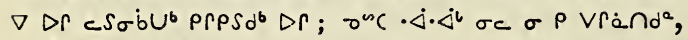

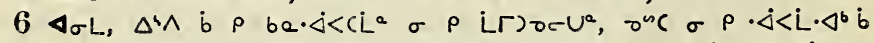

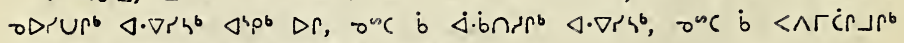

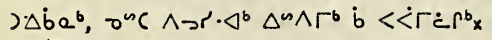

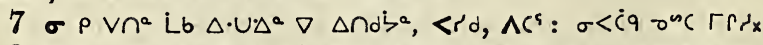

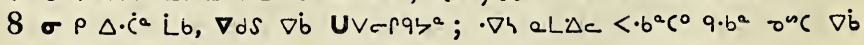
$\dot{b}<\rightarrow b^{b} \Delta \Delta b\left(p \wedge r<c^{0} \sigma\right) \sigma^{b} x$

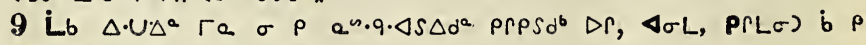
$\left\langle\neg \rho \dot{C} c, \nabla \dot{b} \Delta c \dot{L} \dot{c} c^{a} \Delta S \sigma \dot{b} C_{x}\right.$

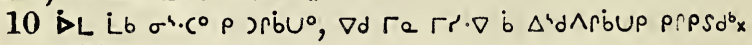
210 


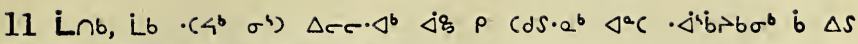
$\Delta \ddot{C}^{a}, \nabla \Delta S \cap S \Delta L b \Delta \zeta^{a} r^{2} \cap \Delta \Delta^{b} \Delta r_{x}$

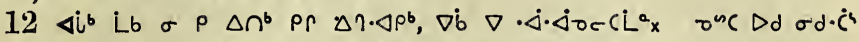

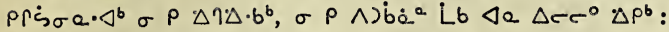

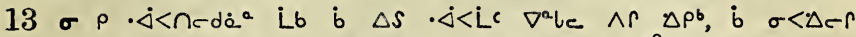

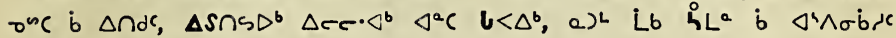
$\wedge c^{5}$;

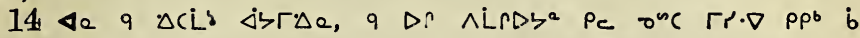
$\triangle C S \operatorname{Ca}^{\mathrm{a} x}$

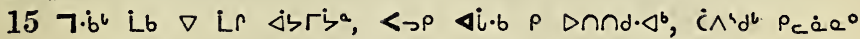
$D^{4} b^{4} x$

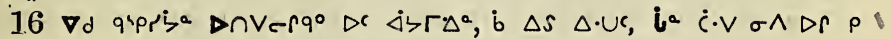

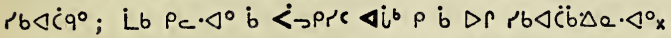

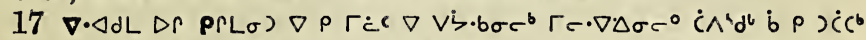

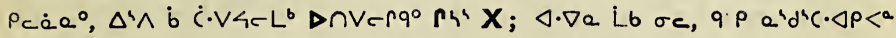
PSL $\sigma)$ ?

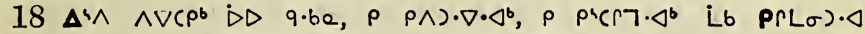

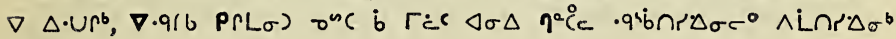
$\Delta S_{x}$

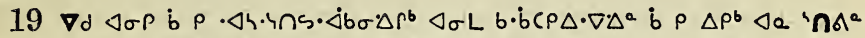

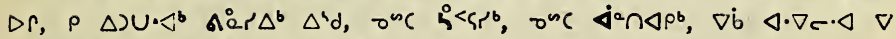

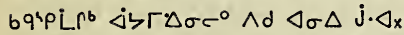

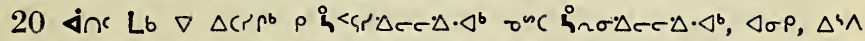

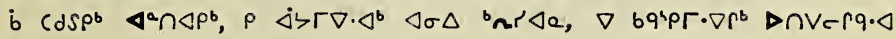
Pitix

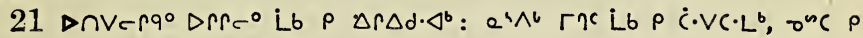
$. q^{\prime} b \cap r^{\prime} c \cdot \nabla \cdot \Delta^{b} \triangleright \cap \vee-r q \cdot \Delta x$

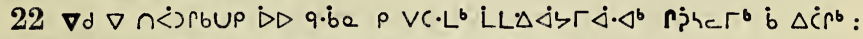

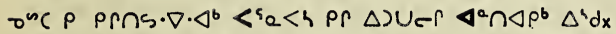

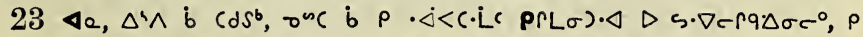

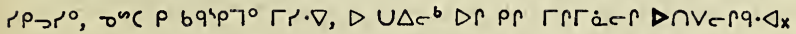

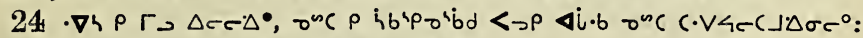

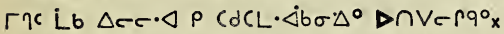

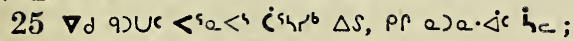

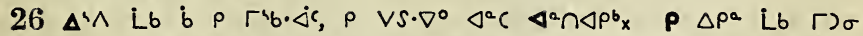

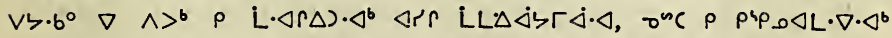

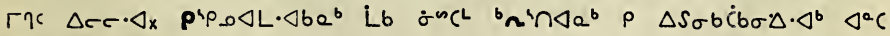
$\Delta^{\circ} \cap \Delta \rho^{b} x$

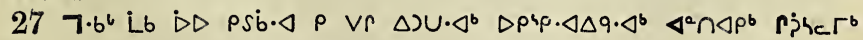
Dix

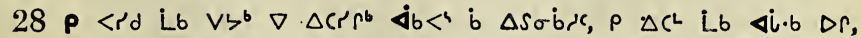

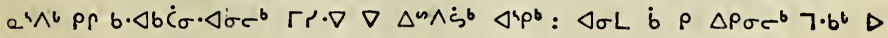

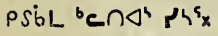

211 


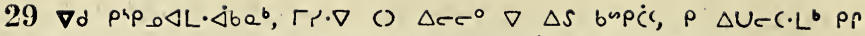

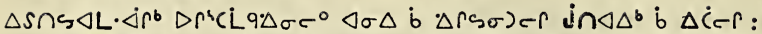

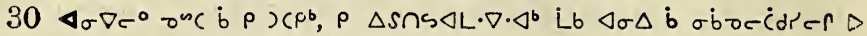

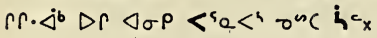

\section{Ápcre $\triangle b^{\circ} 12$.}

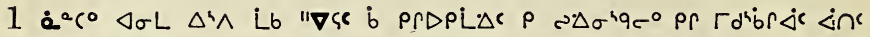
$\triangleleft \sigma \Delta L L \Delta \Delta\rangle \zeta \dot{\Delta} \cdot \Delta x$

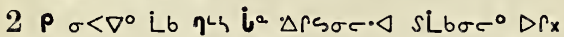

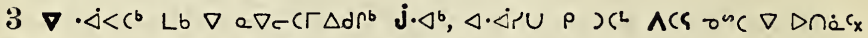
$\nabla d^{h} \wedge \nabla \cdot \triangleleft \cdot b \sigma \Delta \nabla \dot{b} \nabla D \wedge r<\triangleleft c d a^{\circ} \rho S \dot{b} \cdot \Delta x$

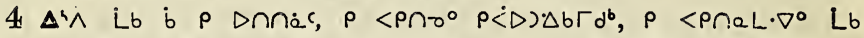

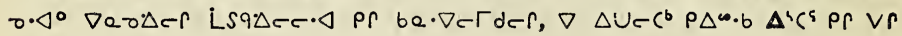
$\cdot \Delta c \Delta() \cdot \triangleleft c \triangleleft \sigma \Delta \Delta c c \cdot \triangleleft x$

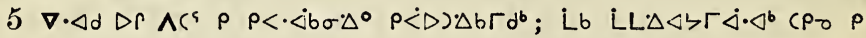
Q.ं) $7 \cdot \triangleleft^{b} P(L \sigma) \cdot \triangleleft \Delta c D r_{x}$

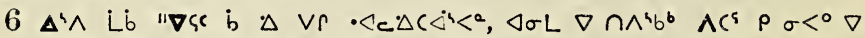

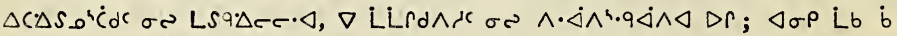
be. $\nabla e\left(\rho^{b} \cdot \Delta c \Delta \cap \Gamma^{b} \Delta^{n} \cdot b U r^{b} \rho\right.$ be. $\nabla e\left(\cdot L^{b} \rho \dot{<} D\right) \Delta b \Gamma d c^{\circ} x$

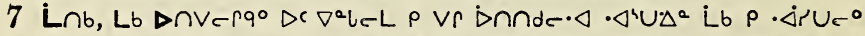

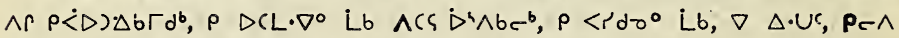

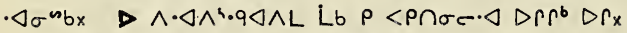

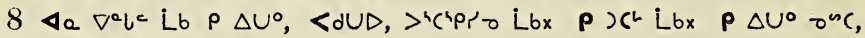
>'CdV, or o Da Lbx

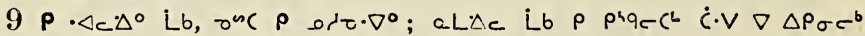
$\Delta \sigma \nabla \sigma^{0}$ b $\left.\rho\right)\left(\Gamma e r \Delta \sigma \Delta \nabla^{2} l_{c}\right.$, Lb $\sigma c \Delta S \sigma^{2} \rho \Delta U-C^{L} x$

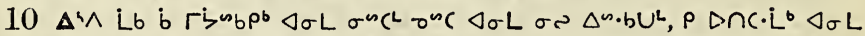

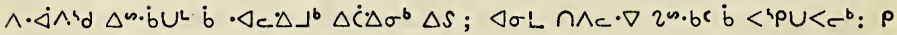

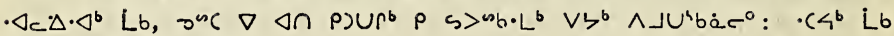
$\triangleleft \sigma \Delta \nabla^{2} l_{e} \rho$ abndx

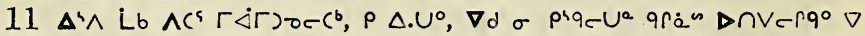

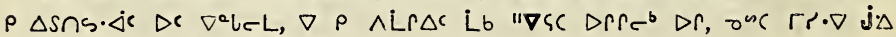
$\Delta c c \cdot \Delta \nabla \Delta S$ a.c. $\nabla e c r e r \Delta r_{x}$

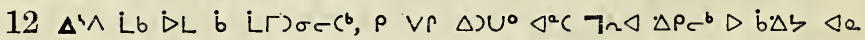

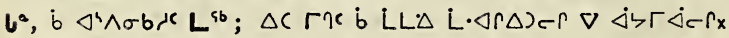

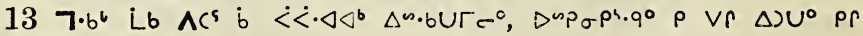
e) $\left.C^{b}\right) \nabla \Delta S \sigma b d^{k} x$

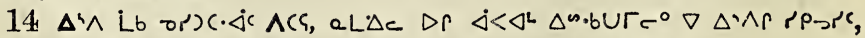

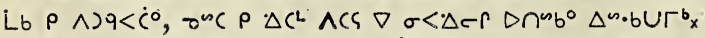

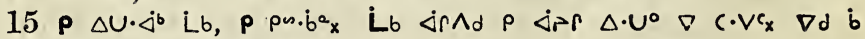
$\Delta \cdot U^{b}, \nabla \cdot \Delta \cdot b \sigma \Delta D^{\circ} \nabla^{\circ} L_{C} L x$ 


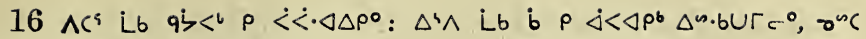

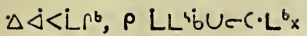

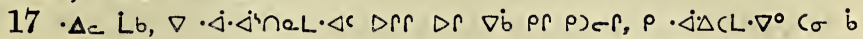

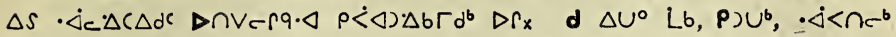

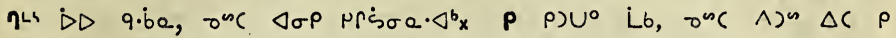
$\triangle U^{\circ} \times$

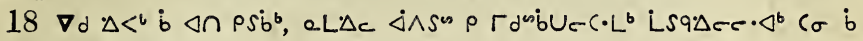
$\Delta \cap e^{-} \triangleleft \sigma \Delta \wedge C \varsigma_{x}$

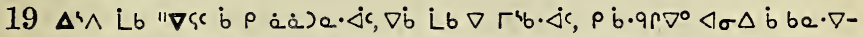

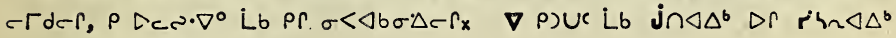
$\rho \Delta) U^{\circ}, \nabla d c$ Lb b $C S q c_{x}$

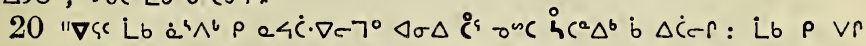

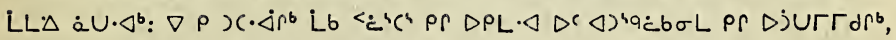

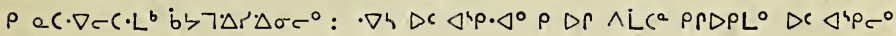
$\Delta r_{x}$

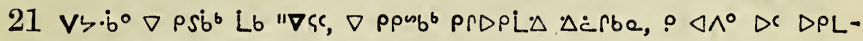
$\left.\cdot \triangleleft \wedge \Delta \sigma^{b}, \rho \cdot \dot{\triangleleft}\right\rangle \Gamma \nabla^{\circ} \mathrm{L} b_{x}$

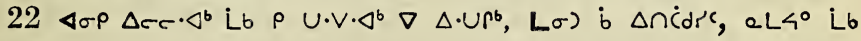
$\Delta e c^{\circ} \mathrm{x}$

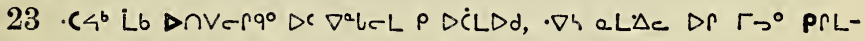

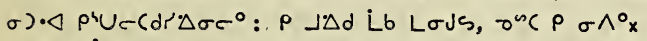

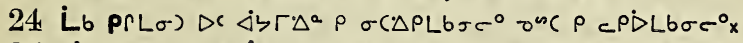

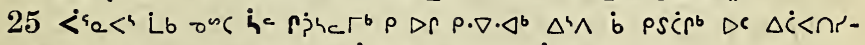

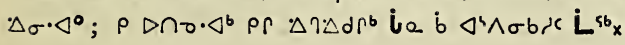

\section{A}

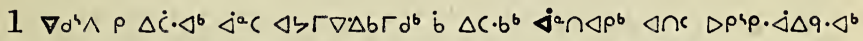

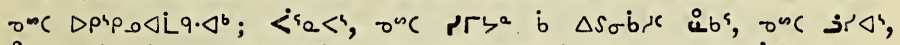

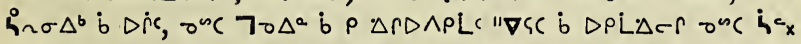

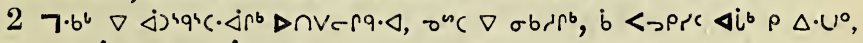

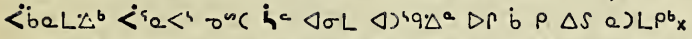

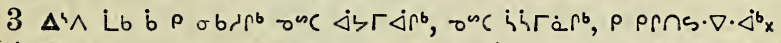

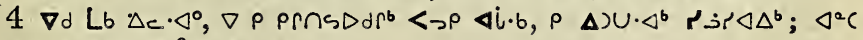

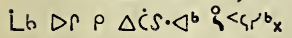

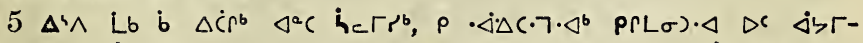

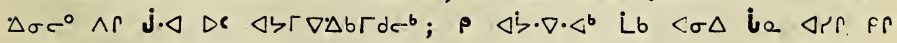

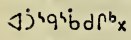

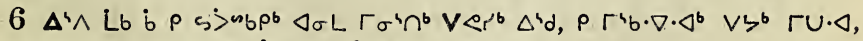

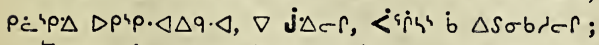

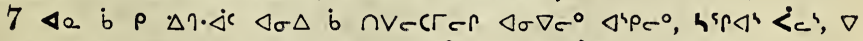

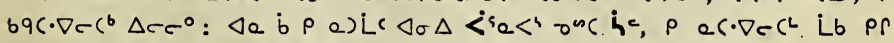

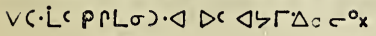




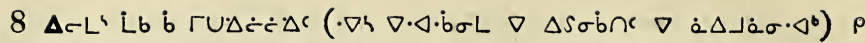

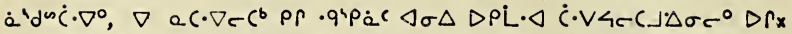

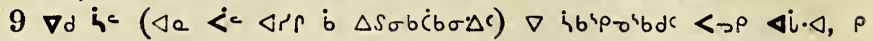
be. $\Delta<7^{\circ}$,

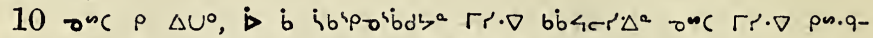

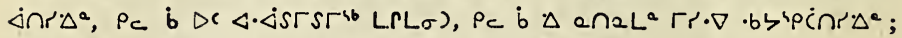

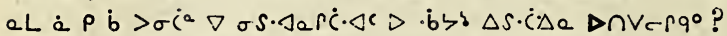

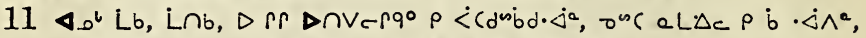

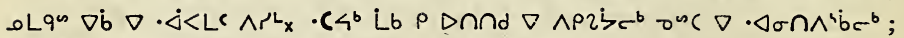

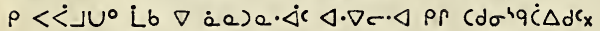

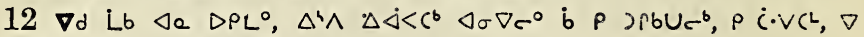
LL'bC.LC $D$ PhP.o $\Delta L q \Delta \sigma \sigma^{\circ} \Delta \cap V c r q \cdot \Delta x$

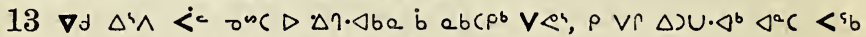
$<$ LAc $\Delta \Delta^{b}: l^{a}$ Lb $\nabla$ abce $p$ p. $\nabla^{\circ}$ píhe $\Gamma^{b} x$

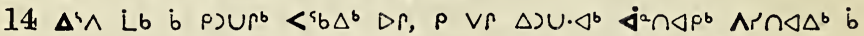

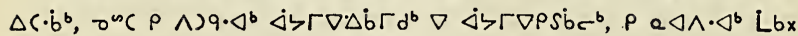

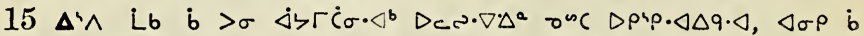

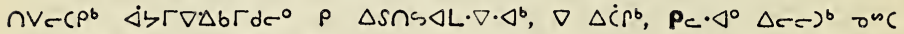

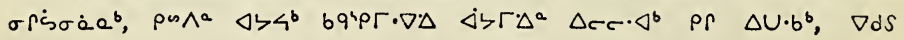
$\Delta \cdot u^{b} \times$

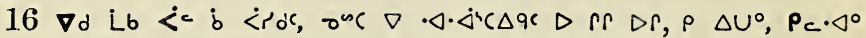

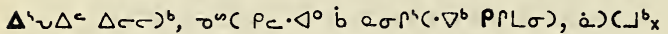

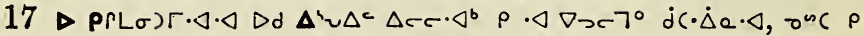

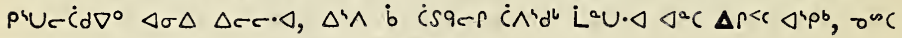

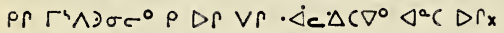

18 a $^{a} C^{\circ}$ Lb or $C^{\circ} \wedge>\cdot a$ a $S V C C \cdot 7^{\circ}$ DC $\Delta r \beta q \Delta \sigma c \cdot \Delta \Delta^{a} C<\cdot b C^{4}-$ $b \Gamma b^{b} \times$

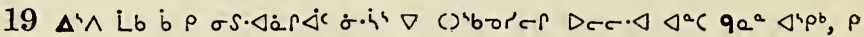
LneL. $\nabla^{\circ} D^{C} \Delta^{4} P c^{\circ} \nabla L \Delta b \dot{Q} \cap d \dot{b} \sigma \cdot \Delta^{b} D r_{x}$

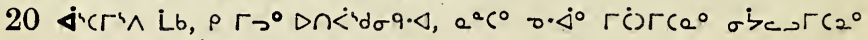

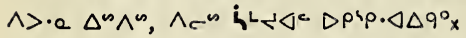

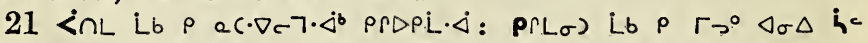

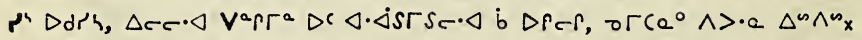

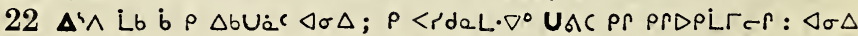

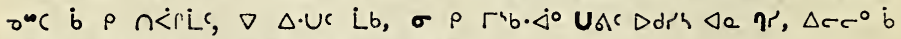

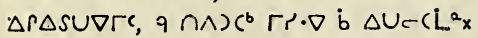

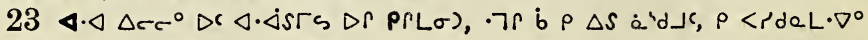
$\Delta \sigma \Delta \Delta^{4} \cup \Delta^{c} D \wedge L \Gamma \Delta \cdot \nabla L, P 4$;

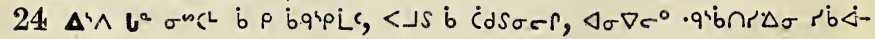

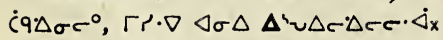

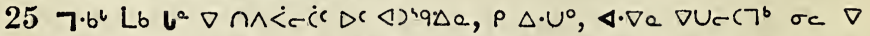

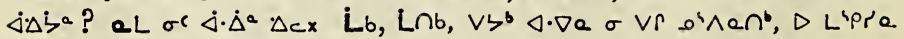

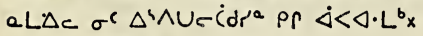

214 


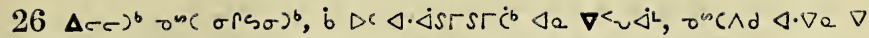

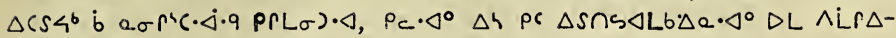
- $\nabla \Delta \sigma \quad \dot{\Delta} \iota \Gamma \Delta^{a} x$

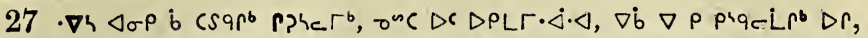

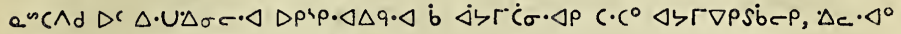
$P \cap \Lambda) C \cdot L^{b} D D \nabla P \sigma S \cdot \Delta \dot{Q}^{\prime} \Gamma L \Gamma^{b}{ }_{x}$

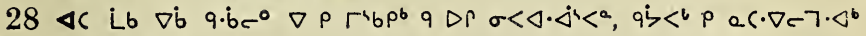

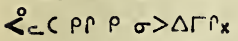

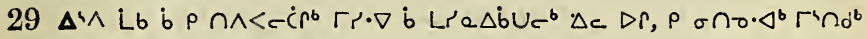

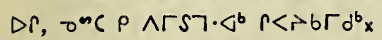

30 PPL $L)$ Lb $P \cdot \triangleleft \sigma^{\infty} b-0^{\circ} \triangleright \sigma \wedge \cdot \Delta \Delta r:$

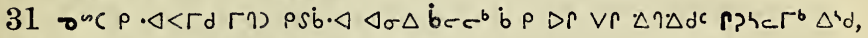

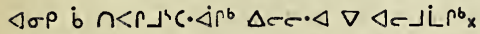

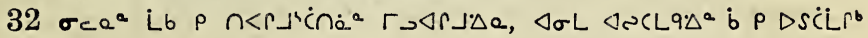
$\Delta \dot{C} \cdot \Delta \dot{L} \cdot \Delta^{b}$,

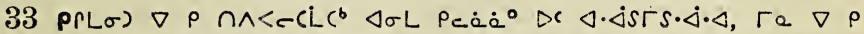

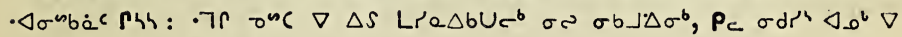
sSib $\rho$ P $\sigma C \Delta P \Delta \Omega^{2} x$

$34 \quad \varangle \sigma L L b$ Dr $\nabla \rho \cdot \Delta \sigma^{m b a c} D_{\sigma} \wedge \cdot \triangleleft \Delta r, \nabla d \quad \nabla \dot{b}$ ra fr $\rho \cdot \nabla \dot{a} c^{b}$

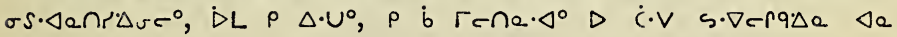
USA $x_{x}$

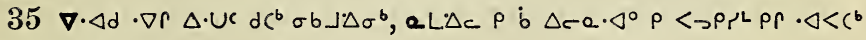
$\sigma s \cdot \Delta \dot{Q} \cap \cdot \Delta \sigma \sigma^{\circ} x$

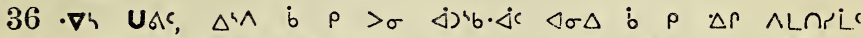

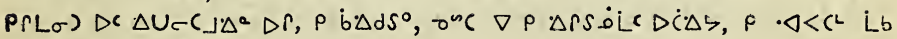
$\sigma s \cdot \Delta \dot{a} \cap r \Delta \sigma c^{\circ}$ :

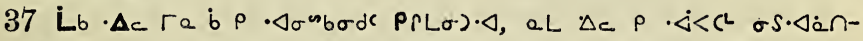
$\Delta \Delta \sigma c^{\circ} \mathrm{x}$

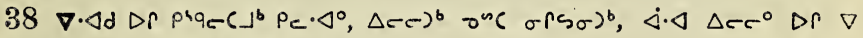
$\triangle C L b \Delta 4^{b} \nabla \cdot \nabla V e r b U P$ Lr.Cं $\Delta a x$

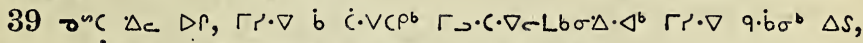

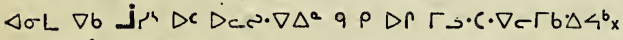

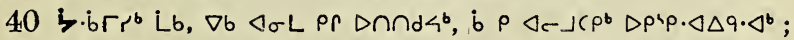

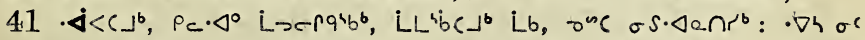

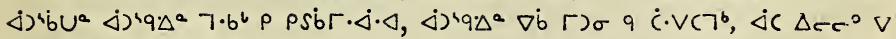
- $\triangle \Delta C L C^{\circ} b_{x}$

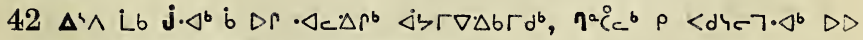

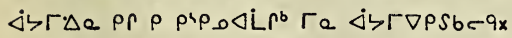

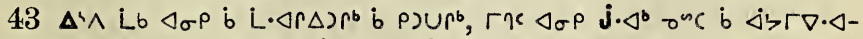

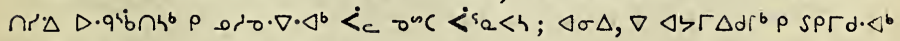

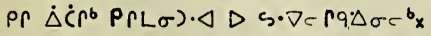

$44 \Gamma_{a}$ Lb $\nabla$ ¿לr $\nabla \rho S b c^{b}$ qbe $r p \cdot \nabla$ b $\Delta C p^{b} \Delta \dot{C} \Delta \sigma^{b} \rho$ Vr LLA

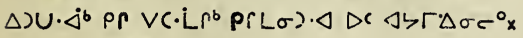

215 


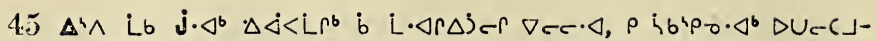

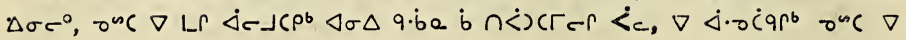
$\dot{<} \dot{c}\lrcorner r^{b} \times$

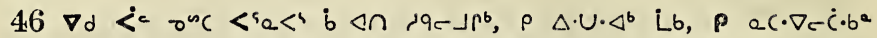

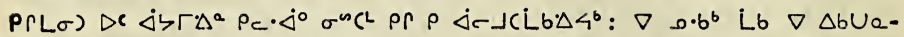

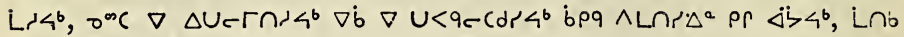
$\sigma \quad \dot{a} C \dot{a} \dot{a}^{b} \eta^{a} c_{c}^{b} c^{b}$

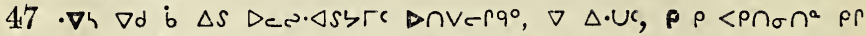

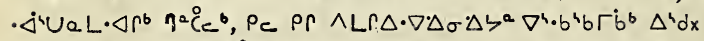

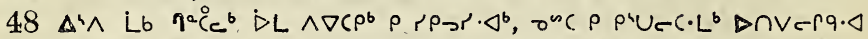

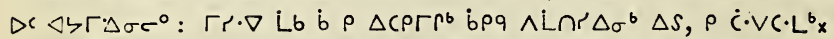

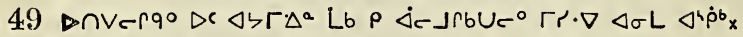

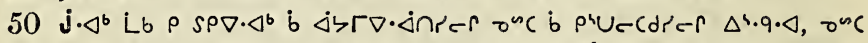

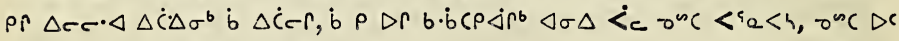

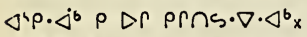

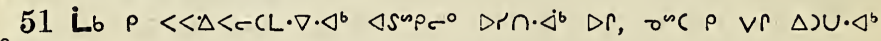
\&ंd $\sigma \triangleleft \Gamma^{\circ} \times$

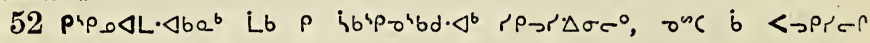
$\triangleleft i \cdot \triangleleft x$

\section{A per a $\triangle b^{\circ} 14$.}

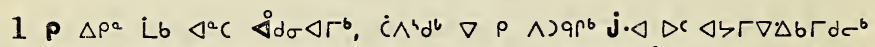

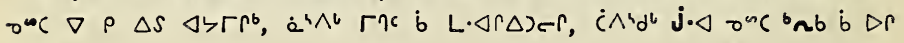
$\dot{c} \cdot \mathrm{VCr} C \mathrm{Crx}_{\mathrm{x}}$

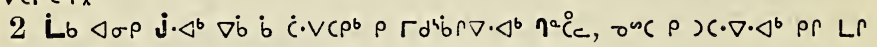

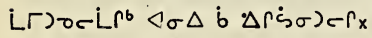

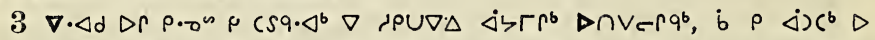

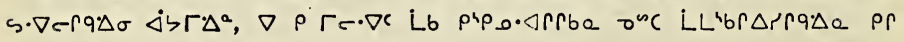

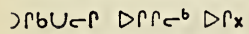

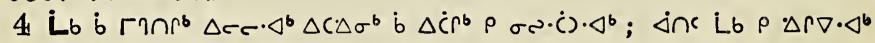

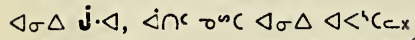

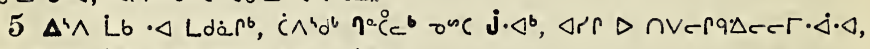

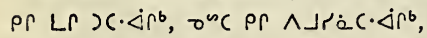

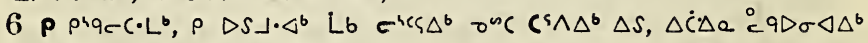

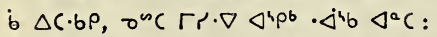

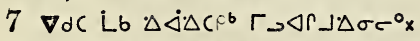

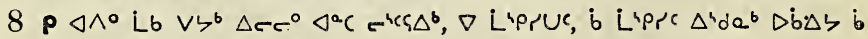
$\sigma \dot{c} \Delta \rho \Delta d c, \nabla \dot{b} \Delta$ bc b $\rho \wedge \wedge u c$ :

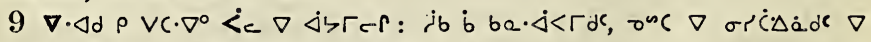

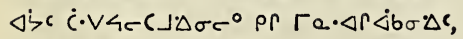

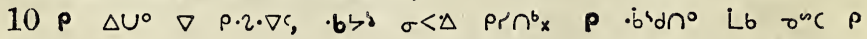
$\wedge\lrcorner \cup^{\circ} \mathrm{x}$

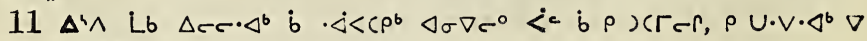
216 


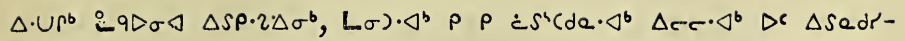
$\Delta \sigma \cdot \dot{\Delta b x}$

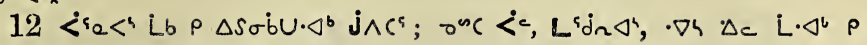
oc $\dot{\Delta} \zeta \Gamma^{\circ} x$

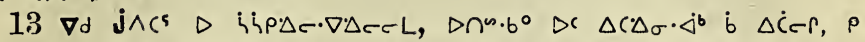

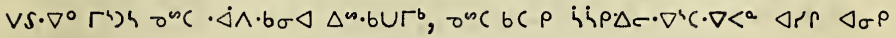
$\Delta c r \cdot \Delta \Delta^{b} x$

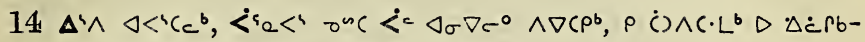

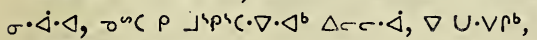

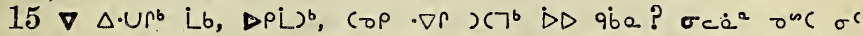

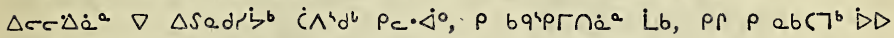

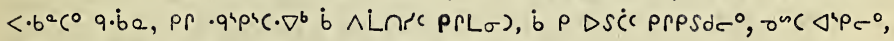

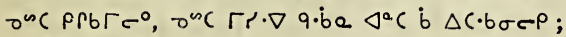

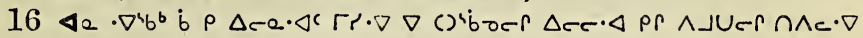
$\Delta C \Delta S \cdot \dot{C} \Delta \sigma \sigma^{b} \mathrm{x}$

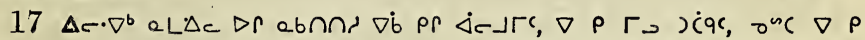

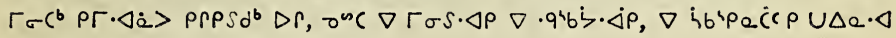

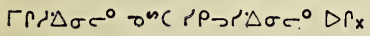

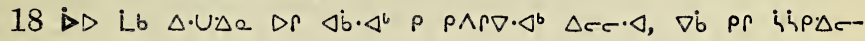
$-\nabla \dot{C} d r^{b} b_{x}$

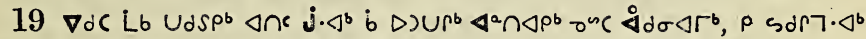

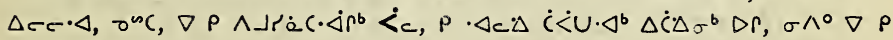
$\Delta U-C^{b} \Gamma^{b} x$

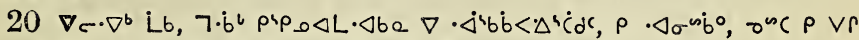

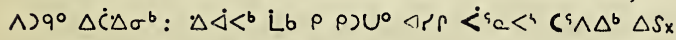

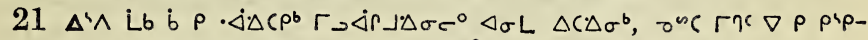

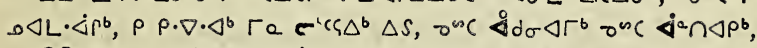

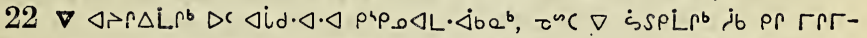

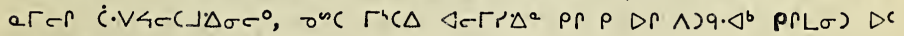
$D P L \Delta \Delta \sigma^{b} x$

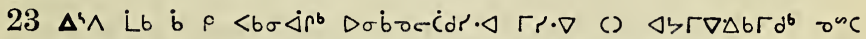

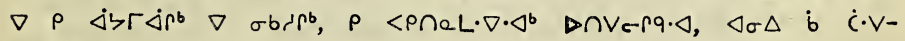
GeLrerx

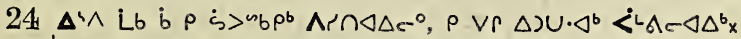

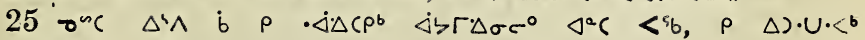
४ं $\cup\left\ulcorner\triangleleft \Delta^{b}\right.$;

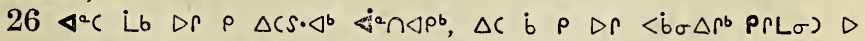

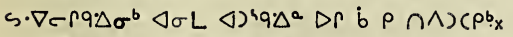

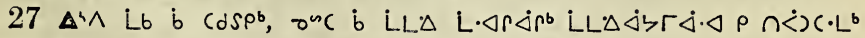

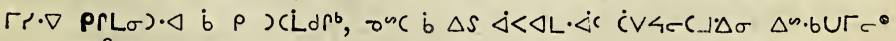
$\triangleleft \sigma \Delta \boldsymbol{\eta}^{\circ} \tilde{C}_{\mathrm{c}} \mathrm{cx}$

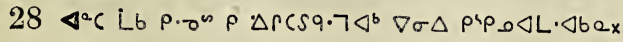




\section{A'pCra $\triangle b^{\circ} 15$.}

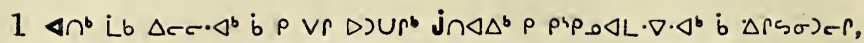

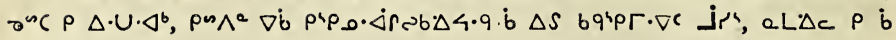
$\rho \wedge \dot{L} \nabla \dot{Q} \cdot \dot{\Delta} \circ_{x}$

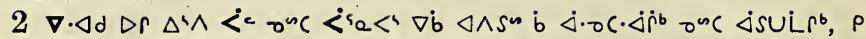

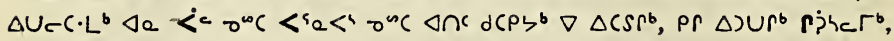

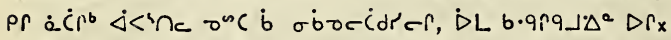

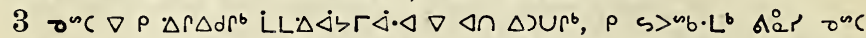

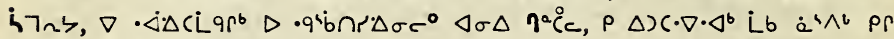
$r \cdot \dot{c}(r e r \Gamma r \cdot \nabla$ b $\Delta r s a) e r x$

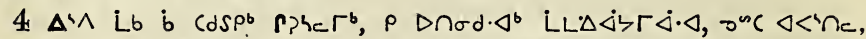

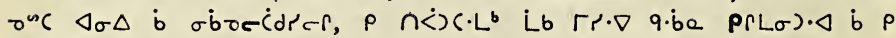
) $c \dot{L} d r^{b} \times$

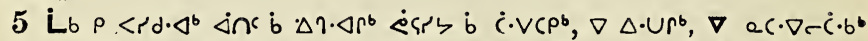

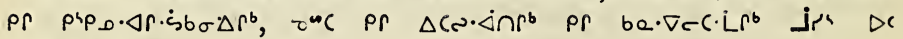
$D_{C} \cdot \nabla \cdot \nabla \sigma \sigma^{\circ} x$

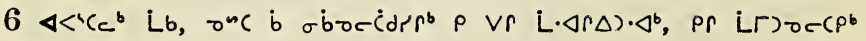
$\Delta\urcorner c^{\circ} \times$

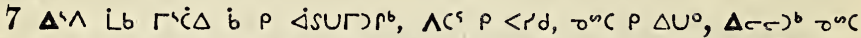

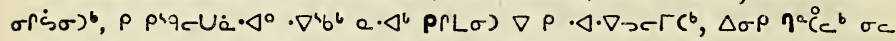

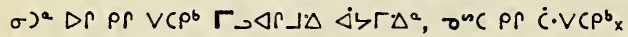

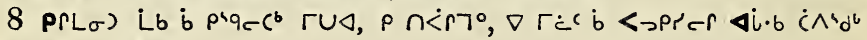
icia $\left.a^{\circ} \dot{b} \rho\right) \dot{i} c^{b}$ :

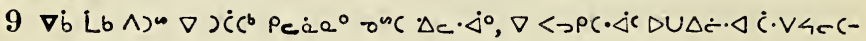
j. $\Delta \sigma^{\circ} \Delta r_{x}$

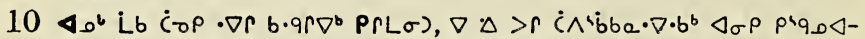
$L \cdot \triangleleft b a b \Delta \sigma L D r, \nabla \dot{b} d \dot{C} \Delta a \cdot \Delta^{b} \sigma^{n C} P_{c} \dot{a}_{a}{ }^{\circ} \dot{b} \rho \wedge \Gamma \Delta \dot{C} \zeta b$ ?

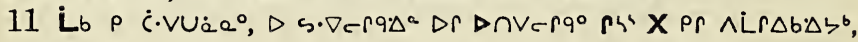
$\dot{c} \wedge^{\wedge} d^{b} \Delta \Delta_{c} \cdot \triangleleft^{\circ}$

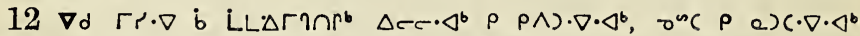

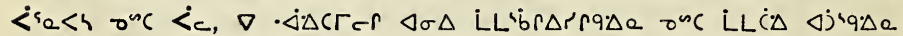

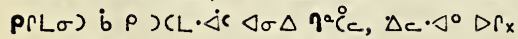

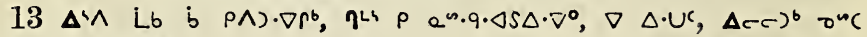
$\left.\sigma \dot{s} \sigma)^{b}, a\right)\left(\cdot \dot{\Delta}^{b}\right.$ :

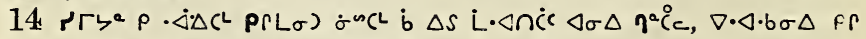

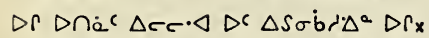

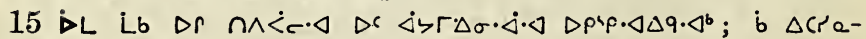
$\Delta b U^{b}$,

$16<\cap L L L$

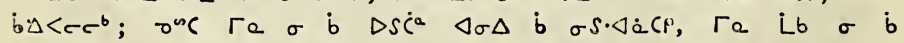
rLPC :

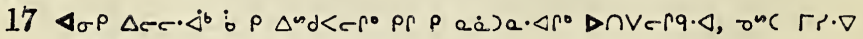
218 


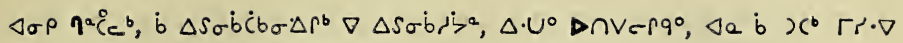
¿D $q \cdot \dot{b}^{2} \times$

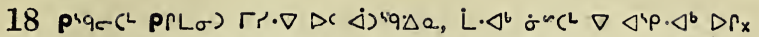

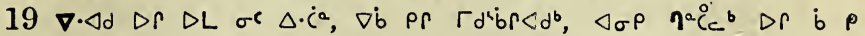

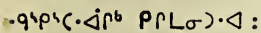

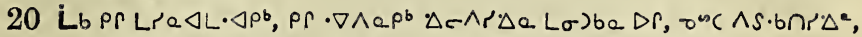

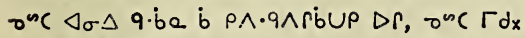

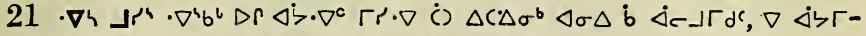
$C \sigma \cdot \Delta \sigma \sigma^{b} c \cdot c^{0} \nabla \Delta b \Gamma \nabla P S b c^{b} \wedge \Gamma \Delta ל \Gamma \nabla \Delta b \Gamma \cdot b x$

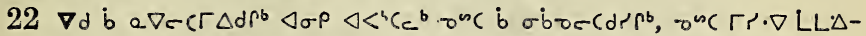

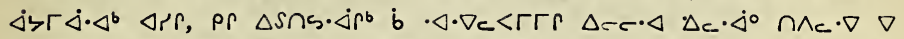

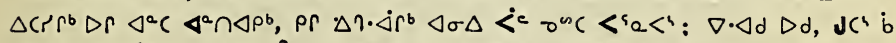

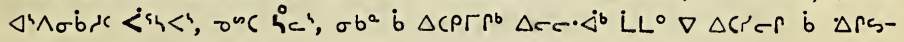
$\sigma) \Gamma$;

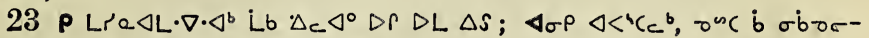

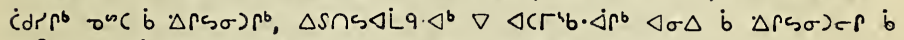

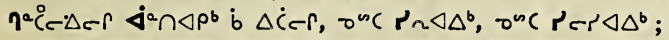

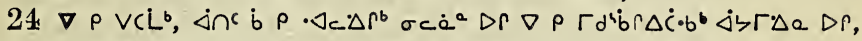

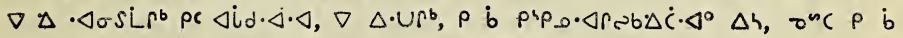

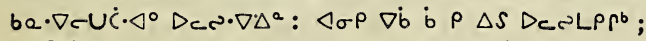

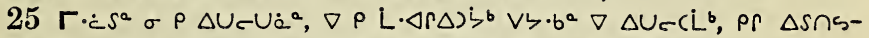

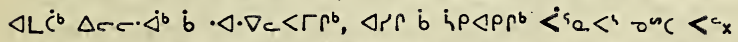

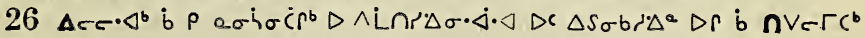
Ps' $X \times$

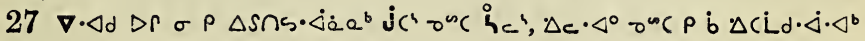
DD $9 \cdot \dot{b} \times x$

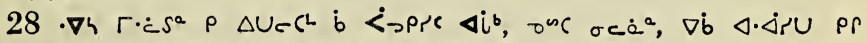

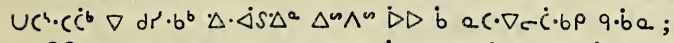

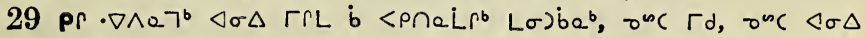

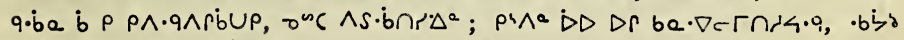
$\rho$ b $) U \dot{Q} \cdot \Delta^{\circ} x \quad \cdot \dot{\Delta} \zeta \zeta x$

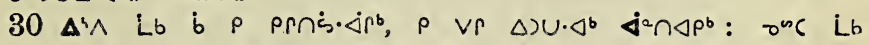

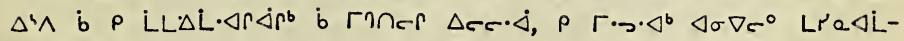
$\nu \nabla \sigma c^{\circ}:$

$31 \varangle \sigma \nabla \sigma^{\circ} \Delta^{h \wedge} \dot{b} \rho \Delta \zeta \Gamma_{C} \Gamma^{b}, \rho \Gamma c \cdot \dot{C} \cdot L^{b} \cdot \nabla h \rho \Gamma \cdot \Delta U \nabla \Delta d \cdot \Delta b x$

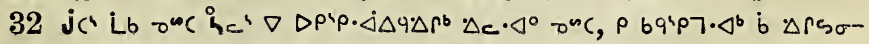

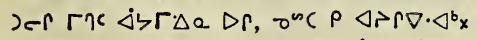

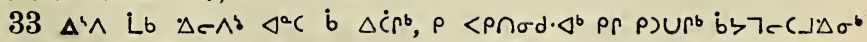

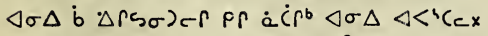

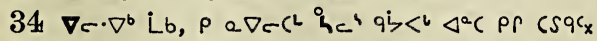

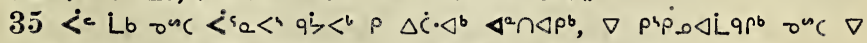

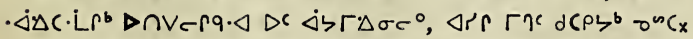

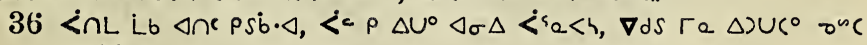
219 


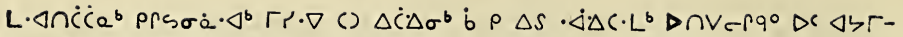

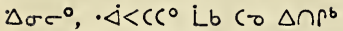

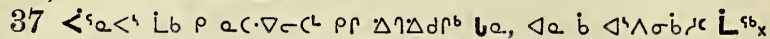

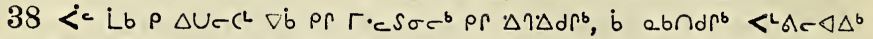

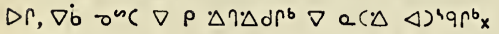

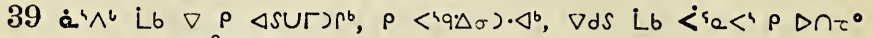

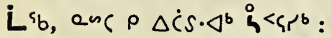

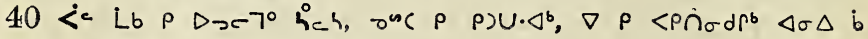

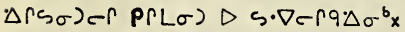

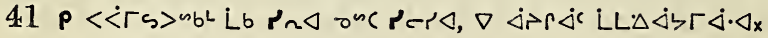

A'pçе $\triangle b^{2} 16$.

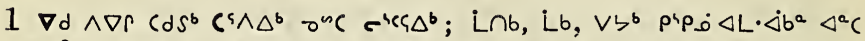

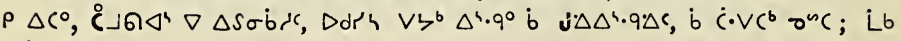
$\nabla \dot{C} \Delta \rightarrow \dot{b} \mathrm{~b} \sim \rho \Delta C r$;

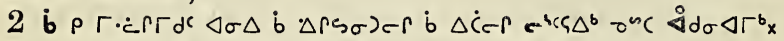

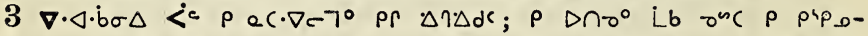

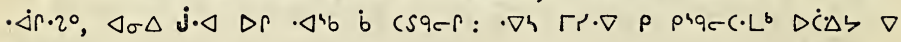

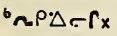

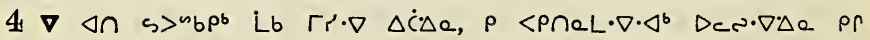

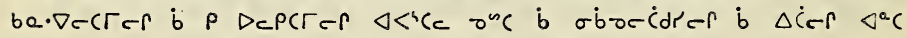
brher $r^{b} x$

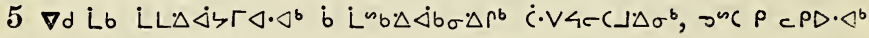

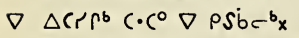

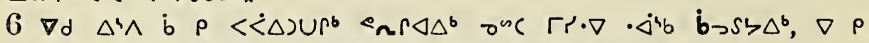

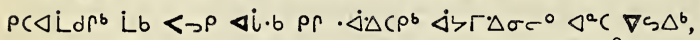

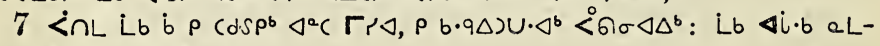

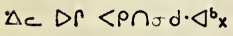

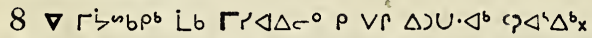

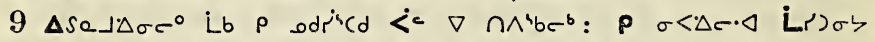

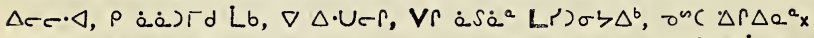

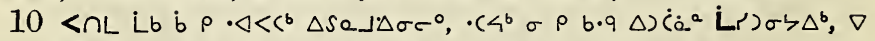

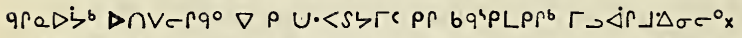

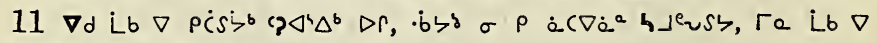
$\varangle \cap \rho^{2} b^{b} \Delta^{a} C \sigma \Delta>c^{4} \Delta^{b}$;

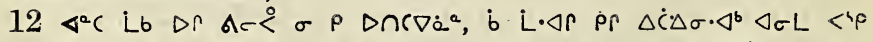

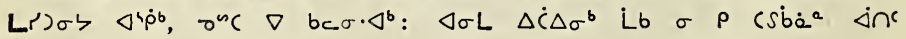
PSi. $\triangleleft x$

$13 \nabla \dot{\triangleleft} \zeta \Gamma \nabla P S b^{b}$ Lb $\sigma \quad \rho \cdot \Delta C \Delta \dot{a}^{a} \Delta \dot{C} \Delta \sigma^{b} \Delta \Gamma \dot{c}=r \wedge^{b}, \Delta C$ b $\Delta S \quad \sigma c$

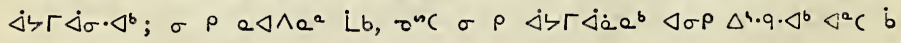
vr $\Delta u r^{b} x_{x}$ 


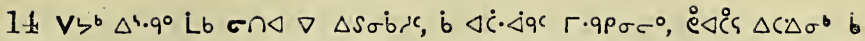

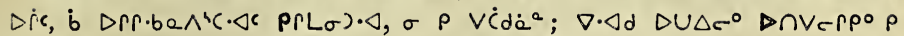

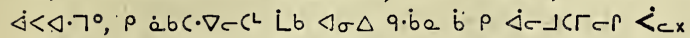

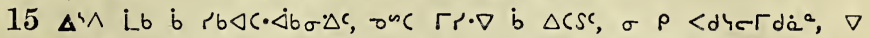

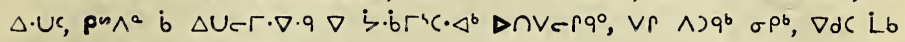

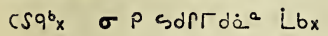

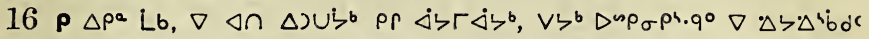

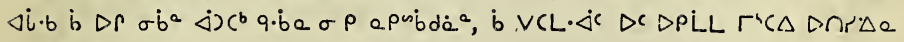
$\nabla \sigma \dot{b} \sigma \Delta C \dot{L} q$ :

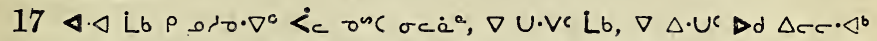

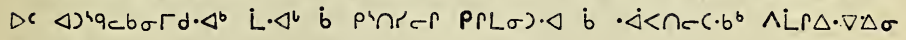
7 'be $x$

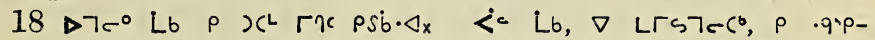

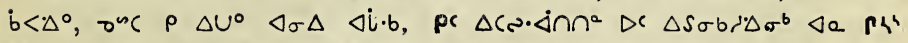
$X$, $p r \quad V r \quad \Delta c \Delta \zeta^{a} \Delta b^{\circ} \Delta^{b} \quad \Delta r_{x} \quad P \quad V r \quad \Delta c \Delta^{\circ}$ Lb $\quad \Delta \sigma L \quad n<\Delta b^{a}$ $\Lambda r\lrcorner \dot{b} \sigma^{b} x$

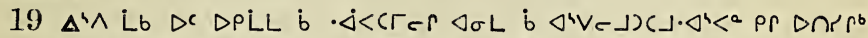

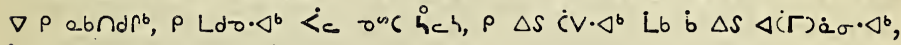

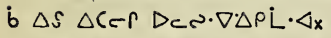

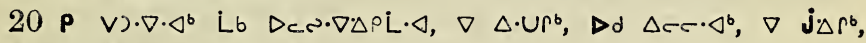

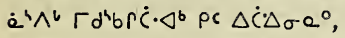

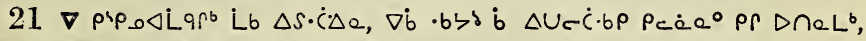

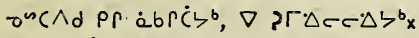

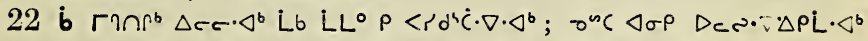

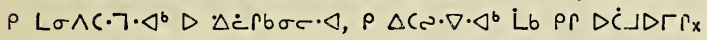

$23 \Delta^{\wedge} \wedge$ Lb $\Gamma^{h}\left(\Delta \quad b \quad \rho<\dot{<} 4 U \cdot \dot{\langle} \Gamma^{b}, \rho \wedge \Gamma \cdot \nabla \wedge \pi \cdot \Delta^{b} \rho<D\right) \Delta b \Gamma d^{b}, \nabla$

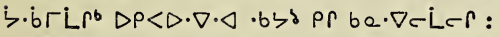

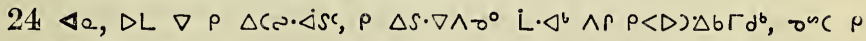
LLirdrUi々 $\wedge^{4} b \cdot \nabla^{\circ} x$

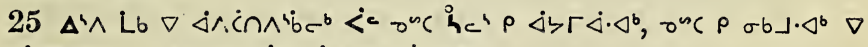

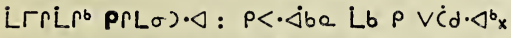

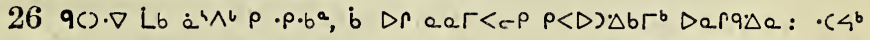
Li $\Gamma r \cdot \nabla \quad \Delta n \cdot b U L$ $\triangleleft \wedge \mathrm{d}<\mathrm{ec} \cdot \Delta \mathrm{x}$

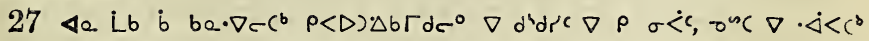

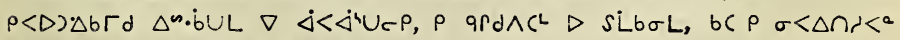

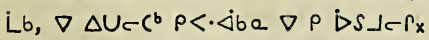

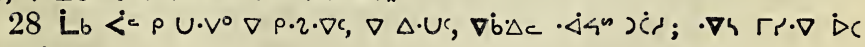
ac $\Delta \dot{\bar{C}} \dot{a}^{a} x$

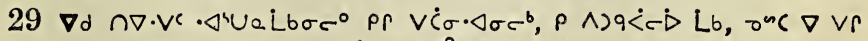

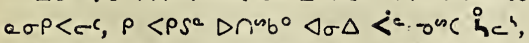

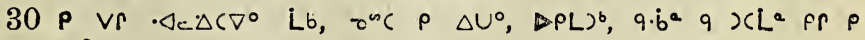
$\triangle L C D i \circ ?$ 
$\varangle<^{\prime} C c^{b} 16,17$.

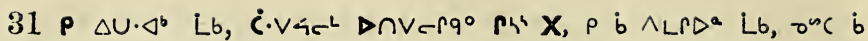
() $93^{\circ} x$

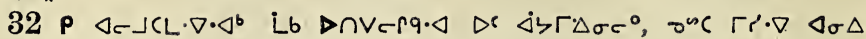
$\Delta \rho^{b}$ bे $\Delta \dot{C}-\rho_{x}$

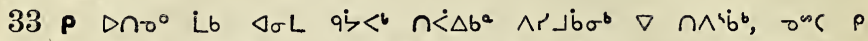

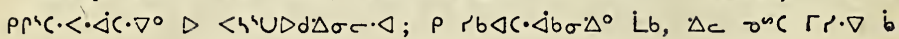
$\triangle C s c \cdot c 4^{b} \mathrm{x}$

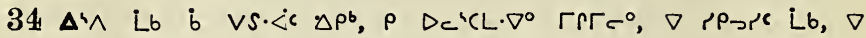

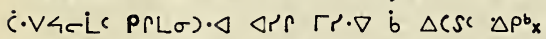

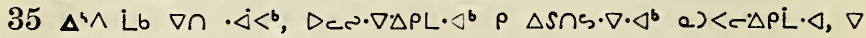
$\triangle \cdot U^{b},\left\langle\rho \cap \sigma^{b} \Delta \sigma \rho \Delta \sigma c \cdot \Delta b x\right.$

36 b be. $\nabla c c^{b}$ Lb $\left.\quad P<D\right) \Delta b \Gamma d c^{\circ} \quad \rho \quad \Delta C L \cdot \nabla^{\circ}<c \quad \Delta 7 c^{\circ} \Delta \cdot U \cdot \Delta \sigma c^{\circ}$,

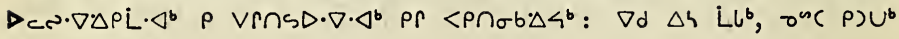
b่ $7-c\lrcorner \Delta \sigma^{b} x$

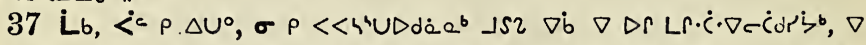

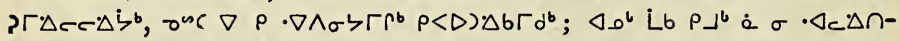

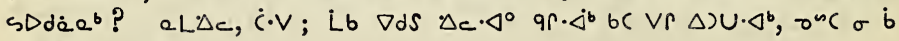
$-\Delta c \Delta c \Delta d \dot{a} e^{b} x$

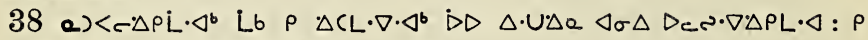

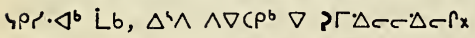

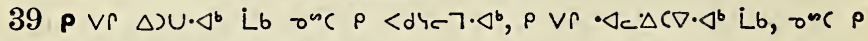

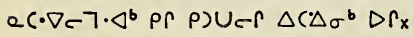

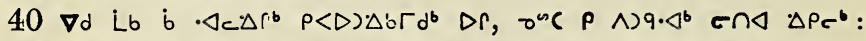

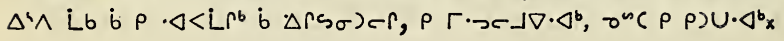

\section{Ápcra $\triangle b^{2} 17$.}

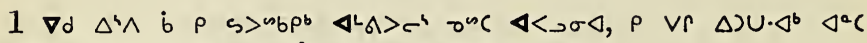
Uh,åb $\Delta^{b}, \Delta c \dot{b} \Delta c \cdot b \sigma c^{b} \mathbf{j} \cdot \Delta^{b} \Delta c \Delta b \Gamma \nabla \Delta b \Gamma d \cdot \Delta^{\circ}:$

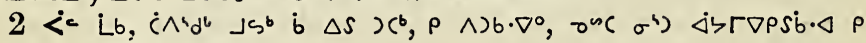
$\Delta r \dot{\Delta} \rightarrow \Gamma]^{\circ}$ prLRe $\triangle 9 \Delta \sigma^{b} \Delta r$,

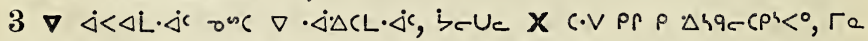

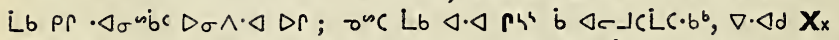

4 inc Lb $\rho$ C.VC.Lb, onc $\rho \cdot \Delta \Delta \eta \cdot \nabla \cdot \Delta^{b}<$ onc heh; $\triangleleft \sigma \rho b$

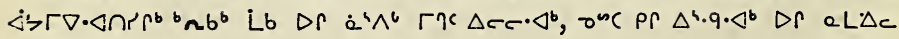
$u b \cdot \triangleleft s \sin ^{x}$

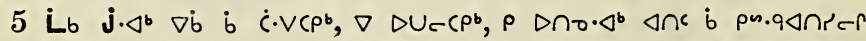

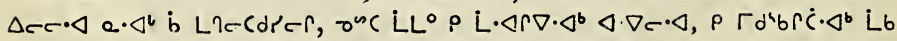

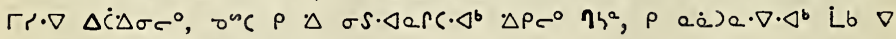
$\Delta V R \cdot \Delta c \Delta C c \cdot \Delta r^{b} \Delta r c \cdot \Delta x$

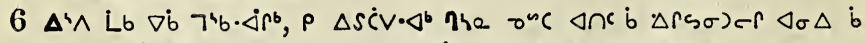

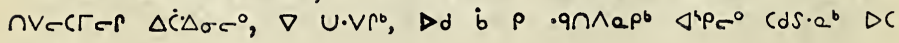
$\triangle$ বr: 


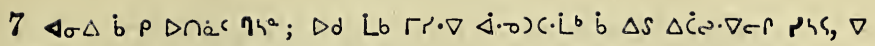

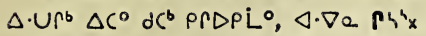

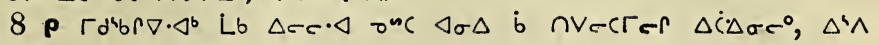
$\wedge \nabla C \Gamma c r$ D $q \cdot b a_{x}$

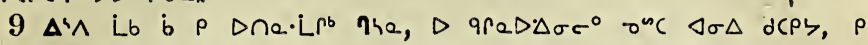
$<P \cap 0 \cdot \Delta^{b} x$

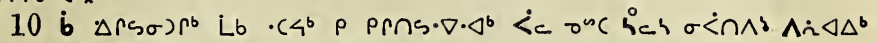

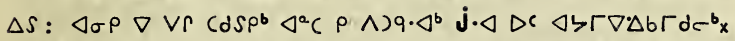

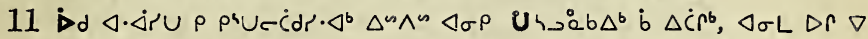

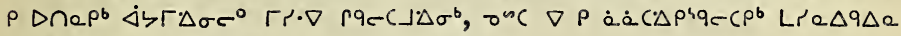

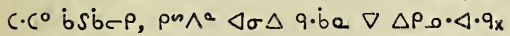

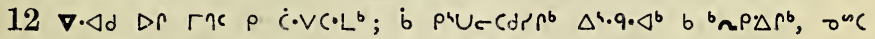
$\Delta c r \cdot \triangleleft^{b} \Delta r, a L \Delta c l \cdot b \cdot \Delta S^{\omega} \omega_{x}$

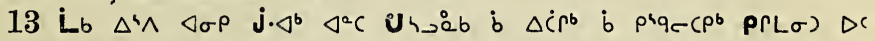

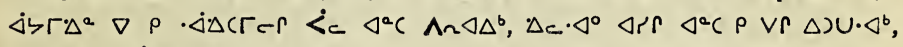
onc $\rho, \Gamma d^{4} \dot{b} r \nabla \cdot \Delta^{b} \Delta \sigma \sigma \cdot \Delta x$

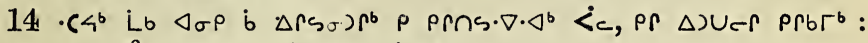

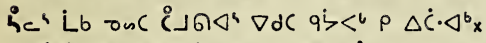

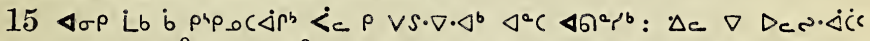

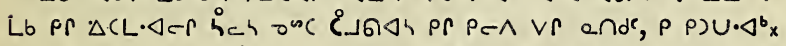

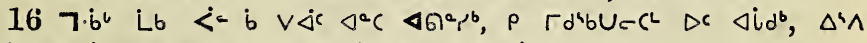

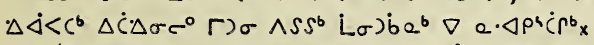

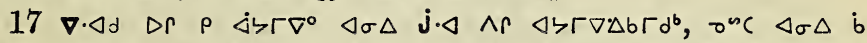

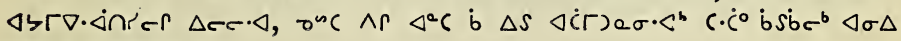
$\checkmark a c$ b epuiddcx

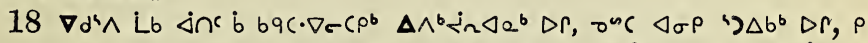

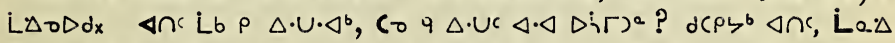

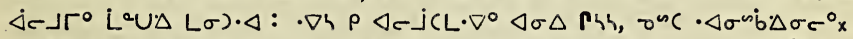

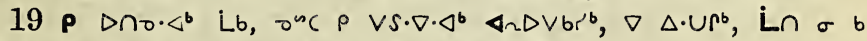

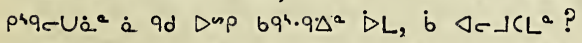

$20 \cdot \nabla 4 \rho \vee \dot{C}^{a} \Delta n k$ LLibr $q \cdot \dot{b} a \quad \sigma c \cdot \Delta \dot{b} \dot{a}^{b}: \nabla \cdot \Delta d$ or $\sigma \Delta \rho^{4} q-U \dot{a}^{a}$ Ca $\nabla \cdot$ iribup $D D$ q.bo.x

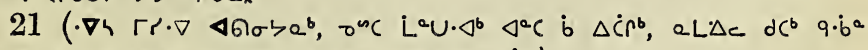

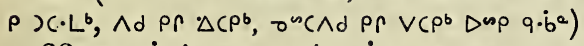

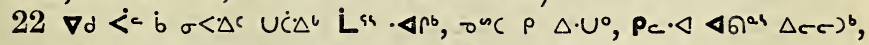

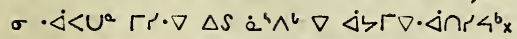

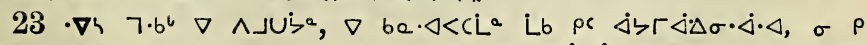

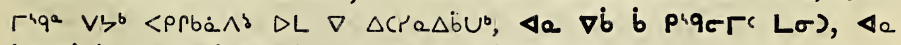

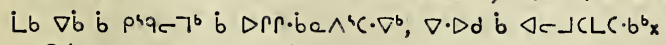

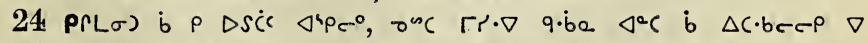

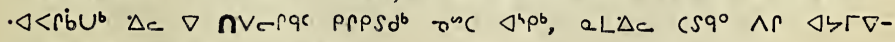

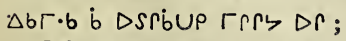

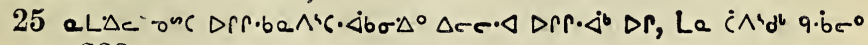
223 


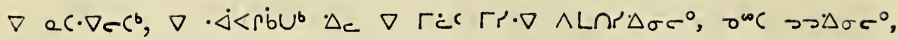

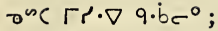

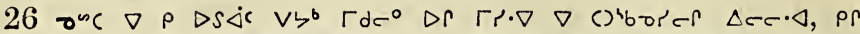

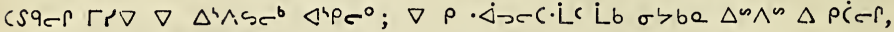
Juc $\Delta C$ q $\Delta$ 'd Csqer;

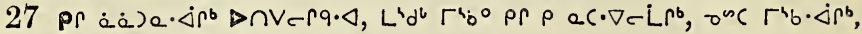
$\Delta C \nabla \dot{b} \nabla \dot{c} \cdot \dot{\Delta}>\Gamma C^{b} \Gamma \cdot \cdot \nabla \nabla \Delta C S \zeta^{b}$;

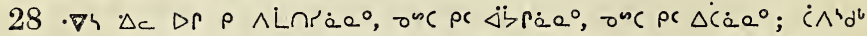

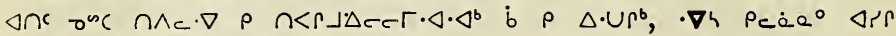
$\nabla \cdot \Delta \cdot \dot{b} \sigma \Delta \triangleright \sigma \dot{c} \Delta \rho \dot{\Delta b e x}$

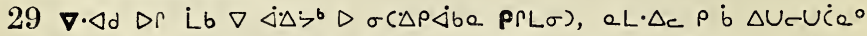

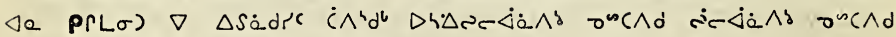
$\Delta\} \sigma, b$ LLCं $\Delta$ LLisSbUb osc $\nabla \Delta U e^{b} \Delta c^{\circ} x$

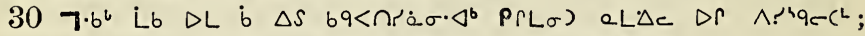

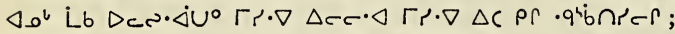

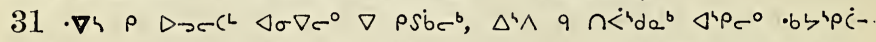

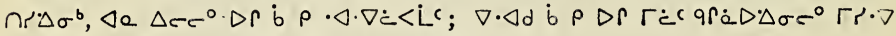
$\Delta c r \cdot \triangleleft, \nabla \rho \cdot \Delta \sigma^{\sim} \dot{b} \dot{a} c \Gamma e \Delta \sigma \wedge \cdot \Delta \nabla \Gamma_{x}$

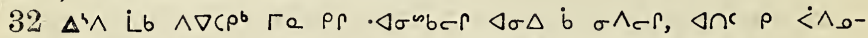

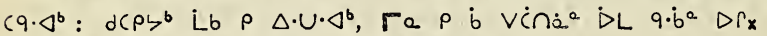

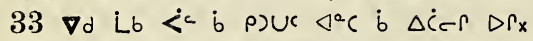

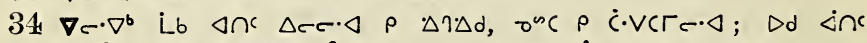

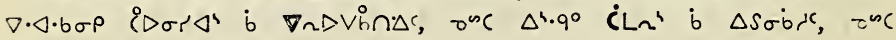

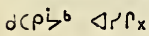

\section{Ápcre $\triangle b^{a} 18$}

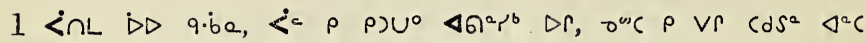
dra ${ }^{2}$;

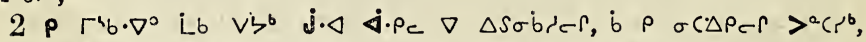

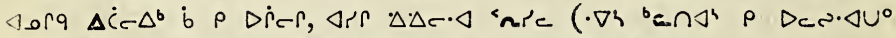

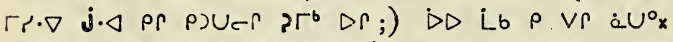

$3 \cdot \Delta c$ Lb onc $\nabla \cdot b_{\sigma} \sigma \Delta \dot{C}<n<c, \rho \cdot \Delta r\left(S q 7^{\circ} \text {, onc } \rho \triangleleft\right)^{4} q^{\circ} ; \cdot \nabla h \quad d C$ $\Delta \dot{c}<n \cdot \Delta \sigma \cdot \dot{\Delta}^{b}$ D $\rho \quad \nabla \Gamma \rho \cdot \Delta \Gamma q \cdot \Delta^{b} x$

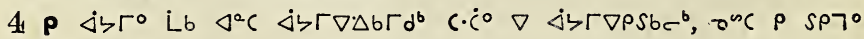

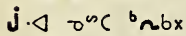

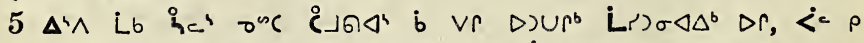

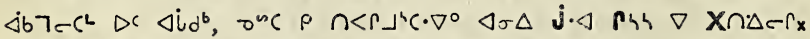

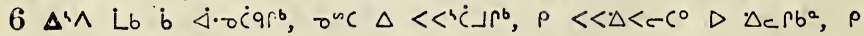

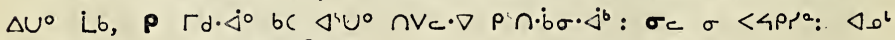
Dr $\sigma \dot{b} \dot{a} \dot{c} \cdot \Delta^{b} \triangleleft \sigma \rho \eta^{a}{ }^{\circ} c^{b} x$

$7 \Delta^{\circ} C$ Lb $\rho$ or $\left.\rho\right) U^{\circ}, \rho \wedge q^{\circ}$ Lb Vלb $\Delta c c \cdot \Delta \Delta P c^{b}, b^{4} c^{b} b$ 224 


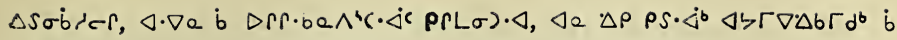
$\Delta c \cdot b \sigma c^{b} x$

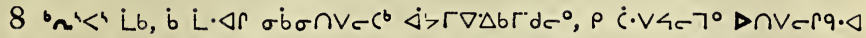

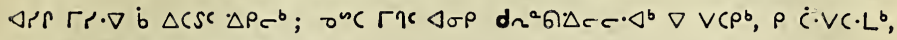
$\operatorname{sic} \rho \cos \Delta C \cdot \Delta b \sigma \cdot \Delta \cdot \Delta b^{x}$

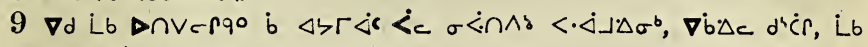

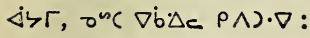

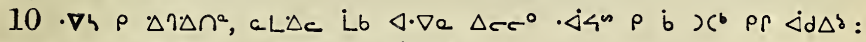

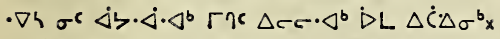

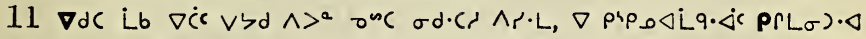
$D: \Delta \zeta \Gamma^{\circ} \Delta \sigma \sigma^{\circ} \Delta c \nabla \Delta c r r_{x}$

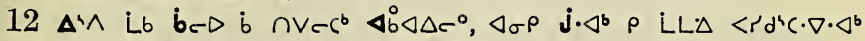
$<c, \rho \vee S \cdot \nabla \cdot \triangleleft^{b} L b \quad \nabla^{a} C \cap<^{i} d \sigma q \Delta \triangleleft \wedge \Delta \sigma^{b}$,

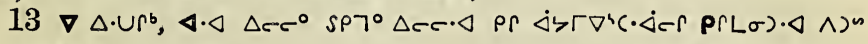
b) $\Delta C^{a} U^{b} D_{C a} \cdot \nabla \Delta \sigma^{b} x$

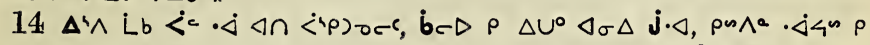

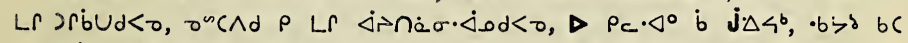
$\triangle U_{-} \dot{C} b \Omega<a$ pr $\wedge S R \Delta C \cdot b^{b}$ :

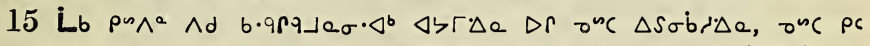

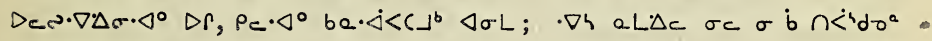
$\triangleleft \sigma \Delta \supset \Delta q \cdot b_{0 x}$

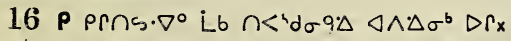

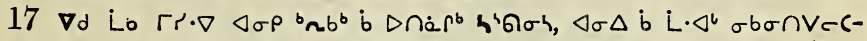

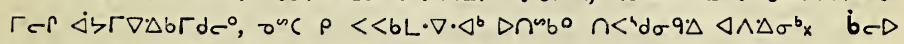

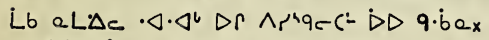

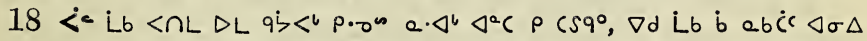

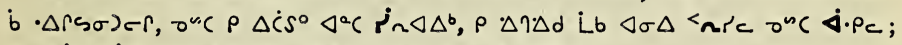

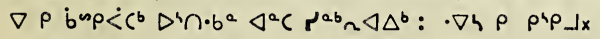

$19 \rho \vee r \Delta U^{\circ}$ Lb $\Delta \Delta h r^{\circ}, \sigma^{m c} \nabla d C F$ abU०: Lb $\left.\Delta c \Lambda_{d} \rho \wedge\right) 9^{\circ}$ $\left.\Delta ל \Gamma \nabla \Delta b \Gamma d^{b}, \sigma^{n C} \rho \Delta r \Delta b \Gamma\right]^{\circ} \triangleleft \sigma \Delta j \cdot \triangleleft x$

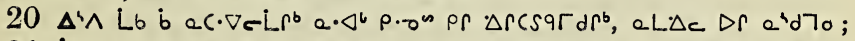

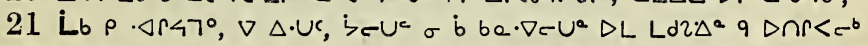

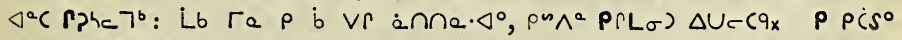
Lb $\Delta \Delta 4$ Pb $^{b} D r_{x}$

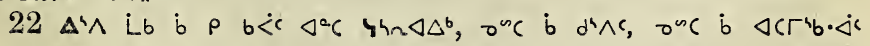
LL $\Delta \dot{\Delta} \zeta \Gamma \dot{<} \cdot \triangleleft, \rho \Delta) U^{\circ} \triangleleft^{a} c \Delta^{a} n \Delta \rho^{b} x$

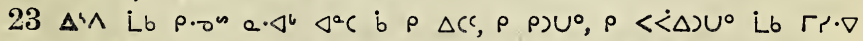

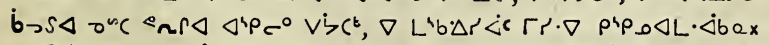

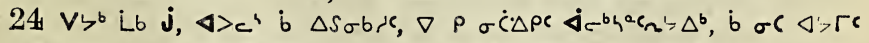

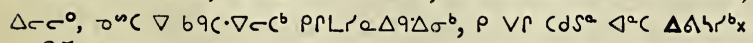

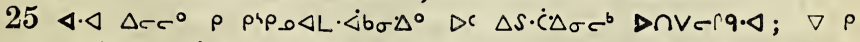

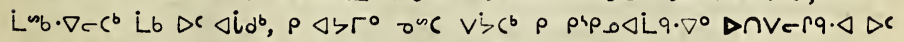

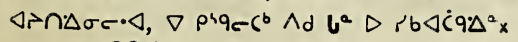




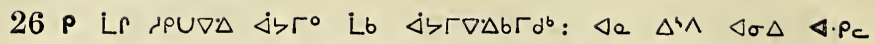

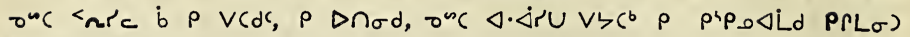
$\Delta c \Delta S \cdot \dot{C} \Delta \sigma c^{\circ} \mathrm{x}$

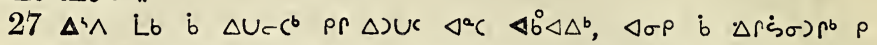

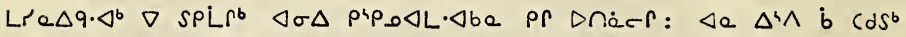
$\Gamma^{h} C \Delta \rho \triangle r \nabla^{\circ} \triangleleft \sigma \Delta$ b $\rho\left(\cdot V C c^{-} c r s \cdot \nabla c r q \Delta \sigma c^{\circ} D r\right.$ :

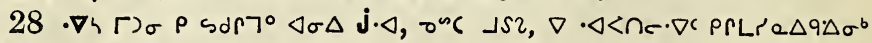

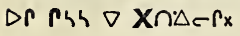

\section{A pere $\Delta b^{a} 19$.}

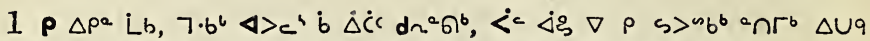

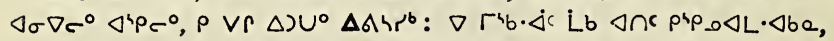

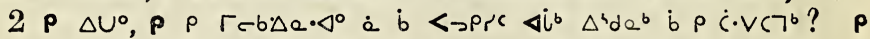

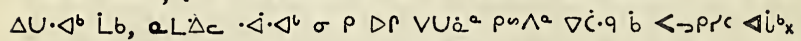

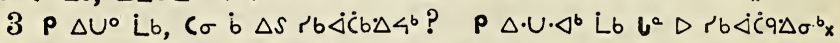

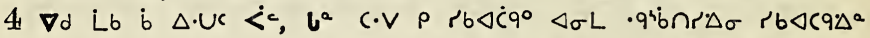
$\Delta r, \nabla \Delta c c \Delta \sigma \Delta \Delta c c \cdot \triangleleft$, pr cं.v4cLer $\Delta \sigma \Delta q \vee r \rho^{4} \wedge \dot{a} n d c, \nabla \cdot \triangleleft \cdot \dot{b} a X$ Prix

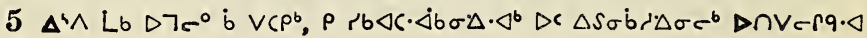
Pin'x

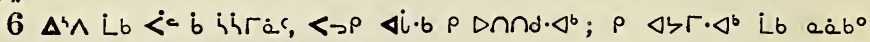
$\Delta S \rho \cdot 2 \Delta e \quad \Delta r$, onc $\rho$ php. $\Delta \Delta q \cdot \Delta^{b} x$

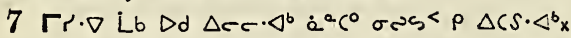

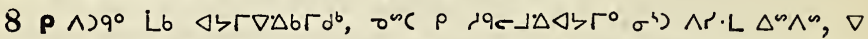
- $\Delta \Delta C L q$, jos $\nabla$ SPr. $\nabla C \quad \sigma \sigma \Delta$ q.be $D r$ b $\Delta(\cdot b \sigma c P$ PrL $\sigma)$ DC $D P L \Delta \Delta \sigma^{b} x$

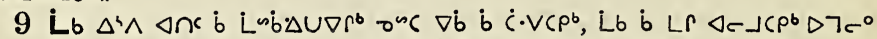

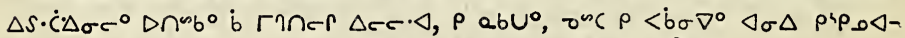

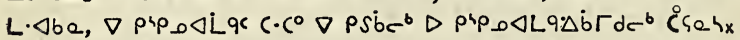

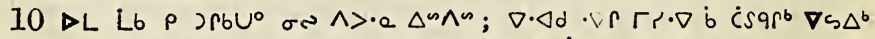

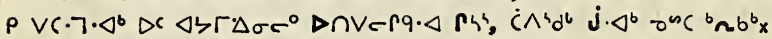

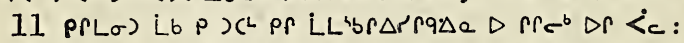

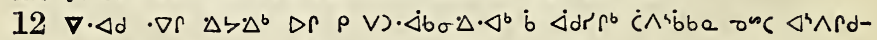

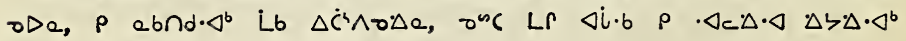
$\Delta r_{x}$

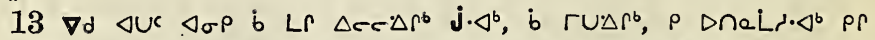

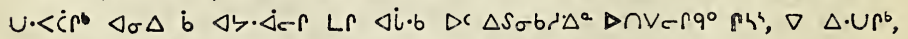

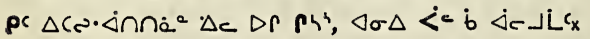

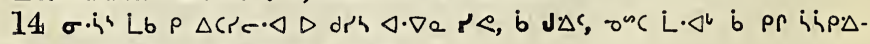
$\left.r \cdot \nabla \Delta r \sigma \Delta c \Delta^{a} C, \Delta \sigma P \Delta 7 \sigma^{\circ} \dot{b}\right)\left(p^{b} x_{x}\right.$

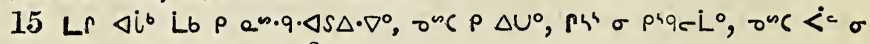
P'qन L०; Lb $\triangleleft \cdot \nabla \sigma P P c \cdot \triangleleft^{\circ}$ ? 
$16 \varangle a \Delta c c^{\circ}$ Lb Lr $\left.\Delta \dot{b} \cdot b \dot{b} \rho \wedge r^{4} b d^{c} \rho \cdot b^{4} d\right) \dot{C} \cdot \nabla^{\circ}, \rho \dot{s} d r \nabla^{\circ}$ Lb, onc $\rho$

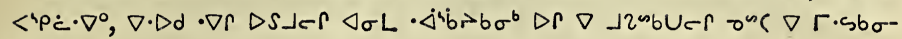
brerx

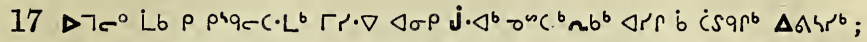

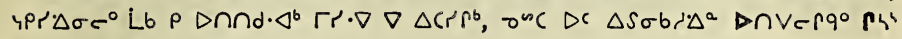

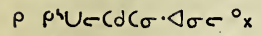

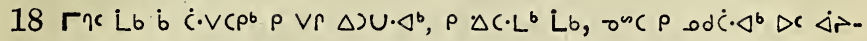
ก. $\cdot \cdot \dot{4} \cdot \triangleleft$

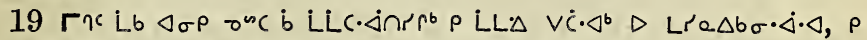

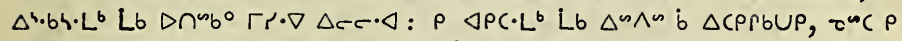

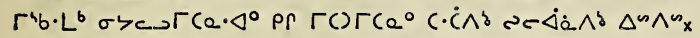

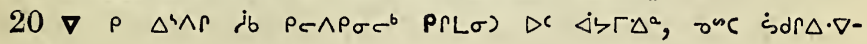
Lbor $\sigma^{b} x$

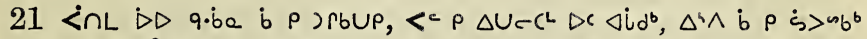

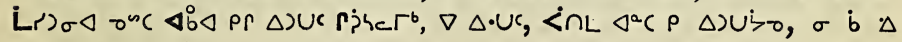
.$<<U_{a}$ bl $\Delta<r_{x}$

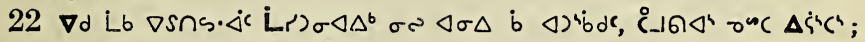
Lb $\Delta c 9 r \cdot j^{b} \Delta-\Lambda^{b} \rho \Delta c^{\circ} \Delta^{a} c \nabla c \Delta^{b} x$

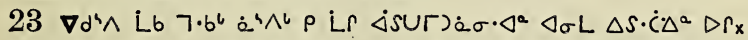

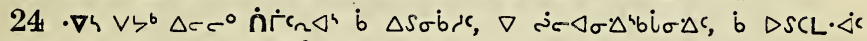

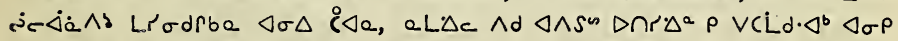
$\triangle r c \cdot \Delta^{b} \Delta \sigma-L \dot{b} \Delta \dot{C}<n r^{b} ;$

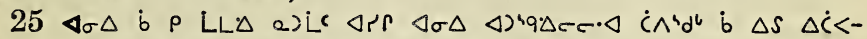

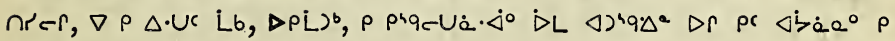

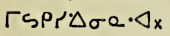

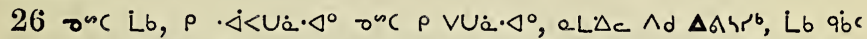

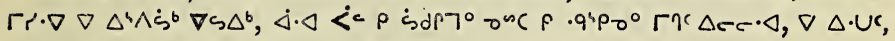

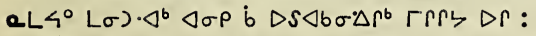

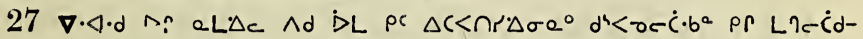

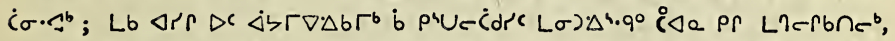

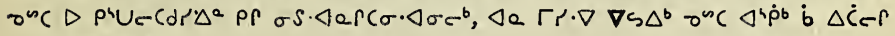
b $\triangleleft 4 \Gamma \nabla^{\prime} \mathrm{Cd}^{\prime} x$

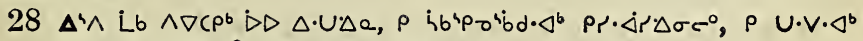

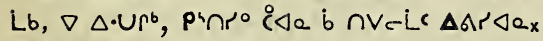

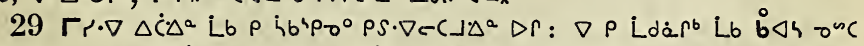

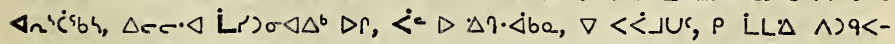
$\rightarrow D \cdot \Delta^{b} \quad \Delta^{a} C \quad C C \cdot \nabla \Delta b \Gamma d^{b} x$

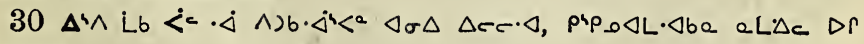
$<p \cap \sigma d x$

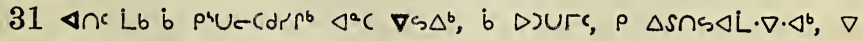

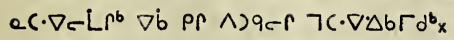

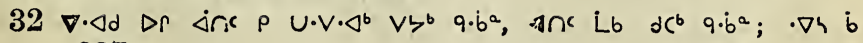
227 


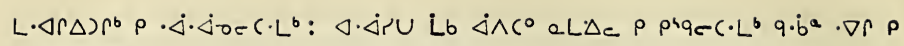
$L \cdot \Delta r \Delta) r^{b} \times$

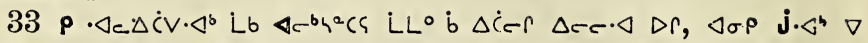

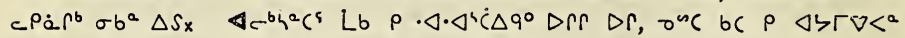
$\triangle e c \cdot \Delta x$

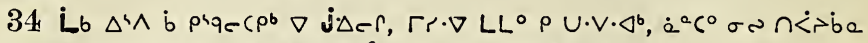

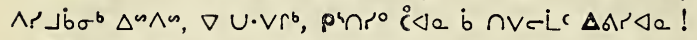

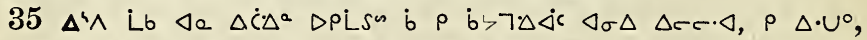

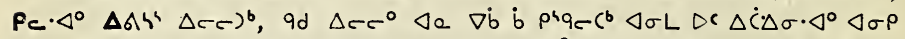

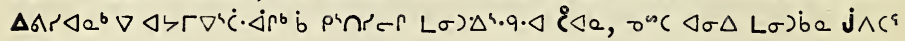
b $\rho D R<\rho S \sigma e r$ ?

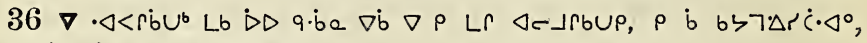
onc $\nabla \dot{b} q \cdot b^{a}$ or $\left.\rho s . \Delta\right)\left(7^{b x}\right.$

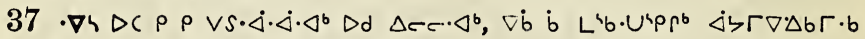

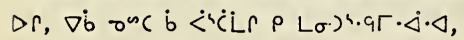

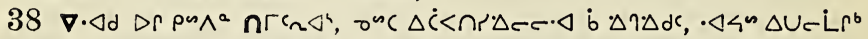

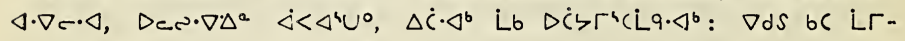
$s \Gamma) \cdot \Delta^{b} x$

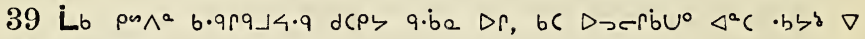
$\dot{L} \cdot \Delta r \Delta) \dot{\alpha} \sigma \cdot \triangleleft^{b} \mathrm{x}$

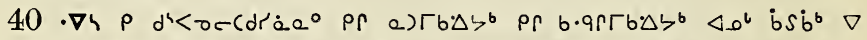

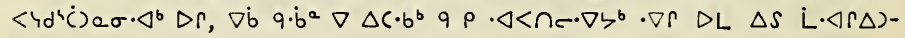
$\dot{\alpha} \sigma \cdot \triangleleft^{b} x$

$41 \Delta^{\prime} \wedge$ Lb $\Delta 7 c^{\circ}$ b $\rho \Delta \cdot U c, \rho$ prns $\left.\nabla \nabla^{\circ} \Delta \sigma \Delta \dot{b} \dot{L} \cdot \Delta r \Delta\right) r_{x}$

\section{Ápcre $\Delta b^{2} 20$.}

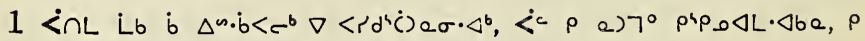

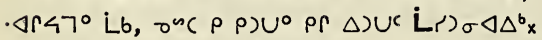

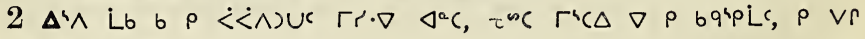
$\Delta U^{\circ} \Delta^{a} c^{b} \sim r^{b}$,

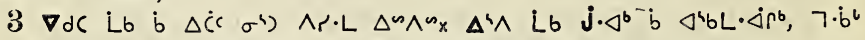

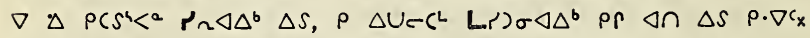

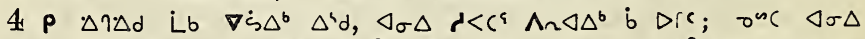

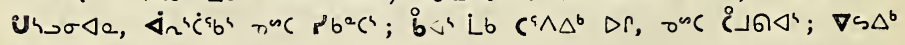
Lib $D S \cap P b_{4}$ JuC CBALLX

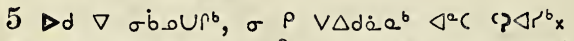

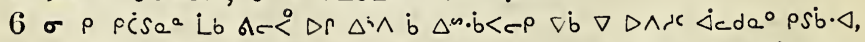

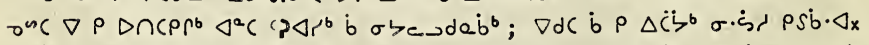

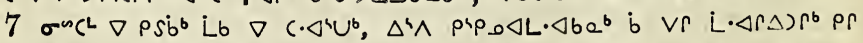

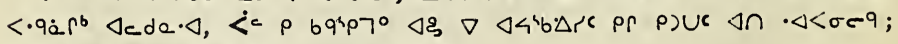
$\rho \triangleleft \hookrightarrow \Gamma^{\circ}$ Lb $\wedge c^{n} \nabla \triangleleft \wedge C \cap \wedge \wedge^{4} b c^{b} x$ 


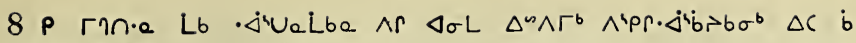
$\Delta S L \cdot \Delta i \Delta r^{b} x_{x}$

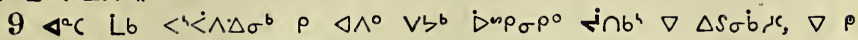

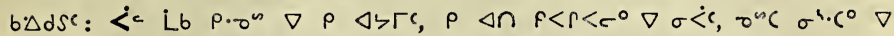

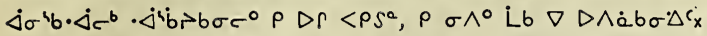

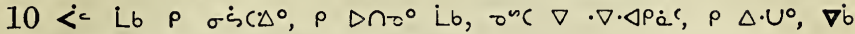

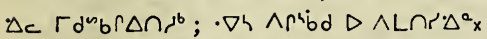

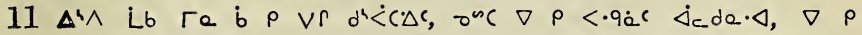

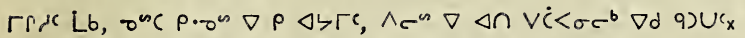

$12 \rho \vee S \cdot \nabla \cdot \Delta^{b}$ Lb $\triangleleft \sigma \Delta \Delta \backsim \rho_{\sigma} \rho \cdot \triangleleft \nabla \wedge L \cap r e r$, eL $\Delta c$ Lb $\Delta \wedge S^{n}$ $\wedge d P \Gamma \cdot s e] \cdot \Delta b x$

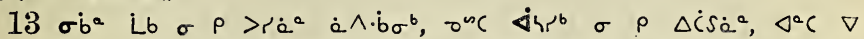

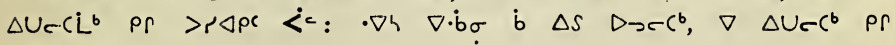
म") $U$ U $\Delta c x$

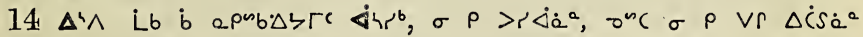
$r \cap c \Delta^{b} x$

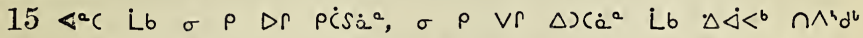

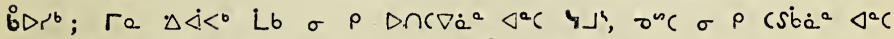

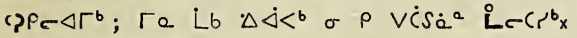

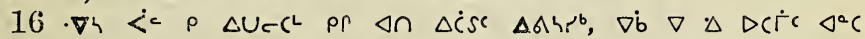

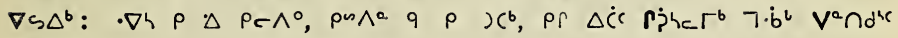
$\nabla$ PSib ${ }^{b} x$

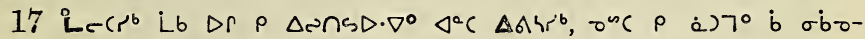
éd.rer $D \dot{C} \zeta[\dot{\Delta} \cdot \Delta x$

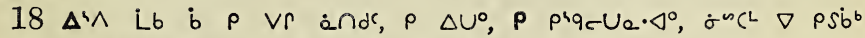

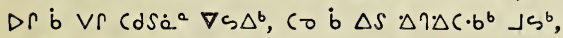

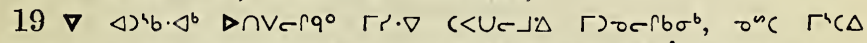

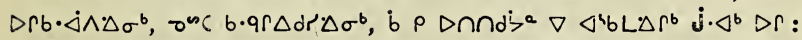

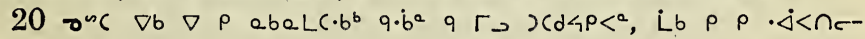

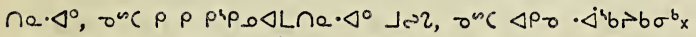

$\left.21 \nabla \cdot \dot{\nabla} \Delta C L \cdot \Delta \rho b j \cdot \Delta^{b}, \sigma^{\infty} C{ }^{b} \sim b^{b} \dot{C} \wedge^{4} d^{b}, \rho \rho \cdot q^{4} b \cap r^{4} C \cdot \Delta \rho^{b} \rho \Gamma L \sigma\right) \cdot \triangleleft$,

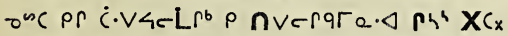

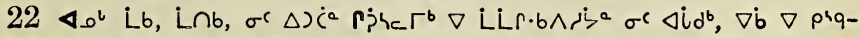

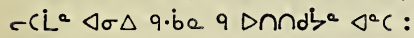

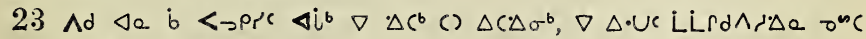
$j e r r \cdot \Delta e \nabla v \Delta d \dot{\zeta}{ }^{a} x$

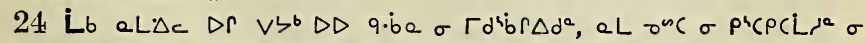

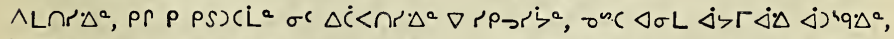

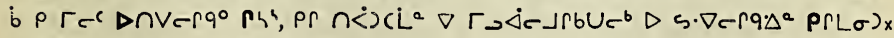

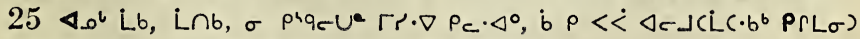

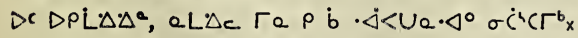

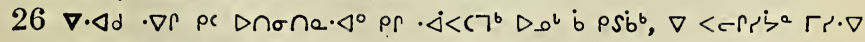
$\triangle r c \cdot \Delta^{b} D \Gamma \mathrm{d} \cdot \Delta^{\circ} \Delta r_{x}$

229 


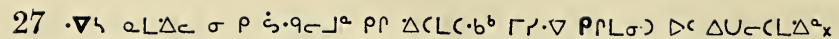

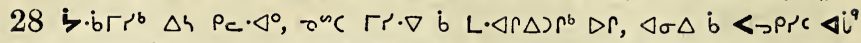

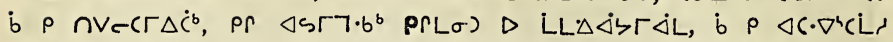
$\cap \wedge_{c} \cdot \nabla \triangleright \Gamma d^{L} \Delta r_{x}$

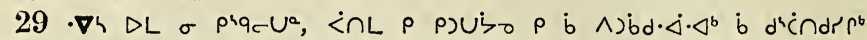

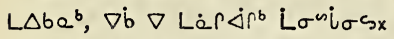

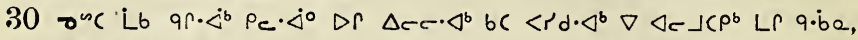

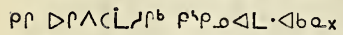

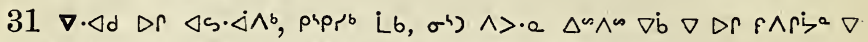

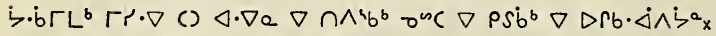

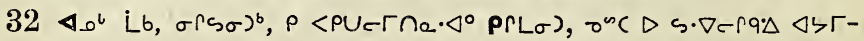

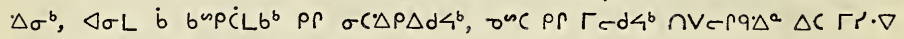
LL $L^{\circ} \dot{b} \Delta \dot{C}^{b} b \dot{b}<>\Delta b \sigma \Delta \Gamma^{b} x$

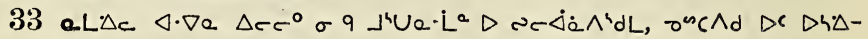

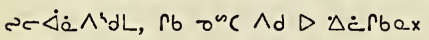

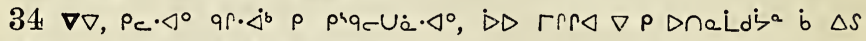

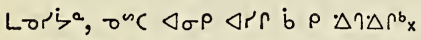

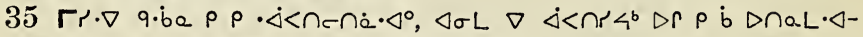

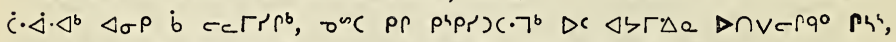

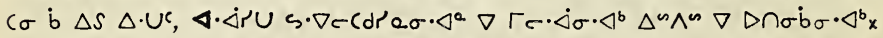

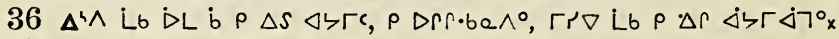

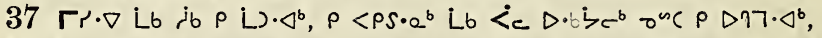

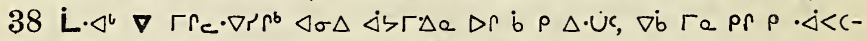
- L $\rho^{b} \Delta \dot{C}^{4} C \Gamma d c^{0} x \rho \Delta \eta \cdot \nabla \cdot \Delta^{6} L b \dot{a} \wedge \cdot b \sigma^{b} \Delta^{4} d x$

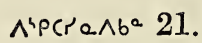

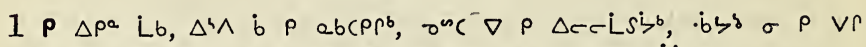

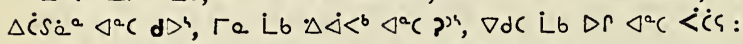

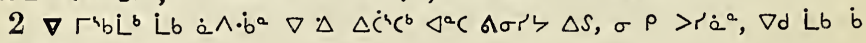
PSPT⿱宀一:

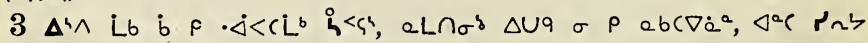

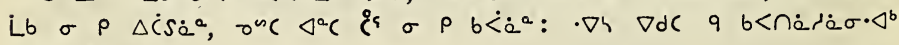
$\dot{\mathrm{a}} \wedge \cdot \dot{b} \sigma^{b} \Delta \Gamma_{x}$

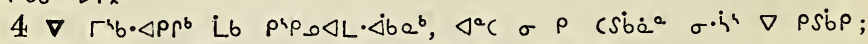

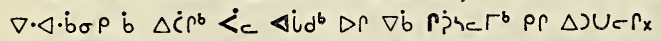

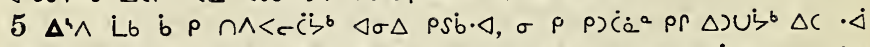

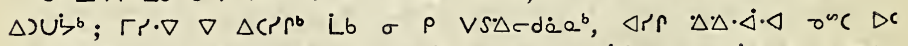

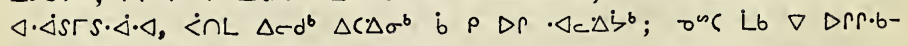

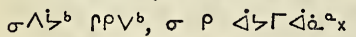

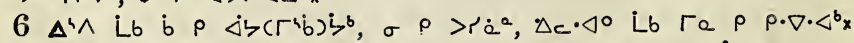

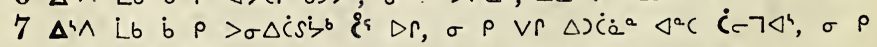

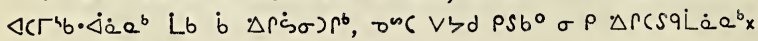

230 


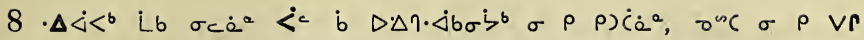

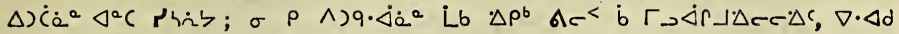

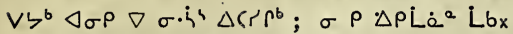

$9 \nabla \cdot \Delta d \Delta c c^{\circ}$ Lb $\rho$ o $\Delta c \cdot \Delta \Delta \dot{C} \sigma h$ b $\rho$ prp. $\Delta \Delta q c \gamma_{x}$

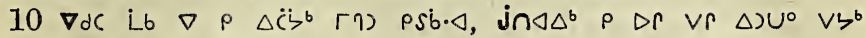

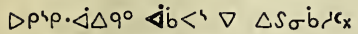

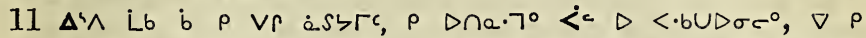

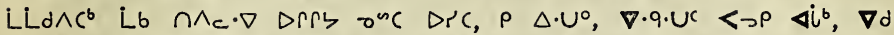

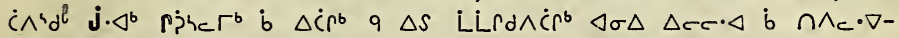

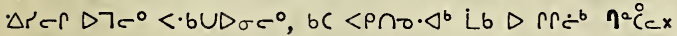

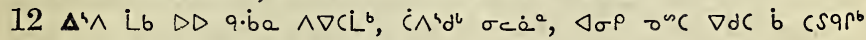

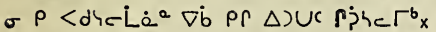

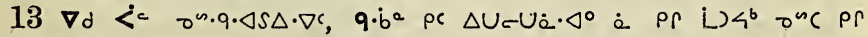

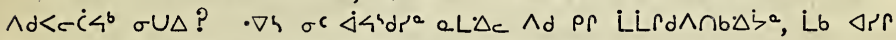

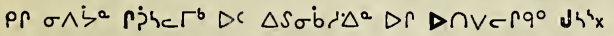

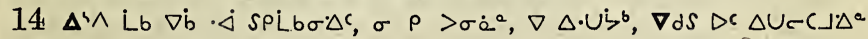
$\triangle \cap V e r q^{\circ} b C \Delta P_{\sigma C^{\circ}} \mathrm{x}$

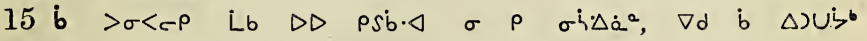
pine $\Gamma^{b} \times$

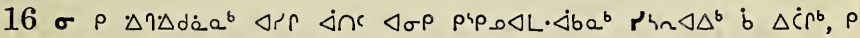

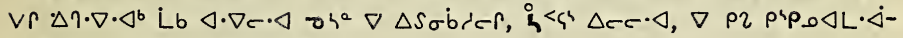
b $\sigma \cdot \Delta r r, \nabla \cdot \triangleleft \cdot b a q \Delta) q L P c_{x}$

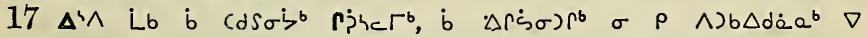
$\Gamma_{c} \cdot \dot{s} \rho^{b} \mathrm{x}$

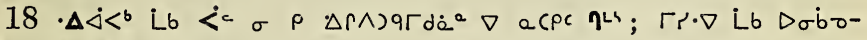
$-\dot{C} \mathrm{~d} r \cdot \Delta^{b} \triangleleft^{a} \mathrm{C} \rho \Delta \dot{C} \cdot \Delta^{b} \mathrm{x}$

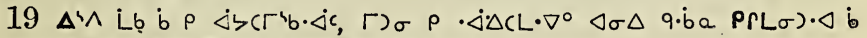

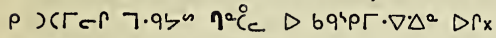

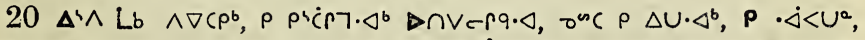

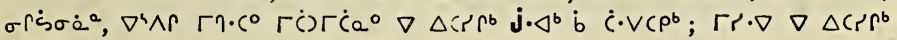
Lb L"

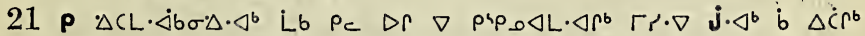

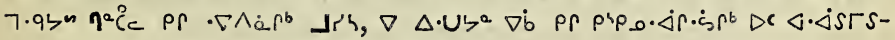

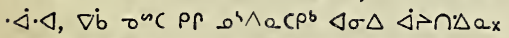

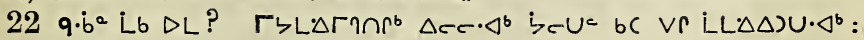
$\cdot \nabla h$ bC VC. Lb $\nabla P C d S a^{a}{ }^{a} x$

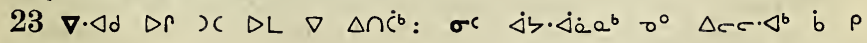
pip」 $\lrcorner \Gamma^{b} ;$

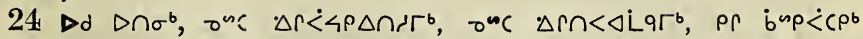

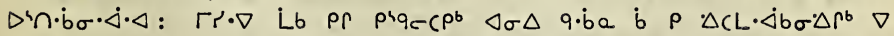

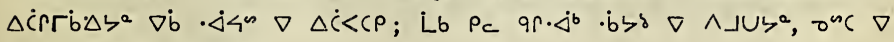
$\mathrm{ba} \cdot \nabla C \mathrm{CL}^{a} D_{C} \cdot \nabla \cdot \Delta^{a} \mathrm{x}$

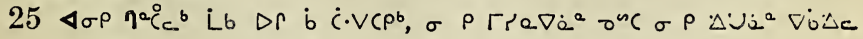
231 


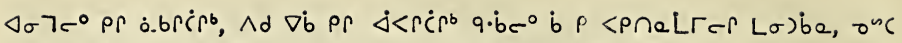

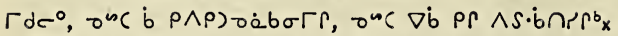

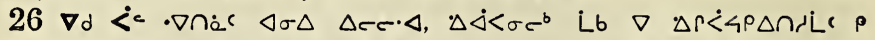

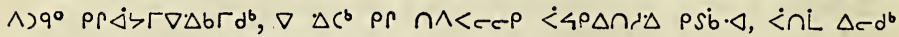

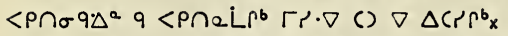

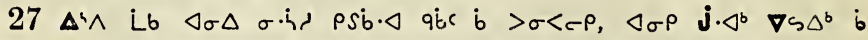

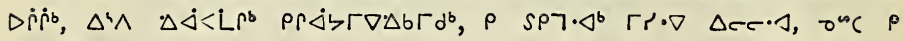
$\Delta \cap \cap 0 \cdot \Delta^{b}$,

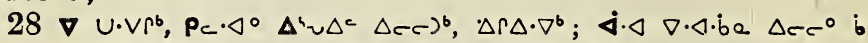

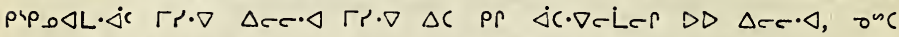

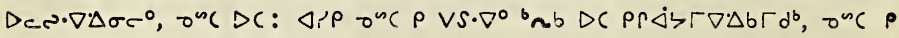
LרciddC $D 7 c^{\circ}<\neg P b \Gamma d c^{\circ} x$

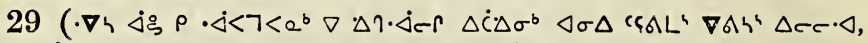
$\Delta \sigma \Delta \dot{<} \rho \wedge) b \nabla^{\circ} \rho \rho \dot{\Delta}\left\langle\Gamma \nabla \Delta b \Gamma d^{b} \dot{b} \Delta U c\left(p b^{x}\right)\right.$

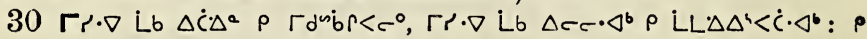

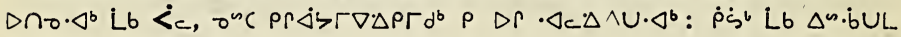
$\rho \rho<\Delta \dot{b U} \cdot \Delta_{x}$

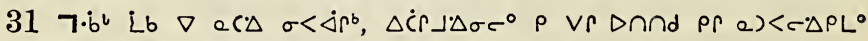

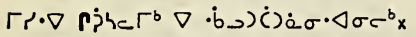

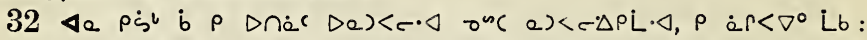

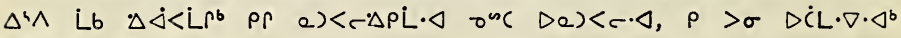
$\dot{i} \mathrm{x}$

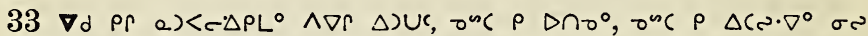

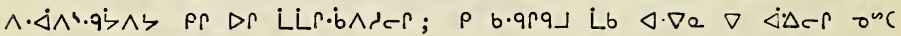
$\left.q \cdot \dot{b} c^{\circ} \dot{b} b\right)\left(r e r_{x}\right.$

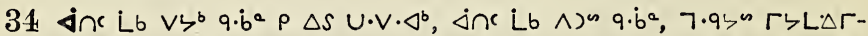

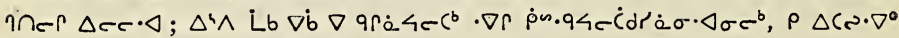
a) $<c \Delta b \Gamma d^{b}$ pr $\left.\Delta\right)(\Delta b \sigma \Delta c r x$

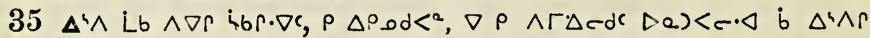
$\dot{\Delta d e \cdot \nabla r e r} \Delta c c \cdot \Delta x$

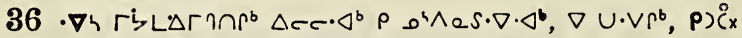

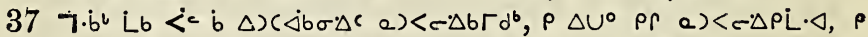
b $\dot{b} r \Delta n^{a} \dot{a} ? \nabla \cdot \Delta d \dot{b} \Delta \cdot U c,{ }^{b} \dot{\sim}^{b} \Delta S \rho \cdot 2 \Delta \sigma^{b}$ a $\rho \rho \Delta b \Gamma^{a}$ ?

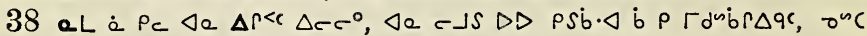

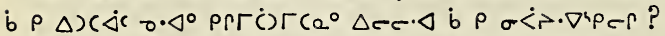

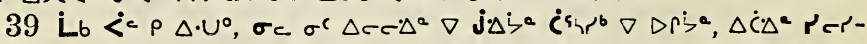

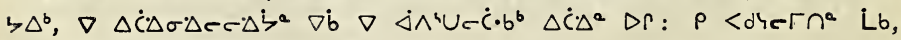
$\Delta r \dot{a} \Delta^{a} \rho r \quad j b \Gamma \Delta \rho^{b} \Delta r c \cdot \Delta^{b} x$

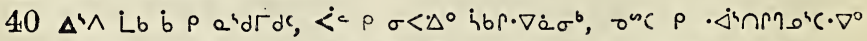

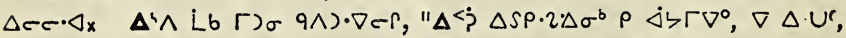




\section{$\Lambda^{\prime} p C r e \triangle b^{\circ} 22$.}

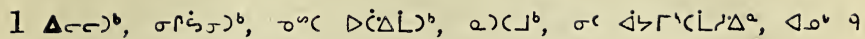
$\dot{\Delta} \zeta \bar{r} \Delta \cdot b^{b} \mathrm{x}$

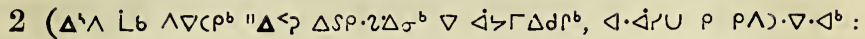
$\left.\Delta \cdot U^{\circ} L b,\right)$

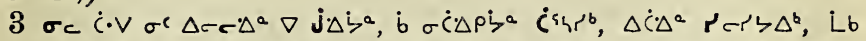

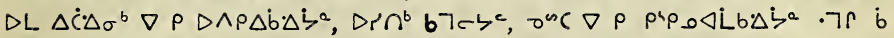

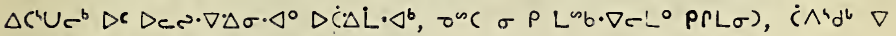

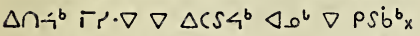

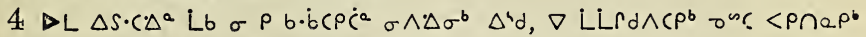
$b<\Delta) \Delta b \Gamma d^{b} \dot{a} V \cdot \Delta^{b} \sigma^{c c}\left(\Delta^{b} \cdot q \cdot \Delta^{b} x\right.$

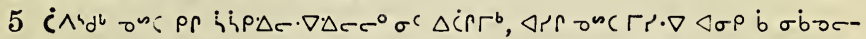

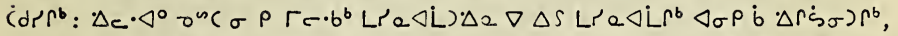

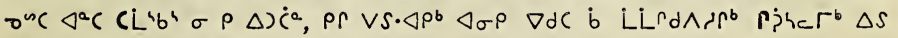
pr $b \cdot b c \rho<j b \sigma \Delta r^{b} b_{x}$

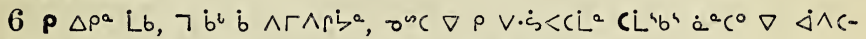

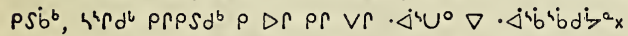

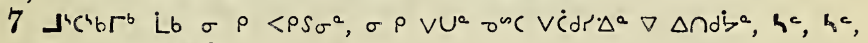
¿ंop $\cdot \nabla r \quad b \cdot \dot{b} C \rho \triangle \zeta^{\circ}$ ?

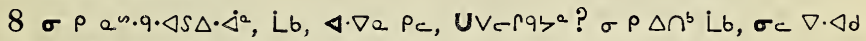

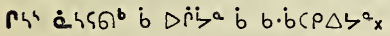

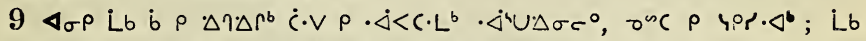

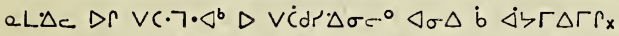

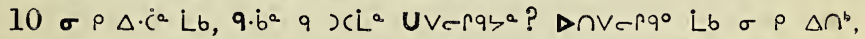

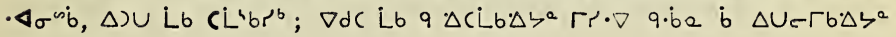
Pr ${ }^{C} L^{\circ} \times$

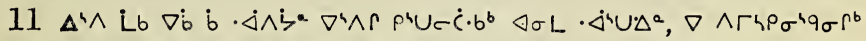

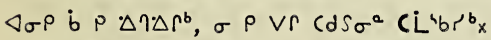

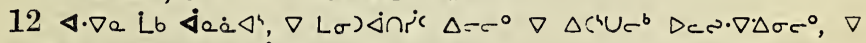

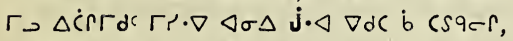

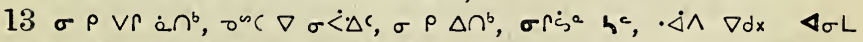

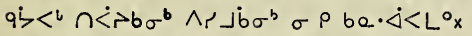

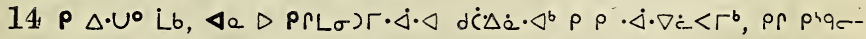

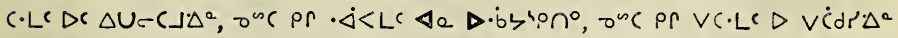
D) $\sigma^{b} x$

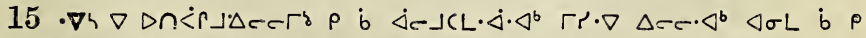
- $\Delta<C L^{2}$ onc b $P V C L^{\circ} x$

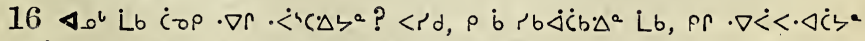

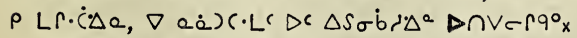

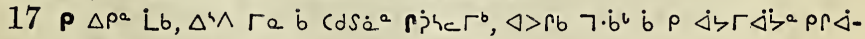
$\zeta \Gamma \nabla \Delta b \Gamma d^{b}, \sigma \rho \Delta S \sigma \cdot \dot{\Delta}^{a}$; 


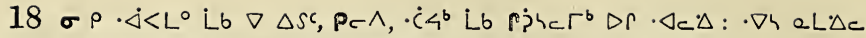
bC $\left.\left.D \cap a \cdot L^{b} \rho \cap \dot{K}\right\lrcorner\right\lrcorner \Delta \sigma c^{\circ} \nabla \Delta \dot{C} r r^{\circ}{ }^{\circ} x$

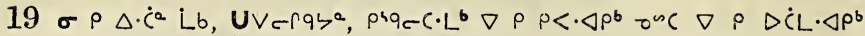
$\Gamma r \cdot \nabla() \triangleleft b \Gamma \nabla \Delta b \Gamma d^{b} \triangleleft \sigma \rho$ bं $\dot{C} \cdot V 4 c \Gamma^{h} \rho^{b}$ :

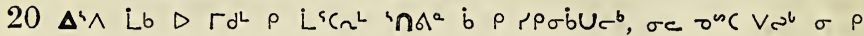

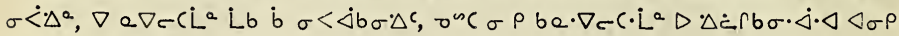
b $\rho \sigma<\Delta \rho^{b} x$

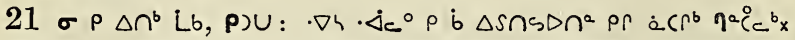

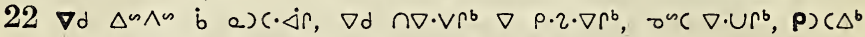

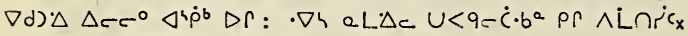

$237 \cdot \dot{b}^{b}$ Lb b b $U \cdot V r^{b}$, osc b $9 r d \cdot \nabla<\Delta \rho^{b} \nabla \Delta \dot{c} \Gamma b \sigma \cdot \dot{\Delta} \cdot \triangleleft$, onc b $\Delta^{n} \dot{<q-}$ $\cdot \nabla<\Delta \rho b$ Lr.bcs,

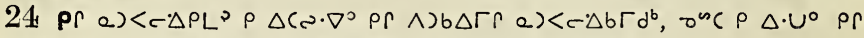

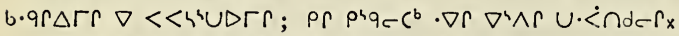

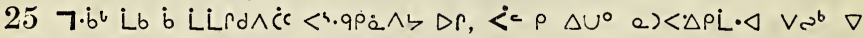

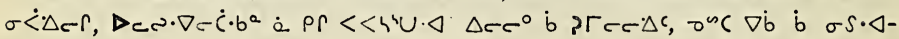

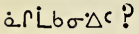

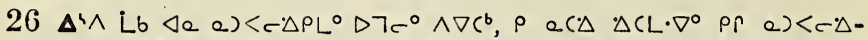

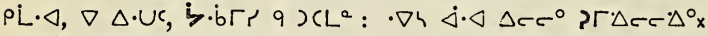

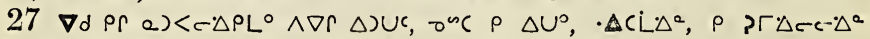
¿. ? $\rho \Delta \cdot \cup^{\circ}, \nabla \nabla x$

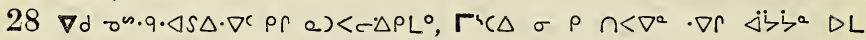

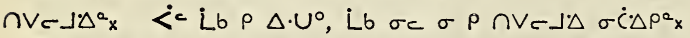

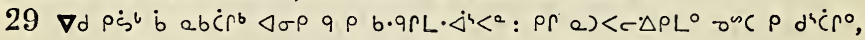

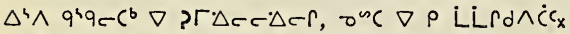

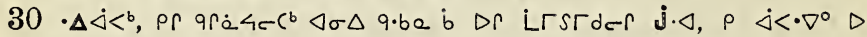

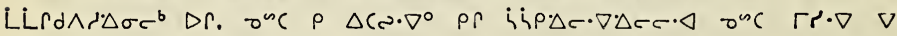

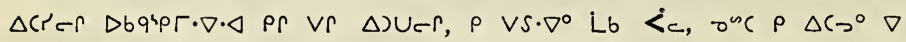
$\dot{C} C L \wedge C r_{x}$

\section{$\Lambda^{4} p c r e \Delta b^{2} 23$.}

$\left.1<\dot{L} L b, \nabla \Delta S \dot{b}<\dot{L}(\dot{b} L \cdot \Delta r \Delta) r r, \rho \Delta \cdot \cup^{0}, \Delta r c\right)^{b}$ onc $\left.\sigma r \dot{s} \sigma\right)^{b}, \sigma \rho$

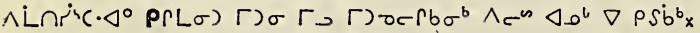

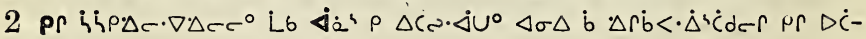
ए) $0 \cdot \dot{\Delta}+\varphi_{x}$

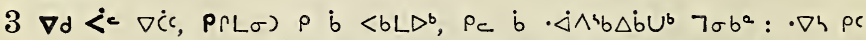

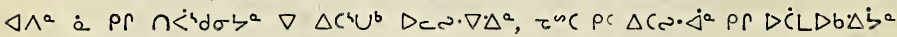
$\nabla \dot{b} \nabla \Delta C^{3} U^{b} D_{C A} \cdot \nabla \Delta^{a}$ ?

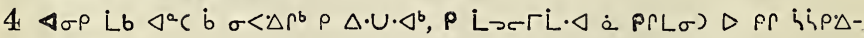
$\leftarrow \cdot \nabla c-L$ ?

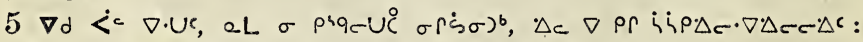

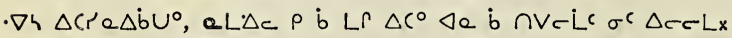

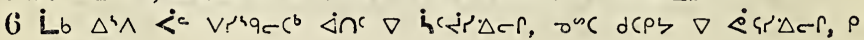
234 


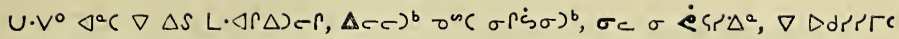

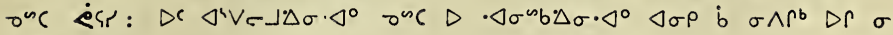
กरंब $\sigma \dot{b} \Delta^{a} x$

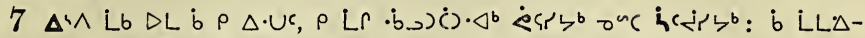

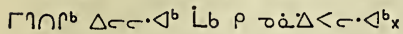

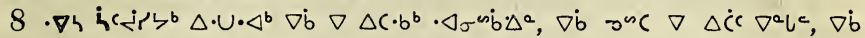

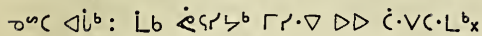

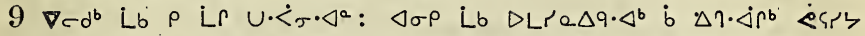

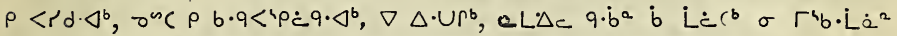

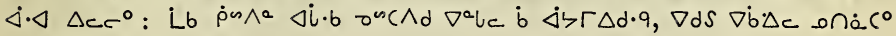
P $(L \sigma)_{x}$

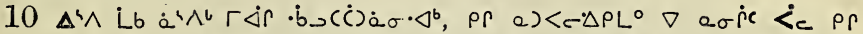

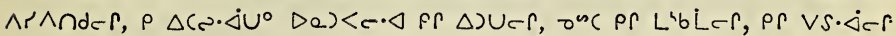
Lb a) $<-\triangle b \Gamma d^{b} x$

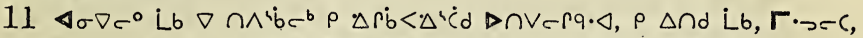

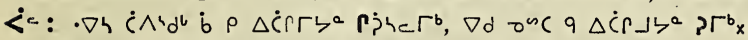

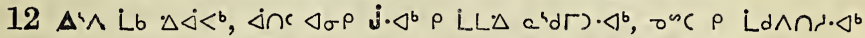

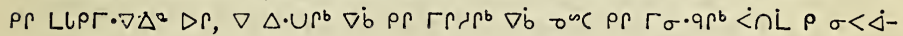
$\cdot \dot{C} \cdot \nabla \dot{<}_{\mathrm{ex}}$

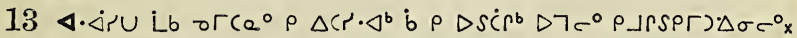

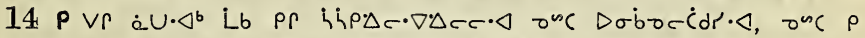

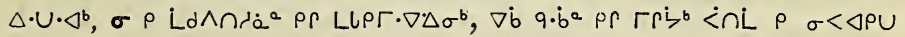
$\dot{<} \mathrm{x}$

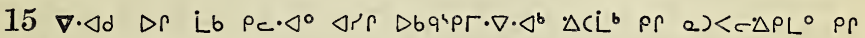

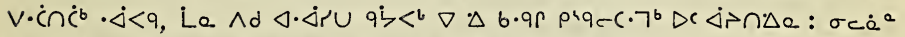

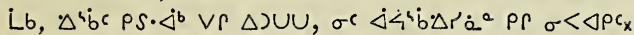

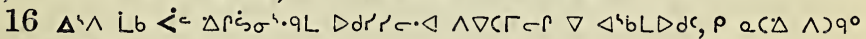

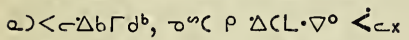

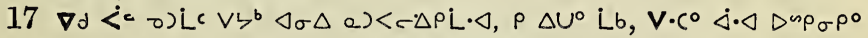

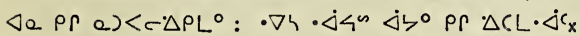

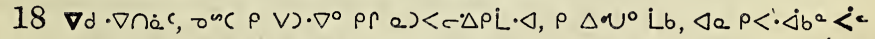

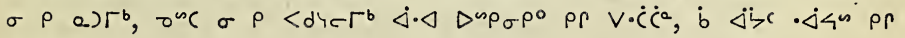
$\triangle C i{ }^{8} x$

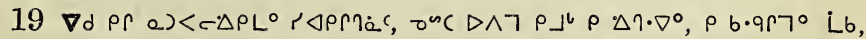

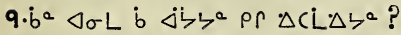

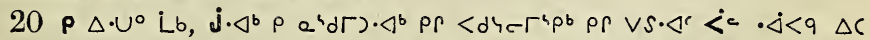

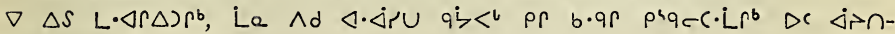
$\Delta \sigma r \cdot \Delta x$

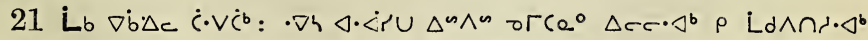

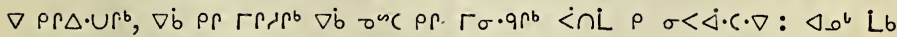

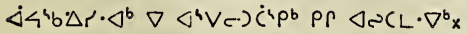

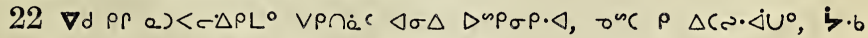

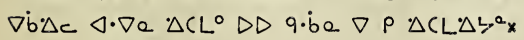




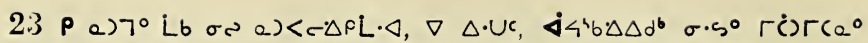

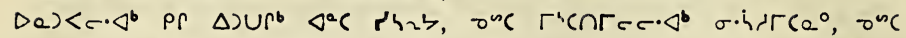

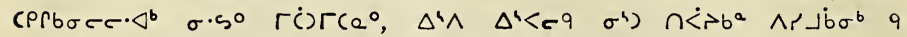

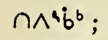

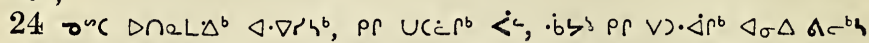
$\Delta \cap \vee-r q \cdot \Delta x$

$25 \Delta L$ Lb $P \Delta S$ Lre $\Delta 9^{\circ}$ :

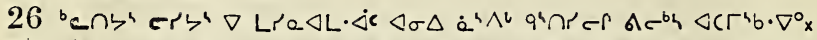

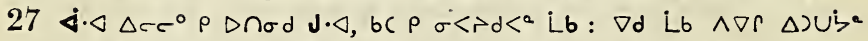

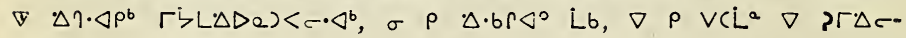
$c \Delta c_{x}$

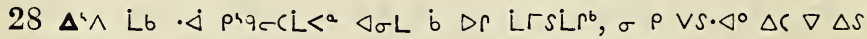
$L \cdot \Delta r \Delta)-r^{\prime}$ :

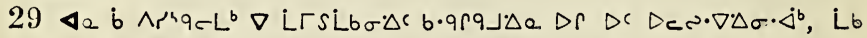

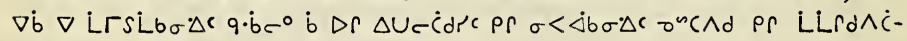
$60 \Delta c_{x}$

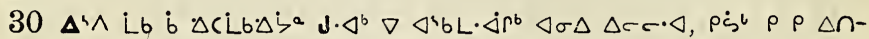

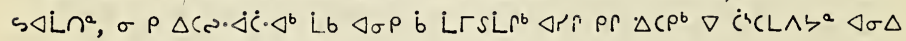

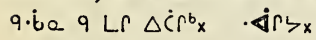

$\left.31 \nabla d D_{a}\right)<c \cdot<^{b}, \dot{b} \rho \Delta\left(\omega \cdot \Delta \dot{c} b \sigma \Delta r^{b}, \nabla n \dot{a} r^{b} \dot{<}_{c}, \sigma^{n} c 7 \cdot \dot{b}^{c} \nabla \cap \Lambda^{b} \dot{b} c^{b}\right.$ $\rho \vee s \cdot \nabla \cdot \triangleleft^{b} \Delta^{a} C \dot{\delta}^{2} \cap V^{c} \sim^{b} x$

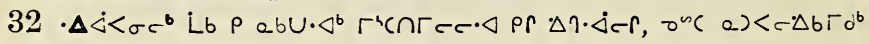
$\rho \Delta S \rho \cdot \nabla \cdot \triangleleft^{b}$ :

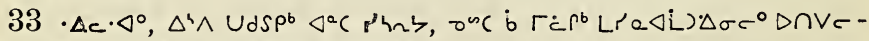

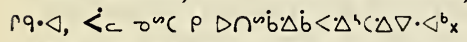

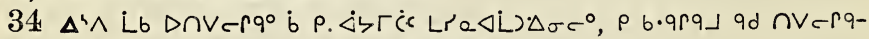

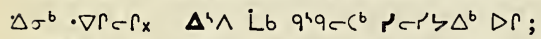

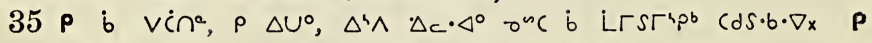

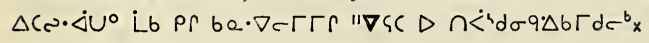

\section{A p pro $\triangle b^{2} 24$.}

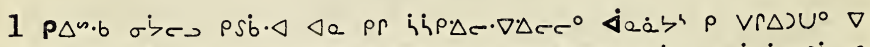

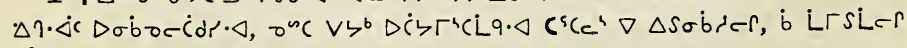
$<\sim_{c} \Delta n^{\circ} b^{\circ} \Delta \cap V e r q \cdot \Delta x$

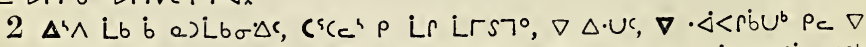

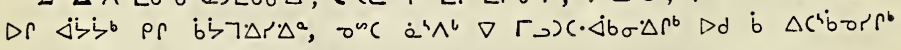

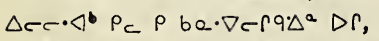

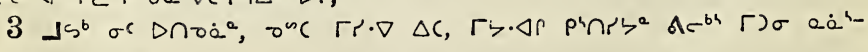
$\mathrm{d}\lrcorner \cdot \Delta q-(\lrcorner \Delta \sigma^{b} x$

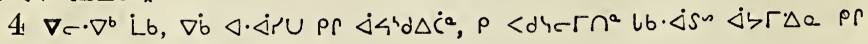

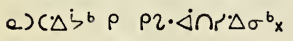

236 


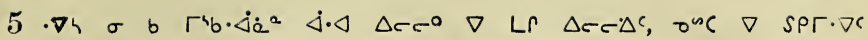

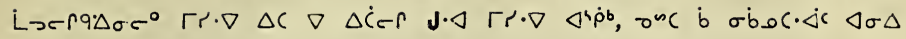
ère:

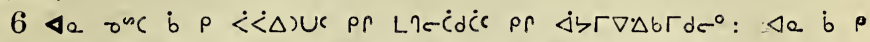

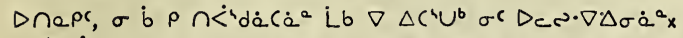

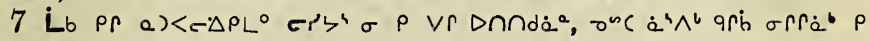
Dr $D \cap 0^{\circ}$,

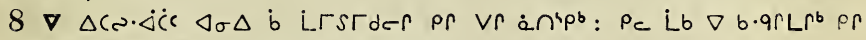

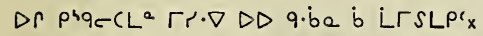

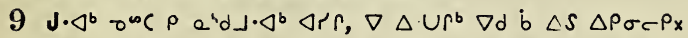

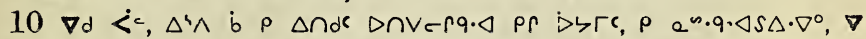

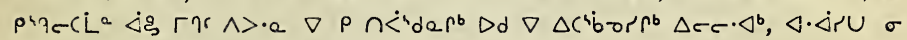
$r \cdot s U^{2} \nabla$ an.q. $\Delta S C L$ Liba :

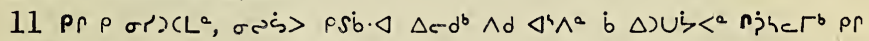

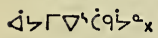

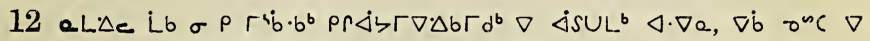

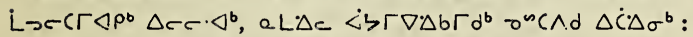

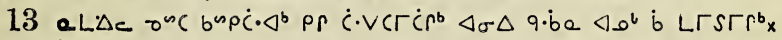

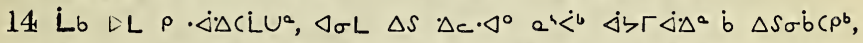

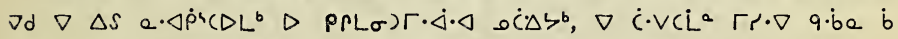

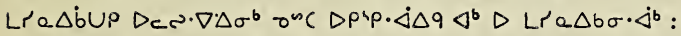

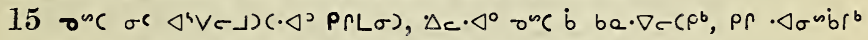

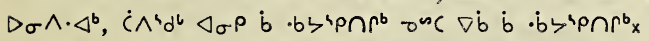

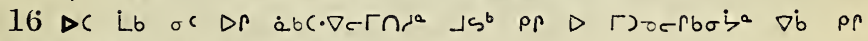

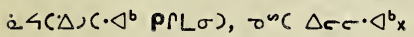

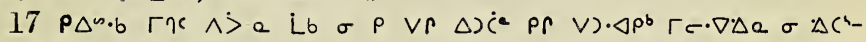
pLbeb, onc $<p \cap \sigma q \Delta e x$

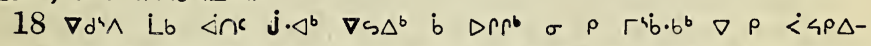

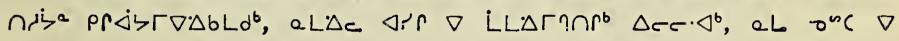
$\Gamma d^{4} b r \Delta q \zeta^{\circ} x$

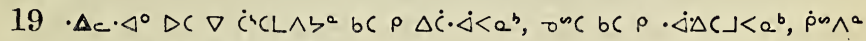

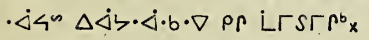

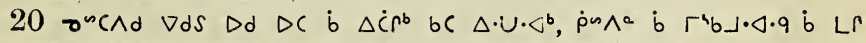

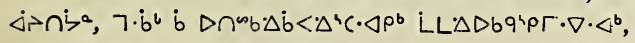

$21 \vee>^{b} \wedge d \quad D L \quad \Delta \cdot U \Delta^{a} \quad \Delta r, \dot{b} \quad \rho \quad U \cdot V b^{a} \nabla \quad \Delta \cap m b \Delta b<\cdot \Delta^{c} c \cdot \Delta \rho^{b}, \nabla$

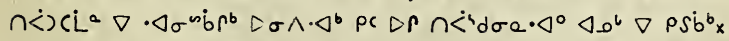

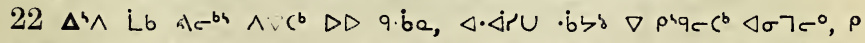

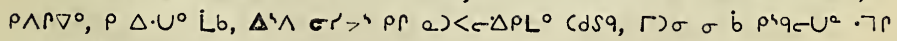
$\nabla \Delta \cap \zeta^{\circ} \mathrm{x}$

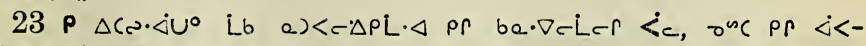

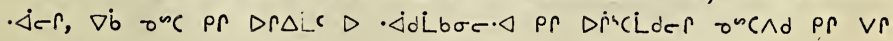
$\dot{c} \cap r^{-} r_{x}$

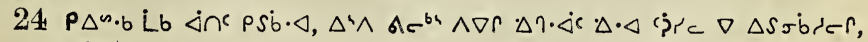
237 


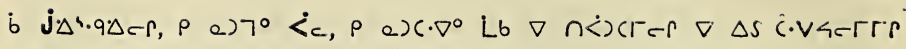
$X(x$

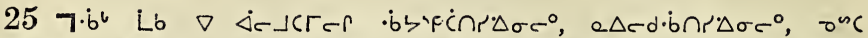

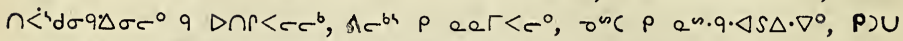
$\Delta a^{l}, \Delta^{4} \wedge a \cdot \Delta^{b} a \Delta<c \Delta d \dot{b} 0, P$ b $\left.a\right) \Gamma \cap^{2} x$

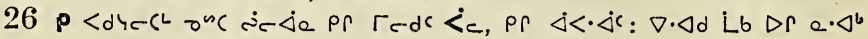
$\Gamma \eta \cdot c^{\circ} \rho$ a) $7^{\circ}, 0^{n c} p \Delta r \Delta b \Gamma 7^{\circ} x$

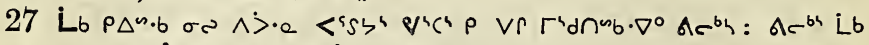

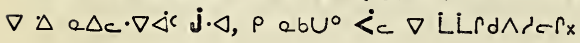

\section{$\Lambda \times p c r a \triangle b^{\circ} 25$.}

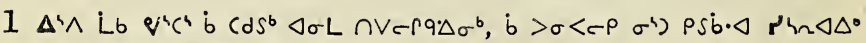

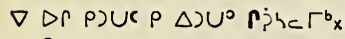

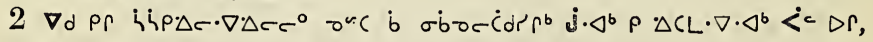
onc $P<$ dyc $7 \cdot \Delta^{b} x$

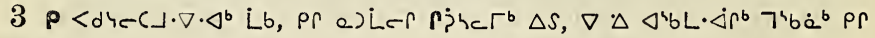
$\sigma<\dot{\Delta} \boldsymbol{r}^{b} \mathrm{x}$

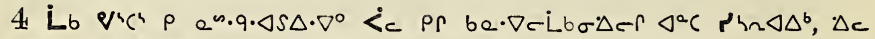

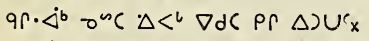

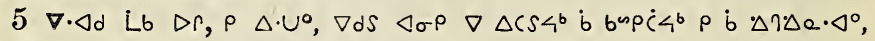

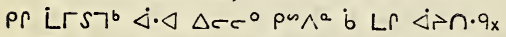

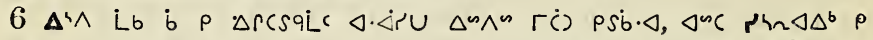

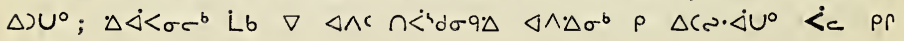
$\operatorname{VST} \Gamma_{x}$

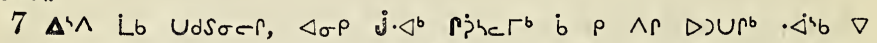

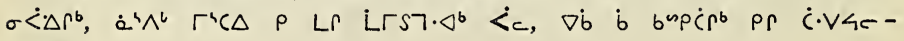
$\dot{C} d \dot{C} \Gamma^{b} x$

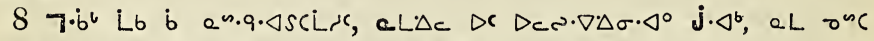

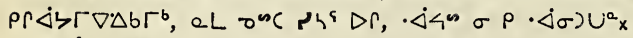

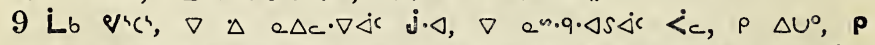

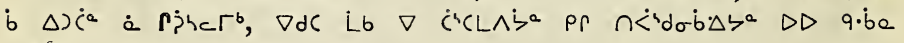
$\Delta r$ ?

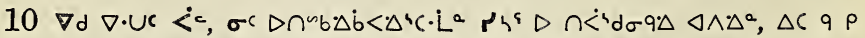

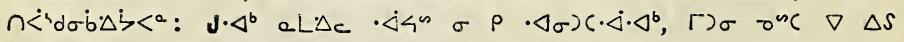
pric CLQ PCX

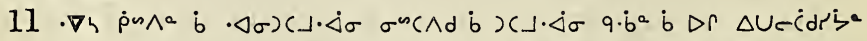

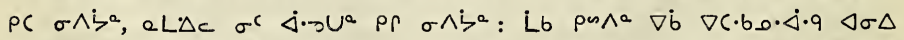

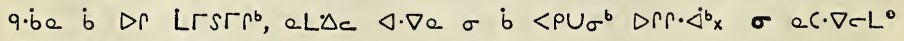

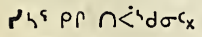

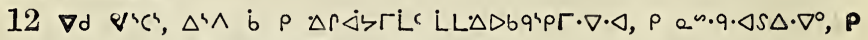

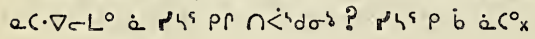




\section{$\varangle<^{4} C^{b} 25,26$.}

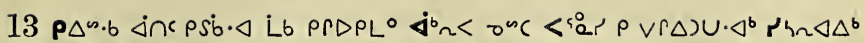

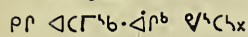

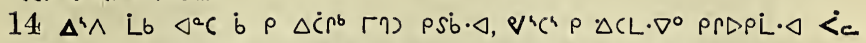

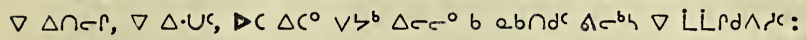

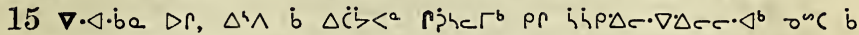

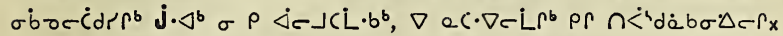

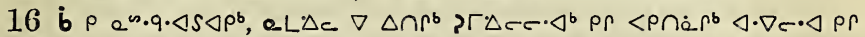

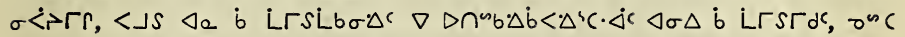

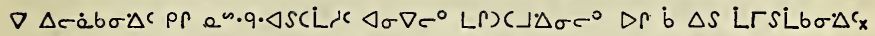

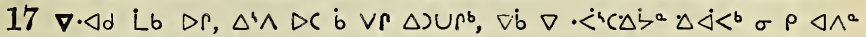

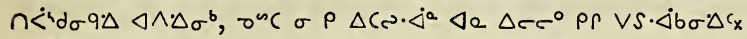

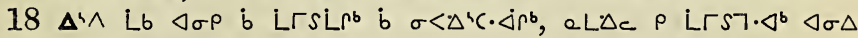
q.be $\operatorname{Dr}$ b $P \Delta U e C L^{\circ}:$

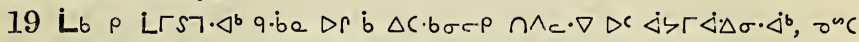

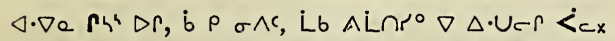

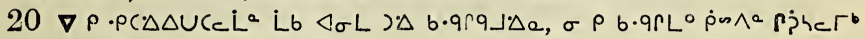

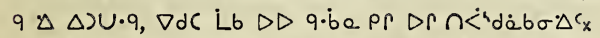

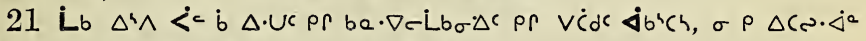
pr be. $\nabla-L b \sigma \Delta c \Delta c d^{b} q \Delta S \cap S \Delta L \cdot \Delta^{b} r^{b} h^{c} x$

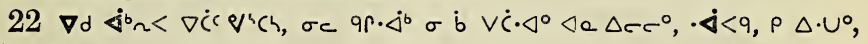
$\rho \dot{b} \vee C \cdot \triangleleft^{\circ} x$

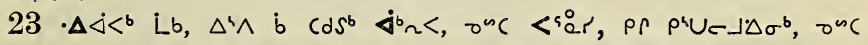

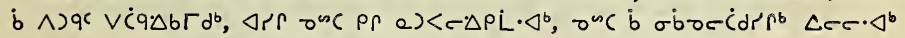

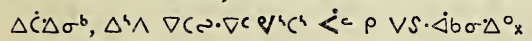

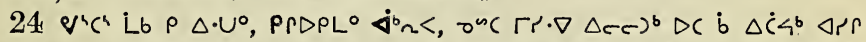

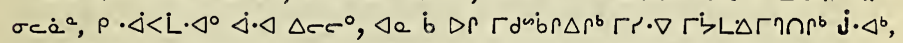

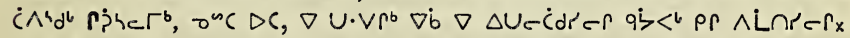

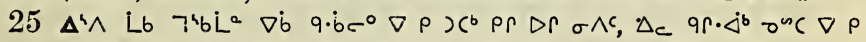

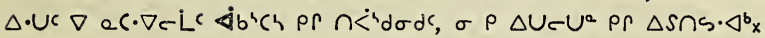

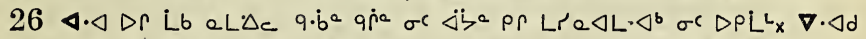

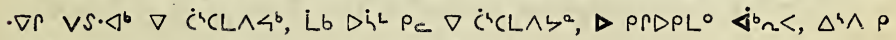

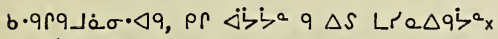

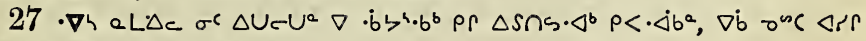

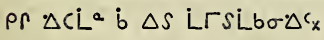

\section{A'pcre $\triangle b^{2} 26$.}

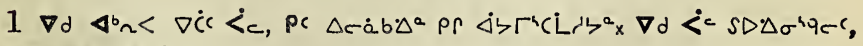
onc $p$ en.q. $\Delta S C L d x$

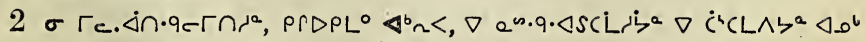

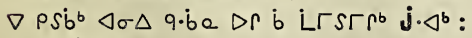

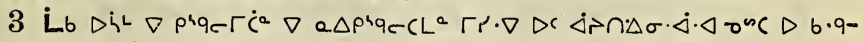

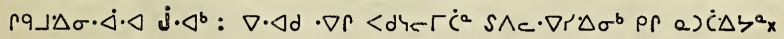




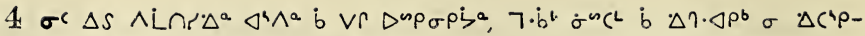

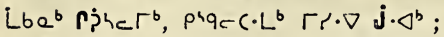

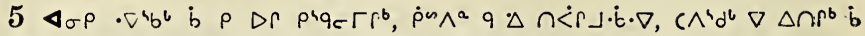

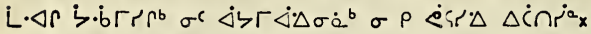

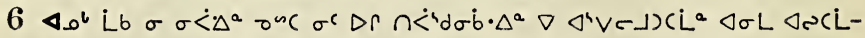
$\left.9 \cdot \Delta^{a} P R L \sigma\right)$ b $P \quad \nabla N(L \cdot \dot{<}<d \dot{C} \Delta a \cdot \Delta$ :

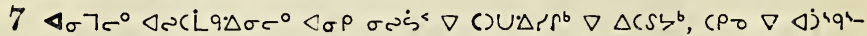

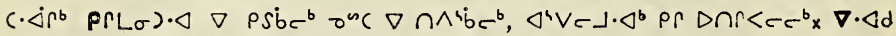

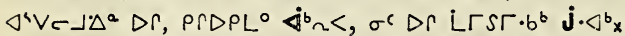

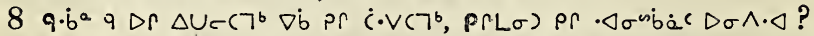

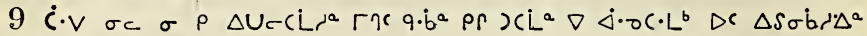
$r^{4} \dot{a} \sin b^{b} \dot{b} \Delta \dot{r i c}_{x}$

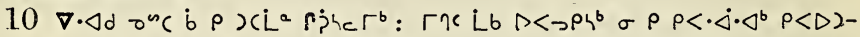

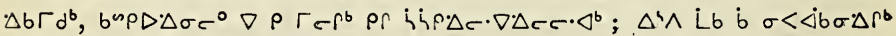

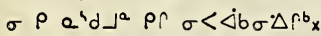

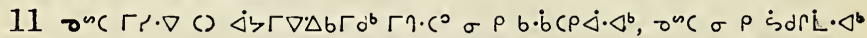

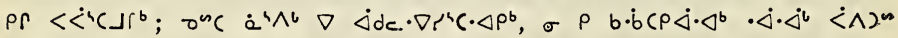
$\Delta \dot{c} \Delta \sigma^{b} x$

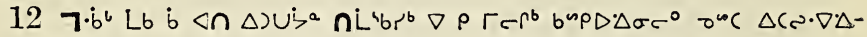
$\sigma c^{\circ}$ pr hip. $\Delta r \cdot \nabla \cdot \Delta c r \cdot \Delta^{b}$,

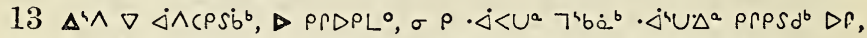

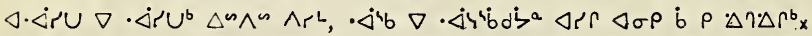

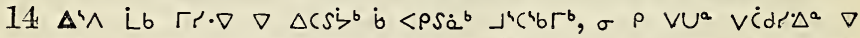

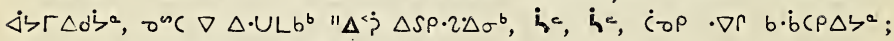

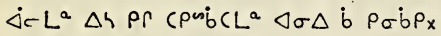

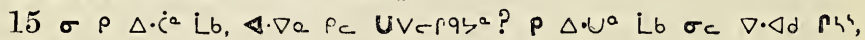
গa $\dot{b} b \cdot \dot{b} c p \triangleleft c_{x}$

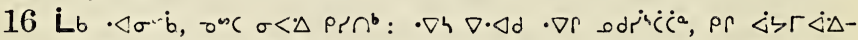

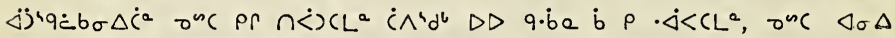
q.be 9 Dr odricic;

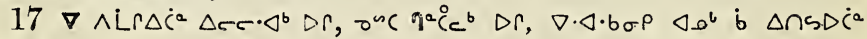
pr a $\operatorname{crb}$,

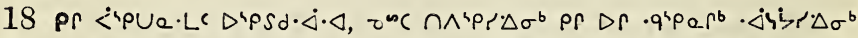

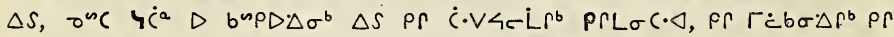

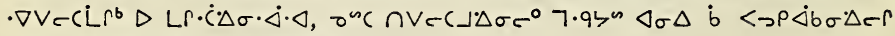
$\dot{c} \cdot V 4 C C\lrcorner \Delta^{a} \Delta r \dot{b} \vee r^{4} \dot{b} d \dot{b} \dot{s}^{a} x$

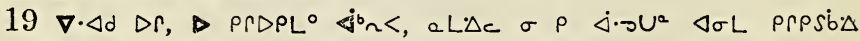
$\triangle S a\lrcorner \Delta^{a}:$

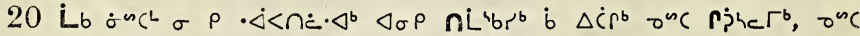

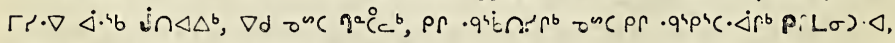

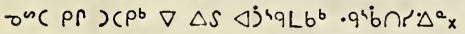

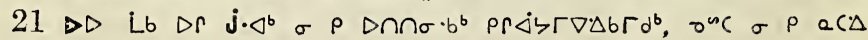
$\sigma \dot{<r} \cdot b^{b} x$ 


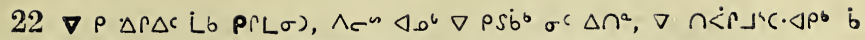

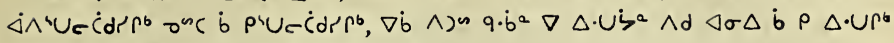

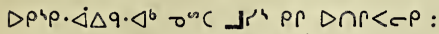

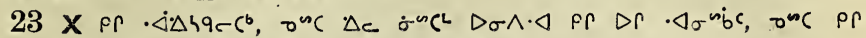

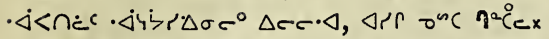

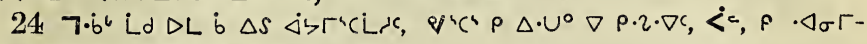
$b \Delta^{a} ; \Gamma^{\prime}\left(\Delta \Delta p^{\prime} q-C\right\lrcorner \cdot \Delta^{a} p$ par.q $\Delta d^{a} x$

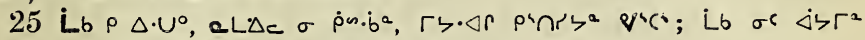

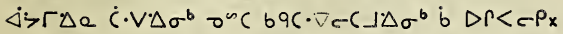

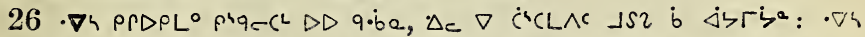

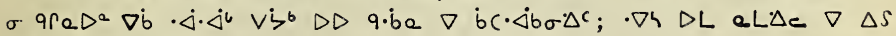

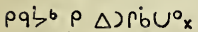

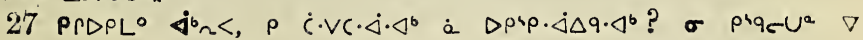
$\dot{C} \cdot V C L^{a} x$

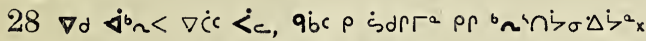

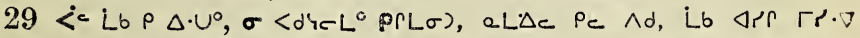

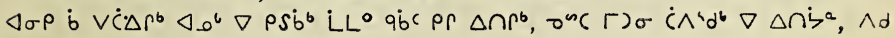

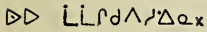

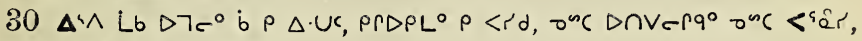
onc $\Delta \sigma \Delta \dot{b} \rho \Delta C \wedge r d r^{b}$ :

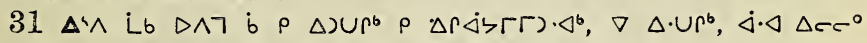

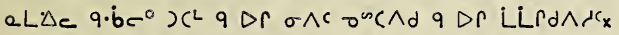

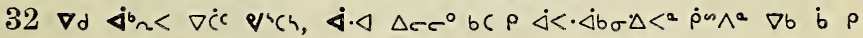
$\Delta \cdot U \cdot q$ or $\cap \dot{<} \cdot d \sigma d c$ risix

\section{Nיpcre $\triangle b^{2} 27$.}

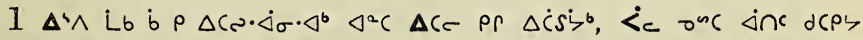

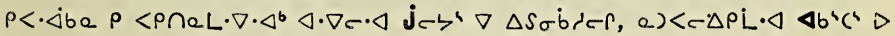
LL $\triangle \longrightarrow \cap \Gamma^{b} x_{x}$

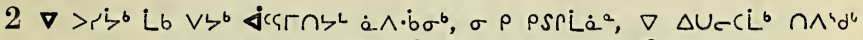

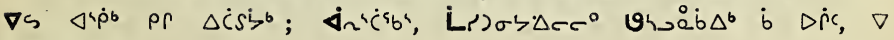
$\Delta\urcorner \Delta \succ \Gamma c_{x}$

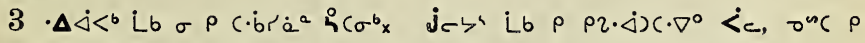

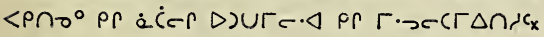

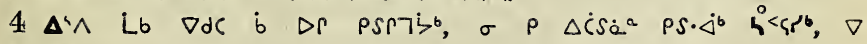
abissibx

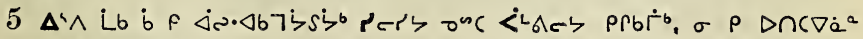
Le, $\Delta \dot{C} \Delta^{a} c r>\Delta^{b} x$

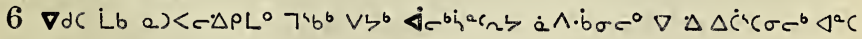
$\Delta C F ; \nabla d C$ Lb $\wedge D i \Delta>\Gamma^{C_{x}}$

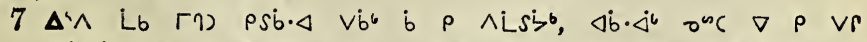

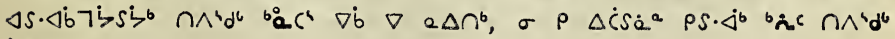
$\dot{i}=\rfloor \sigma$; 
$8 \triangleleft \dot{b} \cdot j^{b} \dot{L} b \nabla \Gamma i, \infty b \Delta L^{b}, \sigma \rho V R \Delta S r \dot{L} a^{a} \Delta C \nabla \Delta S \sigma \dot{b} U^{b} \dot{b} \Gamma \cdot \dot{c} S^{b} \nabla$ $\cap \wedge a \cdot \dot{b}^{b} ; V_{c^{3}} \Delta C \nabla \Delta C \cdot b^{b} \rightarrow r^{b} \Delta \dot{C}^{2} \Delta^{a} x$

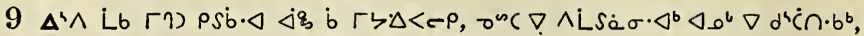

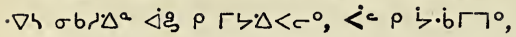

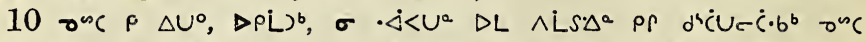

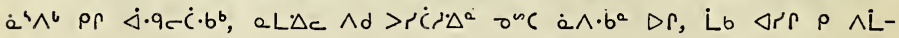
กr. $\Delta \sigma \dot{Q}^{\circ} x$

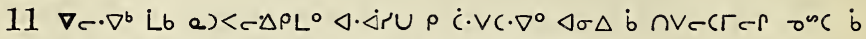

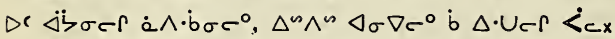

$12 \nabla \dot{b}$ Lb $\triangleleft^{a} c \nabla \cap \wedge a \cdot j^{b} \nabla \Gamma \cdot \dot{c} S^{b} \rho \rho \wedge>\sigma S \dot{a} \sigma \cdot \triangleleft^{b}, a \cdot \triangleleft^{b} \nabla \Gamma \eta \cap r^{b} \rho$

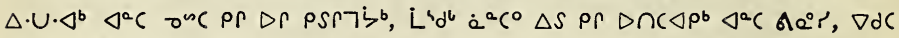

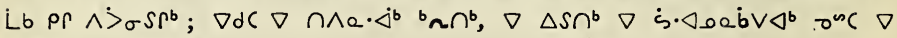
p. $\nabla \cap \rho 0 \dot{0} V \triangleleft^{b} \Delta S_{x}$

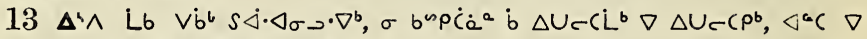

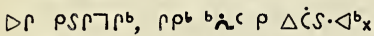

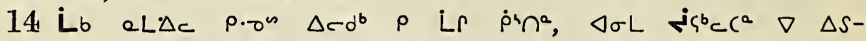
abubx

$15 \Delta^{4} \wedge$ Lb b $\Delta \cap r<c^{b} \dot{a} \wedge \cdot \dot{b}^{a}, \nabla \dot{b} \sigma^{m c} \vee \rho a \Delta<c^{b} \nabla \Delta^{4} \wedge r \rightarrow \cap^{b}, \sigma \rho$ $p r<-\Delta \dot{b} \cdot \Delta \dot{a}{ }^{a} x$

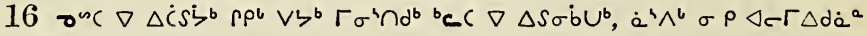
Tindria or $D$ Reib:

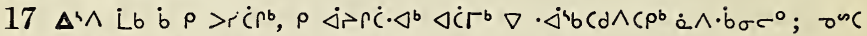

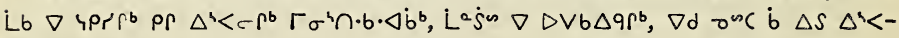
$-\dot{\Delta} b \sigma \Delta \rho^{b} \times$

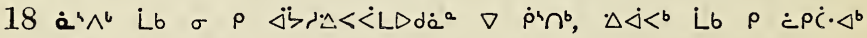
$\dot{a} \wedge \cdot \dot{b} \sigma c^{\circ} ;$

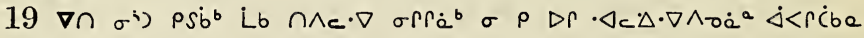
$\dot{a} \wedge \cdot \dot{b} \sigma^{b} \mathrm{x}$

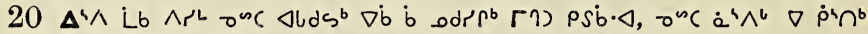

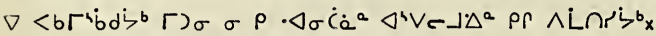

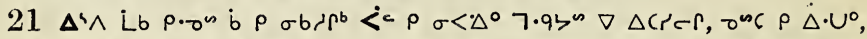

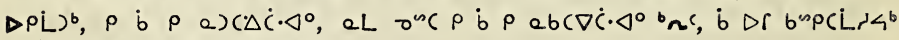

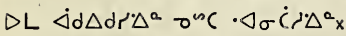

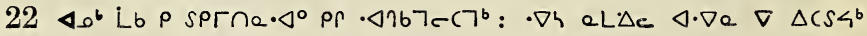
bc $\triangleleft \sigma c^{\circ} \triangleright \wedge$ L $\cap \cdot \Delta^{a}, \wedge d \dot{a} \wedge \cdot \dot{b}^{a} x$

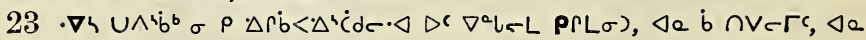
-

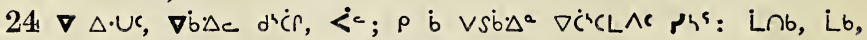

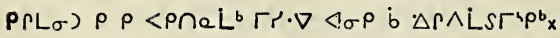

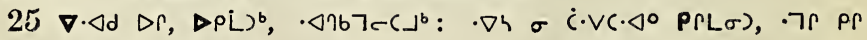
$\triangle \rho b$ b $\rho \Delta S \triangle C L b \Delta b^{\circ}$ a

$26 \nabla c \cdot \nabla^{b} L b \rho b$ b $\left\langle\cdot b i s e \cdot \Delta^{0} \vee \zeta^{b} \Gamma \sigma^{4} \cap d^{b b}\right.$

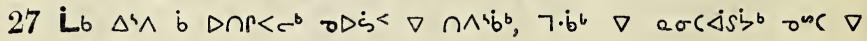
242 


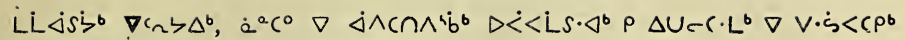
$\dot{a}^{a}{ }^{\circ}{ }^{\circ} \triangleleft^{1} p c^{\circ} ;$

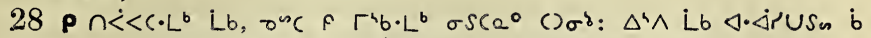

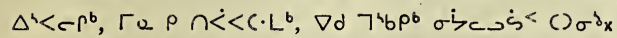

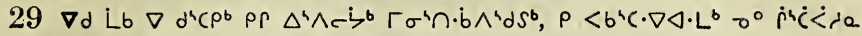

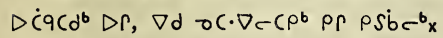

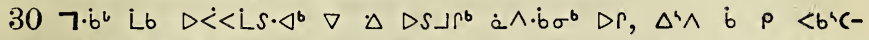

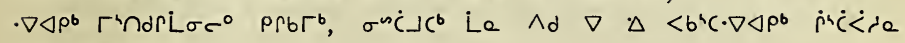
$\dot{\sigma} \backsim \dot{C}\lrcorner \dot{C} d^{b}$

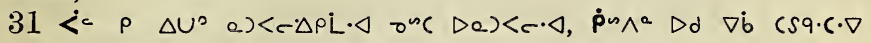
$\dot{a} \wedge \cdot \dot{b} \sigma^{b}, a L \Delta c \rho \dot{b} \wedge \operatorname{Ln} r_{a} \cdot \Delta^{\circ} x$

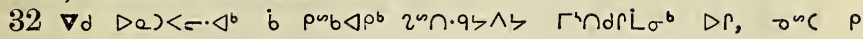
$<P \cap \dot{C} \cdot \triangleleft^{b} x$

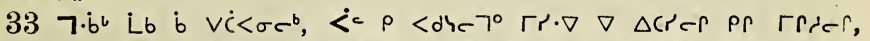

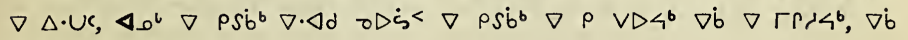
$9 \cdot 6^{a} \nabla P D \cap a 7^{b} x$

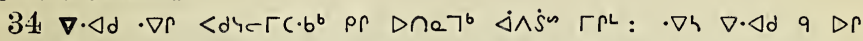

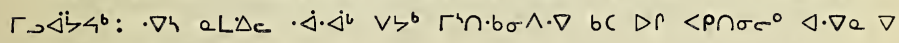
$\Delta C S 4^{b} D^{n} n \cdot b \sigma^{b} x$

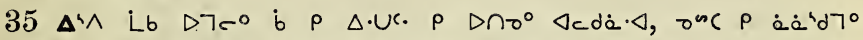

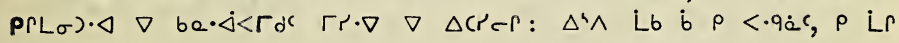
resix

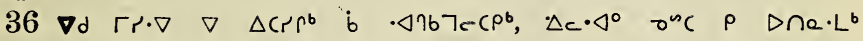
$\operatorname{Lr} \leftarrow c_{0} x$

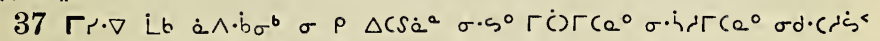
(c) $\Delta i \cdot b^{b} x$

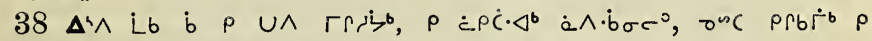
$<b^{4}<\cdot \nabla \cdot \nabla \wedge^{-} \cdot \Delta^{b}<\cdot 92 b \sigma \Gamma a_{x}$

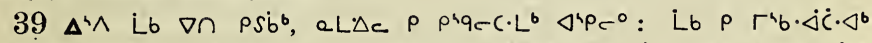

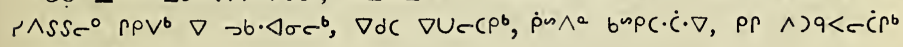
$\dot{a} \wedge \cdot \dot{b}_{\sigma} c^{\circ} \mathrm{x}$

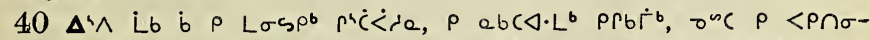

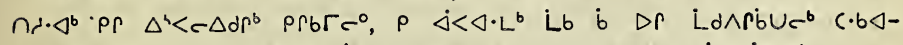

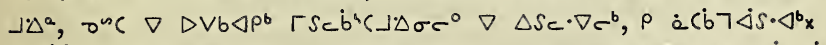

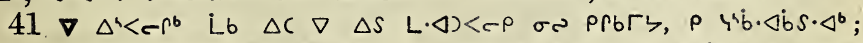
$\Delta \sigma L$ Lb $\left.\left.\dot{\sigma}^{\infty} \dot{C}\right\lrcorner c^{b} \rho \quad \Gamma r\right\lrcorner n^{a}, \nabla \dot{b} \nabla \wedge r r<c^{b}, L b \quad \Delta \sigma L \quad \nabla \dot{C} q c^{b} \rho \wedge d n^{a}$ b $\triangle 4 \wedge r L L b \Delta^{b} x$

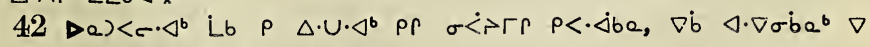

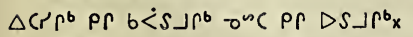

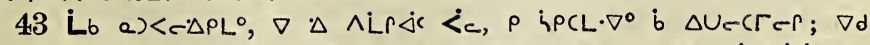

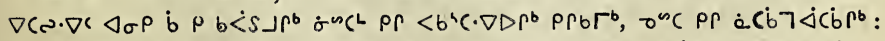

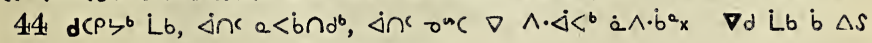

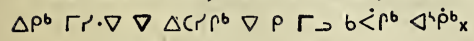

243

$2 \mathrm{R}$ 


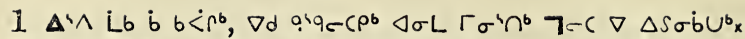

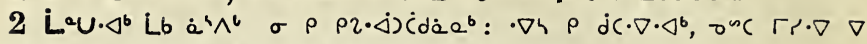
$\left.\Delta C S b^{b} \sigma \rho \wedge\right) b \Delta d \dot{a} a^{b}, \cdot \nabla \zeta \rho P \Gamma \cdot \Delta^{a} \sigma_{a n c} \rho\left(b_{b}{ }^{\circ} x\right.$

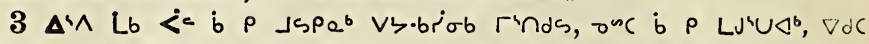

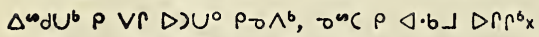

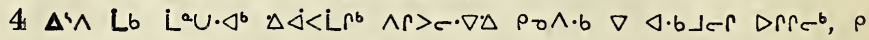

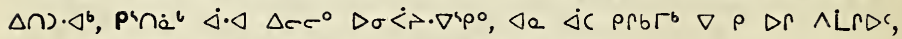

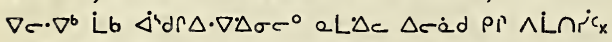

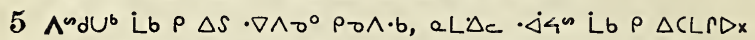

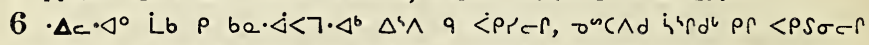

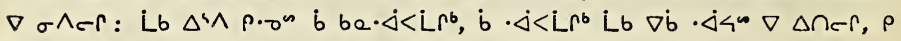

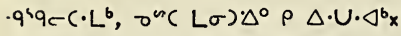

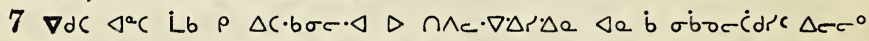

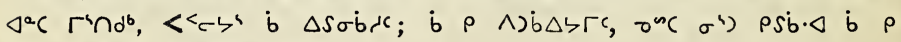

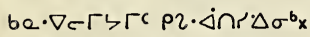

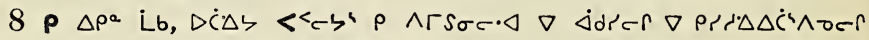

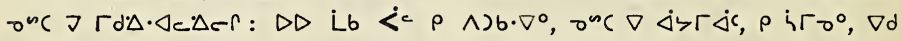
$7 a \cdot \Delta r \dot{\Delta c} c_{x}$

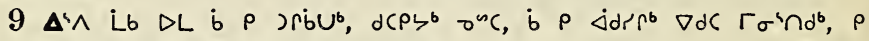
Vr $\Delta J U \cdot \Delta^{b}, \nabla d$ Lb b $r a \cdot \Delta r \Delta b \sigma \Delta r^{b}$;

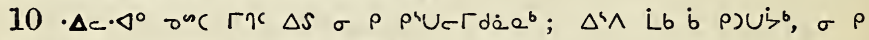

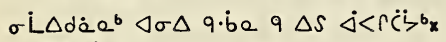

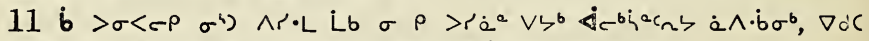

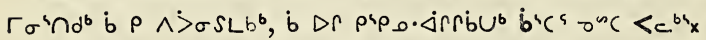

$12 \nabla b<b^{b} L_{b} \varangle^{a} C h s d^{b}, \Delta^{a} C \sigma \rho\left(s \dot{b} \dot{\alpha}^{a} \sigma^{n}\right) \rho s \dot{b} \cdot \Delta x$

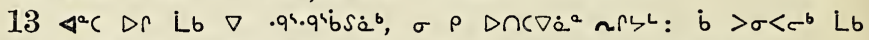

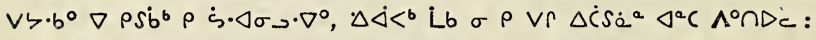

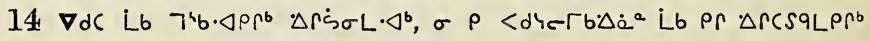

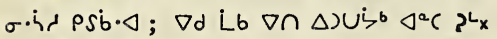

$15 \nabla d C \quad \nabla r \quad L b, \Delta^{4} \wedge \quad \Delta r \dot{s} \sigma \dot{L} \cdot \Delta^{b} \wedge \nabla C \rho b \quad \nabla \quad \Delta \dot{C} r \Gamma \dot{b} \Delta \dot{b} b, \sigma \quad \rho \quad V R$

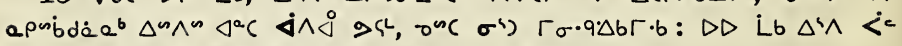

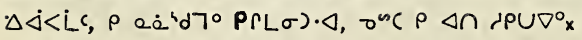

$16 \Delta^{\prime} \wedge$ Lb $\nabla \cap\left(L^{b}{ }^{\prime} L, a\right)<C \Delta P L^{\circ} \rho<P \cap a L \cdot \nabla^{\circ} \rho<\cdot \Delta b a \quad \Delta \sigma \Delta$ b

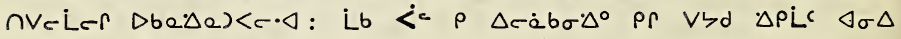
$\nabla e)<c \cdot \triangleleft$ b $b a \cdot \nabla e r d d_{x}$

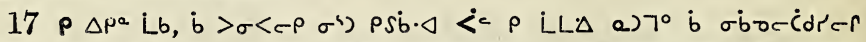

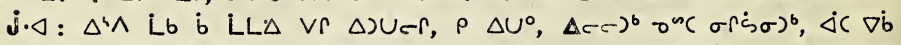

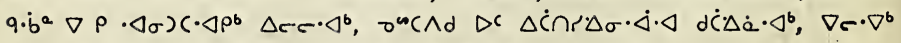

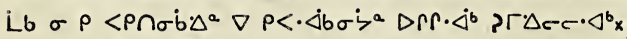

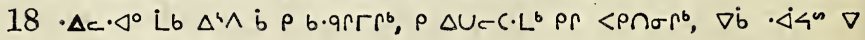
$\Delta \cap \dot{\gamma}^{a}$ q $\Delta r \sigma \dot{<} \Delta \dot{b} \Delta \dot{\zeta}^{\circ} x$

244 


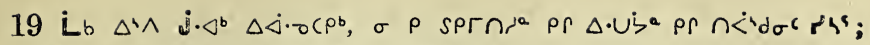

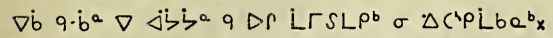

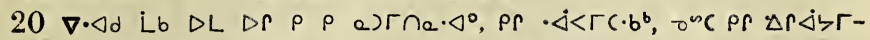

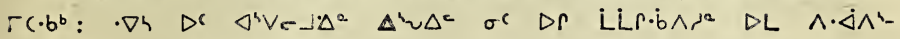
$.9 \dot{ }{ }^{2} \wedge D R_{x}$

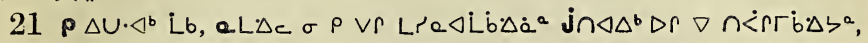

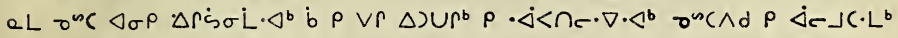

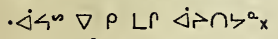

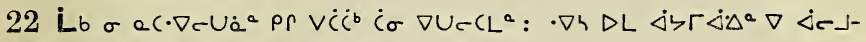

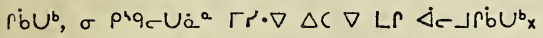

$23 \Delta^{4} \wedge$ Lb b $P$ DCPCL. $\Delta \beta^{b} \vee>^{b} \nabla \rho S \dot{b}^{b}, \Gamma \eta c \rho \vee \dot{Q} U \cdot \Delta^{b} \Delta P c^{b} ; D D$

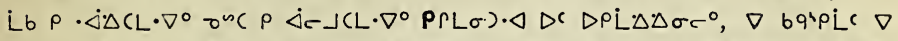

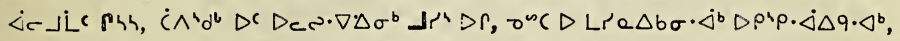
$\nabla \rho \rho z<\ddot{\zeta} c^{b} \Delta \rho \wedge c^{n} \nabla D \dot{C} d S \sigma c^{b} x$

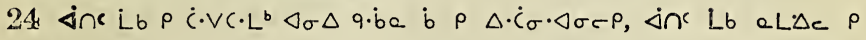
$\dot{C} \cdot V C \cdot L^{b} x$

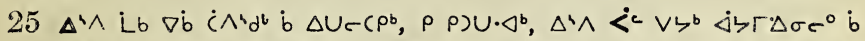

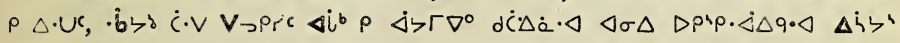
$\Delta R$,

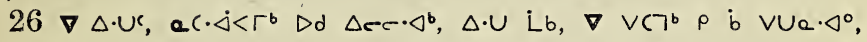

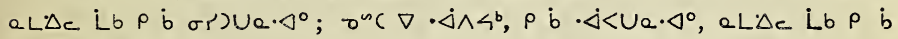
oric $\Delta$ ra. $\triangleleft^{\circ}$ :

$27 \cdot \nabla h \Delta U \Delta \cdot \Delta^{\circ} D_{d} \Delta r c \cdot \Delta^{b} \rho \triangleleft n \Delta r \Delta L b \sigma c^{\circ}, \sigma^{n C} \Delta \dot{C} \cdot \Delta b \cdot \dot{\Delta} \cdot \Delta a L \Delta c$

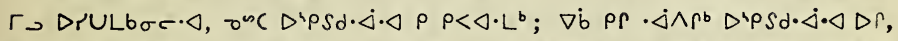

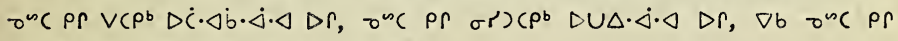

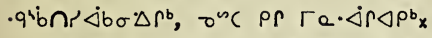

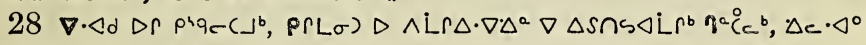
onc b $b$ a $)\left(\cdot 7 \cdot \Delta^{b} x\right.$

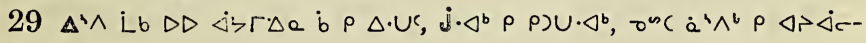
\lrcorner$(\mathrm{L}) \cdot \Delta^{\mathrm{b} x}$

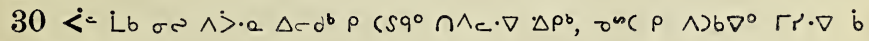
$v r$ ande,

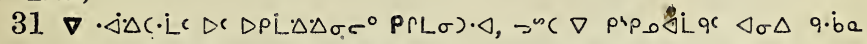

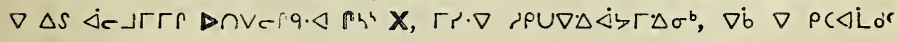
$\triangleleft \cdot \nabla c \cdot \Delta x$ 


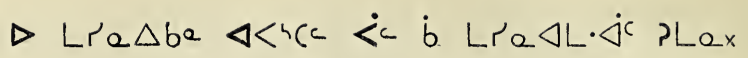

\section{Ápcra $\triangle b^{a} 1$.}

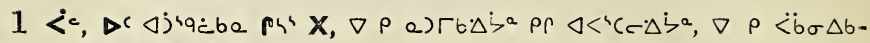
$\left.\Delta \dot{\zeta}^{a} \boldsymbol{P}(L \sigma) \triangleright \Gamma, \Delta \Gamma\right\lrcorner \Delta \sigma^{b} \Delta S$,

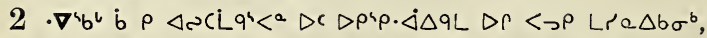

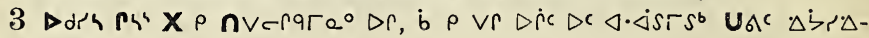
$\Delta \sigma^{b} \Delta S$;

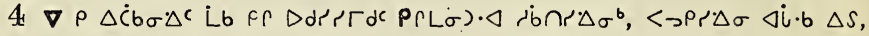
$\nabla \rho \cdot \Delta \sigma^{\omega \dot{b} \dot{\alpha} c} D_{\sigma} \wedge \cdot \triangleleft \Delta \rho:$

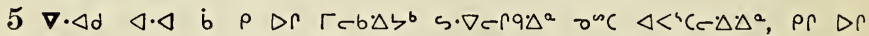

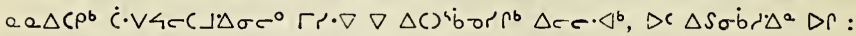

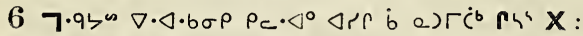

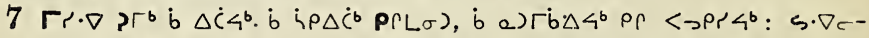

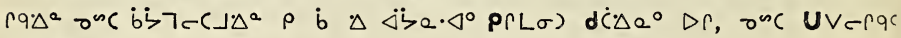
$\mathrm{P}_{4} \mathrm{XX}$

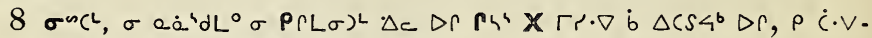

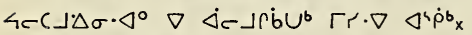

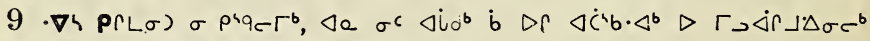

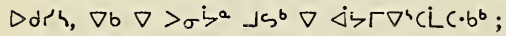

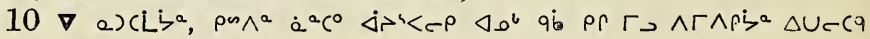
PrL $\sigma)$ Pr $V\} \dot{a} \cap \dot{C} \cdot b^{b} x$

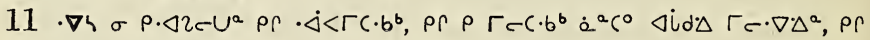
$\Delta r \quad \dot{\nabla r} r \dot{b}<\Delta \Delta b \Delta 4^{b}$;

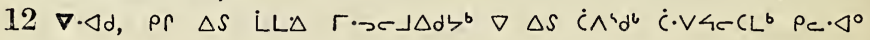
onc $\sigma c x$

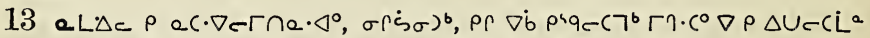

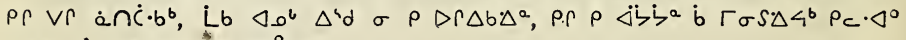

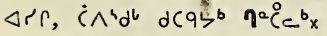

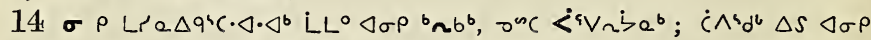

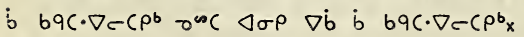

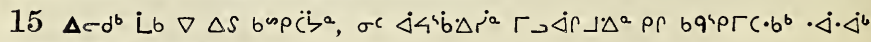

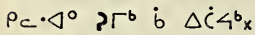

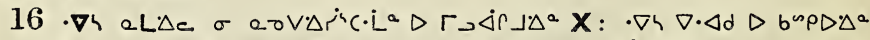

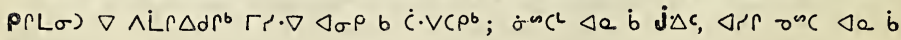
${ }^{b} \cap P \Delta C_{x}$

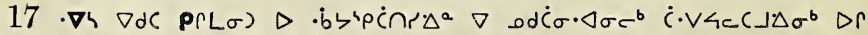

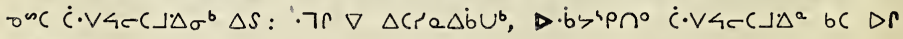
$\wedge \mathrm{Ln} r^{\circ} \mathrm{x}$ 


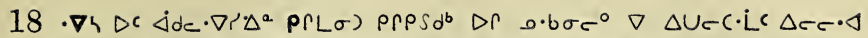

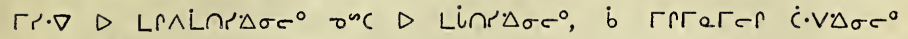
Lins. $\Delta \sigma^{b}$;

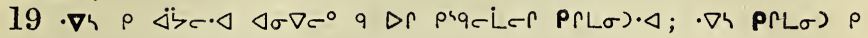
$\cdot \dot{\Delta<n>0} \mathrm{x}$

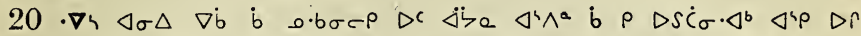

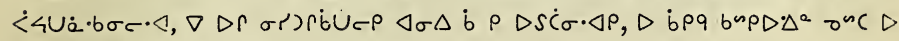

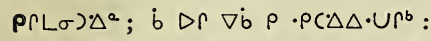

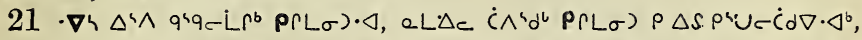

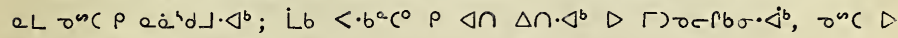

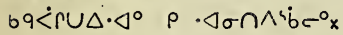

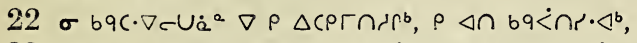

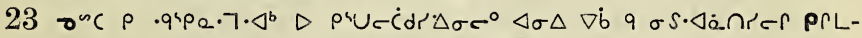

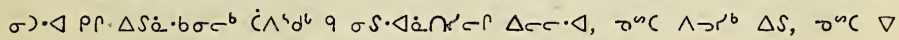

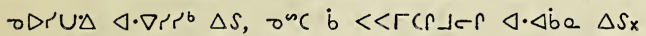

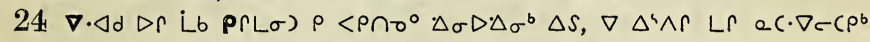

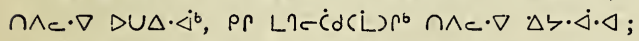

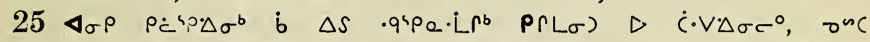

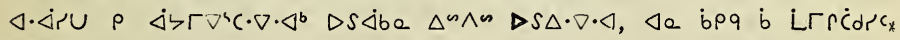
$\nabla 7^{a} \mathrm{x}$

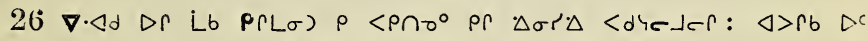

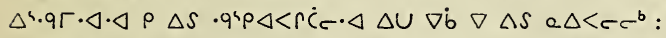

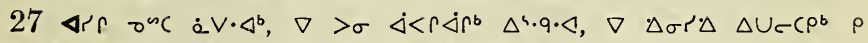

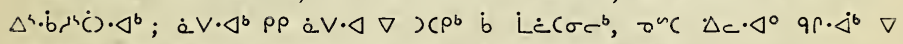
$\nabla n e \rho^{b} \triangleleft \sigma \nabla c^{\circ} \nabla \Delta S$ e $\Delta \cap\left\langle\Delta L\right.$ LLb $\sigma c^{b} D \cdot \triangleleft \sigma \Delta \Delta \sigma \cdot \Delta^{\circ}{ }_{x}$

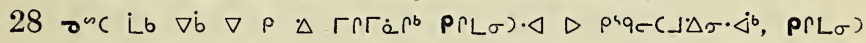

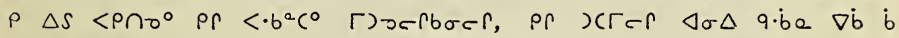
e $\Delta<\epsilon C^{\circ} ;$

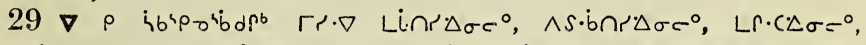

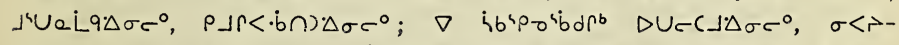

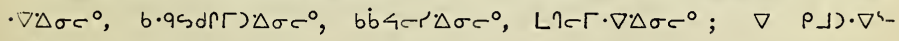
pro,

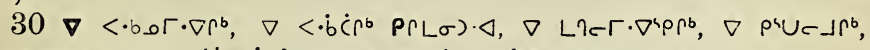

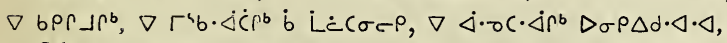

$31 \nabla b \nabla \nabla \sigma r)(\lrcorner \Delta \sigma \rho^{b}, \nabla \wedge d a \rho^{b}$ a $\left.\quad d \Gamma\right) \Delta \sigma c^{\circ}, \nabla \dot{b} \nabla \nabla i \rho \Delta \cdot \nabla \Delta \sigma \rho^{b}$, $\nabla \dot{b} \nabla>_{\sigma} \rho s \cdot \nabla c r \cdot \nabla r^{b}, \nabla \triangleleft \cdot b \cap p^{b}:$

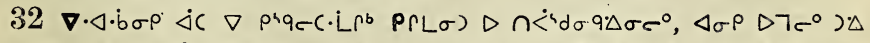

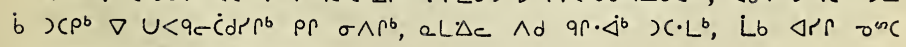
$\left.a \Delta c \cdot \nabla \Delta d \cdot \Delta^{b} \triangleleft \sigma \Delta \dot{b}\right)\left(r-r_{x}\right.$

Ápcre $\triangle b^{2} 2$.

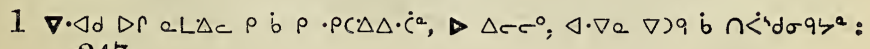
247 


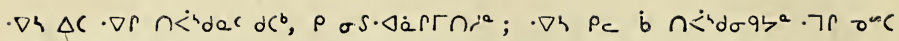
$\nabla \Delta S \Delta r)\left(L^{a} \times\right.$

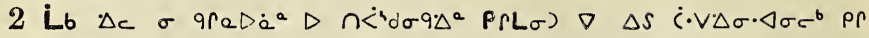
$\left.\Delta^{b}<\epsilon \Delta \rho^{b} \Gamma r \cdot \nabla \Delta \sigma \rho \dot{b}\right)\left(\rho^{b} \Delta D\right) \Delta q \cdot b a x$

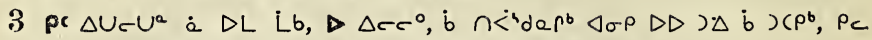

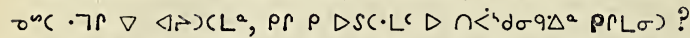

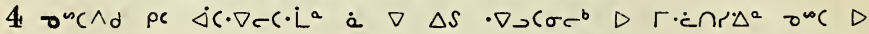

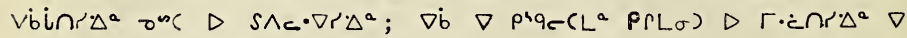
$\triangle x\left(\triangle d \cdot \Delta e\right.$ pr $r C_{C} \cdot \nabla 4 C L^{a}$ ?

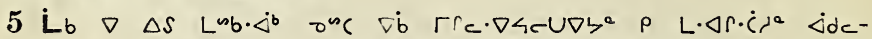

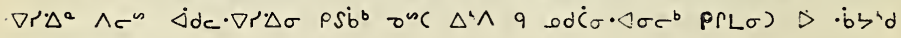
$\cap<\dot{\gamma} \sigma q^{\circ}$;

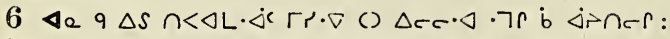

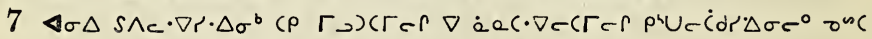

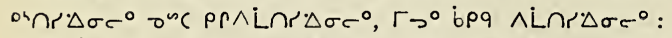

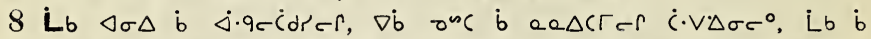

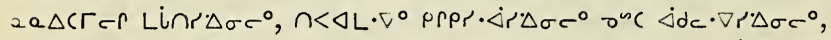

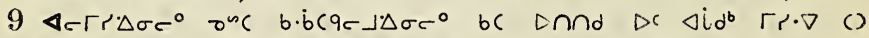

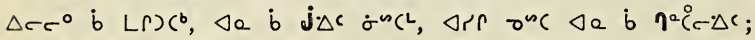

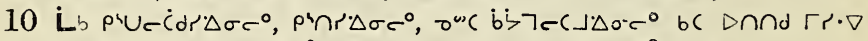

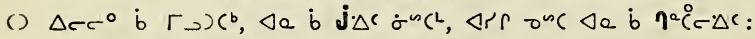

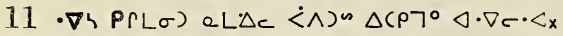

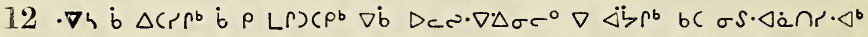

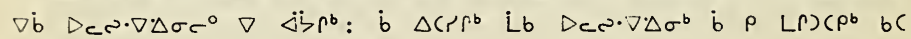
$n<\dot{L} \mathrm{~d} \sigma \mathrm{d} \cdot \Delta^{\mathrm{b}} \nabla c \omega \cdot \nabla \cdot \Delta \sigma c^{\circ}$;

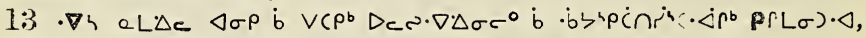

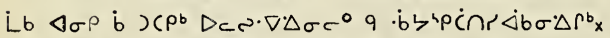

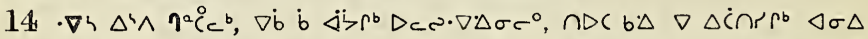

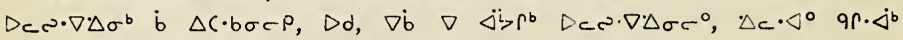
तcल. $\nabla \Delta \sigma \dot{b} \dot{C} \cdot \cdot \Delta^{b}:$

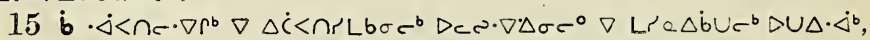

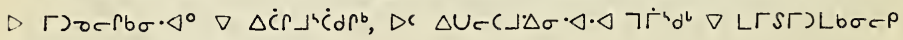

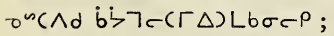

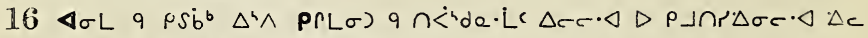

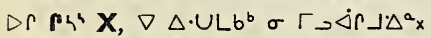

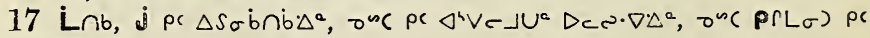
Dr $b \dot{P} \rho\lrcorner \jmath^{a}$,

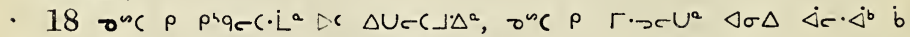

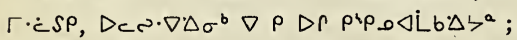

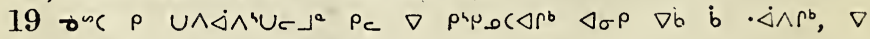

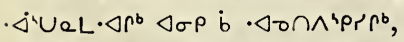

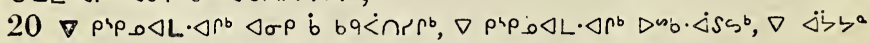

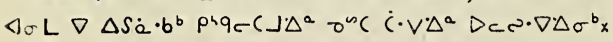




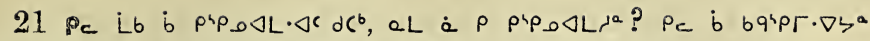

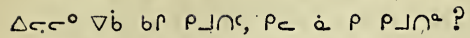

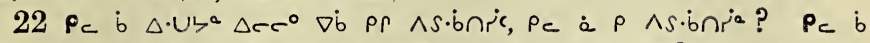

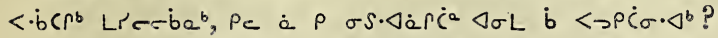

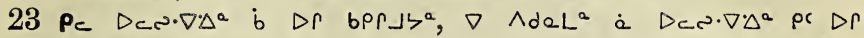
$\triangleleft\left(\cdot \nabla C L^{\circ} P(L \sigma)\right.$ ?

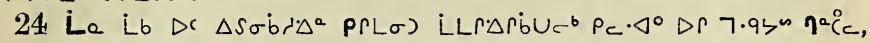
$\nabla \Delta C$ C $\triangle \dot{b} U^{b} \times$

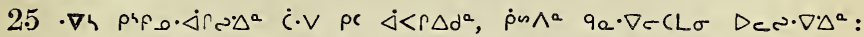

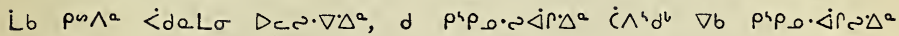
$\Delta S \dot{C} \sigma \cdot \Delta^{a} \mathrm{x}$

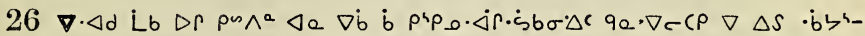

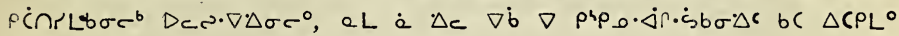

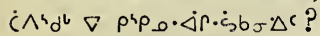

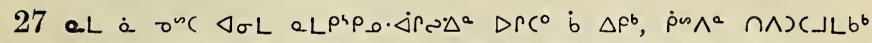

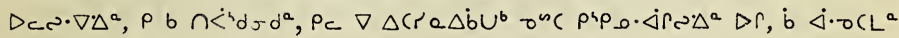
$D_{c} \cdot \nabla \cdot \nabla \cdot \Delta^{a}$ ?

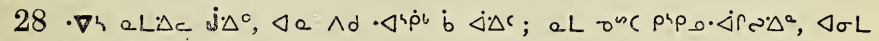

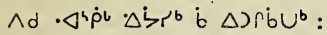

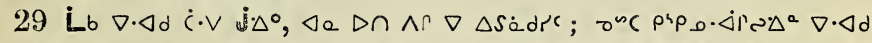

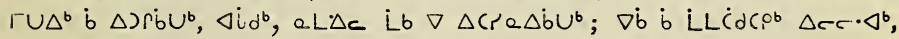
Lb $\wedge d P(L \sigma) \times$

\section{A pera $\triangle b^{a} 3$.}

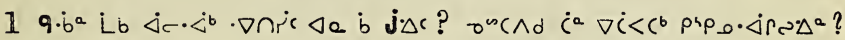

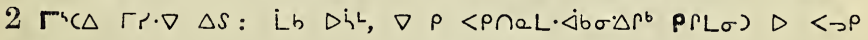
Lre $\Delta q \Delta e^{\circ}$

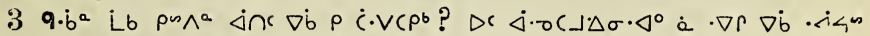
q $\triangle \dot{C}<C \sigma \sigma^{b}$ PrL. $\left.\sigma\right) D \dot{C} \cdot V<C\left(J \Delta^{a}\right.$ ?

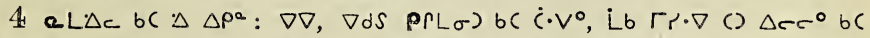

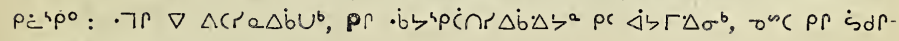
$\Delta \cdot \nabla \zeta^{a} \Delta^{4} \wedge \cap \dot{<}^{\prime} d \sigma b \Delta b \nabla x$

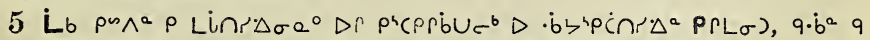

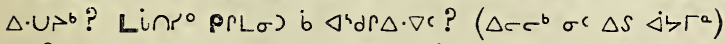

6 aL $\Delta c$ bc $\left.\Delta \Delta \rho^{a}: \cdot \nabla h \nabla d^{4} \wedge \dot{C} \sigma q \Delta S \cap<^{4} d d^{b} \Delta^{4} \rho c^{\circ} P R L \sigma\right)$ ?

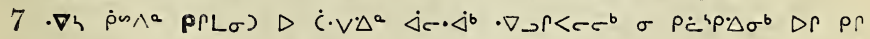

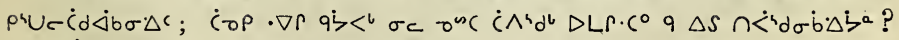

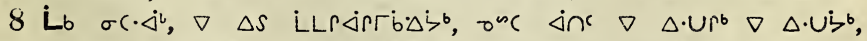

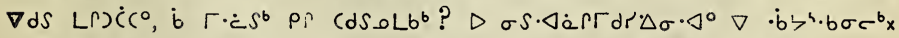

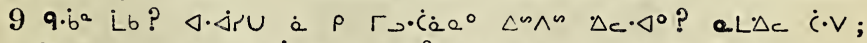

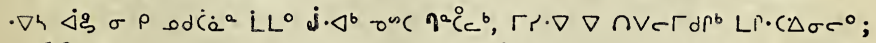

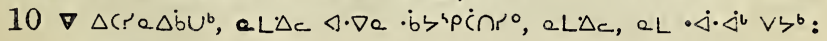
249 


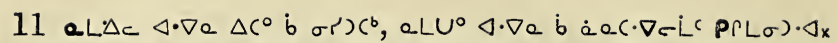

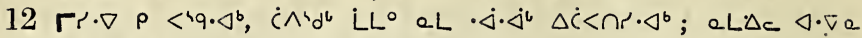
$\left.\Delta C^{\circ} \dot{b} r_{\supset}\right) c^{b}, a L \Delta c, a L \cdot \dot{\Delta} \cdot \Delta^{b} V \zeta^{b} x$

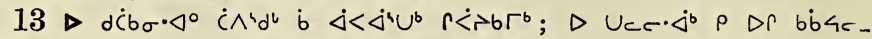

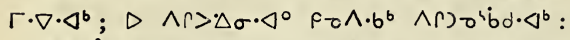

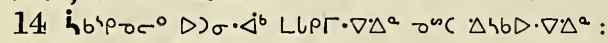

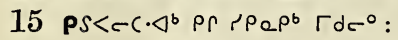

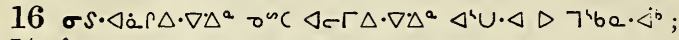

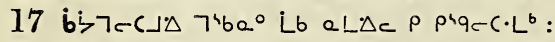

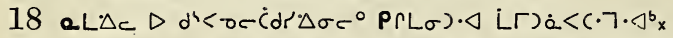

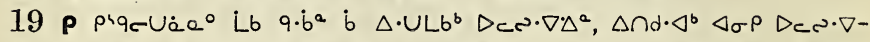

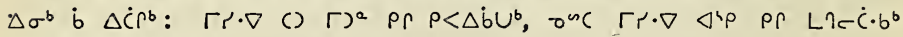
$\nabla \dot{C} C L \wedge C P \Gamma L \sigma)_{x}$

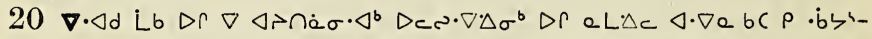

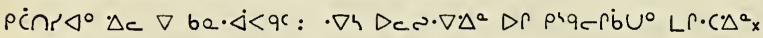

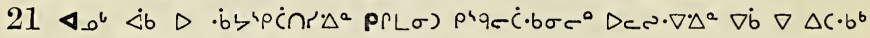

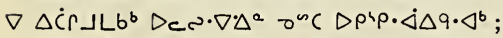

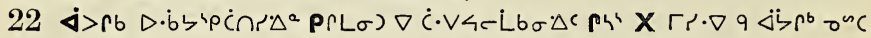

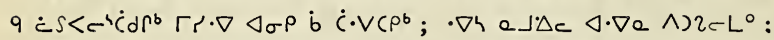

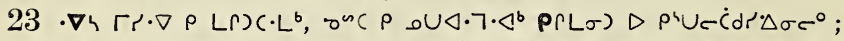

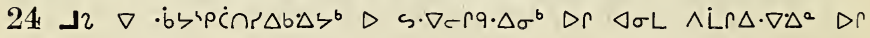
$r_{4} \mathbf{X}$ is $D C C \cdot b^{b}:$

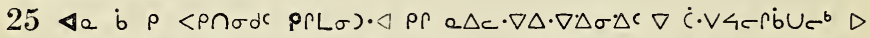

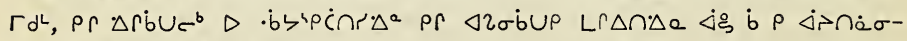

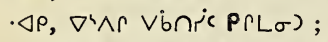

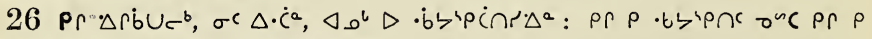

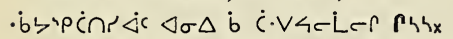

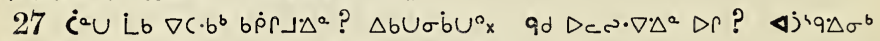

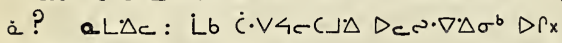

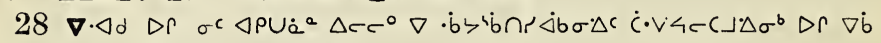

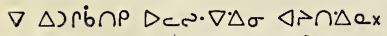

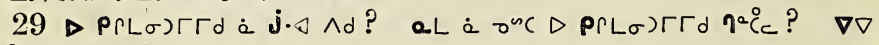

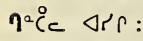

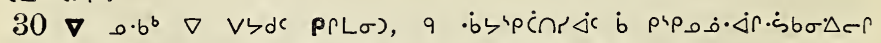

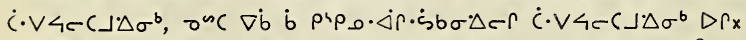

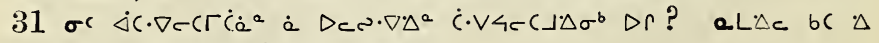

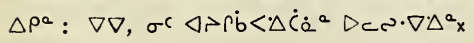

\section{Arpcre $\triangle b^{a} 4$.}

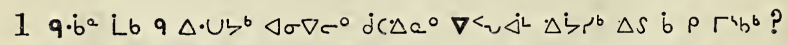

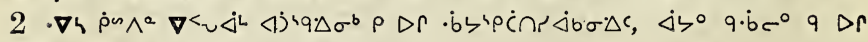

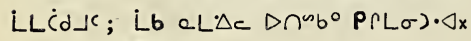




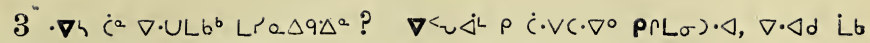

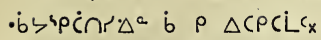

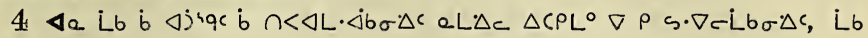
$\nabla \rho \cap<\triangleleft L \cdot \Delta b \sigma \Delta c_{x}$

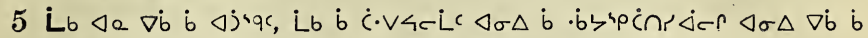

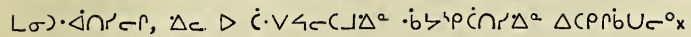

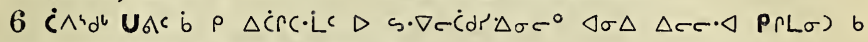
$\triangle C p i c \nabla$. bל

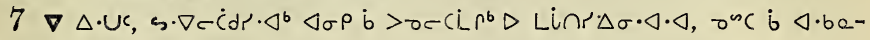
$\Delta$ L $\left.\Gamma^{b} \triangleright \operatorname{Lr}\right)(\lrcorner \Delta \sigma \cdot \triangleleft \cdot \triangleleft x$

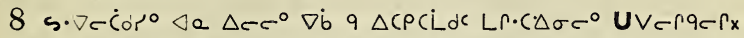

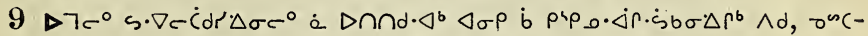

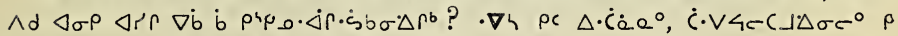

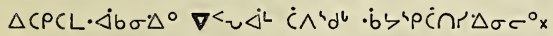

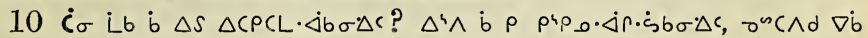

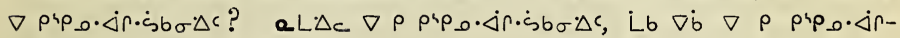
. $\dot{s} b \sigma \Delta c_{x}$

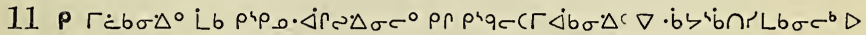

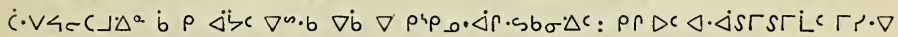

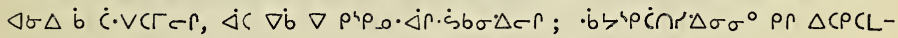

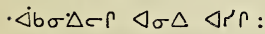

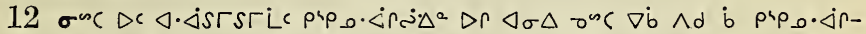

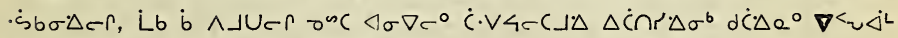

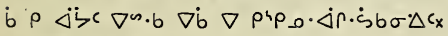

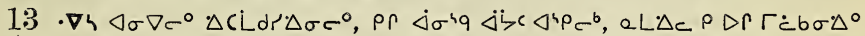

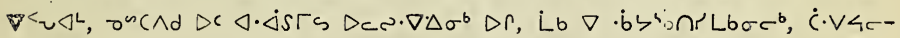
$C\lrcorner \Delta^{a} \Delta r_{x}$

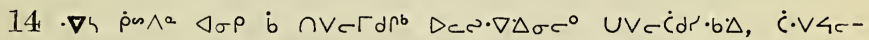

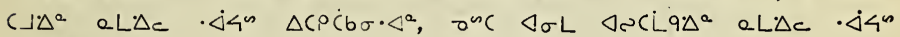
$\Delta \dot{C}<C^{a}$ :

$15 \cdot \nabla h D_{c} \cdot \nabla \cdot \Delta^{a} \dot{\Delta} \dot{d} \cdot \nabla r \cdot \Delta^{a} \Delta S C L_{b} b^{a}: \cdot \nabla h \Delta C \nabla \dot{b} \nabla \Delta C \cdot b^{b} D_{c}{ }^{2} \cdot \nabla \cdot \Delta^{a}$, QL. $\Delta c \Delta\left(\cdot b^{a} \cdot 1 \sigma\right)(\lrcorner \cdot \Delta^{a} x$

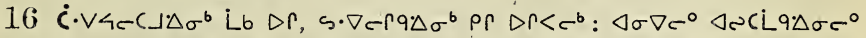

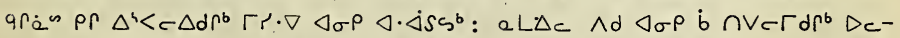

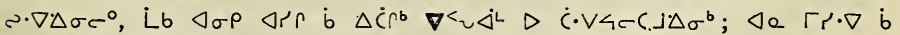
$\triangle C S\rangle^{b}$ bे $\left.\Delta \dot{C} \Delta\right\rangle^{b}$,

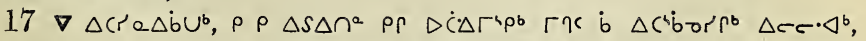

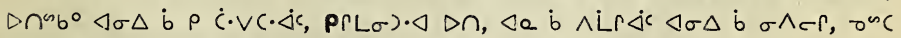
b $\Delta S \sigma \dot{b} c^{b} \triangleleft \sigma \Delta \nabla \dot{b} \dot{b} \Delta c \cdot b \sigma c \rho \dot{c} \wedge^{b} d^{b} \Delta \sigma \Delta \dot{b} \Delta c \cdot b \sigma c P_{x}$

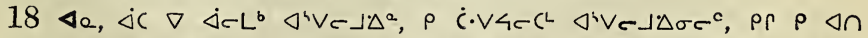

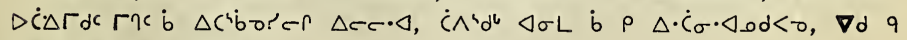

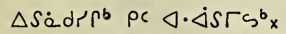

$19 \nabla \dot{b}$ Lb $\nabla$ ce 251 


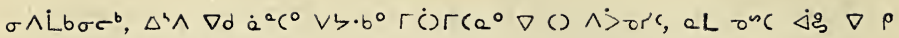
$\sigma \wedge L b \sigma c^{b} D^{4} \dot{<} \zeta 0$ is:

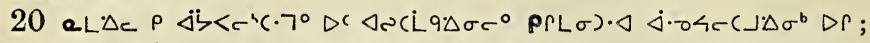

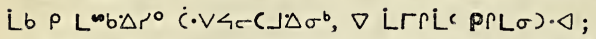

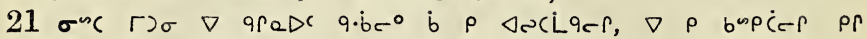
$n n)(r e p x$

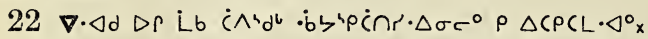

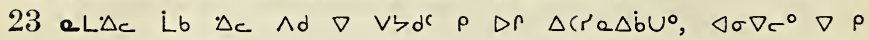
$\triangle C P C L$;

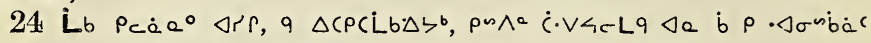

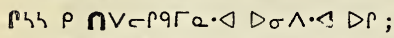

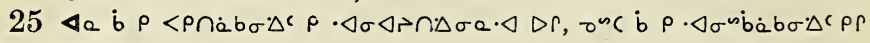

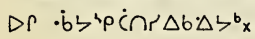

\section{A'pça $\Delta b^{2} 5$.}

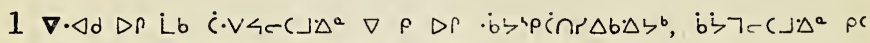

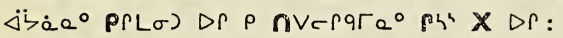

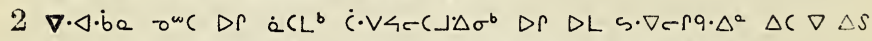

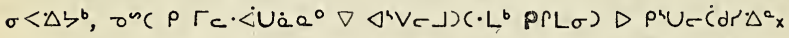

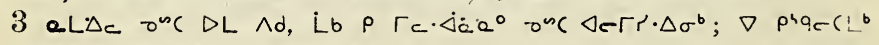
$\Delta c \Gamma \cdot \Delta^{a} \nabla \Delta^{b}<c^{b} s \wedge c \cdot \nabla r \cdot \Delta \sigma^{b}$;

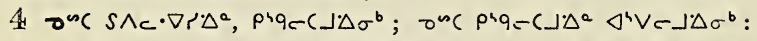

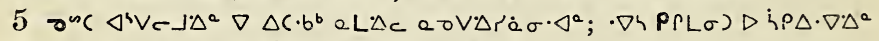

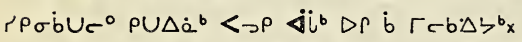

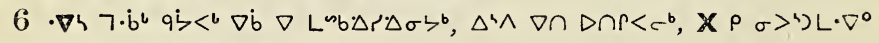
$\triangleleft \sigma \Delta \nabla \dot{b} \dot{b} \quad L \sigma) \cdot \Delta i n \tau-r_{x}$

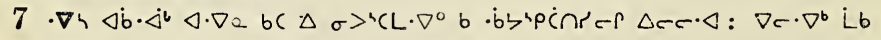

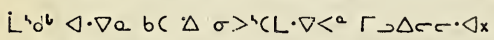

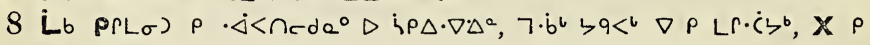
$P \sigma<^{4}\left(L^{\prime} a^{\circ} x\right.$

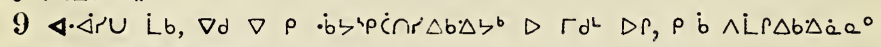
$\dot{\Delta d c} \cdot \nabla r \cdot \Delta \sigma^{b} D \rho \cdot \Delta c \Delta \rho_{x}$

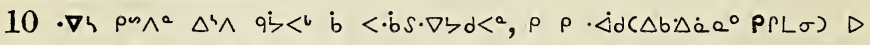

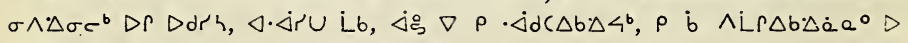
$\wedge \operatorname{Ln} \cdot \Delta \sigma^{b} D P_{x}$

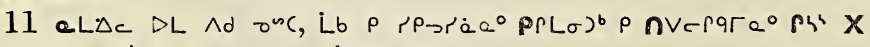
$\Delta r, \Delta a \Delta^{l} \dot{b} \Delta r \quad r-b \Delta b^{b} \cdot \Delta d d c \Delta d r \Delta^{a} x$

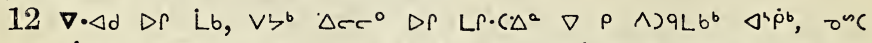
$\sigma \wedge \Delta^{a} L i \cap \Omega \Delta \sigma^{b} \nabla \Delta R<c^{b} ; \sigma^{n c} \sigma \wedge \Delta^{a} \nabla \rho<b \Gamma^{b} b d \rho^{b} \Gamma r \cdot \nabla \Delta c c \cdot \Delta^{b}, \cdot \nabla h$ $r 6 \cdot \nabla \rho L P)\left(\cdot L^{b}:\right.$

$13 \cdot \nabla h \wedge c^{\infty} \dot{b} \vee r \Delta c \cdot b^{b} \quad D_{c} \cdot \cdot \nabla \Delta^{a} L f \cdot c \cdot \Delta^{a} \rho \Delta c \cdot b^{a} \Delta^{a} \dot{p}^{b}: L \rho \cdot c \cdot \Delta^{a} L b$ a $L \Delta C \triangle C P r \dot{b} U^{\circ} \Delta^{4} \wedge \nabla \dot{b} \nabla \Delta C \cdot b^{b} D_{c} \cdot \nabla \cdot \Delta^{a} x$

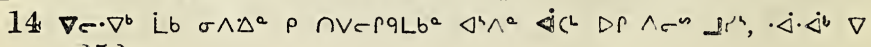
252 


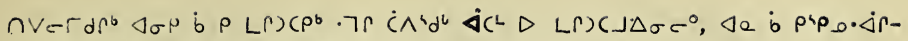
$a^{\wedge} \wedge c \cdot \Delta c \Delta \sigma \Delta \dot{b}\left(d \hat{\sigma} \sigma \sigma^{i}<{ }^{a} x\right.$

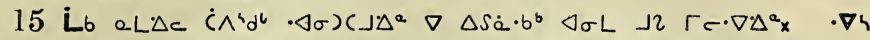

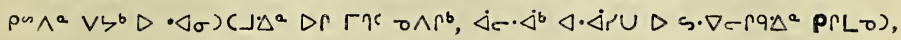

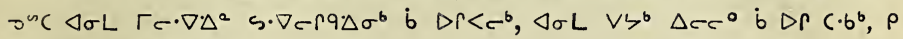
$U \wedge<\epsilon \Delta d \cdot \Delta^{b} r \eta^{x} C_{x}$

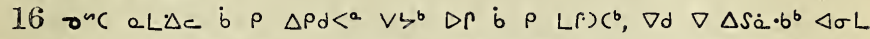

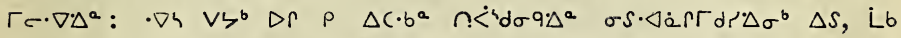

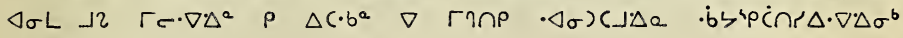
$\Delta S_{x}$

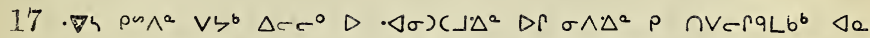

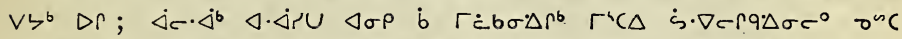

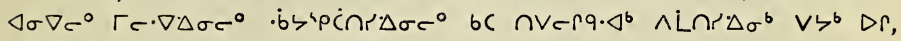
$\min ^{2} \mathrm{Xx}$

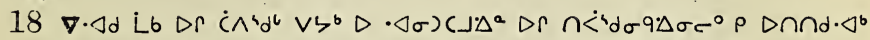

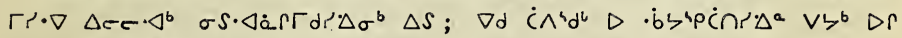

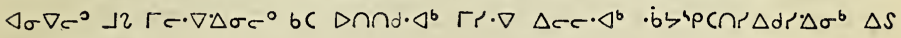
bi $\wedge$ Linters

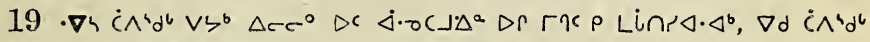

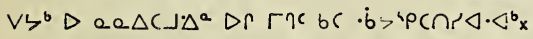

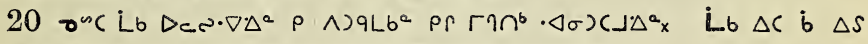

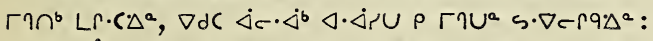

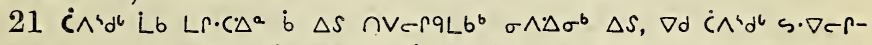

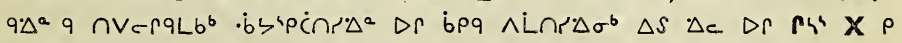
nverar $e^{\circ} x$

\section{Áp $22 \triangle b^{2} 6$.}

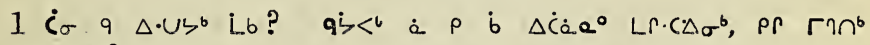
s. $\nabla c r q \Delta^{\circ}$ ?

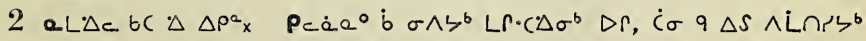
VdC $9 \dot{3}<$ ?

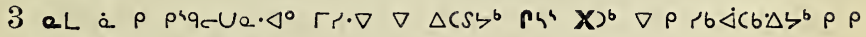
rb $\ddot{C} \dot{C} b \Delta \dot{a} 0^{\circ} \triangleright \sigma \wedge \Delta \sigma^{b}$ ?

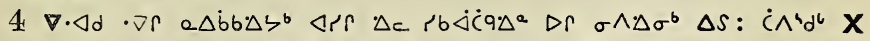

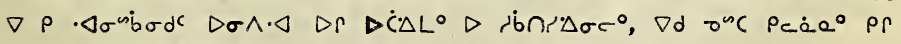

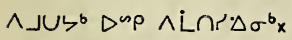

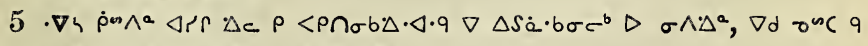
$\triangle S \dot{a} d r^{b} \nabla \Delta S \dot{a} \cdot b \sigma c^{b} \nabla \cdot \triangleleft \sigma^{c b} b \Delta^{a}:$

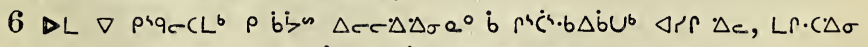

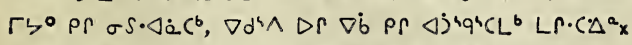

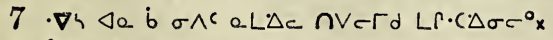

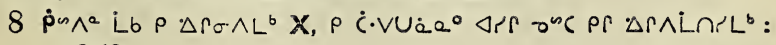
253 


\section{PLa 6,7 .}

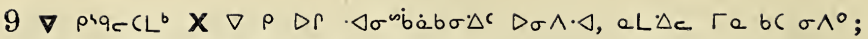

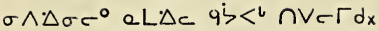

$10 \cdot \nabla h \Delta \sigma \nabla c^{\circ} \nabla \rho \sigma \wedge c, L \Gamma \cdot\left(\Delta \sigma \sigma^{\circ} \vee \zeta \cdot b^{\circ}\right.$ p Dr $\sigma \wedge^{\circ} ;$ Lb $\triangleleft \sigma \nabla \sigma^{\circ} \nabla$

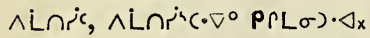

$11 \nabla d \sigma^{n C} \rho_{c} \cdot \Delta^{\circ} \Delta C P \Gamma \cap r^{b} \nabla \sigma \wedge<^{b} L r \cdot C \cdot \Delta \sigma^{b} D r$, Lb $\nabla \wedge L \cap r^{i} C \cdot \nabla^{b}$

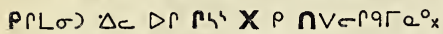

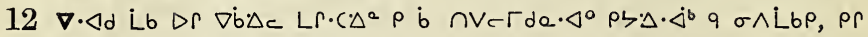
$\triangle e a \Delta C 7^{b} \nabla \Delta S$ LS $\triangle U C C \cdot L L \cdot b^{b x}$

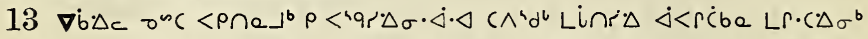

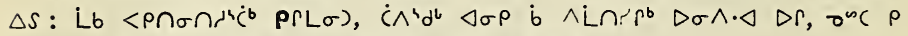

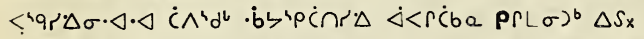

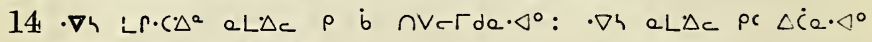
$\nabla c \omega \cdot \nabla \cdot \Delta \sigma^{b}$ Lb $s \cdot \nabla-r q \cdot \Delta \sigma^{b} x$

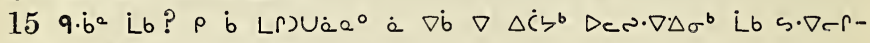
$q \Delta \sigma^{b}$ ? $e L \Delta c b c \Delta \Delta p^{a} x$

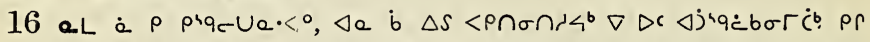

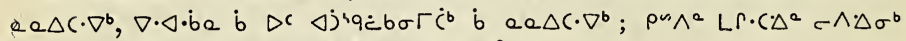

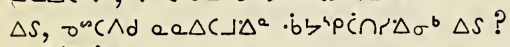

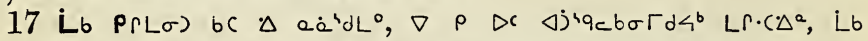

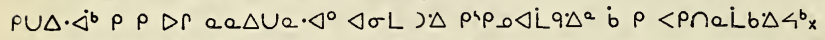

$18 \nabla \rho \Delta \cdot b r \Delta b \Delta 4^{b}$ Lb Lr.c $\Delta \sigma^{b} \Delta r, \rho \Delta \cap \Delta c \quad j^{4} q \dot{c} b \sigma \Gamma d a \cdot \triangleleft^{\circ}$ .bל

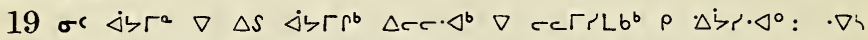

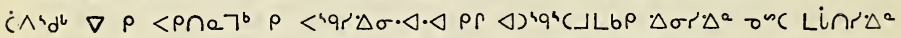

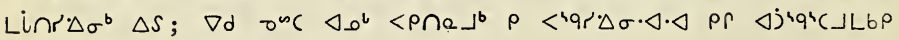
. bל

$20 \cdot \nabla h \Delta^{4} \wedge$ b $\rho \Delta c \Delta^{4} q_{c} b \sigma r d 4^{b} L \Gamma \cdot c \cdot \Delta^{a}, a L \Delta c \rho p \cap V e r d a \cdot \Delta^{0}$ .

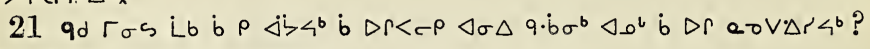
. $\nabla$ \ $\triangleright$ q.be $\Delta C\lrcorner \cdot \triangleleft \sigma \wedge \Delta \sigma^{b x}$

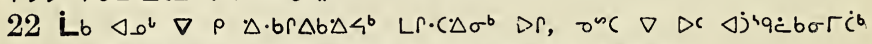

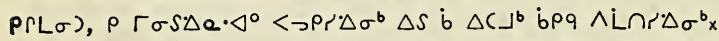

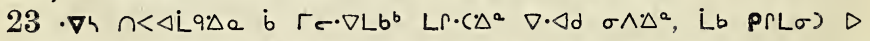

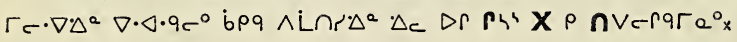

\section{Aspcra $\triangle b^{2} 7$.}

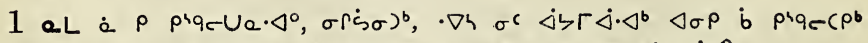

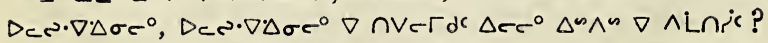

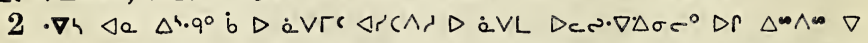

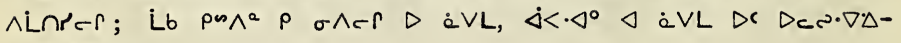
$\sigma \sigma^{\circ} \Delta r_{x}$ 


\section{PLe 7}

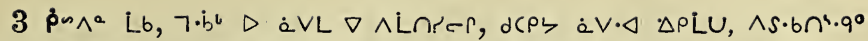

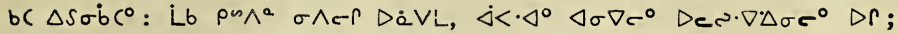

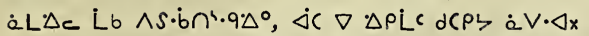

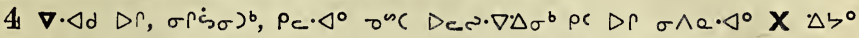

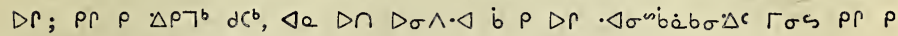
$\sigma\left(\triangle P C L \cdot \Delta^{b} P \Gamma L \sigma\right)_{x}$

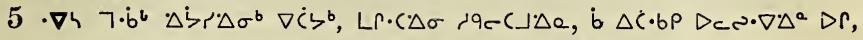

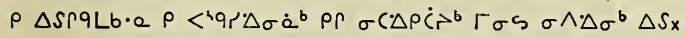

$6 \triangleleft_{0^{6}}$ Lb $\rho \Delta \cdot b r \Delta b \Delta \dot{a} a^{\circ} D c S \cdot \nabla \Delta \sigma^{b} \Delta R, \nabla \sigma \wedge \zeta^{b} \triangleleft \sigma L D r b \dot{b} \rho \cap V e-$

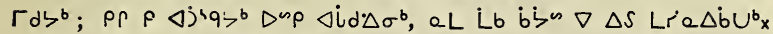

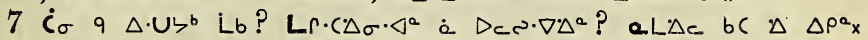

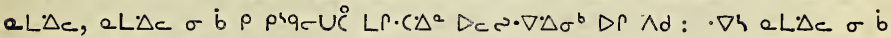

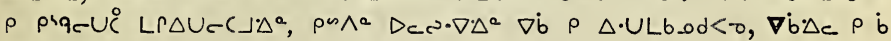
J'UeLiba

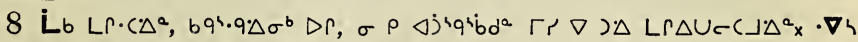
$\nabla \dot{b} \nabla \Delta c \cdot b^{b} D_{c} \cdot \cdot \nabla \cdot \Delta^{a} L C \cdot c \cdot \Delta^{a} \rho \sigma \wedge L b^{a} x$

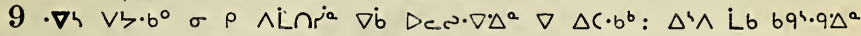

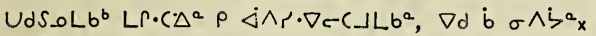

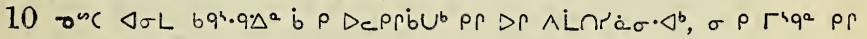
Dr $\sigma \wedge \dot{\alpha} \sigma \cdot \triangleleft^{b} \mathrm{x}$

$11 \cdot \nabla h L \Gamma \cdot C \cdot \Delta^{a}, \Delta \sigma L \quad 69^{a} \cdot 9^{a} \Delta^{a} \Delta \Gamma, \sigma \rho \cdot\left\langle 4 S \Delta d^{a}, \sigma^{m C} \nabla \cdot \Delta d \Delta \Gamma \quad \sigma \rho\right.$ $\sigma \dot{<}>d^{2} x$

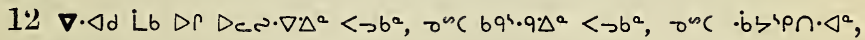
onc $\Gamma \cdot \dot{e} S^{a} x$

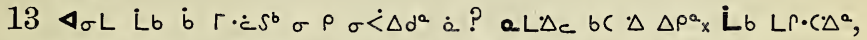

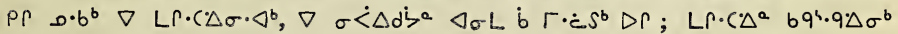

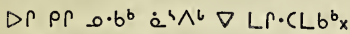

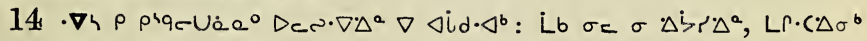

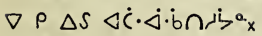

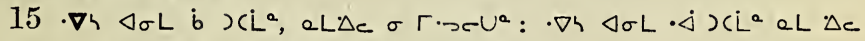
$\sigma \cdot x U^{a} ; \quad L b \triangleleft \sigma L b<\cdot b\left(L^{a}, \nabla \cdot \triangleleft d \nabla\right)\left(L^{a} a x\right.$

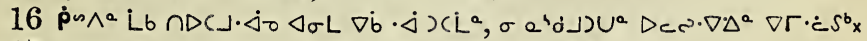

$17 a . L \Delta c$ Lb $\sigma e \dot{b}() L^{a}$, Lb LP. $\left(\Delta^{a} \dot{b} \wedge \mu^{4} b d^{\prime} b^{a} x\right.$

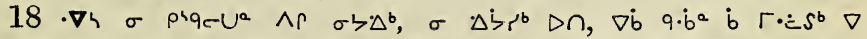
$\wedge \Gamma^{4} b d^{\circ} \zeta^{a}: \cdot \nabla h$ pr $\Delta U e-\left(L^{a} \sigma \rho\right) U^{a}$, Lb q $\left.\Delta S \cap \Lambda\right)\left(L^{a} \triangleleft \sigma L\right.$ b $\Gamma \cdot \dot{c} S^{b}$ aL $\Delta \Delta c \sigma \Gamma^{4} q^{a} x$

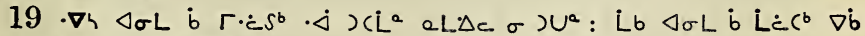
$\cdot \triangleleft x L^{a} \nabla \cdot \Delta d \nabla x L^{a}{ }^{a} x$

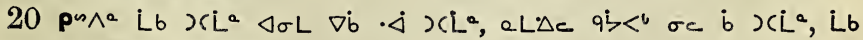
$\triangle \sigma L L \Gamma \cdot C \Delta^{a} \dot{b} \wedge \mu^{3} b d^{2} \zeta^{2} x$

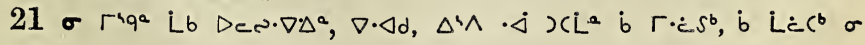
$\Delta \eta \Delta d^{2} x$

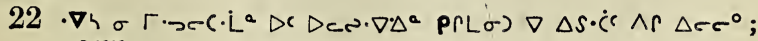

255 


\section{PLe 8.}

23 Lb $\sigma \cdot \cdot \dot{\Delta<U^{2}} d C^{b} \quad D_{c} \cdot \nabla \cdot \nabla \Delta^{2} \sigma \quad\left\langle 49 r^{b} \Delta \sigma^{b}, \nabla\right.$ one JLbb $\Delta \sigma L$

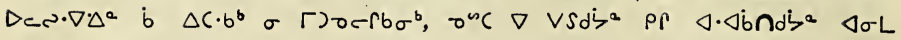
$L \Gamma \cdot C \cdot \Delta \sigma D_{C} \cdot \cdot \nabla \Delta^{a} \dot{b} \Delta C \cdot b^{b} \sigma<^{4} 9 r^{b} \Delta \sigma^{b} x$

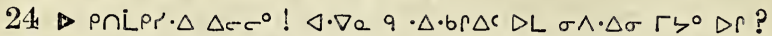

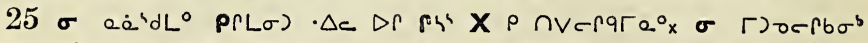

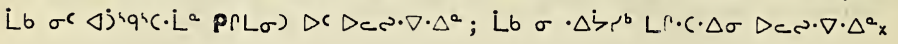

\section{Ápcre $\triangle b^{a} 8$.}

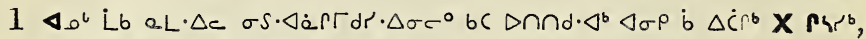
$\nabla \dot{b} \cdot \Delta \dot{b} \cdot \Delta \sigma^{b}$ b $\left.\Delta S \wedge\right\lrcorner U \rho^{b}, \dot{L} b \Delta \dot{L} d^{b} x$

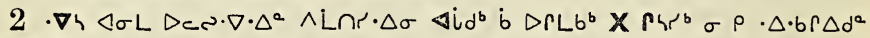

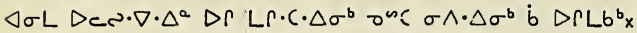

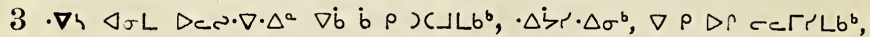

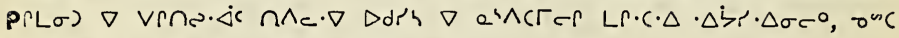
$L \Gamma \cdot C \cdot \Delta^{a} \Delta R, \rho \sigma S \cdot \Delta \dot{a} \Gamma C L L R \cdot C \cdot \Delta \sigma \sigma^{\circ} \cdot \Delta \dot{S} r \cdot \Delta \sigma^{b}$ :

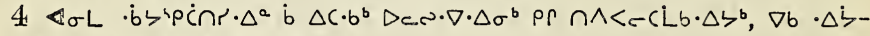
$\left.\left.r \cdot \Delta \sigma^{b} \dot{b} \Delta S \wedge\right\lrcorner U\right\rangle^{b}, L b \Delta i d^{b} x$

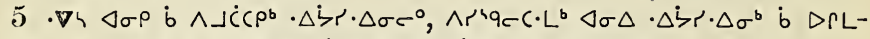

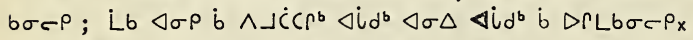

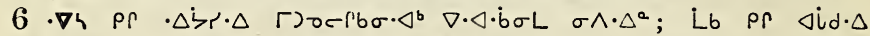
L)

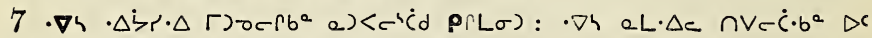

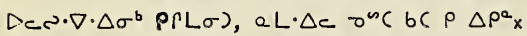

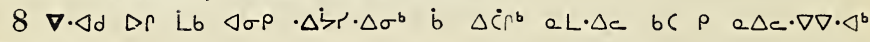
$P(L \sigma) \cdot \triangleleft x$

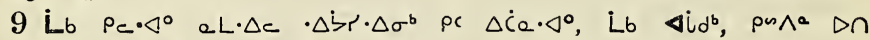

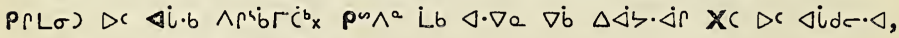

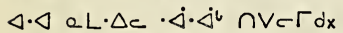

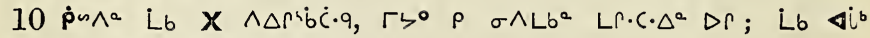

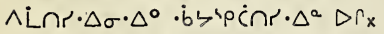

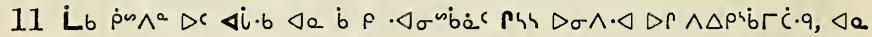

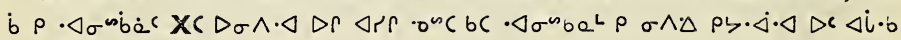
$\Delta r \dot{b} \Delta r^{s} \dot{b} \Gamma c^{b} \times$

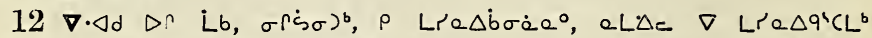
$\Delta \dot{S} \cdot \Delta^{a}, \Delta \dot{\zeta} \cdot \Delta \sigma^{b} \Delta S$ pr $\wedge \dot{L} \cap \zeta^{b} \mathrm{x}$

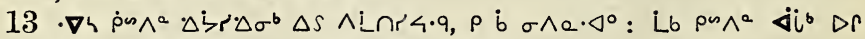

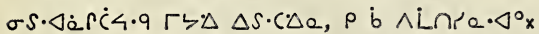

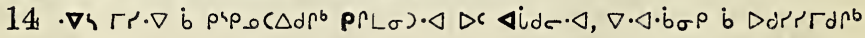
PrL $\sigma) \cdot \triangleleft_{x}$

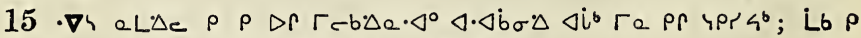

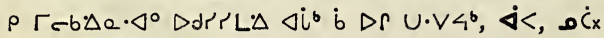




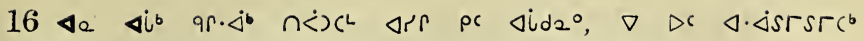
PrL $\sigma)_{x}$

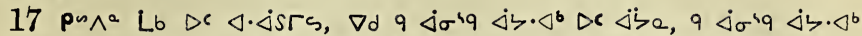

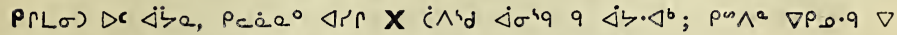

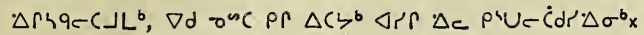

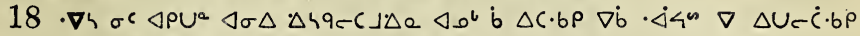

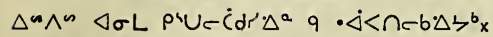

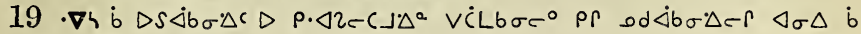

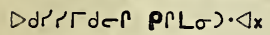

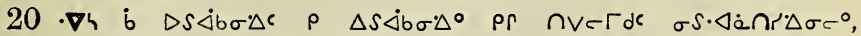

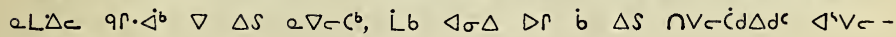
\lrcorner$\Delta \sigma^{b} x$

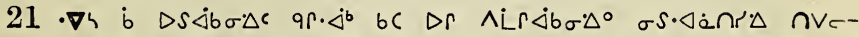

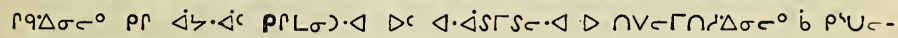
$\dot{c} \cdot b \sigma c^{b x}$

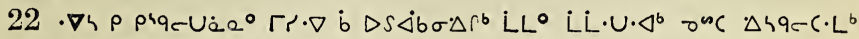

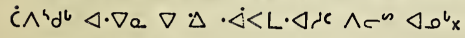

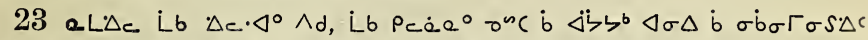

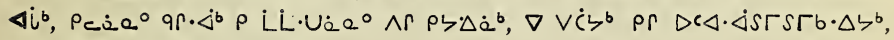
er $\wedge \dot{L} \Gamma \dot{C}_{\sigma} \cdot \triangleleft^{b} \rho b \cdot \Delta e^{0} \nabla \cap x$

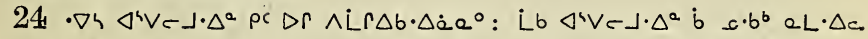

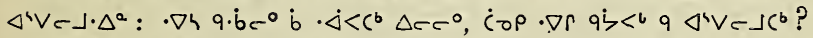

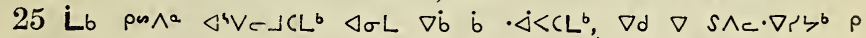
$\mathrm{V} \dot{\mathrm{C}} \mathrm{e}^{\circ} \mathrm{x}$

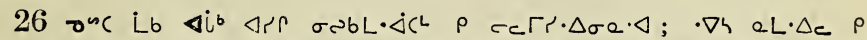

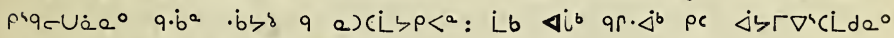
$\dot{L} L \cdot U \cdot \Delta \sigma^{b} \nabla \dot{b} q \rho \Delta \cdot \dot{C} \sigma \cdot \Delta b x$

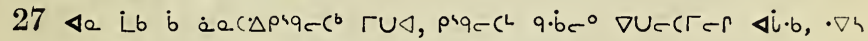

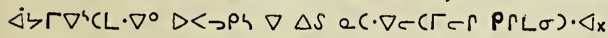

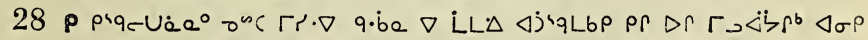

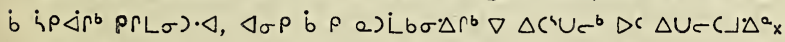

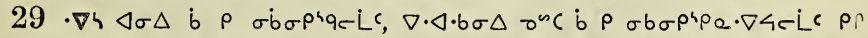

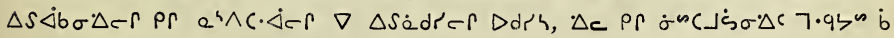
rince $\Delta r \dot{s} e^{x}$

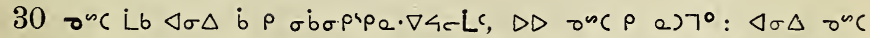

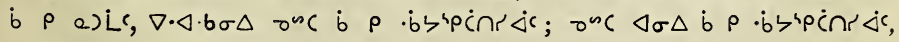
$\nabla \cdot \triangleleft \cdot \dot{b} \sigma \Delta$ foc b $\rho$ prUनcंd $\dot{\Delta} c_{x}$

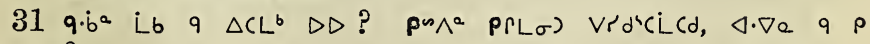
on $\mathrm{C}^{b}$ ?

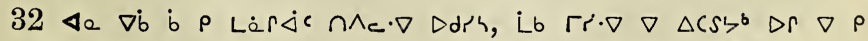

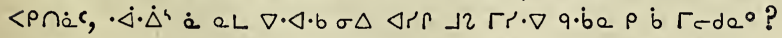

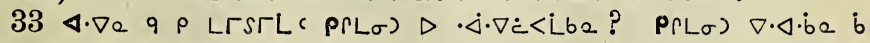
bל 'pcंn $\Delta \cdot \nabla c_{x}$ 


\section{PLe. $8,9$.}

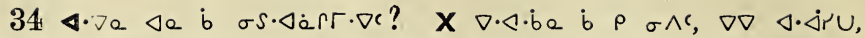

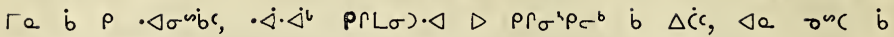
$\dot{\Delta} \ \Gamma \nabla^{4} C \dot{L} c^{b} x$

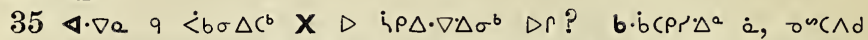

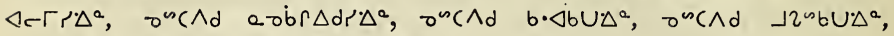

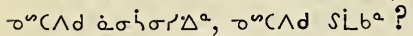

$36 \dot{C} \wedge^{4} d^{b} \nabla \Delta C P a \Delta b U^{b}, P_{c} \quad D \Gamma \sigma \sigma<\Delta b \Delta \dot{a}^{a} \Gamma P \cdot \nabla \nabla^{b} b \sigma \quad P S b^{b} ; \sigma^{c}$

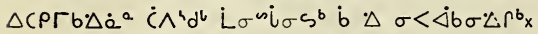

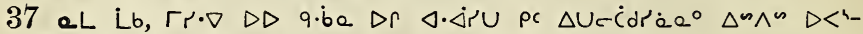
$\rho \dot{c} q \cdot \Delta^{b}, \Delta_{c} \Delta r \dot{b} \rho \dot{\rho} \rho \Delta C^{b} x$

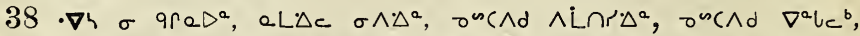

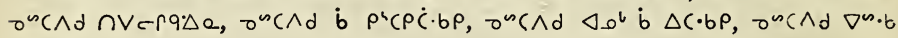
q $\Delta C \cdot b \rho$,

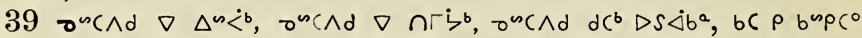

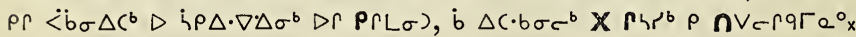

\section{Ápcre $\triangle b^{\circ} 9$.}

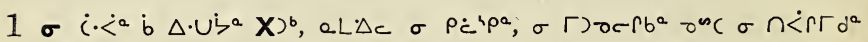

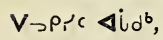

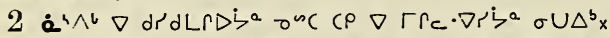

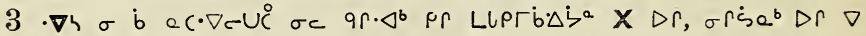
$\Delta C^{3} U^{b} \Delta \dot{S}^{\prime} \Delta^{a}:$

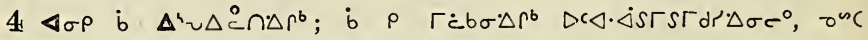

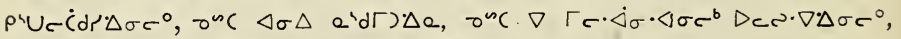

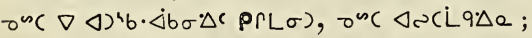

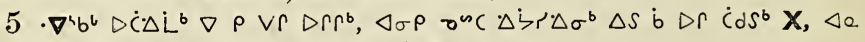
$\Gamma r \cdot \nabla$ b $\cap V-L c, \rho \rho L \sigma)$ b $\rho q$ b $\Gamma \cdot c \rho \dot{C} d r c_{x} \quad \nabla 7^{a}{ }_{x}$

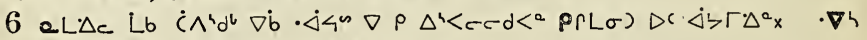

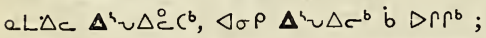

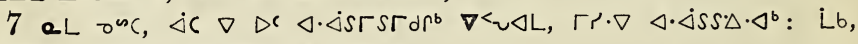

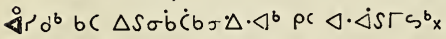

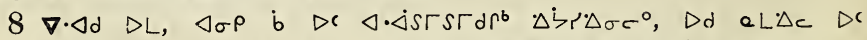

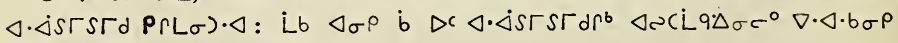
$\triangleleft \cdot \Delta S^{b} S^{b} \dot{b} \Delta C \dot{L} b \sigma \Delta \sigma^{b} x$

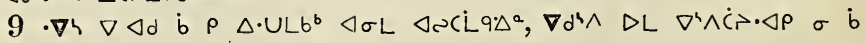
$\cos \sigma^{\circ}$, \s Lb bC $\Delta d d^{\circ} r^{\circ} x$

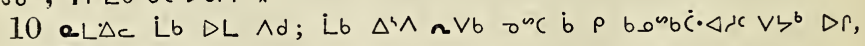
$9 C \Delta \sigma^{\circ} \Delta r^{b} D r D \cap ;$

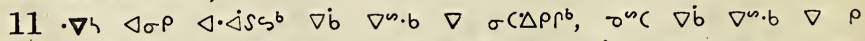

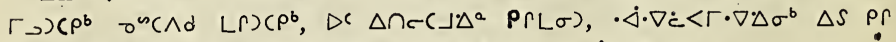
$\left.\dot{\Delta}>r \dot{b}<\Delta L b \sigma c^{b}, a L \Delta c \Delta j^{4} q \Delta \sigma^{b} \Delta r, L b \Delta a \Delta r \dot{b} a\right) \Gamma \cdot \nabla r ;$ 
$12 \rho \triangle \dot{C} b \sigma \Delta^{\circ}, \Delta \cup U L^{\circ} b C \Delta \dot{S}^{4} b \cdot \nabla^{\circ} \triangle S T L \cdot \triangle x$

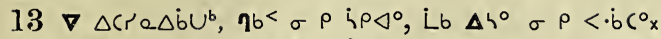

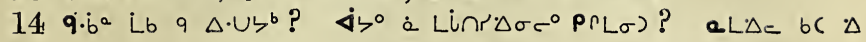
$\triangle p^{a} x$

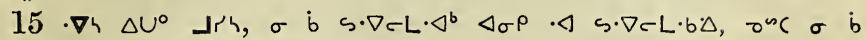
$p \cap L-q L \cdot \triangleleft^{b} \triangleleft \sigma p \cdot \triangleleft \rho \cap L q C L \cdot b \Delta x$

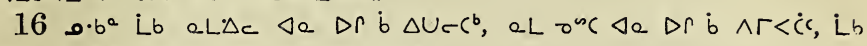
$P \Gamma L \sigma) \Delta \Gamma \dot{b} \cdot \dot{j}\left\langle\Omega c \cdot \nabla\left(s \cdot \nabla c \Gamma q \Delta \sigma \sigma^{\circ} x\right.\right.$

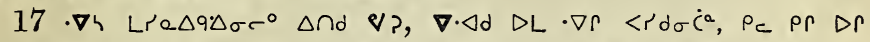

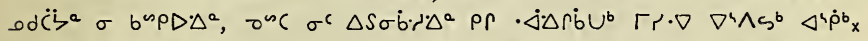

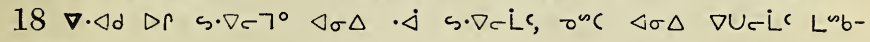
$\triangle \cup \nabla \nabla^{\circ} \mathrm{x}$

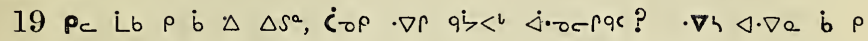
Q'd'C.LC DC $\left.\Delta U C^{\circ} C\right\lrcorner \Delta \sigma \sigma^{\circ}$ ?

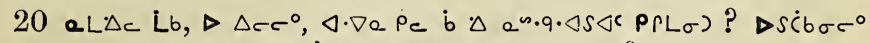

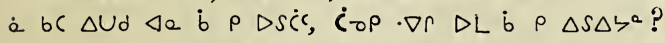

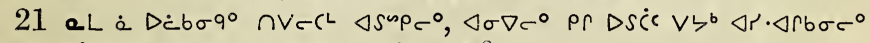

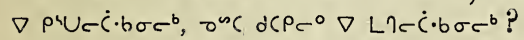

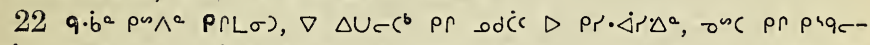

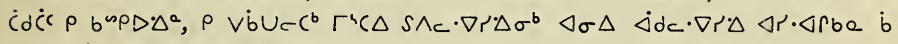
$\rho \triangle C \dot{C} \sigma \cdot \Delta \rho$ Pr $\sigma S \cdot \triangle \dot{L} C P$ :

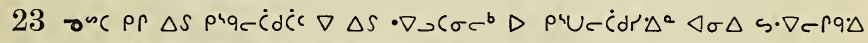
$\Delta r \cdot \Delta r b a, \sigma \dot{b}^{a} \dot{b} \rho \cdot \dot{\Delta} \cdot \nabla S \dot{c} c \rho^{4} \cup c-\dot{c} d r \cdot \Delta \sigma^{b} \Delta S$,

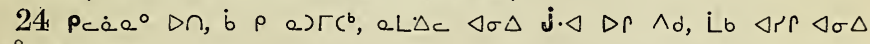

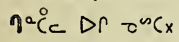

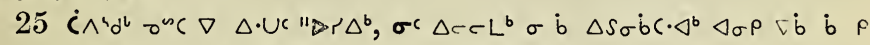

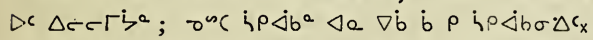

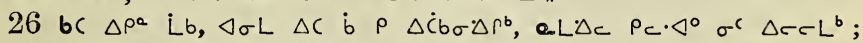

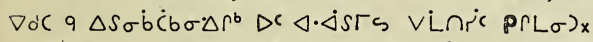

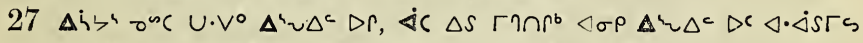

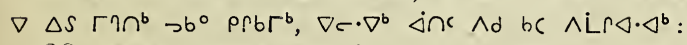

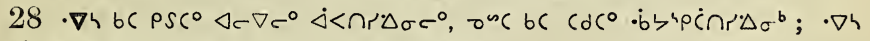

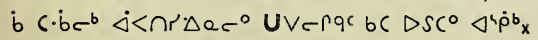

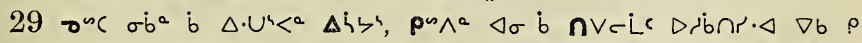

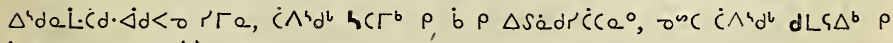
bे $\rho \Delta S \dot{a} d \Delta b \Delta \dot{C} C C^{\circ} x$

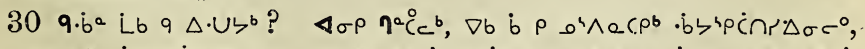

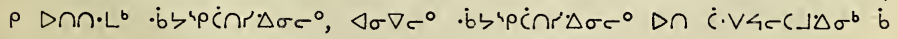
$\Delta R<C^{b}{ }^{b}$

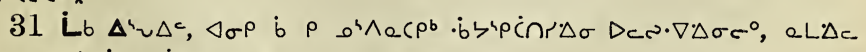

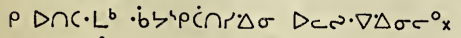

$32 \dot{C} \rho \rho \cdot \nabla \Gamma$ ? $\nabla \dot{b} \nabla \rho$ iec $\nabla e c \rho b \dot{c} \cdot V 4 C C \cdot \Delta \sigma^{b}$, Lb Le $\wedge_{d}$

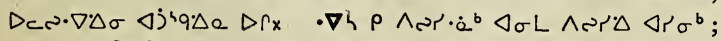




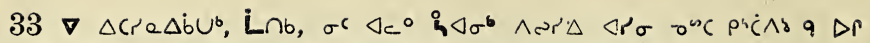
a $4 C \cdot \nabla e r \dot{b} U^{b}: \triangleleft \cdot \nabla e$ Lb $9 \dot{C} \cdot V 4 c L \cdot q$ eL. $\Delta e$ bC erV $\Delta r^{\circ}{ }_{x}$

A $p c r e \triangle b^{2} 10$.

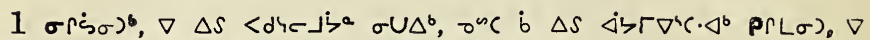

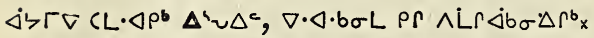

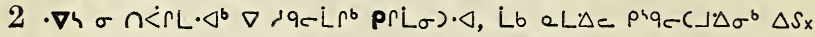

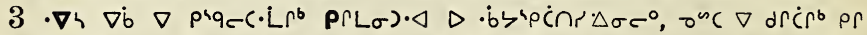

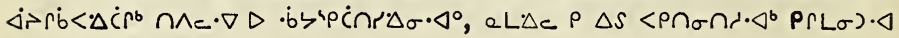
$D \cdot \dot{b}$ ip $\dot{c} \cap \Delta \sigma c^{b} x$

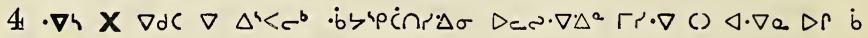
$\dot{c} \cdot \mathrm{Ac} \mathrm{C}^{\mathrm{b}} \mathrm{x}$

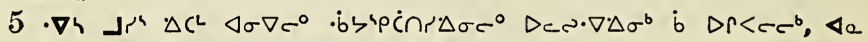

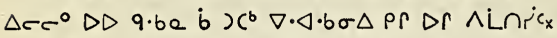

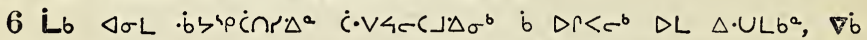

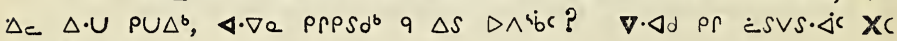
brPSde $D r$ :

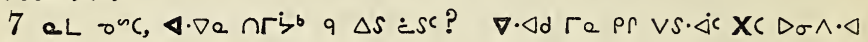
$\Delta r_{x}$

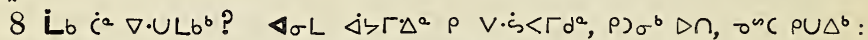
$\nabla \cdot \triangleleft d, \varangle \sigma L \dot{C} \cdot V 4 C C\lrcorner \Delta \sigma \quad \dot{\Delta} \zeta \Gamma \Delta^{a}, \dot{b} \cdot \Delta \cdot \Delta C L^{b} ;$

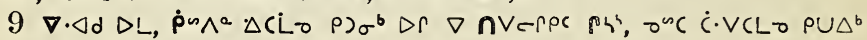
$\left.\Delta \sigma \wedge \cdot \triangleleft \nabla \rho \Delta r \cdot \Delta \sigma^{\omega} \dot{b} \sigma d^{d}(\rho) L \sigma\right) \cdot \triangleleft, \rho \dot{b} \wedge L \Gamma \Delta b \Delta^{\circ} x$

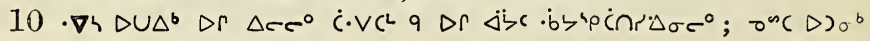
$\Delta C^{L} q \Delta r \Delta \dot{\Delta l C} \wedge \mathrm{L} r \Delta d r \Delta \sigma c^{\circ}{ }^{x}$

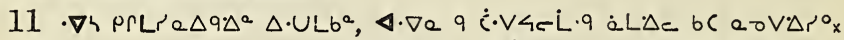

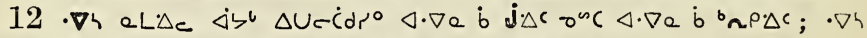

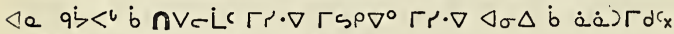

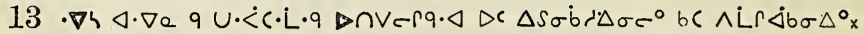

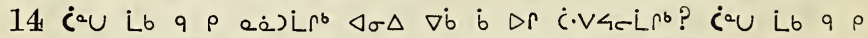

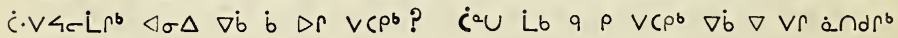
$\triangle 6 q^{h} p r \cdot \nabla \cdot \triangleleft$ ?

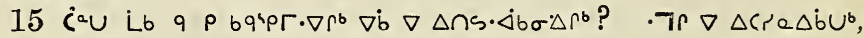

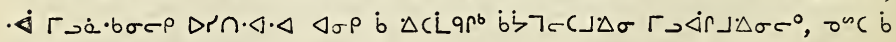

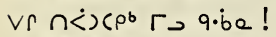

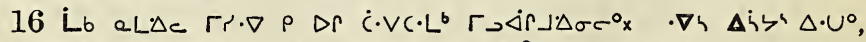

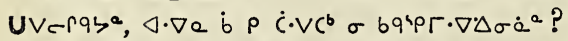

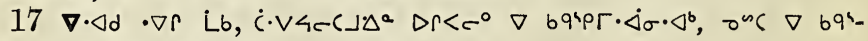

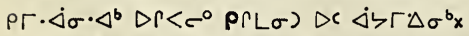

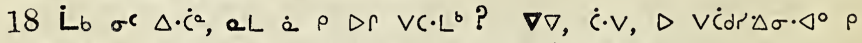

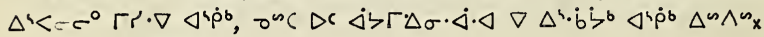
260 


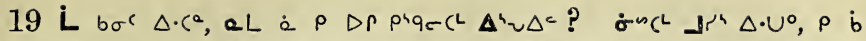

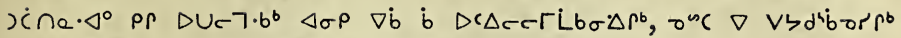

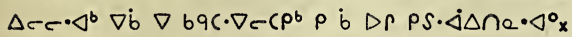

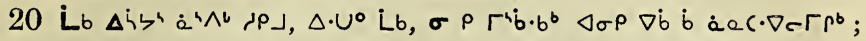

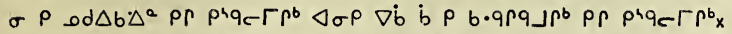

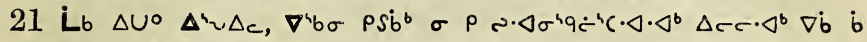
a $\Delta C p^{b} \sigma^{a c} \dot{b} \dot{b} \cdot \sigma_{c} c p^{b} x$

\section{Ápcra $\triangle b^{\circ} 11$.}

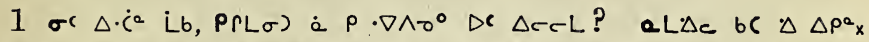

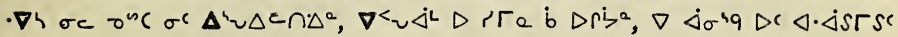
$\mathrm{Verta}_{\mathrm{a}} \mathrm{x}$

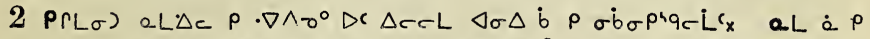

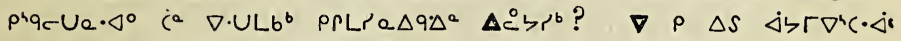
$\rho \rho L \sigma) \cdot \triangleleft \Delta^{h} \cup \Delta c \nabla$ LISLLC, $\nabla \Delta \cdot U c$,

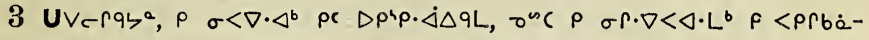

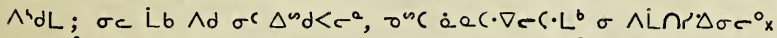

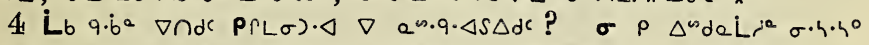

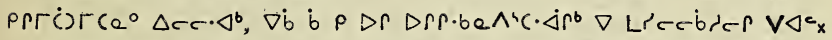

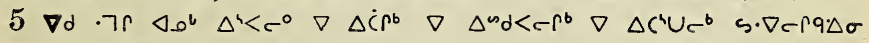
$\cdot \triangleleft \cdot \nabla \dot{c}<r \cdot \nabla \cdot \Delta^{\circ} \cdot x$

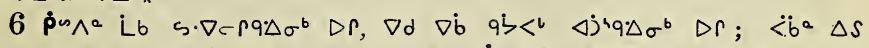

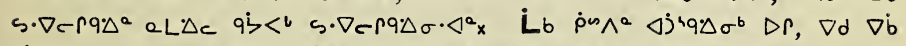

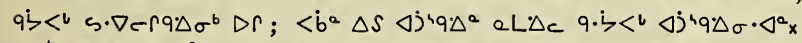

$79 \cdot b^{a}$ Lb? $\Delta^{4} \sim \Delta^{c}$ a L $\Delta c \rho \Gamma \dot{c} b \sigma \Delta^{0} \Delta \sigma \nabla c^{\circ}$ b $\dot{a} a c \cdot \nabla c c^{b}:$ Lb

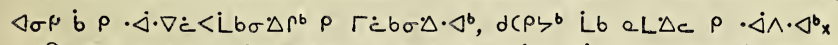

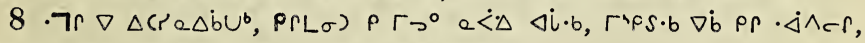

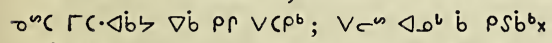

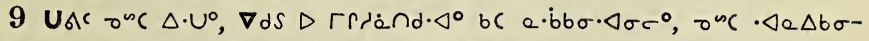

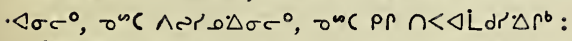

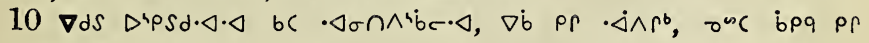

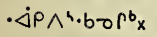

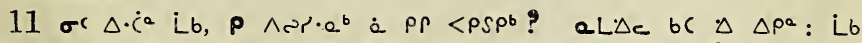

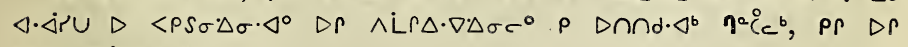

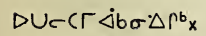

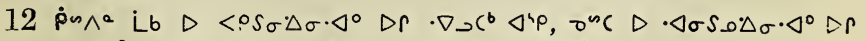

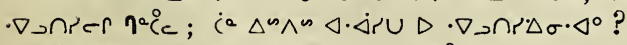

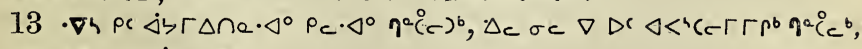

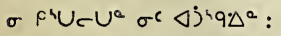

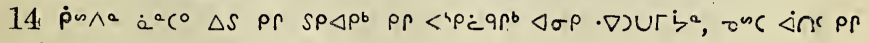
$\Delta S \wedge L r \Delta \rho b_{x}$

261 


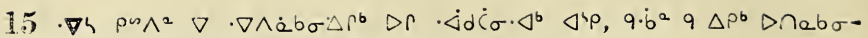

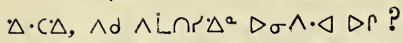

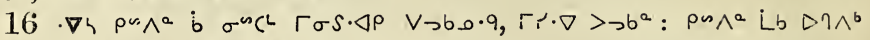

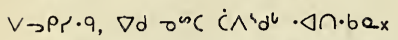

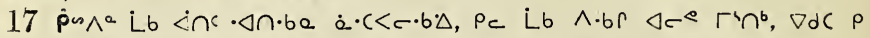

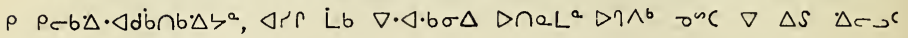
गe $\triangleleft c^{\circ} \Gamma^{4} \cap^{6}$;

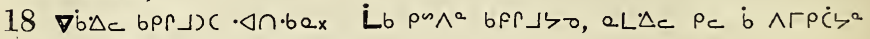

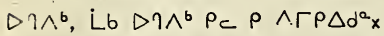

$\left.19 \rho \dot{b} \Delta \cdot \dot{c} a \dot{L} b, \cdot \Delta \cap \cdot b e \rho \dot{a} \cdot \dot{c}<-\dot{C} \sigma \cdot \triangleleft \cdot a, \sigma_{c} \rho \rho \Delta d\right\lrcorner \Delta \dot{b} \Delta \dot{b}{ }^{a} x$

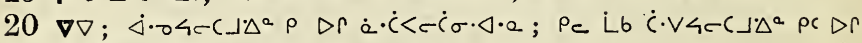

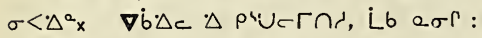

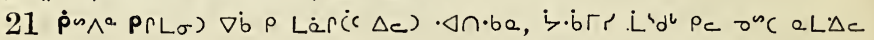
$\rho \dot{b}$ Lér $\Delta^{b} x$

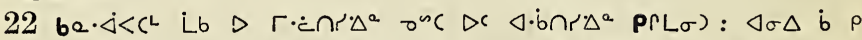

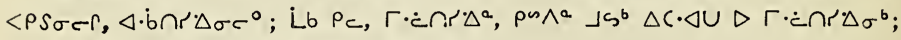

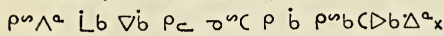

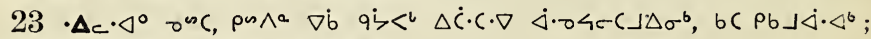

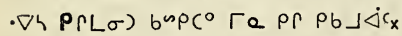

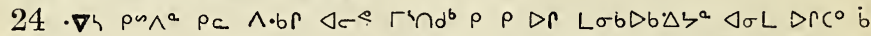

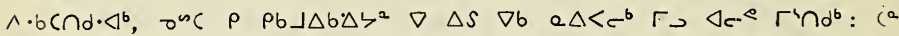

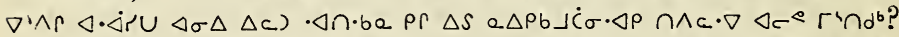

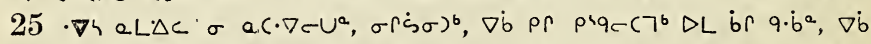

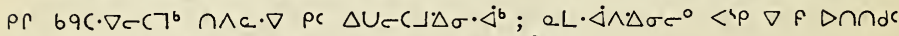

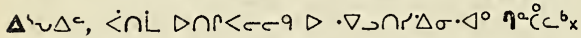

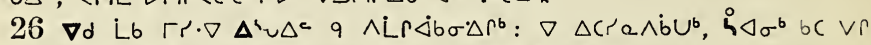

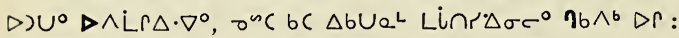

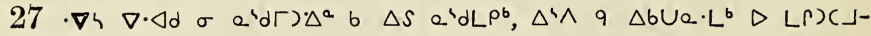
$\Delta \sigma \cdot \dot{\triangleleft} \cdot \triangleleft x$

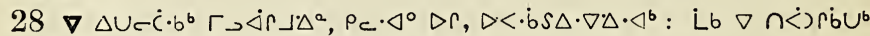

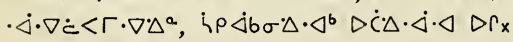

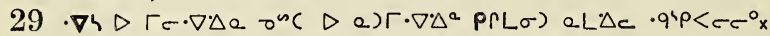

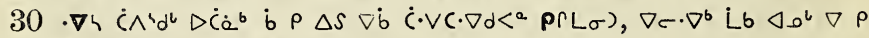

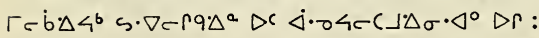

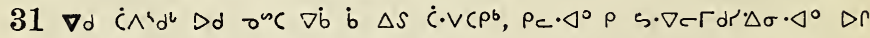

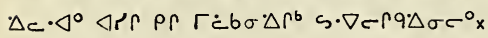

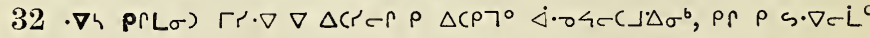
$\Gamma r \cdot \nabla x$

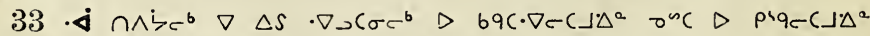

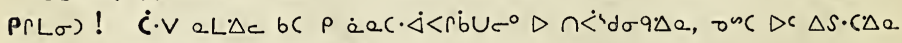
QL. $\Delta c$ bC $\rho \cup \wedge \Gamma^{\top} b \cdot \Delta \dot{B} \dot{b} \cup c^{\circ}$ !

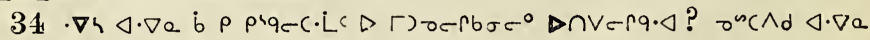
b $P$ bq'pLC?

262 


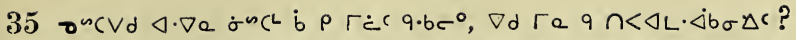

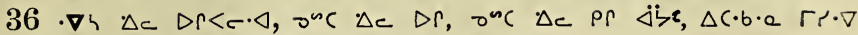

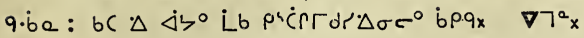

\section{Ápcre $\triangle b^{\circ} 12$.}

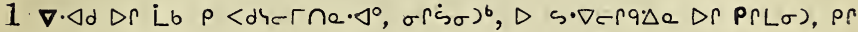

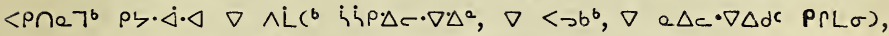

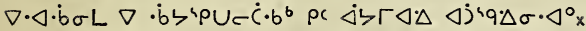

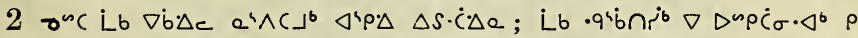

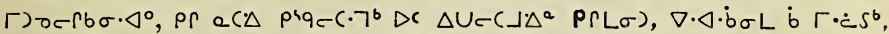

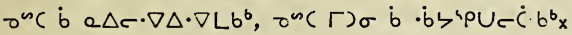

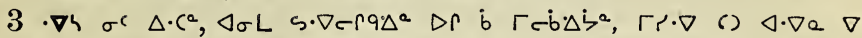

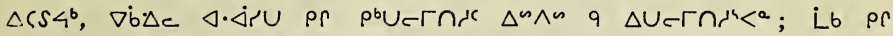

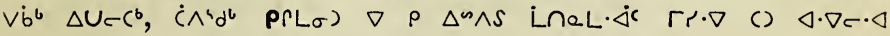
$\dot{C} \cdot V A C C \cdot \Delta \sigma c^{\circ}{ }^{x}$

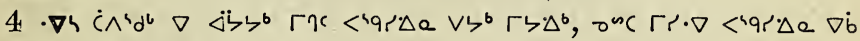
$\dot{C} \Lambda^{4} d^{l} \nabla \dot{\Delta}<\cap<L b^{b}:$

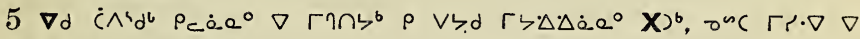

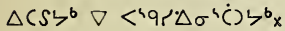

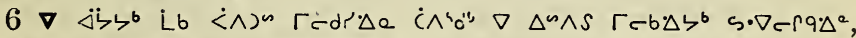

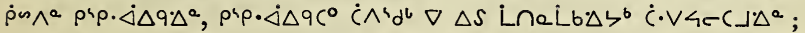

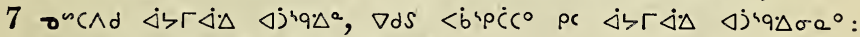

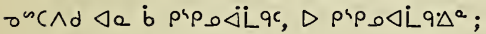

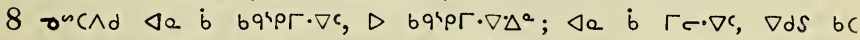

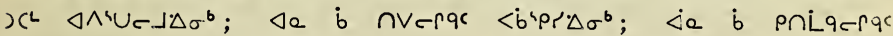
$\Gamma \cdot x-\lrcorner \Delta \sigma^{b} x$

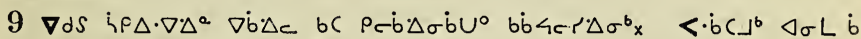
Lé $\left.C^{b} ; \Gamma \Gamma \Gamma \mathrm{a}\right\lrcorner^{b} \triangleleft \sigma L b \Gamma \cdot \dot{c} S^{b} x$

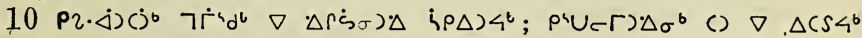

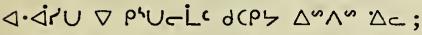

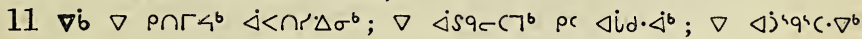
U Verqu;

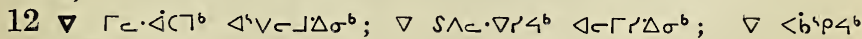
$\dot{\Delta} \zeta\left\ulcorner\dot{\Delta} \Delta \sigma^{b}\right.$;

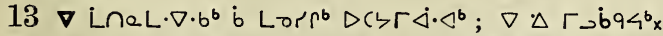

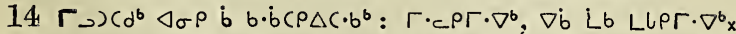

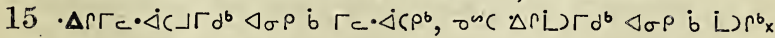

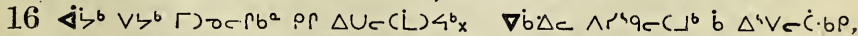

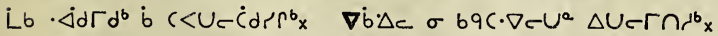

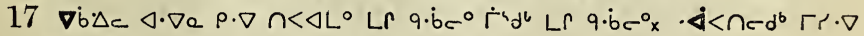
$\Delta c c \cdot \triangleleft^{b} \triangleleft \sigma \Delta q \cdot \dot{b} a$ b $\left.\Gamma_{\supset} \cap \dot{<}\right) \dot{c} \cdot b \rho_{x}$ 
18 рл的 $\triangle r c \cdot \Delta b x$

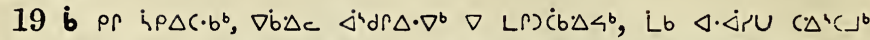

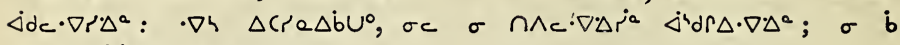
$\rho \cdot \nabla \cap<\Delta L^{a}, \Delta \cdot U^{\circ} U \vee C r q c_{x}$

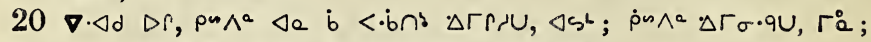

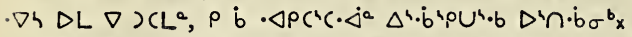

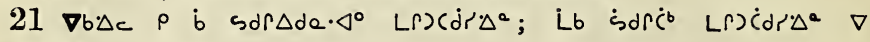
$r \rightarrow)\left(9<r^{b} x\right.$

\section{A'pcre $\triangle b^{\circ} 13$.}

$1 \nabla d S \quad r r \cdot \nabla \quad \Delta \cdot \nabla e$ bC ea $\Delta C \cdot \nabla a \quad \Delta \sigma \Delta$ b $n V e r q_{e} i x \quad \cdot \nabla h$ eld $\Delta c$

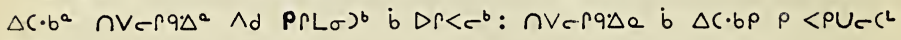
P $\{L \sigma) x$

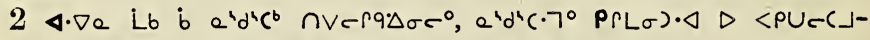

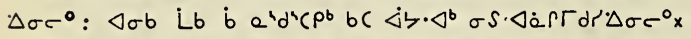

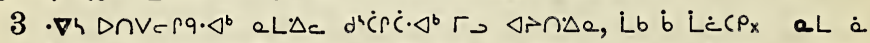

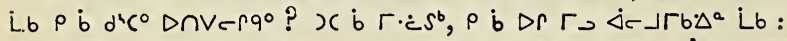

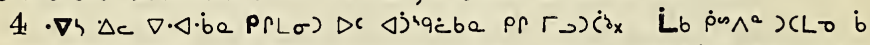

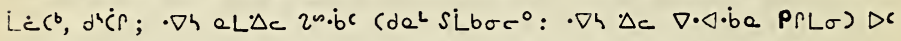

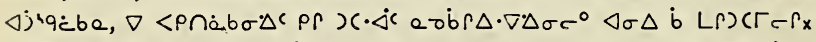

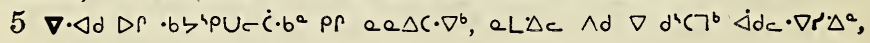
Lig 6 C

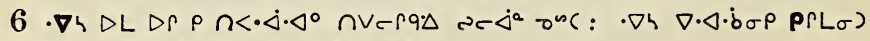
$D C \Delta \dot{j} 4 \dot{c} b e, j s^{b} \Delta 7 c^{\circ} \nabla \Delta \dot{C}<n<r^{b} x$

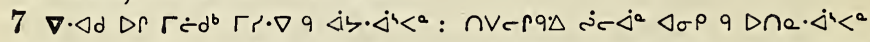

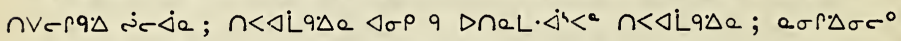

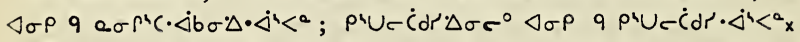

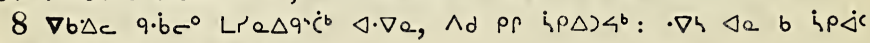
$d(P \zeta P \cap \cap) C^{L} D C c^{2} \cdot \nabla \Delta \sigma c^{\circ} x$

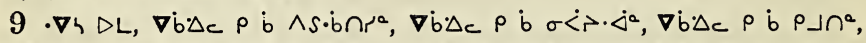

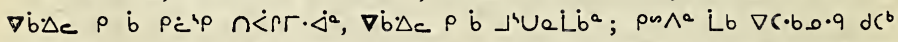
$b q^{b} \cdot 9 \Delta^{a}, \Delta C \cdot b^{a} D C$ b c $b^{b} \Delta \cdot U \Delta \sigma^{b}, D L, p$ b $i p \Delta^{\circ} p r \Delta c c^{\circ} C \Lambda^{4} d^{b} \nabla$ $\triangle S$ hP $\triangle \cap r^{\circ} b^{\circ} x$

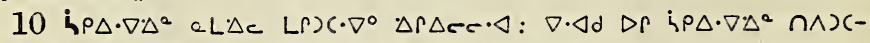
$J L b^{a} D C N \cdot \nabla \Delta^{2} x$

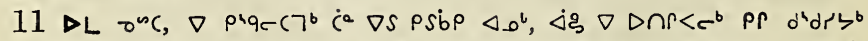

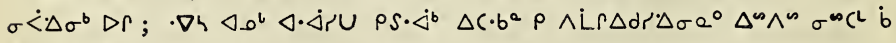
$\dot{C} \cdot V C L P<a x$

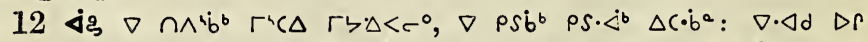

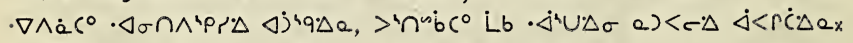

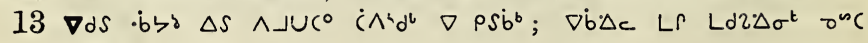
264 


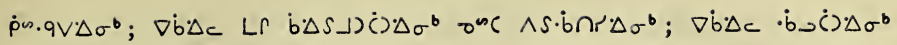
onc $\Delta U C \Gamma) \Delta \sigma^{b} x$

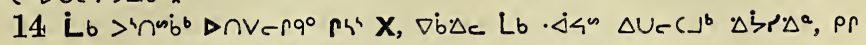
$\cap \wedge)\left(7^{b} \nabla \Delta S\right.$ a $\left.C \cdot \nabla C C\right\lrcorner L_{b} b x$

\section{Ápcre $\triangle b^{a} 14$.}

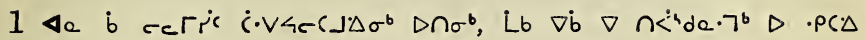
$\triangle U C(\lrcorner \Delta a_{x}$

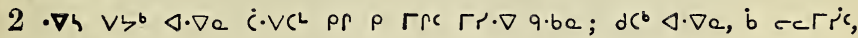
$\sigma \dot{C} \Delta \rho \dot{C} b \sigma s \Gamma \Gamma^{\circ} x$

$3 \nabla d S \nabla \dot{b} \Delta c \triangleleft a b r \rho c b c<c \cdot \nabla c-7^{\circ} \triangleleft \sigma \Delta \nabla b \dot{b} \Gamma f e r ; \nabla d S$ onc $\Delta a$

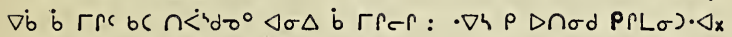

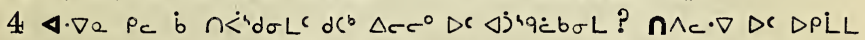

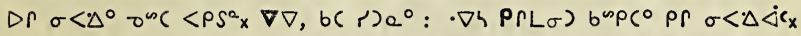

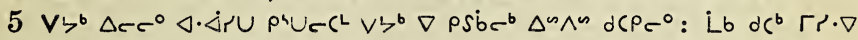

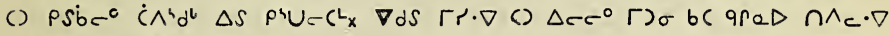
$D\left(>\partial=r b \sigma_{p} x\right.$

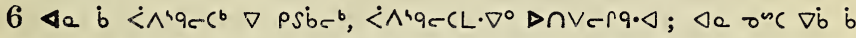

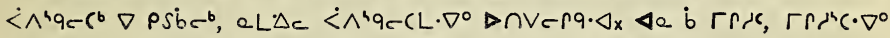

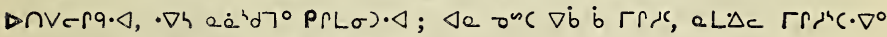

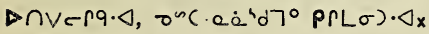

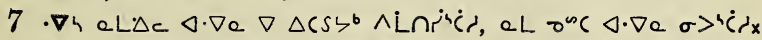

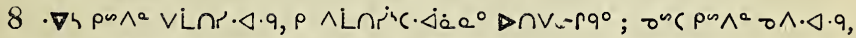

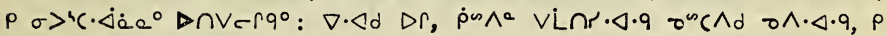

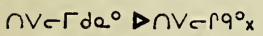

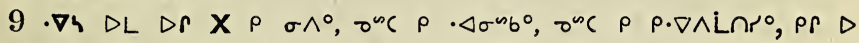

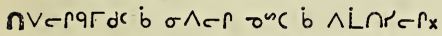

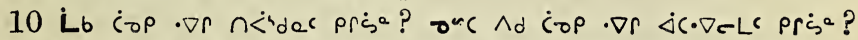

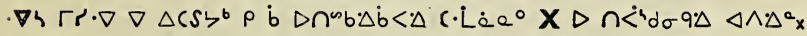

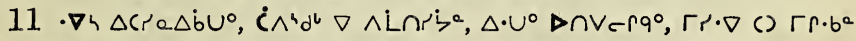

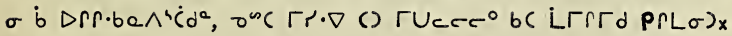

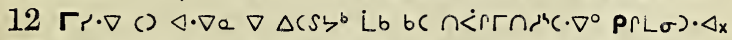

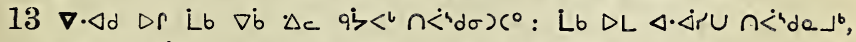

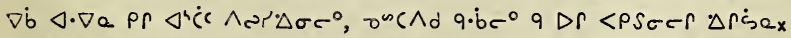

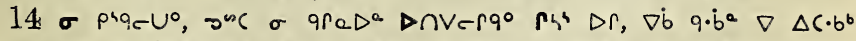

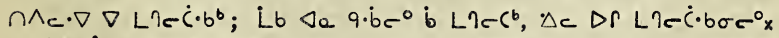

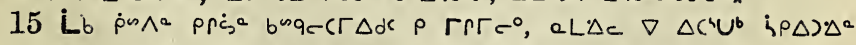

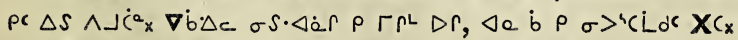

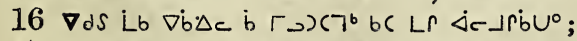

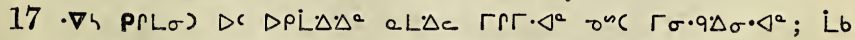

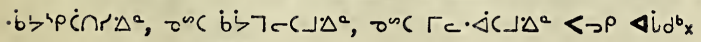




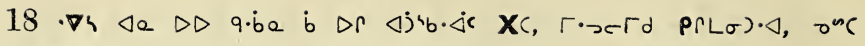
e $\nabla c \Gamma \mathrm{d} \Delta c r \cdot \triangleleft x$

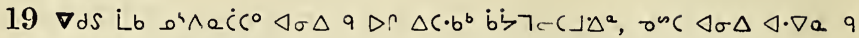

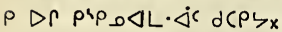

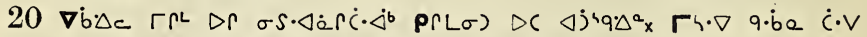

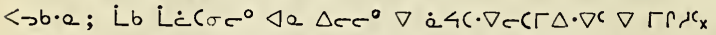

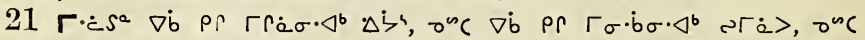

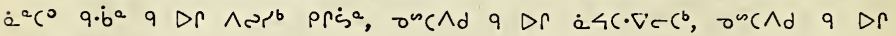

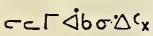

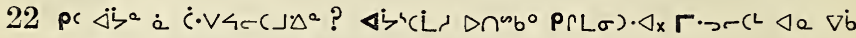
b $\sigma s \cdot \Delta \dot{a} r \Gamma \cap \lambda c \Delta \sigma \nabla c^{\circ} \Delta C \dot{b} \Delta r a^{b} x$

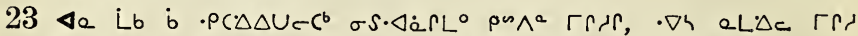

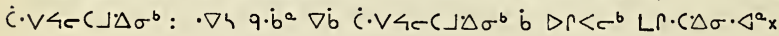

A $p \subset \subset a \triangle b^{a} 15$.

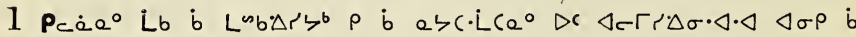

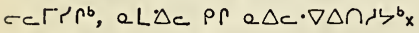

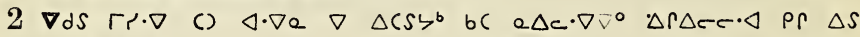

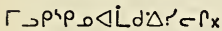

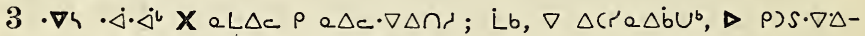
$\sigma \cdot \triangleleft \cdot \triangleleft \triangleleft \sigma \rho$ b $\rho \rho) \cap^{i} \rho^{b} \sigma \rho\left\langle b \Gamma^{i} \dot{b} d \cdot \dot{j}^{a} x\right.$

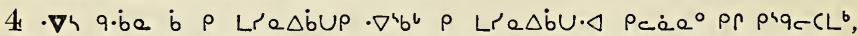

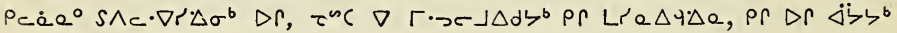
$\Delta \wedge \subset-J \Delta^{a} x$

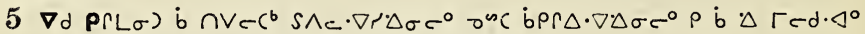

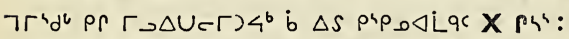

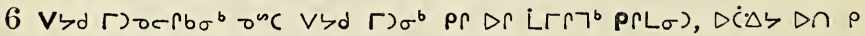

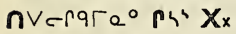

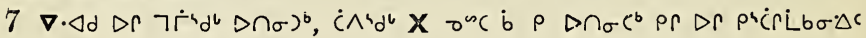
PrL $\sigma)_{x}$

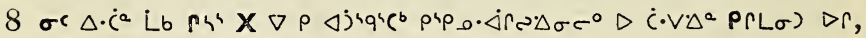

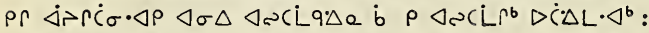

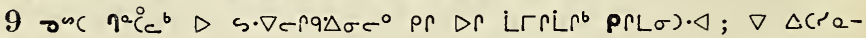

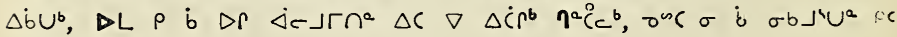
$\Delta S \sigma b i \Delta \Delta^{a} x$

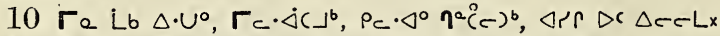

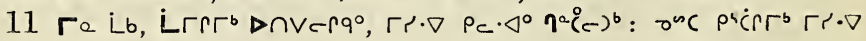
$\left.P_{c} \cdot \triangleleft^{\circ} \Delta-c\right)^{b} x$

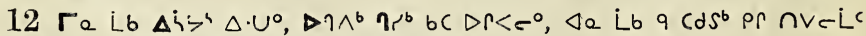

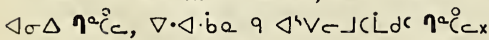

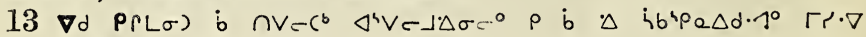
266 


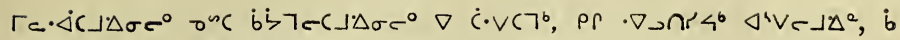

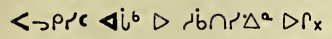

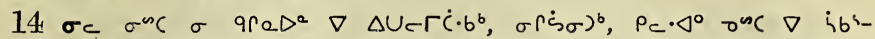

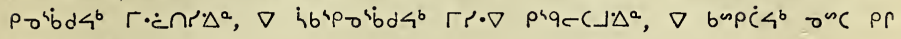
(9)

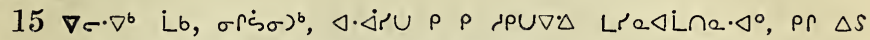

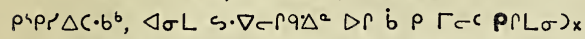

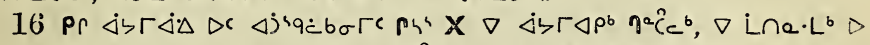

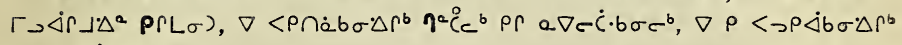
$<\rightarrow P$ \&i.b $\Delta r_{x}$

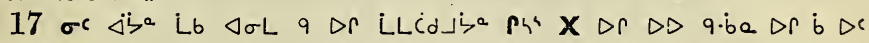
$\ddot{\Delta \zeta \sigma(P(L \sigma) x}$

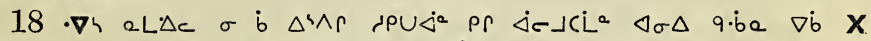

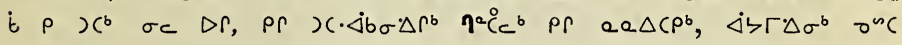
$x\lrcorner \Delta \sigma^{b}$,

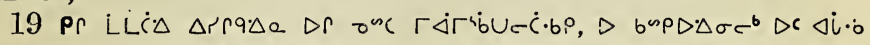

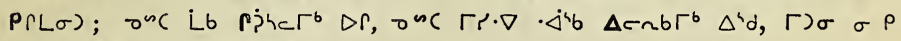

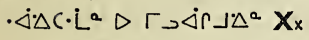

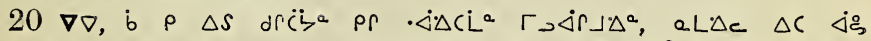

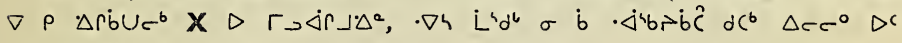
$\nabla^{4} C \Gamma b \sigma^{6}$ :

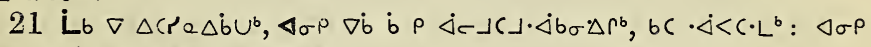
$\sigma^{n} c \nabla \dot{b}$ b $\rho \vee\left(\rho^{b} b c \quad \sigma r\right)\left(\cdot L^{b} x\right.$

$22 \nabla \cdot \Delta d \Delta r \sigma^{n} C \Gamma \eta \cdot c^{\circ} \sigma \rho D C r \Delta \dot{b} \cdot \Delta^{a} \nabla \Delta V r \dot{a} \cap C \cdot b^{b} x$

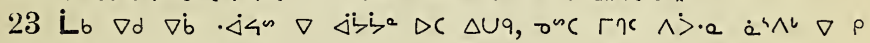

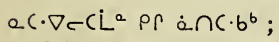

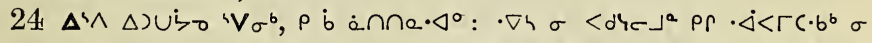

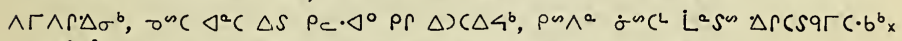

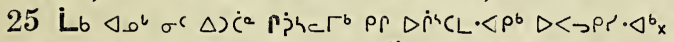

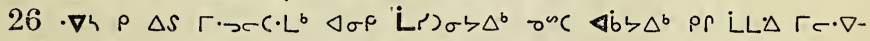

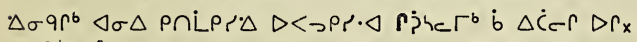

$27 \dot{C} \cdot \vee \rho \Delta S \Gamma \cdot s e C \cdot L^{b} ; \Delta D \nabla \rho$ Lra $\Delta \psi^{4} C \cdot \Delta r^{b} x \quad \cdot \nabla h$ pas $\wedge^{a} \eta^{a}{ }^{\circ} c^{b}$

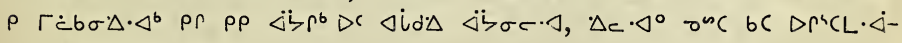

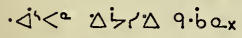

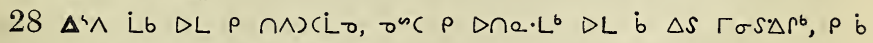

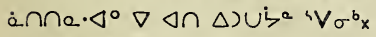

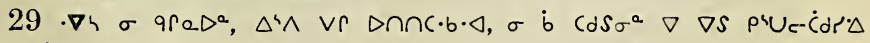
$\left.s \cdot \nabla c \dot{c} \cdot b \sigma c^{b} \nabla \Gamma \sim \Delta \dot{\Delta}\right\lrcorner \Delta^{a} X_{x}$

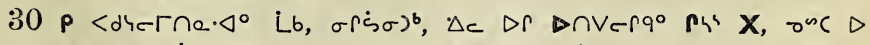

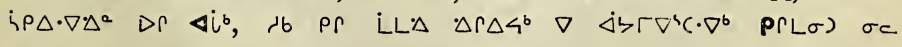
$\Delta r_{x}$

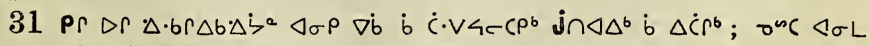

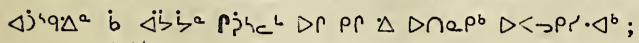




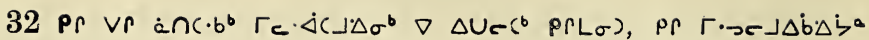
$\operatorname{Ar} \varphi^{c} \cdot \Delta^{\prime}{ }^{x}$

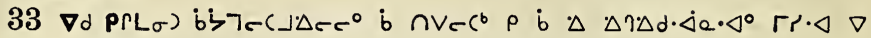
$\Delta C s 4^{b} x \quad \nabla 7^{2} x$

\section{A p}

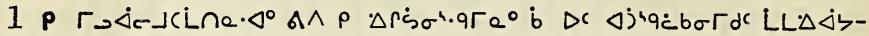
$\Gamma \dot{\Delta} \Delta \dot{b} \Delta \dot{c}^{-r} \varphi^{a b} \sim \Delta \Delta^{b}$ :

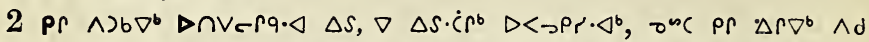

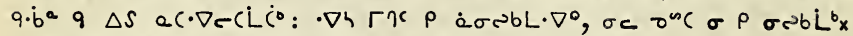

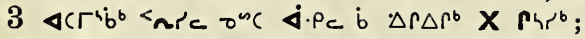

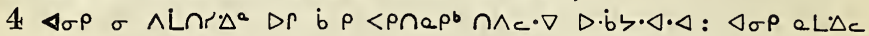

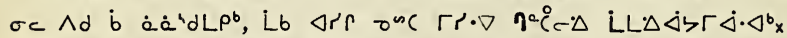

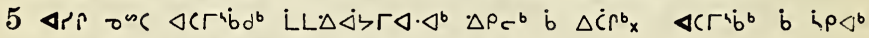
$\Delta \wedge \sigma c^{4}, \Delta a b$ b $\left.\sigma \dot{b} \sigma \Gamma \sigma s \Delta c \Delta^{a} c \quad \Delta b \zeta \Delta^{b} \quad X\right)^{b} \Delta S_{x}$

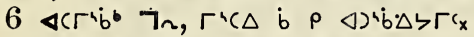

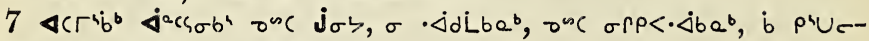

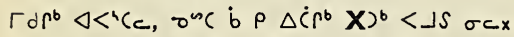

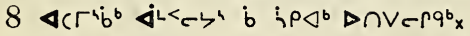

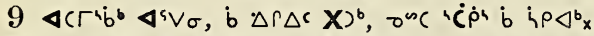

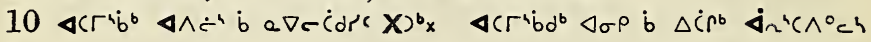
$\Delta P c^{b} x$

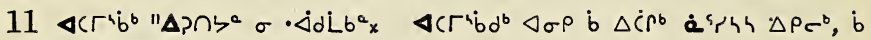

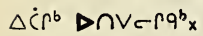

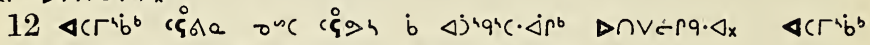

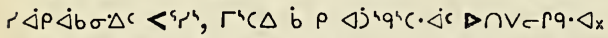

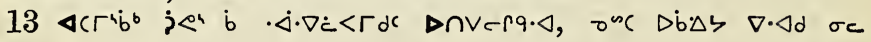
$\sigma \dot{b} \cdot \Delta x$

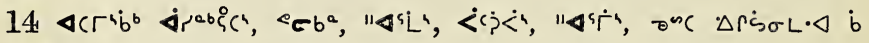
$\Delta \eta \cdot \Delta r^{b} \times$

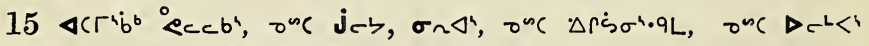

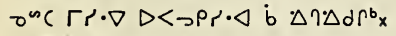

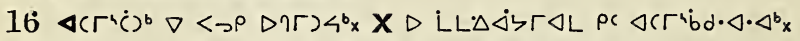

$\left.17 \nabla d \rho<d h e r \cap a \cdot \Delta^{\circ}, \sigma \Gamma \dot{s} \sigma\right)^{b}, \wedge r^{4} q C \Gamma d^{b} \triangleleft_{\sigma} \rho$ b $\Delta \Gamma \Delta(\cdot b q<b \sigma \Delta-$

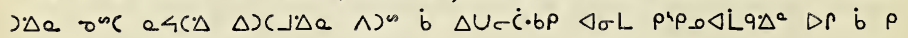

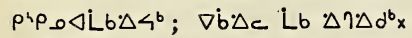

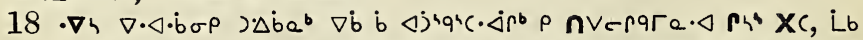

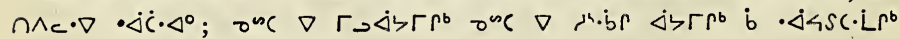
$\Delta \cup \triangle r \cdot \triangleleft \triangleleft \sigma \Delta \nabla \dot{b}$ b $r \cdot \dot{b} b \sigma r_{x}$

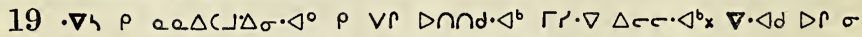

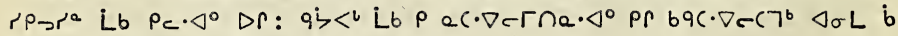

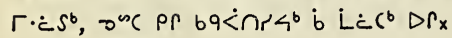

268 


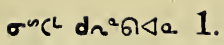

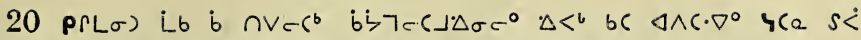

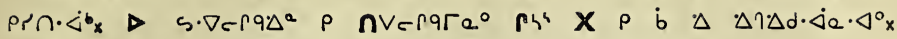
$\nabla\urcorner^{a x}$

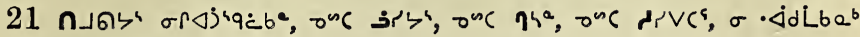
bc $\triangleleft c \Gamma^{i} b \dot{b} \cdot \cdot \triangleleft \cdot \Delta^{b} x$

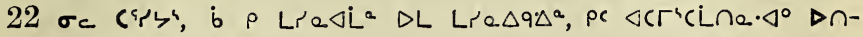
$\operatorname{Ver} q^{b} \mathrm{x}$

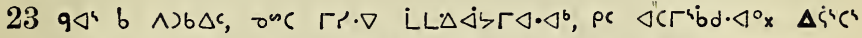

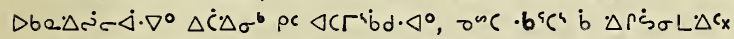

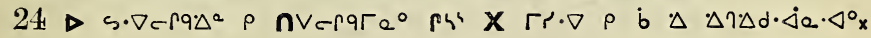
$\nabla 7^{\circ} \mathrm{x}$

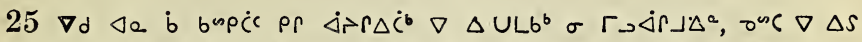

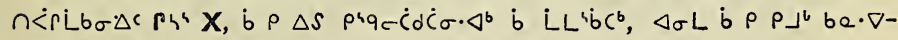
-ribub $\Delta^{4} \wedge^{a} \dot{b} \vee r \Delta^{\prime} \rho \cdot \Delta^{b}$,

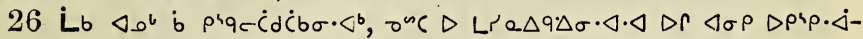

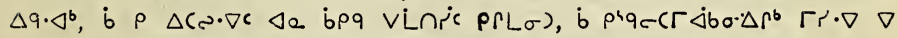

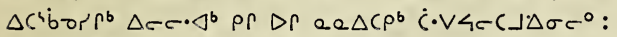

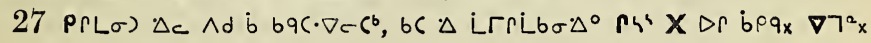

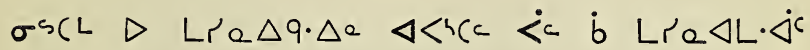 $d \Omega \circ \triangleleft \begin{gathered}x \\ 0\end{gathered}$}

\section{$\Lambda$ Ascra $\triangle b^{a} 1$.}

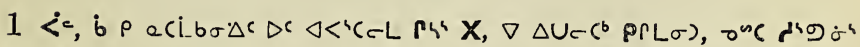
prśä,

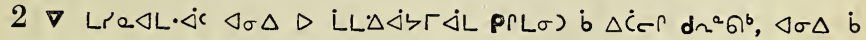

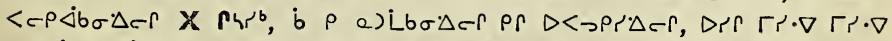

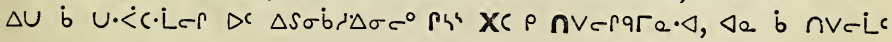

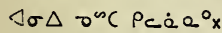

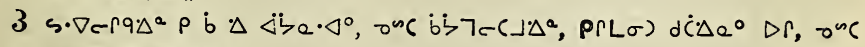

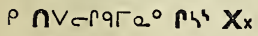

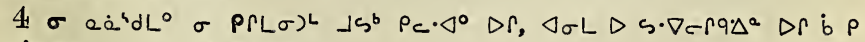

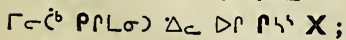

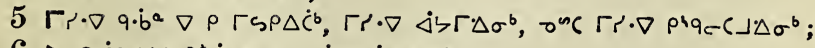

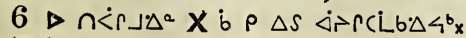

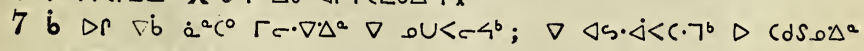

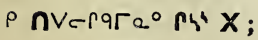




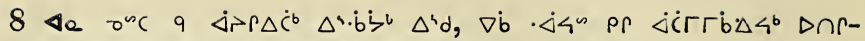

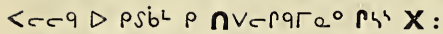

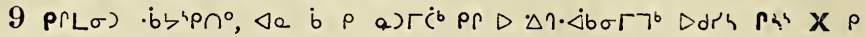

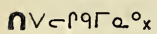

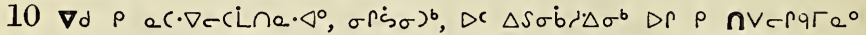

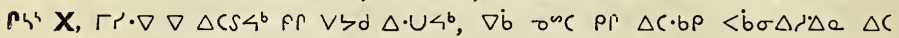

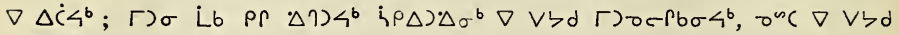

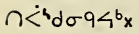

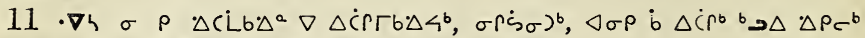
$\nabla \cdot \triangleleft \cdot b \sigma \rho \dot{b} \rho \Delta(\rho b, \nabla<s U \Gamma) \dot{a} \sigma \cdot \triangleleft^{b} \Delta C \nabla \Delta \dot{C} \dot{c}^{b} x$

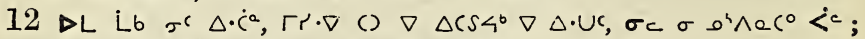
onc $\sigma_{c} \varangle<c^{\prime} ; \sigma^{n c} \sigma_{c} \beta e^{4} ; \sigma^{n} \sigma_{c} X_{x}$

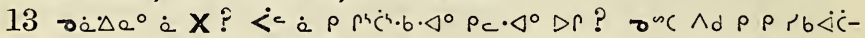

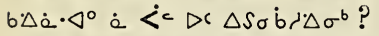

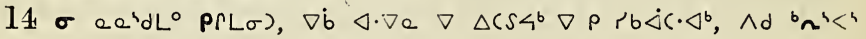
r"c $9 \varangle^{4}$;

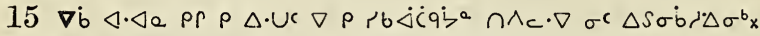

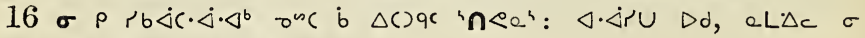

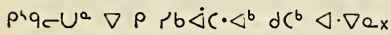

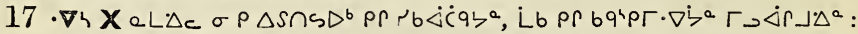

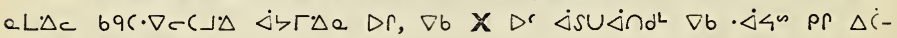
$<C \sigma c^{b} x$

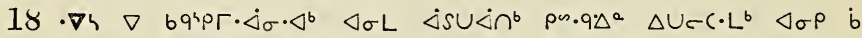

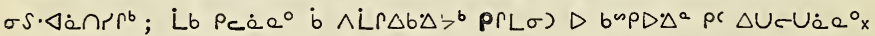

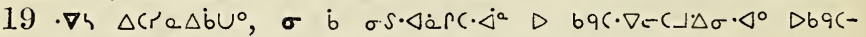

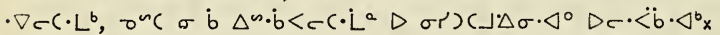

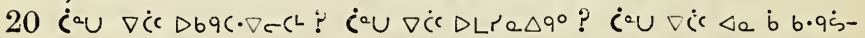

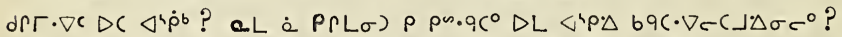

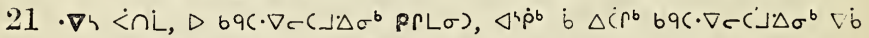

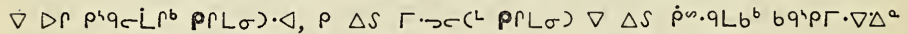

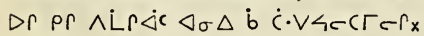

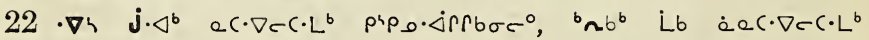
$69 c \cdot \nabla \sigma(\lrcorner \cdot \Delta \sigma \tau^{\circ}:$

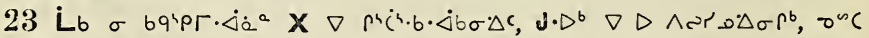
${ }^{b} \sim b^{b}$ bq<ंतn $\Delta^{a}$ b $\triangle U c\left(\rho^{b}\right.$;

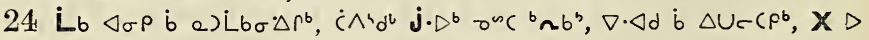

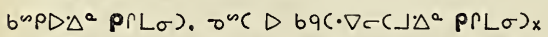

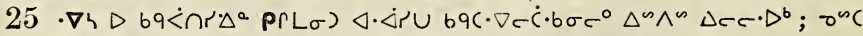

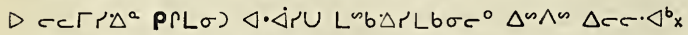

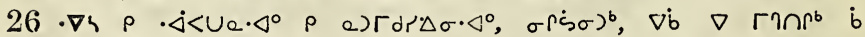

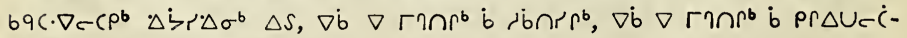
$\operatorname{dr} \rho^{b} \times \nabla$ a)Lbo $\Delta r^{b}$ :

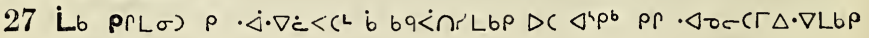
270 


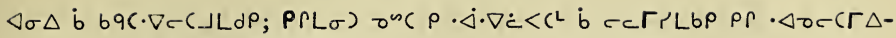
- $\nabla L b P \triangle \sigma \Delta b$ b $L b \triangle P L b P$;

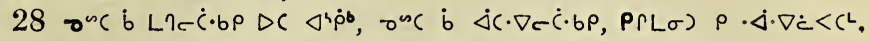
$\nabla \nabla$, onc $q \cdot \dot{b}$ e $\nabla \dot{b}$ b $\Delta c \cdot b \rho$ pr $\sigma-s \cdot \Delta \dot{a} r \dot{c} L b \rho \Delta \sigma \Delta \dot{b} \Delta c \cdot b \rho$ :

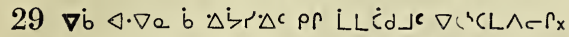

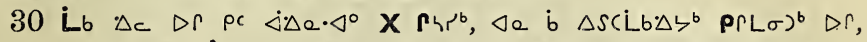

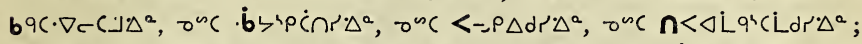

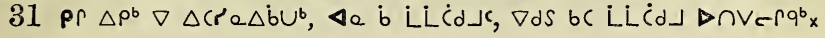

\section{$\Lambda^{\prime} p C r a \triangle b^{\circ} 2$.}

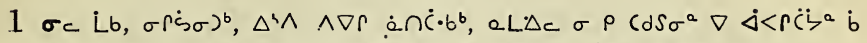

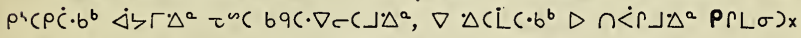

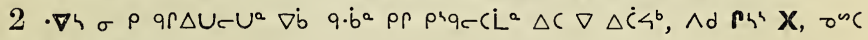
$\Delta c \nabla \rho \operatorname{cic} \cdot b \Delta c_{x}$

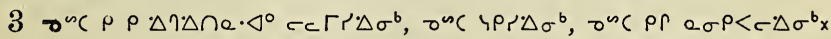

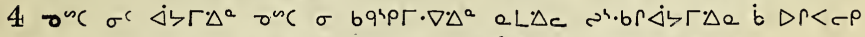

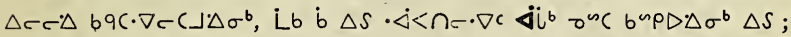

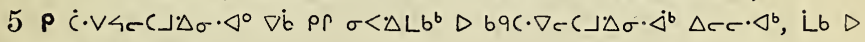
b $\left.\backsim \rho D \triangle \sigma^{b} P \Gamma L \sigma\right) x$

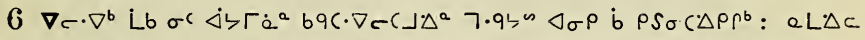

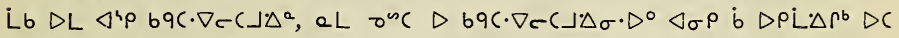
$\triangleleft^{4} \dot{\rho}^{b}, \dot{b} \quad \triangleleft \cap \neg r<\kappa^{b}$ :

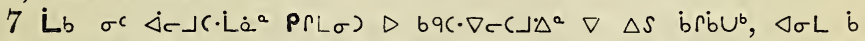

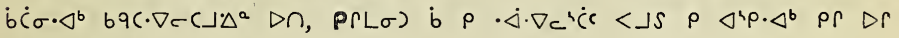

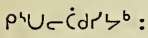

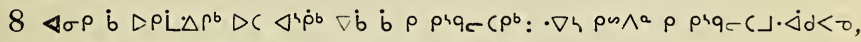

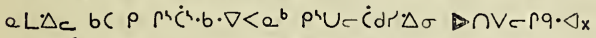

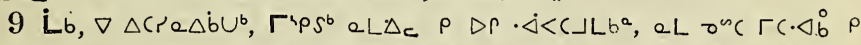

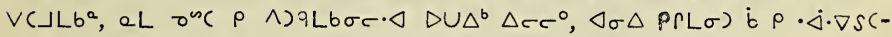
$L \cdot \dot{\Delta c} \triangleleft \sigma \Delta$ b $i \rho \Delta d c_{x}$

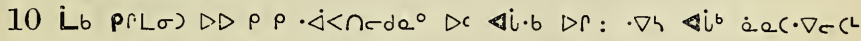

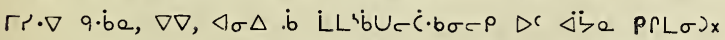

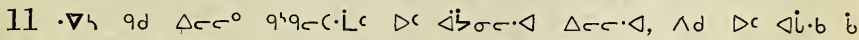

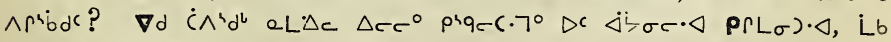
$\wedge$ Ad $D(\Delta i \cdot b \text { PrL })_{x}$

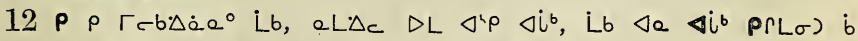

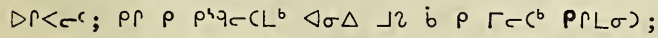

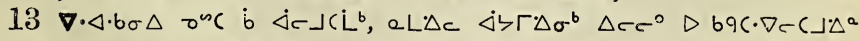

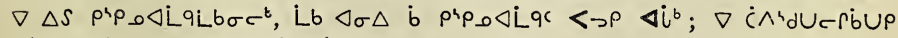
$\Delta i d \Delta q \cdot \dot{b} e \Delta p r \Delta \sigma \Delta \dot{b} \Delta i \dot{d} \cdot \Delta \rho_{x}$

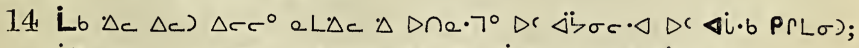

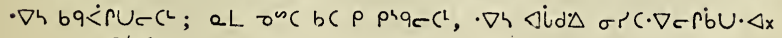
271 


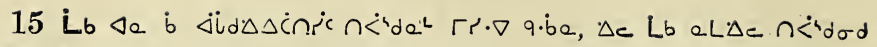
$\triangleleft \cdot \nabla r \cdot \triangleleft x$

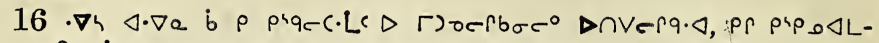

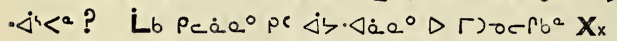

\section{$\Lambda^{4} p\left(r a \triangle b^{2} 3\right.$.}

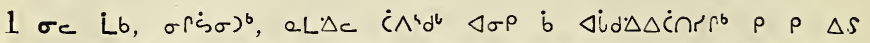

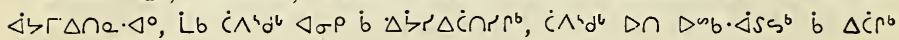
$X)^{b} x$

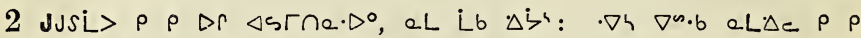

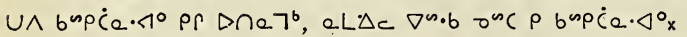

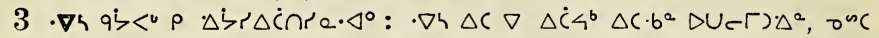

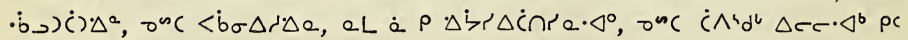
$\Delta S \wedge\lrcorner \dot{C} a \cdot \triangleleft 0$ ?

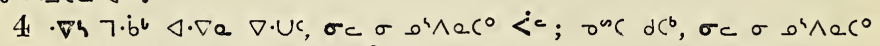

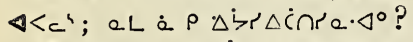

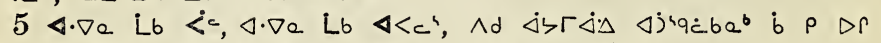

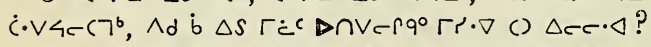

$6 \sigma_{c} \sigma \rho \sigma\left(\Delta \rho \rho b^{\circ}, \Delta<c^{4} \rho \cdot \Delta 4 \cdot \zeta V b \Delta q^{\circ} ;\right.$ Lb $\left.P \rho L \sigma\right) \rho_{c} \rho p q^{\circ} x$

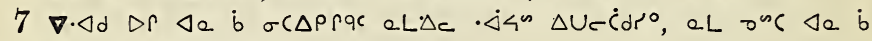
$\cdot \Delta h \cdot \zeta \vee b \Delta q c ; L b$ prL $\sigma) \wedge d$ b c $e p q c_{x}$

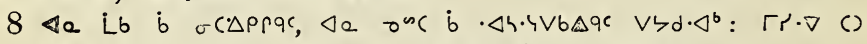
$\Delta c c^{\circ}$ Lb bc $\Gamma \dot{c} b \sigma \Delta^{\circ} \cap \wedge c \cdot \nabla \nabla n<\Delta L d r \cdot \Delta^{a}$ b $\rho \quad \Delta S \Delta j^{4} q c_{x}$

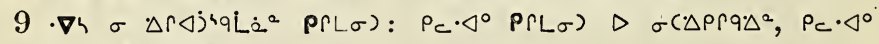
P $(L \sigma) D \cdot \nabla^{4} b r q q^{2} \times$

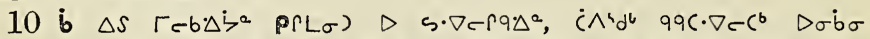

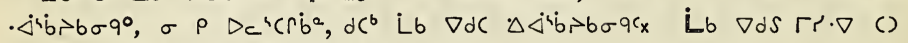

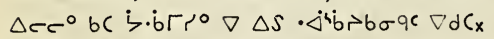

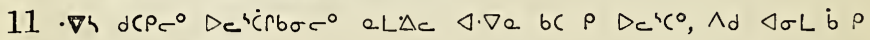
$\nabla_{c}^{\prime} \dot{C} \sigma \cdot \Delta^{b}, \nabla \cdot \Delta d \mu^{4} X_{x}$

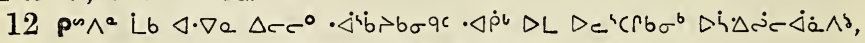

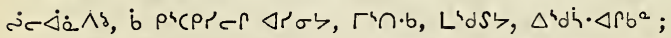

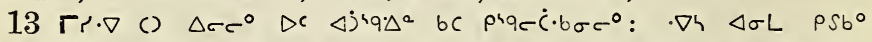

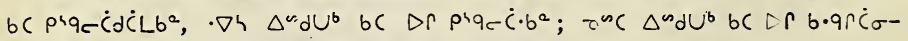

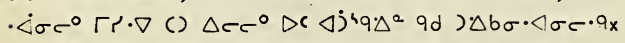

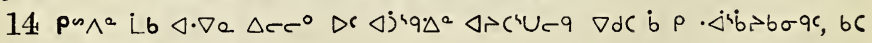

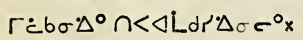

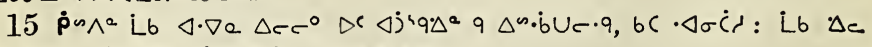

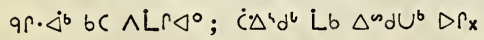

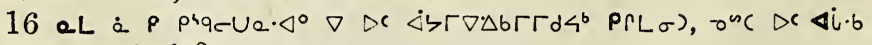
- prlor $\nabla \wedge \mu^{4} \dot{b} \Gamma^{\circ} \dot{c}^{b}$ ? 


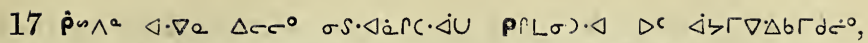

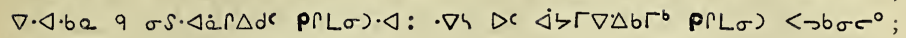
$\rho_{c} \cdot \Delta^{\circ} L_{b} \nabla \cdot \triangleleft d \quad \Delta c \Delta b \Gamma \nabla \Delta b \Gamma^{b}$ x

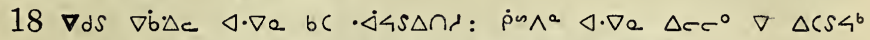

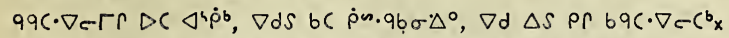

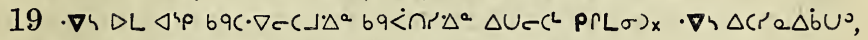

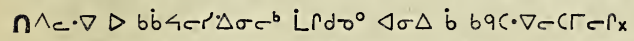

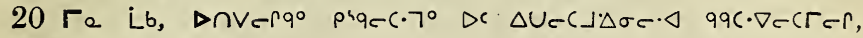
$<\cdot b^{a} c^{\circ} \nabla \Delta U_{-} \dot{c} \cdot b \sigma c P_{x}$

$21 \nabla \cdot \triangleleft d$ Dr $\nabla \dot{b} \Delta c \quad \Delta \cdot \nabla e$ bC LLLCdJ $\Delta r c a^{b} \Delta S ; \quad \nabla h \quad r r \cdot \nabla$ q.be

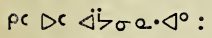

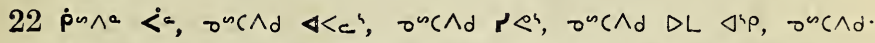

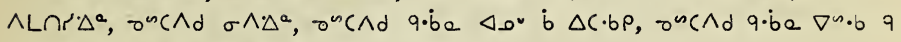

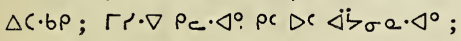

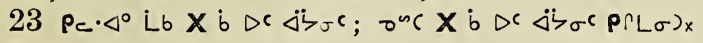

\section{Nipcre $\triangle b^{2} 4$.}

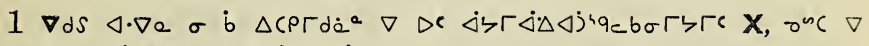
be. $\nabla e n e b \Delta \dot{\zeta} b \operatorname{p}(L \sigma) D$ br $q \cdot \dot{b} e_{x}$

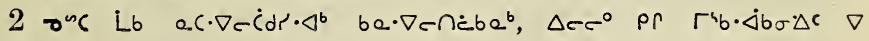
bל 'b?

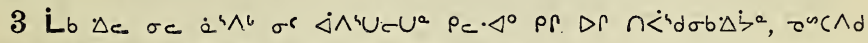

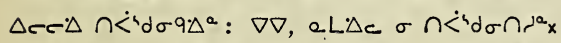

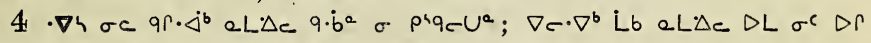

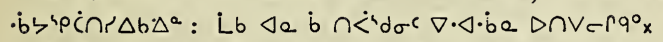

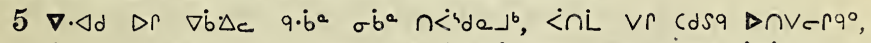

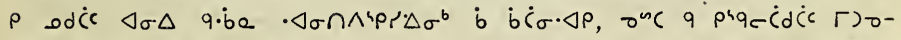

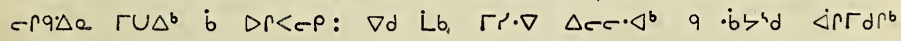
$P(L \sigma) \cdot \triangleleft x$

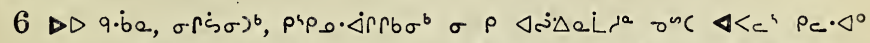

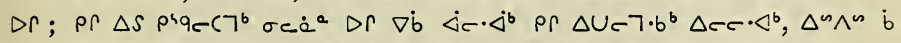

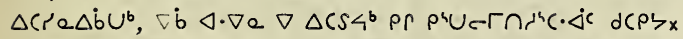

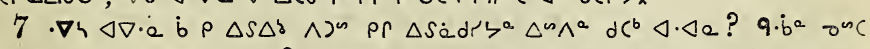

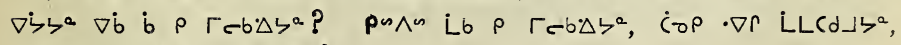
Le $\wedge d \nabla \dot{b} \nabla \rho \Gamma-\dot{b} \Delta \zeta^{a}$ ?

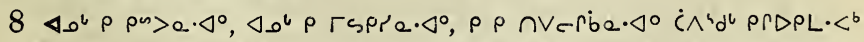

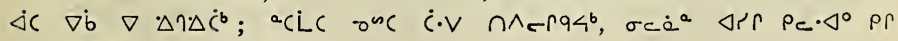
nVerqibs

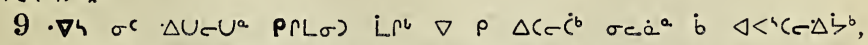

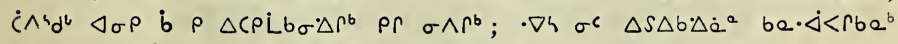

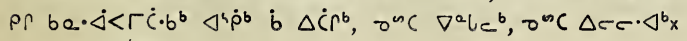




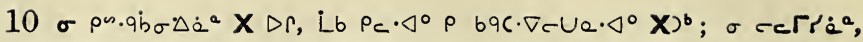

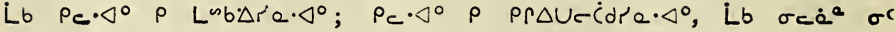

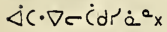

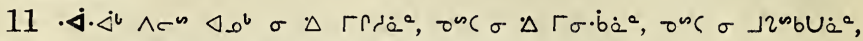

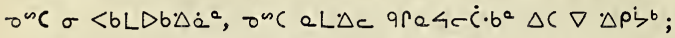

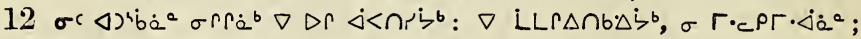

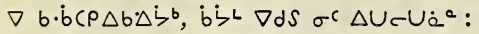

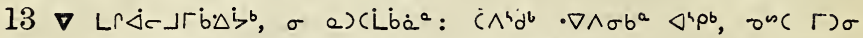
$<\cdot b^{a} c^{0} \dot{b} \cdot \nabla \wedge \sigma \dot{b} U^{b} \sigma^{c} \Delta S \Delta b \Delta \dot{a}^{a} \wedge c^{s} \Delta \sigma^{b} \nabla \rho S \dot{b} b x$

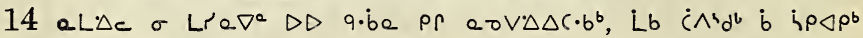
$\sigma d r^{b} \rho c \Delta S$ ל. brr $r \cap a \cdot \Delta^{\circ} x$

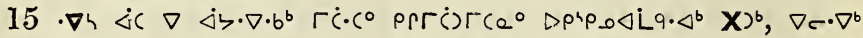

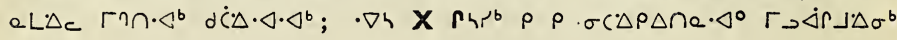
$\Delta r_{x}$

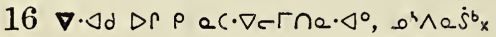

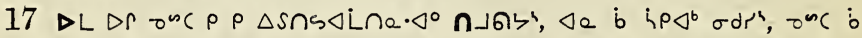

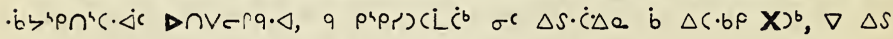

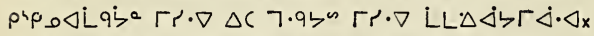

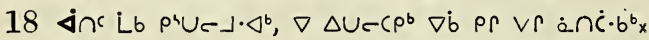

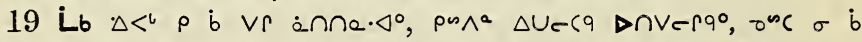

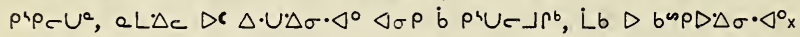

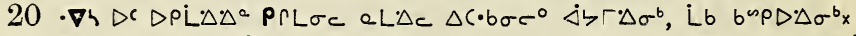

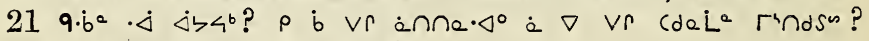
$\operatorname{sic} \wedge d$ i $\rho \cdot \nabla \Delta \sigma^{b}$, onc sibnr $\Delta \sigma^{b}$ ?

\section{Arpcra $\Delta b^{a} 5$,}

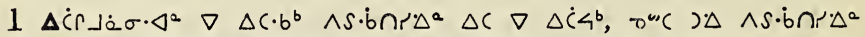

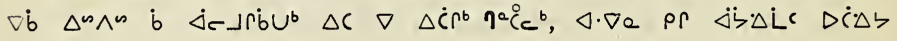
$\Delta \Delta r \cdot \Delta x$

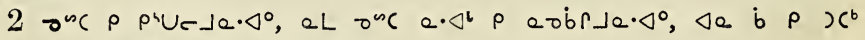

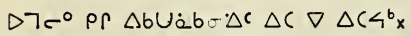

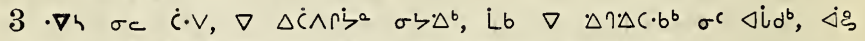

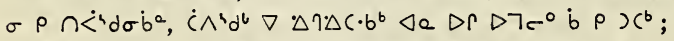

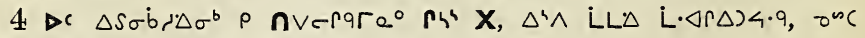

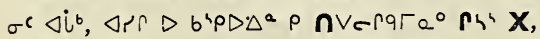

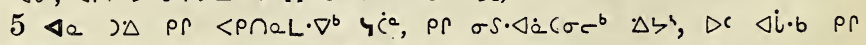

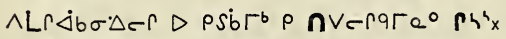

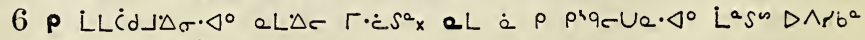

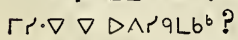

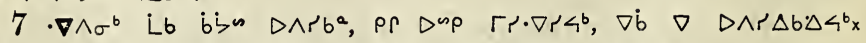

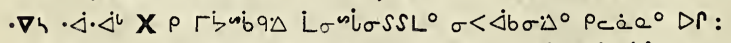

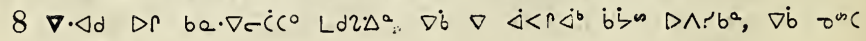
274 


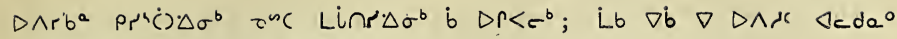

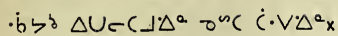

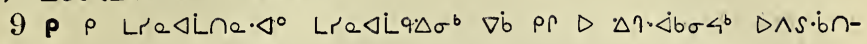
ribl:

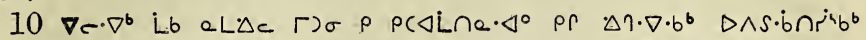

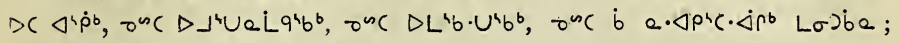

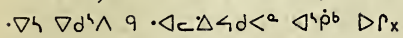

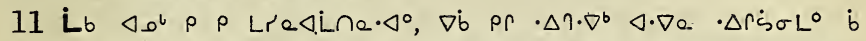

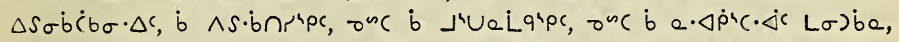

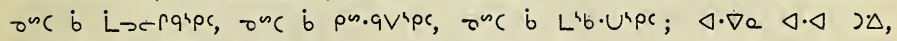

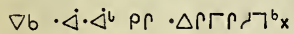

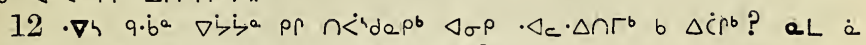

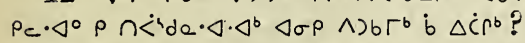

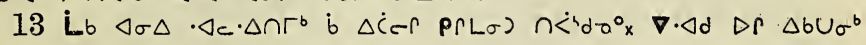
$\Delta C \Delta r \nabla \Delta \dot{C}<^{b} \triangleleft e$ Linr. $\Delta \Delta c c^{\circ} x$

\section{A'pcre $\Delta b^{a} 6$.}

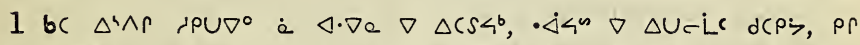

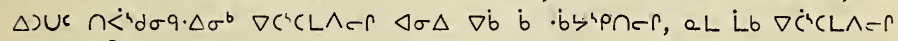
$D<\rightarrow p h$ ?

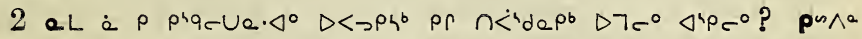

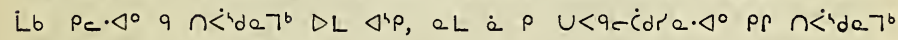
$\triangleleft \sigma \Delta L \cdot \triangleleft^{\natural} \dot{b} \dot{\Delta} \wedge \backsim \sim \dot{C} \cdot b \rho$ ?

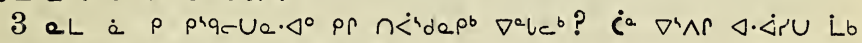
$\triangle \sigma \Delta \Delta L \wedge L \cap \pi \cdot \Delta \sigma^{b} \dot{b} \cap V c-\dot{C} \cdot b \rho$ ?

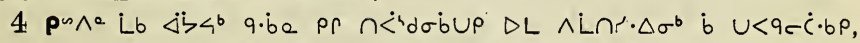

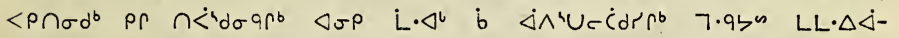
ᄂ $\triangleleft \cdot \triangleleft x$

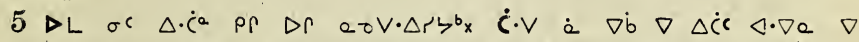

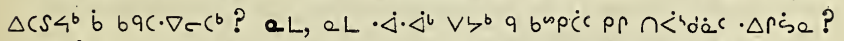

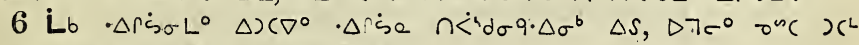

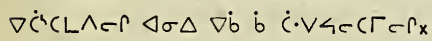

$7 \dot{C} \cdot \wedge$ Lb $\rho \cdot \Delta \sigma)$ Ua. $\left.\Delta^{\circ}, 7 \Gamma^{\cdot} \cdot d^{b} \nabla \Delta\right)(\Delta)<^{b} \cap \dot{<}^{b} d \sigma q \cdot \Delta \sigma^{b} \Delta S_{x} \dot{C}_{0} \rho$

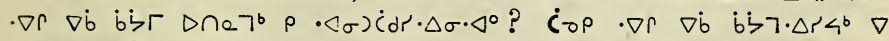
$r s \triangle b \cdot \Delta \zeta^{b} ?$

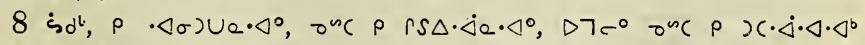
pґsं $\sigma \cdot \triangleleft \cdot \triangleleft^{b} x$

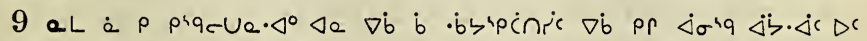
$\Delta P L \cdot \Delta \cdot \Delta \sigma c^{\circ} P(L \sigma) \cdot \Delta$ ? $\nabla \dot{b} \cdot \Delta c \rho \dot{b} \cdot \dot{\Delta}\left\langle S \Delta b \cdot \Delta a \cdot \Delta^{0}:\right.$ e L. $\Delta c \quad \Delta \wedge S \cdot \dot{b}-$

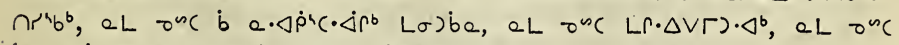

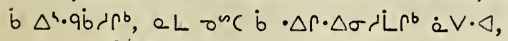




\section{$\sigma^{n C L} d \sim^{2} \rightarrow 0$. 7.}

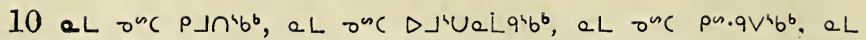

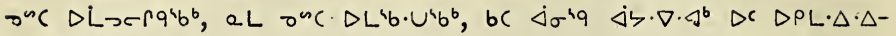
$\sigma c^{\circ} P(L \sigma) \cdot \Delta x$

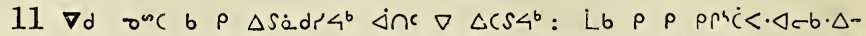

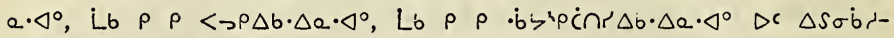

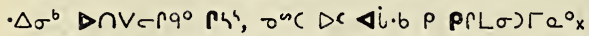

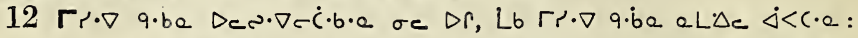

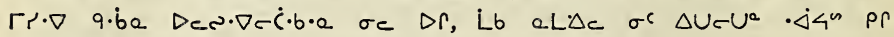
nVerdio $\mathrm{C}$

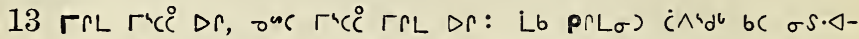

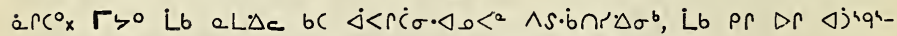

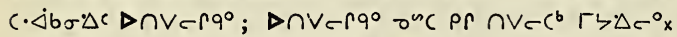

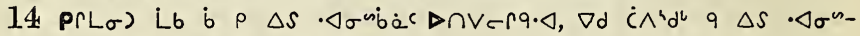
$\dot{b} \sigma c^{b} \cap \wedge c \cdot \nabla \triangleright b^{4} p \Delta \Delta \sigma^{b} \Delta r_{x}$

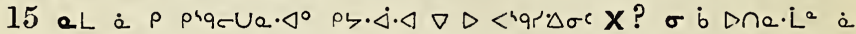

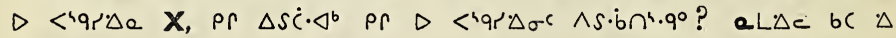
$\Delta p^{a} x$

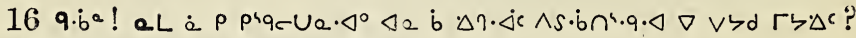

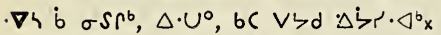

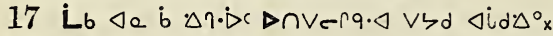

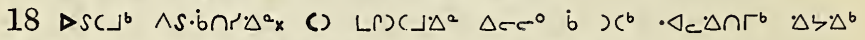
$\Delta)(L$; Lb $\left.\Delta a b) c^{b} \wedge s \cdot b \cap \Delta \sigma c^{\circ} L r\right)\left(L \cap \wedge c \cdot \nabla \Delta b^{\circ}{ }_{x}\right.$

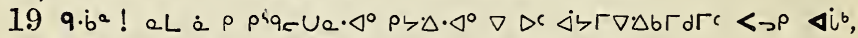

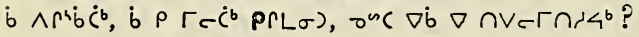

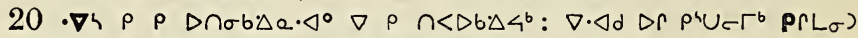

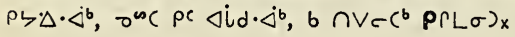

\section{Aspcra $\Delta b^{a} 7$.}

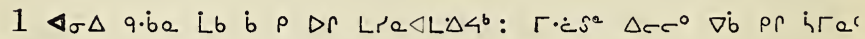
$\Delta 4 \cdot 9 \cdot \Delta x$

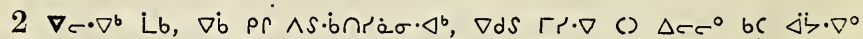

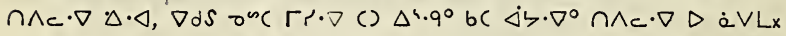

$3 \nabla d S \triangleleft e$ b $\Delta \Delta c$ bc $\Delta \cap \wedge \rho 2 \cdot \Delta)\left(\cdot \nabla^{\circ} \Delta \cdot \Delta: \quad\left(\wedge^{\prime} d^{l}\right.\right.$ onc $<e$ b $\triangleright$

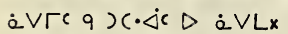

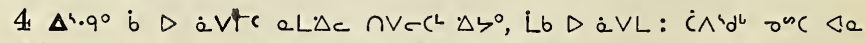
a $V^{\circ}$ b $\Delta \Delta c$ a L $\left.\Delta e \cap V c C^{L} \cap \Lambda_{c} \cdot \nabla \Delta\right\rangle^{\circ}$, Ĺb $\Delta \cdot \Delta x$

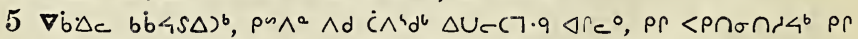

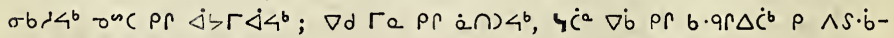
ก. $\Delta \Delta U \subset C-\Delta \sigma \cdot \Delta \cdot \triangleleft \Delta r_{x}$

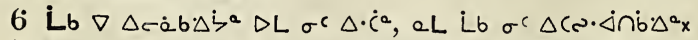

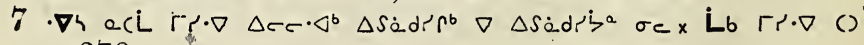
276 


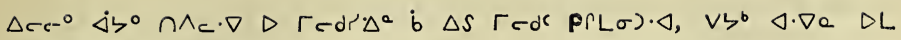
$\triangle S$, d $C^{b}$ Lb $\wedge$ ) $\Delta S_{\mathrm{x}}$

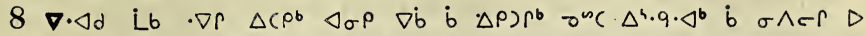

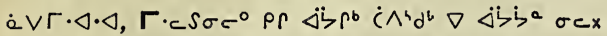

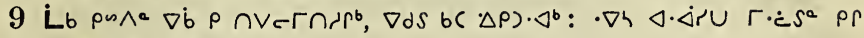

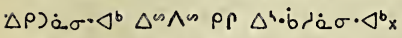

$10 \Delta_{\sigma} \rho$ lb b $\left.\Delta \rho\right) \rho^{b} \sigma^{c} \Delta\left(\sim^{2} L \cdot \triangleleft^{b}\right.$, Lb aL $\Delta c \sigma c$, Lb $\Delta \cap V e r 9^{\circ}, \nabla d S$ $\nabla \dot{b} \Delta_{c} \Delta^{4} \cdot q^{\circ}$ b $D_{\dot{e}} V \Gamma c b C$ abU $D$ àVL:

11 Ĺb pis $\wedge^{a} a b c U, \nabla d S$ a ${ }^{\circ} \mathrm{c} C$ Lb $\nabla \dot{b} \Delta c \Delta a \dot{b} \Delta \Delta c b c \cdot \nabla \wedge \nabla^{\circ} \Delta \cdot \Delta x$

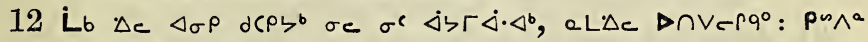

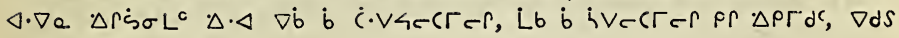
$\nabla b \cdot \Delta e$ bC $\cdot \nabla \wedge_{\sigma^{\circ} x}$

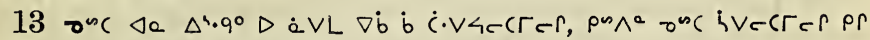
$\triangle P \Gamma d c, \nabla d S \nabla b \Delta c$ bc abU०x

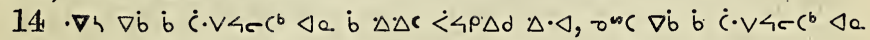

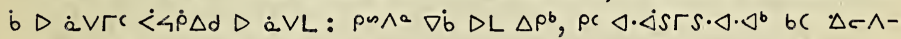

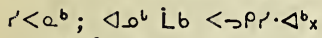

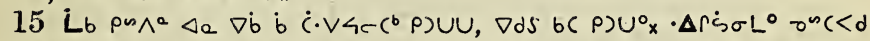

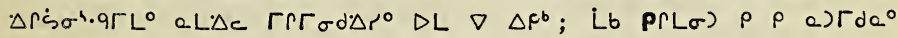
b่า $\neg-(\lrcorner \Delta \sigma^{b} \Delta S_{x}$

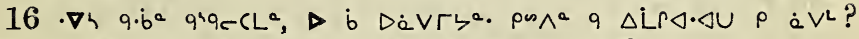

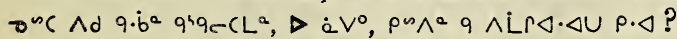

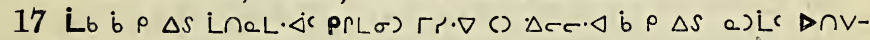

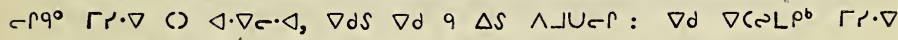
LL $\Delta \dot{j}>r \dot{j \cdot \Delta b x}$

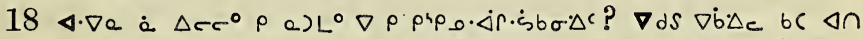

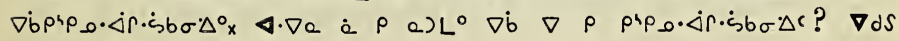
$\nabla \dot{b} \Delta c$ bc paps. $\Delta \dot{\beta} \cdot \dot{s} b \sigma \Delta \Delta^{\circ} x$

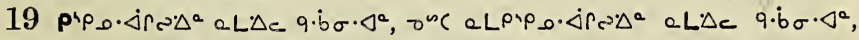

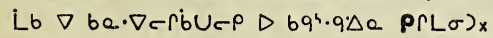

$20 \nabla d S \Gamma r \cdot \nabla() \Delta c^{\circ} \Delta U$ b $p \Delta S$ a) Lb $\sigma^{\circ} \Delta c \nabla d C$ bC $\left(S 9^{\circ} x\right.$

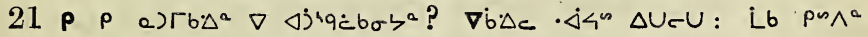
pupciso pP $\left.\cap V \in \Gamma \cap r^{\circ}, D L \varangle \cdot \Delta r U\right)_{x}$

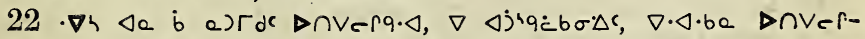

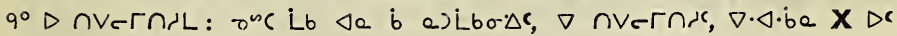
$\Delta \dot{\jmath} 9 \dot{b} b \sigma L_{x}$

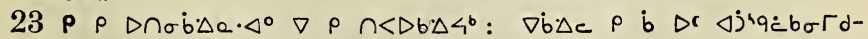
$\cdot \dot{\Delta \cdot \Delta b} \Delta r \cdot \cdot \Delta^{b} x$

$24 \sigma r \dot{s} \sigma)^{b}, \nabla d S \Gamma r \cdot \nabla() \Delta c c^{0}, \Delta c \dot{b} \rho \Delta S$ a) $L b \sigma \Delta c, \nabla j c$ q $\Delta r c-$ SqLi $P \Gamma L \sigma) \cdot \Delta x$

$25 \Delta \backsim \rho_{\sigma} \rho^{4} \cdot q \cdot \Delta^{b} \Delta \Gamma L b, a L \cdot \Delta c \sigma \rho \Delta\left(\omega \Gamma^{b} p \Gamma L \sigma\right): \nabla c \cdot \nabla^{b} L b \sigma \cdot \Delta U^{a}$

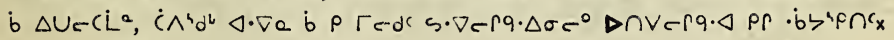
277 


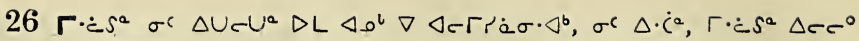
$\nabla d \Delta S$ pr $\Delta \ddot{\zeta c} c_{x}$

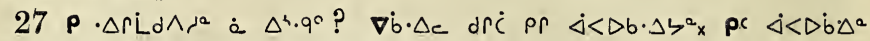

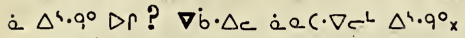

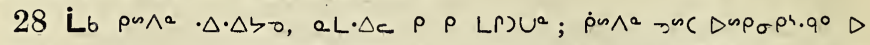

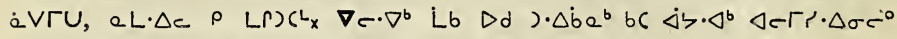
$\cdot \Delta \dot{\zeta} \Gamma \cdot \Delta \sigma^{b}$ : Ĺb $\rho$ Lár $\Delta \cap a \cdot \Delta^{\circ} x$

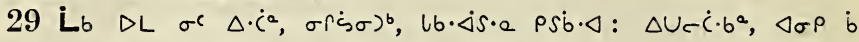

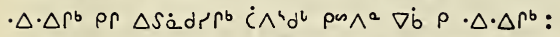

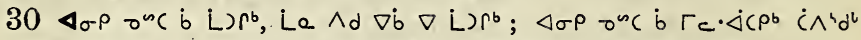

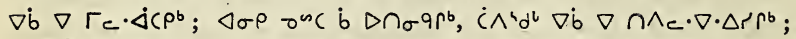

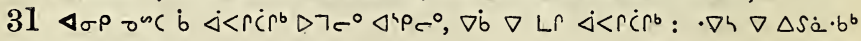
$\nabla L \Delta 4 \rho \cdot \nabla \wedge<\sigma^{\circ} x$

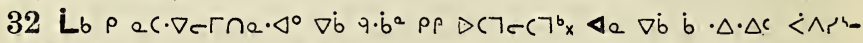

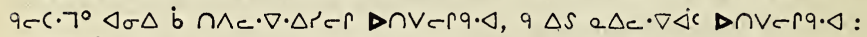

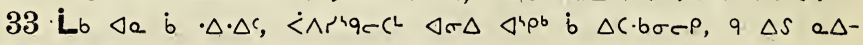
$c \cdot \nabla<i \cdot \Delta \cdot \Delta x$

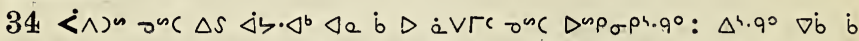

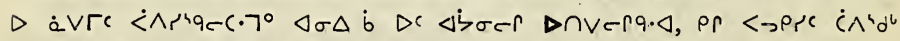

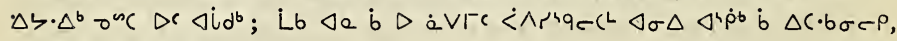
$\rho \Delta S$ e $\Delta c \cdot \nabla \dot{<c} \nabla$ à $V L_{x}$

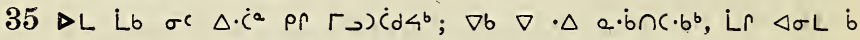

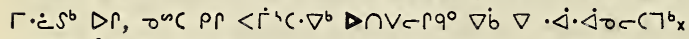

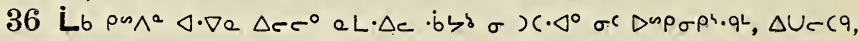

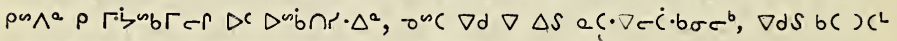
$\cdot \dot{\Delta})(b, a L \cdot \Delta c L \Gamma)(L: \nabla d S b C \cdot \Delta \rho) \cdot \Delta^{b} x$

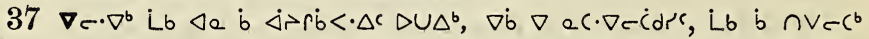

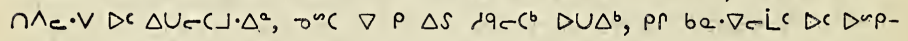
$\left.\sigma P^{4} \cdot q L, \Gamma \dot{\partial}\right)\left(L_{x}\right.$

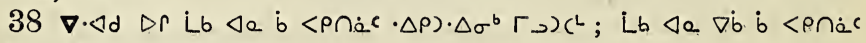
$\cdot \Delta \rho) \cdot \Delta \sigma^{b} \triangleleft \cdot \Delta \dot{\Delta} U \Gamma_{\lrcorner} \rightarrow L_{x}$

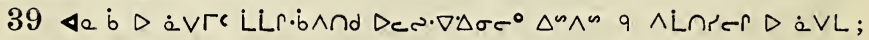

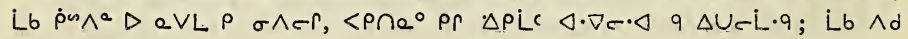
$\Delta \cap \tilde{r}^{\prime} q^{b} \Delta S_{x}$

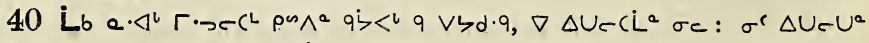
onc $\left.\nabla \Delta \zeta \Delta L^{6} p r L \sigma\right) \Delta(\Delta i \cdot b x$

\section{A pera $\triangle b^{a} 8$}

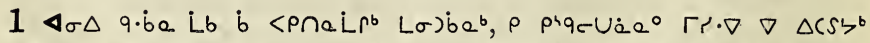

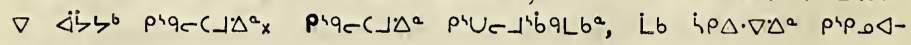
L 


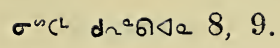

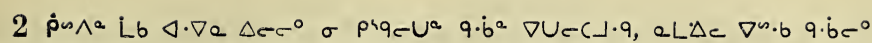
p'qe CL q $\triangle S$ p'qe $c p<a x$

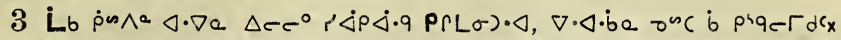

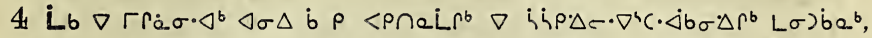

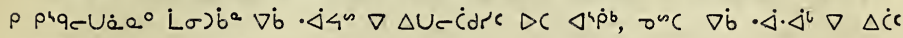
$d\left({ }^{b} P(L \sigma), \wedge d V \nu^{b} x\right.$

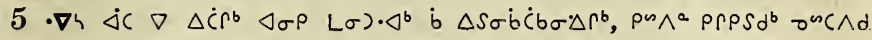

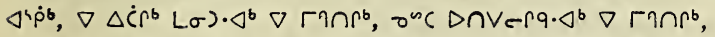

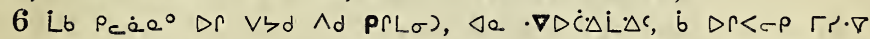

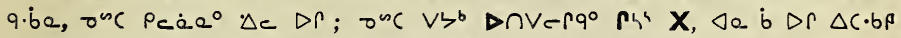

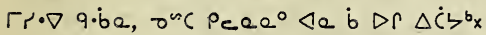

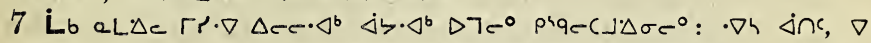

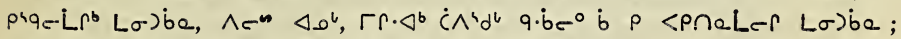

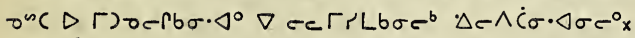

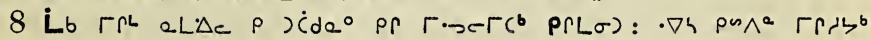

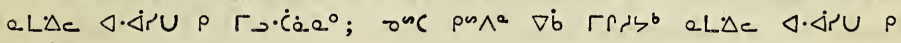
$L \Gamma \cdot \dot{C} e^{\circ} x$

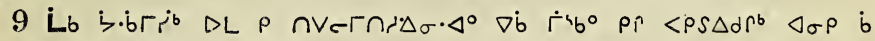
$\operatorname{cetphp^{x}}$

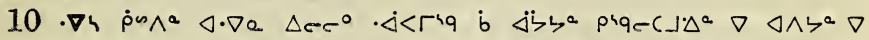

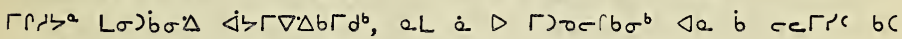
AqeCL pr rre $\Delta \sigma \Delta \dot{b}<$ <naLer Losbe;

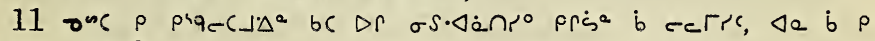

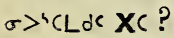

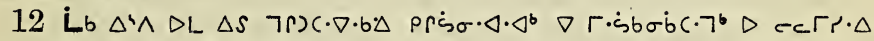

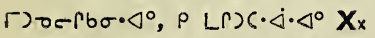

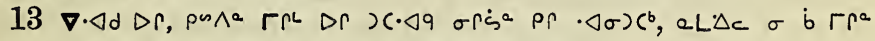

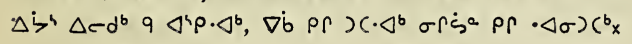

\section{A'pcria $\Delta b^{a} 9$.}

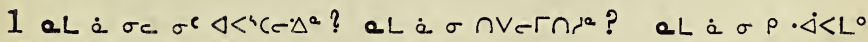

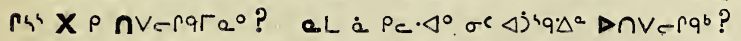

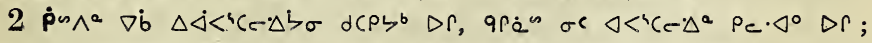

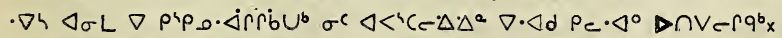

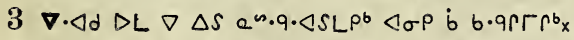

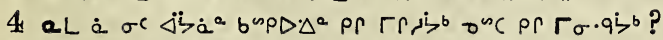

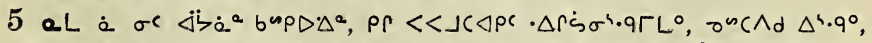

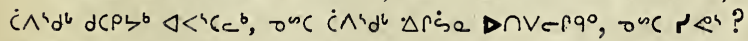

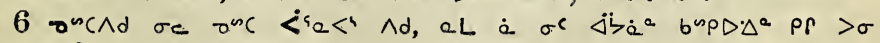
$\triangleleft \dot{)}$ ฯட்?

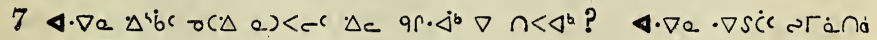
279 


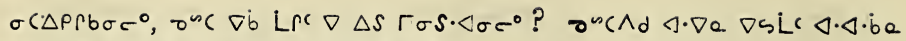

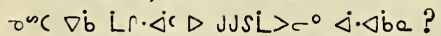

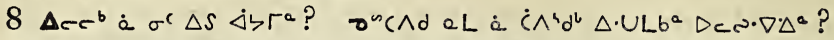

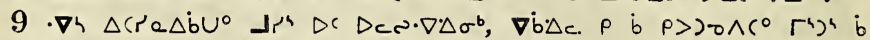

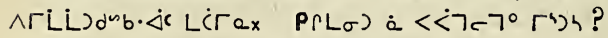

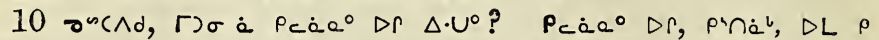

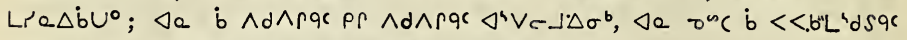

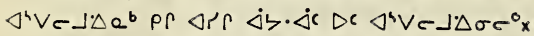

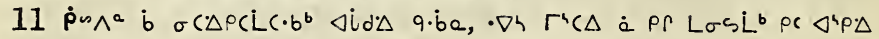
$q \cdot \dot{b} \sigma \cdot \triangleleft \cdot \triangleleft$ ?

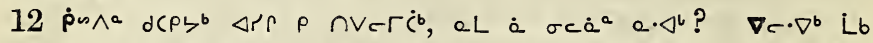

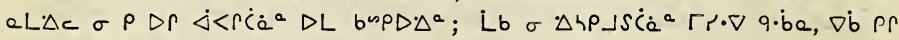
$\left.b \wedge r c \cdot \Delta_{b} \triangleright r \rightarrow \Delta r\right\lrcorner \Delta^{a} X_{x}$

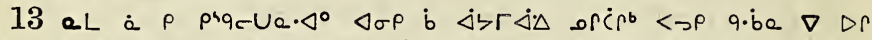

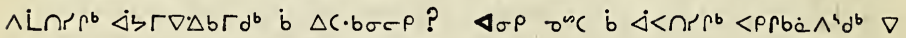

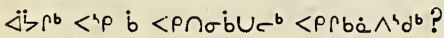

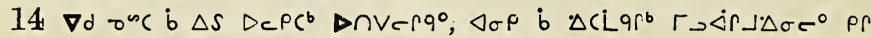

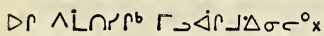

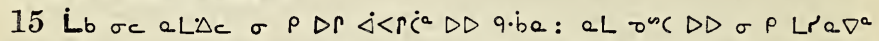

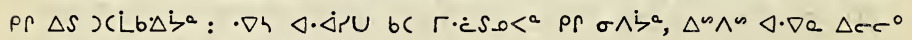

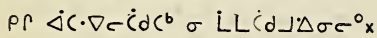

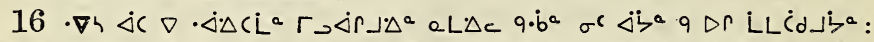

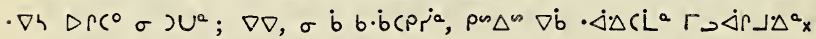

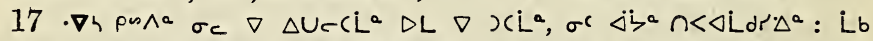

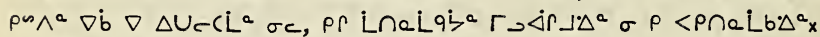

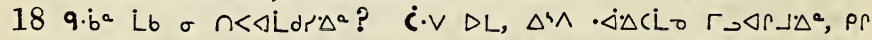

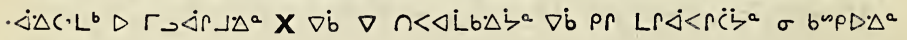
$\Gamma\lrcorner \dot{\Delta r}\lrcorner \Delta \sigma^{b} x$

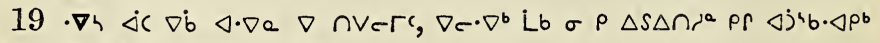
$\Gamma r \cdot \nabla, p \rho \triangle S$ bsp $\Delta \rho^{b} a \cdot \Delta^{l} \Gamma \eta c_{x}$

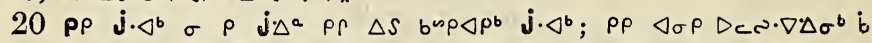

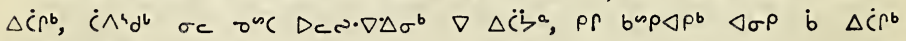
$\nabla c \pi \cdot \nabla \cdot \Delta \sigma^{b}:$

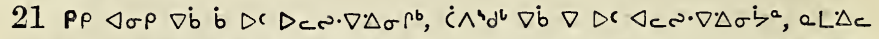

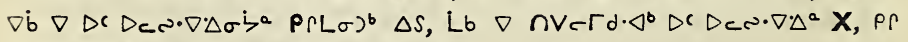
$\triangle S$ bup $\triangleleft \rho b \triangleleft \sigma \rho \nabla \dot{b} \dot{b} \nabla C D C N \cdot \nabla \cdot \Delta \sigma \rho^{b} \times$

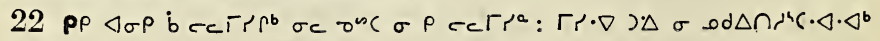

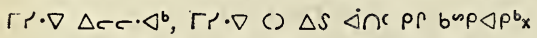

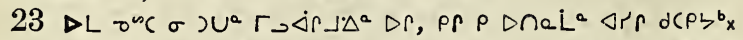

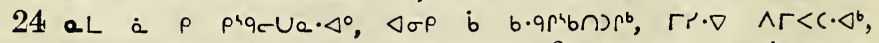

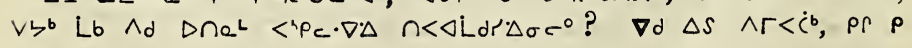
DกQ $77^{b} x$

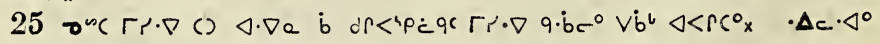
280 


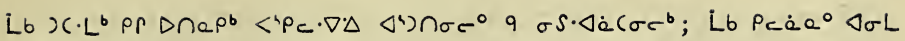
$\nabla \dot{b} 9 \sigma s \cdot \Delta \dot{c} c^{b} x$

$26 \nabla \cdot \Delta d \cdot \nabla r \Delta S \wedge r<\ddot{C} \zeta a \sigma c, a L \Delta c \nabla \dot{b} \nabla .9 \Gamma a \Delta \dot{b} ; \nabla d$ osc $\nabla S$

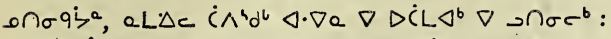

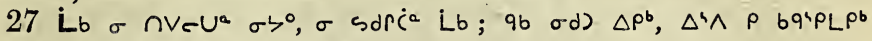

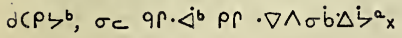

\section{Ápcre $\Delta b^{a} 10$.}

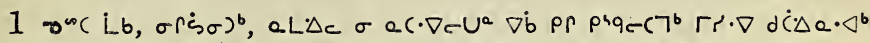

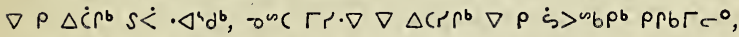

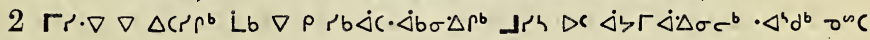
brbit ;

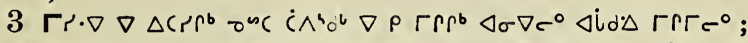

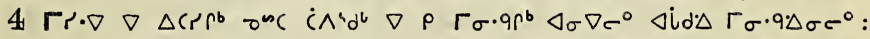

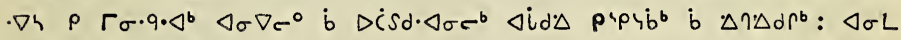
pฯp५íb Lb $\nabla \cdot \Delta b \mathbf{X x}$

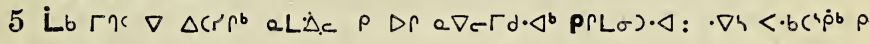

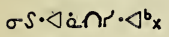

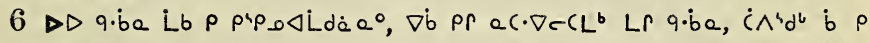
$e c \cdot \nabla c-c p^{b} \Delta c \cdot \triangleleft^{\circ} x$

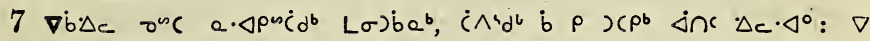

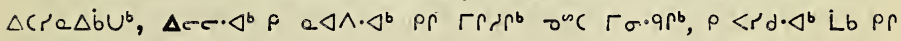
$\neg C \cdot \nabla r^{b} \times$

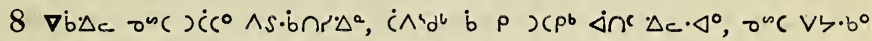

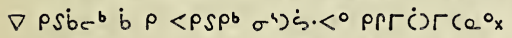

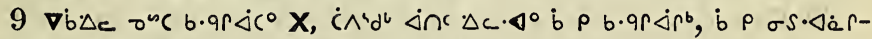

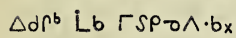

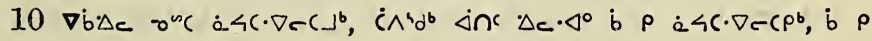
$\sigma \cdot S \cdot \Delta \dot{\alpha} r \Delta d \rho^{b} \dot{L} b \quad \nabla \sigma S \cdot \triangleleft \dot{Q} r \Delta \cdot \nabla \cdot \Delta x$

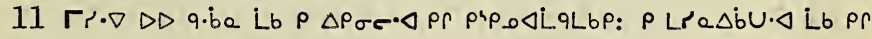

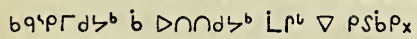

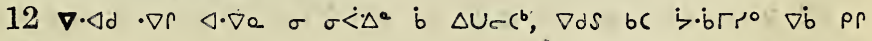
$<P S^{b} x$

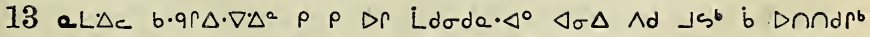

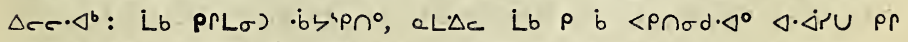

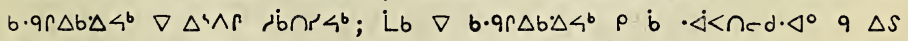

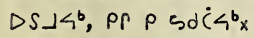

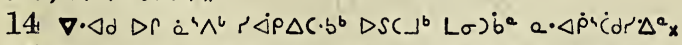

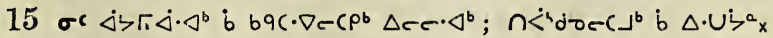

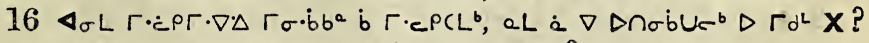
$\left.\checkmark a \Delta c d a^{\circ} b<\cdot 9 a^{b}, a L a \quad \nabla D \cap \sigma b U c^{b} \Delta\right\rangle^{\circ} X$ ? 


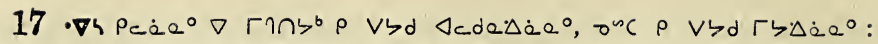

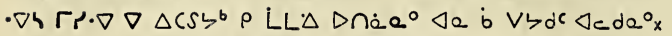

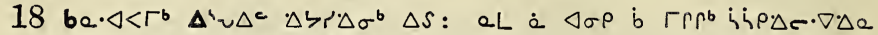

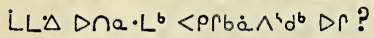

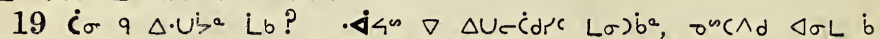

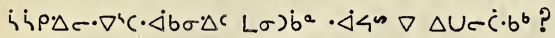

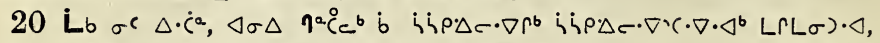

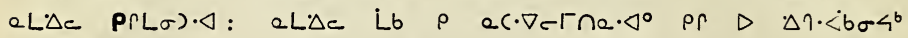
$L \Gamma L \sigma) \cdot \triangleleft^{b} x$

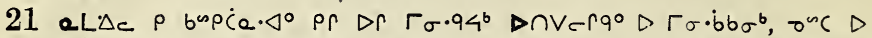

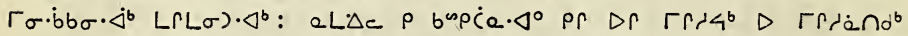

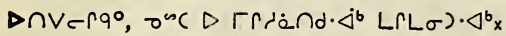

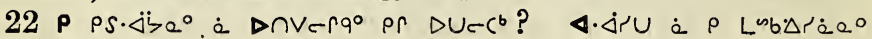
$\triangle \infty \wedge^{\infty} \Delta c$ ?

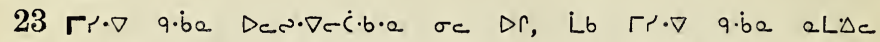

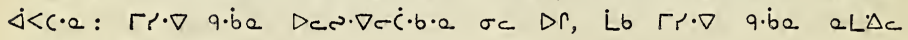
p'p. $\Delta$ LiqLb.Q.

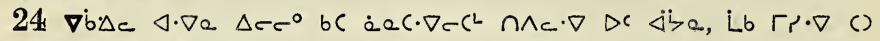

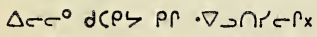

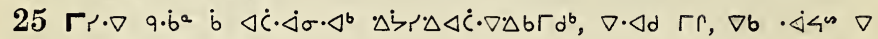

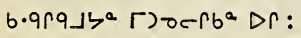

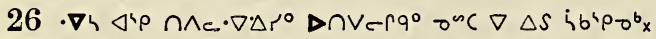

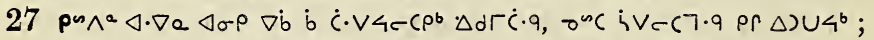

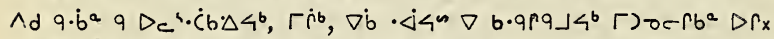

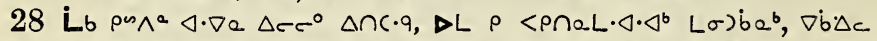

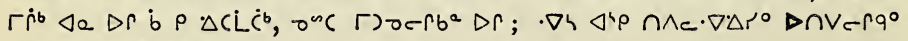
zic $\nabla \Delta S$ íbis ob

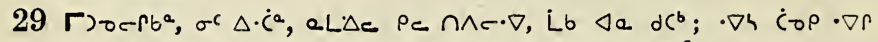

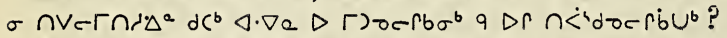

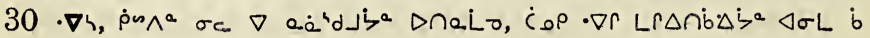

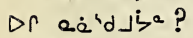

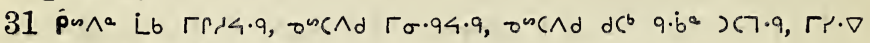

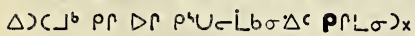

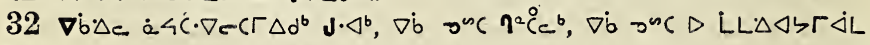
$P(L \sigma)$;

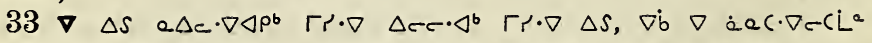

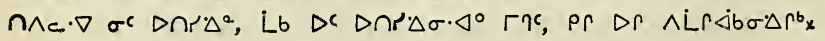

\section{A $P$ Pre $\triangle b^{\circ} 11$.}

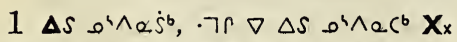

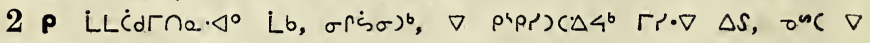
be. $\nabla \sigma c 7^{b} \triangleleft \sigma \Delta \Delta c \cdot \cdot \nabla \Delta a$, b $\Delta S<\rho \cap a L C \cdot b^{b} x$ 


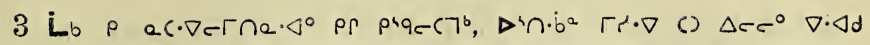

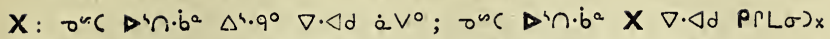

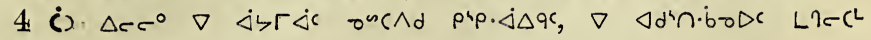
$D^{n} n \cdot b^{a} x$

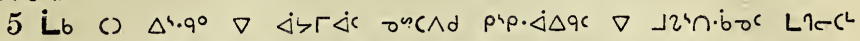

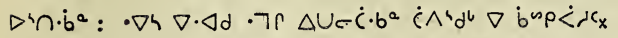

$6 \cdot \nabla h$ pa $\wedge^{a} \Delta^{4} \cdot 9^{\circ} \nabla b \triangleleft \cdot b e \Delta d r, \nabla d S$ bc $<^{4} \cdot b \Delta L^{\circ}$ onc: Lb $\rho a \wedge^{a}$

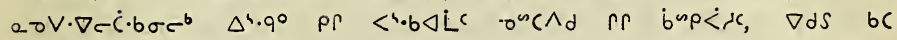
$\Delta \cdot b a \Delta d x$

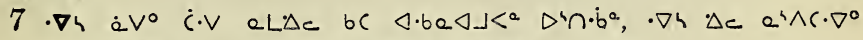

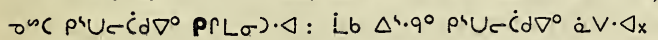

$8 \cdot \nabla 4 \dot{\alpha} V^{0} \dot{a} L \Delta c \Delta^{4} \cdot q^{b} D r$, Lb $\Delta^{4} \cdot q^{\circ} \dot{a} V^{b} \Delta \Gamma_{x}$

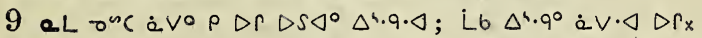

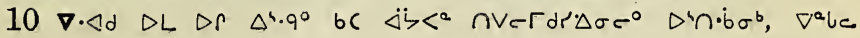
$\Delta r_{x}$

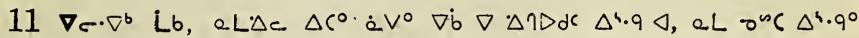
$\nabla \dot{b} \nabla \Delta \cap \Delta d^{d e} \dot{a} \vee \cdot \triangleleft, \Delta \cap V c r q^{b} x$

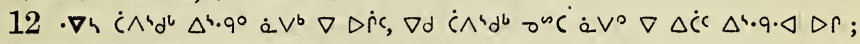
Lb $T$ R. $\nabla$ q.be. $p R L \sigma) D R_{x}$

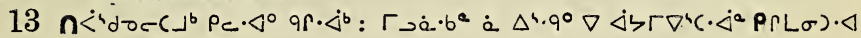
$\nabla \dot{6} \nabla \triangleleft \cdot b a \nabla r$ r ?

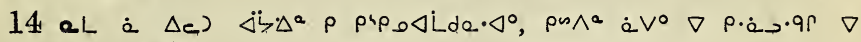
erV $\nabla c \dot{c} \cdot b \sigma c^{b}$ ?

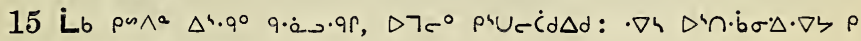

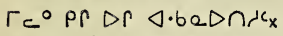

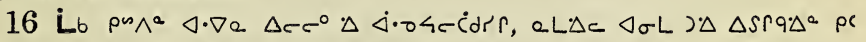

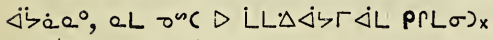

$17 \Delta L$ Lb b $\left.\Delta C L C \cdot b^{b}, a L \Delta c \rho \operatorname{LLC} d r \cap a \cdot \Delta^{\circ}, \nabla \vee \Gamma \quad L \cdot \Delta r \Delta\right) 4^{b}, \nabla b$

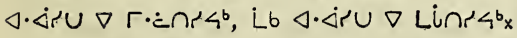

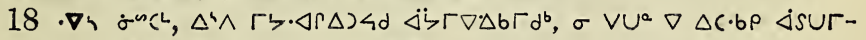
गेe $\Delta C \nabla \Delta \dot{C} \dot{c}^{b} ; a \cdot \Delta^{l} \sigma^{n c} \sigma \dot{c} \cdot V U^{a} x$

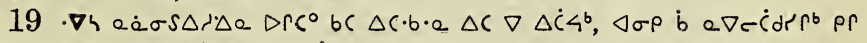
prq- Cंdr pr $\Delta C \nabla \Delta \dot{C}<c^{b} x$

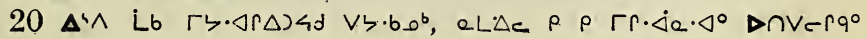
$\Delta \sigma V \cdot 9 \cdot \Delta^{a} x$

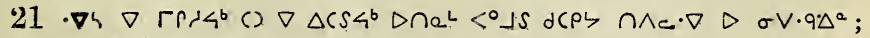

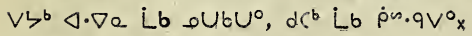

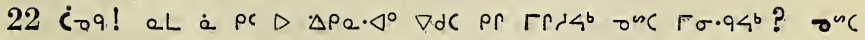

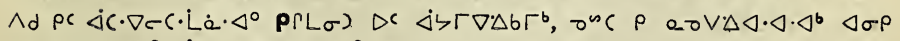

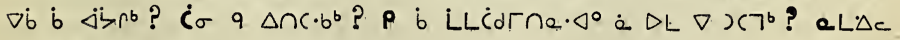

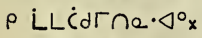

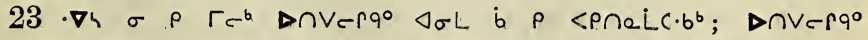

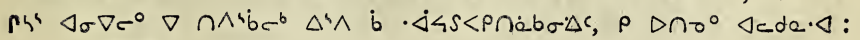
283 
24. $\Delta^{4} \wedge$ Lb b $\rho$ a $\left.\left.a^{4} d\right\lrcorner c, \rho \wedge d \sigma^{\circ}, \rho \quad \Delta \cdot \cup^{0} L b, \Delta \cap \sigma^{b},\right\lrcorner \Delta^{b}:$

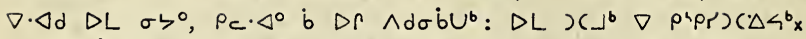

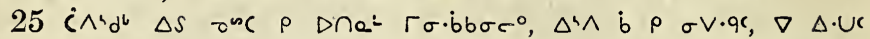
$\Delta L \Gamma \sigma \cdot b b^{a} \nabla \cdot \Delta d$ Dap $\left.\left.a^{b} d \Gamma\right) \Delta^{a} \sigma \Gamma d^{b}: \Delta L\right)(\lrcorner^{b}, c \cdot c^{\circ} 7 \sigma .94 d \nabla$ pips) $\left(\Delta<s^{b} x\right.$

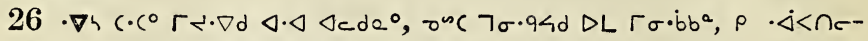

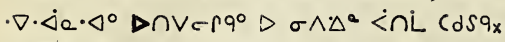

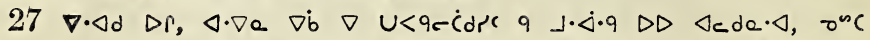

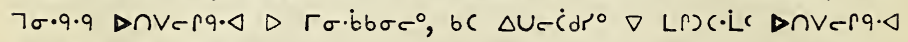
$\triangle \sqcup \Delta c^{\circ}$ onc $D \Gamma d c^{\circ} x$

28 Lb $\nabla d S \Delta c c^{\circ} b c$ abc. $\nabla c r \cap d, \nabla d$ Lb 9 j. $\triangleleft r \triangleleft \sigma \Delta \triangleleft c d a \cdot \triangleleft$, osc q $D \Gamma \Gamma \sigma \cdot q c \Delta \sigma \nabla c^{\circ} \Gamma \sigma \cdot \dot{b} b \sigma c^{\circ} x$

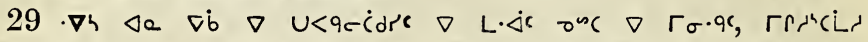

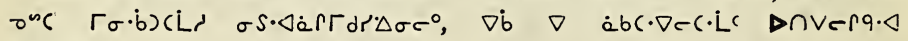
$\Delta \sqcup \Delta c^{\circ} x$

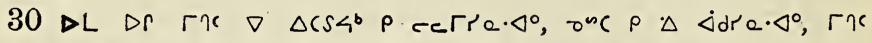
onc $\sigma<\cdot \Delta b^{x}$

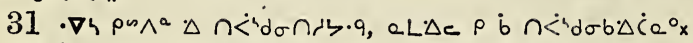

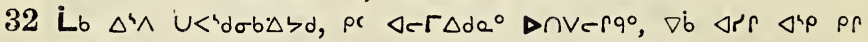
$\sigma S \cdot \Delta \dot{Q} \Gamma \Gamma b \Delta b^{b} \mathrm{x}$

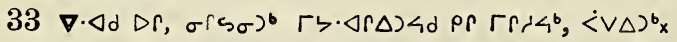

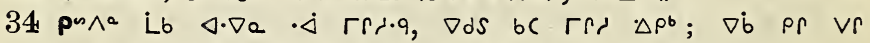

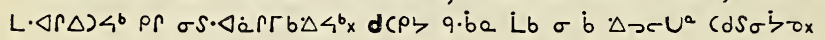

\section{$\Lambda$ A'pcra $\triangle b^{\circ} 12$}

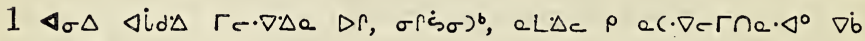
pr prqc $c 7^{b} \times$

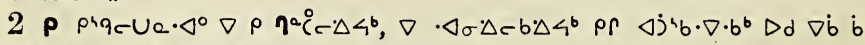

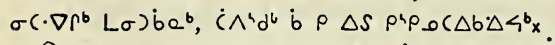

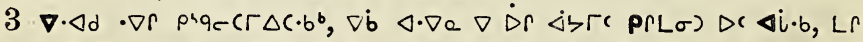

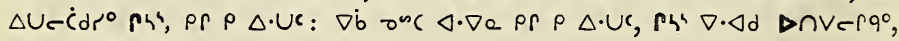
$\wedge_{d}<\rightarrow \rho \varangle i \cdot b \quad r_{x}$

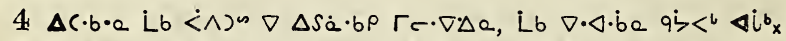

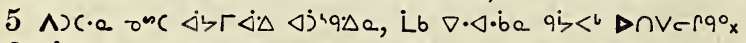

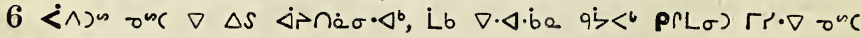
$r+. \nabla b)\left(L q^{\prime} x_{x}\right.$

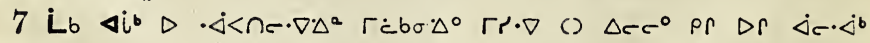
$D n_{i} \cdot x$

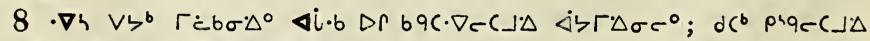
$\Delta ל \Gamma \cdot \Delta \sigma \sigma^{\circ} \triangleleft \sigma \Delta q \dot{b}<\downarrow<i \cdot b \Delta r$;

284 


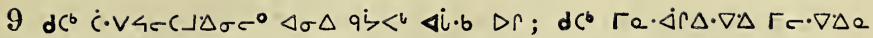
$\triangleleft \sigma \Delta q \dot{\phi}<6<i \cdot b \Delta \Gamma$;

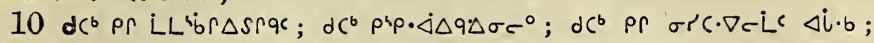

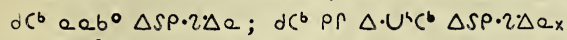

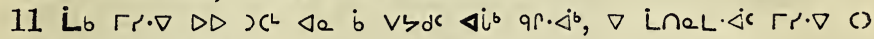
$\triangleleft \cdot \nabla c \cdot \triangleleft \nabla \Delta S \Delta U_{c} c^{b}{ }^{b}$

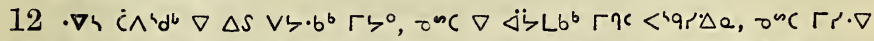

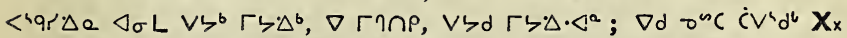

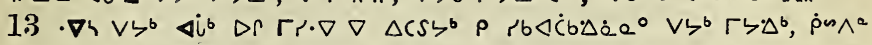

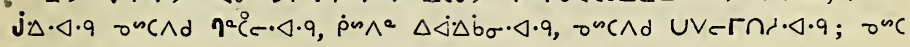

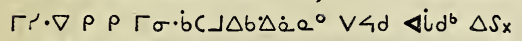

$14 \cdot \nabla h \Gamma \zeta^{\circ}$ e L $\Delta_{c}$ Vלd $<49 r \Delta \sigma \cdot \Delta^{\circ}$, Lb $\Gamma \eta c_{x}$

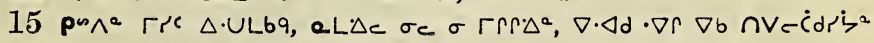

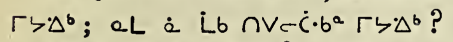

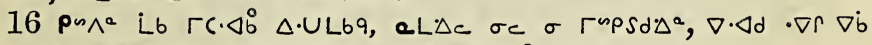

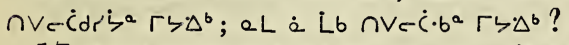

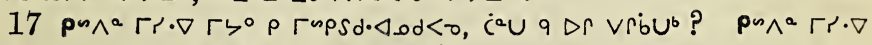

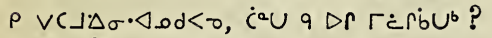

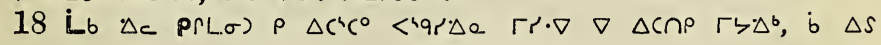
$\Gamma \cdot \sec ^{b} x$

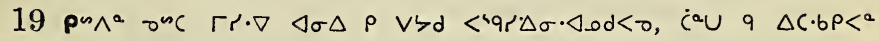
「ל०?

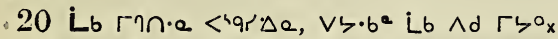

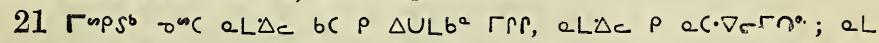

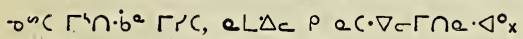

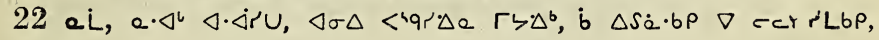
ecc. $\nabla r \dot{c} \cdot b \cdot a_{x}$

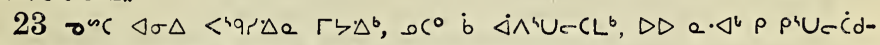

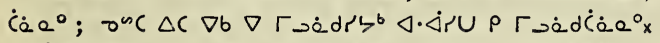

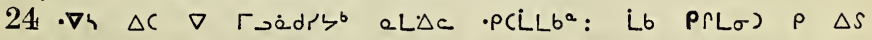

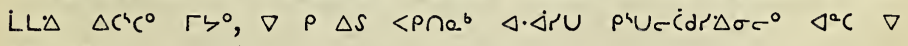
oU< $\sigma^{6}$ :

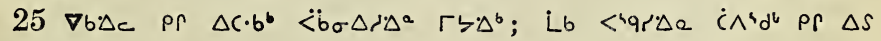
$b e \cdot \nabla r-\Gamma) L b \rho_{x}$

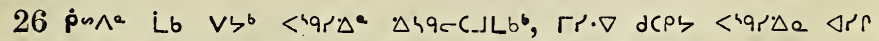

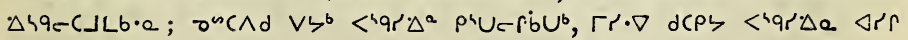
$\left.\Gamma_{C} \cdot \dot{\Delta C}\right\lrcorner L_{b} \cdot Q_{x}$

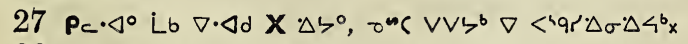

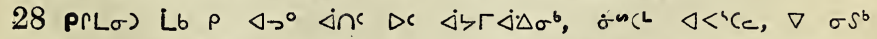

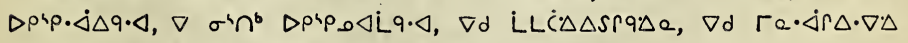
$\Gamma c \cdot \nabla \cdot \Delta e, \sigma a b L q \Delta a, n V e r q \Delta Q, \dot{<} \wedge$ ) $\Delta S P \cdot 2 \Delta e_{x}$

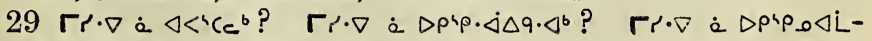

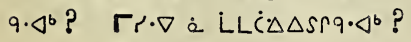




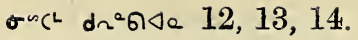

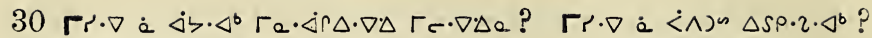
$r_{r} \cdot \nabla$ a $\Delta \cdot U^{\prime} C L q \cdot \Delta^{b}$ ?

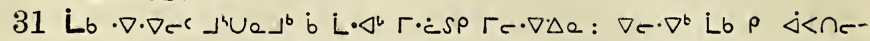
ne. $\triangleleft_{0} \triangleleft \cdot \Delta r u r \neg \Delta s \cdot\left(\Delta^{a} x\right.$

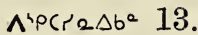

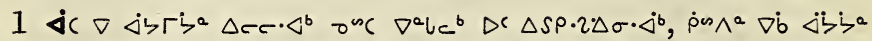

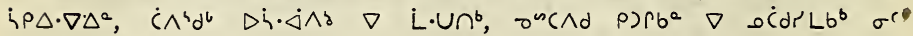
$\triangle U C \dot{C} d^{a} x$

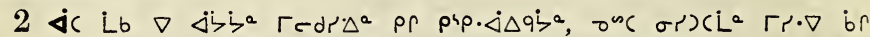

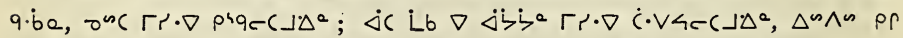

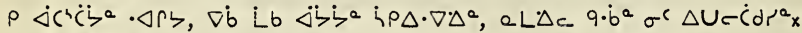

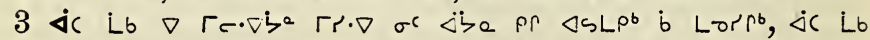

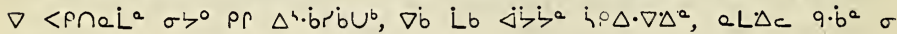
$\operatorname{ses} C \operatorname{Ld}^{2} x$

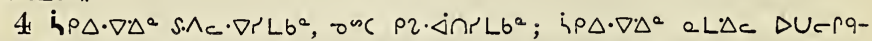

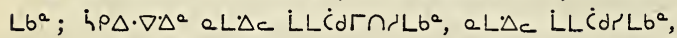

5 aL $\Delta_{c}<\cdot b^{a} c^{\circ} \dot{j} \cap \cap L b^{a}$, aL $L_{c}$ a $a c \cdot \nabla c c J L b^{a} \cap \Lambda_{c} \cdot \nabla q \cdot b_{a} a, a \Delta_{c}$ $\cdot \nabla 9 \Delta c \cdot \nabla r L b^{2}, a L \Delta c$ Lr $\left.\Gamma\right)$ ocraLba :

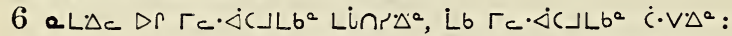

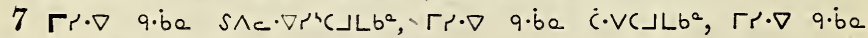

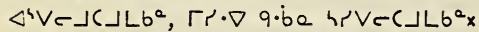

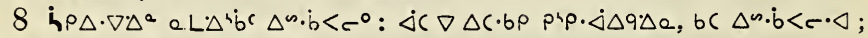

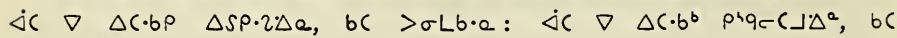
$\dot{<} S d \dot{a} \cdot b^{a} x$

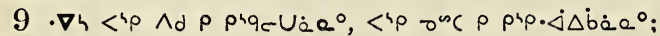

$10 L_{b} \Delta^{4} \wedge \triangleleft \sigma L b L \cdot \Delta r<4 p_{-} q^{b} \Delta \cap r<r q, \nabla d \triangleleft \sigma L b<4 p \Delta U-\dot{c} \cdot b^{b} q$ $\Delta^{n} \cdot b<c^{b} x$

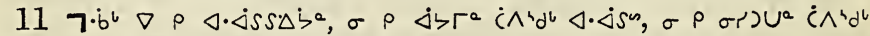

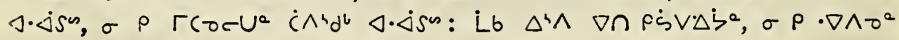
$\triangleleft \cdot \triangleleft s s \Delta q \cdot \dot{b} e_{x}$

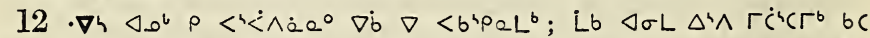

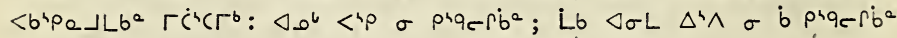

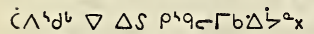

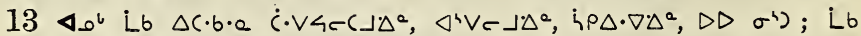
$\triangleleft \sigma L b$ b $L \cdot \Delta \rho$ p $U=\dot{c} \cdot b^{b} \nabla \cdot \Delta d i \rho \Delta \cdot \nabla \cdot \Delta^{a} x$

\section{Ápcra $\triangle b^{a} 14$.}

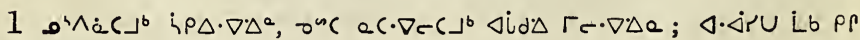
php. $\Delta \Delta q^{b} \mathrm{~b}$ 


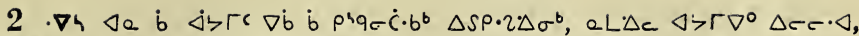

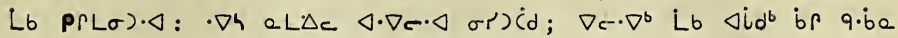
$\dot{\Delta r} \cdot \cup{ }^{\circ} x$

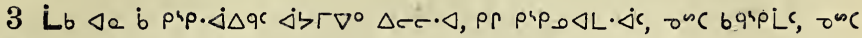
bPr $\dot{A c x}$

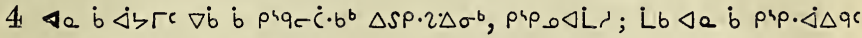
p^p. $\Delta L \cdot \nabla^{\circ} L L \Delta \Delta \zeta \Gamma \dot{\Delta} \cdot \Delta x$

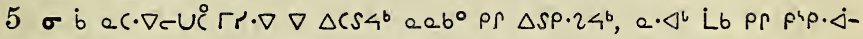

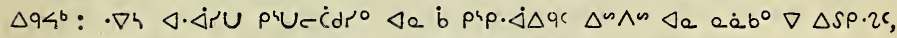

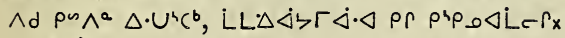

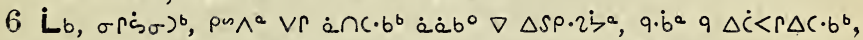

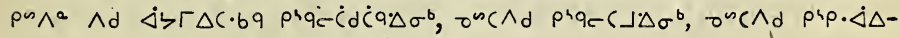

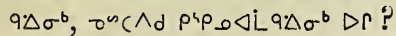

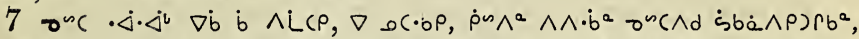

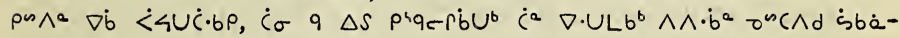
$\wedge P \supset P^{2}$ ?

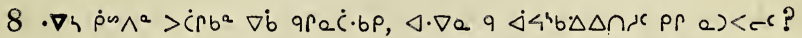

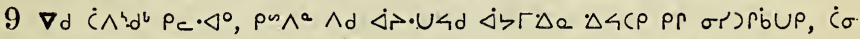

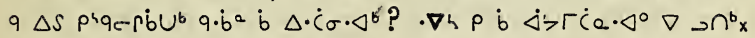

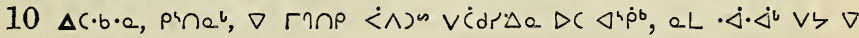
$\triangle C \cap P$ Lb $\nabla \dot{b} \nabla \sigma_{r} C \cdot \nabla e r \dot{b} u^{b}{ }_{x}$

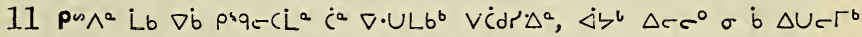

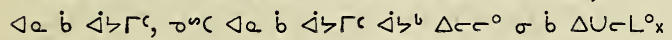

$12 \nabla d \dot{c} \wedge^{4} d^{b} \rho_{c} \cdot \Delta^{0}, \dot{a}^{4} \wedge^{6} \nabla$ ec $\left.\nabla e c 7^{b} \Delta i d \Delta \Gamma c \cdot \nabla \Delta a, \dot{\alpha} \cdot a c \cdot \nabla e c\right\lrcorner^{b}$

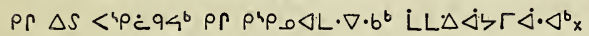

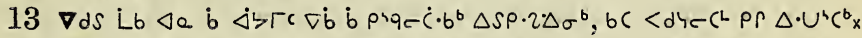

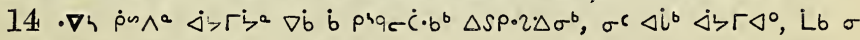
$\sigma r)\left(J \Delta^{a}\right.$ a $L \Delta C \dot{a}^{a} c^{\circ} \Delta \dot{c}<c^{a} x$

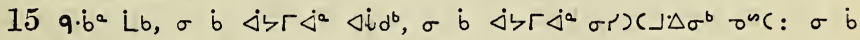
$\sigma b.\lrcorner^{a} \Delta i d^{b}, \sigma b$ b $\left.\left.\left.\sigma\right\lrcorner^{a}\right]^{2} \sigma x\right)(\lrcorner \Delta \sigma^{b} \sigma^{\omega c}(x$

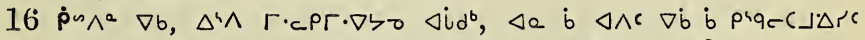

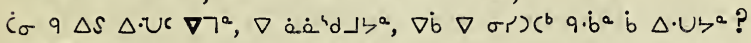

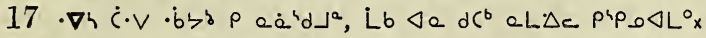

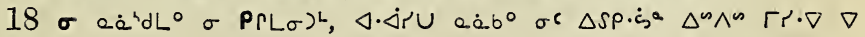
$\Delta C s 4^{\circ}:$

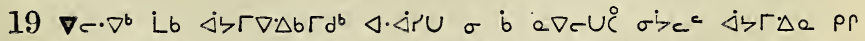

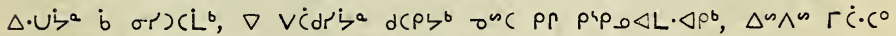

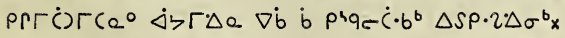

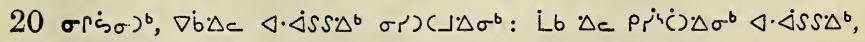
Lb $\left.\sigma \sigma^{\prime}\right)(\lrcorner \Delta \sigma^{b}$ fं́ $V \Delta^{b} x$

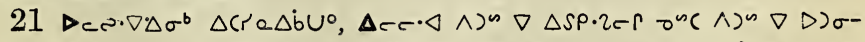

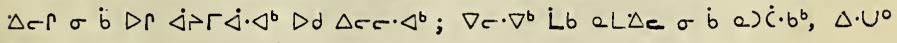
$\triangle \cap \vee-q^{\circ}{ }^{x}$ 


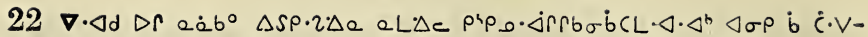

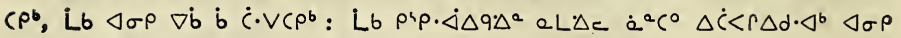
$\nabla \dot{b} \dot{b} \dot{c} \cdot V C \rho^{b}, \dot{L} b \quad \varangle \sigma \rho \dot{b} \dot{c} \cdot V\left(\rho^{b} x\right.$

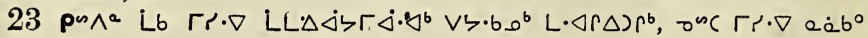

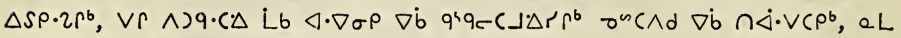
a $b c \Delta \cdot U \cdot \Delta^{b} \nabla$ pr. $q 4^{b}$ ?

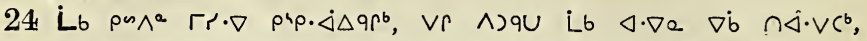

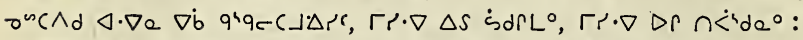

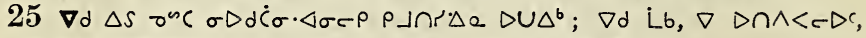

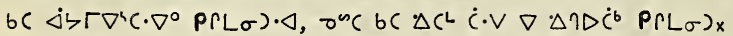

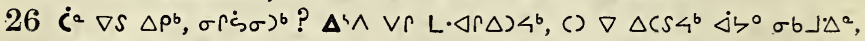

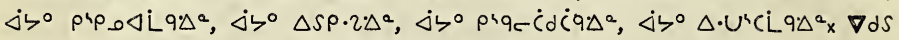

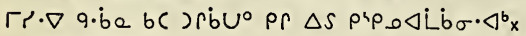

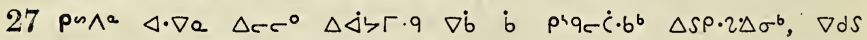
bc $\sigma s \cdot \triangleleft^{b}$, ow(Ad $\left.\Delta i b \sigma^{4}\right)$, onc $7 \Gamma^{4} d^{b} \Delta S ; \nabla d S$ Lb $V b^{b} \triangleleft \cdot \nabla e$ bC $\triangle \cdot U^{\prime} C L^{\circ} q^{\circ}$

$28 \dot{L}_{b} \rho a \wedge^{a} \nabla \dot{b} \nabla \dot{C} \sigma \cdot q \Delta \cap \cdot U^{\prime}\left(\dot{L} q \cdot \triangleleft, \nabla d S \nabla \dot{b} \Delta c b(\rho)\left\langle\dot{b} \Gamma \nabla \Delta b \Gamma d^{b}\right.\right.$;

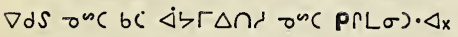

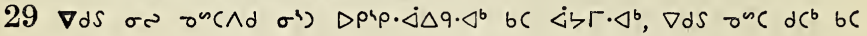
กरंdrec L $\mathrm{L}_{\mathrm{x}}$

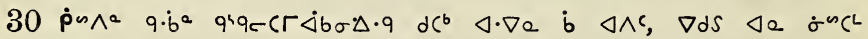
$b(>\sigma \rho) x$

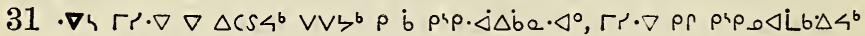

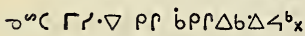

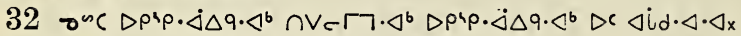

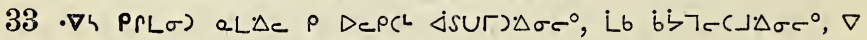

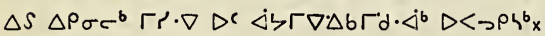

$34 \nabla d S$ pc $\Delta^{h} \cdot \rho \Gamma \cdot \triangleleft \cdot \Delta^{b}$ eL $\Delta c$ bC $\left.\left.\rho\right) \cdot \Delta^{b} \quad \dot{\Delta}\right\rangle \Gamma \nabla \Delta b \Gamma d^{b}: \cdot \nabla h \quad \Delta c \cdot \triangleleft^{0}$

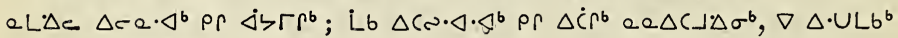
onc $D C a \cdot \nabla \Delta \Delta^{a x}$

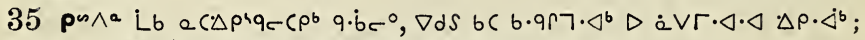

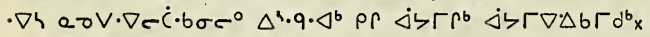

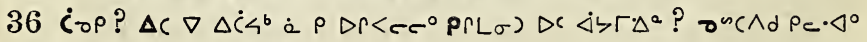
$\wedge d \rho P \vee r \Delta \cap \cap d \cdot \triangleleft a \cdot \triangleleft^{\circ}$ ?

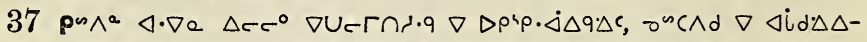

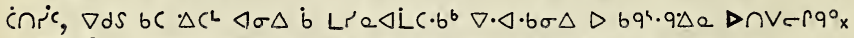

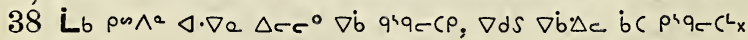

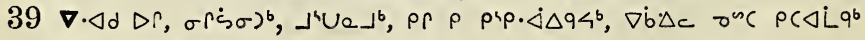

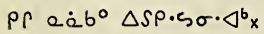

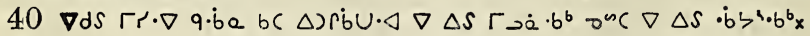




\section{A'pcre $\triangle b^{2} 15$.}

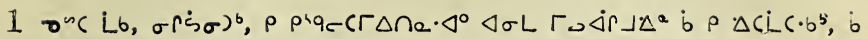
$\rho \nabla \cap e 7^{b} L b, \Delta C$ onc b $\sigma<\Delta<^{b}$;

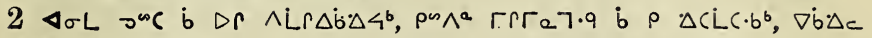
$\left.<\cdot b^{a} c^{\circ} \dot{b} \dot{C} \cdot V C\right\lrcorner \cdot \nabla \cdot q_{x}$

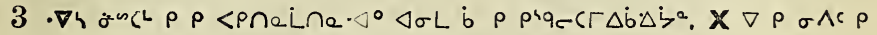

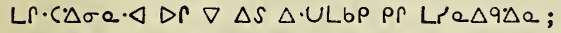

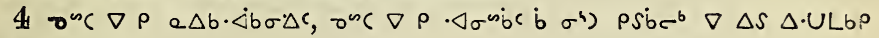
pr Lre $\Delta 9 \Delta \mathrm{Q}$;

$5 \nabla \rho \cdot \Delta<\Gamma d c$ onc $\rho<h, \nabla d \triangleleft \sigma \Delta \dot{b} \sigma \sigma \dot{s} \wedge c r:$

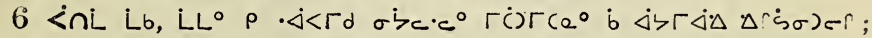

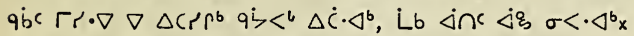

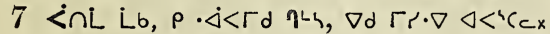

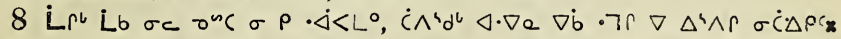

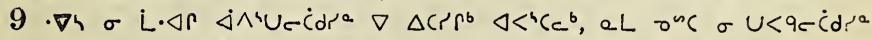

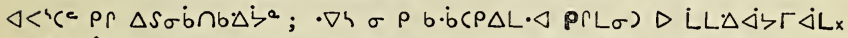

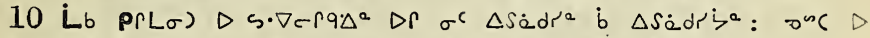

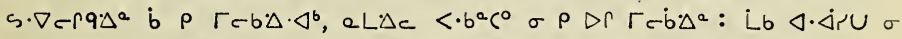

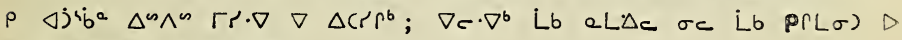
$c \cdot \nabla c r q \Delta^{a}$ bे $\Delta 7 \Delta d \cdot \Delta^{b} x$

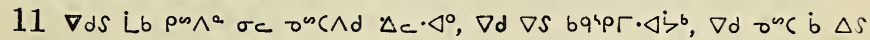
$\dot{c} \cdot \mathrm{V}<7^{b} \mathrm{x}$

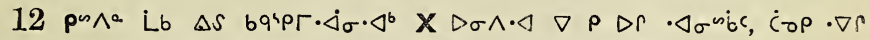

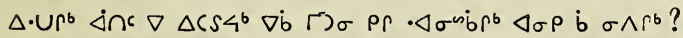

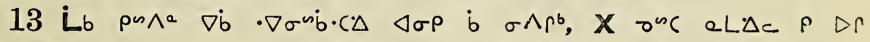
$\cdot \triangleleft \sigma^{\infty} b^{\circ}$ :

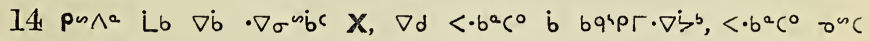
$\rho \dot{C} \cdot \vee 4 c(\lrcorner \cdot \Delta \sigma \cdot \triangleleft^{\circ} x$

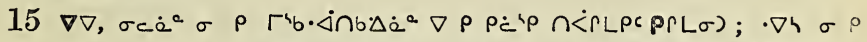

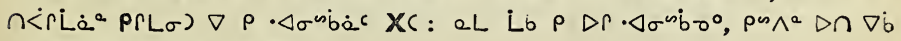
$\cdot \nabla \sigma \omega \dot{b} \cdot\left(\Delta \Delta \triangleleft \sigma \rho \dot{b} \sigma \wedge r^{b} x\right.$

$16 \cdot \nabla h \dot{\rho} \omega \wedge^{a} \nabla \dot{b} \cdot \nabla \sigma^{n \dot{b}} \cdot c \Delta \triangleleft \sigma \rho \dot{b} \sigma \wedge \rho^{b}, \nabla d \nabla \dot{b} \cdot \nabla \sigma^{m b} \dot{b} b \sigma \cdot \Delta c X:$

$\left.17 \dot{\rho} \sim \wedge^{a} \dot{L} b \nabla \dot{b} \cdot \nabla \sigma \omega \dot{b} \dot{a} b \sigma \cdot \Delta \cdot q X, \rho \dot{c} \cdot \vee 4 c c\right\lrcorner \Delta \sigma \cdot \triangleleft^{\circ}<\cdot b^{a} \varsigma^{\circ} \Delta U-\dot{C} \cdot b^{a}$; $q \dot{q}<6$ pc $\Delta \dot{C} \dot{\alpha} \triangleleft^{\circ} \rho L \Gamma \cdot C \cdot \Delta \sigma \cdot \dot{\zeta}^{b} x$

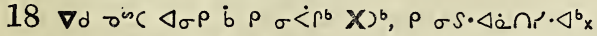

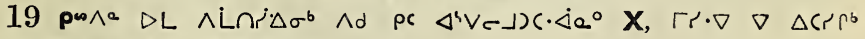

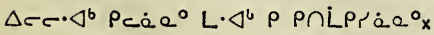

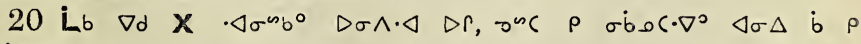
$\sigma \dot{<} \boldsymbol{\Gamma} \dot{x}$

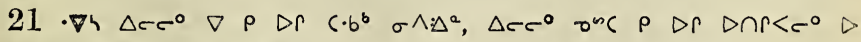
$\cdot \triangleleft \sigma^{\infty} b \Delta \sigma \cdot \triangleleft^{0} \triangleleft \sigma \rho b$ b $\sigma \wedge \rho^{b x}$ 


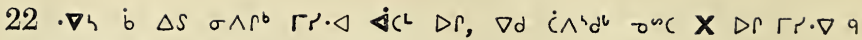
$\wedge i \cap r \Delta b \sigma \Delta r^{b} x$

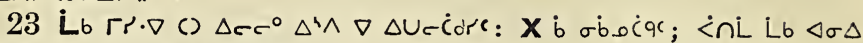
$X$ ib $\cap \wedge c \cdot \nabla \cdot \Delta r<\vee c \cos q_{x}$

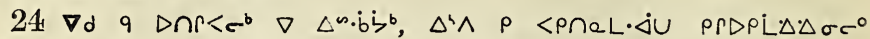

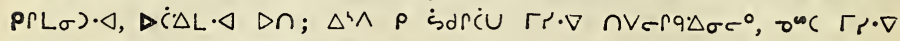
$D P L \Delta \Delta \sigma c^{\circ}, \sigma^{\infty} \mathrm{C}$ bepD $\Delta \sigma \sigma^{\circ} \mathrm{x}$

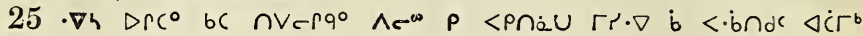
$D$ r ${ }^{b} x$

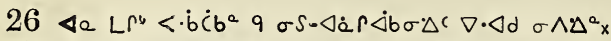

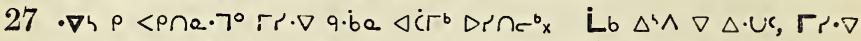

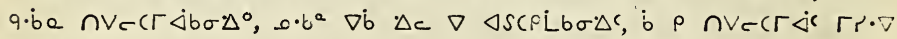
$9 \cdot 62 x$

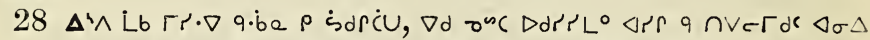

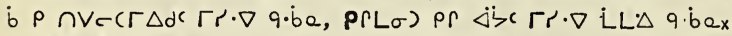

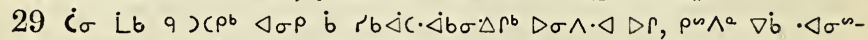

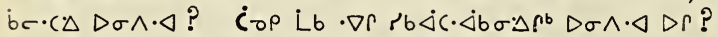

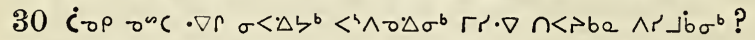

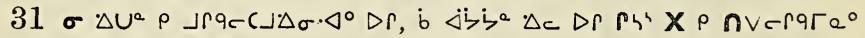
$c \cdot\left(\circ \operatorname{bis} \rho \sigma \wedge^{2} x\right.$

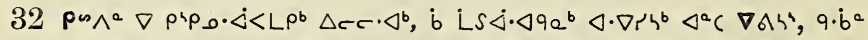

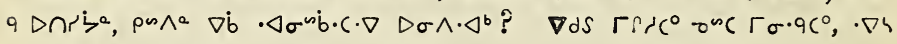
$\cdot<<q \rho \dot{b} \sigma \wedge \dot{a} a^{\circ} x$

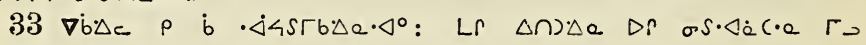
$\triangle S \cdot c \cdot \Delta e^{x}$

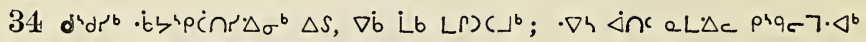

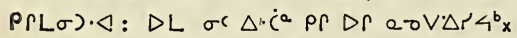

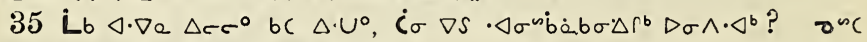
Lb' $9 d \Gamma \zeta \Delta c^{\circ} q \triangleleft \dot{\zeta} \rho^{b} \cdot \triangleleft \sigma^{\omega} \dot{b} \cdot c \cdot \nabla$ ?

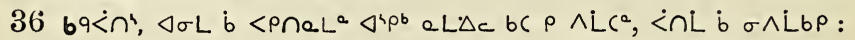

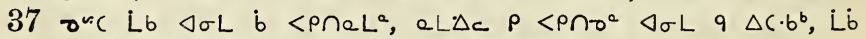

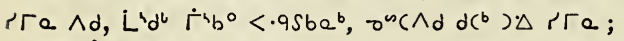

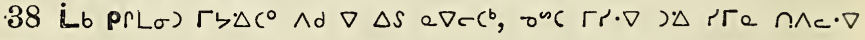
$\triangle \succ^{\circ} \mathrm{x}$

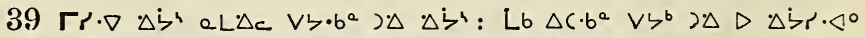

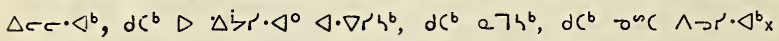

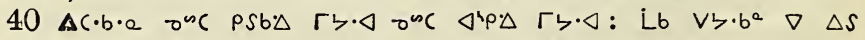

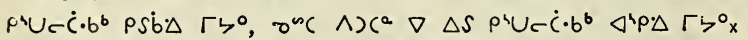

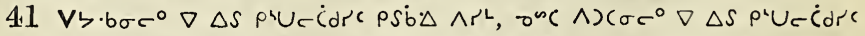

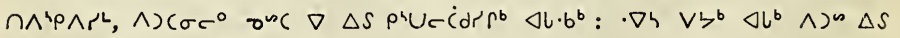

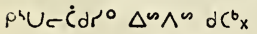

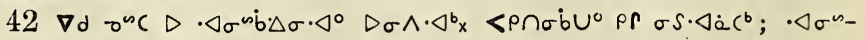
b $\sigma \dot{b} U^{0} \nabla \dot{b}$ Pr $\sigma s \cdot \Delta \dot{a} c^{b}$ :

290 


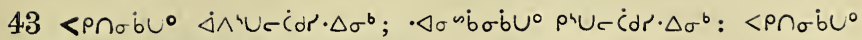

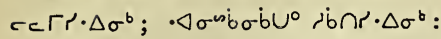

$44\left\langle\rho \cap \sigma \dot{b} U^{\circ} \Delta c\right) \Gamma \zeta^{\circ} ; \cdot \Delta \sigma^{b i b} \sigma \dot{b} U^{\circ} \Delta i d \cdot \Delta \Gamma \zeta^{\circ} x \quad \Delta\left(\cdot b^{a} \Delta c\right) \Gamma \zeta^{\circ}$, $\Delta C \cdot b^{a}$ onc $^{\infty} \Delta i d \cdot \Delta \Gamma \zeta^{\circ} x$

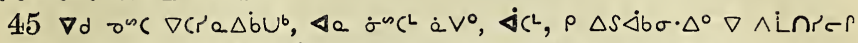

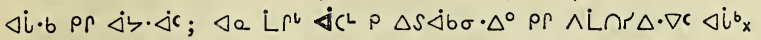

$46 \nabla c \cdot \nabla^{b} L b, a L \cdot \Delta c \dot{\sigma}^{-s}(L) \Delta\left(\cdot b^{a} \triangleleft \sigma L \dot{b} \Delta \dot{L} d \cdot \triangleleft^{b}, L b \triangleleft \sigma L b \Delta c\right) \cdot \triangleleft^{b}$; <nL $L_{b} \triangleleft \sigma L \dot{b} \triangleleft \dot{L} d \cdot \Delta^{b} x$

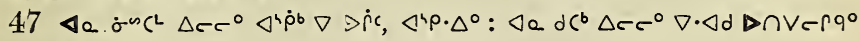
PrPS d $D \rho^{b}$

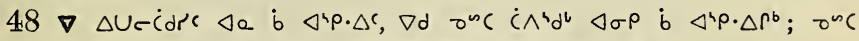

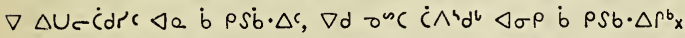

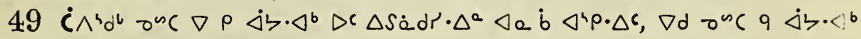
$\triangle a$ b $P S b \cdot \Delta c \quad C C \Delta S \dot{a} d r \cdot \Delta^{a} x$

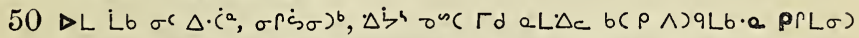

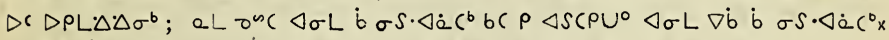

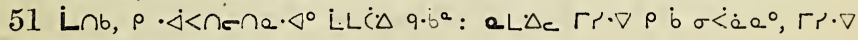
Lb $\rho \dot{b} \cdot q^{\prime} P \dot{\dot{a}} d \Delta b \Delta \dot{a} a^{\circ}$,

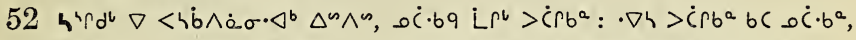

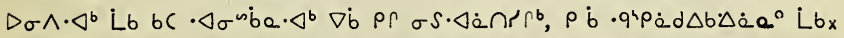

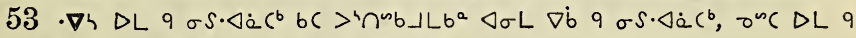
$\sigma \wedge L b^{b} b C>>^{\wedge} \mathrm{b} b J L b^{a} \triangleleft \sigma L \nabla b \quad q \sigma \wedge L b^{b} x$

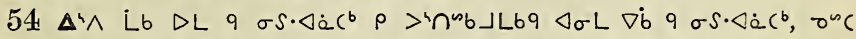
$\Delta L \quad \nabla b \quad q \quad \sigma \wedge L b^{b} \rho>^{4} \cap^{w} b J L b q \quad \varangle \sigma L \quad \nabla b \quad q \quad \sigma \wedge L b^{b}, \nabla d q \cap \wedge<c^{b} \triangleleft \sigma L$ $\Delta \cdot \cup \cdot \Delta^{a} \dot{b}$ Lra $\Delta \dot{b} U^{b}, \sigma \wedge \Delta^{a} \Gamma r \cdot \nabla<-\dot{c} \sigma^{a} \nabla^{a} \dot{s} d r \Delta \cdot \nabla \Delta \sigma^{b}{ }_{x}$

$55 \triangleright \sigma \wedge \Delta^{a}, \dot{c} \sigma \cdot \nabla \rho \wedge \rho>r \cdot \nabla \Delta^{a}$ ? $\triangleright \sigma \cdot b \Delta^{4} \dot{b}^{a}, \dot{c} \sigma \cdot \nabla \rho \dot{s} d r \Delta \cdot \nabla \cdot \Delta^{a}$ ?

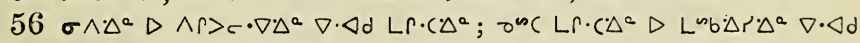
$\nabla c \omega \cdot \nabla \Delta \Delta^{a} x$

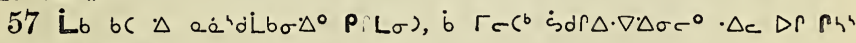

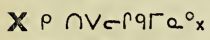

$\left.58 \nabla \cdot \Delta d \Delta r, r \Delta \rho \Delta\left(\cdot b^{b} \sigma \rho \dot{s} \sigma\right)^{b}, \dot{\Delta r} r \dot{b}<\cdot \Delta^{b}, \Gamma r \Gamma \dot{b}<\cdot \Delta^{b}, J s^{b} \nabla \cap \wedge\right)\left(\cdot 7^{b}\right.$

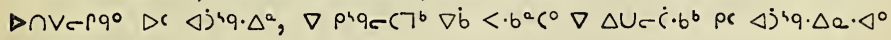
$\Delta \cap \operatorname{Rerq^{b}\mathrm {x}}$

\section{$\Lambda^{4} p c r a \Delta b^{a} 16$.}

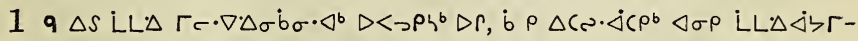
$\varangle \cdot \Delta^{b} b>s \Delta^{b}, \nabla 0 P_{c} \cdot \nabla^{0} \sigma^{a c}(\Delta)(\lrcorner^{b} \times$

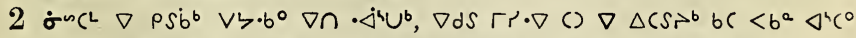
b $\Delta S \Gamma \rightarrow) \dot{C} d(P \Gamma L \sigma) \cdot \triangleleft, \nabla b$ Pr LL $\Delta \Gamma \sigma \cdot \nabla \Delta \sigma \dot{b} \sigma \cdot \Delta^{b} \Delta^{4} \wedge(d S \sigma \dot{b} \sigma x$

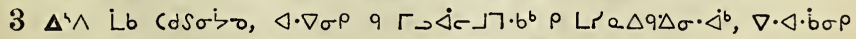

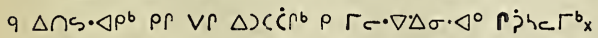

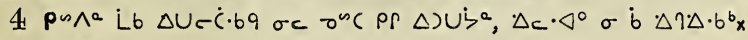
291 


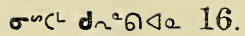

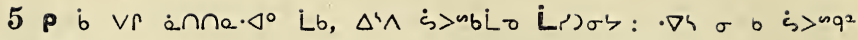
L.P $\sigma$ לx

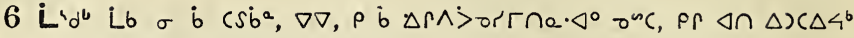
Ad $\Delta U$ q $\Delta U \cdot \dot{\nabla} \sigma_{x}$

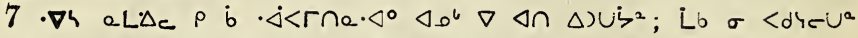

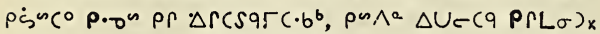

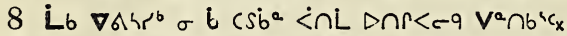

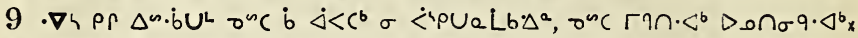

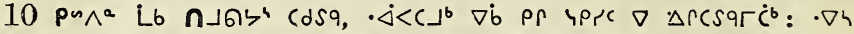

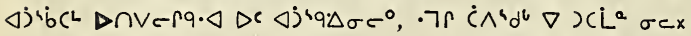

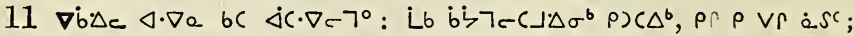

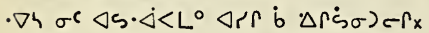

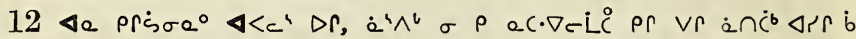

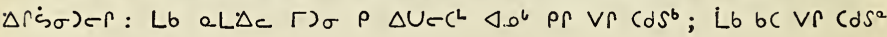
$\Delta \wedge \Delta<c-9 x$

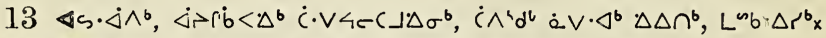

$14 \nabla d S \Gamma \cdot \cdot \nabla \rho)(\lrcorner \Delta \sigma \cdot \dot{\Delta} \cdot \Delta b(\Delta) \mu \dot{b} U \cdot \Delta i \rho \Delta \cdot \nabla \Delta \sigma^{b} x$

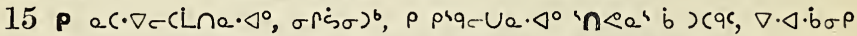

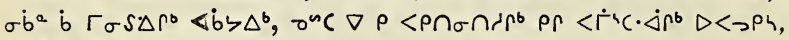

16 pr $\Delta S<\rho \cap \sigma \cap r^{n}\left(\cdot \nabla \cdot b^{b} \nabla \cdot \Delta \cdot \dot{b} \sigma \rho\right) \Delta, \sigma^{n} C \Gamma r \cdot \nabla \Delta \cdot \nabla e$ b $\Delta r \Delta c^{b}$,

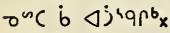

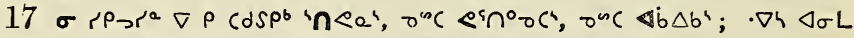

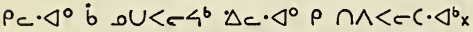

$18 \cdot \nabla h$ o $\rho \Gamma \cdot s c\left(\Gamma \Delta \cdot b^{b} \sigma^{c} \Delta i_{d^{b}} \sigma^{n}\left(\rho_{c} \cdot \Delta^{0}: \nabla \cdot \Delta d \Delta r \nabla \cdot \Delta \cdot b_{\sigma} \rho\right) \Delta\right.$ piqc $\Gamma d^{b} x$

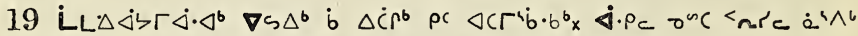

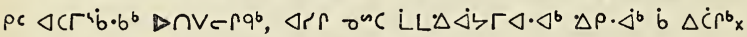

$20 \Gamma \cdot \nabla \dot{b} \Delta(\dot{s} \sigma) \Gamma^{b} \rho c \Delta\left(\Gamma^{i} \dot{b} \cdot b^{b}{ }_{x} \Delta\left(\Gamma^{4}()^{b} \nabla<>\rho \nabla \Gamma\right) \zeta^{b} x\right.$

$21 \sigma^{c} \varangle C \Gamma^{\circ} \dot{b} 9 \Delta^{a} \sigma c<c, \cap \wedge c \cdot \nabla \sigma \rho \uparrow \Delta \Gamma_{x}$

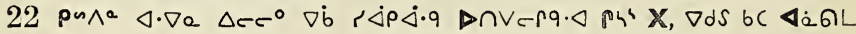
Ĺciex

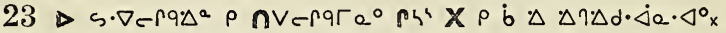

$24 \sigma i \rho \Delta \cdot \nabla \cdot \Delta^{a} \rho \dot{b} \cdot \Delta \cdot \Delta \eta \cdot \Delta d a \cdot \Delta^{0} \Gamma \cdot \cdot \nabla \nabla \Delta C S 4^{b} \times p r^{b} \times \nabla 7^{a} \times$

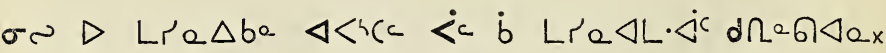

\section{Aspcra $\triangle b^{\circ} 1$.}

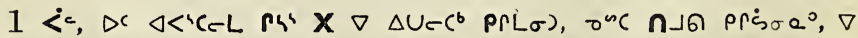

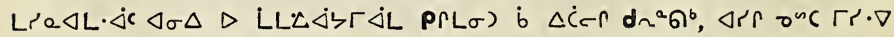
$\Delta \sigma \Delta \Delta<\supset \rho\} \dot{b} \Delta \dot{C}-\Gamma \Gamma r \cdot \nabla \Delta \dot{b}\rangle \Delta^{b}$ :

292 


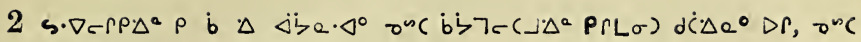

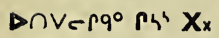

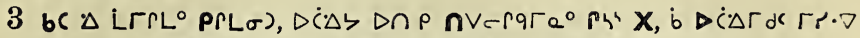

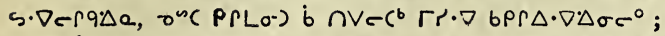

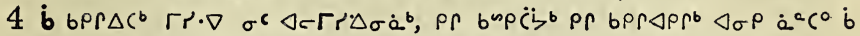

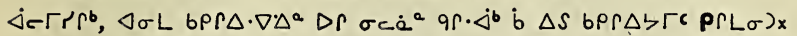

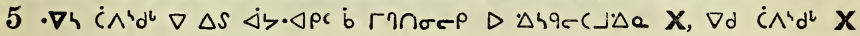

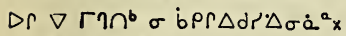

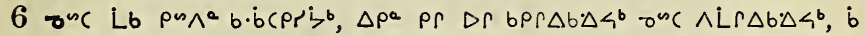

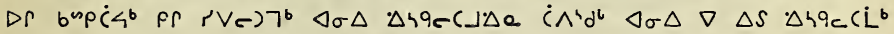

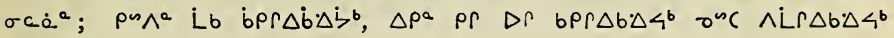
$P_{c} \cdot \nabla^{\circ} x$

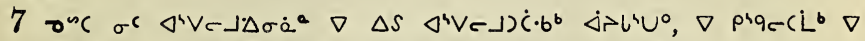

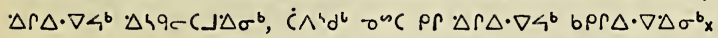

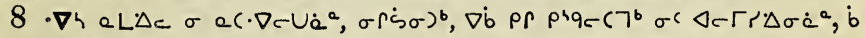

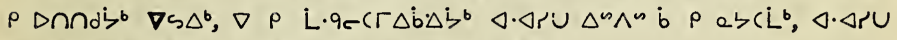

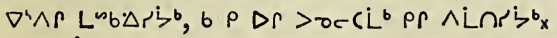

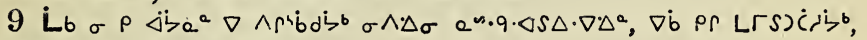
Lb $P$ R L $\sigma) \dot{b} \cdot \Delta \sigma \omega \dot{b} \dot{a}(D \sigma \wedge \triangleleft$;

10 b $\rho \Delta \cdot b r \Delta \zeta \Gamma c$ pr $\sigma \wedge \Delta \sigma^{b} \Delta r, \sigma^{n c} q \dot{b}<b \dot{b} \Delta \cdot b r \Delta b \Gamma c ; \Delta a$ onc b

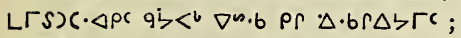

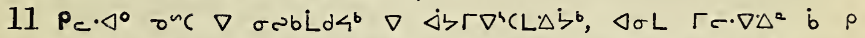

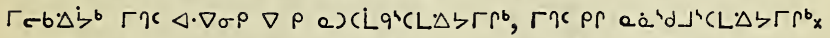

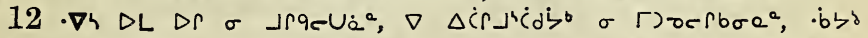

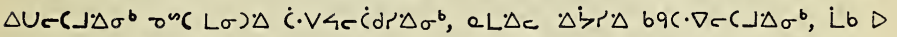

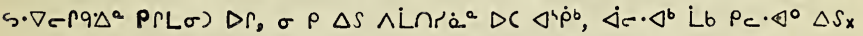

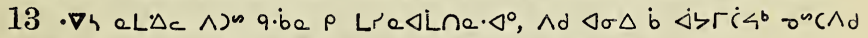
bं Cं.VC

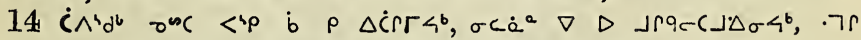

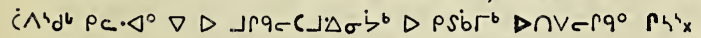

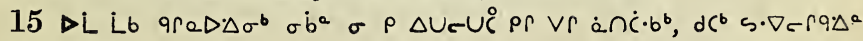

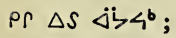

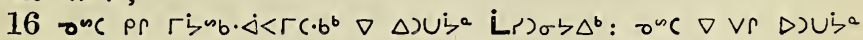

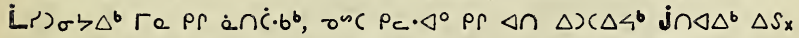

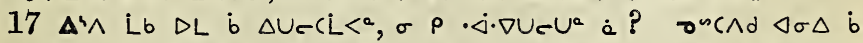

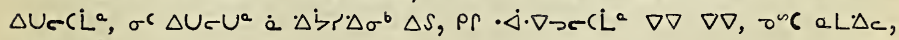
a L $\Delta_{c}$ ?

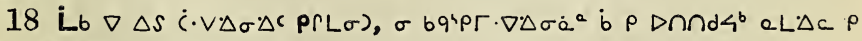
$\triangle \cdot U L b^{a} \nabla \nabla$ onc $a L \Delta C x$

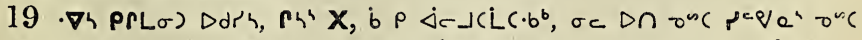

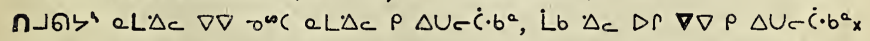

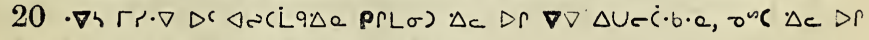
$\nabla 7^{2}$, pr $\triangle S L \Gamma \Gamma L b \sigma \Delta(P \Gamma L \sigma) \sigma c \dot{\alpha}^{2} D \Gamma_{x}$

293 


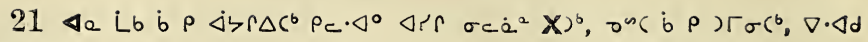
Pr $(\sigma)$;

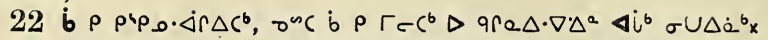

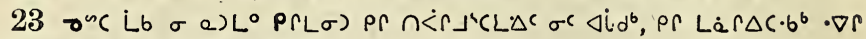

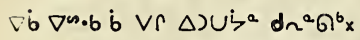

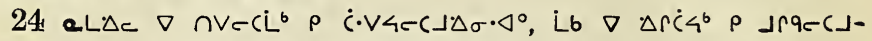
$\left.\Delta \sigma \cdot \triangleleft^{\circ}: \cdot \nabla h \dot{C} \cdot \vee 4 C \dot{C}\right\lrcorner \Delta^{2} \rho c \Delta r \sigma<\Delta a \cdot \Delta^{\circ}{ }_{x}$

\section{A'pcre $\Delta b^{\circ} 2$.}

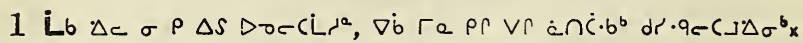

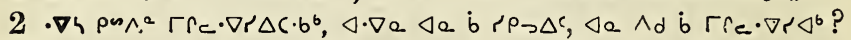

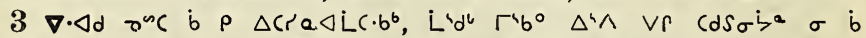

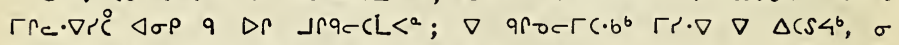

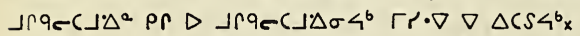

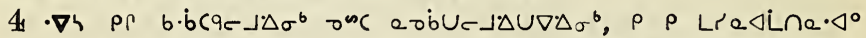

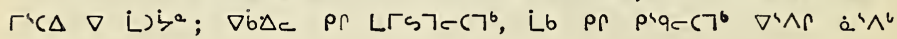
$i p \Delta C \cdot b^{b} x$

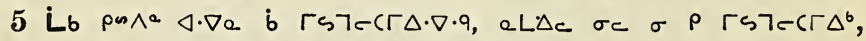

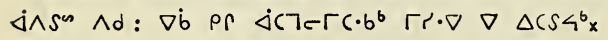

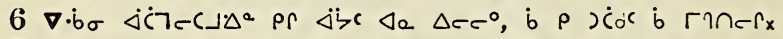

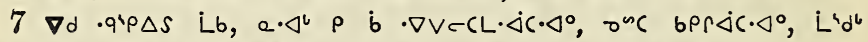

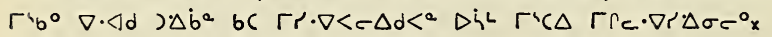

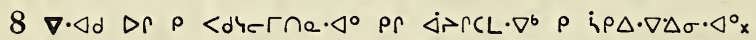

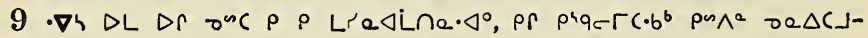
$\cdot \nabla \cdot q \Gamma r \cdot \nabla \Delta s_{x}$

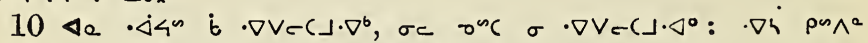

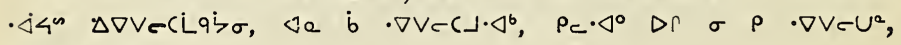
$\Delta n^{\circ} b^{\circ} \mathrm{XC}$;

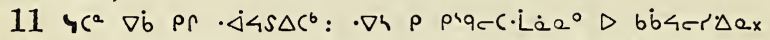

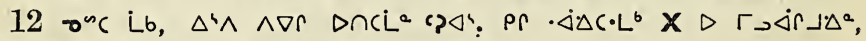

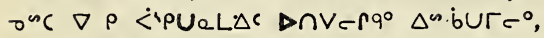

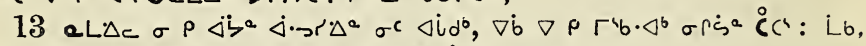

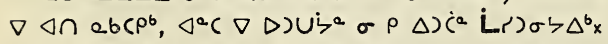

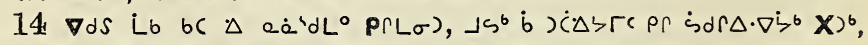

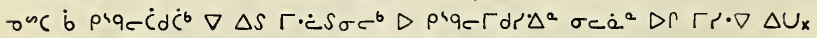

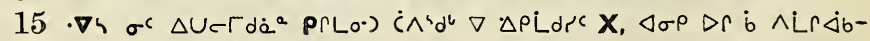

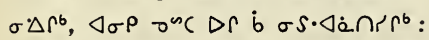

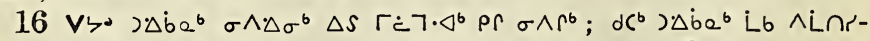

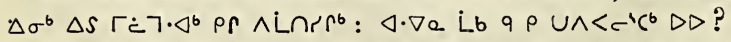

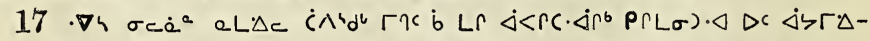

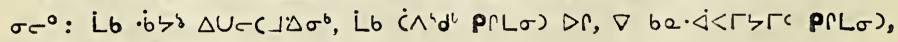
$\left.\sigma^{c} \dot{\Delta} \zeta \Gamma_{a^{a}} X\right)^{b} x$

294 


\section{$\Lambda^{4} p c r a \Delta b^{2} 3$.}

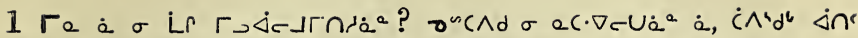

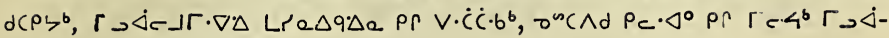
$-J \Gamma \cdot \nabla \Delta$ Lre $\Delta q \Delta \mathrm{Q}$ ? ?

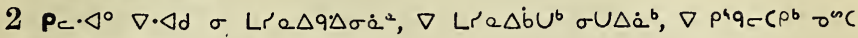

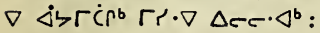

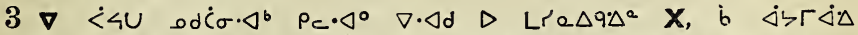

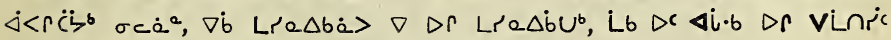

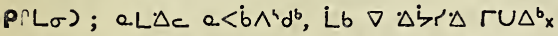

$4 \Delta L \Delta S$ Lb $\sigma L R S)\left(\cdot \Delta \dot{a}^{2} P \Gamma L \sigma\right) \times D r$ :

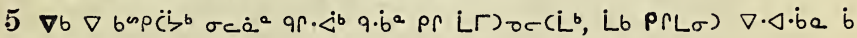
Di bupcisbx

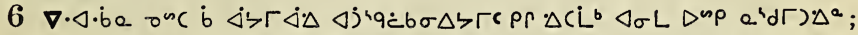
e L $\Delta c$ b $\Delta \cdot U L b^{b}$, Lb b $\Delta i d \Delta L b^{b} ; \cdot \nabla h \dot{b} \Delta \cdot U L b^{b} \sigma \dot{<}>\cdot \nabla L b^{a}$, Lb b $\Delta i d \cdot \Delta L^{b} \Gamma c \cdot \nabla L b^{a} \wedge L \cap R^{\circ} \Delta^{a} x$

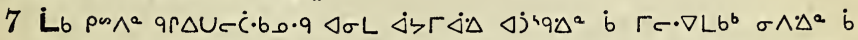

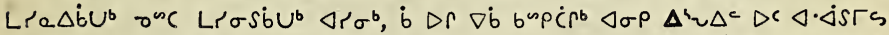

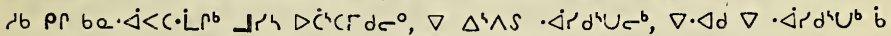
$\varangle \cap \dot{S} S \mathrm{~d} \dot{\mathrm{a}} \cdot \mathrm{b}^{\mathrm{b}}$;

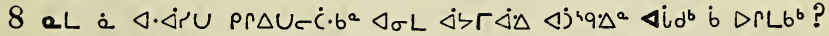

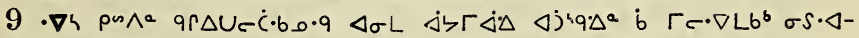

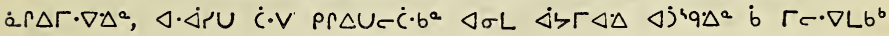

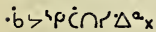

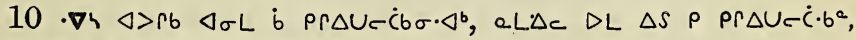
$\Delta \sigma L$ prUcícdr. $\Delta^{a} D r$ b <'peqbx

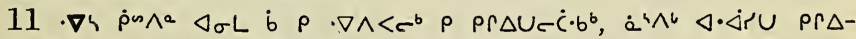
Ue $\dot{C} \cdot b^{a} \triangleleft \sigma L q \dot{L}<b$ b $\wedge L \cap \cap L b^{b} x$

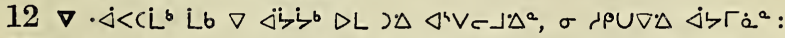

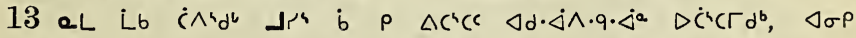

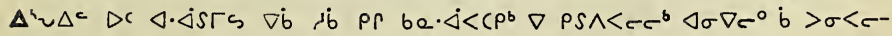
$\dot{C} \sigma \cdot \triangleleft \sigma c^{b}$ :

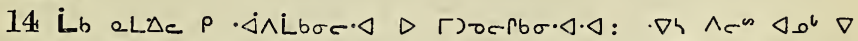

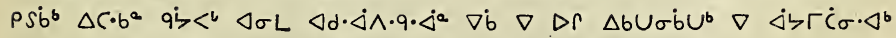

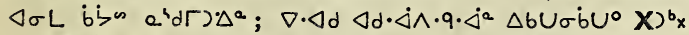

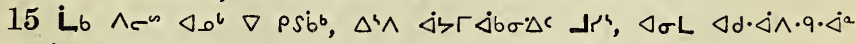
$\triangle U \triangle \cdot \Delta^{b} \Delta \Delta^{4} U c^{\circ} x$

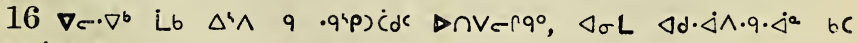
$\triangle 6 \cup \sigma \dot{b} \cup \sigma^{\circ} x$

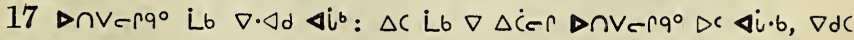
$\Delta C \cdot b^{b} \cap V e r \cap \Delta^{2} \times$

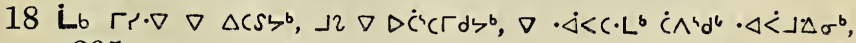
295 


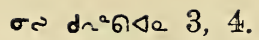

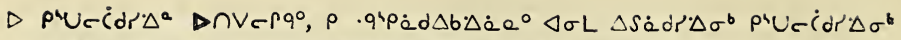

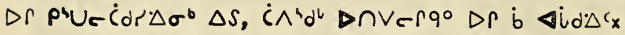

\section{$A^{\prime} p\left(r^{\circ} \circ \triangle b^{2} 4\right.$.}

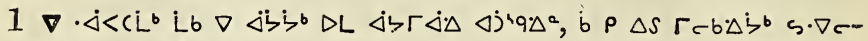
$r q \Delta^{a}, a L \Delta c \sigma$ तidU $\dot{\Delta \dot{a}^{a}}$;

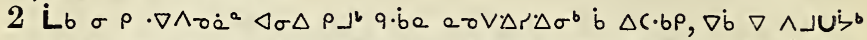

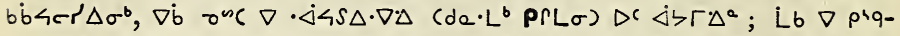

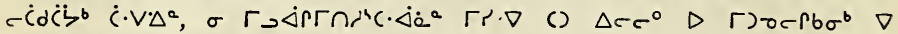
$b e \cdot \dot{\alpha}<\Gamma(P \Gamma L) \times$

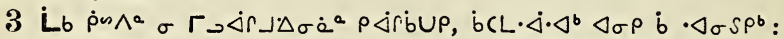

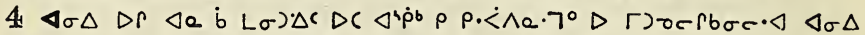

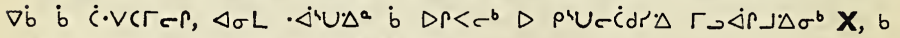

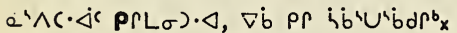

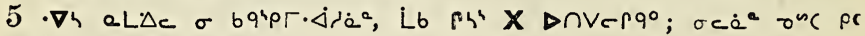

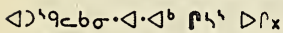

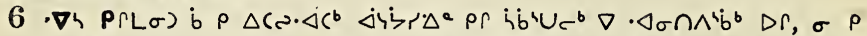

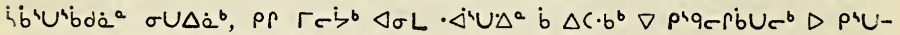

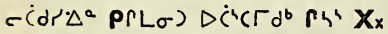

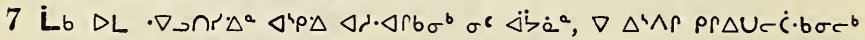

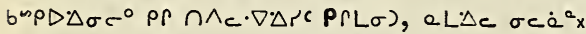

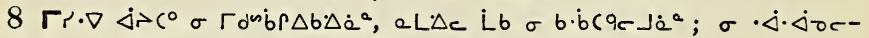

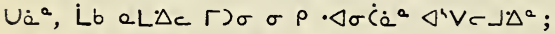

$9 \sigma b \cdot b C P \Delta b \Delta_{a}{ }^{a}$, Lb eL $\Delta c \sigma \cdot \nabla \wedge \sigma b \Delta a^{a} ; \sigma \cdot \nabla \wedge S T b \Delta \dot{a}^{a}$, Lb e $L \Delta_{c}$ $\sigma \sigma S \cdot \Delta \dot{a} \Gamma \Delta b \cdot \dot{a}^{a}$;

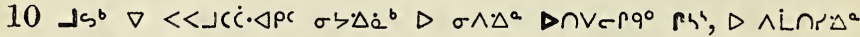

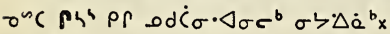

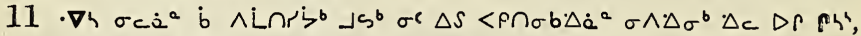

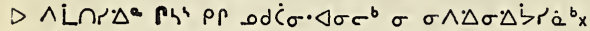

$12 \nabla d \Delta S$ Lb $\sigma \wedge \Delta^{a} \sigma$ or $\Delta d a^{a}$, Lb $P_{c} \cdot \Delta^{\circ} \wedge$ LnR $\Delta^{a} x$

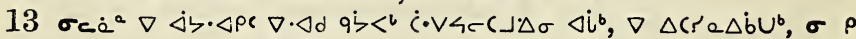

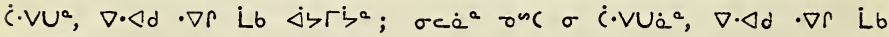
¿לríb;

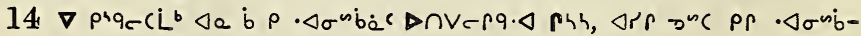

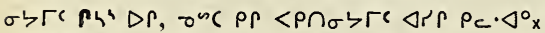

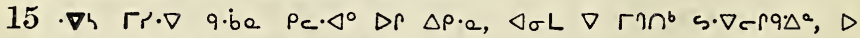

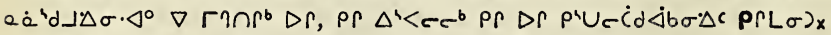

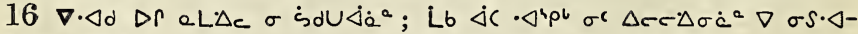
a $c^{b}, \nabla c \cdot \nabla^{b}$ Lb $\wedge r \sigma^{c} \Delta c c \Delta \sigma \dot{a}^{a} \Delta u p<c \dot{C} \sigma \cdot \Delta^{a} c \cdot c^{0} \nabla \rho S b^{b x}$

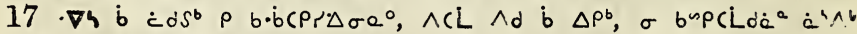

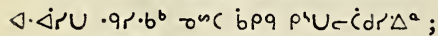




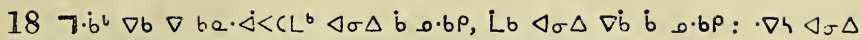
b $0 \cdot b \rho \Delta r_{c}^{\circ} \Delta c \cdot b \cdot a ; i b \triangleleft \sigma \Delta \nabla b$ b.$\cdot b \rho$ b $\rho q \Delta c \cdot b \cdot a_{x}$

$$
\Lambda^{3} p\left(r a-\triangle b^{a} 5 .\right.
$$

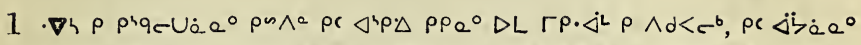

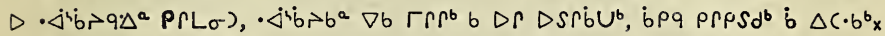

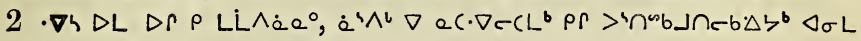

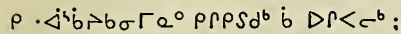

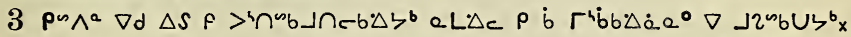

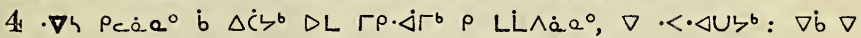

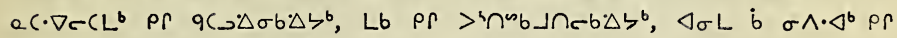

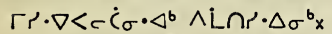

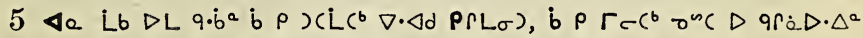
$\Delta i b^{b}$

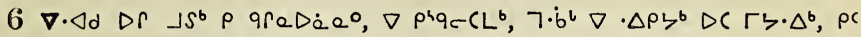

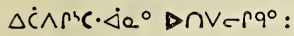

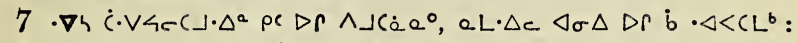

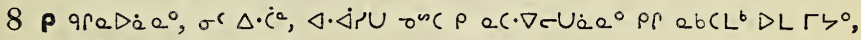
onc $b \rho \cdot \Delta \eta \cdot \Delta^{b} \Delta \cap V-r q^{\circ} x$

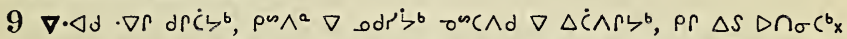

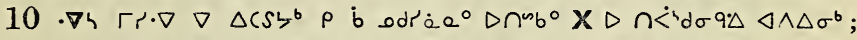

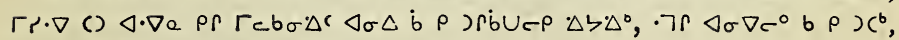

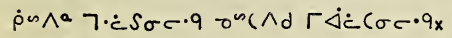

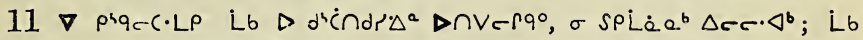

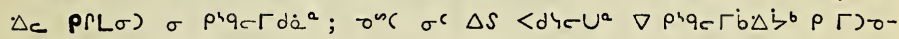

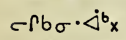

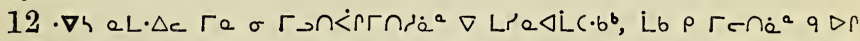

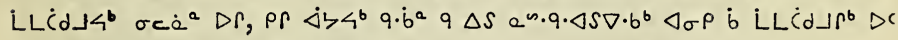

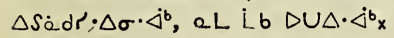

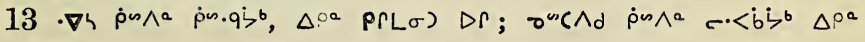
$\rho_{c} \cdot \Delta^{\circ} \nabla r_{x}$

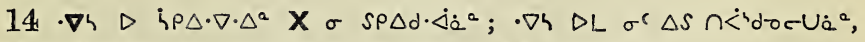

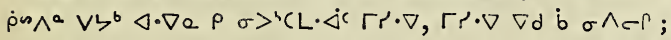

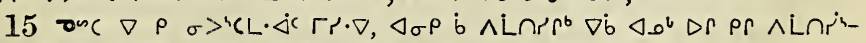

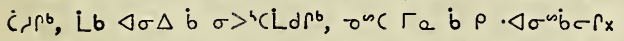

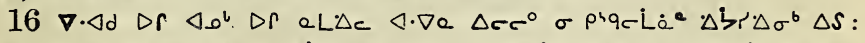

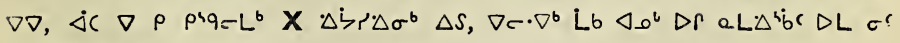
$\triangle S$ prqc Li ${ }^{a} x$

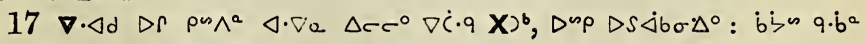
$\cdot \nabla \wedge<c \cdot \triangleleft ;$ L $\cap b, \Gamma r \cdot \nabla q \cdot \dot{b e} \varangle \cap \Delta m b \cdot \varangle x$

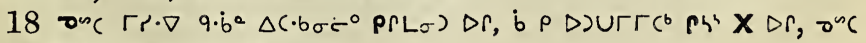

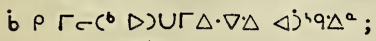

297 


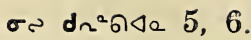

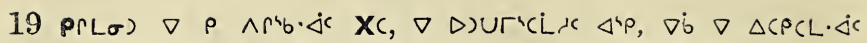

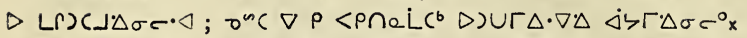

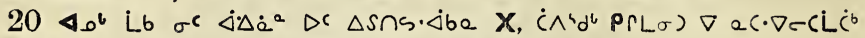

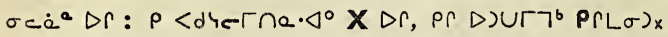

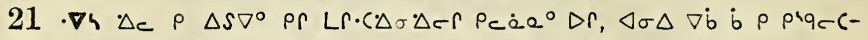

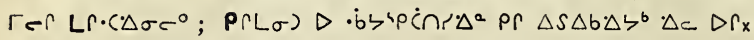

\section{A}

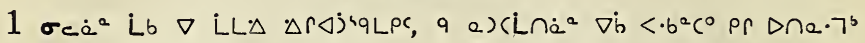
PrLG) D S. $\nabla C r q \Delta^{2} x$

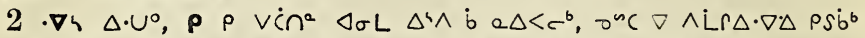

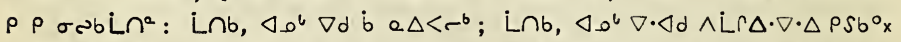

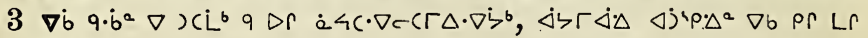

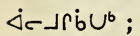

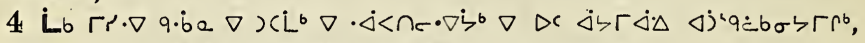

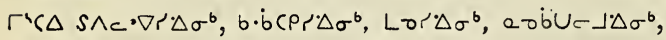

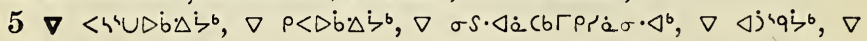
$\Delta s \cdot \dot{\Delta \wedge \dot{\zeta} b}, \nabla \sigma b \dot{\zeta}^{\circ} b$;

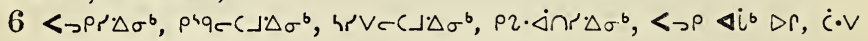
$h_{P} \Delta \cdot \nabla \Delta \sigma^{6}$,

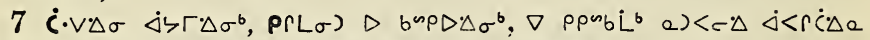

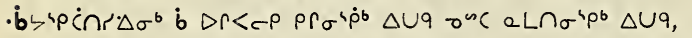

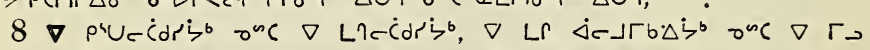

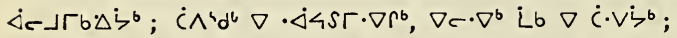

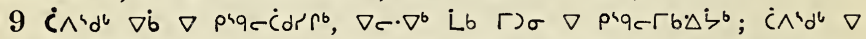

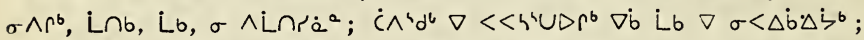

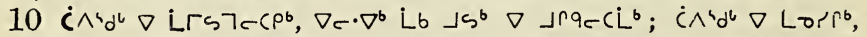

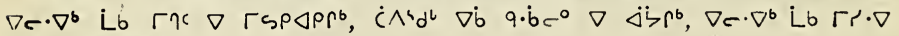

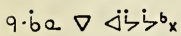

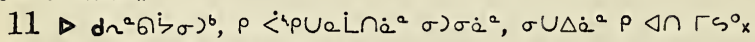

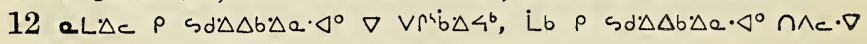
$P \hookrightarrow \triangle \cdot \Delta \Delta^{b} x$

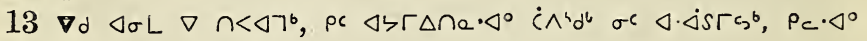
Juc bc $\varangle \cap \Gamma S^{\circ} p U \triangle \cdot \Delta^{\circ} x$

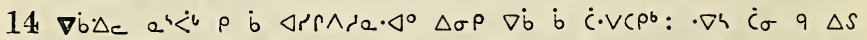

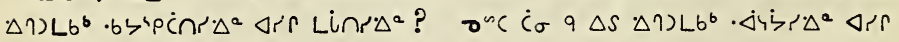
- anNiprac?

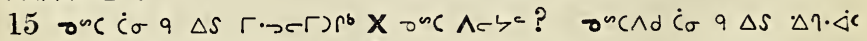
$\triangle a \dot{b} \dot{c} \cdot v c^{b} \triangleleft \sigma \Delta \nabla \dot{b} \dot{b} \dot{c} \cdot v(r e r$ ?

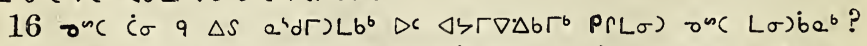

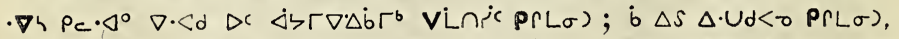

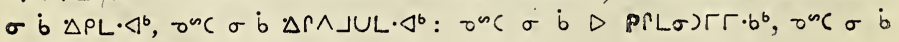
$\Delta C \Delta C r \Gamma L \cdot \Delta^{b} x$ 


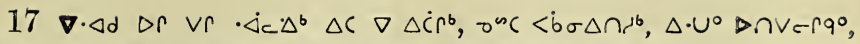

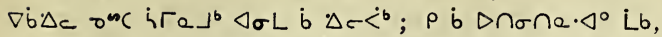

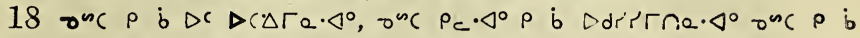

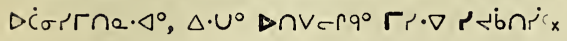

\section{Aipcre $\triangle b^{\circ} 7$.}

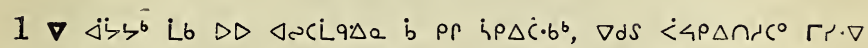

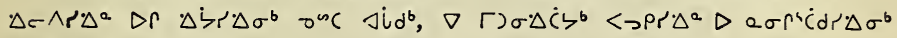
$P(L \sigma) x$.

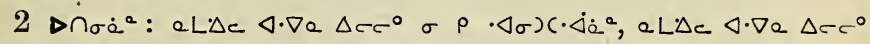
$\sigma P L O \Delta \dot{a}^{a}, Q L \Delta C \triangleleft \cdot \nabla Q \Delta r c^{0} \sigma \rho \cdot \dot{\Delta}\left\langle S \Delta \dot{\Delta}^{a}{ }^{a} x\right.$

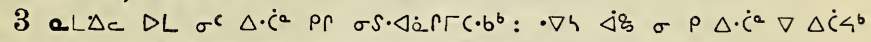

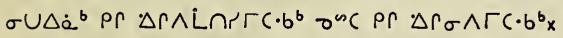

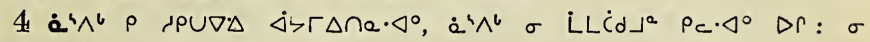

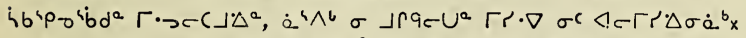

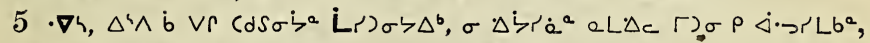

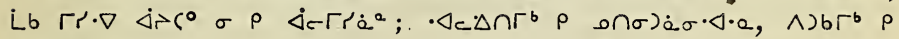

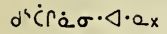

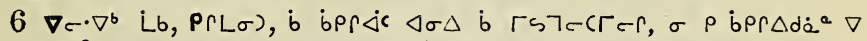
$\operatorname{Vr} \mathrm{CaS}^{\mathrm{b}} \mathrm{C} \mathrm{C}^{\mathrm{s}}$;

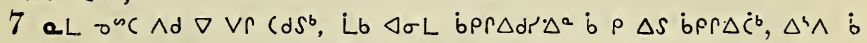

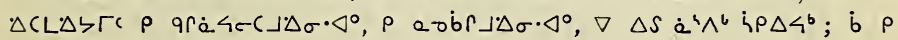

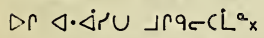

$8 \cdot \nabla h \dot{\Delta C} \nabla \rho \Gamma \Gamma c \cdot \nabla r \Delta C \cdot b^{b} \nabla \rho$ Lre $\Delta L C \cdot \cdot^{b}, a L \Delta c \sigma \Gamma \dot{C} U^{a}, \dot{\Delta l} \nabla \rho$

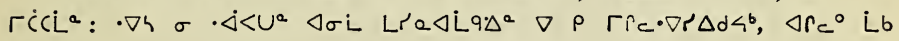
$\wedge d x$

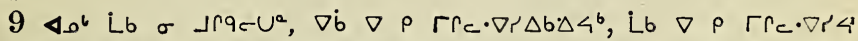

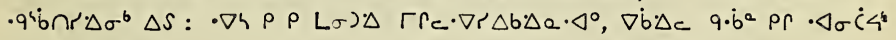
$\sigma c \dot{a}^{a} \Delta r_{x}$

$\left.10 \cdot \nabla h L \sigma) \Delta r r_{c} \cdot \nabla r \cdot \Delta^{a} \Delta S r q L b^{a} \cdot q^{a} b \cap r^{\cdot} \Delta^{a} \dot{b} \Delta C\right\lrcorner^{b} \wedge L \Gamma \Delta d r \Delta \sigma^{3} \nabla \dot{b}$ a $\Gamma \dot{C} \Gamma \dot{b} U^{b}:$ Lb $\Delta^{4} \rho \Delta \Gamma \Gamma_{c} \cdot \nabla r \cdot \Delta^{a} \Delta S \Gamma q L b^{a} \sigma \wedge \Delta^{a} \times$

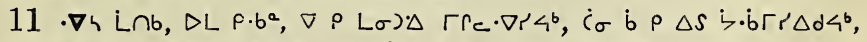

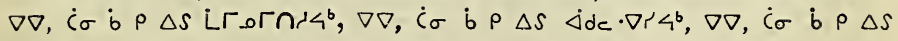

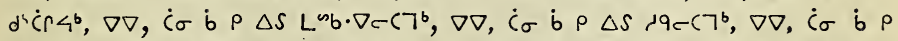

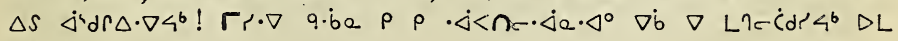
$\Delta \Gamma_{x}$

12 \&c Lb $\nabla \rho$ Lre $\Delta L\left(\cdot b^{b}, a L \Delta c \sigma \rho D r\right) U^{a} \Delta a b \dot{b} \rho$ Lr) $c^{b}, a L$

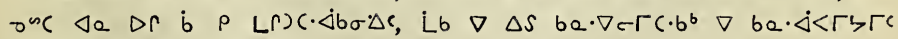
PrL $R$ P P $\triangle S \cdot \triangleleft<C 7^{b} x$

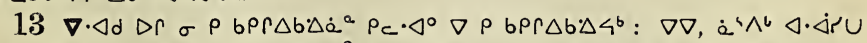

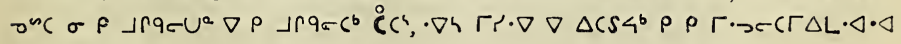
De $\Delta i \cdot b x$ 


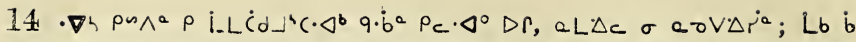
$p \quad \Delta S \Delta C L C \cdot b^{b} \Gamma r \cdot \nabla$ q.be $\left.\dot{c} \cdot V \Delta \sigma^{b}, \nabla d \dot{C} \wedge^{b} d^{b} \sigma L L \dot{C} d\right\lrcorner \Delta \sigma \dot{a}^{a}{ }^{b} b \quad \rho \quad \Delta S$

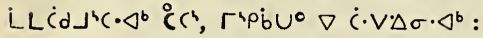

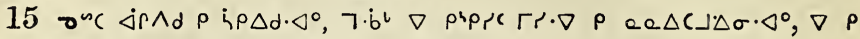

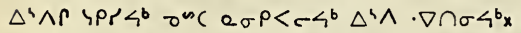

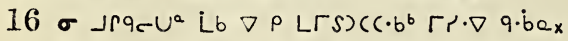

\section{Arpcre $\triangle b^{a} 8$.}

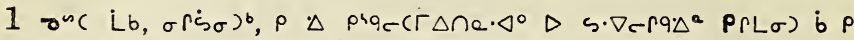

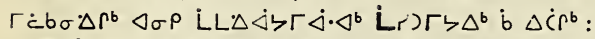

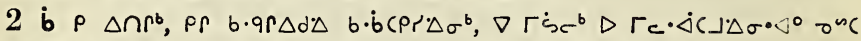

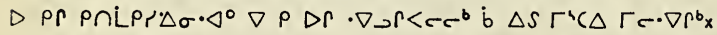

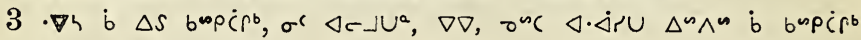
$\Delta=\cdot \Delta^{\circ} a r \cdot<^{b} \rho$ e $\nabla c C \cdot L^{b}$;

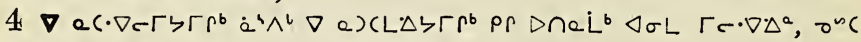

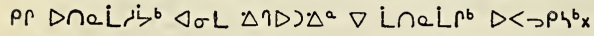

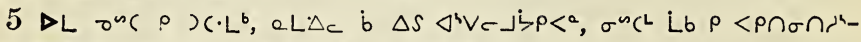

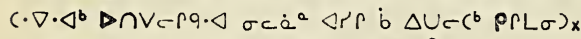

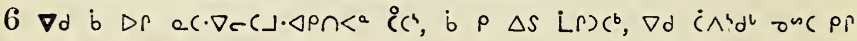
PSCLC $\dot{C}^{b} \triangleleft \sigma L S \cdot \nabla C \Gamma q^{\circ} \Delta^{a} x$

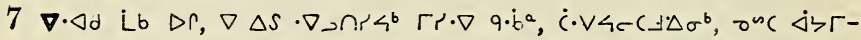

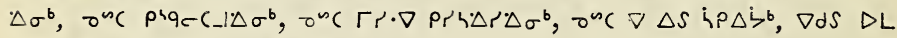
$\Delta S \mathrm{~s} \cdot \nabla e r q \Delta \sigma^{b} \cdot \nabla \rightarrow \cap r^{b} \sigma^{n c} C_{x}$

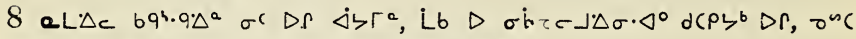

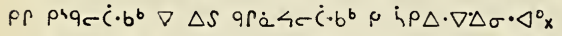

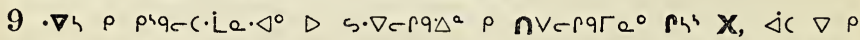

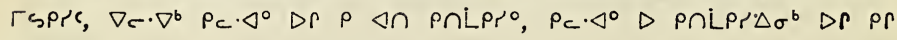
Lsprsis

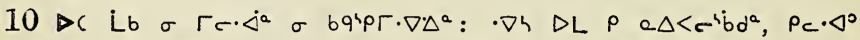

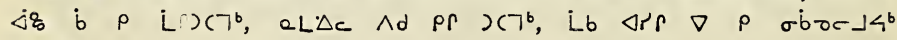
$\wedge>0^{b} x$

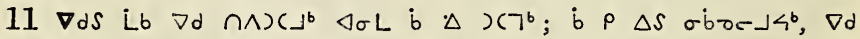

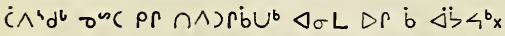

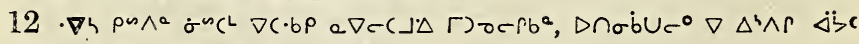
$\triangleleft \cdot \nabla a \Delta c c^{\circ}$, aL Lb $\nabla b$ b $\Delta S \quad \dot{\zeta b c_{x}}$

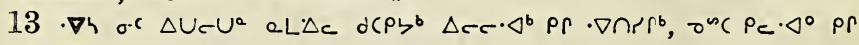
$\cdot<\cdot \triangleleft U<4^{b}$ :

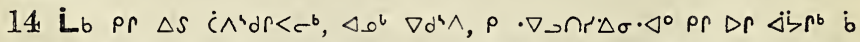

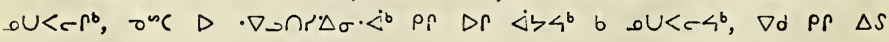

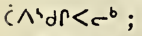

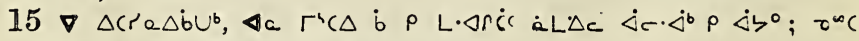

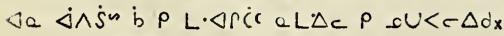




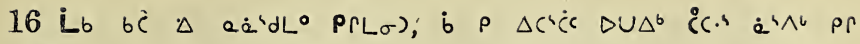
$\triangle U C \Gamma \dot{C}^{b} x_{x}$

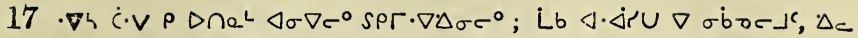
$a^{2} \cdot \dot{\Delta}^{b} \nabla \Delta U C^{b}, \rho \rho \dot{a} \cap d \cdot \triangleleft^{\circ} x$

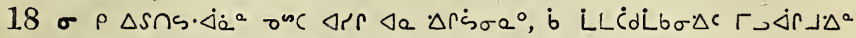

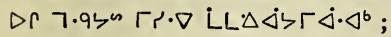

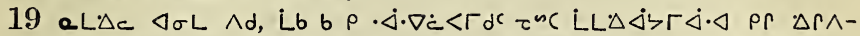

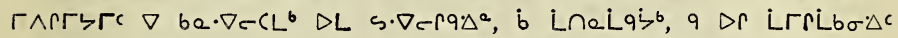

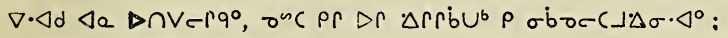

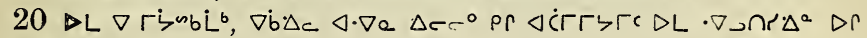
b L LQL Liخb:

$21 \nabla$ od

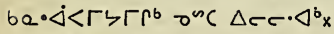

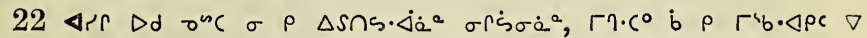
brharc tr. $L C S)\left(\cdot b^{b} D r_{x}\right.$

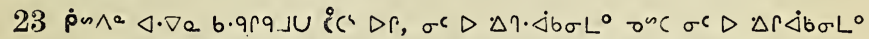

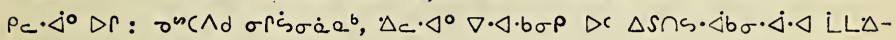

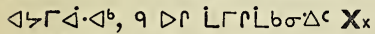

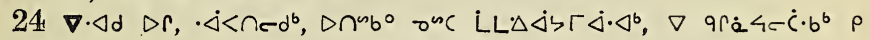

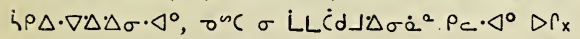

\section{Ápcreaba 9.}

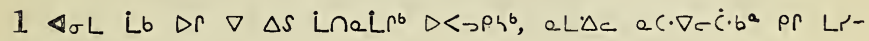
e $\triangle \mathrm{L} C \cdot \mathrm{b}^{6}$;

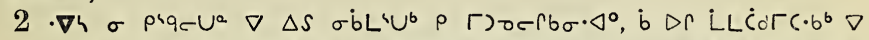

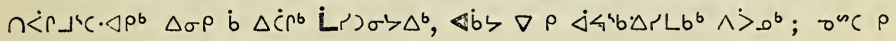

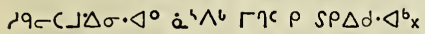

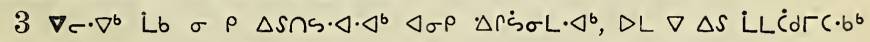

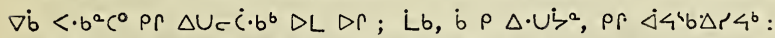

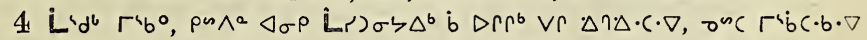

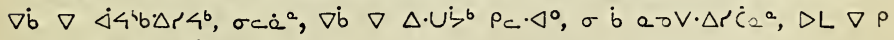
$\triangle S$ ire $D \cdot \Delta L L \dot{L} \dot{L} d \dot{b} b_{x}$

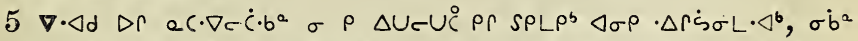

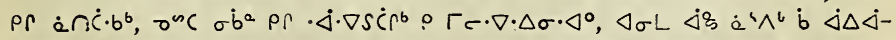

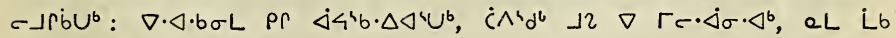

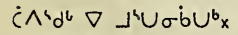

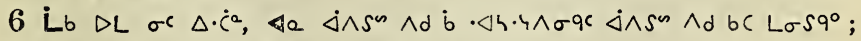

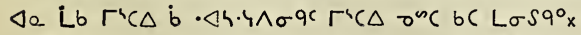

$7 \nabla d S$ b $\Delta C S c \Delta c c^{0} \nabla \Delta S \Delta U c^{b} \Delta U \Delta^{b}, \nabla d a \Delta S \Gamma c \cdot \nabla c ; \nabla b \cdot \Delta c \nabla$

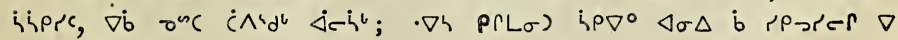
$\Gamma-\nabla-r_{x}$ 


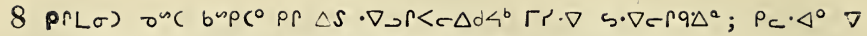

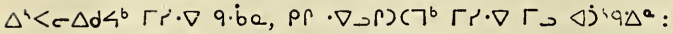

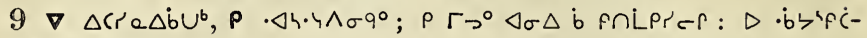
$n \pi \Delta^{a}$ b $p q \Delta c \cdot b \sigma c^{\circ} \times$

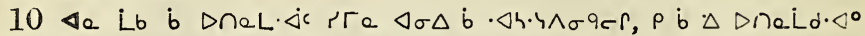

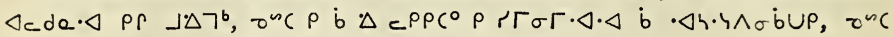

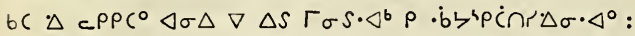

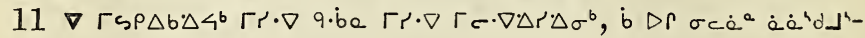
$\left(\cdot \Delta P(P \Gamma L \sigma)_{x}\right.$

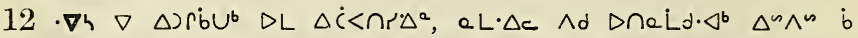

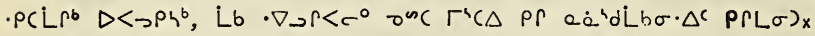

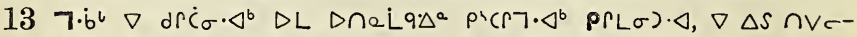

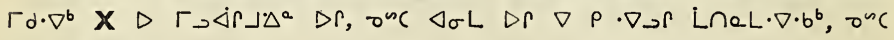
$\Gamma r \cdot \nabla \Delta r c \cdot \Delta l ;$

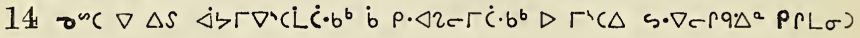
b $\wedge r^{2} \dot{b} \cdot \nabla^{\circ} \cdot \nabla^{\circ} D r_{x}$

$15 b\left(\Delta a \dot{a}^{b} d L^{\circ} \rho r L \sigma\right) D \Gamma c \cdot \nabla \Delta^{a} \Delta r \nabla b q \rho \Delta r \dot{b} U c^{b} x$

\section{A'pcra $\triangle b^{a} 10$.}

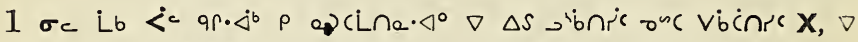

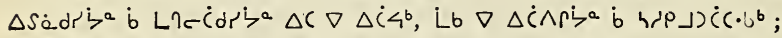

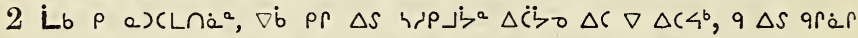

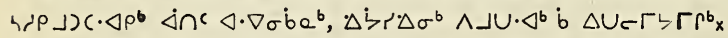

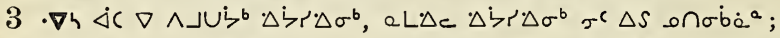

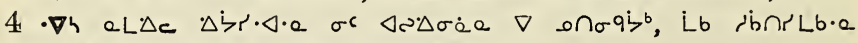

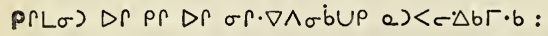

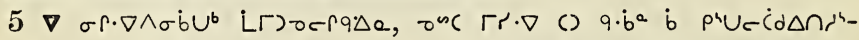

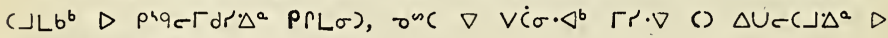
Q.e $\Delta \dot{C} d r \Delta \sigma^{b} X$

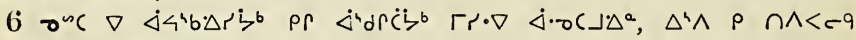
$\rho_{C} \cdot \triangleleft^{\circ} \rho$ e e $\left.\Delta C\right\lrcorner \Delta \sigma \cdot \Delta^{\circ} x$

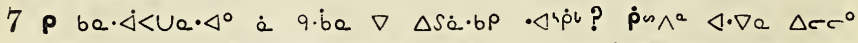

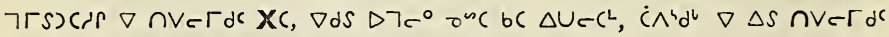

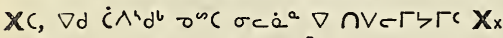

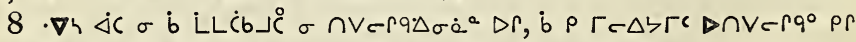

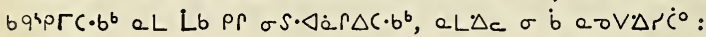

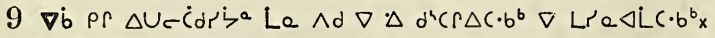

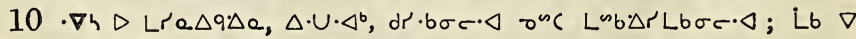

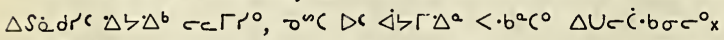

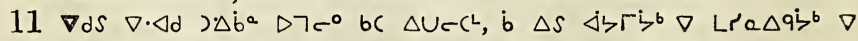

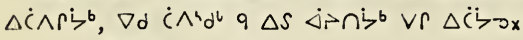




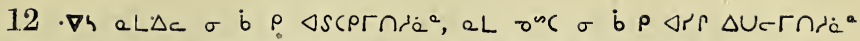

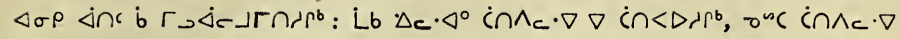

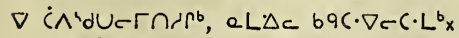

13 Lb a LAc $\sigma \dot{b}$ LLCंd $\dot{a}^{a}{ }^{a} \nabla \dot{b} \nabla \Delta S \cap<\Delta \dot{b} U^{b}, \dot{L} b \quad \nabla \Delta S \cap<\Delta \dot{b} U^{b}$

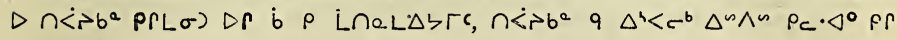
$D \cap \cap \zeta^{b} x$

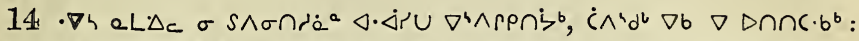

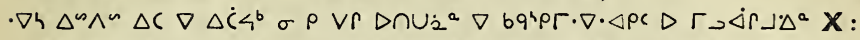

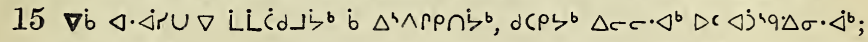

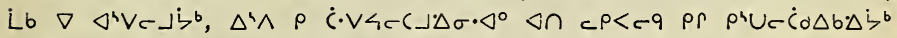
$\rho_{c} \cdot \triangleleft^{\circ} D \Gamma \dot{b} \Delta C^{4} U^{b} D L \sigma \cap \dot{<}>b \sigma a^{c}$,

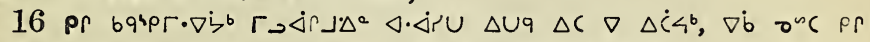

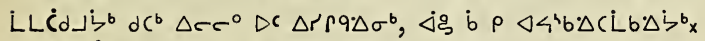

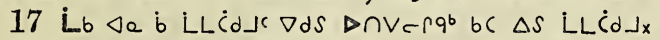

$18 \cdot \nabla 4$ a L $\triangle \cap \vee-\cap 9 \cdot \triangleleft_{x}$

\section{A'pcra $\triangle b^{a} 11$.}

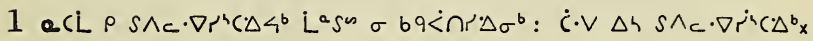

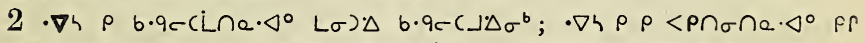

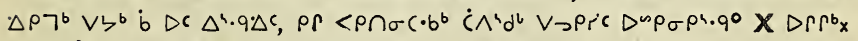

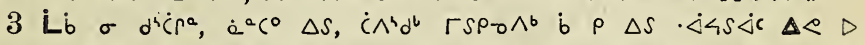

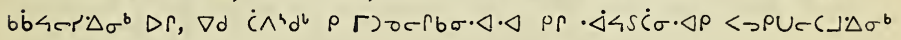
$\Delta r X)^{b}$ bे $\Delta C \cdot b \sigma \sigma^{b} x$

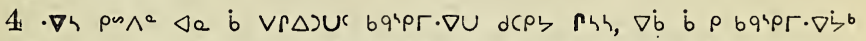

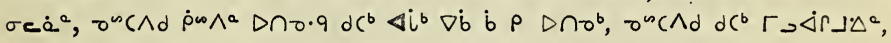
$\nabla \dot{b} \dot{b} \rho \Delta \cap \sigma^{b}, \dot{c} \cdot \nabla \rho \dot{b} S \Lambda c \cdot \nabla r^{\prime} c \cdot \dot{\Delta} \cdot \dot{c} \cdot \Delta^{\circ} x$

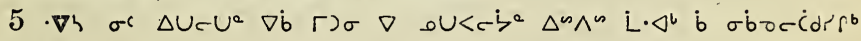
$\triangleleft<c^{\prime} c^{b} x$

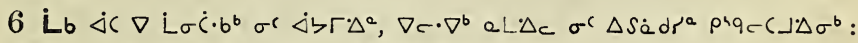

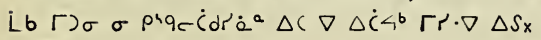

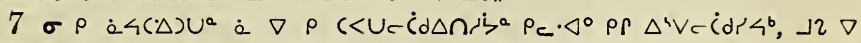

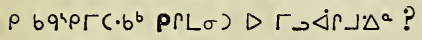

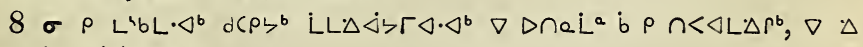
$\dot{\Delta \succ}\left\ulcorner\dot{\Delta} \Delta \Delta \dot{b} \dot{b} b \cdot b^{b} \mathrm{x}\right.$

$9 \Delta^{\prime} \wedge$ Lb b $\Delta \eta \Delta C \cdot b^{b}, \nabla \rho \cdot \rho C L \zeta^{a}$ Lb, aL $\Delta c \Delta \cdot \nabla a \Delta c c^{0} \sigma \rho$

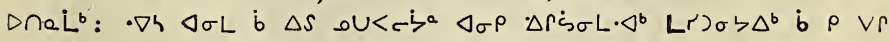

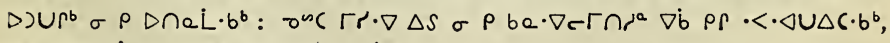
$\nabla d$ onc 9 ib<b a $\Delta S$ be. $\nabla c r n$ ribax

$10 \dot{c} \wedge^{4} d^{b} \nabla \Delta S \wedge r^{4} b d \cdot \Delta^{b} X \nabla \dot{c} \cdot V \Delta^{a}, a L \Delta c \triangleleft \cdot \nabla a \Delta r c^{0} \sigma b$ b $P \wedge r \Delta^{b}$

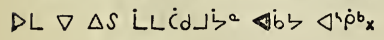
303 


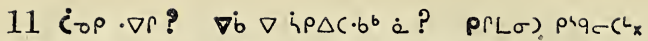

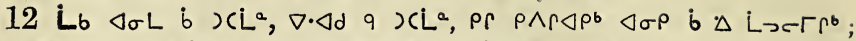

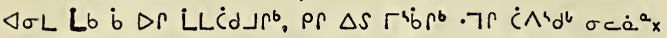

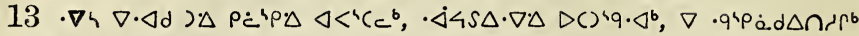

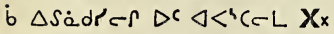

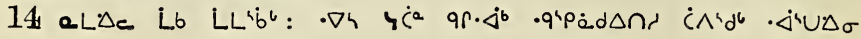
$\nabla^{a} \iota^{c} x$

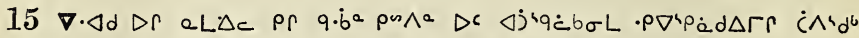

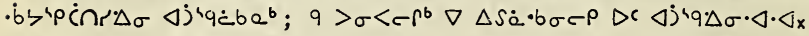

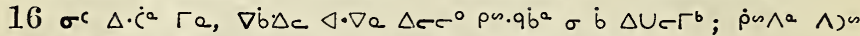

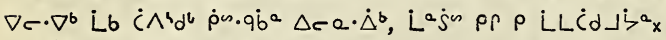

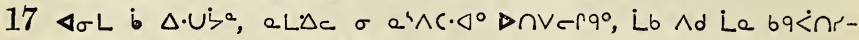
$\Delta \sigma^{b} \Delta S, \nabla$ 9ra $\Delta \dot{\zeta}^{a} \Delta L \nabla$ LLCंd $\lrcorner \dot{\zeta}^{\circ} x$

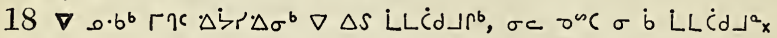

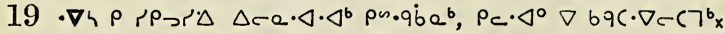

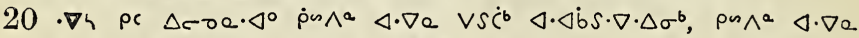

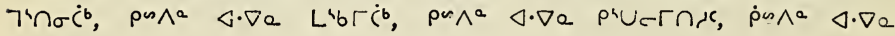
$\left\langle<\cdot 9 D C^{b} x\right.$

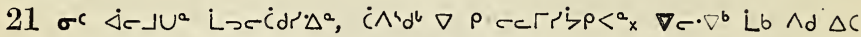

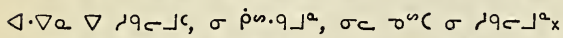

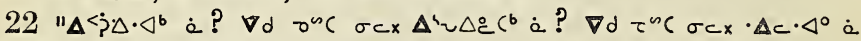

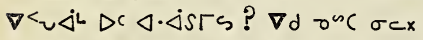

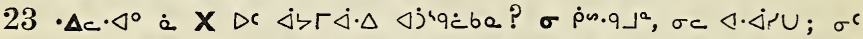

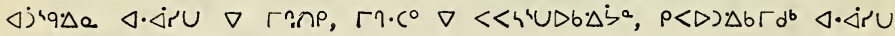
$\Gamma \eta \cdot c^{\circ}, \sigma \wedge \Delta \sigma^{b} \Gamma \eta \cdot c^{\circ} x$

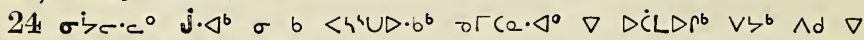
oU $U<c^{b} x$

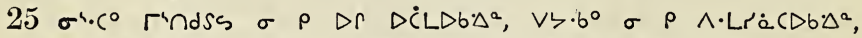

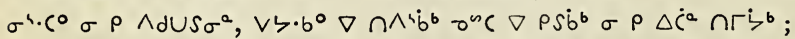

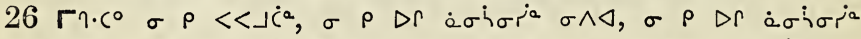

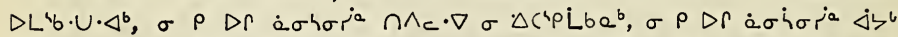

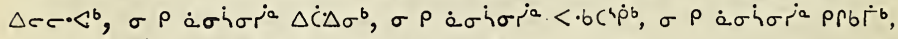

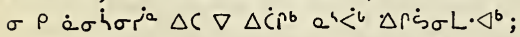

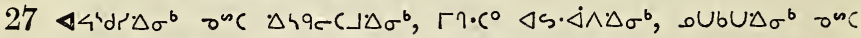

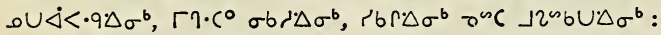

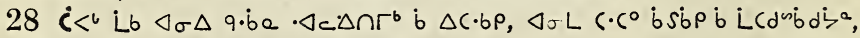

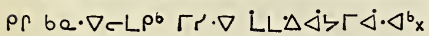

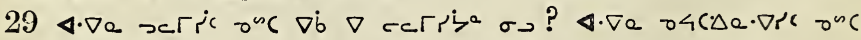
$\nabla \dot{b} \nabla \Delta \cdot \dot{b} \dot{b} \bar{b}^{a} \sigma_{c}$ ?

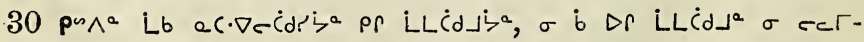
r. $\Delta e \Delta r_{x}$

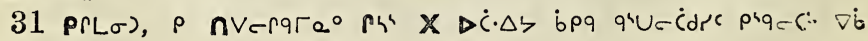
$\nabla \rho \dot{c} \rho \dot{C}^{\circ} \mathrm{x}$ 


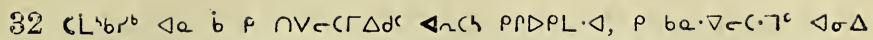

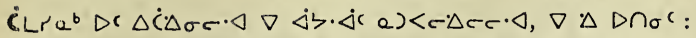

$\left.33 \sigma^{n} c \dot{s}\right\rangle\left\langle 4<\wedge \Delta \sigma^{b} \sigma P D \Gamma \dot{a} i \wedge 9 \sigma b \Delta^{a}, \nabla d\right.$ Lb b $D S L^{b} x$

\section{$A^{4} p\left(r a \Delta b^{\circ} 12\right.$.}

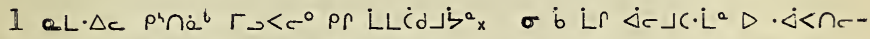

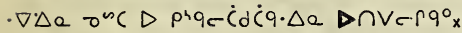

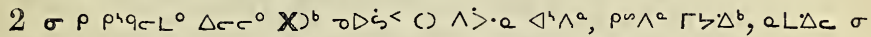

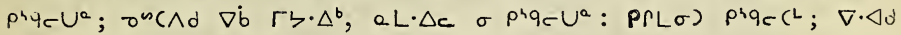
$\triangleleft \cdot \triangleleft P D \wedge 0^{\circ} \Delta\left(\nabla \sigma^{4}\right) \triangleleft \cap P S d \cdot \Delta^{b} x$

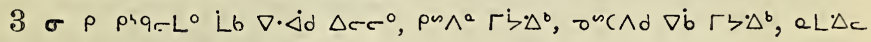
$\left.\sigma p^{4} q_{C} U^{2}: p r L \sigma\right)$ prqe $(L$,

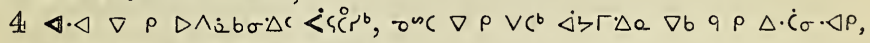

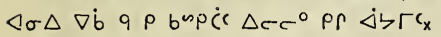

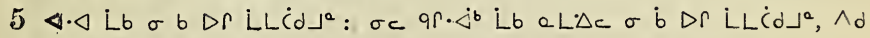
$\sigma \tau c \Gamma r \Delta \sigma^{b} x$

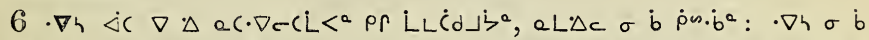

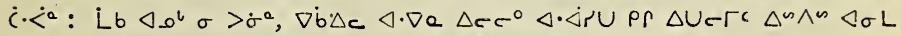

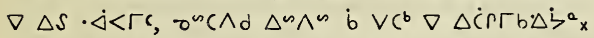

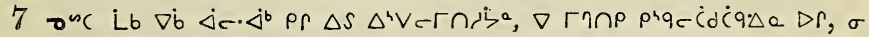

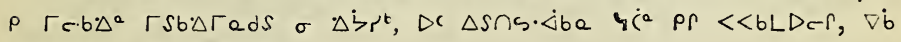

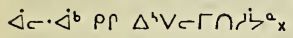

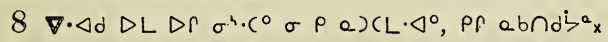

$9 \sigma \rho \Delta \cap^{b} L b, \sigma s \cdot \nabla c \beta 7 \Delta^{a} \rho \dot{b} \cup \wedge<c \Delta d^{a} ; \cdot \nabla h \sigma L \omega b \Delta \cdot \Delta^{a} p S \dot{C} \sigma \cdot \varangle^{a}$

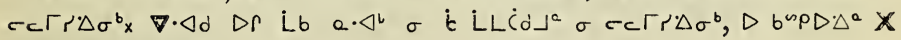
$\operatorname{si} \cdot U D)\left(d \cdot \Delta x^{\prime} x\right.$

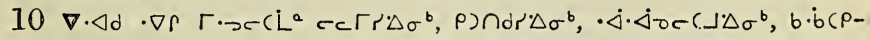

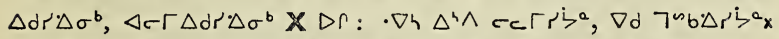

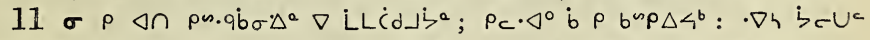

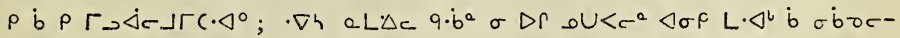
$\dot{c} \mathrm{dr} b c^{b} \triangleleft<^{4} c c^{b} x$

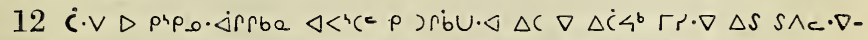

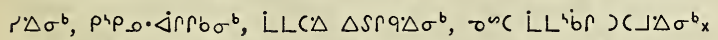

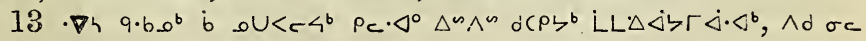
$\nabla \dot{b} \nabla P \Delta r \cdot<\cdot \Delta U \Delta C \cdot b^{b}$ ? $>\sigma_{c} C C L \Delta^{b} D L \cdot \Delta \sigma \Delta \cap \Delta^{a}{ }^{a}$

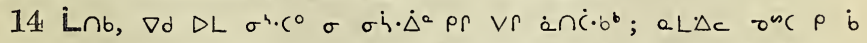

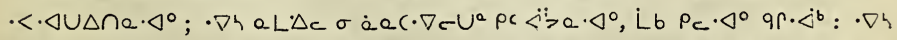

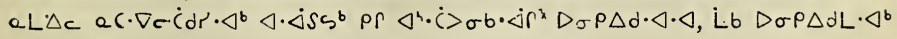
$\triangleleft \cdot \triangleleft s s_{x}$

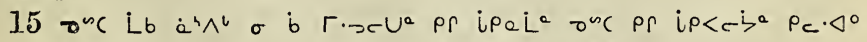

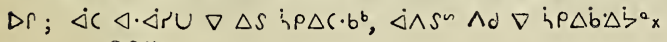




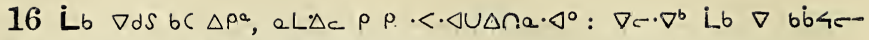

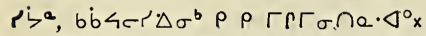

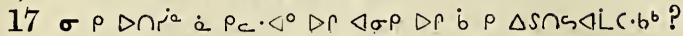

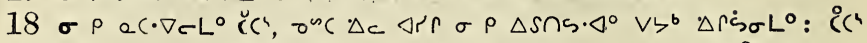

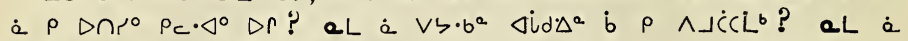
$V \zeta \cdot b^{a}$ bे $\rho \Delta C d^{\prime} q \dot{b} b$ ?

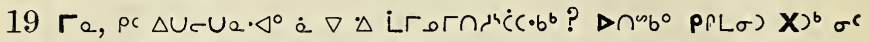

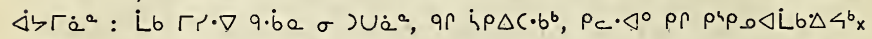

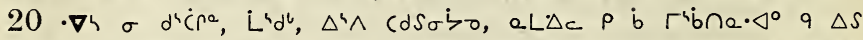

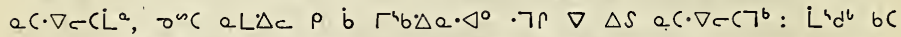

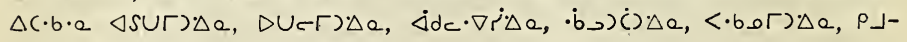

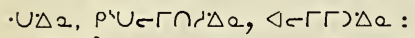

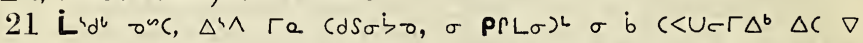

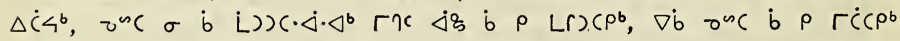

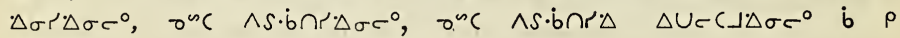
$\dot{\Delta r})\left(\rho^{b} x\right.$

\section{Aיpcrà $\triangle b^{a} 13$.}

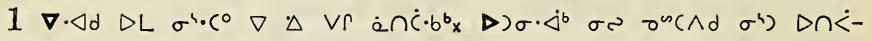

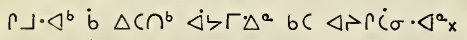

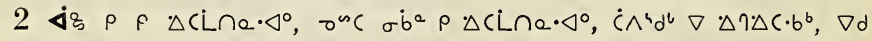

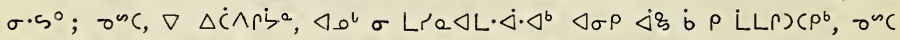

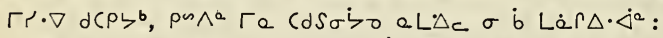

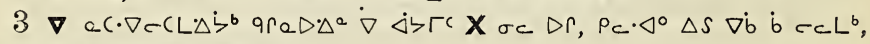
L.b b jibnr $L b^{b} \nabla \wedge \Gamma^{4} b d \zeta^{b} x$

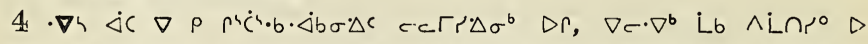

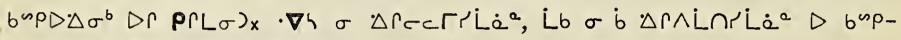
$\nabla \cdot \Delta \sigma^{b} D \Gamma P(L \sigma) P_{c} \cdot \Delta^{\circ} \Delta S_{x}$

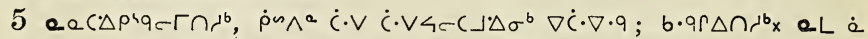

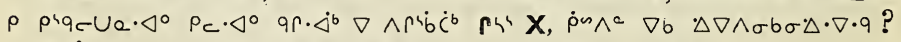

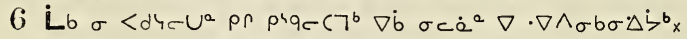

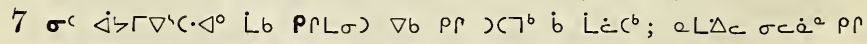

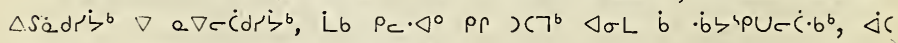
$\sigma C \dot{c}^{a} \dot{C} \Lambda^{b} d^{b} \cdot \nabla \wedge \sigma b a^{b} \nabla \Delta C P \Gamma b \Delta \dot{b} b x$

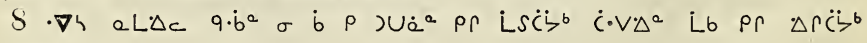
$\dot{c} \cdot \vee \Delta \Delta^{2} x$

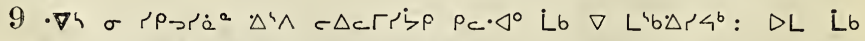

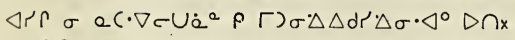

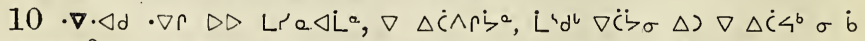

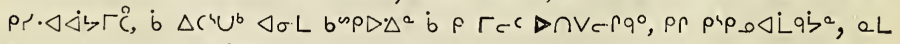

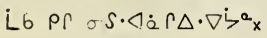




\section{brsषa 1.}

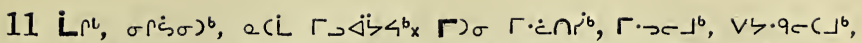

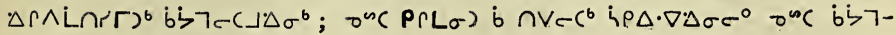
$\tau\left(\mu \Delta \sigma \sigma^{\circ} b\right.$ b $\Delta \lambda \Delta \Delta d^{\circ} \cdot \Delta^{\circ} x$

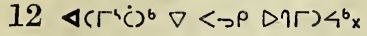

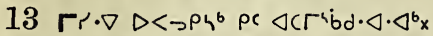

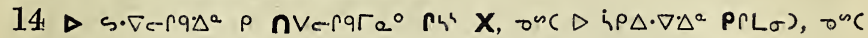

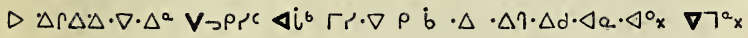

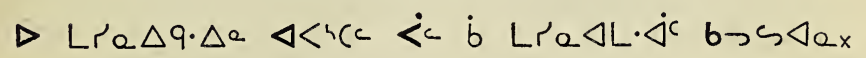

\section{N'pcre $\triangle b^{a} 1$.}

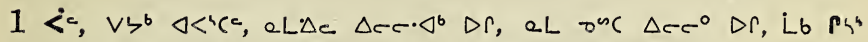

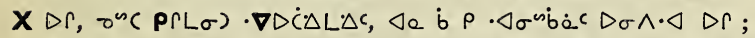

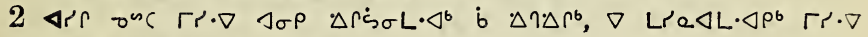

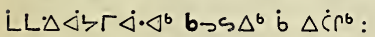

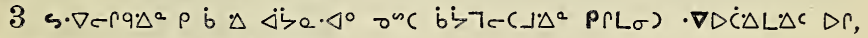

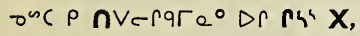

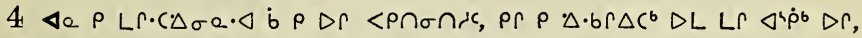
b $\Delta U C^{6}{ }^{b} p(L \sigma) d \dot{d} \Delta a^{c}$;

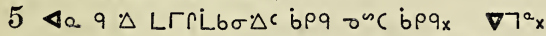

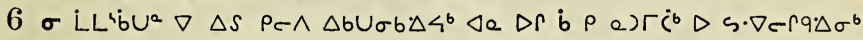

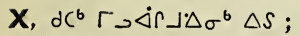

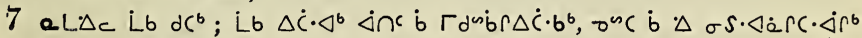
$\nabla \Gamma \rightarrow \Delta r\lrcorner \Delta \sigma \sigma^{\circ} \mathbf{X} C_{x}$

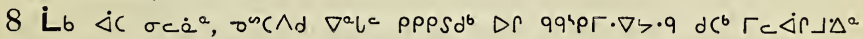
$\triangle^{u} \wedge^{n} \triangleleft \sigma L \sigma c \dot{a}^{a}$ bे 9 b

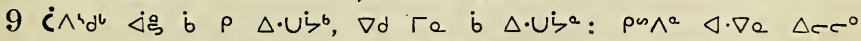

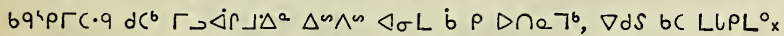

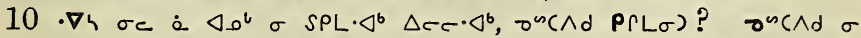

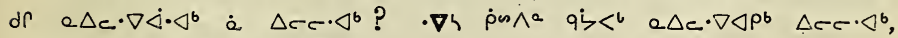
e L $\Delta \Delta_{c} \sigma$ b $\Delta C \Delta j \wedge q_{c} b \sigma \Delta i \mathbf{X} \times$

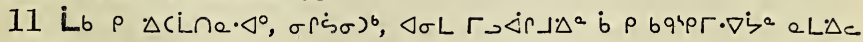
$\triangle r c^{6} \rho \Delta r<r^{\circ} x$

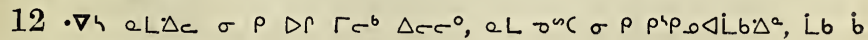
$\triangle S \rho^{i} q C C r \Delta C \boldsymbol{P S}^{i} \mathbf{X}_{K}$

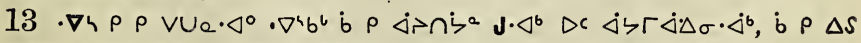

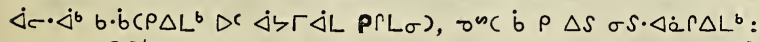
307 


$$
\text { bsc }<\text { e } 1,2 \text {. }
$$

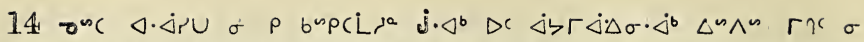

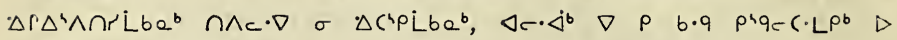
$\cap \dot{r}\lrcorner \cdot \Delta \sigma \cdot \Delta \cdot \triangleleft \rho(\Delta\rangle^{b} \times$

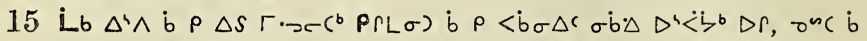
$b$ a) $R C D S \cdot \nabla C r q \Delta^{a} D r$,

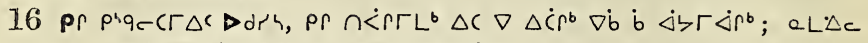

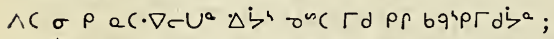

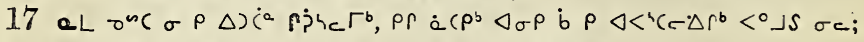

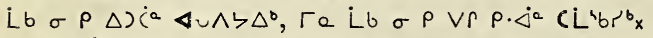

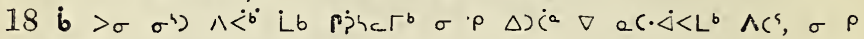
$\triangle r C S 9 L^{\circ} L b \quad \sigma \dot{L} c-\dot{S}>P S b \cdot \Delta x$

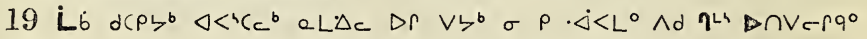
$\Delta$ risax

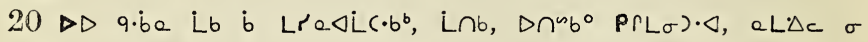
$\rho \dot{c}^{\prime} \rho^{\prime} a_{x}$

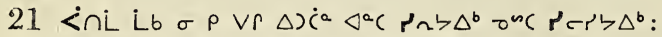

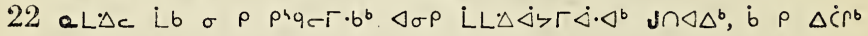
$x^{6}$ :

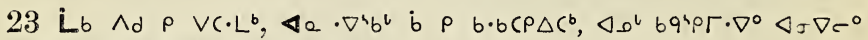

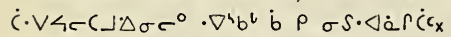

$24 \rho L \Gamma \Gamma\urcorner \cdot \triangleleft^{b}$ Lb $\rho \Gamma(\sigma) \cdot \triangleleft \sigma_{c} \Delta r_{x}$

\section{A $p\left(r c \Delta b^{\circ} 2\right.$.}

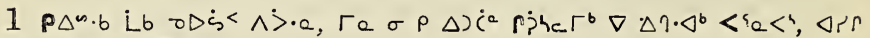
The $\sigma$ P $P \cap 0^{\circ}{ }^{\circ} \mathrm{C}\left(c^{4} \mathrm{x}\right.$

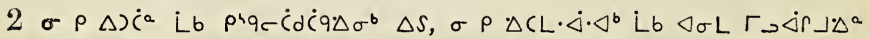

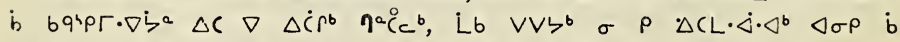

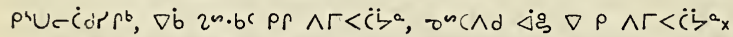

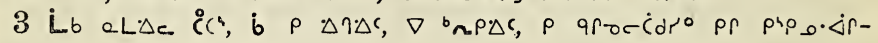
$\dot{s} b \sigma \Delta c$ :

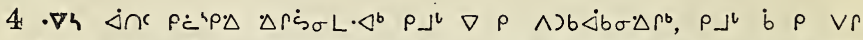

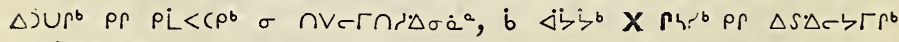
$\varangle \cdot \Delta \dot{b} \cap d \Delta \sigma^{b}$ :

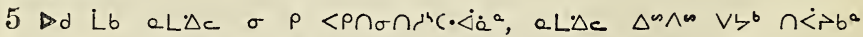

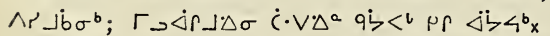

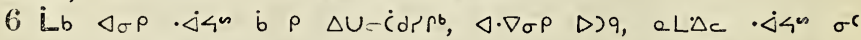

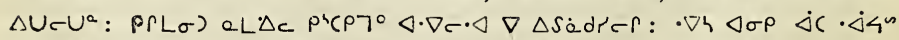
b $\rho \Delta U c \dot{c}\left(d^{\prime} \rho^{b}, \nabla \Delta \zeta \zeta \Gamma \Delta\right) \dot{a} \sigma \cdot J^{b}$ a L $\Delta c a \cdot \dot{b}^{a} \sigma \rho \Delta S \Delta r \Delta \cdot b^{b}$ :

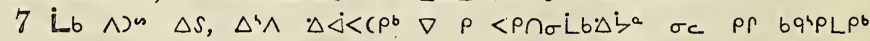

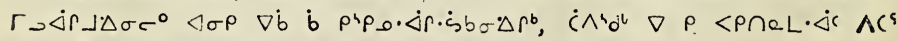
$\Delta \sigma \Delta \dot{b}$ php.o. $\Delta r \cdot \dot{s} b \sigma \Delta r r$;

308 


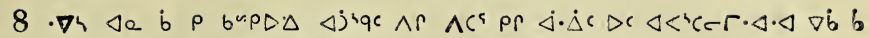

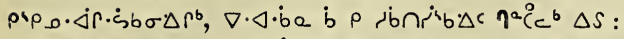

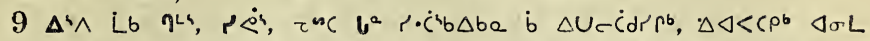

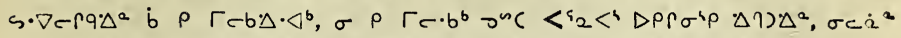

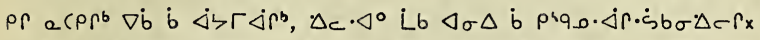

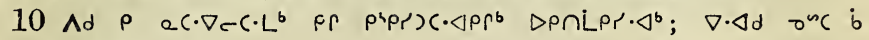
obor Jía $p r)\left(L^{a} x\right.$

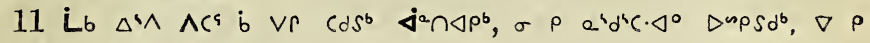
$\Delta U e \dot{C} d p e$ of $\Delta \dot{C} \Gamma \dot{L} b \sigma \Delta c_{x}$

$12 \cdot \nabla 4<0\lrcorner S \triangleleft \cdot \nabla \sigma b a^{b} \rho$ cospb qub $\Delta r, \rho \Delta r r r r^{\circ} \triangleleft \sigma \Delta \eta^{2} c_{c}^{\circ}$ :

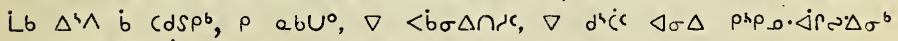
i nRecidicix

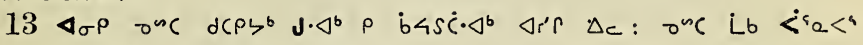

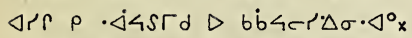

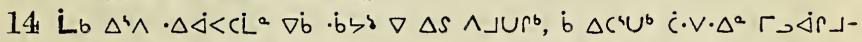

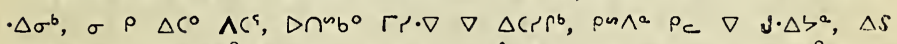

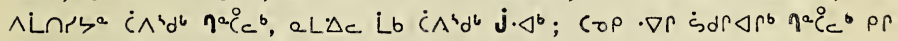

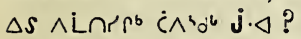

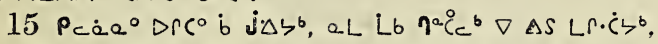

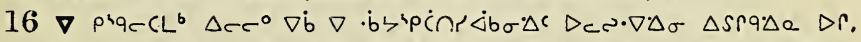

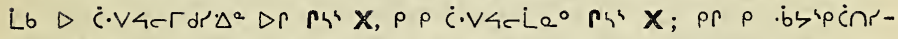

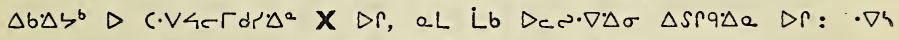

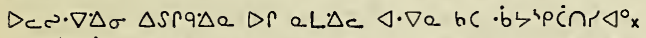

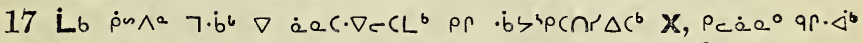

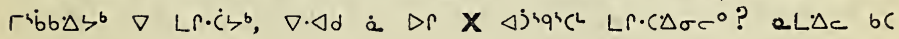
$\Delta \Delta p^{a} x$

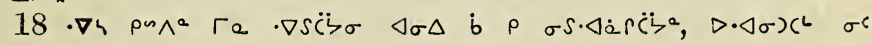
$\Delta s \Delta \cap r^{a{ }^{a} \mathrm{x}}$

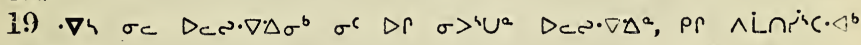
Pr $L \sigma)_{x}$

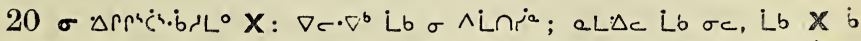

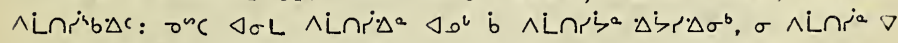

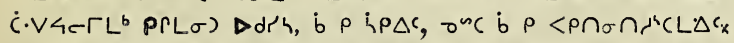

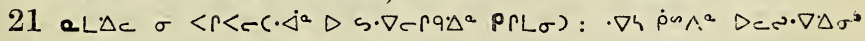

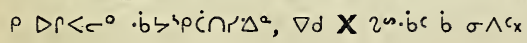

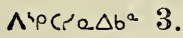

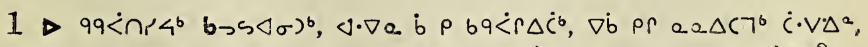

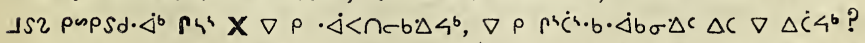

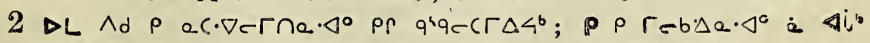

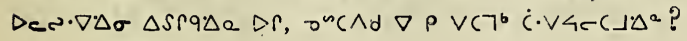
309 


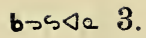

$3 \nabla d$ a $\nabla^{4} \wedge r$ bq<ंก $b \Delta a \cdot \Delta^{\circ} \Delta \dot{b} \cdot \Delta \sigma^{b} \Delta r, ?$

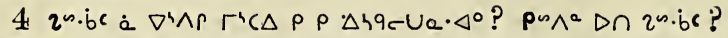

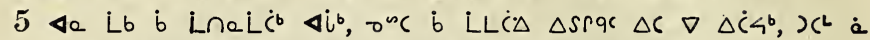

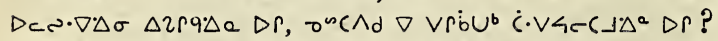

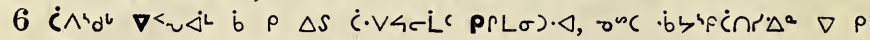
$\triangle C P L C$ ?

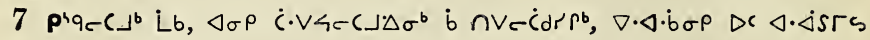
$\boldsymbol{\nabla}<v<j \times$

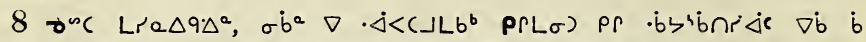

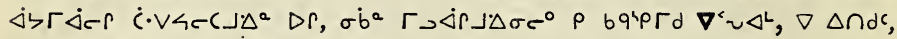

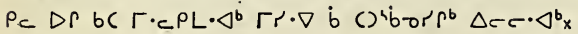

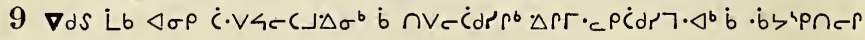
$\nabla<$ ข

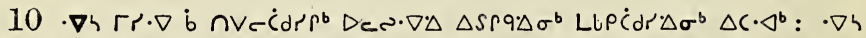

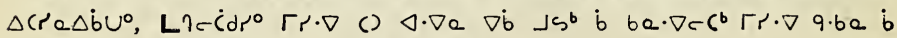
Lra $\Delta \dot{b U C r} \nabla c \omega \cdot \nabla \Delta \sigma$ Lra $\Delta b \sigma^{b}$ pr $)^{b}{ }^{b}$

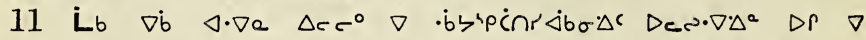

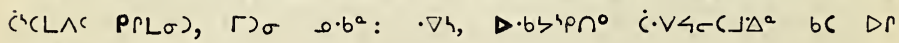
$\wedge \operatorname{Ln} r^{\circ} x$

$12 \Delta c \omega \cdot \nabla \Delta^{a}$ Lb aL $\left.\Delta e \dot{C} \cdot V A_{c} c\right\lrcorner \Delta \sigma^{b} U<q-\dot{C} \cdot b^{a}:$ Lb $\triangleleft a \Delta c c^{0} \dot{b}$ ) $c^{b} \nabla d<$ a $\triangle S \wedge$ L $\cap r^{\circ}$

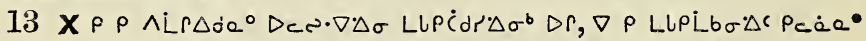

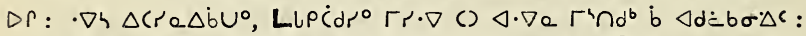

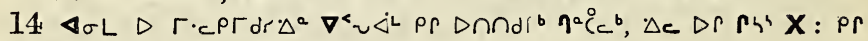

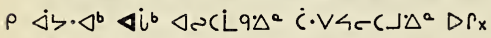

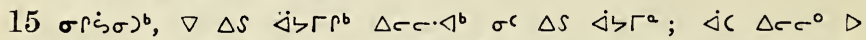

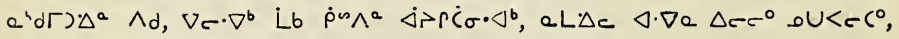

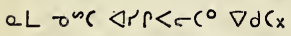

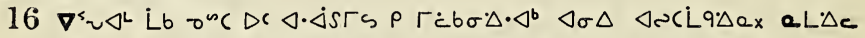

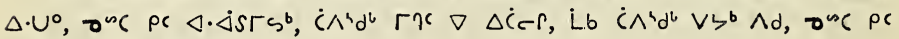
$\Delta \cdot \dot{\Delta s} r^{\infty}, \nabla \cdot \Delta d \mathrm{X}$

$17 \Delta L \sigma^{n}\left(\sigma^{c} \Delta \cdot \dot{c}^{a}, 4 \sigma L a^{4} d \Gamma\right) \Delta^{a}, \sigma^{a}$ b $\rho$ jrric $\left.\left.\rho \Gamma L \sigma\right) X\right)^{b}$,

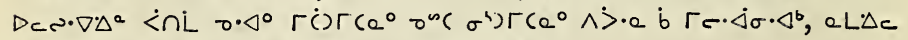

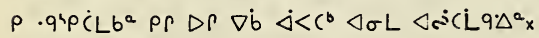

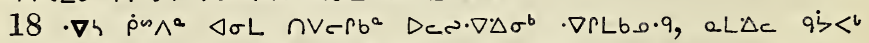

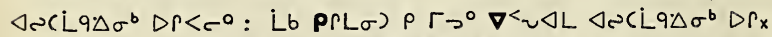

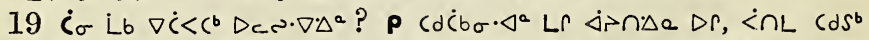

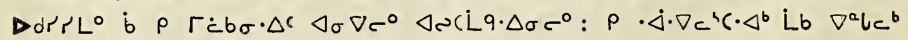

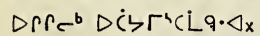

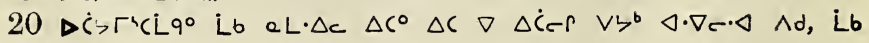
PrL $\sigma)$ Vלdx

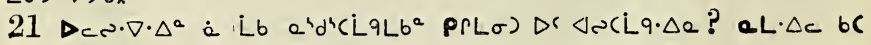
310 


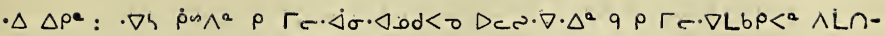

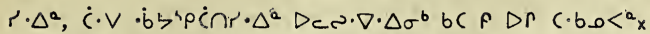

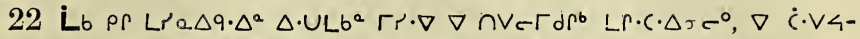

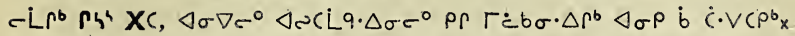

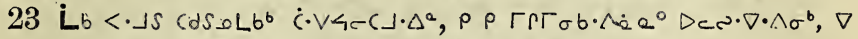

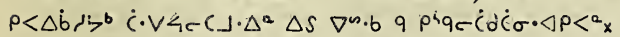

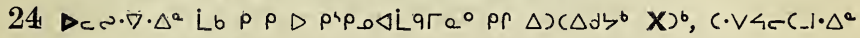
pr $D r \cdot b b^{4} p i n r \Delta b \cdot \Delta b^{b} x$

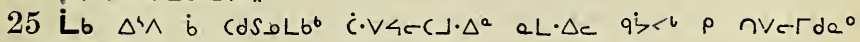
DPhP_o $\Delta \mathrm{L}^{\circ} \mathrm{x}$

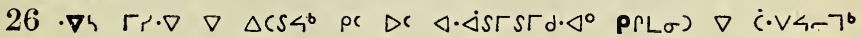
$\mathrm{X}$ mix

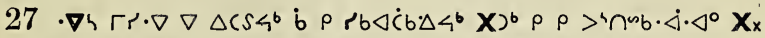

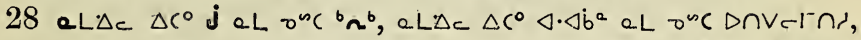

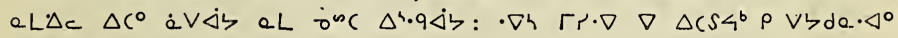

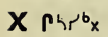

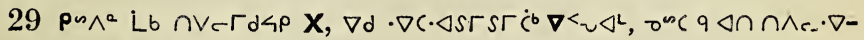

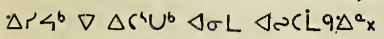

A'pc?a $\triangle b^{a} 4$.

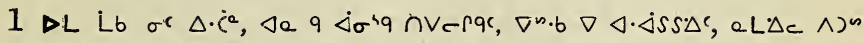

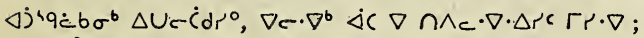

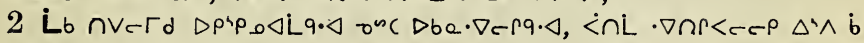
$\triangle C P C L$ Ld $D \dot{C} \triangle \triangle \mathrm{Lx}$

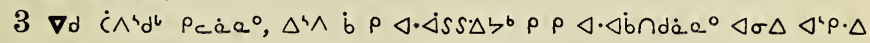
onch piposiquae :

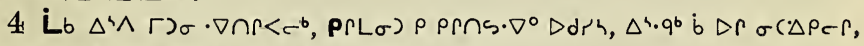

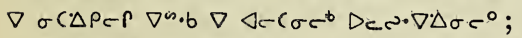

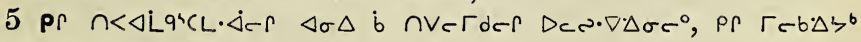
$D \operatorname{dr} t L \Delta C<\mathrm{g} \Gamma \cdot \nabla \cdot \Delta^{2} x$

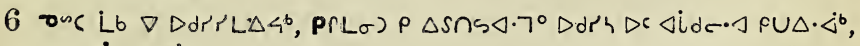
$\nabla U \cdot \operatorname{ver} \dot{\Delta}<, \quad \rho \dot{c} x$

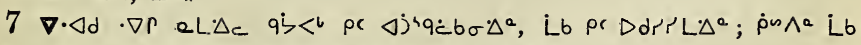

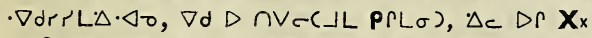

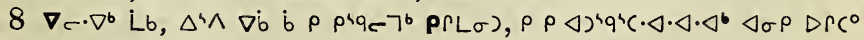
$\nabla \dot{b} \dot{b} L \sigma) \Delta \rho^{b} \times$

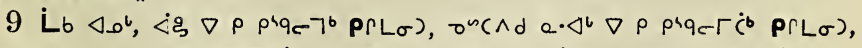

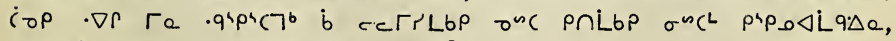

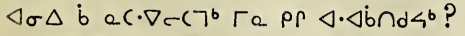

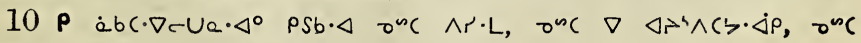
ব'p $\Delta e_{x}$ 


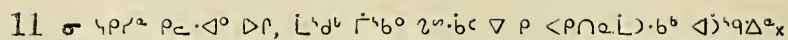

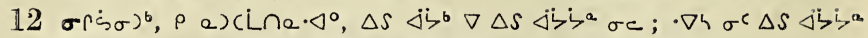

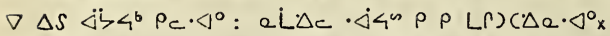

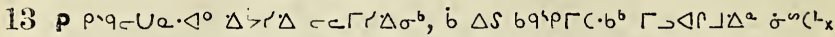

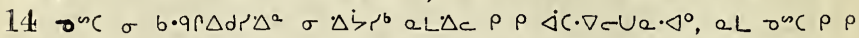

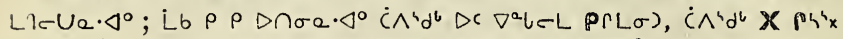

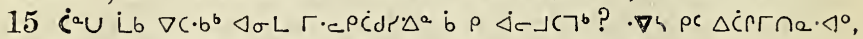

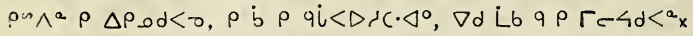

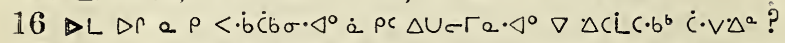

17 pc $\Delta C h \rho \Delta d \cdot \Delta \cdot \Delta^{b}$, Lo eL $\Delta c \cdot b^{b b} ; \nabla \nabla, \rho$ b $\Delta b \cup \sigma d \cdot \Delta c \cdot \Delta \cdot \Delta^{b}$, p饣 $i \rho \nabla \cdot b^{b} x$

18 Lb $\Gamma \cdot \dot{e} S^{a} \rho \rho$ db $i \rho \dot{C}_{\sigma} \cdot \Delta^{b}$ b $\Gamma \cdot \dot{c} S^{b}$, aLAc Lb $\wedge d 7 \cdot \dot{b}^{b} \nabla$ $\triangle 1 \Delta c \cdot b^{b} x$

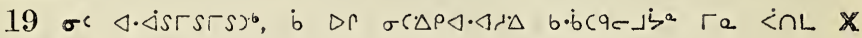
$\wedge \operatorname{mib} \dot{c}$;

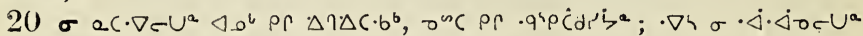
$\rho_{C} \cdot \Delta^{\circ} \nabla r_{\mathrm{x}}$

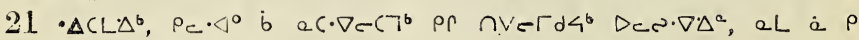
$V U^{a} \cdot \Delta^{\circ} \nabla_{c} \cdot \nabla \cdot \Delta^{a}$ ?

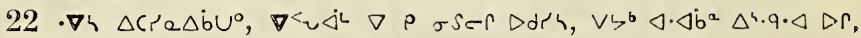

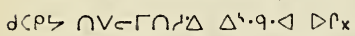

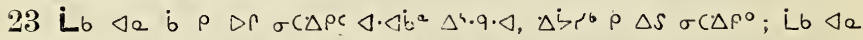

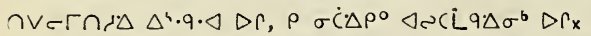

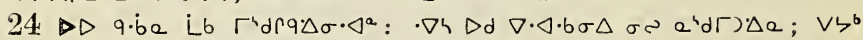
i $\sigma \cdot \Delta \dot{r}^{b} \Delta r, \dot{b} \Delta \triangleleft \cdot \Delta \dot{b} \sigma \Delta \cdot \nabla L b^{b}, \nabla \cdot \Delta d \nabla \dot{b}^{4} x$

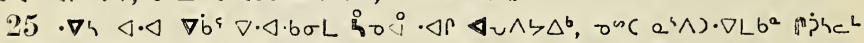
$\Delta a^{b} \dot{b} \Delta c \cdot b^{b}, \sigma^{n} c \Delta \cdot \Delta \dot{b} s \cdot \nabla \cdot \Delta \sigma^{b} b \Delta \dot{c} \Delta r f \Delta c \Delta \cdot \Delta s \Gamma c_{x}$

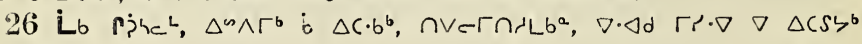
$\rho \mathrm{b} \cdot \Delta \mathrm{e}^{\circ} \mathrm{x}$

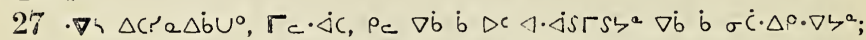

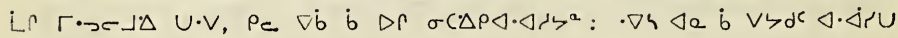

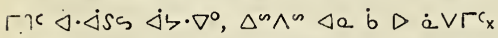

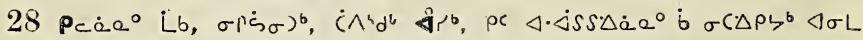
$\operatorname{coc} \mathrm{L} 9 \Delta \sigma^{\circ} \Delta r_{\mathrm{x}}$

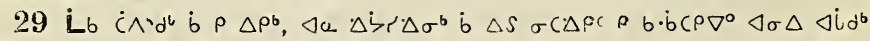

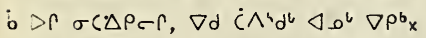

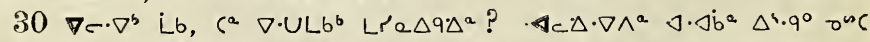

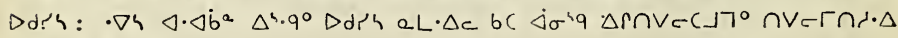

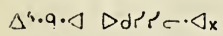

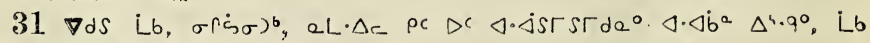
กverni. $\triangle \Delta^{i} \cdot 9^{\circ} \mathrm{x}$ 


$$
\text { bssde } 5 \text {. }
$$

\section{$A^{4} p c r a \Delta b^{a} 5$.}

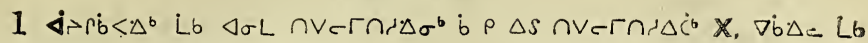
Te $a \cdot \dot{b} C D r^{b} \Delta \cdot \Delta \dot{b} s \cdot \nabla \cdot \Delta \dot{C} \wedge \dot{b} b \sigma^{b} x$

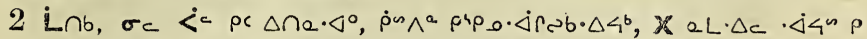
b) $\Delta \dot{C}<r \Delta d \cdot \Delta^{\circ} x$

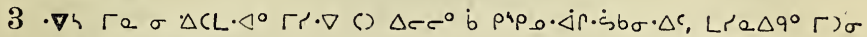
Pr $\cap \wedge)\left(b \cos \nabla \cdot \Delta \sigma c^{\circ} x\right.$

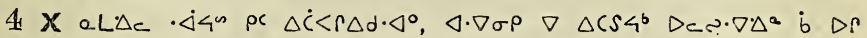

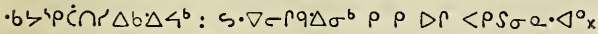

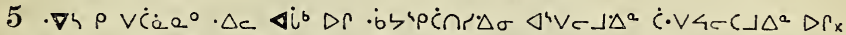

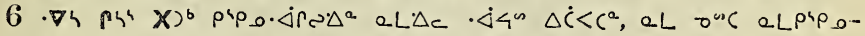

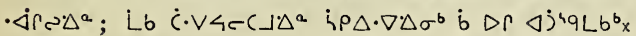

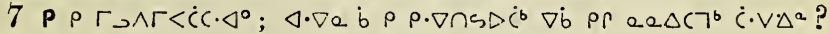

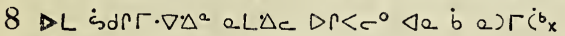

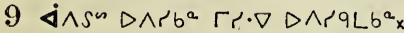

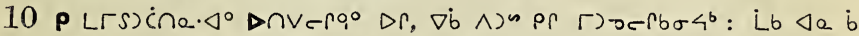

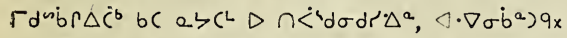

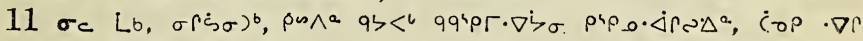

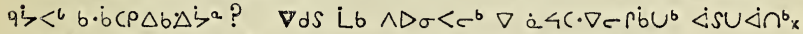

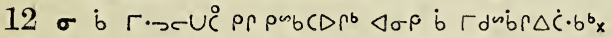

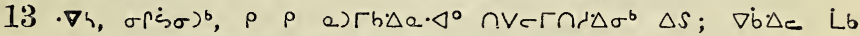

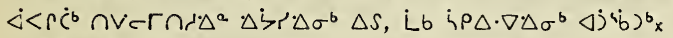

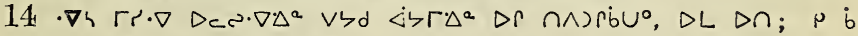

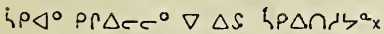

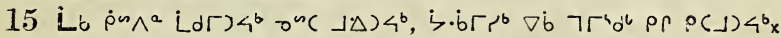

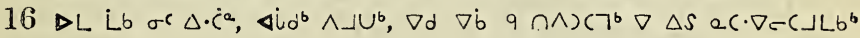
$\Delta i \Delta \Delta \Delta^{a} x$

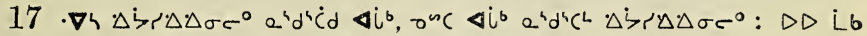
$\dot{<} \wedge)^{n}$ ec. $\left.\nabla c(\lrcorner L b \cdot a: \dot{b} \Delta r \nabla \dot{b} \rho\right)\left(7^{b} \Delta \sigma \Delta q \cdot \dot{b} a \dot{b} \Delta\right)\left(7^{b} x\right.$

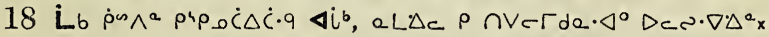

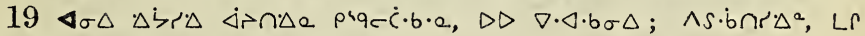
LL $\Delta \Delta) \cdot \Delta^{a}, \Delta \sigma r \cdot \Delta^{a}, \wedge s \cdot b \cup \sim(\lrcorner \Delta^{a}$,

$20 \nabla a \cdot \Delta \rho^{a}\left(\cdot \Delta b \sigma \Delta^{c}(\sigma \sigma) b^{a}\right.$, di $\left.\wedge r q \Delta^{a},<\cdot b s \cdot \nabla \cdot \Delta^{a}, \rho b r\right) \cdot \Delta^{a}, b \cdot q \sigma(\lrcorner \Delta e$,

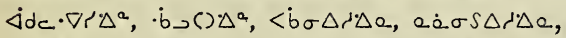

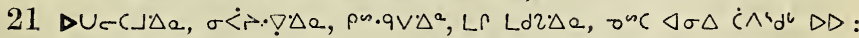

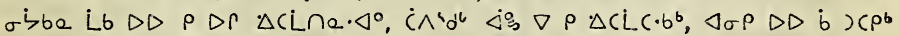

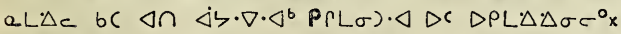

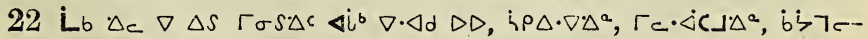

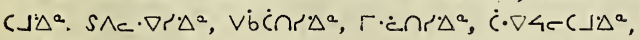

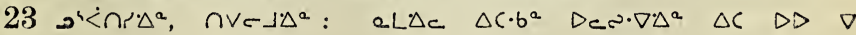
P'́Ci $\sigma \cdot \triangleleft P_{x}$

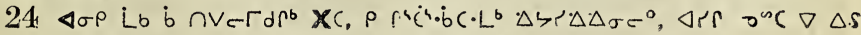

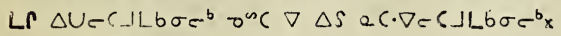




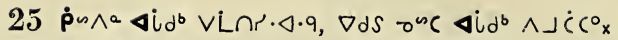

$\left.26 \nabla d S \nabla \dot{b} \Delta c a\left(\cdot \nabla-\dot{C}\left(c^{\circ}<\cdot b^{a} c^{\circ} L L \dot{C} d \Gamma d r^{\circ} \Delta^{a}, \nabla \rho S \cdot \Delta \Delta\right)\right)^{b}, \nabla \Delta U C r\right) b^{b x}$

\section{$\Lambda^{4} p C r a \Delta b^{a} 6$.}

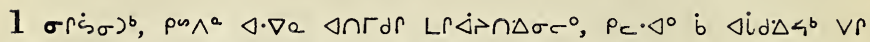

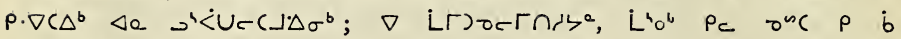
$b \cdot q r \Delta b \cdot \Delta^{a} x$

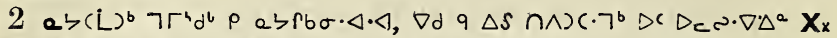

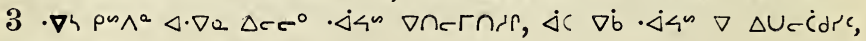

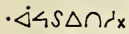

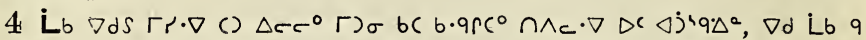

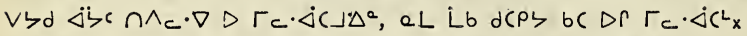

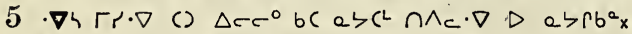

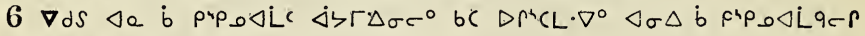

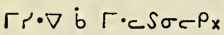

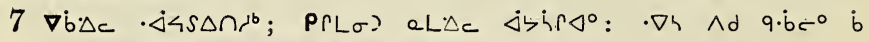

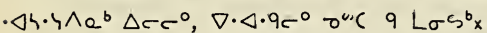

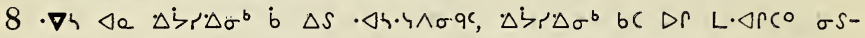

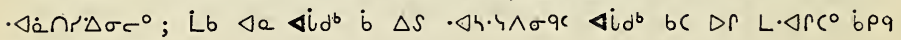
$\wedge \mathrm{L} \cap \ln ^{\prime} \Delta \sigma r^{\circ} \mathrm{x}$

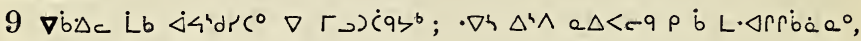
$\nabla \dot{b} . \cup \cup \sigma \dot{\varphi} 9 x$

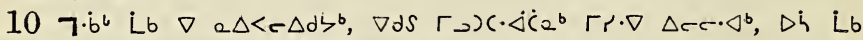
$\triangleleft \sigma \rho \dot{b}\left\langle S C \rho L b \sigma \Delta \Gamma^{b} \triangleleft \sigma \Delta \dot{b} \dot{C} \cdot V 4-C \Gamma-\Gamma_{x}\right.$

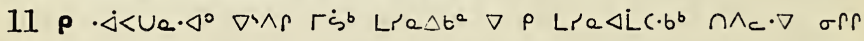
$\Delta \Gamma_{x}$

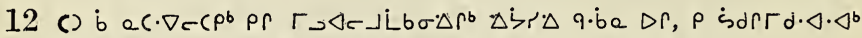

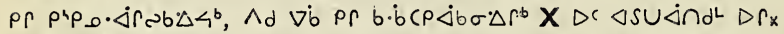

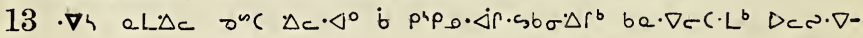

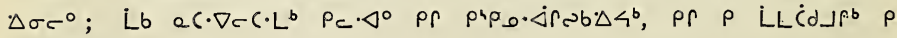
$\Delta b_{r} \cdot \Delta^{b} b_{x}$

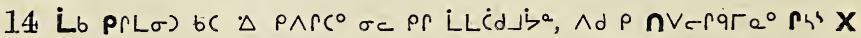

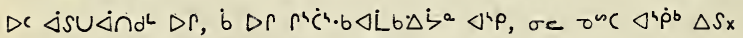

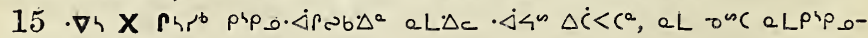

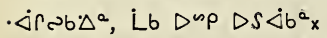

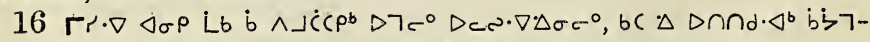

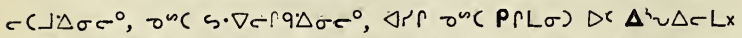

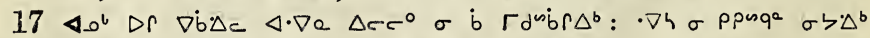

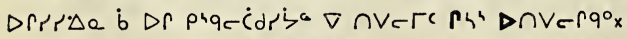

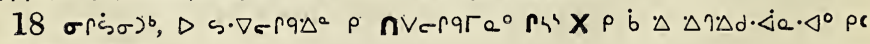
$\Delta i d \cdot \triangleleft b^{b} \quad \nabla 7^{2} x$ 


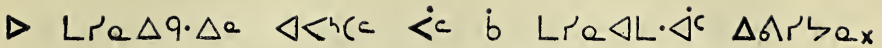

\section{Aspcra $\triangle b^{a} 1$.}

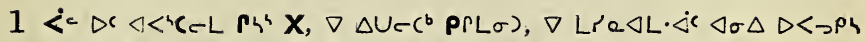
b $\Delta C e r \Delta \Delta 4 r^{b}$, onc $\Delta \sigma \Delta \dot{b}$ bb'pner $X$ phrb:

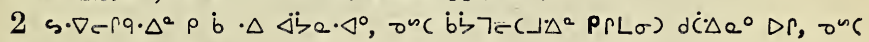

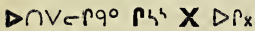

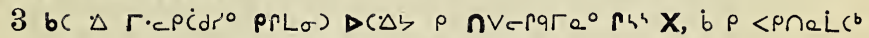
$[r \cdot \nabla \Delta i d \Delta s \cdot \nabla e \dot{C} d r \cdot \Delta e \text { prpsb. } 9 \cdot \dot{b} \text { e } \Delta S \mathbf{X})^{b}$ :

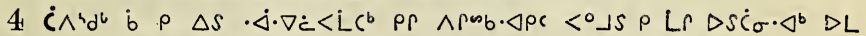

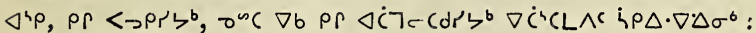

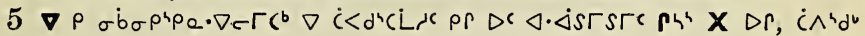
$\nabla \Delta S \Gamma_{2} \Delta U e^{b}$,

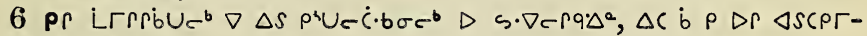
$b \Delta b^{b} r \Delta \rho \dot{\Delta b \sigma \Delta c:}$

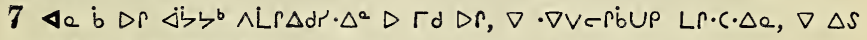
$\cdot \nabla \rightarrow \sigma^{2} c^{b} D s \cdot \nabla e r q \cdot \Delta^{a}$;

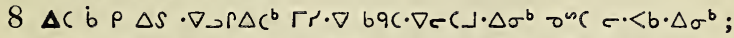

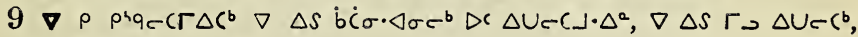
$\Delta c a r \cdot \dot{\beta}^{b}$ b $\rho \Delta U c^{b}$;

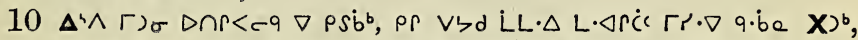

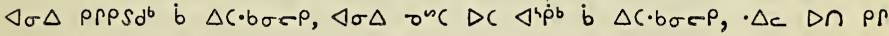
Apribde:

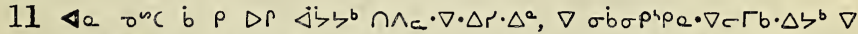

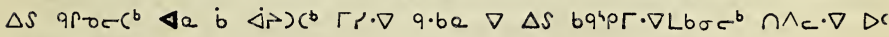
$\triangle U \sim C \cdot \cdot \Delta^{a}:$

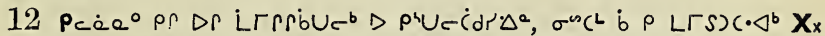

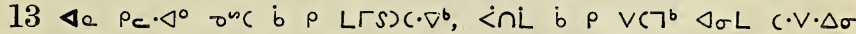

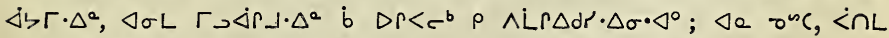

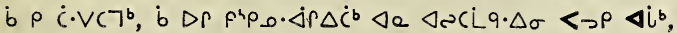

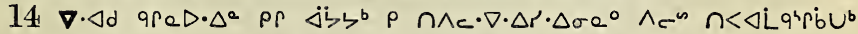

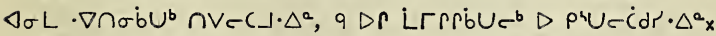

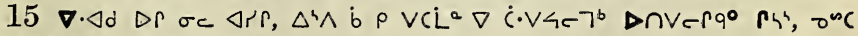
$\nabla \triangle S$ i $\beta \nabla \cdot b^{b} \Gamma r \cdot \nabla D<>\rho h^{b}$,

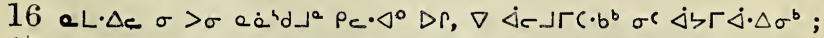

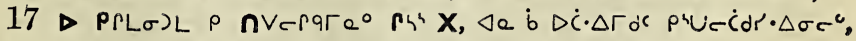

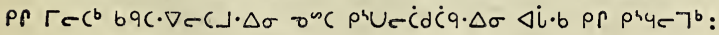




\section{Ab:ל 1, 2.}

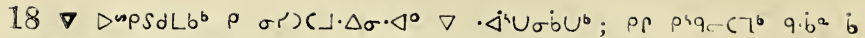

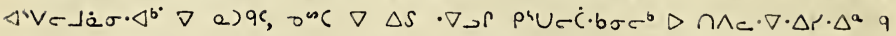

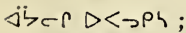

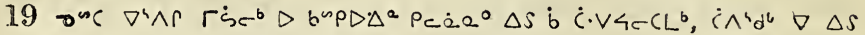

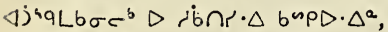

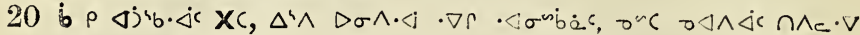
$\triangle P \Gamma \sigma^{4} \dot{\rho}^{b} \rho \Gamma \rho S d^{b}$,

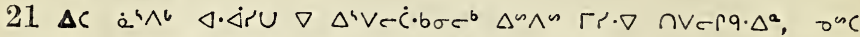

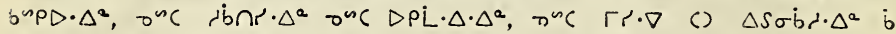

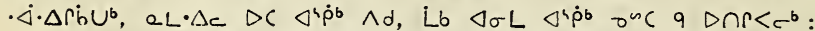

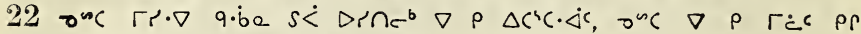

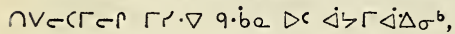

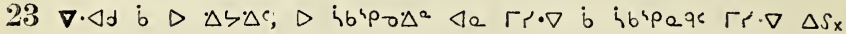

\section{Appre $\triangle b^{a} 2$.}

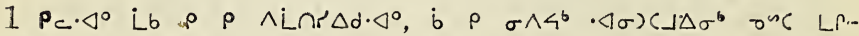
$\cdot\left(\Delta \sigma^{b}\right.$

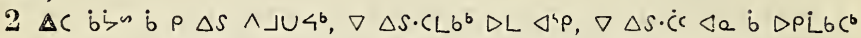

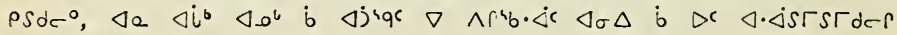
$S \wedge C\lrcorner \Delta \sigma C^{\circ}:$

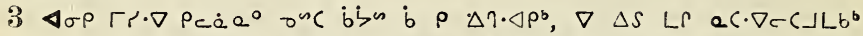

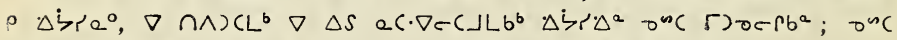

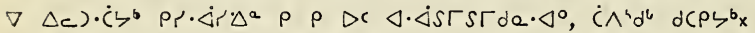

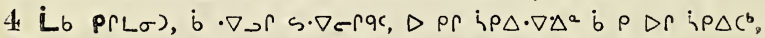

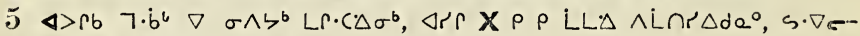

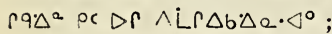

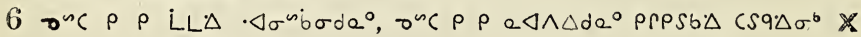
ant? :

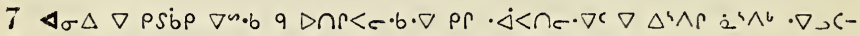

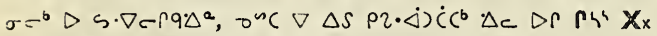

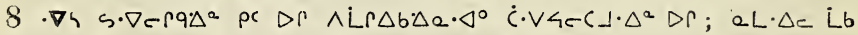
$\left.\rho_{C} \cdot \Delta^{0} \cap \wedge_{C} \cdot \nabla \Delta r ; \nabla \cdot \Delta d P r L \sigma\right) \nabla \Gamma c \cdot \nabla \cdot \Delta^{a}:$

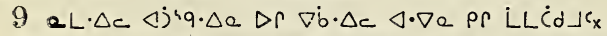

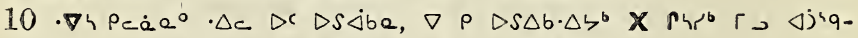
- $\left.\Delta \sigma^{b} \Delta S, \Delta \sigma \Delta P \rho L \sigma\right)$ b $P$ DeP(b $\left.P \rho \wedge\right\lrcorner \dot{C}\left(L^{b} x\right.$

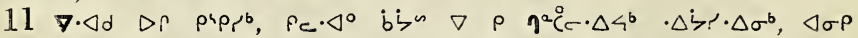

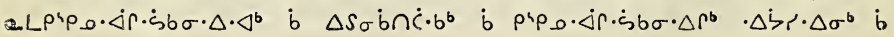

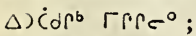

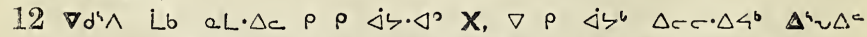

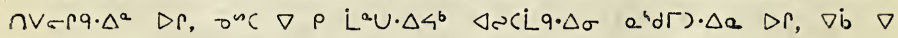

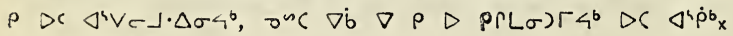




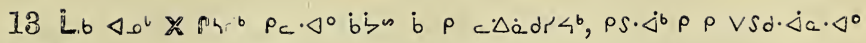
$X \triangleright \Gamma d L^{x}$

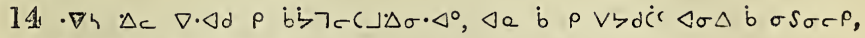
onc b $\rho \sigma \rho \cdot \nabla<\Delta^{b} \wedge$ is $\rho \sigma \sigma \sigma \sigma c^{\circ}$;

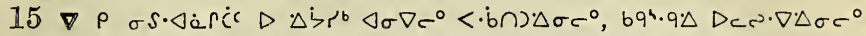

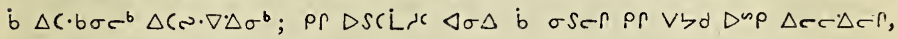
$\nabla d S \nabla$ bi $>$ Cec $\lrcorner \Delta \sigma q c ;$

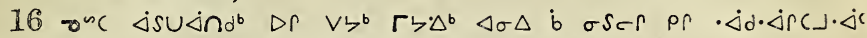
$P(L \sigma) \cdot \triangleleft, \nabla d\left(\nabla P D S \sigma<i\left(c \Delta \sigma \nabla \Gamma^{\circ}<\cdot \dot{b} \cap\right) \Delta \sigma \sigma^{\circ}\right.$;

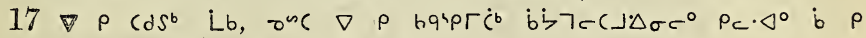

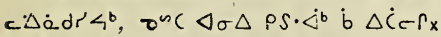

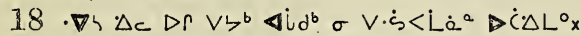

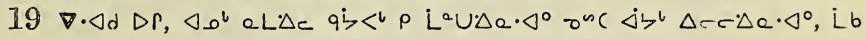

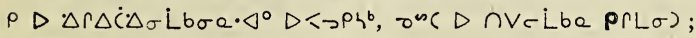

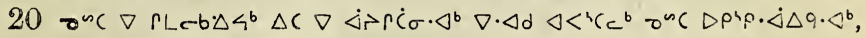

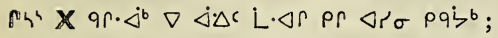

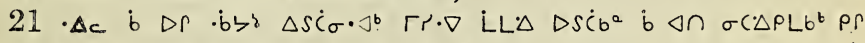

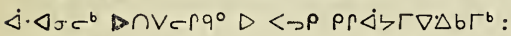

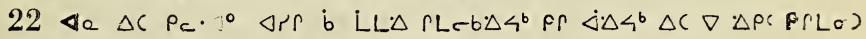
$\Delta c \Delta i^{b} \Delta r_{x}$

\section{$\Lambda^{3} p c r a \triangle b^{a} 3$.}

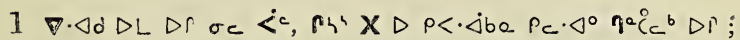

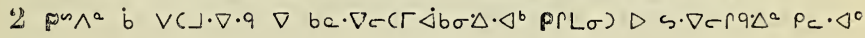
Dr:

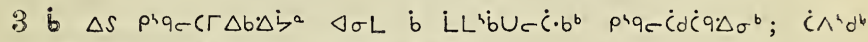

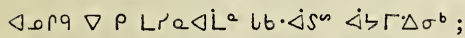

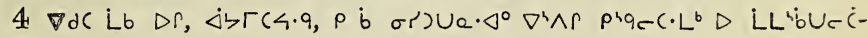
dr. $\Delta^{a} X$

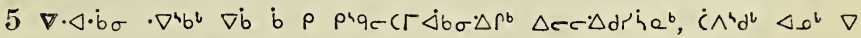

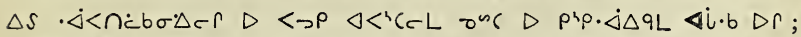

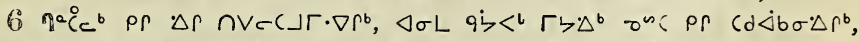

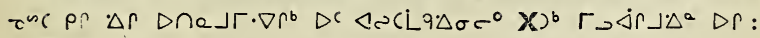

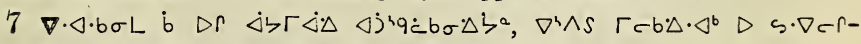

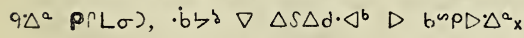

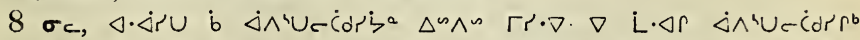

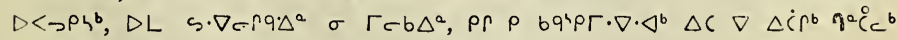

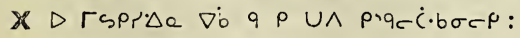

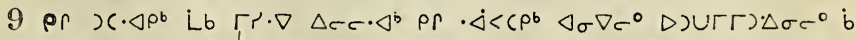

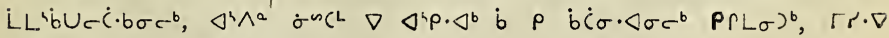
q.be $b$ P DScich' X Dr:

\section{7}




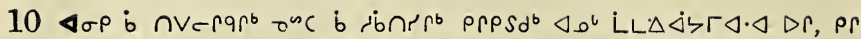

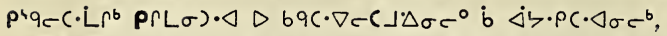

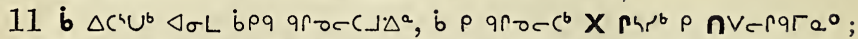

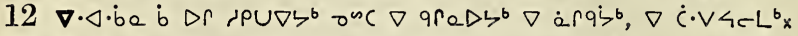

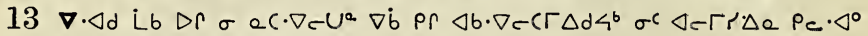
b $\Delta r \Delta \cap \cap d^{b} \dot{b}^{a}, \dot{b} \Delta^{4} V-\dot{c} d \Delta d \zeta^{b} x$

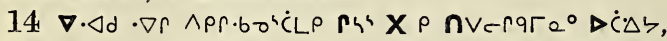

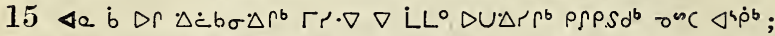

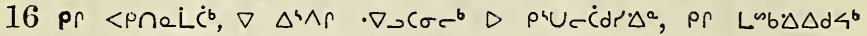

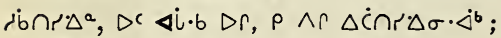

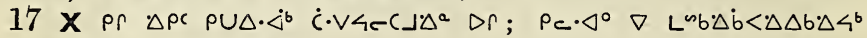

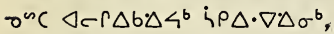

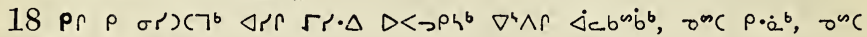
$\rho^{4}<b^{b}, \partial^{n C} \Delta^{n} \dot{<}^{b} ;$

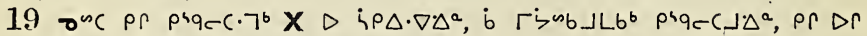

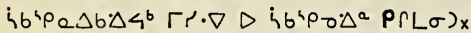

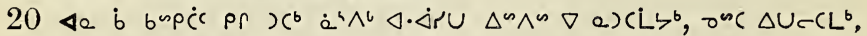

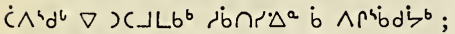

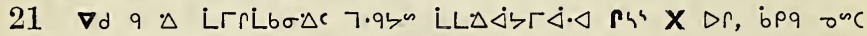
bpq, $\triangleleft^{4} \dot{p}^{b} \nabla \dot{b} \quad \Delta^{b} \dot{b} c q \Delta^{c} \cdot \dot{b}<c^{b} x \quad \nabla 7^{a} x$

$\Lambda^{r} p\left(r a \Delta b^{a} 4\right.$.

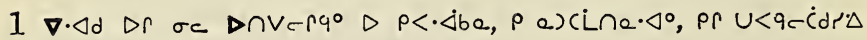
$\wedge\lrcorner U\left\langle^{b} \triangleleft \sigma L\right.$ a) $\Gamma^{d} d r^{\prime} \Delta \sigma^{b}$ b $\Delta S$ a) $\Gamma b \Delta<^{b}$;

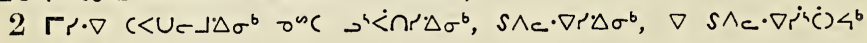
i $P \triangle \cdot \nabla \cdot \Delta \sigma^{b}$;

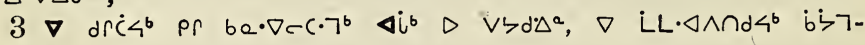
$-C\lrcorner \Delta^{a} x$

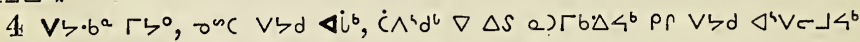
$\rho$ e) $\Gamma \mathrm{d} r \cdot \Delta \sigma \cdot \dot{\Delta}^{b}$;

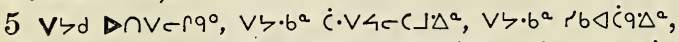

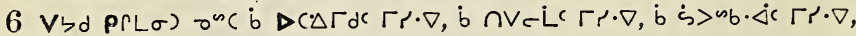

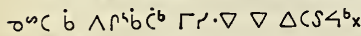

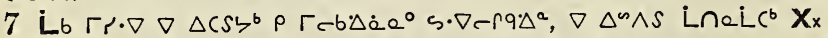

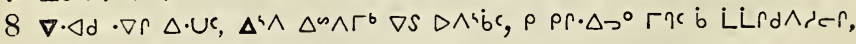
J"C $\rho \Gamma \sim^{\circ} \Delta c c \cdot \triangleleft \Gamma c \cdot \nabla \cdot \Delta e_{x}$

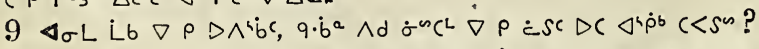

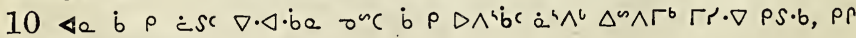

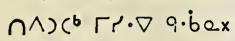

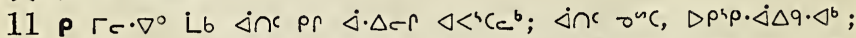

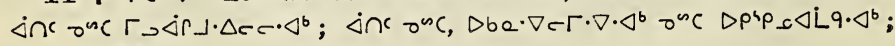
318 
ASThO 4 .

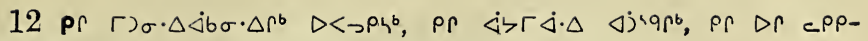
$\sigma c^{b} X \Delta \iota^{\circ}$ :

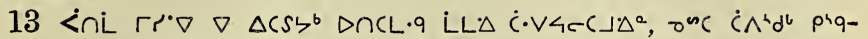

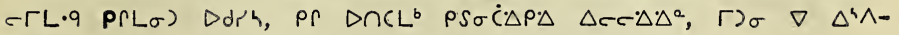
nPกC X:

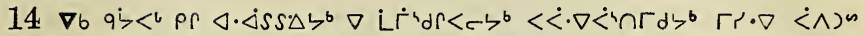

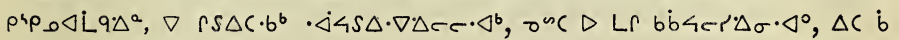
$D r \Delta \cdot \dot{s} \Delta q^{b}$ pr $\cdot \dot{\varangle}\left\langle s \Delta \cdot \nabla r^{b}\right.$;

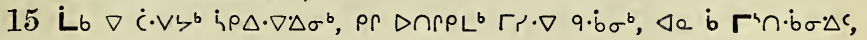
$x \triangleright \cap:$

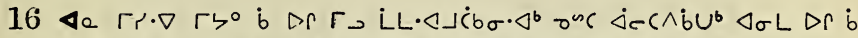

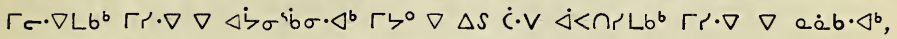

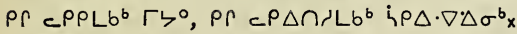

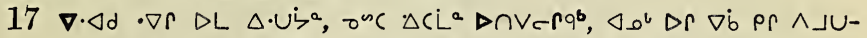

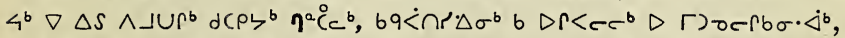

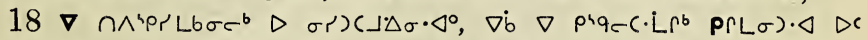

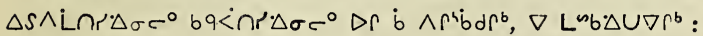

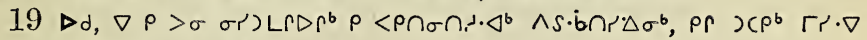
$\left.\Delta \sigma r \Delta \sigma \sigma^{0} \nabla \Delta r \cdot \zeta c\right\lrcorner r^{b} x_{x}$

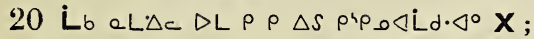

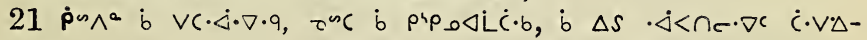
$\sigma c^{\circ} p^{4}$ :

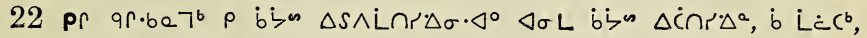
$\dot{C} \wedge^{4} d^{l} \nabla \Delta S \cdot \triangleleft\langle S \Delta \cdot \nabla L b P$ LS QC $\nabla C \cdot C\lrcorner \Delta Q$;

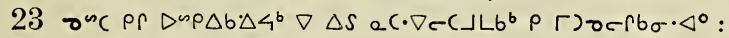

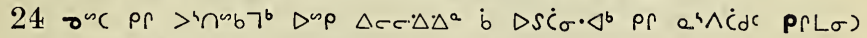
bל

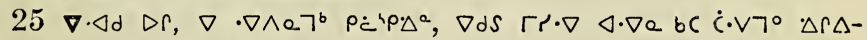
$\tau \cdot \Delta: \cdot \nabla h \rho$ Vלó $U \Delta r \dot{a} 0^{\circ} x$

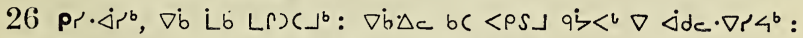

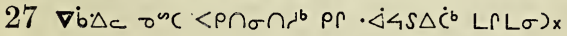

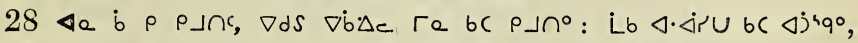

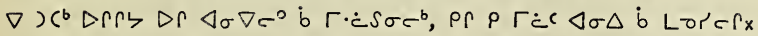

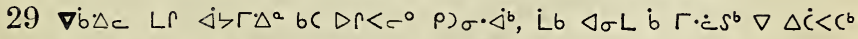

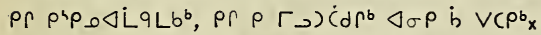

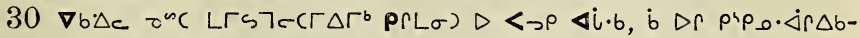

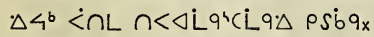

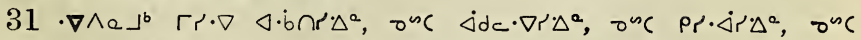

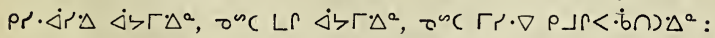

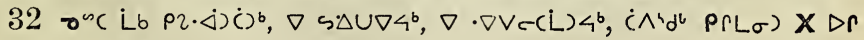
$\nabla P \cdot \nabla V e C L \dot{C}^{b}{ }_{x}$ 


\section{$A^{4} p C r a \triangle b^{a} 5$.}

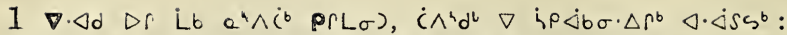

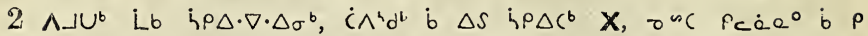

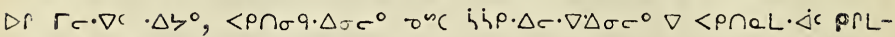
$\sigma) \cdot \triangleleft,\left\langle P \cap \sigma q \cdot \Delta \tau c^{\circ} \dot{b} \cdot \Delta P L \cdot b \sigma c^{b} x\right.$

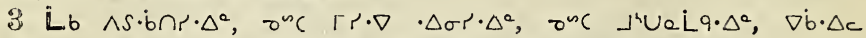

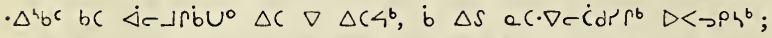

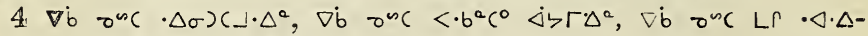

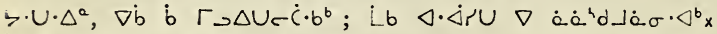

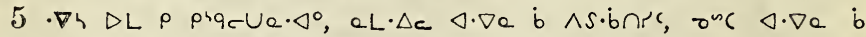

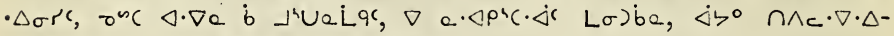
$r \cdot \Delta^{a}(L \sigma) \sigma^{n} C \times \Delta C D P L \cdot \Delta \cdot \Delta \sigma^{b} \times$

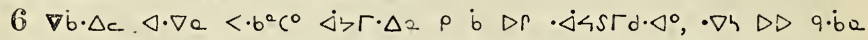

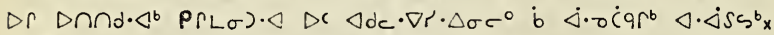

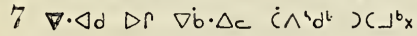

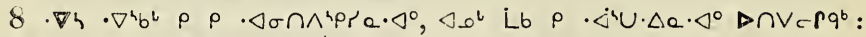

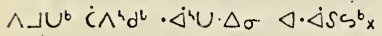

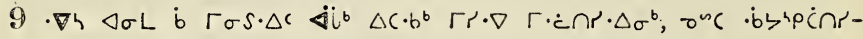
$\cdot \Delta \sigma^{b}$, onc $\dot{c} \cdot \nabla \cdot \Delta \sigma^{b}$;

$10 \nabla$ or placc $7^{b} \quad \varangle \sigma L$ b $a \Delta c \cdot \nabla \Delta d^{c} \Delta \cap V e r q^{\circ} x$

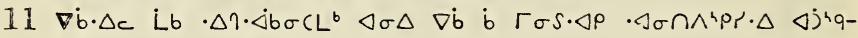
$\cdot \Delta a$, Lb $\Delta \cdot \Delta r U P)(\lrcorner^{b} x$

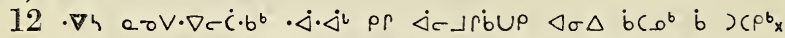

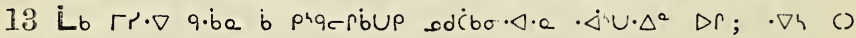
$a \cdot b^{a} \dot{b}$ odcं $q L b^{b} \cdot \dot{j} U \cdot \Delta \sigma \cdot \Delta^{a} *$

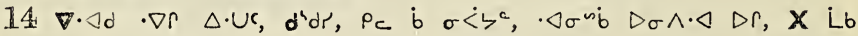
$\rho$ b $\Gamma c^{b} \cdot \dot{\gamma} U \cdot \Delta \sigma c^{\circ} x$

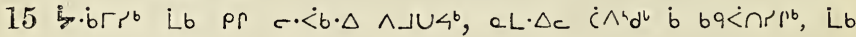
$\dot{c} \wedge$ म db bे $69 c \cdot \nabla c c p b$,

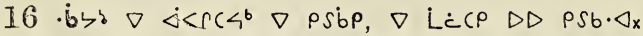

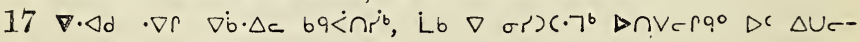
(\lrcorner$\cdot \Delta^{a} \times$

$18 \nabla \dot{b} \cdot \Delta c$ onc ju.qVb $\omega \Gamma \dot{a}>\Delta r, \nabla \cdot \Delta d \Delta \dot{h} \Gamma \Delta r \cdot \Delta^{a}:$ Lb $\rho \dot{b} \cdot \Delta$

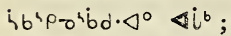

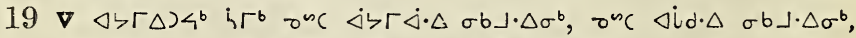

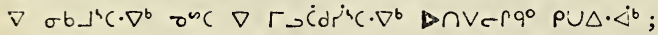

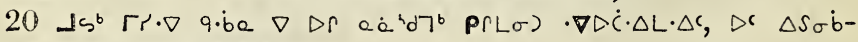

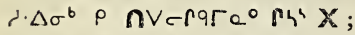

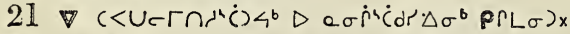

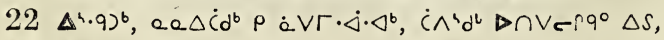

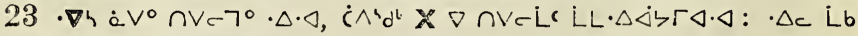

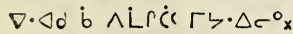




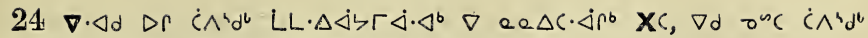
$\Delta^{4} \cdot 9 \cdot \Delta^{b}$ bc ee $\Delta C \cdot \nabla \cdot \Delta^{b} \triangleright \dot{a} \vee \Gamma \cdot \triangleleft \cdot \triangleleft \Gamma r \cdot \nabla \Delta S_{x}$

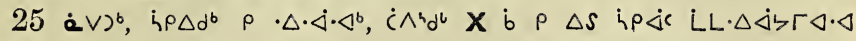

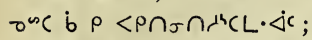

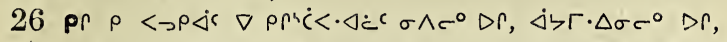

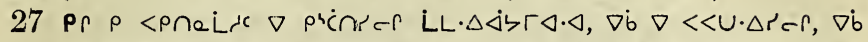

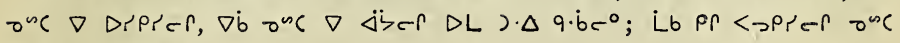
Di be Listert

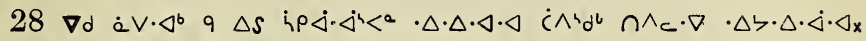

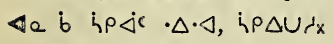

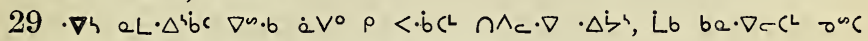

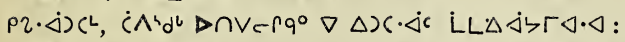

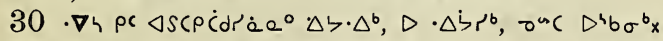

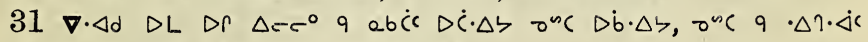

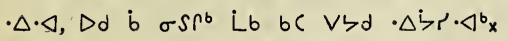

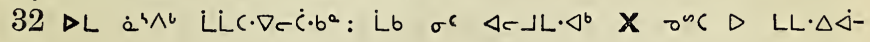
$\succ \Gamma \triangleleft \dot{L x}$

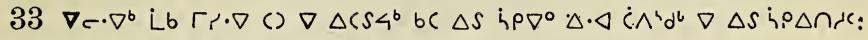

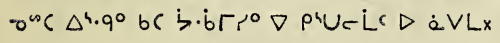

Ápcre $\Delta b^{2} 6$.

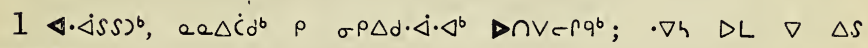
. bל '. b6x

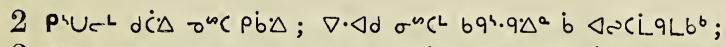

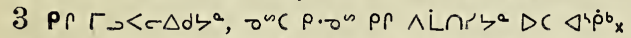

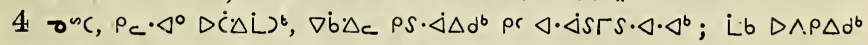

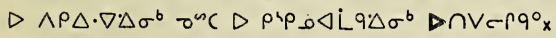

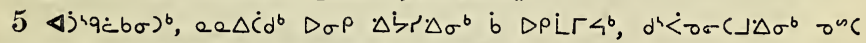

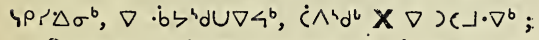

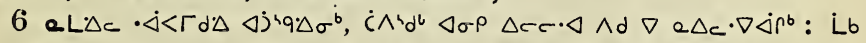

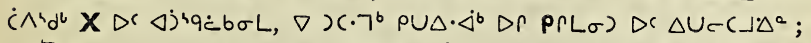

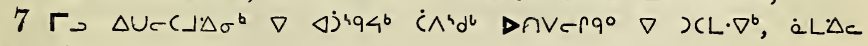
$\triangle c c \cdot \Delta^{b} \mathrm{x}$

$8 \nabla p^{b} q-c 7^{b} \wedge d q \cdot b^{a} \nabla \Gamma \cdot c \cdot s^{b} \triangleleft \cdot \nabla e \Delta c c^{0} \nabla x c^{b}, \nabla \cdot \triangleleft \cdot q_{c} c^{0} q \Gamma c d s$

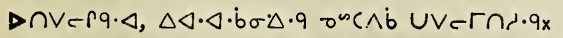

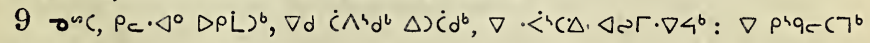

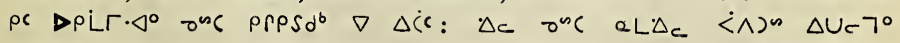
$\triangle r c \cdot \Delta x$

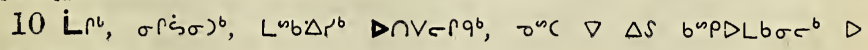
Jibne. $\Delta^{a} \times$ 
ArAbe 1.

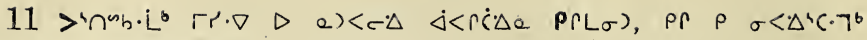
$L(L \sigma) D \cdot \dot{j}\left\langle S \Delta \cdot \nabla \Delta e^{2}\right.$

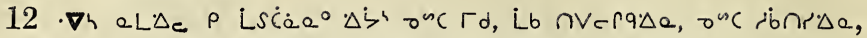

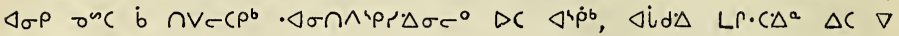
$\Delta \mathrm{V} V \subset \dot{C} \cdot b^{b} \mathrm{x}$

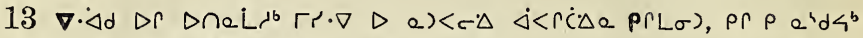

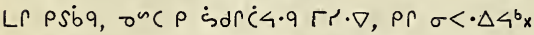

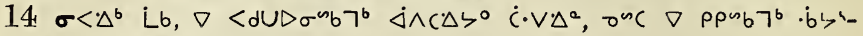

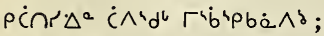

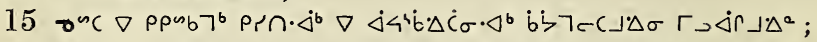

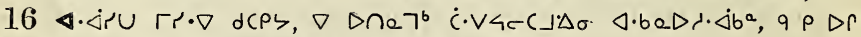

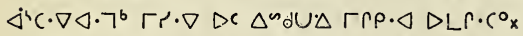

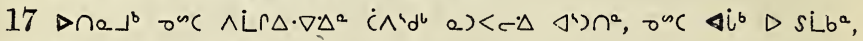
$\nabla \cdot \Delta d P(L \sigma) \triangleright C \quad \dot{\Delta} \zeta \Gamma \Delta^{a}:$

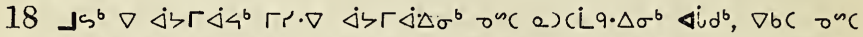

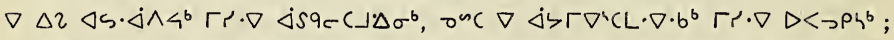

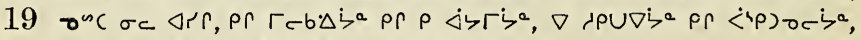

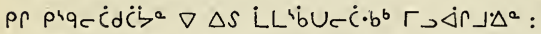

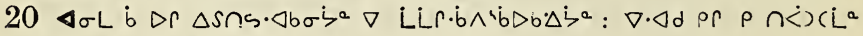

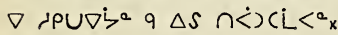

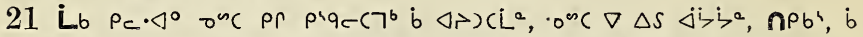

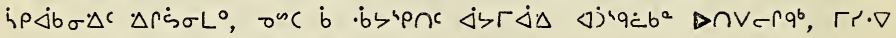
q. be $\rho$ b plqcec $\triangle \Delta d \cdot \triangleleft^{\circ}$ :

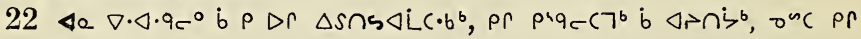
bPr $\triangle \dot{C}^{b}$ p $\triangle \triangle \cdot \dot{\Delta b}^{b} x$

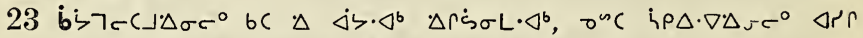

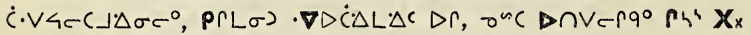

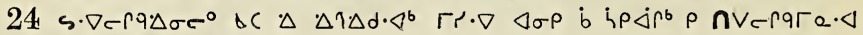

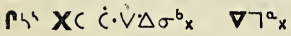

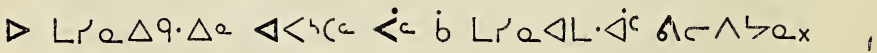

$\Lambda^{\prime} p\left(r e \triangle b^{a} 1\right.$.

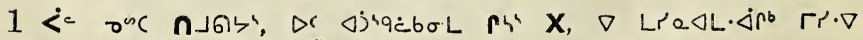

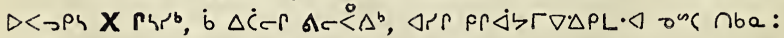

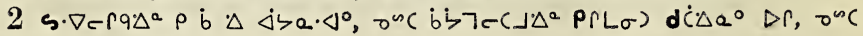
$\Delta \cap V=r q^{\circ} P_{4} \cdot X_{x}$

322 


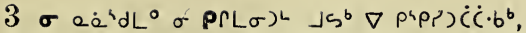

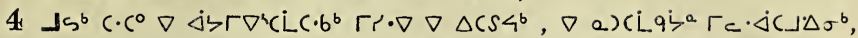

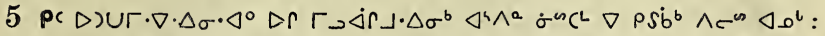

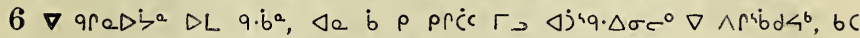

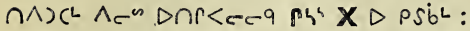

$7 \boldsymbol{\nabla} \cdot \dot{b}^{b} \cdot b^{b} D L$ Pf $\Delta S \Delta U C \Gamma\left(\cdot b^{b} \Gamma r \cdot \nabla \nabla \Delta\right) S 4^{b}, \nabla \Delta \zeta \Delta C \cdot b^{b} \sigma U \Delta^{b}$;

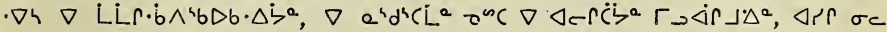
b $\rho$ L L

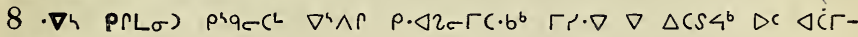
$\checkmark \triangle^{\mathrm{b}} \mathrm{PL}^{4} \mathrm{XX}$

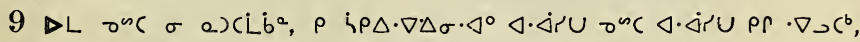

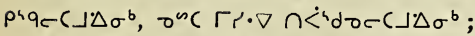

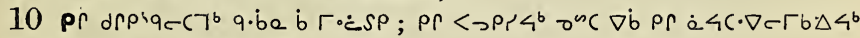
$\wedge c^{n} D \cap R<c c q D P S b^{2} \mathbf{X}$;

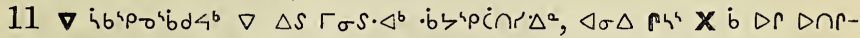

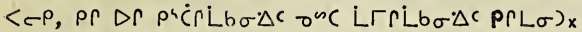

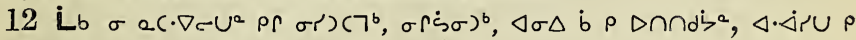

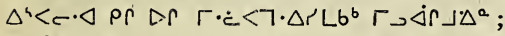

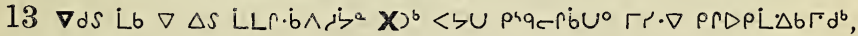

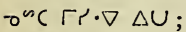

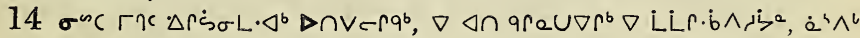

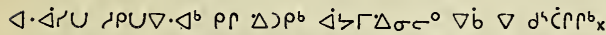

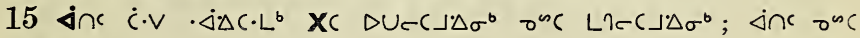
$\Gamma \cdot s e c\lrcorner \Delta \sigma^{b}$ :

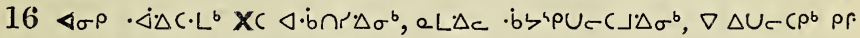

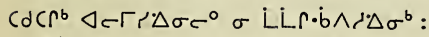

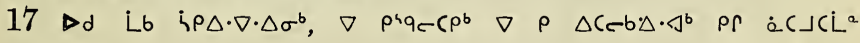
$\Gamma \rightarrow \Delta r\lrcorner \Delta \Delta^{2} x$

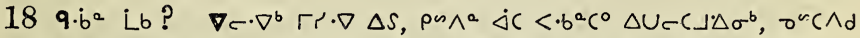

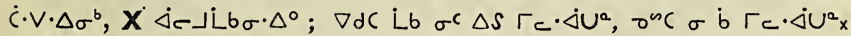

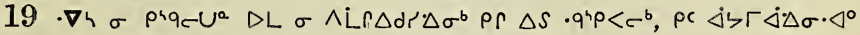

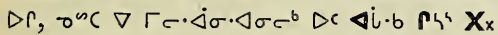

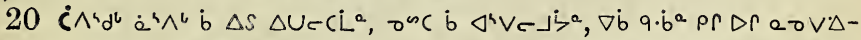

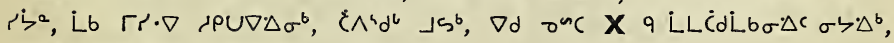

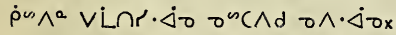

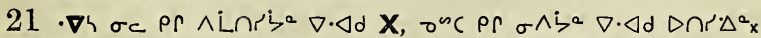

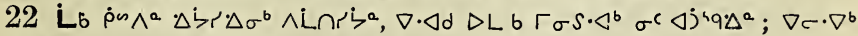

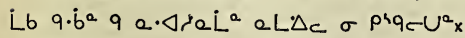

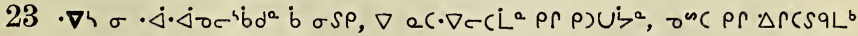

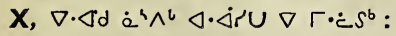

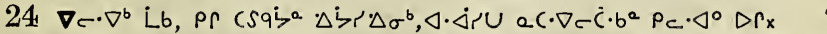

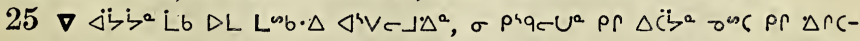

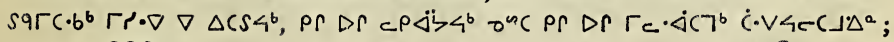




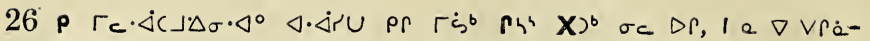
$n \dot{c} \cdot b^{b}{ }^{b}$

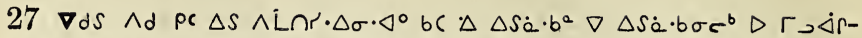

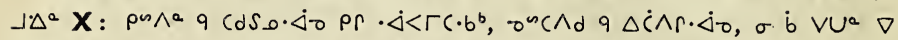

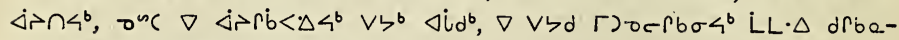

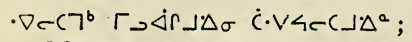

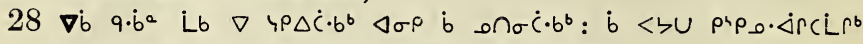

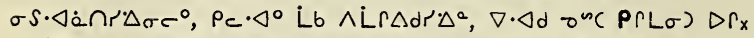

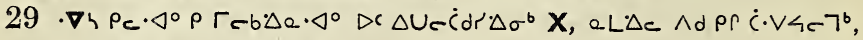
Lb $\triangleleft$ ? $\triangle$ r $\Delta r$ pr $\triangle 49 c C 7^{\circ}$;

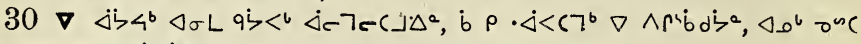
b $\left.\vee C 7^{b} \nabla \wedge r^{4} b d^{\circ}\right)^{\circ} x$

\section{Aipcra $\triangle b^{2} 2$,}

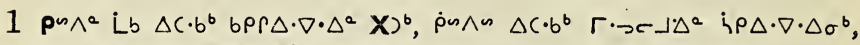

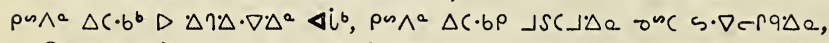

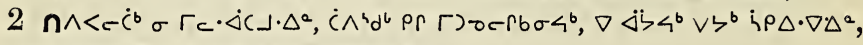

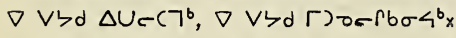

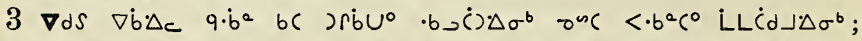

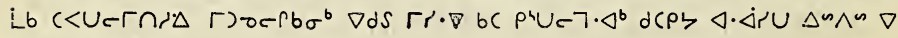
pruernibstax

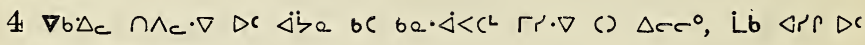
$\ddot{\Delta i} \sigma c \cdot \triangleleft d C \rho\rangle \triangleleft \cdot \nabla c \cdot \triangleleft x$

$5 \nabla d S D L \Gamma$ ) or $r b^{a} \rho$ b $\Delta \wedge r^{4} b d a \cdot \Delta^{\circ}, \dot{b} \rho \wedge r^{4} b d s$ onc $\mathbf{X} r^{4}$;

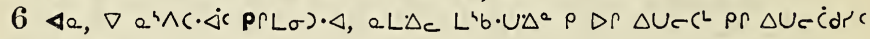
$\left.\dot{C} \wedge^{4} d^{l} P R L \sigma\right) \cdot \Delta$;

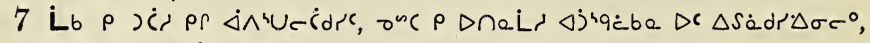

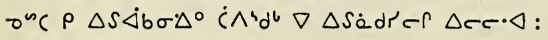

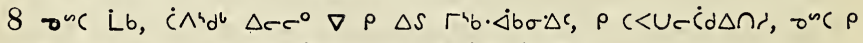

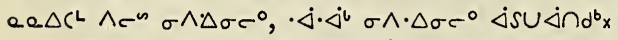

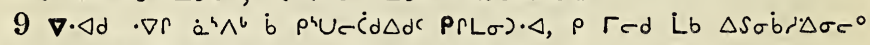

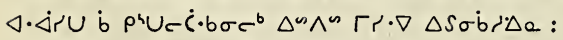

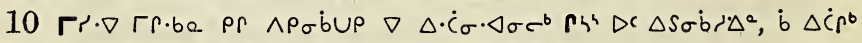

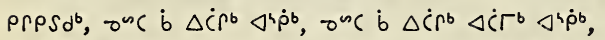

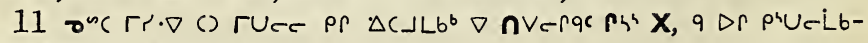
$\sigma \Delta(P \Gamma L \sigma) \cdot \nabla D C \dot{C} \Delta \Delta \Delta C_{x}$

$12 \nabla \cdot \Delta d \quad D r, r\left\langle\rho \Delta C \cdot b^{b}, \quad i \wedge^{b} d^{b} J s^{b}\right.$ b $\rho$ ea $\Delta C 7^{b}, a L \Delta c \wedge d \nabla$

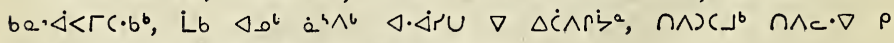

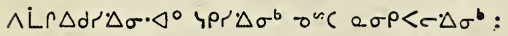

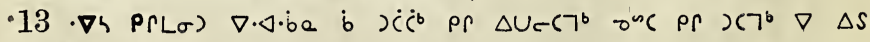
$r \cdot x+c_{b}:$

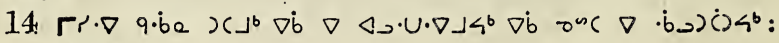
324 


\section{ヘヘヘ๖৫ 2,3 .}

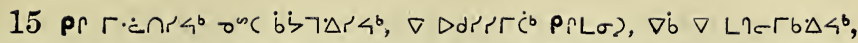

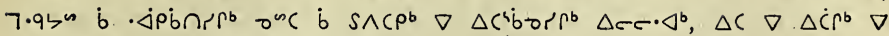

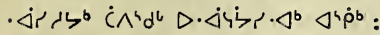

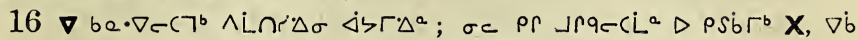
$<\cdot b^{a} c^{\circ} \nabla \rho \wedge \Gamma<\dot{C}<b, \nabla \dot{b} \sigma^{n c}<<\cdot b^{a} c^{\circ} \nabla \rho \triangleleft \dot{j}^{i} \rho<^{b} x$

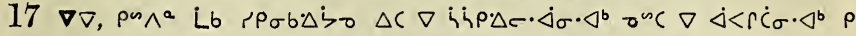

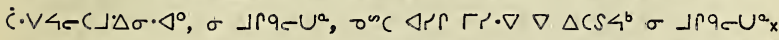

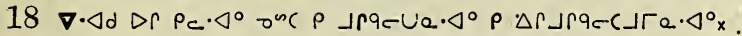

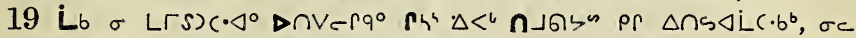

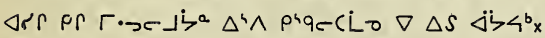

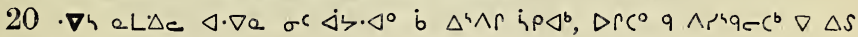
$\Delta ל \zeta^{b}:$

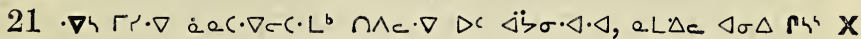

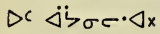

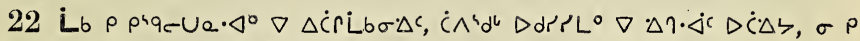
$\left.\Delta S \Delta r \Delta)^{4} a r^{b} r \Delta \Delta r\right\lrcorner \Delta \sigma^{b} \times$

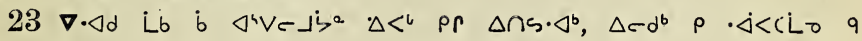
$\Delta^{4}<\epsilon \Delta d^{2} \dot{\zeta}^{2} x$

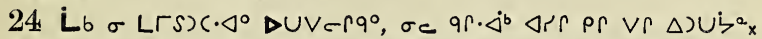

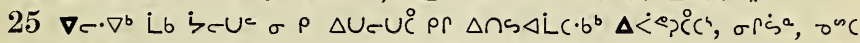

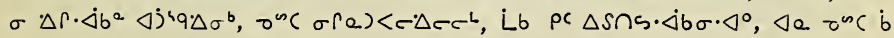
$\left.\rho D \mu^{\prime} C L q C \sigma \cdot P C\right\lrcorner \Delta Q_{x}$

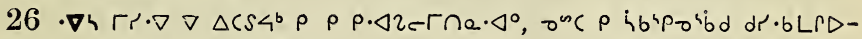

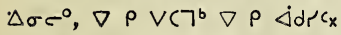

$27 \cdot \nabla h \dot{c} \cdot \vee \rho \quad \dot{\triangleleft} d \rho^{\circ} q \dot{b}\left(\sigma \wedge \Delta \sigma^{b} \Delta S:\right.$ Lb $\rho$ s. $\left.\nabla c \Gamma d \rho \rho L \sigma\right) \cdot \triangleleft:$ : Q L $\Delta c$.

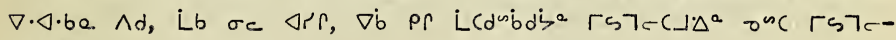
(\lrcorner$\Delta^{a} \mathrm{x}$

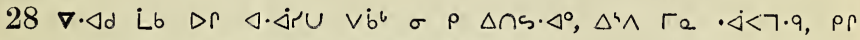

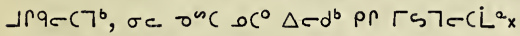

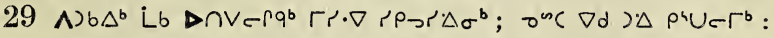

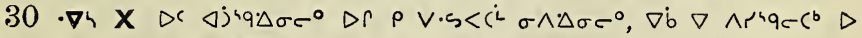

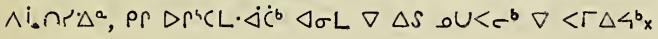

\section{$\Lambda^{4} p\left(p a \triangle b^{\circ} 3\right.$.}

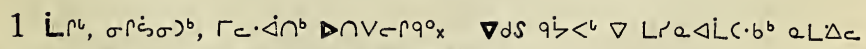
$\sigma c \Delta c \Gamma \Delta d^{2}$, Lb $P_{c} \cdot \triangleleft^{\circ}$ Dr $\wedge$ LrUecं. $b^{2} x$

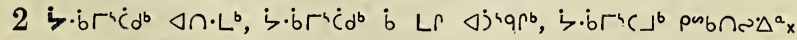

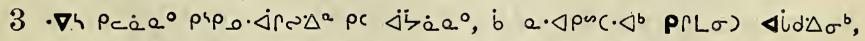

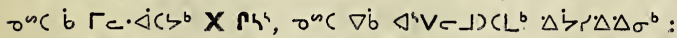

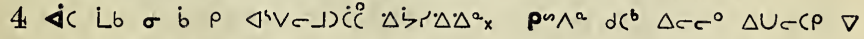

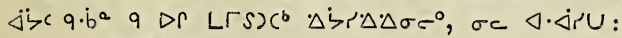

325 


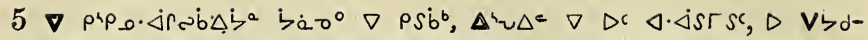

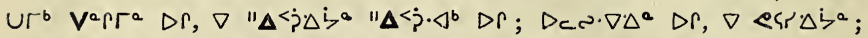

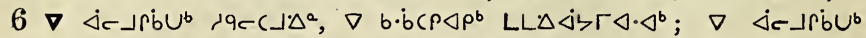

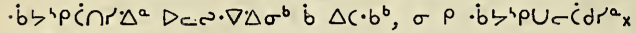

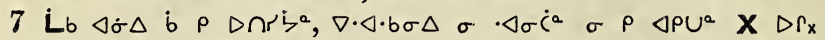

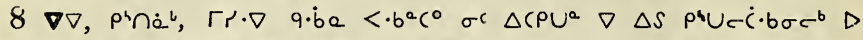

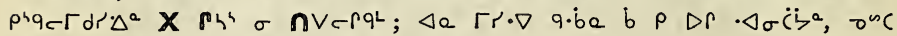

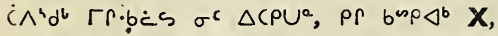

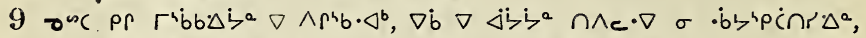

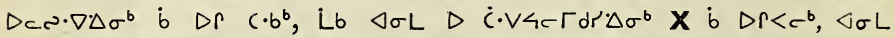

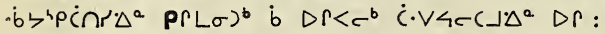

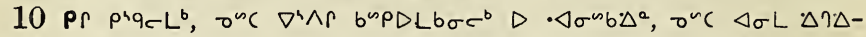

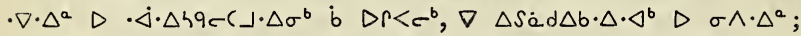

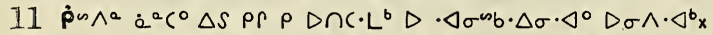

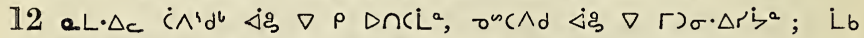

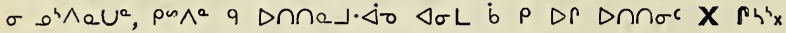

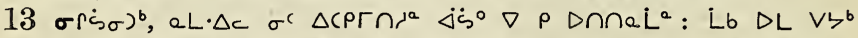
$\left.\left.q \cdot \dot{b}^{a} \sigma\right) U^{a} ; \nabla \cdot \Delta \sigma \rho^{h} \rho r\right)\left(L^{a} \triangleleft \sigma \Delta \Delta \dot{C}^{a}{ }^{b} b \Delta\left(\cdot b \rho, \sigma^{c}\left(\nabla b \cdot q r \cap a L^{a} \triangleleft \sigma \Delta\right.\right.\right.$ $\sigma \dot{b} \sigma^{b} \dot{b} \Delta c \cdot b \rho$,

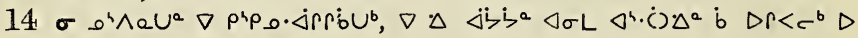

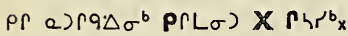

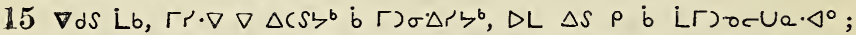

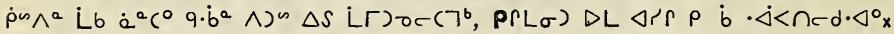

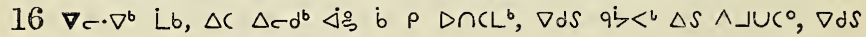
$\nabla \cdot \triangleleft d 9 \dot{\zeta}>\iota \dot{L} r) \operatorname{doc}\left({ }^{\circ} x\right.$

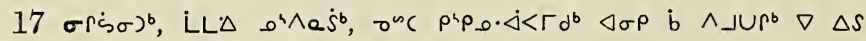

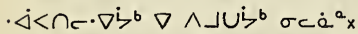

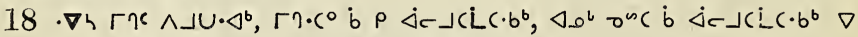

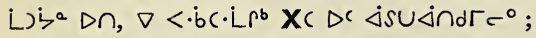

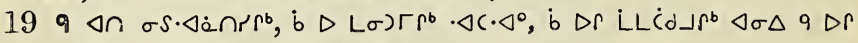

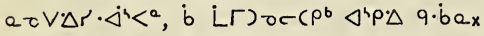

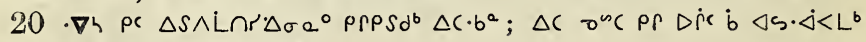

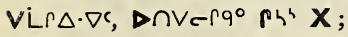

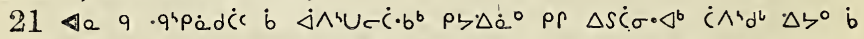

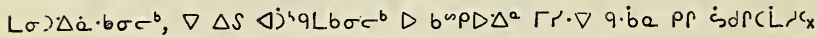

\section{$A^{2} p c r a \triangle b^{\circ} 4$}

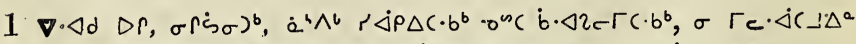

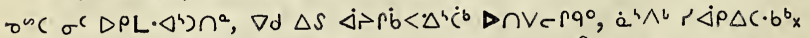

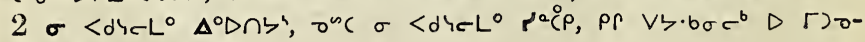

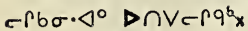




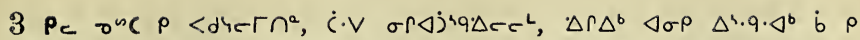

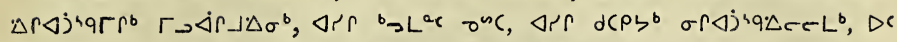

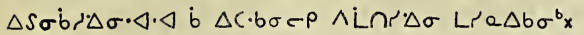

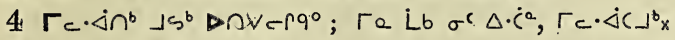

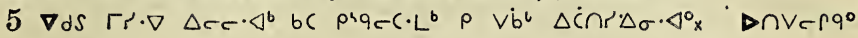
$\rho s \cdot<^{\circ} \Delta C^{\circ}{ }^{x}$

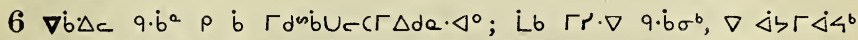

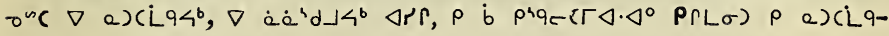
$\Delta \sigma \cdot \triangleleft \cdot \triangleleft$;

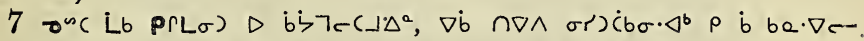

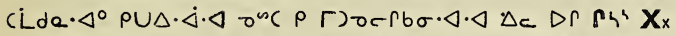

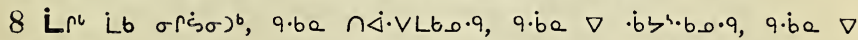

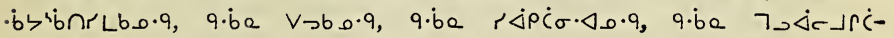

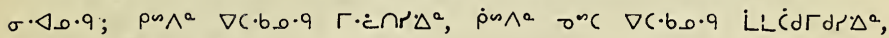

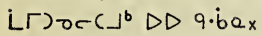

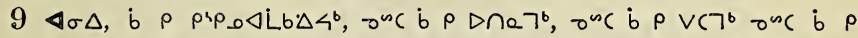

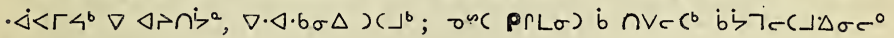
$\rho$ b $\Delta i i^{\circ} \Delta d \cdot \Delta^{\circ} x$

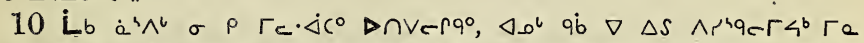

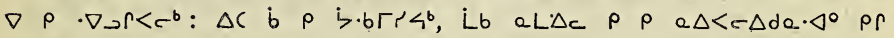
$x\urcorner^{b} \times$

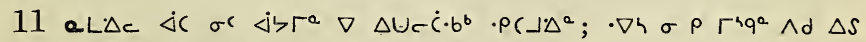
$\triangleleft \dot{\zeta} \zeta \sigma, \nabla d C$ PR UVCCL ${ }^{\circ} \times$

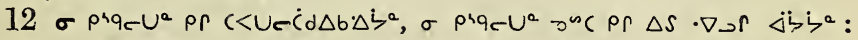

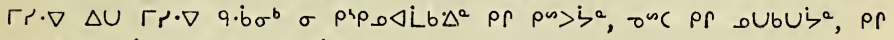

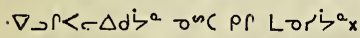

$13 \Gamma \cdot \nabla$ q.be $\sigma \dot{b} \rho) u^{a} \Delta_{c} D r X \dot{b}$ L"b. $\Delta r \Delta c_{x}$

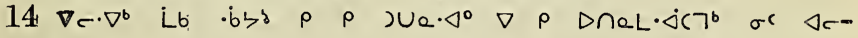
$\Gamma \cdot \Delta^{a} x$

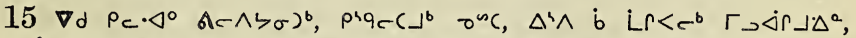

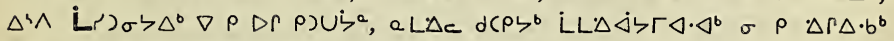

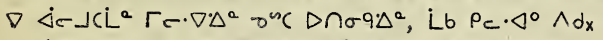

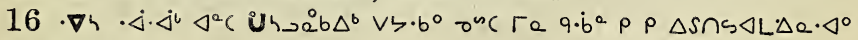

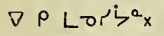

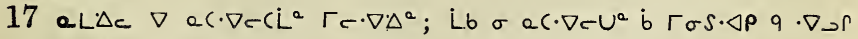
$\triangle C P C L b \triangle S^{b} \mathrm{x}$

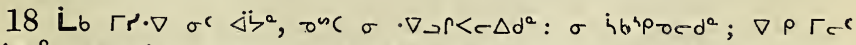

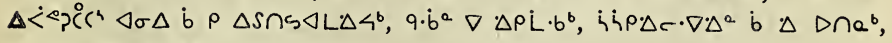
$\nabla$ e $\Delta c \cdot \nabla \Delta d(p(L \sigma) x$

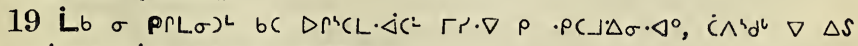

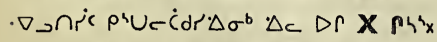

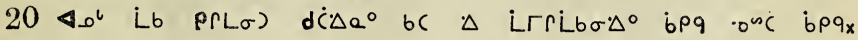
$\nabla 7^{2} \times$ 


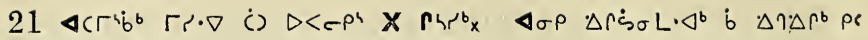
$\triangle c r^{-} b \dot{b} \cdot \cdot \triangleleft \cdot \Delta^{b} x$

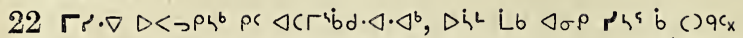

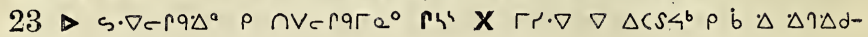
$\cdot \dot{\cdot a} \cdot \Delta 0^{\circ} \times \quad \nabla 7^{a} x$

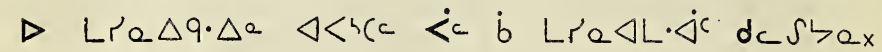

\section{nipcre $\triangle b_{i} 1$.}

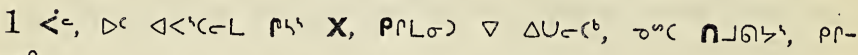
$\dot{s} \sigma 0^{\circ}$,

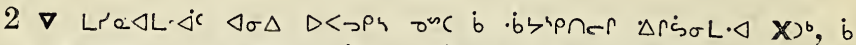

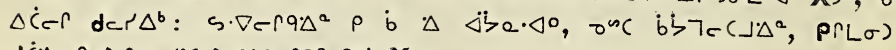

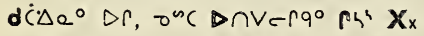

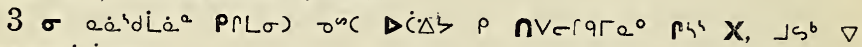
$\dot{\Delta} \succ \Gamma \nabla^{\top} C \dot{C} \dot{C} \cdot b^{b} x$

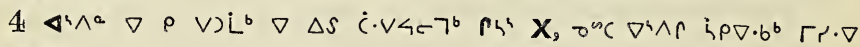
$D<$ TPL, ;

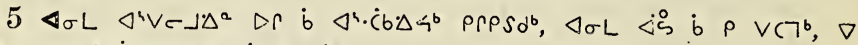
$\left.\triangle S \triangle \cdot \cup L b^{b} \dot{C} \cdot V \Delta^{a} \Gamma \Delta \Delta r\right\lrcorner \Delta \sigma^{b}$;

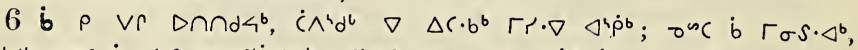

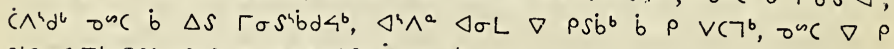

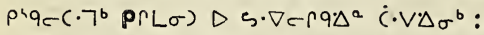

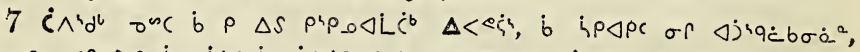

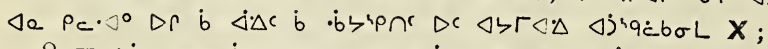

$8 \nabla \cdot \triangleleft \cdot \dot{b} a$ occ b f $\Delta C L \Delta>\Gamma c \rho$ h $\rho \Delta \cdot \nabla \Delta_{\sigma} \cdot \triangleleft^{\circ} \triangleleft \dot{i} d^{b} x$

$9 \Delta L \quad D C \sigma c a^{a} \nabla^{\omega C}, \Delta^{4} \Lambda^{a} \Delta \sigma L \nabla \rho S b^{b} \dot{b} \rho V C L^{b}, a L \Delta c \sigma$

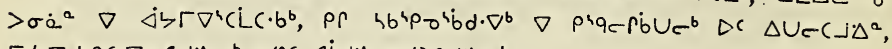

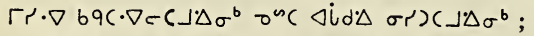

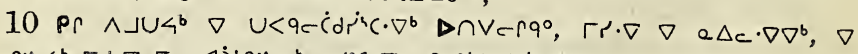

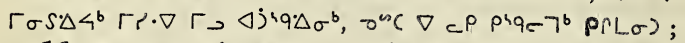

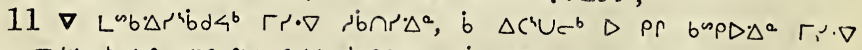

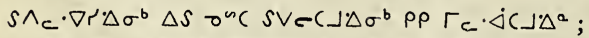

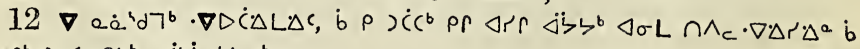

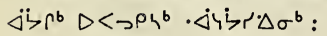




\section{dcsbe $1,2$.}

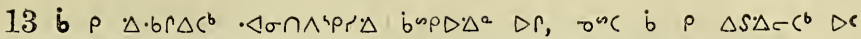

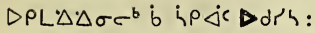

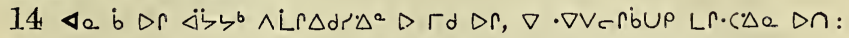

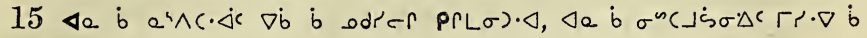
$\Delta S \triangle \Gamma c$ :

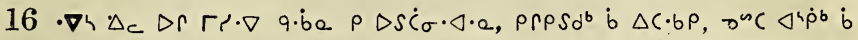

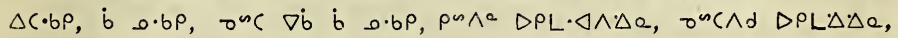

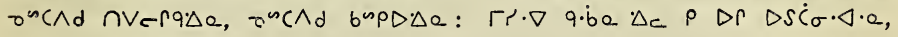

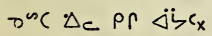

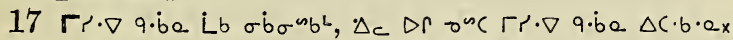

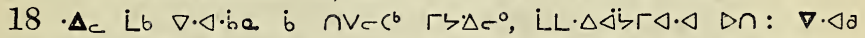

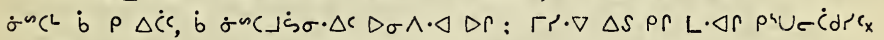

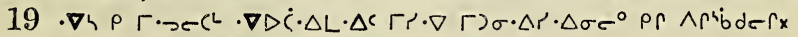

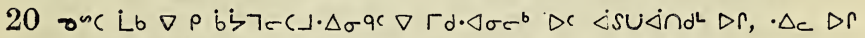

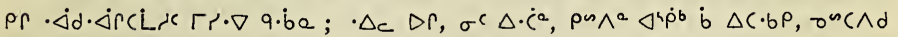
Pr:PSd b $\triangle C \cdot b \rho_{x}$

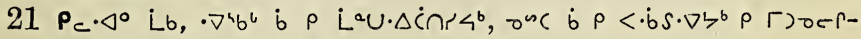

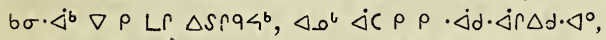

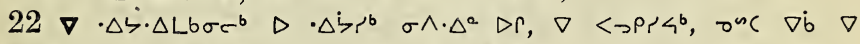

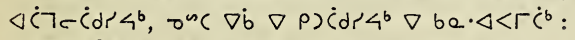

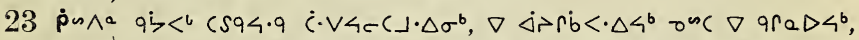

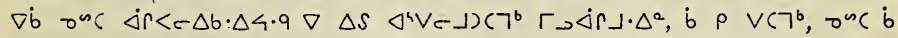
$\rho \cdot \Delta C L \cdot \Delta b \sigma \cdot \Delta c \Gamma r \cdot \nabla \quad \Delta \cdot \nabla e s \dot{<} \rho \rho \rho S d^{b} \dot{b} \Delta \dot{c} ; \nabla \cdot \Delta d \dot{b}$ or $\sigma_{c}<\dot{c} \nabla$

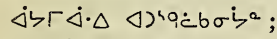

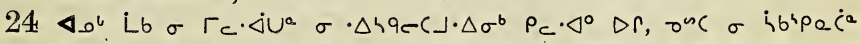

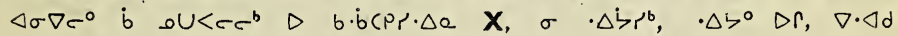
LL. $\Delta \dot{\Delta} \zeta \Gamma \triangleleft \cdot \Delta^{b}$;

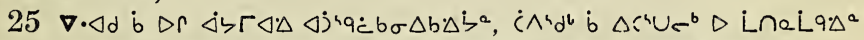

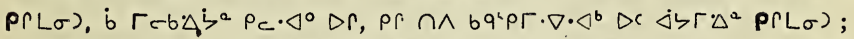

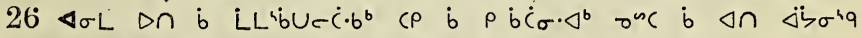

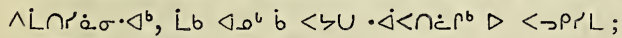

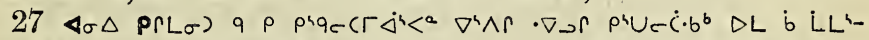

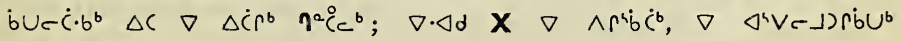

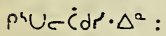

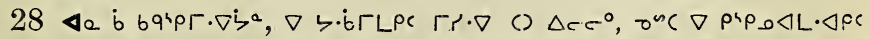

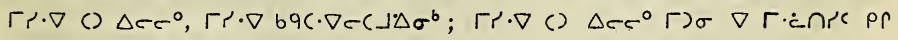
$<P \cap Q L \cdot \triangle P C \times$ PL $^{4}$ :

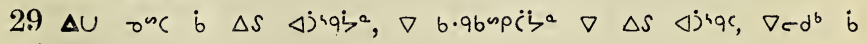
$\Delta)^{4} q^{b} b d \cdot \Delta^{b} x$

\section{A $p c r a-\triangle b^{a} 2$.}

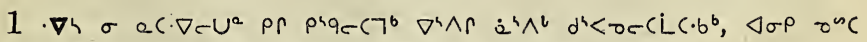

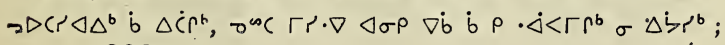




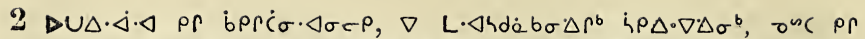

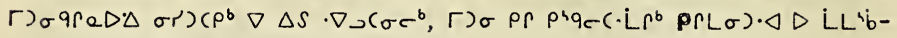

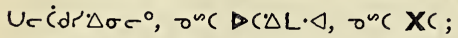

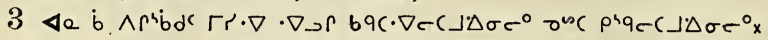

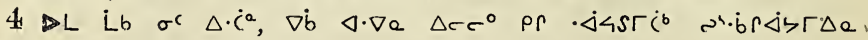
$\Delta r_{x}$

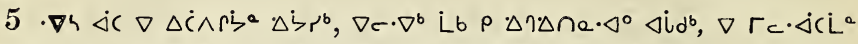

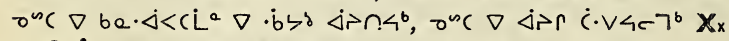

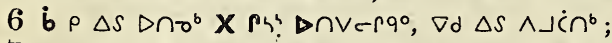

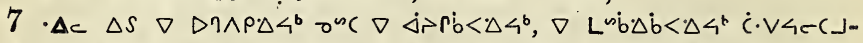

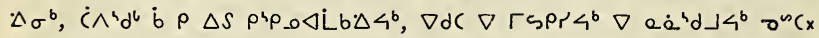

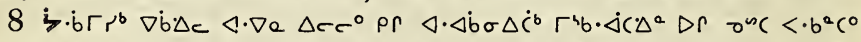

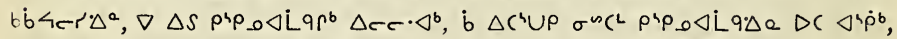

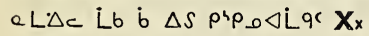

$9 \cdot \nabla h \Delta c \Delta \zeta \Delta$ wibd $\nabla \Delta S$ LL. $\left.\nabla c \dot{c} \cdot b \sigma c^{b} p \rho L \sigma\right) \Delta\left(\Delta U r \dot{C} d r \cdot \Delta^{a} x\right.$

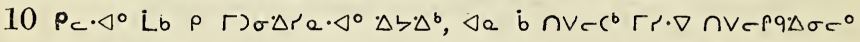
O"C b"PD $\triangle \sigma \sigma^{\circ}$ :

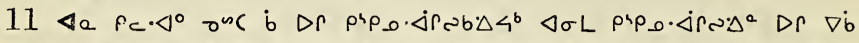

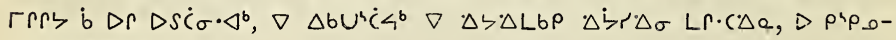
- $\Delta r r^{\circ} \Delta^{a} X \Delta r$ :

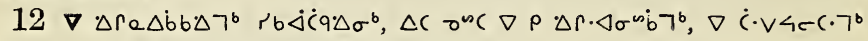

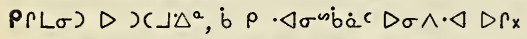

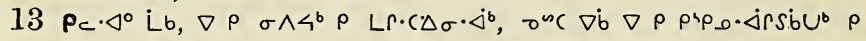

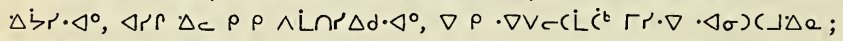

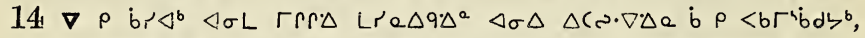

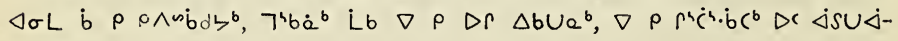
nd $\Gamma^{\mathrm{b}}$;

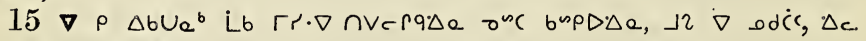

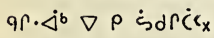

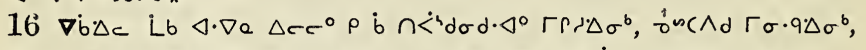

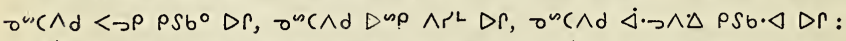

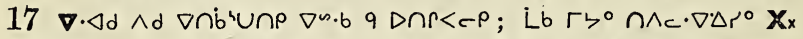

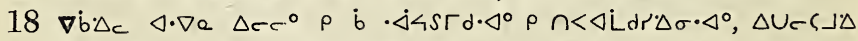

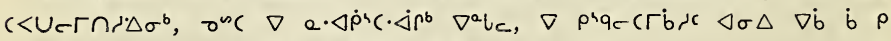

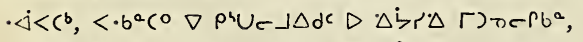

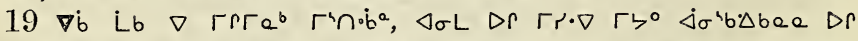

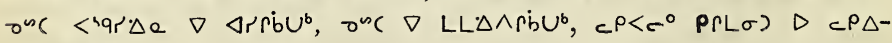
$\cdot \nabla \cdot \Delta^{a} \Delta r_{x}$

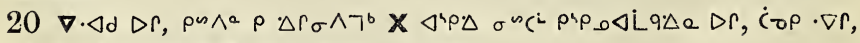

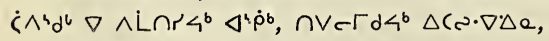

$\left.\left.21\left(\nabla b \Delta_{c} 4 \Gamma_{e}\right\lrcorner^{b}, \nabla b \Delta c d r^{4} c\right\lrcorner^{b}, \nabla b \Delta_{c} \Gamma r \Gamma e\right\lrcorner^{b}$,

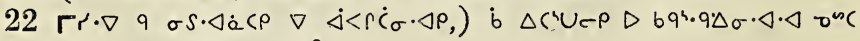

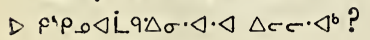

330 


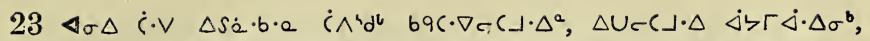

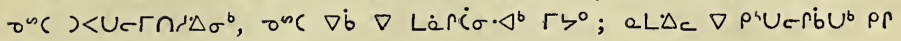
UVeríub $\triangle \dot{S}^{4} x$

\section{Nipcre $\triangle b^{a} 3$.}

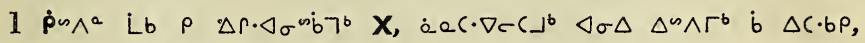
$\Delta\left(\mathbf{X} \nabla \triangleleft \wedge \cdot \dot{\Delta}(\boldsymbol{P} \rho L \sigma) \cdot \triangleleft D \rho \Gamma \sigma^{4} \rho c^{b} \mathrm{x}\right.$

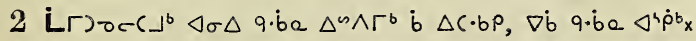

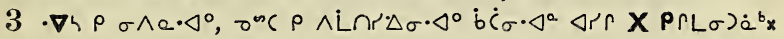

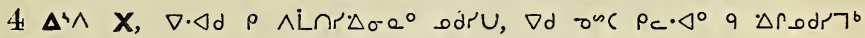
$L \sigma) \cdot \dot{\psi}^{4} U \Delta \sigma^{b} \mathrm{x}$

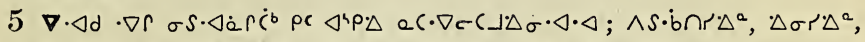

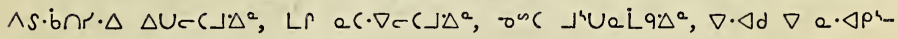
$(\cdot \dot{b} b \sigma \cdot \Delta c L \sigma)^{a}{ }^{a}$ :

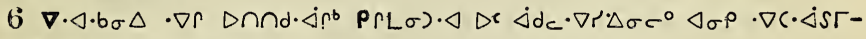

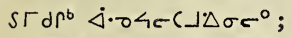

$\left.7 \nabla \cdot \triangleleft \cdot b \sigma \Delta \cdot \nabla^{h} b^{b} \dot{b} \rho \wedge\right\lrcorner \dot{C} C 7^{b}, 7 \cdot \dot{b}^{b} \nabla \rho \wedge L \cap r^{4} C 7^{b} x$

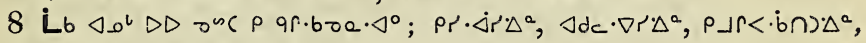

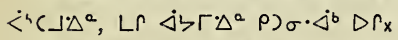

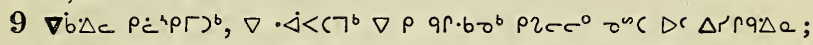

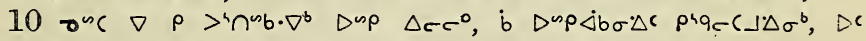
$\Delta S \dot{a} d r \cdot \Delta \sigma c^{b} \triangleleft \sigma \Delta \dot{b} \rho D S \Delta d c$;

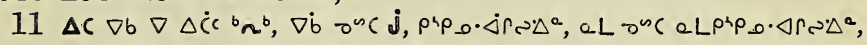

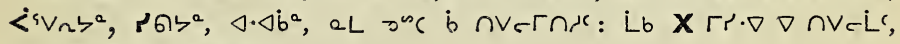

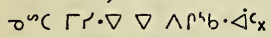

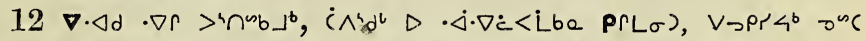

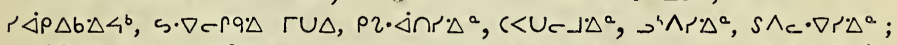

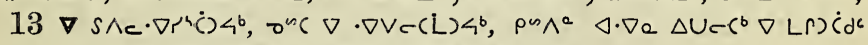

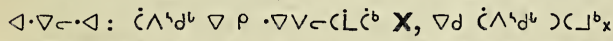

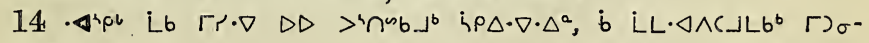
$\cdot \Delta r \cdot \Delta \Delta^{a} \times$

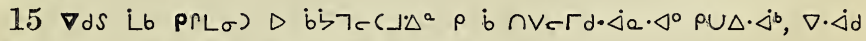

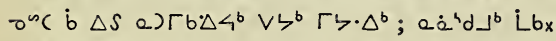

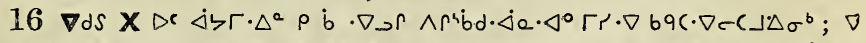

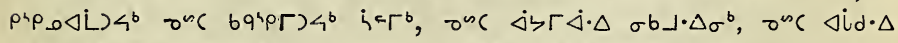

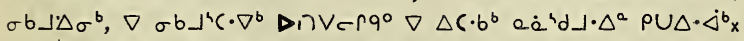

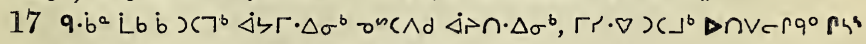
$\left.\Delta\left(\Delta S \sigma \dot{b} r \cdot \Delta \sigma^{b}, \nabla \text { a. }{ }^{b} d\right]^{b} P R L_{\sigma}\right) \cdot \nabla D \dot{C} \cdot \Delta L \cdot \Delta c \cdot \Delta c \Delta r_{x}$

$\left.18 \Delta^{4} \cdot 9\right)^{b}, 2 e \Delta \dot{C}^{b} \cap \wedge c \cdot \nabla \rho \dot{a} \vee \Gamma \cdot \triangleleft \cdot \triangleleft, \nabla \Delta S \Delta U C \Gamma \dot{C}^{b} \Delta \cap V-q^{\circ}{ }^{\circ}$

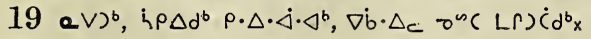

$20 \varangle \cdot \Delta S S)^{b}$, ea $\Delta \dot{C} d^{b} \quad \rho \quad \sigma \rho \Delta d \cdot \triangleleft \cdot \Delta^{b} \quad r r \cdot \nabla \quad \Delta S, \cdot \nabla h \nabla \cdot \triangleleft \cdot 9 c^{\circ} \quad a^{\natural} \wedge \cdot$ $r \cdot \sec$ L $D$ nera $9^{\circ}$

331. 
$21 \Delta \dot{C} \cdot \Delta \dot{L})^{b}, \nabla \dot{b} \cdot \Delta c \rho s \cdot \Delta \Delta d^{b} p c \triangleleft \cdot \Delta s \Gamma s \cdot \Delta \cdot \triangleleft^{b}, \nabla \dot{b}$ pr $\dot{a}\left\langle C \cdot \nabla c\left(p^{b} x\right.\right.$

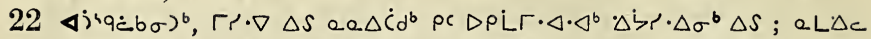

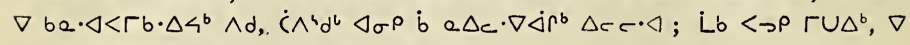
e $\sigma \Gamma^{4}\left(\cdot \nabla^{b} P \Gamma(L)\right.$ :

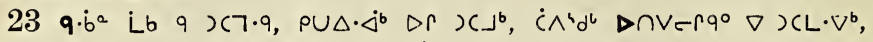
QL. $\Delta_{c}$ Lb $\Delta c c \cdot \Delta^{b} \wedge d$;

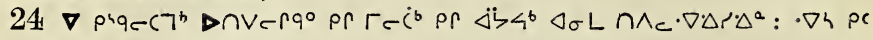
$\triangleleft)^{4} q^{\circ} C \cdot \triangleleft \cdot \triangleleft^{\circ} \triangleright \cap V-r q^{\circ} X_{x}$

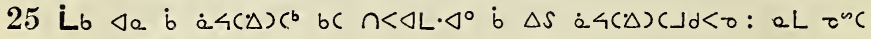
$\dot{<\wedge} \Delta)^{n} \Delta U r L \cdot \Delta^{b} \Delta r c \cdot \Delta^{b} x$

\section{A $P C c_{2} \triangle b^{a} 4$.}

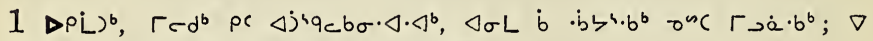

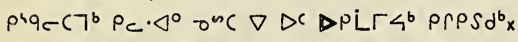

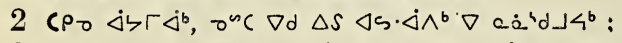

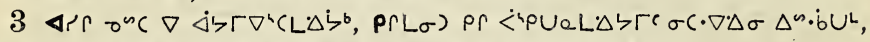

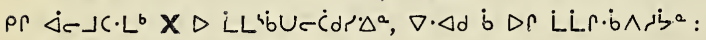

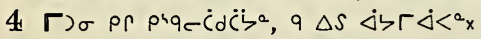

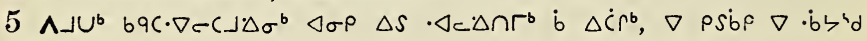
$\dot{\Delta}<\hat{C} \dot{C} \dot{\zeta}^{b} \mathrm{x}$

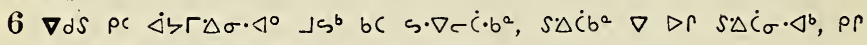

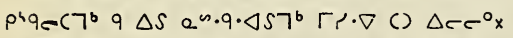

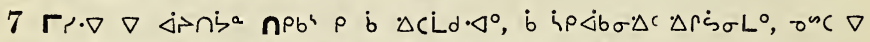

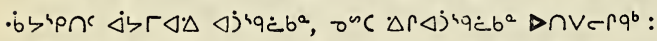

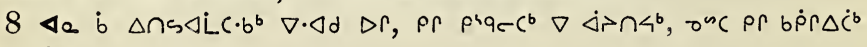
$\rho \cup \triangle \cdot \Delta^{b}$;

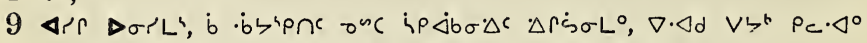

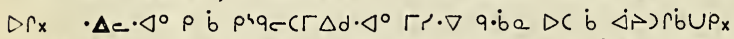

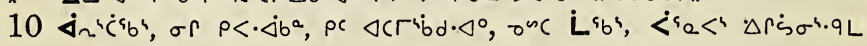

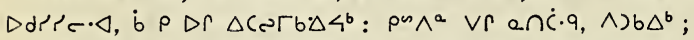

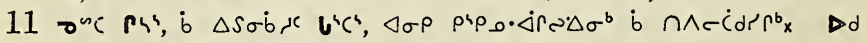

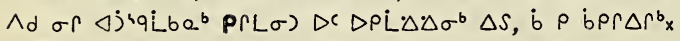

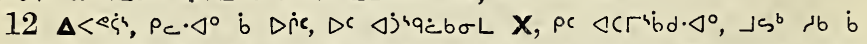

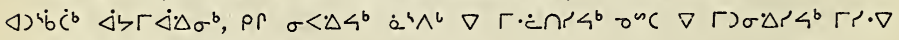
$\Delta C \Delta U \leftarrow\left(J \Delta \sigma^{b} \text { PrL } \sigma\right)_{x}$

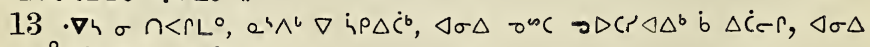

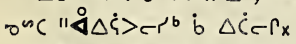

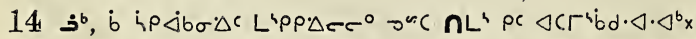

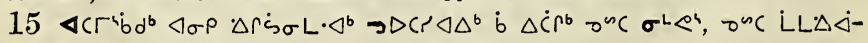
$\iota \Gamma \Delta \cdot \Delta^{b} \Delta \rho^{b} \dot{b} \Delta \dot{C}^{b} b_{x}$

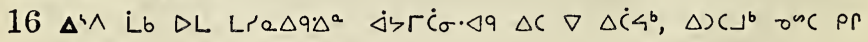
332 


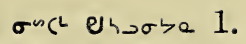

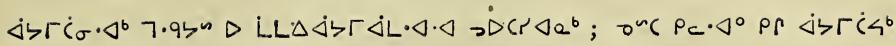
$\triangle \sigma L \rightarrow D C r \Delta \Delta^{b} D \Gamma_{x}$

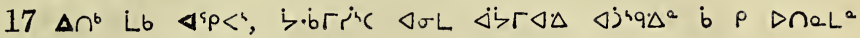
$\left.\Delta \cap V \in q^{b}, \operatorname{pr} \cap \wedge\right)\left(L^{\circ} x\right.$

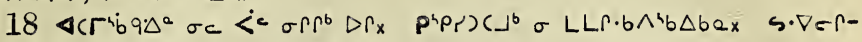
$q \Delta^{a} \rho b \Delta \Delta \eta \Delta d a \cdot<^{\circ} x \quad \nabla 7^{a} x$

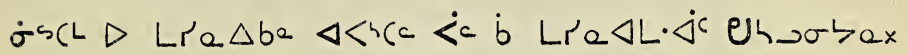

\section{A'pcra $\triangle b^{a} 1$.}

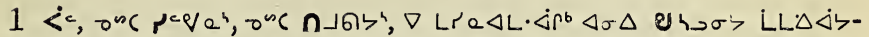

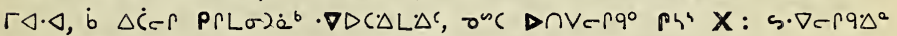

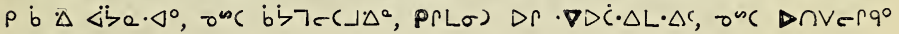
$\mathrm{PS}^{4} \mathrm{X}$ *

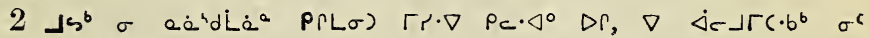
$\dot{\Delta} \zeta \Gamma \dot{\Delta} \cdot \Delta \sigma \dot{a}^{\mathrm{b}}$;

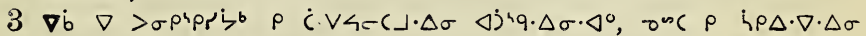

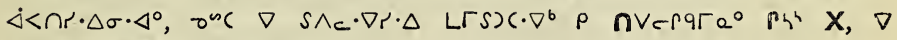
ba. $\left.\dot{\langle}<\Gamma \dot{C}^{b} P \rho L \sigma\right) d \dot{C} \cdot \Delta a^{\circ}$;

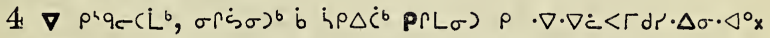

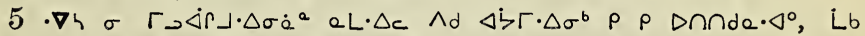

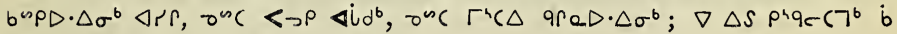
$\rho \Delta S \cdot \dot{C}^{b} \Delta C \nabla \Delta \dot{C} \angle_{1}^{b} P_{C} \cdot \triangleleft^{\circ} \Delta \Gamma_{x}$

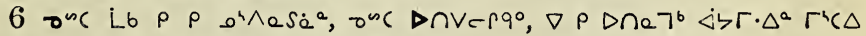

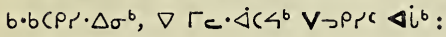

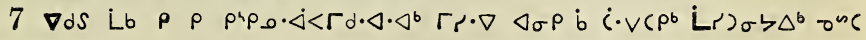
$\Delta b \succ \Delta \Delta^{b} x$

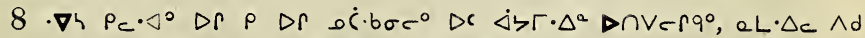

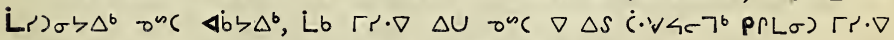

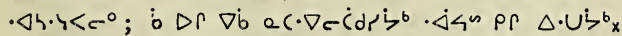

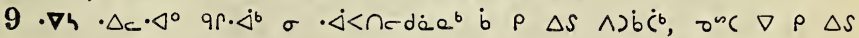

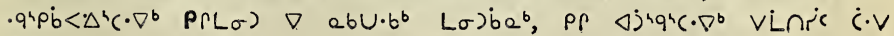
$P(L \sigma)$;

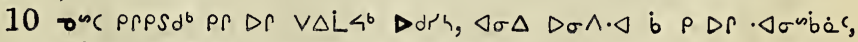

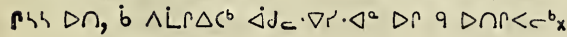




\section{A p $P$ C $a \triangle b^{a} 2$.}

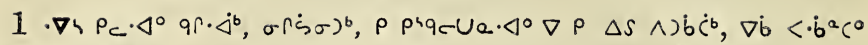
$\nabla \rho \Delta U_{c} \dot{C} \cdot b^{b}:$

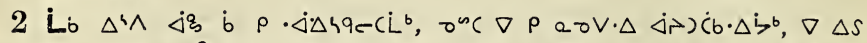

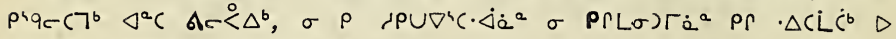

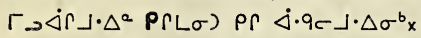

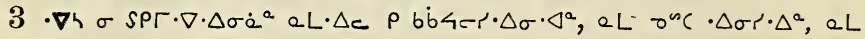
$\nabla^{s C} \cdot \dot{\triangleleft}\left\langle s \Delta \cdot \nabla \cdot \Delta^{a}:\right.$

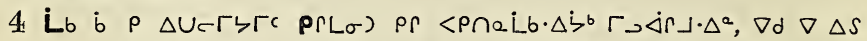

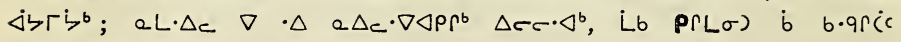
$\sigma \cup \Delta \dot{a} \mathbf{x}$

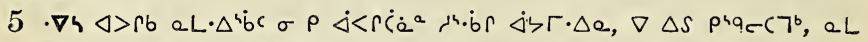
on JUaLq. $\Delta \sigma \varangle \cdot b \sigma \cdot \Delta^{a} ; P(L \sigma) \rho^{4} q c C^{L}$ :

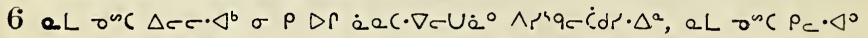

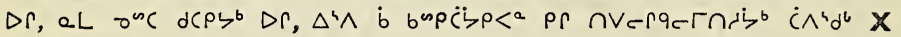
$D C \triangleleft<^{h} C L_{x}$

7 Ĺb $\rho$ P د

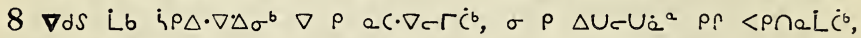

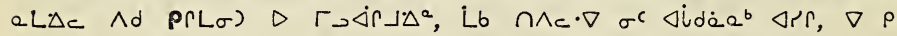
$i p \Delta \dot{C}^{b} x$

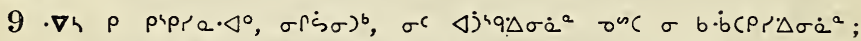

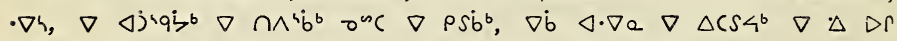

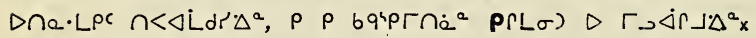

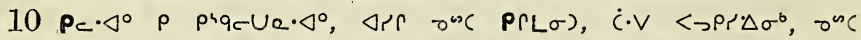

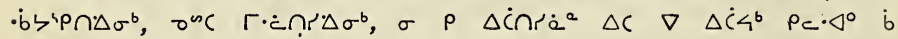
$\dot{c} \cdot \vee C 7^{b}$;

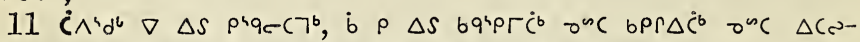

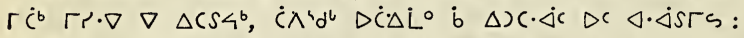

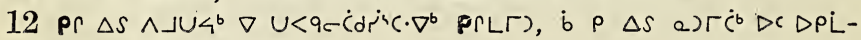
$\Delta \Delta \sigma^{b}$ osc $D \rho^{b} \cup c \dot{C} d r \Delta \sigma^{b} x$

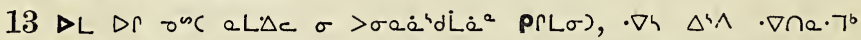

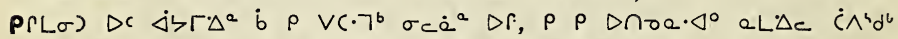

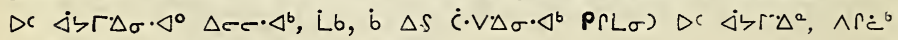
$\dot{b}$ bepD $\triangle \triangle)^{b} \dot{b} d^{\circ}<^{b} \rho_{c} \cdot \triangleleft^{\circ} \dot{b} \dot{c} \cdot V C 7^{b} x$

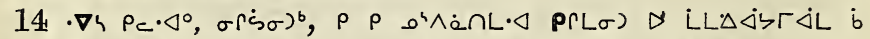

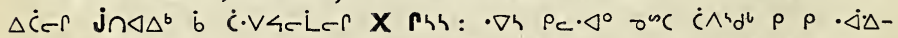

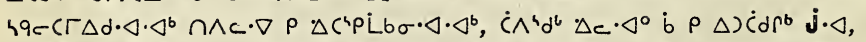

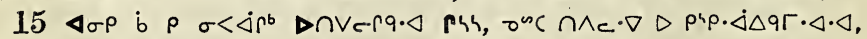

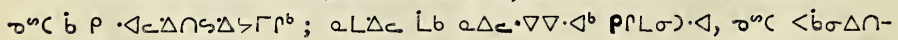

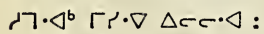

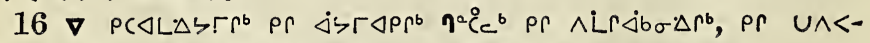

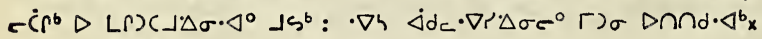




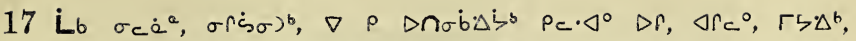

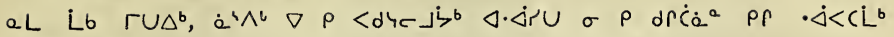
bंic $C\left\ulcorner\mathrm{~d} \cdot \triangleleft^{\circ} \mathrm{x}\right.$

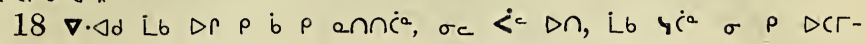
$\Delta \mathrm{di}^{\mathrm{a}} \mathrm{x}$

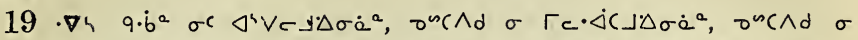

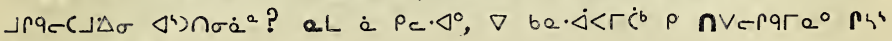
$\mathrm{X} \vee r \cos s$ ?

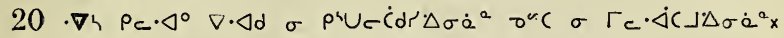

\section{Aspcre $\triangle b^{\circ} 3$.}

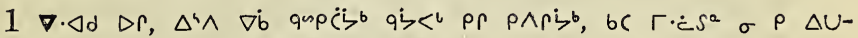

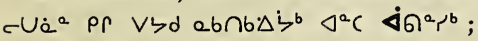

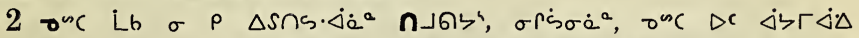

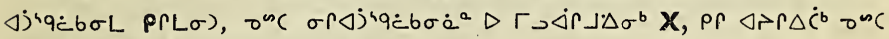

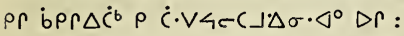

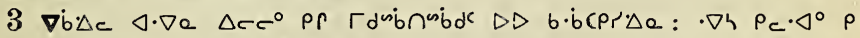
prqcUe. $\left.\Delta^{0} \nabla d C \nabla<p U C \dot{C} d d^{2}\right\rangle^{b} x$

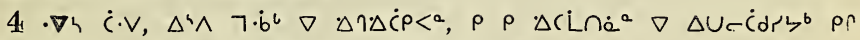

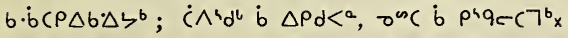

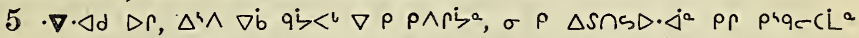

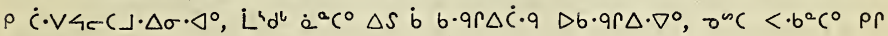

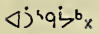

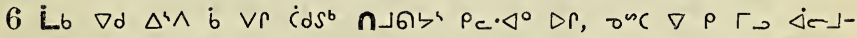

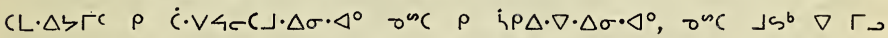

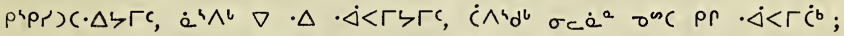

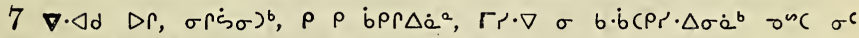
$\left.\Delta C \Gamma r \cdot \Delta \sigma \dot{\alpha}^{b}, \rho \dot{c} \cdot V<C C\right\lrcorner \cdot \Delta \sigma \cdot \nabla^{\circ} \Delta \Gamma:$

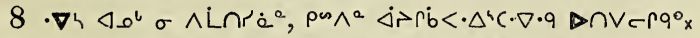

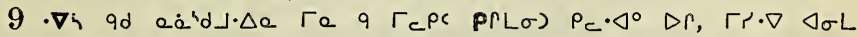

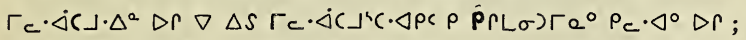

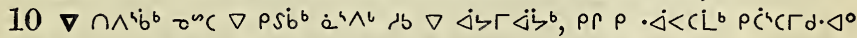

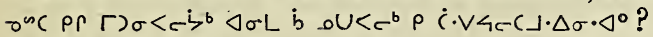

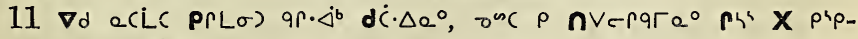
oC $\triangle S T C P R$ ancibx

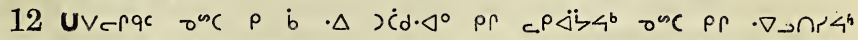

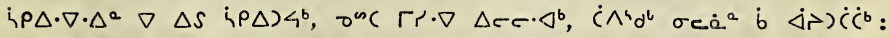

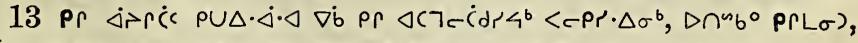

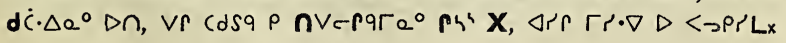




\section{$\Lambda^{4} p\left(r a \Delta b^{2} 4\right.$.}

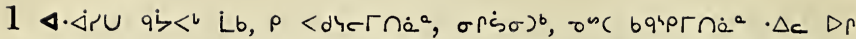

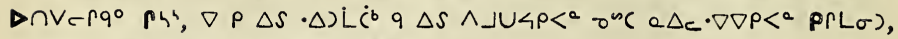

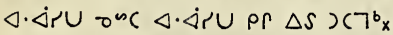

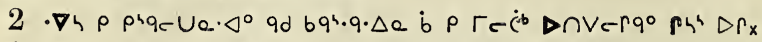

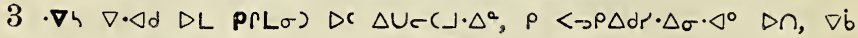
br $x 7^{b} \wedge s \cdot b \cap r \cdot \Delta a$;

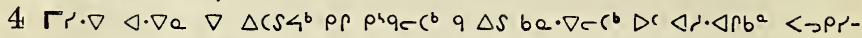
$\cdot \triangle \sigma^{b}$ onc pinc $C$ dr $\cdot \triangle \sigma^{b}$;

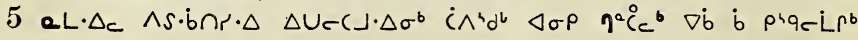
$P \Gamma L \sigma) \cdot \Delta x$

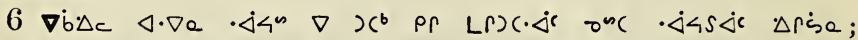

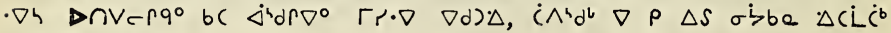
onc $\dot{\Delta} \sigma] c \dot{L} \dot{c} b_{x}$

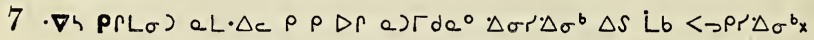

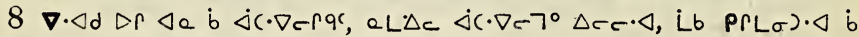

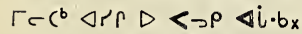

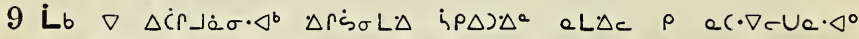

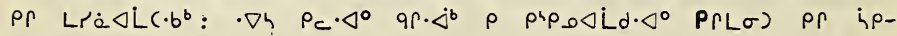
$\triangle \supset b^{b} x$

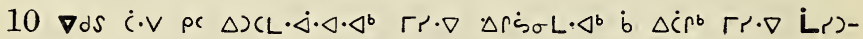

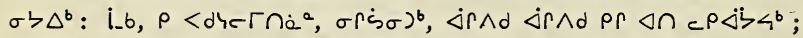

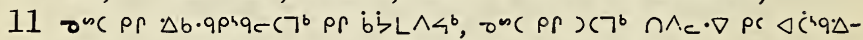

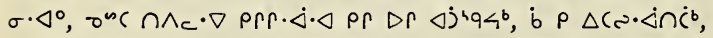

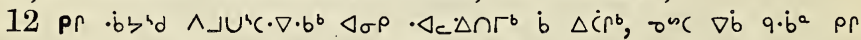
- $P C L \zeta^{b} \mathrm{x}$

13 Ĺb aLde $\sigma$ e $\left(\cdot \nabla c U^{a} \nabla \dot{b}\right.$ pr prqe $\left(7^{b}, \sigma \rho \dot{s} \sigma\right)^{b}, \nabla \Delta \dot{C} \dot{L}-$

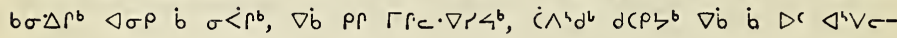
\lrcorner$\sigma \rho^{b} \mathrm{x}$

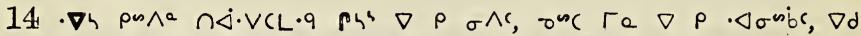

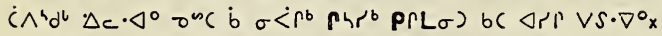

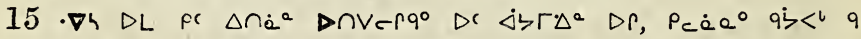

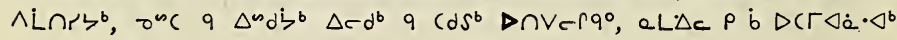
$\Delta \sigma \rho$ b $\sigma \dot{<} \rho^{b} x$

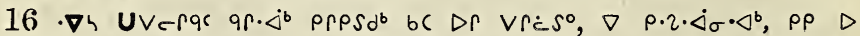
$V \dot{C} d \Delta^{a} \rho \Gamma \nabla^{a} l e, \rho \rho \sigma^{n C} \nabla>\left(r b^{a} \rho \Gamma L \sigma\right): \Delta \sigma \rho$ Lb $\left.\dot{b} \sigma \wedge \rho^{b} X\right)^{b}$

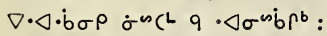

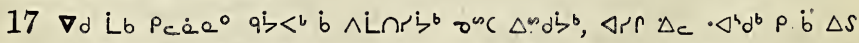

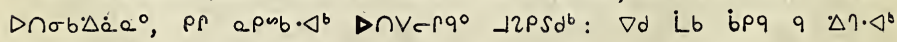
D N V r r $^{\circ} \mathrm{x}$

$18 \nabla \cdot \triangleleft d \Delta r, b \rho r \Delta)^{b} D D<\zeta r \cdot \Delta a \Delta r_{x}$ 


\section{$A^{4} p c r a \triangle b^{2} 5$.}

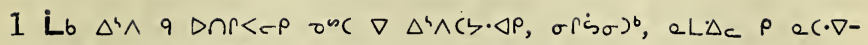
-Ue. $\Delta_{0} \operatorname{tr} \operatorname{Lr} e \Delta L\left(\cdot b_{b} x\right.$

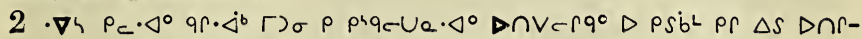

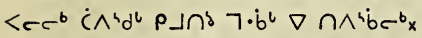

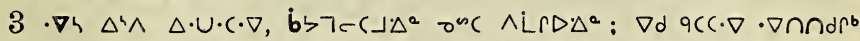

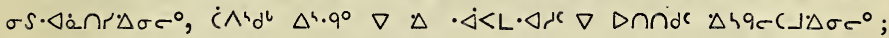
a L $\Delta_{c}$ L $L b$ bC $\left.\triangleright S\right\lrcorner \cdot \triangleleft^{b} x$

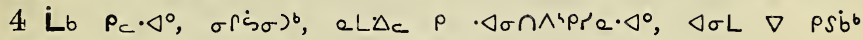

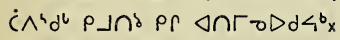

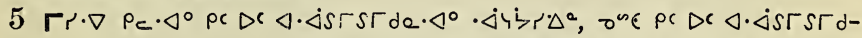

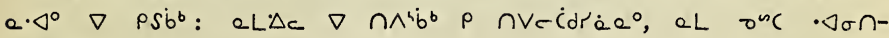
Aist. $\Delta^{2} x$

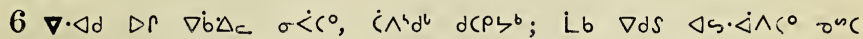
$\operatorname{vichin}\left(0^{\circ} x\right.$

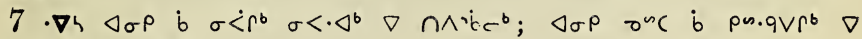
$\cap \wedge \dot{b} b r^{b} \dot{p} \backsim \cdot q \vee \cdot \Delta^{b} x$

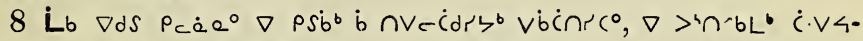

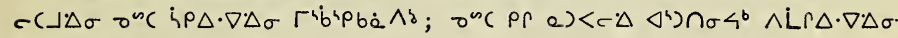
$\triangleleft^{4} \vee\ulcorner\lrcorner \Delta^{a}$ :

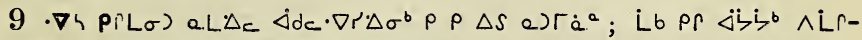

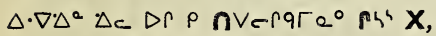

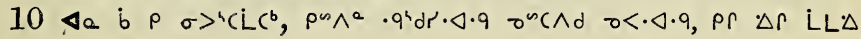
$\wedge \operatorname{LnRLbx}$

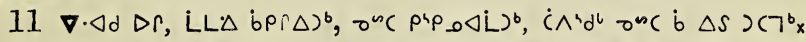

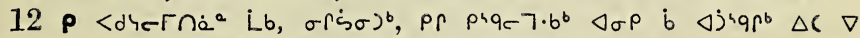

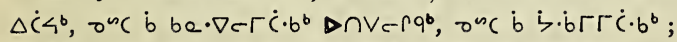

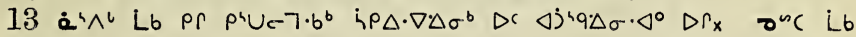
b่ $>\triangle \nabla r^{i}(\dot{C}) \times$

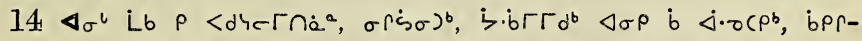

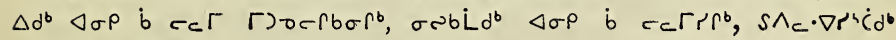
$r t \cdot \nabla \cdot \Delta c c \cdot \Delta$ bx

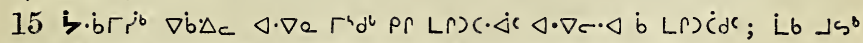

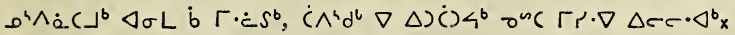

16 bрq $\left.\Gamma_{c} \cdot \dot{\Delta c}\right\lrcorner^{b} \mathrm{x}$

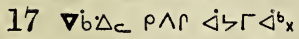

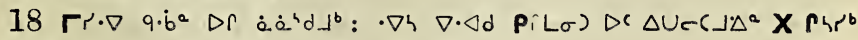
$\rho_{c} \cdot \triangleleft^{\circ} \Delta r_{x}$

$19 \nabla \dot{b} \cdot \Delta_{c} \Delta^{4} c \cdot \nabla 4 c \Gamma^{b} \Delta i^{b} x$

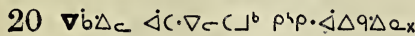

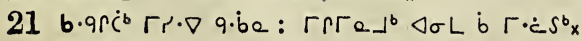
337 


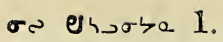

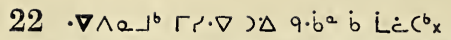

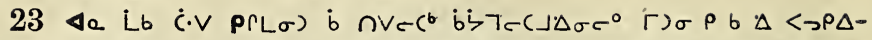

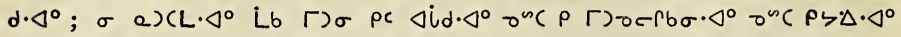

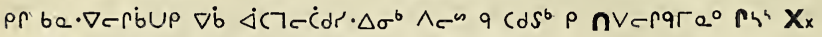

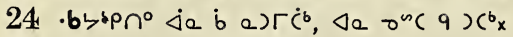

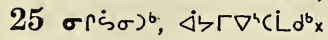

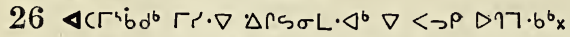

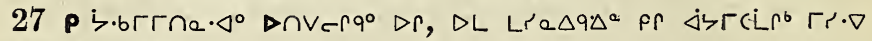
$\langle\rightarrow P \Gamma \dot{S} \sigma L \cdot \triangleleft b x$

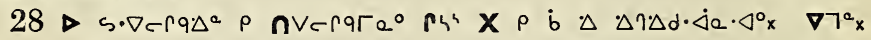

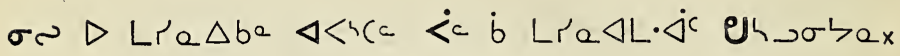

A'pcra $\triangle b^{\circ} 1$.

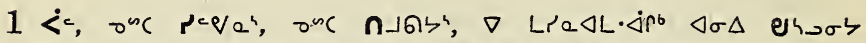

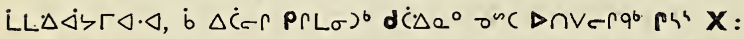

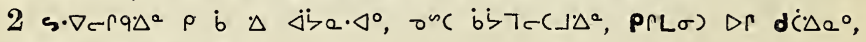

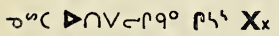

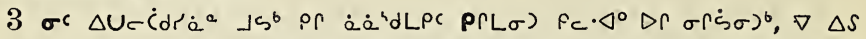

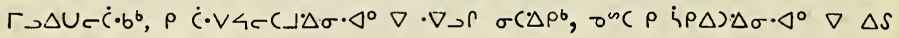

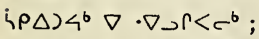

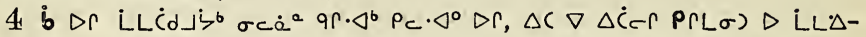

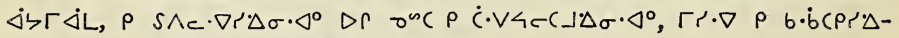

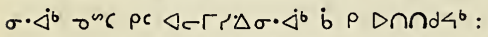

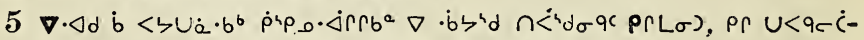
$\left.d \Gamma b \Delta \zeta^{b} P \Gamma L \sigma\right) \Delta\left(\Delta P L \Delta \Delta \sigma^{b}, \nabla \cdot \Delta d\right.$ b $\Delta \Gamma \cdot \Delta 49 c\left(7^{b}:\right.$

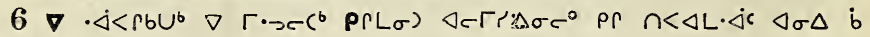
$r \operatorname{du}^{\circ} \dot{b} \Delta C \cdot b^{b}$;

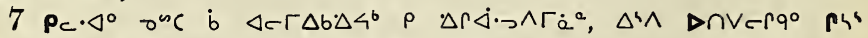

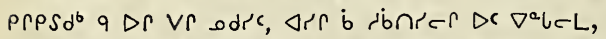

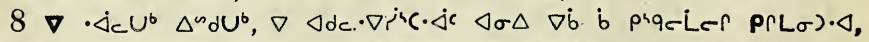

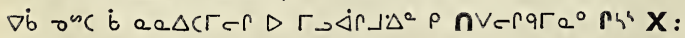

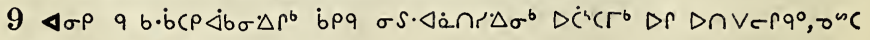

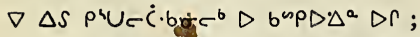

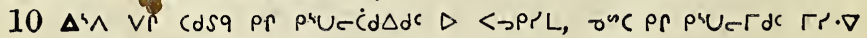
338 


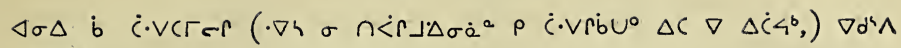
$\nabla P S b^{b} x$

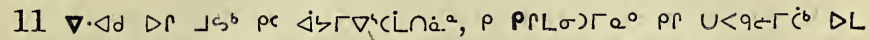

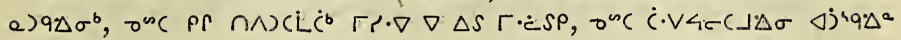
b"P $\triangle \triangle \sigma^{6}$;

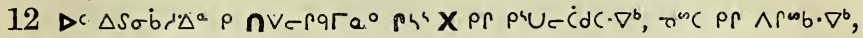

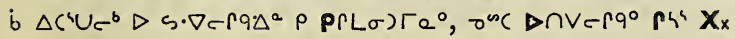

\section{A'pcre $\triangle b^{2} 2$.}

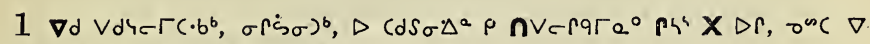
LL $\Delta \Delta) \zeta^{b} \nabla \Delta \cap m b \Delta \dot{b}<\Delta^{4}\left(\cdot \Delta^{b}\right.$,

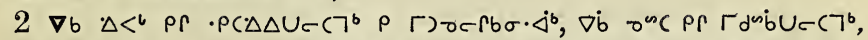

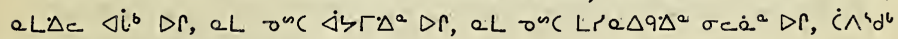
$\nabla \vee v \dot{\alpha} \cdot b \sigma c^{b} X \triangleright P S \dot{b}^{L} \mathbf{X}$

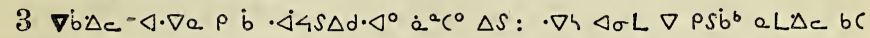

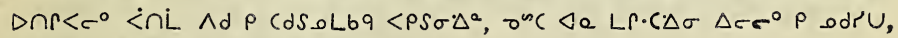

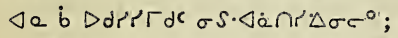

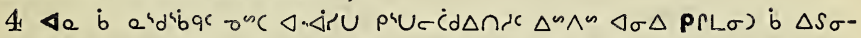

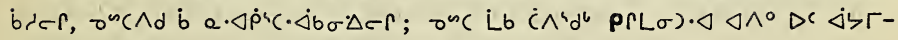
$\nabla \Delta b \Gamma d c^{b} P(L \sigma) \cdot \triangleleft, \nabla \cdot \Delta<n c \cdot \nabla(\nabla \rho \rho L \sigma) \Delta c_{x}$

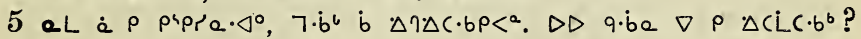

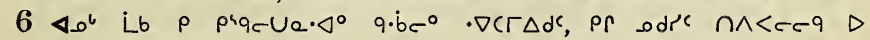
psib $x$

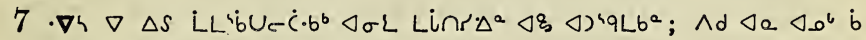
$\Delta C \Gamma \Delta \cdot \nabla^{c} b C D C \Gamma \Delta \cdot \nabla^{\circ},<i n \dot{L} \Delta b U_{\dot{\alpha}} b \sigma \Delta U_{x}$

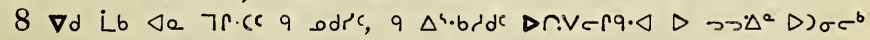

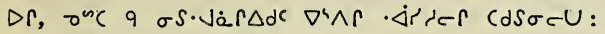

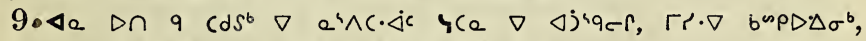

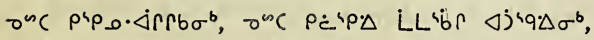

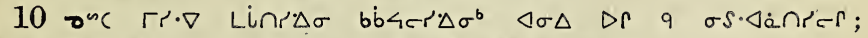

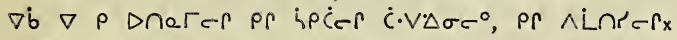

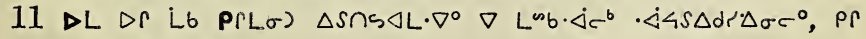
c.verer besparaco:

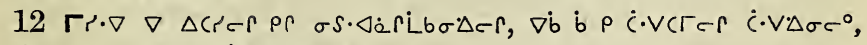
Le b 6 b.xecter Lind $\triangle 0^{\circ} c^{\circ} x$

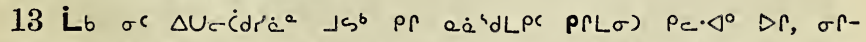

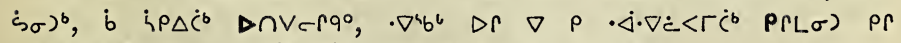

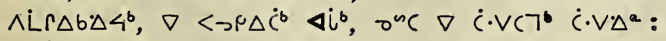

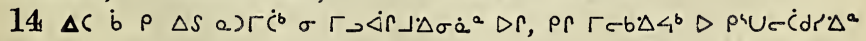

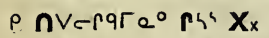

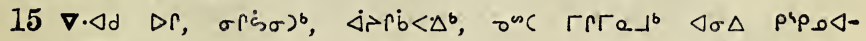
339 


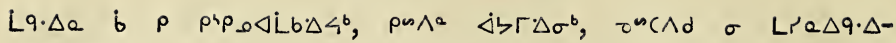
$\sigma \alpha^{a} \Delta r_{x}$

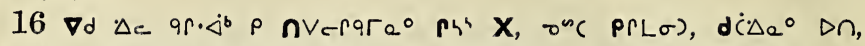

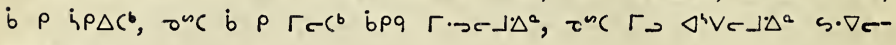
rq. $\triangle^{\circ} D r_{x}$

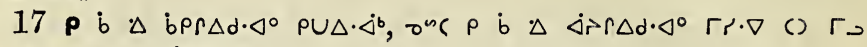

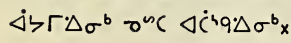

\section{Aipcra $\triangle b^{a} 3$.}

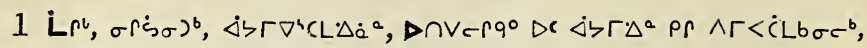

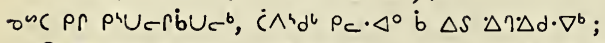

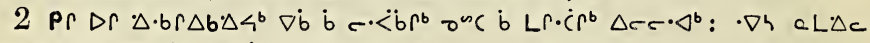

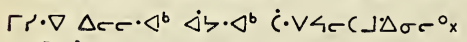

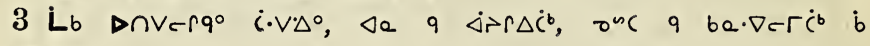
$\operatorname{Le} c^{b} D \Gamma_{x}$

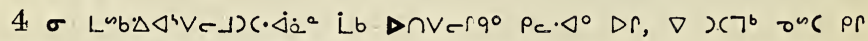
$x<7^{b} \Delta \sigma \Delta q \cdot \dot{b} e$ b $\triangle c a \Gamma \dot{c} \cdot b^{b x}$

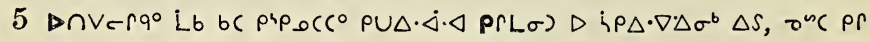

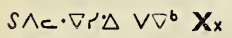

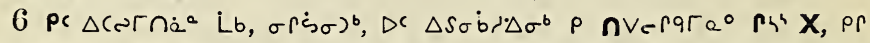

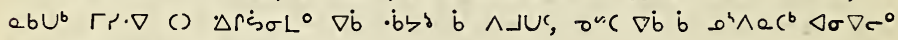

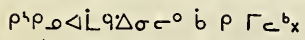

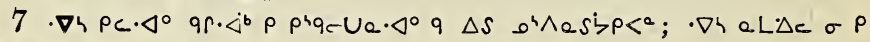
Linráa $\triangle C$ b $\Delta \dot{C} \dot{c}^{b}$;

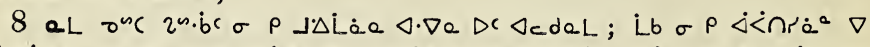

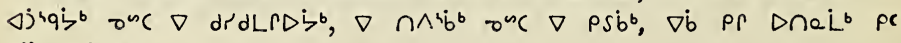

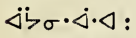

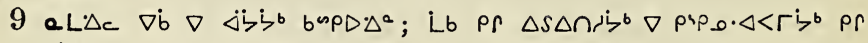
$\Omega^{4} \wedge a s b^{b} x$

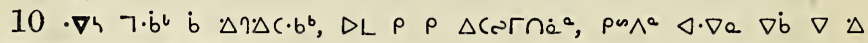

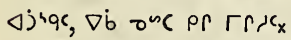

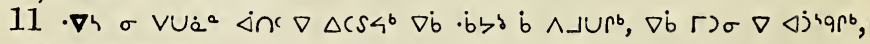

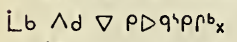

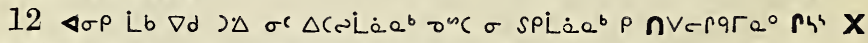

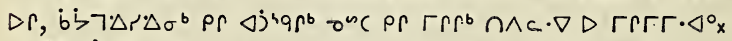

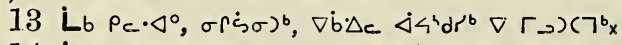

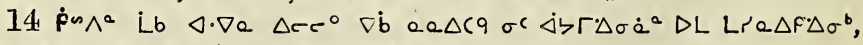

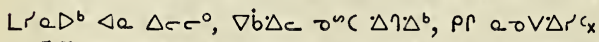

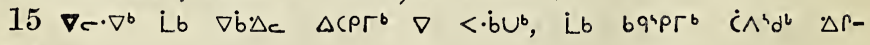
$\dot{s} \sigma L^{\circ} x$

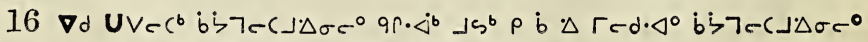

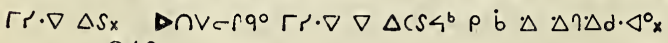
340 


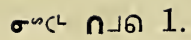

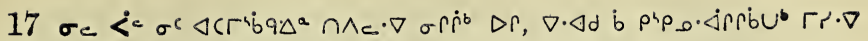

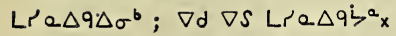

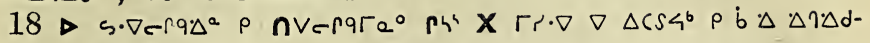
$\cdot \dot{\cdot} a \cdot \dot{\Delta}^{0} \mathrm{x} \quad \nabla 7^{a} \mathrm{x}$

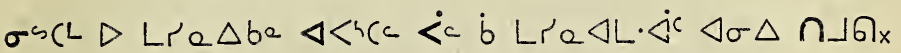

\section{A'pcra $\triangle b^{a} 1$.}

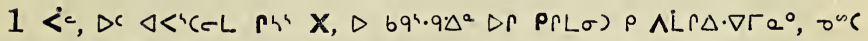

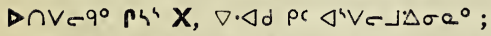

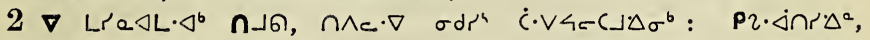

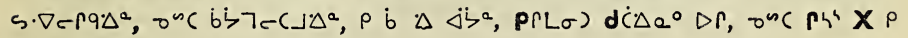
$n \in-6 C^{\circ} e^{\circ} x$

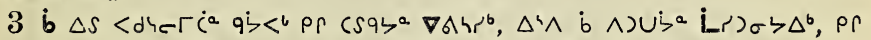

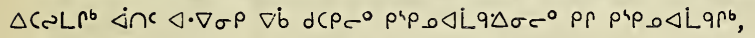

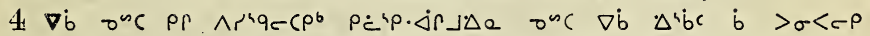

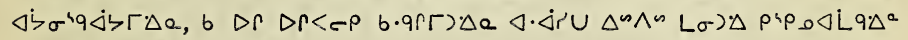
$\dot{c} \cdot V 4 C C\lrcorner \Delta \sigma^{b} \dot{b} \Delta C \cdot b^{b} x$

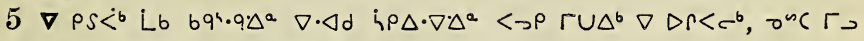

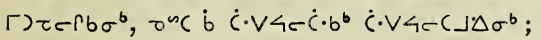

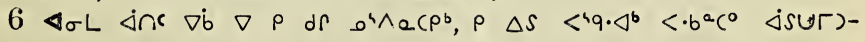
$\Delta \sigma^{b}$;

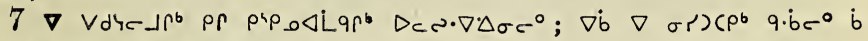
$\triangle \cdot U p^{b}$ oncAd b $\varangle-\lambda C c^{b} x$

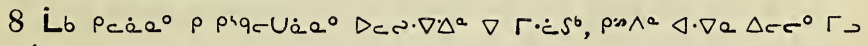
¿<rír ;

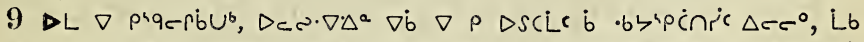

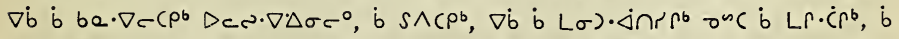

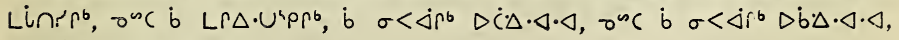
onc b $\quad \sigma<\Delta \beta^{\circ} \Delta r c \cdot \Delta$,

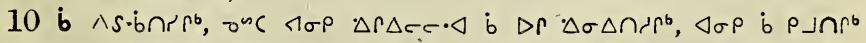

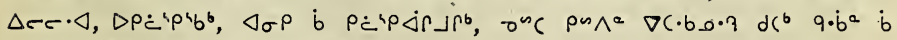

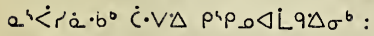

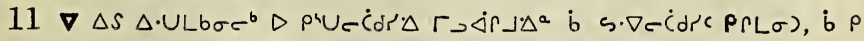
$<P \cap a L b \Delta \cdot \Delta^{b}$ Pr be. $\nabla C C \cdot L^{b} x$

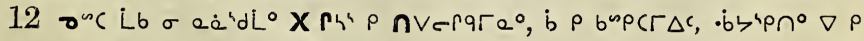

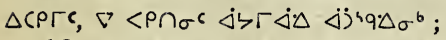

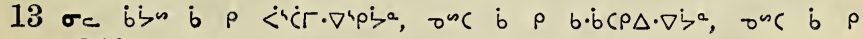
341 


\section{$\operatorname{\sigma oc} n$ กงด 1, 2.}

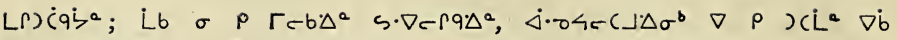
$\nabla$ p prqc CLiax

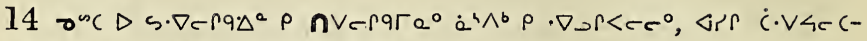

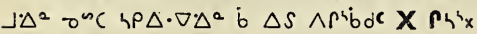

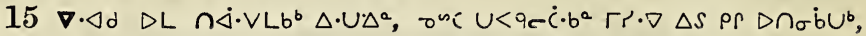

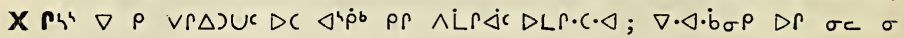
$\sigma \dot{b} \gamma-\dot{c} d p^{a} x$

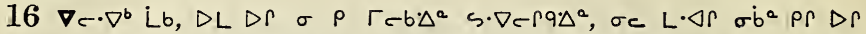

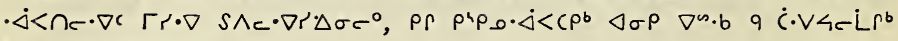
bрq $\wedge$ L $\cap \cdot \Delta \sigma^{b} \Delta S_{x}$

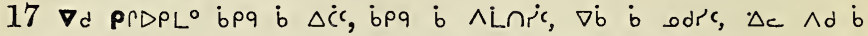

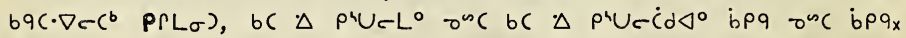
$\nabla 7^{2} x$

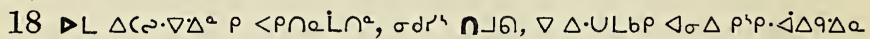

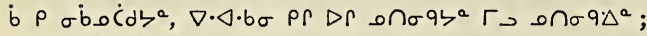

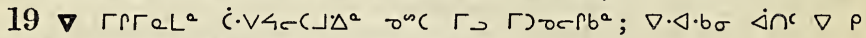

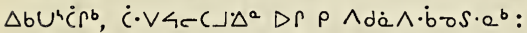

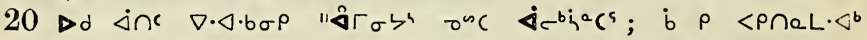
$y c^{a}$, pp $\Delta b \cdot q$ piqec $p^{b} \nabla \dot{b}$ pp $\left.\dot{<}^{4} C\right\lrcorner \rho^{b} x$

\section{A $p c r a \Delta b^{\circ} 2$.}

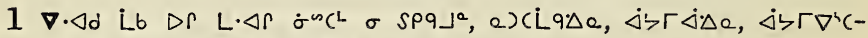

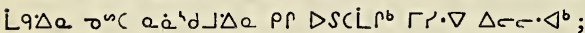

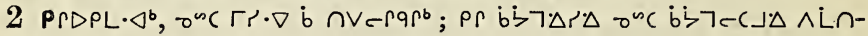

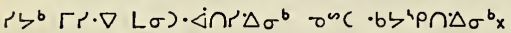

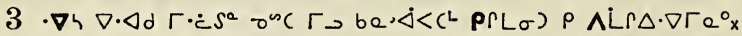

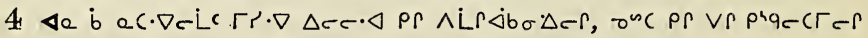
$\dot{c} \cdot \vee \Delta \sigma \sigma^{\circ} x$

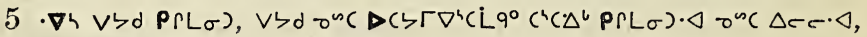

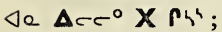

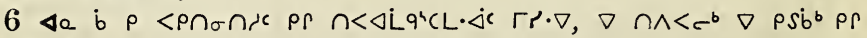
กरंगribu $u^{b}$

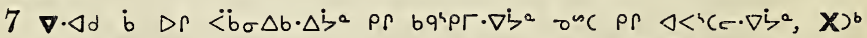

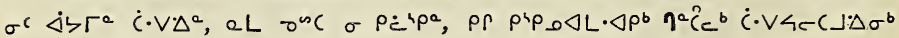
onc $\dot{C} \cdot V \cdot \Delta \sigma^{b} x$

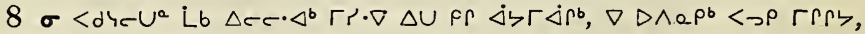

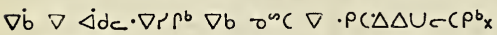

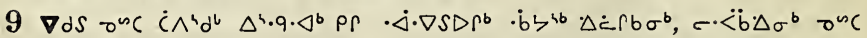

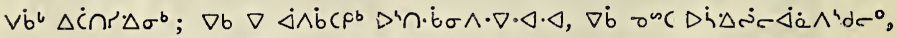

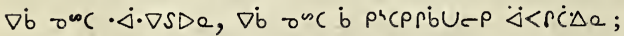

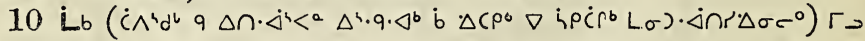
$\Delta \dot{j}^{4} q \Delta e \quad \Delta r_{x}$ 


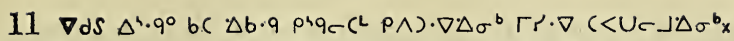

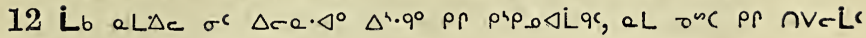
$\dot{a} \vee \triangleleft$, Lb $\operatorname{si} \rho \wedge J \cdot \nabla c^{\prime} x$

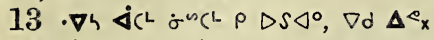

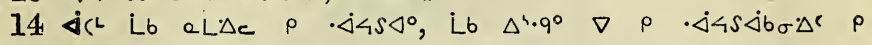
$\cdot \triangleleft \sigma)\left(L_{x}\right.$

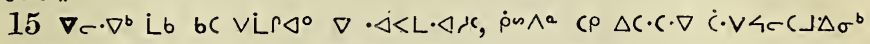

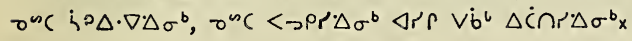

\section{A $p\left(\right.$ C $a \Delta b^{a} 3$.}

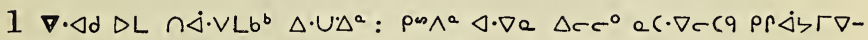
$\triangle P L^{\circ} D C \Delta \dot{C}<\cap r \cdot \Delta^{\circ}$, e.C. $\nabla e C^{L} \Gamma_{2} \Delta \dot{j}^{4} 9 \Delta \sigma \sigma^{\circ} x$

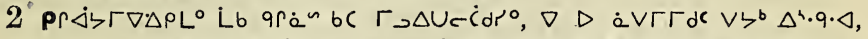

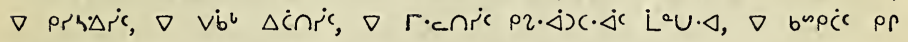
pho.ouLis;

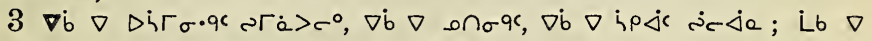

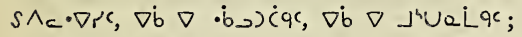

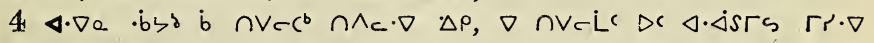
$r \cdot \ddot{i b} \cdot \dot{\Delta n} \wedge \cdot \Delta \sigma^{b}$;

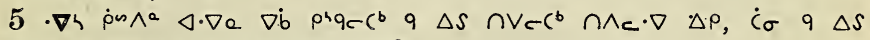

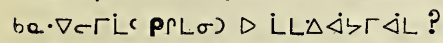

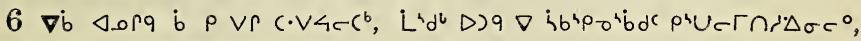
$b\left(<\rho S^{a} \nabla \sigma S \cdot \Delta \dot{L} \Gamma \Gamma \cdot \nabla \cdot \Delta \sigma c^{b} L \Gamma L \sigma\right) \cdot \Delta x$

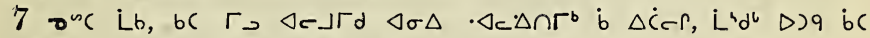

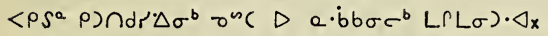

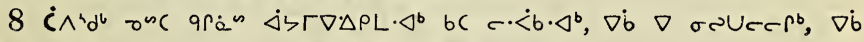

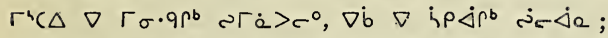

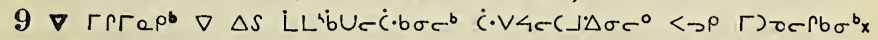

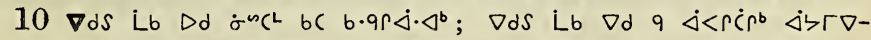

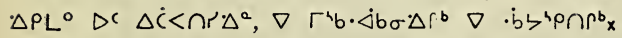

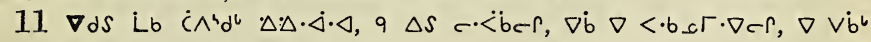

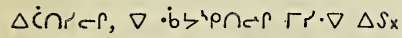

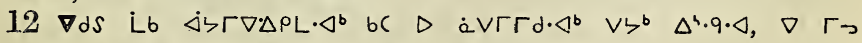

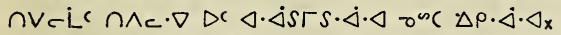

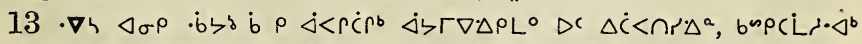

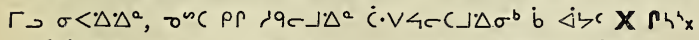

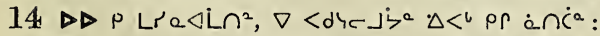

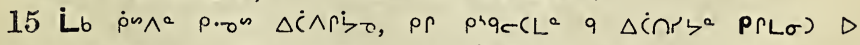

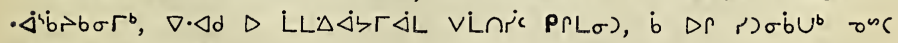
$\dot{\Delta} \rightarrow \Gamma \dot{C} \sigma \cdot \Delta^{b} \dot{C} \cdot \nabla \cdot \Delta^{a} x$

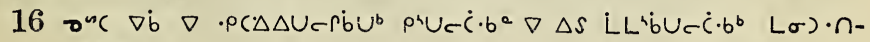
343 


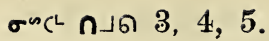

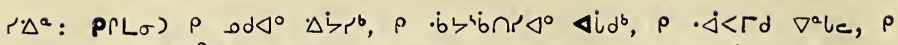

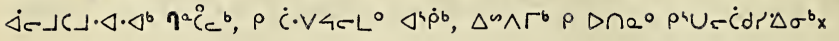

\section{A pcra $\triangle b^{2} 4$.}

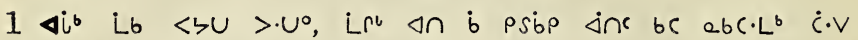

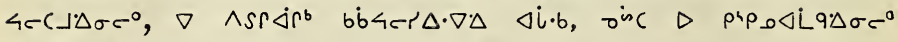
$L(L \sigma) \cdot \triangleleft ;$

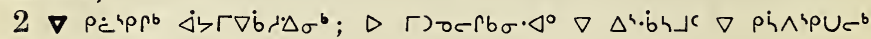
$\Lambda \cdot \dot{\Delta} \wedge \cdot \nabla r ;$

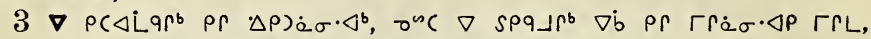

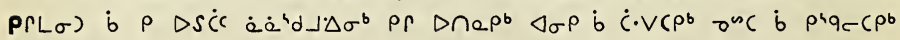
$\dot{c} \cdot V \Delta \sigma \sigma^{\circ} x$

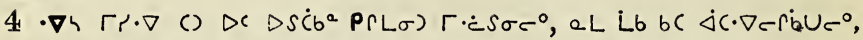

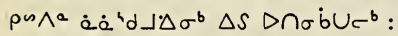

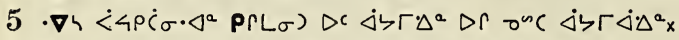

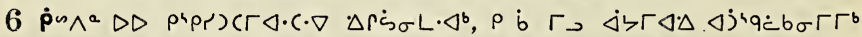

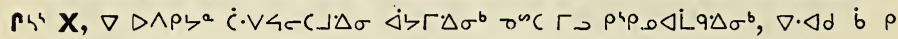
$\rho^{4} \wedge \dot{a} C L^{a} x$

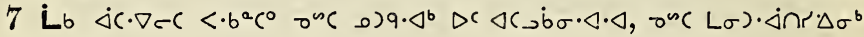
$\Delta S \Delta j+9 \Delta \cap i x$

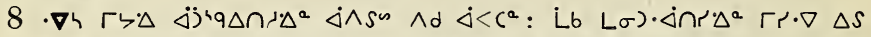

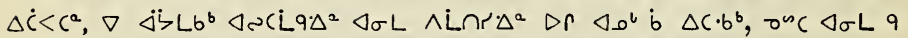
$<<c^{b} x$

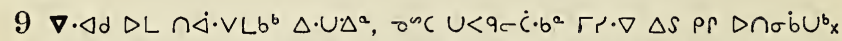

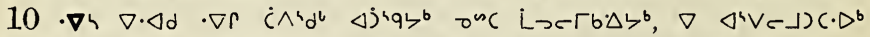

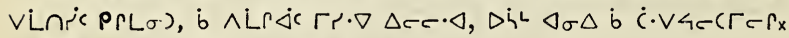

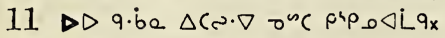

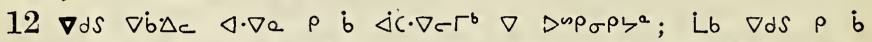

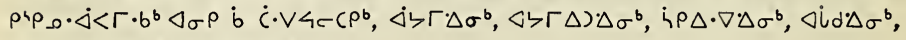

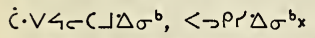

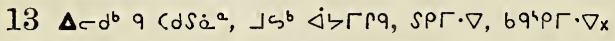

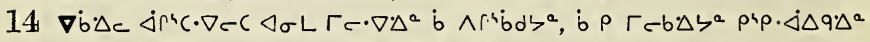

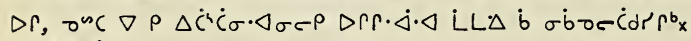

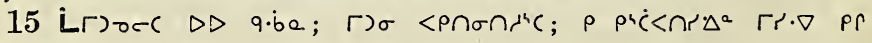

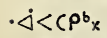

16 ל. לا.

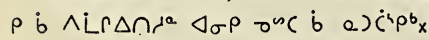

\section{$\Lambda^{\top} p\left(\mathcal{P}^{\circ} \circ \triangle b^{\circ} 5\right.$.}

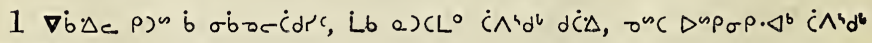
prisab; 


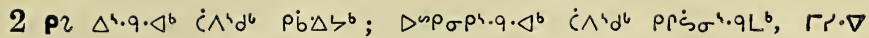
$<\rho \rho \cdot \Delta \sigma^{b} \Delta S_{x}$

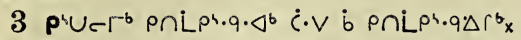

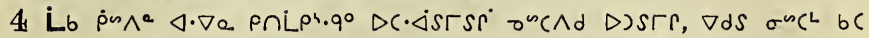

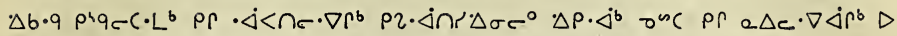

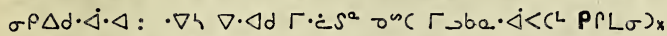

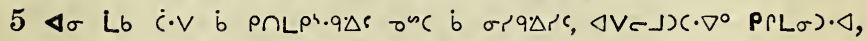

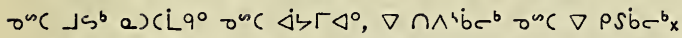

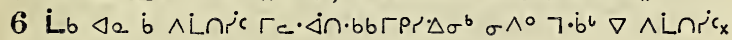

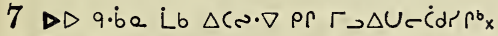

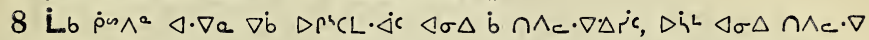

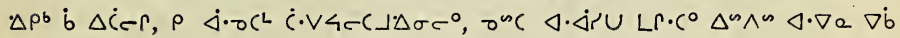
$\dot{b} \dot{\Delta} \zeta r \dot{\Delta} c_{x}$

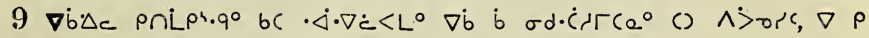
$\triangle P \Gamma d^{C} \vee b^{b} \dot{a} V \cdot \triangleleft$;

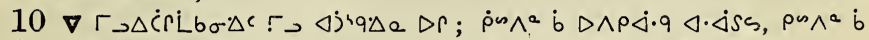

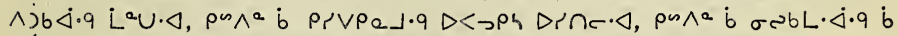

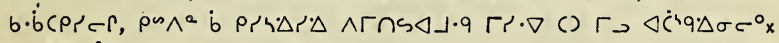

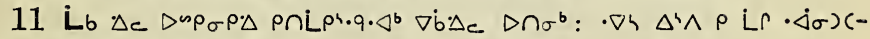
$\cdot \dot{\Delta} \cdot C \cdot \nabla \times C$ bC $\triangleright \dot{a} \vee \Gamma \cdot \Delta^{b} ;$

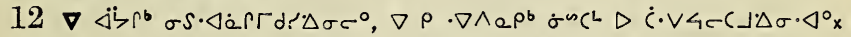

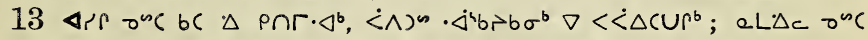

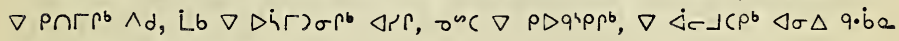

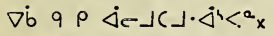

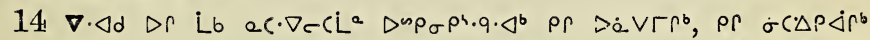

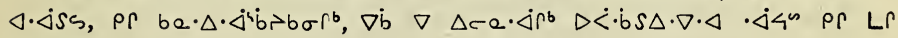
$\triangle \cdot U-\Gamma:$

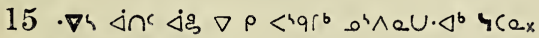

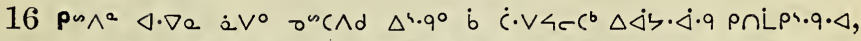

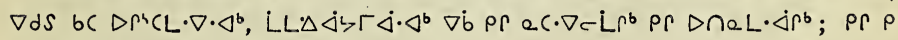
$\Delta \rho^{\top} C L \cdot \Delta \rho^{b} \triangleleft \sigma \Delta \dot{C} \cdot V \dot{b} \rho \cap L \rho^{4} \cdot q \Delta r r_{x}$

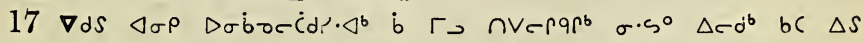

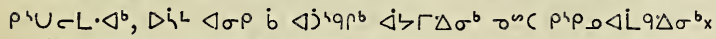

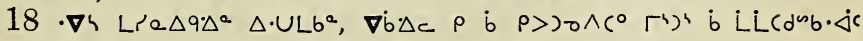

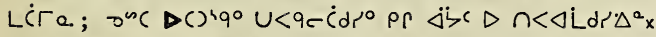

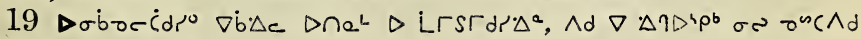
$\left.\sigma^{i)} \triangleright \cap<\dot{r}\right\lrcorner \cdot \triangleleft^{b} x$

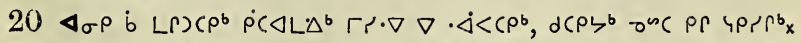

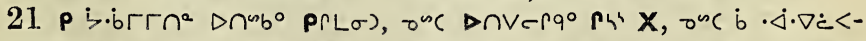

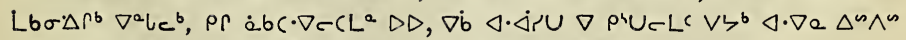
$\left.d c^{b}, \nabla \dot{b} q \cdot \dot{b}^{a} \nabla\right) C^{a} \nabla \dot{b} \nabla \Delta S \cdot b b^{b} \cap \dot{<}^{b} d \sigma c r b u^{b} \times$

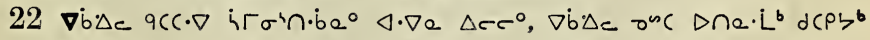

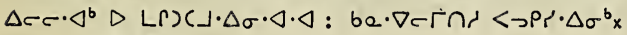




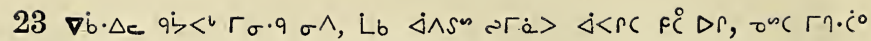
$\nabla$ ¿drín $x$

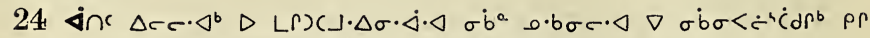

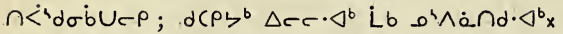

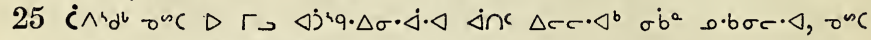

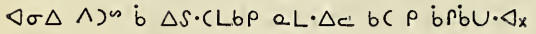

\section{$\Lambda^{4} p\left(\uparrow \circ \triangle b^{\circ} 6\right.$.}

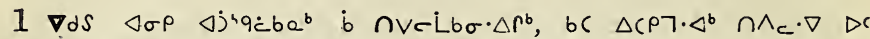

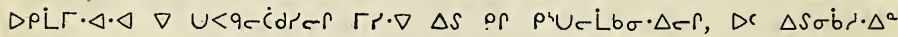

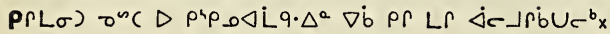

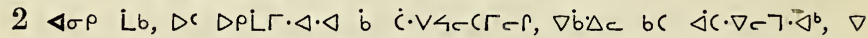

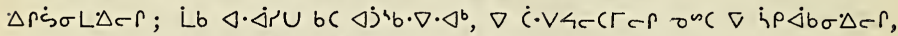

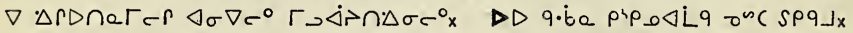

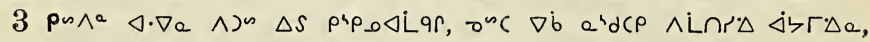

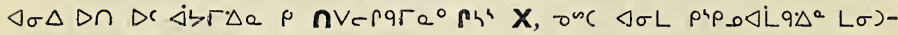
- $\Delta$ in $\Delta \sigma^{b}$ b $\Delta c^{\mathrm{b}} \mathrm{u}^{\mathrm{b}}$ :

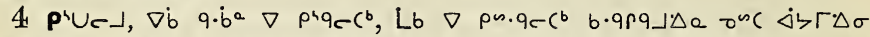

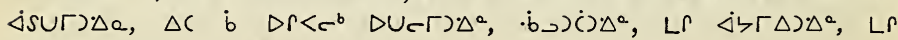
$\Delta U C r) \Delta e$,

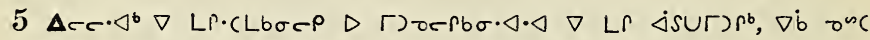

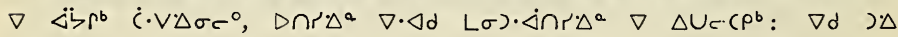
$a b S^{b} x$

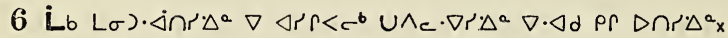

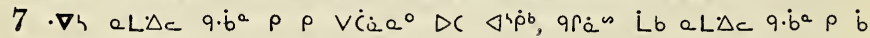
pr. $\Delta \dot{C} \dot{a} Q^{\circ} x$

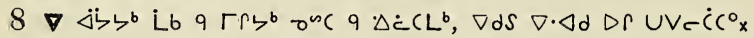

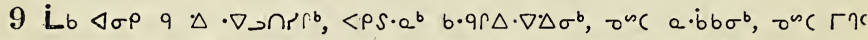

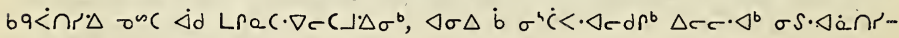
$\Delta \sigma^{b} \sigma^{\omega \prime} \mathrm{C} \cdot \triangleleft \sigma S \sigma^{-} \Delta \sigma^{b} \mathrm{x}$

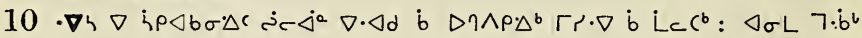

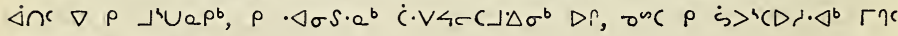
$r \Gamma_{c} \cdot \nabla r^{\prime} \Delta a \Delta r_{x}$

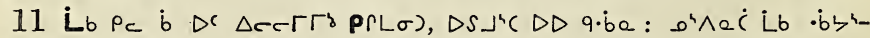

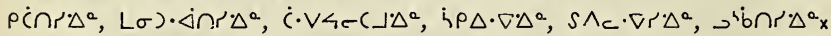

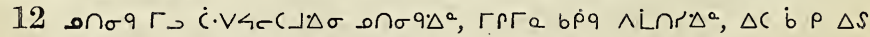

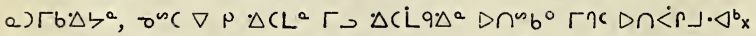

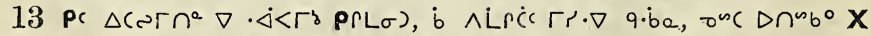

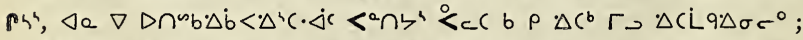

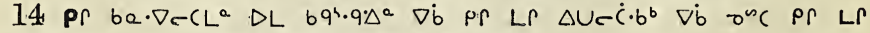

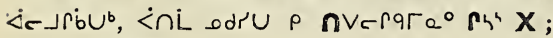
346 


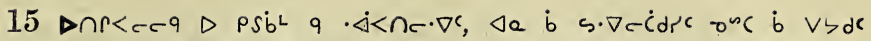

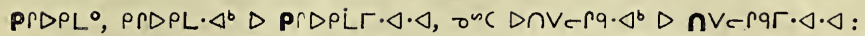

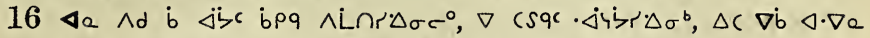
$\Delta c c^{\circ} q \rho \quad \dot{a} c^{b} ; \Delta a \quad \nabla \dot{b} b \cdot \rho \cdot \Delta<r d c \Delta c c \cdot \Delta, \nabla \dot{b}$ occ b bupcer $\rho p$

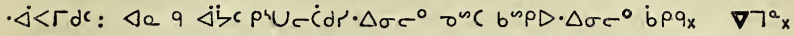

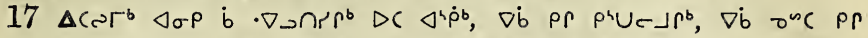

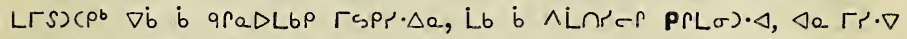

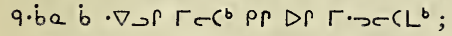

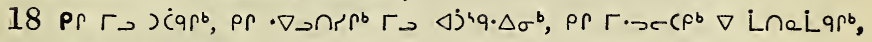

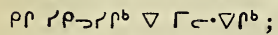

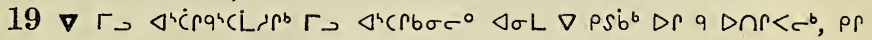
$\rho$ Lirdepb b́pq $\wedge$ L $\cap r \cdot \Delta \sigma c^{\circ}$ x

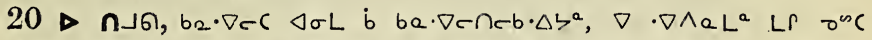

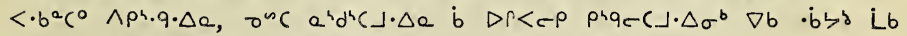
DL $\nabla \Delta S \sigma \dot{b} U^{b}$;

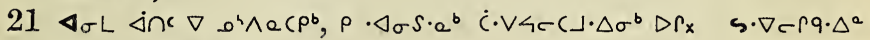
$\rho$ b $\left.\Delta \cdot \Delta \eta \cdot \Delta d^{a} x \quad \nabla\right\urcorner^{a} x$

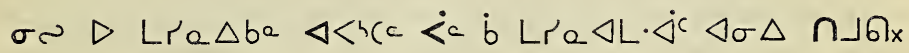

\section{$A_{h} p c r a \Delta b^{a} 1$.}

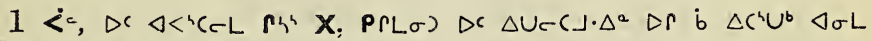

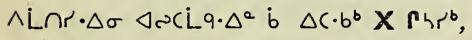

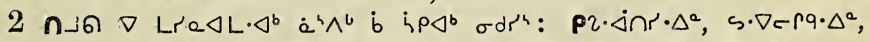

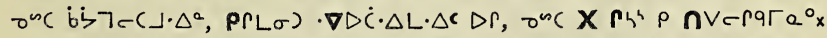

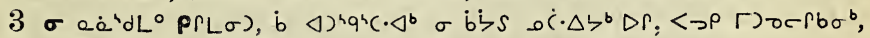

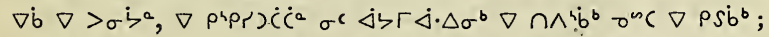

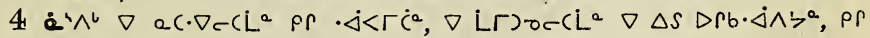
$\left.i \rho_{0} b_{b} d \zeta^{a} r_{c} \cdot \Delta c\right\lrcorner \cdot \Delta^{a}$;

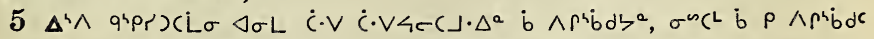

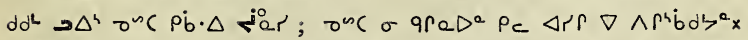

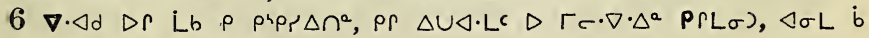
$\wedge \Gamma^{4} \dot{b} d \zeta^{a} \nabla \rho h \Gamma \sigma^{4} \cap \cdot \dot{b} \sigma \dot{C}^{a} \Delta R x$

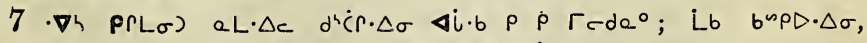

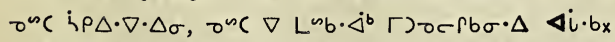

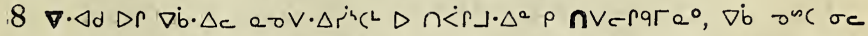

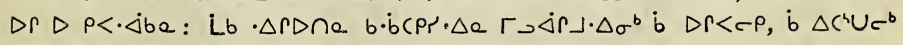
$P \Gamma L \sigma) \triangle b^{\prime \prime} P D \cdot \triangle^{\circ}$; 


\section{ซ๐ ก」ด $1,2$.}

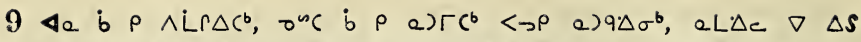

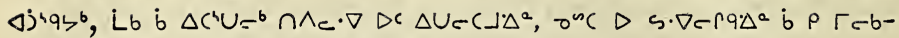

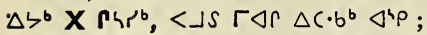

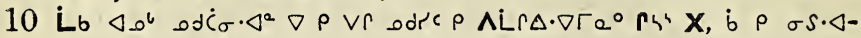

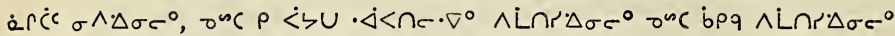
$r \rightarrow \Delta r-\Delta^{a} \Delta r:$

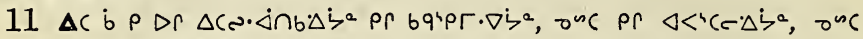

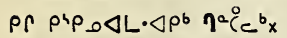

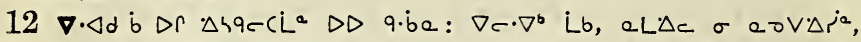

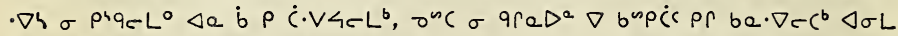

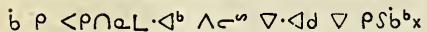

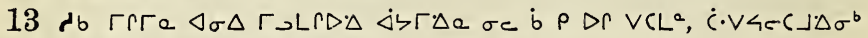

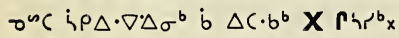

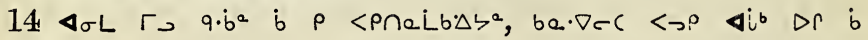
$\wedge r^{i b} C^{b} x$

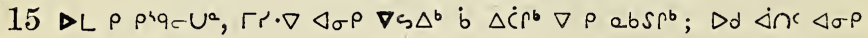

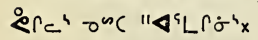

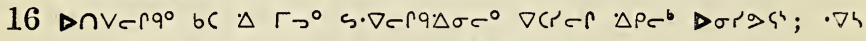

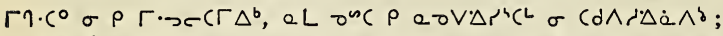

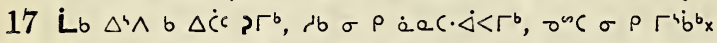

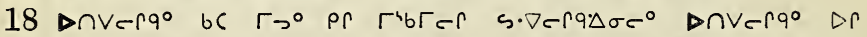

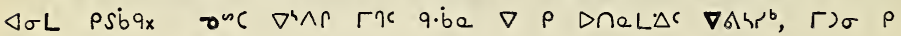
prqa $u^{2} x$

\section{$\Lambda^{\prime} p\left(P^{\circ} \triangle \Delta b^{a} 2\right.$.}

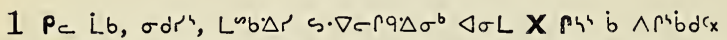

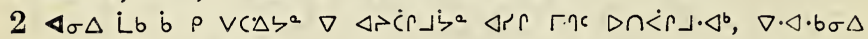

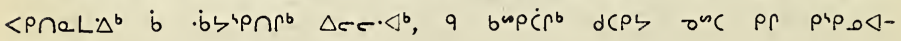
$L \cdot \dot{\Delta r^{b} x}$

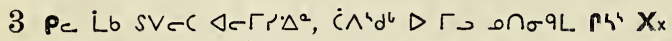

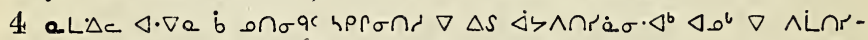
$\dot{\alpha} \sigma \cdot \Delta^{b} ;$ pr e $\Delta c \cdot \nabla \dot{\Delta c} \Delta \sigma \Delta \dot{b} \rho \cdot \dot{\Delta} \cdot \nabla \dot{c}<r d c$ pr $\nabla \rho n_{\sigma}-q \Delta c_{x}$

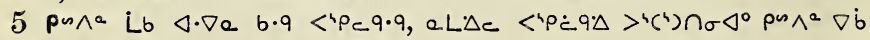
. bל b. 6 . $95 \cdot 9 x$

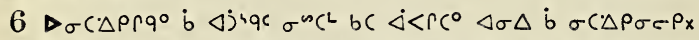

7 Lr)oec b $\Delta \cdot U^{\circ}$; $\Delta \cap V e r q^{\circ}$ Lb $\rho$ b $\Delta \Gamma c^{b}$ pr or $)\left(L^{a}\right.$

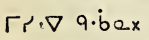

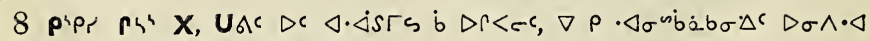
$\left.\Delta r, \nabla \triangle \cdot U L b^{b} \sigma r \Delta \Delta r\right\lrcorner \Delta^{a}:$

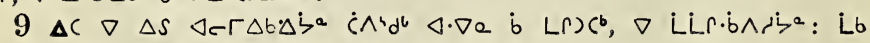

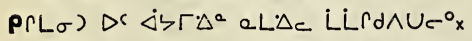

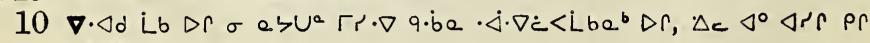
348 


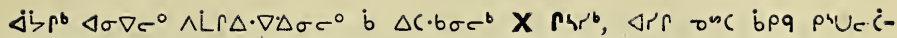
dr $\Delta \sigma \sigma^{\circ} x$

$11 \dot{C} \cdot V L b^{a} \Delta \cdot U \Delta^{2}, \cdot \nabla h$ pas $\wedge^{a} \rho \quad \Delta R \sigma \wedge L^{b}, \Delta \rho f$ fonc $\rho$ b $\Delta r \wedge L-$ กría $0^{\circ}$ :

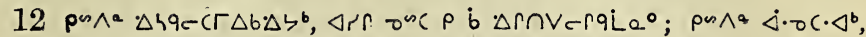
$\Delta c \operatorname{vesc} \rho$ b $\dot{\Delta} \cdot \sigma_{c} \dot{c} d a^{\circ}$ :

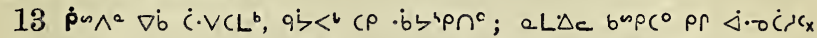

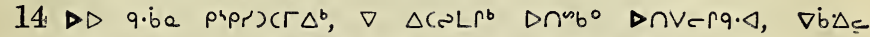

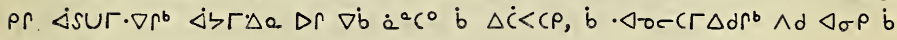
a) $\operatorname{cob}^{x}$

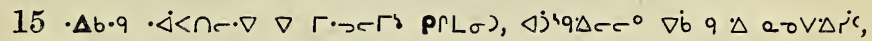

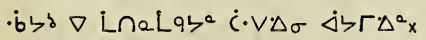

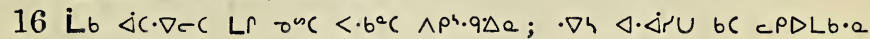
$\operatorname{Lin} \pi \cdot \Delta \sigma^{b} \Delta S_{x}$

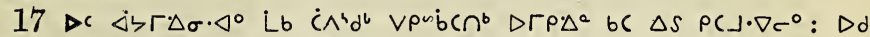

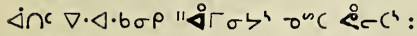

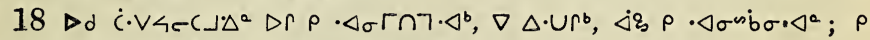

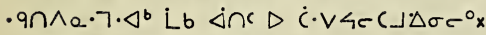

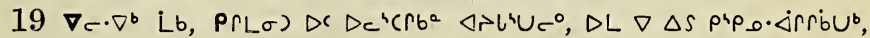

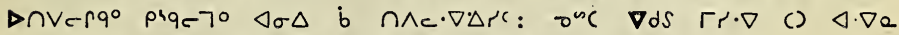

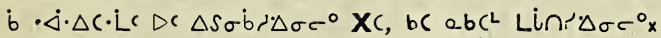

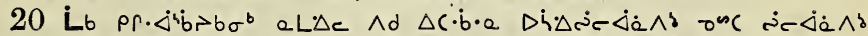

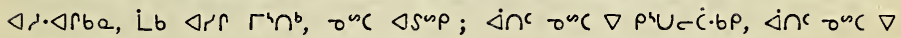
$\dot{\Delta c} \cdot \nabla-\dot{c} \cdot b p_{x}$

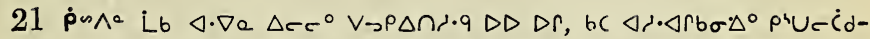

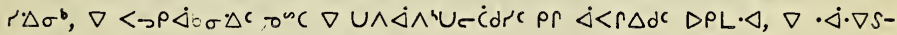

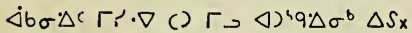

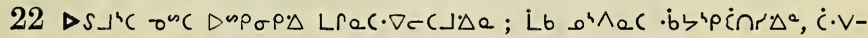

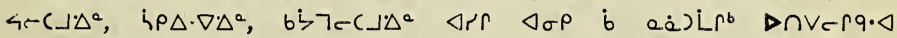
$\left\langle\rightarrow \Gamma U \triangle^{b} x\right.$

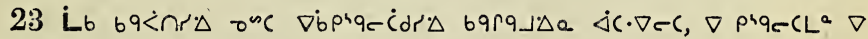
$\sigma(\triangle P \dot{C}(b P$. b $\rightarrow) \dot{C}) \triangle e$;

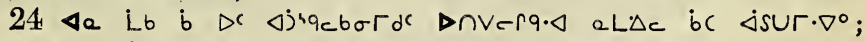

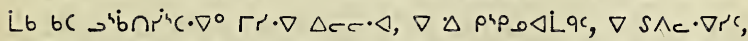

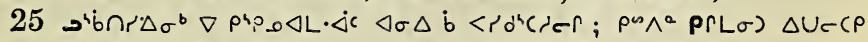

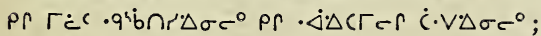

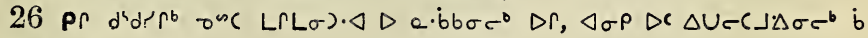
$\triangleleft \cdot \triangleleft b \cap r^{b} r_{x}$

\section{Aipcra $\Delta b^{\circ} 3$.}

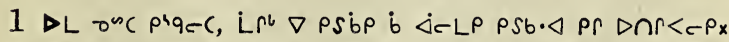

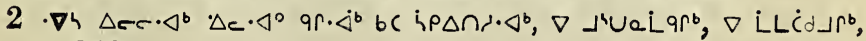
349 


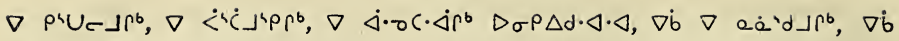
$\nabla<>\rho \rho^{b}$,

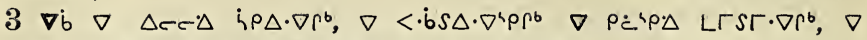
$\Delta \sigma r \rho^{b}, \nabla \triangleleft \cdot \dot{b} \cap \rho^{b}, \nabla \Delta c \cdot \nabla-\dot{L} r^{b} \Delta \sigma \Delta \dot{b} r \cdot \dot{c} \cap r e r$,

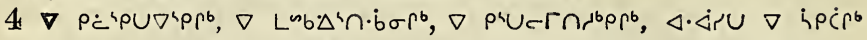

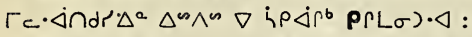

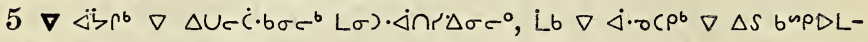
$\left.b \sigma c^{b}: \Delta d\right) \Delta$ Lb ebs $S^{b}$

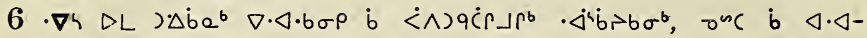

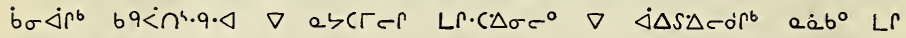
e $C \cdot \nabla \subset C\lrcorner \Delta e$,

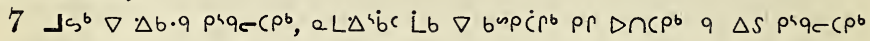
$\dot{c} \cdot \nabla \sigma \sigma^{\circ} \mathrm{x}$

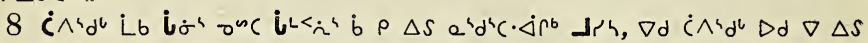

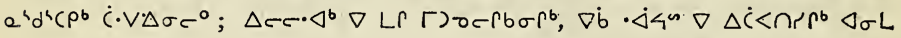
$\dot{C} \cdot \vee 4 \subset C\lrcorner \Delta^{a} \Delta \Gamma_{x}$

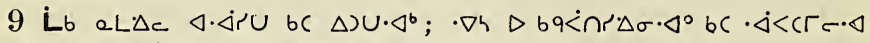
$\Gamma \cdot \nabla \Delta c c \cdot \triangleleft, \dot{c} \Lambda^{b} d^{l} \Delta c \cdot \triangleleft^{0} \nabla \rho \Delta \rho_{\sigma} \sigma c^{b} x$

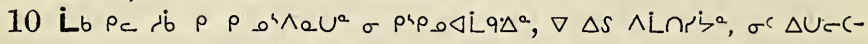

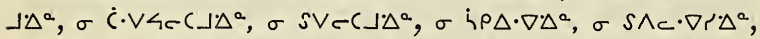

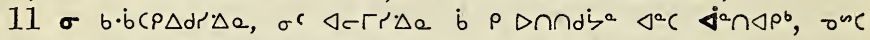

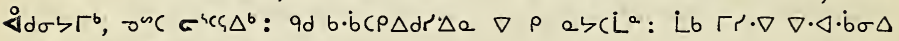
$\Delta r \Delta \cap V \in r^{\circ} \sigma \rho \cdot \Delta \cdot b r \Delta^{b} x$

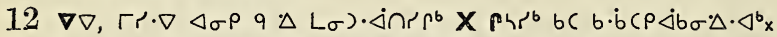

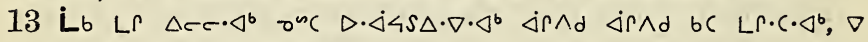

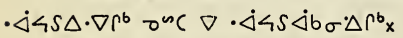

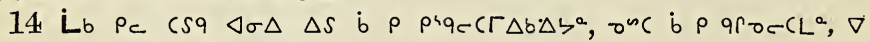
prqc CLa $\triangleleft \cdot \nabla$ e. iे p prpo $\Delta$ L's;

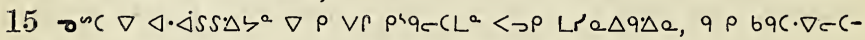

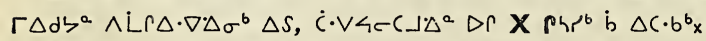

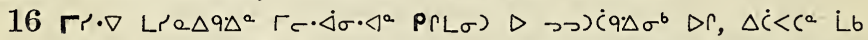

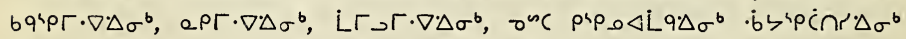
$\Delta S:$

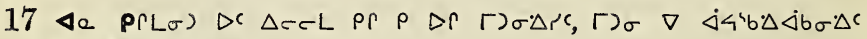

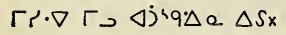

\section{A p p r e $\triangle b^{a} 4$.}

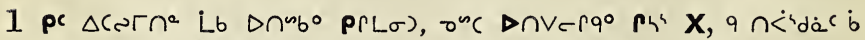

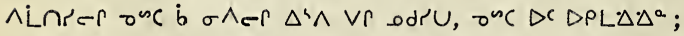

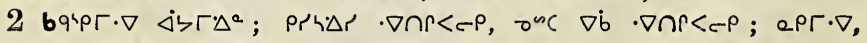

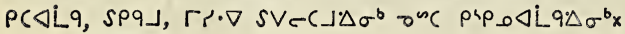

\section{0}




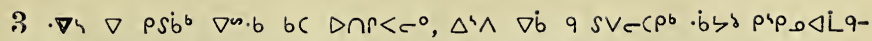

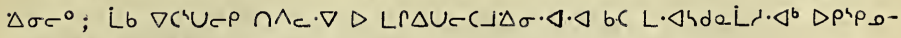
$\triangleleft L q \cdot \triangleleft, \nabla$ P५P) $\triangleleft q \rho^{b}$ :

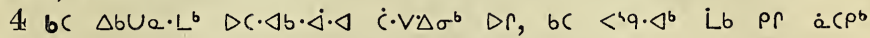
$\Delta c \rightarrow b e x$

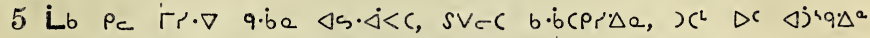

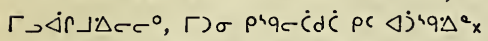

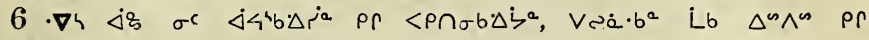
9) Uं००

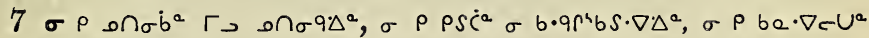
$\triangle \sigma L \dot{C} \cdot V 4 C C\lrcorner \Delta^{a}$ :

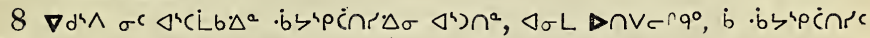

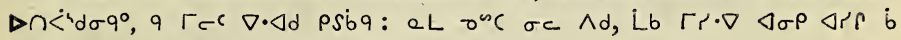
$\left\langle P C \cdot \Delta \beta^{b} D\right.$ odr. $\Delta^{a} x$

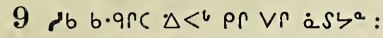

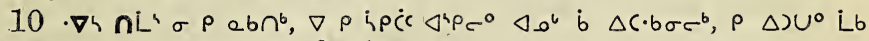

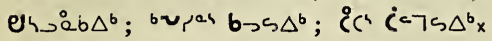

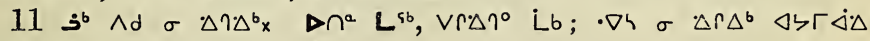
$\Delta)^{4} q \Delta \sigma^{b} x$

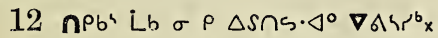

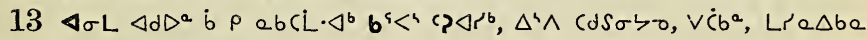

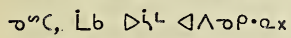

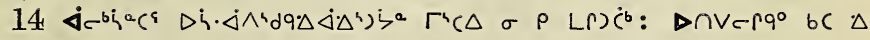
$n<\Delta L \cdot \nabla^{\circ}$ b $\Delta S \Delta j 49 c r$ :

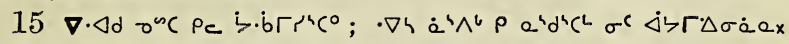

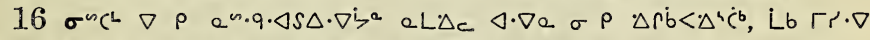

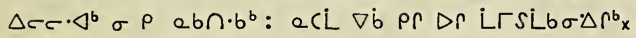

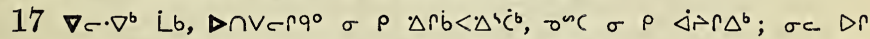

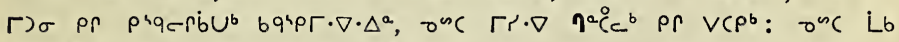
$\left.\Gamma S \wedge S^{\circ} D\right) \sigma^{b} \sigma \rho D r \cdot \Delta \cdot b r \Delta b \cdot \Delta^{a} x$

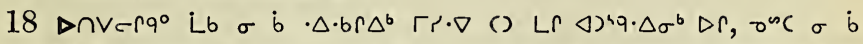

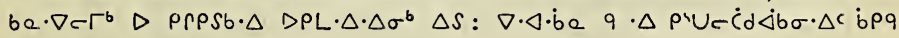
osc bpqx $\nabla 7^{a x}$

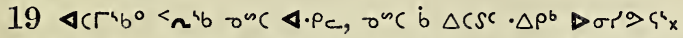

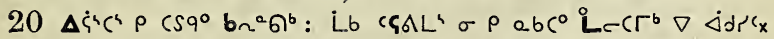

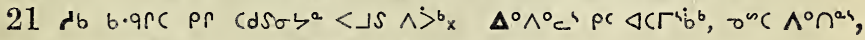

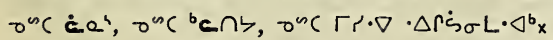

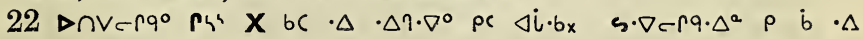
$\cdot \Delta \eta \cdot \Delta d^{a} \times \quad \nabla 7^{a} \times$ 


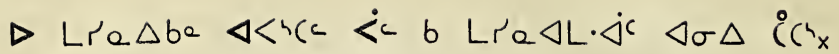

\section{Ápcre $\Delta b^{2} 1$.}

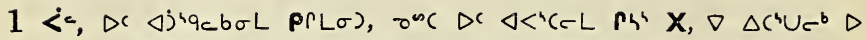

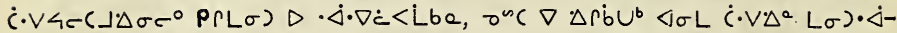
ni. $\Delta \sigma^{b} \dot{b} \Delta C^{b} U^{b}$;

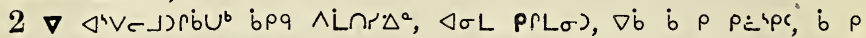
$\triangle \sim C L$ L $q<<J S \rho$ L. $<\Delta^{4} \rho \cdot \Delta^{b}:$

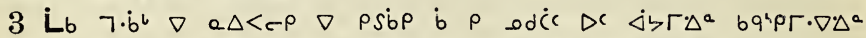

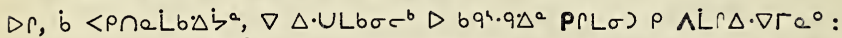

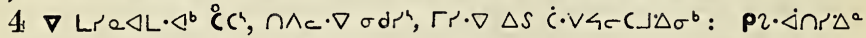

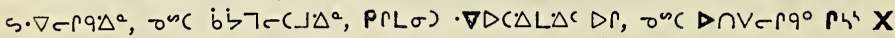
b $\Lambda$ LL $\triangle \cdot \nabla \Gamma e^{\circ}{ }^{x}$

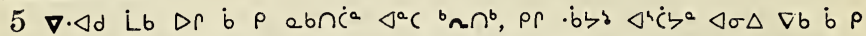

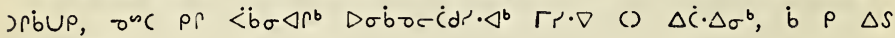
DePTCं ;

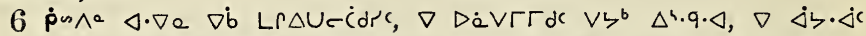

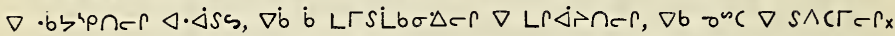

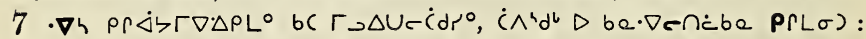

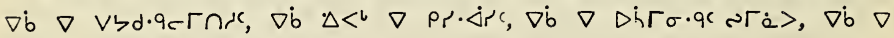
on

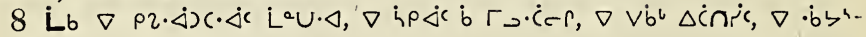

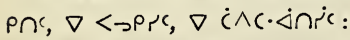

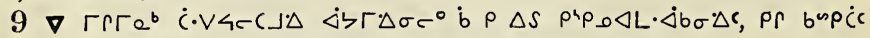

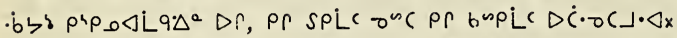

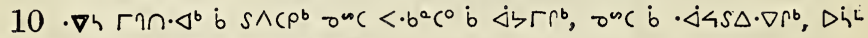

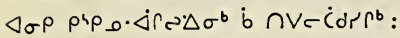

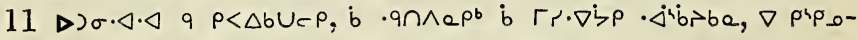

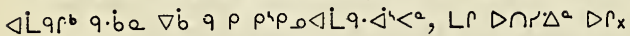

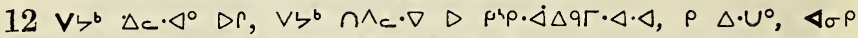

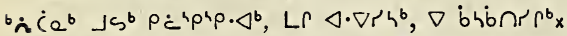

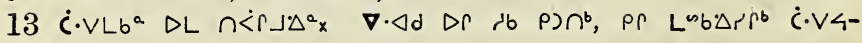
-(\lrcorner$\Delta \sigma^{b} ;$

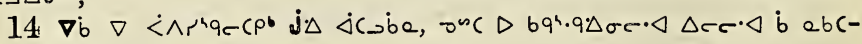
$\operatorname{rer} \dot{C} \cdot V \cdot \Delta \sigma \sigma^{\circ} x$

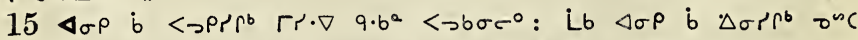

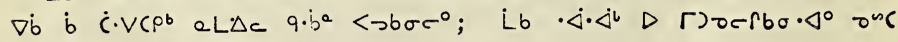
$\Delta U \Delta \cdot \Delta^{\circ} \Delta r<r c^{\circ} x$

352 


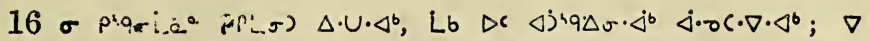

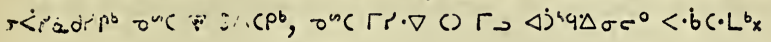

\section{Ápcra $\triangle b^{a} 2$.}

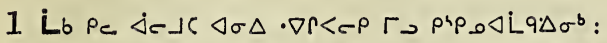

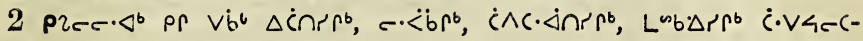
$J \Delta \sigma^{b}, i \rho \Delta \cdot \nabla \Delta \sigma^{b}, s \wedge c \cdot \nabla r^{b} \Delta \sigma^{b} x$

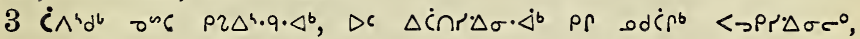

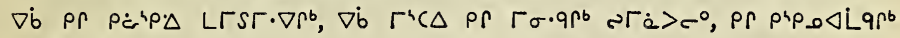
r. $q \cdot \dot{b e}$ :

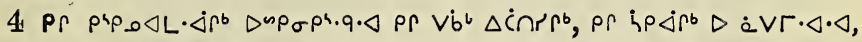

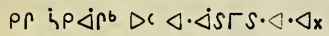

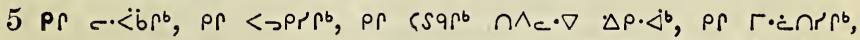

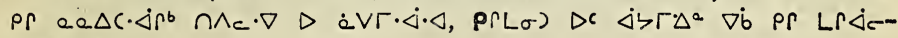
$\mathrm{Jrib} U \mathrm{c}^{\mathrm{s} x}$

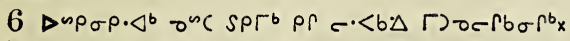

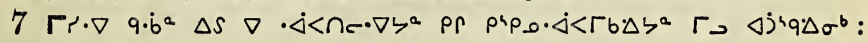

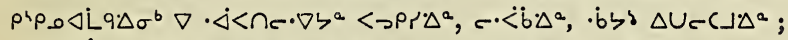

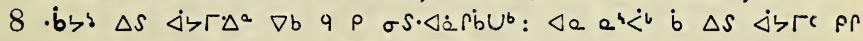

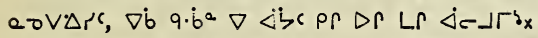

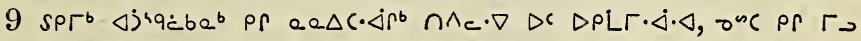

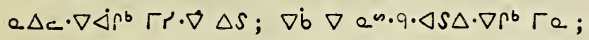

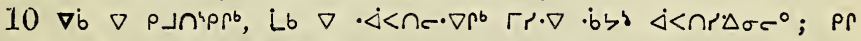

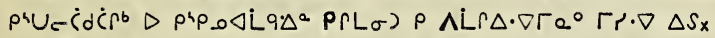

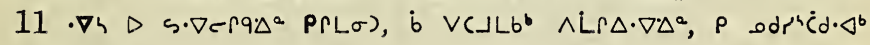
$r+\cdot \nabla \Delta r \cdot \cdot \Delta^{b}$,

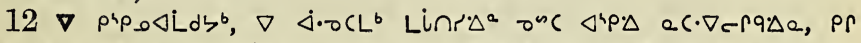

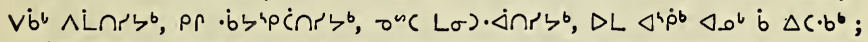

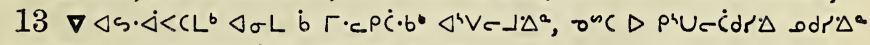

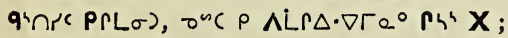

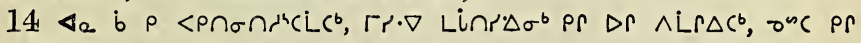

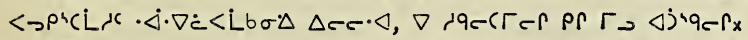

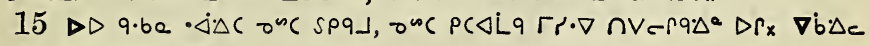
$\triangleleft \cdot \nabla e \rho$ b $\left\langle c \cdot \nabla e \Gamma^{b} x\right.$

\section{A'pcre $\Delta b^{\circ} 3$.}

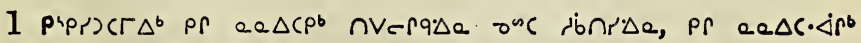

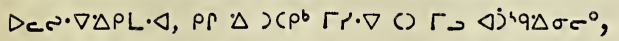

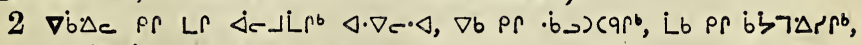

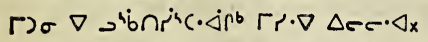

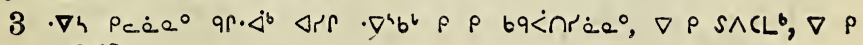
353 


\section{¿c' 3.}

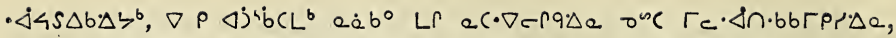

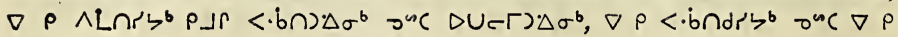
$<\cdot b n>)^{b} x$

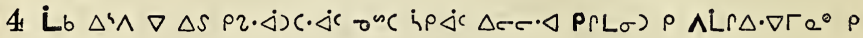
$\rho \cdot b \sigma \sigma^{\circ}$;

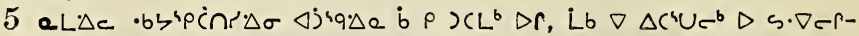

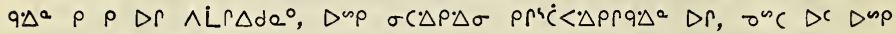

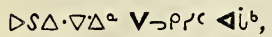

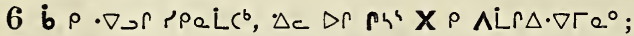

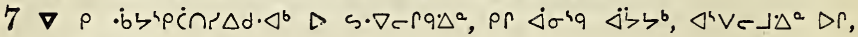
bpq $\wedge$ L $\operatorname{R} \cdot \Delta^{a} x$

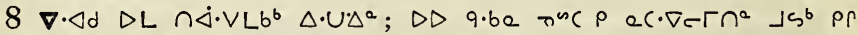

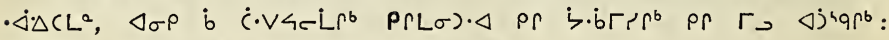

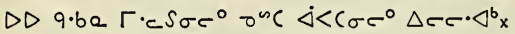

9 Lb $\nabla \dot{b}$ N

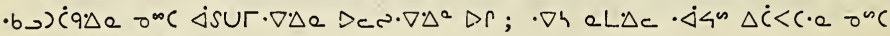
$<\cdot b^{\circ} c^{\circ} \Delta U-\dot{c} \cdot b \cdot a_{x}$

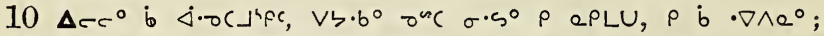

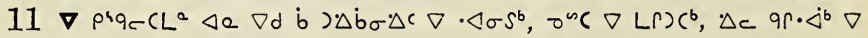

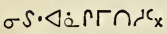

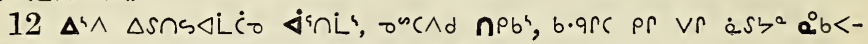

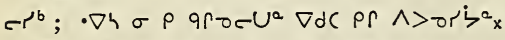

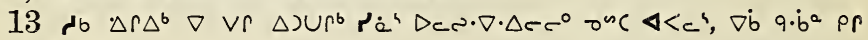
.PCL $r^{b} x$

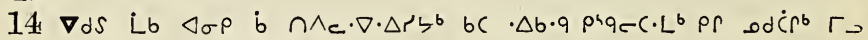
$\Delta j 49 \cdot \Delta e \nabla \Delta S$ e $\Delta<c \rho, \nabla b$ pr $\nabla b \Gamma \sigma S \cdot \Delta r^{b} \times$

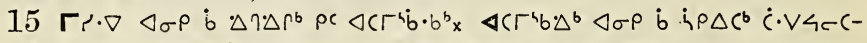

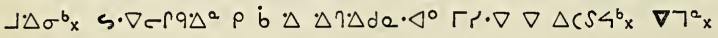

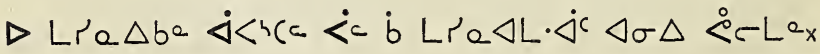

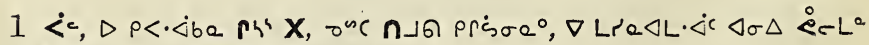

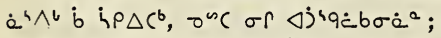

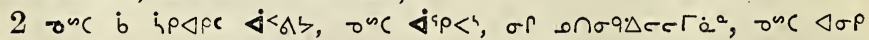

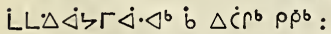

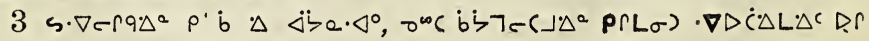

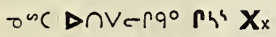

354 


\section{écLax}

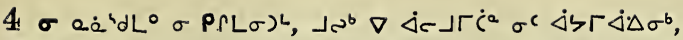

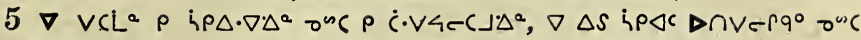
$\Gamma r \cdot \nabla D<>P S^{2} ;$

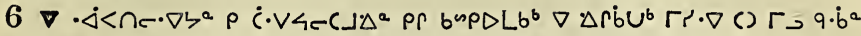

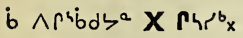

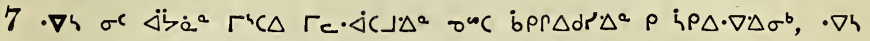

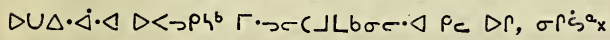

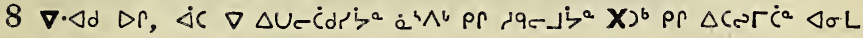
b $\propto \Delta<c^{b}$,

$9 \nabla-\cdot \nabla^{b}$ Lb $i \rho \Delta \cdot \nabla \Delta^{a} \quad \Delta r, a \cdot \Delta^{b} \rho<d h e r \cap^{a}, \nabla \Delta \Delta \zeta^{a} \varangle a<c b$

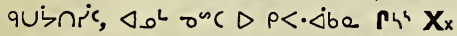

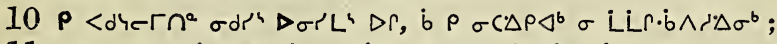

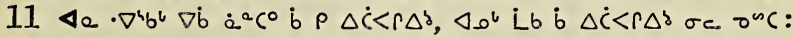

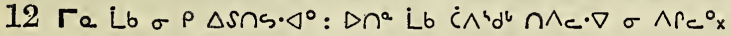

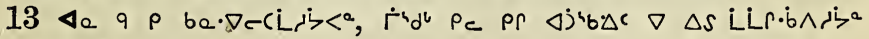
$\Gamma \rightarrow \Delta r\lrcorner \Delta \sigma^{b} x$

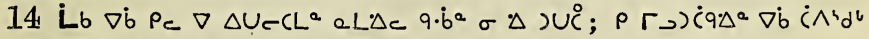

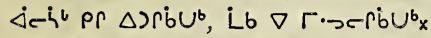

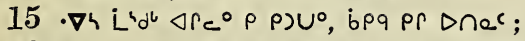

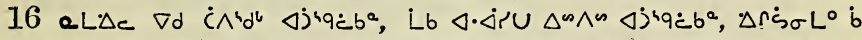

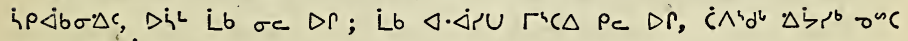
$\Delta \cap \vee-\Gamma q^{b}$ !

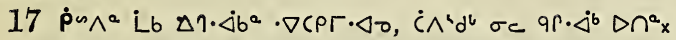

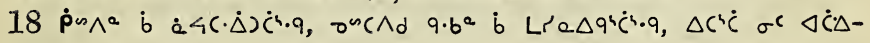
LRe $\Delta b \sigma^{\mathrm{b}}$ :

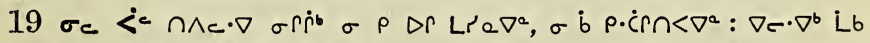

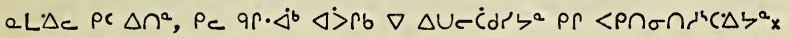

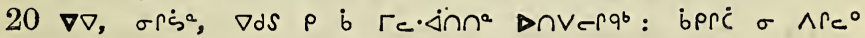

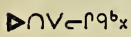

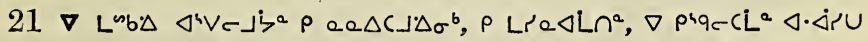
PP $) L^{a} \Delta \omega \wedge \omega \Delta \sigma L \dot{b} \Delta \cdot U^{\circ} \zeta^{a} x$

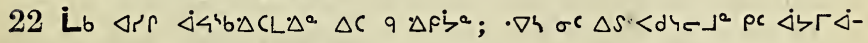
$\Delta \sigma \cdot \Delta \cdot \triangleleft \Delta r, \sigma c \rho r<P \cap a L b \Delta \zeta^{b} x$

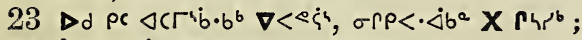

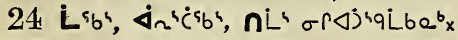

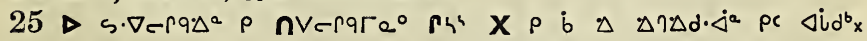
$\nabla 7^{2} x$ 
$\triangleright$ Lre $\Delta b e$ j<ice <c b Lre $\Delta L \cdot \dot{<c} " \Delta s \dot{p} \cdot \Delta_{x}$

A $p$ cre $\triangle b^{\circ} .1$.

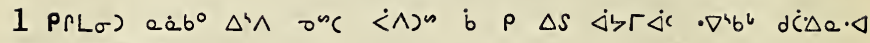

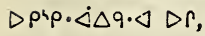

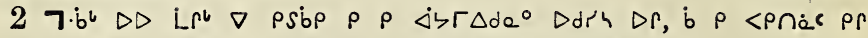

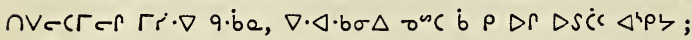

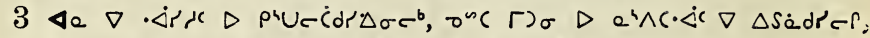

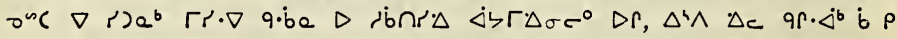

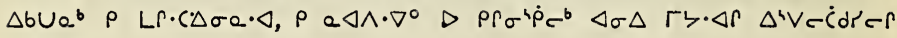
$\triangle M \wedge r^{b} ;$

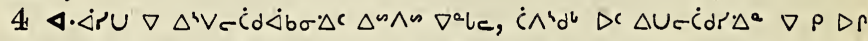

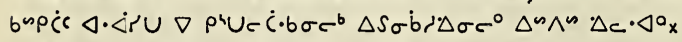

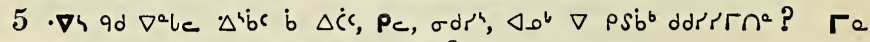
Lb, $\sigma \dot{b} \Delta \dot{C} \Delta \Gamma^{b}, \Delta c \tau^{n} c \sigma$ b $D d r T L^{\circ}$ ?

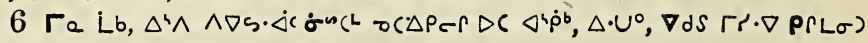
$\Delta c \nabla^{a} l e L$ bc $a \cdot \Delta \dot{p}^{h} C \cdot \nabla c \cdot \Delta x$

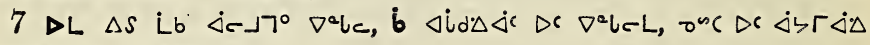
बj rqंba b $\cdot \Delta c U \Delta \Delta c_{x}$

8 Lb $\triangleright d r R L \cdot \triangleleft \Delta U^{\circ}$, pe $\left.D P L \cdot \triangleleft \wedge \triangle a, \triangleright p \rho L \sigma\right)$, bpq o"c bpq bC

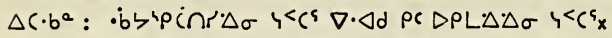

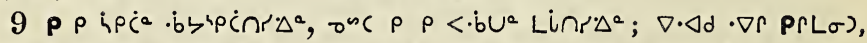

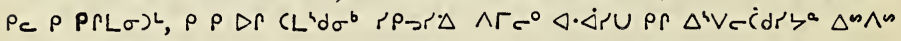
$\rho \Delta \eta \cdot \dot{\Delta b a^{b} x}$

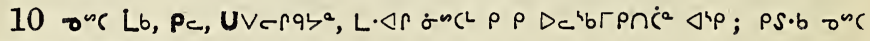
PRPS P DSCLb.ex

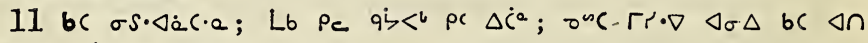
$\dot{b}\rangle s \cdot \triangleleft \cdot a \dot{C} \wedge^{\prime} d^{l} \triangleleft d \nabla^{\circ} ;$

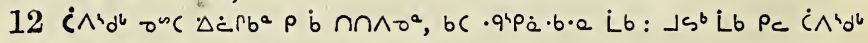

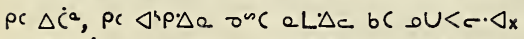

$13 \dot{L}_{b}$ 9d $\nabla^{a} l_{c} \Delta b \dot{b} \dot{b} \Delta \dot{c} c, \varangle \wedge \rho_{c} \sigma \rho \rho \sigma^{4} \dot{p} b, \dot{K} \cap \rho \Delta S \Delta \cdot b \cdot \nabla \triangleleft \sigma \rho$

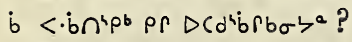

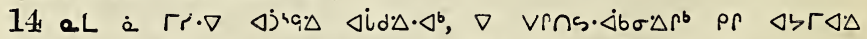

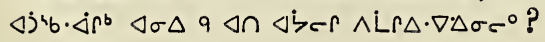

A pera $\triangle b^{2} 2$.

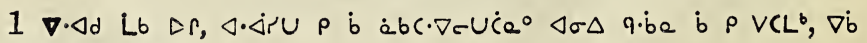
$\triangle$ bi $p r$ asdeLbx 


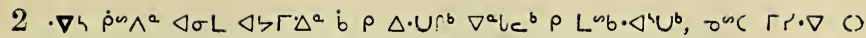

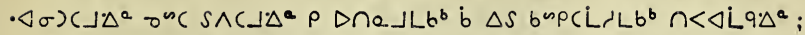

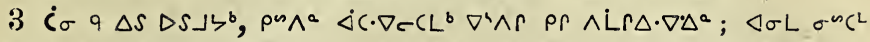

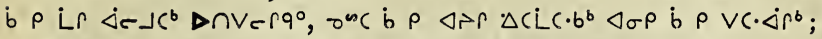

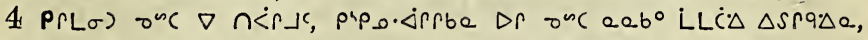

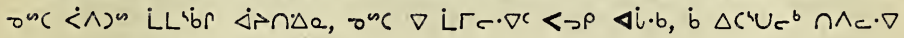
$\Delta C \Delta U C C \cdot \Delta^{\circ}$ ?

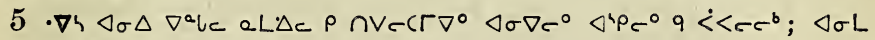
b $\dot{\Delta} \cdot J C L^{b} x$

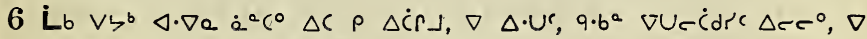

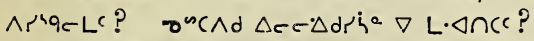

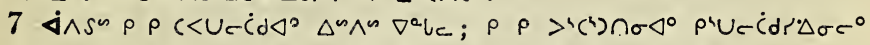

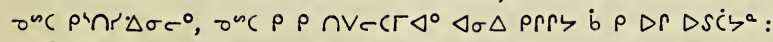

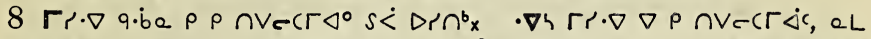

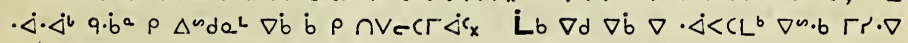
$q \cdot \dot{b} a \nabla \cap V e c r \Delta b \sigma \cdot \Delta c_{x}$

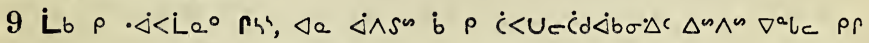

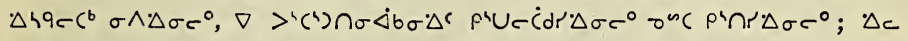

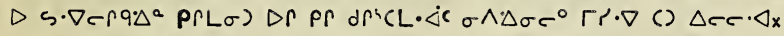

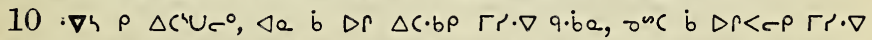

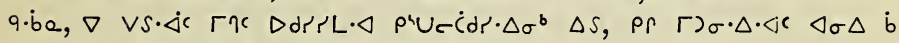

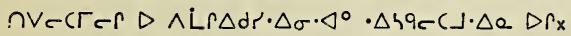

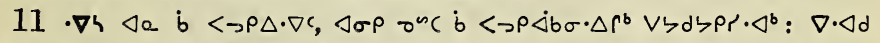
Dr aL. $\Delta c$ a $\sigma V \cdot \Delta r^{\circ} \sigma r \dot{s} a^{b}$ pr $\Delta S \sigma \dot{b} \dot{c}$,

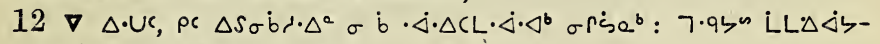

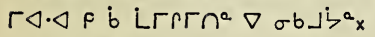

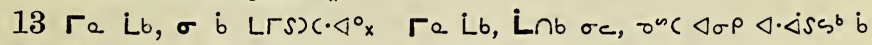
$p \Gamma e(P R L \sigma)_{x}$

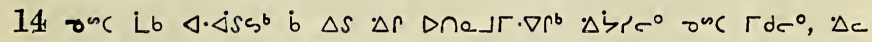

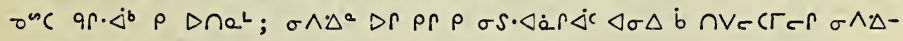
$\sigma \sigma^{\circ}, \nabla \cdot \triangleleft d L(L \sigma)$;

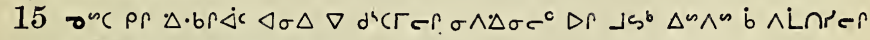

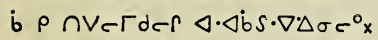

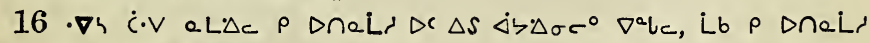

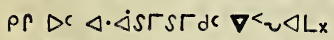

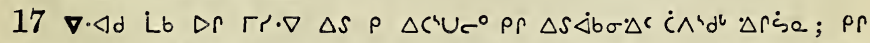

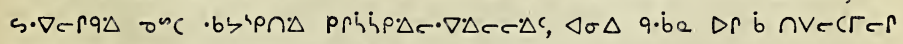

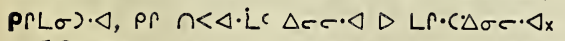

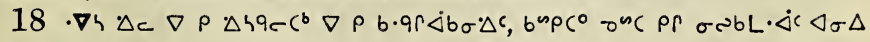
b $b \cdot 9 r \Delta b \sigma \Delta-r x$ 


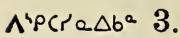

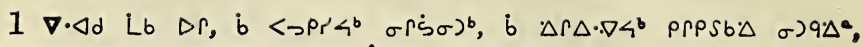

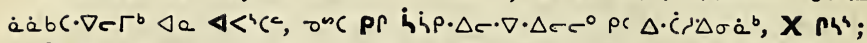

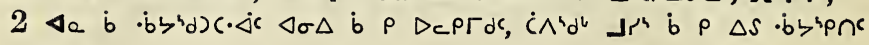
b) $\Delta C s c \Delta p b_{x}$

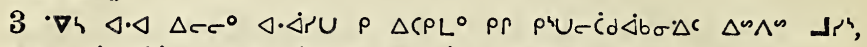

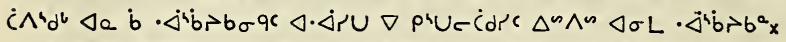

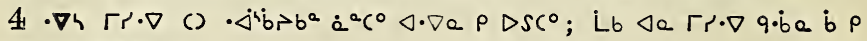
$D S(C \nabla \cdot \Delta d \text { P } P L \sigma)_{x}$

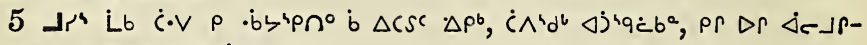

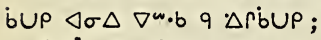

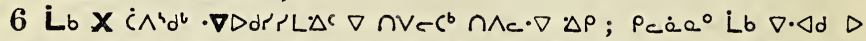

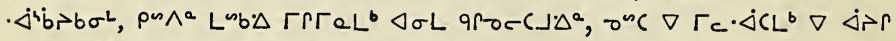

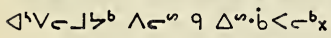

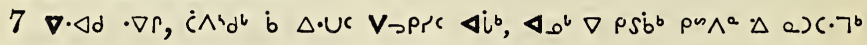
b) $\Delta \cdot u r$,

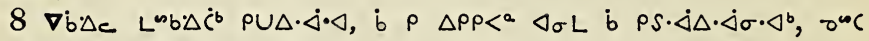

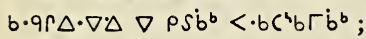

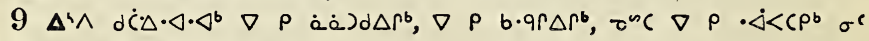
$\Delta j \cdot q \cdot \Delta e$ or(a $) \wedge\rangle \cdot e^{x}$

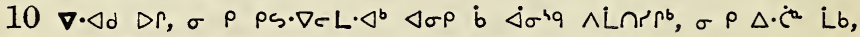

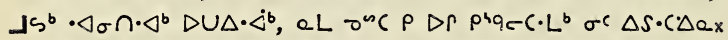

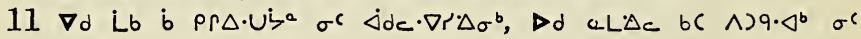
$\dot{\Delta \cdot s r \cdot \Delta \sigma x}$

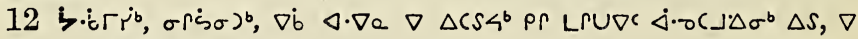

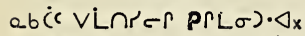

13 Lb 75b b9?

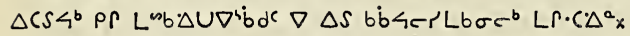

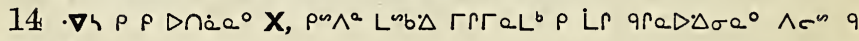
$\Delta n \cdot b<c^{b} ;$

$\left.157 \cdot \dot{b}^{b} \nabla \Delta \cdot \dot{c} \sigma^{\circ} \Delta^{b}, \Delta \sigma^{b} \nabla \rho S \dot{b}^{b}, \rho a \wedge^{a} \Delta a\right) c \cdot 7^{b} \dot{b} \Delta \cdot U^{c}, \nabla \dot{b} \Delta c$

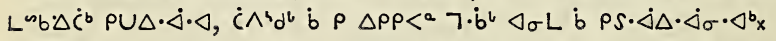

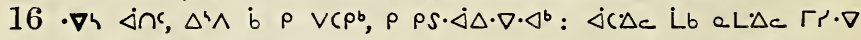
$\triangle \sigma \rho \Delta r^{<}<\Lambda^{\circ}$ b $\left.\rho \nabla\right)\left(\Delta d r^{b}\right]$

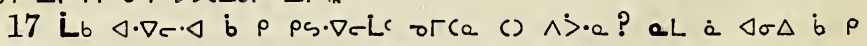

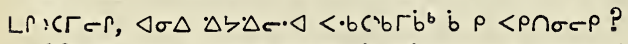

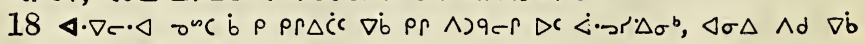
b $\rho \dot{C} \cdot V C r<r$ ?

$19 \rho \cdot \dot{<}\left\langle U \dot{a} c^{\circ}\right.$ Lb $\left.\nabla \dot{b} \nabla \rho \wedge\right) q \rho^{b} \nabla \dot{b} \nabla \rho \dot{c} \cdot V\left(\rho b^{b}\right.$ 


\section{$\Lambda^{\top} p c ? a \Delta b^{2} 4$.}

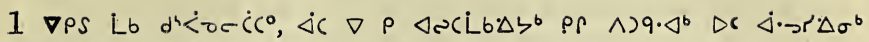
$\triangle \cdot \nabla \sigma b a^{b} \nabla \triangle C s \zeta^{b} P f$. $U^{m b} b p^{b}$

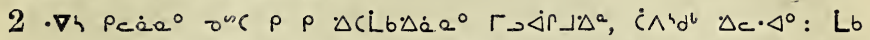

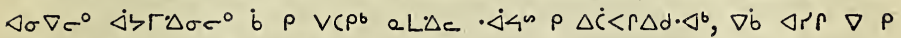
$\dot{c} \cdot V A_{C} C \rho b \quad \nabla_{\sigma} \rho$ b $\rho \vee C \rho^{b}{ }_{x}$

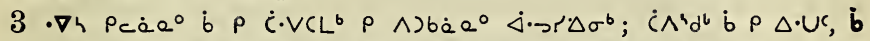

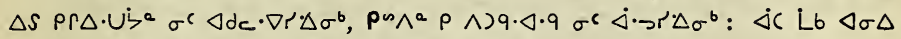

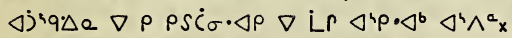

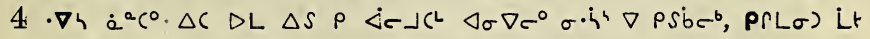

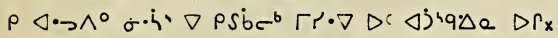

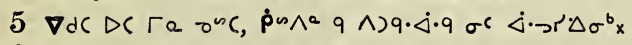

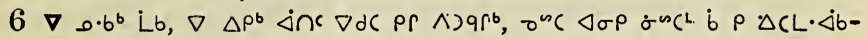

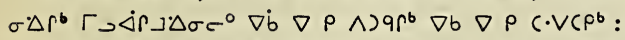

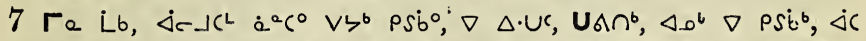

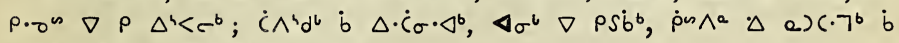
$\Delta \cdot U c, \nabla \dot{b} \Delta c L^{\omega b} \Delta \cdot \dot{C}^{b}$ PU $\Delta \cdot \dot{j} \cdot \Delta_{x}$

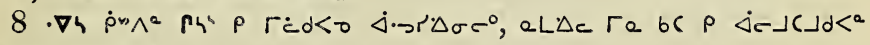
$d C P C^{\circ} \nabla P S \operatorname{Sic} C^{b}$

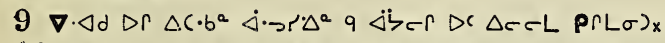

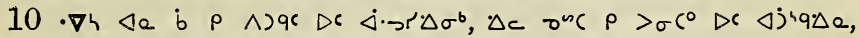

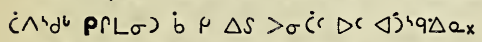

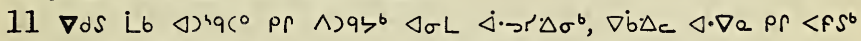
$\triangle \sigma L \dot{b} P \Delta S<P S \dot{a} \sigma \cdot \triangleleft^{b} \nabla \dot{b} \nabla \rho \dot{C} \cdot V 4 c r \dot{b} U^{b} x$

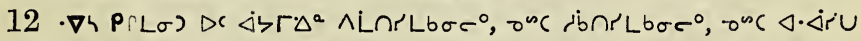

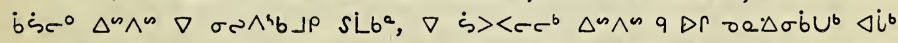

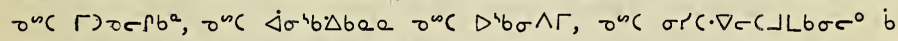

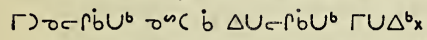

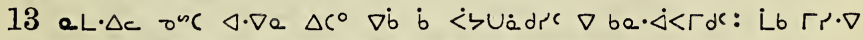

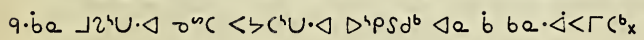

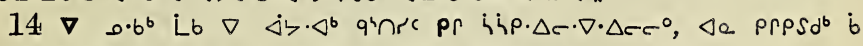

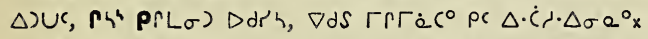

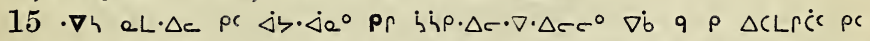

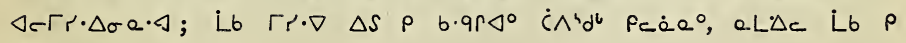
$L \Gamma)\left(L^{2} x\right.$

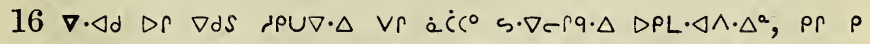

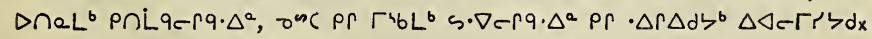

\section{$\Lambda^{4} p c r a \triangle b^{a} 5$.}

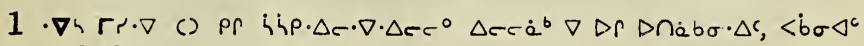
359 


\section{$" \Delta<j \cdot \triangleleft 5,6$.}

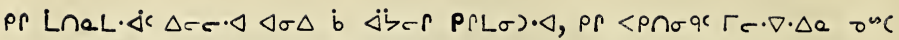
i i $p \cdot \Delta \tau \cdot \nabla \cdot \Delta e$ LS) (\lrcorner$\cdot \Delta e \Delta R$ :

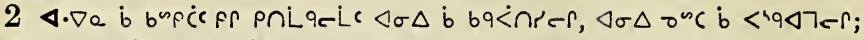

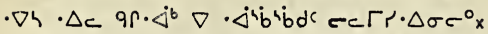

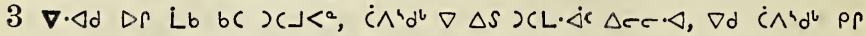

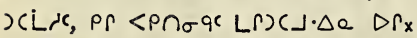

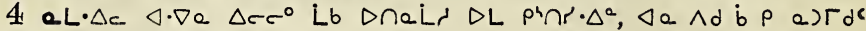
P $L \sigma) \cdot \triangleleft, \dot{C} \wedge^{4} d^{l} \nabla \zeta^{2} x$

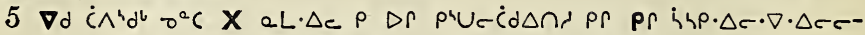

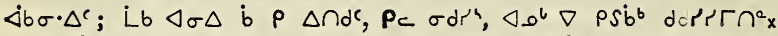

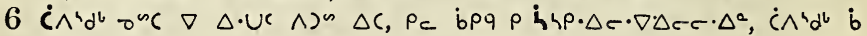

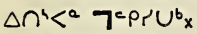

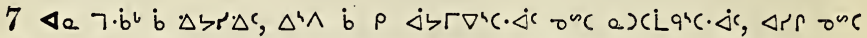

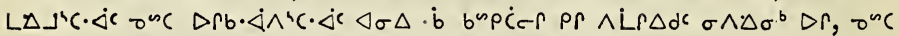

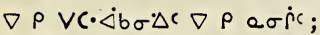

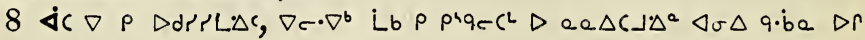

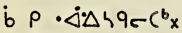

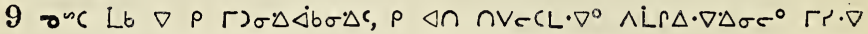
$\Delta \sigma \Delta$ b e $\Delta \Delta \dot{c} d^{c}$;

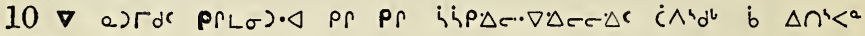
T<prubx

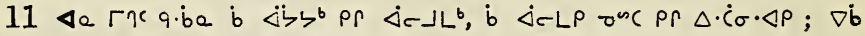
$\nabla<\dot{<} \cup \vee C 7^{b} x$

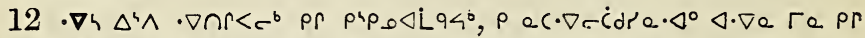

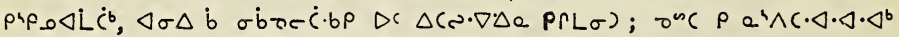

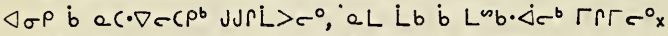

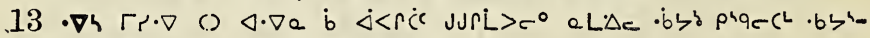

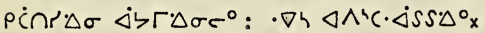

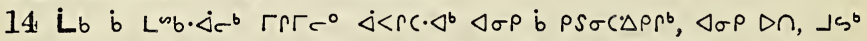

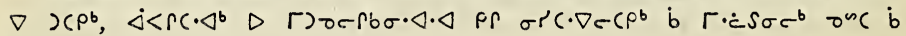
$\dot{L} \dot{c}\left(\sigma \sigma^{b} x\right.$

\section{$A^{4} p c r a \Delta b^{\circ} 6$.}

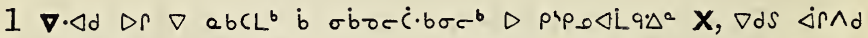

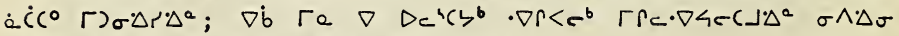
$\Delta j 4 q \Delta \sigma^{b} \Delta r, \sigma^{\infty}(\nabla \dot{C} \cdot V 4-L b \sigma \Delta(\rho) L \sigma)$;

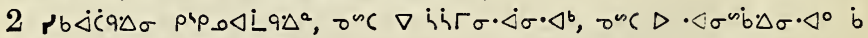
$\sigma \wedge r^{b}, \sigma^{n C}$ b $p q \cap \dot{<}^{\prime} d \sigma q \Delta^{a} x$

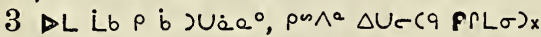

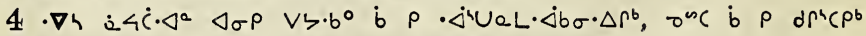

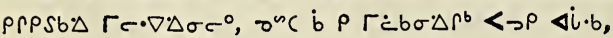

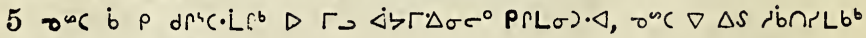
$\triangleleft \sigma L \triangleleft^{4} p q<<c^{b}$, 


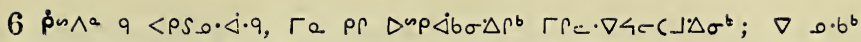

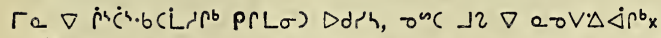

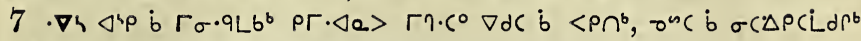
$\sigma\left(\Delta P \dot{C} b \sigma-s \triangleleft \sigma \rho \dot{b}\right.$ orCं $\left.{ }^{b}, \Gamma \cdot C \cdot P(L) P R L \sigma\right)$ :

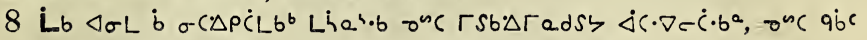

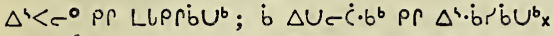

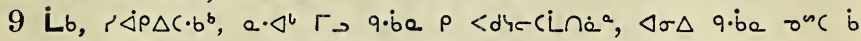

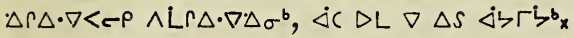

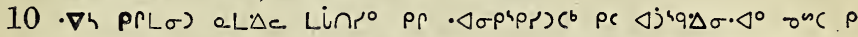

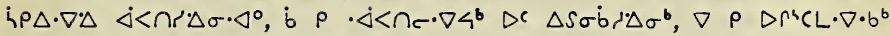

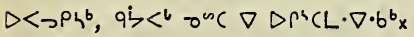

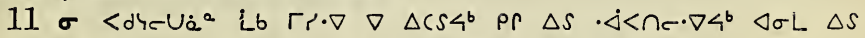

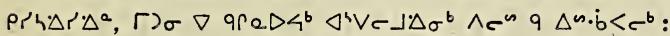

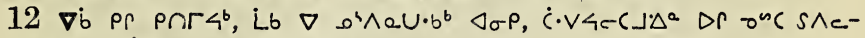

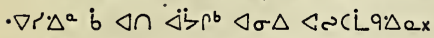

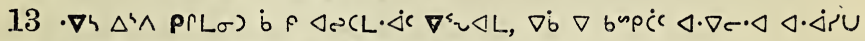

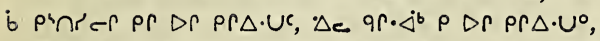

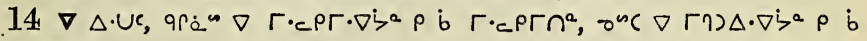
$\Gamma \eta) \Delta n^{2} \times$

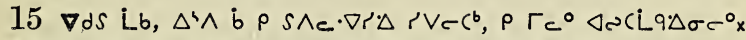

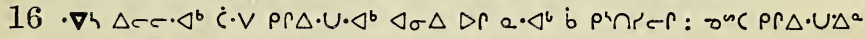

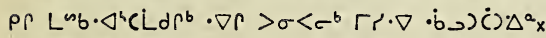

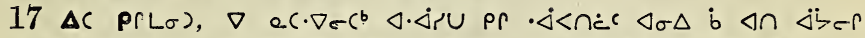

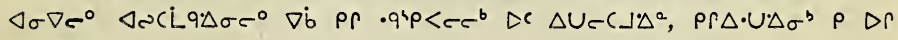
$\triangle r C^{\circ}$;

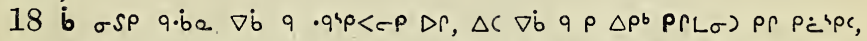

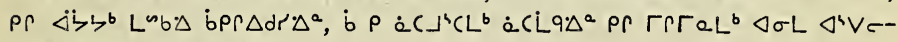

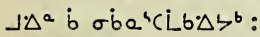

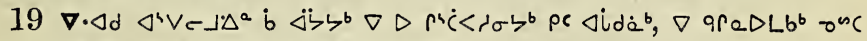
$\nabla L^{a b} \cdot \Delta$ bUb, चnc b $\left.\wedge\right) q L b^{b} \wedge r \Delta d \Delta b \sigma^{b}$;

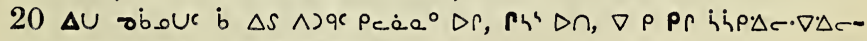

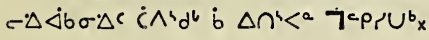

\section{A $p c r a \triangle b^{a} 7$.}

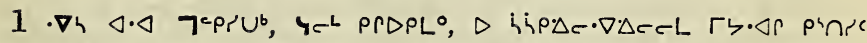

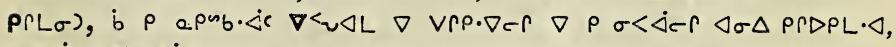
Onc b P T.cPlc;

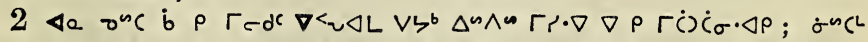

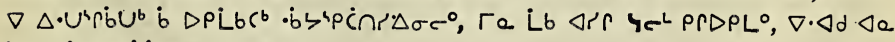
b $\triangle P L b C^{b}$ bं 7 CCL $\triangle \sigma \sigma^{\circ}$;

$3 \nabla \dot{b} \nabla \nabla c \Delta c, \nabla \dot{b} \nabla \Delta \dot{b} \Delta c, \nabla b \nabla \dot{j}^{4} 9 \Delta \dot{c} r \Gamma c, \nabla \dot{b} \nabla$ Lr $\Delta P S \dot{b} \Gamma c, \nabla \dot{b}$ 361 


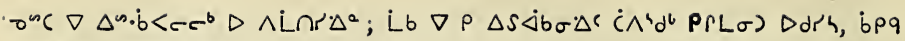
$\nabla$ iT $\Delta \cdot \Delta r \cdot \nabla \Delta r-\Delta c_{x}$

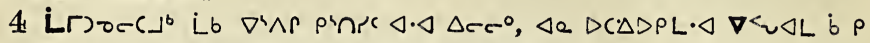

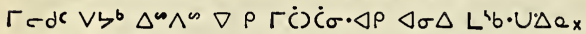

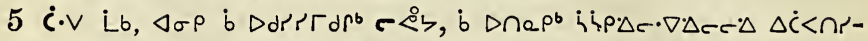

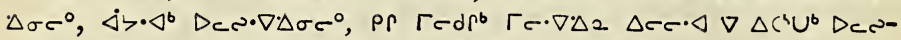

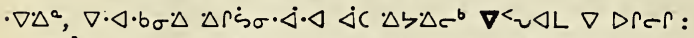

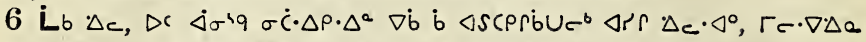

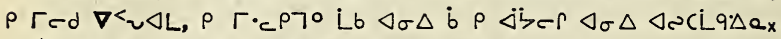

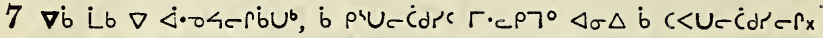

$8 \Delta C$ Lb $\Delta c c \cdot \triangleleft^{b}$ b $\sigma \wedge \rho^{b} \Delta \cap a \cdot L^{b} \Gamma c \cdot \nabla \Delta a$ : Lb $\triangleleft^{a} C \cdot \Delta c \Delta \cap_{a} L \triangleleft a$ b $\Delta \dot{C} \dot{L} L b \sigma \Delta c \nabla \wedge \dot{L} \cap \dot{r} c_{x}$

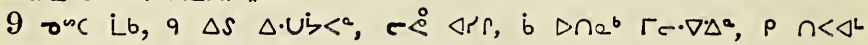
$\Gamma c \cdot \nabla \Delta a \nabla^{<} u \triangleleft \Gamma^{b}:$

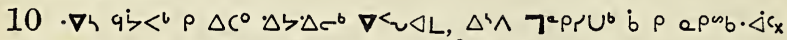

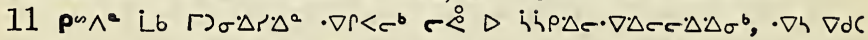

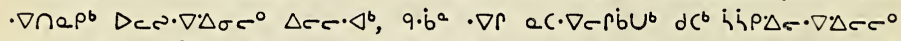

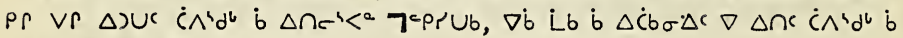
$\Delta \cap c^{i}<{ }^{2} \nabla \varsigma_{e}$ ?

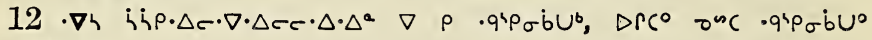
$\operatorname{DC} \cdot \nabla \cdot \Delta \Delta^{2} \mathrm{x}$

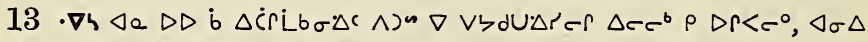
$\nabla \dot{b} \triangleleft \cdot \nabla a \nabla \Delta \dot{c} c \dot{b}<j<n\} c<\rho r b \dot{a} \wedge{ }^{4} d^{b} x$

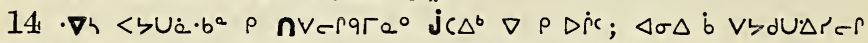

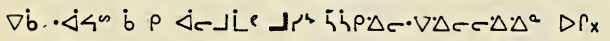

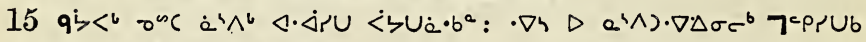

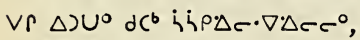

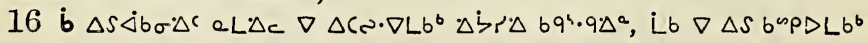
$\Delta \sigma L \nabla \dot{b} q>\sigma<\sigma^{b} \wedge \dot{L} \cap \Delta^{a} x$

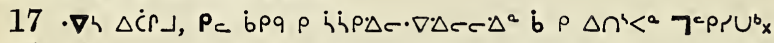

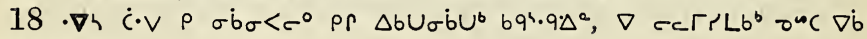

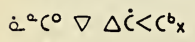

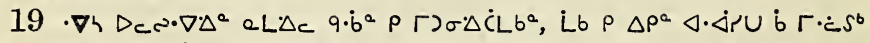
$\left.\left.\Delta^{4} \vee c\right\lrcorner \Delta^{a} \nabla \rho \vee \dot{C}_{\sigma} \cdot \Delta^{b} ; \nabla \sigma L \dot{b} \Delta \rho V \cdot \dot{s}<L^{b} \rho \rho L \sigma\right)_{x}$

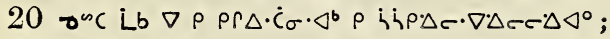

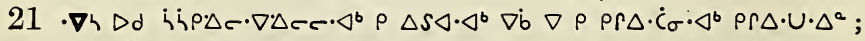

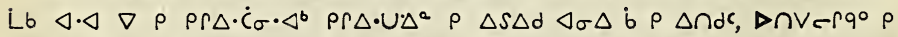

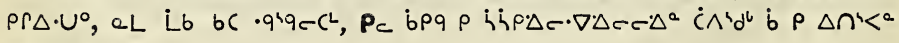

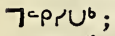

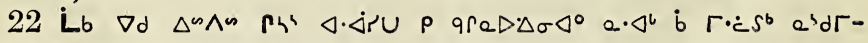
$\partial \Delta^{\circ} \Delta r_{x}$

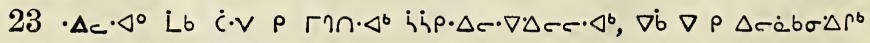
bpq pp $\triangle \dot{C} \beta^{b} \nabla \rho \sigma \wedge p^{b}$ :

362 


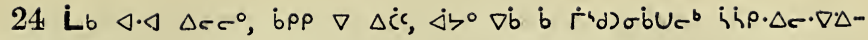
$c-\Delta \Delta \sigma \sigma^{\circ} x$

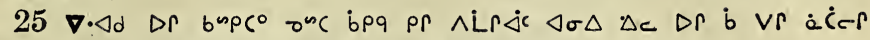

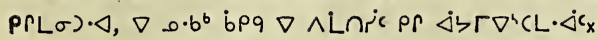

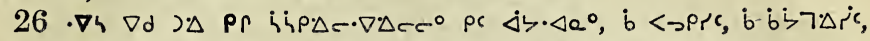

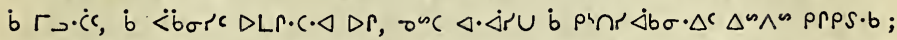

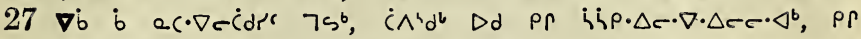

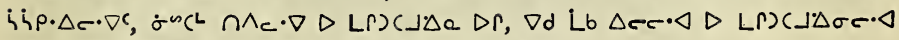

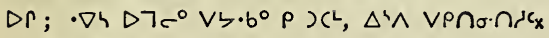

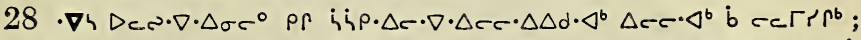

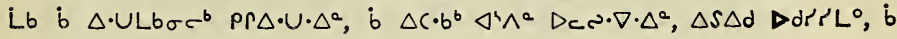
г) $\sigma \Delta \Delta \dot{b} b \sigma \cdot \Delta c \dot{b} p q_{x}$

\section{$\Lambda$ A $p\left(r a \triangle b^{2} 8\right.$.}

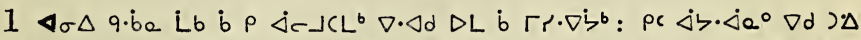

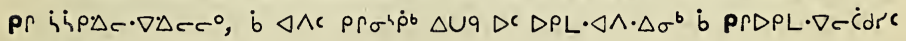
PrPSd ;

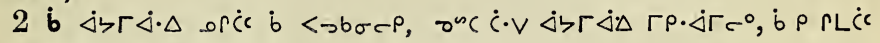
$\Delta \cap V \in \rho^{\circ}$, aL Lb $\Delta c c^{\circ} x$

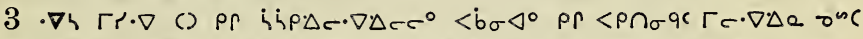

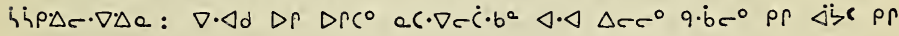
$<P \cap \sigma q G_{X}$

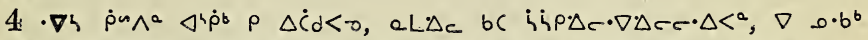

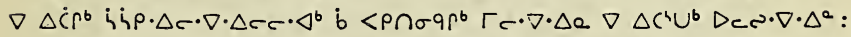

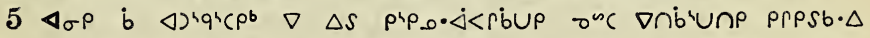

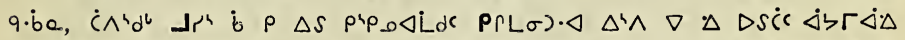

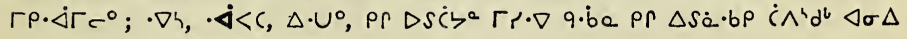

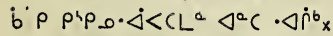

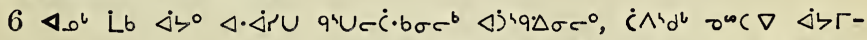

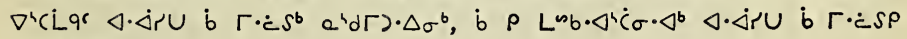

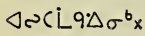

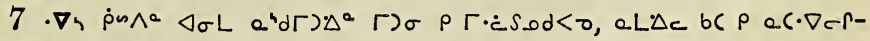
bU $<a \triangleleft \sigma L d c^{b} x$

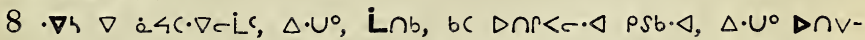

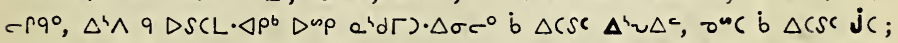

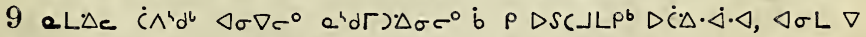

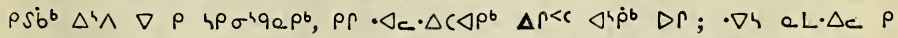

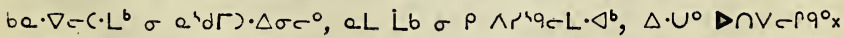

$10 \cdot \nabla h \nabla \cdot \Delta d \quad D L$ a $d r) \cdot \Delta^{2} q \quad D S\left(L \cdot \Delta \rho^{b}\right.$ b $\Delta\left(S^{c} \Delta^{2} v \Delta^{c} \rho>\sigma<\sigma \cdot b \cdot \nabla\right.$

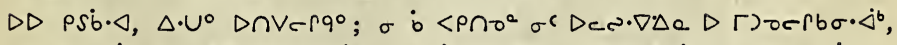

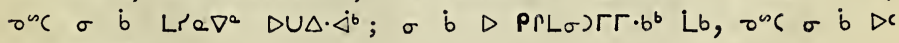
$\triangle c-\Gamma L \cdot \Delta^{b}$; 


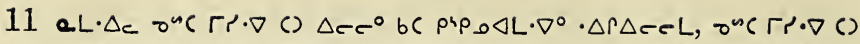

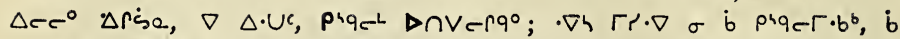

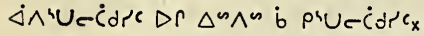

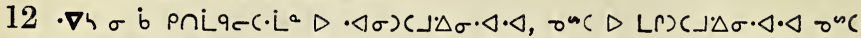

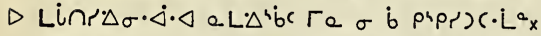

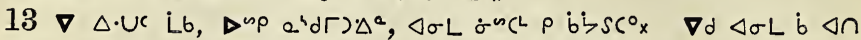

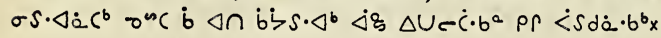

\section{$\Lambda^{\top} p c r a \Delta b^{a} 9$.}

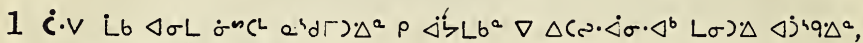

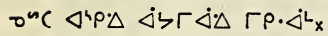

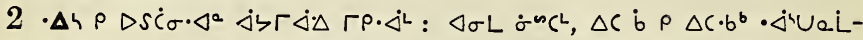

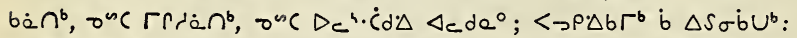

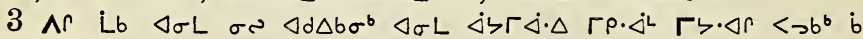
$\triangle S \sigma \dot{b} U^{b}$;

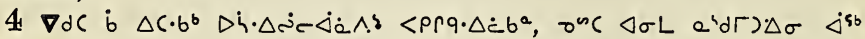

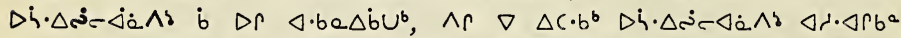

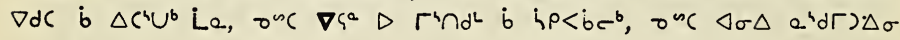
$a<b r \sigma\rangle$;

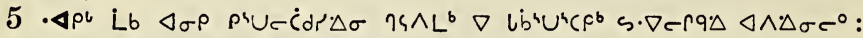

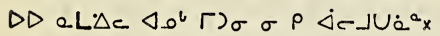

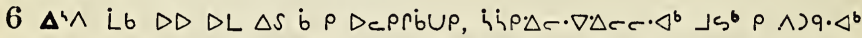

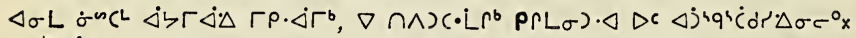

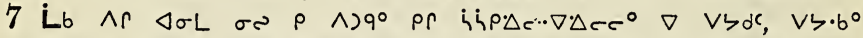

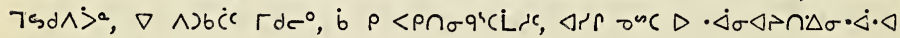
$\triangle r c \cdot \Delta b D r:$

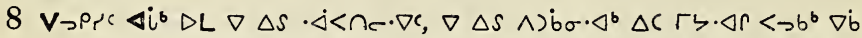

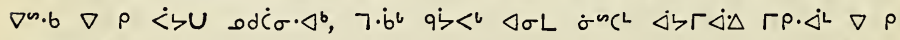
$\sigma<\Delta L b^{b} ;$

$9 \nabla \cdot \Delta d p^{4} p_{\Omega} \cdot \Delta r r b^{a} \nabla d^{4} \wedge \operatorname{pr} \Delta C \cdot b^{b}, \nabla d C \dot{b} \Delta S<p \cap \sigma \cdot b \sigma \cdot \Delta \rho \Gamma c \cdot \nabla \cdot \Delta e$

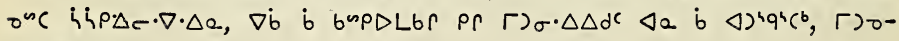
$-\Gamma b \sigma^{b} \Delta S$;

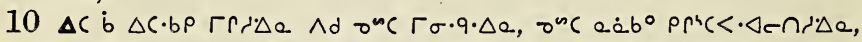

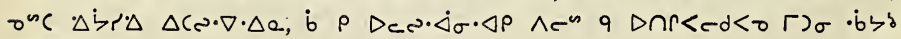
$\dot{\Delta} \succ r \dot{\Delta} \cdot \Delta^{a} x$

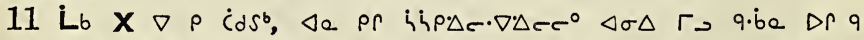

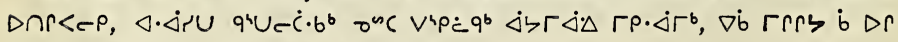
$\nabla S \dot{C} \sigma \cdot \triangleleft^{b}, \nabla \cdot \triangleleft d, \nabla b \Delta L \nabla \triangle S D S \dot{C} \sigma \cdot \triangleleft^{b}$;

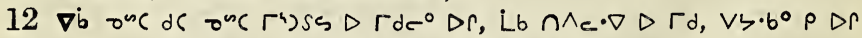

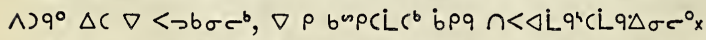

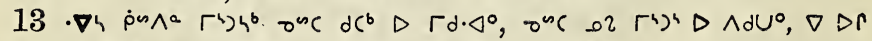

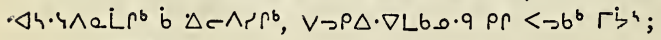

364 


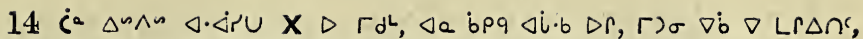

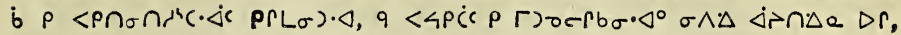
pr $\Delta j s \cdot \nabla^{b} \vee \operatorname{LinT}(\operatorname{Pr} L \sigma)$ ?

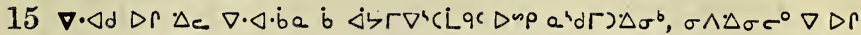

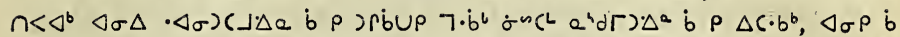

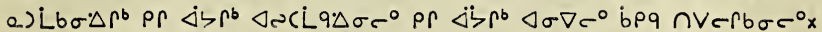

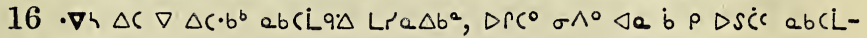
$9 \circ \mathrm{Lr} Q \Delta b \sigma \sigma^{\circ} \mathrm{x}$

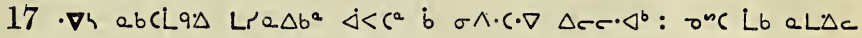

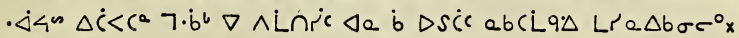

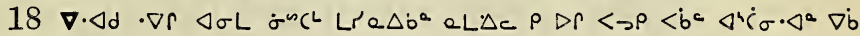
$\nabla$ rb $\Delta \dot{b} U^{b} \Gamma d:$

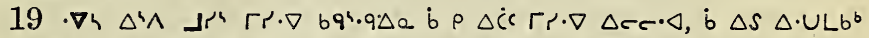

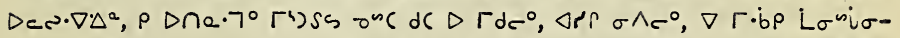

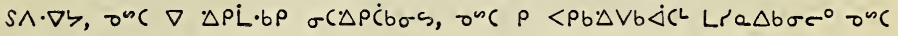
$[r \cdot \nabla \Delta c c \cdot \triangleleft$,

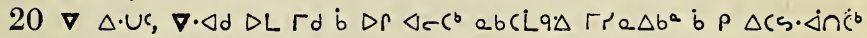
PrL $\sigma)_{x}$

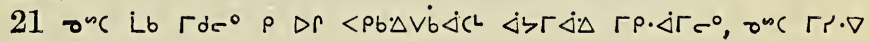

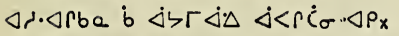

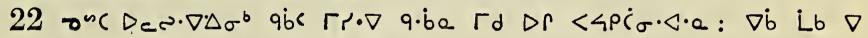
$r b \Delta b^{b}{ }^{b} \Gamma d$ a L $\Delta c \Delta C \cdot b^{a} \cdot \Delta 2 a L q \Delta^{a} x$

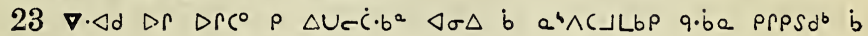

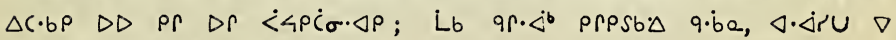

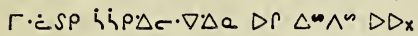

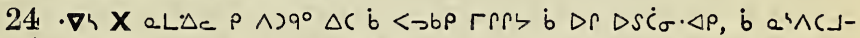

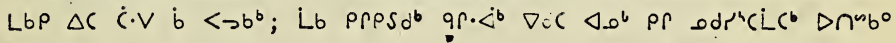
$P \Gamma L \sigma) \cdot \triangleleft:$

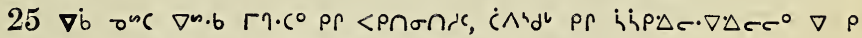

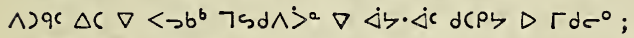

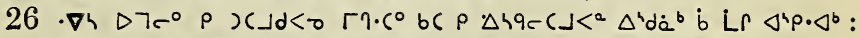

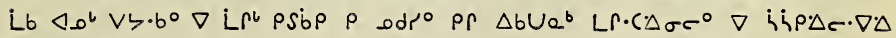

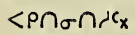

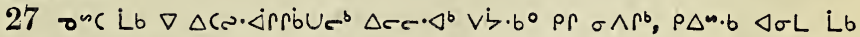
or $\cap \dot{<} d \dot{d} b \sigma \cdot \Delta \rho^{b}$;

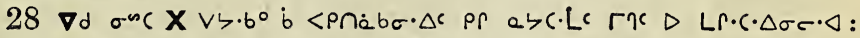

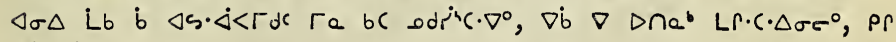
$\wedge L \wedge \dot{\Delta} c_{x}$

\section{$\Lambda^{\wedge} p C r a \triangle b^{2} 10$.}

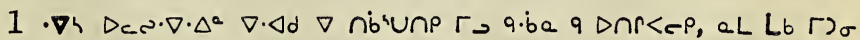

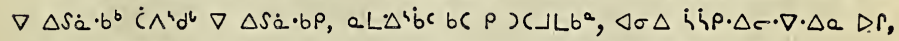

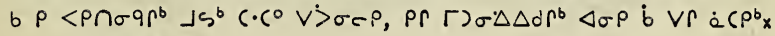


$\left.\left.2 \cdot \nabla 4 \rho a \wedge^{a} \rho\right) x\right\lrcorner L b^{b}, a L \dot{a} b C \rho>\sigma<\rho \cap \sigma b U d<a ? \cdot \nabla h \quad D C b \Gamma \triangleleft \cdot \triangleleft^{b}$,

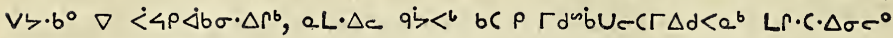
$D$ D)

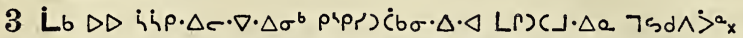

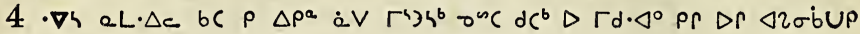
LP) $\left(J \cdot \Delta 0_{-x}\right.$

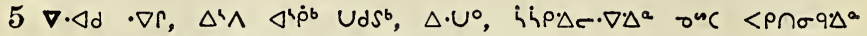

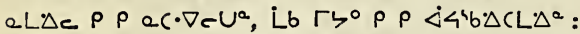

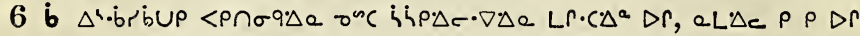
$r \cdot \operatorname{sen}^{2} x$

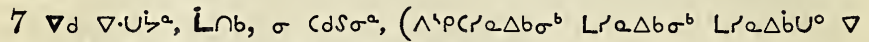

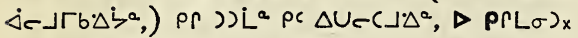

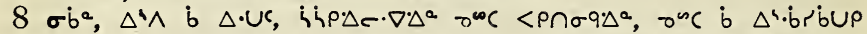

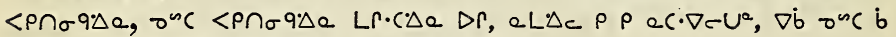
Dr $\Gamma \cdot \operatorname{seC}^{a} ;\left(\dot{b}<P \cap \sigma \dot{b} U p\right.$ DeN. $\left.\nabla \Delta \sigma^{b} ;\right)$

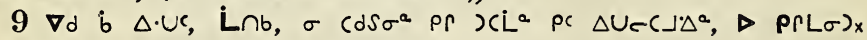

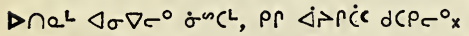

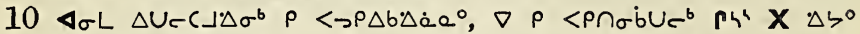
$\mathrm{V} \zeta \cdot 6^{\circ} \wedge d \nabla U \wedge<e^{b} \mathrm{C}$

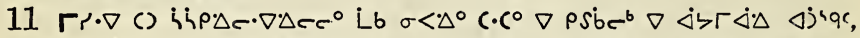

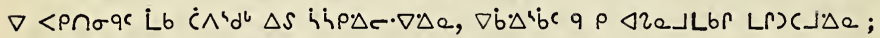

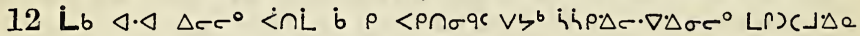

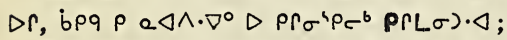

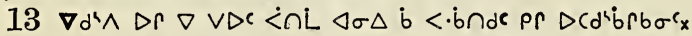

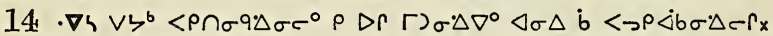

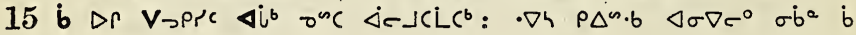
$P \Delta \cdot U c$,

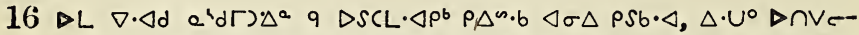

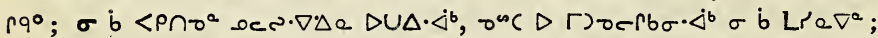

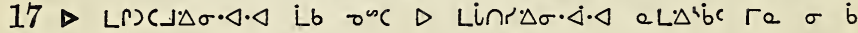

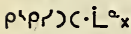

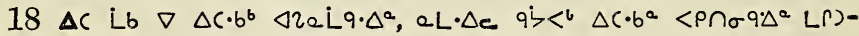
(\lrcorner$\cdot \Delta^{a} \Delta r_{x}$

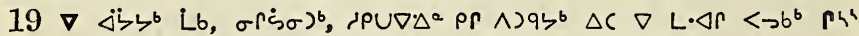
$\triangle \Gamma d D r$,

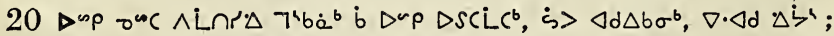

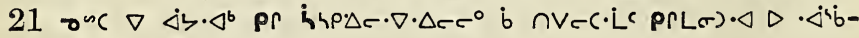
$>b \sigma \Gamma r^{\circ}$;

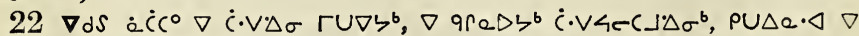

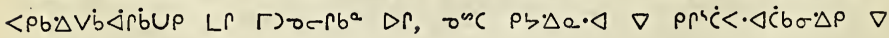
$<4 \mathrm{~b} b \Gamma^{\mathrm{b}} \sigma \wedge \mathrm{b}_{\mathrm{x}}$

$\left.23 \nabla d S \Gamma \Gamma \Gamma \dot{a} C^{\circ} \nabla \cdot \dot{\triangleleft} \Delta C L^{b} \rho \dot{C} \cdot V 4 e C\right\lrcorner \Delta \sigma a^{0} \nabla \dot{b} \nabla \dot{b} \rho \cdot \nabla 4 e C L^{b} ; \cdot \nabla h$ -bל 


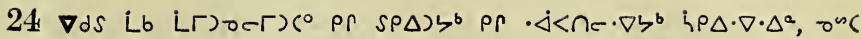
$r \rightarrow \Delta j 49 \cdot \Delta e ;$

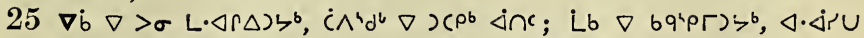
Lb $\left.9 \dot{\zeta}<<^{b} \nabla x^{b} \nabla \cdot \dot{<}<C\right]^{b} \triangleleft \sigma L \nabla \rho L^{b} b^{b} \nabla<<<c^{b} x$

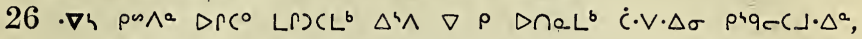

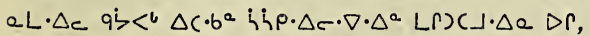

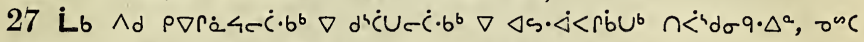

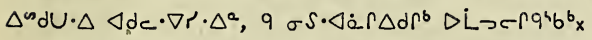

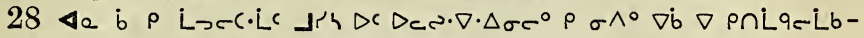

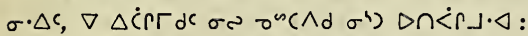

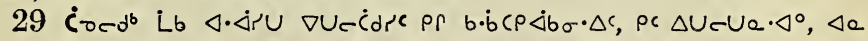

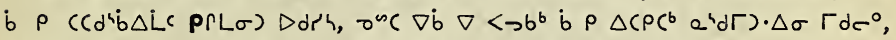

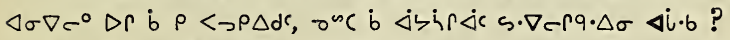

$30 \cdot \nabla h \rho$ prqe Le ${ }^{\circ} \triangleleft a$ b $\rho \Delta \cdot U c, \Delta^{4} d r \Delta \cdot \nabla \cdot \Delta^{a} \sigma c \sigma \cap \wedge_{c} \cdot \nabla \cdot \Delta r^{a}$;

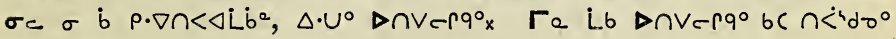
$\Delta C \Delta r-L_{x}$

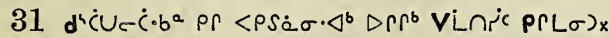

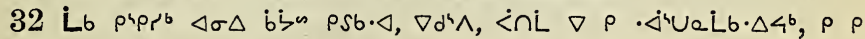

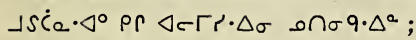

$33<<\rho, 7 \cdot b^{b} \nabla \rho \Delta s \Delta b \cdot \Delta \zeta^{b} \rho \rho<\dot{<} \wedge \Delta b \cdot \Delta \zeta^{b}, \nabla \rho \rho b \Gamma b \cdot \Delta \zeta^{b}$ occ $\nabla$

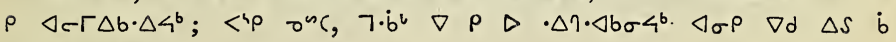
$x \cdot \Delta b \sigma \cdot \Delta \varphi^{b} x$

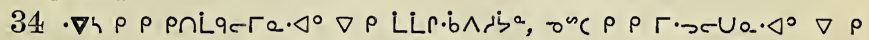

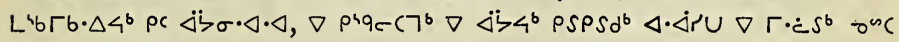
bpq $\nabla \rightarrow \cap 0 \cdot \Delta a^{2} x$

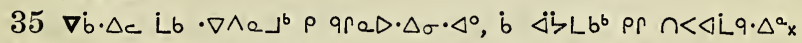

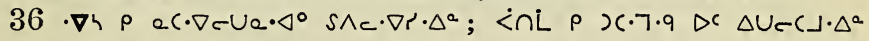
PSL $\sigma)$, PR $\Gamma-b \cdot \Delta 4^{b} \triangleleft \sigma L \triangleleft N\left(L q \cdot \Delta^{a} x\right.$

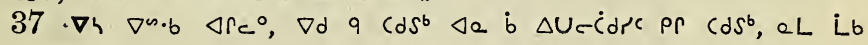
bc $\cdot \dot{<} c \cdot \Delta^{\circ}$

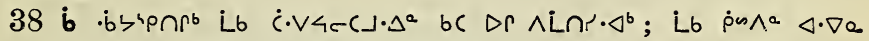
$\Delta c c^{\circ} \triangleleft 2 \dot{b}<\cdot \Delta U$, aL. $\Delta c$ bc iVerd oc $\Delta i \cdot b x$

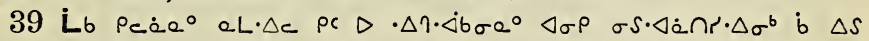

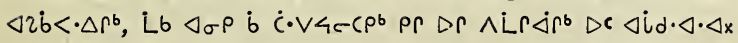

\section{Ápcra $\triangle b^{a} 11$.}

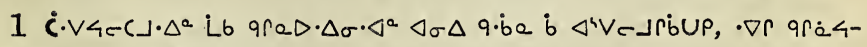
rríup $\nabla \sigma \Delta \nabla \dot{b} \dot{b}$ - $0.6 P_{x}$

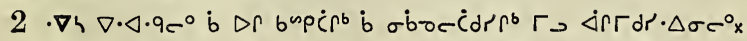

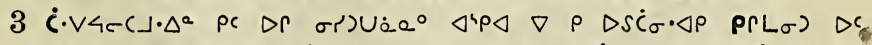

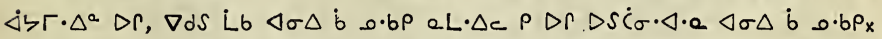
$4 \dot{C} \cdot V 4 c-(\lrcorner \cdot \Delta^{a}$ Dr $\left.\nabla \wedge<\rho<P \cap Q L \cdot \nabla^{\circ} \rho \Gamma L \sigma\right) \cdot \triangleleft \Delta \cdot \Delta r U \quad 7 \cdot \dot{C} S \sigma c^{b}$ 367 


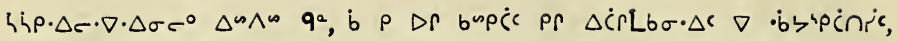

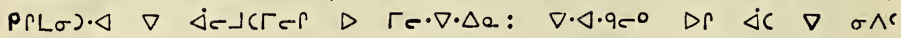
$9 \dot{i}<6 \dot{\Delta}>\Gamma^{\circ} \mathrm{x}$

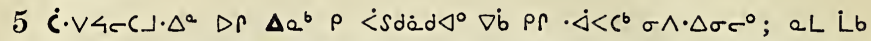

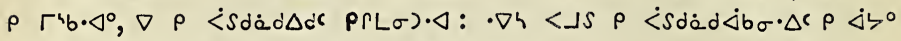
$\nabla 7 \sigma^{\circ} \Delta(\rho\lrcorner \cdot \Delta \sigma \sigma^{\circ}, \nabla \rho$ a $\left.\Delta c \cdot \nabla \dot{\Delta c} \rho \Gamma L \sigma\right) \cdot \Delta x$

$6 \nabla \dot{b}$ Lb $\left.\left.\nabla \Delta c \cdot b^{b} \dot{C} \cdot V 4 c\right)\right\lrcorner \cdot \Delta^{a} \dot{a} L \cdot \Delta c b c \rho \Delta \rho^{a}$ pr $a \Delta c \cdot \nabla \Delta b \sigma \cdot \Delta c$

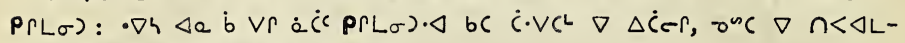
- $\Delta$ er $\triangleleft \sigma \Delta$ bl b àcc. $\nabla e r d e r x$

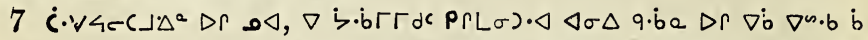

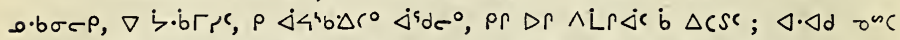

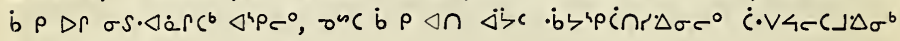
b) $D r<e c^{b} x$

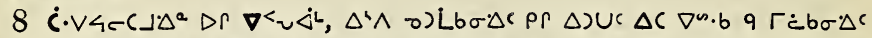

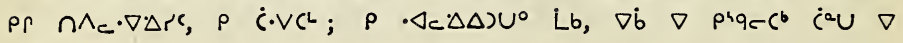
$\Delta U C_{x}$

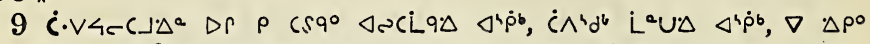

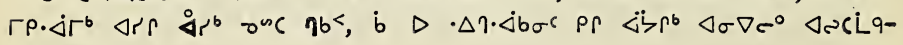
$\Delta \sigma \sigma^{\circ}$ :

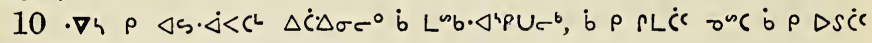
P(L $\sigma)_{x}$

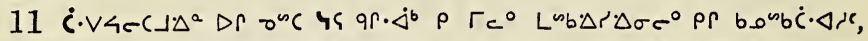

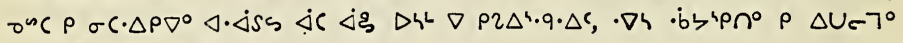
$\triangleleft \sigma \Delta \dot{b} \rho \triangleleft \sigma C L d C_{x}$

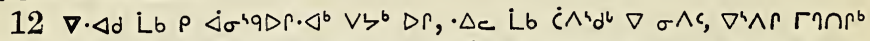

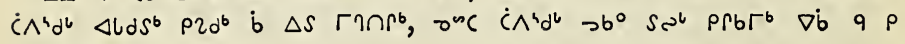

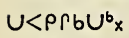

$\left.13 \Gamma r \cdot \nabla D d \rho \sigma V \cdot \triangleleft^{b} \dot{C} \cdot V 4-C\right\lrcorner \Delta \sigma^{b}, \nabla b \nabla \rho D \cap a \rho^{b} \triangleleft \sigma C L q \Delta a$, Lb $\nabla$

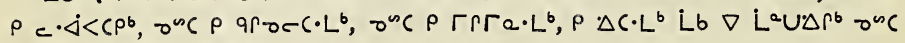

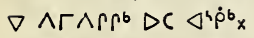

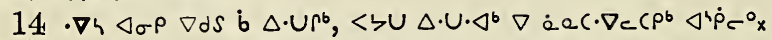

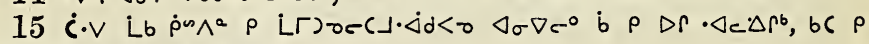

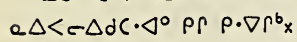

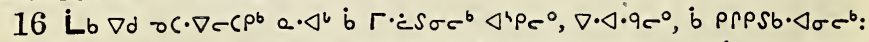

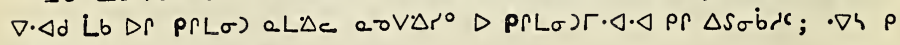
$\dot{\Delta} \iota^{4} b \Delta C L \cdot \nabla^{\circ} \Delta C \Delta \sigma \sigma^{\circ} x$

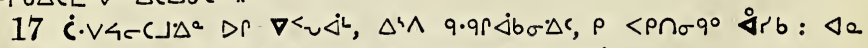

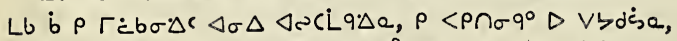

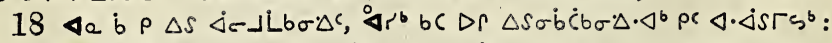

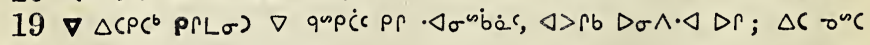

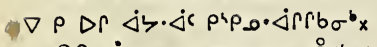

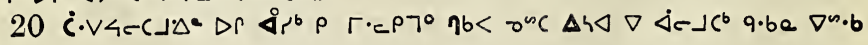
q $\triangle P_{\sigma} \sigma P_{X}$ 


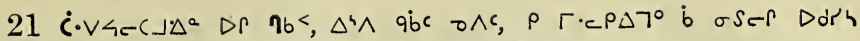

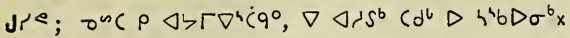

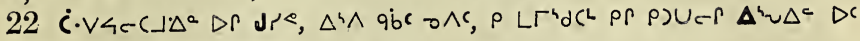
$\triangle \cdot \Delta S T S:$ onc $\rho \Delta C \omega \cdot \nabla^{\circ} D^{4} b e \Delta r_{x}$

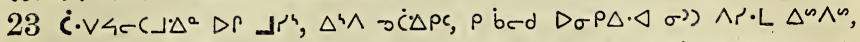

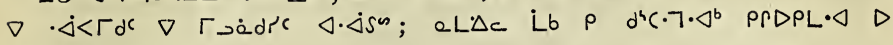
$69^{4} \cdot 9 \cdot \Delta \sigma \sigma^{\circ} x$

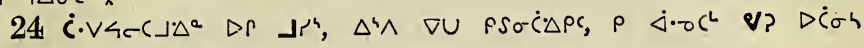
$\nabla$ drtc. $\triangle \operatorname{pr} \triangle S_{\sigma} \dot{b} \dot{c} b \sigma \cdot \Delta c$;

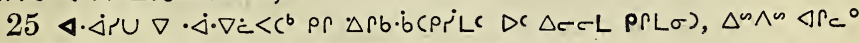

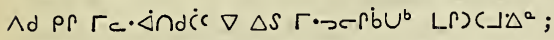

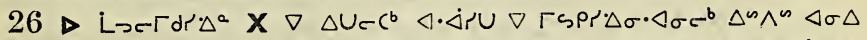

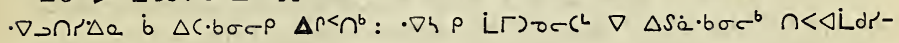
$\Delta \sigma \sigma^{\circ} \mathrm{x}$

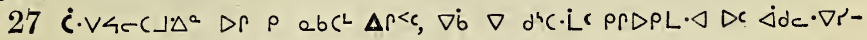

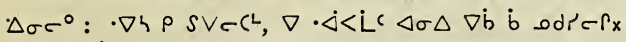

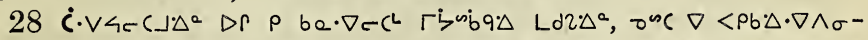

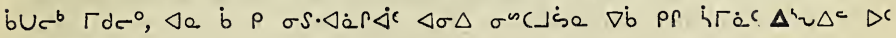
$\triangleleft \cdot \Delta S \Gamma \mathrm{sx}_{\mathrm{x}}$

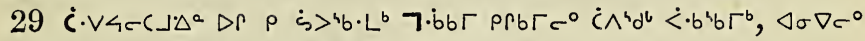
$\left.\Delta r^{<} \cap \Delta a^{b} \nabla d r\right)\left(\beta^{b} \rho \sigma^{h}\left(<\cdot \nabla \cdot \nabla^{b} x\right.\right.$

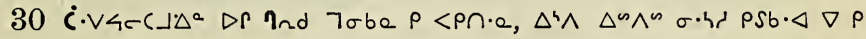
$\cdot \dot{<} \dot{b} b \Delta \dot{b} \cup p_{x}$

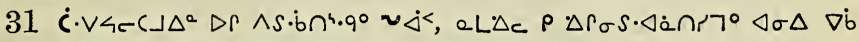

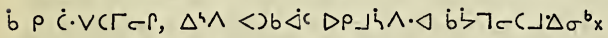

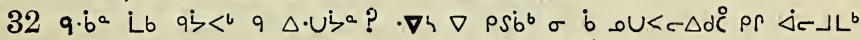

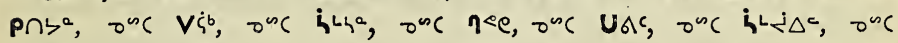
$\nabla \rho^{4} \rho \cdot \Delta \triangle q \cdot \triangleleft^{\mathrm{b}}$ :

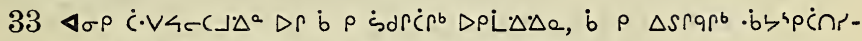

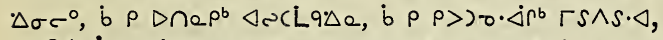

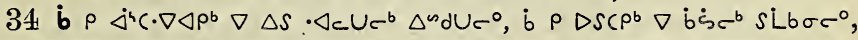

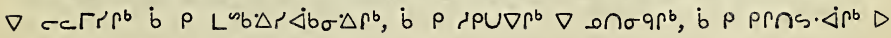

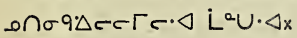

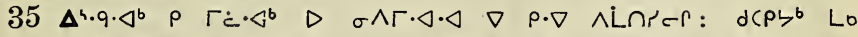

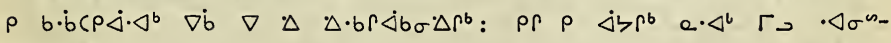
$b \Delta \sigma \sigma^{\circ} \mathrm{x}$

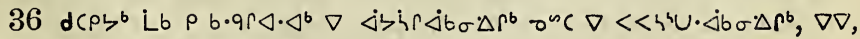

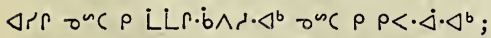

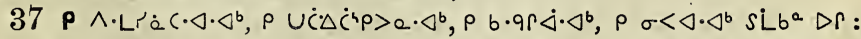

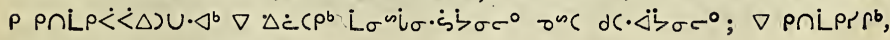

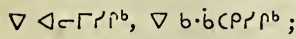

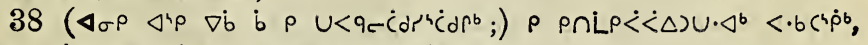

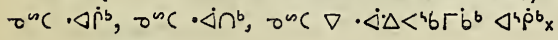




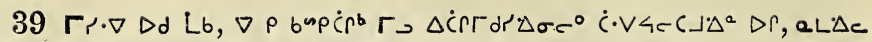

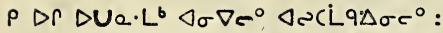

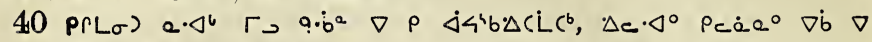

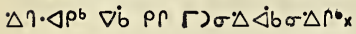

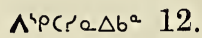

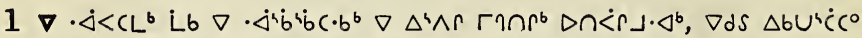

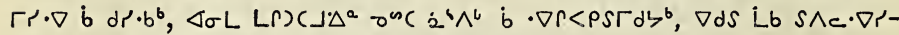

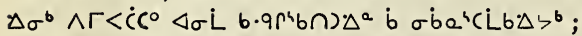

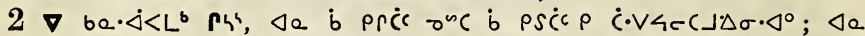

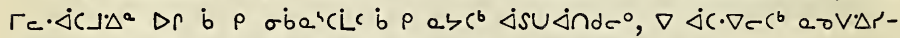

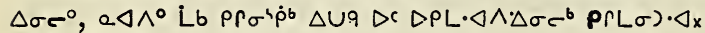

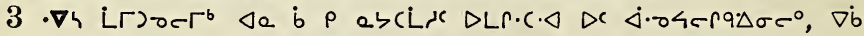

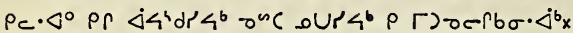

4 aL. $\Delta c \nabla^{a} \cdot b \Gamma d^{b} \Delta S \rho \rho$ LLSibe. $\Delta^{\circ}, \nabla a^{b} d^{4} C 7^{b} L \Gamma \cdot C \cdot \Delta^{a} x$

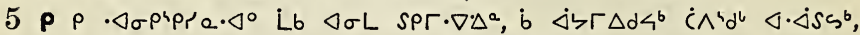

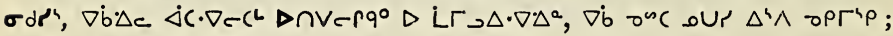

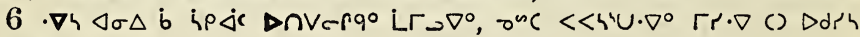
b) $D \cap \dot{a}^{-} c_{x}$

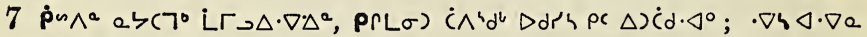
$\nabla d r L^{\circ} \nabla \dot{b}$ b $L \Gamma \Delta \Delta d^{6} \Delta \dot{C} \Delta \zeta$ ?

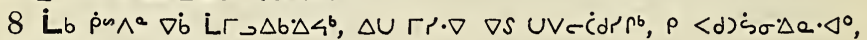

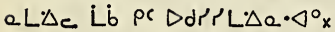

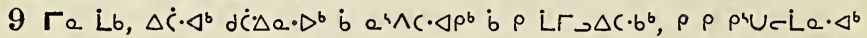

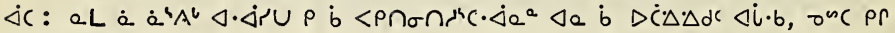
$\wedge \mathrm{L} \cap \mathrm{C}^{\circ}$ ?

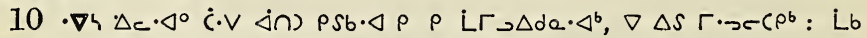

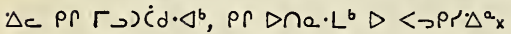

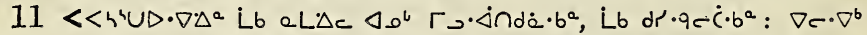

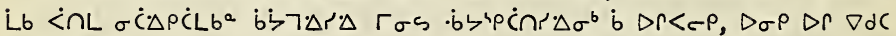
b orrarb $^{2}$

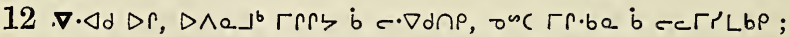

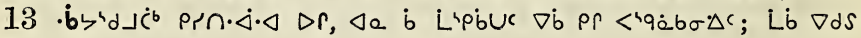
$a \cdot \Delta l b c r e \cdot \Delta r \Delta{ }^{\circ} x$

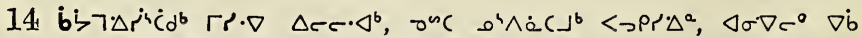

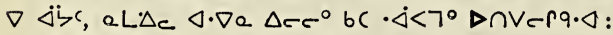

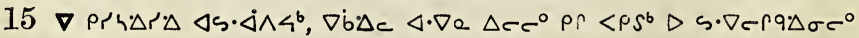

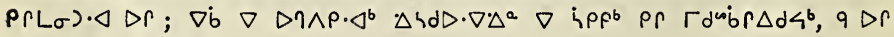

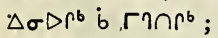

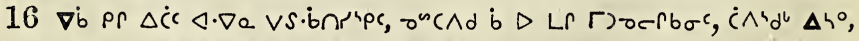

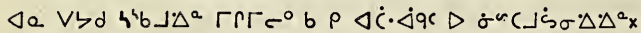

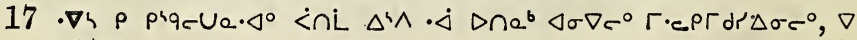
370 


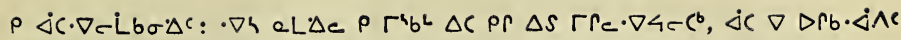
$V \vee b c^{b} \nabla \rho$ a e $c \cdot \nabla c c^{b} x$

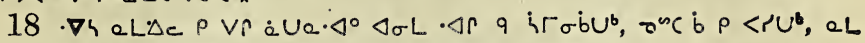

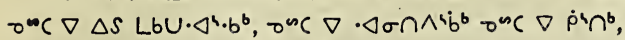

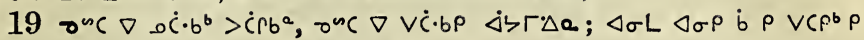

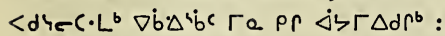

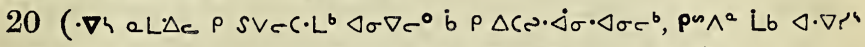

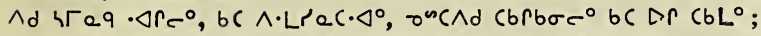

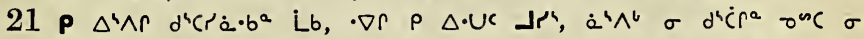
$\left.\operatorname{cor} r<e^{a}:\right)$

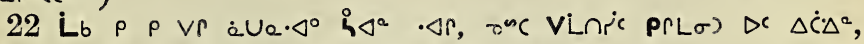

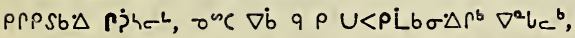

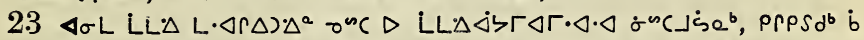

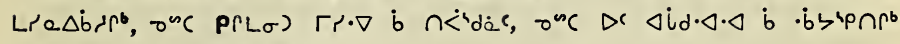
$\left.\Delta c c \cdot \Delta^{b} \nabla \Gamma\right) \sigma \Delta b \sigma \Delta \Gamma^{b}$,

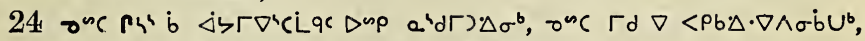

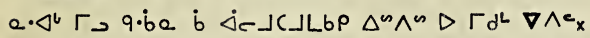

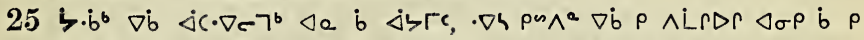

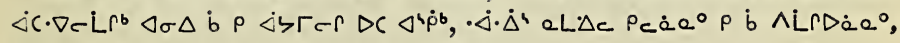

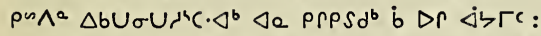

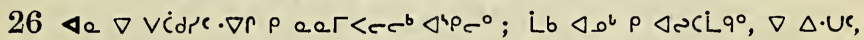

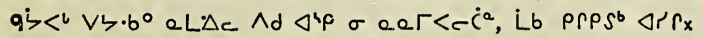

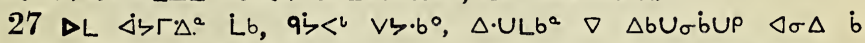
e $\sigma P<C P, \dot{C} \Lambda^{\prime} d^{l} \triangleleft \sigma \Delta \dot{b} P \Delta S \dot{C}_{\sigma} \cdot \triangleleft P, \triangleleft \sigma \Delta \nabla \dot{b} q \rho$ ee $\Gamma<C P$ P $\Delta C \cdot b P_{x}$

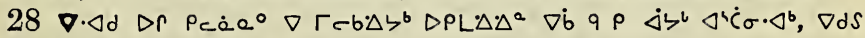

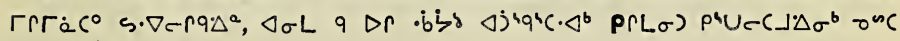
L $\sigma) \triangle$ Q $\sigma \Gamma \Delta \sigma^{b}$;

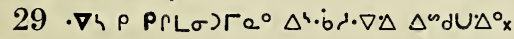

\section{A'pcra $\Delta b^{\circ} 13$.}

$1 \nabla d S \Delta r \dot{s} \sigma) \Delta h \rho \Delta) \Delta^{a}(\rho) b c \Delta c \cdot b^{a} x$

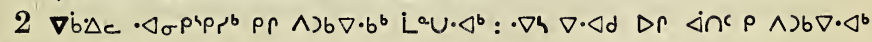
$\nabla$ ale $\nabla b \nabla p^{4} q_{c}-c b^{b} x$

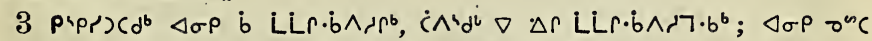

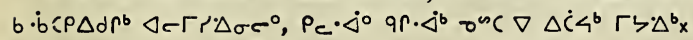

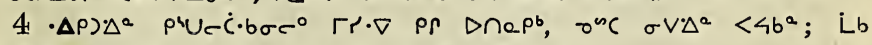

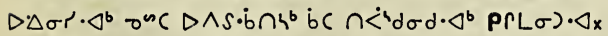

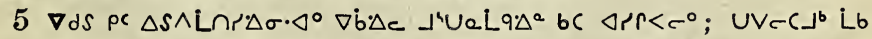

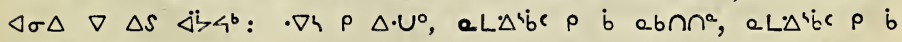
$\cdot \nabla \wedge \sigma \cap^{2} \mathrm{x}$

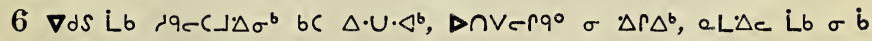

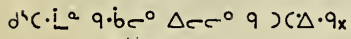

371 


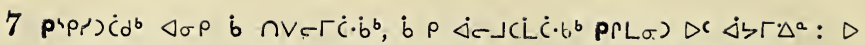

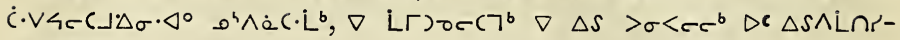
$\Delta \sigma \cdot \Delta^{\circ} x$

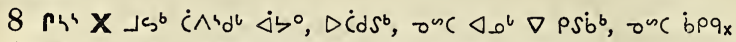

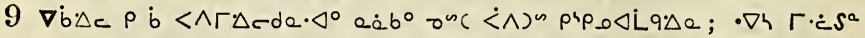

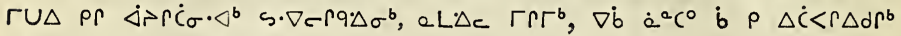
$\triangle \sigma \rho \nabla d C \dot{b} \rho \wedge\lrcorner U r^{b} x_{x}$

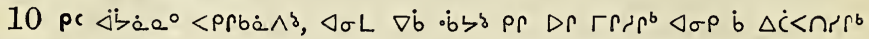

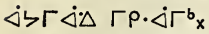

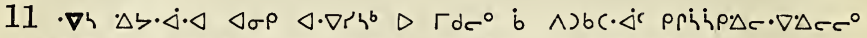
$\left\langle\supset \rho \Delta b \Gamma d^{b} L R\right)(\lrcorner \Delta^{a} \Delta r, \cdot \Delta c \Delta \cap \Gamma^{b} b V S \Delta \sigma^{b} \rho \Delta^{4} \cdot b r^{b} b U c \cdot \Delta x$

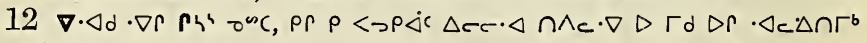

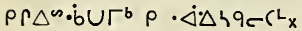

$13 \nabla \cdot \triangleleft d \quad D R \quad \nabla d S \quad \triangleleft c \Delta \cap \Gamma^{b} \quad b V S \Delta \sigma^{b}$ ac $\Delta \quad \dot{c} C C^{\circ}, \nabla \quad a b C \cdot L^{b} \quad D$ e. $V \Delta \Delta \Delta d d^{2} \Delta^{a} x$

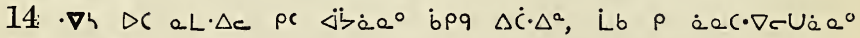
$V \zeta^{b} q \dot{<}<c^{b} x$

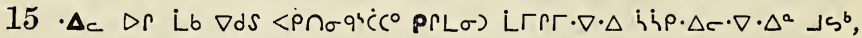
$\nabla \cdot \triangleleft d$, b $\left.D R<\sigma^{b} \rho\right) \sigma a^{b}, \nabla$ a $\dot{a}^{b} d\left(\cdot L^{b} D C \Delta S \sigma \dot{b} r \cdot \Delta^{a} x\right.$

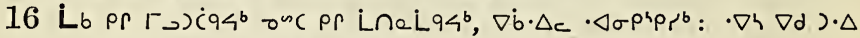

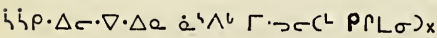

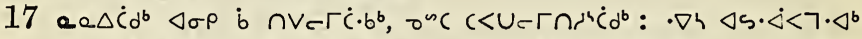

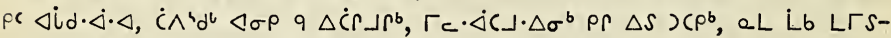

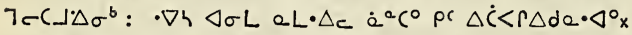

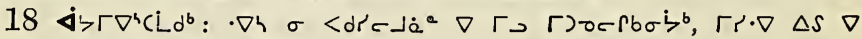

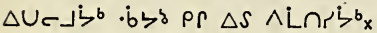

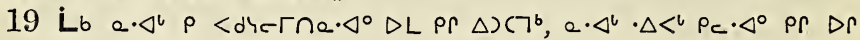
$<P \cap \sigma \dot{b} \cdot \Delta \dot{b}{ }^{a} x$

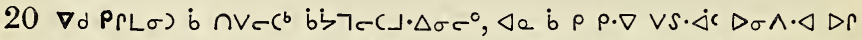

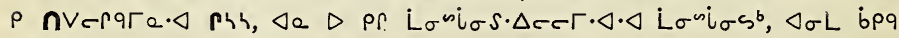
2bCLq. $\triangle$ Lra $\triangle b \sigma \Gamma d D r$,

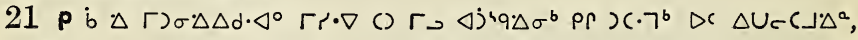

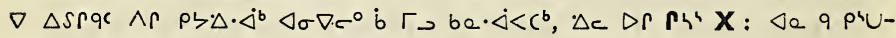

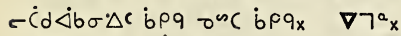

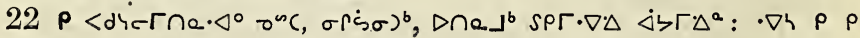

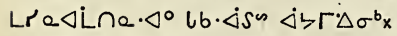

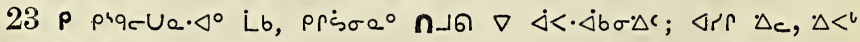
CdSq, $\rho \dot{b} \cdot \dot{\Delta}<\Gamma \cap \dot{Q} \cdot \Delta^{\circ} x$

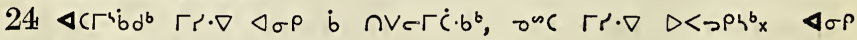

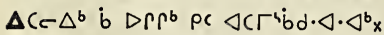

25 s. $\nabla e r q \Delta^{a} \Gamma r \cdot \nabla \rho b \Delta \Delta 7 \Delta d a \cdot \Delta^{\circ} x \quad \nabla 7^{a} x$ 


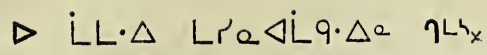

Ápcra $\triangle b^{a} 1$.

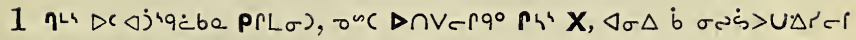
b e $\dot{\alpha} \sigma^{4} \Delta \dot{C}-r, \Delta \dot{C} r^{4} b \cdot \nabla^{\circ} x$

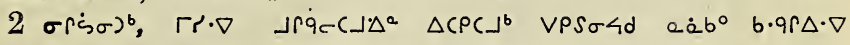
$\Delta \sigma^{b}$;

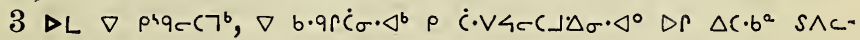
$\cdot \nabla r \cdot \Delta^{a} x$

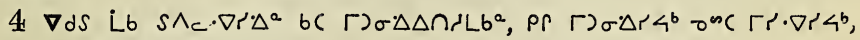
$\nabla \dot{b} q \cdot b^{a} \nabla \cdot p c L \zeta^{b}{ }_{x}$

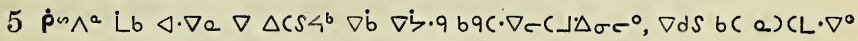

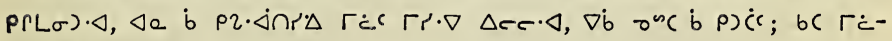
$b \sigma \cdot \Delta^{\circ} L b_{x}$

6 Lb $\nabla d S$ bc a) $\left.\left.C L^{\circ} \dot{C} \cdot V 4_{c}(\lrcorner \dot{\Delta} \sigma^{b}, \nabla \dot{b} \Gamma\right) \sigma \nabla \dot{j} \cdot q_{c}\right\lrcorner c^{b}: \cdot \nabla h \triangleleft a b$

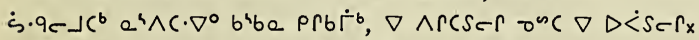

$7 \cdot \nabla h \nabla b \Delta_{c} \triangleleft a \Delta c c^{\circ}$ bc $\Delta U c c^{L} q \cdot b c^{\circ}$ pr $\Gamma e d c \Delta \cap V e r q \cdot \Delta x$

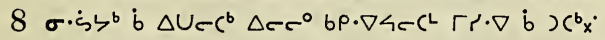

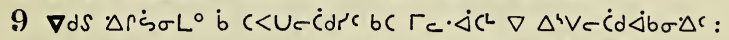

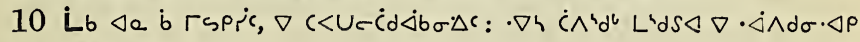
bc $\Delta S \cdot \nabla \wedge<c^{\circ} \mathrm{x}$

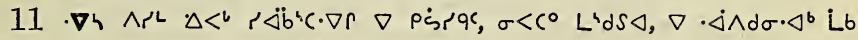

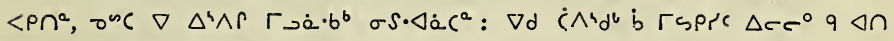
<sdadrc $\Delta C \Delta s \cdot C \Delta \sigma^{b} x$

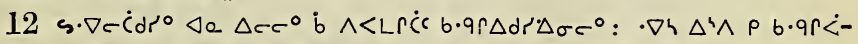

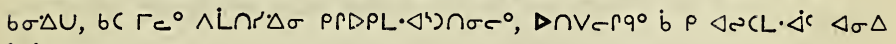
b h $P \Delta d c_{x}$

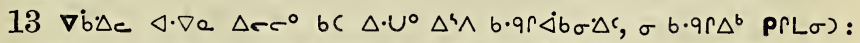

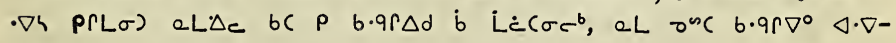
$\ulcorner\cdot \triangleleft$ :

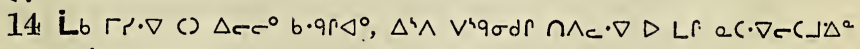
onc $s \triangle p \Delta b \sigma \Delta r x$

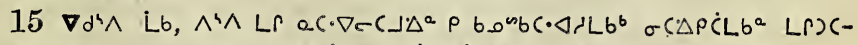

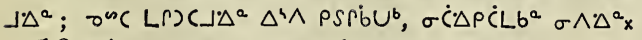

$16 \nabla \dot{b} \Delta c \cdot \triangleleft \sigma U \Delta_{c}(\lrcorner^{b}, r\left\langle\rho \Delta\left(\cdot b^{b} \sigma \rho \dot{s} \sigma\right)^{b} x\right.$

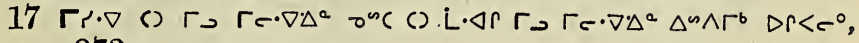
373 


\section{q4 1, 2。}

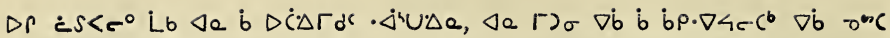

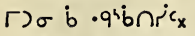

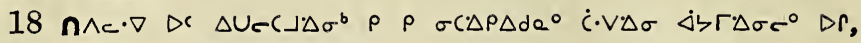

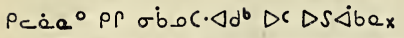

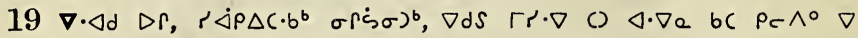

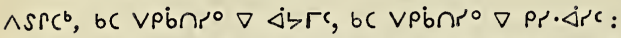

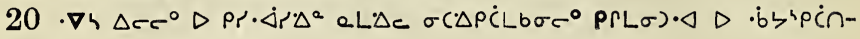
$\Delta \cdot \Delta \sigma c^{\circ} x$

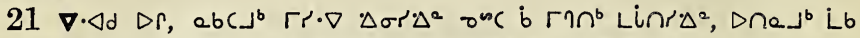

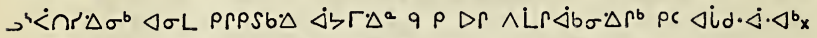

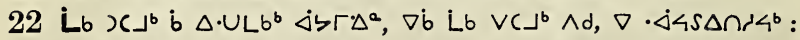

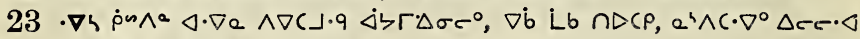
$\nabla$ be $\cdot \dot{\Delta}<C \Gamma-r \Delta \dot{C}^{b}\left(\Gamma^{b} \cdot \dot{\Delta}<\right\lrcorner \Delta \sigma^{b}:$

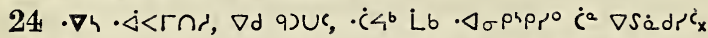

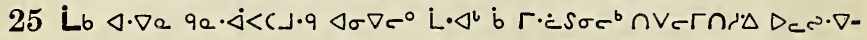

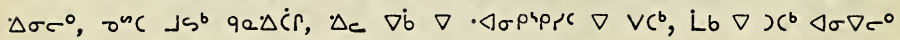

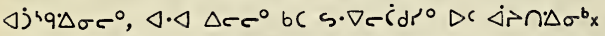

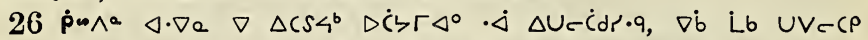

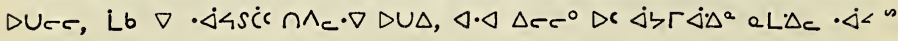
$\Delta \dot{C}<C \sigma c^{\circ} \mathrm{x}$

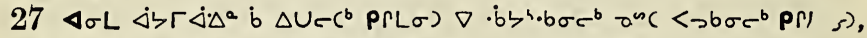

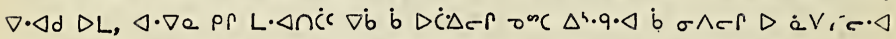

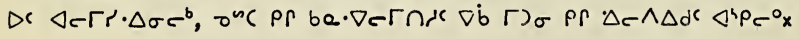

\section{$A^{4} p C r a \triangle b^{a} 2$,}

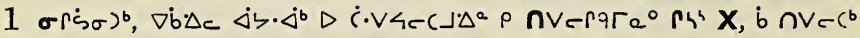

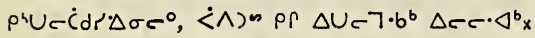

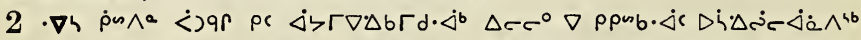

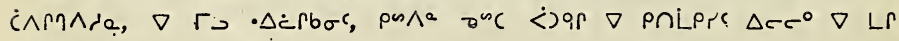
$\triangle \dot{c} b \sigma^{c}$;

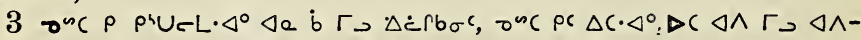

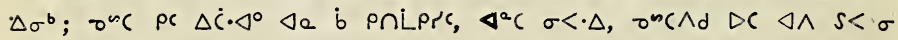
(dibib $\sigma^{b}$ :

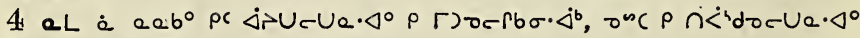
LC LJoredise?

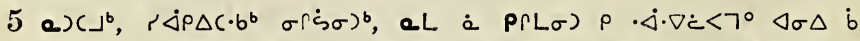

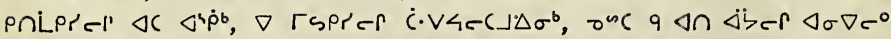

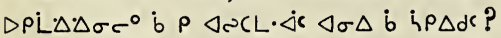

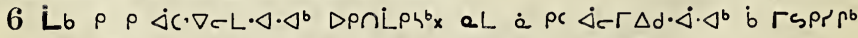

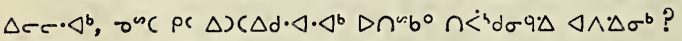

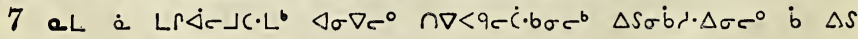
$\triangle S \sigma \dot{b} \cap{ }^{\circ} \Delta \zeta^{\circ}$ ?

374 


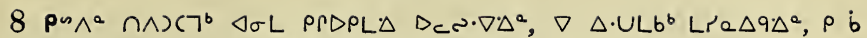

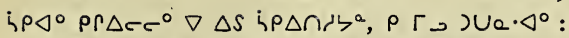

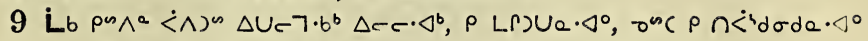
$\left.\Delta c \sigma^{\circ} \nabla \cdot \Delta^{a} \dot{(} \Lambda^{4} d^{b} D \cdot \Delta \sigma\right)\left(\mu^{b} b^{b} x\right.$

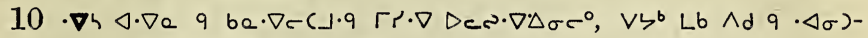

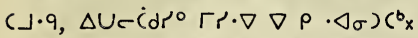

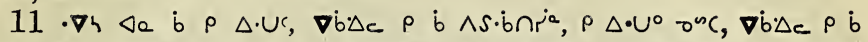

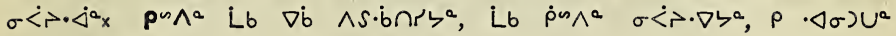
$\operatorname{Den} \nabla \Delta \Delta^{a} \mathrm{x}$

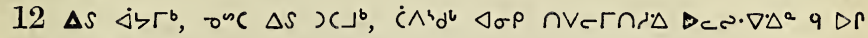
กर́ंdàb $\sigma \Delta r^{b} b_{x}$

$13 \cdot \nabla h \Delta c b c<b 0 n\left\langle\dot{<} d \sigma d r \Delta \sigma c^{\circ} \nabla \dot{b} \nabla \Delta r<<c^{b} s \cdot \nabla c r q \Delta \sigma c^{\circ}, \nabla b\right.$

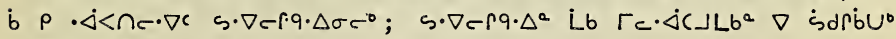

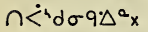

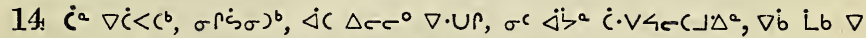

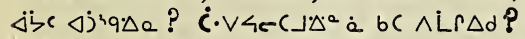

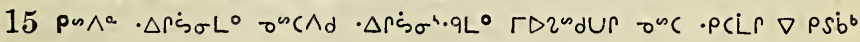
LrT

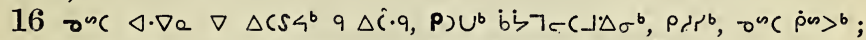
$\nabla b$ Lb $\nabla \Gamma_{\supset} \cdot b^{b} \Delta \sigma \Delta q \cdot \dot{b} a q \dot{<}<r \Delta \cdot \nabla^{b} \Gamma^{\circ} ; c^{a} \nabla \dot{C}<c^{b}$ ?

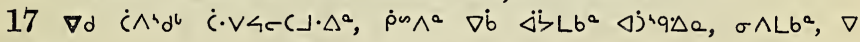
$V ל \cdot b^{b} x$

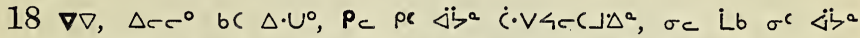

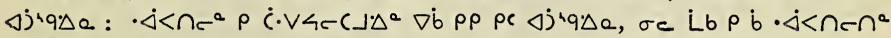
$\sigma \dot{c} \cdot V\left\langle e(\lrcorner \Delta^{a}\right.$ oc $\Delta \dot{j}+q \Delta a \Delta \Gamma_{x}$

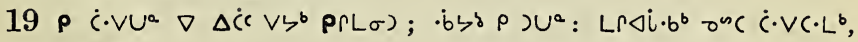
$0 . \Gamma<c \cdot \Delta_{b} L_{b x}$

20 Lb $\rho$ b prqcue, $\Delta$ pa.q. $\Delta c c^{\circ}, \dot{c} \cdot V 4-C \cdot \Delta^{a} \nabla \dot{b} \nabla \Delta r r<c^{b}$

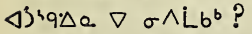

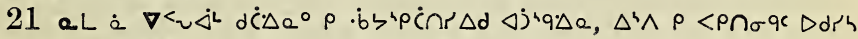

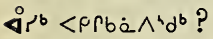

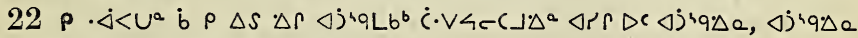
Lb $\left.D \Gamma \dot{C} \cdot V A c(\lrcorner \Delta^{a} \rho \Gamma\right) \sigma \Delta \Delta \dot{b} U^{\circ} x$

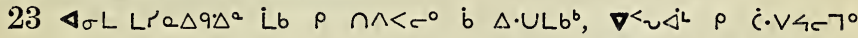

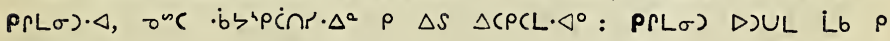
$\triangle S \sigma \dot{b} c^{\circ} \times$

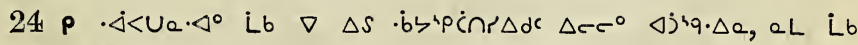
$\dot{C} \cdot V\langle e C\lrcorner \Delta^{a} \wedge d x$

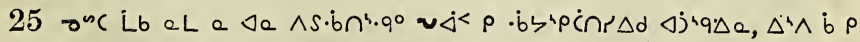

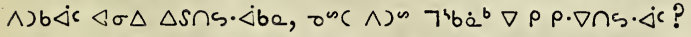

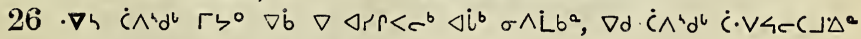

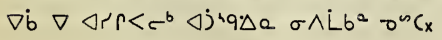


Arpcra $\triangle b^{a} 3$.

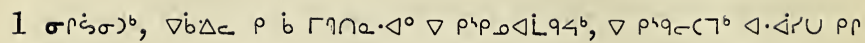
กरं'ं $\sigma b \Delta \zeta^{b} \times$

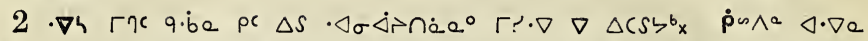

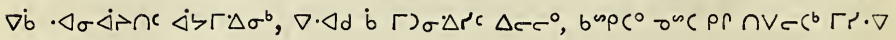
$\Gamma \zeta \cdot \Delta c^{\circ} x$

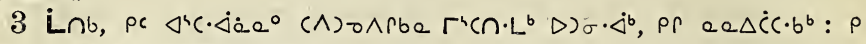

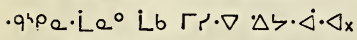

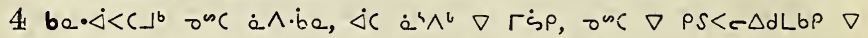

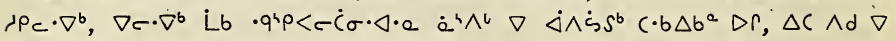
$\Delta U=c^{b} \triangleleft a b$ b $\cap V c^{c} c^{b} x$

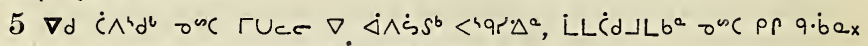

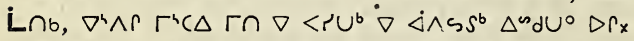

6 rUer Lb $\Delta^{a} d U \cdot \Delta^{a}, \nabla \Gamma r \cdot \nabla^{b} b \Gamma d \cdot \Delta^{b}$ Linr. $\Delta^{a} ; \nabla d s \Delta c \cdot b^{a} \Gamma U_{c}$

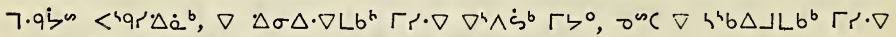

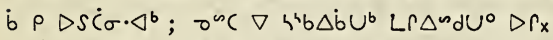

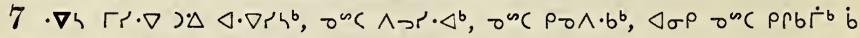

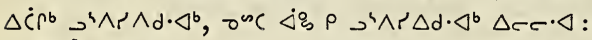

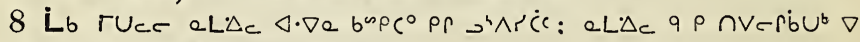

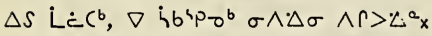

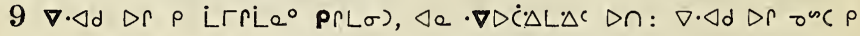

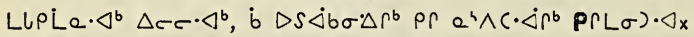

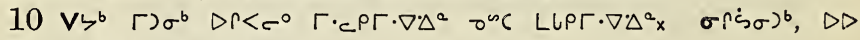

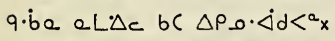

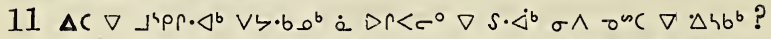

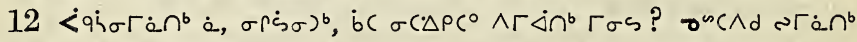

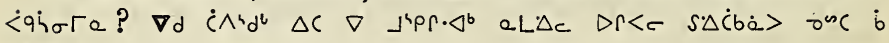
$\triangle b S^{b} \sigma \wedge x$

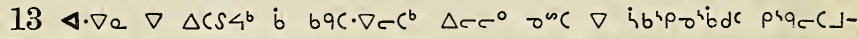

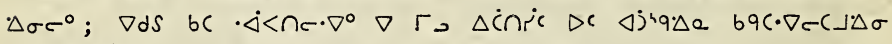
sibnt $\Delta \sigma^{b} x$

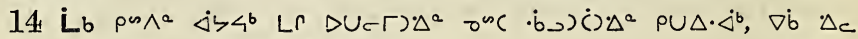

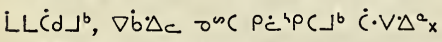

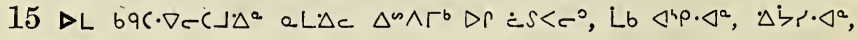
$L \Gamma(\sigma) \cdot \Delta^{a} x$

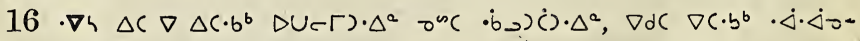

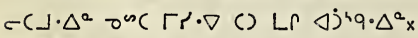

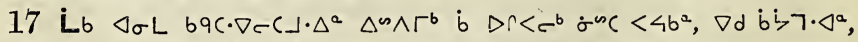

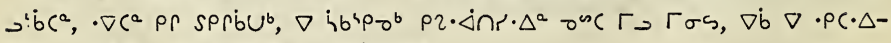

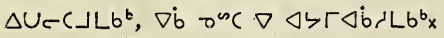




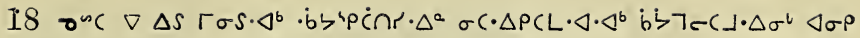
$\dot{b} \dot{b} \succ \neg-C\lrcorner \cdot \Delta \sigma q \sigma^{b} \mathrm{x}$

\section{Aspcra $\Delta b^{\circ} 4$.}

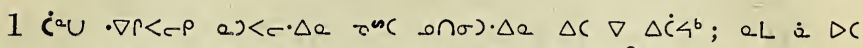

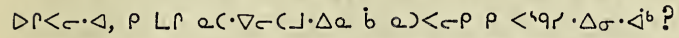

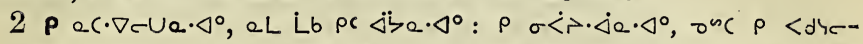

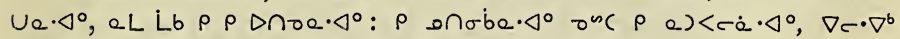

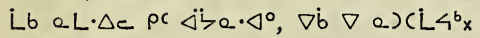

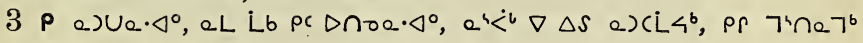
$\rho \operatorname{LC} e C \cdot \nabla \in C\lrcorner \cdot \Delta \sigma \cdot \nabla^{b} x$

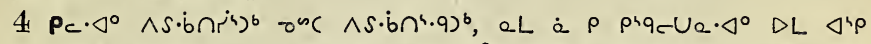

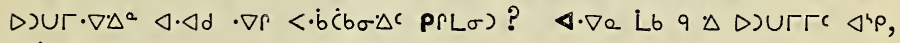
$<\cdot \dot{b} U^{\circ}$ P $(L \sigma) \cdot \Delta x$

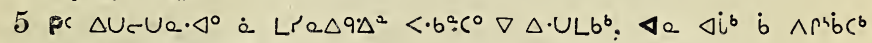
$\triangleleft b \cdot \Delta C L \Delta U C C\lrcorner \Delta \sigma c^{c}$ ?

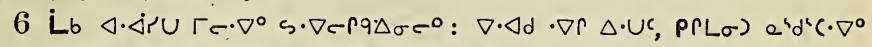

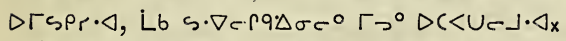

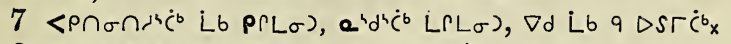

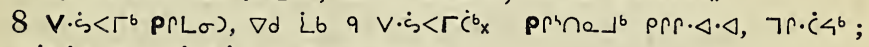

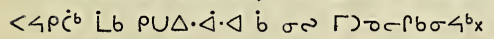

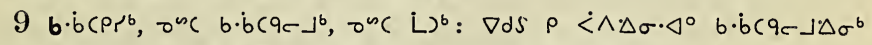

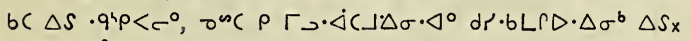

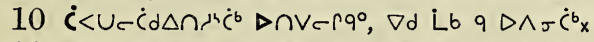

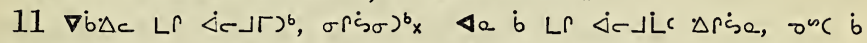

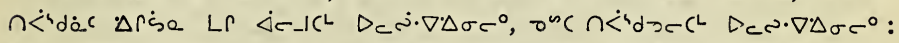

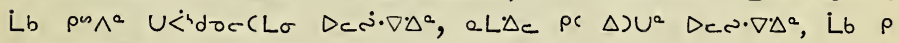
กं่doc $U^{a} x$

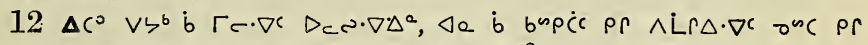
$\sigma S \cdot \Delta \dot{a} \Gamma \Delta \cdot \nabla^{c}: \triangleleft \cdot \nabla e \rho_{c}$ b $\cap \dot{<}^{4} d e^{c} d^{b} C^{b} \triangleleft \cdot \nabla e$ ?

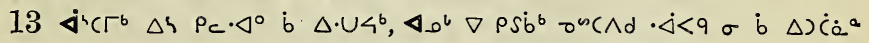

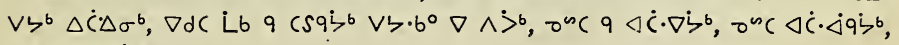
onC $\Delta$ níb:

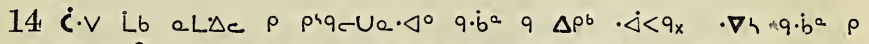

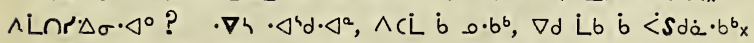

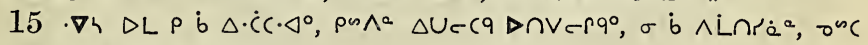
$\Delta L \sigma^{n}(\Delta \sigma L \sigma \dot{b}) \cup \dot{a}^{a} x$

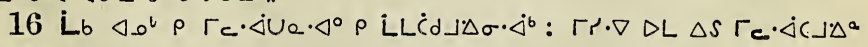
Ĺغ $C^{a} \cdot x$

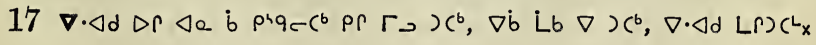




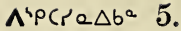

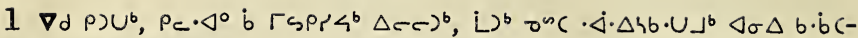

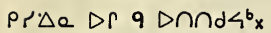

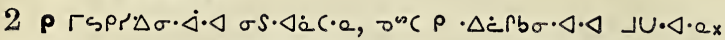

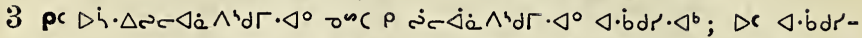

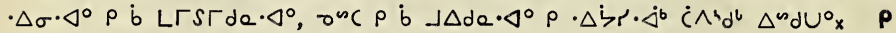

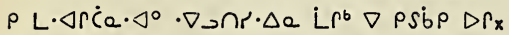

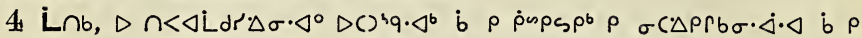

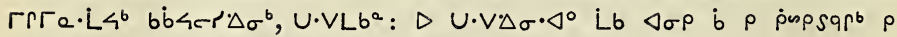

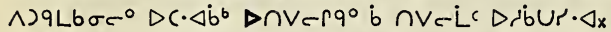

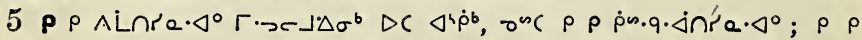

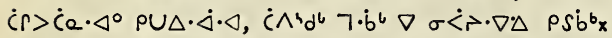

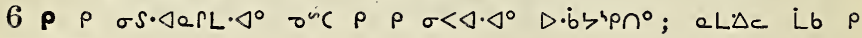
os dic $\dot{c} \cdot \triangleleft_{0} x$

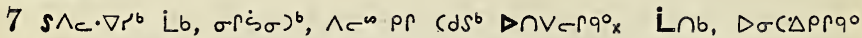

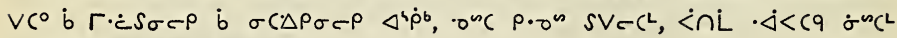

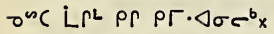

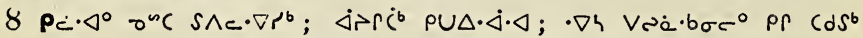
DnV $-r^{\circ} \mathrm{x}$

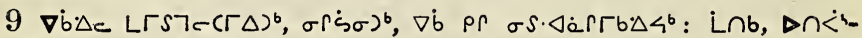
d $\sigma q^{\circ} \sigma<\Delta^{0} \nabla n^{\infty} b^{\circ} \Delta^{\omega} \cdot \dot{b} U \Gamma^{b} x$

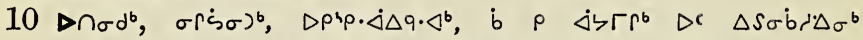

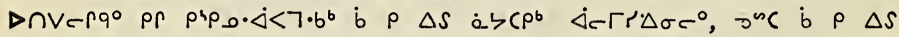
$s \wedge c \cdot \nabla r r^{b} x$

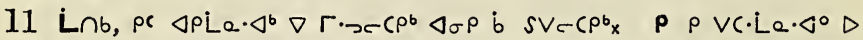

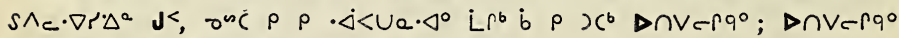

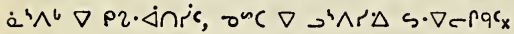

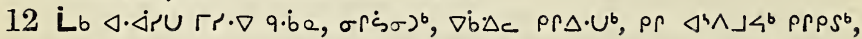

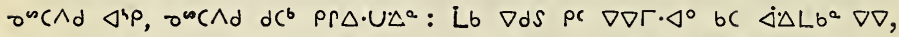

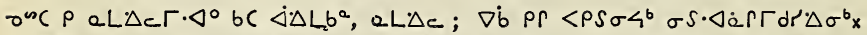

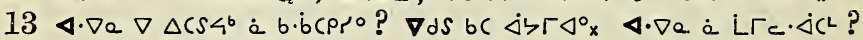
$\nabla d S$ bC $\sigma b\lrcorner\langle\dot{b} \Gamma \dot{\zeta} \Delta \sigma b\lrcorner \Delta a_{x}$

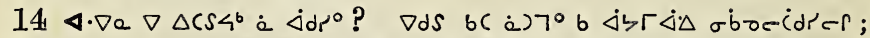

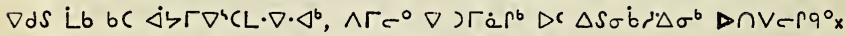

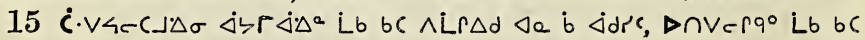

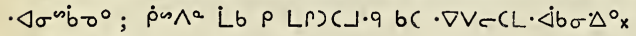

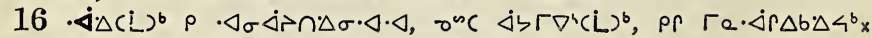

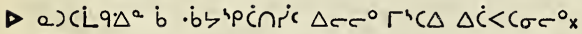

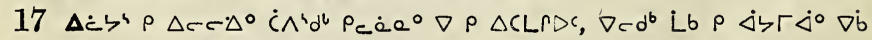

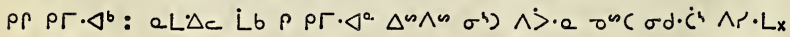

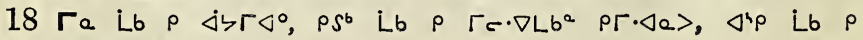
$\sigma C \triangle P \dot{C} L b^{a} \triangleright \sigma \dot{C} \triangle P \dot{C} b a x$ 


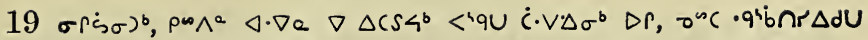
$\triangleleft \cdot \nabla c \cdot \triangleleft ;$

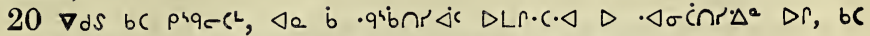

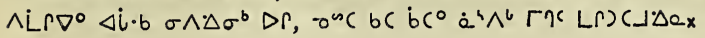

\section{$\dot{\sigma} s C L \quad L L L \cdot \Delta L i e \Delta L q \cdot \Delta e \quad \Lambda \varsigma_{x}$}

\section{$\Lambda^{\prime} p c r a \triangle b^{\circ} 1$.}

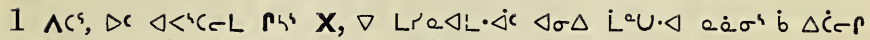

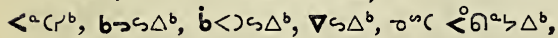

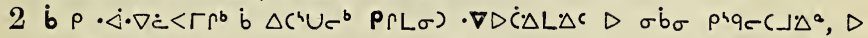

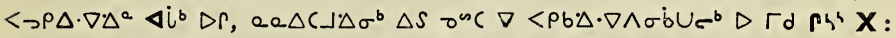

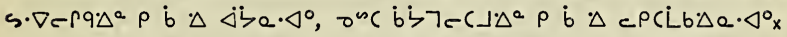

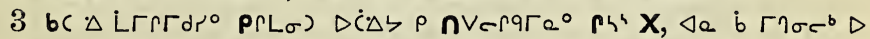

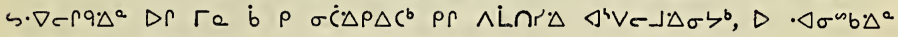
$r^{\prime} \times D_{\sigma} \wedge \cdot \triangleleft \Delta r$,

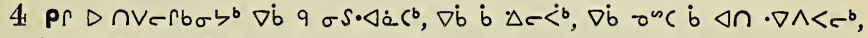
$\nabla \triangle^{b} \dot{C} L b \triangle S^{b}$ PrPS db,

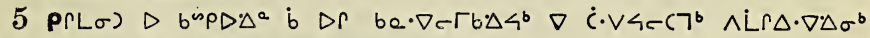

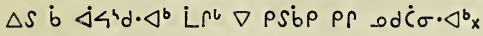

$6 \Delta C \dot{b} \Delta S \dot{a}^{4} \wedge^{b} \Gamma_{c} \cdot \dot{\Delta} C 7^{b}, \Delta C \Delta_{0^{b}} \wedge C L, \dot{\rho} \omega \wedge^{a} \Delta U-\dot{c} \cdot b^{b} \rho \rho \Delta \rho b, \rho$

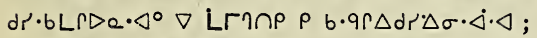

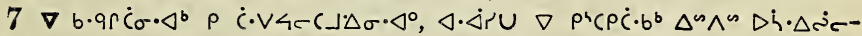

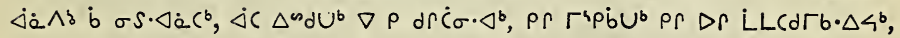

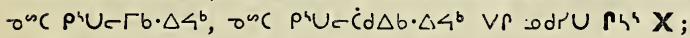

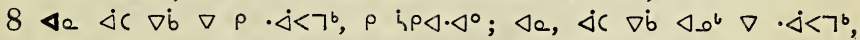

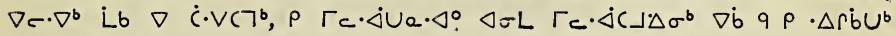

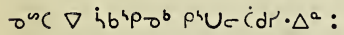

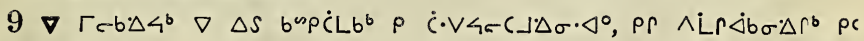
$\Delta i d \cdot \Delta \cdot \triangleleft^{b} \Delta \cap_{x}$

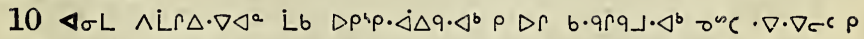

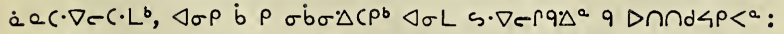

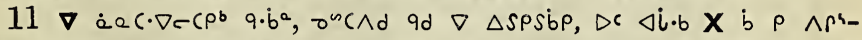

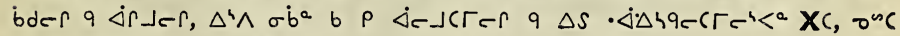

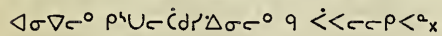




\section{$\operatorname{\sigma } \backsim C^{\circ} \wedge c^{s} 1,2$.}

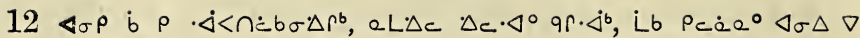

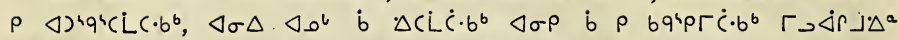

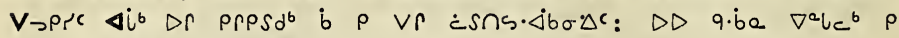

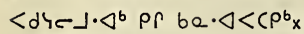

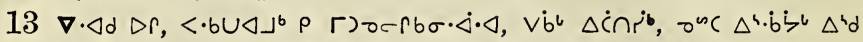

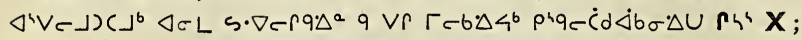

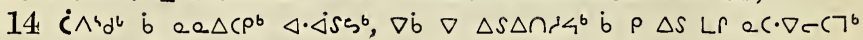
- $\nabla$ b b $\Delta^{4} \wedge \nabla \dot{b}$ b $\rho$ piqन $c 7^{b}$;

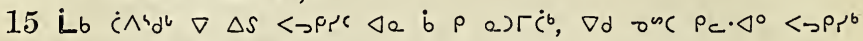
$\Gamma r \cdot \nabla \nabla \Delta S \wedge \mathrm{L} \cap>\mathrm{e} \sigma \cdot \Delta^{b} ;$

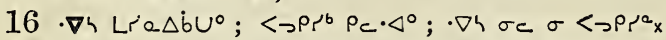

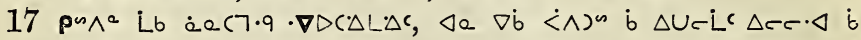

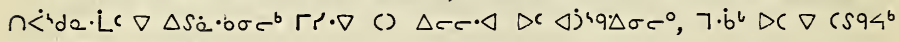
$\wedge L \cap r^{b}$ e $\sigma r \Delta \sigma^{b}$ :

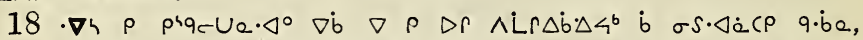

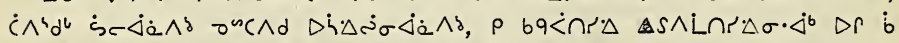

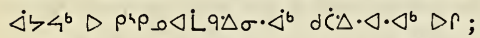

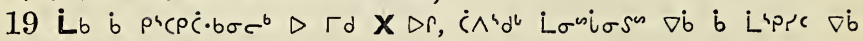
onc b $\triangle r \wedge r c$ :

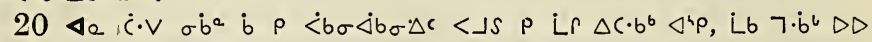

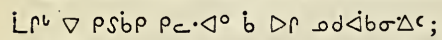

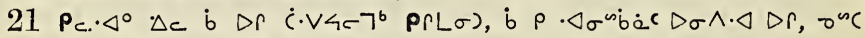

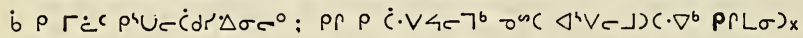

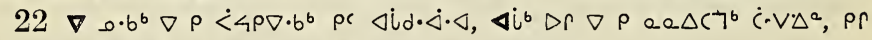
$\left.\dot{c} \cdot \vee i \rho \nabla \cdot b^{b} \Delta r \dot{\zeta} \sigma L \cdot \triangleleft^{b},<>\rho \Gamma U \Delta^{b} \cdot \nabla \cdot \nabla \tau^{c} i \rho \Delta\right)^{b}$ :

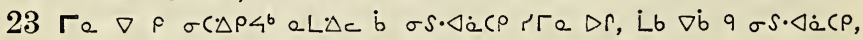

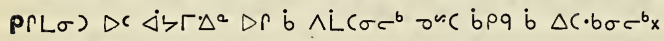

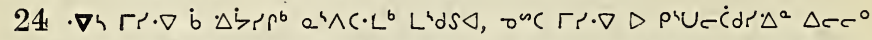

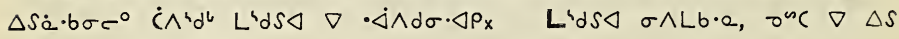

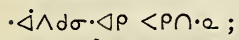

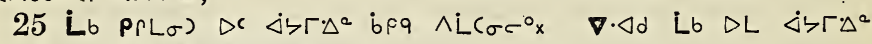
$\Gamma د \Delta r\lrcorner \Delta \sigma^{b} \dot{b} \Delta r$ bqu $\Gamma b \Delta 4^{b} x$

\section{$\Lambda^{4} p c r e \triangle b^{a} 2$.}

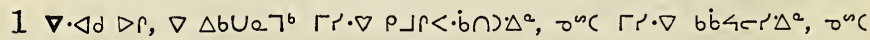

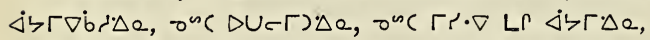

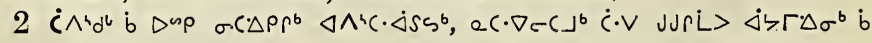
$\Delta R<r^{b}, \rho C D R D \wedge P \angle^{b} ;$

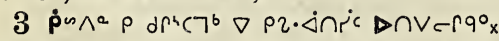

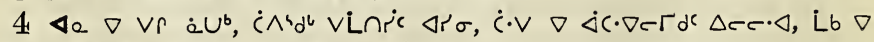

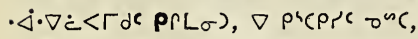

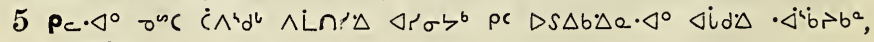
380 


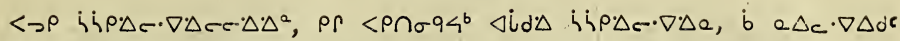
P $P(\sigma) \Delta c \Delta \Gamma \boldsymbol{P h}^{4} \mathbf{X} \mathrm{X}$

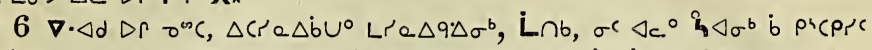

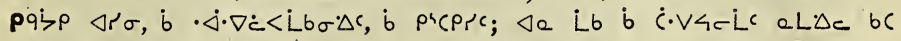

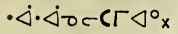

$7 P_{c .} \cdot \triangleleft^{\circ}$ Lb b $\dot{C} \cdot V C 7^{b} \rho$ P'CPL. $\triangleleft^{\circ}$, Lb $\triangleleft \sigma \rho$ b $S \wedge C \rho b$ Dr $\triangleleft \sigma \Delta$

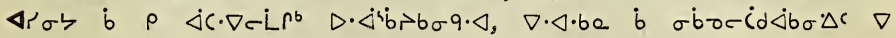
pqל० ;

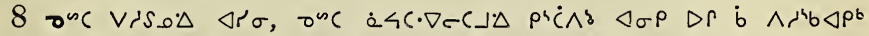

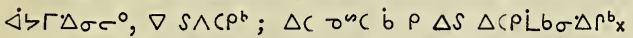

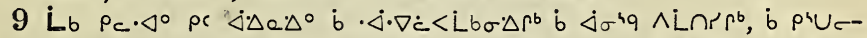

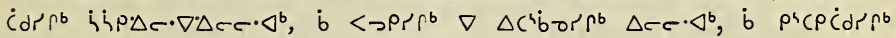

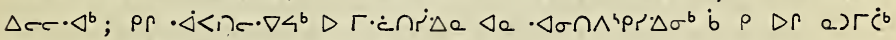

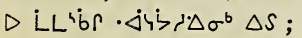

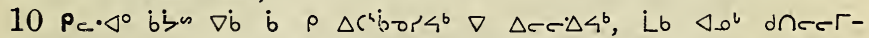

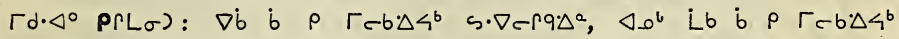
$c \cdot \nabla c r q \cdot \Delta_{a} x$

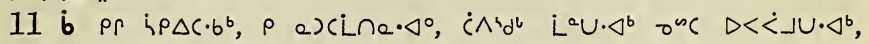

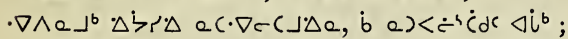

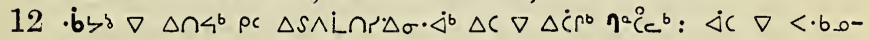

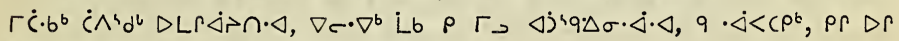

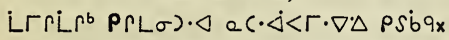

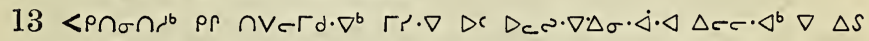

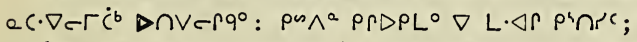

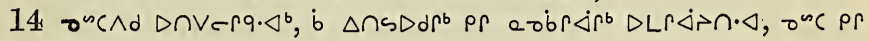

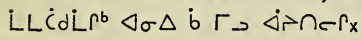

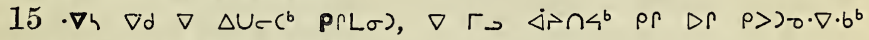
$\triangle 6 q<\dot{<} 6 \cdot \Delta^{b} \nabla$ bq<ंกr. $\Delta \Delta \cdot \cup r^{b}:$

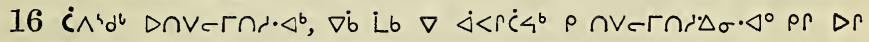

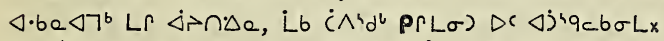

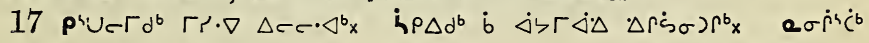

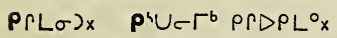

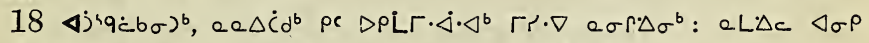

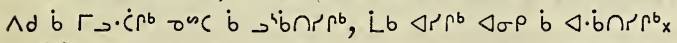

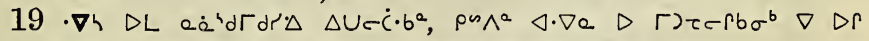

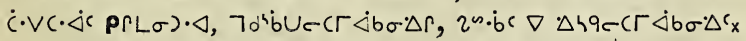

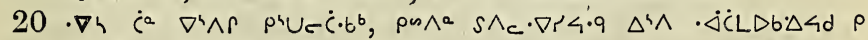

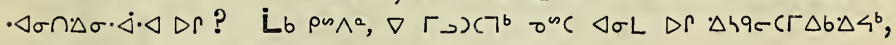
$S \wedge c \cdot \nabla r\left\langle\cdot 9\right.$ Lb, $D 7 c^{\circ} a \Delta c \cdot \nabla \Delta d$ PRLC) $x$

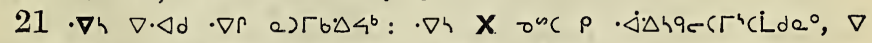

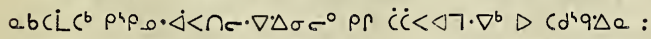

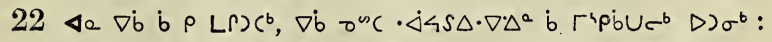

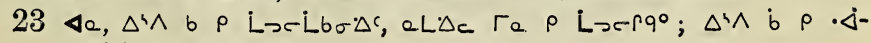
381 


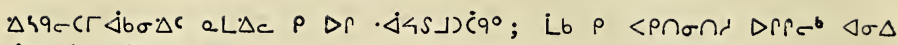
.bेל bे $\cap<4 d \sigma q c r$ :

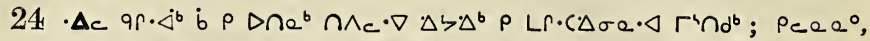

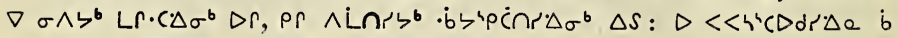
$\Delta r \Gamma e \cdot \Delta r \Delta b \Delta \zeta^{b} x$

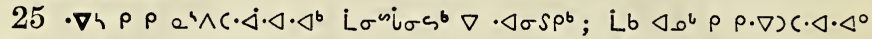

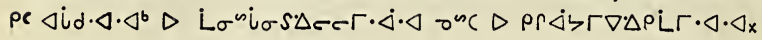

\section{$\Lambda^{\prime} \rho C \beta a \triangle b^{\circ} 3$.}

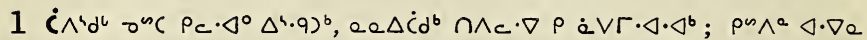

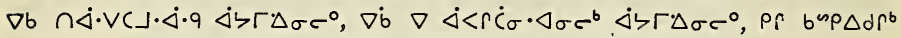
$D C \Delta S \wedge \mathrm{L} \cap \Omega^{\prime} \Delta \sigma \sigma^{\circ} \Delta \Delta \cdot \Delta \cdot \Delta$ :

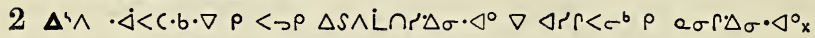

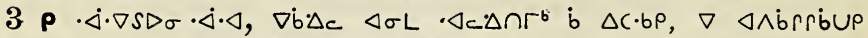

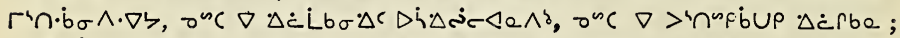

4 Lb $\nabla d S \Delta_{\sigma L} \Delta \dot{C} \Gamma^{b} \Delta \dot{C} \cap r^{b} \Delta^{a} \Gamma U \Delta^{b} \dot{b} \quad \Delta C \cdot b^{b}, \Delta \sigma L \quad \nabla \dot{b} \quad 9 \sigma S \cdot \Delta_{\dot{a}} C^{b}$;

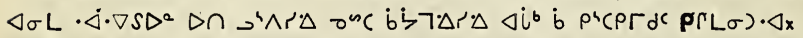

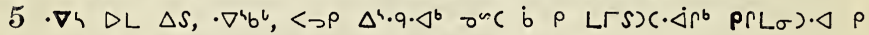

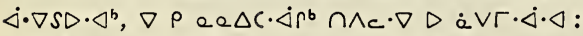

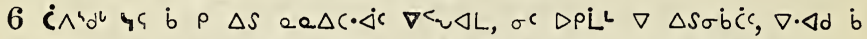

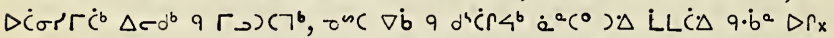

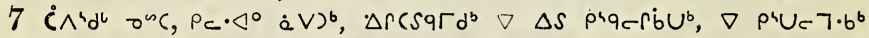

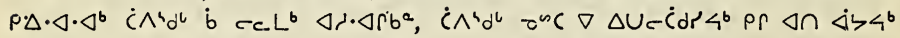

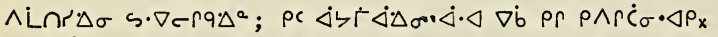

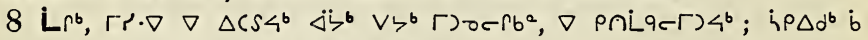

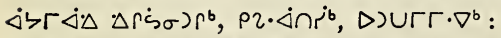

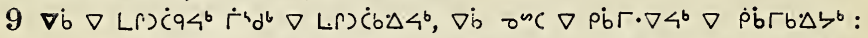

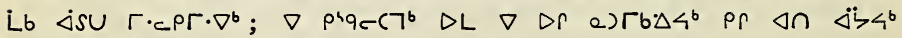
$r \cdot c b r 9 r \cdot \Delta a x$

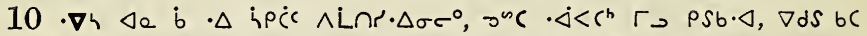

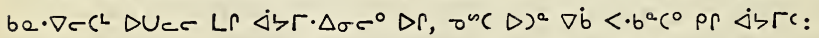

$11 \nabla d S$ br $\nabla \wedge a^{L} \dot{b}$ Lec $\left(\sigma c^{b}, b c\right) c^{L}$ Lb b $\Gamma \cdot \dot{c} S \sigma c^{b} ; \nabla d S$ bc

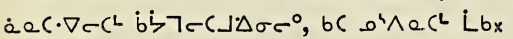

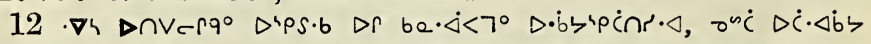

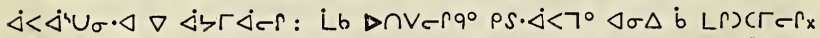

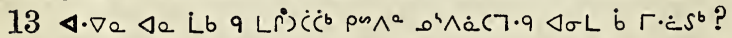

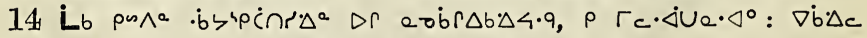

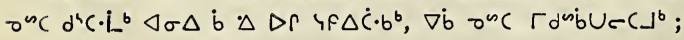

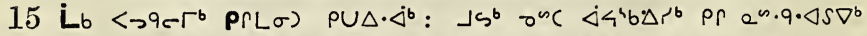

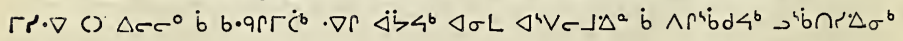
$\sigma^{n c} e \sigma r \Delta \sigma^{b}$ : 


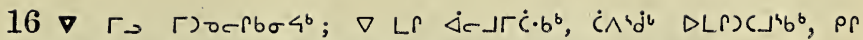

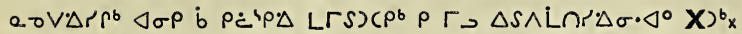

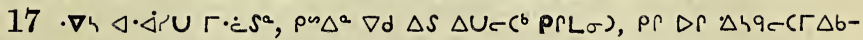
$\left.\Delta 4^{b} \nabla \Gamma \rightarrow\right)\left(7^{b} \Delta^{n} \wedge \omega \nabla L \beta\right)\left(7^{b} \mathrm{x}\right.$

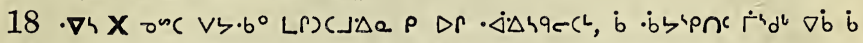

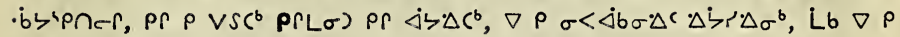

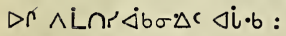

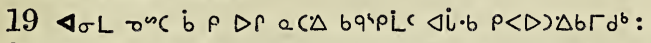

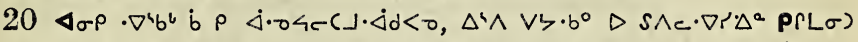

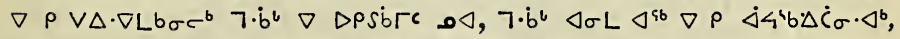

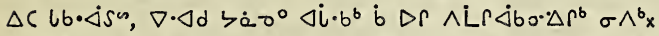

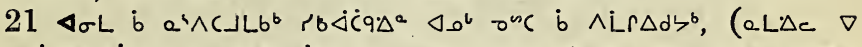

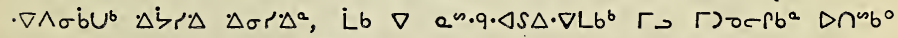
$\boldsymbol{P}(L \sigma)), D \cdot \triangleleft \sigma^{\infty} b \Delta^{2} D r \quad \boldsymbol{P}^{4} \mathbf{X}$ :

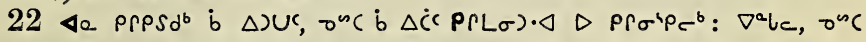

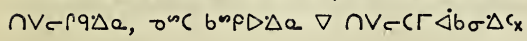

A

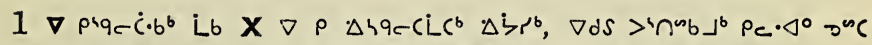

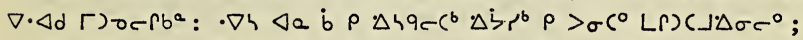

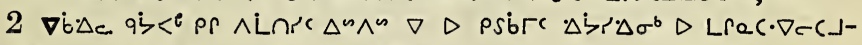
$\Delta \sigma c^{b} \Delta c c \cdot \triangleleft$, Lb $D\left(\Delta U=(\lrcorner \Delta \sigma c^{b} P \Gamma L \sigma\right) \cdot \Delta x$

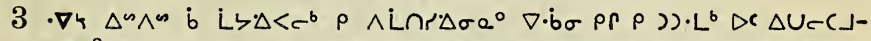

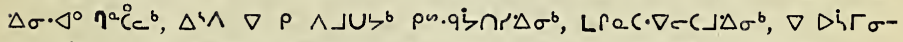

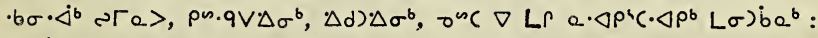

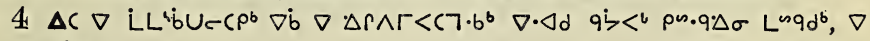
$<\cdot b \_\Gamma \dot{c} \cdot b^{b}$ :

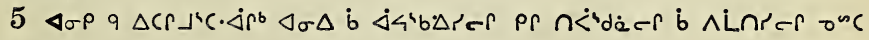
b) $\sigma \wedge-r_{x}$

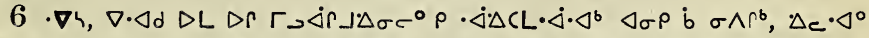

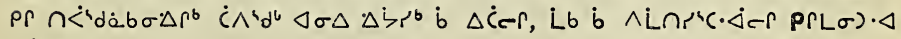
$\Delta i d^{b} x$

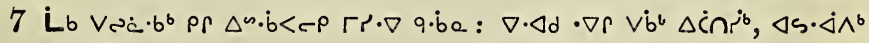
L.b $\nabla \Delta \triangleleft\left\ulcorner\Delta\left\langle^{b} x\right.\right.$

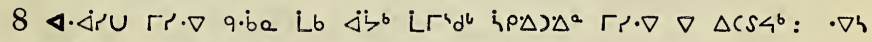

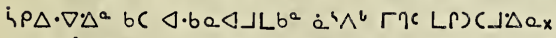

$\left.\left.9 \dot{L} \dot{r^{*}} d^{b} a \dot{a} \Delta \dot{b}\right)^{b}, \nabla \dot{b} \nabla \Delta \zeta q c\left(7^{b} \dot{b}\right) \dot{c}\right)<^{b} x$

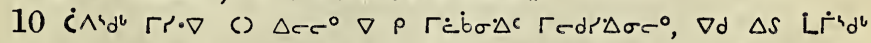

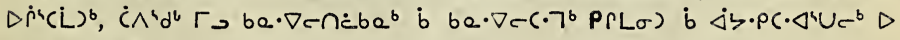
c. $\nabla e r q \Delta^{a} x$

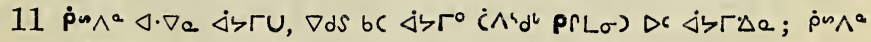
383 


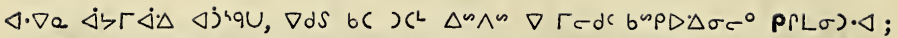

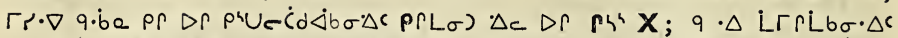
onc $\cap V$ - $p q c$ bpq onc bpqx $\nabla 7^{2} x$

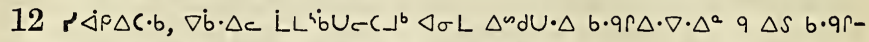
$\Delta b \cdot \Delta \zeta^{b}, \dot{C} \wedge^{b} d^{b} \nabla \Delta \cap \cap d^{b} q \cdot b^{a}$ b $L^{b} L^{b} b \cup c \dot{c} \cdot b^{b}$ :

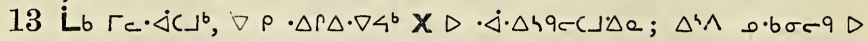

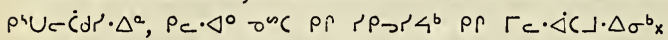

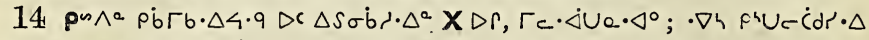

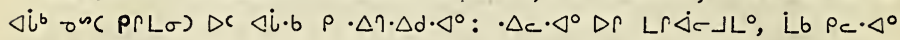
Dr pruc $\dot{C} d \triangleleft^{\circ} x$

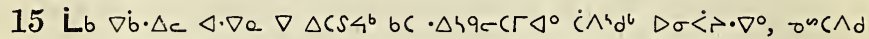

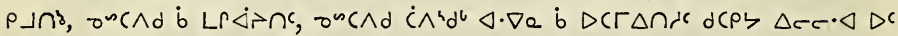

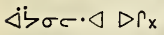

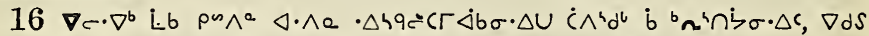

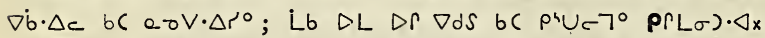

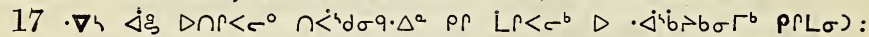

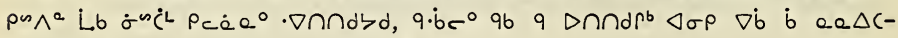

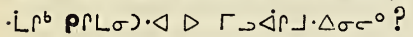

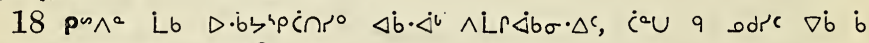
Le). ¿nRc onC DLR.Co?

$19 \nabla \cdot \Delta d \Delta r, \nabla d S \Delta \sigma \Delta \dot{b} \cdot \Delta h q c\left(p b\right.$ b $\Delta c^{a} \cup c^{b} p(i j \sigma) \Delta c \Delta \cup c\left(d \cdot \Delta^{a}\right.$,

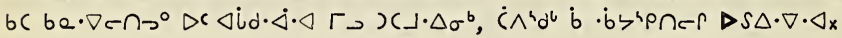

$\Lambda^{4} p C_{P} a \Delta b^{2} 5$.

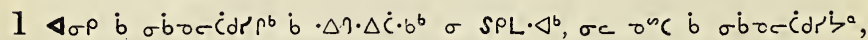

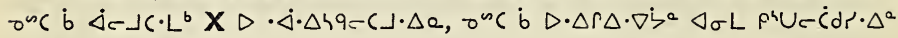
$9 \vee r \rho d \dot{C} \sigma \cdot \Delta^{b}$ :

$\left.2 \Delta S \Gamma d^{b} \nabla L_{\sigma^{u}} i_{\sigma} S L P \Gamma L \sigma\right)$ b $\Delta \eta \Delta \dot{c} \cdot b^{b}, \nabla \dot{a} \dot{b} b \cdot \dot{\Delta}<7 \cdot b^{b}, a L \cdot \Delta c \wedge d$

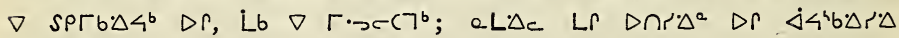
r) 0 rerbo ${ }^{b}$ :

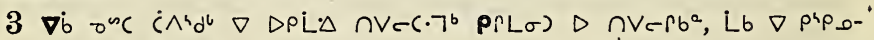

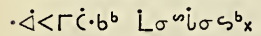

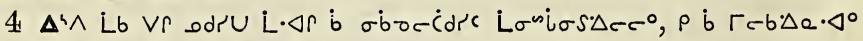
$p^{n} U c \dot{C}\left(d r \Delta D P L \cdot \Delta^{b}\right) \cap^{a} \nabla \dot{b} \dot{b} \Delta \cap \sigma>\Delta \dot{a} \cdot b^{b} x$

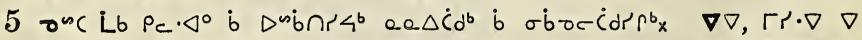

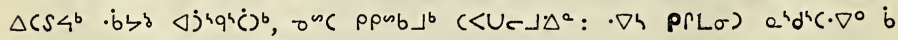

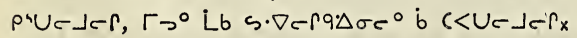

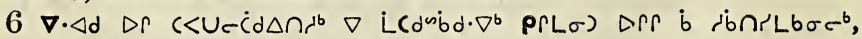

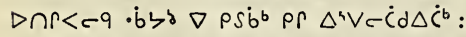

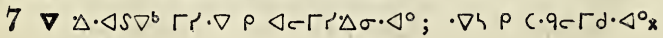
384 


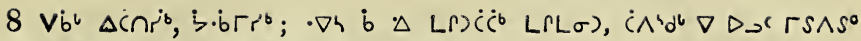

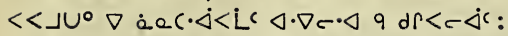

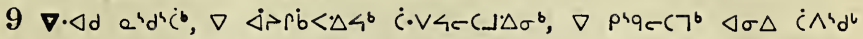

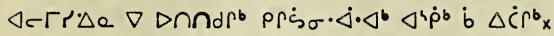

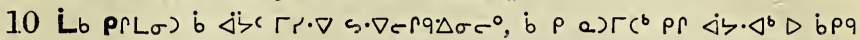

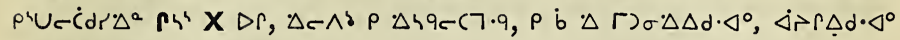
$L " b \Delta \Delta \Delta \cdot \Delta^{\circ}, \quad s p b<\Delta \Delta d \cdot \Delta^{\circ} x$

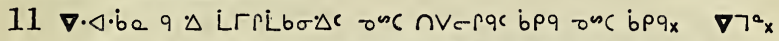

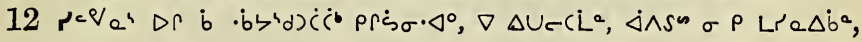

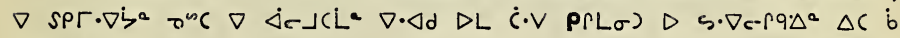
$\sigma<\Delta<s^{b} x$

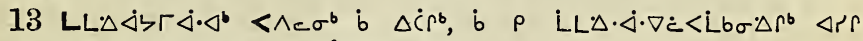

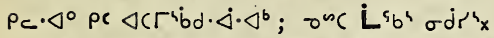

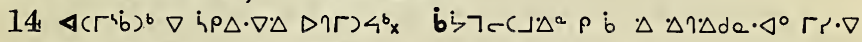

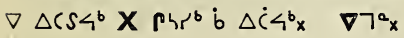

\section{$\sigma \sim \triangleright L L \cdot \Delta L^{\prime} e \Delta L q \cdot \Delta e \wedge c_{x}$}

\section{A'pcra $\triangle b^{2} 1$.}

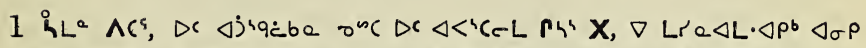

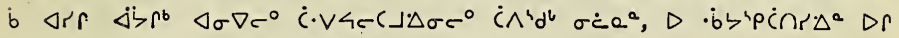

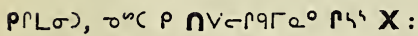

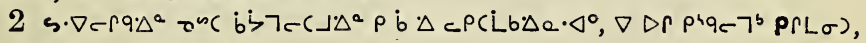

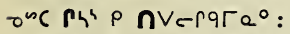

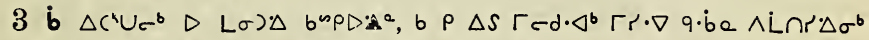

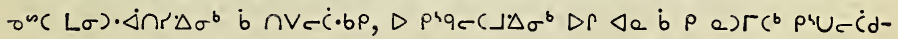

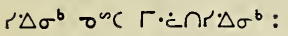

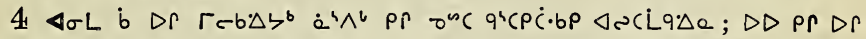

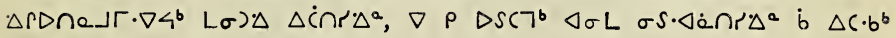
$\triangle 4 p b \operatorname{Lr} a c \cdot \nabla e C \cdot \Delta^{2} D \Gamma_{x}$

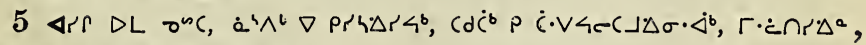

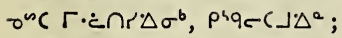

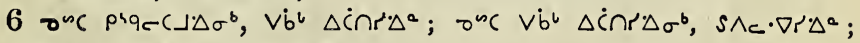

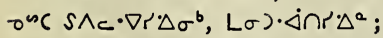

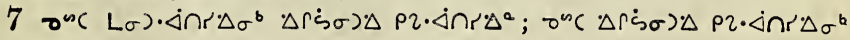
ip $\triangle \cdot \nabla \cdot \Delta^{2} x$

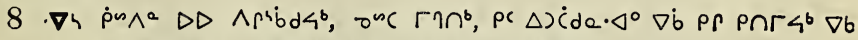

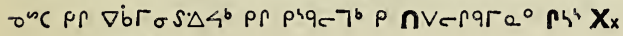




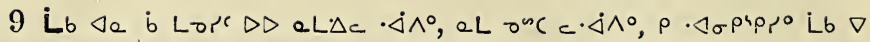

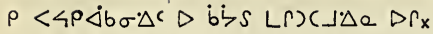

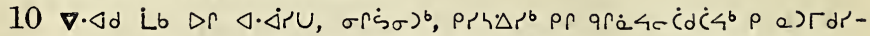

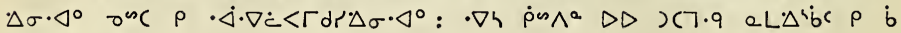
$\left\langle\rho S \sigma e \cdot \Delta^{\circ}\right.$ :

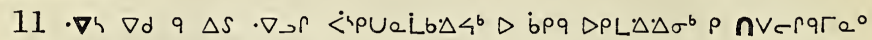

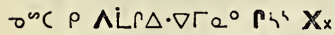

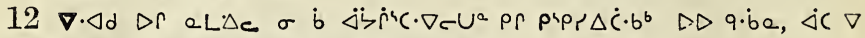

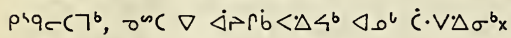

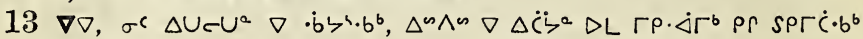
$\nabla$ pipr $\triangle \dot{C} \cdot b^{b}$;

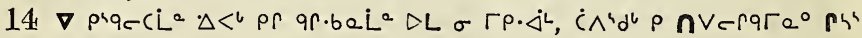
$\mathbf{X} \nabla \rho \cdot \dot{\Delta<n \kappa^{-} x}$

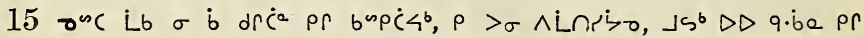
pips<ib

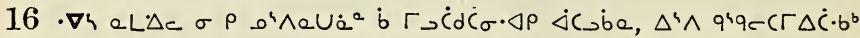

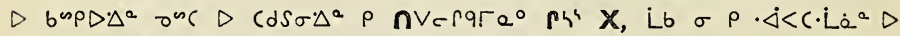
PCDPL. $\nabla C \dot{C} \mathrm{C} P \cdot A^{\circ} x$

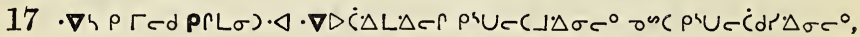

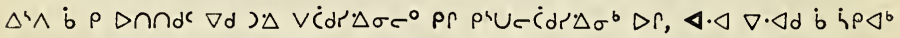
$\sigma d d^{\prime}, \dot{a}^{4} \wedge^{l} \dot{b} a \Delta_{c} \cdot \nabla \Delta c_{x}$

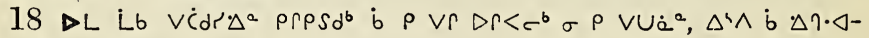
$p \cap<0<\neg \rho \cdot \Delta r^{b} x$

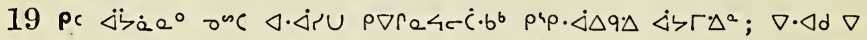

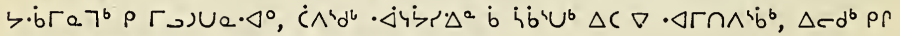

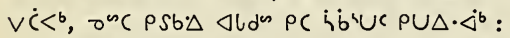

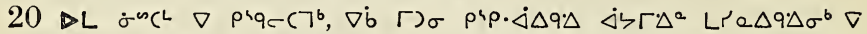

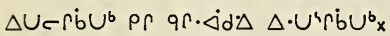

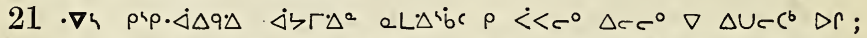

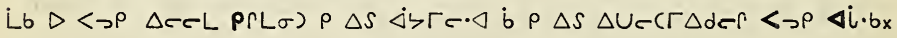

\section{$\Lambda \wedge c ̧ \circ \triangle b^{2} 2$.}

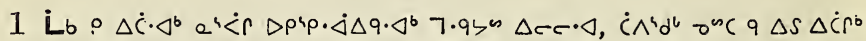

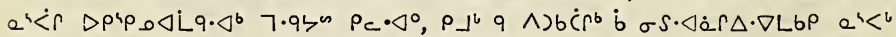

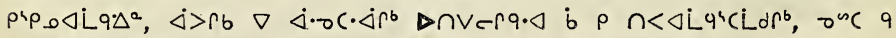

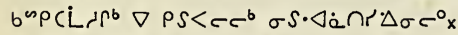

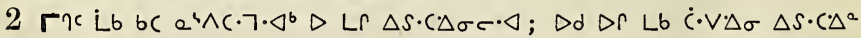

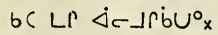

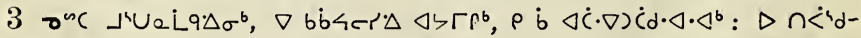

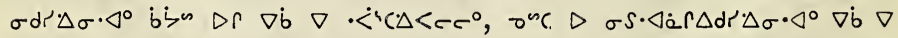
$\sigma \dot{L} L b \sigma c^{b} x$

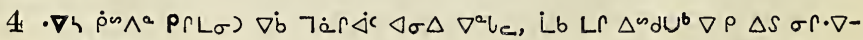
386 


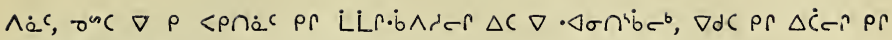

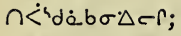

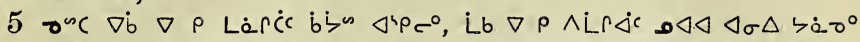

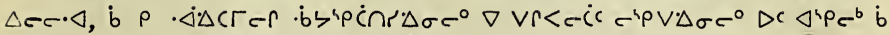
Lr. $\dot{C} C$;

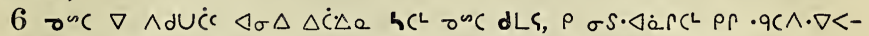

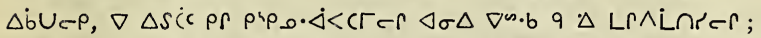

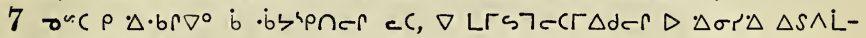

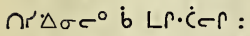

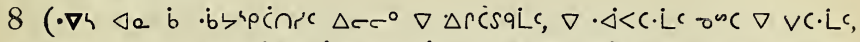

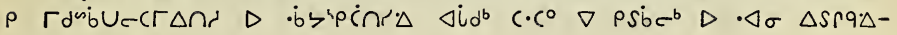
$\sigma c \cdot \Delta \nabla r:)$

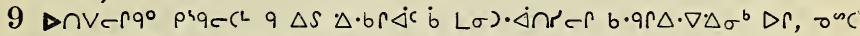

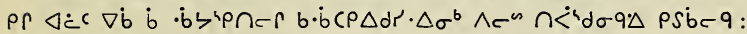

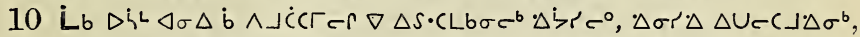

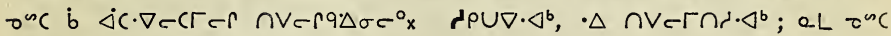

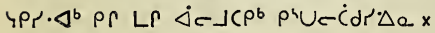

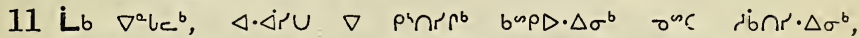

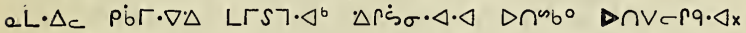

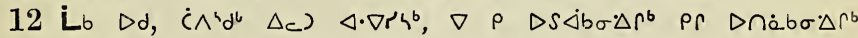

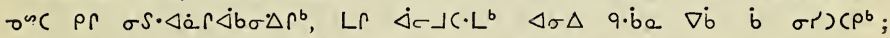
$\Gamma)$ Lb bC $\sigma S \cdot \Delta \dot{a} \cap \cdot \cdot \Delta^{b} \cap \wedge_{c} \cdot \nabla D$ Lind $\Delta \sigma \cdot \dot{\Delta}^{b}$;

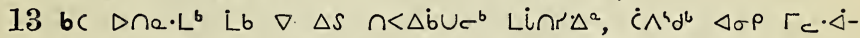

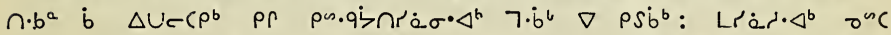
$\left.\Delta \Delta c \wedge r^{\prime} \cdot \Delta^{b}, \nabla \Gamma_{c} \cdot \dot{\Delta} n d \Delta \cap r^{b} \nabla b \dot{b}<-r \cdot \Delta \sigma \cdot j^{b} 7 \cdot b^{b} \nabla \quad \Delta r \Delta d\right) \Gamma \dot{c} \cdot b^{b} ;$

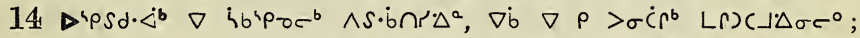

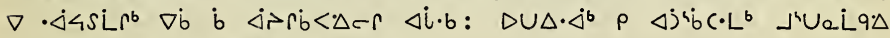

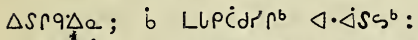

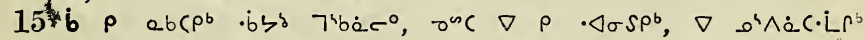

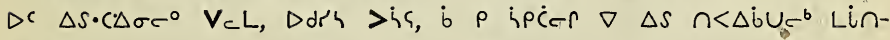

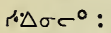

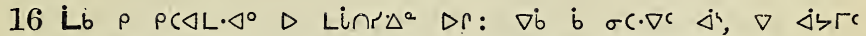

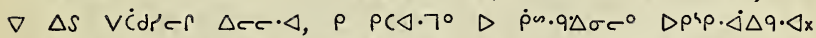

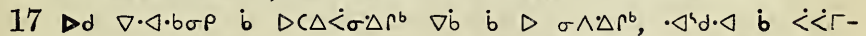
$\cdot \nabla \dot{<}^{3} C \rho \nabla \dot{\rho}^{4} \cap^{b} ; \Delta \sigma \rho \nabla \wedge P 2 \dot{\zeta}^{b} \nabla \cdot \Delta \sigma \cap \wedge^{b} b^{b}$ b $\rho q$ b $\Delta C^{4} C L \cdot \Delta b \sigma \Delta \rho^{b} x$

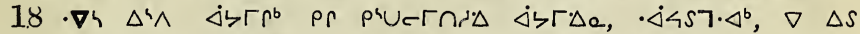

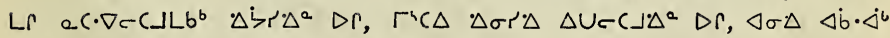

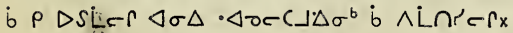

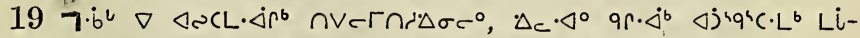

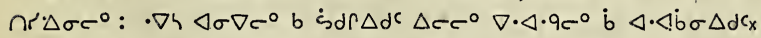

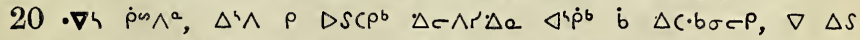

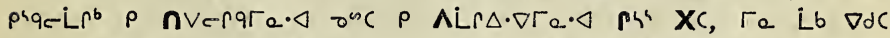

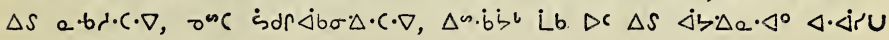
LंटC $\sigma c^{\circ} \Delta m \wedge^{n} \sigma^{n} C_{x}$ 


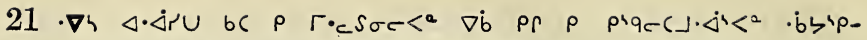

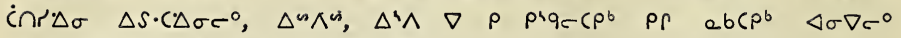
$<\rightarrow \rho \quad b q^{4} \cdot 9 \Delta \sigma \sigma^{\circ}$ b $\rho \quad<p \cap_{0} L \rho^{b} x$

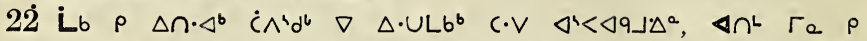

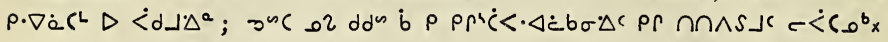

\section{A'pcra $\triangle b^{\circ} 3$.}

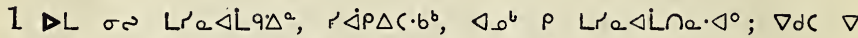

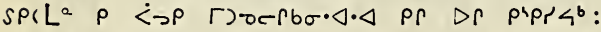

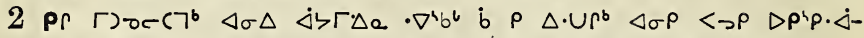

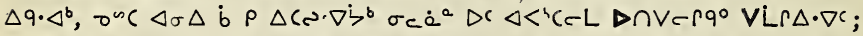

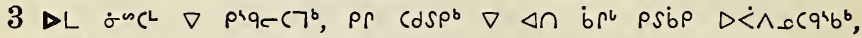
$\nabla \wedge\lrcorner \dot{C}\left(\rho^{b} \cap \wedge c \cdot \nabla D L R\right.$ a $\left.C \cdot \nabla-C\right\lrcorner \cdot \Delta \sigma \cdot \dot{\Delta} \cdot \triangleleft$,

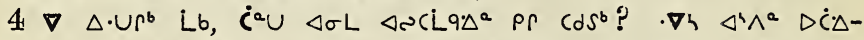

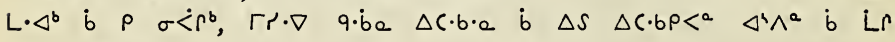
$\Delta S \dot{C} \cdot \Delta^{b} \Delta \Gamma_{x}$

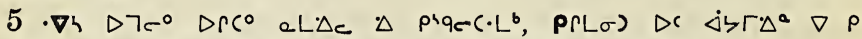

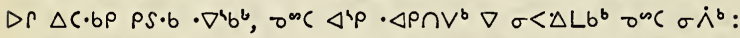

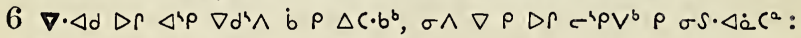

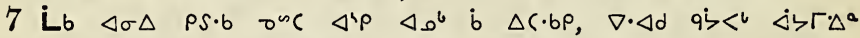

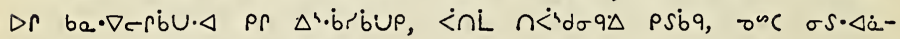

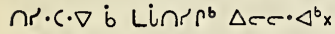

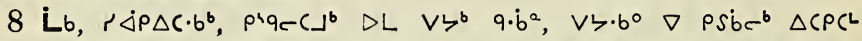

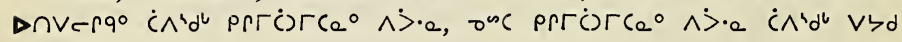
PSLOX

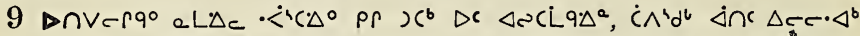

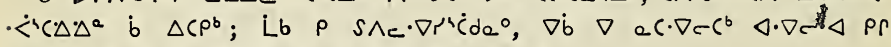

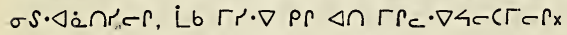

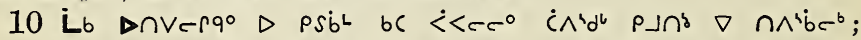

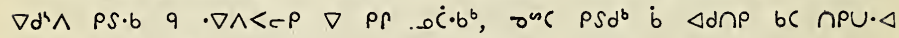

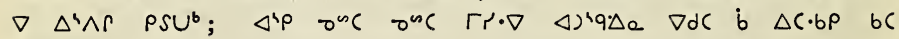
ib'pu. $\triangleleft x$

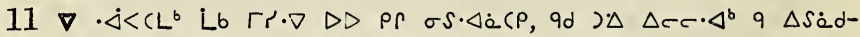

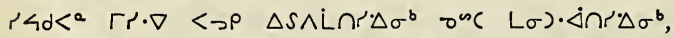

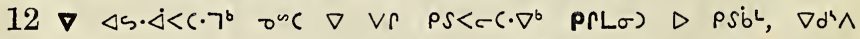

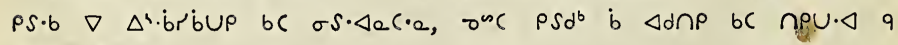
$\triangle A R r$ bSUix

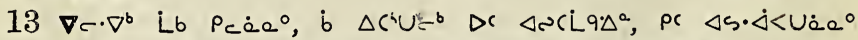

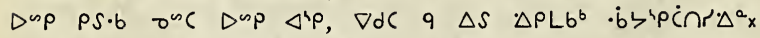

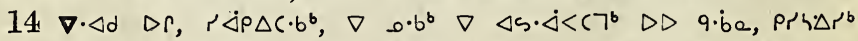

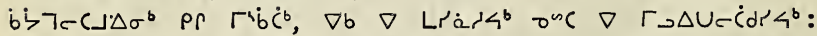

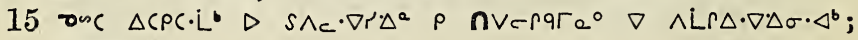
388 
\&ัs $\wedge C^{s}$

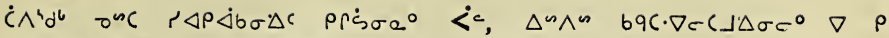

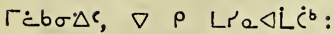

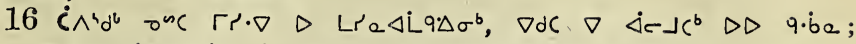

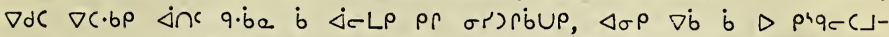

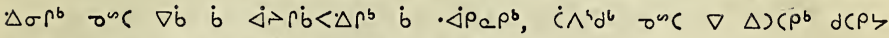

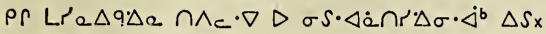

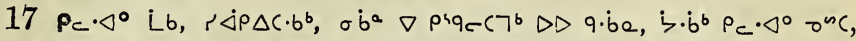

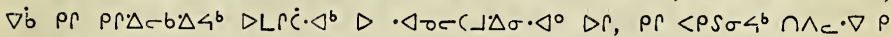
$L " b \cdot \nabla c C\lrcorner \Delta \sigma \cdot \Delta^{b} \Delta r_{x}$

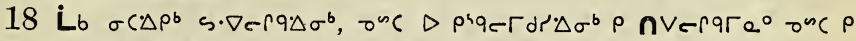

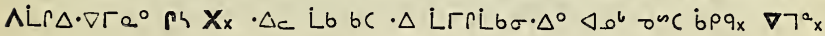

$$
\begin{aligned}
& \dot{\sigma} s C L \quad L L \cdot \Delta \text { Lre } \Delta \dot{L} q \cdot \Delta e^{2} \text { lex }_{x} \\
& \text { A pera } \Delta b^{a} 1 .
\end{aligned}
$$

$1 \varangle \sigma L L \cdot \Delta r \dot{\sigma}^{n C L}$ b $\rho \Delta \dot{r}^{b}, \dot{b} \rho V C L^{b}, \sigma^{n p s d a} e$ b $\rho \Delta r \cdot j<C L^{b}, \dot{b} \rho$

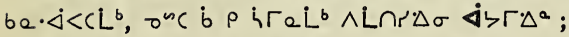

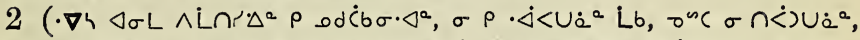

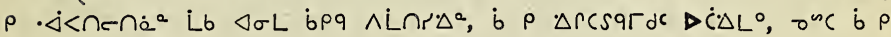
$\cdot \dot{\Delta}<n-b \Delta\rangle^{b}$;)

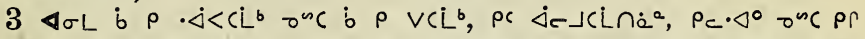

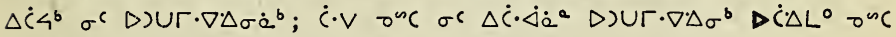
Ddrh Ph' $X_{x}$

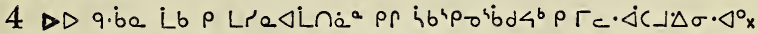

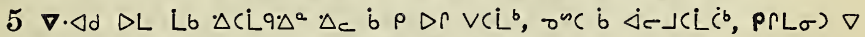

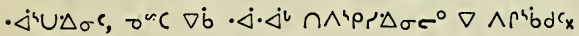

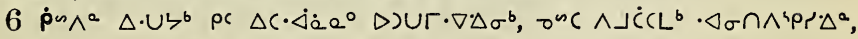

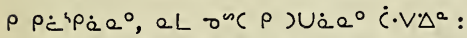

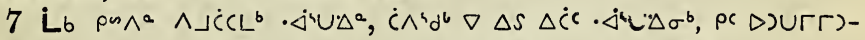

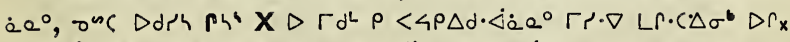

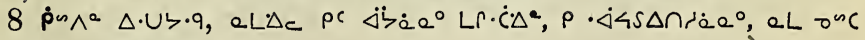
$\dot{C} \cdot \vee \cdot \Delta \cdot \rho \wedge r^{\circ} \dot{b} \dot{a} a^{\circ} x$

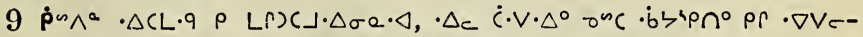

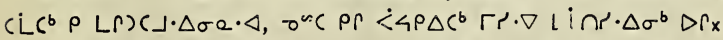

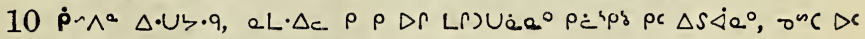
$\dot{\Delta b i \cdot \Delta a} a L \cdot \Delta c \rho \wedge r^{<i b d \cdot} \dot{\Delta} a_{a}{ }^{\circ} x$

389 


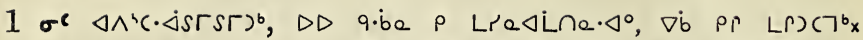

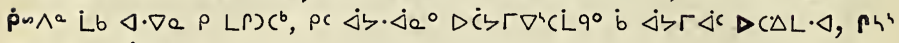

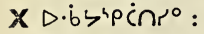

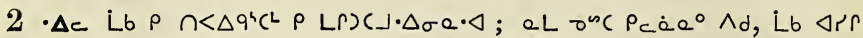
$\triangle L \Gamma)(\lrcorner \Delta \sigma \cdot \dot{\Delta} \cdot \triangleleft \triangleleft \sigma \rho \Gamma \rho \cdot \nabla \triangleleft 4 \dot{\rho} b \dot{b} \Delta \dot{C} \Gamma^{b} x$

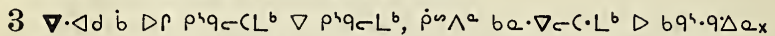

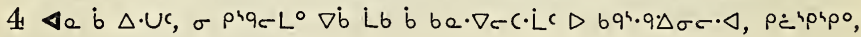

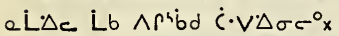

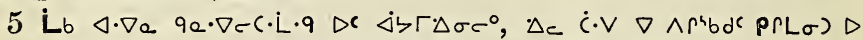

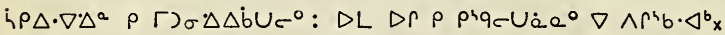

$6 \varangle a \sigma \wedge r^{4} b \cdot \triangleleft^{\circ}$ b $\Delta \cdot U c, \nabla d S$ bc $\left.\Delta S \wedge\right\lrcorner U<^{a}, \cdot 7 r^{b}$ b $\left.\rho \Delta S \wedge\right\lrcorner U \Gamma_{x}$

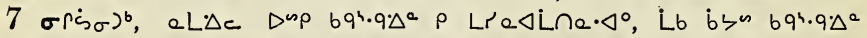

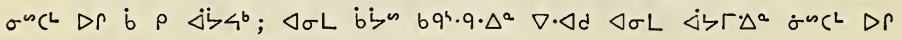
b) $\rho \vee C 7^{b} x$

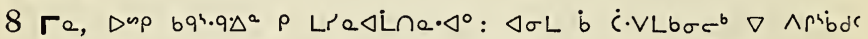

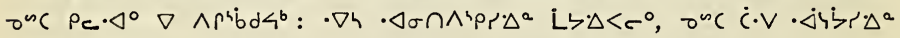
$\triangle \Omega^{\circ} \operatorname{lib} q^{\circ} x$

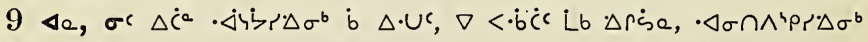
$\Delta c^{\circ} \wedge \kappa^{\infty} \triangleleft \Delta^{b} \mathrm{x}$

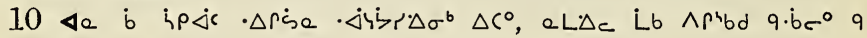
$\Delta r \wedge r s^{b} \times$

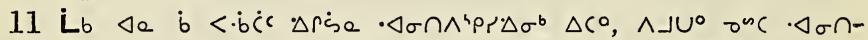

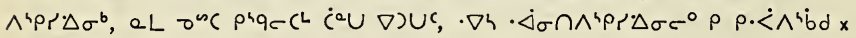

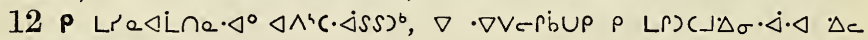
De $\Delta S \sigma \dot{b} \cdot \Delta^{2} \Delta R_{x}$

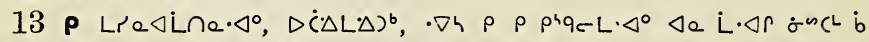

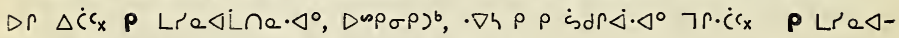

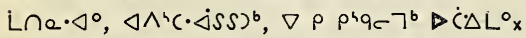

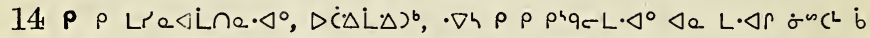

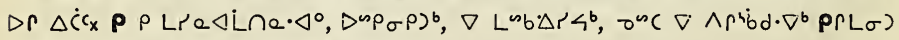
$\Delta C \dot{\Delta}>\Gamma \Delta^{a}, \sigma^{\operatorname{sic}} \nabla \rho \dot{s} d r \nabla^{b} 7 r \cdot \dot{c}_{x}$

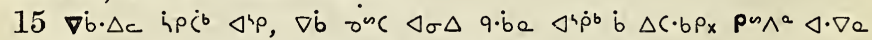

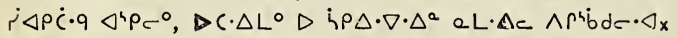

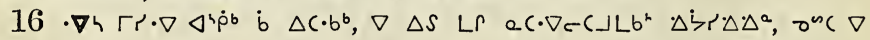

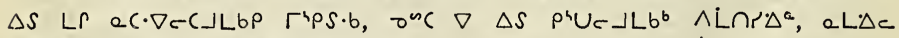

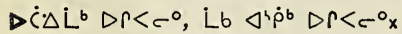

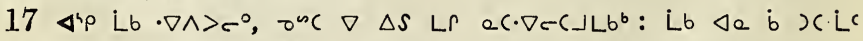
PrL $\sigma) \cdot \triangleleft D\left(\Delta U \sigma(\lrcorner \Delta \sigma \tau^{\circ}\right.$ bPq $\Delta C^{\circ} x$

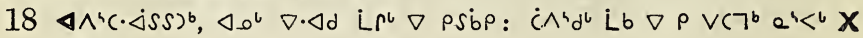
390 


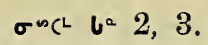

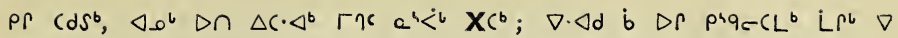
pSibPx

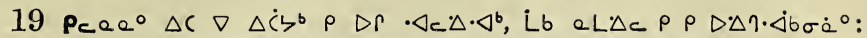

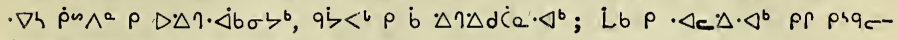
$\dot{C} d r \rho^{b} \nabla \dot{b} \Gamma r \cdot \nabla \nabla \rho \Delta \Delta \eta \cdot \dot{\Delta b \sigma \zeta b} x$

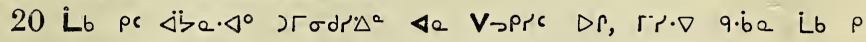
prq-Ue. $\triangleleft^{\circ} x$

21 eL $\Delta c$ p $\rho$ Lre $\Delta \dot{L} \cap a \cdot \Delta^{\circ} \nabla \dot{b} \nabla$ prqc $C 7^{b} \dot{C} \cdot V \Delta^{c}$, Lb $\nabla \dot{C} \cdot V C 7^{b}$, $\sigma^{n} \mathrm{C} \nabla \dot{b} \nabla \rho \dot{c} s \cdot \Delta \sigma \cdot \Delta^{b} \dot{C} \cdot V \cdot \Delta^{a} x$

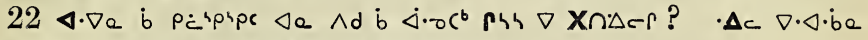

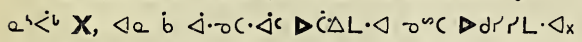

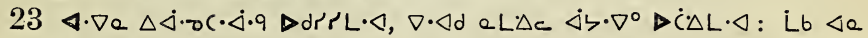

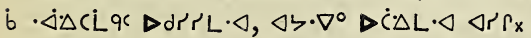

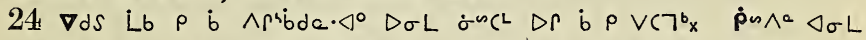

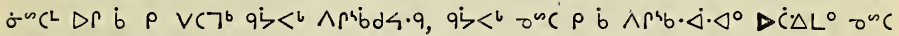
$\Delta$ drtiox

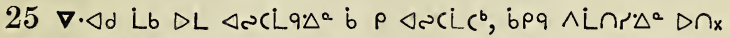

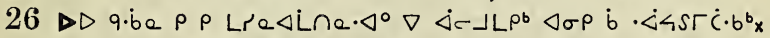

27 Lb $\Delta \sigma L) \Gamma \sigma d r \cdot \Delta^{a} \dot{b} \rho \Gamma_{c} \dot{c}^{b} \rho \wedge r^{b} b d a \cdot \Delta^{\circ}$, aL $\Delta c$ Lb $\rho$ a $C \cdot \nabla c U-$

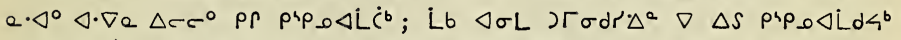

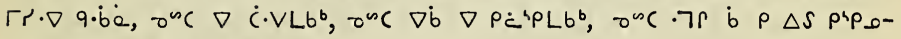
$\Delta \dot{L} d^{b}<^{b}, \nabla d q \Delta S \wedge P^{b} b \cdot \nabla^{b} x$

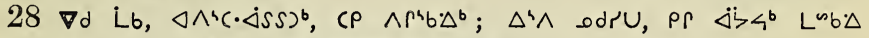

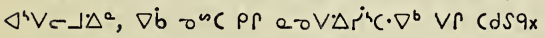

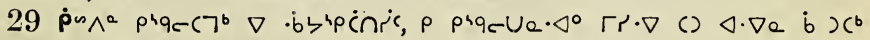

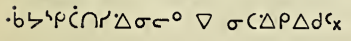

\section{$\Lambda^{4} p C^{\circ} a \triangle b^{\circ} 3$.}

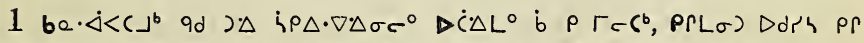

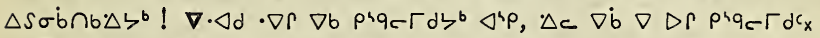

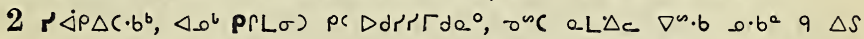

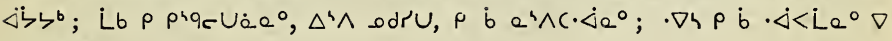

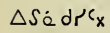

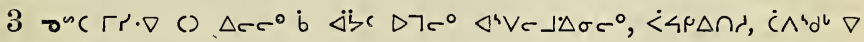

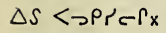

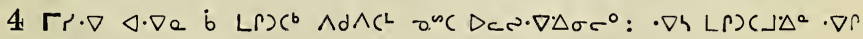

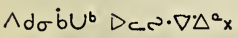

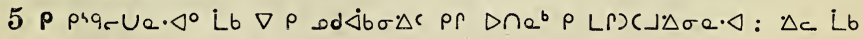

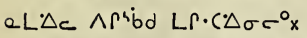

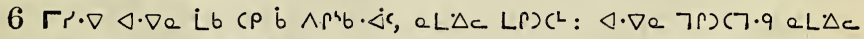
$\Delta r \cdot \dot{\delta}<7^{\circ}$, aL ons $\Delta r$ prqc $7^{\circ} x$

391 


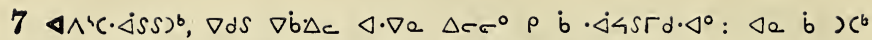

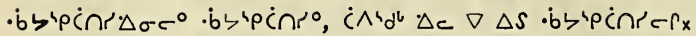

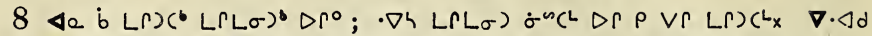

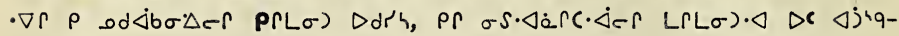
$\Delta \sigma r \cdot \Delta x$

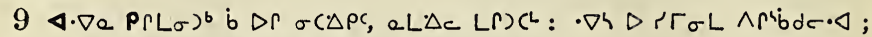

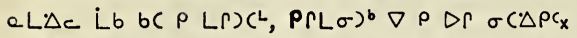

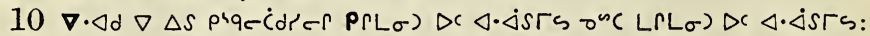

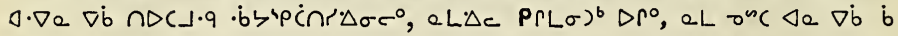
ip<ic $\Delta r \dot{s} a x$

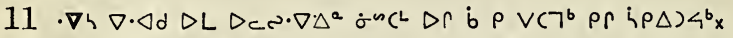

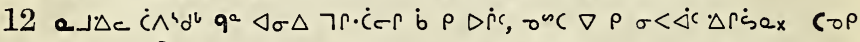

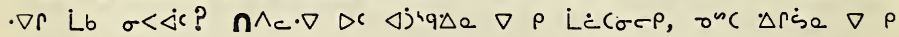

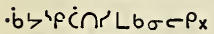

$\left.13 \nabla \dot{b} \Delta_{c} \dot{L} L^{b} b(\lrcorner^{b}, \sigma \rho \dot{s} \sigma\right)^{b}, \dot{\rho} n \wedge^{a}<\cdot b \cap d^{b} \zeta^{b} \Delta^{b} \rho_{x}$

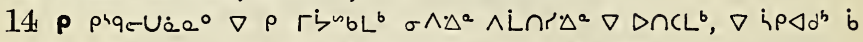

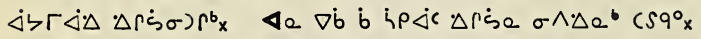

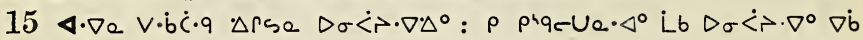
$\nabla \wedge r^{4} \dot{b} d c$ b $\rho q \wedge i \cap \Omega \cdot \Delta \sigma c^{\circ} x$

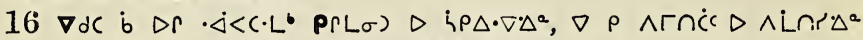

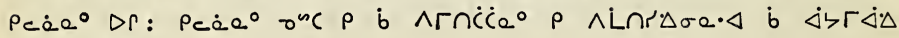
$\Delta r \dot{s} \sigma>r^{b} \Delta r_{x}$

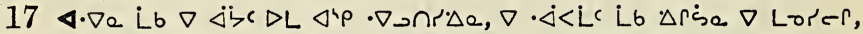

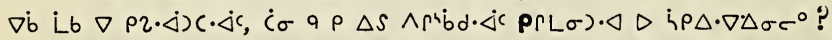

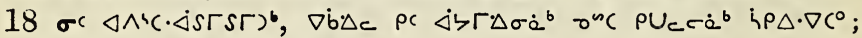
Lb $\rho c \Delta)(\lrcorner \Delta \sigma \sigma^{b}{ }^{b} \sigma^{n} c \dot{C} \cdot V \cdot \Delta \sigma^{b} x$

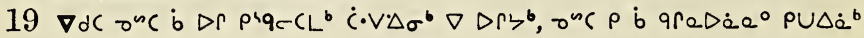
$\mathrm{q}^{\mathrm{a} \cdot} \cdot \Delta<\mathrm{r} \mathrm{C} \mathrm{dx}$

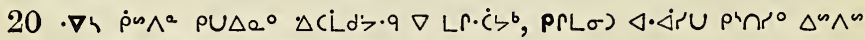

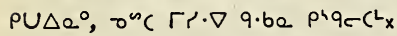

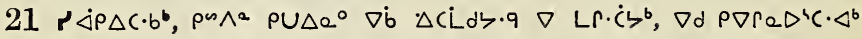
PRLO)x

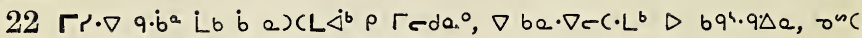
$\nabla)\left(L^{*} \Delta \sigma \Delta q \cdot b e\right.$ b $\Gamma_{\supset} \rightarrow b e \cdot \Delta<c^{b}$ :

$23 \Delta L$ Lb $\nabla \cdot \Delta d \quad b \quad b 9 \cdot 9 \cdot \Delta^{a}$, pr $\dot{C} \cdot V 4 C C \cdot L^{b} \quad \Delta C \quad \Delta S \sigma \dot{b} \lambda \cdot \Delta \sigma c^{\circ}$

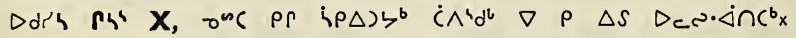

24 ४a Lb b be. $\nabla c c \cdot L c \quad \triangleright \quad b q^{4} \cdot 9 \cdot \Delta \sigma c \cdot \triangleleft, \wedge \Gamma^{4} b \cdot \nabla^{\circ}$, osc $\wedge \Gamma^{4} b d x$

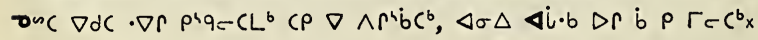

\section{Ápcre $\triangle b^{2} 4$.}

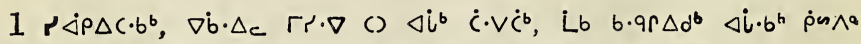

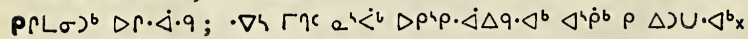
392 


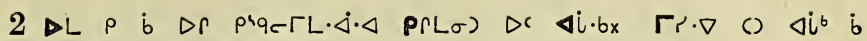

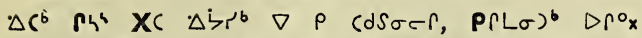

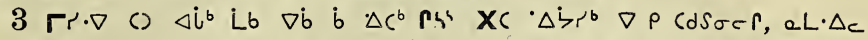

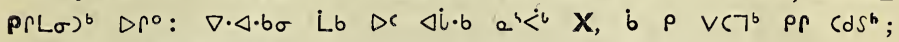

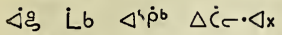

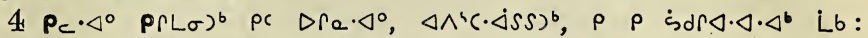

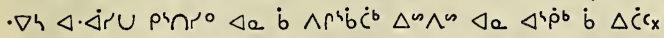

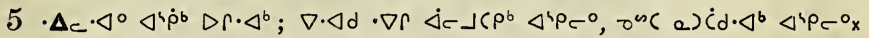

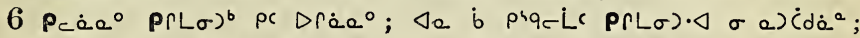

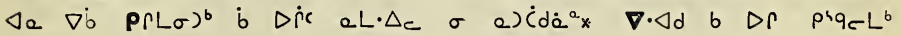

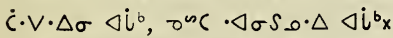

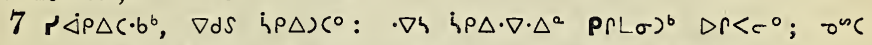

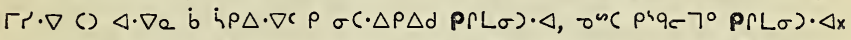

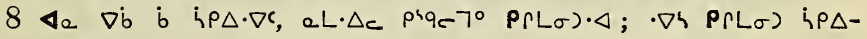
$\cdot \nabla \cdot \Delta \sigma \cdot \Delta{ }^{\circ} \mathrm{x}$

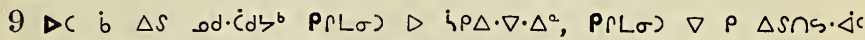

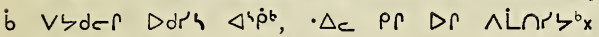

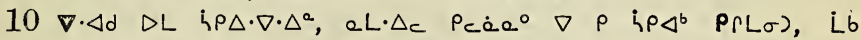

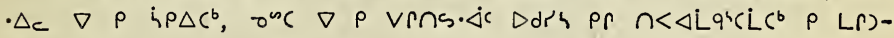
(\lrcorner$\cdot \triangle \sigma a \cdot \Delta x$

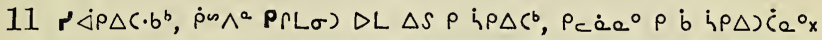

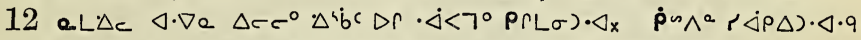

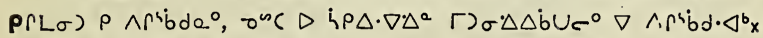

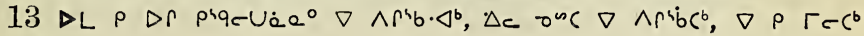
DC $\triangleleft i \cdot b_{x}$

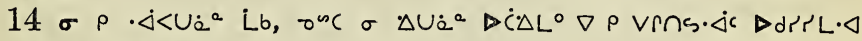

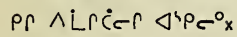

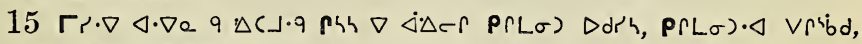

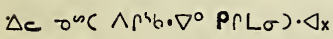

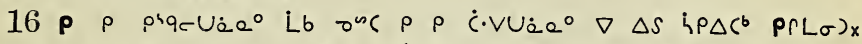

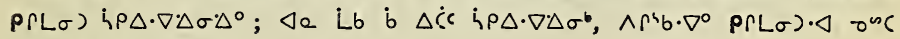
$\wedge \Gamma^{(} \dot{b} d P \Gamma(\sigma) \cdot \triangleleft x$

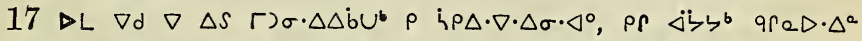

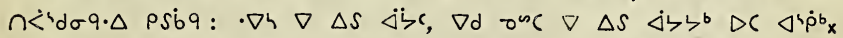

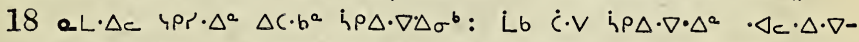

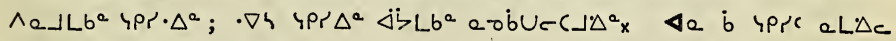
$\rho \Gamma \supset \sigma \Delta \Delta^{\circ} i \rho \Delta \cdot \nabla \Delta \sigma^{b} x$

$19 \rho i \rho\left\langle\sigma^{\circ} \Delta c^{i} c^{L} \nabla \rho i \rho \Delta C^{b}\right.$

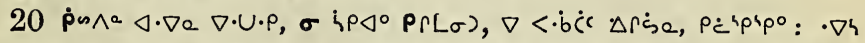

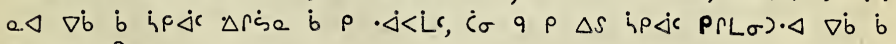
P. $\cdot \dot{\Delta<L C ?}$

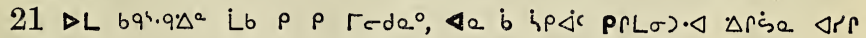
Pr $i \rho \dot{\Delta c_{x}}$ 


\section{A $p c r a \triangle b^{2} 5$.}

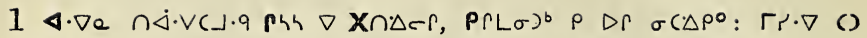

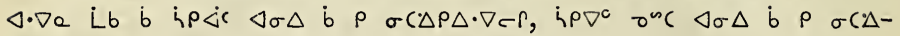
$\rho \triangle \mathrm{d} c \Gamma_{\mathrm{x}}$

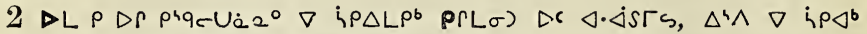

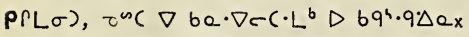

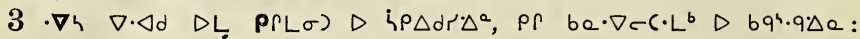

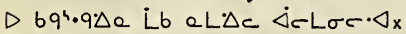

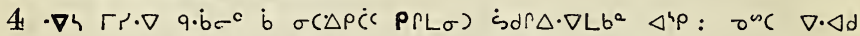

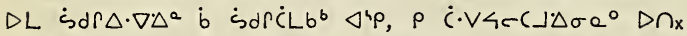

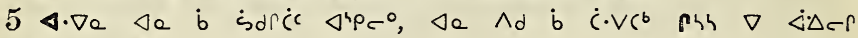
PrL $\sigma) D d r h$ ?

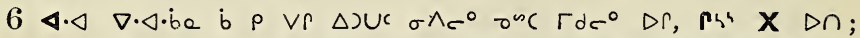

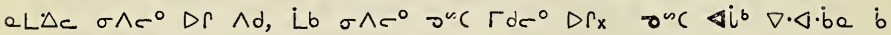
$n \dot{<}) c^{b}, \Delta i^{b} \nabla \dot{c} \cdot v \Delta \sigma \Delta c_{x}$

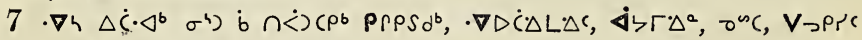

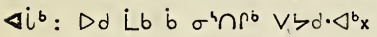

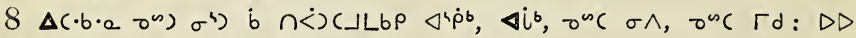
Lb $\quad$ b $\left.\left.\quad \sigma^{4} \cap P \quad V\right\rangle^{b} \Delta S \cap \dot{K}\right)(J L b \cdot a x$

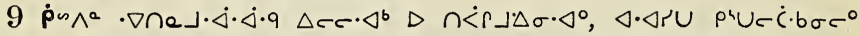

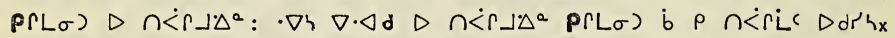

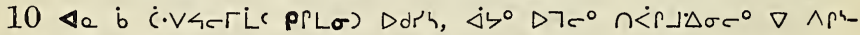

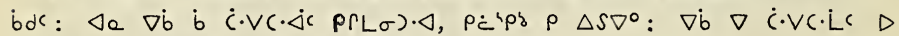

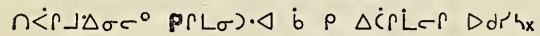

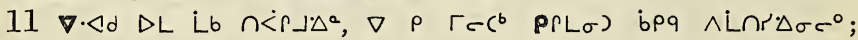

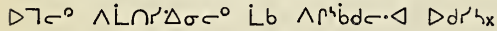

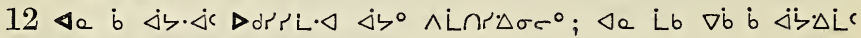

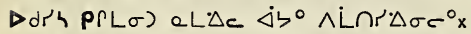

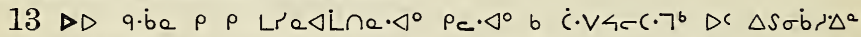

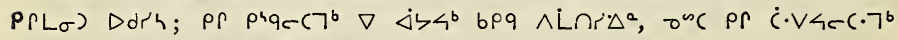
$D\left(\triangle S \sigma \dot{b} r \Delta^{a} P(L \sigma) \quad D d r h_{x}\right.$

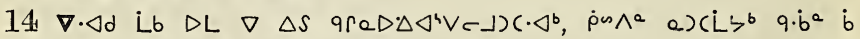
$\Delta C^{4} U c^{b} \Delta C \Delta U C(\lrcorner \Delta^{a}, \rho$ a) $\dot{C} d e^{\circ}:$

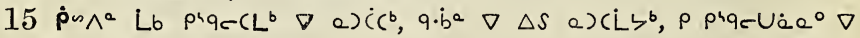

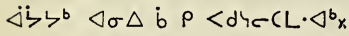

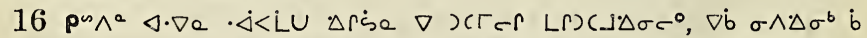

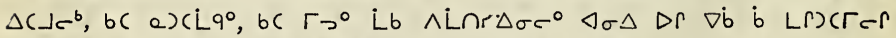
$\left.\sigma \wedge \Delta \sigma^{b} b \quad \Delta C\right\lrcorner c^{b} x \quad \Delta\left(\cdot b^{a} L \Gamma\right)(\lrcorner \Delta^{a} \sigma \wedge \Delta \sigma^{b} b \Delta C J^{b}:$ a $L \Delta c \sigma^{c} \Delta \cdot \dot{c}^{a} p \rho$ $\dot{\Delta} \succ \Gamma \nabla^{4} C 7^{b} \triangleleft \sigma L x$

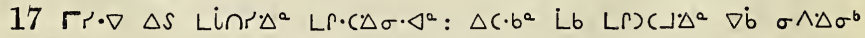
b $\Delta C\lrcorner^{b} \mathrm{x}$ 


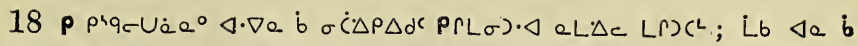

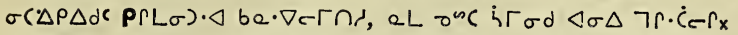

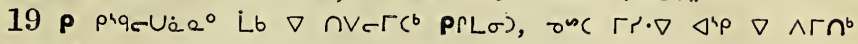
Lin? $\Delta \sigma^{b} x$

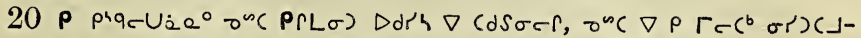

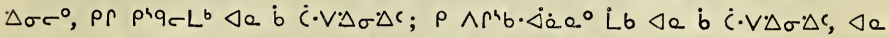

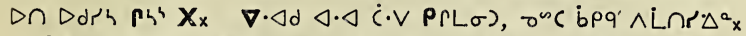

$21 \varangle \wedge^{4}(\cdot \Delta s S)^{b}$, be $\left.\nabla e \Gamma \cap r^{b} L \sigma\right) b a^{b} D r_{x} \nabla 7^{a} x$

\section{$\sigma \sim i L \cdot \Delta L^{\prime} e \Delta L q \cdot \Delta e$ lex}

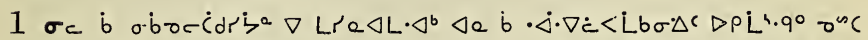

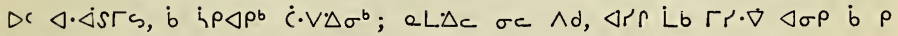
prqc $C \rho^{\circ} \dot{C} \cdot V \cdot \Delta \sigma \sigma^{\circ}$;

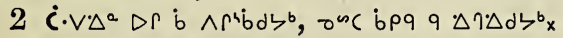

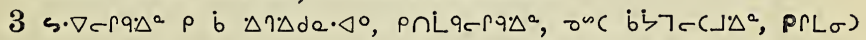

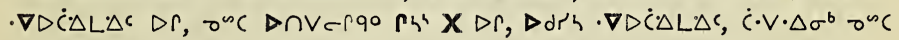
iP $\triangle \cdot \nabla \cdot \Delta \sigma^{b}{ }^{b}$

4. $\dot{a}^{4} \wedge b \sigma \rho \Gamma c \cdot \Delta U^{a} \nabla \rho \Gamma^{b} b \cdot \Delta \rho^{b} \rho c \triangleleft \cdot\left\langle s \Gamma S^{b} \nabla \wedge\right\lrcorner U \rho^{b} \dot{c} \cdot V \cdot \Delta \sigma^{b}, \dot{b}$ $\rho \Delta C \sigma^{\circ} \cdot \Delta \Omega^{b} \nabla \dot{C} \cdot \Delta L^{\circ} x$

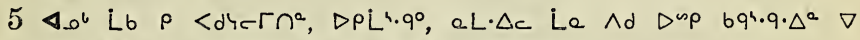

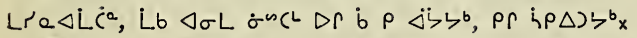

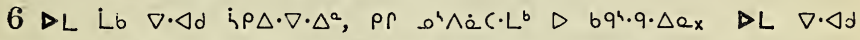

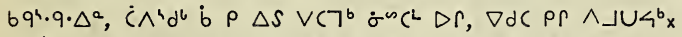

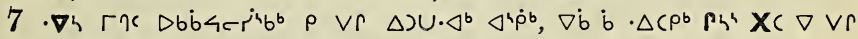

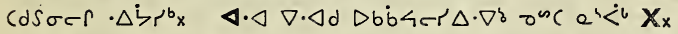

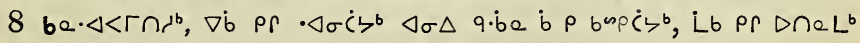
r) $\sigma \cap<\Delta L d r \cdot \Delta \Delta^{2} x$

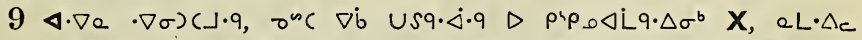

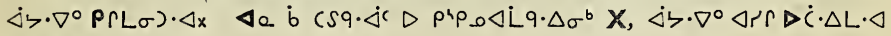
onc $\triangle$ drth $\Delta \cdot \triangle x$

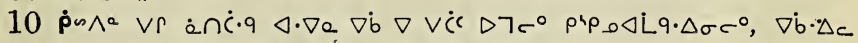

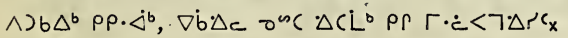

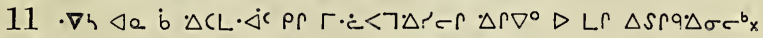

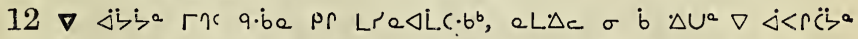

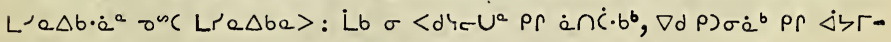

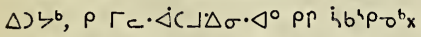

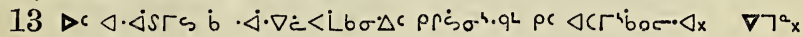
395 


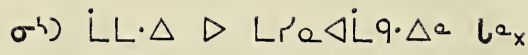

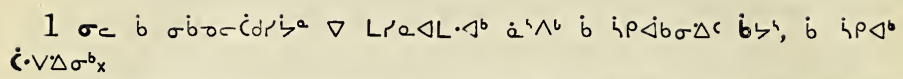

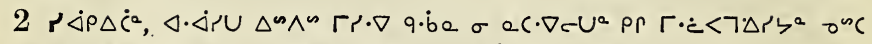

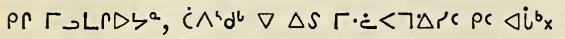

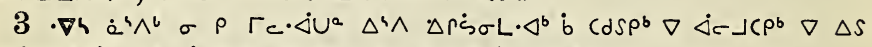

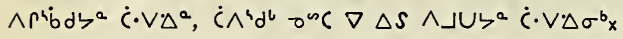

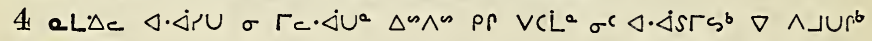
$\dot{c} \cdot v \triangle \sigma^{b} x$

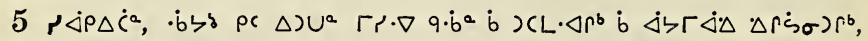
onc La $U \cdot \Delta^{b} ;$

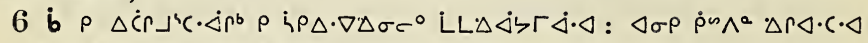

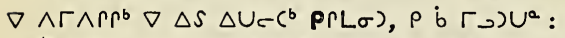

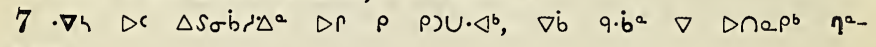
$C^{b} D r_{x}$

$8 \nabla \cdot \Delta d$ Dr $\rho \dot{b} \Delta \cap \dot{a}\left(a \cdot \triangleleft^{b} \nabla d\right) \triangle \rho r \Delta r \Delta r \Delta \cdot \nabla r^{b} \dot{c} \cdot v \cdot \Delta \sigma^{b} x$

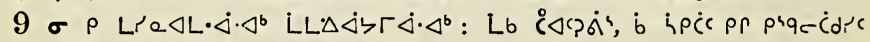
$\Delta C \nabla \Delta \dot{C} e r, \quad Q L i \Delta c$ or $D \cap \sigma d \dot{a}^{a} x$

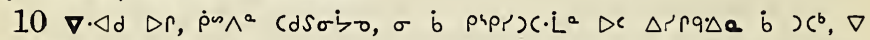

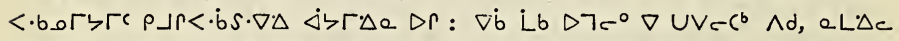

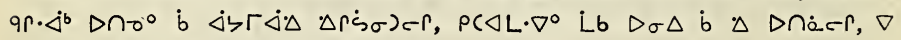

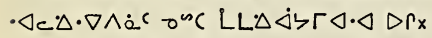

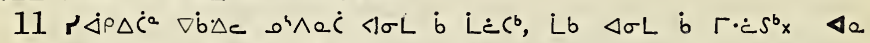

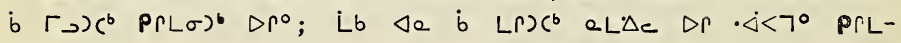
$\sigma) \cdot \triangleleft x$

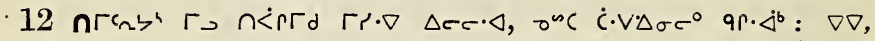

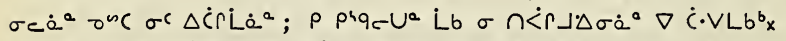

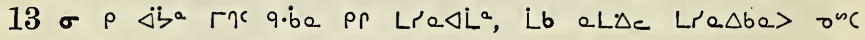
Lre $\triangle$ bey $\rho$ b $\triangleright r$ Lre $\Delta$ L $n^{2}$ :

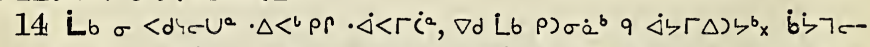

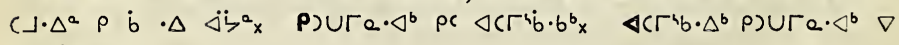

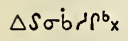




\section{$\triangleright L L \cdot \Delta L r e \Delta L q \cdot \Delta e$ je}

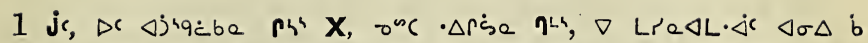

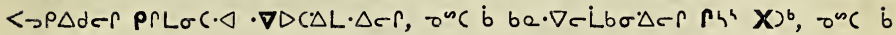
a.) $L b \sigma \cdot \Delta r r_{x}$

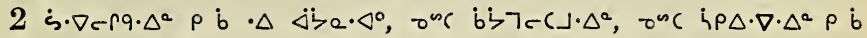
$\triangle c P C L b \cdot \Delta a \cdot \Delta 0^{\circ} x$

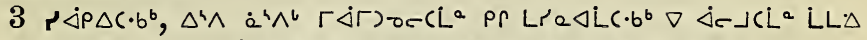

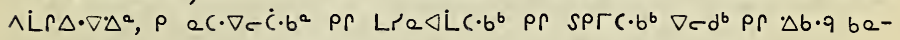

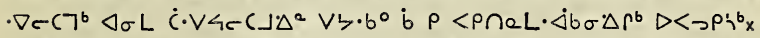

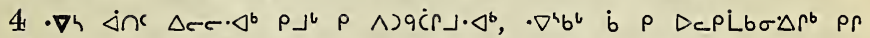

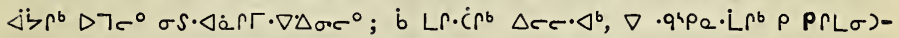

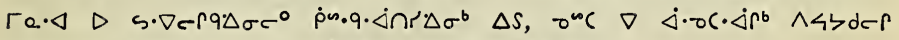

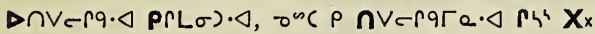

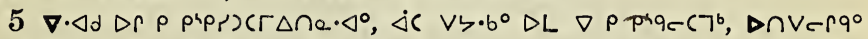

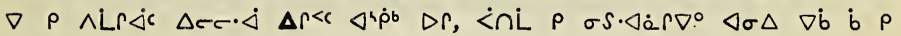

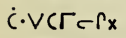

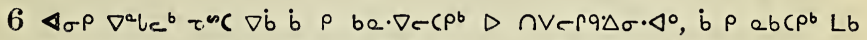

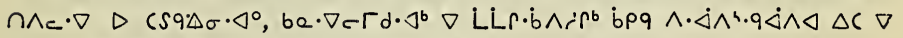

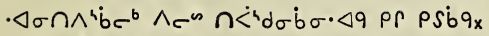

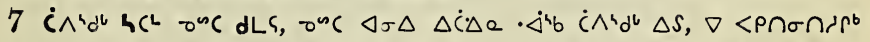

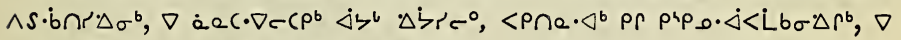

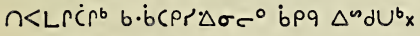

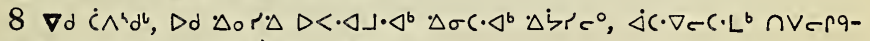

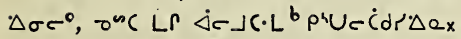

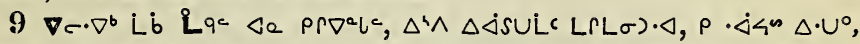

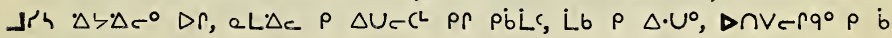
$\Delta P C \Delta L^{b} x$

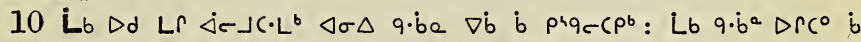

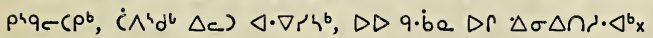

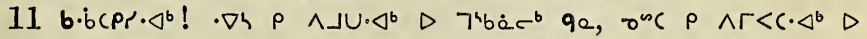

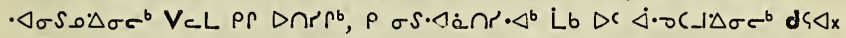

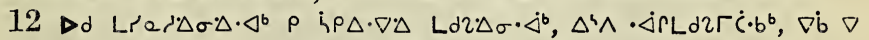

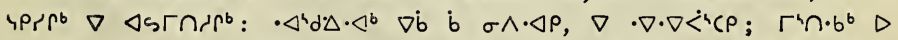
$\Gamma \sigma S \Gamma \cdot \Delta \cdot \triangleleft \dot{b}$ o.s. $\triangleleft \dot{a} C \sigma c P, \nabla b \quad \nabla \Gamma \sigma s \Delta r^{b}, \sigma \cdot s^{\circ} \nabla \sigma \wedge r^{b}, \nabla r r<b r-$ $\cdot \nabla \wedge c \dot{c} b \cdot \Delta r^{b} ;$

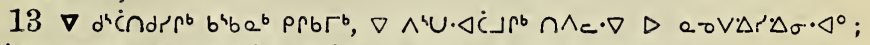

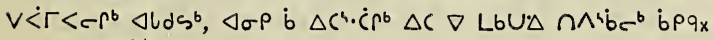
397 


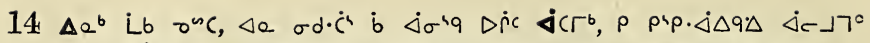

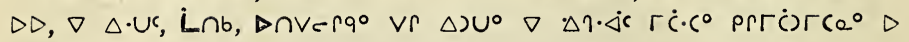
$<\rightarrow P$ L,

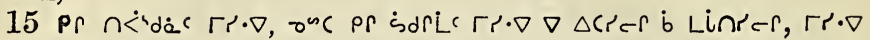

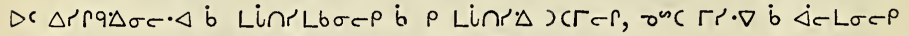

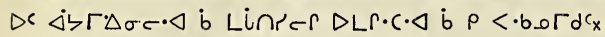

$16 \triangleright d \nabla \cdot \triangleleft \cdot b \sigma \rho \quad \nabla \Gamma C \cdot \nabla^{4} b^{b}, \quad \nabla C \dot{C} \Gamma \Gamma \cdot \nabla^{4} b^{b}, \nabla$ ob $\wedge e c p^{b} \cap \wedge c \cdot \nabla D L R$

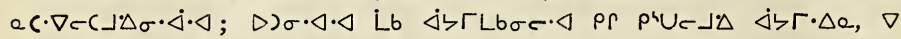

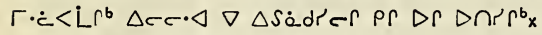

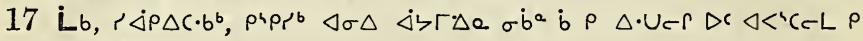

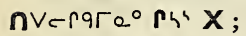

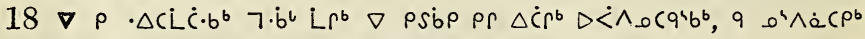

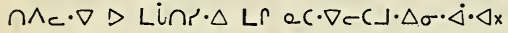

$\left.19 \triangleright d \nabla \cdot \triangleleft \cdot \dot{b} \sigma \rho \dot{b}<\ddot{b} \sigma \Delta n r^{b}, \nabla \Delta c\right) \dot{c} n \rho^{b}, \nabla \dot{b} \nabla \dot{\Delta b} \cdot \dot{\Delta} \rho^{b} \Delta i \cdot b x$

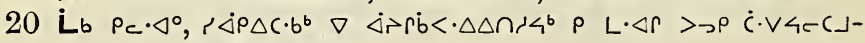

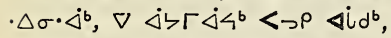

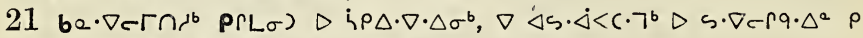

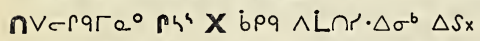

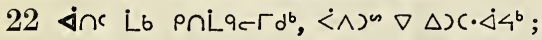

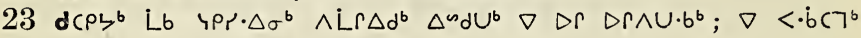

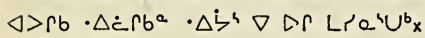

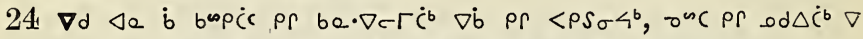

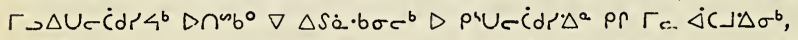

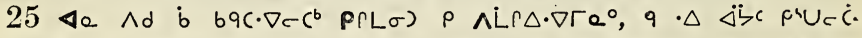

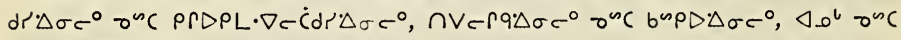
b $\left.\rho q_{x} \nabla\right\urcorner^{\circ} x$

$\Delta$ prqe $\dot{C} d \dot{C} q \cdot \Delta e \quad \Delta<>p h$ lex

\section{$\Lambda^{4} p c r a \Delta b^{\circ} 1$.}

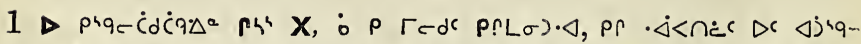

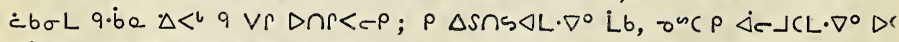
$\triangle 34 q c b \sigma L$ le, $\Delta C \nabla^{2} L C L D r$;

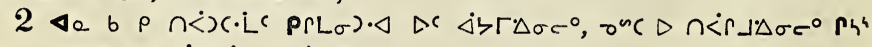
Xc, onc $r i \cdot \nabla q \cdot \dot{b}$ b $b \cdot \cdot \dot{<}<c^{b} x$

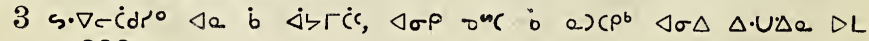
398 


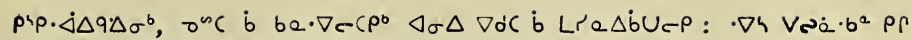
DnR $<c^{b} x$

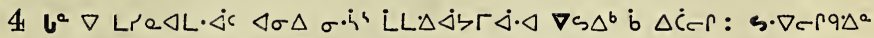

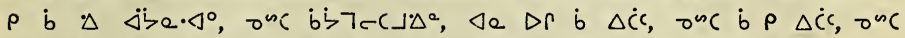

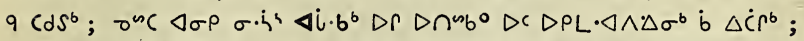

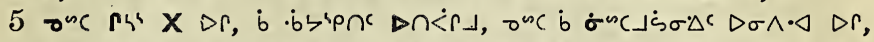

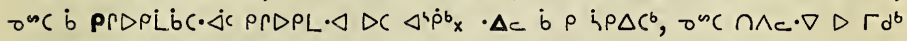

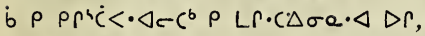

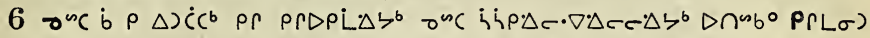

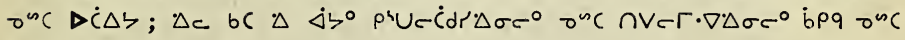
bpqx $\nabla\urcorner^{a} x$

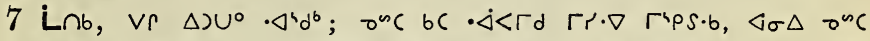

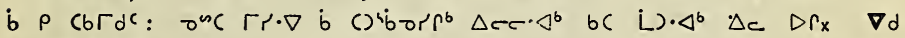
q $\triangle \rho^{b} \times \nabla 7^{a} \times$

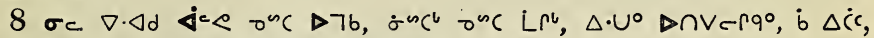

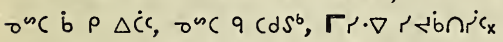

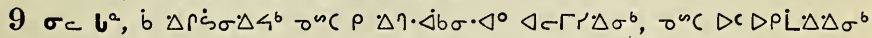

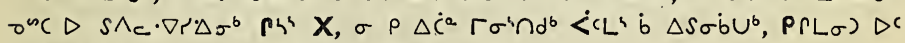

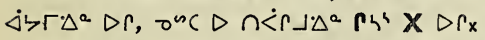

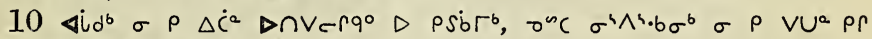
$v \dot{C} d r^{*} \Delta^{a}, \dot{C} \wedge^{4} d^{l}>\dot{C} r b^{a}$,

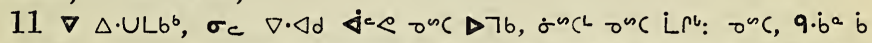

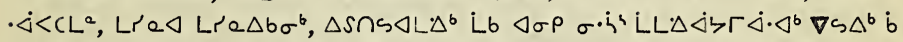

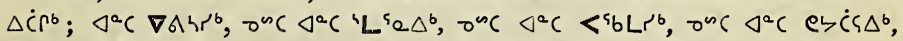

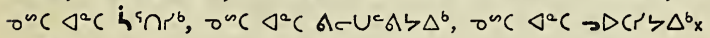

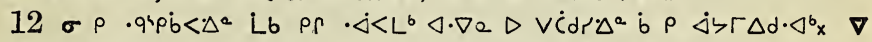

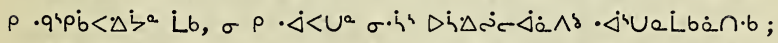

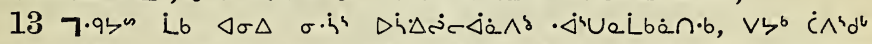

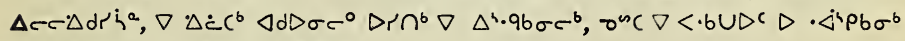

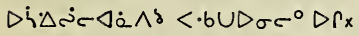

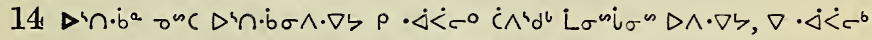

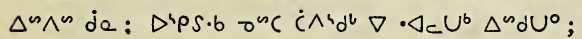

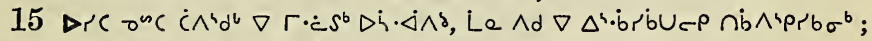

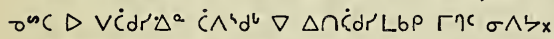

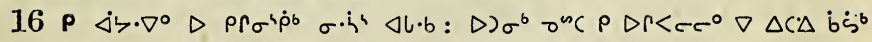

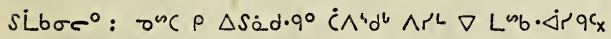

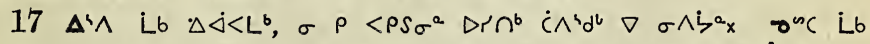

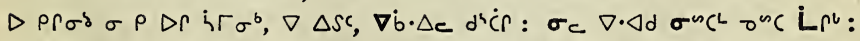

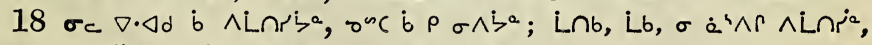

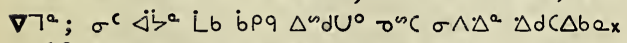

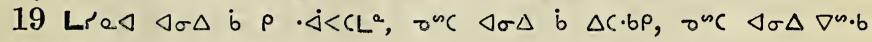
$9 \Delta C \cdot b p$;

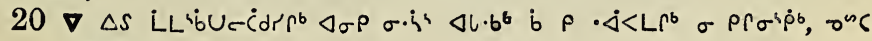
399 


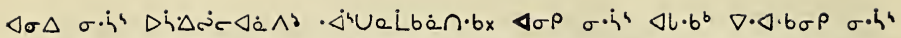

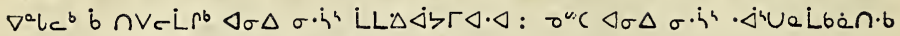

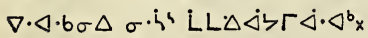

\section{Aipcra $\Delta b^{a} 2$.}

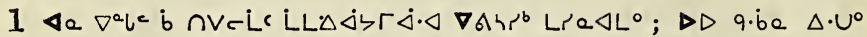

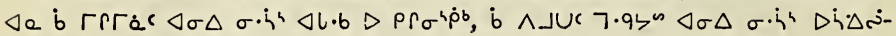

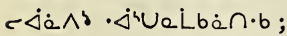

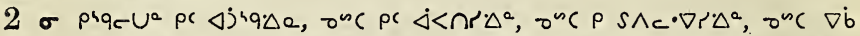

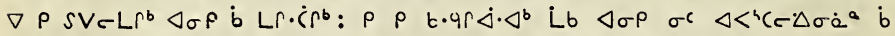
$\Delta \cdot u^{b}, \nabla \dot{b}$ Lb $\left.\nabla \cdot \Delta d \dot{b}\right) \Delta \dot{b} \sigma \Delta r^{b}, \rho \rho \Gamma^{b} b \cdot \dot{\Delta} \cdot \triangleleft^{b}$ Lb $\nabla \rho \dot{c}^{4} \rho \rho \rho \rho^{b}$ :

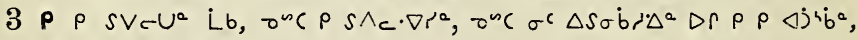
QL TnC $P$ P OUS

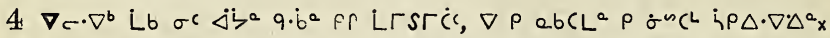

$5 \nabla \cdot \Delta d$ Dr phpr, i ${ }^{a} \cup$ b $p \Delta r<p S \sigma b^{a}$, onc $r r_{c} \cdot \nabla 4 c c, x$ Lb $\Delta \sigma \Delta$

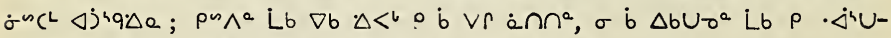

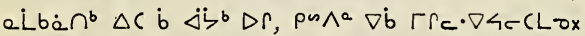

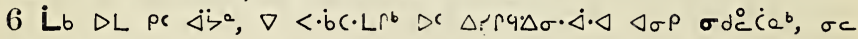
$\operatorname{Ars} \dot{b}<\cdot \dot{b} c L^{a} x$

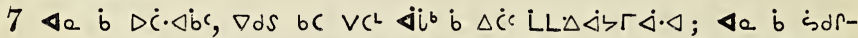

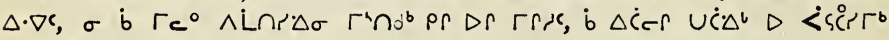
PRLG)x

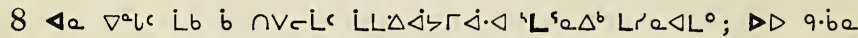

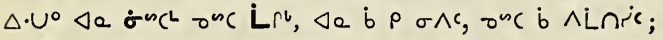

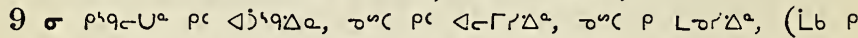

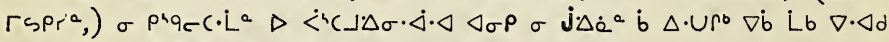

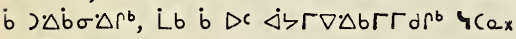

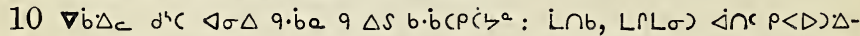

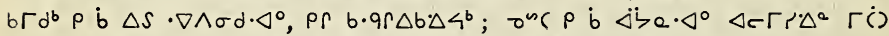

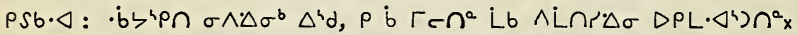

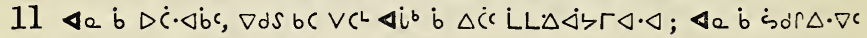
e L $\Delta c$ bc $\Delta d \Delta d \triangleleft \sigma \nabla c^{\circ} \sigma r \sigma \wedge \Delta \sigma c^{\circ} x$

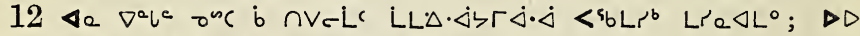
q.be $\Delta \cdot v^{\circ} \triangleleft a$ b $\ddot{j \zeta c} \dot{b} \Delta c \Delta \dot{b} \dot{s} c^{b}$ síb $\sigma c^{\circ}$;

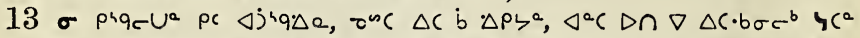

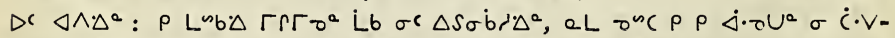

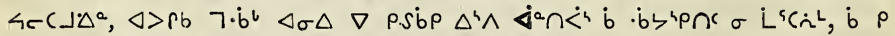

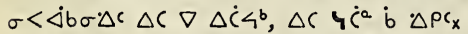

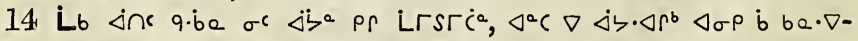

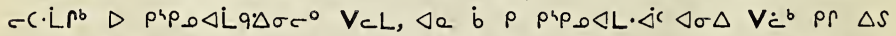

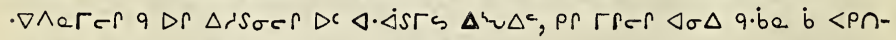

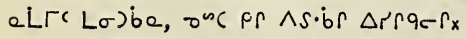




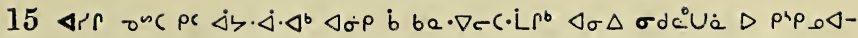
L $9 \Delta \sigma c \cdot \triangleleft, \triangleleft \sigma L b<\cdot \dot{b} C L^{\circ} x$

$16 r r_{c} \cdot \nabla 4 c c ;$ pa $\wedge^{a}$ Lb $\nabla b \Delta<b \rho$ b Vr anna, $\nabla d$ Lb 9 onepb $\nabla$ $\dot{\Delta}\left\langle\beta \ddot{C} \dot{\zeta}^{a} \triangleleft \sigma L S \dot{L} b^{a} \sigma>\sigma^{b} \dot{b} \quad D R<c^{b} x\right.$

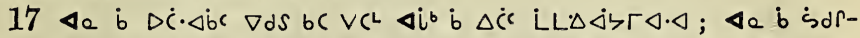

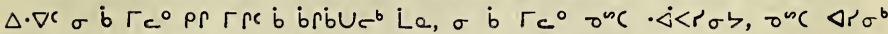

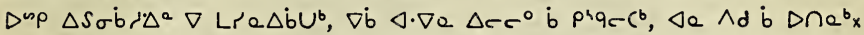

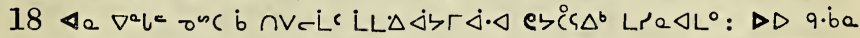

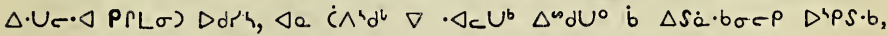

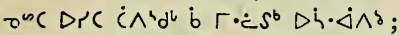

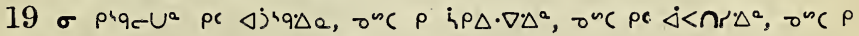

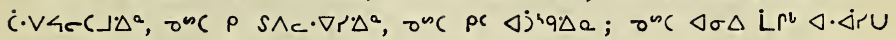

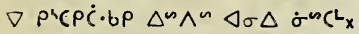

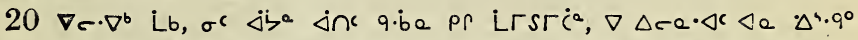

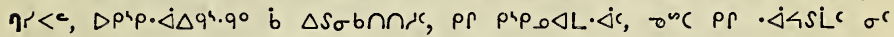

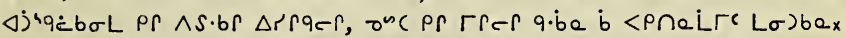

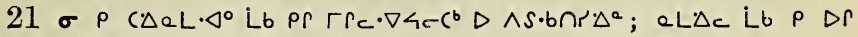
$\Gamma \Gamma_{C} \cdot \nabla 4 C C^{2} x$

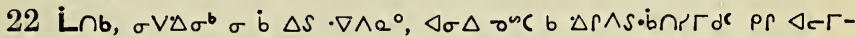

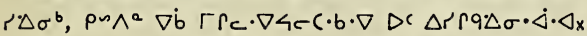

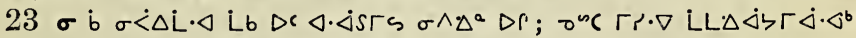

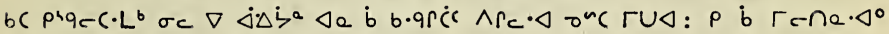

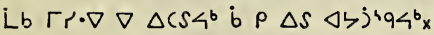

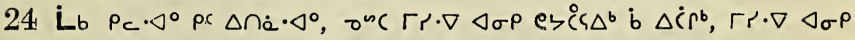

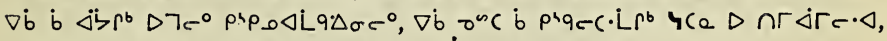
$\nabla \Delta S \sigma \dot{b} C \rho^{b} ; a L \Delta c d c^{b} a b \rho b^{a} \rho \dot{b}<\dot{<} \cap a L \cap \cap \dot{a} \cdot<^{\circ}$ :

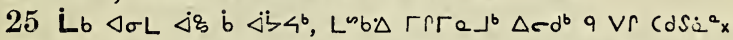

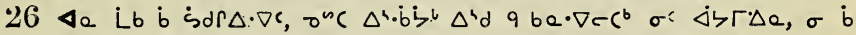

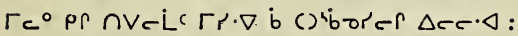

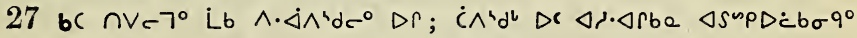

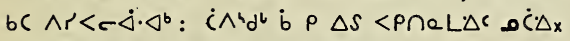

$28 \sigma b \Gamma_{c}{ }^{\circ} \dot{L} b \cdot \dot{\Delta}<a \triangleleft u d e_{2 x}$

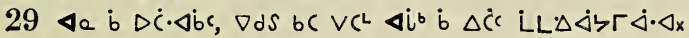

A

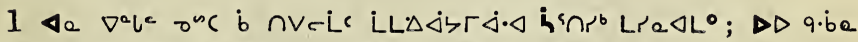

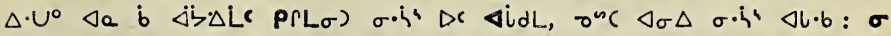

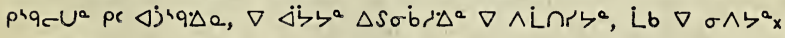

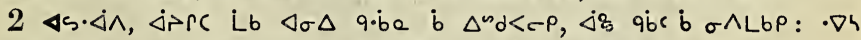

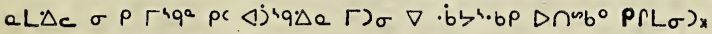

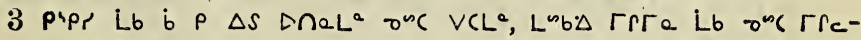
401

D D 


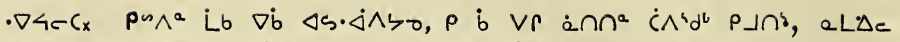

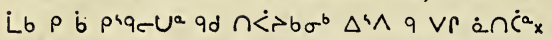

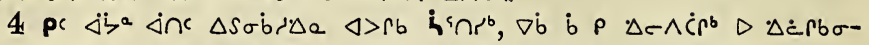

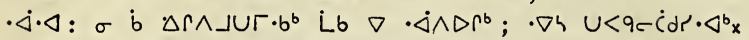

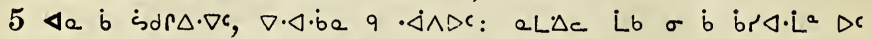

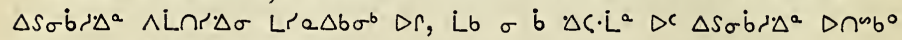

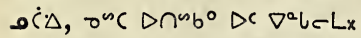

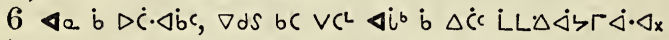

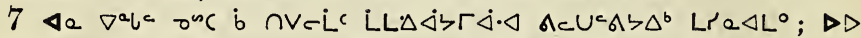

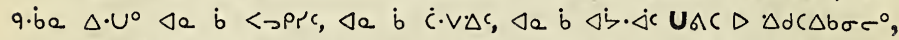

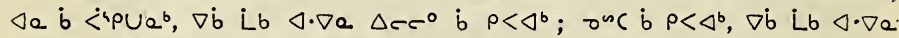

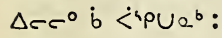

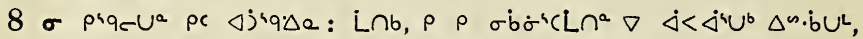

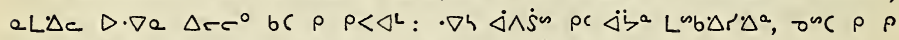

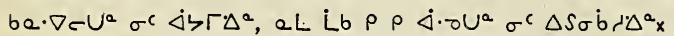

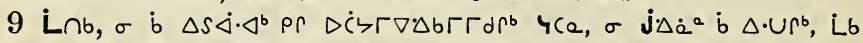

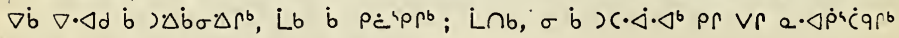

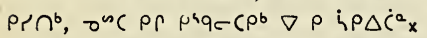

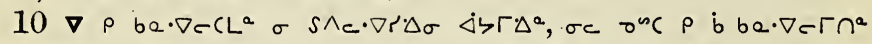

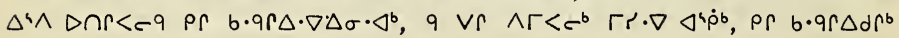
$\triangle \sigma \rho \triangleleft^{b} \rho^{b} b \dot{b} c s q \rho^{b} x$

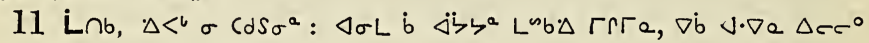
on $\left.\Delta U Q^{b} p C D P L \cdot \Delta^{b}\right) \cap \sigma \sigma^{\circ} \mathrm{x}$

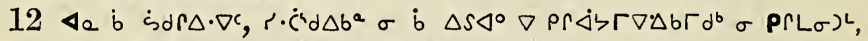

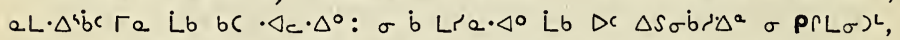

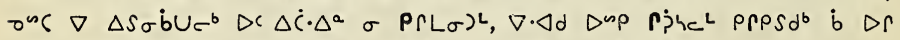

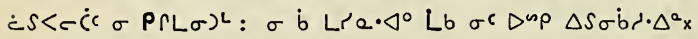

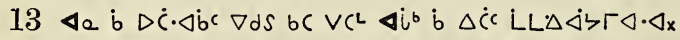

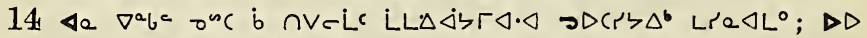

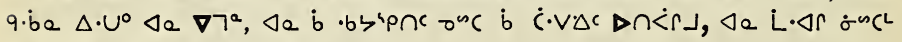
b) $\rho \Delta \dot{C}(\nabla \rho$ L $\nabla S P(q(P \Gamma L \sigma)$;

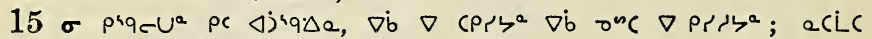
cbrלa guc

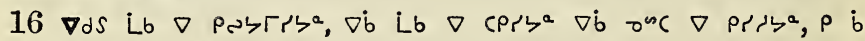

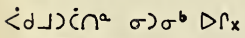

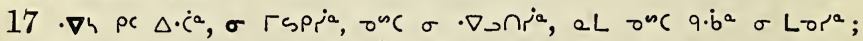
aL Lb $\rho$ prqqua $\nabla$ b.bc

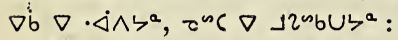

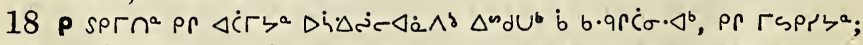

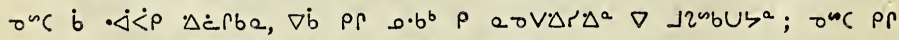

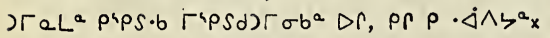

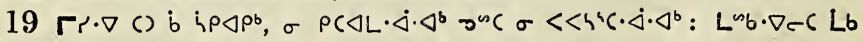
onc rre. $\nabla 4-C_{x}$ 


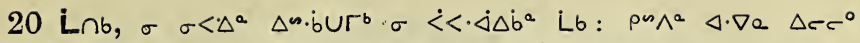

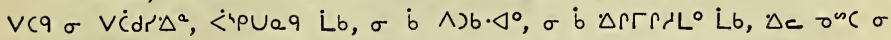

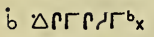

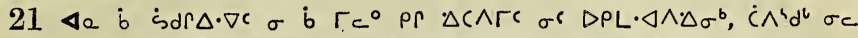

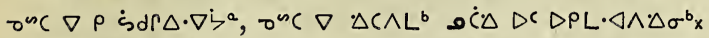

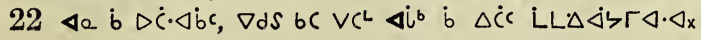

\section{Ápcra $\triangle b^{a} 4$.}

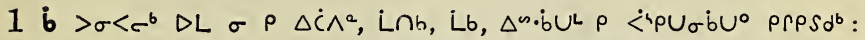

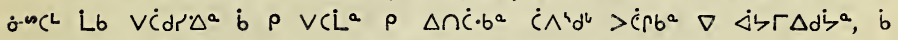

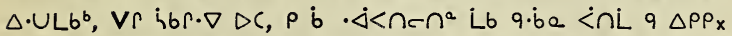

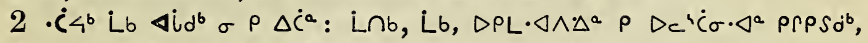
$V b^{b} L b P \Delta \wedge^{\circ} \Delta P L \cdot \triangleleft \wedge \Delta \sigma^{b} x$

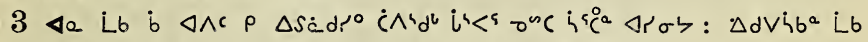
$\rho \Delta c \cdot b^{a} \cdot \dot{j}^{\mathrm{b} b} \Delta P L \cdot \Delta \wedge \Delta \sigma^{b}, \nabla \Delta S \dot{a} \cdot b^{b} \dot{c} \wedge^{4} d^{b} \nabla r \tau^{a c} \Delta r \sigma x$

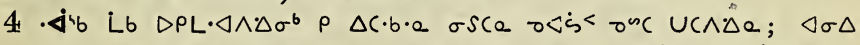

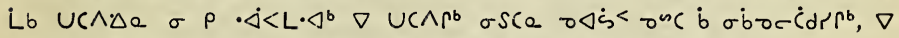

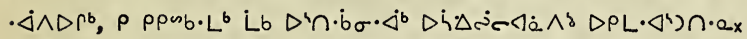

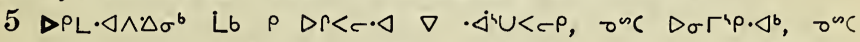

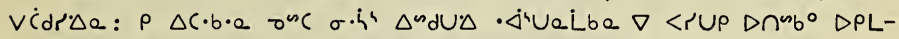
$\left.\cdot \Delta \Lambda \Delta \sigma^{b}, \nabla \cdot \triangleleft \cdot b \sigma \Delta \rho \rho L \sigma\right) \Delta c \Delta i \cdot b \dot{b} \quad \sigma \cdot h \cdot c \Gamma x$

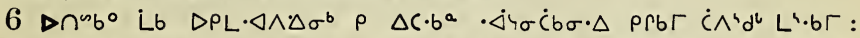

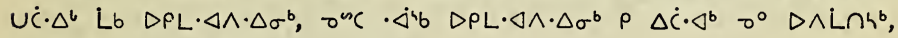

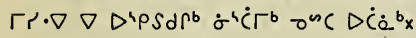

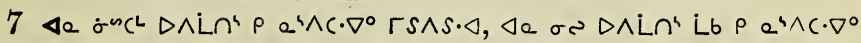

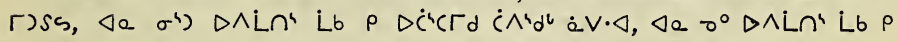

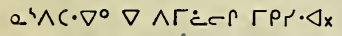

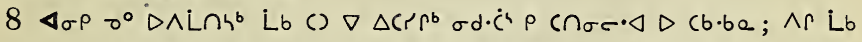

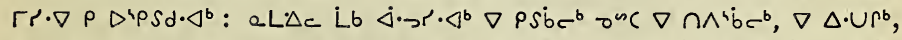

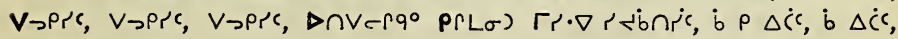
-ons $q \Delta \dot{C}_{\mathrm{x}}$

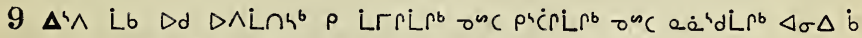

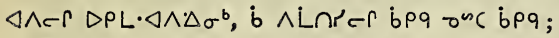

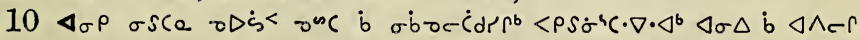

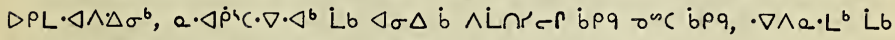

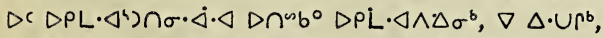

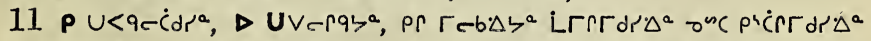

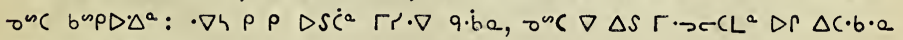
onc $P$ SSRGU. $\Delta x$ 
Ápcra $\triangle b^{\circ} 5$.

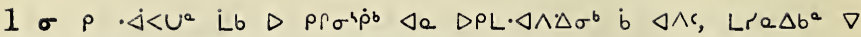

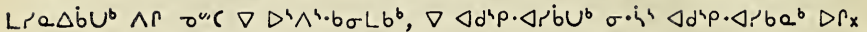

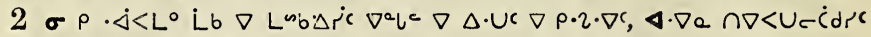

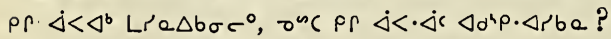

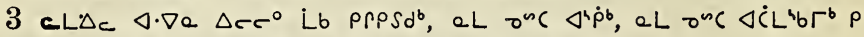

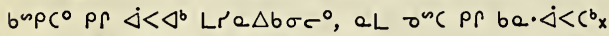

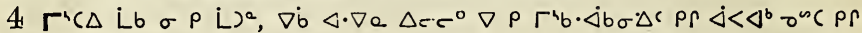

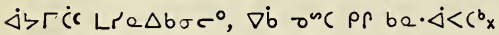

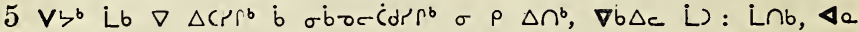

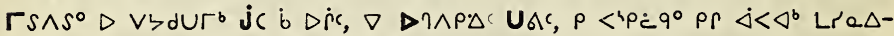

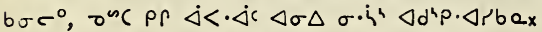

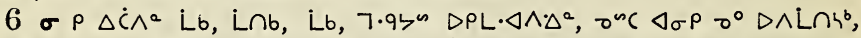

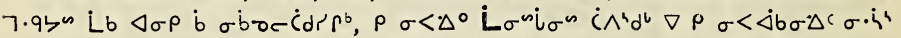

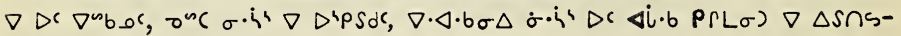

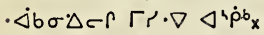

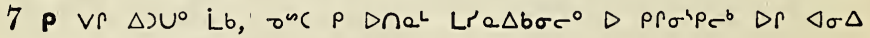
$D P L \cdot \triangleleft \wedge \Delta \sigma^{b}$ b $\triangleleft \wedge c \Gamma_{x}$

$8 \Delta^{4} \wedge$ Lb $\nabla \cap a^{b}$ Lre $\Delta b \sigma \sigma^{\circ}, \triangleleft \sigma P \sigma^{\circ} D \wedge L \cap h^{b}$ onc $\Delta \sigma P \sigma S C a$ onc

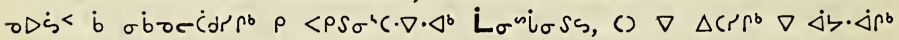

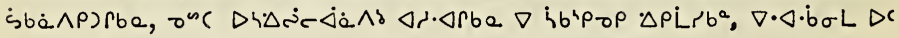

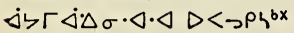

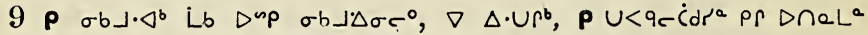

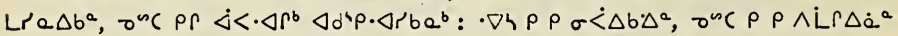

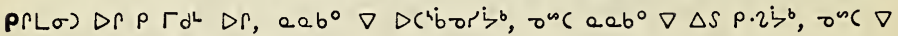

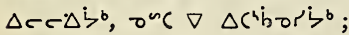

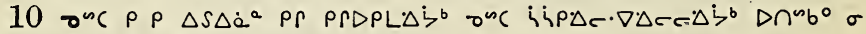

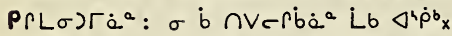

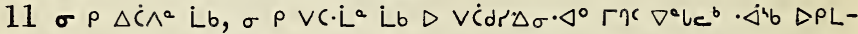

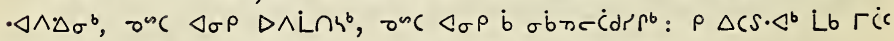

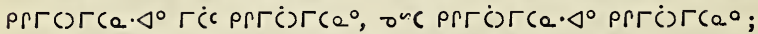

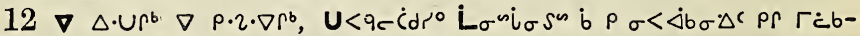

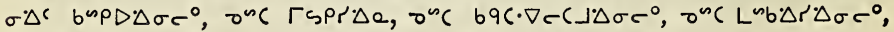

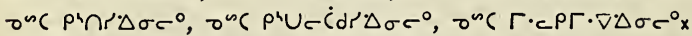

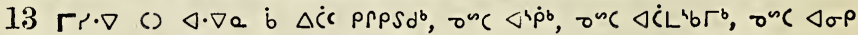

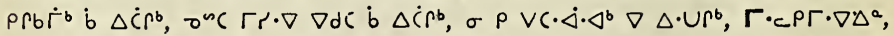

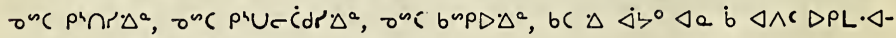

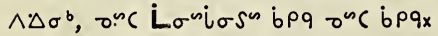

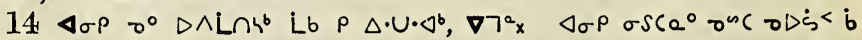

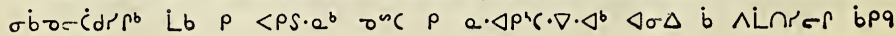
onc bpqx 


\section{$\Lambda^{\prime} \rho C\left\ulcorner a \triangle b^{\circ} 6\right.$.}

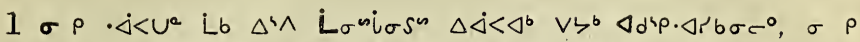

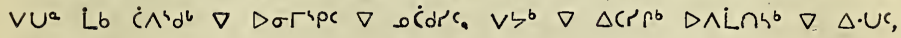
$\mathrm{V} r \cdot \dot{\Delta}<\mathrm{C} x^{2}$

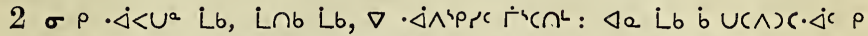

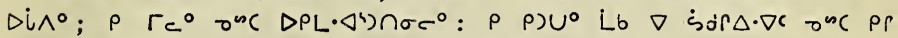
$\dot{s} d r \Delta \cdot \nabla c_{x}$

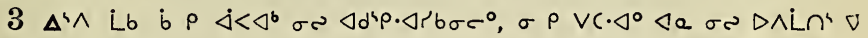
$\Delta \cdot U c, V r \cdot \dot{j}<C_{x}$

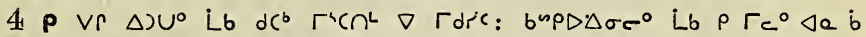

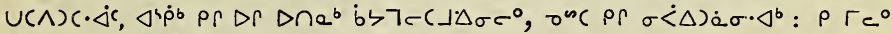
Lo $\cos \operatorname{sib\sigma \sigma ^{\circ }x}$

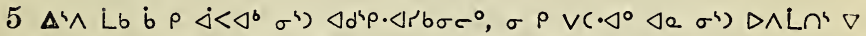

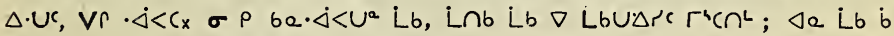

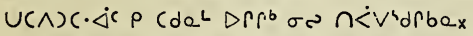

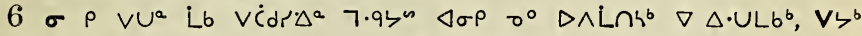

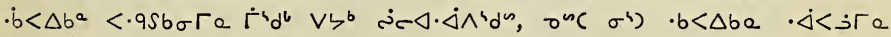

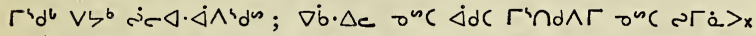

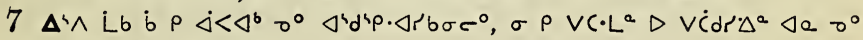
$\nabla \wedge L^{\prime} \nabla \Delta \cdot U c, V r \cdot \dot{S}<C x$

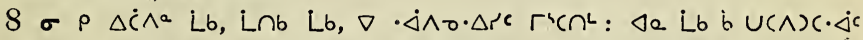

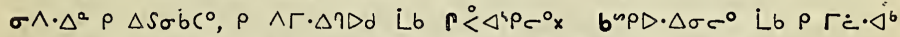

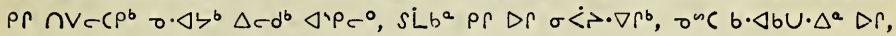

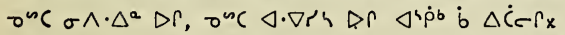

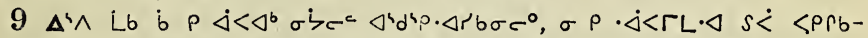

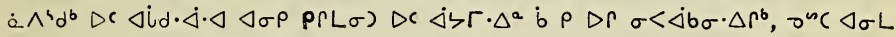

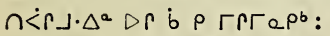

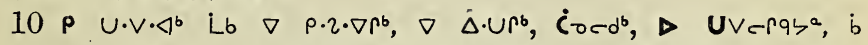

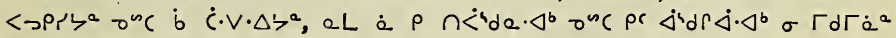
$\operatorname{Dr} \varangle \sigma \rho \dot{b} C S q \rho^{b} \triangleleft^{4} \dot{\rho} b$ ?

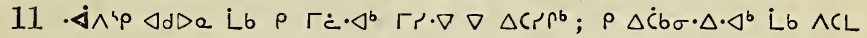

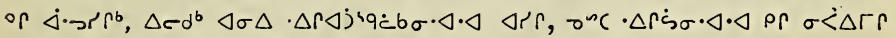
$\dot{c} \wedge \cdot d^{b} \cdot \Delta c \cdot \Delta^{\circ} a r \cdot \dot{j}^{b}, \operatorname{sr} \cap \wedge<c^{b} \mathrm{x}$

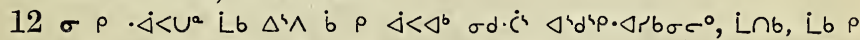

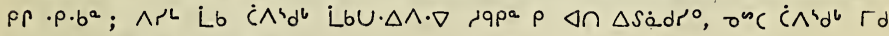

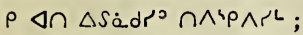

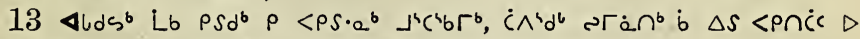
ras $\nabla \dot{b}$ b $\triangleleft \cap \cup c \rho, \Delta^{4} \Lambda \cdot \nabla \cdot \nabla \dot{<S C} \nabla \lambda \rho_{c} \cdot \nabla c^{b}$;

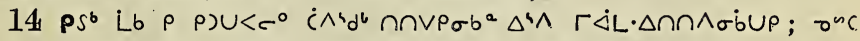

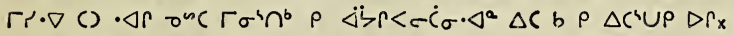

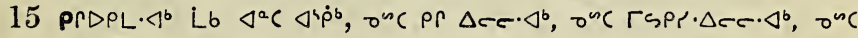
405 


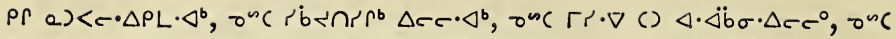

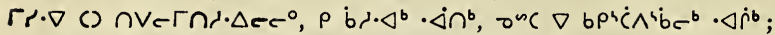

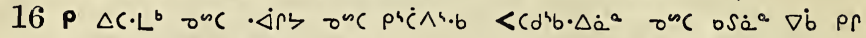

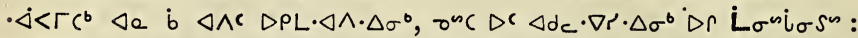

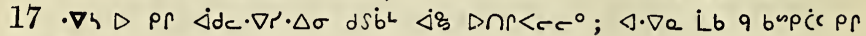
$\sigma<\cdot \Delta r ?$

\section{$\Lambda^{4} p\left(r a \triangle b^{a} 7\right.$.}

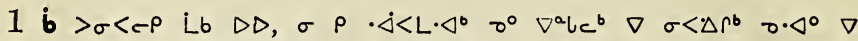

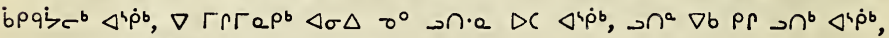
aL onc $P R b \Gamma^{b}$, aL onc $\sigma^{n} \Gamma^{n} \cap d^{b} x$

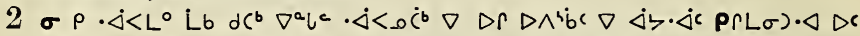

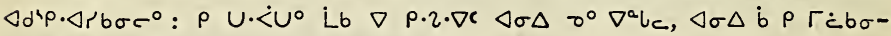

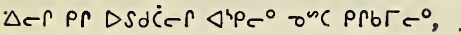

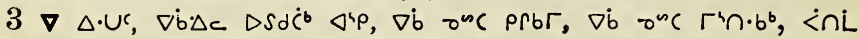

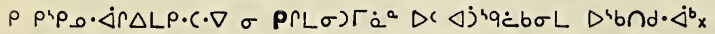

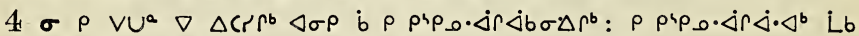

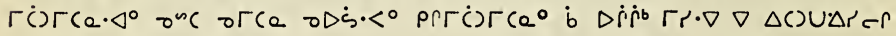
$\Delta^{4} \sim \Delta^{c} \Delta r \Delta \cdot \Delta s \Gamma S_{x}$

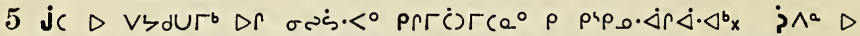

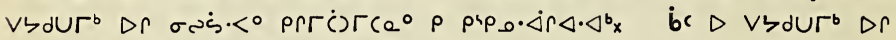

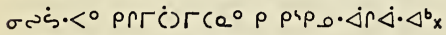

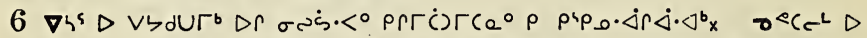

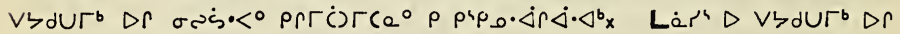

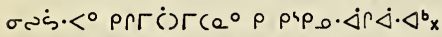

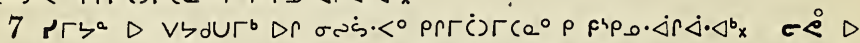

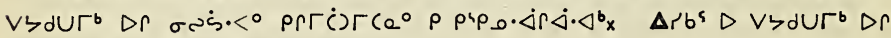

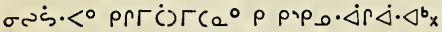

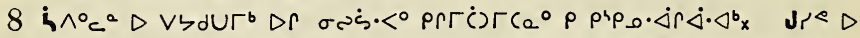

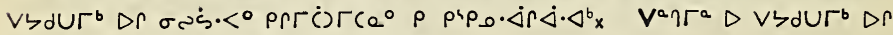

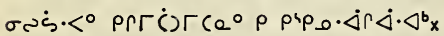

9 b $>\sigma<c^{b} \nabla L \sigma \rho \cdot \dot{j}<U^{a}$, L $\cap b$ Lb $a^{4} \wedge^{b} \nabla \Gamma \eta \cap r^{b} \Delta c c \cdot \triangleleft^{b} \nabla b$ b $p$

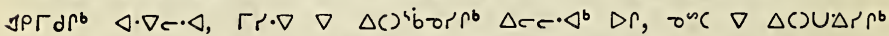

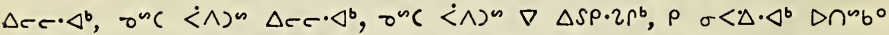

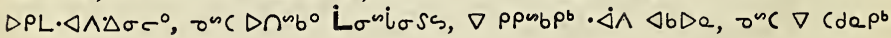
$<L \cdot \triangleleft \cap \cdot b e$;

$10 \rho U \cdot V \cdot \triangleleft^{b} L b \nabla \rho \cdot 2 \cdot \nabla \Gamma^{b}, \nabla \Delta \cdot U \Gamma^{b}, \Lambda L \Gamma \Delta \cdot \nabla \Delta \sigma \sigma^{0}\left\langle\zeta^{\circ} \rho \rho \Gamma L \sigma\right) \Gamma e^{0}$

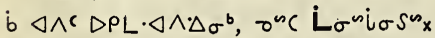

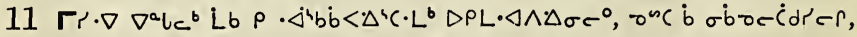

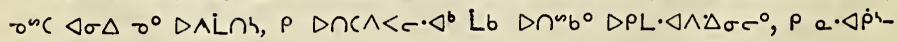
$\left(\cdot \nabla \cdot \triangleleft^{b} L \mathrm{~L} b \rho(L \sigma) \cdot \triangleleft\right.$,

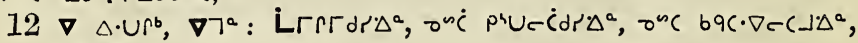
406 


\section{$p^{\prime} q-\dot{C} \dot{d} q^{\circ} \Delta^{2} 7,8$.}

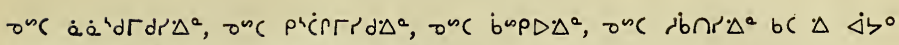
$\sigma$ prLG)

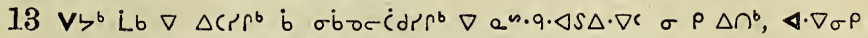

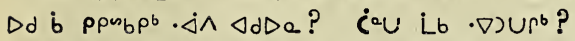

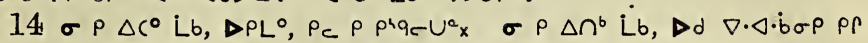

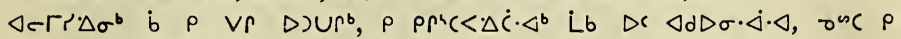

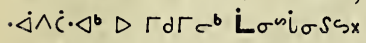

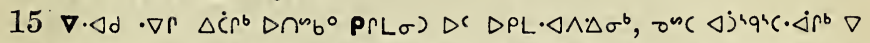

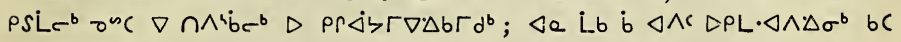
$\triangle r C S 97^{\circ} \times$

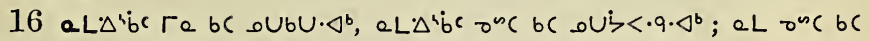
$\cdot \triangleleft \zeta^{4} b L d \cdot \triangleleft^{b} \wedge \cdot \cdot L, Q L J^{n C} \nabla$ PSUcb;

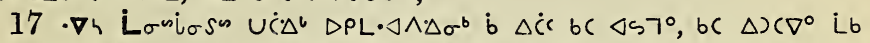

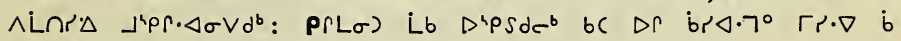
$\Delta r b \cdot \Delta \Lambda_{c} r_{x}$

\section{A $p c r a \Delta b^{a} 8$.}

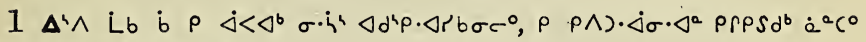
$\left.\dot{\Delta} \wedge c^{\circ} \cap \dot{<} \vec{r} b^{a} \wedge r\right\lrcorner b \sigma^{b} x$

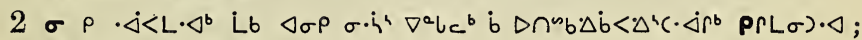
$\mu \Gamma \dot{c} \cdot \Delta^{b}$ Lb $\sigma \cdot \dot{h}^{4}>\dot{C} r b a_{x}$

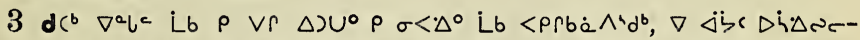

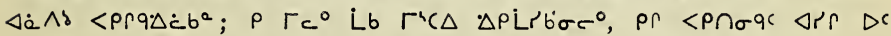

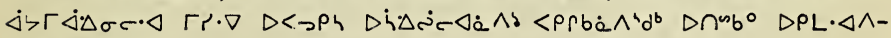
$\Delta \sigma^{b}$ b $\Delta C \cdot b^{b} x$

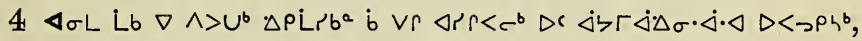

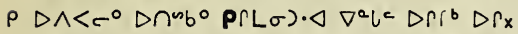

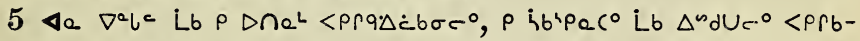

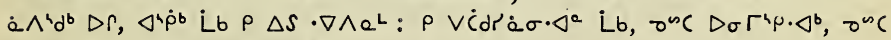

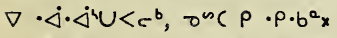

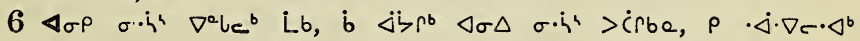
of $\rho) \dot{C}^{\mathrm{b}} \mathrm{x}$

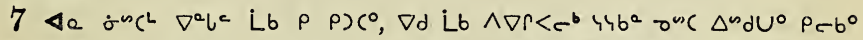
$\Gamma d^{b}, \Delta^{4} \dot{p}^{b}$ Lb $\rho \Delta S \cdot \nabla \wedge \sigma \dot{b} U \cdot \triangleleft: \sigma^{4} \cdot c^{\circ} \Delta-d^{b}$ Lb $\Gamma^{4} \cap \cdot b^{b} \rho i b^{4} \rho d \cdot \Delta^{b}, \sigma^{n C}$

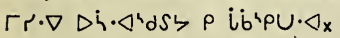

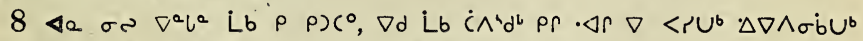

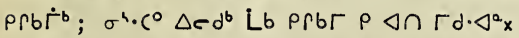

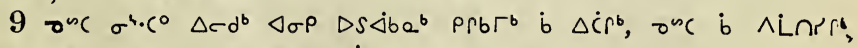
$\rho \sigma \wedge \cdot \Delta^{b} ; \sigma^{4} \cdot c^{0} \Delta r d^{b} \partial^{n c} \dot{a} \wedge \cdot \dot{b} a \rho \sigma s \cdot \Delta \dot{a}\left(\cdot a_{x}\right.$

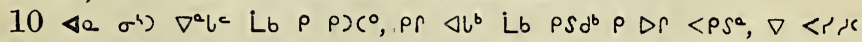

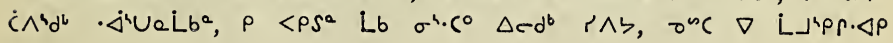
$\sigma \wedge$ ケ 


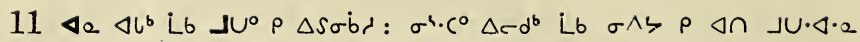
$\Gamma \nmid \Delta c c \cdot \triangleleft^{b} L b \sigma \wedge \zeta P D \Gamma \sigma \wedge \cdot \triangleleft^{b}, \nabla \Delta 4 b b \Gamma \rho_{x}$

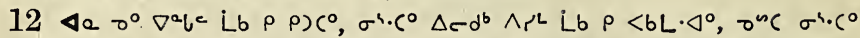

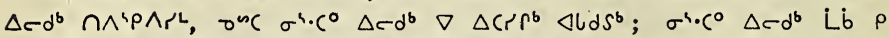

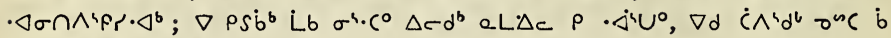
$\Delta S \dot{a} \cdot b^{b} \nabla \cap \wedge{ }^{4} b^{b} \mathrm{x}$

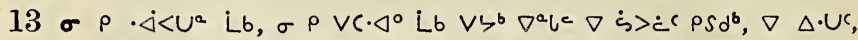

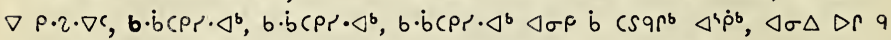

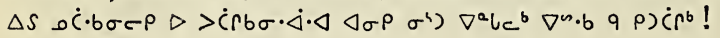

\section{ヘ}

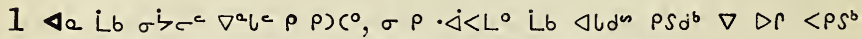

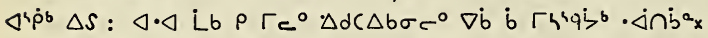

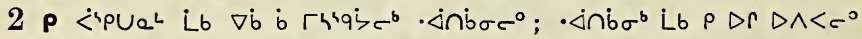

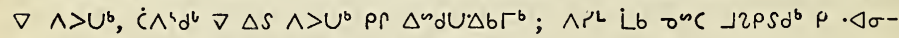

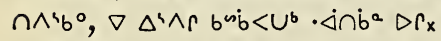

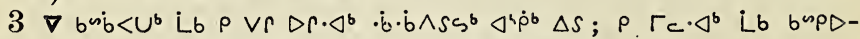

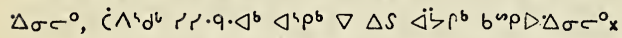

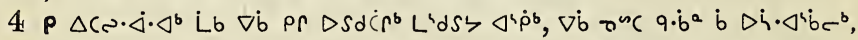

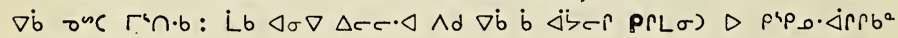
$\Delta$ ib $\cap d \cdot \Delta^{b} x$

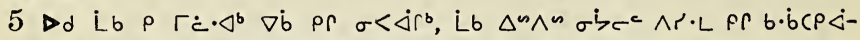

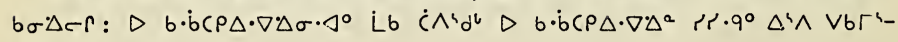
$b \cdot \Delta r \Delta r \cdot \Delta x$

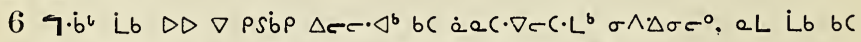

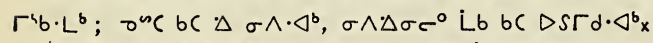

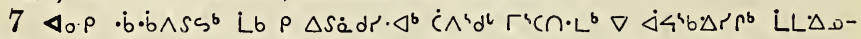

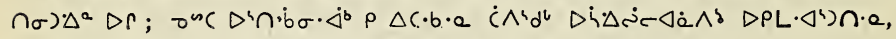

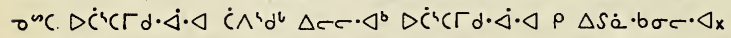

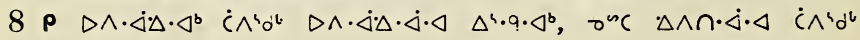
$\left\ulcorner S \wedge S \cdot \Delta^{b} \Delta \wedge \cap \cdot \Delta \cdot \Delta x\right.$

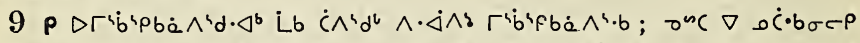

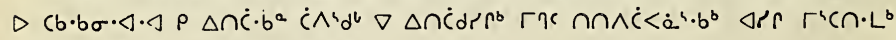

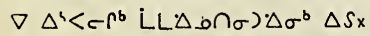

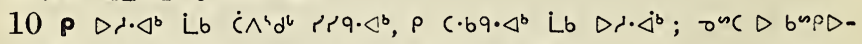

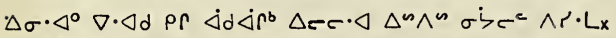

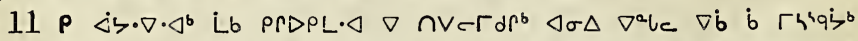

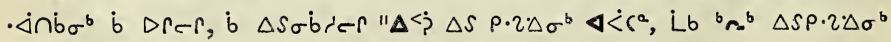

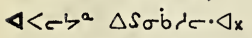

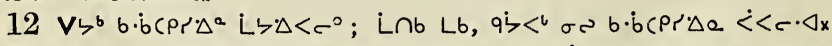

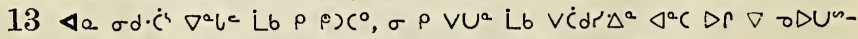

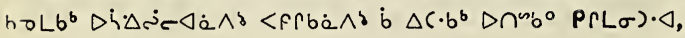

408 


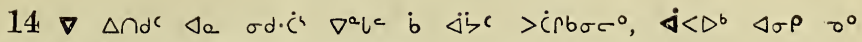

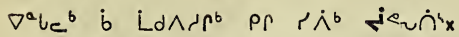

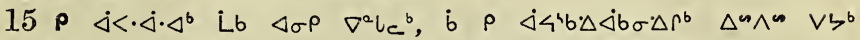

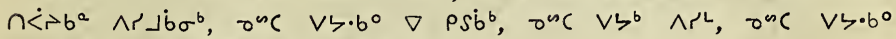
$\nabla \wedge>^{b}, \sigma^{4} \cdot c^{0} \Delta r^{b} \rho \rho \sigma<\Delta \rho^{b} \Delta r c^{\circ} \Delta x$

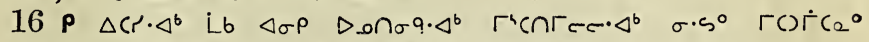

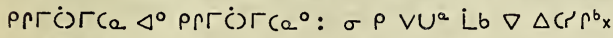

$17 \Delta L \quad \Delta S \quad L b \quad \sigma \quad \rho \cdot \dot{i}<L \cdot \Delta^{b} \Gamma^{b} C \cap \cdot L^{b} \quad \Delta S a J \cdot \Delta \sigma^{b}, \Delta \sigma \rho$ oac b

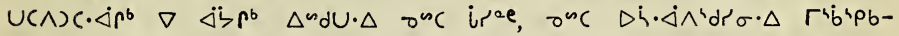

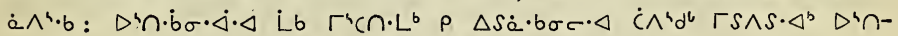

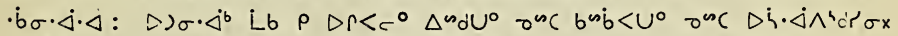

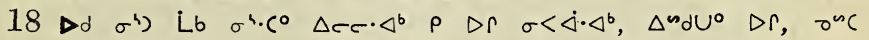

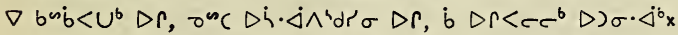

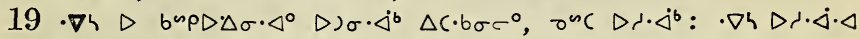
$\rho \quad \Delta S \dot{a} \cdot b \sigma c \cdot \triangleleft \quad \dot{c} \wedge^{4} d^{b} \Gamma S \rho_{0} \wedge \cdot b^{b}, \rho \quad D^{h} \cap \cdot \dot{b} \sigma \cdot<^{b}, \nabla \cdot \Delta \cdot b \sigma \Delta$ onc $\cdot \nabla R$ $\triangle 49-C \Gamma \triangle \cdot \nabla \rho^{6} x$

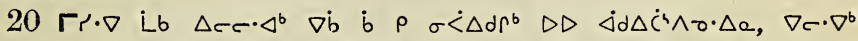

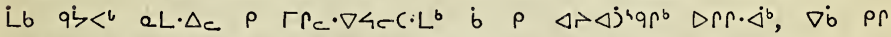

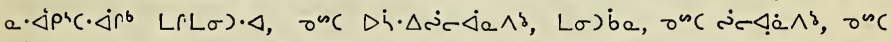

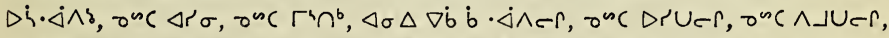

21 aL onc $\rho \Gamma \Gamma_{C} \cdot \nabla 4 c C \cdot L^{b} D \quad \sigma \dot{<}>\cdot \nabla \cdot \Delta \sigma \cdot \dot{\Delta} \cdot \Delta$, onc $D \Gamma U \Delta \cdot \Delta-$

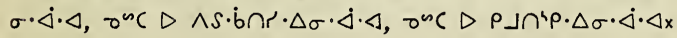

\section{Ápça $\triangle b^{a} 10$.}

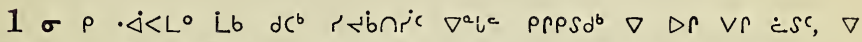

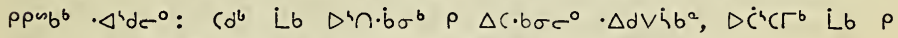

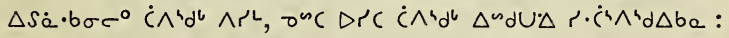

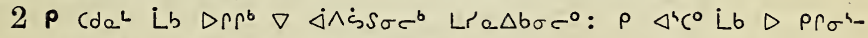

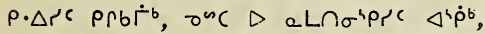

3 P U.V L Lb $\nabla \rho \cdot 2 \cdot \nabla^{c}, \dot{C} \wedge^{4} d^{b} \Gamma s \wedge s^{\circ} \nabla D_{\mathcal{A}^{c}}: \Delta^{4} \wedge$ Lb b $\rho U \cdot V^{c}$, $\left.\sigma \cdot h^{4} \nabla \sigma \Gamma^{4} \rho \cdot \Delta^{b} \rho \rho\right) \cdot \Delta^{b} x$

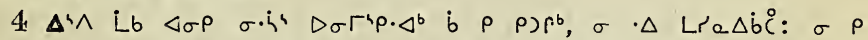

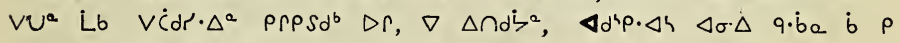

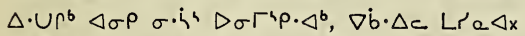

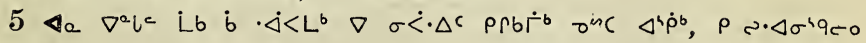
Prps d $\Delta S$,

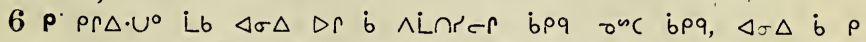

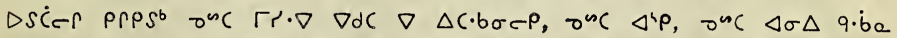

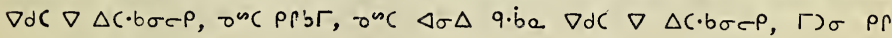
$>\sigma<-\rho \nabla \rho S \operatorname{sig}:$

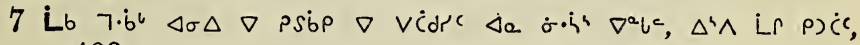
409 


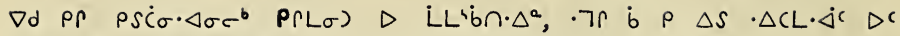

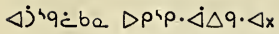

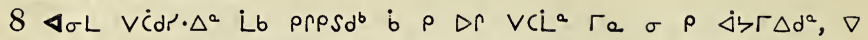

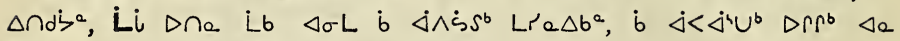

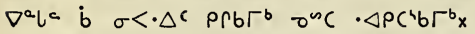

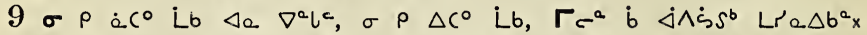
$\nabla d \nabla S c, \nabla \cap a, \Gamma \Gamma$ Lb; $\rho$ b $\rho S \cdot \Delta C<^{b} b d^{a} \rho \cdot \Delta \dot{C}^{b}$ Lb, Lb $\left.\rho\right) \sigma^{b} b c s \cdot \triangleleft^{\circ}$

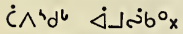

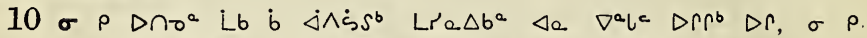

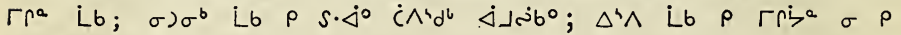
PS. $\triangleleft$ " $b \mathrm{C}^{\mathrm{a}} \mathrm{x}$

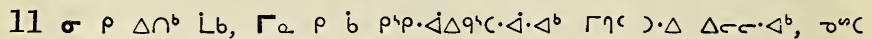

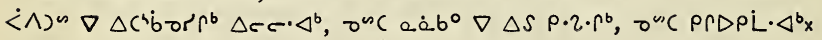

\section{Ápcra $\Delta b^{a} 11$.}

$1 \sigma \rho \Gamma-\dot{b} \cdot \Delta^{a}$ Lb $\cdot \Delta \dot{<}^{b} \dot{C} \wedge^{4} d^{b} \Gamma^{4} \cap^{b} \nabla \Delta S \dot{a} \cdot b^{b}: \nabla^{a} l^{c}$ Lb $\rho \sigma<\cdot \Delta^{0}$,

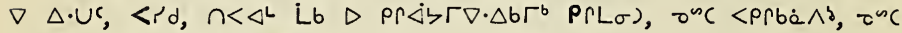
$\triangle \sigma \rho \nabla d C \dot{b} \quad \dot{\zeta} \Gamma \nabla \cdot c \dot{c} q r^{b} x$

2 i $b \quad \Delta \sigma L \quad L \cdot \Delta r \Delta) \cdot \Delta b \Gamma^{b} b \quad \Delta C \cdot b^{b} \cdot \Delta c \cdot \Delta n \Gamma^{b} \quad \rho \Gamma \Delta \zeta \Gamma \nabla \cdot \Delta b \Gamma d^{b} \rho$ b

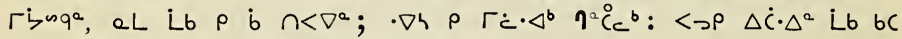

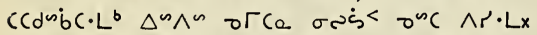

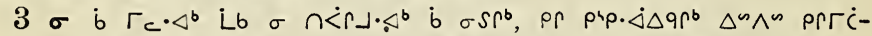

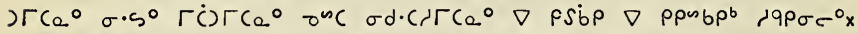

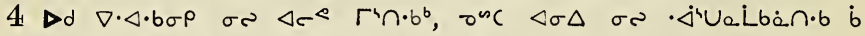
$\sigma<\cdot \Delta L b P \quad \Delta \cap \omega b^{\circ} P(L \sigma)$ b $\cap V \in c^{b} \Delta^{4} P c^{0} x$

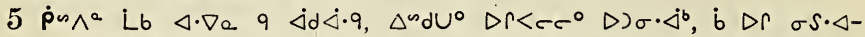

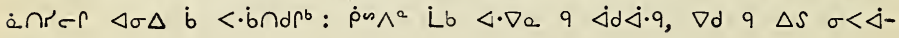
$b \sigma \cdot \Delta c_{x}$

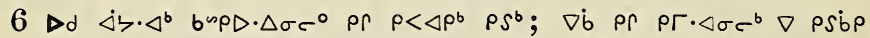

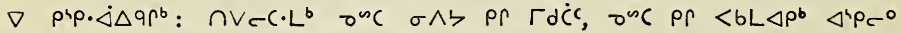

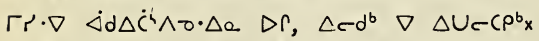

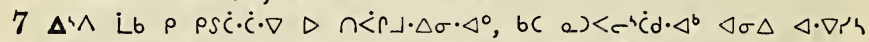

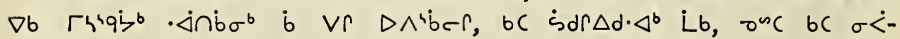
$\Delta d \cdot \Delta^{b} x$

$8 \cdot \Delta \dot{b} \cdot \dot{\Delta} \cdot \triangleleft$ Lb bC $\wedge \Gamma \cap \sigma c \cdot \triangleleft \wedge\lrcorner u^{b} b \dot{a}^{b}$ pr $\Delta \dot{C} \cdot \Delta \sigma^{b}, \dot{b} \quad \Delta i d \cdot \Delta \Delta S \sigma=$

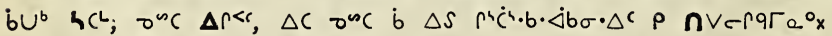

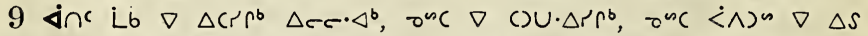

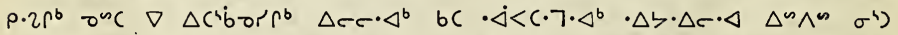

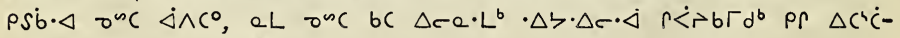
$\sigma \cdot \triangleleft \sigma c P_{X}$

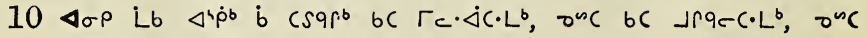
410 


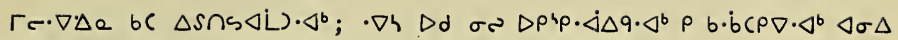
$\Delta$ sipb $\dot{b}$ csqcri

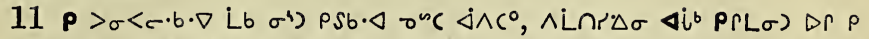

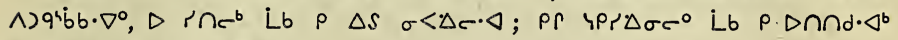
$r \cdot \cdot \nabla \triangleleft \sigma^{\prime} \rho \dot{b} \rho \cdot \dot{\Delta}<\dot{L} r^{b} x_{x}$

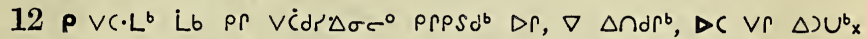

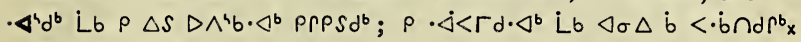

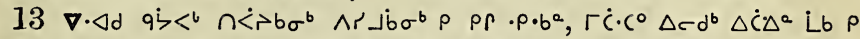

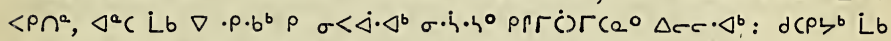

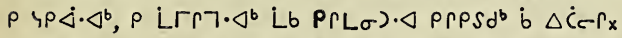

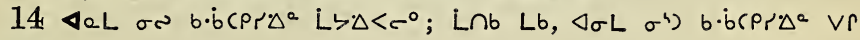
PS $\angle C^{\circ} \mathrm{x}$

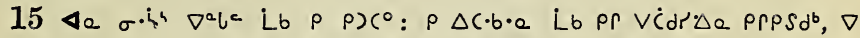

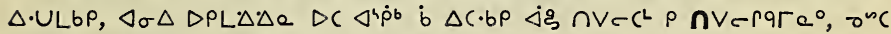
$\triangle X \cap L ; b C \cap V e r q^{\circ} L b$ b $p q$ z $^{\circ C} \mathrm{~b} b \rho q_{\mathrm{X}}$

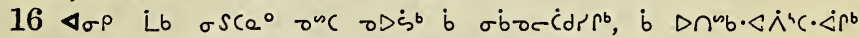

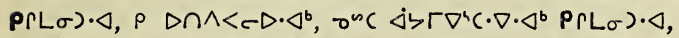

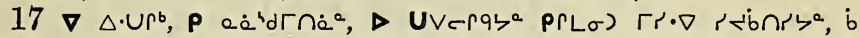

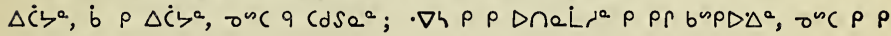
nV $\sim \operatorname{rib}^{\mathrm{a}} \mathrm{x}$

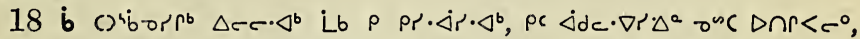

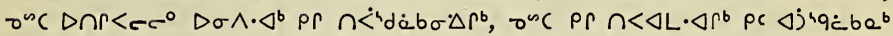

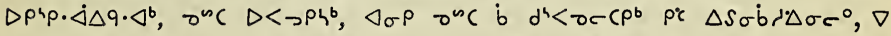

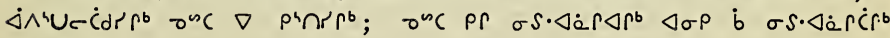
$\triangle 4 p c^{\circ} \mathrm{x}$

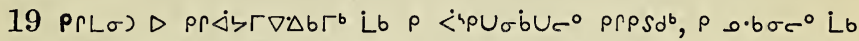

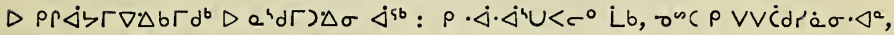

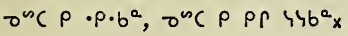

\section{$\Lambda^{4} p c r a \triangle b^{e} 12$.}

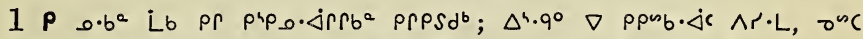

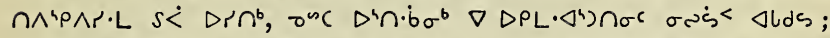

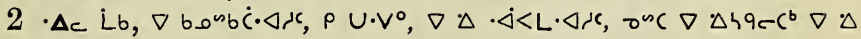
$\sigma\left(\Delta P \Delta \cdot \nabla c_{x}\right.$

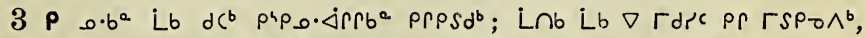

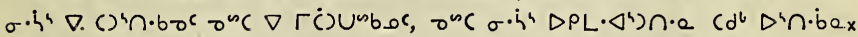

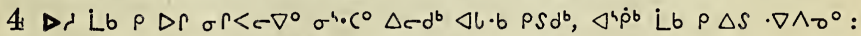

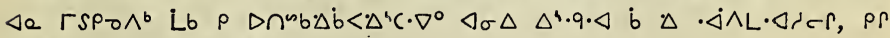
\lrcorner$\Delta \dot{L} C D C \Delta \cdot \Delta S T S \rho \dot{S} \downarrow \sigma C \Delta P C \Gamma_{X}$

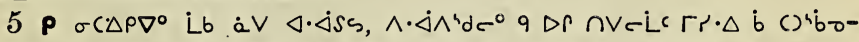

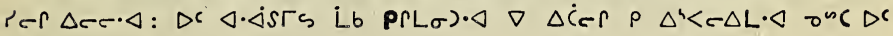
$\Delta P L \cdot \triangleleft \wedge \Delta \sigma \sigma^{\circ} x$ 


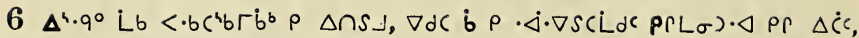

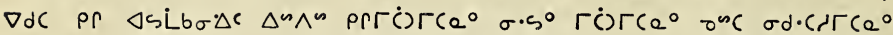
$P S b \cdot \triangleleft x$

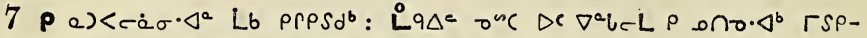

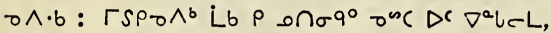

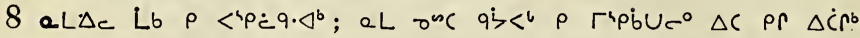
presd dix

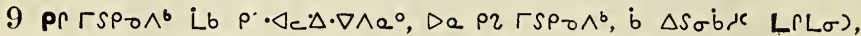

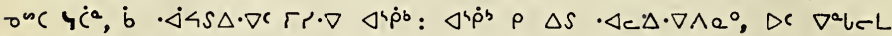
$\Delta r \zeta \rho \cdot \Delta c \Delta \cdot \nabla \Lambda_{\dot{L}} b \sigma \cdot \Delta r \cdot \Delta x$

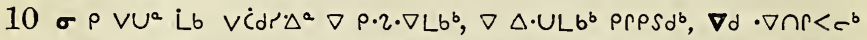

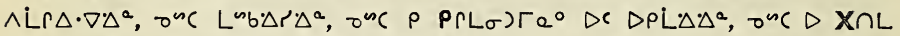

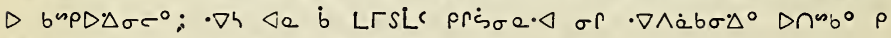

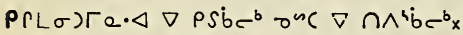

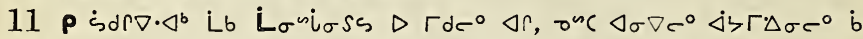

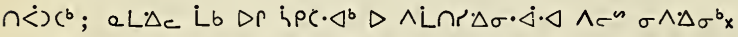

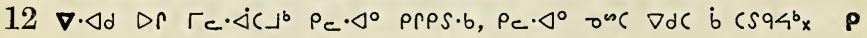

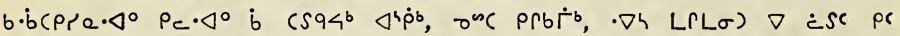

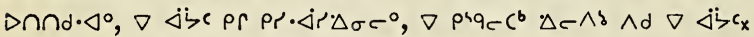

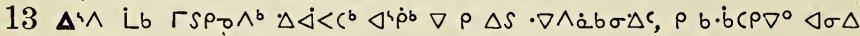

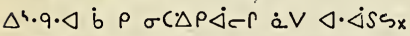

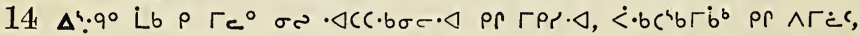

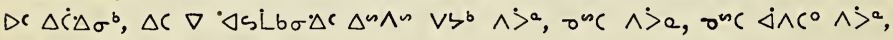

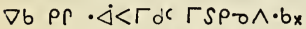

$\left.15 \Gamma S \rho_{0} \wedge^{b} L b \Delta\right) \sigma^{b} \rho \Delta R \cdot \Delta c \Delta \cdot \nabla \wedge Q L \cdot \nabla^{\circ} \sigma \wedge \sigma^{\circ} \triangleleft \sigma \Delta \Delta^{4} \cdot q \cdot \triangleleft \dot{C} \wedge^{4} d^{l}$

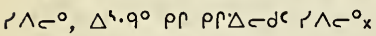

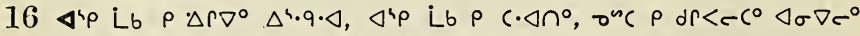

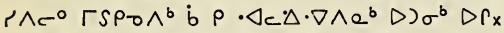

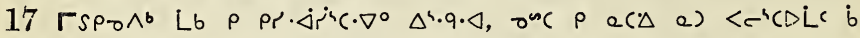

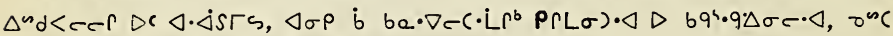

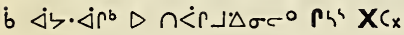

\section{Ápcre $\triangle b^{a} 13$.}

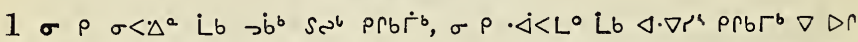

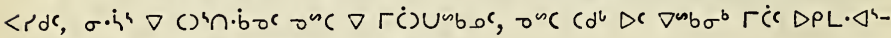
)n.e, onc $\left.\Delta^{n} n \cdot \dot{b} \sigma^{b}<\dot{c}\right\lrcorner \Delta \Delta S \sigma \dot{b} \dot{i} \Delta e_{x}$

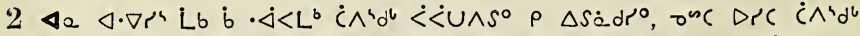

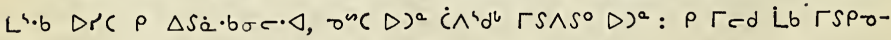

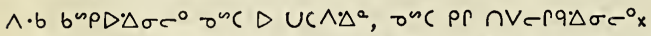

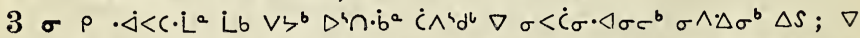

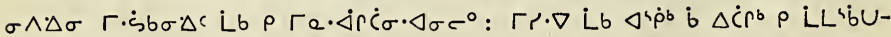
$-7 \cdot \Delta^{b} \triangleleft \sigma \Delta \triangleleft \cdot \nabla r h x$

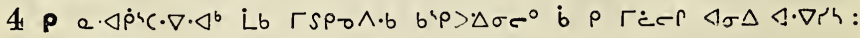
412 


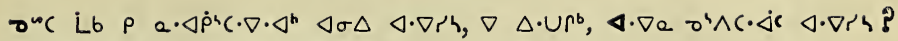

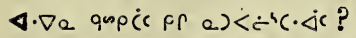

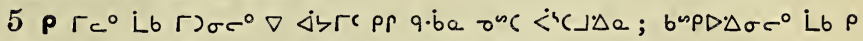

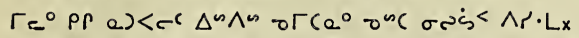

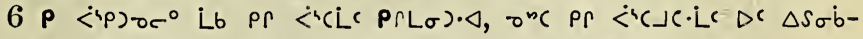

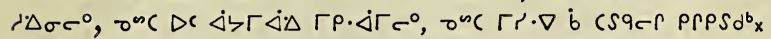

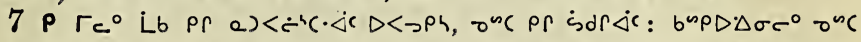

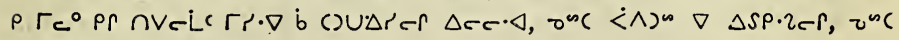
i cosigrer $a c c \cdot \neg x$

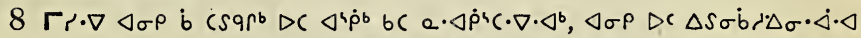

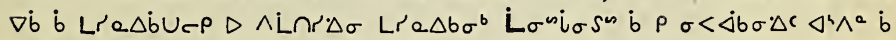
Lr $\triangleleft^{4} p \cdot \Delta^{b} x$

$9 \dot{\rho} \backsim \wedge a \triangleleft \cdot \nabla e \cdot \nabla \dot{C} \cdot \triangleleft \dot{h} \cdot q, \nabla d S b c \vee\left(L^{2}\right.$

$10 \varangle$ a $\triangleleft \cdot \triangleleft \dot{b} s \cdot \nabla \Delta \sigma^{b} \dot{b} \Delta x \Delta \cdot \nabla c, \triangleleft \cdot \Delta \dot{b} s \cdot \nabla \Delta \sigma^{b}$ bc $\left.\Delta\right) U^{\circ} ; \triangleleft a s \dot{L} b \sigma \sigma^{\circ}$

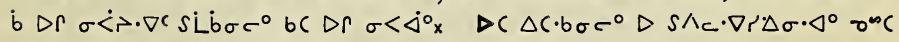

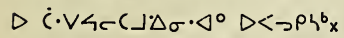

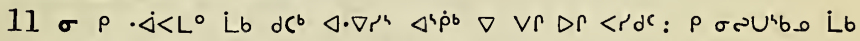

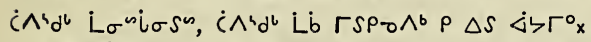

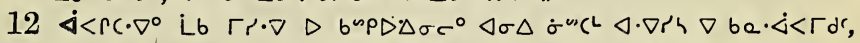

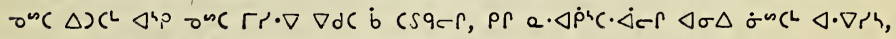
$\Delta a \nabla \sigma \wedge \Delta \sigma \Gamma \cdot \dot{s} b \sigma \cdot \Delta c \dot{b} \Gamma e \cdot \Delta r \dot{c} \sigma \cdot \Delta \sigma c^{b} x$

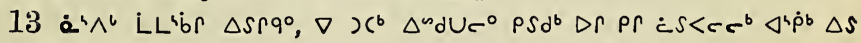
$\nabla$ be. $\dot{\Delta}<C \Gamma e r \Delta c c \cdot \Delta x$

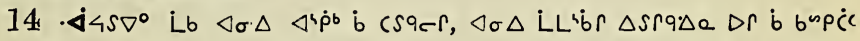

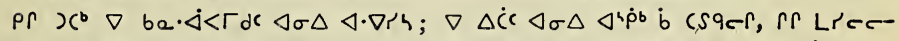

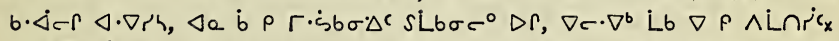

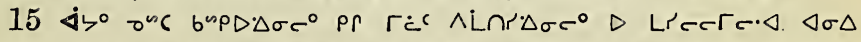

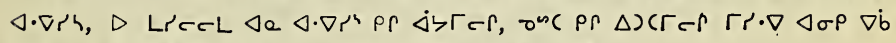
b $\triangle a \cdot \Delta \rho^{b} C D L r^{b} D$ LrecL $\triangleleft \cdot \nabla r^{h}$ fr $\sigma<\Delta b \sigma \cdot \Delta r^{b} x$

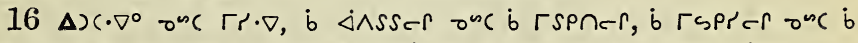

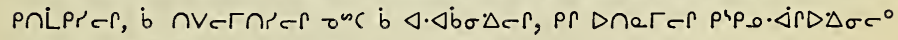

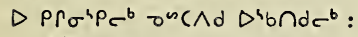

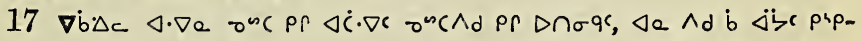

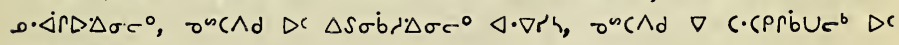
$\Delta S \sigma \dot{b} \gamma^{\prime} \Delta^{2} \mathrm{x}$

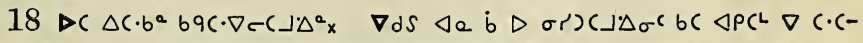

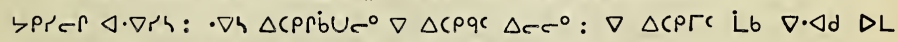

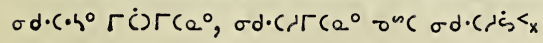

\section{Ápcre $\triangle b^{\circ} 14$.}

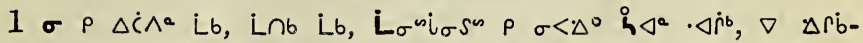

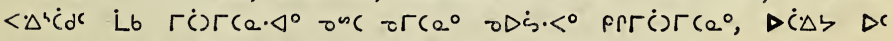

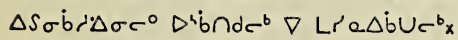

413 


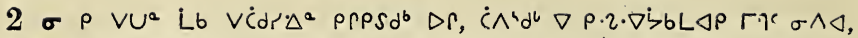

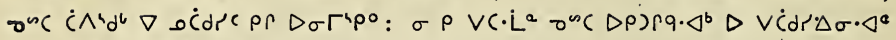

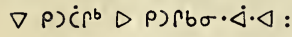

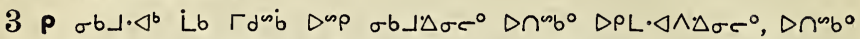

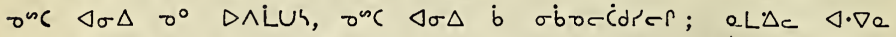

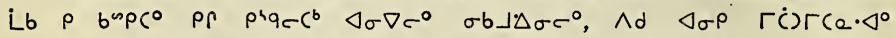

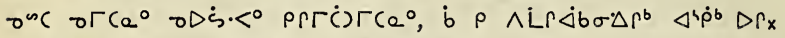

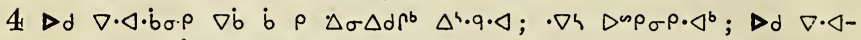

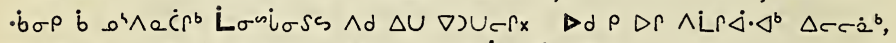

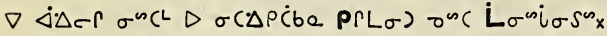

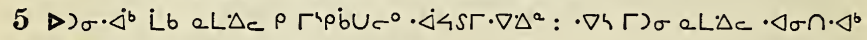
$\Delta \cap n^{\infty} b^{\circ} P(L \sigma) \cdot \triangleleft \sigma^{n}\left(D P L \cdot \Delta \wedge \Delta \sigma c^{\circ} x\right.$

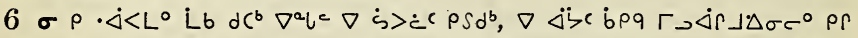

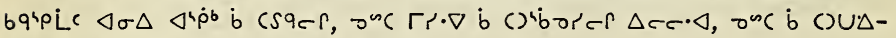

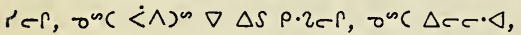

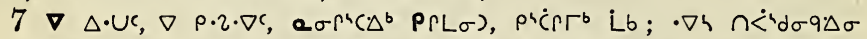

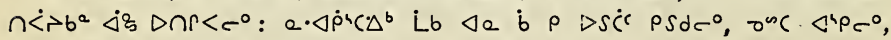
onc

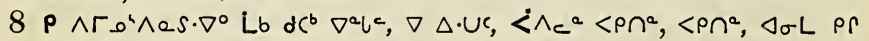

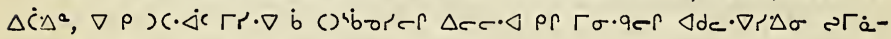
$>c^{\circ} \Delta \wedge S \cdot \dot{b} \cap 4 \Delta \sigma^{b} \dot{b} \quad \Delta R<c c^{b} x$

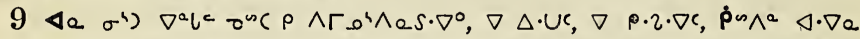

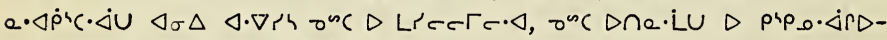
$\Delta \sigma \sigma^{\circ} \Delta^{b} b \cap d^{b}, \sigma^{\omega}<\wedge d \Delta \dot{r}^{b}$,

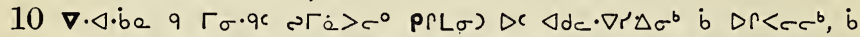

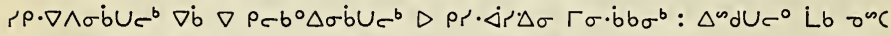

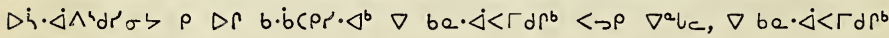
onc Lonioss:

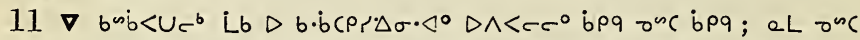

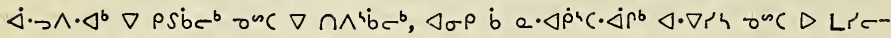

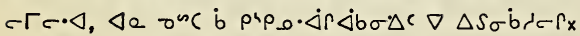

$12 \Delta C \Delta C \cdot b \sigma c^{\circ} \nabla S \wedge c \cdot \nabla r \cdot \Delta \sigma \cdot \Delta^{\circ} D<\neg \rho h^{b}: \Delta C \Delta \dot{C} \cdot \Delta^{b} \Delta \sigma \rho b$ b be. $\nabla-$

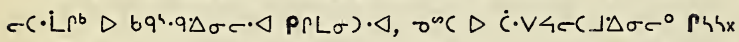

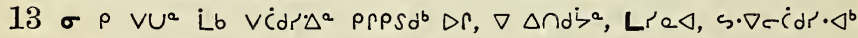

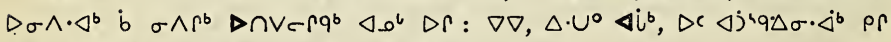

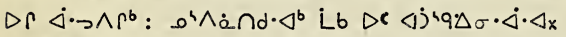

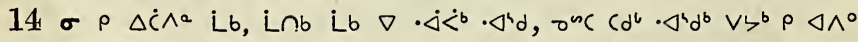

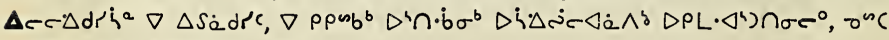
$\nabla R r^{b} \nabla \dot{b} \dot{s} \sigma^{b}$ pupsbar $\sigma^{\circ} x$

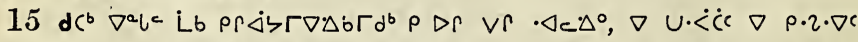

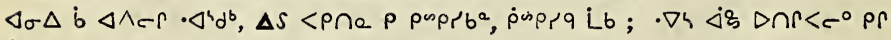

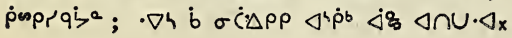




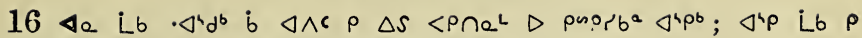
pussibuox $x$

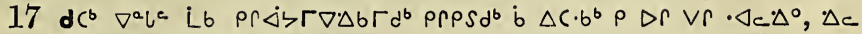

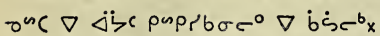

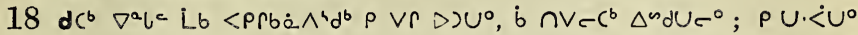

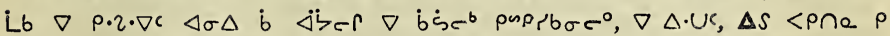

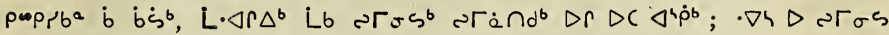
pesnic. $\triangle x$

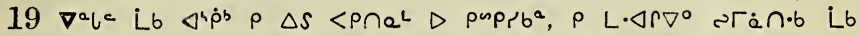

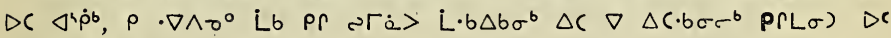

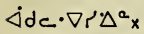

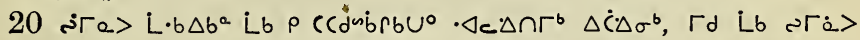

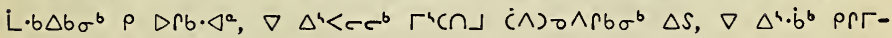

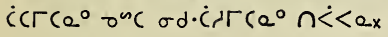

\section{$\Lambda^{\wedge} \rho C\left\ulcorner\propto \triangle b^{\circ} 15\right.$.}

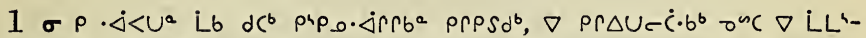

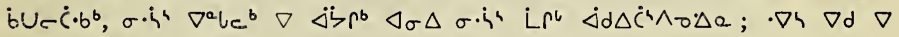

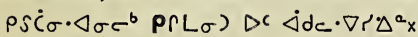

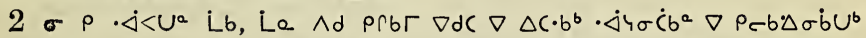

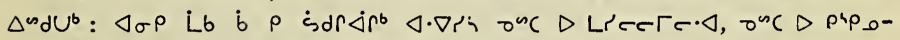

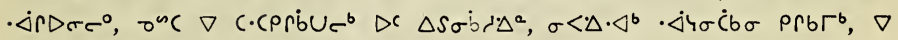
$\left.\Delta ל \cdot \Delta \beta^{b} P(L \sigma) \cdot \Delta D P\right)(b \sigma e \cdot \Delta x$

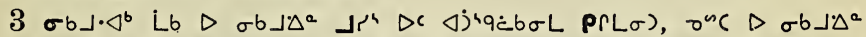

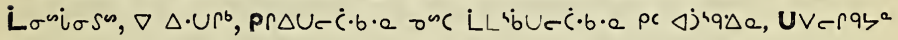

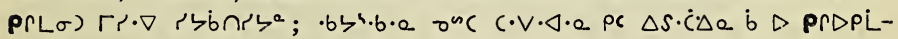

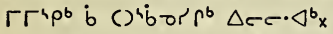

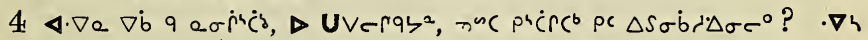

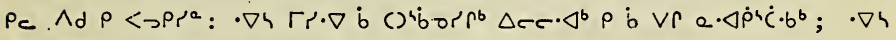
$\rho \cap \dot{<} d \sigma q \Delta a<\dot{<}\rangle$ od $\dot{C} \sigma \cdot \triangleleft \cdot e_{x}$

$5 \dot{b}>\sigma<\Gamma^{b} D L \quad \sigma \quad \rho \Delta \dot{C} \wedge a$, Lnb Lb, $\rho \Gamma \dot{\Delta} \zeta \Gamma \nabla \Delta b \Gamma^{b} \nabla \cap \dot{C} \mu \Delta \sigma$

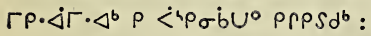

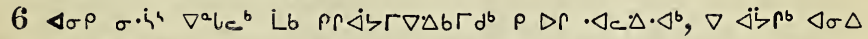

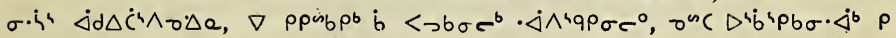

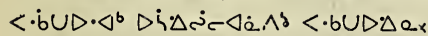

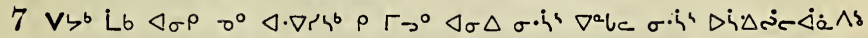

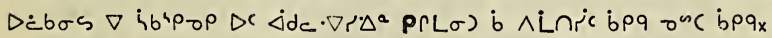

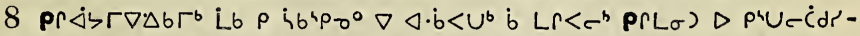

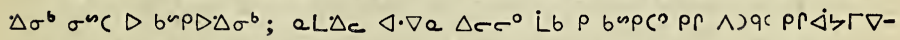

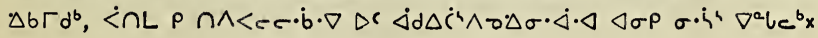




\section{$\Lambda^{\prime} p\left(r a \triangle b^{\circ} 16\right.$.}

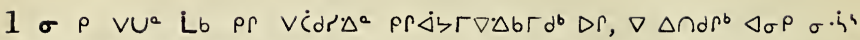

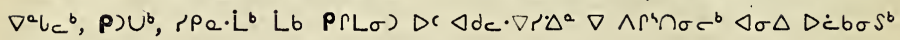
$\cdot \triangleleft P\left(c^{b} b \Gamma^{b} \times\right.$

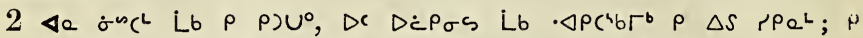

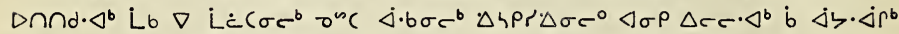

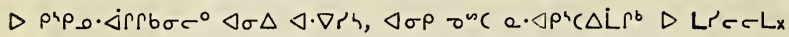

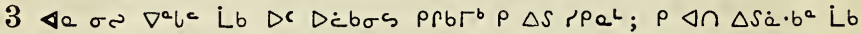

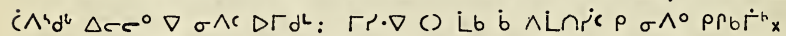

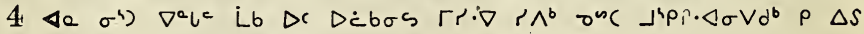
PPQL; $\nabla d$ Lb b $\left\ulcorner d \cdot \triangleleft P_{x}\right.$

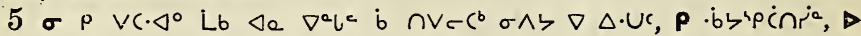

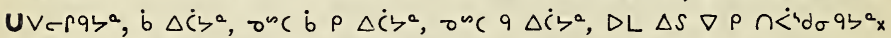

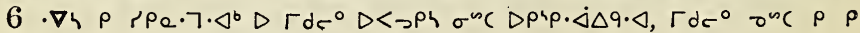

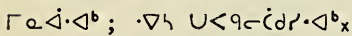

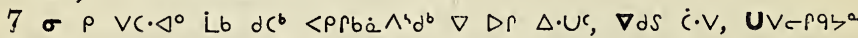

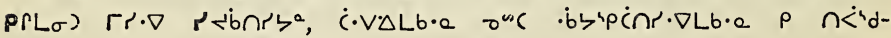
$\sigma 9 \Delta a x$

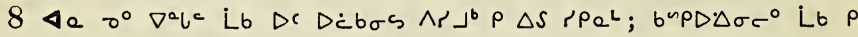

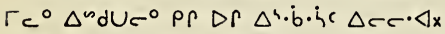

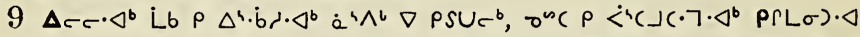

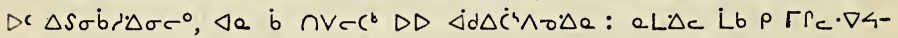
$C C \cdot L^{b} \operatorname{PC} \operatorname{LrCLL} \mathrm{L}^{\mathrm{b} x}$

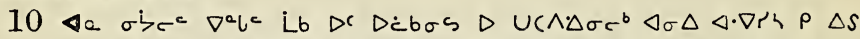

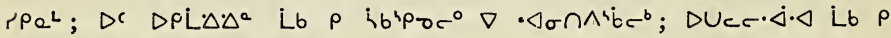
LLdd $\cdot L^{b}$ b $\Delta^{4} \wedge \Gamma \cdot \Delta^{4} q C C \rho^{b}$,

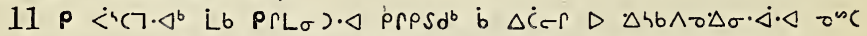

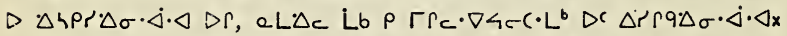

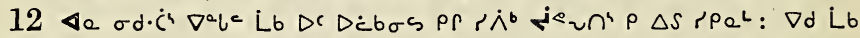

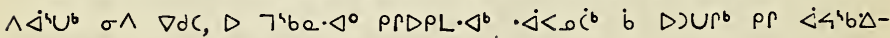
$\dot{c} \sigma \cdot \triangleleft \sigma c^{b} x$

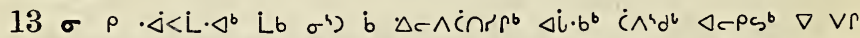

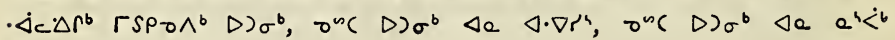
$\Delta \rho^{\top} \rho \cdot \Delta \Delta q^{\circ} \mathrm{x}$

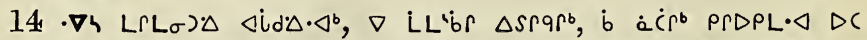

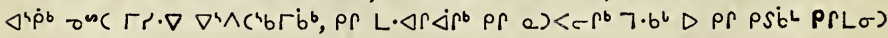

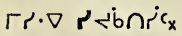

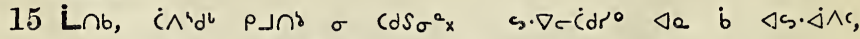

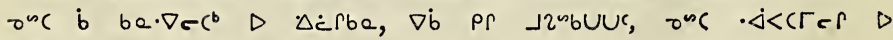
ervar. $\Delta a x$

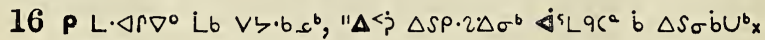
416 


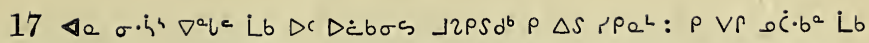

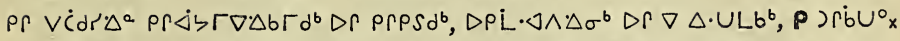

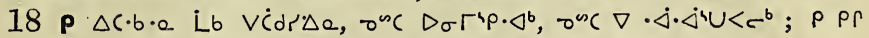

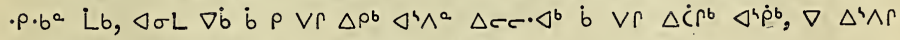

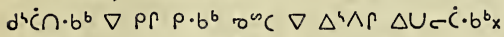

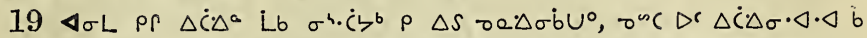

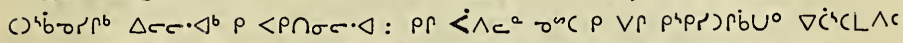

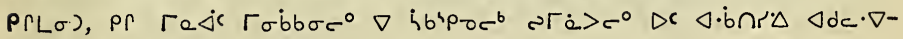
$r \Delta \sigma^{b} \dot{b} \quad D r<e c^{b} x$

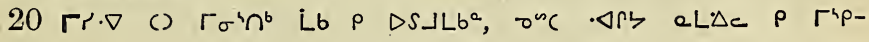
bu. $\Delta x$

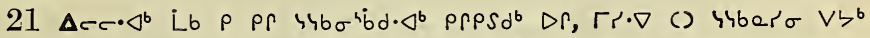

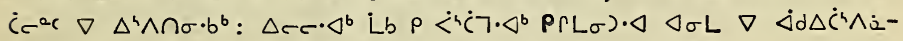

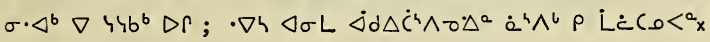

\section{$\Lambda^{4} p C \leftrightarrow a \triangle b^{\circ} 17$.}

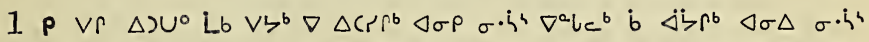

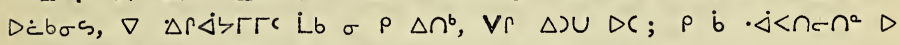

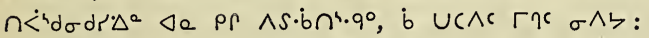

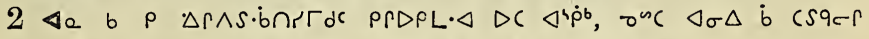

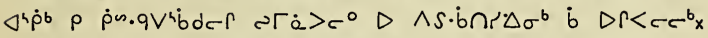

$3 \nabla d \nabla S \Delta c^{c} \Delta i d^{b}<\cdot b c^{b} b \Gamma b^{b}: \sigma \quad \rho \cdot\left\langle<L^{\circ}\right.$ Lb $\Delta^{4} \cdot q^{\circ} \Delta U(\wedge) c \cdot<c$

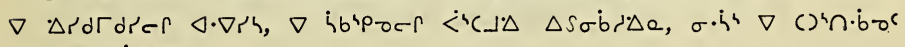

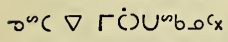

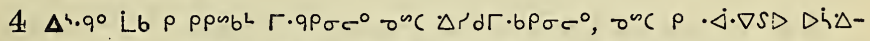

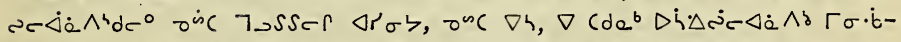

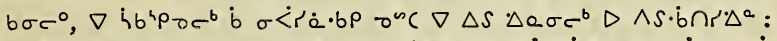

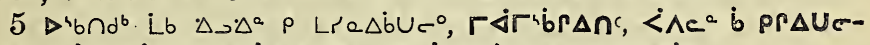

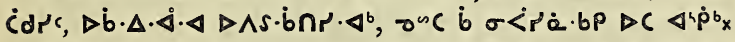

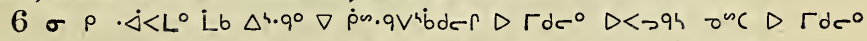

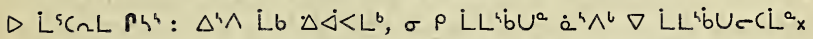

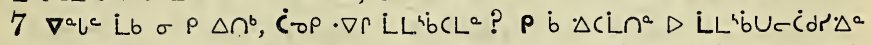

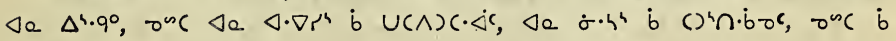
reunbosx

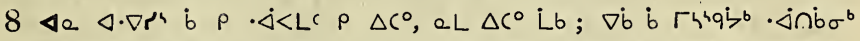

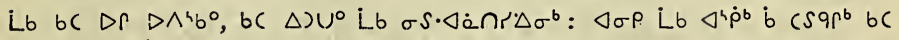

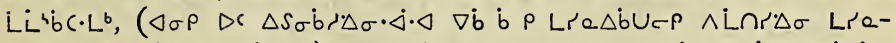
$\left.\Delta b \sigma^{b} \Delta^{4} \wedge^{c} \dot{b} \dot{L} r \Delta^{4} \dot{p} \cdot \Delta^{b},\right) \Delta^{4} \wedge \cdot \dot{\dot{L}}<\dot{L} \cdot c \cdot \nabla \Delta \sigma \Delta \Delta \cdot \nabla r h \dot{b} \rho \Delta \dot{C} c r, \nabla \dot{b} \dot{L} b$ $\nabla \Delta \dot{C}-r, \nabla c \cdot \nabla^{b}$ Ĺb $\nabla \Delta \dot{C}-r_{x}$

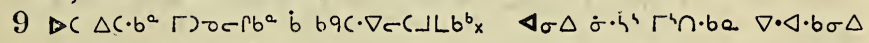
$\sigma^{4} h^{4} \cdot \Delta \Gamma \zeta, \Delta \sigma \Delta b \cup C \wedge c \Delta^{4} \cdot 9^{\circ} x$ 


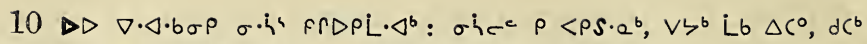
Lb a L $\Delta c \nabla^{n} \cdot b C d S^{a} ; \Delta^{4} \wedge$ Lb $C d S 9, b c \Delta c^{\circ} \Delta r_{c}{ }^{\circ} \wedge d x$

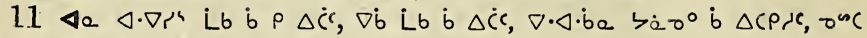

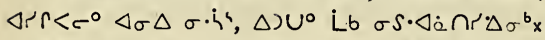

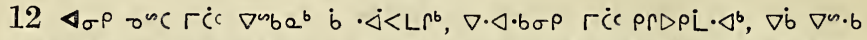

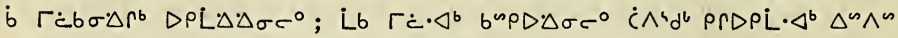

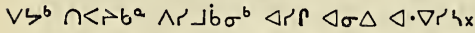

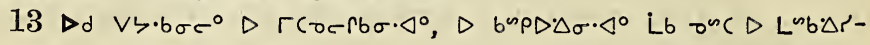
$\Delta \sigma \cdot \triangleleft^{0} b C \Gamma_{\supset} \cdot \triangleleft^{b} \triangleleft \sigma \Delta \triangleleft \cdot \nabla r h x$

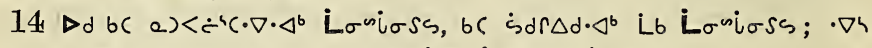

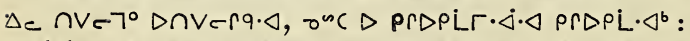

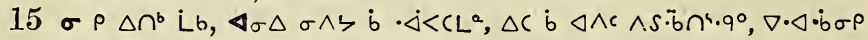

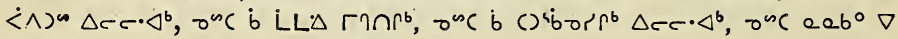
$\triangle S \rho \cdot 2 r^{b}{ }^{x}$

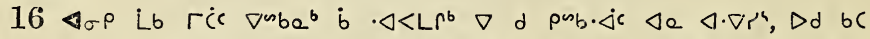

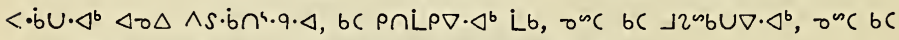

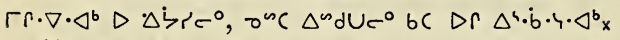

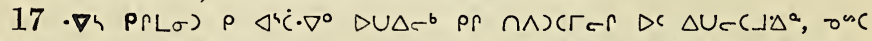

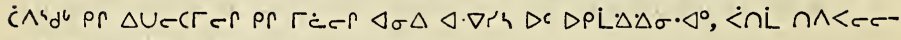
$\cdot b \cdot \nabla D C \Delta b \Gamma \Delta a \operatorname{Pr} L \sigma)_{x}$

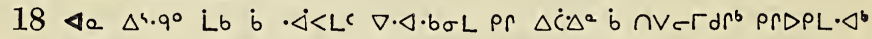
$\triangle C \triangleleft \dot{s} b_{x}$

\section{$\Lambda^{\wedge} p\left(r a \triangle b^{a} 18\right.$.}

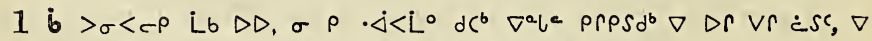

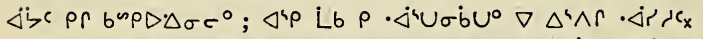

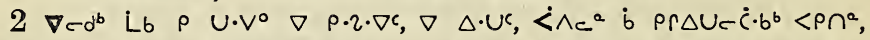

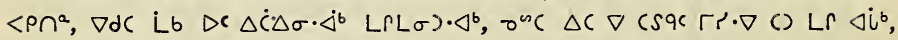

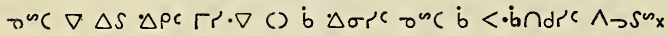

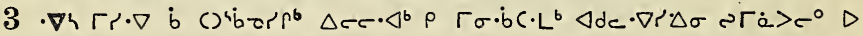

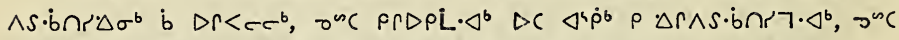

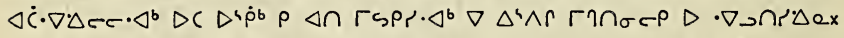

$4 \sigma \rho \vee U^{a}$ Lb $d C^{b} V \dot{C} d r \Delta^{a}$ PrPS db $D r, \nabla \Delta \cdot U L b^{b}, V R$ ab $\cap^{b} \sigma^{c}$

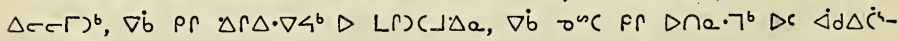
Aode:

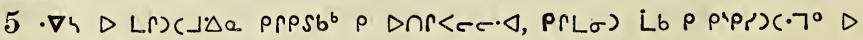
Lin $\Delta \sigma \sigma \cdot \Delta x$

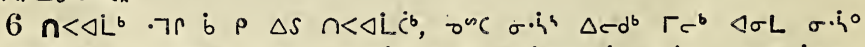

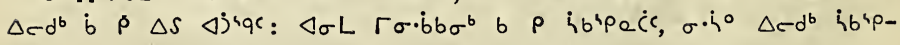
e. Lbx

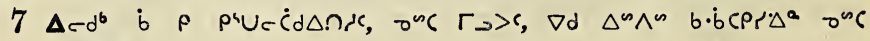
418 


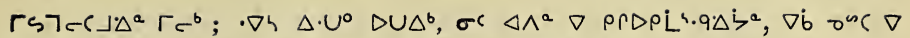

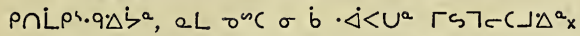

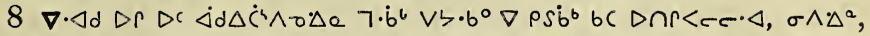
$\sigma^{n c}$ L.) $\left.\Delta^{a}, \sigma^{n c} b \cdot \Delta b U \Delta^{a}, \Gamma\right) \sigma$ Lb bC ibipr $\Delta^{n d U c^{\circ}} \Delta \Gamma: \cdot \nabla h$ L"b $\Delta r^{\circ}$

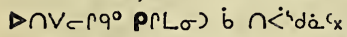

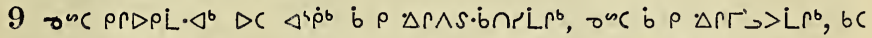

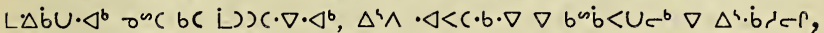

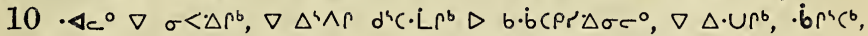

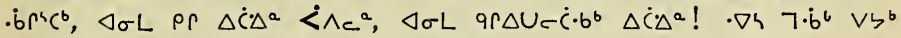
$\left.\dot{<}>b^{a} \wedge r\right\lrcorner \dot{b} \sigma^{b} \Delta \cap r<\sigma^{0} \rho \cap<\dot{<} d \sigma d r \cdot \Delta^{a} x$

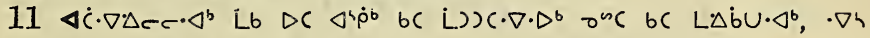
e L $\Delta c \triangleleft \cdot \nabla a \triangleleft \dot{C} \cdot \nabla^{\circ} \Delta C \triangleleft \dot{C} \cdot \dot{\Delta b} \sigma c \cdot \triangleleft$ :

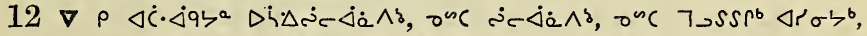

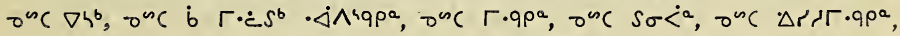

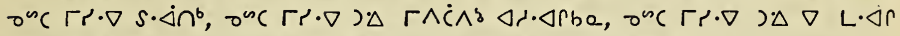

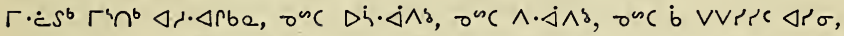

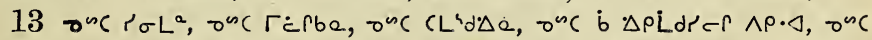

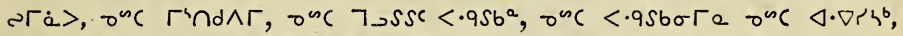

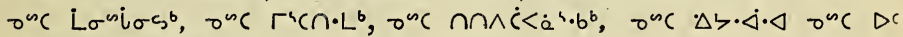
$\Delta \mathrm{id} \cdot \triangleleft \triangleleft \Delta r c \cdot \triangleleft^{b} \mathrm{x}$

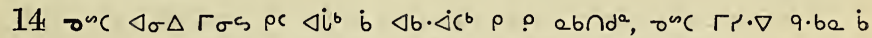

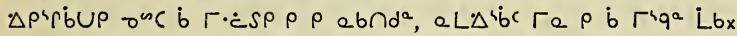

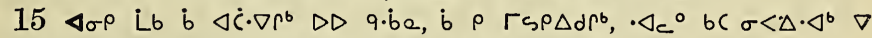

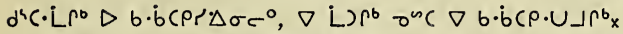

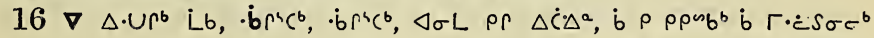

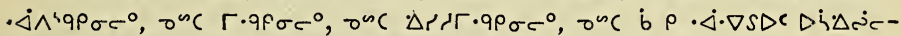

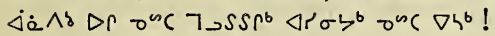

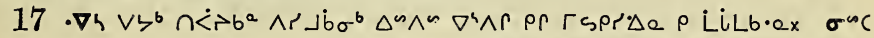

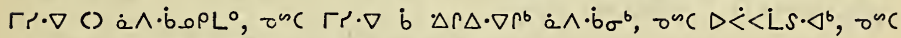

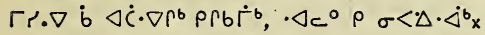

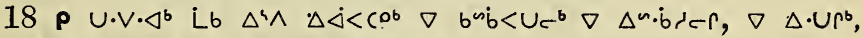
qd $\Delta \dot{C} \Delta^{a} \nabla U c \dot{C} \cdot b^{b} \dot{C} \wedge^{b} d^{b} D L$ or $\Delta \dot{C} \Delta^{a}$ !

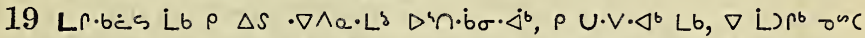

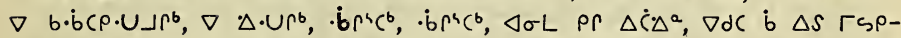

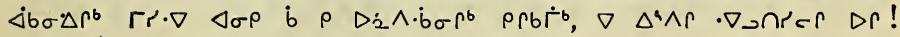

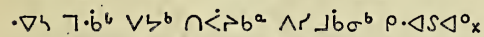

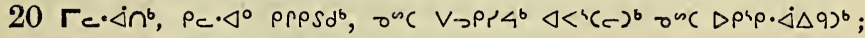

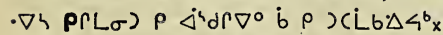

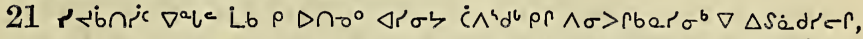

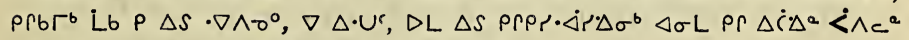
bc $\sigma r \cdot \nabla \wedge \sigma b \cap^{\circ}, a L \cdot \dot{\Delta}^{4} b c \Gamma a$ bc $\Gamma^{\prime} \rho b U^{\circ}$ Lbx

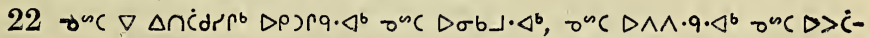

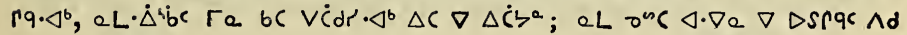
419

EE 2 


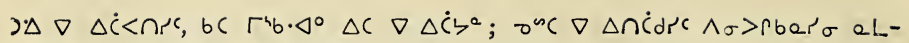

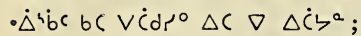

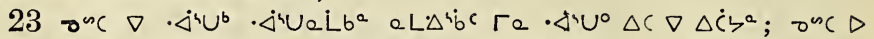

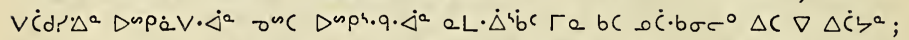

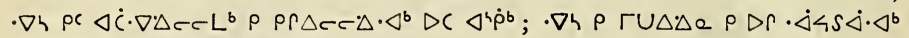

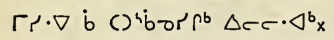

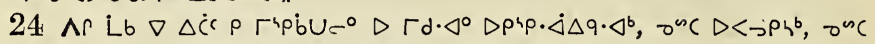
$\Gamma r \cdot \nabla \triangleleft \sigma \rho \Delta C \Delta^{4} \dot{\rho}^{b} b \dot{b} \rho \sigma<\dot{\Delta b \sigma} \Delta \rho^{b} b_{x}$

\section{Ápcra $\triangle b^{\circ} 19$.}

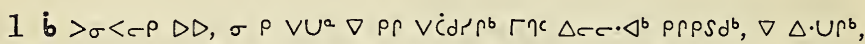

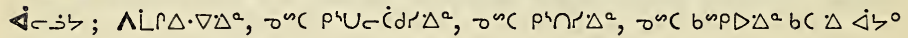
$\left.\Delta \cap V C P Q^{\circ} \rho P R L \sigma\right) \Gamma \mathrm{Q}^{\circ}$ :

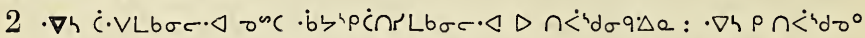

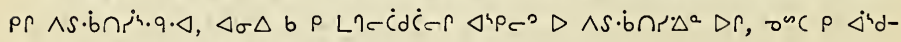

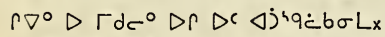

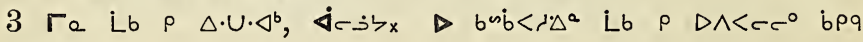
osc $b p q_{x}$

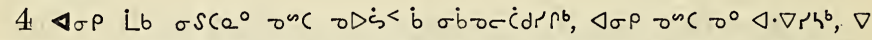

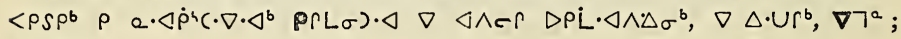

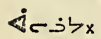

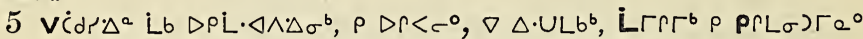

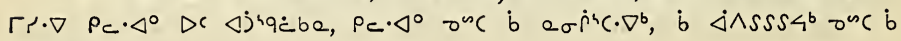
$\operatorname{rsph}<^{b} x$

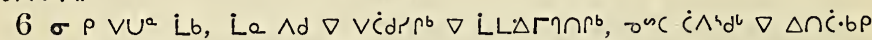

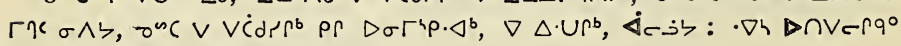

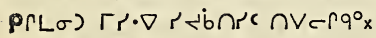

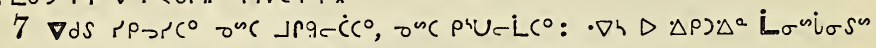

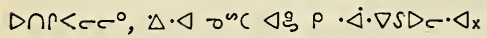

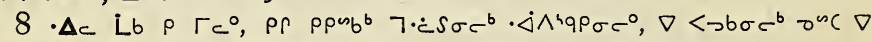

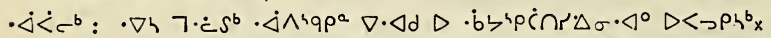

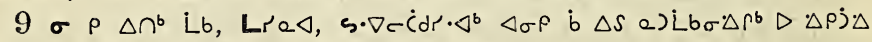

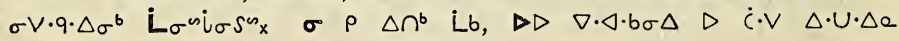
$P \Gamma L \sigma)_{x}$

$10 \sigma \rho<d S \sigma^{a}$ Lb $\left.D^{\prime} R^{b} \rho \rho a \cdot \Delta \dot{\rho}^{4} c \cdot \Delta^{b} x \quad \sigma \rho \Delta \cap^{b}, \dot{b} \cdot b \nabla b\right) \dot{C}: \sigma c$

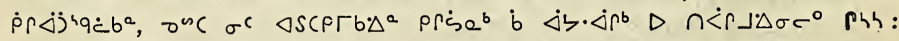

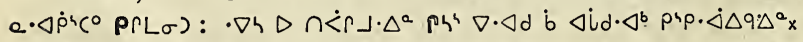

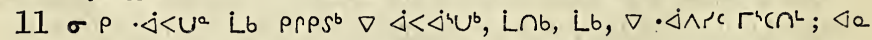

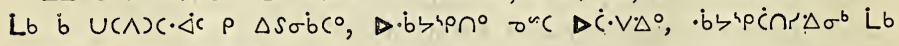
$\cap \dot{<}^{\circ} d \sigma q^{\circ} \sigma^{n}(a)<c^{\circ}{ }_{x}$

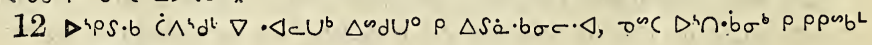
420 


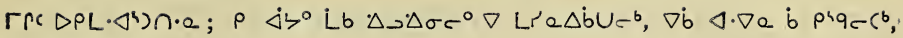
$\Delta c a c \cdot<_{b} \wedge d x$

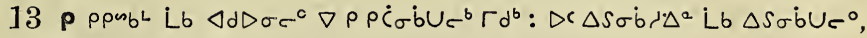
$\Delta\left(\dot{\Delta} \zeta \Gamma \Delta^{a} p \rho L \sigma\right)_{x}$

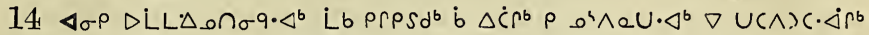

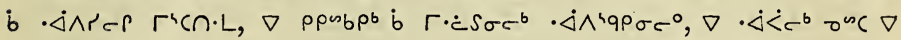
$<\rightarrow b \sigma c^{b} x$

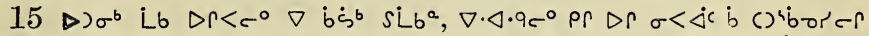

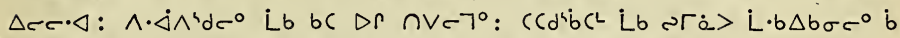

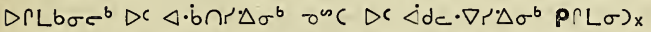

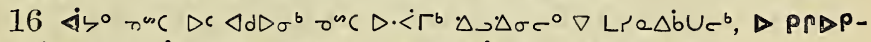

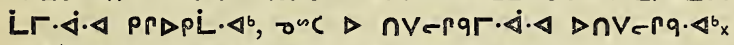

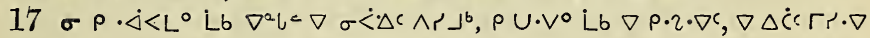

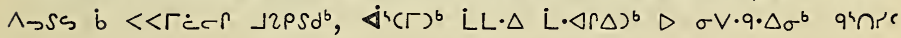
PPLO);

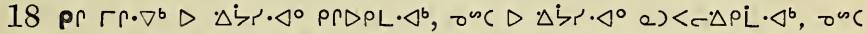

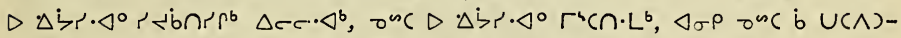

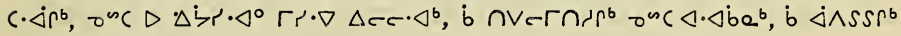
$\cos \dot{b} \operatorname{sen} h^{b} x$

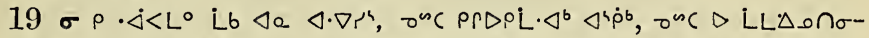

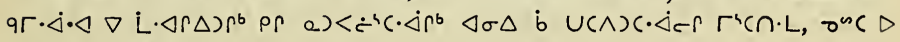
LL $\Delta \circ \cap \sigma a \Gamma \tau \cdot \Delta x$

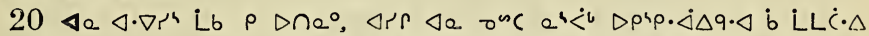

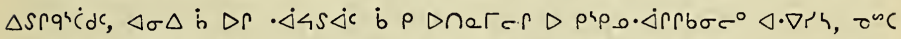

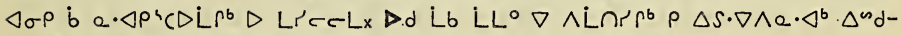
$U \cdot \Delta \dot{i} b \Delta b \sigma^{b} \nabla \cdot \Delta c U^{b} \Delta \dot{h} \cdot \dot{\Delta} \Lambda^{h} d r \sigma_{x}$

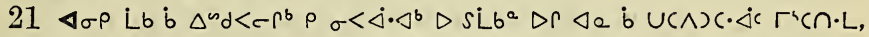

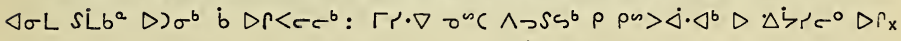

\section{$\Lambda^{4} p C r a \triangle b^{a} 20$.}

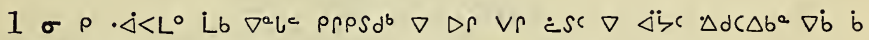

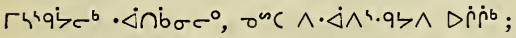

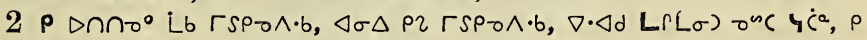

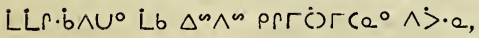

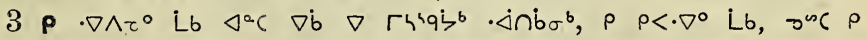

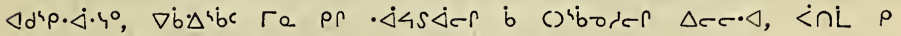

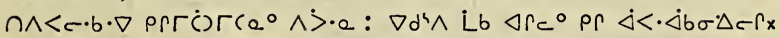

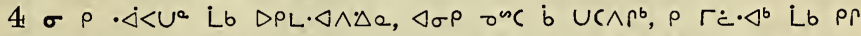

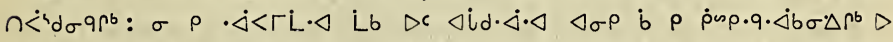

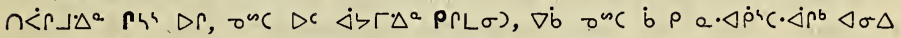

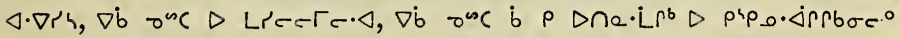
421 


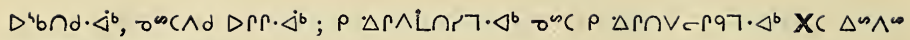

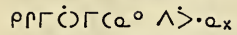

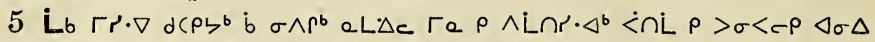

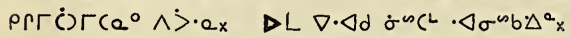

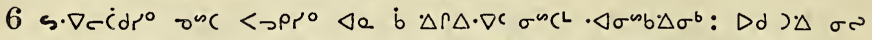

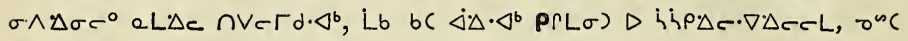

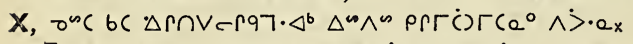

$7 \Delta^{i} \wedge$ Lb $\left.\rho \cap \wedge<c \cdot b \cdot \nabla p R \Gamma \dot{C}\right) \Gamma\left(a^{\circ} \wedge>\cdot a, 4 C^{a} b C<<\cdot \triangleleft^{\circ} D \rho<D\right) \Delta b \Gamma^{b}$ $\Delta r$,

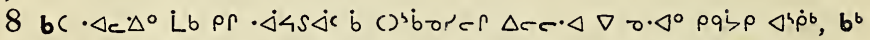

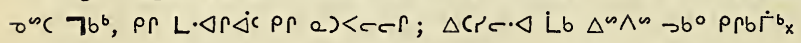

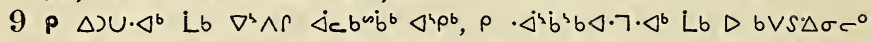

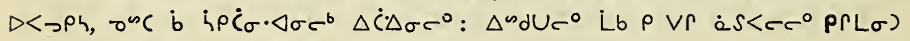
Dr PrPSd $d^{b}, \dot{b} \sigma S \cdot \Delta \dot{a} \Gamma \Delta d \rho^{b} x$

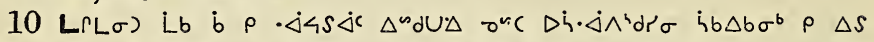

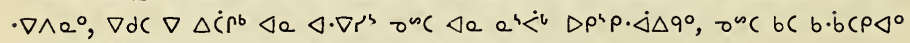
$\nabla \rho s \dot{b} c^{b}$ occ $^{n} \nabla \cap \Lambda^{4} \dot{b} c^{b}$, b $b q$ onc b $\rho q_{x}$

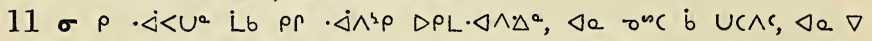

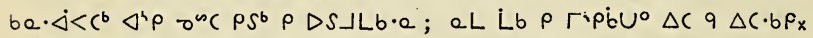

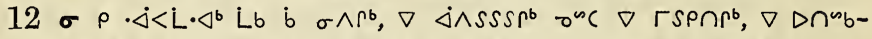

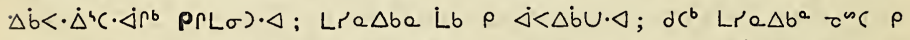

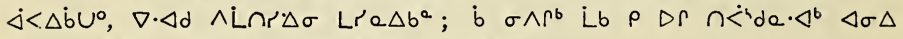
Lra $\Delta b \sigma^{b}$ b Lra $\Delta \dot{b} U c e, \cdot 7 r$ b $\rho \Delta S \Delta j^{4} q b^{b} b_{x}$

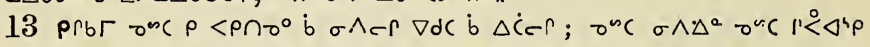

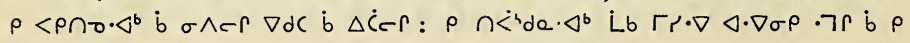
$\Delta S \Delta j 4 a r^{6} x$

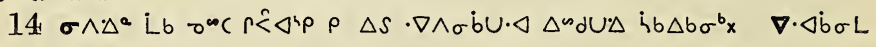
$\alpha \leadsto \sigma \wedge \Delta^{a} x$

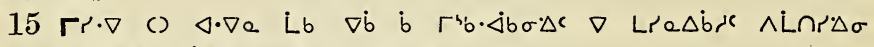
Lra $\Delta b \sigma^{b} \Delta^{w} d U \Delta \dot{b} b \Delta b \sigma^{b} \rho \Delta S \cdot \nabla \wedge a^{\circ} \times$

\section{$\Lambda^{4} p c r a \triangle b^{2} 21$.}

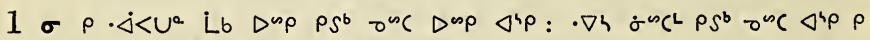

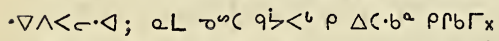

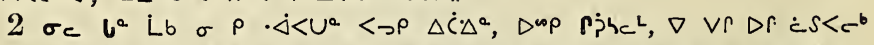

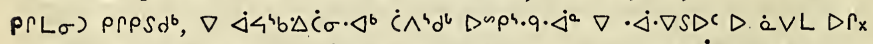

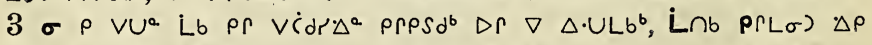
$\Delta C \cdot b \sigma \sigma^{\circ} \Delta C \nabla \Delta \dot{C}-r \Delta c c \cdot \triangleleft, b C \Delta r\left(S 97^{\circ} L b, b C \Delta C \Delta c c \Gamma^{\circ} L b, P \Gamma L \sigma\right)$

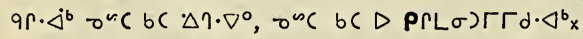

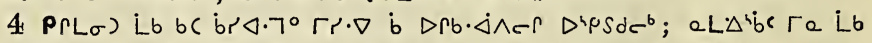
bc $\Delta\left(\cdot b^{a} \sigma \wedge \Delta^{a}\right.$, aL osc $\left.\Gamma s\right] c\left(J^{a} \Delta^{a}\right.$, aL osc $\left.L\right) \Delta^{a}$, aL osc $\Gamma a b c$ $\left.\Delta c \cdot b^{a} \Delta 49 c c\right\lrcorner \cdot \Delta^{a}: \cdot \nabla h$ bं $s$ s $q \cdot b e \rho \cdot \nabla \wedge<c \cdot \Delta x$ 
5 ४e Lb b $\Delta \wedge c \quad \Delta \rho L \cdot \Delta \wedge \Delta \sigma^{b} \rho \quad \Delta \cdot \cup^{\circ}, \quad L \cap b, \Gamma r \cdot \nabla \quad q \cdot b e$ oc

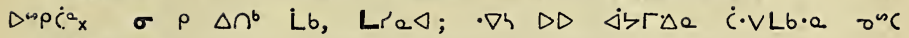
b. ל'P $P$ Lb.ax

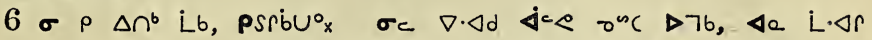

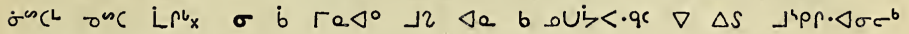
$\wedge \operatorname{Ln} \cap \Delta \sigma \sigma \wedge c^{\circ} x$

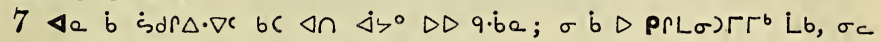
$\operatorname{Toc}^{n} \sigma$ b $D$ drtL L ${ }^{\circ}$

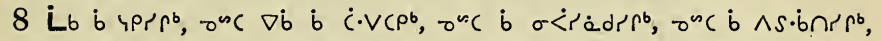

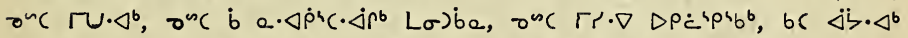

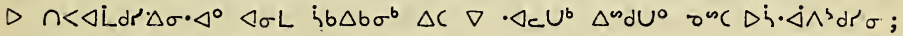
$\nabla \cdot \Delta \cdot \dot{b} \sigma L \sigma a \sigma \wedge \Delta^{a} x$

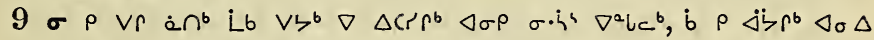

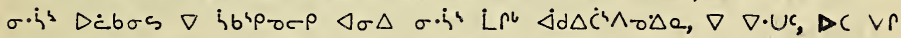

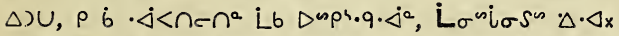

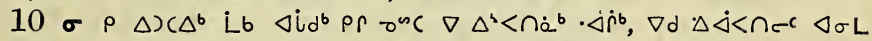

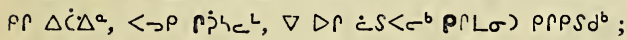

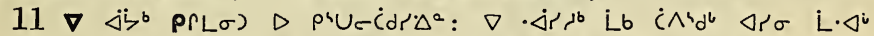

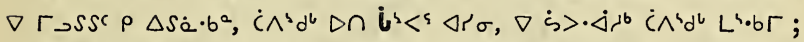

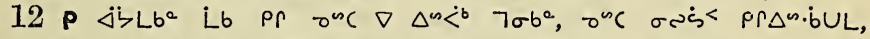

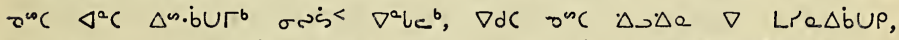

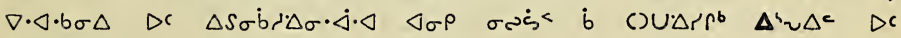
$\triangleleft \cdot \triangleleft s \Gamma s_{x}$

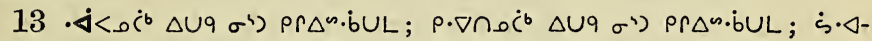

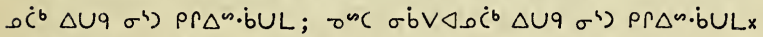

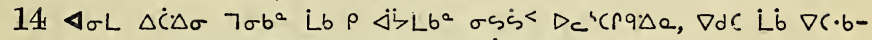

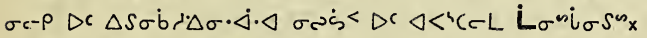

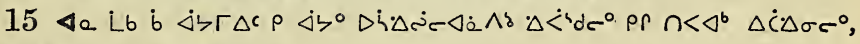
onc $\Delta n$. bUL onc $7 \sigma b \sigma c^{\circ} \mathrm{x}$

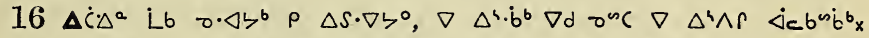

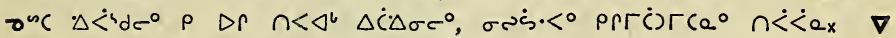

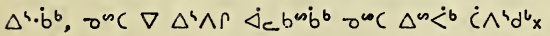

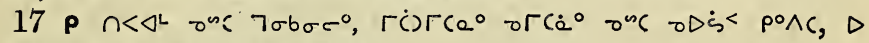
$\cap<\dot{r} b \sigma^{b} \Delta r c^{\circ} \Delta S \nabla \cdot \triangleleft \cdot b e \nabla^{a} c^{c} x$

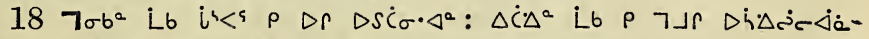

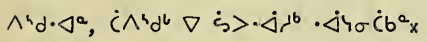

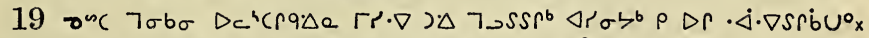

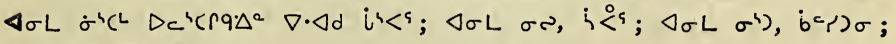
$\triangle \sigma L 0^{\circ}, \nabla \Gamma \zeta^{\circ} c$;

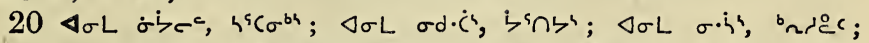

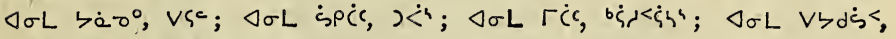
irac; $\triangleleft \sigma L \sigma \sigma^{2} \dot{s}<\Delta \Gamma Q^{4} \times$

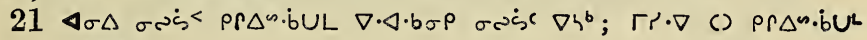
423 


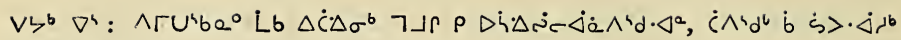
$\cdot \Delta 4 \sigma \dot{c} b^{a} x$

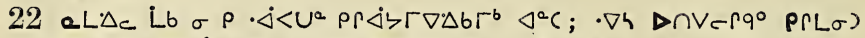

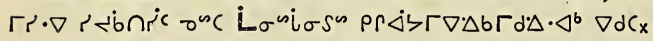

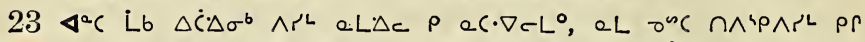

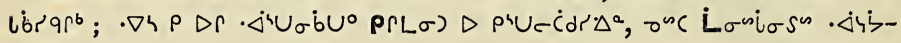
$r \Delta \sigma \cdot \Delta^{\circ} \nabla \partial C_{x}$

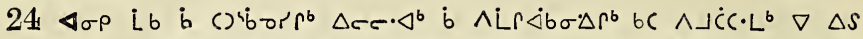

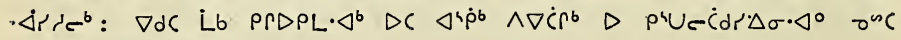
$\nabla \sin \cdot \Delta \sigma \cdot \Delta^{\circ} x$

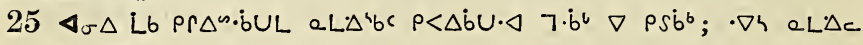
$b C \cap \wedge$ " $b^{\circ} \triangleleft^{\circ} C x$

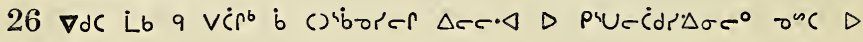
pine. $\Delta \sigma r^{\circ} \times$

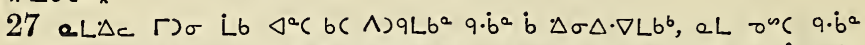

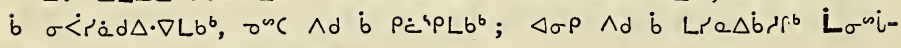

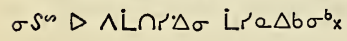

\section{Ápcre $\triangle b^{a} 22$.}

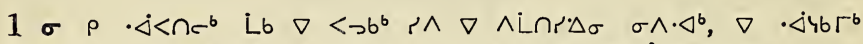

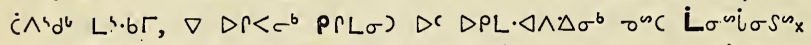

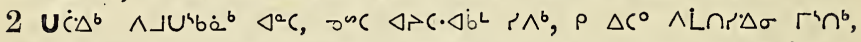

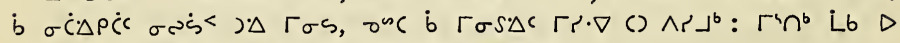

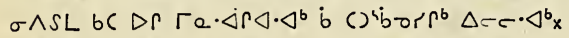

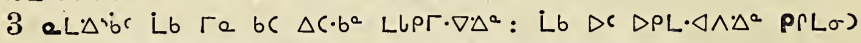

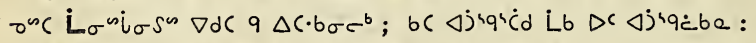

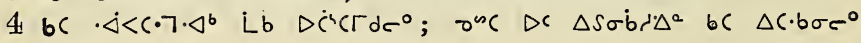
$\Delta$ ibnd. $\dot{b} \mathrm{x}_{\mathrm{x}}$

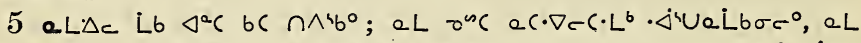

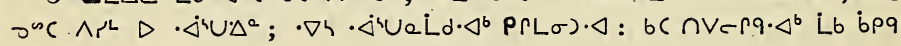
onc $\dot{b} p q_{x}$

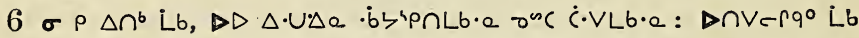

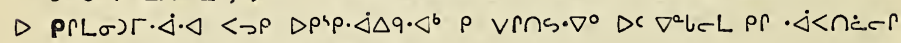
$\Delta C \Delta j$ 'qं be $\triangleleft \sigma \Delta \Delta<6 q . \Delta \rho_{\sigma}+\rho_{x}$

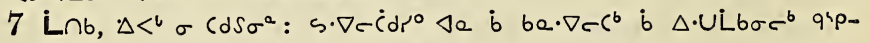
- $\dot{\Delta} \Delta q \Delta^{a} \Delta C$ Lra $a b \sigma^{b} x$

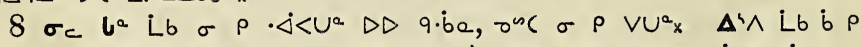

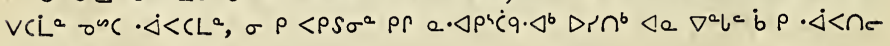
$\triangle D q \cdot \dot{b} e_{x}$

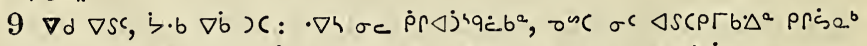

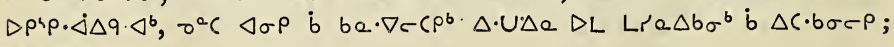
Q. $\triangleleft \dot{\rho} \wedge\left({ }^{\circ} \rho(L \sigma)_{x}\right.$

424 


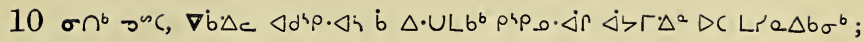
- $\nabla 49 \dot{b}<\Delta \cap r<c^{\circ} x$

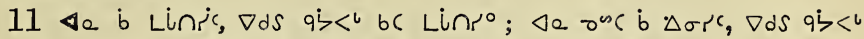

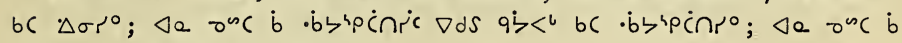

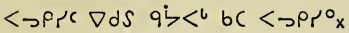

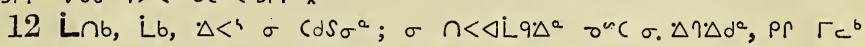
$\Gamma r \cdot \nabla() \Delta c c^{\circ} q \Delta S \dot{\alpha} \cdot b \sigma c^{b} \Delta C \Delta j^{4} q \cdot \Delta^{a} x$

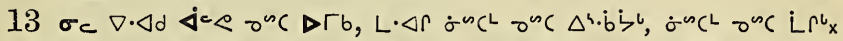

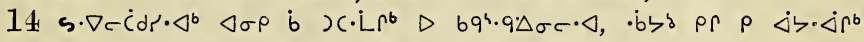

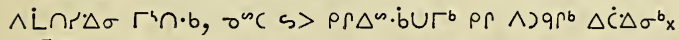

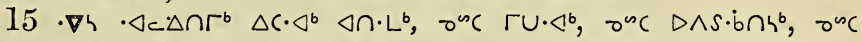

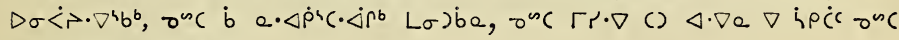

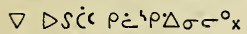

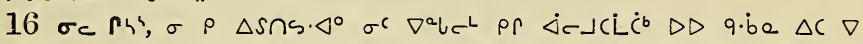

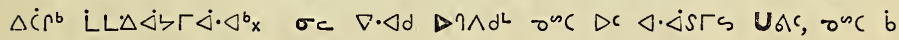

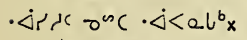

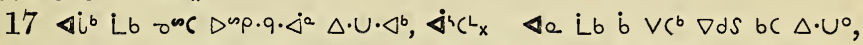

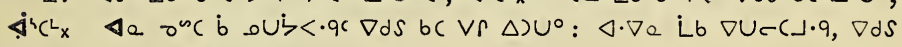

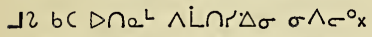

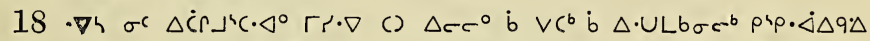

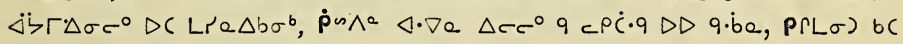

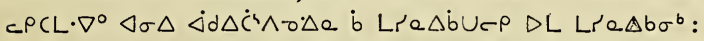

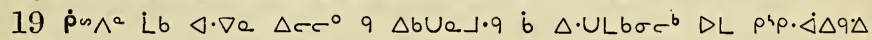

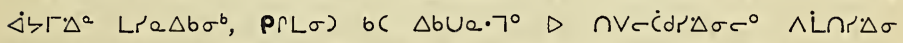

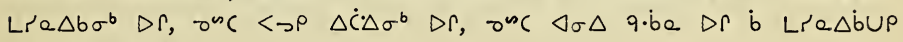
$\nabla L$ LRe $\Delta b \sigma^{b} x$

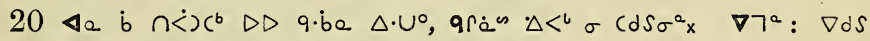
$\triangle S C S^{2}, U \vee C r q \zeta^{\circ} r^{\prime} x$

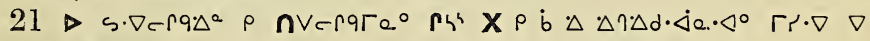
$\Delta c s 4^{b} \times \quad \nabla 7^{a} x$

Gilbert \& Rivington, 52, St. John's Square and 28, Whitefriars Street, Lond on.

425 


5 



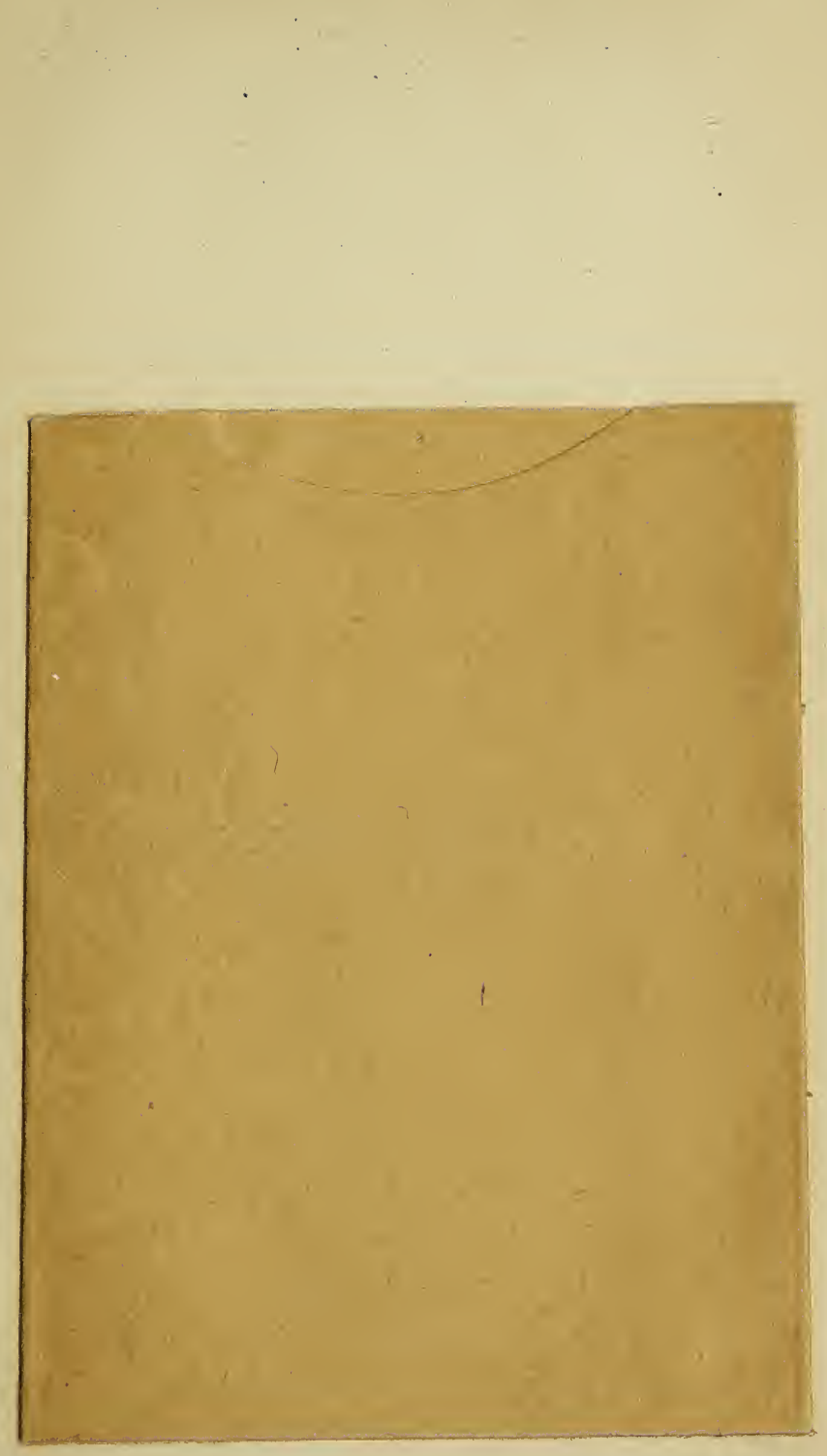


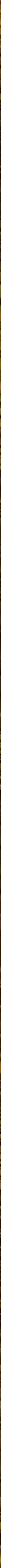

